\title{
Summary of FY11 Sulfate Retention Studies for Defense Waste Processing Facility Glass
}

K. M. Fox

T. B. Edwards

March 2012

Savannah River National Laboratory Savannah River Nuclear Solutions, LLC Aiken, SC 29808

Prepared for the U.S. Department of Energy under contract number DE-AC09-08SR22470. 
SRNL-STI-2012-00152

Revision 0

\section{DISCLAIMER}

This work was prepared under an agreement with and funded by the U.S. Government. Neither the U.S. Government or its employees, nor any of its contractors, subcontractors or their employees, makes any express or implied:

1. warranty or assumes any legal liability for the accuracy, completeness, or for the use or results of such use of any information, product, or process disclosed; or

2. representation that such use or results of such use would not infringe privately owned rights; or

3. endorsement or recommendation of any specifically identified commercial product, process, or service.

Any views and opinions of authors expressed in this work do not necessarily state or reflect those of the United States Government, or its contractors, or subcontractors.

\section{Printed in the United States of America \\ Prepared for U.S. Department of Energy}


Keywords: Sulfate, Waste Glass, DWPF

Retention: Permanent

\section{Summary of FY11 Sulfate Retention Studies for Defense Waste Processing Facility Glass}

K. M. Fox

T. B. Edwards

March 2012

Savannah River National Laboratory Savannah River Nuclear Solutions, LLC Aiken, SC 29808

Prepared for the U.S. Department of Energy under contract number DE-AC09-08SR22470. 
SRNL-STI-2012-00152

Revision 0

\section{REVIEWS AND APPROVALS}

AUTHORS:

K. M. Fox, Process Technology Programs

Date

T. B. Edwards, Applied Computational Engineering and Statistics

Date

TECHNICAL REVIEW:

D. K. Peeler, Process Technology Programs

Date

APPROVAL:

C. C. Herman, Manager

Date

Process Technology Programs

S. L. Marra, Manager

Date

Environmental \& Chemical Process Technology Research Programs

J. E. Occhipinti, Manager

Date

Waste Solidification Engineering 


\section{EXECUTIVE SUMMARY}

This report describes the results of studies related to the incorporation of sulfate in high level waste (HLW) borosilicate glass produced at the Savannah River Site (SRS) Defense Waste Processing Facility (DWPF). A group of simulated HLW glasses produced for earlier sulfate retention studies was selected for full chemical composition measurements to determine whether there is any clear link between composition and sulfate retention over the compositional region evaluated. In addition, the viscosity of several glasses was measured to support future efforts in modeling sulfate solubility as a function of predicted viscosity. The intent of these studies was to develop a better understanding of sulfate retention in borosilicate HLW glass to allow for higher loadings of sulfate containing waste.

Based on the results of these and other studies, the ability to improve sulfate solubility in DWPF borosilicate glasses lies in reducing the connectivity of the glass network structure. This can be achieved, as an example, by increasing the concentration of alkali species in the glass. However, this must be balanced with other effects of reduced network connectivity, such as reduced viscosity, potentially lower chemical durability, and in the case of higher sodium and aluminum concentrations, the propensity for nepheline crystallization. Future DWPF processing is likely to target higher waste loadings and higher sludge sodium concentrations, meaning that alkali concentrations in the glass will already be relatively high. It is therefore unlikely that there will be the ability to target significantly higher total alkali concentrations in the glass solely to support increased sulfate solubility without the increased alkali concentration causing failure of other Product Composition Control System (PCCS) constraints, such as low viscosity and durability.

No individual components were found to provide a significant improvement in sulfate retention (i.e., an increase of the magnitude necessary to have a dramatic impact on blending, washing, or waste loading strategies for DWPF) for the glasses studied here. In general, the concentrations of those species that significantly improve sulfate solubility in a borosilicate glass must be added in relatively large concentrations (e.g., 13 to $38 \mathrm{wt} \%$ or more of the frit) in order to have a substantial impact. For DWPF, these concentrations would constitute too large of a portion of the frit to be practical. Therefore, it is unlikely that specific additives may be introduced into the DWPF glass via the frit to significantly improve sulfate solubility.

The results presented here continue to show that sulfate solubility or retention is a function of individual glass compositions, rather than a property of a broad glass composition region. It would therefore be inappropriate to set a single sulfate concentration limit for a range of DWPF glass compositions. Sulfate concentration limits should continue to be identified and implemented for each sludge batch. The current PCCS limit is $0.4 \mathrm{wt} \% \mathrm{SO}_{4}{ }^{2-}$ in glass, although frit development efforts have led to an increased limit of $0.6 \mathrm{wt} \%$ for recent sludge batches. Slightly higher limits (perhaps 0.7-0.8 wt \%) may be possible for future sludge batches.

An opportunity for allowing a higher sulfate concentration limit at DWPF may lay lie in improving the laboratory experiments used to set this limit. That is, there are several differences between the crucible-scale testing currently used to define a limit for DWPF operation and the actual conditions within the DWPF melter. In particular, no allowance is currently made for sulfur partitioning (volatility versus retention) during melter processing as the sulfate limit is set for a specific sludge batch. A better understanding of the partitioning of sulfur in a bubbled melter operating with a cold cap as well as the impacts of sulfur on the off-gas system may allow a higher sulfate concentration limit to be established for the melter feed. This approach would 
SRNL-STI-2012-00152

Revision 0

have to be taken carefully to ensure that a sulfur salt layer is not formed on top of the melt pool while allowing higher sulfur based feeds to be processed through DWPF. 


\section{TABLE OF CONTENTS}

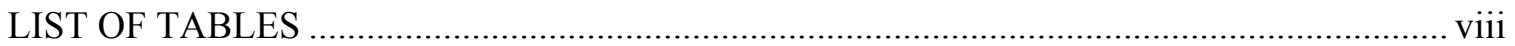

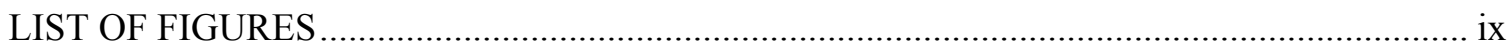

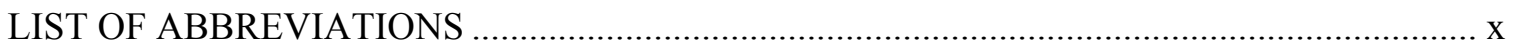

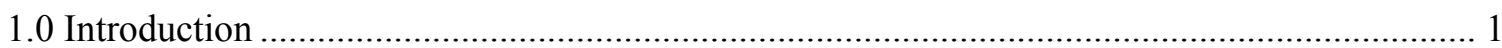

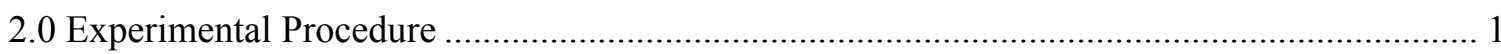

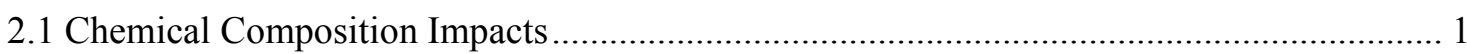

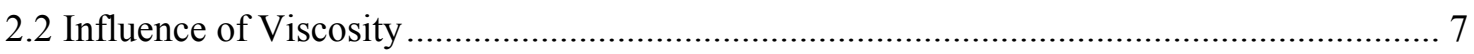

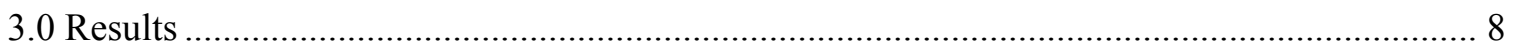

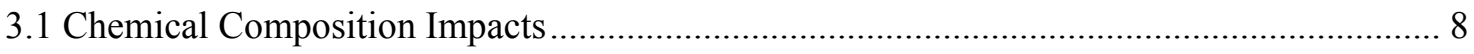

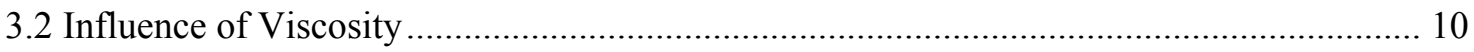

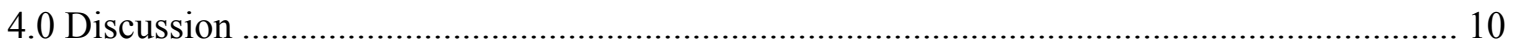

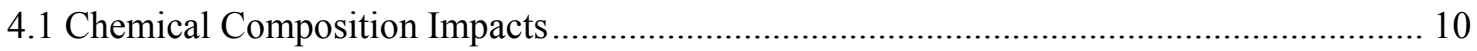

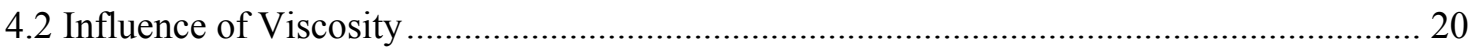

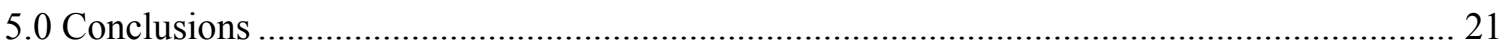

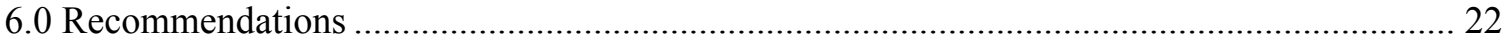

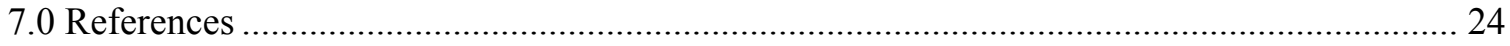


SRNL-STI-2012-00152

Revision 0

\section{LIST OF TABLES}

Table 2-1. Distribution of Targeted Sulfate Concentrations in 31 Selected Glasses...................... 3

Table 4-1. Predicted and Measured Viscosities and Sulfate Concentrations of the Study Glasses. 21 


\section{LIST OF FIGURES}

Figure 2-1. Scatterplot of Major Oxides (wt \%) in 41 Selected Glass Compositions. Green Data Points Indicate Those Glasses with Visible Sulfate Gall on the Surface after Melting. 2

Figure 2-2. Scatterplot of Major Oxides (wt \%) in 50 Selected Glass Compositions. Orange Data

Points Indicate Those Glasses with Visible Sulfate Gall on the Surface after Melting. 4

Figure 2-3. Partitioning of 50 Glasses by Targeted Composition Based on Visual Observations of Yellow Sulfate Layer. 5

Figure 2-4. Partitioning of 41 Glasses Targeting $1.2 \mathrm{wt} \% \mathrm{SO}_{4}{ }^{2-}$ by Targeted Composition Based on Visual Observations of Yellow Sulfate Layer. 6

Figure 2-5. Distribution of Targeted $\mathrm{CaO}$ Concentrations in 50 Selected Glasses. 7

Figure 4-1. Partitioning of Glass Compositions Based on Measured Sulfate Concentration and Visual Observations of Sulfate Surface Layers. 11

Figure 4-2. Partitioning of Glass Compositions Based on Measured $\mathrm{CaO}$ Concentration and Visual Observations of Sulfate Surface Layers. 13

Figure 4-3. Partitioning of Glass Compositions Based on Measured Concentrations of Major Oxides and Visual Observations of Sulfate Surface Layers. 14

Figure 4-4. Plots of the Ratio of Measured to Targeted Sulfate Concentration versus (a) $\mathrm{MnO}$ Concentration and (b) $\mathrm{SiO}_{2}$ Concentration. The Red Data Points Represent Glasses with a Visible Sulfate Surface Layer after Melting. The Blue Data Points Represent Glasses with No Visible Sulfate Layer.

Figure 4-5. Results of Stepwise Regression Analysis of the Influence of the Concentrations of the Major Oxides on the Ratio of Measured to Targeted Sulfate Concentration. 17

Figure 4-6. Graphical Results of Stepwise Regression Analysis of the Influence of the Concentrations of the Major Oxides on the Ratio of Measured to Targeted Sulfate Concentration.

Figure 4-7. Partitioning of Glass Compositions Based on Measured Concentrations of Major Oxides with Statistically Significant Impacts on Sulfate Retention and Visual Observations of Sulfate Surface Layers. 19

Figure 4-8. Plot of Measured and Model Predicted Viscosities of the Study Glasses. 21 


\section{LIST OF ABBREVIATIONS}

$\begin{array}{ll}\text { ANOVA } & \text { Analysis of Variance } \\ \text { DWPF } & \text { Defense Waste Processing Facility } \\ \text { HLW } & \text { High Level Waste } \\ \text { ICP-AES } & \text { Inductively Coupled Plasma - Atomic Emission Spectroscopy } \\ \text { LM } & \text { Lithium Metaborate } \\ \text { PCCS } & \text { Product Composition Control System } \\ \text { PF } & \text { Peroxide Fusion } \\ \text { SB7b } & \text { Sludge Batch 7b } \\ \text { SRNL } & \text { Savannah River National Laboratory } \\ \text { SRS } & \text { Savannah River Site }\end{array}$




\subsection{Introduction}

Jantzen and Smith provide a review of the potential impacts of sulfur to the Defense Waste Processing Facility (DWPF) melting process. ${ }^{1}$ In summary, sulfur has a low solubility in borosilicate glass. This low solubility can result in the formation of a molten sulfate salt gall layer on top of the melt pool to which cesium and strontium can partition. This sulfate gall layer can accelerate corrosion of the melter components, can reduce the effectiveness of Joule heating due to its low resistance, and can potentially lead to steam excursions. As the glass cools, the gall layer can form inclusions or a separate phase in the glass, which can impact radionuclide release. A sulfate concentration limit is set for each sludge batch processed at the DWPF in order to avoid these issues.

This report describes the results of studies related to the incorporation of sulfate in high level waste (HLW) borosilicate glass produced at the DWPF. A group of simulated HLW glasses produced for earlier sulfate retention studies was selected for full chemical composition measurements to determine whether there is any clear link between composition and sulfate retention over the compositional region evaluated. These results are evaluated, and comparisons are made both within the data set and among several reports available in the literature on sulfate solubility in borosilicate glass. In addition, the viscosity of several glasses was measured to enhance efforts to model sulfate solubility as a function of predicted viscosity. The intent of these studies was to develop a better understanding of sulfate retention in borosilicate HLW glass to allow for higher loadings of sulfate containing waste.

This work was initiated by Technical Task Requests ${ }^{2,3}$ and performed following Task Technical and Quality Assurance Plans. ${ }^{4,5}$

\subsection{Experimental Procedure}

\subsection{Chemical Composition Impacts}

A database of 199 simulated waste glasses ${ }^{a}$ fabricated at the Savannah River National Laboratory (SRNL) in support of sulfate retention studies was used as the basis for selecting a group of glass compositions for further analysis. The database consists of targeted compositions, visual observations of sulfate retention, and measured sulfate concentration values. No other chemical composition data had been measured for these glasses. These glasses were fabricated in support of earlier sulfate retention guidance produced for the DWPF. ${ }^{6-8}$

A down-selection process was performed as follows to select a reasonably sized subset of the 199 glass compositions for further analysis. JMP software ${ }^{9}$ was used to support the selection process and the subsequent analyses presented in this report. First, those glass compositions that targeted a sulfate $\left(\mathrm{SO}_{4}^{2-}\right)$ concentration of $1.2 \mathrm{wt} \%$ were selected as being of interest since this concentration is twice the limit set for the current processing of Sludge Batches $7 \mathrm{a}$ and $7 \mathrm{~b},{ }^{8,10}$ as well as previous batches, at the DWPF. This yielded 41 compositions. A scatterplot of the concentrations of the major oxides in these 41 glasses showed a fairly good distribution across the compositional space, as shown in Figure 2-1. The visual observations of the glasses are represented on the plot as follows: blue points represent glasses with clean surfaces (no visible sulfate or crystallization) after cooling, red points represent glasses with a surface layer of spinel crystallization after cooling, green points represent glasses with a yellow sulfate gall surface layer after cooling, and orange points represent glasses with a white surface film (assumed to be sulfate

\footnotetext{
${ }^{a}$ See SRNL-NB-2011-00056 for a complete listing of these data.
} 
salts) after cooling. The visual observations did not affect the selection process, but will be used later to aid in the analysis of the composition data.

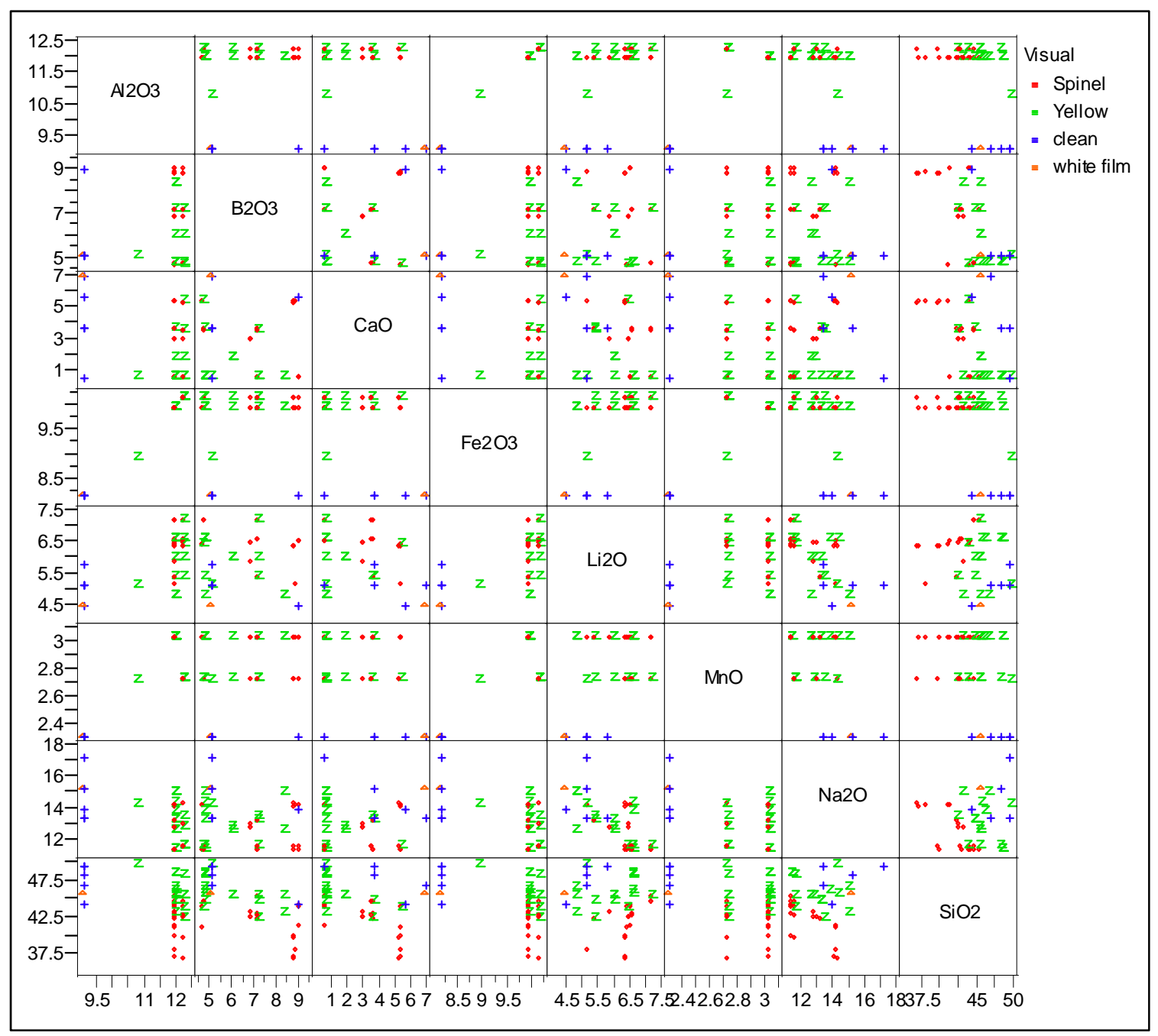

Figure 2-1. Scatterplot of Major Oxides (wt \%) in 41 Selected Glass Compositions. Green Data Points Indicate Those Glasses with Visible Sulfate Gall on the Surface after Melting.

Further compositional variation was sought by selecting those glasses in the database of 199 with the minimum and maximum targeted concentrations of $\mathrm{Al}_{2} \mathrm{O}_{3}, \mathrm{~B}_{2} \mathrm{O}_{3}, \mathrm{CaO}, \mathrm{Fe}_{2} \mathrm{O}_{3}, \mathrm{~K}_{2} \mathrm{O}, \mathrm{Li}_{2} \mathrm{O}$, $\mathrm{MnO}, \mathrm{Na}_{2} \mathrm{O}$, and $\mathrm{SiO}_{2}$. This yielded 31 glasses from the database. The targeted sulfate concentrations of these glasses are given in Table 2-1. 
SRNL-STI-2012-00152

Revision 0

Table 2-1. Distribution of Targeted Sulfate Concentrations in 31 Selected Glasses.

\begin{tabular}{|c|c|}
\hline $\mathrm{SO}_{\mathbf{4}}{ }^{2-}$ (wt \%) & $\begin{array}{c}\text { Number of } \\
\text { Glasses }\end{array}$ \\
\hline 0.074 & 1 \\
\hline 0.104 & 1 \\
\hline 0.6 & 2 \\
\hline 0.9 & 2 \\
\hline 1 & 5 \\
\hline 1.2 & 12 \\
\hline 2 & 8 \\
\hline
\end{tabular}

The 12 glasses with targeted sulfate concentrations of $1.2 \mathrm{wt} \%$ are included in the group of 41 selected earlier. Those glasses with targeted sulfate concentrations of greater than $1.2 \mathrm{wt} \%$ or less than $0.6 \mathrm{wt} \%$ are outside the range of interest for this study. Therefore, nine additional glass compositions were added to the group of 41 selected earlier. A scatterplot of the 50 selected glass compositions, Figure 2-2, shows additional coverage in concentrations of the major oxides as well as sulfate. 


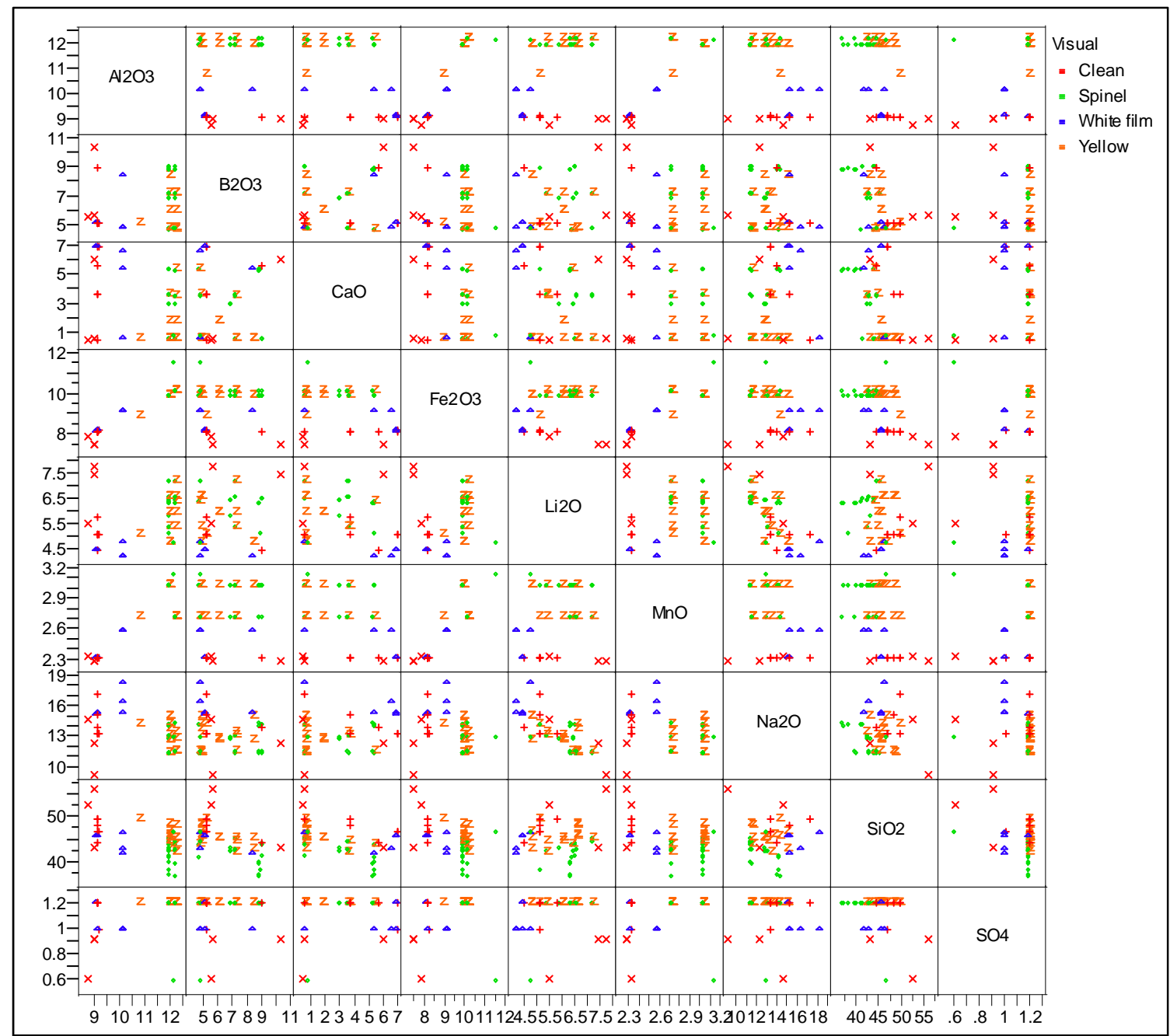

Figure 2-2. Scatterplot of Major Oxides (wt \%) in 50 Selected Glass Compositions. Orange Data Points Indicate Those Glasses with Visible Sulfate Gall on the Surface after Melting.

A statistical partitioning routine was used to identify potential trends among the major oxides of targeted glass compositions and the visual observations of a yellow sulfate layer on some of the glasses after melting. The results are shown in Figure 2-3. This preliminary analysis shows that higher $\mathrm{CaO}$ concentrations may play a role in improved retention of sulfate in the glasses. Similar results are shown in Figure 2-4 for the 41 glasses that all targeted $1.2 \mathrm{wt} \% \mathrm{SO}_{4}{ }^{2-}$. The distribution of targeted $\mathrm{CaO}$ concentrations in the 50 glasses, Figure 2-5, shows that the partitioning results are not simply a result of a skewed distribution. The distribution also shows that the range of $\mathrm{CaO}$ concentrations included in this data set is likely sufficient to indicate any true trends among $\mathrm{CaO}$ concentrations and sulfate retention. Measuring the full chemical composition of the 50 selected glasses will allow for further analysis. 


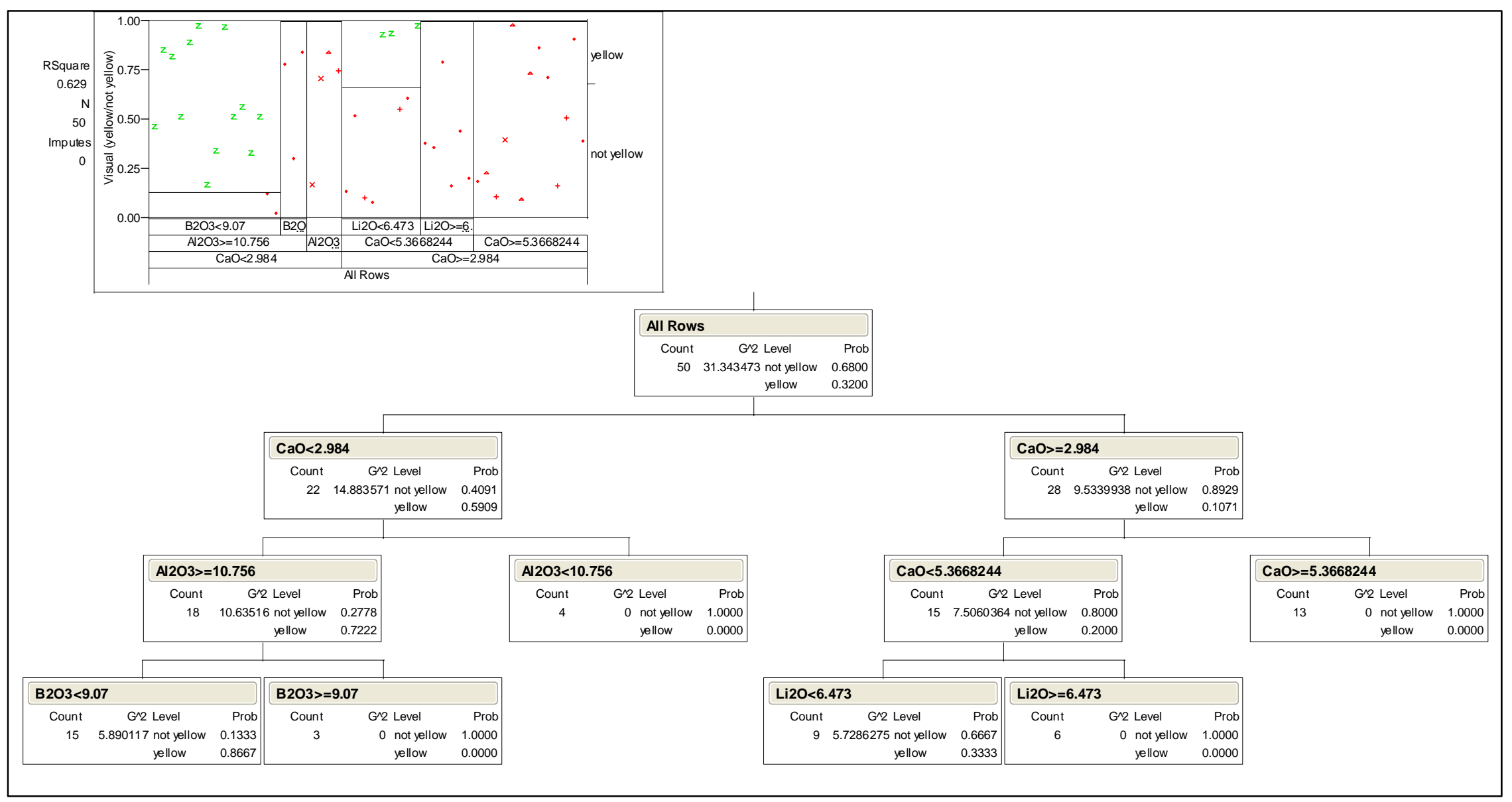

Figure 2-3. Partitioning of 50 Glasses by Targeted Composition Based on Visual Observations of Yellow Sulfate Layer. 


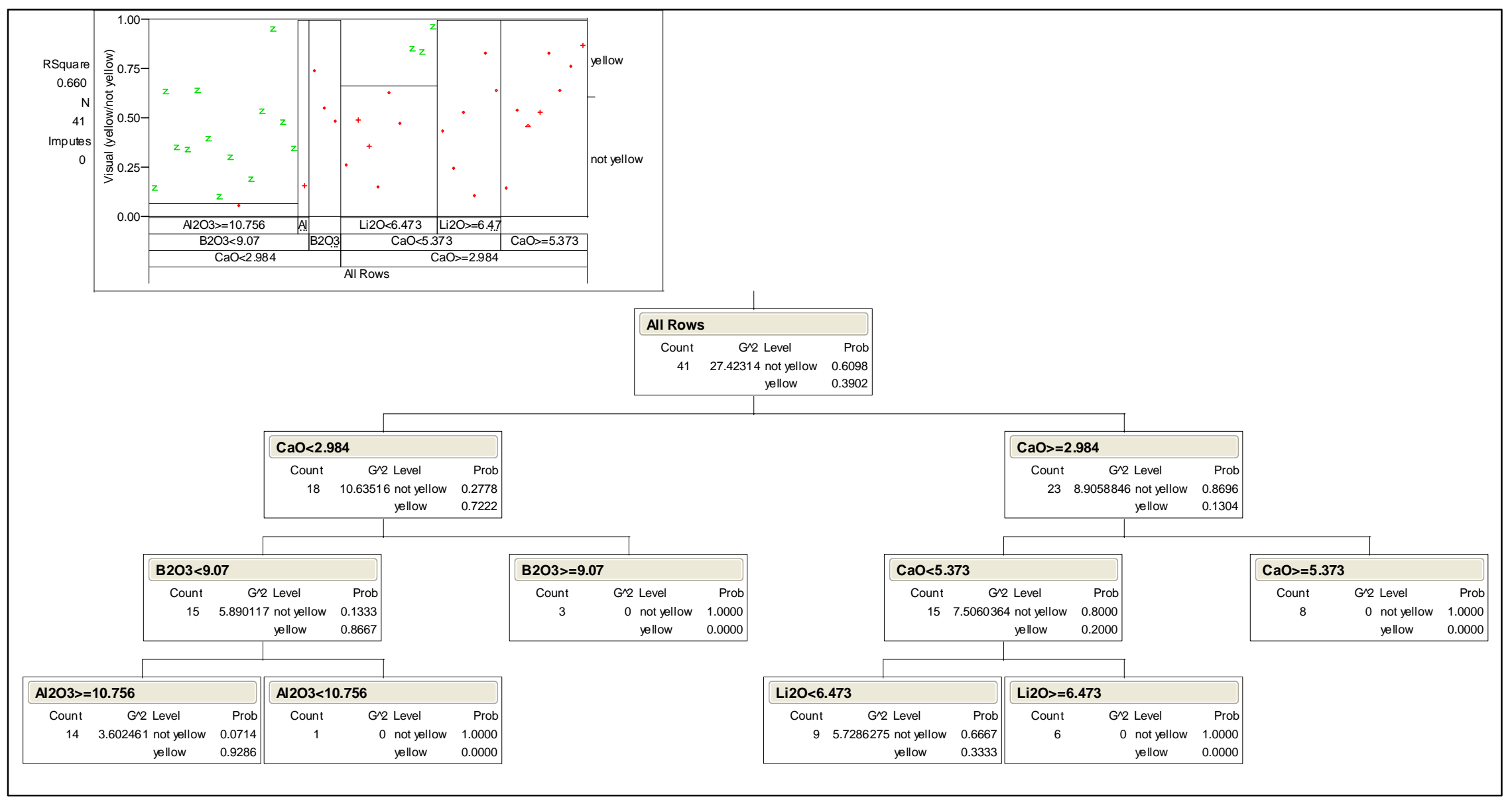

Figure 2-4. Partitioning of 41 Glasses Targeting $1.2 \mathrm{wt} \% \mathrm{SO}_{4}{ }^{2-}$

by Targeted Composition Based on Visual Observations of Yellow Sulfate Layer. 


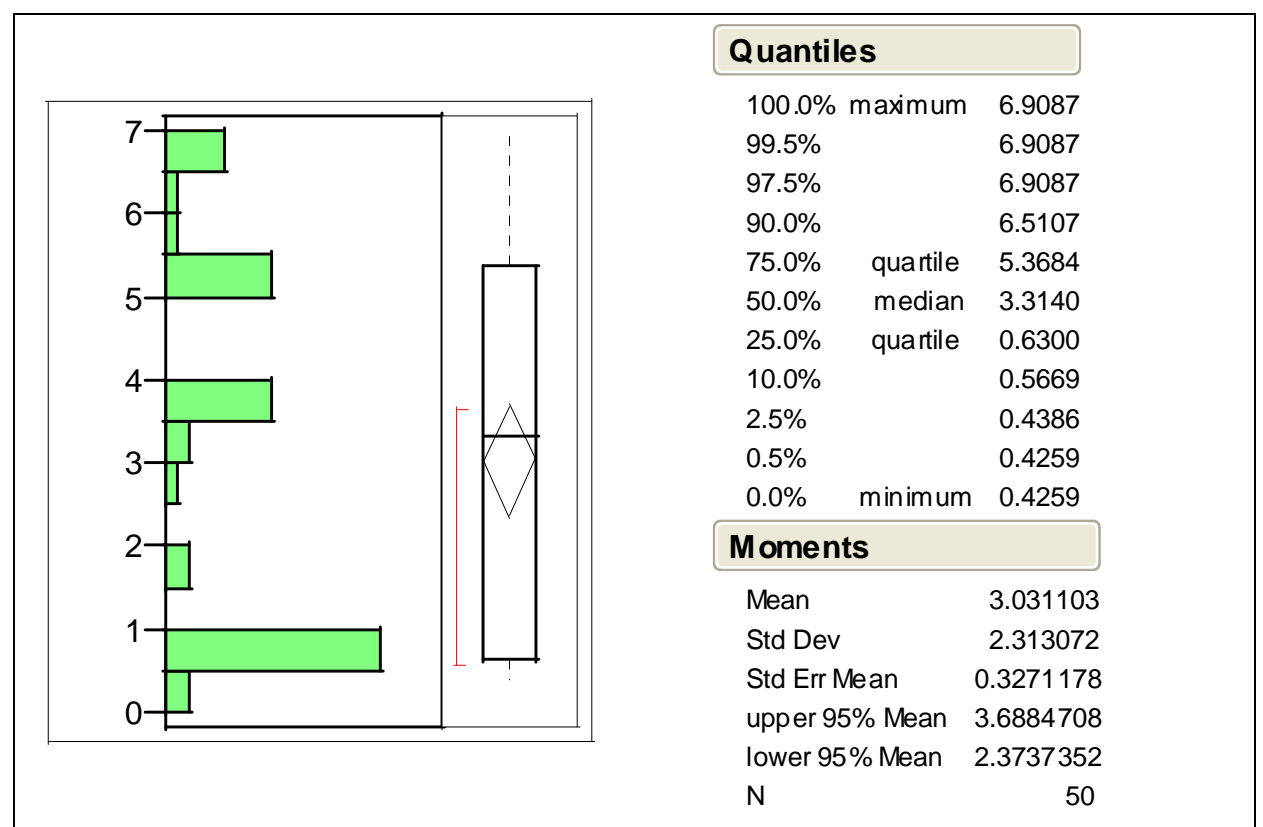

Figure 2-5. Distribution of Targeted CaO Concentrations in 50 Selected Glasses.

An analytical plan was prepared and issued to support the chemical composition analysis of the 50 selected glass compositions. ${ }^{11}$ In summary, representative samples of each glass were prepared for analysis in duplicate by sodium peroxide fusion (PF) and lithium-metaborate fusion (LM). Each of the samples was analyzed, twice for each element of interest, by Inductively Coupled Plasma - Atomic Emission Spectroscopy (ICP-AES). Glass standards were also intermittently measured to assess the performance of the ICP-AES instrument over the course of these analyses.

Concurrent with this task, a modeling study was undertaken at the University of Sheffield with the goal of predicting sulfate solubility in waste glasses using cation field strength relationships. An extensive data set of 290 glasses was provided to Sheffield by SRNL to support this task. ${ }^{\text {a }}$ The outcome of the modeling work will be summarized and compared with the experimental results in Section 4.0.

\subsection{Influence of Viscosity}

Jantzen and Smith have proposed a relationship between sulfate solubility and predicted viscosity for DWPF-type glass compositions. ${ }^{1}$ Viscosity data were measured for several of the glasses included in the present study to support future efforts in modeling sulfate solubility as a function of predicted viscosity. However, an evaluation of these models is outside the scope of the present task. The data will be documented in this report for future use.

The viscosity of the selected glasses was measured following Procedure A of the ASTM C 965 standard. ${ }^{12}$ Orton high temperature rotating spindle viscometers were used with platinum crucibles and spindles. The crucibles were specially designed to operate with small quantities of glass to support measurements of radioactive glasses when necessary. ${ }^{13,14}$ A well characterized standard glass was used to determine the appropriate spindle constants. ${ }^{14,15}$ Measurements were taken over a range of temperatures from 1050 to $1250{ }^{\circ} \mathrm{C}$ in $50{ }^{\circ} \mathrm{C}$ intervals. Measurements at

${ }^{\text {a }}$ See data in SRNL-L3100-2011-00038, transmitted by email to the University of Sheffield March 1, 2011. 
$1150{ }^{\circ} \mathrm{C}$ were taken at three different times during the procedure to provide an opportunity to identify the effects of any crystallization or volatilization that may have occurred during the test. The data were fit to a Fulcher equation ${ }^{16,17}$ to provide a measured viscosity value at the nominal DWPF melt temperature of $1150^{\circ} \mathrm{C}$.

\subsection{Results}

\subsection{Chemical Composition Impacts}

The measured versus targeted compositions of the study glasses are presented and compared in this section. Measurements for samples of the Batch $1^{18}$ and the low-activity reference material $(\mathrm{LRM})^{19}$ standard glasses that were included in the analytical plan along with the study glasses are also discussed. The elemental concentrations were converted to oxide concentrations by multiplying the values for each element by the gravimetric factor for the corresponding oxide. During this process, an elemental concentration that was determined to be below the detection limit of the analytical procedures used was reduced to half of that detection limit as the oxide concentration was determined. ${ }^{20}$ In the discussions that follow, the analytical sequences of the measurements are explored, the measurements of the standards are investigated, and the measurements for each glass are reviewed. In addition, the average chemical composition for each glass is determined, and comparisons are made between the measurements and the targeted compositions of the glasses.

Table A-1 and Table A-2 Appendix A provide the elemental concentration measurements from the study glasses that were digested using LM, and Table A-3 in Appendix A provides the measurements from the samples of these glasses digested using PF. Measurements for samples of the standard Batch 1 and LRM glasses that were included in the analytical plan along with the study glasses are also provided in these tables. Exhibit A-1 in Appendix A provides plots of the sample measurements for each oxide over both preparation methods. The plots are in analytical sequence with different symbols and colors being used to represent each of the study glasses and the standard glass. In general, there does not appear to be any gross patterns or trends due to the analytical sequence. Further opportunity for a review of the measurements for each glass is provided in the discussions that follow.

Exhibit A-2 in Appendix A provides plots of the oxide concentration measurements by Glass ID (including the Batch 1 and LRM standards) by analytical solution or Lab ID for both preparation methods for the study glasses. The different symbols and colors being used to represent the glasses are discernible in this exhibit. These plots show the individual measurements across the duplicates of each preparation method and the two ICP-AES calibrations for each glass for each oxide. The results are grouped by analytical block and arranged by targeted concentration to facilitate the interpretation of the measurements. A review of the plots presented in this exhibit reveals the repeatability of the four individual values for each oxide for each glass. In general, there appears to be good repeatability of these measurements for each of the oxides for each of the glasses. There is one slightly high measurement for $\mathrm{Al}_{2} \mathrm{O}_{3}$ for glass $\mathrm{BS}-01$. The first $\mathrm{LM}$ preparation for glass $\mathrm{BC}-05$ resulted in a low measurement for $\mathrm{CaO}$. The first $\mathrm{PF}$ preparation for glass $\mathrm{BC}-05$ resulted in a slightly high measurement for $\mathrm{SiO}_{2}$. There were slightly high measurements for $\mathrm{Ce}_{2} \mathrm{O}_{3}$ and $\mathrm{La}_{2} \mathrm{O}_{3}$ for glass BS-08. There were slightly high measurements for $\mathrm{BaO}, \mathrm{Ce}_{2} \mathrm{O}_{3}$, and $\mathrm{La}_{2} \mathrm{O}_{3}$ for glass QB-35. Scatter in the $\mathrm{B}_{2} \mathrm{O}_{3}$ and $\mathrm{SiO}_{2}$ measurements for the glasses measured in the $\mathrm{W}$ series appears somewhat greater than that of the other four series. Also, the data for the LM preparations of glasses QB-22 and QB-37 appear to have been reversed. This issue was corrected for the following analyses. The $\mathrm{Na}_{2} \mathrm{O}$ values for glass QB-37 are high. The values of $\mathrm{CaO}$ for glasses QB-33 and QB-37 (after swapping with QB-22) are unusually low. These values were replaced by the $\mathrm{CaO}$ measurements from the $\mathrm{PF}$ preparation for these two 
glasses in the following analyses. All of the other data were used as reported. The data suggest no other significant issues in the batching of the study glasses or in the analytical process used to provide representative measurements of their compositions.

Exhibit A-3 in Appendix A provides statistical analyses of the results for the Batch 1 and LRM standards that were included with the study glasses by analytical block/sub-block for each oxide of interest over both preparation methods. The results include analysis of variance (ANOVA) investigations looking for statistically significant differences among the means of these groups for each of the oxides. The reference values for the oxide concentrations of the standards are given in the header for each set of measurements in the exhibit. The results from the statistical tests for the Batch 1 standard included with the study glasses may be summarized as follows: $\mathrm{Al}_{2} \mathrm{O}_{3}$ in Series $\mathrm{W}$ and $\mathrm{X}, \mathrm{B}_{2} \mathrm{O}_{3}$ in Series $\mathrm{X}, \mathrm{BaO}$ in Series $\mathrm{V}, \mathrm{CaO}$ in Series $\mathrm{W}$ and $\mathrm{X}, \mathrm{Cr}_{2} \mathrm{O}_{3}$ in Series $\mathrm{V}$ and $\mathrm{W}, \mathrm{Fe}_{2} \mathrm{O}_{3}$ in Series $\mathrm{X}, \mathrm{K}_{2} \mathrm{O}$ in Series $\mathrm{T}, \mathrm{Li}_{2} \mathrm{O}$ in Series $\mathrm{X}, \mathrm{MnO}$ in Series $\mathrm{W}$ and $\mathrm{X}, \mathrm{Na}_{2} \mathrm{O}$ in Series $\mathrm{W}$ and $\mathrm{X}, \mathrm{SiO}_{2}$ in Series $\mathrm{W}$ and $\mathrm{X}, \mathrm{SO}_{4}$ in Series $\mathrm{U}, \mathrm{TiO}_{2}$ in Series W, and $\mathrm{ZrO}_{2}$ in Series V and $\mathrm{X}$ have measurements that indicate an ICP-AES calibration effect on the block averages at the 5\% significance level. The results from the statistical tests for the LRM standard included with the study glasses may be summarized as follows: $\mathrm{Al}_{2} \mathrm{O}_{3}$ in Series $\mathrm{T}$ and $\mathrm{X}, \mathrm{B}_{2} \mathrm{O}_{3}$ in Series $\mathrm{X}$, $\mathrm{CaO}$ in Series T, V, W, and $\mathrm{X}, \mathrm{Cr}_{2} \mathrm{O}_{3}$ in Series $\mathrm{T}$ and $\mathrm{W}, \mathrm{Fe}_{2} \mathrm{O}_{3}$ in Series $\mathrm{W}, \mathrm{K}_{2} \mathrm{O}$ in Series $\mathrm{T}$ and $\mathrm{X}, \mathrm{Li}_{2} \mathrm{O}$ in Series $\mathrm{X}, \mathrm{MgO}$ in Series T, V, and W, MnO in Series V, W, and $\mathrm{X}, \mathrm{Na}_{2} \mathrm{O}$ in Series T, $\mathrm{W}$, and $\mathrm{X}, \mathrm{NiO}$ in Series $\mathrm{V}$ and $\mathrm{W}, \mathrm{PbO}$ in Series $\mathrm{V}$ and $\mathrm{W}, \mathrm{SiO}_{2}$ in Series $\mathrm{V}$ and $\mathrm{X}, \mathrm{SO}_{4}$ in Series $\mathrm{T}, \mathrm{TiO}_{2}$ in Series $\mathrm{W}$ and $\mathrm{X}$, and $\mathrm{ZrO}_{2}$ in Series $\mathrm{W}$ have measurements that indicate an ICP-AES calibration effect on the block averages at the 5\% significance level. Note that some of these effects are artifacts of the detection limits where the standards do not contain the particular element of interest. While statistically significant, the practical impact of these calibration effects is minimal.

All of the measurements for each oxide for each of the 50 study glasses (i.e., all of the measurements in Appendix A Table A-1, Table A-2, and Table A-3) were averaged to determine a representative chemical composition for each glass. A sum of oxides was also computed for each glass based upon the measured values. Exhibit A-4 in Appendix A provides plots showing results for each glass for each oxide to help highlight the comparisons among the measured and targeted values. Some observations from the plots of Exhibit A-4 are offered: $\mathrm{The} \mathrm{Cr}_{2} \mathrm{O}_{3}$ values are slightly high for glasses LT-04, LT-10, and QB-03. The $\mathrm{Na}_{2} \mathrm{O}$ values are high for glass QB-37 and low for QB-33. There is some scatter in the $\mathrm{SiO}_{2}$ data. The $\mathrm{SO}_{4}{ }^{2-}$ values are generally low for the study glasses, which may indicate volatility during melting since the measured $\mathrm{SO}_{4}{ }^{2-}$ concentration for the LRM standard glass is very close to the reference value. There are some small differences between measured and targeted concentrations of the minor $(<1 \mathrm{wt} \%)$ components. The sums of oxides are generally low for the study glasses, although they remain within the DWPF Product Composition Control System (PCCS) acceptable range of 95-105 wt \%. In general, there appear to have been only minor difficulties in meeting the targeted concentrations for the study glasses.

Table A-4 in Appendix A provides a summary of the average measured compositions as well as the targeted compositions and the associated differences and relative differences. Note that the targeted sums of oxides for the Batch 1 and LRM reference glasses do not sum to $100 \%$ due to an incomplete coverage of the oxides in these glasses. All of the sums of oxides for the study glasses fall within the PCCS acceptable interval of 95 to $105 \mathrm{wt} \%$. Entries in Table A-4 show the relative differences between the measured values and the targeted values. These differences are shaded when they are greater than or equal to $5 \%$. Overall, these comparisons between the measured and targeted compositions again suggest only minor difficulties in meeting the targeted compositions for the study glasses. 


\subsection{Influence of Viscosity}

Viscosity data were collected for 14 of the study glasses. A review of the measured data showed no evidence of crystallization or significant volatilization occurring during the viscosity measurements. That is, there was no hysteresis in the measured viscosity as a function of temperature. Glass LL03 had measured viscosity values that were similar at temperatures of $1200{ }^{\circ} \mathrm{C}$ and $1250{ }^{\circ} \mathrm{C}$. Fulcher fits were made both with and without the $1250{ }^{\circ} \mathrm{C}$ data. This was not found to have a significant impact on the Fulcher fit, thus all of the data were included in calculating a measured viscosity for LL03 at $1150{ }^{\circ} \mathrm{C}$. Complete data from the fitting of Fulcher equations to the measured viscosity data are included as Appendix B.

\subsection{Discussion}

\subsection{Chemical Composition Impacts}

The measured composition data for the 50 glasses were reviewed to identify any impacts on sulfate retention in the glasses. A comparison of the targeted and measured sulfate concentrations of the 50 glasses showed that the mean difference was $0.038 \mathrm{wt} \%$, with a standard error of 0.005 $\mathrm{wt} \%$. This indicates that there were no serious issues with sulfate retention for most all of the glasses.

Partitioning of the glass compositions based on the visual observations and the measured sulfate concentrations reveals mixed results, as shown in Figure 4-1. Of the 25 glasses with measured sulfate concentrations greater than $1.089 \mathrm{wt} \%, 21$ glasses were free of a visible yellow sulfate surface layer after melting. This indicates that there is a glass composition region that is able to retain considerably more sulfate than the current DWPF limit of $0.6 \mathrm{wt} \%$. However, note also that for the 25 glasses with measured sulfate concentrations of less than $1.089 \mathrm{wt} \%, 12$ glasses exhibited a sulfate surface layer after melting. Sulfate solubility in this series of glasses appears to be a function of the individual compositions, rather than a general property applicable to the entire region. This indicates that it would be inappropriate to set a single sulfate concentration limit for a broad range of DWPF glass compositions. 


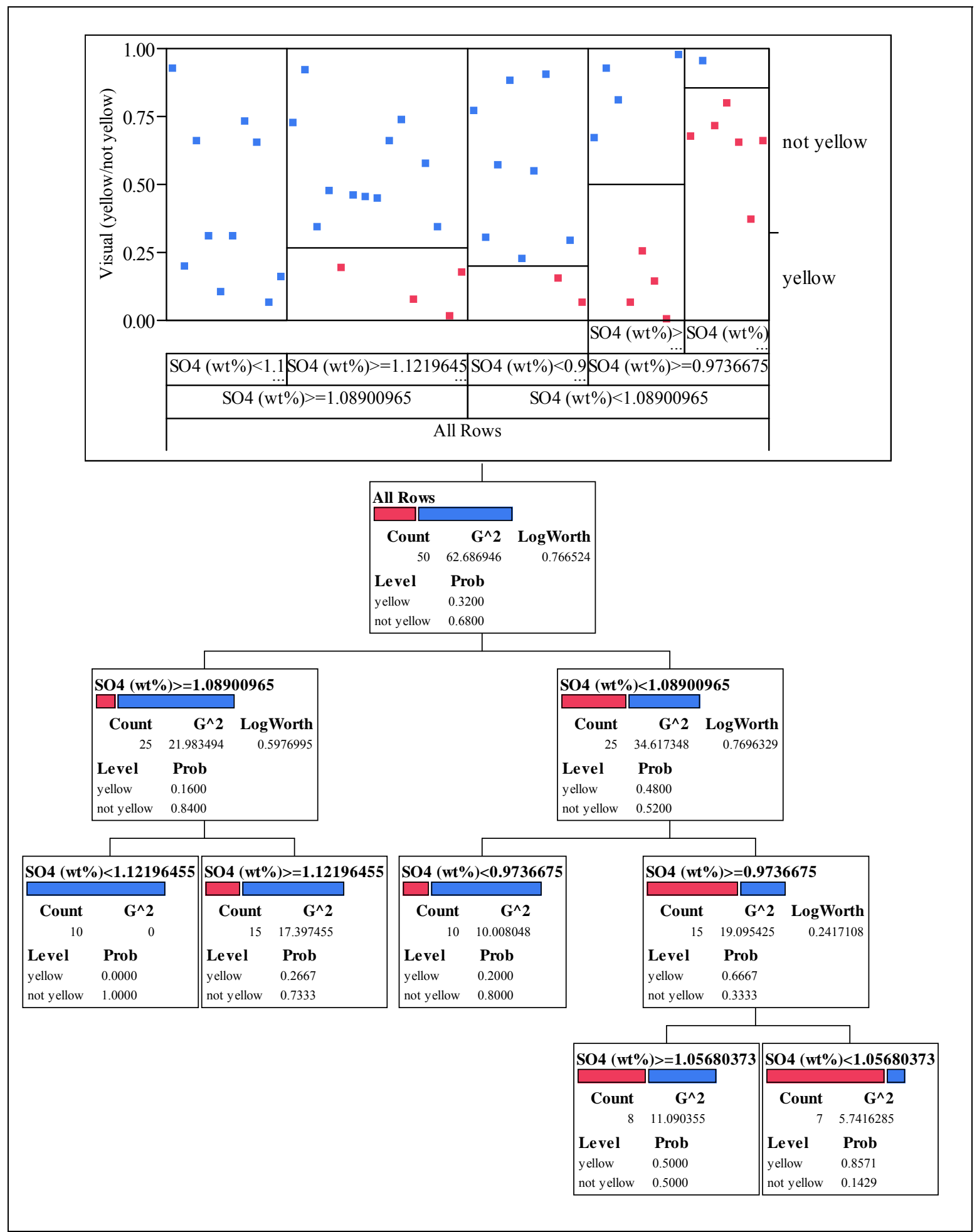

Figure 4-1. Partitioning of Glass Compositions Based on Measured Sulfate Concentration and Visual Observations of Sulfate Surface Layers. 
As discussed earlier in Section 2.1, partitioning of the glasses based on their targeted compositions and visual observations showed that higher $\mathrm{CaO}$ concentrations may play a role in improved retention of sulfate in the glasses (see Figure 2-3 and Figure 2-4). To determine whether this observation would hold true using the measured compositions, the glasses were partitioned by measured $\mathrm{CaO}$ concentration using the visual observations of the presence or absence of sulfate surface layers. The results are shown in Figure 4-2. Based on the measured $\mathrm{CaO}$ data, there again appears to be a relationship between increased $\mathrm{CaO}$ concentrations and increased sulfate retention (i.e., lack of a yellow sulfate surface layer). However, this observation is confounded in that further partitioning shows that all of the glasses with $\mathrm{CaO}$ concentrations of less than $0.61 \mathrm{wt} \%$ were also free of any sulfate surface layer, despite their relatively high sulfate concentrations. While these results provide some indication that $\mathrm{CaO}$ may improve sulfate retention, the effect is not well defined for the glasses in this study. 


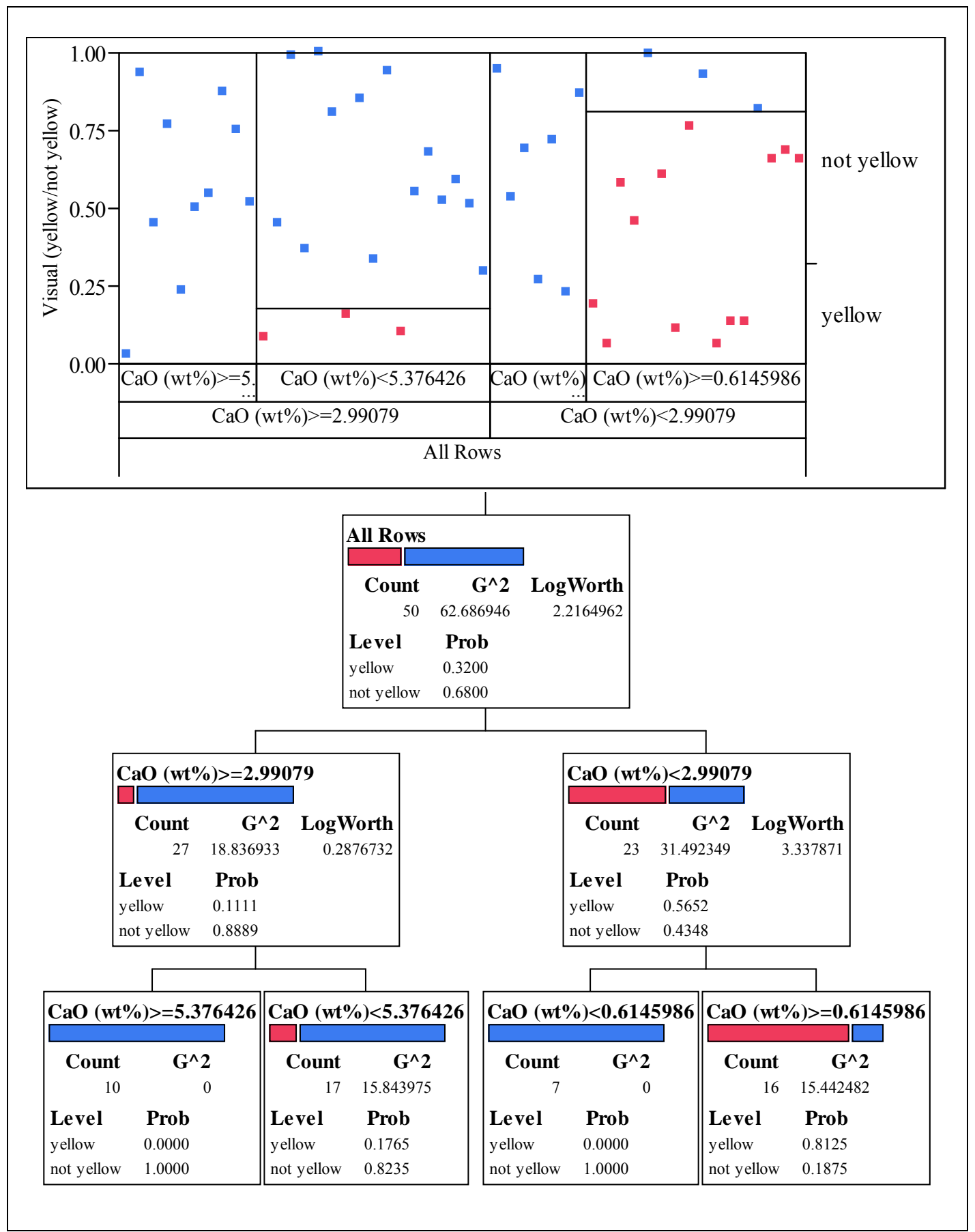

Figure 4-2. Partitioning of Glass Compositions Based on Measured CaO Concentration and Visual Observations of Sulfate Surface Layers.

Next, a partitioning routine was used to evaluate the glasses based on the measured concentrations of all the major oxides and the visual observations. The results are shown in Figure 4-3. At the first split, all 15 of the glasses with measured $\mathrm{MnO}$ concentrations below 2.67 $\mathrm{wt} \%$ were free of a sulfate surface layer after melting. Of the remaining 35 glasses, the majority of those 15 glasses with $\mathrm{SiO}_{2}$ concentrations of less than $43.64 \mathrm{wt} \%$ were also free of a sulfate surface layer after melting. 


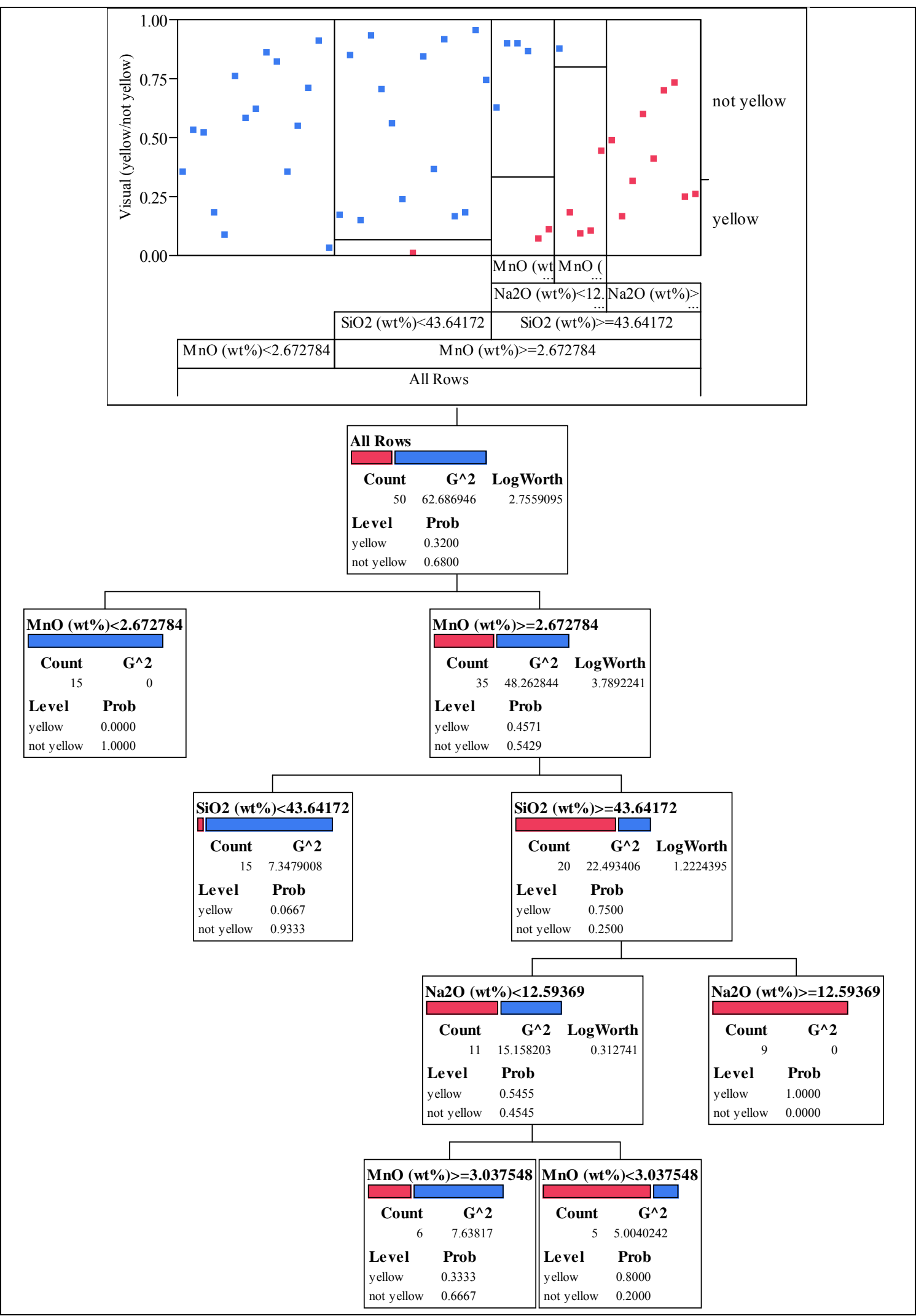

Figure 4-3. Partitioning of Glass Compositions Based on Measured Concentrations of Major Oxides and Visual Observations of Sulfate Surface Layers. 
Thus far, these evaluations have been based on the observation of the presence or absence of a yellow sulfate layer on the surface of the glasses after melting. Another way to evaluate the impact of composition on sulfate retention is to look for trends between the ratio of the measured to the targeted sulfate concentration and the overall composition of each glass. For example, consider Figure 4-4, which plots the ratio of measured to targeted sulfate concentration on the vertical axis and the measured $\mathrm{MnO}$ concentration (a), or the measured $\mathrm{SiO}_{2}$ concentration (b) on the horizontal axis. A ratio of 1.0 on the vertical axis would indicate that all of the targeted sulfate was retained in the glass. A review of the plots in Figure 4-4 shows that, in general, the amount of sulfate retained in the glasses is somewhat reduced as the concentration of either $\mathrm{MnO}$ or $\mathrm{SiO}_{2}$ in the glasses increases. This appears to be in agreement with the partitioning results shown earlier in Figure 4-3.

(a)

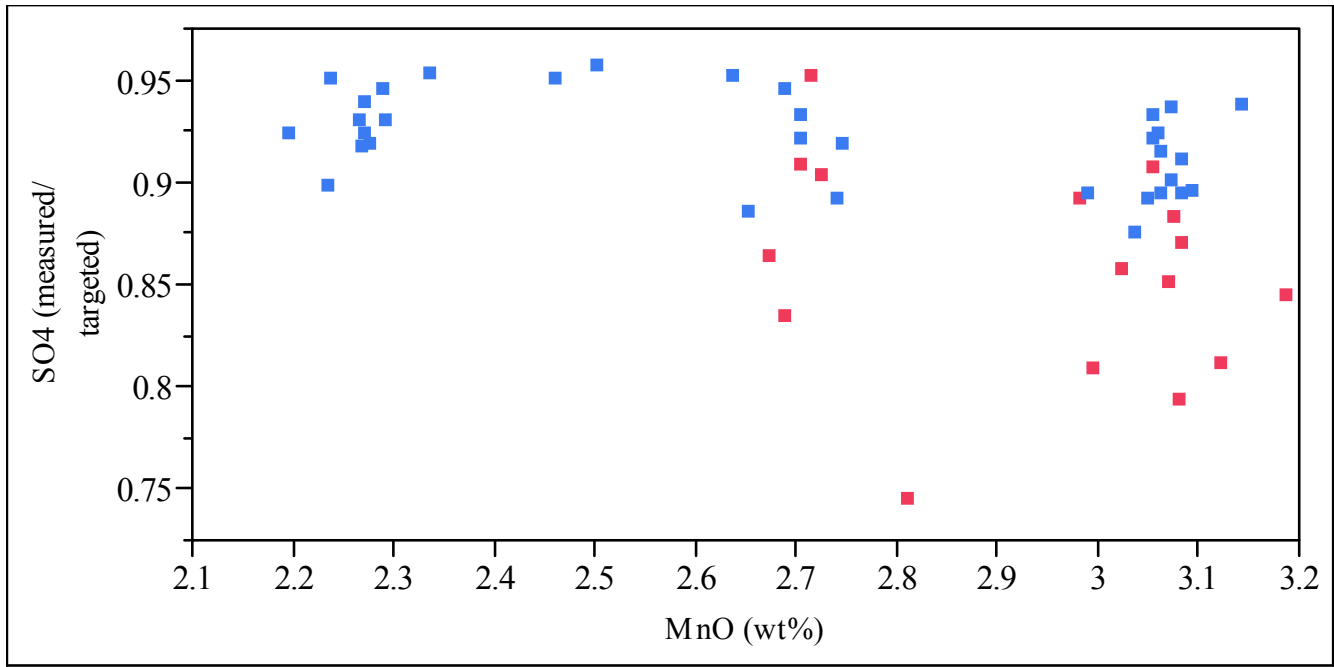

(b)

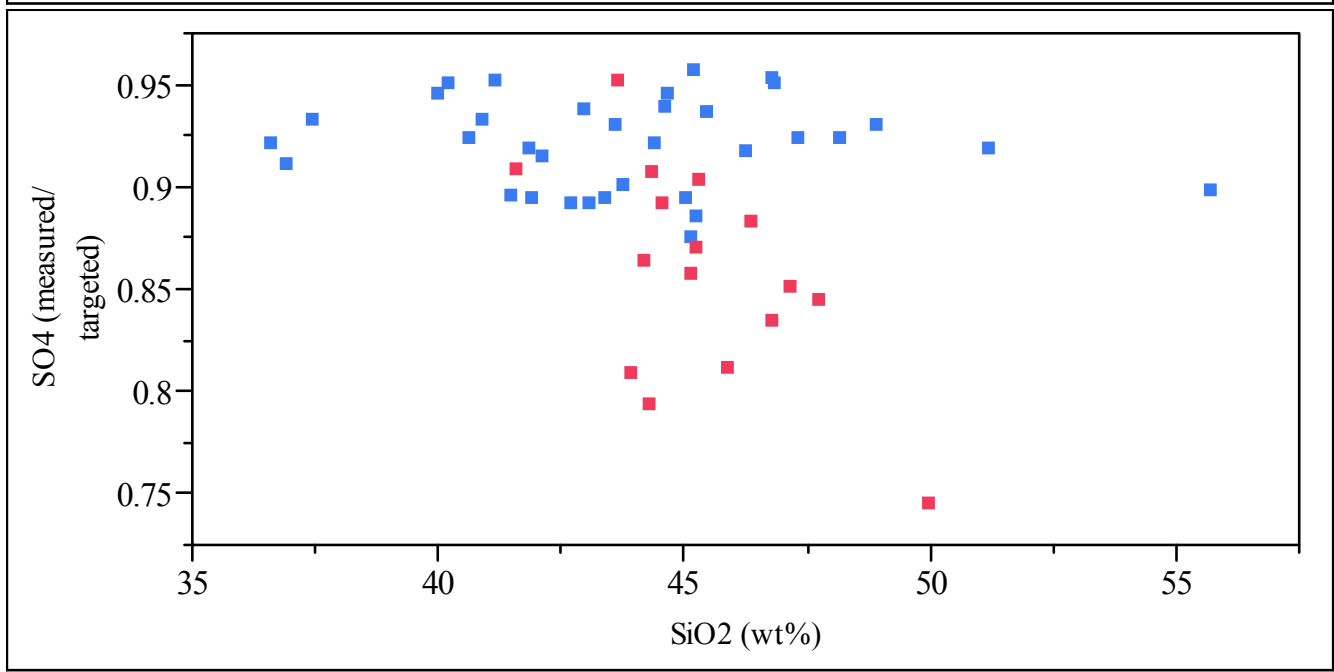

Figure 4-4. Plots of the Ratio of Measured to Targeted Sulfate Concentration versus (a) MnO Concentration and (b) $\mathrm{SiO}_{2}$ Concentration. The Red Data Points Represent Glasses with a Visible Sulfate Surface Layer after Melting. The Blue Data Points Represent Glasses with No Visible Sulfate Layer. 
The relationships between the measured concentrations of the major oxides and the ratio of measured to targeted sulfate concentration were evaluated to determine which of those oxides had statistically significant impacts. The results of a stepwise regression analysis, as shown in Figure 4-5, indicated that $\mathrm{Al}_{2} \mathrm{O}_{3}, \mathrm{~B}_{2} \mathrm{O}_{3}, \mathrm{Li}_{2} \mathrm{O}, \mathrm{MnO}, \mathrm{NiO}$, and $\mathrm{SiO}_{2}$ had statistically significant impacts on the ratio of measured to targeted sulfate concentration. The oxides $\mathrm{CaO}, \mathrm{Fe}_{2} \mathrm{O}_{3}$, and $\mathrm{Na}_{2} \mathrm{O}$ were also included in the analysis but were found not to have a statistically significant impact for the compositions studied. This may be an unintended consequence of the selection process, since $\mathrm{CaO}, \mathrm{Fe}_{2} \mathrm{O}_{3}$, and $\mathrm{Na}_{2} \mathrm{O}$ are typically shown to influence sulfate retention (see the summaries provided by Jantzen and coauthors ${ }^{1,21}$ ). The influences of $\mathrm{Li}_{2} \mathrm{O}$ and $\mathrm{NiO}$ were positive in the stepwise regression analysis, indicating that they improved the amount of sulfate retained. The influences of $\mathrm{Al}_{2} \mathrm{O}_{3}, \mathrm{~B}_{2} \mathrm{O}_{3}, \mathrm{MnO}$, and $\mathrm{SiO}_{2}$ were negative, indicating that they reduced the amount of sulfate retained. These relationships are shown graphically in the plots of Figure 4-6. 


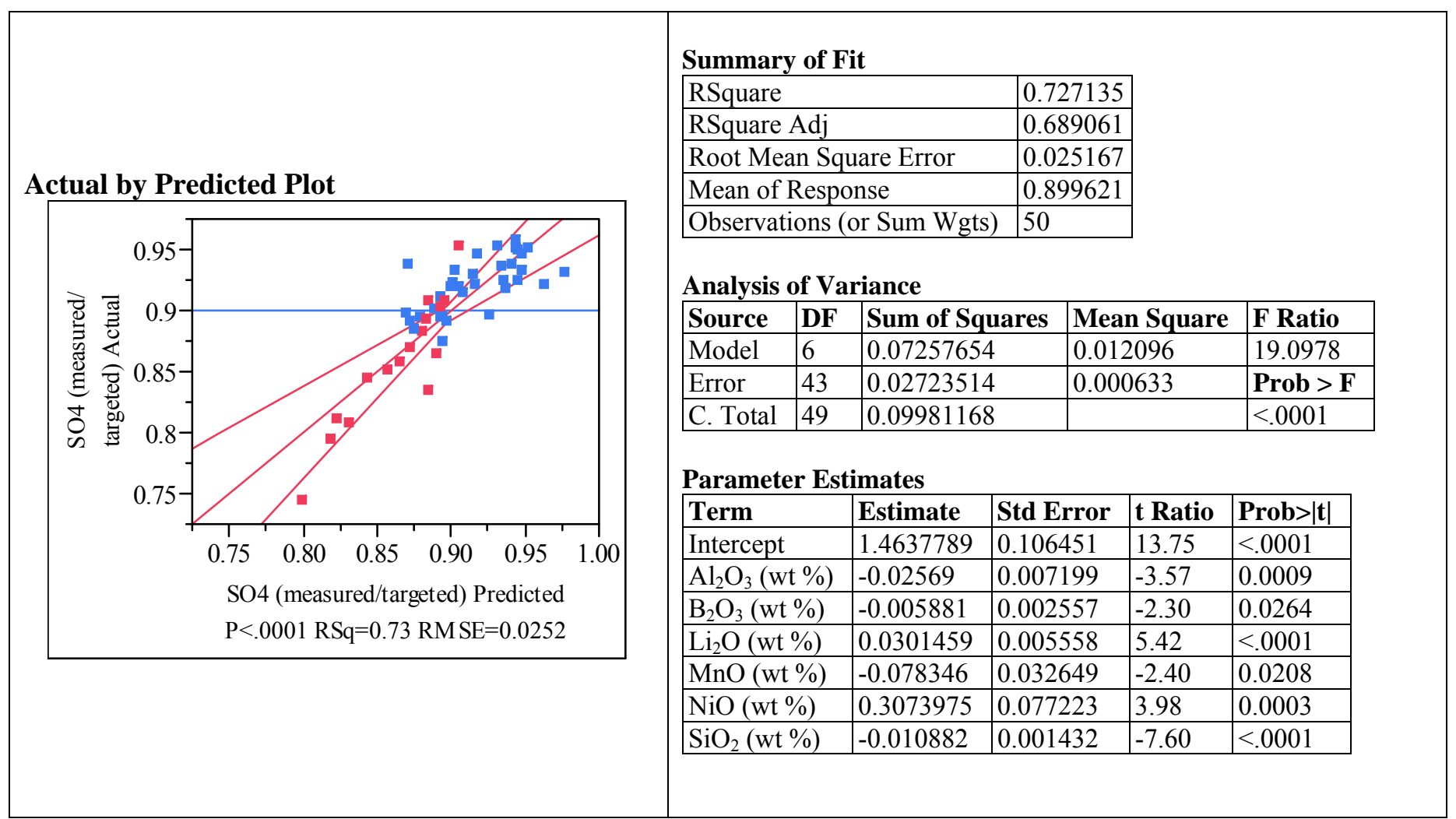

Figure 4-5. Results of Stepwise Regression Analysis of the Influence of the Concentrations of the Major Oxides on the Ratio of Measured to Targeted Sulfate Concentration. 

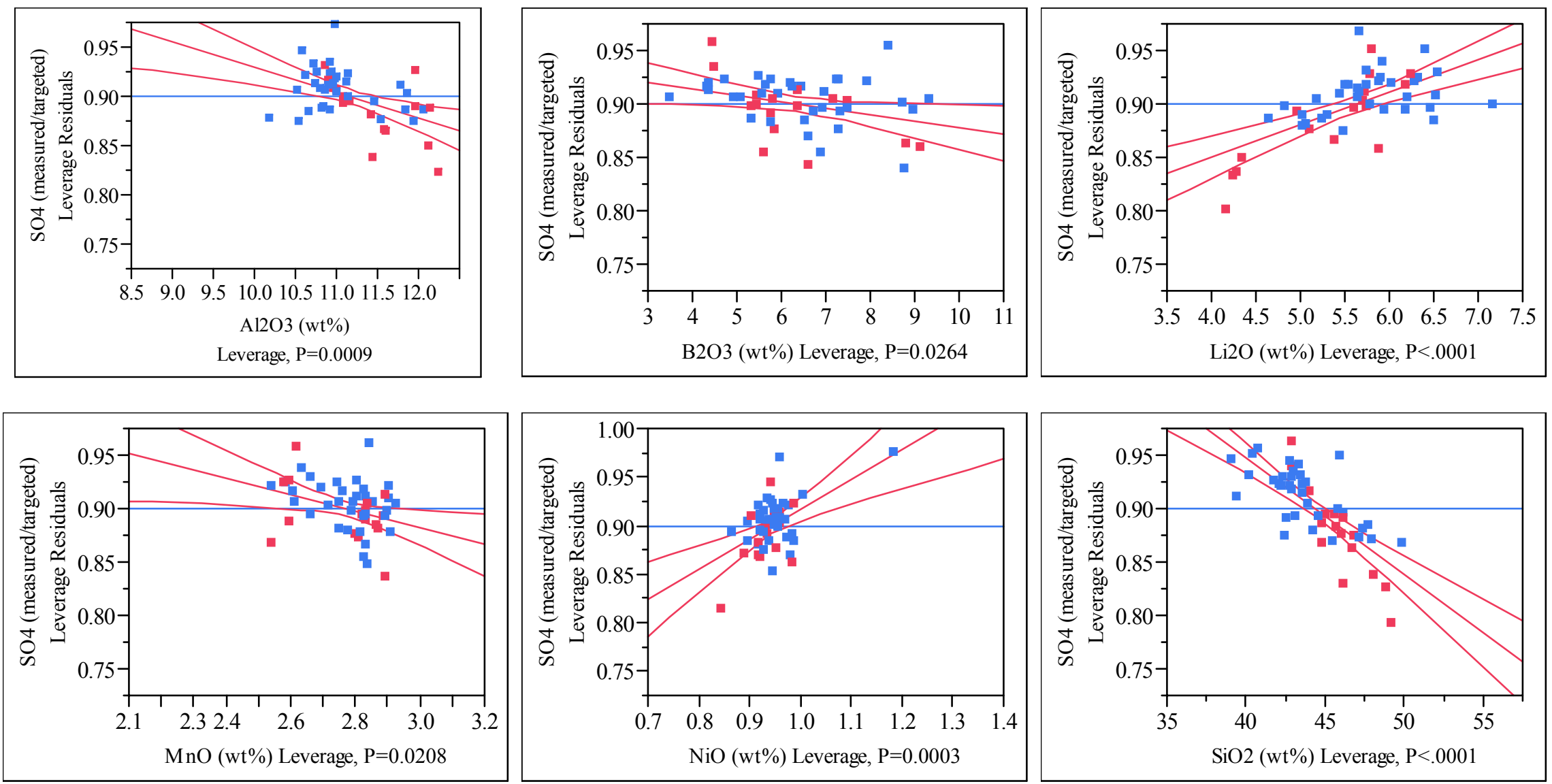

Figure 4-6. Graphical Results of Stepwise Regression Analysis of the Influence of the Concentrations of the Major Oxides on the Ratio of Measured to Targeted Sulfate Concentration. 
These results are consistent with those represented in Figure 4-3 and Figure 4-4 in that increased $\mathrm{MnO}$ and $\mathrm{SiO}_{2}$ concentrations appear to lead to reduced sulfate retention. Partitioning of the measured glass compositions using only those oxides that had a statistically significant impact on sulfate retention and the visual observations, as shown in Figure 4-7, again reinforces the finding that higher concentrations of $\mathrm{MnO}, \mathrm{SiO}_{2}$, and $\mathrm{Al}_{2} \mathrm{O}_{3}$ lead to reduced sulfate retention, while higher $\mathrm{Li}_{2} \mathrm{O}$ concentrations can increase sulfate retention.

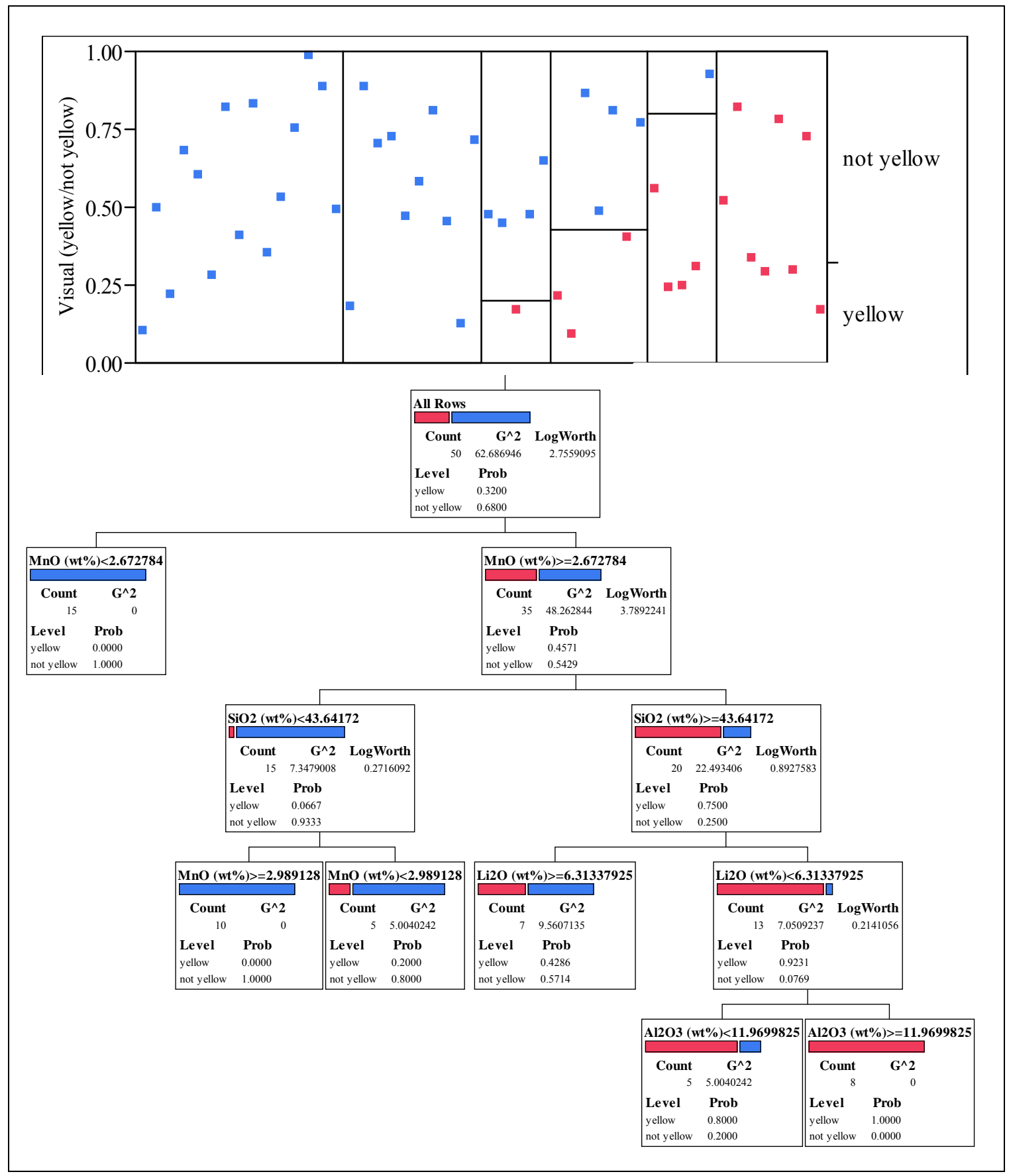

Figure 4-7. Partitioning of Glass Compositions Based on Measured Concentrations of Major Oxides with Statistically Significant Impacts on Sulfate Retention and Visual Observations of Sulfate Surface Layers. 
These results, particularly for the glass network former $\mathrm{SiO}_{2}$, are consistent with the recent findings by Manara et al. that the incorporation of sulfates in borosilicate glasses is favored by network depolymerization (i.e., a reduction in the concentration of the glass forming oxides). ${ }^{22}$ This is also consistent with previous sulfate solubility work on DWPF glasses by Jantzen and coauthors. ${ }^{1,21}$ Although it was not found to be statistically significant for the glasses studied here, the potential impact of $\mathrm{CaO}$ in improving sulfate retention is also in agreement with these findings, as $\mathrm{CaO}$ is a glass network modifier that reduces network polymerization. Schreiber and coauthors have shown improved sulfate retention with $\mathrm{CaO}$ additions, while Feng and coauthors observed the opposite effect. ${ }^{1}$ The results of the present study seem to show a reduced sulfate solubility with increased concentrations of $\mathrm{MnO}$ and $\mathrm{Al}_{2} \mathrm{O}_{3}$, which are considered intermediate glass network formers. ${ }^{23}$ Langowski has reported decreased sulfate retention with increased $\mathrm{Al}_{2} \mathrm{O}_{3}$ concentrations. ${ }^{24}$

Further corroboration of these results is provided by the findings of the cation field strength modeling effort completed at the University of Sheffield. The final report of the Sheffield study is included as Appendix C. In summary, the model demonstrated that enhanced sulfate solubility occurs at low values of cation field strength, with $\mathrm{SiO}_{2}$ concentration exhibiting the strongest effect. Of the data set modeled by Sheffield, the glasses with less than approximately $45 \mathrm{~mol} \%$ $\mathrm{SiO}_{2}$ did not exhibit the formation of a yellow sulfate surface layer. Their report recommends that additional data be obtained for glasses in this compositional region to enable further experimental validation of the model results.

\subsection{Influence of Viscosity}

The measured viscosity of each of the 14 glasses is given in Table 4-1. The model predicted viscosity values for each glass along with their upper and lower $95 \%$ confidence intervals are also given in the table. These values are presented graphically in Figure 4-8. Four of the glasses have measured viscosities that fall below the lower 95\% confidence bound, and are therefore not predictable by the current model. It is important to note that some of the study glasses have measured $\mathrm{B}_{2} \mathrm{O}_{3}, \mathrm{CaO}, \mathrm{Cr}_{2} \mathrm{O}_{3}, \mathrm{Li}_{2} \mathrm{O}, \mathrm{MgO}, \mathrm{SiO}_{2}$, and $\mathrm{ZnO}$ concentrations that are outside of the development region for the current PCCS viscosity model. ${ }^{25}$ Some of the study glasses also have measured $\mathrm{B}_{2} \mathrm{O}_{3}, \mathrm{CaO}, \mathrm{Li}_{2} \mathrm{O}$, and $\mathrm{SiO}_{2}$ concentrations that fall outside of the range of the model validation region. ${ }^{25}$ Therefore, the current model is not necessarily expected to correctly predict the viscosities of these glasses. The measured viscosity data, along with the measured compositions discussed earlier, should be used in future efforts for modeling sulfate retention as a function of predicted viscosity. 
Table 4-1. Predicted and Measured Viscosities and Sulfate Concentrations of the Study Glasses.

\begin{tabular}{||c|c|c|c|c|c||}
\hline Glass ID & $\begin{array}{c}\text { Measured } \\
\text { Viscosity } \\
\text { (Fit at } \\
\mathbf{1 1 5 0} \mathbf{~} \mathbf{C})^{\mathbf{( P )}}\end{array}$ & $\begin{array}{c}\text { Predicted } \\
\text { Viscosity (P) }\end{array}$ & $\begin{array}{c}\text { Lower } \\
\mathbf{9 5 \%} \mathbf{C I}(\mathbf{P})\end{array}$ & $\begin{array}{c}\text { Upper } \\
\mathbf{9 5 \% C I}(\mathbf{P})\end{array}$ & Predictable? \\
\hline AB-06 & 69.69 & 51.87 & 35.39 & 76.04 & Yes \\
\hline AF-04 & 13.91 & 13.63 & 9.24 & 20.11 & Yes \\
\hline BC-05 & 13.78 & 32.45 & 22.11 & 47.62 & No \\
\hline BC-06 & 18.78 & 13.65 & 9.25 & 20.14 & Yes \\
\hline BC-07 & 9.03 & 7.85 & 5.29 & 11.64 & Yes \\
\hline BC-08 & 16.54 & 19.74 & 13.42 & 29.05 & Yes \\
\hline BC-09 & 22.2 & 26.32 & 17.92 & 38.67 & Yes \\
\hline LL-03 & 8.54 & 14.00 & 9.49 & 20.65 & No \\
\hline LL-05 & 21.29 & 24.99 & 17.01 & 36.72 & Yes \\
\hline LL-06 & 8.99 & 9.52 & 6.43 & 14.09 & Yes \\
\hline LL-10 & 27.82 & 38.81 & 26.46 & 56.93 & Yes \\
\hline LL-11 & 27.51 & 43.34 & 29.56 & 63.55 & No \\
\hline LL-12 & 13.17 & 22.16 & 15.07 & 32.58 & No \\
\hline LT-01 & 73.94 & 91.65 & 62.53 & 134.34 & Yes \\
\hline \hline
\end{tabular}

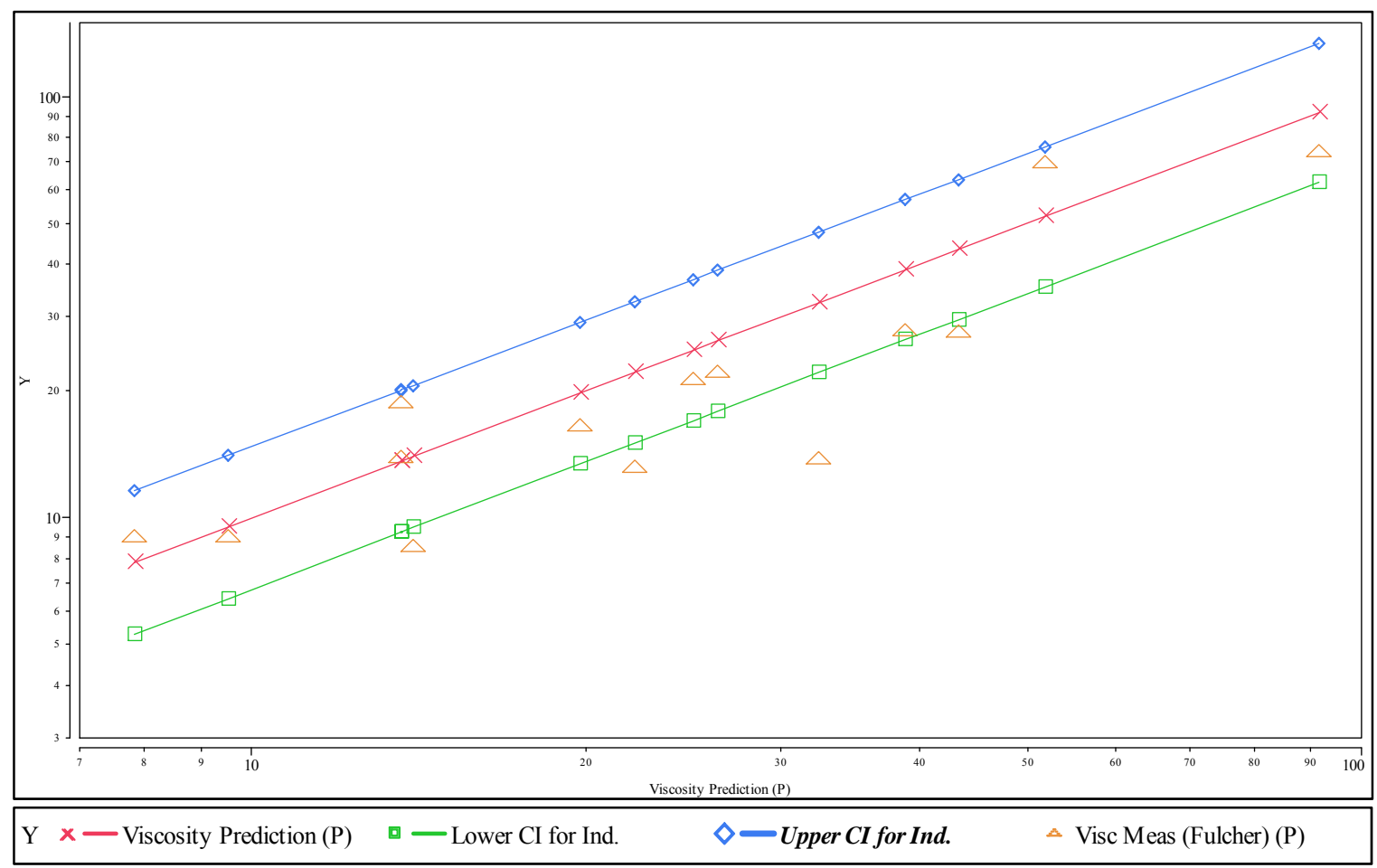

Figure 4-8. Plot of Measured and Model Predicted Viscosities of the Study Glasses.

\subsection{Conclusions}

Partitioning of the study glass compositions based on the visual observations and the measured sulfate concentrations revealed mixed results in terms of the impact of glass composition on 
sulfate retention. There is a composition region among the glasses studied here that is able to retain considerably more sulfate $(>1.0 \mathrm{wt} \%)$ than the current DWPF limit of $0.6 \mathrm{wt} \%$. However, for the 25 glasses with measured sulfate concentrations of less than $1.089 \mathrm{wt} \%, 12$ glasses exhibited a sulfate surface layer after melting. This indicates that it would be inappropriate to try to identify an acceptable sulfate concentration for a broad range of glass compositions (e.g., a single sulfate concentration limit for all glass compositions to be processed at the DWPF). In other words, sulfate solubility in this series of glasses, as well as other DWPF-type compositions, ${ }^{1}$ appears to be a function of the individual compositions, rather than a general property applicable to a large compositional region.

Partitioning of the glasses based on their targeted compositions and visual observations showed that higher $\mathrm{CaO}$ concentrations may play a role in improved retention of sulfate in the glasses. Based on the measured $\mathrm{CaO}$ data, there indeed appears to be a relationship between increased $\mathrm{CaO}$ concentrations and increased sulfate retention (i.e., lack of a yellow sulfate surface layer). However, this observation is confounded in that further partitioning shows that all of the glasses with $\mathrm{CaO}$ concentrations of less than $0.61 \mathrm{wt} \%$ were also free of any sulfate surface layer, despite their relatively high sulfate concentrations. While these results provide some indication that $\mathrm{CaO}$ may improve sulfate retention, the effect is not well defined for the compositional region studied.

The results of a stepwise regression analysis indicated that $\mathrm{Al}_{2} \mathrm{O}_{3}, \mathrm{~B}_{2} \mathrm{O}_{3}, \mathrm{Li}_{2} \mathrm{O}, \mathrm{MnO}, \mathrm{NiO}$, and $\mathrm{SiO}_{2}$ had statistically significant impacts on the ratio of measured to targeted sulfate concentration. The oxides $\mathrm{CaO}, \mathrm{Fe}_{2} \mathrm{O}_{3}$, and $\mathrm{Na}_{2} \mathrm{O}$ were included in the analysis but were found not to have a statistically significant impact for these compositions. The influences of $\mathrm{Li}_{2} \mathrm{O}$ and $\mathrm{NiO}$ were positive, indicating that they improved the amount of sulfate retained. The influences of $\mathrm{Al}_{2} \mathrm{O}_{3}, \mathrm{~B}_{2} \mathrm{O}_{3}, \mathrm{MnO}$, and $\mathrm{SiO}_{2}$ were negative, indicating that they reduced the amount of sulfate retained. The influences of all these components are generally consistent with findings described by others in the technical literature (see for example the summaries provided by Jantzen and coauthors ${ }^{1,21}$ ). Further corroboration of these results is provided by the findings of the cation field strength modeling effort completed at the University of Sheffield (see Appendix C). The model demonstrated that enhanced sulfate solubility occurs at low values of cation field strength, with $\mathrm{SiO}_{2}$ concentration exhibiting the strongest effect.

\subsection{Recommendations}

Based on the results of these and other studies, the ability to improve sulfate solubility in DWPF borosilicate glasses lies in reducing the connectivity of the glass network structure. This can be achieved, as an example, by increasing the concentration of alkali species in the glass. However, this must be balanced with other effects of reduced network connectivity, such as reduced viscosity, potentially lower chemical durability, and in the case of higher sodium and aluminum concentrations, the propensity for nepheline crystallization. Future DWPF processing is likely to target higher waste loadings and higher sludge sodium concentrations, meaning that alkali concentrations in the glass will already be relatively high. It is therefore unlikely that there will be the ability to target significantly higher total alkali concentrations in the glass solely to support increased sulfate solubility without the increased alkali concentration causing failure of other PCCS constraints, such as low viscosity and durability.

No individual components were found to provide a significant improvement in sulfate retention (i.e., an increase of the magnitude necessary to have a dramatic impact on blending, washing, or waste loading strategies for DWPF) for the glasses studied here. In general, the concentrations of those species that improve sulfate solubility in a borosilicate glass must be added in relatively 
large concentrations in order to have a substantial impact. For example, additions of $\mathrm{BaO}$ at 15 wt $\%$ of the glass, ${ }^{26} \mathrm{CaO}$ at 5 to $10 \mathrm{wt} \%$ of the glass, ${ }^{27}$ and $\mathrm{V}_{2} \mathrm{O}_{5}$ at 5 to $10 \mathrm{wt} \%$ of the glass ${ }^{22,27}$ have been shown to significantly improve sulfate solubility in borosilicate glasses. For DWPF, these concentrations would equate to about 13 to $38 \mathrm{wt} \%$ of the frit composition at $40 \%$ waste loading, which is far too high to be practical. Therefore, it is unlikely that specific additives may be introduced into the DWPF glass via the frit to significantly improve sulfate solubility.

The results presented here continue to show that sulfate solubility or retention is a function of individual glass compositions, rather than a property of a broad glass composition region. It would therefore be inappropriate to set a single sulfate concentration limit for a range of DWPF glass compositions. Sulfate concentration limits should continue to be identified and implemented for each sludge batch. The current PCCS limit is $0.4 \mathrm{wt} \% \mathrm{SO}_{4}{ }^{2-}$ in glass, although frit development efforts have led to an increased limit of $0.6 \mathrm{wt} \%$ for recent sludge batches. Slightly higher limits (perhaps 0.7-0.8 wt \%) may be possible for future sludge batches.

An opportunity for allowing a higher sulfate concentration limit at DWPF may lay lie in improving the laboratory experiments used to set this limit. That is, there are several differences between the crucible-scale testing currently used to define a limit for DWPF operation and the actual conditions within the DWPF melter. In particular, no allowance is currently made for sulfur partitioning (volatility versus retention) during melter processing as the sulfate limit is set for a specific sludge batch. A better understanding of the partitioning of sulfur in a bubbled melter operating with a cold cap as well as the impacts of sulfur on the off-gas system may allow a higher sulfate concentration limit to be established for the melter feed. This approach would have to be taken carefully to ensure that a sulfur salt layer is not formed on top of the melt pool while allowing higher sulfur concentration feeds to be processed through DWPF. 


\subsection{References}

1. Jantzen, C. M. and M. E. Smith, "Revision of the Defense Waste Processing Facility (DWPF) Sulfate Solubility Limit," U.S. Department of Energy Report WSRC-TR-2003-00518, Revision 0, Westinghouse Savannah River Company, Aiken, SC (2003).

2. Bricker, J. M., “Technical Task Request: Sludge Batch 6 Sulfate Solubility Study," U.S. Department of Energy Report HLW-DWPF-TTR-2009-0013, Savannah River Remediation, Aiken, SC (2009).

3. Fellinger, T. L., "Technical Task Request: Increasing the Sulfate Limit in Defense Waste Processing Facility (DWPF) Glass," U.S. Department of Energy Report HLW-DWPF-TTR-20090018, Savannah River Remediation, Aiken, SC (2009).

4. Billings, A. L., "Sulfate Solubility Studies for DWPF Glass," U.S. Department of Energy Report SRNL-RP-2009-00560, Revision 1, Savannah River National Laboratory, Aiken, SC (2009).

5. Fox, K. M., A. L. Billings and J. W. Amoroso, "Updated Experimental Plans for Sulfate Studies," U.S. Department of Energy Memorandum SRNL-L3100-2011-00055, Savannah River National Laboratory, Aiken, SC (2011).

6. Billings, A. L., "DWPF Sulfate Limit Verification for SB6," U.S. Department of Energy Report SRNL-STI-2010-00191, Revision 0, Savannah River National Laboratory, Aiken, SC (2010).

7. Billings, A. L. and K. M. Fox, "Retention of Sulfate in Savannah River Site High-Level Radioactive Waste Glass," International Journal of Applied Glass Science, 1 [4] 388-400 (2010).

8. Billings, A. L., "Sulfate Solubility Limit Verification for DWPF Sludge Batch 7a," U.S. Department of Energy Report SRNL-STI-2011-00197, Revision 0, Savannah River National Laboratory, Aiken, SC (2011).

9. JMP $^{\mathrm{TM}}$, Ver. 7.0.2, [Computer Software] SAS Institute Inc., Cary, NC (2007).

10. Billings, A. L., "Sulfate Solubility Limit Verification for DWPF Sludge Batch 7b," U.S. Department of Energy Memorandum SRNL-L3100-2011-00159, Savannah River National Laboratory, Aiken, SC (2011).

11. Edwards, T. B., "Analytical Plans for Measuring the Chemical Compositions of Sulfate Retention Study Glasses," U.S. Department of Energy Memorandum SRNL-L4221-2011-00007, Savannah River National Laboratory, Aiken, SC (2011).

12. ASTM, "Standard Practice for Measuring Viscosity of Glass Above the Softening Point," ASTM C-965, (2007).

13. Schumacher, R. F. and D. K. Peeler, "Establishment of Harrop, High-Temperature Viscometer," U.S. Department of Energy Report WSRC-RP-98-00737, Revision 0, Westinghouse Savannah River Company, Aiken, SC (1998). 
14. Schumacher, R. F., R. J. Workman and T. B. Edwards, "Calibration and Measurement of the Viscosity of DWPF Start-Up Glass," U.S. Department of Energy Report WSRC-RP-2000-00874, Revision 0, Westinghouse Savannah River Company, Aiken, SC (2001).

15. Crum, J. V., R. L. Russell, M. J. Schweiger, D. E. Smith, J. D. Vienna, T. B. Edwards, C. M. Jantzen, D. K. Peeler, R. F. Schumacher and R. J. Workman, "DWPF Startup Frit Viscosity Measurement Round Robin Results," Pacific Northwest National Laboratory, (Unpublished).

16. Fulcher, G. S., "Analysis of Recent Measurements of the Viscosity of Glasses," Journal of the American Ceramic Society, 8 [6] 339-355 (1925).

17. Fulcher, G. S., "Analysis of Recent Measurements of the Viscosity of Glasses, II," Journal of the American Ceramic Society, 8 [12] 789-794 (1925).

18. Jantzen, C. M., J. B. Pickett, K. G. Brown, T. B. Edwards and D. C. Beam, "Process/Product Models for the Defense Waste Processing Facility (DWPF): Part I. Predicting Glass Durability from Composition Using a Thermodynamic Hydration Energy Reaction Model (THERMO ${ }^{\mathrm{TM}}$ )," U.S. Department of Energy Report WSRC-TR-93-673, Revision 1, Westinghouse Savannah River Company, Aiken, South Carolina (1995).

19. Ebert, W. L. and S. F. Wolfe, "Round-robin Testing of a Reference Glass for Low-Activity Waste Forms," U.S. Department of Energy Report ANL-99/22, Argonne National Laboratory, Argonne, IL (1999).

20. U.S. Environmental Protection Agency, "Statistical Analysis of Ground-Water Monitoring Data at RCRA Facilities: Interim Final Guidance,” 530-SW-89-026, (1989).

21. Jantzen, C. M., M. E. Smith and D. K. Peeler, "Dependency of Sulfate Solubility on Melt Composition and Melt Polymerization"; pp. 141-152 in Ceramic Transactions, Vol. 168, Environmental Issues and Waste Management Technologies in the Ceramic and Nuclear Industries X. Edited by J. D. Vienna, C. C. Herman and S. Marra. The American Ceramic Society, Westerville, OH, 2005.

22. Manara, D., A. Grandjean, O. Pinet, J. L. Dussossoy and D. R. Neuville, "Sulfur Behavior in Silicate Glasses and Melts: Implications for Sulfate Incorporation in Nuclear Waste Glasses as a Function of Alkali Cation and $\mathrm{V}_{2} \mathrm{O}_{5}$ Content," Journal of Non-Crystalline Solids, 353 [1] 12-23 (2007).

23. Dietzel, A., Z. Electrochem., 48 9-23 (1942).

24. Langowski, M. H., "The Incorporation of P, S, Cr, F, Cl, I, Mn, Ti, U, and Bi into Simulated Nuclear Waste Glasses: Literature Study," U.S. Department of Energy Report PNNL-10980 UC512, Pacific Northwest National Laboratory, Richland, WA (1996).

25. Jantzen, C. M., "The Impacts of Uranium and Thorium on the Defense Waste Processing Facility (DWPF) Viscosity Model," U.S. Department of Energy Report WSRC-TR-2004-00311, Revision 0, Westinghouse Savannah River Company, Aiken, SC (2005).

26. Kaushik, C. P., R. K. Mishra, P. Sengupta, A. Kumar, D. Das, G. B. Kale and K. Raj, "Barium Borosilicate Glass - A Potential Matrix for Immobilization of Sulfate Bearing Highlevel Radioactive Liquid Waste,” Journal of Nuclear Materials, 358 129-138 (2006). 
SRNL-STI-2012-00152

Revision 0

27. Youchak-Billings, A. L., "A Scoping Study Examining the Possibility of a Frit Additive to Increase Sulfur Solubility in DWPF Glass," U.S. Department of Energy Report SRNL-PSE-200800173, Savannah River National Laboratory, Aiken, SC (2008). 
SRNL-STI-2012-00152

Revision 0

Appendix A. Tables and Exhibits Supporting the Analysis of the Chemical Composition Measurements of the Glasses Selected to Identify Compositional Influences on Sulfate Retention 
Table A-1. Measured Elemental Concentrations for Glasses Prepared Using Lithium Metaborate (part 1)

\begin{tabular}{|c|c|c|c|c|c|c|c|c|c|c|c|c|c|c|}
\hline Series & Glass ID & Block & Sub-Blk & Sequence & Lab ID & Al (wt \%) & Ba (wt \%) & Ca (wt \%) & $\mathrm{Ce}$ (wt \%) & Cr (wt \%) & Cu (wt \%) & $\mathrm{Fe}(\mathrm{wt} \%)$ & K (wt \%) & La (wt \%) \\
\hline $\mathrm{T}$ & Batch 1 & 1 & 1 & 1 & BCHLM111 & 2.59 & 0.126 & 0.943 & $<0.010$ & 0.077 & 0.312 & 9.03 & 3.03 & $\begin{array}{c}<010 \\
\end{array}$ \\
\hline $\mathrm{T}$ & LRM & 1 & 1 & 2 & LRMLM111 & 5.32 & $<0.010$ & 0.438 & $<0.010$ & 0.137 & $<0.010$ & 1.04 & 1.28 & $<0.010$ \\
\hline $\mathrm{T}$ & $\begin{array}{l}\mathrm{BC}-03 \\
\end{array}$ & 1 & 1 & 3 & t10LM21 & 6.49 & 0.040 & 0.545 & 0.042 & 0.089 & 0.037 & 6.86 & $<0.100$ & 0.033 \\
\hline $\mathrm{T}$ & BC-01 & 1 & 1 & 4 & t04LM11 & 6.52 & 0.037 & 0.519 & 0.040 & 0.089 & 0.034 & 6.73 & $<0.100$ & 0.028 \\
\hline $\mathrm{T}$ & BC-02 & 1 & 1 & 5 & t02LM11 & 6.45 & 0.037 & 0.516 & 0.040 & 0.095 & 0.034 & 6.73 & $<0.100$ & 0.029 \\
\hline $\mathrm{T}$ & AF-02 & 1 & 1 & 6 & t03LM11 & 6.44 & 0.036 & 0.498 & 0.043 & 0.093 & 0.033 & 7.02 & $<0.100$ & 0.028 \\
\hline $\mathrm{T}$ & AF-05 & 1 & 1 & 7 & t06LM21 & 6.37 & 0.038 & 2.25 & 0.039 & 0.094 & 0.037 & 6.82 & $<0.100$ & 0.028 \\
\hline $\mathrm{T}$ & Batch 1 & 1 & 1 & 8 & BCHLM112 & 2.54 & 0.124 & 0.928 & $<0.010$ & 0.077 & 0.312 & 8.88 & 2.96 & $<0.010$ \\
\hline $\mathrm{T}$ & LRM & 1 & 1 & 9 & LRMLM112 & 5.22 & $<0.010$ & 0.430 & $<0.010$ & 0.138 & $<0.010$ & 1.02 & 1.24 & $<0.010$ \\
\hline $\mathrm{T}$ & BC-01 & 1 & 1 & 10 & t04LM21 & 6.23 & 0.037 & 0.492 & 0.040 & 0.089 & 0.034 & 6.47 & $<0.100$ & 0.028 \\
\hline $\mathrm{T}$ & AF-05 & 1 & 1 & 11 & t06LM11 & 6.34 & 0.038 & 2.24 & 0.039 & 0.094 & 0.038 & 6.78 & $<0.100$ & 0.028 \\
\hline $\mathrm{T}$ & BC-02 & 1 & 1 & 12 & t02LM21 & 6.13 & 0.036 & 0.488 & 0.045 & 0.093 & 0.034 & 6.44 & $<0.100$ & 0.029 \\
\hline $\mathrm{T}$ & BC- 03 & 1 & 1 & 13 & t10LM11 & 6.15 & 0.038 & 0.502 & 0.041 & 0.089 & 0.037 & 6.55 & $<0.100$ & 0.033 \\
\hline $\mathrm{T}$ & AF-02 & 1 & 1 & 14 & t03LM21 & 6.18 & 0.035 & 0.483 & 0.040 & 0.091 & 0.033 & 6.75 & $<0.100$ & 0.027 \\
\hline $\mathrm{T}$ & Batch 1 & 1 & 1 & 15 & BCHLM113 & 2.46 & 0.121 & 0.889 & $<0.010$ & 0.075 & 0.301 & 8.62 & 2.78 & $<0.010$ \\
\hline $\mathrm{T}$ & LRM & 1 & 1 & 16 & LRMLM113 & 5.07 & $<0.010$ & 0.417 & $<0.010$ & 0.135 & $<0.010$ & 0.99 & 1.19 & $<0.010$ \\
\hline $\mathrm{T}$ & Batch 1 & 1 & 2 & 1 & BCHLM121 & 2.44 & 0.125 & 0.874 & $<0.010$ & 0.077 & 0.310 & 8.55 & 2.82 & $<0.010$ \\
\hline $\mathrm{T}$ & LRM & 1 & 2 & 2 & LRMLM121 & 5.22 & $<0.010$ & 0.400 & $<0.010$ & 0.139 & $<0.010$ & 1.02 & 1.23 & $<0.010$ \\
\hline $\mathrm{T}$ & AF-05 & 1 & 2 & 3 & t06LM22 & 6.29 & 0.036 & 2.19 & 0.037 & 0.095 & 0.037 & 6.74 & $<0.100$ & 0.028 \\
\hline $\mathrm{T}$ & AF-05 & 1 & 2 & 4 & t06LM12 & 6.44 & 0.037 & 2.24 & 0.037 & 0.096 & 0.037 & 6.89 & $<0.100$ & 0.027 \\
\hline $\mathrm{T}$ & AF-02 & 1 & 2 & 5 & t03LM12 & 6.57 & 0.034 & 0.485 & 0.040 & 0.095 & 0.033 & 7.14 & $<0.100$ & 0.027 \\
\hline $\mathrm{T}$ & BC-01 & 1 & 2 & 6 & t04LM12 & 6.67 & 0.035 & 0.505 & 0.038 & 0.090 & 0.034 & 6.89 & $<0.100$ & 0.028 \\
\hline $\mathrm{T}$ & BC-02 & 1 & 2 & 7 & t02LM12 & 6.53 & 0.036 & 0.495 & 0.038 & 0.097 & 0.034 & 6.82 & $<0.100$ & 0.029 \\
\hline $\mathrm{T}$ & Batch 1 & 1 & 2 & 8 & BCHLM122 & 2.62 & 0.125 & 0.930 & $<0.010$ & 0.077 & 0.313 & 9.14 & 2.70 & $<0.010$ \\
\hline $\mathrm{T}$ & LRM & 1 & 2 & 9 & LRMLM122 & 5.26 & $<0.010$ & 0.403 & $<0.010$ & 0.140 & $<0.010$ & 1.02 & 1.24 & $<0.010$ \\
\hline $\mathrm{T}$ & BC-02 & 1 & 2 & 10 & t02LM22 & 6.43 & 0.036 & 0.493 & 0.042 & 0.096 & 0.034 & 6.72 & $<0.100$ & 0.029 \\
\hline $\mathrm{T}$ & BC- 03 & 1 & 2 & 11 & t10LM22 & 6.38 & 0.039 & 0.508 & 0.040 & 0.091 & 0.037 & 6.75 & $<0.100$ & 0.033 \\
\hline $\mathrm{T}$ & AF-02 & 1 & 2 & 12 & t03LM22 & 6.40 & 0.035 & 0.475 & 0.038 & 0.095 & 0.033 & 7.00 & $<0.100$ & 0.028 \\
\hline $\mathrm{T}$ & BC- 03 & 1 & 2 & 13 & t10LM12 & 6.47 & 0.037 & 0.506 & 0.038 & 0.091 & 0.037 & 6.86 & $<0.100$ & 0.033 \\
\hline $\mathrm{T}$ & BC-01 & 1 & 2 & 14 & t04LM22 & 6.40 & 0.036 & 0.478 & 0.038 & 0.091 & 0.034 & 6.63 & $<0.100$ & 0.028 \\
\hline $\mathrm{T}$ & Batch 1 & 1 & 2 & 15 & BCHLM123 & 2.55 & 0.125 & 0.906 & $<0.010$ & 0.077 & 0.314 & 8.95 & 2.74 & $<0.010$ \\
\hline $\mathrm{T}$ & LRM & 1 & 2 & 16 & LRMLM123 & 5.10 & $<0.010$ & 0.388 & $<0.010$ & 0.140 & $<0.010$ & 1.00 & 1.22 & $<0.010$ \\
\hline $\mathrm{T}$ & Batch 1 & 2 & 1 & 1 & BCHLM211 & 2.63 & 0.123 & 0.881 & $<0.010$ & 0.076 & 0.309 & 9.23 & 2.70 & $<0.010$ \\
\hline $\mathrm{T}$ & LRM & 2 & 1 & 2 & LRMLM211 & 5.38 & $<0.010$ & 0.354 & $<0.010$ & 0.136 & $<0.010$ & 1.02 & 1.18 & $<0.010$ \\
\hline $\mathrm{T}$ & AF-04 & 2 & 1 & 3 & t01LM21 & 6.55 & 0.029 & 3.99 & 0.037 & 0.089 & 0.032 & 6.92 & $<0.100$ & 0.031 \\
\hline $\mathrm{T}$ & BC-04 & 2 & 1 & 4 & t05LM21 & 6.52 & 0.033 & 3.98 & 0.035 & 0.096 & 0.031 & 6.90 & $<0.100$ & 0.031 \\
\hline $\mathrm{T}$ & BC-04 & 2 & 1 & 5 & t05LM11 & 6.56 & 0.033 & 4.00 & 0.035 & 0.100 & 0.031 & 7.01 & $<0.100$ & 0.032 \\
\hline $\mathrm{T}$ & AF-01 & 2 & 1 & 6 & t07LM21 & 6.61 & 0.041 & 0.439 & 0.033 & 0.098 & 0.036 & 7.15 & $<0.100$ & 0.027 \\
\hline $\mathrm{T}$ & AB-06 & 2 & 1 & 7 & t08LM11 & 6.02 & 0.025 & 0.408 & 0.031 & 0.085 & 0.035 & 6.39 & $<0.100$ & 0.021 \\
\hline $\mathrm{T}$ & Batch 1 & 2 & 1 & 8 & BCHLM212 & 2.67 & 0.121 & 0.911 & $<0.010$ & 0.075 & 0.303 & 9.37 & 2.62 & $<0.010$ \\
\hline $\mathrm{T}$ & LRM & 2 & 1 & 9 & LRMLM212 & 5.53 & $<0.010$ & 0.371 & $<0.010$ & 0.134 & $<0.010$ & 1.05 & 1.15 & $<0.010$ \\
\hline $\mathrm{T}$ & AB-06 & 2 & 1 & 10 & t08LM21 & 6.12 & 0.026 & 0.425 & 0.033 & 0.087 & 0.050 & 6.47 & $<0.100$ & 0.022 \\
\hline
\end{tabular}


Table A-1. Measured Elemental Concentrations for Glasses Prepared Using Lithium Metaborate (part 1) (continued)

\begin{tabular}{|c|c|c|c|c|c|c|c|c|c|c|c|c|c|c|}
\hline Series & Glass ID & Block & Sub-Blk & Sequence & Lab ID & Al (wt \%) & Ba (wt \%) & Ca (wt \%) & Ce (wt \%) & Cr (wt \%) & Cu (wt \%) & $\mathrm{Fe}$ (wt \%) & K (wt \%) & La (wt \%) \\
\hline $\mathrm{T}$ & AF-04 & 2 & 1 & 11 & t01LM11 & 6.75 & 0.030 & 4.12 & 0.038 & 0.092 & 0.033 & 7.09 & $<<0.100$ & 0.032 \\
\hline $\mathrm{T}$ & AF-03 & 2 & 1 & 12 & t09LM11 & 6.68 & 0.040 & 0.445 & 0.036 & 0.096 & 0.044 & 7.05 & $<0.100$ & 0.027 \\
\hline $\mathrm{T}$ & AF-01 & 2 & 1 & 13 & t07LM11 & 6.56 & 0.042 & 0.442 & 0.034 & 0.097 & 0.036 & 7.08 & $<0.100$ & 0.028 \\
\hline $\mathrm{T}$ & AF-03 & 2 & 1 & 14 & t09LM21 & 6.57 & 0.041 & 0.455 & 0.036 & 0.097 & 0.040 & 6.92 & $<0.100$ & 0.027 \\
\hline $\mathrm{T}$ & Batch 1 & 2 & 1 & 15 & BCHLM213 & 2.62 & 0.125 & 0.879 & $<0.010$ & 0.078 & 0.313 & 9.21 & 2.73 & $<0.010$ \\
\hline $\mathrm{T}$ & LRM & 2 & 1 & 16 & LRMLM213 & 5.40 & $<0.010$ & 0.359 & $<0.010$ & 0.138 & $<0.010$ & 1.02 & 1.19 & $<0.010$ \\
\hline $\mathrm{T}$ & Batch 1 & 2 & 2 & 1 & BCHLM 221 & 2.65 & 0.123 & 0.950 & $<0.010$ & 0.076 & 0.305 & 9.22 & 2.60 & $<0.010$ \\
\hline $\mathrm{T}$ & LRM & 2 & 2 & 2 & LRMLM221 & 5.35 & $<0.010$ & 0.422 & $<0.010$ & 0.137 & $<0.010$ & 1.05 & 1.15 & $<0.010$ \\
\hline $\mathrm{T}$ & AB-06 & 2 & 2 & 3 & t08LM22 & 5.82 & 0.028 & 0.464 & 0.032 & 0.086 & 0.051 & 6.23 & $<0.100$ & 0.023 \\
\hline $\mathrm{T}$ & AF-04 & 2 & 2 & 4 & t01LM22 & 6.30 & 0.030 & 3.88 & 0.036 & 0.088 & 0.032 & 6.69 & $<0.100$ & 0.031 \\
\hline $\mathrm{T}$ & AB-06 & 2 & 2 & 5 & t08LM12 & 5.87 & 0.027 & 0.463 & 0.032 & 0.087 & 0.037 & 6.29 & $<0.100$ & 0.023 \\
\hline $\mathrm{T}$ & BC-04 & 2 & 2 & 6 & t05LM12 & 6.51 & 0.035 & 4.04 & 0.035 & 0.102 & 0.033 & 6.97 & $<0.100$ & 0.033 \\
\hline $\mathrm{T}$ & $\mathrm{AF}-01$ & 2 & 2 & 7 & t07LM22 & 6.45 & 0.043 & 0.495 & 0.033 & 0.100 & 0.037 & 7.02 & $<0.100$ & 0.029 \\
\hline $\mathrm{T}$ & Batch 1 & 2 & 2 & 8 & BCHLM 222 & 2.55 & 0.124 & 0.903 & $<0.010$ & 0.077 & 0.306 & 8.90 & 2.64 & $<0.010$ \\
\hline $\mathrm{T}$ & LRM & 2 & 2 & 9 & LRMLM222 & 5.24 & $<0.010$ & 0.412 & $<0.010$ & 0.136 & $<0.010$ & 1.04 & 1.16 & $<0.010$ \\
\hline $\mathrm{T}$ & AF-03 & 2 & 2 & 10 & t09LM12 & 6.47 & 0.040 & 0.498 & 0.034 & 0.095 & 0.044 & 6.84 & $<0.100$ & 0.027 \\
\hline $\mathrm{T}$ & AF-04 & 2 & 2 & 11 & t01LM12 & 6.38 & 0.031 & 3.95 & 0.037 & 0.091 & 0.033 & 6.79 & $<0.100$ & 0.032 \\
\hline $\mathrm{T}$ & AF-03 & 2 & 2 & 12 & t09LM22 & 6.48 & 0.041 & 0.518 & 0.035 & 0.096 & 0.039 & 6.84 & $<0.100$ & 0.027 \\
\hline $\mathrm{T}$ & BC-04 & 2 & 2 & 13 & t05LM22 & 6.38 & 0.035 & 3.97 & 0.034 & 0.096 & 0.032 & 6.74 & $<0.100$ & 0.032 \\
\hline $\mathrm{T}$ & AF-01 & 2 & 2 & 14 & t07LM12 & 6.55 & 0.043 & 0.513 & 0.033 & 0.096 & 0.036 & 7.07 & $<0.100$ & 0.028 \\
\hline $\mathrm{T}$ & Batch 1 & 2 & 2 & 15 & BCHLM 223 & 2.64 & 0.123 & 0.943 & $<0.010$ & 0.076 & 0.305 & 9.13 & 2.59 & $<0.010$ \\
\hline $\mathrm{T}$ & LRM & 2 & 2 & 16 & LRMLM223 & 5.34 & $<0.010$ & 0.421 & $<0.010$ & 0.136 & $<0.010$ & 1.05 & 1.15 & $<0.010$ \\
\hline $\mathrm{U}$ & Batch 1 & 1 & 1 & 1 & BCHLM111 & 2.50 & 0.125 & 0.825 & $<0.010$ & 0.076 & 0.313 & 8.80 & 2.71 & $<0.010$ \\
\hline $\mathrm{U}$ & LRM & 1 & 1 & 2 & LRMLM111 & 5.04 & $<0.010$ & 0.333 & $<0.010$ & 0.137 & $<0.010$ & 0.99 & 1.20 & $<0.010$ \\
\hline $\mathrm{U}$ & BC-09 & 1 & 1 & 3 & u10LM21 & 6.13 & 0.035 & 2.07 & 0.034 & 0.093 & 0.058 & 6.52 & $<0.100$ & 0.027 \\
\hline $\mathrm{U}$ & BS-05 & 1 & 1 & 4 & u04LM11 & 6.29 & 0.032 & 1.25 & 0.038 & 0.103 & 0.040 & 6.60 & $<0.100$ & 0.028 \\
\hline $\mathrm{U}$ & BS-01 & 1 & 1 & 5 & u02LM11 & 6.22 & 0.037 & 0.440 & 0.038 & 0.092 & 0.038 & 6.67 & $<0.100$ & 0.026 \\
\hline $\mathrm{U}$ & BC-05 & 1 & 1 & 6 & u03LM11 & 6.30 & 0.032 & 1.25 & 0.038 & 0.103 & 0.047 & 6.62 & $<0.100$ & 0.028 \\
\hline $\mathrm{U}$ & BS-02 & 1 & 1 & 7 & u06LM21 & 6.29 & 0.036 & 2.50 & 0.037 & 0.087 & 0.038 & 6.75 & $<0.100$ & 0.029 \\
\hline $\mathrm{U}$ & Batch 1 & 1 & 1 & 8 & BCHLM112 & 2.56 & 0.125 & 0.837 & $<0.010$ & 0.077 & 0.316 & 8.74 & 2.75 & $<0.010$ \\
\hline $\mathrm{U}$ & LRM & 1 & 1 & 9 & LRMLM112 & 5.16 & $<0.010$ & 0.340 & $<0.010$ & 0.138 & $<0.010$ & 1.08 & 1.21 & $<0.010$ \\
\hline $\mathrm{U}$ & BS-05 & 1 & 1 & 10 & u04LM21 & 6.29 & 0.031 & 1.25 & 0.038 & 0.104 & 0.042 & 6.61 & $<0.100$ & 0.028 \\
\hline $\mathrm{U}$ & BS-02 & 1 & 1 & 11 & u06LM11 & 6.62 & 0.035 & 2.63 & 0.036 & 0.086 & 0.047 & 7.11 & $<0.100$ & 0.028 \\
\hline $\mathrm{U}$ & BS-01 & 1 & 1 & 12 & u02LM21 & 7.12 & 0.039 & 0.447 & 0.039 & 0.097 & 0.038 & 7.65 & $<0.100$ & 0.027 \\
\hline $\mathrm{U}$ & BC-09 & 1 & 1 & 13 & u10LM11 & 6.24 & 0.036 & 2.11 & 0.035 & 0.094 & 0.045 & 6.62 & $<0.100$ & 0.028 \\
\hline $\mathrm{U}$ & BC-05 & 1 & 1 & 14 & u03LM21 & 6.61 & 0.035 & 3.95 & 0.037 & 0.096 & 0.034 & 6.87 & $<0.100$ & 0.032 \\
\hline $\mathrm{U}$ & Batch 1 & 1 & 1 & 15 & BCHLM113 & 2.52 & 0.130 & 0.878 & $<0.010$ & 0.079 & 0.329 & 8.85 & 2.89 & $<0.010$ \\
\hline $\mathrm{U}$ & LRM & 1 & 1 & 16 & LRMLM113 & 5.07 & $<0.010$ & 0.354 & $<0.010$ & 0.143 & $<0.010$ & 1.00 & 1.28 & $<0.010$ \\
\hline $\mathrm{U}$ & Batch 1 & 1 & 2 & 1 & BCHLM121 & 2.57 & 0.123 & 0.823 & $<0.010$ & 0.075 & 0.310 & 8.93 & 2.71 & $<0.010$ \\
\hline $\mathrm{U}$ & LRM & 1 & 2 & 2 & LRMLM121 & 5.16 & $<0.010$ & 0.333 & $<0.010$ & 0.136 & $<0.010$ & 1.09 & 1.19 & $<0.010$ \\
\hline $\mathrm{U}$ & BS-02 & 1 & 2 & 3 & u06LM22 & 6.41 & 0.033 & 2.60 & 0.034 & 0.084 & 0.045 & 6.86 & $<0.100$ & 0.027 \\
\hline $\mathrm{U}$ & BS-02 & 1 & 2 & 4 & u06LM12 & 6.31 & 0.032 & 2.55 & 0.033 & 0.084 & 0.035 & 6.80 & $<0.100$ & 0.026 \\
\hline $\mathrm{U}$ & BC-05 & 1 & 2 & 5 & u03LM12 & 6.40 & 0.029 & 1.31 & 0.035 & 0.102 & 0.044 & 6.76 & $<0.100$ & 0.026 \\
\hline
\end{tabular}


Table A-1. Measured Elemental Concentrations for Glasses Prepared Using Lithium Metaborate (part 1) (continued)

\begin{tabular}{|c|c|c|c|c|c|c|c|c|c|c|c|c|c|c|}
\hline Series & Glass ID & Block & Sub-Blk & Sequence & Lab ID & Al (wt \%) & Ba (wt \%) & Ca (wt \%) & Ce (wt \%) & Cr (wt \%) & Cu (wt \%) & $\mathrm{Fe}$ (wt \%) & K (wt \%) & La (wt \%) \\
\hline $\mathrm{U}$ & BS-05 & 1 & 2 & 6 & u04LM12 & 6.52 & 0.030 & 1.34 & 0.036 & 0.103 & 0.038 & 6.86 & $<<0.100$ & 0.026 \\
\hline $\mathrm{U}$ & BS-01 & 1 & 2 & 7 & u02LM12 & 6.41 & 0.035 & 0.434 & 0.036 & 0.092 & 0.037 & 6.92 & $<0.100$ & 0.024 \\
\hline $\mathrm{U}$ & Batch 1 & 1 & 2 & 8 & BCHLM122 & 2.66 & 0.125 & 0.837 & $<0.010$ & 0.076 & 0.316 & 9.22 & 2.76 & $<0.010$ \\
\hline $\mathrm{U}$ & LRM & 1 & 2 & 9 & LRMLM122 & 5.28 & $<0.010$ & 0.335 & $<0.010$ & 0.137 & $<0.010$ & 1.18 & 1.21 & $<0.010$ \\
\hline $\mathrm{U}$ & BS-01 & 1 & 2 & 10 & u02LM22 & 6.49 & 0.036 & 0.436 & 0.036 & 0.096 & 0.037 & 7.00 & $<0.100$ & 0.025 \\
\hline $\mathrm{U}$ & BC-09 & 1 & 2 & 11 & u10LM22 & 6.42 & 0.034 & 2.20 & 0.033 & 0.094 & 0.058 & 6.84 & $<0.100$ & 0.026 \\
\hline $\mathrm{U}$ & BC-05 & 1 & 2 & 12 & u03LM22 & 6.45 & 0.032 & 3.87 & 0.034 & 0.095 & 0.032 & 6.74 & $<0.100$ & 0.029 \\
\hline $\mathrm{U}$ & BC-09 & 1 & 2 & 13 & u10LM12 & 6.34 & 0.033 & 2.17 & 0.033 & 0.092 & 0.043 & 6.76 & $<0.100$ & 0.026 \\
\hline $\mathrm{U}$ & BS-05 & 1 & 2 & 14 & u04LM22 & 6.40 & 0.030 & 1.31 & 0.036 & 0.105 & 0.042 & 6.79 & $<0.100$ & 0.027 \\
\hline $\mathrm{U}$ & Batch 1 & 1 & 2 & 15 & BCHLM123 & 2.64 & 0.126 & 0.849 & $<0.010$ & 0.077 & 0.319 & 9.08 & 2.78 & $<0.010$ \\
\hline $\mathrm{U}$ & LRM & 1 & 2 & 16 & LRMLM123 & 5.27 & $<0.010$ & 0.342 & $<0.010$ & 0.139 & $<0.010$ & 1.07 & 1.22 & $<0.010$ \\
\hline $\mathrm{U}$ & Batch 1 & 2 & 1 & 1 & BCHLM211 & 2.50 & 0.127 & 0.823 & $<0.010$ & 0.077 & 0.311 & 8.80 & 2.70 & $<0.010$ \\
\hline $\mathrm{U}$ & LRM & 2 & 1 & 2 & LRMLM211 & 5.15 & $<0.010$ & 0.333 & $<0.010$ & 0.137 & $<0.010$ & 1.08 & $\begin{array}{l}1.19 \\
\end{array}$ & $<0.010$ \\
\hline $\mathrm{U}$ & BC-08 & 2 & 1 & 3 & u01LM21 & 6.25 & 0.036 & 3.80 & 0.035 & 0.103 & 0.038 & 6.81 & $<0.100$ & 0.030 \\
\hline $\mathrm{U}$ & BC-06 & 2 & 1 & 4 & u05LM21 & 6.27 & 0.043 & 0.442 & 0.036 & 0.092 & 0.036 & 6.65 & $<0.100$ & 0.027 \\
\hline $\mathrm{U}$ & BC-06 & 2 & 1 & 5 & u05LM11 & 6.26 & 0.044 & 0.439 & 0.036 & 0.094 & 0.045 & 6.64 & $<0.100$ & 0.028 \\
\hline $\mathrm{U}$ & BC-07 & 2 & 1 & 6 & u07LM21 & 6.36 & 0.038 & 3.86 & 0.037 & 0.097 & 0.043 & 6.92 & $<0.100$ & 0.030 \\
\hline $\mathrm{U}$ & BS-04 & 2 & 1 & 7 & u08LM11 & 6.37 & 0.041 & 2.56 & 0.038 & 0.116 & 0.039 & 6.86 & $<0.100$ & 0.033 \\
\hline $\mathrm{U}$ & Batch 1 & 2 & 1 & 8 & BCHLM212 & 2.60 & 0.128 & 0.842 & $<0.010$ & 0.078 & 0.318 & 9.12 & 2.75 & $<0.010$ \\
\hline $\mathrm{U}$ & LRM & 2 & 1 & 9 & LRMLM212 & 5.22 & $<0.010$ & 0.342 & $<0.010$ & 0.140 & $<0.010$ & 1.04 & 1.22 & $<0.010$ \\
\hline $\mathrm{U}$ & BS-04 & 2 & 1 & 10 & u08LM21 & 6.40 & 0.041 & 2.60 & 0.038 & 0.114 & 0.038 & 6.89 & $<0.100$ & 0.032 \\
\hline $\mathrm{U}$ & BC-08 & 2 & 1 & 11 & u01LM11 & 6.39 & 0.036 & 3.93 & 0.036 & 0.102 & 0.036 & 6.92 & $<0.100$ & 0.030 \\
\hline $\mathrm{U}$ & BS-03 & 2 & 1 & 12 & u09LM11 & 6.32 & 0.033 & 0.425 & 0.034 & 0.097 & 0.039 & 7.07 & $<0.100$ & 0.024 \\
\hline $\mathrm{U}$ & BC-07 & 2 & 1 & 13 & u07LM11 & 6.43 & 0.038 & 3.88 & 0.036 & 0.096 & 0.038 & 7.50 & $<0.100$ & 0.029 \\
\hline $\mathrm{U}$ & BS-03 & 2 & 1 & 14 & u09LM21 & 6.37 & 0.034 & 0.447 & 0.034 & 0.100 & 0.051 & 7.33 & $<0.100$ & 0.024 \\
\hline $\mathrm{U}$ & Batch 1 & 2 & 1 & 15 & BCHLM213 & 2.65 & 0.126 & 0.827 & $<0.010$ & 0.077 & 0.312 & 9.20 & 2.67 & $<0.010$ \\
\hline $\mathrm{U}$ & LRM & 2 & 1 & 16 & LRMLM213 & 5.23 & $<0.010$ & 0.341 & $<0.010$ & 0.139 & $<0.010$ & 1.04 & 1.20 & $<0.010$ \\
\hline $\mathrm{U}$ & Batch 1 & 2 & 2 & 1 & BCHLM221 & 2.58 & 0.124 & 0.819 & $<0.010$ & 0.075 & 0.309 & 8.89 & 2.68 & $<0.010$ \\
\hline $\mathrm{U}$ & LRM & 2 & 2 & 2 & LRMLM221 & 5.18 & $<0.010$ & 0.334 & $<0.010$ & 0.137 & $<0.010$ & 1.03 & 1.20 & $<0.010$ \\
\hline $\mathrm{U}$ & BS-04 & 2 & 2 & 3 & u08LM22 & 6.30 & 0.039 & 2.54 & 0.036 & 0.112 & 0.036 & 6.78 & $<0.100$ & 0.030 \\
\hline $\mathrm{U}$ & BC-08 & 2 & 2 & 4 & u01LM22 & 6.22 & 0.035 & 3.77 & 0.034 & 0.102 & 0.036 & 6.74 & $<0.100$ & 0.028 \\
\hline $\mathrm{U}$ & BS-04 & 2 & 2 & 5 & u08LM12 & 6.28 & 0.040 & 2.52 & 0.037 & 0.114 & 0.037 & 6.76 & $<0.100$ & 0.030 \\
\hline $\mathrm{U}$ & BC-06 & 2 & 2 & 6 & u05LM12 & 6.28 & 0.043 & 0.442 & 0.035 & 0.093 & 0.043 & 6.66 & $<0.100$ & 0.026 \\
\hline $\mathrm{U}$ & BC-07 & 2 & 2 & 7 & u07LM22 & 6.27 & 0.037 & 3.80 & 0.035 & 0.096 & 0.041 & 6.82 & $<0.100$ & 0.028 \\
\hline $\mathrm{U}$ & Batch 1 & 2 & 2 & 8 & BCHLM222 & 2.56 & 0.125 & 0.831 & $<0.010$ & 0.076 & 0.312 & 8.81 & 2.73 & $<0.010$ \\
\hline $\mathrm{U}$ & LRM & 2 & 2 & 9 & LRMLM222 & 5.17 & $<0.010$ & 0.340 & $<0.010$ & 0.138 & $<0.010$ & 1.11 & 1.22 & $<0.010$ \\
\hline $\mathrm{U}$ & BS- 03 & 2 & 2 & 10 & u09LM12 & 6.25 & 0.032 & 0.421 & 0.033 & 0.096 & 0.037 & 6.99 & $<0.100$ & 0.022 \\
\hline $\mathrm{U}$ & BC-08 & 2 & 2 & 11 & u01LM12 & 6.32 & 0.035 & 3.87 & 0.035 & 0.100 & 0.034 & 6.86 & $<0.100$ & 0.028 \\
\hline $\mathrm{U}$ & BS-03 & 2 & 2 & 12 & u09LM22 & 6.31 & 0.033 & 0.455 & 0.033 & 0.099 & 0.050 & 7.22 & $<0.100$ & 0.023 \\
\hline $\mathrm{U}$ & BC-06 & 2 & 2 & 13 & $\mathrm{u} 05 \mathrm{LM} 22$ & 6.30 & 0.042 & 0.446 & 0.035 & 0.091 & 0.034 & 6.66 & $<0.100$ & 0.025 \\
\hline $\mathrm{U}$ & BC-07 & 2 & 2 & 14 & u07LM12 & 6.31 & 0.036 & 3.79 & 0.035 & 0.095 & 0.036 & 7.35 & $<0.100$ & 0.028 \\
\hline $\mathrm{U}$ & Batch 1 & 2 & 2 & 15 & BCHLM 223 & 2.54 & 0.126 & 0.834 & $<0.010$ & 0.076 & 0.314 & 8.91 & 2.77 & $<0.010$ \\
\hline $\mathrm{U}$ & LRM & 2 & 2 & 16 & LRMLM223 & 5.28 & $<0.010$ & 0.344 & $<0.010$ & 0.138 & $<0.010$ & 1.13 & 1.24 & $<0.010$ \\
\hline
\end{tabular}


Table A-1. Measured Elemental Concentrations for Glasses Prepared Using Lithium Metaborate (part 1) (continued)

\begin{tabular}{|c|c|c|c|c|c|c|c|c|c|c|c|c|c|c|}
\hline Series & Glass ID & Block & Sub-Blk & Sequence & Lab ID & Al (wt \%) & Ba (wt \%) & Ca (wt \%) & Ce (wt \%) & Cr (wt \%) & Cu (wt \%) & $\mathrm{Fe}$ (wt \%) & K (wt \%) & La (wt \%) \\
\hline $\mathrm{V}$ & Batch 1 & 1 & 1 & 1 & BCHLM111 & 2.56 & 0.124 & 0.895 & $<0.010$ & 0.077 & 0.309 & 8.95 & 2.65 & $<0.010$ \\
\hline $\mathrm{V}$ & LRM & 1 & 1 & 2 & LRMLM111 & 5.16 & $<0.010$ & 0.410 & $<0.010$ & 0.130 & $<0.010$ & 1.02 & 1.31 & $<0.010$ \\
\hline $\mathrm{V}$ & LL-06 & 1 & 1 & 3 & v10LM21 & 6.37 & 0.028 & 3.85 & 0.032 & 0.087 & 0.036 & 6.82 & $<0.100$ & 0.025 \\
\hline $\mathrm{V}$ & LL-10 & 1 & 1 & 4 & v04LM11 & 6.26 & 0.028 & 0.438 & 0.034 & 0.086 & 0.031 & 6.61 & $<0.100$ & 0.024 \\
\hline $\mathrm{V}$ & BS-08 & 1 & 1 & 5 & v02LM11 & 6.29 & 0.037 & 0.502 & 0.040 & 0.086 & 0.032 & 6.38 & $<0.100$ & 0.027 \\
\hline $\mathrm{V}$ & LL-05 & 1 & 1 & 6 & v03LM11 & 6.57 & 0.031 & 2.24 & 0.035 & 0.080 & 0.031 & 7.00 & $<0.100$ & 0.025 \\
\hline $\mathrm{V}$ & LL-12 & 1 & 1 & 7 & v06LM21 & 6.61 & 0.026 & 3.99 & 0.033 & 0.080 & 0.027 & 7.00 & $<0.100$ & 0.025 \\
\hline $\mathrm{V}$ & Batch 1 & 1 & 1 & 8 & BCHLM112 & 2.59 & 0.124 & 0.908 & $<0.010$ & 0.077 & 0.309 & 9.03 & 2.64 & $<0.010$ \\
\hline $\mathrm{V}$ & LRM & 1 & 1 & 9 & LRMLM112 & 5.25 & $<0.010$ & 0.412 & $<0.010$ & 0.133 & $<0.010$ & 1.04 & 1.30 & $<0.010$ \\
\hline $\mathrm{V}$ & LL-10 & 1 & 1 & 10 & v04LM21 & 6.22 & 0.028 & 0.429 & 0.034 & 0.085 & 0.029 & 6.60 & $<0.100$ & 0.024 \\
\hline $\mathrm{V}$ & LL-12 & 1 & 1 & 11 & v06LM11 & 6.07 & 0.025 & 3.68 & 0.034 & 0.080 & 0.027 & 6.47 & $<0.100$ & 0.025 \\
\hline $\mathrm{V}$ & BS-08 & 1 & 1 & 12 & $\mathrm{v} 02 \mathrm{LM} 21$ & 6.04 & 0.037 & 0.471 & 0.041 & 0.085 & 0.031 & 6.13 & $<0.100$ & 0.027 \\
\hline $\mathrm{V}$ & LL-06 & 1 & 1 & 13 & v10LM11 & 6.12 & 0.028 & 3.70 & 0.032 & 0.088 & 0.035 & 6.54 & $<0.100$ & 0.024 \\
\hline $\mathrm{V}$ & LL-05 & 1 & 1 & 14 & v03LM21 & 6.58 & 0.031 & 2.20 & 0.034 & 0.081 & 0.031 & 6.99 & $<0.100$ & 0.024 \\
\hline $\mathrm{V}$ & Batch 1 & 1 & 1 & 15 & BCHLM113 & 2.45 & 0.125 & 0.857 & $<0.010$ & 0.078 & 0.313 & 8.49 & 2.70 & $<0.010$ \\
\hline $\mathrm{V}$ & LRM & 1 & 1 & 16 & LRMLM113 & 4.99 & $<0.010$ & 0.389 & $<0.010$ & 0.134 & $<0.010$ & 0.974 & 1.31 & $<0.010$ \\
\hline $\mathrm{V}$ & Batch 1 & 1 & 2 & 1 & BCHLM121 & 2.53 & 0.127 & 0.887 & $<0.010$ & 0.077 & 0.310 & $\begin{array}{l}8.84 \\
\end{array}$ & 2.66 & $<0.010$ \\
\hline $\mathrm{V}$ & LRM & 1 & 2 & 2 & LRMLM121 & 5.10 & $<0.010$ & 0.404 & $<0.010$ & 0.133 & $<0.010$ & 1.00 & 1.28 & $<0.010$ \\
\hline $\mathrm{V}$ & LL-12 & 1 & 2 & 3 & v06LM22 & 6.30 & 0.028 & 3.77 & 0.032 & 0.080 & 0.027 & 6.70 & $<0.100$ & 0.026 \\
\hline $\mathrm{V}$ & LL-12 & 1 & 2 & 4 & v06LM12 & 6.22 & 0.029 & 3.75 & 0.032 & 0.081 & 0.028 & 6.64 & $<0.100$ & 0.026 \\
\hline $\mathrm{V}$ & LL-05 & 1 & 2 & 5 & v03LM12 & 6.29 & 0.034 & 2.13 & 0.033 & 0.081 & 0.032 & 6.75 & $<0.100$ & 0.025 \\
\hline $\mathrm{V}$ & LL-10 & 1 & 2 & 6 & v04LM12 & 6.22 & 0.031 & 0.425 & 0.032 & 0.087 & 0.030 & 6.62 & $<0.100$ & 0.025 \\
\hline $\mathrm{V}$ & BS-08 & 1 & 2 & 7 & v02LM12 & 6.07 & 0.047 & 0.480 & 0.062 & 0.087 & 0.033 & 6.21 & $<0.100$ & 0.054 \\
\hline $\mathrm{V}$ & Batch 1 & 1 & 2 & 8 & BCHLM122 & 2.51 & 0.127 & 0.871 & $<0.010$ & 0.078 & 0.311 & 8.84 & 2.68 & $<0.010$ \\
\hline $\mathrm{V}$ & LRM & 1 & 2 & 9 & LRMLM122 & 5.09 & $<0.010$ & 0.395 & $<0.010$ & 0.134 & $<0.010$ & 1.01 & 1.30 & $<0.010$ \\
\hline $\mathrm{V}$ & BS-08 & 1 & 2 & 10 & v02LM22 & 6.15 & 0.040 & 0.475 & 0.040 & 0.086 & 0.032 & 6.30 & $<0.100$ & 0.028 \\
\hline $\mathrm{V}$ & LL-06 & 1 & 2 & 11 & v10LM22 & 6.34 & 0.031 & 3.75 & 0.031 & 0.088 & 0.038 & 6.85 & $<0.100$ & 0.026 \\
\hline $\mathrm{V}$ & LL-05 & 1 & 2 & 12 & v03LM22 & 6.20 & 0.034 & 2.04 & 0.032 & 0.081 & 0.032 & 6.72 & $<0.100$ & 0.026 \\
\hline $\mathrm{V}$ & LL-06 & 1 & 2 & 13 & $\begin{array}{l}\text { v10LM12 } \\
\end{array}$ & 6.30 & 0.030 & 3.70 & 0.030 & 0.088 & 0.036 & 6.85 & $<0.100$ & 0.025 \\
\hline $\mathrm{V}$ & LL-10 & 1 & 2 & 14 & v04LM22 & 6.10 & 0.031 & 0.410 & 0.032 & 0.085 & 0.030 & 6.60 & $<0.100$ & 0.025 \\
\hline $\mathrm{V}$ & Batch 1 & 1 & 2 & 15 & BCHLM123 & 2.51 & 0.128 & 0.860 & $<0.010$ & 0.077 & 0.313 & 8.91 & 2.68 & $<0.010$ \\
\hline $\mathrm{V}$ & LRM & 1 & 2 & 16 & LRMLM123 & 5.06 & $<0.010$ & 0.387 & $<0.010$ & 0.134 & $<0.010$ & 1.04 & 1.31 & $<0.010$ \\
\hline $\mathrm{V}$ & Batch 1 & 2 & 1 & 1 & BCHLM211 & 2.49 & 0.125 & 0.832 & $<0.010$ & 0.077 & 0.311 & 8.81 & 2.66 & $<0.010$ \\
\hline $\mathrm{V}$ & LRM & 2 & 1 & 2 & LRMLM211 & 5.14 & $<0.010$ & 0.355 & $<0.010$ & 0.134 & $<0.010$ & 0.98 & 1.30 & $<0.010$ \\
\hline $\mathrm{V}$ & LL-11 & 2 & 1 & 3 & v01LM21 & 6.26 & 0.033 & 3.76 & 0.034 & 0.099 & 0.029 & 6.90 & $<0.100$ & 0.029 \\
\hline $\mathrm{V}$ & BS-09 & 2 & 1 & 4 & v05LM21 & 6.14 & 0.032 & 2.54 & 0.041 & 0.107 & 0.036 & 6.69 & $<0.100$ & 0.029 \\
\hline $\mathrm{V}$ & BS-09 & 2 & 1 & 5 & v05LM11 & 6.15 & 0.033 & 2.53 & 0.035 & 0.108 & 0.037 & 6.67 & $<0.100$ & 0.029 \\
\hline $\mathrm{V}$ & BS-07 & 2 & 1 & 6 & v07LM21 & 6.26 & 0.037 & 2.57 & 0.037 & 0.099 & 0.034 & 6.72 & $<0.100$ & 0.030 \\
\hline $\mathrm{V}$ & $\begin{array}{l}\text { LL-03 } \\
\end{array}$ & 2 & 1 & 7 & v08LM11 & 4.73 & 0.021 & 4.30 & 0.029 & 0.078 & 0.036 & 5.10 & $<0.100$ & 0.025 \\
\hline $\mathrm{V}$ & Batch 1 & 2 & 1 & 8 & BCHLM212 & 2.57 & 0.125 & 0.859 & $<0.010$ & 0.077 & 0.313 & 9.19 & 2.70 & $<0.010$ \\
\hline $\mathrm{V}$ & LRM & 2 & 1 & 9 & LRMLM212 & 5.32 & $<0.010$ & 0.369 & $<0.010$ & 0.134 & $<0.010$ & 1.02 & 1.33 & $<0.010$ \\
\hline $\mathrm{V}$ & LL-03 & 2 & 1 & 10 & v08LM21 & 4.86 & 0.021 & 4.42 & 0.029 & 0.078 & 0.031 & 5.25 & $<0.100$ & 0.025 \\
\hline $\mathrm{V}$ & LL-11 & 2 & 1 & 11 & v01LM11 & 6.49 & 0.033 & 3.90 & 0.034 & 0.099 & 0.029 & 7.15 & $<0.100$ & 0.028 \\
\hline
\end{tabular}


Table A-1. Measured Elemental Concentrations for Glasses Prepared Using Lithium Metaborate (part 1) (continued)

\begin{tabular}{|c|c|c|c|c|c|c|c|c|c|c|c|c|c|c|}
\hline Series & Glass ID & Block & Sub-Blk & Sequence & Lab ID & Al (wt \%) & Ba (wt \%) & Ca (wt \%) & Ce (wt \%) & Cr (wt \%) & Cu (wt \%) & $\mathrm{Fe}$ (wt \%) & K (wt \%) & La (wt \%) \\
\hline $\mathrm{V}$ & BS-06 & 2 & 1 & 12 & v09LM11 & 6.28 & 0.029 & 0.427 & 0.037 & 0.100 & 0.032 & 6.44 & $<<0.100$ & 0.024 \\
\hline $\mathrm{V}$ & BS-07 & 2 & 1 & 13 & v07LM11 & 6.34 & 0.036 & 2.61 & 0.037 & 0.098 & 0.036 & 6.87 & $<0.100$ & 0.029 \\
\hline $\mathrm{V}$ & BS-06 & 2 & 1 & 14 & v09LM21 & 6.54 & 0.030 & 0.448 & 0.037 & 0.100 & 0.031 & 6.70 & $<0.100$ & 0.025 \\
\hline $\mathrm{V}$ & Batch 1 & 2 & 1 & 15 & BCHLM213 & 2.50 & 0.123 & 0.839 & $<0.010$ & 0.076 & 0.307 & 8.96 & 2.63 & $<0.010$ \\
\hline $\mathrm{V}$ & LRM & 2 & 1 & 16 & LRMLM213 & 5.15 & $<0.010$ & 0.352 & $<0.010$ & 0.132 & $<0.010$ & 0.994 & 1.28 & $<0.010$ \\
\hline $\mathrm{V}$ & Batch 1 & 2 & 2 & 1 & BCHLM221 & 2.45 & 0.124 & 0.866 & $<0.010$ & 0.076 & 0.309 & $\begin{array}{l}8.54 \\
\end{array}$ & 2.67 & $<0.010$ \\
\hline $\mathrm{V}$ & LRM & 2 & 2 & 2 & LRMLM221 & 5.14 & $<0.010$ & 0.418 & $<0.010$ & 0.131 & $<0.010$ & 1.03 & 1.29 & $<0.010$ \\
\hline $\mathrm{V}$ & LL-03 & 2 & 2 & 3 & v08LM22 & 4.66 & 0.020 & 4.28 & 0.028 & 0.077 & 0.030 & 4.97 & $<0.100$ & 0.025 \\
\hline $\mathrm{V}$ & LL-11 & 2 & 2 & 4 & v01LM22 & 6.25 & 0.032 & 3.81 & 0.033 & 0.097 & 0.028 & 6.84 & $<0.100$ & 0.028 \\
\hline $\mathrm{V}$ & LL-03 & 2 & 2 & 5 & v08LM12 & 4.65 & 0.020 & 4.28 & 0.029 & 0.077 & 0.034 & 4.96 & $<0.100$ & 0.024 \\
\hline $\mathrm{V}$ & BS-09 & 2 & 2 & 6 & v05LM12 & 6.16 & 0.032 & 2.60 & 0.035 & 0.106 & 0.035 & 6.63 & $<0.100$ & 0.029 \\
\hline $\mathrm{V}$ & BS-07 & 2 & 2 & 7 & $\mathrm{v} 07 \mathrm{LM} 22$ & 6.20 & 0.036 & 2.61 & 0.037 & 0.098 & 0.033 & 6.62 & $<0.100$ & 0.029 \\
\hline $\mathrm{V}$ & Batch 1 & 2 & 2 & 8 & BCHLM222 & 2.55 & 0.124 & 0.903 & $<0.010$ & 0.075 & 0.309 & 8.89 & 2.67 & $<0.010$ \\
\hline $\mathrm{V}$ & LRM & 2 & 2 & 9 & LRMLM222 & 5.14 & $<0.010$ & 0.418 & $<0.010$ & 0.132 & $<0.010$ & 1.03 & 1.30 & $<0.010$ \\
\hline $\mathrm{V}$ & BS-06 & 2 & 2 & 10 & v09LM12 & 6.29 & 0.029 & 0.498 & 0.038 & 0.100 & 0.031 & 6.37 & $<0.100$ & 0.025 \\
\hline $\mathrm{V}$ & LL-11 & 2 & 2 & 11 & v01LM12 & 6.40 & 0.032 & 3.89 & 0.034 & 0.098 & 0.028 & 6.99 & $<0.100$ & 0.029 \\
\hline $\mathrm{V}$ & BS-06 & 2 & 2 & 12 & v09LM22 & 6.44 & 0.030 & 0.502 & 0.038 & 0.100 & 0.030 & 6.54 & $<0.100$ & 0.025 \\
\hline $\mathrm{V}$ & BS-09 & 2 & 2 & 13 & v05LM22 & 6.31 & 0.032 & 2.67 & 0.040 & 0.106 & 0.035 & 6.84 & $<0.100$ & 0.029 \\
\hline $\mathrm{V}$ & BS-07 & 2 & 2 & 14 & v07LM12 & 6.23 & 0.036 & 2.62 & 0.037 & 0.097 & 0.035 & 6.86 & $<0.100$ & 0.030 \\
\hline $\mathrm{V}$ & Batch 1 & 2 & 2 & 15 & BCHLM223 & 2.60 & 0.124 & 0.918 & $<0.010$ & 0.075 & 0.310 & 9.02 & 2.70 & $<0.010$ \\
\hline $\mathrm{V}$ & LRM & 2 & 2 & 16 & LRMLM223 & 5.24 & $<0.010$ & 0.423 & $<0.010$ & 0.132 & $<0.010$ & 1.05 & 1.31 & $<0.010$ \\
\hline $\mathrm{W}$ & Batch 1 & 1 & 1 & 1 & BCHLM111 & 2.61 & 0.122 & 0.858 & $<0.010$ & 0.075 & 0.301 & 8.70 & 2.78 & $<0.010$ \\
\hline $\mathrm{W}$ & LRM & 1 & 1 & 2 & LRMLM111 & 5.07 & $<0.010$ & 0.432 & $<0.010$ & 0.133 & $<0.010$ & 1.19 & 1.29 & $<0.010$ \\
\hline $\mathrm{W}$ & LT-12 & 1 & 1 & 3 & w10LM21 & 6.30 & 0.029 & 2.51 & 0.031 & 0.094 & 0.033 & 6.68 & $<0.100$ & 0.027 \\
\hline $\mathrm{W}$ & LT-01 & 1 & 1 & 4 & w04LM11 & 4.68 & 0.029 & 0.353 & 0.028 & 0.069 & 0.028 & 4.89 & $<0.100$ & 0.020 \\
\hline $\mathrm{W}$ & LT-11 & 1 & 1 & 5 & w02LM11 & 6.22 & 0.028 & 0.416 & 0.036 & 0.085 & 0.033 & 6.83 & $<0.100$ & 0.024 \\
\hline $\mathrm{W}$ & LT-10 & 1 & 1 & 6 & w03LM11 & 6.25 & 0.027 & 2.52 & 0.034 & 0.146 & 0.033 & 6.68 & $<0.100$ & 0.026 \\
\hline $\mathrm{W}$ & QB-03 & 1 & 1 & 7 & w06LM21 & 4.55 & 0.024 & 4.73 & 0.030 & 0.167 & 0.036 & 5.31 & 0.201 & 0.023 \\
\hline $\mathrm{W}$ & Batch 1 & 1 & 1 & 8 & BCHLM112 & 2.58 & 0.126 & 0.854 & $<0.010$ & 0.077 & 0.316 & 8.60 & 2.73 & $<0.010$ \\
\hline $\mathrm{W}$ & LRM & 1 & 1 & 9 & LRMLM112 & 5.04 & $<0.010$ & 0.430 & $<0.010$ & 0.134 & $<0.010$ & 1.14 & 1.31 & $<0.010$ \\
\hline $\mathrm{W}$ & LT-01 & 1 & 1 & 10 & w04LM21 & 4.62 & 0.030 & 0.351 & 0.028 & 0.071 & 0.030 & 4.81 & $<0.100$ & 0.021 \\
\hline $\mathrm{W}$ & QB-03 & 1 & 1 & 11 & w06LM11 & 4.69 & 0.025 & 4.83 & 0.030 & 0.172 & 0.039 & 5.42 & 0.204 & 0.024 \\
\hline $\mathrm{W}$ & LT-11 & 1 & 1 & 12 & w02LM21 & 6.29 & 0.028 & 0.440 & 0.035 & 0.088 & 0.037 & 6.90 & $<0.100$ & 0.025 \\
\hline W & LT-12 & 1 & 1 & 13 & w10LM11 & 6.27 & 0.030 & 2.52 & 0.032 & 0.095 & 0.037 & 6.65 & $<0.100$ & 0.027 \\
\hline $\mathrm{W}$ & $\begin{array}{l}\text { LT-10 } \\
\end{array}$ & 1 & 1 & 14 & w03LM21 & 6.16 & 0.025 & 2.47 & 0.034 & 0.140 & 0.030 & 6.57 & $<0.100$ & 0.026 \\
\hline $\mathrm{W}$ & Batch 1 & 1 & 1 & 15 & BCHLM113 & 2.58 & 0.126 & 0.849 & $<0.010$ & 0.077 & 0.313 & 8.56 & 2.75 & $<0.010$ \\
\hline $\mathrm{W}$ & LRM & 1 & 1 & 16 & LRMLM113 & 5.10 & $<0.010$ & 0.435 & $<0.010$ & 0.135 & $<0.010$ & 1.16 & 1.30 & $<0.010$ \\
\hline $\mathrm{W}$ & Batch 1 & 1 & 2 & 1 & BCHLM121 & 2.69 & 0.124 & 0.904 & $<0.010$ & 0.076 & 0.307 & 9.04 & 2.65 & $<0.010$ \\
\hline $\mathrm{W}$ & LRM & 1 & 2 & 2 & LRMLM121 & 5.25 & $<0.010$ & 0.460 & $<0.010$ & 0.132 & $<0.010$ & 1.19 & 1.27 & $<0.010$ \\
\hline $\mathrm{W}$ & QB-03 & 1 & 2 & 3 & w06LM22 & 4.78 & 0.026 & 4.89 & 0.029 & 0.165 & 0.036 & 5.59 & 0.199 & 0.024 \\
\hline $\mathrm{W}$ & $\overline{\text { QB-03 }}$ & 1 & 2 & 4 & w06LM12 & 4.89 & 0.027 & 5.01 & 0.029 & 0.170 & 0.036 & 5.70 & 0.205 & 0.023 \\
\hline $\mathrm{W}$ & LT-10 & 1 & 2 & 5 & w03LM12 & 6.53 & 0.027 & 2.66 & 0.033 & 0.142 & 0.032 & 6.99 & $<0.100$ & 0.026 \\
\hline $\mathrm{W}$ & LT-01 & 1 & 2 & 6 & w04LM12 & 4.85 & 0.031 & 0.379 & 0.027 & 0.070 & 0.028 & 5.05 & $<0.100$ & 0.020 \\
\hline
\end{tabular}


Table A-1. Measured Elemental Concentrations for Glasses Prepared Using Lithium Metaborate (part 1) (continued)

\begin{tabular}{|c|c|c|c|c|c|c|c|c|c|c|c|c|c|c|}
\hline Series & Glass ID & Block & Sub-Blk & Sequence & Lab ID & Al (wt \%) & Ba (wt \%) & Ca (wt \%) & Ce (wt \%) & Cr (wt \%) & Cu (wt \%) & $\mathrm{Fe}$ (wt \%) & K (wt \%) & La (wt \%) \\
\hline $\mathrm{W}$ & LT-11 & 1 & 2 & 7 & w02LM12 & 6.46 & 0.029 & 0.448 & 0.034 & 0.085 & 0.032 & 7.08 & $<0.100$ & 0.024 \\
\hline $\mathrm{W}$ & Batch 1 & 1 & 2 & 8 & BCHLM122 & 2.67 & 0.123 & 0.899 & $<0.010$ & 0.076 & 0.304 & 8.92 & 2.72 & $<0.010$ \\
\hline $\mathrm{W}$ & LRM & 1 & 2 & 9 & LRMLM122 & 5.29 & $<0.010$ & 0.467 & $<0.010$ & 0.132 & $<0.010$ & 1.19 & 1.27 & $<0.010$ \\
\hline $\mathrm{W}$ & LT-11 & 1 & 2 & 10 & w02LM22 & 6.48 & 0.030 & 0.469 & 0.034 & 0.088 & 0.037 & 7.12 & $<0.100$ & 0.025 \\
\hline $\mathrm{W}$ & LT-12 & 1 & 2 & 11 & w10LM22 & 6.50 & 0.031 & 2.64 & 0.030 & 0.094 & 0.033 & 6.88 & $<0.100$ & 0.026 \\
\hline $\mathrm{W}$ & LT-10 & 1 & 2 & 12 & w03LM22 & 6.34 & 0.026 & 2.57 & 0.033 & 0.137 & 0.030 & 6.73 & $<0.100$ & 0.025 \\
\hline $\mathrm{W}$ & LT-12 & 1 & 2 & 13 & w10LM12 & 6.48 & 0.031 & 2.63 & 0.031 & 0.093 & 0.035 & 6.85 & $<0.100$ & 0.026 \\
\hline $\mathrm{W}$ & LT-01 & 1 & 2 & 14 & w04LM22 & 4.76 & 0.031 & 0.373 & 0.027 & 0.071 & 0.030 & 4.96 & $<0.100$ & 0.020 \\
\hline $\mathrm{W}$ & Batch 1 & 1 & 2 & 15 & BCHLM123 & 2.64 & 0.123 & 0.888 & $<0.010$ & 0.075 & 0.304 & 8.77 & 2.83 & $<0.010$ \\
\hline $\mathrm{W}$ & LRM & 1 & 2 & 16 & LRMLM123 & 5.12 & $<0.010$ & 0.451 & $<0.010$ & 0.133 & $<0.010$ & 1.15 & 1.30 & $<0.010$ \\
\hline $\mathrm{W}$ & Batch 1 & 2 & 1 & 1 & BCHLM211 & 2.62 & 0.124 & 0.859 & $<0.010$ & 0.076 & 0.306 & 8.88 & 2.86 & $<0.010$ \\
\hline $\mathrm{W}$ & LRM & 2 & 1 & 2 & LRMLM211 & 5.11 & $<0.010$ & 0.450 & $<0.010$ & 0.132 & $<0.010$ & 1.15 & 1.28 & $<0.010$ \\
\hline $\mathrm{W}$ & $\begin{array}{l}\text { LT-04 } \\
\end{array}$ & 2 & 1 & 3 & w01LM21 & 6.21 & 0.027 & 0.406 & 0.031 & 0.109 & 0.041 & 6.53 & $<0.100$ & 0.023 \\
\hline $\mathrm{W}$ & LOL-12 & 2 & 1 & 4 & w05LM21 & 4.52 & 0.030 & 0.316 & 0.060 & 0.037 & 0.036 & 5.34 & $<0.100$ & 0.025 \\
\hline $\mathrm{W}$ & LOL-12 & 2 & 1 & 5 & w05LM11 & 4.37 & 0.030 & 0.301 & 0.060 & 0.037 & 0.042 & 5.16 & $<0.100$ & 0.025 \\
\hline $\mathrm{W}$ & LT-05 & 2 & 1 & 6 & w07LM21 & 6.21 & 0.028 & 1.227 & 0.035 & 0.097 & 0.036 & 6.58 & $<0.100$ & 0.024 \\
\hline$\overline{\mathrm{W}}$ & QB-04 & 2 & 1 & 7 & w08LM11 & 4.69 & 0.032 & 4.75 & 0.024 & 0.096 & 0.024 & 5.47 & 0.196 & 0.021 \\
\hline $\mathrm{W}$ & Batch 1 & 2 & 1 & 8 & BCHLM212 & 2.59 & 0.124 & 0.851 & $<0.010$ & 0.076 & 0.306 & 8.70 & 2.87 & $<0.010$ \\
\hline $\mathrm{W}$ & LRM & 2 & 1 & 9 & LRMLM212 & 5.12 & $<0.010$ & 0.425 & $<0.010$ & 0.135 & $<0.010$ & 1.14 & 1.32 & $<0.010$ \\
\hline $\mathrm{W}$ & QB-04 & 2 & 1 & 10 & w08LM21 & 4.68 & 0.032 & 4.74 & 0.024 & 0.096 & 0.025 & 5.44 & 0.198 & 0.022 \\
\hline $\mathrm{W}$ & LT-04 & 2 & 1 & 11 & w01LM11 & 6.26 & 0.028 & 0.412 & 0.032 & 0.114 & 0.048 & 6.50 & $<0.100$ & 0.024 \\
\hline $\mathrm{W}$ & LT-06 & 2 & 1 & 12 & w09LM11 & 6.15 & 0.029 & 2.47 & 0.031 & 0.102 & 0.031 & 6.73 & $<0.100$ & 0.027 \\
\hline $\mathrm{W}$ & LT-05 & 2 & 1 & 13 & w07LM11 & 6.13 & 0.029 & 1.205 & 0.035 & 0.097 & 0.032 & 6.46 & $<0.100$ & 0.024 \\
\hline $\mathrm{W}$ & $\begin{array}{l}\text { LT-06 } \\
\end{array}$ & 2 & 1 & 14 & w09LM21 & 6.23 & 0.030 & 2.49 & 0.031 & 0.103 & 0.035 & 6.84 & $<0.100$ & 0.027 \\
\hline $\mathrm{W}$ & Batch 1 & 2 & 1 & 15 & BCHLM213 & 2.57 & 0.128 & 0.846 & $<0.010$ & 0.078 & 0.319 & 8.62 & 2.77 & $<0.010$ \\
\hline $\mathrm{W}$ & LRM & 2 & 1 & 16 & LRMLM213 & 4.99 & $<0.010$ & 0.412 & $<0.010$ & 0.136 & $<0.010$ & 1.11 & 1.32 & $<0.010$ \\
\hline $\mathrm{W}$ & Batch 1 & 2 & 2 & 1 & BCHLM221 & 2.62 & 0.123 & 0.900 & $<0.010$ & 0.075 & 0.307 & 8.68 & 2.66 & $<0.010$ \\
\hline $\mathrm{W}$ & LRM & 2 & 2 & 2 & LRMLM221 & 5.11 & $<0.010$ & 0.482 & $<0.010$ & 0.131 & $<0.010$ & 1.19 & 1.28 & $<0.010$ \\
\hline $\mathrm{W}$ & QB-04 & 2 & 2 & 3 & w08LM22 & 4.73 & 0.030 & 4.79 & 0.023 & 0.094 & 0.023 & 5.52 & 0.193 & 0.020 \\
\hline $\mathrm{W}$ & LT-04 & 2 & 2 & 4 & w01LM22 & 6.31 & 0.026 & 0.472 & 0.031 & 0.109 & 0.038 & 6.64 & $<0.100$ & 0.022 \\
\hline $\mathrm{W}$ & QB-04 & 2 & 2 & 5 & w08LM12 & 4.75 & 0.030 & 4.80 & 0.023 & 0.094 & 0.022 & 5.53 & 0.193 & 0.020 \\
\hline $\mathrm{W}$ & LOL-12 & 2 & 2 & 6 & w05LM12 & 4.62 & 0.028 & 0.382 & 0.059 & 0.036 & 0.041 & 5.46 & $<0.100$ & 0.024 \\
\hline $\mathrm{W}$ & LT-05 & 2 & 2 & 7 & w07LM22 & 6.36 & 0.027 & 1.31 & 0.034 & 0.094 & 0.035 & 6.77 & $<0.100$ & 0.023 \\
\hline W & Batch 1 & 2 & 2 & 8 & BCHLM222 & 2.67 & 0.120 & 0.918 & $<0.010$ & 0.073 & 0.296 & 8.90 & 2.76 & $<0.010$ \\
\hline $\mathrm{W}$ & LRM & 2 & 2 & 9 & LRMLM222 & 5.22 & $<0.010$ & 0.493 & $<0.010$ & 0.131 & $<0.010$ & 1.22 & 1.28 & $<0.010$ \\
\hline $\mathrm{W}$ & LT-06 & 2 & 2 & 10 & w09LM12 & 6.36 & 0.027 & 2.60 & 0.030 & 0.101 & 0.029 & 7.04 & $<0.100$ & 0.026 \\
\hline $\mathrm{W}$ & LT-04 & 2 & 2 & 11 & w01LM12 & 6.56 & 0.026 & 0.498 & 0.031 & 0.110 & 0.046 & 6.86 & $<0.100$ & 0.023 \\
\hline $\mathrm{W}$ & LT-06 & 2 & 2 & 12 & w09LM22 & 6.47 & 0.028 & 2.62 & 0.030 & 0.100 & 0.033 & 7.17 & $<0.100$ & 0.026 \\
\hline $\mathrm{W}$ & LOL-12 & 2 & 2 & 13 & w05LM22 & 4.57 & 0.028 & 0.381 & 0.059 & 0.036 & 0.034 & 5.42 & $<0.100$ & 0.024 \\
\hline $\mathrm{W}$ & LT-05 & 2 & 2 & 14 & w07LM12 & 6.28 & 0.027 & 1.29 & 0.034 & 0.095 & 0.030 & 6.68 & $<0.100$ & 0.023 \\
\hline $\mathrm{W}$ & Batch 1 & 2 & 2 & 15 & BCHLM223 & 2.63 & 0.121 & 0.904 & $<0.010$ & 0.074 & 0.300 & 8.75 & 2.69 & $<0.010$ \\
\hline $\mathrm{W}$ & LRM & 2 & 2 & 16 & LRMLM 223 & 5.24 & $<0.010$ & 0.495 & $<0.010$ & 0.129 & $<0.010$ & 1.22 & 1.26 & $<0.010$ \\
\hline $\mathrm{X}$ & Batch 1 & 1 & 1 & 1 & BCHLM111 & 2.63 & 0.122 & 0.873 & $<0.010$ & 0.074 & 0.299 & 8.84 & 2.78 & $<0.010$ \\
\hline
\end{tabular}


Table A-1. Measured Elemental Concentrations for Glasses Prepared Using Lithium Metaborate (part 1) (continued)

\begin{tabular}{|c|c|c|c|c|c|c|c|c|c|c|c|c|c|c|}
\hline Series & Glass ID & Block & Sub-Blk & Sequence & Lab ID & Al (wt \%) & Ba (wt \%) & Ca (wt \%) & Ce (wt \%) & Cr (wt \%) & Cu (wt \%) & $\mathrm{Fe}$ (wt \%) & K (wt \%) & La (wt \%) \\
\hline $\mathrm{X}$ & LRM & 1 & 1 & 2 & LRMLM111 & 5.16 & $<0.010$ & 0.440 & $<0.010$ & 0.132 & $<0.010$ & 1.40 & 1.23 & $<0.010$ \\
\hline $\mathrm{X}$ & QB-34 & 1 & 1 & 3 & x10LM21 & 4.71 & 0.025 & 4.84 & 0.028 & 0.102 & 0.026 & 5.94 & 0.192 & 0.022 \\
\hline $\mathrm{X}$ & QB-37 & 1 & 1 & 4 & x04LM11 & 5.44 & 0.029 & 4.72 & 0.030 & 0.118 & 0.029 & 6.28 & 0.223 & 0.027 \\
\hline $\mathrm{X}$ & QB-32 & 1 & 1 & 5 & $\begin{array}{l}\mathrm{x} 02 \mathrm{LM} 11 \\
\end{array}$ & 4.64 & 0.024 & 0.366 & 0.027 & 0.099 & 0.025 & 5.47 & 0.197 & 0.016 \\
\hline $\mathrm{X}$ & QB-22 & 1 & 1 & 6 & $\begin{array}{l}\mathrm{x} 03 \mathrm{LM} 111 \\
\end{array}$ & 4.71 & 0.025 & 0.395 & 0.027 & 0.101 & 0.026 & 5.57 & 0.201 & 0.016 \\
\hline $\mathrm{X}$ & QB-35 & 1 & 1 & 7 & x06LM21 & 4.68 & 0.021 & $\begin{array}{ll}4.78 \\
\end{array}$ & 0.028 & 0.086 & 0.025 & 5.45 & 0.188 & 0.023 \\
\hline $\mathrm{X}$ & Batch 1 & 1 & 1 & 8 & BCHLM112 & 2.66 & 0.122 & 0.882 & $<0.010$ & 0.074 & 0.300 & 8.91 & 2.80 & $<0.010$ \\
\hline $\mathrm{X}$ & LRM & 1 & 1 & 9 & LRMLM112 & 5.24 & $<0.010$ & 0.449 & $<0.010$ & 0.133 & $<0.010$ & 1.17 & 1.23 & $<0.010$ \\
\hline $\mathrm{X}$ & QB-37 & 1 & 1 & 10 & x04LM21 & 5.44 & 0.029 & 4.73 & 0.029 & 0.118 & 0.028 & 6.27 & 0.224 & 0.026 \\
\hline $\mathrm{X}$ & QB-35 & 1 & 1 & 11 & x06LM11 & 4.83 & 0.021 & 4.93 & 0.028 & 0.086 & 0.025 & 5.58 & 0.198 & 0.023 \\
\hline $\mathrm{X}$ & QB-32 & 1 & 1 & 12 & x02LM21 & 4.82 & 0.025 & 0.393 & 0.027 & 0.101 & 0.026 & 5.68 & 0.203 & 0.016 \\
\hline $\mathrm{X}$ & QB-34 & 1 & 1 & 13 & x10LM11 & 4.74 & 0.024 & 4.90 & 0.028 & 0.102 & 0.030 & 5.98 & 0.201 & 0.022 \\
\hline $\mathrm{X}$ & QB-22 & 1 & 1 & 14 & x03LM21 & 4.75 & 0.025 & 0.378 & 0.027 & 0.101 & 0.026 & 5.58 & 0.207 & 0.016 \\
\hline $\mathrm{X}$ & Batch 1 & 1 & 1 & 15 & BCHLM113 & 2.62 & 0.124 & 0.869 & $<0.010$ & 0.075 & 0.307 & 8.79 & 2.72 & $<0.010$ \\
\hline $\mathrm{X}$ & LRM & 1 & 1 & 16 & LRMLM113 & 5.18 & $<0.010$ & 0.441 & $<0.010$ & 0.134 & $<0.010$ & 1.16 & 1.25 & $<0.010$ \\
\hline $\mathrm{X}$ & Batch 1 & 1 & 2 & 1 & BCHLM121 & 2.60 & 0.125 & 0.885 & $<0.010$ & 0.077 & 0.309 & 8.66 & 2.78 & $<0.010$ \\
\hline $\mathrm{X}$ & LRM & 1 & 2 & 2 & LRMLM121 & 5.06 & $<0.010$ & 0.463 & $<0.010$ & 0.136 & $<0.010$ & 1.16 & 1.27 & $<0.010$ \\
\hline $\mathrm{X}$ & QB-35 & 1 & 2 & 3 & x06LM22 & 4.50 & 0.039 & 4.59 & 0.083 & 0.088 & 0.027 & 5.24 & 0.195 & 0.081 \\
\hline $\mathrm{X}$ & QB-35 & 1 & 2 & 4 & x06LM12 & 4.68 & 0.023 & 4.78 & 0.030 & 0.089 & 0.026 & 5.55 & 0.204 & 0.024 \\
\hline $\mathrm{X}$ & QB-22 & 1 & 2 & 5 & $\begin{array}{l}\mathrm{x} 03 \mathrm{LM} 12 \\
\end{array}$ & 4.62 & 0.027 & 0.420 & 0.028 & 0.103 & 0.028 & 5.45 & 0.207 & 0.017 \\
\hline $\mathrm{X}$ & QB-37 & 1 & 2 & 6 & x04LM12 & 5.44 & 0.031 & 4.72 & 0.031 & 0.120 & 0.030 & 6.28 & 0.228 & 0.028 \\
\hline $\mathrm{X}$ & QB-32 & 1 & 2 & 7 & $\mathrm{x} 02 \mathrm{LM} 12$ & 4.74 & 0.027 & 0.411 & 0.028 & 0.102 & 0.027 & 5.60 & 0.206 & 0.017 \\
\hline $\mathrm{X}$ & Batch 1 & 1 & 2 & 8 & BCHLM122 & 2.64 & 0.124 & 0.902 & $<0.010$ & 0.076 & 0.304 & 8.78 & 2.82 & $<0.010$ \\
\hline $\mathrm{X}$ & LRM & 1 & 2 & 9 & LRMLM122 & 5.16 & $<0.010$ & 0.477 & $<0.010$ & 0.135 & $<0.010$ & 1.18 & 1.25 & $<0.010$ \\
\hline $\mathrm{X}$ & QB-32 & 1 & 2 & 10 & x02LM22 & 4.65 & 0.027 & 0.411 & 0.028 & 0.102 & 0.027 & 5.47 & 0.204 & 0.017 \\
\hline $\mathrm{X}$ & QB-34 & 1 & 2 & 11 & x10LM22 & 4.72 & 0.026 & 4.85 & 0.029 & 0.103 & 0.028 & 5.93 & 0.191 & 0.023 \\
\hline $\mathrm{X}$ & QB-22 & 1 & 2 & 12 & x03LM22 & 4.64 & 0.026 & 0.407 & 0.027 & 0.101 & 0.027 & 5.45 & 0.203 & 0.016 \\
\hline $\mathrm{X}$ & QB-34 & 1 & 2 & 13 & x10LM12 & 4.77 & 0.025 & 4.71 & 0.029 & 0.102 & 0.030 & 5.76 & 0.196 & 0.023 \\
\hline $\mathrm{X}$ & QB-37 & 1 & 2 & 14 & x04LM22 & 5.36 & 0.030 & 4.67 & 0.030 & 0.119 & 0.029 & 6.18 & 0.222 & 0.027 \\
\hline $\mathrm{X}$ & Batch 1 & 1 & 2 & 15 & BCHLM123 & 2.61 & 0.122 & 0.892 & $<0.010$ & 0.074 & 0.299 & 8.63 & $\begin{array}{ll}2.77 \\
\end{array}$ & $<0.010$ \\
\hline $\mathrm{X}$ & LRM & 1 & 2 & 16 & LRMLM123 & 5.10 & $<0.010$ & 0.472 & $<0.010$ & 0.133 & $<0.010$ & 1.17 & 1.26 & $<0.010$ \\
\hline $\mathrm{X}$ & Batch 1 & 2 & 1 & 1 & BCHLM211 & 2.59 & 0.124 & 0.861 & $<0.010$ & 0.077 & 0.309 & 8.69 & 2.90 & $<0.010$ \\
\hline $\mathrm{X}$ & LRM & 2 & 1 & 2 & LRMLM211 & 4.99 & $<0.010$ & 0.450 & $<0.010$ & 0.136 & $<0.010$ & 1.13 & 1.26 & $<0.010$ \\
\hline $\mathrm{X}$ & QB-19 & 2 & 1 & 3 & x01LM21 & 5.11 & 0.028 & 0.409 & 0.031 & 0.110 & 0.030 & 5.91 & 0.223 & 0.022 \\
\hline $\mathrm{X}$ & QB-33 & 2 & 1 & 4 & $\begin{array}{l}\mathrm{x} 05 \mathrm{LM} 21 \\
\end{array}$ & 4.48 & 0.023 & 2.49 & 0.027 & 0.084 & 0.023 & 5.10 & 0.217 & 0.023 \\
\hline $\mathrm{X}$ & QB-33 & 2 & 1 & 5 & $\begin{array}{l}\mathrm{x} 05 \mathrm{~L} \text { L11 } \\
\end{array}$ & 4.55 & 0.024 & 2.54 & 0.028 & 0.086 & 0.023 & 5.21 & 0.212 & 0.024 \\
\hline $\mathrm{X}$ & QB-36 & 2 & 1 & 6 & x07LM21 & 4.52 & 0.021 & 2.53 & 0.025 & 0.100 & 0.041 & 5.35 & 0.202 & 0.022 \\
\hline $\mathrm{X}$ & QB-20 & 2 & 1 & 7 & x08LM11 & 5.09 & 0.026 & 3.67 & 0.030 & 0.114 & 0.032 & 5.94 & 0.224 & 0.027 \\
\hline $\mathrm{X}$ & Batch 1 & 2 & 1 & 8 & BCHLM212 & 2.55 & 0.124 & 0.844 & $<0.010$ & 0.076 & 0.308 & 8.49 & 2.89 & $<0.010$ \\
\hline $\mathrm{X}$ & LRM & 2 & 1 & 9 & LRMLM212 & 4.92 & $<0.010$ & 0.421 & $<0.010$ & 0.137 & $<0.010$ & 1.11 & 1.29 & $<0.010$ \\
\hline $\mathrm{X}$ & QB-20 & 2 & 1 & 10 & x08LM21 & 5.15 & 0.027 & 3.71 & 0.030 & 0.116 & 0.031 & 6.00 & 0.229 & 0.026 \\
\hline $\mathrm{X}$ & QB-19 & 2 & 1 & 11 & $\begin{array}{l}\mathrm{x} 01 \mathrm{LM} 11 \\
\end{array}$ & 5.06 & 0.028 & 0.408 & 0.031 & 0.111 & 0.030 & 5.88 & 0.228 & 0.022 \\
\hline $\mathrm{X}$ & SPS-02 & 2 & 1 & 12 & x09LM11 & 6.15 & 0.045 & 0.602 & 0.093 & 0.052 & 0.051 & 7.23 & $<0.100$ & 0.032 \\
\hline
\end{tabular}


Table A-1. Measured Elemental Concentrations for Glasses Prepared Using Lithium Metaborate (part 1) (continued)

\begin{tabular}{|c|c|c|c|c|c|c|c|c|c|c|c|c|c|c|}
\hline Series & Glass ID & Block & Sub-Blk & Sequence & Lab ID & Al (wt \%) & $\mathrm{Ba}(\mathrm{wt} \%)$ & Ca (wt \%) & Ce (wt \%) & Cr (wt \%) & Cu (wt \%) & $\mathrm{Fe}$ (wt \%) & K (wt \%) & La (wt \%) \\
\hline $\mathrm{X}$ & QB-36 & 2 & 1 & 13 & x07LM11 & 4.64 & 0.021 & 2.60 & 0.025 & 0.101 & 0.034 & 5.38 & 0.204 & 0.022 \\
\hline $\mathrm{X}$ & SPS-02 & 2 & 1 & 14 & x09LM21 & 6.15 & 0.044 & 0.604 & 0.093 & 0.052 & 0.051 & 7.19 & $<0.100$ & 0.032 \\
\hline $\mathrm{X}$ & Batch 1 & 2 & 1 & 15 & BCHLM213 & 2.56 & 0.125 & 0.851 & $<0.010$ & 0.077 & 0.316 & 8.60 & 2.74 & $<0.010$ \\
\hline $\mathrm{X}$ & LRM & 2 & 1 & 16 & LRMLM213 & 4.97 & $<0.010$ & 0.426 & $<0.010$ & 0.136 & $<0.010$ & 1.12 & 1.29 & $<0.010$ \\
\hline $\mathrm{X}$ & Batch 1 & 2 & 2 & 1 & BCHLM221 & 2.63 & 0.125 & 0.867 & $<0.010$ & 0.076 & 0.310 & 8.85 & 2.68 & $<0.010$ \\
\hline $\mathrm{X}$ & LRM & 2 & 2 & 2 & LRMLM221 & 5.11 & $<0.010$ & 0.429 & $<0.010$ & 0.131 & 0.004 & 1.15 & 1.28 & $<0.010$ \\
\hline $\mathrm{X}$ & QB-20 & 2 & 2 & 3 & x08LM22 & 5.26 & 0.027 & 3.78 & 0.030 & 0.113 & 0.030 & 6.15 & 0.225 & 0.025 \\
\hline $\mathrm{X}$ & QB-19 & 2 & 2 & 4 & x01LM22 & 5.32 & 0.029 & 0.424 & 0.031 & 0.109 & 0.029 & 6.17 & 0.223 & 0.021 \\
\hline $\mathrm{X}$ & QB-20 & 2 & 2 & 5 & x08LM12 & 5.20 & 0.027 & 3.76 & 0.030 & 0.113 & 0.031 & 6.10 & 0.222 & 0.027 \\
\hline $\mathrm{X}$ & QB-33 & 2 & 2 & 6 & x05LM12 & 4.60 & 0.025 & 2.56 & 0.029 & 0.085 & 0.022 & 5.28 & 0.213 & 0.023 \\
\hline $\mathrm{X}$ & QB-36 & 2 & 2 & 7 & x07LM22 & 4.65 & 0.022 & 2.61 & 0.025 & 0.100 & 0.041 & 5.50 & 0.202 & 0.022 \\
\hline $\mathrm{X}$ & Batch 1 & 2 & 2 & 8 & BCHLM222 & 2.60 & 0.124 & 0.860 & $<0.010$ & 0.076 & 0.307 & 8.74 & 2.88 & $<0.010$ \\
\hline $\mathrm{X}$ & LRM & 2 & 2 & 9 & LRMLM222 & 5.07 & $<0.010$ & 0.427 & $<0.010$ & 0.134 & 0.006 & 1.24 & 1.29 & $<0.010$ \\
\hline $\mathrm{X}$ & $\begin{array}{l}\text { SPS-02 } \\
\end{array}$ & 2 & 2 & 10 & x09LM12 & 6.26 & 0.045 & 0.619 & 0.092 & 0.052 & 0.050 & 7.35 & $<0.100$ & 0.031 \\
\hline $\mathrm{X}$ & QB-19 & 2 & 2 & 11 & x01LM12 & 5.25 & 0.029 & 0.414 & 0.032 & 0.110 & 0.031 & 6.10 & 0.228 & 0.021 \\
\hline $\mathrm{X}$ & SPS-02 & 2 & 2 & 12 & x09LM22 & 6.26 & 0.045 & 0.610 & 0.093 & 0.052 & 0.051 & 7.37 & $<0.100$ & 0.031 \\
\hline $\mathrm{X}$ & QB-33 & 2 & 2 & 13 & $\mathrm{x} 05 \mathrm{LM} 22$ & 4.60 & 0.024 & 2.58 & 0.027 & 0.083 & 0.023 & 5.24 & 0.218 & 0.023 \\
\hline $\mathrm{X}$ & QB-36 & 2 & 2 & 14 & x07LM12 & 4.67 & 0.022 & 2.62 & 0.026 & 0.101 & 0.034 & 5.44 & 0.207 & 0.022 \\
\hline $\mathrm{X}$ & Batch 1 & 2 & 2 & 15 & BCHLM223 & 2.60 & 0.124 & 0.860 & $<0.010$ & 0.076 & 0.309 & 8.77 & $\begin{array}{ll}2.84 \\
\end{array}$ & $<0.010$ \\
\hline $\mathrm{X}$ & LRM & 2 & 2 & 16 & LRMLM223 & 5.02 & $<0.010$ & 0.423 & $<0.010$ & 0.135 & 0.007 & 1.12 & 1.31 & $<0.010$ \\
\hline
\end{tabular}


Table A-2. Measured Elemental Concentrations for the Glasses Prepared Using Lithium Metaborate (part 2)

\begin{tabular}{|c|c|c|c|c|c|c|c|c|c|c|c|c|c|c|}
\hline Series & Glass ID & Block & Sub-Blk & Sequence & Lab ID & Mg (wt \%) & Mn (wt \%) & Na (wt \%) & Ni (wt \%) & Pb (wt \%) & S (wt \%) & $\mathrm{Ti}$ (wt \%) & Zn (wt \%) & $\mathrm{Zr}$ (wt \%) \\
\hline $\mathrm{T}$ & Batch 1 & 1 & 1 & 1 & BCHLM111 & 0.860 & 1.35 & 6.79 & 0.535 & $<<0.020$ & $<0.050$ & 0.384 & $<<0.010$ & 0.067 \\
\hline $\mathrm{T}$ & LRM & 1 & 1 & 2 & LRMLM111 & 0.062 & 0.060 & 16.6 & 0.137 & 0.083 & 0.089 & 0.061 & $<0.010$ & 0.642 \\
\hline $\mathrm{T}$ & $\begin{array}{l}\mathrm{BC}-03 \\
\end{array}$ & 1 & 1 & 3 & t10LM21 & 0.222 & 2.40 & 11.1 & 0.737 & $<0.020$ & 0.370 & $<0.010$ & 0.027 & 0.086 \\
\hline $\mathrm{T}$ & BC-01 & 1 & 1 & 4 & t04LM11 & 0.171 & 2.40 & 8.73 & 0.760 & $<0.020$ & 0.347 & $<0.010$ & 0.028 & 0.076 \\
\hline $\mathrm{T}$ & BC-02 & 1 & 1 & 5 & t02LM11 & 0.194 & 2.39 & 8.68 & 0.764 & $<0.020$ & 0.360 & $<0.010$ & 0.025 & 0.087 \\
\hline $\mathrm{T}$ & AF-02 & 1 & 1 & 6 & t03LM11 & 0.194 & 2.33 & 11.3 & 0.767 & $<0.020$ & 0.333 & $<0.010$ & 0.024 & 0.090 \\
\hline $\mathrm{T}$ & AF-05 & 1 & 1 & 7 & t06LM21 & 0.197 & 2.36 & 9.69 & 0.742 & $<0.020$ & 0.372 & $<0.010$ & 0.028 & 0.089 \\
\hline $\mathrm{T}$ & Batch 1 & 1 & 1 & 8 & BCHLM112 & 0.843 & 1.32 & 6.67 & 0.529 & $<0.020$ & $<0.050$ & 0.377 & $<0.010$ & 0.067 \\
\hline $\mathrm{T}$ & LRM & 1 & 1 & 9 & LRMLM112 & 0.063 & 0.060 & 16.1 & 0.136 & 0.082 & 0.089 & 0.061 & $<0.010$ & 0.643 \\
\hline $\mathrm{T}$ & BC-01 & 1 & 1 & 10 & t04LM21 & 0.171 & 2.30 & 8.25 & 0.752 & $<0.020$ & 0.357 & $<0.010$ & 0.027 & 0.081 \\
\hline $\mathrm{T}$ & AF-05 & 1 & 1 & 11 & t06LM11 & 0.199 & 2.36 & 9.67 & 0.738 & $<0.020$ & 0.377 & $<0.010$ & 0.028 & 0.088 \\
\hline $\mathrm{T}$ & BC-02 & 1 & 1 & 12 & t02LM21 & 0.192 & 2.29 & 8.20 & 0.755 & $<0.020$ & 0.369 & $<0.010$ & 0.024 & 0.088 \\
\hline $\mathrm{T}$ & BC- 03 & 1 & 1 & 13 & t10LM11 & 0.223 & 2.30 & 10.4 & 0.734 & $<0.020$ & 0.389 & $<0.010$ & 0.033 & 0.088 \\
\hline $\mathrm{T}$ & AF-02 & 1 & 1 & 14 & t03LM21 & 0.192 & 2.25 & 10.7 & 0.751 & $<0.020$ & 0.344 & $<0.010$ & 0.024 & 0.092 \\
\hline $\mathrm{T}$ & Batch 1 & 1 & 1 & 15 & BCHLM113 & 0.817 & 1.28 & 6.38 & 0.512 & $<0.020$ & $<0.050$ & 0.360 & $<0.010$ & 0.065 \\
\hline $\mathrm{T}$ & LRM & 1 & 1 & 16 & LRMLM113 & 0.061 & 0.058 & 15.6 & 0.133 & 0.080 & 0.090 & 0.059 & $<0.010$ & 0.628 \\
\hline $\mathrm{T}$ & Batch 1 & 1 & 2 & 1 & BCHLM121 & 0.818 & 1.27 & 6.35 & 0.533 & $<0.020$ & $<0.050$ & 0.378 & $<0.010$ & 0.066 \\
\hline $\mathrm{T}$ & LRM & 1 & 2 & 2 & LRMLM121 & 0.062 & 0.059 & 16.13 & 0.137 & 0.082 & 0.086 & 0.061 & $<0.010$ & 0.649 \\
\hline $\mathrm{T}$ & AF-05 & 1 & 2 & 3 & t06LM22 & 0.198 & 2.34 & 9.54 & 0.750 & $<0.020$ & 0.356 & $<0.010$ & 0.028 & 0.089 \\
\hline $\mathrm{T}$ & AF-05 & 1 & 2 & 4 & t06LM12 & 0.200 & 2.39 & 9.77 & 0.758 & $<0.020$ & 0.359 & $<0.010$ & 0.029 & 0.089 \\
\hline $\mathrm{T}$ & AF-02 & 1 & 2 & 5 & t03LM12 & 0.197 & 2.38 & 11.5 & 0.777 & $<0.020$ & 0.321 & $<0.010$ & 0.025 & 0.091 \\
\hline $\mathrm{T}$ & BC-01 & 1 & 2 & 6 & t04LM12 & 0.173 & 2.45 & 8.89 & 0.779 & $<0.020$ & 0.339 & $<0.010$ & 0.029 & 0.077 \\
\hline $\mathrm{T}$ & BC-02 & 1 & 2 & 7 & t02LM12 & 0.196 & 2.42 & 8.76 & 0.784 & $<0.020$ & 0.352 & $<0.010$ & 0.025 & 0.088 \\
\hline $\mathrm{T}$ & Batch 1 & 1 & 2 & 8 & BCHLM122 & 0.872 & 1.36 & 6.86 & 0.534 & $<0.020$ & $<0.050$ & 0.377 & $<0.010$ & 0.066 \\
\hline $\mathrm{T}$ & LRM & 1 & 2 & 9 & LRMLM122 & 0.062 & 0.059 & 16.3 & 0.138 & 0.083 & 0.081 & 0.061 & $<0.010$ & 0.654 \\
\hline $\mathrm{T}$ & BC-02 & 1 & 2 & 10 & t02LM22 & 0.196 & 2.39 & 8.60 & 0.778 & $<0.020$ & 0.354 & $<0.010$ & 0.025 & 0.089 \\
\hline $\mathrm{T}$ & BC- 03 & 1 & 2 & 11 & t10LM22 & 0.227 & 2.36 & 10.8 & 0.755 & $<0.020$ & 0.370 & $<0.010$ & 0.028 & 0.088 \\
\hline $\mathrm{T}$ & AF-02 & 1 & 2 & 12 & t03LM22 & 0.198 & 2.32 & 11.1 & 0.789 & $<0.020$ & 0.334 & $<0.010$ & 0.025 & 0.094 \\
\hline $\mathrm{T}$ & BC- 03 & 1 & 2 & 13 & t10LM12 & 0.227 & 2.40 & 10.9 & 0.755 & $<0.020$ & 0.374 & $<0.010$ & 0.034 & 0.089 \\
\hline $\mathrm{T}$ & BC-01 & 1 & 2 & 14 & t04LM22 & 0.173 & 2.36 & 8.47 & 0.772 & $<0.020$ & 0.347 & $<0.010$ & 0.028 & 0.082 \\
\hline $\mathrm{T}$ & Batch 1 & 1 & 2 & 15 & BCHLM123 & 0.857 & 1.33 & 6.66 & 0.534 & $<0.020$ & $<0.050$ & 0.383 & $<0.010$ & 0.067 \\
\hline $\mathrm{T}$ & LRM & 1 & 2 & 16 & LRMLM123 & 0.062 & 0.059 & 15.7 & 0.139 & 0.082 & 0.084 & 0.061 & $<0.010$ & 0.655 \\
\hline $\mathrm{T}$ & Batch 1 & 2 & 1 & 1 & BCHLM211 & 0.850 & 1.35 & 6.94 & 0.531 & $<0.020$ & $<0.050$ & 0.386 & $<0.010$ & 0.065 \\
\hline $\mathrm{T}$ & LRM & 2 & 1 & 2 & LRMLM211 & 0.061 & 0.058 & 16.7 & 0.135 & 0.081 & 0.082 & 0.060 & $<0.010$ & 0.646 \\
\hline $\mathrm{T}$ & AF-04 & 2 & 1 & 3 & t01LM21 & 0.180 & 2.39 & 11.0 & 0.741 & $<0.020$ & 0.366 & $<0.010$ & 0.021 & 0.090 \\
\hline $\mathrm{T}$ & BC-04 & 2 & 1 & 4 & t05LM21 & 0.188 & 2.36 & 8.83 & 0.742 & $<0.020$ & 0.357 & $<0.010$ & 0.030 & 0.098 \\
\hline $\mathrm{T}$ & BC-04 & 2 & 1 & 5 & t05LM11 & 0.194 & 2.40 & 8.88 & 0.777 & $<0.020$ & 0.371 & $<0.010$ & 0.031 & 0.099 \\
\hline $\mathrm{T}$ & AF-01 & 2 & 1 & 6 & t07LM21 & 0.194 & 2.43 & 11.9 & 0.781 & $<0.020$ & 0.324 & $<0.010$ & 0.026 & 0.091 \\
\hline $\mathrm{T}$ & AB-06 & 2 & 1 & 7 & t08LM11 & 0.162 & 2.18 & 11.4 & 0.675 & $<0.020$ & 0.298 & $<0.010$ & 0.019 & 0.068 \\
\hline $\mathrm{T}$ & Batch 1 & 2 & 1 & 8 & BCHLM212 & 0.862 & 1.37 & 7.11 & 0.524 & $<0.020$ & $<0.050$ & 0.376 & $<0.010$ & 0.064 \\
\hline $\mathrm{T}$ & LRM & 2 & 1 & 9 & LRMLM212 & 0.060 & 0.059 & 17.3 & 0.132 & 0.079 & 0.084 & 0.058 & $<0.010$ & 0.633 \\
\hline $\mathrm{T}$ & AB-06 & 2 & 1 & 10 & t08LM21 & 0.168 & 2.21 & 11.8 & 0.696 & $<0.020$ & 0.309 & $<0.010$ & 0.020 & 0.071 \\
\hline
\end{tabular}


Table A-2. Measured Elemental Concentrations for the Glasses Prepared Using Lithium Metaborate (part 2) (continued)

\begin{tabular}{|c|c|c|c|c|c|c|c|c|c|c|c|c|c|c|}
\hline Series & Glass ID & Block & Sub-Blk & Sequence & Lab ID & Mg (wt \%) & Mn (wt \%) & Na (wt \%) & Ni (wt \%) & $\mathrm{Pb}(\mathrm{wt} \%)$ & S (wt \%) & Ti (wt \%) & Zn (wt \%) & $\operatorname{Zr}$ (wt \%) \\
\hline $\mathrm{T}$ & AF-04 & 2 & 1 & 11 & t01LM11 & 0.185 & 2.45 & 11.4 & 0.771 & $<0.020$ & 0.376 & $<0.010$ & 0.022 & 0.097 \\
\hline $\mathrm{T}$ & $\mathrm{AF}-03$ & 2 & 1 & 12 & t09LM11 & 0.199 & 2.41 & 10.0 & 0.793 & $<0.020$ & 0.319 & $<0.010$ & 0.025 & 0.087 \\
\hline $\mathrm{T}$ & AF-01 & 2 & 1 & 13 & t07LM11 & 0.197 & 2.40 & 11.7 & 0.800 & $<0.020$ & 0.331 & $<0.010$ & 0.024 & 0.099 \\
\hline $\mathrm{T}$ & AF-03 & 2 & 1 & 14 & t09LM21 & 0.200 & 2.37 & 9.79 & 0.798 & $<0.020$ & 0.329 & $<0.010$ & 0.026 & 0.100 \\
\hline $\mathrm{T}$ & Batch 1 & 2 & 1 & 15 & BCHLM213 & 0.848 & 1.34 & 6.95 & 0.541 & $<0.020$ & $<0.050$ & 0.390 & $<0.010$ & 0.066 \\
\hline $\mathrm{T}$ & LRM & 2 & 1 & 16 & LRMLM213 & 0.061 & 0.058 & 16.9 & 0.136 & 0.081 & 0.083 & 0.059 & $<0.010$ & 0.654 \\
\hline $\mathrm{T}$ & Batch 1 & 2 & 2 & 1 & BCHLM221 & 0.889 & 1.38 & 6.92 & 0.525 & $<0.020$ & $<0.050$ & 0.378 & $<0.010$ & 0.065 \\
\hline $\mathrm{T}$ & LRM & 2 & 2 & 2 & LRMLM221 & 0.061 & 0.059 & 16.5 & 0.136 & 0.082 & 0.087 & 0.061 & $<0.010$ & 0.641 \\
\hline $\mathrm{T}$ & AB-06 & 2 & 2 & 3 & t08LM22 & 0.164 & 2.15 & 11.0 & 0.678 & $<0.020$ & 0.303 & $<0.010$ & 0.020 & 0.071 \\
\hline $\mathrm{T}$ & AF-04 & 2 & 2 & 4 & t01LM22 & 0.177 & 2.34 & 10.5 & 0.729 & $<0.020$ & 0.359 & $<0.010$ & 0.022 & 0.089 \\
\hline $\mathrm{T}$ & AB-06 & 2 & 2 & 5 & t08LM12 & 0.163 & 2.17 & 11.0 & 0.678 & $<0.020$ & 0.299 & $<0.010$ & 0.020 & 0.070 \\
\hline $\mathrm{T}$ & BC-04 & 2 & 2 & 6 & t05LM12 & 0.196 & 2.41 & 8.78 & 0.779 & $<0.020$ & 0.366 & $<0.010$ & 0.032 & 0.101 \\
\hline $\mathrm{T}$ & AF-01 & 2 & 2 & 7 & t07LM22 & 0.196 & 2.41 & 11.5 & 0.779 & $<0.020$ & 0.325 & $<0.010$ & 0.027 & 0.092 \\
\hline $\mathrm{T}$ & Batch 1 & 2 & 2 & 8 & BCHLM222 & 0.856 & 1.33 & 6.60 & 0.523 & $<0.020$ & $<0.050$ & 0.377 & $<0.010$ & 0.066 \\
\hline $\mathrm{T}$ & LRM & 2 & 2 & 9 & LRMLM222 & 0.061 & 0.058 & 16.2 & 0.134 & 0.080 & 0.080 & 0.060 & $<0.010$ & 0.638 \\
\hline $\mathrm{T}$ & $\mathrm{AF}-03$ & 2 & 2 & 10 & t09LM12 & 0.193 & 2.38 & 9.69 & 0.764 & $<0.020$ & 0.311 & $<0.010$ & 0.025 & 0.084 \\
\hline $\mathrm{T}$ & AF-04 & 2 & 2 & 11 & t01LM12 & 0.180 & 2.37 & 10.7 & 0.744 & $<0.020$ & 0.358 & $<0.010$ & 0.022 & 0.094 \\
\hline $\mathrm{T}$ & $\mathrm{AF}-03$ & 2 & 2 & 12 & t09LM22 & 0.195 & 2.38 & 9.67 & 0.771 & $<0.020$ & 0.313 & $<0.010$ & 0.026 & 0.098 \\
\hline $\mathrm{T}$ & BC-04 & 2 & 2 & 13 & t05LM22 & 0.187 & 2.35 & 8.65 & 0.726 & $<0.020$ & 0.349 & $<0.010$ & 0.030 & 0.096 \\
\hline $\mathrm{T}$ & AF-01 & 2 & 2 & 14 & t07LM12 & 0.192 & 2.43 & 11.6 & 0.772 & $<0.020$ & 0.320 & $<0.010$ & 0.024 & 0.097 \\
\hline $\mathrm{T}$ & Batch 1 & 2 & 2 & 15 & BCHLM223 & 0.881 & 1.38 & 6.95 & 0.522 & $<0.020$ & $<0.050$ & 0.378 & $<0.010$ & 0.065 \\
\hline $\mathrm{T}$ & LRM & 2 & 2 & 16 & LRMLM223 & 0.061 & 0.058 & 16.6 & 0.134 & 0.079 & 0.083 & 0.060 & $<0.010$ & 0.637 \\
\hline $\mathrm{U}$ & Batch 1 & 1 & 1 & 1 & BCHLM111 & 0.808 & 1.32 & 6.56 & 0.536 & $<0.020$ & $<0.050$ & 0.394 & $<0.010$ & 0.066 \\
\hline $\mathrm{U}$ & LRM & 1 & 1 & 2 & LRMLM111 & 0.061 & 0.058 & 15.3 & 0.135 & 0.080 & 0.086 & 0.059 & $<0.010$ & 0.652 \\
\hline $\mathrm{U}$ & BC-09 & 1 & 1 & 3 & u10LM21 & 0.179 & 2.30 & 9.15 & 0.749 & $<0.020$ & 0.371 & $<0.010$ & 0.022 & 0.088 \\
\hline $\mathrm{U}$ & BS-05 & 1 & 1 & 4 & u04LM11 & 0.206 & 2.34 & 9.19 & 0.805 & $<0.020$ & 0.364 & $<0.010$ & 0.027 & 0.091 \\
\hline $\mathrm{U}$ & BS-01 & 1 & 1 & 5 & u02LM11 & 0.196 & 2.32 & 8.28 & 0.770 & $<0.020$ & 0.345 & $<0.010$ & 0.028 & 0.089 \\
\hline $\mathrm{U}$ & BC-05 & 1 & 1 & 6 & u03LM11 & 0.206 & 2.34 & 9.20 & 0.807 & $<0.020$ & 0.356 & $<0.010$ & 0.027 & 0.092 \\
\hline $\mathrm{U}$ & BS-02 & 1 & 1 & 7 & u06LM21 & 0.196 & 2.33 & 8.35 & 0.766 & $<0.020$ & 0.376 & $<0.010$ & 0.026 & 0.092 \\
\hline $\mathrm{U}$ & Batch 1 & 1 & 1 & 8 & BCHLM112 & 0.810 & 1.36 & 6.49 & 0.538 & $<0.020$ & $<0.050$ & 0.393 & $<0.010$ & 0.067 \\
\hline $\mathrm{U}$ & LRM & 1 & 1 & 9 & LRMLM112 & 0.061 & 0.058 & 15.5 & 0.137 & 0.081 & 0.089 & 0.059 & $<0.010$ & 0.660 \\
\hline $\mathrm{U}$ & BS-05 & 1 & 1 & 10 & u04LM21 & 0.205 & 2.35 & 9.25 & 0.806 & $<0.020$ & 0.362 & $<0.010$ & 0.027 & 0.091 \\
\hline $\mathrm{U}$ & BS-02 & 1 & 1 & 11 & u06LM11 & 0.193 & 2.45 & 8.78 & 0.747 & $<0.020$ & 0.373 & $<0.010$ & 0.025 & 0.087 \\
\hline $\mathrm{U}$ & BS-01 & 1 & 1 & 12 & u02LM21 & 0.201 & 2.66 & 9.46 & 0.795 & $<0.020$ & 0.354 & $<0.010$ & 0.029 & 0.091 \\
\hline $\mathrm{U}$ & BC-09 & 1 & 1 & 13 & u10LM11 & 0.184 & 2.34 & 9.25 & 0.779 & $<0.020$ & 0.381 & $<0.010$ & 0.022 & 0.096 \\
\hline $\mathrm{U}$ & BC-05 & 1 & 1 & 14 & u03LM21 & 0.206 & 2.45 & 8.68 & 0.796 & $<0.020$ & 0.388 & $<0.010$ & 0.024 & 0.101 \\
\hline $\mathrm{U}$ & Batch 1 & 1 & 1 & 15 & BCHLM113 & 0.838 & 1.32 & 6.55 & 0.558 & $<0.020$ & $<0.050$ & 0.412 & $<0.010$ & 0.069 \\
\hline $\mathrm{U}$ & LRM & 1 & 1 & 16 & LRMLM113 & 0.063 & 0.060 & 15.4 & 0.141 & 0.084 & 0.082 & 0.063 & $<0.010$ & 0.686 \\
\hline $\mathrm{U}$ & Batch 1 & 1 & 2 & 1 & BCHLM121 & 0.803 & 1.37 & 6.68 & 0.531 & $<0.020$ & $<0.050$ & 0.381 & $<0.010$ & 0.064 \\
\hline $\mathrm{U}$ & LRM & 1 & 2 & 2 & LRMLM121 & 0.059 & 0.058 & 15.4 & 0.135 & 0.079 & 0.078 & 0.058 & $<0.010$ & 0.650 \\
\hline $\mathrm{U}$ & BS-02 & 1 & 2 & 3 & u06LM22 & 0.191 & 2.40 & 8.50 & 0.741 & $<0.020$ & 0.356 & $<0.010$ & 0.024 & 0.086 \\
\hline $\mathrm{U}$ & BS-02 & 1 & 2 & 4 & u06LM12 & 0.190 & 2.37 & 8.34 & 0.735 & $<0.020$ & 0.354 & $<0.010$ & 0.024 & 0.087 \\
\hline $\mathrm{U}$ & BC-05 & 1 & 2 & 5 & u03LM12 & 0.202 & 2.42 & 9.34 & 0.793 & $<0.020$ & 0.344 & $<0.010$ & 0.025 & 0.090 \\
\hline
\end{tabular}


Table A-2. Measured Elemental Concentrations for the Glasses Prepared Using Lithium Metaborate (part 2) (continued)

\begin{tabular}{|c|c|c|c|c|c|c|c|c|c|c|c|c|c|c|}
\hline Series & Glass ID & Block & Sub-Blk & Sequence & Lab ID & Mg (wt \%) & Mn (wt \%) & Na (wt \%) & Ni (wt \%) & $\mathrm{Pb}(\mathrm{wt} \%)$ & S (wt \%) & Ti (wt \%) & Zn (wt \%) & $\operatorname{Zr}$ (wt \%) \\
\hline $\mathrm{U}$ & BS-05 & 1 & 2 & 6 & u04LM12 & 0.206 & 2.45 & 9.61 & 0.798 & $<0.020$ & 0.348 & $<0.010$ & 0.026 & 0.090 \\
\hline $\mathrm{U}$ & BS-01 & 1 & 2 & 7 & u02LM12 & 0.195 & 2.43 & 8.51 & 0.765 & $<0.020$ & 0.338 & $<0.010$ & 0.027 & 0.088 \\
\hline $\mathrm{U}$ & Batch 1 & 1 & 2 & 8 & BCHLM122 & 0.817 & 1.41 & 6.86 & 0.536 & $<0.020$ & $<0.050$ & 0.388 & $<0.010$ & 0.065 \\
\hline $\mathrm{U}$ & LRM & 1 & 2 & 9 & LRMLM122 & 0.060 & 0.058 & 15.7 & 0.135 & 0.080 & 0.080 & 0.058 & $<0.010$ & 0.651 \\
\hline $\mathrm{U}$ & BS-01 & 1 & 2 & 10 & u02LM22 & 0.199 & 2.46 & 8.56 & 0.783 & $<0.020$ & 0.344 & $<0.010$ & 0.027 & 0.088 \\
\hline $\mathrm{U}$ & BC-09 & 1 & 2 & 11 & u10LM22 & 0.183 & 2.44 & 9.52 & 0.769 & $<0.020$ & 0.384 & $<0.010$ & 0.022 & 0.089 \\
\hline $\mathrm{U}$ & BC-05 & 1 & 2 & 12 & u03LM22 & 0.202 & 2.42 & 8.44 & 0.779 & $<0.020$ & 0.379 & $<0.010$ & 0.022 & 0.098 \\
\hline $\mathrm{U}$ & BC-09 & 1 & 2 & 13 & u10LM12 & 0.181 & 2.41 & 9.35 & 0.770 & $<0.020$ & 0.363 & $<0.010$ & 0.021 & 0.094 \\
\hline $\mathrm{U}$ & BS-05 & 1 & 2 & 14 & u04LM22 & 0.207 & 2.41 & 9.32 & 0.813 & $<0.020$ & 0.360 & $<0.010$ & 0.026 & 0.092 \\
\hline $\mathrm{U}$ & Batch 1 & 1 & 2 & 15 & BCHLM123 & 0.824 & 1.40 & 6.73 & 0.544 & $<0.020$ & $<0.050$ & 0.393 & $<0.010$ & 0.067 \\
\hline $\mathrm{U}$ & LRM & 1 & 2 & 16 & LRMLM123 & 0.061 & 0.059 & 16.0 & 0.137 & 0.081 & 0.081 & 0.059 & $<0.010$ & 0.669 \\
\hline $\mathrm{U}$ & Batch 1 & 2 & 1 & 1 & BCHLM211 & 0.805 & 1.32 & 6.53 & 0.532 & $<0.020$ & $<0.050$ & 0.382 & $<0.010$ & 0.067 \\
\hline $\mathrm{U}$ & LRM & 2 & 1 & 2 & LRMLM211 & 0.061 & 0.059 & 15.4 & 0.136 & 0.082 & 0.078 & 0.060 & $<0.010$ & 0.651 \\
\hline $\mathrm{U}$ & BC-08 & 2 & 1 & 3 & u01LM21 & 0.215 & 2.36 & 10.3 & 0.779 & $<0.020$ & 0.370 & $<0.010$ & 0.026 & 0.092 \\
\hline $\mathrm{U}$ & BC-06 & 2 & 1 & 4 & u05LM21 & 0.198 & 2.36 & 10.5 & 0.734 & $<0.020$ & 0.371 & $<0.010$ & 0.026 & 0.090 \\
\hline $\mathrm{U}$ & BC-06 & 2 & 1 & 5 & u05LM11 & 0.203 & 2.36 & 10.4 & 0.762 & $<0.020$ & 0.378 & $<0.010$ & 0.026 & 0.092 \\
\hline $\mathrm{U}$ & BC-07 & 2 & 1 & 6 & u07LM21 & 0.197 & 2.38 & 10.4 & 0.768 & $<0.020$ & 0.374 & $<0.010$ & 0.024 & 0.085 \\
\hline $\mathrm{U}$ & BS-04 & 2 & 1 & 7 & u08LM11 & 0.198 & 2.41 & 8.47 & 0.817 & $<0.020$ & 0.371 & $<0.010$ & 0.030 & 0.079 \\
\hline $\mathrm{U}$ & Batch 1 & 2 & 1 & 8 & BCHLM212 & 0.813 & 1.37 & 6.77 & 0.541 & $<0.020$ & $<0.050$ & 0.396 & $<0.010$ & 0.068 \\
\hline $\mathrm{U}$ & LRM & 2 & 1 & 9 & LRMLM212 & 0.062 & 0.060 & 15.7 & 0.139 & 0.084 & 0.084 & 0.062 & $<0.010$ & 0.668 \\
\hline $\mathrm{U}$ & BS-04 & 2 & 1 & 10 & u08LM21 & 0.196 & 2.42 & 8.51 & 0.807 & $<0.020$ & 0.371 & $<0.010$ & 0.029 & 0.087 \\
\hline $\mathrm{U}$ & BC-08 & 2 & 1 & 11 & u01LM11 & 0.212 & 2.40 & 10.7 & 0.756 & $<0.020$ & 0.372 & $<0.010$ & 0.028 & 0.087 \\
\hline $\mathrm{U}$ & BS-03 & 2 & 1 & 12 & u09LM11 & 0.213 & 2.35 & 8.41 & 0.750 & $<0.020$ & 0.348 & $<0.010$ & 0.024 & 0.091 \\
\hline $\mathrm{U}$ & BC-07 & 2 & 1 & 13 & u07LM11 & 0.197 & 2.39 & 10.5 & 0.824 & $<0.020$ & 0.372 & $<0.010$ & 0.024 & 0.106 \\
\hline $\mathrm{U}$ & BS-03 & 2 & 1 & 14 & u09LM21 & 0.219 & 2.38 & 8.58 & 0.790 & $<0.020$ & 0.358 & $<0.010$ & 0.025 & 0.092 \\
\hline $\mathrm{U}$ & Batch 1 & 2 & 1 & 15 & BCHLM213 & 0.802 & 1.39 & 6.84 & 0.532 & $<0.020$ & $<0.050$ & 0.382 & $<0.010$ & 0.067 \\
\hline $\mathrm{U}$ & LRM & 2 & 1 & 16 & LRMLM213 & 0.062 & 0.060 & 15.8 & 0.138 & 0.082 & 0.084 & 0.061 & $<0.010$ & 0.664 \\
\hline $\mathrm{U}$ & Batch 1 & 2 & 2 & 1 & BCHLM221 & 0.799 & 1.35 & 6.61 & 0.529 & $<0.020$ & 0.017 & 0.382 & $<0.010$ & 0.065 \\
\hline $\mathrm{U}$ & LRM & 2 & 2 & 2 & LRMLM221 & 0.060 & 0.058 & 15.7 & 0.135 & 0.080 & 0.083 & 0.060 & $<0.010$ & 0.667 \\
\hline $\mathrm{U}$ & BS-04 & 2 & 2 & 3 & u08LM22 & 0.194 & 2.38 & 8.37 & 0.801 & $<0.020$ & 0.361 & $<0.010$ & 0.027 & 0.086 \\
\hline $\mathrm{U}$ & BC-08 & 2 & 2 & 4 & u01LM22 & 0.214 & 2.34 & 10.3 & 0.782 & $<0.020$ & 0.372 & $<0.010$ & 0.024 & 0.090 \\
\hline $\mathrm{U}$ & BS-04 & 2 & 2 & 5 & u08LM12 & 0.196 & 2.37 & 8.35 & 0.811 & $<0.020$ & 0.367 & $<0.010$ & 0.028 & 0.076 \\
\hline $\mathrm{U}$ & BC-06 & 2 & 2 & 6 & u05LM12 & 0.203 & 2.37 & 10.5 & 0.765 & $<0.020$ & 0.380 & $<0.010$ & 0.025 & 0.091 \\
\hline $\mathrm{U}$ & BC-07 & 2 & 2 & 7 & u07LM22 & 0.197 & 2.35 & 10.3 & 0.770 & $<0.020$ & 0.372 & $<0.010$ & 0.022 & 0.084 \\
\hline $\mathrm{U}$ & Batch 1 & 2 & 2 & 8 & BCHLM222 & 0.805 & 1.34 & 6.54 & 0.532 & $<0.020$ & 0.009 & 0.381 & $<0.010$ & 0.066 \\
\hline $\mathrm{U}$ & LRM & 2 & 2 & 9 & LRMLM222 & 0.060 & 0.058 & 15.5 & 0.136 & 0.082 & 0.082 & 0.060 & $<0.010$ & 0.669 \\
\hline $\mathrm{U}$ & BS-03 & 2 & 2 & 10 & u09LM12 & 0.213 & 2.32 & 8.28 & 0.754 & $<0.020$ & 0.347 & $<0.010$ & 0.022 & 0.090 \\
\hline $\mathrm{U}$ & BC-08 & 2 & 2 & 11 & u01LM12 & 0.212 & 2.38 & 10.5 & 0.755 & $<0.020$ & 0.374 & $<0.010$ & 0.026 & 0.086 \\
\hline $\mathrm{U}$ & BS-03 & 2 & 2 & 12 & u09LM22 & 0.220 & 2.36 & 8.36 & 0.794 & $<0.020$ & 0.358 & $<0.010$ & 0.024 & 0.092 \\
\hline $\mathrm{U}$ & BC-06 & 2 & 2 & 13 & u05LM22 & 0.197 & 2.37 & 10.6 & 0.738 & $<0.020$ & 0.376 & $<0.010$ & 0.024 & 0.089 \\
\hline $\mathrm{U}$ & BC-07 & 2 & 2 & 14 & u07LM12 & 0.195 & 2.34 & 10.3 & 0.822 & $<0.020$ & 0.375 & $<0.010$ & 0.022 & 0.105 \\
\hline $\mathrm{U}$ & Batch 1 & 2 & 2 & 15 & BCHLM223 & 0.811 & 1.34 & 6.58 & 0.536 & $<0.020$ & 0.014 & 0.390 & $<0.010$ & 0.067 \\
\hline $\mathrm{U}$ & LRM & 2 & 2 & 16 & LRMLM223 & 0.061 & 0.059 & 15.7 & 0.138 & 0.082 & 0.084 & 0.061 & $<0.010$ & 0.663 \\
\hline
\end{tabular}


Table A-2. Measured Elemental Concentrations for the Glasses Prepared Using Lithium Metaborate (part 2) (continued)

\begin{tabular}{|c|c|c|c|c|c|c|c|c|c|c|c|c|c|c|}
\hline Series & Glass ID & Block & Sub-Blk & Sequence & Lab ID & $M g$ (wt \%) & Mn (wt \%) & $\mathrm{Na}(\mathrm{wt} \%)$ & Ni (wt \%) & $\mathrm{Pb}(\mathrm{wt} \%)$ & S (wt \%) & Ti (wt \%) & Zn (wt \%) & Zr (wt \%) \\
\hline $\mathrm{V}$ & Batch 1 & 1 & 1 & 1 & BCHLM111 & 0.803 & 1.35 & 6.70 & 0.530 & $<0.020$ & $<0.050$ & 0.380 & $<0.010$ & 0.065 \\
\hline $\mathrm{V}$ & LRM & 1 & 1 & 2 & LRMLM111 & 0.060 & 0.056 & 15.6 & 0.130 & 0.079 & 0.078 & 0.060 & $<0.010$ & 0.630 \\
\hline $\mathrm{V}$ & LL-06 & 1 & 1 & 3 & v10LM21 & 0.224 & 2.12 & 10.5 & 0.693 & $<0.020$ & 0.373 & $<0.010$ & 0.023 & 0.078 \\
\hline $\mathrm{V}$ & LL-10 & 1 & 1 & 4 & v04LM11 & 0.163 & 2.07 & 8.20 & 0.699 & $<0.020$ & 0.354 & $<0.010$ & 0.020 & 0.089 \\
\hline $\mathrm{V}$ & BS-08 & 1 & 1 & 5 & v02LM11 & 0.202 & 2.36 & 9.73 & 0.777 & $<0.020$ & 0.360 & $<0.010$ & 0.023 & 0.083 \\
\hline $\mathrm{V}$ & LL-05 & 1 & 1 & 6 & v03LM11 & 0.177 & 2.18 & 9.79 & 0.695 & $<0.020$ & 0.366 & $<0.010$ & 0.022 & 0.077 \\
\hline $\mathrm{V}$ & LL-12 & 1 & 1 & 7 & v06LM21 & 0.172 & 2.17 & 8.73 & 0.698 & $<0.020$ & 0.375 & $<0.010$ & 0.022 & 0.100 \\
\hline $\mathrm{V}$ & Batch 1 & 1 & 1 & 8 & BCHLM112 & 0.803 & 1.36 & 6.82 & 0.529 & $<0.020$ & $<0.050$ & 0.380 & $<0.010$ & 0.066 \\
\hline $\mathrm{V}$ & LRM & 1 & 1 & 9 & LRMLM112 & 0.059 & 0.056 & 15.9 & 0.130 & 0.079 & 0.083 & 0.058 & $<0.010$ & 0.622 \\
\hline $\mathrm{V}$ & LL-10 & 1 & 1 & 10 & v04LM21 & 0.163 & 2.06 & 8.17 & 0.706 & $<0.020$ & 0.356 & $<0.010$ & 0.021 & 0.089 \\
\hline $\mathrm{V}$ & LL-12 & 1 & 1 & 11 & v06LM11 & 0.172 & 2.01 & 8.06 & 0.688 & $<0.020$ & 0.376 & $<0.010$ & 0.022 & 0.079 \\
\hline $\mathrm{V}$ & BS-08 & 1 & 1 & 12 & v02LM21 & 0.201 & 2.27 & 9.36 & 0.773 & $<0.020$ & 0.364 & $<0.010$ & 0.023 & 0.084 \\
\hline $\mathrm{V}$ & LL-06 & 1 & 1 & 13 & v10LM11 & 0.223 & 2.04 & 10.2 & 0.695 & $<0.020$ & 0.380 & $<0.010$ & 0.023 & 0.078 \\
\hline $\mathrm{V}$ & LL-05 & 1 & 1 & 14 & v03LM21 & 0.177 & 2.17 & 9.80 & 0.698 & $<0.020$ & 0.369 & $<0.010$ & 0.021 & 0.079 \\
\hline $\mathrm{V}$ & Batch 1 & 1 & 1 & 15 & BCHLM113 & 0.811 & 1.28 & 6.45 & 0.535 & $<0.020$ & $<0.050$ & 0.388 & $<0.010$ & 0.066 \\
\hline $\mathrm{V}$ & LRM & 1 & 1 & 16 & LRMLM113 & 0.059 & 0.056 & 15.1 & 0.131 & 0.079 & 0.077 & 0.059 & $<0.010$ & 0.628 \\
\hline $\mathrm{V}$ & Batch 1 & 1 & 2 & 1 & BCHLM121 & 0.804 & 1.34 & 6.63 & 0.529 & $<0.020$ & $<0.050$ & 0.381 & $<0.010$ & 0.066 \\
\hline $\mathrm{V}$ & LRM & 1 & 2 & 2 & LRMLM121 & 0.059 & 0.057 & 15.3 & 0.130 & 0.079 & 0.084 & 0.058 & $<0.010$ & 0.618 \\
\hline $\mathrm{V}$ & LL-12 & 1 & 2 & 3 & v06LM22 & 0.171 & 2.08 & 8.23 & 0.684 & $<0.020$ & 0.376 & $<0.010$ & 0.023 & 0.080 \\
\hline $\mathrm{V}$ & LL-12 & 1 & 2 & 4 & v06LM12 & 0.173 & 2.07 & 8.14 & 0.699 & $<0.020$ & 0.375 & $<0.010$ & 0.023 & 0.101 \\
\hline $\mathrm{V}$ & LL-05 & 1 & 2 & 5 & v03LM12 & 0.178 & 2.09 & 9.19 & 0.691 & $<0.020$ & 0.373 & $<0.010$ & 0.022 & 0.079 \\
\hline $\mathrm{V}$ & LL-10 & 1 & 2 & 6 & v04LM12 & 0.165 & 2.06 & 7.99 & 0.705 & $<0.020$ & 0.355 & $<0.010$ & 0.022 & 0.091 \\
\hline $\mathrm{V}$ & BS-08 & 1 & 2 & 7 & v02LM12 & 0.202 & 2.29 & 9.21 & 0.776 & $<0.020$ & 0.363 & $<0.010$ & 0.024 & 0.093 \\
\hline $\mathrm{V}$ & Batch 1 & 1 & 2 & 8 & BCHLM122 & 0.805 & 1.33 & 6.44 & 0.530 & $<0.020$ & $<0.050$ & 0.382 & $<0.010$ & 0.067 \\
\hline $\mathrm{V}$ & LRM & 1 & 2 & 9 & LRMLM122 & 0.059 & 0.057 & 15.1 & 0.132 & 0.079 & 0.082 & 0.059 & $<0.010$ & 0.626 \\
\hline $\mathrm{V}$ & BS-08 & 1 & 2 & 10 & v02LM22 & 0.202 & 2.32 & 9.16 & 0.778 & $<0.020$ & 0.366 & $<0.010$ & 0.024 & 0.085 \\
\hline $\mathrm{V}$ & LL-06 & 1 & 2 & 11 & v10LM22 & 0.226 & 2.12 & 10.1 & 0.699 & $<0.020$ & 0.381 & $<0.010$ & 0.024 & 0.079 \\
\hline $\mathrm{V}$ & LL-05 & 1 & 2 & 12 & v03LM22 & 0.178 & 2.07 & 8.87 & 0.692 & $<0.020$ & 0.374 & $<0.010$ & 0.022 & 0.081 \\
\hline $\mathrm{V}$ & LL-06 & 1 & 2 & 13 & v10LM12 & 0.223 & 2.10 & 9.96 & 0.698 & $<0.020$ & 0.374 & $<0.010$ & 0.024 & 0.079 \\
\hline $\mathrm{V}$ & $\begin{array}{l}\text { LL-10 } \\
\end{array}$ & 1 & 2 & 14 & v04LM22 & 0.164 & 2.03 & 7.62 & 0.707 & $<0.020$ & 0.354 & $<0.010$ & 0.021 & 0.090 \\
\hline $\mathrm{V}$ & Batch 1 & 1 & 2 & 15 & BCHLM123 & 0.808 & 1.33 & 6.30 & 0.535 & $<0.020$ & $<0.050$ & 0.390 & $<0.010$ & 0.067 \\
\hline $\mathrm{V}$ & LRM & 1 & 2 & 16 & LRMLM123 & 0.059 & 0.057 & 14.5 & 0.132 & 0.079 & 0.081 & 0.060 & $<0.010$ & 0.628 \\
\hline $\mathrm{V}$ & Batch 1 & 2 & 1 & 1 & BCHLM211 & 0.804 & 1.29 & 6.56 & 0.531 & $<0.020$ & $<0.050$ & 0.380 & $<0.010$ & 0.066 \\
\hline $\mathrm{V}$ & LRM & 2 & 1 & 2 & LRMLM211 & 0.059 & 0.056 & 15.5 & 0.130 & 0.078 & 0.078 & 0.058 & $<0.010$ & 0.622 \\
\hline $\mathrm{V}$ & LL-11 & 2 & 1 & 3 & v01LM21 & 0.175 & 2.06 & 8.25 & 0.715 & $<0.020$ & 0.395 & $<0.010$ & 0.022 & 0.088 \\
\hline $\mathrm{V}$ & BS-09 & 2 & 1 & 4 & v05LM21 & 0.190 & 2.28 & 9.38 & 0.790 & $<0.020$ & 0.367 & $<0.010$ & 0.024 & 0.090 \\
\hline $\mathrm{V}$ & BS-09 & 2 & 1 & 5 & v05LM11 & 0.192 & 2.28 & 9.36 & 0.801 & $<0.020$ & 0.369 & $<0.010$ & 0.024 & 0.091 \\
\hline $\mathrm{V}$ & BS-07 & 2 & 1 & 6 & v07LM21 & 0.197 & 2.32 & 9.42 & 0.781 & $<0.020$ & 0.357 & $<0.010$ & 0.026 & 0.089 \\
\hline $\mathrm{V}$ & $\begin{array}{l}\text { LL-03 } \\
\end{array}$ & 2 & 1 & 7 & v08LM11 & 0.149 & 1.73 & 8.84 & 0.593 & $<0.020$ & 0.292 & $<0.010$ & 0.020 & 0.070 \\
\hline $\mathrm{V}$ & Batch 1 & 2 & 1 & 8 & BCHLM212 & 0.805 & 1.33 & 6.72 & 0.533 & $<0.020$ & $<0.050$ & 0.389 & $<0.010$ & 0.067 \\
\hline $\mathrm{V}$ & LRM & 2 & 1 & 9 & LRMLM212 & 0.059 & 0.056 & 15.9 & 0.131 & 0.079 & 0.086 & 0.059 & $<0.010$ & 0.631 \\
\hline $\mathrm{V}$ & LL-03 & 2 & 1 & 10 & v08LM21 & 0.149 & 1.79 & 9.08 & 0.606 & $<0.020$ & 0.288 & $<0.010$ & 0.020 & 0.069 \\
\hline $\mathrm{V}$ & LL-11 & 2 & 1 & 11 & v01LM11 & 0.175 & 2.13 & 8.52 & 0.713 & $<0.020$ & 0.401 & $<0.010$ & 0.022 & 0.093 \\
\hline
\end{tabular}


Table A-2. Measured Elemental Concentrations for the Glasses Prepared Using Lithium Metaborate (part 2) (continued)

\begin{tabular}{|c|c|c|c|c|c|c|c|c|c|c|c|c|c|c|}
\hline Series & Glass ID & Block & Sub-Blk & Sequence & Lab ID & Mg (wt \%) & Mn (wt \%) & Na (wt \%) & Ni (wt \%) & $\mathrm{Pb}(\mathrm{wt} \%)$ & S (wt \%) & Ti (wt \%) & Zn (wt \%) & $\operatorname{Zr}$ (wt \%) \\
\hline $\mathrm{V}$ & BS-06 & 2 & 1 & 12 & v09LM11 & 0.190 & 2.33 & 9.84 & 0.770 & $<0.020$ & 0.357 & $<<0.010$ & 0.026 & 0.098 \\
\hline $\mathrm{V}$ & BS-07 & 2 & 1 & 13 & v07LM11 & 0.195 & 2.36 & 9.51 & 0.779 & $<0.020$ & 0.346 & $<0.010$ & 0.026 & 0.089 \\
\hline $\mathrm{V}$ & BS-06 & 2 & 1 & 14 & v09LM21 & 0.194 & 2.42 & 10.2 & 0.788 & $<0.020$ & 0.364 & $<0.010$ & 0.027 & 0.107 \\
\hline $\mathrm{V}$ & Batch 1 & 2 & 1 & 15 & BCHLM213 & 0.794 & 1.30 & 6.52 & 0.525 & $<0.020$ & $<0.050$ & 0.375 & $<0.010$ & 0.065 \\
\hline $\mathrm{V}$ & LRM & 2 & 1 & 16 & LRMLM213 & 0.058 & 0.055 & 15.3 & 0.128 & 0.077 & 0.079 & 0.057 & $<0.010$ & 0.616 \\
\hline $\mathrm{V}$ & Batch 1 & 2 & 2 & 1 & BCHLM221 & 0.801 & 1.30 & 6.40 & 0.530 & $<0.020$ & $<0.050$ & 0.381 & $<0.010$ & 0.064 \\
\hline $\mathrm{V}$ & LRM & 2 & 2 & 2 & LRMLM221 & 0.058 & 0.055 & 15.4 & 0.128 & 0.076 & 0.075 & 0.057 & $<0.010$ & 0.618 \\
\hline $\mathrm{V}$ & LL-03 & 2 & 2 & 3 & v08LM22 & 0.149 & 1.75 & 8.76 & 0.601 & $<0.020$ & 0.282 & $<0.010$ & 0.018 & 0.069 \\
\hline $\mathrm{V}$ & LL-11 & 2 & 2 & 4 & v01LM22 & 0.174 & 2.09 & 8.27 & 0.711 & $<0.020$ & 0.394 & $<0.010$ & 0.021 & 0.085 \\
\hline $\mathrm{V}$ & LL-03 & 2 & 2 & 5 & v08LM12 & 0.148 & 1.75 & 8.77 & 0.589 & $<0.020$ & 0.277 & $<0.010$ & 0.018 & 0.068 \\
\hline $\mathrm{V}$ & BS-09 & 2 & 2 & 6 & v05LM12 & 0.190 & 2.33 & 9.46 & 0.794 & $<0.020$ & 0.365 & $<0.010$ & 0.023 & 0.089 \\
\hline $\mathrm{V}$ & BS-07 & 2 & 2 & 7 & v07LM22 & 0.195 & 2.34 & 9.41 & 0.774 & $<0.020$ & 0.341 & $<0.010$ & 0.025 & 0.087 \\
\hline $\mathrm{V}$ & Batch 1 & 2 & 2 & 8 & BCHLM222 & 0.801 & 1.35 & 6.65 & 0.530 & $<0.020$ & $<0.050$ & 0.381 & $<0.010$ & 0.064 \\
\hline $\mathrm{V}$ & LRM & 2 & 2 & 9 & LRMLM222 & 0.058 & 0.055 & 15.4 & 0.128 & 0.076 & 0.078 & 0.057 & $<0.010$ & 0.622 \\
\hline $\mathrm{V}$ & BS-06 & 2 & 2 & 10 & v09LM12 & 0.192 & 2.36 & 9.95 & 0.777 & $<0.020$ & 0.363 & $<0.010$ & 0.026 & 0.098 \\
\hline $\mathrm{V}$ & LL-11 & 2 & 2 & 11 & v01LM12 & 0.175 & 2.13 & 8.46 & 0.717 & $<0.020$ & 0.404 & $<0.010$ & 0.021 & 0.092 \\
\hline $\mathrm{V}$ & BS-06 & 2 & 2 & 12 & v09LM22 & 0.196 & 2.42 & 10.2 & 0.801 & $<0.020$ & 0.366 & $<0.010$ & 0.026 & 0.107 \\
\hline $\mathrm{V}$ & BS-09 & 2 & 2 & 13 & v05LM22 & 0.189 & 2.37 & 9.66 & 0.789 & $<0.020$ & 0.373 & $<0.010$ & 0.023 & 0.089 \\
\hline $\mathrm{V}$ & BS-07 & 2 & 2 & 14 & v07LM12 & 0.197 & 2.35 & 9.48 & 0.787 & $<0.020$ & 0.351 & $<0.010$ & 0.025 & 0.088 \\
\hline $\mathrm{V}$ & Batch 1 & 2 & 2 & 15 & BCHLM 223 & 0.803 & 1.37 & 6.75 & 0.530 & $<0.020$ & $<0.050$ & 0.389 & $<0.010$ & 0.065 \\
\hline $\mathrm{V}$ & LRM & 2 & 2 & 16 & LRMLM223 & 0.058 & 0.055 & 15.7 & 0.129 & 0.078 & 0.081 & 0.058 & $<0.010$ & 0.626 \\
\hline $\mathrm{W}$ & Batch 1 & 1 & 1 & 1 & BCHLM111 & 0.789 & 1.35 & 6.58 & 0.520 & $<0.020$ & $<0.050$ & 0.387 & $<0.010$ & 0.065 \\
\hline $\mathrm{W}$ & LRM & 1 & 1 & 2 & LRMLM111 & 0.059 & 0.056 & 15.0 & 0.130 & 0.078 & 0.082 & 0.059 & $<0.010$ & 0.625 \\
\hline $\mathrm{W}$ & LT-12 & 1 & 1 & 3 & w10LM21 & 0.185 & 2.09 & 8.26 & 0.719 & $<0.020$ & 0.376 & $<0.010$ & 0.021 & 0.088 \\
\hline $\mathrm{W}$ & LT-01 & 1 & 1 & 4 & w04LM11 & 0.123 & 1.72 & 6.34 & 0.586 & $<0.020$ & 0.276 & $<0.010$ & 0.020 & 0.072 \\
\hline $\mathrm{W}$ & LT-11 & 1 & 1 & 5 & w02LM11 & 0.179 & 2.06 & 8.16 & 0.711 & $<0.020$ & 0.377 & $<0.010$ & 0.020 & 0.082 \\
\hline $\mathrm{W}$ & LT-10 & 1 & 1 & 6 & w03LM11 & 0.170 & 2.08 & 8.20 & 0.737 & $<0.020$ & 0.405 & $<0.010$ & 0.022 & 0.078 \\
\hline $\mathrm{W}$ & QB-03 & 1 & 1 & 7 & w06LM21 & 0.164 & 1.75 & 9.24 & 0.703 & $<0.020$ & 0.327 & $<0.010$ & 0.023 & 0.078 \\
\hline $\mathrm{W}$ & Batch 1 & 1 & 1 & 8 & BCHLM112 & 0.817 & 1.34 & 6.50 & 0.545 & $<0.020$ & $<0.050$ & 0.399 & $<0.010$ & 0.068 \\
\hline $\mathrm{W}$ & LRM & 1 & 1 & 9 & LRMLM112 & 0.060 & 0.057 & 14.8 & 0.133 & 0.079 & 0.093 & 0.060 & $<0.010$ & 0.638 \\
\hline $\mathrm{W}$ & $\begin{array}{l}\text { LT-01 } \\
\end{array}$ & 1 & 1 & 10 & w04LM21 & 0.125 & 1.69 & 6.23 & 0.590 & $<0.020$ & 0.292 & $<0.010$ & 0.021 & 0.067 \\
\hline $\mathrm{W}$ & QB-03 & 1 & 1 & 11 & w06LM11 & 0.166 & 1.79 & 9.57 & 0.705 & $<0.020$ & 0.331 & $<0.010$ & 0.024 & 0.084 \\
\hline W & LT-11 & 1 & 1 & 12 & w02LM21 & 0.183 & 2.09 & 8.27 & 0.735 & $<0.020$ & 0.395 & $<0.010$ & 0.021 & 0.085 \\
\hline $\mathrm{W}$ & LT-12 & 1 & 1 & 13 & w10LM11 & 0.187 & 2.09 & 8.21 & 0.726 & $<0.020$ & 0.400 & $<0.010$ & 0.022 & 0.087 \\
\hline $\mathrm{W}$ & LT-10 & 1 & 1 & 14 & w03LM21 & 0.164 & 2.05 & 8.03 & 0.708 & $<0.020$ & 0.395 & $<0.010$ & 0.022 & 0.076 \\
\hline $\mathrm{W}$ & Batch 1 & 1 & 1 & 15 & BCHLM113 & 0.817 & 1.33 & 6.44 & 0.542 & $<0.020$ & $<0.050$ & 0.398 & $<0.010$ & 0.068 \\
\hline $\mathrm{W}$ & LRM & 1 & 1 & 16 & LRMLM113 & 0.060 & 0.057 & 14.9 & 0.132 & 0.079 & 0.083 & 0.061 & $<0.010$ & 0.640 \\
\hline $\mathrm{W}$ & Batch 1 & 1 & 2 & 1 & BCHLM121 & 0.798 & 1.38 & 6.78 & 0.528 & $<0.020$ & $<0.050$ & 0.381 & $<0.010$ & 0.065 \\
\hline $\mathrm{W}$ & LRM & 1 & 2 & 2 & LRMLM121 & 0.059 & 0.056 & 15.6 & 0.129 & 0.077 & 0.076 & 0.057 & $<0.010$ & 0.615 \\
\hline $\mathrm{W}$ & QB-03 & 1 & 2 & 3 & w06LM22 & 0.162 & 1.83 & 9.72 & 0.685 & $<0.020$ & 0.311 & $<0.010$ & 0.024 & 0.076 \\
\hline $\mathrm{W}$ & QB-03 & 1 & 2 & 4 & w06LM12 & 0.165 & 1.87 & 10.1 & 0.695 & $<0.020$ & 0.320 & $<0.010$ & 0.024 & 0.083 \\
\hline $\mathrm{W}$ & LT-10 & 1 & 2 & 5 & w03LM12 & 0.166 & 2.16 & 8.59 & 0.713 & $<0.020$ & 0.383 & $<0.010$ & 0.022 & 0.076 \\
\hline $\mathrm{W}$ & LT-01 & 1 & 2 & 6 & w04LM12 & 0.123 & 1.77 & 6.60 & 0.581 & $<0.020$ & 0.270 & $<0.010$ & 0.021 & 0.071 \\
\hline
\end{tabular}


Table A-2. Measured Elemental Concentrations for the Glasses Prepared Using Lithium Metaborate (part 2) (continued)

\begin{tabular}{|c|c|c|c|c|c|c|c|c|c|c|c|c|c|c|}
\hline Series & Glass ID & Block & Sub-Blk & Sequence & Lab ID & Mg (wt \%) & Mn (wt \%) & Na (wt \%) & Ni (wt \%) & $\mathrm{Pb}(\mathrm{wt} \%)$ & S (wt \%) & Ti (wt \%) & Zn (wt \%) & $\operatorname{Zr}$ (wt \%) \\
\hline $\mathrm{W}$ & LT-11 & 1 & 2 & 7 & w02LM12 & 0.177 & 2.14 & 8.53 & 0.699 & $<0.020$ & 0.366 & $<<0.010$ & 0.020 & 0.080 \\
\hline $\mathrm{W}$ & Batch 1 & 1 & 2 & 8 & BCHLM122 & 0.791 & 1.37 & 6.78 & 0.521 & $<0.020$ & $<0.050$ & 0.377 & $<0.010$ & 0.065 \\
\hline $\mathrm{W}$ & LRM & 1 & 2 & 9 & LRMLM122 & 0.059 & 0.056 & 15.7 & 0.130 & 0.077 & 0.077 & 0.057 & $<0.010$ & 0.614 \\
\hline $\mathrm{W}$ & LT-11 & 1 & 2 & 10 & w02LM22 & 0.180 & 2.15 & 8.59 & 0.718 & $<0.020$ & 0.370 & $<0.010$ & 0.021 & 0.083 \\
\hline $\mathrm{W}$ & LT-12 & 1 & 2 & 11 & w10LM22 & 0.183 & 2.16 & 8.58 & 0.710 & $<0.020$ & 0.376 & $<0.010$ & 0.022 & 0.085 \\
\hline $\mathrm{W}$ & LT-10 & 1 & 2 & 12 & w03LM22 & 0.161 & 2.09 & 8.38 & 0.687 & $<0.020$ & 0.372 & $<0.010$ & 0.022 & 0.073 \\
\hline $\mathrm{W}$ & LT-12 & 1 & 2 & 13 & w10LM12 & 0.183 & 2.15 & 8.60 & 0.702 & $<0.020$ & 0.374 & $<0.010$ & 0.022 & 0.084 \\
\hline $\mathrm{W}$ & $\begin{array}{l}\text { LT-01 } \\
\end{array}$ & 1 & 2 & 14 & w04LM22 & 0.123 & 1.74 & 6.49 & 0.575 & $<0.020$ & 0.273 & $<0.010$ & 0.021 & 0.065 \\
\hline $\mathrm{W}$ & Batch 1 & 1 & 2 & 15 & BCHLM123 & 0.795 & 1.36 & 6.69 & 0.521 & $<0.020$ & $<0.050$ & 0.382 & $<0.010$ & 0.066 \\
\hline $\mathrm{W}$ & LRM & 1 & 2 & 16 & LRMLM123 & 0.060 & 0.057 & 15.2 & 0.131 & 0.077 & 0.082 & 0.058 & $<0.010$ & 0.622 \\
\hline $\mathrm{W}$ & Batch 1 & 2 & 1 & 1 & BCHLM211 & 0.795 & 1.34 & 6.63 & 0.525 & $<0.020$ & $<0.050$ & 0.384 & $<0.010$ & 0.065 \\
\hline $\mathrm{W}$ & LRM & 2 & 1 & 2 & LRMLM211 & 0.058 & 0.056 & 15.1 & 0.130 & 0.080 & 0.085 & 0.058 & $<0.010$ & 0.619 \\
\hline $\mathrm{W}$ & LT-04 & 2 & 1 & 3 & w01LM21 & 0.171 & 2.03 & 8.12 & 0.733 & $<0.020$ & 0.350 & $<0.010$ & 0.021 & 0.083 \\
\hline $\mathrm{W}$ & LOL-12 & 2 & 1 & 4 & w05LM21 & 0.131 & 1.74 & 10.5 & 0.703 & $<0.020$ & 0.204 & 0.260 & 0.025 & 0.062 \\
\hline $\mathrm{W}$ & LOL-12 & 2 & 1 & 5 & w05LM11 & 0.131 & 1.69 & 10.2 & 0.699 & $<0.020$ & 0.201 & 0.262 & 0.025 & 0.063 \\
\hline $\mathrm{W}$ & LT-05 & 2 & 1 & 6 & w07LM21 & 0.189 & 2.04 & 8.98 & 0.732 & $<0.020$ & 0.367 & $<0.010$ & 0.018 & 0.098 \\
\hline $\mathrm{W}$ & QB-04 & 2 & 1 & 7 & w08LM11 & 0.167 & 1.75 & 11.1 & 0.712 & $<0.020$ & 0.323 & $<0.010$ & 0.022 & 0.091 \\
\hline $\mathrm{W}$ & Batch 1 & 2 & 1 & 8 & BCHLM212 & 0.800 & 1.32 & 6.56 & 0.525 & $<0.020$ & $<0.050$ & 0.381 & $<0.010$ & 0.066 \\
\hline $\mathrm{W}$ & LRM & 2 & 1 & 9 & LRMLM212 & 0.059 & 0.057 & 15.2 & 0.132 & 0.078 & 0.078 & 0.059 & $<0.010$ & 0.629 \\
\hline $\mathrm{W}$ & QB-04 & 2 & 1 & 10 & w08LM21 & 0.167 & 1.74 & 11.0 & 0.714 & $<0.020$ & 0.328 & $<0.010$ & 0.022 & 0.094 \\
\hline $\mathrm{W}$ & $\begin{array}{l}\text { LT-04 } \\
\text { L }\end{array}$ & 2 & 1 & 11 & w01LM11 & 0.176 & 2.03 & 8.20 & 0.752 & $<0.020$ & 0.359 & $<0.010$ & 0.023 & 0.088 \\
\hline $\mathrm{W}$ & LT-06 & 2 & 1 & 12 & w09LM11 & 0.181 & 2.02 & 9.49 & 0.771 & $<0.020$ & 0.380 & $<0.010$ & 0.020 & 0.085 \\
\hline $\mathrm{W}$ & LT-05 & 2 & 1 & 13 & w07LM11 & 0.189 & 2.01 & 8.87 & 0.730 & $<0.020$ & 0.370 & $<0.010$ & 0.018 & 0.097 \\
\hline $\mathrm{W}$ & LT-06 & 2 & 1 & 14 & w09LM21 & 0.182 & 2.05 & 9.56 & 0.737 & $<0.020$ & 0.377 & $<0.010$ & 0.020 & 0.083 \\
\hline $\mathrm{W}$ & Batch 1 & 2 & 1 & 15 & BCHLM213 & 0.824 & 1.31 & 6.57 & 0.547 & $<0.020$ & $<0.050$ & 0.401 & $<0.010$ & 0.068 \\
\hline $\mathrm{W}$ & LRM & 2 & 1 & 16 & LRMLM213 & 0.060 & 0.058 & 14.8 & 0.134 & 0.080 & 0.081 & 0.060 & $<0.010$ & 0.635 \\
\hline $\mathrm{W}$ & Batch 1 & 2 & 2 & 1 & BCHLM 221 & 0.799 & 1.36 & 6.59 & 0.529 & $<0.020$ & $<0.050$ & 0.379 & $<0.010$ & 0.064 \\
\hline $\mathrm{W}$ & LRM & 2 & 2 & 2 & LRMLM221 & 0.057 & 0.055 & 15.1 & 0.128 & 0.076 & 0.075 & 0.058 & $<0.010$ & 0.615 \\
\hline $\mathrm{W}$ & QB-04 & 2 & 2 & 3 & w08LM22 & 0.164 & 1.80 & 11.0 & 0.705 & $<0.020$ & 0.311 & $<0.010$ & 0.021 & 0.092 \\
\hline $\mathrm{W}$ & $\begin{array}{l}\text { LT-04 } \\
\end{array}$ & 2 & 2 & 4 & w01LM22 & 0.170 & 2.10 & 8.28 & 0.732 & $<0.020$ & 0.345 & $<0.010$ & 0.020 & 0.082 \\
\hline $\mathrm{W}$ & QB-04 & 2 & 2 & 5 & w08LM12 & 0.164 & 1.80 & 11.2 & 0.705 & $<0.020$ & 0.312 & $<0.010$ & 0.021 & 0.089 \\
\hline $\mathrm{W}$ & LOL-12 & 2 & 2 & 6 & w05LM12 & 0.129 & 1.82 & 10.7 & 0.690 & $<0.020$ & 0.191 & 0.258 & 0.024 & 0.061 \\
\hline W & LT-05 & 2 & 2 & 7 & w07LM22 & 0.186 & 2.13 & 9.22 & 0.726 & $<0.020$ & 0.352 & $<0.010$ & 0.017 & 0.096 \\
\hline $\mathrm{W}$ & Batch 1 & 2 & 2 & 8 & BCHLM222 & 0.781 & 1.39 & 6.72 & 0.513 & $<0.020$ & $<0.050$ & 0.370 & $<0.010$ & 0.064 \\
\hline $\mathrm{W}$ & LRM & 2 & 2 & 9 & LRMLM222 & 0.057 & 0.055 & 15.4 & 0.128 & 0.076 & 0.078 & 0.057 & $<0.010$ & 0.614 \\
\hline $\mathrm{W}$ & LT-06 & 2 & 2 & 10 & w09LM12 & 0.177 & 2.14 & 9.76 & 0.755 & $<0.020$ & 0.370 & $<0.010$ & 0.019 & 0.082 \\
\hline $\mathrm{W}$ & LT-04 & 2 & 2 & 11 & w01LM12 & 0.170 & 2.17 & 8.56 & 0.729 & $<0.020$ & 0.346 & $<0.010$ & 0.021 & 0.085 \\
\hline $\mathrm{W}$ & LT-06 & 2 & 2 & 12 & w09LM22 & 0.179 & 2.17 & 9.88 & 0.725 & $<0.020$ & 0.371 & $<0.010$ & 0.019 & 0.080 \\
\hline $\mathrm{W}$ & LOL-12 & 2 & 2 & 13 & w05LM22 & 0.129 & 1.80 & 10.6 & 0.690 & $<0.020$ & 0.196 & 0.256 & 0.024 & 0.061 \\
\hline $\mathrm{W}$ & LT-05 & 2 & 2 & 14 & w07LM12 & 0.185 & 2.10 & 9.06 & 0.723 & $<0.020$ & 0.361 & $<0.010$ & 0.017 & 0.095 \\
\hline $\mathrm{W}$ & Batch 1 & 2 & 2 & 15 & BCHLM 223 & 0.787 & 1.37 & 6.59 & 0.521 & $<0.020$ & $<0.050$ & 0.377 & $<0.010$ & 0.064 \\
\hline $\mathrm{W}$ & LRM & 2 & 2 & 16 & LRMLM223 & 0.057 & 0.055 & 15.4 & 0.128 & 0.076 & 0.085 & 0.057 & $<0.010$ & 0.610 \\
\hline $\mathrm{X}$ & Batch 1 & 1 & 1 & 1 & BCHLM111 & 0.785 & 1.34 & 6.69 & 0.515 & $<0.020$ & $<0.050$ & 0.374 & $<0.010$ & 0.064 \\
\hline
\end{tabular}


Table A-2. Measured Elemental Concentrations for the Glasses Prepared Using Lithium Metaborate (part 2) (continued)

\begin{tabular}{|c|c|c|c|c|c|c|c|c|c|c|c|c|c|c|}
\hline Series & Glass ID & Block & Sub-Blk & Sequence & Lab ID & Mg (wt \%) & Mn (wt \%) & Na (wt \%) & Ni (wt \%) & $\mathrm{Pb}(\mathrm{wt} \%)$ & S (wt \%) & Ti (wt \%) & Zn (wt \%) & $\operatorname{Zr}$ (wt \%) \\
\hline $\mathrm{X}$ & LRM & 1 & 1 & 2 & LRMLM111 & 0.059 & 0.056 & 15.4 & 0.134 & 0.078 & 0.083 & 0.057 & $<0.010$ & 0.631 \\
\hline $\mathrm{X}$ & QB-34 & 1 & 1 & 3 & x10LM21 & 0.156 & 1.76 & 9.73 & 0.698 & $<0.020$ & 0.368 & $<0.010$ & 0.020 & 0.068 \\
\hline $\mathrm{X}$ & QB-37 & 1 & 1 & 4 & x04LM11 & 0.152 & 2.04 & 12.5 & 0.795 & $<0.020$ & 0.320 & $<0.010$ & 0.024 & 0.082 \\
\hline $\mathrm{X}$ & QB-32 & 1 & 1 & 5 & $\begin{array}{l}\mathrm{x} 02 \mathrm{LM} 11 \\
\end{array}$ & 0.204 & 1.74 & 12.4 & 0.693 & $<0.020$ & 0.373 & $<0.010$ & 0.018 & 0.068 \\
\hline $\mathrm{X}$ & QB-22 & 1 & 1 & 6 & $\begin{array}{l}\mathrm{x} 03 \mathrm{LM} 11 \\
\end{array}$ & 0.204 & 1.76 & 12.6 & 0.701 & $<0.020$ & 0.375 & $<0.010$ & 0.019 & 0.069 \\
\hline $\mathrm{X}$ & QB-35 & 1 & 1 & 7 & x06LM21 & 0.151 & 1.76 & 11.0 & 0.678 & $<0.020$ & 0.369 & $<0.010$ & 0.018 & 0.069 \\
\hline $\mathrm{X}$ & Batch 1 & 1 & 1 & 8 & BCHLM112 & 0.787 & 1.35 & 6.77 & 0.516 & $<0.020$ & $<0.050$ & 0.375 & $<0.010$ & 0.065 \\
\hline $\mathrm{X}$ & LRM & 1 & 1 & 9 & LRMLM112 & 0.059 & 0.056 & 15.6 & 0.133 & 0.078 & 0.082 & 0.057 & $<0.010$ & 0.631 \\
\hline $\mathrm{X}$ & QB-37 & 1 & 1 & 10 & x04LM21 & 0.151 & 2.04 & 12.4 & 0.781 & $<0.020$ & 0.319 & $<0.010$ & 0.023 & 0.081 \\
\hline $\mathrm{X}$ & QB-35 & 1 & 1 & 11 & x06LM11 & 0.151 & 1.80 & 11.3 & 0.677 & $<0.020$ & 0.378 & $<0.010$ & 0.018 & 0.069 \\
\hline $\mathrm{X}$ & QB-32 & 1 & 1 & 12 & x02LM21 & 0.206 & 1.80 & 12.8 & 0.710 & $<0.020$ & 0.383 & $<0.010$ & 0.018 & 0.069 \\
\hline $\mathrm{X}$ & QB-34 & 1 & 1 & 13 & x10LM11 & 0.155 & 1.76 & 9.85 & 0.680 & $<0.020$ & 0.363 & $<0.010$ & 0.020 & 0.067 \\
\hline $\mathrm{X}$ & QB-22 & 1 & 1 & 14 & x03LM21 & 0.204 & 1.77 & 12.6 & 0.701 & $<0.020$ & 0.378 & $<0.010$ & 0.018 & 0.070 \\
\hline $\mathrm{X}$ & Batch 1 & 1 & 1 & 15 & BCHLM113 & 0.799 & 1.34 & 6.68 & 0.526 & $<0.020$ & $<0.050$ & 0.378 & $<0.010$ & 0.065 \\
\hline $\mathrm{X}$ & LRM & 1 & 1 & 16 & LRMLM113 & 0.059 & 0.056 & 15.4 & 0.135 & 0.077 & 0.082 & 0.057 & $<0.010$ & 0.641 \\
\hline $\mathrm{X}$ & Batch 1 & 1 & 2 & 1 & BCHLM121 & 0.802 & 1.34 & 6.57 & 0.530 & $<0.020$ & $<0.050$ & 0.382 & $<0.010$ & 0.066 \\
\hline $\mathrm{X}$ & LRM & 1 & 2 & 2 & LRMLM121 & 0.060 & 0.058 & 15.0 & 0.136 & 0.079 & 0.083 & 0.060 & $<0.010$ & 0.644 \\
\hline $\mathrm{X}$ & QB-35 & 1 & 2 & 3 & x06LM22 & 0.153 & 1.71 & 10.5 & 0.692 & $<0.020$ & 0.376 & $<0.010$ & 0.019 & 0.093 \\
\hline $\mathrm{X}$ & QB-35 & 1 & 2 & 4 & x06LM12 & 0.152 & 1.77 & 10.9 & 0.680 & $<0.020$ & 0.379 & $<0.010$ & 0.019 & 0.071 \\
\hline $\mathrm{X}$ & QB-22 & 1 & 2 & 5 & $\begin{array}{l}\mathrm{x} 03 \mathrm{LM} 12 \\
\end{array}$ & 0.208 & 1.75 & 12.2 & 0.715 & $<0.020$ & 0.382 & $<0.010$ & 0.020 & 0.071 \\
\hline $\mathrm{X}$ & QB-37 & 1 & 2 & 6 & x04LM12 & 0.154 & 2.06 & 12.4 & 0.800 & $<0.020$ & 0.328 & $<0.010$ & 0.025 & 0.083 \\
\hline $\mathrm{X}$ & QB-32 & 1 & 2 & 7 & x02LM12 & 0.209 & 1.80 & 12.6 & 0.709 & $<0.020$ & 0.374 & $<0.010$ & 0.020 & 0.070 \\
\hline $\mathrm{X}$ & Batch 1 & 1 & 2 & 8 & BCHLM122 & 0.794 & 1.36 & 6.69 & 0.521 & $<0.020$ & $<0.050$ & 0.382 & $<0.010$ & 0.066 \\
\hline $\mathrm{X}$ & LRM & 1 & 2 & 9 & LRMLM122 & 0.060 & 0.057 & 15.3 & 0.135 & 0.079 & 0.087 & 0.059 & $<0.010$ & 0.637 \\
\hline $\mathrm{X}$ & QB-32 & 1 & 2 & 10 & $\mathrm{x} 02 \mathrm{LM} 22$ & 0.206 & 1.76 & 12.3 & 0.705 & $<0.020$ & 0.372 & $<0.010$ & 0.019 & 0.070 \\
\hline $\mathrm{X}$ & QB-34 & 1 & 2 & 11 & x10LM22 & 0.156 & 1.78 & 9.68 & 0.692 & $<0.020$ & 0.366 & $<0.010$ & 0.020 & 0.068 \\
\hline $\mathrm{X}$ & QB-22 & 1 & 2 & 12 & x03LM22 & 0.201 & 1.76 & 12.3 & 0.688 & $<0.020$ & 0.368 & $<0.010$ & 0.018 & 0.069 \\
\hline $\mathrm{X}$ & QB-34 & 1 & 2 & 13 & x10LM12 & 0.153 & 1.73 & 9.42 & 0.671 & $<0.020$ & 0.357 & $<0.010$ & 0.020 & 0.067 \\
\hline $\mathrm{X}$ & QB-37 & 1 & 2 & 14 & x04LM22 & 0.150 & 2.03 & 12.2 & 0.779 & $<0.020$ & 0.314 & $<0.010$ & 0.024 & 0.081 \\
\hline $\mathrm{X}$ & Batch 1 & 1 & 2 & 15 & BCHLM123 & 0.781 & 1.34 & 6.57 & 0.511 & $<0.020$ & $<0.050$ & 0.372 & $<0.010$ & 0.065 \\
\hline $\mathrm{X}$ & LRM & 1 & 2 & 16 & LRMLM123 & 0.059 & 0.057 & 15.1 & 0.131 & 0.077 & 0.080 & 0.058 & $<0.010$ & 0.619 \\
\hline $\mathrm{X}$ & Batch 1 & 2 & 1 & 1 & BCHLM211 & 0.802 & 1.33 & 6.58 & 0.527 & $<0.020$ & $<0.050$ & 0.384 & $<0.010$ & 0.067 \\
\hline $\mathrm{X}$ & LRM & 2 & 1 & 2 & LRMLM211 & 0.060 & 0.058 & 14.8 & 0.132 & 0.080 & 0.082 & 0.060 & $<0.010$ & 0.641 \\
\hline $\mathrm{X}$ & QB-19 & 2 & 1 & 3 & $\mathrm{x} 01 \mathrm{LM} 21$ & 0.185 & 1.91 & 12.9 & 0.790 & $<0.020$ & 0.327 & $<0.010$ & 0.024 & 0.091 \\
\hline $\mathrm{X}$ & QB-33 & 2 & 1 & 4 & x05LM21 & 0.144 & 1.67 & 9.17 & 0.682 & $<0.020$ & 0.364 & $<0.010$ & 0.023 & 0.072 \\
\hline $\mathrm{X}$ & QB-33 & 2 & 1 & 5 & $\begin{array}{l}\mathrm{x} 05 \mathrm{LM} 11 \\
\end{array}$ & 0.148 & 1.70 & 9.29 & 0.699 & $<0.020$ & 0.375 & $<0.010$ & 0.023 & 0.073 \\
\hline $\mathrm{X}$ & QB-36 & 2 & 1 & 6 & x07LM21 & 0.153 & 1.70 & 10.5 & 0.711 & $<0.020$ & 0.387 & $<0.010$ & 0.023 & 0.072 \\
\hline $\mathrm{X}$ & QB-20 & 2 & 1 & 7 & x08LM11 & 0.156 & 1.88 & 10.7 & 0.777 & $<0.020$ & 0.314 & $<0.010$ & 0.024 & 0.097 \\
\hline $\mathrm{X}$ & Batch 1 & 2 & 1 & 8 & BCHLM212 & 0.802 & 1.30 & 6.45 & 0.526 & $<0.020$ & $<0.050$ & 0.380 & $<0.010$ & 0.067 \\
\hline $\mathrm{X}$ & LRM & 2 & 1 & 9 & LRMLM212 & 0.059 & 0.058 & 14.6 & 0.131 & 0.079 & 0.083 & 0.059 & $<0.010$ & 0.640 \\
\hline $\mathrm{X}$ & QB-20 & 2 & 1 & 10 & $\mathrm{x} 08 \mathrm{LM} 21$ & 0.155 & 1.89 & 10.8 & 0.776 & $<0.020$ & 0.318 & $<0.010$ & 0.024 & 0.101 \\
\hline $\mathrm{X}$ & QB-19 & 2 & 1 & 11 & $\begin{array}{l}\mathrm{x} 01 \mathrm{LM} 11 \\
\end{array}$ & 0.186 & 1.90 & 12.8 & 0.805 & $<0.020$ & 0.337 & $<0.010$ & 0.024 & 0.080 \\
\hline $\mathrm{X}$ & SPS-02 & 2 & 1 & 12 & x09LM11 & 0.170 & 2.36 & 9.03 & 1.033 & $<0.020$ & 0.187 & $<0.010$ & 0.026 & 0.105 \\
\hline
\end{tabular}


Table A-2. Measured Elemental Concentrations for the Glasses Prepared Using Lithium Metaborate (part 2) (continued)

\begin{tabular}{|c|c|c|c|c|c|c|c|c|c|c|c|c|c|c|}
\hline Series & Glass ID & Block & Sub-Blk & Sequence & Lab ID & Mg (wt \%) & Mn (wt \%) & $\mathrm{Na}(\mathrm{wt} \%)$ & Ni (wt \%) & Pb (wt \%) & S (wt \%) & Ti (wt \%) & Zn (wt \%) & $\mathrm{Zr}$ (wt \%) \\
\hline $\mathrm{X}$ & QB-36 & 2 & 1 & 13 & x07LM11 & 0.154 & 1.74 & 10.8 & 0.701 & $<0.020$ & 0.386 & $<0.010$ & 0.023 & 0.073 \\
\hline $\mathrm{X}$ & SPS-02 & 2 & 1 & 14 & x09LM21 & 0.170 & 2.36 & 9.03 & 1.025 & $<0.020$ & 0.189 & $<0.010$ & 0.028 & 0.104 \\
\hline $\mathrm{X}$ & Batch 1 & 2 & 1 & 15 & BCHLM213 & 0.812 & 1.32 & 6.51 & 0.536 & $<0.020$ & $<0.050$ & 0.384 & $<0.010$ & 0.067 \\
\hline $\mathrm{X}$ & LRM & 2 & 1 & 16 & LRMLM213 & 0.060 & 0.057 & 14.7 & 0.136 & 0.080 & 0.082 & 0.059 & $<0.010$ & 0.643 \\
\hline $\mathrm{X}$ & Batch 1 & 2 & 2 & 1 & BCHLM221 & 0.802 & 1.34 & 6.72 & 0.533 & $<0.020$ & $<0.050$ & 0.384 & $<0.010$ & 0.065 \\
\hline $\mathrm{X}$ & LRM & 2 & 2 & 2 & LRMLM221 & 0.058 & 0.055 & 15.1 & 0.128 & 0.076 & 0.081 & 0.056 & $<0.010$ & 0.614 \\
\hline $\mathrm{X}$ & QB-20 & 2 & 2 & 3 & x08LM22 & 0.152 & 1.93 & 11.1 & 0.756 & $<0.020$ & 0.307 & $<0.010$ & 0.023 & 0.100 \\
\hline $\mathrm{X}$ & QB-19 & 2 & 2 & 4 & x01LM22 & 0.182 & 1.98 & 13.5 & 0.776 & $<0.020$ & 0.318 & $<0.010$ & 0.024 & 0.090 \\
\hline $\mathrm{X}$ & QB-20 & 2 & 2 & 5 & $\begin{array}{l}\mathrm{x} 08 \mathrm{LM} 12 \\
\end{array}$ & 0.154 & 1.92 & 11.0 & 0.771 & $<0.020$ & 0.316 & $<0.010$ & 0.024 & 0.096 \\
\hline $\mathrm{X}$ & QB-33 & 2 & 2 & 6 & x05LM12 & 0.146 & 1.72 & 9.43 & 0.692 & $<0.020$ & 0.370 & $<0.010$ & 0.023 & 0.073 \\
\hline $\mathrm{X}$ & QB-36 & 2 & 2 & 7 & x07LM22 & 0.152 & 1.74 & 10.9 & 0.705 & $<0.020$ & 0.376 & $<0.010$ & 0.022 & 0.071 \\
\hline $\mathrm{X}$ & Batch 1 & 2 & 2 & 8 & BCHLM222 & 0.797 & 1.33 & 6.64 & 0.523 & $<0.020$ & $<0.050$ & 0.378 & $<0.010$ & 0.066 \\
\hline $\mathrm{X}$ & LRM & 2 & 2 & 9 & LRMLM222 & 0.059 & 0.056 & 15.1 & 0.134 & 0.078 & 0.081 & 0.057 & $<0.010$ & 0.633 \\
\hline $\mathrm{X}$ & $\begin{array}{l}\text { SPS-02 } \\
\end{array}$ & 2 & 2 & 10 & x09LM12 & 0.168 & 2.40 & 9.25 & 1.021 & $<0.020$ & 0.186 & $<0.010$ & 0.026 & 0.104 \\
\hline $\mathrm{X}$ & QB-19 & 2 & 2 & 11 & $\mathrm{x} 01 \mathrm{LM} 12$ & 0.183 & 1.96 & 13.3 & 0.797 & $<0.020$ & 0.330 & $<0.010$ & 0.024 & 0.079 \\
\hline $\mathrm{X}$ & SPS-02 & 2 & 2 & 12 & $\begin{array}{l}\text { x09LM22 } \\
\end{array}$ & 0.169 & 2.40 & 9.22 & 1.018 & $<0.020$ & 0.186 & $<0.010$ & 0.026 & 0.103 \\
\hline $\mathrm{X}$ & QB-33 & 2 & 2 & 13 & x05LM22 & 0.142 & 1.71 & 9.50 & 0.670 & $<0.020$ & 0.364 & $<0.010$ & 0.022 & 0.071 \\
\hline $\mathrm{X}$ & QB-36 & 2 & 2 & 14 & $\mathrm{x} 07 \mathrm{LM} 12$ & 0.153 & 1.75 & 10.9 & 0.699 & $<0.020$ & 0.377 & $<0.010$ & 0.022 & 0.073 \\
\hline $\mathrm{X}$ & Batch 1 & 2 & 2 & 15 & BCHLM223 & 0.798 & 1.33 & 6.65 & 0.523 & $<0.020$ & $<0.050$ & 0.377 & $<0.010$ & 0.066 \\
\hline $\mathrm{X}$ & LRM & 2 & 2 & 16 & LRMLM223 & 0.059 & 0.056 & 14.9 & 0.136 & 0.078 & 0.083 & 0.058 & $<0.010$ & 0.641 \\
\hline
\end{tabular}


SRNL-STI-2012-00152

Revision 0

Table A-3. Measured Elemental Concentrations for the Glasses Prepared Using Peroxide Fusion

\begin{tabular}{|c|c|c|c|c|c|c|c|c|}
\hline Series & \begin{tabular}{|l|l|} 
Glass ID \\
\end{tabular} & Block & Sub-Blk & \begin{tabular}{|l|} 
Sequence \\
\end{tabular} & \begin{tabular}{|l|} 
Lab ID \\
\end{tabular} & B (wt \%) & Li (wt \%) & Si (wt \%) \\
\hline $\mathrm{T}$ & Batch 1 & 1 & 1 & 1 & BCHPF111 & 2.45 & 1.99 & 22.4 \\
\hline $\mathrm{T}$ & LRM & 1 & 1 & 2 & LRMPF111 & 2.63 & $<0.100$ & 25.5 \\
\hline $\mathrm{T}$ & BC-04 & 1 & 1 & 3 & t05PF21 & 1.64 & 2.98 & 20.2 \\
\hline $\mathrm{T}$ & AB-06 & 1 & 1 & 4 & t08PF21 & 1.77 & 2.43 & 23.5 \\
\hline $\mathrm{T}$ & AF-01 & 1 & 1 & 5 & t07PF21 & 1.59 & 2.25 & 21.7 \\
\hline $\mathrm{T}$ & AF-01 & 1 & 1 & 6 & t07PF11 & 1.57 & 2.24 & 21.4 \\
\hline $\mathrm{T}$ & AF-03 & 1 & 1 & 7 & t09PF21 & 2.74 & 2.20 & 20.6 \\
\hline $\mathrm{T}$ & Batch 1 & 1 & 1 & 8 & BCHPF112 & 2.53 & 2.11 & 24.3 \\
\hline $\mathrm{T}$ & LRM & 1 & 1 & 9 & LRMPF112 & 2.57 & $<0.100$ & 25.9 \\
\hline $\mathrm{T}$ & BC-04 & 1 & 1 & 10 & t05PF11 & 1.67 & 3.01 & 20.6 \\
\hline $\mathrm{T}$ & AB-06 & 1 & 1 & 11 & t08PF11 & 1.75 & 2.43 & 23.7 \\
\hline $\mathrm{T}$ & AF-02 & 1 & 1 & 12 & t03PF11 & 2.77 & 2.21 & 20.5 \\
\hline $\mathrm{T}$ & AF-02 & 1 & 1 & 13 & t03PF21 & 2.66 & 2.15 & 20.6 \\
\hline $\mathrm{T}$ & AF-03 & 1 & 1 & 14 & t09PF11 & 2.77 & 2.22 & 21.0 \\
\hline $\mathrm{T}$ & Batch 1 & 1 & 1 & 15 & BCHPF113 & 2.43 & 2.06 & 23.7 \\
\hline $\mathrm{T}$ & LRM & 1 & 1 & 16 & LRMPF113 & 2.51 & $<<0.100$ & 25.4 \\
\hline $\mathrm{T}$ & Batch 1 & 1 & 2 & 1 & BCHPF121 & 2.46 & 1.97 & 23.0 \\
\hline $\mathrm{T}$ & LRM & 1 & 2 & 2 & LRMPF121 & 2.34 & $<0.100$ & 24.2 \\
\hline $\mathrm{T}$ & AF-02 & 1 & 2 & 3 & t03PF12 & 2.65 & 2.10 & 20.7 \\
\hline $\mathrm{T}$ & BC-04 & 1 & 2 & 4 & t05PF12 & 1.47 & 2.85 & 20.8 \\
\hline $\mathrm{T}$ & AF-03 & 1 & 2 & 5 & t09PF22 & 2.65 & 2.14 & 20.5 \\
\hline $\mathrm{T}$ & AF-03 & 1 & 2 & 6 & t09PF12 & 2.65 & 2.13 & 20.7 \\
\hline $\mathrm{T}$ & AB-06 & 1 & 2 & 7 & t08PF22 & 1.62 & 2.33 & 23.1 \\
\hline $\mathrm{T}$ & Batch 1 & 1 & 2 & 8 & BCHPF122 & 2.34 & 2.00 & 23.7 \\
\hline $\mathrm{T}$ & LRM & 1 & 2 & 9 & LRMPF122 & 2.31 & $<0.100$ & 24.9 \\
\hline $\mathrm{T}$ & AF-01 & 1 & 2 & 10 & \begin{tabular}{|l|}
$\mathrm{t} 07 \mathrm{PF} 22$ \\
\end{tabular} & 1.49 & 2.15 & 21.3 \\
\hline $\mathrm{T}$ & AF-02 & 1 & 2 & 11 & t03PF22 & 2.54 & 2.08 & 20.3 \\
\hline $\mathrm{T}$ & AF-01 & 1 & 2 & 12 & t07PF12 & 1.47 & 2.17 & 21.4 \\
\hline $\mathrm{T}$ & BC-04 & 1 & 2 & 13 & t05PF22 & 1.47 & 2.89 & 20.2 \\
\hline $\mathrm{T}$ & AB-06 & 1 & 2 & 14 & \begin{tabular}{|l|}
$\mathrm{t} 08 \mathrm{PF} 12$ \\
\end{tabular} & 1.57 & 2.32 & 23.1 \\
\hline $\mathrm{T}$ & Batch 1 & 1 & 2 & 15 & BCHPF123 & 2.36 & 2.02 & 24.1 \\
\hline $\mathrm{T}$ & LRM & 1 & 2 & 16 & LRMPF123 & 2.34 & $<0.100$ & 25.1 \\
\hline $\mathrm{T}$ & Batch 1 & 2 & 1 & 1 & BCHPF211 & 2.36 & 1.93 & 22.4 \\
\hline $\mathrm{T}$ & LRM & 2 & 1 & 2 & LRMPF211 & 2.39 & $<0.100$ & 24.5 \\
\hline $\mathrm{T}$ & BC-03 & 2 & 1 & 3 & t10PF21 & 1.46 & 2.86 & 20.7 \\
\hline $\mathrm{T}$ & AF-04 & 2 & 1 & 4 & t01PF21 & 2.62 & 2.22 & 17.3 \\
\hline $\mathrm{T}$ & AF-05 & 2 & 1 & 5 & t06PF11 & 2.10 & 2.57 & 19.9 \\
\hline $\mathrm{T}$ & BC-01 & 2 & 1 & 6 & t04PF21 & 1.40 & 2.84 & 21.7 \\
\hline $\mathrm{T}$ & BC-01 & 2 & 1 & 7 & t04PF11 & 1.40 & 2.85 & 21.9 \\
\hline $\mathrm{T}$ & Batch 1 & 2 & 1 & 8 & BCHPF212 & 2.36 & 2.01 & 23.5 \\
\hline $\mathrm{T}$ & LRM & 2 & 1 & 9 & LRMPF212 & 2.45 & $<0.100$ & 25.4 \\
\hline $\mathrm{T}$ & BC-02 & 2 & 1 & 10 & t02PF21 & 2.80 & 2.88 & 20.3 \\
\hline $\mathrm{T}$ & AF-04 & 2 & 1 & 11 & t01PF11 & 2.65 & 2.24 & 17.4 \\
\hline $\mathrm{T}$ & BC-02 & 2 & 1 & 12 & t02PF11 & 2.85 & 2.96 & 20.8 \\
\hline $\mathrm{T}$ & BC-03 & 2 & 1 & 13 & t10PF11 & 1.43 & 2.88 & 20.8 \\
\hline $\mathrm{T}$ & AF-05 & 2 & 1 & 14 & t06PF21 & 2.05 & 2.55 & 19.8 \\
\hline $\mathrm{T}$ & Batch 1 & 2 & 1 & 15 & BCHPF213 & 2.30 & 1.98 & 23.1 \\
\hline $\mathrm{T}$ & LRM & 2 & 1 & 16 & LRMPF213 & 2.39 & $<0.100$ & 25.2 \\
\hline $\mathrm{T}$ & Batch 1 & 2 & 2 & 1 & BCHPF221 & 2.46 & 2.01 & 22.7 \\
\hline $\mathrm{T}$ & LRM & 2 & 2 & 2 & LRMPF221 & 2.51 & $<0.100$ & 24.6 \\
\hline $\mathrm{T}$ & BC-02 & 2 & 2 & 3 & $\mathrm{t} 02 \mathrm{PF} 12$ & 2.87 & 2.96 & 20.3 \\
\hline $\mathrm{T}$ & AF-04 & 2 & 2 & 4 & t01PF12 & 2.63 & 2.24 & 16.9 \\
\hline $\mathrm{T}$ & BC-03 & 2 & 2 & 5 & t10PF22 & 1.50 & 2.93 & 20.7 \\
\hline $\mathrm{T}$ & BC-01 & 2 & 2 & 6 & $\mathrm{t} 04 \mathrm{PF} 12$ & 1.51 & 2.98 & 22.4 \\
\hline $\mathrm{T}$ & AF-05 & 2 & 2 & 7 & t06PF12 & 2.16 & 2.63 & 20.1 \\
\hline $\mathrm{T}$ & Batch 1 & 2 & 2 & 8 & BCHPF 222 & 2.28 & 1.98 & 22.3 \\
\hline $\mathrm{T}$ & LRM & 2 & 2 & 9 & LRMPF222 & 2.26 & $<0.100$ & 23.5 \\
\hline $\mathrm{T}$ & AF-04 & 2 & 2 & 10 & t01PF22 & 2.75 & 2.33 & 17.4 \\
\hline $\mathrm{T}$ & $\mathrm{BC}-02$ & 2 & 2 & 11 & t02PF22 & 2.73 & 2.86 & 19.7 \\
\hline $\mathrm{T}$ & $\mathrm{BC}-03$ & 2 & 2 & 12 & t10PF12 & 1.48 & 2.92 & 20.7 \\
\hline $\mathrm{T}$ & BC-01 & 2 & 2 & 13 & t04PF22 & 1.49 & 2.96 & 22.2 \\
\hline $\mathrm{T}$ & AF-05 & 2 & 2 & 14 & t06PF22 & 2.14 & 2.64 & 20.0 \\
\hline $\mathrm{T}$ & Batch 1 & 2 & 2 & 15 & BCHPF223 & 2.41 & 2.06 & 23.3 \\
\hline $\mathrm{T}$ & LRM & 2 & 2 & 16 & LRMPF223 & 2.58 & $<0.100$ & 26.4 \\
\hline $\mathrm{U}$ & Batch 1 & 1 & 1 & 1 & BCHPF111 & 2.48 & 1.97 & 23.3 \\
\hline $\mathrm{U}$ & LRM & 1 & 1 & 2 & LRMPF111 & 2.46 & $<0.100$ & 24.8 \\
\hline
\end{tabular}


SRNL-STI-2012-00152

Revision 0

Table A-3. Measured Elemental Concentrations for the Glasses Prepared Using Peroxide Fusion (continued)

\begin{tabular}{|c|c|c|c|c|c|c|c|c|}
\hline Series & \begin{tabular}{|l|l|} 
Glass ID \\
\end{tabular} & Block & Sub-Blk & Sequence & Lab ID & B (wt \%) & Li (wt \%) & Si (wt \%) \\
\hline $\mathrm{U}$ & BC-06 & 1 & \begin{tabular}{|l|}
1 \\
\end{tabular} & \begin{tabular}{|l|}
3 \\
\end{tabular} & u05PF21 & 2.91 & 2.90 & 19.1 \\
\hline $\mathrm{U}$ & BS-04 & 1 & 1 & 4 & u08PF21 & 2.31 & 2.90 & 19.9 \\
\hline $\mathrm{U}$ & BC-07 & 1 & 1 & 5 & u07PF21 & 2.74 & 2.79 & 16.9 \\
\hline $\mathrm{U}$ & BC-07 & 1 & 1 & 6 & $\mathrm{u} 07 \mathrm{PF} 11$ & 2.67 & 2.73 & 17.5 \\
\hline $\mathrm{U}$ & BS-03 & 1 & 1 & 7 & u09PF21 & 2.28 & 3.18 & 21.3 \\
\hline $\mathrm{U}$ & Batch 1 & 1 & 1 & 8 & BCHPF112 & 2.33 & 1.93 & 23.1 \\
\hline $\mathrm{U}$ & LRM & 1 & 1 & 9 & LRMPF112 & 2.40 & $<0.100$ & 25.2 \\
\hline $\mathrm{U}$ & BC-06 & 1 & 1 & 10 & u05PF11 & 2.81 & 2.82 & 19.1 \\
\hline $\mathrm{U}$ & BS-04 & 1 & 1 & 11 & u08PF11 & 2.21 & 2.82 & 19.2 \\
\hline $\mathrm{U}$ & BC-05 & 1 & 1 & 12 & u03PF11 & 2.68 & 2.76 & 20.9 \\
\hline $\mathrm{U}$ & BC-05 & 1 & 1 & 13 & u03PF21 & 2.71 & 2.77 & 19.3 \\
\hline $\mathrm{U}$ & BS-03 & 1 & 1 & 14 & u09PF11 & 2.29 & 3.19 & 21.1 \\
\hline $\mathrm{U}$ & Batch 1 & 1 & 1 & 15 & BCHPF113 & 2.31 & 1.93 & 23.3 \\
\hline $\mathrm{U}$ & LRM & 1 & 1 & 16 & LRMPF113 & 2.40 & $<0.100$ & 24.9 \\
\hline $\mathrm{U}$ & Batch 1 & 1 & 2 & 1 & BCHPF121 & 2.38 & 1.90 & 23.5 \\
\hline $\mathrm{U}$ & LRM & 1 & 2 & 2 & LRMPF121 & 2.34 & $<0.100$ & 25.1 \\
\hline $\mathrm{U}$ & BC-05 & 1 & 2 & 3 & u03PF12 & 2.73 & 2.83 & 21.3 \\
\hline $\mathrm{U}$ & BC-06 & 1 & 2 & 4 & $\mathrm{u} 05 \mathrm{PF} 12$ & 2.78 & 2.88 & 19.1 \\
\hline $\mathrm{U}$ & BS-03 & 1 & 2 & 5 & $\mathrm{u} 09 \mathrm{PF} 22$ & 2.16 & 3.15 & 21.1 \\
\hline $\mathrm{U}$ & BS-03 & 1 & 2 & 6 & u09PF12 & 2.18 & 3.17 & 20.9 \\
\hline $\mathrm{U}$ & BS-04 & 1 & 2 & 7 & u08PF22 & 2.17 & 2.89 & 19.5 \\
\hline $\mathrm{U}$ & Batch 1 & 1 & 2 & 8 & BCHPF122 & 2.24 & 1.92 & 24.2 \\
\hline $\mathrm{U}$ & LRM & 1 & 2 & 9 & LRMPF122 & 2.30 & $<0.100$ & 25.5 \\
\hline $\mathrm{U}$ & BC-07 & 1 & 2 & 10 & u07PF22 & 2.88 & 2.95 & 16.8 \\
\hline $\mathrm{U}$ & BC-05 & 1 & 2 & 11 & u03PF22 & 2.65 & 2.77 & 18.8 \\
\hline $\mathrm{U}$ & BC-07 & 1 & 2 & 12 & $\mathrm{u} 07 \mathrm{PF} 12$ & 2.84 & 2.95 & 17.2 \\
\hline $\mathrm{U}$ & BC-06 & 1 & 2 & 13 & $\mathrm{u} 05 \mathrm{PF} 22$ & 2.88 & 2.99 & 19.2 \\
\hline $\mathrm{U}$ & BS-04 & 1 & 2 & 14 & u08PF12 & 2.27 & 3.00 & 18.9 \\
\hline $\mathrm{U}$ & Batch 1 & 1 & 2 & 15 & BCHPF123 & 2.44 & 2.03 & 23.9 \\
\hline $\mathrm{U}$ & LRM & 1 & 2 & 16 & LRMPF123 & 2.37 & $<0.100$ & 25.7 \\
\hline $\mathrm{U}$ & Batch 1 & 2 & 1 & 1 & BCHPF211 & 2.43 & 1.94 & 23.1 \\
\hline $\mathrm{U}$ & LRM & 2 & 1 & 2 & LRMPF211 & 2.29 & $<0.100$ & 25.2 \\
\hline $\mathrm{U}$ & BC-09 & 2 & 1 & 3 & u10PF21 & 2.08 & 2.84 & 19.3 \\
\hline $\mathrm{U}$ & BC-08 & 2 & 1 & 4 & u01PF21 & 1.43 & 2.83 & 18.9 \\
\hline $\mathrm{U}$ & BS-02 & 2 & 1 & 5 & u06PF11 & 1.47 & 3.23 & 21.8 \\
\hline $\mathrm{U}$ & BS-05 & 2 & 1 & 6 & $\mathrm{u} 04 \mathrm{PF} 21$ & 1.83 & 2.67 & 21.0 \\
\hline $\mathrm{U}$ & BS-05 & 2 & 1 & 7 & u04PF11 & 1.72 & 2.55 & 20.9 \\
\hline $\mathrm{U}$ & Batch 1 & 2 & 1 & 8 & BCHPF212 & 2.35 & 1.97 & 23.9 \\
\hline $\mathrm{U}$ & LRM & 2 & 1 & 9 & LRMPF212 & 2.44 & $<0.100$ & 25.7 \\
\hline $\mathrm{U}$ & BS-01 & 2 & 1 & 10 & u02PF21 & 1.53 & 2.95 & 22.1 \\
\hline $\mathrm{U}$ & BC-08 & 2 & 1 & 11 & u01PF11 & 1.47 & 2.93 & 19.3 \\
\hline $\mathrm{U}$ & BS-01 & 2 & 1 & 12 & $\mathrm{u} 02 \mathrm{PF} 11$ & 1.55 & 3.02 & 21.9 \\
\hline $\mathrm{U}$ & BC-09 & 2 & 1 & 13 & u10PF11 & 2.19 & 2.99 & 19.6 \\
\hline $\mathrm{U}$ & BS-02 & 2 & 1 & 14 & u06PF21 & 1.43 & 3.23 & 20.7 \\
\hline $\mathrm{U}$ & Batch 1 & 2 & 1 & 15 & BCHPF213 & 2.33 & 1.97 & 24.2 \\
\hline $\mathrm{U}$ & LRM & 2 & 1 & 16 & LRMPF213 & 2.43 & $<0.100$ & 25.6 \\
\hline $\mathrm{U}$ & Batch 1 & 2 & 2 & 1 & BCHPF221 & 2.50 & 1.99 & 23.5 \\
\hline $\mathrm{U}$ & LRM & 2 & 2 & 2 & LRMPF221 & 2.39 & $<0.100$ & 25.4 \\
\hline $\mathrm{U}$ & BS-01 & 2 & 2 & 3 & u02PF12 & 1.49 & 2.91 & 22.6 \\
\hline $\mathrm{U}$ & BC-08 & 2 & 2 & 4 & $\mathrm{u} 01 \mathrm{PF} 12$ & 1.47 & 2.91 & 19.1 \\
\hline $\mathrm{U}$ & BC-09 & 2 & 2 & 5 & u10PF22 & 1.95 & 2.75 & 20.0 \\
\hline $\mathrm{U}$ & BS-05 & 2 & 2 & 6 & u04PF12 & 1.77 & 2.61 & 21.5 \\
\hline $\mathrm{U}$ & BS-02 & 2 & 2 & 7 & u06PF12 & 1.36 & 3.10 & 20.7 \\
\hline $\mathrm{U}$ & Batch 1 & 2 & 2 & 8 & BCHPF222 & 2.22 & 1.90 & 23.3 \\
\hline $\mathrm{U}$ & LRM & 2 & 2 & 9 & LRMPF222 & 2.34 & $<0.100$ & 25.3 \\
\hline $\mathrm{U}$ & BC-08 & 2 & 2 & 10 & u01PF22 & 1.39 & 2.77 & 18.7 \\
\hline $\mathrm{U}$ & BS-01 & 2 & 2 & 11 & $\mathrm{u} 02 \mathrm{PF} 22$ & 1.44 & 2.88 & 22.7 \\
\hline $\mathrm{U}$ & BC-09 & 2 & 2 & 12 & u10PF12 & 2.04 & 2.86 & 19.8 \\
\hline $\mathrm{U}$ & BS-05 & 2 & 2 & 13 & $\mathrm{u} 04 \mathrm{PF} 22$ & 1.79 & 2.65 & 21.2 \\
\hline $\mathrm{U}$ & BS-02 & 2 & 2 & 14 & u06PF22 & 1.34 & 3.09 & 21.0 \\
\hline $\mathrm{U}$ & Batch 1 & 2 & 2 & 15 & BCHPF223 & 2.20 & 1.88 & 23.5 \\
\hline $\mathrm{U}$ & LRM & 2 & 2 & 16 & LRMPF223 & 2.22 & $<0.100$ & 25.7 \\
\hline $\mathrm{V}$ & Batch 1 & 1 & 1 & 1 & BCHPF111 & 2.42 & 1.95 & 22.8 \\
\hline $\mathrm{V}$ & LRM & 1 & 1 & 2 & LRMPF111 & 2.44 & $<0.100$ & 24.6 \\
\hline $\mathrm{V}$ & BS-09 & 1 & 1 & 3 & v05PF21 & 2.30 & 2.38 & 19.8 \\
\hline $\mathrm{V}$ & LL-03 & 1 & 1 & 4 & v08PF21 & 3.24 & 3.39 & 20.4 \\
\hline
\end{tabular}


SRNL-STI-2012-00152

Revision 0

Table A-3. Measured Elemental Concentrations for the Glasses Prepared Using Peroxide Fusion (continued)

\begin{tabular}{|c|c|c|c|c|c|c|c|c|}
\hline Series & \begin{tabular}{|l|l|} 
Glass ID \\
\end{tabular} & Block & Sub-Blk & Sequence & Lab ID & B (wt \%) & Li (wt \%) & Si (wt \%) \\
\hline $\mathrm{V}$ & BS-07 & 1 & \begin{tabular}{|l|}
1 \\
\end{tabular} & \begin{tabular}{|l|}
5 \\
\end{tabular} & v07PF21 & 1.58 & 2.48 & 22.0 \\
\hline $\mathrm{V}$ & BS-07 & 1 & 1 & 6 & v07PF11 & 1.51 & 2.42 & 21.3 \\
\hline $\mathrm{V}$ & BS-06 & 1 & 1 & 7 & v09PF21 & 1.52 & 2.99 & 21.8 \\
\hline $\mathrm{V}$ & Batch 1 & 1 & 1 & 8 & BCHPF112 & 2.33 & 1.93 & 22.7 \\
\hline $\mathrm{V}$ & LRM & 1 & 1 & 9 & LRMPF112 & 2.39 & $<0.100$ & 24.2 \\
\hline $\mathrm{V}$ & BS-09 & 1 & 1 & 10 & v05PF11 & 2.30 & 2.36 & 19.9 \\
\hline $\mathrm{V}$ & LL-03 & 1 & 1 & 11 & v08PF11 & 3.28 & 3.41 & 20.7 \\
\hline $\mathrm{V}$ & LL-05 & 1 & 1 & 12 & v03PF11 & 2.12 & 2.83 & 19.5 \\
\hline $\mathrm{V}$ & LL-05 & 1 & 1 & 13 & v03PF21 & 2.06 & 2.79 & 19.2 \\
\hline $\mathrm{V}$ & BS-06 & 1 & 1 & 14 & v09PF11 & 1.54 & 3.03 & 22.1 \\
\hline $\mathrm{V}$ & Batch 1 & 1 & 1 & 15 & BCHPF113 & 2.34 & 1.93 & 22.9 \\
\hline $\mathrm{V}$ & LRM & 1 & 1 & 16 & LRMPF113 & 2.36 & $<0.100$ & 23.8 \\
\hline $\mathrm{V}$ & Batch 1 & 1 & 2 & 1 & BCHPF121 & 2.39 & 1.97 & 22.7 \\
\hline $\mathrm{V}$ & LRM & 1 & 2 & 2 & LRMPF121 & 2.38 & $<0.100$ & 23.8 \\
\hline $\mathrm{V}$ & LL-05 & 1 & 2 & 3 & v03PF12 & 2.16 & 2.91 & 19.8 \\
\hline $\mathrm{V}$ & BS-09 & 1 & 2 & 4 & v05PF12 & 2.22 & 2.36 & 19.5 \\
\hline $\mathrm{V}$ & BS-06 & 1 & 2 & 5 & v09PF22 & 1.46 & 2.96 & 21.3 \\
\hline $\mathrm{V}$ & BS-06 & 1 & 2 & 6 & v09PF12 & 1.47 & 2.99 & 21.5 \\
\hline $\mathrm{V}$ & LL-03 & 1 & 2 & 7 & v08PF22 & 3.39 & 3.56 & 20.4 \\
\hline $\mathrm{V}$ & Batch 1 & 1 & 2 & 8 & BCHPF122 & 2.23 & 1.91 & 22.1 \\
\hline $\mathrm{V}$ & LRM & 1 & 2 & 9 & LRMPF122 & 2.30 & $<0.100$ & 23.8 \\
\hline $\mathrm{V}$ & BS-07 & 1 & 2 & 10 & \begin{tabular}{|l|}
$\mathrm{v} 07 \mathrm{PF} 22$ \\
\end{tabular} & 1.44 & 2.36 & 20.4 \\
\hline $\mathrm{V}$ & LL-05 & 1 & 2 & 11 & v03PF22 & 2.12 & 2.89 & 19.7 \\
\hline $\mathrm{V}$ & BS-07 & 1 & 2 & 12 & v07PF12 & 1.44 & 2.40 & 20.7 \\
\hline $\mathrm{V}$ & BS-09 & 1 & 2 & 13 & v05PF22 & 2.17 & 2.35 & 19.1 \\
\hline $\mathrm{V}$ & LL-03 & 1 & 2 & 14 & v08PF12 & 3.11 & 3.36 & 20.0 \\
\hline $\mathrm{V}$ & Batch 1 & 1 & 2 & 15 & BCHPF123 & 2.34 & 1.99 & 23.0 \\
\hline $\mathrm{V}$ & LRM & 1 & 2 & 16 & LRMPF123 & 2.37 & $<0.100$ & 24.2 \\
\hline $\mathrm{V}$ & Batch 1 & 2 & 1 & 1 & BCHPF211 & 2.53 & 2.07 & 23.3 \\
\hline $\mathrm{V}$ & LRM & 2 & 1 & 2 & LRMPF211 & 2.48 & $<0.100$ & 24.2 \\
\hline $\mathrm{V}$ & LL-06 & 2 & 1 & 3 & v10PF21 & 2.82 & 2.93 & 17.4 \\
\hline $\mathrm{V}$ & LL-11 & 2 & 1 & 4 & v01PF21 & 1.54 & 2.87 & 20.0 \\
\hline $\mathrm{V}$ & LL-12 & 2 & 1 & 5 & v06PF11 & 2.79 & 2.91 & 18.4 \\
\hline $\mathrm{V}$ & LL-10 & 2 & 1 & 6 & v04PF21 & 2.77 & 2.92 & 21.0 \\
\hline $\mathrm{V}$ & LL-10 & 2 & 1 & 7 & v04PF11 & 2.72 & 2.86 & 21.6 \\
\hline $\mathrm{V}$ & Batch 1 & 2 & 1 & 8 & BCHPF212 & 2.29 & 1.96 & 21.8 \\
\hline $\mathrm{V}$ & LRM & 2 & 1 & 9 & LRMPF212 & 2.35 & $<0.100$ & 23.5 \\
\hline $\mathrm{V}$ & BS-08 & 2 & 1 & 10 & v02PF21 & 2.33 & 2.71 & 20.5 \\
\hline $\mathrm{V}$ & LL-11 & 2 & 1 & 11 & v01PF11 & 1.55 & 2.92 & 20.3 \\
\hline $\mathrm{V}$ & BS-08 & 2 & 1 & 12 & v02PF11 & 2.32 & 2.73 & 20.6 \\
\hline $\mathrm{V}$ & LL-06 & 2 & 1 & 13 & v10PF11 & 2.77 & 2.89 & 17.2 \\
\hline $\mathrm{V}$ & LL-12 & 2 & 1 & 14 & v06PF21 & 2.75 & 2.87 & 18.2 \\
\hline $\mathrm{V}$ & Batch 1 & 2 & 1 & 15 & BCHPF213 & 2.42 & 2.04 & 22.9 \\
\hline $\mathrm{V}$ & LRM & 2 & 1 & 16 & LRMPF213 & 2.45 & $<0.100$ & 23.9 \\
\hline $\mathrm{V}$ & Batch 1 & 2 & 2 & 1 & BCHPF221 & 2.35 & 1.94 & 23.2 \\
\hline $\mathrm{V}$ & LRM & 2 & 2 & 2 & LRMPF221 & 2.42 & $<0.100$ & 25.6 \\
\hline $\mathrm{V}$ & BS-08 & 2 & 2 & 3 & v02PF12 & 2.23 & 2.69 & 21.1 \\
\hline $\mathrm{V}$ & LL-11 & 2 & 2 & 4 & v01PF12 & 1.45 & 2.92 & 21.1 \\
\hline $\mathrm{V}$ & LL-06 & 2 & 2 & 5 & v10PF22 & 2.73 & 2.89 & 17.9 \\
\hline $\mathrm{V}$ & LL-10 & 2 & 2 & 6 & v04PF12 & 2.85 & 2.97 & 21.3 \\
\hline $\mathrm{V}$ & LL-12 & 2 & 2 & 7 & v06PF12 & 2.77 & 2.91 & 19.4 \\
\hline $\mathrm{V}$ & Batch 1 & 2 & 2 & 8 & BCHPF222 & 2.36 & 2.00 & 23.8 \\
\hline $\mathrm{V}$ & LRM & 2 & 2 & 9 & LRMPF222 & 2.41 & $<0.100$ & 25.4 \\
\hline $\mathrm{V}$ & LL-11 & 2 & 2 & 10 & v01PF22 & 1.39 & 2.78 & 20.2 \\
\hline $\mathrm{V}$ & BS-08 & 2 & 2 & 11 & v02PF22 & 2.20 & 2.65 & 21.1 \\
\hline $\mathrm{V}$ & LL-06 & 2 & 2 & 12 & v10PF12 & 2.65 & 2.83 & 17.5 \\
\hline $\mathrm{V}$ & LL-10 & 2 & 2 & 13 & v04PF22 & 2.73 & 2.92 & 20.7 \\
\hline $\mathrm{V}$ & LL-12 & 2 & 2 & 14 & v06PF22 & 2.68 & 2.84 & 18.8 \\
\hline $\mathrm{V}$ & Batch 1 & 2 & 2 & 15 & BCHPF223 & 2.35 & 1.97 & 23.6 \\
\hline $\mathrm{V}$ & LRM & 2 & 2 & 16 & LRMPF223 & 2.41 & $<0.100$ & 25.7 \\
\hline $\mathrm{W}$ & Batch 1 & 1 & 1 & 1 & BCHPF111 & 2.45 & 1.99 & 23.5 \\
\hline $\mathrm{W}$ & LRM & 1 & 1 & 2 & LRMPF111 & 2.48 & $<0.100$ & 24.9 \\
\hline $\mathrm{W}$ & LOL-12 & 1 & 1 & 3 & w05PF21 & 1.64 & 2.41 & 24.1 \\
\hline $\mathrm{W}$ & QB-04 & 1 & 1 & 4 & w08PF21 & 1.50 & 1.96 & 20.9 \\
\hline $\mathrm{W}$ & LT-05 & 1 & 1 & 5 & w07PF21 & 1.76 & 2.63 & 20.7 \\
\hline $\mathrm{W}$ & LT-05 & 1 & 1 & 6 & w07PF11 & 1.78 & 2.63 & 20.4 \\
\hline
\end{tabular}


SRNL-STI-2012-00152

Revision 0

Table A-3. Measured Elemental Concentrations for the Glasses Prepared Using Peroxide Fusion (continued)

\begin{tabular}{|c|c|c|c|c|c|c|c|c|}
\hline Series & \begin{tabular}{|l|l|} 
Glass ID \\
\end{tabular} & Block & Sub-Blk & Sequence & Lab ID & B (wt \%) & Li (wt \%) & Si (wt \%) \\
\hline $\mathrm{W}$ & LT-06 & \begin{tabular}{|l|}
1 \\
\end{tabular} & \begin{tabular}{|l|}
1 \\
\end{tabular} & $\begin{array}{l}7 \\
\end{array}$ & w09PF21 & 2.05 & 2.31 & 19.3 \\
\hline $\mathrm{W}$ & Batch 1 & 1 & 1 & 8 & BCHPF112 & 2.14 & 1.91 & 23.5 \\
\hline W & LRM & 1 & 1 & 9 & LRMPF112 & 2.18 & $<0.100$ & 24.9 \\
\hline $\mathrm{W}$ & LOL-12 & 1 & 1 & 10 & w05PF11 & 1.66 & 2.42 & 23.4 \\
\hline $\mathrm{W}$ & QB-04 & 1 & 1 & 11 & w08PF11 & 1.53 & 2.05 & 21.0 \\
\hline W & LT-10 & 1 & 1 & 12 & w03PF11 & 1.37 & 3.12 & 20.4 \\
\hline $\mathrm{W}$ & LT-10 & 1 & 1 & 13 & w03PF21 & 1.36 & 3.13 & 20.2 \\
\hline $\mathrm{W}$ & LT-06 & 1 & 1 & 14 & w09PF11 & 2.05 & 2.29 & 19.2 \\
\hline $\mathrm{W}$ & Batch 1 & 1 & 1 & 15 & BCHPF113 & 2.22 & 1.94 & 23.6 \\
\hline $\mathrm{W}$ & LRM & 1 & 1 & 16 & LRMPF113 & 2.25 & $<0.100$ & 25.3 \\
\hline $\mathrm{W}$ & Batch 1 & 1 & 2 & 1 & BCHPF121 & 2.42 & 1.96 & 24.5 \\
\hline $\mathrm{W}$ & LRM & 1 & 2 & 2 & LRMPF121 & 2.34 & $<0.100$ & 26.1 \\
\hline $\mathrm{W}$ & LT-10 & 1 & 2 & 3 & w03PF12 & 1.52 & 3.14 & 21.5 \\
\hline $\mathrm{W}$ & LOL-12 & 1 & 2 & 4 & w05PF12 & 1.65 & 2.36 & 24.3 \\
\hline $\mathrm{W}$ & LT-06 & 1 & 2 & 5 & w09PF22 & 2.18 & 2.33 & 19.7 \\
\hline $\mathrm{W}$ & LT-06 & 1 & 2 & 6 & w09PF12 & 2.13 & 2.28 & 19.5 \\
\hline $\mathrm{W}$ & QB-04 & 1 & 2 & 7 & w08PF22 & 1.53 & 1.94 & 20.8 \\
\hline $\mathrm{W}$ & Batch 1 & 1 & 2 & 8 & BCHPF122 & 2.21 & 1.88 & 24.6 \\
\hline $\mathrm{W}$ & LRM & 1 & 2 & 9 & LRMPF122 & 2.37 & $<0.100$ & 26.2 \\
\hline $\mathrm{W}$ & LT-05 & 1 & 2 & 10 & w07PF22 & 1.81 & 2.57 & 20.9 \\
\hline $\mathrm{W}$ & LT-10 & 1 & 2 & 11 & w03PF22 & 1.44 & 3.15 & 20.9 \\
\hline $\mathrm{W}$ & LT-05 & 1 & 2 & 12 & w07PF12 & 1.83 & 2.60 & 20.6 \\
\hline $\mathrm{W}$ & LOL-12 & 1 & 2 & 13 & w05PF22 & 1.55 & 2.31 & 23.9 \\
\hline $\mathrm{W}$ & QB-04 & 1 & 2 & 14 & w08PF12 & 1.58 & 2.01 & 20.8 \\
\hline $\mathrm{W}$ & Batch 1 & 1 & 2 & 15 & BCHPF123 & 2.41 & 1.95 & 24.3 \\
\hline $\mathrm{W}$ & LRM & 1 & 2 & 16 & LRMPF123 & 2.41 & $<0.100$ & 25.4 \\
\hline $\mathrm{W}$ & Batch 1 & 2 & 1 & 1 & BCHPF211 & 2.52 & 1.98 & 24.0 \\
\hline $\mathrm{W}$ & LRM & 2 & 1 & 2 & LRMPF211 & 2.53 & $<0.100$ & 25.4 \\
\hline $\mathrm{W}$ & LT-12 & 2 & 1 & 3 & w10PF21 & 2.39 & 2.98 & 19.6 \\
\hline W & LT-04 & 2 & 1 & 4 & w01PF21 & 1.62 & 2.97 & 22.0 \\
\hline $\mathrm{W}$ & QB-03 & 2 & 1 & 5 & w06PF11 & 1.64 & 2.28 & 21.6 \\
\hline $\mathrm{W}$ & LT-01 & 2 & 1 & 6 & w04PF21 & 1.85 & 3.50 & 25.4 \\
\hline $\mathrm{W}$ & LT-01 & 2 & 1 & 7 & w04PF11 & 1.82 & 3.45 & 25.6 \\
\hline $\mathrm{W}$ & Batch 1 & 2 & 1 & 8 & BCHPF212 & 2.41 & 1.96 & 23.8 \\
\hline $\mathrm{W}$ & LRM & 2 & 1 & 9 & LRMPF212 & 2.41 & $<0.100$ & 25.3 \\
\hline $\mathrm{W}$ & LT-11 & 2 & 1 & 10 & w02PF21 & 2.32 & 3.21 & 20.9 \\
\hline $\mathrm{W}$ & LT-04 & 2 & 1 & 11 & w01PF11 & 1.58 & 2.97 & 22.0 \\
\hline $\mathrm{W}$ & LT-11 & 2 & 1 & 12 & w02PF11 & 2.31 & 3.25 & 20.6 \\
\hline $\mathrm{W}$ & LT-12 & 2 & 1 & 13 & w10PF11 & 2.35 & 2.97 & 19.8 \\
\hline $\mathrm{W}$ & QB-03 & 2 & 1 & 14 & w06PF21 & 1.65 & 2.32 & 21.0 \\
\hline $\mathrm{W}$ & Batch 1 & 2 & 1 & 15 & BCHPF213 & 2.38 & 2.00 & 23.5 \\
\hline $\mathrm{W}$ & LRM & 2 & 1 & 16 & LRMPF213 & 2.48 & $<0.100$ & 24.6 \\
\hline $\mathrm{W}$ & Batch 1 & 2 & 2 & 1 & BCHPF221 & 2.31 & 1.89 & 23.3 \\
\hline $\mathrm{W}$ & LRM & 2 & 2 & 2 & LRMPF221 & 2.25 & $<0.100$ & 24.9 \\
\hline $\mathrm{W}$ & LT-11 & 2 & 2 & 3 & w02PF12 & 2.09 & 3.09 & 21.6 \\
\hline $\mathrm{W}$ & LT-04 & 2 & 2 & 4 & w01PF12 & 1.38 & 2.85 & 21.6 \\
\hline $\mathrm{W}$ & LT-12 & 2 & 2 & 5 & w10PF22 & 2.07 & 2.83 & 20.6 \\
\hline $\mathrm{W}$ & LT-01 & 2 & 2 & 6 & w04PF12 & 1.56 & 3.26 & 26.8 \\
\hline $\mathrm{W}$ & QB-03 & 2 & 2 & 7 & w06PF12 & 1.39 & 2.15 & 22.7 \\
\hline $\mathrm{W}$ & Batch 1 & 2 & 2 & 8 & BCHPF222 & 2.17 & 1.88 & 23.7 \\
\hline $\mathrm{W}$ & LRM & 2 & 2 & 9 & LRMPF222 & 2.15 & $<0.100$ & 25.4 \\
\hline $\mathrm{W}$ & LT-04 & 2 & 2 & 10 & w01PF22 & 1.40 & 2.82 & 21.9 \\
\hline $\mathrm{W}$ & LT-11 & 2 & 2 & 11 & w02PF22 & 2.14 & 3.18 & 21.6 \\
\hline $\mathrm{W}$ & LT-12 & 2 & 2 & 12 & w10PF12 & 2.18 & 2.91 & 20.5 \\
\hline $\mathrm{W}$ & LT-01 & 2 & 2 & 13 & w04PF22 & 1.63 & 3.39 & 26.3 \\
\hline $\mathrm{W}$ & QB-03 & 2 & 2 & 14 & w06PF22 & 1.48 & 2.25 & 22.2 \\
\hline $\mathrm{W}$ & Batch 1 & 2 & 2 & 15 & BCHPF223 & 2.25 & 1.93 & 23.4 \\
\hline $\mathrm{W}$ & LRM & 2 & 2 & 16 & LRMPF223 & 2.27 & $<0.100$ & 25.4 \\
\hline $\mathrm{X}$ & Batch 1 & 1 & 1 & 1 & BCHPF111 & 2.43 & 1.95 & 23.9 \\
\hline $\mathrm{X}$ & LRM & 1 & 1 & 2 & LRMPF111 & 2.42 & 0.03 & 25.4 \\
\hline $\mathrm{X}$ & QB-33 & 1 & 1 & 3 & x05PF21 & 2.67 & 2.01 & 21.7 \\
\hline $\mathrm{X}$ & QB-20 & 1 & 1 & 4 & x08PF21 & 2.52 & 1.78 & 18.7 \\
\hline $\mathrm{X}$ & QB-36 & 1 & 1 & 5 & $\mathrm{x} 07 \mathrm{PF} 21$ & 1.52 & 2.22 & 21.4 \\
\hline $\mathrm{X}$ & QB-36 & 1 & 1 & 6 & $\mathrm{x} 07 \mathrm{PF} 11$ & 1.59 & 2.23 & 22.0 \\
\hline $\mathrm{X}$ & SPS-02 & 1 & 1 & 7 & x09PF21 & 1.51 & 2.06 & 21.1 \\
\hline $\mathrm{X}$ & Batch 1 & 1 & 1 & 8 & BCHPF112 & 2.39 & 1.96 & 24.1 \\
\hline
\end{tabular}


SRNL-STI-2012-00152

Revision 0

Table A-3. Measured Elemental Concentrations for the Glasses Prepared Using Peroxide Fusion (continued)

\begin{tabular}{|c|c|c|c|c|c|c|c|c|}
\hline Series & Glass ID & Block & Sub-Blk & Sequence & Lab ID & B (wt \%) & Li (wt \%) & Si (wt \%) \\
\hline $\mathrm{X}$ & LRM & 1 & 1 & 9 & LRMPF112 & 2.39 & 0.03 & 25.8 \\
\hline $\mathrm{X}$ & QB-33 & 1 & 1 & 10 & $\mathrm{x} 05 \mathrm{PF} 11$ & 2.73 & 1.95 & 22.1 \\
\hline $\mathrm{X}$ & QB-20 & 1 & 1 & 11 & x08PF11 & 2.46 & 1.76 & 18.5 \\
\hline $\mathrm{X}$ & QB-22 & 1 & 1 & 12 & $\mathrm{x} 03$ PF11 & 1.46 & 1.79 & 19.2 \\
\hline $\mathrm{X}$ & QB-22 & 1 & 1 & 13 & $\mathrm{x} 03 \mathrm{PF} 21$ & 1.47 & 1.82 & 19.6 \\
\hline $\mathrm{X}$ & SPS-02 & 1 & 1 & 14 & x09PF11 & 1.50 & 2.07 & 21.0 \\
\hline $\mathrm{X}$ & Batch 1 & 1 & 1 & 15 & BCHPF113 & 2.39 & 1.96 & 23.8 \\
\hline $\mathrm{X}$ & LRM & 1 & 1 & 16 & LRMPF113 & 2.48 & 0.04 & 25.5 \\
\hline $\mathrm{X}$ & Batch 1 & 1 & 2 & 1 & BCHPF121 & 2.32 & 1.93 & 23.4 \\
\hline $\mathrm{X}$ & LRM & 1 & 2 & 2 & LRMPF121 & 2.38 & 0.02 & 24.7 \\
\hline $\mathrm{X}$ & QB-22 & 1 & 2 & 3 & x03PF12 & 1.35 & 1.74 & 18.8 \\
\hline $\mathrm{X}$ & QB-33 & 1 & 2 & 4 & $\mathrm{x} 05 \mathrm{PF} 12$ & 2.63 & 1.92 & 22.3 \\
\hline $\mathrm{X}$ & SPS-02 & 1 & 2 & 5 & x09PF22 & 1.43 & 2.05 & 21.4 \\
\hline $\mathrm{X}$ & SPS-02 & 1 & 2 & 6 & $\mathrm{x} 09 \mathrm{PF} 12$ & 1.38 & 2.03 & 21.5 \\
\hline $\mathrm{X}$ & QB-20 & 1 & 2 & 7 & x08PF22 & 2.31 & 1.70 & 19.1 \\
\hline $\mathrm{X}$ & Batch 1 & 1 & 2 & 8 & BCHPF122 & 2.22 & 1.89 & 23.8 \\
\hline $\mathrm{X}$ & LRM & 1 & 2 & 9 & LRMPF122 & 2.31 & 0.02 & 25.3 \\
\hline $\mathrm{X}$ & QB-36 & 1 & 2 & 10 & x07PF22 & 1.47 & 2.22 & 22.0 \\
\hline $\mathrm{X}$ & QB-22 & 1 & 2 & 11 & $\mathrm{x} 03$ PF22 & 1.38 & 1.80 & 19.4 \\
\hline $\mathrm{X}$ & QB-36 & 1 & 2 & 12 & $\mathrm{x} 07 \mathrm{PF} 12$ & 1.47 & 2.19 & 22.2 \\
\hline $\mathrm{X}$ & QB-33 & 1 & 2 & 13 & $\mathrm{x} 05 \mathrm{PF} 22$ & 2.54 & 1.97 & 22.4 \\
\hline $\mathrm{X}$ & QB-20 & 1 & 2 & 14 & x08PF12 & 2.46 & 1.80 & 18.9 \\
\hline $\mathrm{X}$ & Batch 1 & 1 & 2 & 15 & BCHPF123 & 2.31 & 1.94 & 23.6 \\
\hline $\mathrm{X}$ & LRM & 1 & 2 & 16 & LRMPF123 & 2.40 & 0.02 & 24.9 \\
\hline $\mathrm{X}$ & Batch 1 & 2 & 1 & 1 & BCHPF211 & 2.47 & 2.02 & 23.5 \\
\hline $\mathrm{X}$ & LRM & 2 & 1 & 2 & LRMPF211 & 2.44 & 0.05 & 24.5 \\
\hline $\mathrm{X}$ & QB-34 & 2 & 1 & 3 & x10PF21 & 1.64 & 2.29 & 21.7 \\
\hline $\mathrm{X}$ & QB-19 & 2 & 1 & 4 & $\mathrm{x} 01 \mathrm{PF} 21$ & 1.51 & 2.17 & 20.8 \\
\hline $\mathrm{X}$ & QB-35 & 2 & 1 & 5 & x06PF11 & 1.58 & 2.00 & 21.6 \\
\hline $\mathrm{X}$ & QB-37 & 2 & 1 & 6 & $\mathrm{x} 04 \mathrm{PF} 21$ & 1.55 & 2.57 & 22.8 \\
\hline $\mathrm{X}$ & QB-37 & 2 & 1 & 7 & x04PF11 & 1.55 & 2.58 & 22.7 \\
\hline $\mathrm{X}$ & Batch 1 & 2 & 1 & 8 & BCHPF212 & 2.49 & 2.05 & 23.1 \\
\hline $\mathrm{X}$ & LRM & 2 & 1 & 9 & LRMPF212 & 2.48 & 0.05 & 24.3 \\
\hline $\mathrm{X}$ & QB-32 & 2 & 1 & 10 & x02PF21 & 1.68 & 2.33 & 23.4 \\
\hline $\mathrm{X}$ & QB-19 & 2 & 1 & 11 & $\mathrm{x} 01 \mathrm{PF} 11$ & 1.48 & 2.13 & 20.7 \\
\hline $\mathrm{X}$ & QB-32 & 2 & 1 & 12 & $\mathrm{x} 02 \mathrm{PF} 11$ & 1.59 & 2.28 & 22.5 \\
\hline $\mathrm{X}$ & QB-34 & 2 & 1 & 13 & x10PF11 & 1.61 & 2.33 & 21.9 \\
\hline $\mathrm{X}$ & QB-35 & 2 & 1 & 14 & x06PF21 & 1.64 & 2.08 & 21.0 \\
\hline $\mathrm{X}$ & Batch 1 & 2 & 1 & 15 & BCHPF213 & 2.50 & 2.08 & 23.4 \\
\hline $\mathrm{X}$ & LRM & 2 & 1 & 16 & LRMPF213 & 2.54 & 0.05 & 24.7 \\
\hline $\mathrm{X}$ & Batch 1 & 2 & 2 & 1 & BCHPF221 & 2.41 & 1.98 & 23.8 \\
\hline $\mathrm{X}$ & LRM & 2 & 2 & 2 & LRMPF221 & 2.38 & 0.07 & 25.0 \\
\hline $\mathrm{X}$ & QB-32 & 2 & 2 & 3 & x02PF12 & 1.42 & 2.14 & 23.0 \\
\hline $\mathrm{X}$ & QB-19 & 2 & 2 & 4 & $\mathrm{x} 01 \mathrm{PF} 12$ & 1.36 & 2.02 & 21.5 \\
\hline $\mathrm{X}$ & QB-34 & 2 & 2 & 5 & $\mathrm{x} 10 \mathrm{PF} 22$ & 1.47 & 2.17 & 21.8 \\
\hline $\mathrm{X}$ & QB-37 & 2 & 2 & 6 & $\mathrm{x} 04 \mathrm{PF} 12$ & 1.50 & 2.52 & 22.2 \\
\hline $\mathrm{X}$ & QB-35 & 2 & 2 & 7 & x06PF12 & 1.54 & 1.96 & 20.8 \\
\hline $\mathrm{X}$ & Batch 1 & 2 & 2 & 8 & BCHPF222 & 2.29 & 1.94 & 23.7 \\
\hline $\mathrm{X}$ & LRM & 2 & 2 & 9 & LRMPF222 & 2.30 & 0.06 & 25.0 \\
\hline $\mathrm{X}$ & QB-19 & 2 & 2 & 10 & x01PF22 & 1.51 & 2.14 & 21.5 \\
\hline $\mathrm{X}$ & QB-32 & 2 & 2 & 11 & $\mathrm{x} 02 \mathrm{PF} 22$ & 1.53 & 2.20 & 22.5 \\
\hline $\mathrm{X}$ & QB-34 & 2 & 2 & 12 & x10PF12 & 1.48 & 2.20 & 21.1 \\
\hline $\mathrm{X}$ & QB-37 & 2 & 2 & 13 & $\mathrm{x} 04 \mathrm{PF} 22$ & 1.50 & 2.52 & 22.3 \\
\hline $\mathrm{X}$ & QB-35 & 2 & 2 & 14 & x06PF22 & 1.50 & 1.96 & 20.0 \\
\hline $\mathrm{X}$ & Batch 1 & 2 & 2 & 15 & BCHPF223 & 2.29 & 1.95 & 23.6 \\
\hline $\mathrm{X}$ & LRM & 2 & 2 & 16 & LRMPF223 & 2.37 & 0.06 & 24.6 \\
\hline
\end{tabular}


Table A-4. Average Measured Chemical Compositions versus Targeted Compositions by Oxide by Glass ID

\begin{tabular}{|c|c|c|c|c|c|}
\hline Glass ID & Oxide & $\begin{array}{c}\text { Measured } \\
\text { (wt \%) }\end{array}$ & $\begin{array}{c}\text { Targeted } \\
\text { (wt \%) }\end{array}$ & $\begin{array}{c}\text { Diff of } \\
\text { Measured }\end{array}$ & $\begin{array}{l}\text { \% Diff of } \\
\text { Measured }\end{array}$ \\
\hline AB-06 & $\mathrm{Al}_{2} \mathrm{O}_{3}$ & 11.2567 & 10.7560 & 0.5007 & $4.7 \%$ \\
\hline AB-06 & $\mathrm{B}_{2} \mathrm{O}_{3}$ & 5.4014 & 5.1200 & 0.2814 & $5.5 \%$ \\
\hline AB-06 & $\mathrm{BaO}$ & 0.0296 & 0.0420 & -0.0124 & $-29.5 \%$ \\
\hline AB-06 & $\mathrm{CaO}$ & 0.6156 & 0.5650 & 0.0506 & $9.0 \%$ \\
\hline AB-06 & $\mathrm{Ce}_{2} \mathrm{O}_{3}$ & 0.0375 & 0.0350 & 0.0025 & $7.1 \%$ \\
\hline AB-06 & $\mathrm{Cr}_{2} \mathrm{O}_{3}$ & 0.1261 & 0.1370 & -0.0109 & $-8.0 \%$ \\
\hline AB-06 & $\mathrm{CuO}$ & 0.0541 & 0.0370 & 0.0171 & $46.2 \%$ \\
\hline AB-06 & $\mathrm{Fe}_{2} \mathrm{O}_{3}$ & 9.0714 & 8.9250 & 0.1464 & $1.6 \%$ \\
\hline AB-06 & $\mathrm{K}_{2} \mathrm{O}$ & 0.0602 & 0.0090 & 0.0512 & $568.9 \%$ \\
\hline AB-06 & $\mathrm{La}_{2} \mathrm{O}_{3}$ & 0.0261 & 0.0310 & -0.0049 & $-15.8 \%$ \\
\hline AB-06 & $\mathrm{Li}_{2} \mathrm{O}$ & 5.1185 & 5.1200 & -0.0015 & $0.0 \%$ \\
\hline AB-06 & $\mathrm{MgO}$ & 0.2724 & 0.3220 & -0.0496 & $-15.4 \%$ \\
\hline AB-06 & $\mathrm{MnO}$ & 2.8116 & 2.7170 & 0.0946 & $3.5 \%$ \\
\hline AB-06 & $\mathrm{Na}_{2} \mathrm{O}$ & 15.2324 & 14.2750 & 0.9574 & $6.7 \%$ \\
\hline AB-06 & $\mathrm{NiO}$ & 0.8675 & 0.9990 & -0.1315 & $-13.2 \%$ \\
\hline AB-06 & $\mathrm{PbO}$ & 0.0108 & 0.0080 & 0.0028 & $35.0 \%$ \\
\hline AB-06 & $\mathrm{SiO}_{2}$ & 49.9527 & 49.5680 & 0.3847 & $0.8 \%$ \\
\hline AB-06 & $\mathrm{SO}_{4}$ & 0.9055 & 1.2000 & -0.2945 & $-24.5 \%$ \\
\hline AB-06 & $\mathrm{TiO}_{2}$ & 0.0083 & 0.0000 & 0.0083 & \\
\hline AB-06 & $\mathrm{ZnO}$ & 0.0246 & 0.0230 & 0.0016 & $7.0 \%$ \\
\hline AB-06 & $\mathrm{ZrO}_{2}$ & 0.0946 & 0.1110 & -0.0164 & $-14.8 \%$ \\
\hline AB-06 & Sum & 101.9776 & 100.0000 & 1.9776 & $2.0 \%$ \\
\hline AF-01 & $\mathrm{Al}_{2} \mathrm{O}_{3}$ & 12.3621 & 11.9930 & 0.3691 & $3.1 \%$ \\
\hline AF-01 & $\mathrm{B}_{2} \mathrm{O}_{3}$ & 4.9264 & 4.8000 & 0.1264 & $2.6 \%$ \\
\hline AF-01 & $\mathrm{BaO}$ & 0.0472 & 0.0470 & 0.0002 & $0.4 \%$ \\
\hline AF-01 & $\mathrm{CaO}$ & 0.6608 & 0.6300 & 0.0308 & $4.9 \%$ \\
\hline AF-01 & $\mathrm{Ce}_{2} \mathrm{O}_{3}$ & 0.0389 & 0.0390 & -0.0001 & $-0.3 \%$ \\
\hline AF-01 & $\mathrm{Cr}_{2} \mathrm{O}_{3}$ & 0.1429 & 0.1520 & -0.0091 & $-6.0 \%$ \\
\hline AF-01 & $\mathrm{CuO}$ & 0.0454 & 0.0420 & 0.0034 & $8.1 \%$ \\
\hline AF-01 & $\mathrm{Fe}_{2} \mathrm{O}_{3}$ & 10.1223 & 9.9510 & 0.1713 & $1.7 \%$ \\
\hline AF-01 & $\mathrm{K}_{2} \mathrm{O}$ & 0.0602 & 0.0100 & 0.0502 & $502.0 \%$ \\
\hline AF-01 & $\mathrm{La}_{2} \mathrm{O}_{3}$ & 0.0328 & 0.0340 & -0.0012 & $-3.5 \%$ \\
\hline AF-01 & $\mathrm{Li}_{2} \mathrm{O}$ & 4.7418 & 4.8000 & -0.0582 & $-1.2 \%$ \\
\hline AF-01 & $\mathrm{MgO}$ & 0.3230 & 0.3590 & -0.0360 & $-10.0 \%$ \\
\hline AF-01 & $\mathrm{MnO}$ & 3.1215 & 3.0300 & 0.0915 & $3.0 \%$ \\
\hline AF-01 & $\mathrm{Na}_{2} \mathrm{O}$ & 15.7379 & 15.0070 & 0.7309 & $4.9 \%$ \\
\hline AF-01 & $\mathrm{NiO}$ & 0.9964 & 1.1140 & -0.1176 & $-10.6 \%$ \\
\hline AF-01 & $\mathrm{PbO}$ & 0.0108 & 0.0090 & 0.0018 & $20.0 \%$ \\
\hline AF-01 & $\mathrm{SiO}_{2}$ & 45.8880 & 46.6340 & -0.7460 & $-1.6 \%$ \\
\hline AF-01 & $\mathrm{SO}_{4}$ & 0.9737 & 1.2000 & -0.2263 & $-18.9 \%$ \\
\hline AF-01 & $\mathrm{TiO}_{2}$ & 0.0083 & 0.0000 & 0.0083 & \\
\hline AF-01 & $\mathrm{ZnO}$ & 0.0314 & 0.0260 & 0.0054 & $20.8 \%$ \\
\hline AF-01 & $\mathrm{ZrO}_{2}$ & 0.1280 & 0.1240 & 0.0040 & $3.2 \%$ \\
\hline AF-01 & Sum & 100.3996 & 100.0010 & 0.3986 & $0.4 \%$ \\
\hline AF-02 & $\mathrm{Al}_{2} \mathrm{O}_{3}$ & 12.0881 & 11.9930 & 0.0951 & $0.8 \%$ \\
\hline AF-02 & $\mathrm{B}_{2} \mathrm{O}_{3}$ & 8.5488 & 8.4000 & 0.1488 & $1.8 \%$ \\
\hline AF-02 & $\mathrm{BaO}$ & 0.0391 & 0.0470 & -0.0079 & $-16.8 \%$ \\
\hline AF-02 & $\mathrm{CaO}$ & 0.6790 & 0.6300 & 0.0490 & $7.8 \%$ \\
\hline AF-02 & $\mathrm{Ce}_{2} \mathrm{O}_{3}$ & 0.0471 & 0.0390 & 0.0081 & $20.8 \%$ \\
\hline AF-02 & $\mathrm{Cr}_{2} \mathrm{O}_{3}$ & 0.1367 & 0.1520 & -0.0153 & $-10.1 \%$ \\
\hline AF-02 & $\mathrm{CuO}$ & 0.0413 & 0.0420 & -0.0007 & $-1.7 \%$ \\
\hline AF-02 & $\mathrm{Fe}_{2} \mathrm{O}_{3}$ & 9.9757 & 9.9510 & 0.0247 & $0.2 \%$ \\
\hline AF-02 & $\mathrm{K}_{2} \mathrm{O}$ & 0.0602 & 0.0100 & 0.0502 & $502.0 \%$ \\
\hline $\mathrm{AF}-02$ & $\mathrm{La}_{2} \mathrm{O}_{3}$ & 0.0323 & 0.0340 & -0.0017 & $-5.0 \%$ \\
\hline AF-02 & $\mathrm{Li}_{2} \mathrm{O}$ & 4.5964 & 4.8000 & -0.2036 & $-4.2 \%$ \\
\hline AF-02 & $\mathrm{MgO}$ & 0.3238 & 0.3590 & -0.0352 & $-9.8 \%$ \\
\hline AF-02 & $\mathrm{MnO}$ & 2.9956 & 3.0300 & -0.0344 & $-1.1 \%$ \\
\hline
\end{tabular}


SRNL-STI-2012-00152

Revision 0

Table A-4. Average Measured Chemical Compositions versus Targeted Compositions by Oxide by Glass ID (continued)

\begin{tabular}{|c|c|c|c|c|c|}
\hline Glass ID & Oxide & $\begin{array}{c}\text { Measured } \\
\text { (wt \%) }\end{array}$ & $\begin{array}{c}\text { Targeted } \\
(\text { wt \%) }\end{array}$ & $\begin{array}{c}\text { Diff of } \\
\text { Measured }\end{array}$ & $\begin{array}{l}\text { \% Diff of } \\
\text { Measured }\end{array}$ \\
\hline AF-02 & $\mathrm{Na}_{2} \mathrm{O}$ & 15.0302 & 15.0070 & 0.0232 & $0.2 \%$ \\
\hline AF-02 & $\mathrm{NiO}$ & 0.9811 & 1.1140 & -0.1329 & $-11.9 \%$ \\
\hline AF-02 & $\mathrm{PbO}$ & 0.0108 & 0.0090 & 0.0018 & $20.0 \%$ \\
\hline AF-02 & $\mathrm{SiO}_{2}$ & 43.9091 & 43.0340 & 0.8751 & $2.0 \%$ \\
\hline AF-02 & $\mathrm{SO}_{4}$ & 0.9976 & 1.2000 & -0.2024 & $-16.9 \%$ \\
\hline AF-02 & $\mathrm{TiO}_{2}$ & 0.0083 & 0.0000 & 0.0083 & \\
\hline AF-02 & $\mathrm{ZnO}$ & 0.0305 & 0.0260 & 0.0045 & $17.3 \%$ \\
\hline AF-02 & $\mathrm{ZrO}_{2}$ & 0.1239 & 0.1240 & -0.0001 & $-0.1 \%$ \\
\hline AF-02 & Sum & 100.6557 & 100.0010 & 0.6547 & $0.7 \%$ \\
\hline AF-03 & $\mathrm{Al}_{2} \mathrm{O}_{3}$ & 12.3762 & 11.9930 & 0.3832 & $3.2 \%$ \\
\hline AF-03 & $\mathrm{B}_{2} \mathrm{O}_{3}$ & 8.7018 & 8.4000 & 0.3018 & $3.6 \%$ \\
\hline AF-03 & $\mathrm{BaO}$ & 0.0452 & 0.0470 & -0.0018 & $-3.8 \%$ \\
\hline AF-03 & $\mathrm{CaO}$ & 0.6702 & 0.6300 & 0.0402 & $6.4 \%$ \\
\hline AF-03 & $\mathrm{Ce}_{2} \mathrm{O}_{3}$ & 0.0413 & 0.0390 & 0.0023 & $5.9 \%$ \\
\hline AF-03 & $\mathrm{Cr}_{2} \mathrm{O}_{3}$ & 0.1403 & 0.1520 & -0.0117 & $-7.7 \%$ \\
\hline AF-03 & $\mathrm{CuO}$ & 0.0523 & 0.0420 & 0.0103 & $24.5 \%$ \\
\hline AF-03 & $\mathrm{Fe}_{2} \mathrm{O}_{3}$ & 9.8828 & 9.9510 & -0.0682 & $-0.7 \%$ \\
\hline AF-03 & $\mathrm{K}_{2} \mathrm{O}$ & 0.0602 & 0.0100 & 0.0502 & $502.0 \%$ \\
\hline AF-03 & $\mathrm{La}_{2} \mathrm{O}_{3}$ & 0.0317 & 0.0340 & -0.0023 & $-6.8 \%$ \\
\hline AF-03 & $\mathrm{Li}_{2} \mathrm{O}$ & 4.6772 & 4.8000 & -0.1228 & $-2.6 \%$ \\
\hline AF-03 & $\mathrm{MgO}$ & 0.3263 & 0.3590 & -0.0327 & $-9.1 \%$ \\
\hline AF-03 & $\mathrm{MnO}$ & 3.0795 & 3.0300 & 0.0495 & $1.6 \%$ \\
\hline AF-03 & $\mathrm{Na}_{2} \mathrm{O}$ & 13.1936 & 12.6070 & 0.5866 & $4.7 \%$ \\
\hline AF-03 & $\mathrm{NiO}$ & 0.9945 & 1.1140 & -0.1195 & $-10.7 \%$ \\
\hline AF-03 & $\mathrm{PbO}$ & 0.0108 & 0.0090 & 0.0018 & $20.0 \%$ \\
\hline AF-03 & $\mathrm{SiO}_{2}$ & 44.2835 & 45.4340 & -1.1505 & $-2.5 \%$ \\
\hline AF-03 & $\mathrm{SO}_{4}$ & 0.9527 & 1.2000 & -0.2473 & $-20.6 \%$ \\
\hline AF-03 & $\mathrm{TiO}_{2}$ & 0.0083 & 0.0000 & 0.0083 & \\
\hline AF-03 & $\mathrm{ZnO}$ & 0.0317 & 0.0260 & 0.0057 & $21.9 \%$ \\
\hline AF-03 & $\mathrm{ZrO}_{2}$ & 0.1246 & 0.1240 & 0.0006 & $0.5 \%$ \\
\hline AF-03 & Sum & 99.6846 & 100.0010 & -0.3164 & $-0.3 \%$ \\
\hline AF-04 & $\mathrm{Al}_{2} \mathrm{O}_{3}$ & 12.2723 & 11.9930 & 0.2793 & $2.3 \%$ \\
\hline AF-04 & $\mathrm{B}_{2} \mathrm{O}_{3}$ & 8.5730 & 8.9300 & -0.3570 & $-4.0 \%$ \\
\hline AF-04 & $\mathrm{BaO}$ & 0.0335 & 0.0470 & -0.0135 & $-28.7 \%$ \\
\hline AF-04 & $\mathrm{CaO}$ & 5.5758 & 5.4250 & 0.1508 & $2.8 \%$ \\
\hline AF-04 & $\mathrm{Ce}_{2} \mathrm{O}_{3}$ & 0.0433 & 0.0390 & 0.0043 & $11.0 \%$ \\
\hline AF-04 & $\mathrm{Cr}_{2} \mathrm{O}_{3}$ & 0.1315 & 0.1520 & -0.0205 & $-13.5 \%$ \\
\hline AF-04 & $\mathrm{CuO}$ & 0.0407 & 0.0420 & -0.0013 & $-3.1 \%$ \\
\hline AF-04 & $\mathrm{Fe}_{2} \mathrm{O}_{3}$ & 9.8256 & 9.9510 & -0.1254 & $-1.3 \%$ \\
\hline AF-04 & $\mathrm{K}_{2} \mathrm{O}$ & 0.0602 & 0.0100 & 0.0502 & $502.0 \%$ \\
\hline AF-04 & $\mathrm{La}_{2} \mathrm{O}_{3}$ & 0.0369 & 0.0340 & 0.0029 & $8.5 \%$ \\
\hline AF-04 & $\mathrm{Li}_{2} \mathrm{O}$ & 4.8602 & 5.1690 & -0.3088 & $-6.0 \%$ \\
\hline AF-04 & $\mathrm{MgO}$ & 0.2993 & 0.3590 & -0.0597 & $-16.6 \%$ \\
\hline AF-04 & $\mathrm{MnO}$ & 3.0827 & 3.0300 & 0.0527 & $1.7 \%$ \\
\hline AF-04 & $\mathrm{Na}_{2} \mathrm{O}$ & 14.6932 & 14.1820 & 0.5112 & $3.6 \%$ \\
\hline AF-04 & $\mathrm{NiO}$ & 0.9496 & 1.1140 & -0.1644 & $-14.8 \%$ \\
\hline AF-04 & $\mathrm{PbO}$ & 0.0108 & 0.0090 & 0.0018 & $20.0 \%$ \\
\hline AF-04 & $\mathrm{SiO}_{2}$ & 36.9029 & 38.1650 & -1.2621 & $-3.3 \%$ \\
\hline AF-04 & $\mathrm{SO}_{4}$ & 1.0928 & 1.2000 & -0.1072 & $-8.9 \%$ \\
\hline AF-04 & $\mathrm{TiO}_{2}$ & 0.0083 & 0.0000 & 0.0083 & \\
\hline AF-04 & $\mathrm{ZnO}$ & 0.0271 & 0.0260 & 0.0011 & $4.2 \%$ \\
\hline AF-04 & $\mathrm{ZrO}_{2}$ & 0.1249 & 0.1240 & 0.0009 & $0.7 \%$ \\
\hline AF-04 & Sum & 98.6448 & 100.0010 & -1.3562 & $-1.4 \%$ \\
\hline AF-05 & $\mathrm{Al}_{2} \mathrm{O}_{3}$ & 12.0172 & 11.9930 & 0.0242 & $0.2 \%$ \\
\hline AF-05 & $\mathrm{B}_{2} \mathrm{O}_{3}$ & 6.8020 & 6.8930 & -0.0910 & $-1.3 \%$ \\
\hline AF-05 & $\mathrm{BaO}$ & 0.0416 & 0.0470 & -0.0054 & $-11.5 \%$ \\
\hline AF-05 & $\mathrm{CaO}$ & 3.1202 & 3.0600 & 0.0602 & $2.0 \%$ \\
\hline
\end{tabular}


SRNL-STI-2012-00152

Revision 0

Table A-4. Average Measured Chemical Compositions versus Targeted Compositions by Oxide by Glass ID (continued)

\begin{tabular}{|c|c|c|c|c|c|}
\hline Glass ID & Oxide & $\begin{array}{c}\text { Measured } \\
\text { (wt \%) }\end{array}$ & $\begin{array}{c}\text { Targeted } \\
(\text { wt \%) }\end{array}$ & $\begin{array}{c}\text { Diff of } \\
\text { Measured }\end{array}$ & $\begin{array}{l}\text { \% Diff of } \\
\text { Measured }\end{array}$ \\
\hline AF-05 & $\mathrm{Ce}_{2} \mathrm{O}_{3}$ & 0.0445 & 0.0390 & 0.0055 & $14.1 \%$ \\
\hline AF-05 & $\mathrm{Cr}_{2} \mathrm{O}_{3}$ & 0.1385 & 0.1520 & -0.0135 & $-8.9 \%$ \\
\hline AF-05 & $\mathrm{CuO}$ & 0.0466 & 0.0420 & 0.0046 & $11.0 \%$ \\
\hline AF-05 & $\mathrm{Fe}_{2} \mathrm{O}_{3}$ & 9.7327 & 9.9510 & -0.2183 & $-2.2 \%$ \\
\hline AF-05 & $\mathrm{K}_{2} \mathrm{O}$ & 0.0602 & 0.0100 & 0.0502 & $502.0 \%$ \\
\hline AF-05 & $\mathrm{La}_{2} \mathrm{O}_{3}$ & 0.0325 & 0.0340 & -0.0015 & $-4.4 \%$ \\
\hline AF-05 & $\mathrm{Li}_{2} \mathrm{O}$ & 5.5922 & 5.8750 & -0.2828 & $-4.8 \%$ \\
\hline AF-05 & $\mathrm{MgO}$ & 0.3292 & 0.3590 & -0.0298 & $-8.3 \%$ \\
\hline AF-05 & $\mathrm{MnO}$ & 3.0505 & 3.0300 & 0.0205 & $0.7 \%$ \\
\hline AF-05 & $\mathrm{Na}_{2} \mathrm{O}$ & 13.0318 & 12.8120 & 0.2198 & $1.7 \%$ \\
\hline AF-05 & $\mathrm{NiO}$ & 0.9506 & 1.1140 & -0.1634 & $-14.7 \%$ \\
\hline AF-05 & $\mathrm{PbO}$ & 0.0108 & 0.0090 & 0.0018 & $20.0 \%$ \\
\hline AF-05 & $\mathrm{SiO}_{2}$ & 42.6790 & 43.2310 & -0.5520 & $-1.3 \%$ \\
\hline AF-05 & $\mathrm{SO}_{4}$ & 1.0965 & 1.2000 & -0.1035 & $-8.6 \%$ \\
\hline AF-05 & $\mathrm{TiO}_{2}$ & 0.0083 & 0.0000 & 0.0083 & \\
\hline AF-05 & $\mathrm{ZnO}$ & 0.0352 & 0.0260 & 0.0092 & $35.4 \%$ \\
\hline AF-05 & $\mathrm{ZrO}_{2}$ & 0.1199 & 0.1240 & -0.0041 & $-3.3 \%$ \\
\hline AF-05 & Sum & 98.9400 & 100.0010 & -1.0610 & $-1.1 \%$ \\
\hline Batch 1 & $\mathrm{Al}_{2} \mathrm{O}_{3}$ & 4.8784 & 4.8770 & 0.0014 & $0.0 \%$ \\
\hline Batch 1 & $\mathrm{~B}_{2} \mathrm{O}_{3}$ & 7.6043 & 7.7770 & -0.1727 & $-2.2 \%$ \\
\hline Batch 1 & $\mathrm{BaO}$ & 0.1389 & 0.1510 & -0.0121 & $-8.0 \%$ \\
\hline Batch 1 & $\mathrm{CaO}$ & 1.2227 & 1.2200 & 0.0027 & $0.2 \%$ \\
\hline Batch 1 & $\mathrm{Ce}_{2} \mathrm{O}_{3}$ & 0.0059 & 0.0000 & 0.0059 & \\
\hline Batch 1 & $\mathrm{Cr}_{2} \mathrm{O}_{3}$ & 0.1114 & 0.1070 & 0.0044 & $4.1 \%$ \\
\hline Batch 1 & $\mathrm{CuO}$ & 0.3873 & 0.3990 & -0.0117 & $-2.9 \%$ \\
\hline Batch 1 & $\mathrm{Fe}_{2} \mathrm{O}_{3}$ & 12.6748 & 12.8390 & -0.1642 & $-1.3 \%$ \\
\hline Batch 1 & $\mathrm{~K}_{2} \mathrm{O}$ & 3.3030 & 3.3270 & -0.0240 & $-0.7 \%$ \\
\hline Batch 1 & $\mathrm{La}_{2} \mathrm{O}_{3}$ & 0.0059 & 0.0000 & 0.0059 & \\
\hline Batch 1 & $\mathrm{Li}_{2} \mathrm{O}$ & 4.2351 & 4.4290 & -0.1939 & $-4.4 \%$ \\
\hline Batch 1 & $\mathrm{MgO}$ & 1.3483 & 1.4190 & -0.0707 & $-5.0 \%$ \\
\hline Batch 1 & $\mathrm{MnO}$ & 1.7328 & 1.7260 & 0.0068 & $0.4 \%$ \\
\hline Batch 1 & $\mathrm{Na}_{2} \mathrm{O}$ & 8.9599 & 9.0030 & -0.0431 & $-0.5 \%$ \\
\hline Batch 1 & $\mathrm{NiO}$ & 0.6740 & 0.7510 & -0.0770 & $-10.3 \%$ \\
\hline Batch 1 & $\mathrm{PbO}$ & 0.0108 & 0.0000 & 0.0108 & \\
\hline Batch 1 & $\mathrm{SiO}_{2}$ & 50.1131 & 50.2200 & -0.1069 & $-0.2 \%$ \\
\hline Batch 1 & $\mathrm{SO}_{4}$ & 0.0731 & 0.0000 & 0.0731 & \\
\hline Batch 1 & $\mathrm{TiO}_{2}$ & 0.6388 & 0.6770 & -0.0382 & $-5.6 \%$ \\
\hline Batch 1 & $\mathrm{ZnO}$ & 0.0062 & 0.0000 & 0.0062 & \\
\hline Batch 1 & $\mathrm{ZrO}_{2}$ & 0.0890 & 0.0980 & -0.0090 & $-9.2 \%$ \\
\hline Batch 1 & Sum & 98.2137 & 99.0200 & -0.8063 & $-0.8 \%$ \\
\hline BC-01 & $\mathrm{Al}_{2} \mathrm{O}_{3}$ & 12.1967 & 11.9930 & 0.2037 & $1.7 \%$ \\
\hline BC-01 & $\mathrm{B}_{2} \mathrm{O}_{3}$ & 4.6689 & 4.8000 & -0.1311 & $-2.7 \%$ \\
\hline BC-01 & $\mathrm{BaO}$ & 0.0405 & 0.0470 & -0.0065 & $-13.8 \%$ \\
\hline BC-01 & $\mathrm{CaO}$ & 0.6975 & 0.6300 & 0.0675 & $10.7 \%$ \\
\hline BC-01 & $\mathrm{Ce}_{2} \mathrm{O}_{3}$ & 0.0457 & 0.0390 & 0.0067 & $17.2 \%$ \\
\hline BC-01 & $\mathrm{Cr}_{2} \mathrm{O}_{3}$ & 0.1312 & 0.1520 & -0.0208 & $-13.7 \%$ \\
\hline BC-01 & $\mathrm{CuO}$ & 0.0426 & 0.0420 & 0.0006 & $1.4 \%$ \\
\hline BC-01 & $\mathrm{Fe}_{2} \mathrm{O}_{3}$ & 9.5504 & 9.9510 & -0.4006 & $-4.0 \%$ \\
\hline BC-01 & $\mathrm{K}_{2} \mathrm{O}$ & 0.0602 & 0.0100 & 0.0502 & $502.0 \%$ \\
\hline BC-01 & $\mathrm{La}_{2} \mathrm{O}_{3}$ & 0.0328 & 0.0340 & -0.0012 & $-3.5 \%$ \\
\hline BC-01 & $\mathrm{Li}_{2} \mathrm{O}$ & 6.2596 & 6.5990 & -0.3394 & $-5.1 \%$ \\
\hline BC-01 & $\mathrm{MgO}$ & 0.2852 & 0.3590 & -0.0738 & $-20.6 \%$ \\
\hline BC-01 & $\mathrm{MnO}$ & 3.0698 & 3.0300 & 0.0398 & $1.3 \%$ \\
\hline BC-01 & $\mathrm{Na}_{2} \mathrm{O}$ & 11.5726 & 11.4070 & 0.1656 & $1.5 \%$ \\
\hline BC-01 & $\mathrm{NiO}$ & 0.9744 & 1.1140 & -0.1396 & $-12.5 \%$ \\
\hline BC-01 & $\mathrm{PbO}$ & 0.0108 & 0.0090 & 0.0018 & $20.0 \%$ \\
\hline BC-01 & $\mathrm{SiO}_{2}$ & 47.1716 & 48.4350 & -1.2634 & $-2.6 \%$ \\
\hline
\end{tabular}


SRNL-STI-2012-00152

Revision 0

Table A-4. Average Measured Chemical Compositions versus Targeted Compositions by Oxide by Glass ID (continued)

\begin{tabular}{|c|c|c|c|c|c|}
\hline Glass ID & Oxide & $\begin{array}{c}\text { Measured } \\
\text { (wt \%) }\end{array}$ & $\begin{array}{c}\text { Targeted } \\
\text { (wt \%) }\end{array}$ & $\begin{array}{c}\text { Diff of } \\
\text { Measured }\end{array}$ & $\begin{array}{l}\text { \% Diff of } \\
\text { Measured }\end{array}$ \\
\hline BC-01 & $\mathrm{SO}_{4}$ & 1.0411 & 1.2000 & -0.1589 & $-13.2 \%$ \\
\hline BC-01 & $\mathrm{TiO}_{2}$ & 0.0083 & 0.0000 & 0.0083 & \\
\hline BC-01 & $\mathrm{ZnO}$ & 0.0349 & 0.0260 & 0.0089 & $34.2 \%$ \\
\hline BC-01 & $\mathrm{ZrO}_{2}$ & 0.1067 & 0.1240 & -0.0173 & $-14.0 \%$ \\
\hline BC-01 & Sum & 98.0014 & 100.0010 & -1.9996 & $-2.0 \%$ \\
\hline BC-02 & $\mathrm{Al}_{2} \mathrm{O}_{3}$ & 12.0645 & 11.9930 & 0.0715 & $0.6 \%$ \\
\hline BC-02 & $\mathrm{B}_{2} \mathrm{O}_{3}$ & 9.0560 & 9.0830 & -0.0270 & $-0.3 \%$ \\
\hline BC-02 & $\mathrm{BaO}$ & 0.0405 & 0.0470 & -0.0065 & $-13.8 \%$ \\
\hline BC-02 & $\mathrm{CaO}$ & 0.6968 & 0.6300 & 0.0668 & $10.6 \%$ \\
\hline BC-02 & $\mathrm{Ce}_{2} \mathrm{O}_{3}$ & 0.0483 & 0.0390 & 0.0093 & $23.8 \%$ \\
\hline BC-02 & $\mathrm{Cr}_{2} \mathrm{O}_{3}$ & 0.1392 & 0.1520 & -0.0128 & $-8.4 \%$ \\
\hline BC-02 & $\mathrm{CuO}$ & 0.0426 & 0.0420 & 0.0006 & $1.4 \%$ \\
\hline BC-02 & $\mathrm{Fe}_{2} \mathrm{O}_{3}$ & 9.5468 & 9.9510 & -0.4042 & $-4.1 \%$ \\
\hline BC-02 & $\mathrm{K}_{2} \mathrm{O}$ & 0.0602 & 0.0100 & 0.0502 & $502.0 \%$ \\
\hline BC-02 & $\mathrm{La}_{2} \mathrm{O}_{3}$ & 0.0340 & 0.0340 & 0.0000 & $0.0 \%$ \\
\hline BC-02 & $\mathrm{Li}_{2} \mathrm{O}$ & 6.2757 & 6.5320 & -0.2563 & $-3.9 \%$ \\
\hline BC-02 & $\mathrm{MgO}$ & 0.3225 & 0.3590 & -0.0365 & $-10.2 \%$ \\
\hline BC-02 & $\mathrm{MnO}$ & 3.0634 & 3.0300 & 0.0334 & $1.1 \%$ \\
\hline BC-02 & $\mathrm{Na}_{2} \mathrm{O}$ & 11.5389 & 11.3930 & 0.1459 & $1.3 \%$ \\
\hline BC-02 & $\mathrm{NiO}$ & 0.9801 & 1.1140 & -0.1339 & $-12.0 \%$ \\
\hline BC-02 & $\mathrm{PbO}$ & 0.0108 & 0.0090 & 0.0018 & $20.0 \%$ \\
\hline BC-02 & $\mathrm{SiO}_{2}$ & 43.3743 & 44.2330 & -0.8587 & $-1.9 \%$ \\
\hline BC-02 & $\mathrm{SO}_{4}$ & 1.0748 & 1.2000 & -0.1252 & $-10.4 \%$ \\
\hline BC-02 & $\mathrm{TiO}_{2}$ & 0.0083 & 0.0000 & 0.0083 & \\
\hline BC-02 & $\mathrm{ZnO}$ & 0.0308 & 0.0260 & 0.0048 & $18.5 \%$ \\
\hline BC-02 & $\mathrm{ZrO}_{2}$ & 0.1189 & 0.1240 & -0.0051 & $-4.1 \%$ \\
\hline BC-02 & Sum & 98.5274 & 100.0010 & -1.4736 & $-1.5 \%$ \\
\hline BC-03 & $\mathrm{Al}_{2} \mathrm{O}_{3}$ & 12.0408 & 11.9930 & 0.0478 & $0.4 \%$ \\
\hline BC-03 & $\mathrm{B}_{2} \mathrm{O}_{3}$ & 4.7252 & 4.7940 & -0.0688 & $-1.4 \%$ \\
\hline BC-03 & $\mathrm{BaO}$ & 0.0430 & 0.0470 & -0.0040 & $-8.5 \%$ \\
\hline BC-03 & $\mathrm{CaO}$ & 0.7209 & 0.6300 & 0.0909 & $14.4 \%$ \\
\hline BC-03 & $\mathrm{Ce}_{2} \mathrm{O}_{3}$ & 0.0471 & 0.0390 & 0.0081 & $20.8 \%$ \\
\hline BC-03 & $\mathrm{Cr}_{2} \mathrm{O}_{3}$ & 0.1315 & 0.1520 & -0.0205 & $-13.5 \%$ \\
\hline BC-03 & $\mathrm{CuO}$ & 0.0463 & 0.0420 & 0.0043 & $10.2 \%$ \\
\hline BC-03 & $\mathrm{Fe}_{2} \mathrm{O}_{3}$ & 9.6576 & 9.9510 & -0.2934 & $-2.9 \%$ \\
\hline BC-03 & $\mathrm{K}_{2} \mathrm{O}$ & 0.0602 & 0.0100 & 0.0502 & $502.0 \%$ \\
\hline BC-03 & $\mathrm{La}_{2} \mathrm{O}_{3}$ & 0.0387 & 0.0340 & 0.0047 & $13.8 \%$ \\
\hline BC-03 & $\mathrm{Li}_{2} \mathrm{O}$ & 6.2380 & 6.5880 & -0.3500 & $-5.3 \%$ \\
\hline BC-03 & $\mathrm{MgO}$ & 0.3727 & 0.3590 & 0.0137 & $3.8 \%$ \\
\hline BC-03 & $\mathrm{MnO}$ & 3.0537 & 3.0300 & 0.0237 & $0.8 \%$ \\
\hline BC-03 & $\mathrm{Na}_{2} \mathrm{O}$ & 14.5584 & 14.2850 & 0.2734 & $1.9 \%$ \\
\hline BC-03 & $\mathrm{NiO}$ & 0.9483 & 1.1140 & -0.1657 & $-14.9 \%$ \\
\hline BC-03 & $\mathrm{PbO}$ & 0.0108 & 0.0090 & 0.0018 & $20.0 \%$ \\
\hline BC-03 & $\mathrm{SiO}_{2}$ & 44.3370 & 45.5750 & -1.2380 & $-2.7 \%$ \\
\hline BC-03 & $\mathrm{SO}_{4}$ & 1.1257 & 1.2000 & -0.0743 & $-6.2 \%$ \\
\hline BC-03 & $\mathrm{TiO}_{2}$ & 0.0083 & 0.0000 & 0.0083 & \\
\hline BC-03 & $\mathrm{ZnO}$ & 0.0380 & 0.0260 & 0.0120 & $46.2 \%$ \\
\hline BC-03 & $\mathrm{ZrO}_{2}$ & 0.1185 & 0.1240 & -0.0055 & $-4.4 \%$ \\
\hline BC-03 & Sum & 98.3210 & 100.0020 & -1.6810 & $-1.7 \%$ \\
\hline BC-04 & $\mathrm{Al}_{2} \mathrm{O}_{3}$ & 12.2676 & 11.9930 & 0.2746 & $2.3 \%$ \\
\hline BC-04 & $\mathrm{B}_{2} \mathrm{O}_{3}$ & 5.0311 & 4.6740 & 0.3571 & $7.6 \%$ \\
\hline BC-04 & $\mathrm{BaO}$ & 0.0380 & 0.0470 & -0.0090 & $-19.1 \%$ \\
\hline BC-04 & $\mathrm{CaO}$ & 5.5933 & 5.4260 & 0.1673 & $3.1 \%$ \\
\hline BC-04 & $\mathrm{Ce}_{2} \mathrm{O}_{3}$ & 0.0407 & 0.0390 & 0.0017 & $4.4 \%$ \\
\hline BC-04 & $\mathrm{Cr}_{2} \mathrm{O}_{3}$ & 0.1440 & 0.1520 & -0.0080 & $-5.3 \%$ \\
\hline BC-04 & $\mathrm{CuO}$ & 0.0397 & 0.0420 & -0.0023 & $-5.5 \%$ \\
\hline BC-04 & $\mathrm{Fe}_{2} \mathrm{O}_{3}$ & 9.8721 & 9.9510 & -0.0789 & $-0.8 \%$ \\
\hline
\end{tabular}


SRNL-STI-2012-00152

Revision 0

Table A-4. Average Measured Chemical Compositions versus Targeted Compositions by Oxide by Glass ID (continued)

\begin{tabular}{|c|c|c|c|c|c|}
\hline Glass ID & Oxide & $\begin{array}{c}\text { Measured } \\
\text { (wt \%) }\end{array}$ & $\begin{array}{c}\text { Targeted } \\
\text { (wt \%) }\end{array}$ & $\begin{array}{c}\text { Diff of } \\
\text { Measured }\end{array}$ & $\begin{array}{l}\text { \% Diff of } \\
\text { Measured }\end{array}$ \\
\hline BC-04 & $\mathrm{K}_{2} \mathrm{O}$ & 0.0602 & 0.0100 & 0.0502 & $502.0 \%$ \\
\hline BC-04 & $\mathrm{La}_{2} \mathrm{O}_{3}$ & 0.0375 & 0.0340 & 0.0035 & $10.3 \%$ \\
\hline BC-04 & $\mathrm{Li}_{2} \mathrm{O}$ & 6.3134 & 6.4230 & -0.1096 & $-1.7 \%$ \\
\hline BC-04 & $\mathrm{MgO}$ & 0.3171 & 0.3590 & -0.0419 & $-11.7 \%$ \\
\hline BC-04 & $\mathrm{MnO}$ & 3.0731 & 3.0300 & 0.0431 & $1.4 \%$ \\
\hline BC-04 & $\mathrm{Na}_{2} \mathrm{O}$ & 11.8422 & 11.3730 & 0.4692 & $4.1 \%$ \\
\hline BC-04 & $\mathrm{NiO}$ & 0.9620 & 1.1140 & -0.1520 & $-13.6 \%$ \\
\hline BC-04 & $\mathrm{PbO}$ & 0.0108 & 0.0090 & 0.0018 & $20.0 \%$ \\
\hline BC-04 & $\mathrm{SiO}_{2}$ & 43.7487 & 43.9750 & -0.2263 & $-0.5 \%$ \\
\hline BC-04 & $\mathrm{SO}_{4}$ & 1.0808 & 1.2000 & -0.1192 & $-9.9 \%$ \\
\hline BC-04 & $\mathrm{TiO}_{2}$ & 0.0083 & 0.0000 & 0.0083 & \\
\hline BC-04 & $\mathrm{ZnO}$ & 0.0383 & 0.0260 & 0.0123 & $47.3 \%$ \\
\hline BC-04 & $\mathrm{ZrO}_{2}$ & 0.1331 & 0.1240 & 0.0091 & $7.3 \%$ \\
\hline BC-04 & Sum & 100.6519 & 100.0010 & 0.6509 & $0.7 \%$ \\
\hline BC-05 & $\mathrm{Al}_{2} \mathrm{O}_{3}$ & 12.3384 & 11.9930 & 0.3454 & $2.9 \%$ \\
\hline BC-05 & $\mathrm{B}_{2} \mathrm{O}_{3}$ & 8.6696 & 8.8450 & -0.1754 & $-2.0 \%$ \\
\hline BC-05 & $\mathrm{BaO}$ & 0.0374 & 0.0470 & -0.0096 & $-20.4 \%$ \\
\hline BC-05 & $\mathrm{CaO}$ & 5.4709 & 5.3800 & 0.0909 & $1.7 \%$ \\
\hline BC-05 & $\mathrm{Ce}_{2} \mathrm{O}_{3}$ & 0.0416 & 0.0390 & 0.0026 & $6.7 \%$ \\
\hline BC-05 & $\mathrm{Cr}_{2} \mathrm{O}_{3}$ & 0.1396 & 0.1520 & -0.0124 & $-8.2 \%$ \\
\hline BC-05 & $\mathrm{CuO}$ & 0.0413 & 0.0420 & -0.0007 & $-1.7 \%$ \\
\hline BC-05 & $\mathrm{Fe}_{2} \mathrm{O}_{3}$ & 9.7291 & 9.9510 & -0.2219 & $-2.2 \%$ \\
\hline BC-05 & $\mathrm{K}_{2} \mathrm{O}$ & 0.0602 & 0.0100 & 0.0502 & $502.0 \%$ \\
\hline BC-05 & $\mathrm{La}_{2} \mathrm{O}_{3}$ & 0.0358 & 0.0340 & 0.0018 & $5.3 \%$ \\
\hline BC-05 & $\mathrm{Li}_{2} \mathrm{O}$ & 5.9904 & 6.3610 & -0.3706 & $-5.8 \%$ \\
\hline BC-05 & $\mathrm{MgO}$ & 0.3383 & 0.3590 & -0.0207 & $-5.8 \%$ \\
\hline BC-05 & $\mathrm{MnO}$ & 3.1441 & 3.0300 & 0.1141 & $3.8 \%$ \\
\hline BC-05 & $\mathrm{Na}_{2} \mathrm{O}$ & 11.5389 & 11.3620 & 0.1769 & $1.6 \%$ \\
\hline BC-05 & $\mathrm{NiO}$ & 1.0021 & 1.1140 & -0.1119 & $-10.0 \%$ \\
\hline BC-05 & $\mathrm{PbO}$ & 0.0108 & 0.0090 & 0.0018 & $20.0 \%$ \\
\hline BC-05 & $\mathrm{SiO}_{2}$ & 42.9464 & 39.9220 & 3.0244 & $7.6 \%$ \\
\hline BC-05 & $\mathrm{SO}_{4}$ & 1.1489 & 1.2000 & -0.0511 & $-4.3 \%$ \\
\hline BC-05 & $\mathrm{TiO}_{2}$ & 0.0083 & 0.0000 & 0.0083 & \\
\hline BC-05 & $\mathrm{ZnO}$ & 0.0286 & 0.0260 & 0.0026 & $10.0 \%$ \\
\hline BC-05 & $\mathrm{ZrO}_{2}$ & 0.1344 & 0.1240 & 0.0104 & $8.4 \%$ \\
\hline BC-05 & Sum & 102.8552 & 100.0000 & 2.8552 & $2.9 \%$ \\
\hline BC-06 & $\mathrm{Al}_{2} \mathrm{O}_{3}$ & 11.8613 & 11.9930 & -0.1317 & $-1.1 \%$ \\
\hline BC-06 & $\mathrm{B}_{2} \mathrm{O}_{3}$ & 9.1606 & 9.0700 & 0.0906 & $1.0 \%$ \\
\hline BC-06 & $\mathrm{BaO}$ & 0.0480 & 0.0470 & 0.0010 & $2.1 \%$ \\
\hline BC-06 & $\mathrm{CaO}$ & 0.6188 & 0.6300 & -0.0112 & $-1.8 \%$ \\
\hline BC-06 & $\mathrm{Ce}_{2} \mathrm{O}_{3}$ & 0.0416 & 0.0390 & 0.0026 & $6.7 \%$ \\
\hline BC-06 & $\mathrm{Cr}_{2} \mathrm{O}_{3}$ & 0.1352 & 0.1520 & -0.0168 & $-11.1 \%$ \\
\hline BC-06 & $\mathrm{CuO}$ & 0.0494 & 0.0420 & 0.0074 & $17.6 \%$ \\
\hline BC-06 & $\mathrm{Fe}_{2} \mathrm{O}_{3}$ & 9.5111 & 9.9510 & -0.4399 & $-4.4 \%$ \\
\hline BC-06 & $\mathrm{K}_{2} \mathrm{O}$ & 0.0602 & 0.0100 & 0.0502 & $502.0 \%$ \\
\hline BC-06 & $\mathrm{La}_{2} \mathrm{O}_{3}$ & 0.0311 & 0.0340 & -0.0029 & $-8.5 \%$ \\
\hline BC-06 & $\mathrm{Li}_{2} \mathrm{O}$ & 6.2380 & 6.5230 & -0.2850 & $-4.4 \%$ \\
\hline BC-06 & $\mathrm{MgO}$ & 0.3321 & 0.3590 & -0.0269 & $-7.5 \%$ \\
\hline BC-06 & $\mathrm{MnO}$ & 3.0537 & 3.0300 & 0.0237 & $0.8 \%$ \\
\hline BC-06 & $\mathrm{Na}_{2} \mathrm{O}$ & 14.1540 & 14.2440 & -0.0900 & $-0.6 \%$ \\
\hline BC-06 & $\mathrm{NiO}$ & 0.9541 & 1.1140 & -0.1599 & $-14.4 \%$ \\
\hline BC-06 & $\mathrm{PbO}$ & 0.0108 & 0.0090 & 0.0018 & $20.0 \%$ \\
\hline BC-06 & $\mathrm{SiO}_{2}$ & 40.9141 & 41.4050 & -0.4909 & $-1.2 \%$ \\
\hline BC-06 & $\mathrm{SO}_{4}$ & 1.1272 & 1.2000 & -0.0728 & $-6.1 \%$ \\
\hline BC-06 & $\mathrm{TiO}_{2}$ & 0.0083 & 0.0000 & 0.0083 & \\
\hline BC-06 & $\mathrm{ZnO}$ & 0.0314 & 0.0260 & 0.0054 & $20.8 \%$ \\
\hline BC-06 & $\mathrm{ZrO}_{2}$ & 0.1222 & 0.1240 & -0.0018 & $-1.5 \%$ \\
\hline
\end{tabular}


SRNL-STI-2012-00152

Revision 0

Table A-4. Average Measured Chemical Compositions versus Targeted Compositions by Oxide by Glass ID (continued)

\begin{tabular}{|c|c|c|c|c|c|}
\hline Glass ID & Oxide & $\begin{array}{c}\text { Measured } \\
\text { (wt \%) }\end{array}$ & $\begin{array}{c}\text { Targeted } \\
(\text { wt \%) }\end{array}$ & $\begin{array}{c}\text { Diff of } \\
\text { Measured }\end{array}$ & $\begin{array}{l}\text { \% Diff of } \\
\text { Measured }\end{array}$ \\
\hline BC-06 & Sum & 98.4633 & 100.0020 & -1.5387 & $-1.5 \%$ \\
\hline BC-07 & $\mathrm{Al}_{2} \mathrm{O}_{3}$ & 11.9842 & 11.9930 & -0.0088 & $-0.1 \%$ \\
\hline BC-07 & $\mathrm{B}_{2} \mathrm{O}_{3}$ & 8.9594 & 8.8330 & 0.1264 & $1.4 \%$ \\
\hline BC-07 & $\mathrm{BaO}$ & 0.0416 & 0.0470 & -0.0054 & $-11.5 \%$ \\
\hline BC-07 & $\mathrm{CaO}$ & 5.3624 & 5.3730 & -0.0106 & $-0.2 \%$ \\
\hline BC-07 & $\mathrm{Ce}_{2} \mathrm{O}_{3}$ & 0.0419 & 0.0390 & 0.0029 & $7.4 \%$ \\
\hline BC-07 & $\mathrm{Cr}_{2} \mathrm{O}_{3}$ & 0.1403 & 0.1520 & -0.0117 & $-7.7 \%$ \\
\hline BC-07 & $\mathrm{CuO}$ & 0.0494 & 0.0420 & 0.0074 & $17.6 \%$ \\
\hline BC-07 & $\mathrm{Fe}_{2} \mathrm{O}_{3}$ & 10.2188 & 9.9510 & 0.2678 & $2.7 \%$ \\
\hline BC-07 & $\mathrm{K}_{2} \mathrm{O}$ & 0.0602 & 0.0100 & 0.0502 & $502.0 \%$ \\
\hline BC-07 & $\mathrm{La}_{2} \mathrm{O}_{3}$ & 0.0337 & 0.0340 & -0.0003 & $-0.9 \%$ \\
\hline BC-07 & $\mathrm{Li}_{2} \mathrm{O}$ & 6.1465 & 6.3520 & -0.2055 & $-3.2 \%$ \\
\hline BC-07 & $\mathrm{MgO}$ & 0.3259 & 0.3590 & -0.0331 & $-9.2 \%$ \\
\hline BC-07 & $\mathrm{MnO}$ & 3.0537 & 3.0300 & 0.0237 & $0.8 \%$ \\
\hline BC-07 & $\mathrm{Na}_{2} \mathrm{O}$ & 13.9855 & 14.1390 & -0.1535 & $-1.1 \%$ \\
\hline BC-07 & $\mathrm{NiO}$ & 1.0129 & 1.1140 & -0.1011 & $-9.1 \%$ \\
\hline BC-07 & $\mathrm{PbO}$ & 0.0108 & 0.0090 & 0.0018 & $20.0 \%$ \\
\hline BC-07 & $\mathrm{SiO}_{2}$ & 36.5820 & 37.1740 & -0.5920 & $-1.6 \%$ \\
\hline BC-07 & $\mathrm{SO}_{4}$ & 1.1182 & 1.2000 & -0.0818 & $-6.8 \%$ \\
\hline BC-07 & $\mathrm{TiO}_{2}$ & 0.0083 & 0.0000 & 0.0083 & \\
\hline BC-07 & $\mathrm{ZnO}$ & 0.0286 & 0.0260 & 0.0026 & $10.0 \%$ \\
\hline BC-07 & $\mathrm{ZrO}_{2}$ & 0.1283 & 0.1240 & 0.0043 & $3.5 \%$ \\
\hline BC-07 & Sum & 99.2927 & 100.0010 & -0.7083 & $-0.7 \%$ \\
\hline BC-08 & $\mathrm{Al}_{2} \mathrm{O}_{3}$ & 11.8944 & 11.9930 & -0.0986 & $-0.8 \%$ \\
\hline BC-08 & $\mathrm{B}_{2} \mathrm{O}_{3}$ & 4.6367 & 4.6670 & -0.0303 & $-0.6 \%$ \\
\hline BC-08 & $\mathrm{BaO}$ & 0.0396 & 0.0470 & -0.0074 & $-15.7 \%$ \\
\hline BC-08 & $\mathrm{CaO}$ & 5.3764 & 5.4190 & -0.0426 & $-0.8 \%$ \\
\hline BC-08 & $\mathrm{Ce}_{2} \mathrm{O}_{3}$ & 0.0410 & 0.0390 & 0.0020 & $5.1 \%$ \\
\hline BC-08 & $\mathrm{Cr}_{2} \mathrm{O}_{3}$ & 0.1487 & 0.1520 & -0.0033 & $-2.2 \%$ \\
\hline BC-08 & $\mathrm{CuO}$ & 0.0451 & 0.0420 & 0.0031 & $7.4 \%$ \\
\hline BC-08 & $\mathrm{Fe}_{2} \mathrm{O}_{3}$ & 9.7684 & 9.9510 & -0.1826 & $-1.8 \%$ \\
\hline BC-08 & $\mathrm{K}_{2} \mathrm{O}$ & 0.0602 & 0.0100 & 0.0502 & $502.0 \%$ \\
\hline BC-08 & $\mathrm{La}_{2} \mathrm{O}_{3}$ & 0.0340 & 0.0340 & 0.0000 & $0.0 \%$ \\
\hline BC-08 & $\mathrm{Li}_{2} \mathrm{O}$ & 6.1573 & 6.4140 & -0.2567 & $-4.0 \%$ \\
\hline BC-08 & $\mathrm{MgO}$ & 0.3536 & 0.3590 & -0.0054 & $-1.5 \%$ \\
\hline BC-08 & $\mathrm{MnO}$ & 3.0601 & 3.0300 & 0.0301 & $1.0 \%$ \\
\hline BC-08 & $\mathrm{Na}_{2} \mathrm{O}$ & 14.0866 & 14.1770 & -0.0904 & $-0.6 \%$ \\
\hline BC-08 & $\mathrm{NiO}$ & 0.9773 & 1.1140 & -0.1367 & $-12.3 \%$ \\
\hline BC-08 & $\mathrm{PbO}$ & 0.0108 & 0.0090 & 0.0018 & $20.0 \%$ \\
\hline BC-08 & $\mathrm{SiO}_{2}$ & 40.6467 & 41.1940 & -0.5473 & $-1.3 \%$ \\
\hline BC-08 & $\mathrm{SO}_{4}$ & 1.1145 & 1.2000 & -0.0855 & $-7.1 \%$ \\
\hline BC-08 & $\mathrm{TiO}_{2}$ & 0.0083 & 0.0000 & 0.0083 & \\
\hline BC-08 & $\mathrm{ZnO}$ & 0.0324 & 0.0260 & 0.0064 & $24.6 \%$ \\
\hline BC-08 & $\mathrm{ZrO}_{2}$ & 0.1199 & 0.1240 & -0.0041 & $-3.3 \%$ \\
\hline BC-08 & Sum & 98.6121 & 100.0010 & -1.3889 & $-1.4 \%$ \\
\hline BC-09 & $\mathrm{Al}_{2} \mathrm{O}_{3}$ & 11.8708 & 11.9930 & -0.1222 & $-1.0 \%$ \\
\hline BC-09 & $\mathrm{B}_{2} \mathrm{O}_{3}$ & 6.6491 & 6.8550 & -0.2059 & $-3.0 \%$ \\
\hline BC-09 & $\mathrm{BaO}$ & 0.0385 & 0.0470 & -0.0085 & $-18.1 \%$ \\
\hline BC-09 & $\mathrm{CaO}$ & 2.9908 & 3.0460 & -0.0552 & $-1.8 \%$ \\
\hline BC-09 & $\mathrm{Ce}_{2} \mathrm{O}_{3}$ & 0.0395 & 0.0390 & 0.0005 & $1.3 \%$ \\
\hline BC-09 & $\mathrm{Cr}_{2} \mathrm{O}_{3}$ & 0.1363 & 0.1520 & -0.0157 & $-10.3 \%$ \\
\hline BC-09 & $\mathrm{CuO}$ & 0.0638 & 0.0420 & 0.0218 & $51.9 \%$ \\
\hline BC-09 & $\mathrm{Fe}_{2} \mathrm{O}_{3}$ & 9.5575 & 9.9510 & -0.3935 & $-4.0 \%$ \\
\hline BC-09 & $\mathrm{K}_{2} \mathrm{O}$ & 0.0602 & 0.0100 & 0.0502 & $502.0 \%$ \\
\hline BC-09 & $\mathrm{La}_{2} \mathrm{O}_{3}$ & 0.0314 & 0.0340 & -0.0026 & $-7.6 \%$ \\
\hline BC-09 & $\mathrm{Li}_{2} \mathrm{O}$ & 6.1573 & 6.4730 & -0.3157 & $-4.9 \%$ \\
\hline BC-09 & $\mathrm{MgO}$ & 0.3014 & 0.3590 & -0.0576 & $-16.0 \%$ \\
\hline
\end{tabular}


SRNL-STI-2012-00152

Revision 0

Table A-4. Average Measured Chemical Compositions versus Targeted Compositions by Oxide by Glass ID (continued)

\begin{tabular}{|c|c|c|c|c|c|}
\hline Glass ID & Oxide & $\begin{array}{c}\text { Measured } \\
\text { (wt \%) }\end{array}$ & $\begin{array}{c}\text { Targeted } \\
\text { (wt \%) }\end{array}$ & $\begin{array}{c}\text { Diff of } \\
\text { Measured }\end{array}$ & $\begin{array}{l}\text { \% Diff of } \\
\text { Measured }\end{array}$ \\
\hline BC-09 & $\mathrm{MnO}$ & 3.0634 & 3.0300 & 0.0334 & $1.1 \%$ \\
\hline BC-09 & $\mathrm{Na}_{2} \mathrm{O}$ & 12.5600 & 12.7980 & -0.2380 & $-1.9 \%$ \\
\hline BC-09 & $\mathrm{NiO}$ & 0.9757 & 1.1140 & -0.1383 & $-12.4 \%$ \\
\hline BC-09 & $\mathrm{PbO}$ & 0.0108 & 0.0090 & 0.0018 & $20.0 \%$ \\
\hline BC-09 & $\mathrm{SiO}_{2}$ & 42.0907 & 42.6990 & -0.6083 & $-1.4 \%$ \\
\hline BC-09 & $\mathrm{SO}_{4}$ & 1.1227 & 1.2000 & -0.0773 & $-6.4 \%$ \\
\hline BC-09 & $\mathrm{TiO}_{2}$ & 0.0083 & 0.0000 & 0.0083 & \\
\hline BC-09 & $\mathrm{ZnO}$ & 0.0271 & 0.0260 & 0.0011 & $4.2 \%$ \\
\hline BC-09 & $\mathrm{ZrO}_{2}$ & 0.1239 & 0.1240 & -0.0001 & $-0.1 \%$ \\
\hline BC-09 & Sum & 97.8793 & 100.0010 & -2.1217 & $-2.1 \%$ \\
\hline BS-01 & $\mathrm{Al}_{2} \mathrm{O}_{3}$ & 12.3951 & 11.9930 & 0.4021 & $3.4 \%$ \\
\hline BS-01 & $\mathrm{B}_{2} \mathrm{O}_{3}$ & 4.8379 & 4.8000 & 0.0379 & $0.8 \%$ \\
\hline BS-01 & $\mathrm{BaO}$ & 0.0410 & 0.0470 & -0.0060 & $-12.8 \%$ \\
\hline BS-01 & $\mathrm{CaO}$ & 0.6146 & 0.6300 & -0.0154 & $-2.4 \%$ \\
\hline BS-01 & $\mathrm{Ce}_{2} \mathrm{O}_{3}$ & 0.0436 & 0.0390 & 0.0046 & $11.8 \%$ \\
\hline BS-01 & $\mathrm{Cr}_{2} \mathrm{O}_{3}$ & 0.1378 & 0.1520 & -0.0142 & $-9.3 \%$ \\
\hline BS-01 & $\mathrm{CuO}$ & 0.0469 & 0.0420 & 0.0049 & $11.7 \%$ \\
\hline BS-01 & $\mathrm{Fe}_{2} \mathrm{O}_{3}$ & 10.0937 & 9.9510 & 0.1427 & $1.4 \%$ \\
\hline BS-01 & $\mathrm{K}_{2} \mathrm{O}$ & 0.0602 & 0.0100 & 0.0502 & $502.0 \%$ \\
\hline BS-01 & $\mathrm{La}_{2} \mathrm{O}_{3}$ & 0.0299 & 0.0340 & -0.0041 & $-12.1 \%$ \\
\hline BS-01 & $\mathrm{Li}_{2} \mathrm{O}$ & 6.3295 & 6.5990 & -0.2695 & $-4.1 \%$ \\
\hline BS-01 & $\mathrm{MgO}$ & 0.3279 & 0.3590 & -0.0311 & $-8.7 \%$ \\
\hline BS-01 & $\mathrm{MnO}$ & 3.1860 & 3.0300 & 0.1560 & $5.1 \%$ \\
\hline BS-01 & $\mathrm{Na}_{2} \mathrm{O}$ & 11.7310 & 11.4070 & 0.3240 & $2.8 \%$ \\
\hline BS-01 & $\mathrm{NiO}$ & 0.9903 & 1.1140 & -0.1237 & $-11.1 \%$ \\
\hline BS-01 & $\mathrm{PbO}$ & 0.0108 & 0.0090 & 0.0018 & $20.0 \%$ \\
\hline BS-01 & $\mathrm{SiO}_{2}$ & 47.7599 & 48.4350 & -0.6751 & $-1.4 \%$ \\
\hline BS-01 & $\mathrm{SO}_{4}$ & 1.0343 & 1.2000 & -0.1657 & $-13.8 \%$ \\
\hline BS-01 & $\mathrm{TiO}_{2}$ & 0.0083 & 0.0000 & 0.0083 & \\
\hline BS-01 & $\mathrm{ZnO}$ & 0.0345 & 0.0260 & 0.0085 & $32.7 \%$ \\
\hline BS-01 & $\mathrm{ZrO}_{2}$ & 0.1202 & 0.1240 & -0.0038 & $-3.1 \%$ \\
\hline BS-01 & Sum & 99.8337 & 100.0010 & -0.1673 & $-0.2 \%$ \\
\hline BS-02 & $\mathrm{Al}_{2} \mathrm{O}_{3}$ & 12.1070 & 11.9930 & 0.1140 & $1.0 \%$ \\
\hline BS-02 & $\mathrm{B}_{2} \mathrm{O}_{3}$ & 4.5079 & 4.8000 & -0.2921 & $-6.1 \%$ \\
\hline BS-02 & $\mathrm{BaO}$ & 0.0380 & 0.0470 & -0.0090 & $-19.1 \%$ \\
\hline BS-02 & $\mathrm{CaO}$ & 3.5959 & 3.6300 & -0.0341 & $-0.9 \%$ \\
\hline BS-02 & $\mathrm{Ce}_{2} \mathrm{O}_{3}$ & 0.0410 & 0.0390 & 0.0020 & $5.1 \%$ \\
\hline BS-02 & $\mathrm{Cr}_{2} \mathrm{O}_{3}$ & 0.1246 & 0.1520 & -0.0274 & $-18.0 \%$ \\
\hline BS-02 & $\mathrm{CuO}$ & 0.0516 & 0.0420 & 0.0096 & $22.9 \%$ \\
\hline BS-02 & $\mathrm{Fe}_{2} \mathrm{O}_{3}$ & 9.8363 & 9.9510 & -0.1147 & $-1.2 \%$ \\
\hline BS-02 & $\mathrm{K}_{2} \mathrm{O}$ & 0.0602 & 0.0100 & 0.0502 & $502.0 \%$ \\
\hline BS-02 & $\mathrm{La}_{2} \mathrm{O}_{3}$ & 0.0323 & 0.0340 & -0.0017 & $-5.0 \%$ \\
\hline BS-02 & $\mathrm{Li}_{2} \mathrm{O}$ & 6.8085 & 7.2000 & -0.3915 & $-5.4 \%$ \\
\hline BS-02 & $\mathrm{MgO}$ & 0.3192 & 0.3590 & -0.0398 & $-11.1 \%$ \\
\hline BS-02 & $\mathrm{MnO}$ & 3.0827 & 3.0300 & 0.0527 & $1.7 \%$ \\
\hline BS-02 & $\mathrm{Na}_{2} \mathrm{O}$ & 11.4479 & 11.4070 & 0.0409 & $0.4 \%$ \\
\hline BS-02 & $\mathrm{NiO}$ & 0.9509 & 1.1140 & -0.1631 & $-14.6 \%$ \\
\hline BS-02 & $\mathrm{PbO}$ & 0.0108 & 0.0090 & 0.0018 & $20.0 \%$ \\
\hline BS-02 & $\mathrm{SiO}_{2}$ & 45.0323 & 44.8340 & 0.1983 & $0.4 \%$ \\
\hline BS-02 & $\mathrm{SO}_{4}$ & 1.0928 & 1.2000 & -0.1072 & $-8.9 \%$ \\
\hline BS-02 & $\mathrm{TiO}_{2}$ & 0.0083 & 0.0000 & 0.0083 & \\
\hline BS-02 & $\mathrm{ZnO}$ & 0.0308 & 0.0260 & 0.0048 & $18.5 \%$ \\
\hline BS-02 & $\mathrm{ZrO}_{2}$ & 0.1189 & 0.1240 & -0.0051 & $-4.1 \%$ \\
\hline BS-02 & Sum & 99.2979 & 100.0010 & -0.7031 & $-0.7 \%$ \\
\hline BS-03 & $\mathrm{Al}_{2} \mathrm{O}_{3}$ & 11.9275 & 11.9930 & -0.0655 & $-0.5 \%$ \\
\hline BS-03 & $\mathrm{B}_{2} \mathrm{O}_{3}$ & 7.1723 & 7.2000 & -0.0277 & $-0.4 \%$ \\
\hline BS-03 & $\mathrm{BaO}$ & 0.0368 & 0.0470 & -0.0102 & $-21.7 \%$ \\
\hline
\end{tabular}


SRNL-STI-2012-00152

Revision 0

Table A-4. Average Measured Chemical Compositions versus Targeted Compositions by Oxide by Glass ID (continued)

\begin{tabular}{|c|c|c|c|c|c|}
\hline Glass ID & Oxide & $\begin{array}{c}\text { Measured } \\
\text { (wt \%) }\end{array}$ & $\begin{array}{c}\text { Targeted } \\
(\text { wt \%) }\end{array}$ & $\begin{array}{c}\text { Diff of } \\
\text { Measured }\end{array}$ & $\begin{array}{l}\text { \% Diff of } \\
\text { Measured }\end{array}$ \\
\hline BS-03 & $\mathrm{CaO}$ & 0.6115 & 0.6300 & -0.0185 & $-2.9 \%$ \\
\hline BS-03 & $\mathrm{Ce}_{2} \mathrm{O}_{3}$ & 0.0392 & 0.0390 & 0.0002 & $0.5 \%$ \\
\hline BS-03 & $\mathrm{Cr}_{2} \mathrm{O}_{3}$ & 0.1432 & 0.1520 & -0.0088 & $-5.8 \%$ \\
\hline BS-03 & $\mathrm{CuO}$ & 0.0554 & 0.0420 & 0.0134 & $31.9 \%$ \\
\hline BS-03 & $\mathrm{Fe}_{2} \mathrm{O}_{3}$ & 10.2259 & 9.9510 & 0.2749 & $2.8 \%$ \\
\hline BS-03 & $\mathrm{K}_{2} \mathrm{O}$ & 0.0602 & 0.0100 & 0.0502 & $502.0 \%$ \\
\hline BS-03 & $\mathrm{La}_{2} \mathrm{O}_{3}$ & 0.0273 & 0.0340 & -0.0067 & $-19.7 \%$ \\
\hline BS-03 & $\mathrm{Li}_{2} \mathrm{O}$ & 6.8301 & 7.2000 & -0.3699 & $-5.1 \%$ \\
\hline BS-03 & $\mathrm{MgO}$ & 0.3586 & 0.3590 & -0.0004 & $-0.1 \%$ \\
\hline BS-03 & $\mathrm{MnO}$ & 3.0375 & 3.0300 & 0.0075 & $0.2 \%$ \\
\hline BS-03 & $\mathrm{Na}_{2} \mathrm{O}$ & 11.3333 & 11.4070 & -0.0737 & $-0.6 \%$ \\
\hline BS-03 & $\mathrm{NiO}$ & 0.9824 & 1.1140 & -0.1316 & $-11.8 \%$ \\
\hline BS-03 & $\mathrm{PbO}$ & 0.0108 & 0.0090 & 0.0018 & $20.0 \%$ \\
\hline BS-03 & $\mathrm{SiO}_{2}$ & 45.1392 & 45.4340 & -0.2948 & $-0.6 \%$ \\
\hline BS-03 & $\mathrm{SO}_{4}$ & 1.0568 & 1.2000 & -0.1432 & $-11.9 \%$ \\
\hline BS-03 & $\mathrm{TiO}_{2}$ & 0.0083 & 0.0000 & 0.0083 & \\
\hline BS-03 & $\mathrm{ZnO}$ & 0.0296 & 0.0260 & 0.0036 & $13.8 \%$ \\
\hline BS-03 & $\mathrm{ZrO}_{2}$ & 0.1233 & 0.1240 & -0.0007 & $-0.6 \%$ \\
\hline BS-03 & Sum & 99.2093 & 100.0010 & -0.7917 & $-0.8 \%$ \\
\hline BS-04 & $\mathrm{Al}_{2} \mathrm{O}_{3}$ & 11.9747 & 11.9930 & -0.0183 & $-0.2 \%$ \\
\hline BS-04 & $\mathrm{B}_{2} \mathrm{O}_{3}$ & 7.2126 & 7.2000 & 0.0126 & $0.2 \%$ \\
\hline BS-04 & $\mathrm{BaO}$ & 0.0449 & 0.0470 & -0.0021 & $-4.5 \%$ \\
\hline BS-04 & $\mathrm{CaO}$ & 3.5750 & 3.6300 & -0.0550 & $-1.5 \%$ \\
\hline BS-04 & $\mathrm{Ce}_{2} \mathrm{O}_{3}$ & 0.0436 & 0.0390 & 0.0046 & $11.8 \%$ \\
\hline BS-04 & $\mathrm{Cr}_{2} \mathrm{O}_{3}$ & 0.1666 & 0.1520 & 0.0146 & $9.6 \%$ \\
\hline BS-04 & $\mathrm{CuO}$ & 0.0469 & 0.0420 & 0.0049 & $11.7 \%$ \\
\hline BS-04 & $\mathrm{Fe}_{2} \mathrm{O}_{3}$ & 9.7541 & 9.9510 & -0.1969 & $-2.0 \%$ \\
\hline BS-04 & $\mathrm{K}_{2} \mathrm{O}$ & 0.0602 & 0.0100 & 0.0502 & $502.0 \%$ \\
\hline BS-04 & $\mathrm{La}_{2} \mathrm{O}_{3}$ & 0.0367 & 0.0340 & 0.0027 & $7.9 \%$ \\
\hline BS-04 & $\mathrm{Li}_{2} \mathrm{O}$ & 6.2488 & 6.6000 & -0.3512 & $-5.3 \%$ \\
\hline BS-04 & $\mathrm{MgO}$ & 0.3250 & 0.3590 & -0.0340 & $-9.5 \%$ \\
\hline BS-04 & $\mathrm{MnO}$ & 3.0924 & 3.0300 & 0.0624 & $2.1 \%$ \\
\hline BS-04 & $\mathrm{Na}_{2} \mathrm{O}$ & 11.3569 & 11.4070 & -0.0501 & $-0.4 \%$ \\
\hline BS-04 & $\mathrm{NiO}$ & 1.0295 & 1.1140 & -0.0845 & $-7.6 \%$ \\
\hline BS-04 & $\mathrm{PbO}$ & 0.0108 & 0.0090 & 0.0018 & $20.0 \%$ \\
\hline BS-04 & $\mathrm{SiO}_{2}$ & 41.4489 & 43.0340 & -1.5851 & $-3.7 \%$ \\
\hline BS-04 & $\mathrm{SO}_{4}$ & 1.1010 & 1.2000 & -0.0990 & $-8.3 \%$ \\
\hline BS-04 & $\mathrm{TiO}_{2}$ & 0.0083 & 0.0000 & 0.0083 & \\
\hline BS-04 & $\mathrm{ZnO}$ & 0.0355 & 0.0260 & 0.0095 & $36.5 \%$ \\
\hline BS-04 & $\mathrm{ZrO}_{2}$ & 0.1108 & 0.1240 & -0.0132 & $-10.6 \%$ \\
\hline BS-04 & Sum & 97.6833 & 100.0010 & -2.3177 & $-2.3 \%$ \\
\hline BS-05 & $\mathrm{Al}_{2} \mathrm{O}_{3}$ & 12.0456 & 11.9930 & 0.0526 & $0.4 \%$ \\
\hline BS-05 & $\mathrm{B}_{2} \mathrm{O}_{3}$ & 5.7234 & 6.0000 & -0.2766 & $-4.6 \%$ \\
\hline BS-05 & $\mathrm{BaO}$ & 0.0343 & 0.0470 & -0.0127 & $-27.0 \%$ \\
\hline BS-05 & $\mathrm{CaO}$ & 1.8015 & 1.8300 & -0.0285 & $-1.6 \%$ \\
\hline BS-05 & $\mathrm{Ce}_{2} \mathrm{O}_{3}$ & 0.0433 & 0.0390 & 0.0043 & $11.0 \%$ \\
\hline BS-05 & $\mathrm{Cr}_{2} \mathrm{O}_{3}$ & 0.1516 & 0.1520 & -0.0004 & $-0.3 \%$ \\
\hline BS-05 & $\mathrm{CuO}$ & 0.0507 & 0.0420 & 0.0087 & $20.7 \%$ \\
\hline BS-05 & $\mathrm{Fe}_{2} \mathrm{O}_{3}$ & 9.6004 & 9.9510 & -0.3506 & $-3.5 \%$ \\
\hline BS-05 & $\mathrm{K}_{2} \mathrm{O}$ & 0.0602 & 0.0100 & 0.0502 & $502.0 \%$ \\
\hline BS-05 & $\mathrm{La}_{2} \mathrm{O}_{3}$ & 0.0320 & 0.0340 & -0.0020 & $-5.9 \%$ \\
\hline BS-05 & $\mathrm{Li}_{2} \mathrm{O}$ & 5.6406 & 6.0000 & -0.3594 & $-6.0 \%$ \\
\hline BS-05 & $\mathrm{MgO}$ & 0.3416 & 0.3590 & -0.0174 & $-4.8 \%$ \\
\hline BS-05 & $\mathrm{MnO}$ & 3.0827 & 3.0300 & 0.0527 & $1.7 \%$ \\
\hline BS-05 & $\mathrm{Na}_{2} \mathrm{O}$ & 12.5937 & 12.6070 & -0.0133 & $-0.1 \%$ \\
\hline BS-05 & $\mathrm{NiO}$ & 1.0250 & 1.1140 & -0.0890 & $-8.0 \%$ \\
\hline BS-05 & $\mathrm{PbO}$ & 0.0108 & 0.0090 & 0.0018 & $20.0 \%$ \\
\hline
\end{tabular}


SRNL-STI-2012-00152

Revision 0

Table A-4. Average Measured Chemical Compositions versus Targeted Compositions by Oxide by Glass ID (continued)

\begin{tabular}{|c|c|c|c|c|c|}
\hline Glass ID & Oxide & $\begin{array}{c}\text { Measured } \\
\text { (wt \%) }\end{array}$ & $\begin{array}{c}\text { Targeted } \\
\text { (wt \%) }\end{array}$ & $\begin{array}{c}\text { Diff of } \\
\text { Measured }\end{array}$ & $\begin{array}{l}\text { \% Diff of } \\
\text { Measured }\end{array}$ \\
\hline BS-05 & $\mathrm{SiO}_{2}$ & 45.2462 & 45.4340 & -0.1878 & $-0.4 \%$ \\
\hline BS-05 & $\mathrm{SO}_{4}$ & 1.0740 & 1.2000 & -0.1260 & $-10.5 \%$ \\
\hline BS-05 & $\mathrm{TiO}_{2}$ & 0.0083 & 0.0000 & 0.0083 & \\
\hline BS-05 & $\mathrm{ZnO}$ & 0.0330 & 0.0260 & 0.0070 & $26.9 \%$ \\
\hline BS-05 & $\mathrm{ZrO}_{2}$ & 0.1229 & 0.1240 & -0.0011 & $-0.9 \%$ \\
\hline BS-05 & Sum & 98.7219 & 100.0010 & -1.2791 & $-1.3 \%$ \\
\hline BS-06 & $\mathrm{Al}_{2} \mathrm{O}_{3}$ & 12.0692 & 11.9930 & 0.0762 & $0.6 \%$ \\
\hline BS-06 & $\mathrm{B}_{2} \mathrm{O}_{3}$ & 4.8218 & 4.8000 & 0.0218 & $0.5 \%$ \\
\hline BS-06 & $\mathrm{BaO}$ & 0.0329 & 0.0470 & -0.0141 & $-30.0 \%$ \\
\hline BS-06 & $\mathrm{CaO}$ & 0.6559 & 0.6300 & 0.0259 & $4.1 \%$ \\
\hline BS-06 & $\mathrm{Ce}_{2} \mathrm{O}_{3}$ & 0.0439 & 0.0390 & 0.0049 & $12.6 \%$ \\
\hline BS-06 & $\mathrm{Cr}_{2} \mathrm{O}_{3}$ & 0.1462 & 0.1520 & -0.0058 & $-3.8 \%$ \\
\hline BS-06 & $\mathrm{CuO}$ & 0.0388 & 0.0420 & -0.0032 & $-7.6 \%$ \\
\hline BS-06 & $\mathrm{Fe}_{2} \mathrm{O}_{3}$ & 9.3109 & 9.9510 & -0.6401 & $-6.4 \%$ \\
\hline BS-06 & $\mathrm{K}_{2} \mathrm{O}$ & 0.0602 & 0.0100 & 0.0502 & $502.0 \%$ \\
\hline BS-06 & $\mathrm{La}_{2} \mathrm{O}_{3}$ & 0.0290 & 0.0340 & -0.0050 & $-14.7 \%$ \\
\hline BS-06 & $\mathrm{Li}_{2} \mathrm{O}$ & 6.4426 & 6.6000 & -0.1574 & $-2.4 \%$ \\
\hline BS-06 & $\mathrm{MgO}$ & 0.3201 & 0.3590 & -0.0389 & $-10.8 \%$ \\
\hline BS-06 & $\mathrm{MnO}$ & 3.0763 & 3.0300 & 0.0463 & $1.5 \%$ \\
\hline BS-06 & $\mathrm{Na}_{2} \mathrm{O}$ & 13.5440 & 13.8070 & -0.2630 & $-1.9 \%$ \\
\hline BS-06 & $\mathrm{NiO}$ & 0.9976 & 1.1140 & -0.1164 & $-10.4 \%$ \\
\hline BS-06 & $\mathrm{PbO}$ & 0.0108 & 0.0090 & 0.0018 & $20.0 \%$ \\
\hline BS-06 & $\mathrm{SiO}_{2}$ & 46.3693 & 46.0340 & 0.3353 & $0.7 \%$ \\
\hline BS-06 & $\mathrm{SO}_{4}$ & 1.0860 & 1.2000 & -0.1140 & $-9.5 \%$ \\
\hline BS-06 & $\mathrm{TiO}_{2}$ & 0.0083 & 0.0000 & 0.0083 & \\
\hline BS-06 & $\mathrm{ZnO}$ & 0.0327 & 0.0260 & 0.0067 & $25.8 \%$ \\
\hline BS-06 & $\mathrm{ZrO}_{2}$ & 0.1385 & 0.1240 & 0.0145 & $11.7 \%$ \\
\hline BS-06 & Sum & 99.2350 & 100.0010 & -0.7660 & $-0.8 \%$ \\
\hline BS-07 & $\mathrm{Al}_{2} \mathrm{O}_{3}$ & 11.8235 & 11.9930 & -0.1695 & $-1.4 \%$ \\
\hline BS-07 & $\mathrm{B}_{2} \mathrm{O}_{3}$ & 4.8057 & 4.8000 & 0.0057 & $0.1 \%$ \\
\hline BS-07 & $\mathrm{BaO}$ & 0.0405 & 0.0470 & -0.0065 & $-13.8 \%$ \\
\hline BS-07 & $\mathrm{CaO}$ & 3.6414 & 3.6300 & 0.0114 & $0.3 \%$ \\
\hline BS-07 & $\mathrm{Ce}_{2} \mathrm{O}_{3}$ & 0.0433 & 0.0390 & 0.0043 & $11.0 \%$ \\
\hline BS-07 & $\mathrm{Cr}_{2} \mathrm{O}_{3}$ & 0.1432 & 0.1520 & -0.0088 & $-5.8 \%$ \\
\hline BS-07 & $\mathrm{CuO}$ & 0.0432 & 0.0420 & 0.0012 & $2.9 \%$ \\
\hline BS-07 & $\mathrm{Fe}_{2} \mathrm{O}_{3}$ & 9.6755 & 9.9510 & -0.2755 & $-2.8 \%$ \\
\hline BS-07 & $\mathrm{K}_{2} \mathrm{O}$ & 0.0602 & 0.0100 & 0.0502 & $502.0 \%$ \\
\hline BS-07 & $\mathrm{La}_{2} \mathrm{O}_{3}$ & 0.0346 & 0.0340 & 0.0006 & $1.8 \%$ \\
\hline BS-07 & $\mathrm{Li}_{2} \mathrm{O}$ & 5.1993 & 5.4000 & -0.2007 & $-3.7 \%$ \\
\hline BS-07 & $\mathrm{MgO}$ & 0.3250 & 0.3590 & -0.0340 & $-9.5 \%$ \\
\hline BS-07 & $\mathrm{MnO}$ & 3.0246 & 3.0300 & -0.0054 & $-0.2 \%$ \\
\hline BS-07 & $\mathrm{Na}_{2} \mathrm{O}$ & 12.7453 & 13.2070 & -0.4617 & $-3.5 \%$ \\
\hline BS-07 & $\mathrm{NiO}$ & 0.9929 & 1.1140 & -0.1211 & $-10.9 \%$ \\
\hline BS-07 & $\mathrm{PbO}$ & 0.0108 & 0.0090 & 0.0018 & $20.0 \%$ \\
\hline BS-07 & $\mathrm{SiO}_{2}$ & 45.1392 & 44.8340 & 0.3052 & $0.7 \%$ \\
\hline BS-07 & $\mathrm{SO}_{4}$ & 1.0448 & 1.2000 & -0.1552 & $-12.9 \%$ \\
\hline BS-07 & $\mathrm{TiO}_{2}$ & 0.0083 & 0.0000 & 0.0083 & \\
\hline BS-07 & $\mathrm{ZnO}$ & 0.0317 & 0.0260 & 0.0057 & $21.9 \%$ \\
\hline BS-07 & $\mathrm{ZrO}_{2}$ & 0.1192 & 0.1240 & -0.0048 & $-3.9 \%$ \\
\hline BS-07 & Sum & 98.9525 & 100.0010 & -1.0485 & $-1.0 \%$ \\
\hline BS-08 & $\mathrm{Al}_{2} \mathrm{O}_{3}$ & 11.5968 & 11.9930 & -0.3962 & $-3.3 \%$ \\
\hline BS-08 & $\mathrm{B}_{2} \mathrm{O}_{3}$ & 7.3092 & 7.2000 & 0.1092 & $1.5 \%$ \\
\hline BS-08 & $\mathrm{BaO}$ & 0.0449 & 0.0470 & -0.0021 & $-4.5 \%$ \\
\hline BS-08 & $\mathrm{CaO}$ & 0.6744 & 0.6300 & 0.0444 & $7.0 \%$ \\
\hline BS-08 & $\mathrm{Ce}_{2} \mathrm{O}_{3}$ & 0.0536 & 0.0390 & 0.0146 & $37.4 \%$ \\
\hline BS-08 & $\mathrm{Cr}_{2} \mathrm{O}_{3}$ & 0.1257 & 0.1520 & -0.0263 & $-17.3 \%$ \\
\hline BS-08 & $\mathrm{CuO}$ & 0.0401 & 0.0420 & -0.0019 & $-4.5 \%$ \\
\hline
\end{tabular}


SRNL-STI-2012-00152

Revision 0

Table A-4. Average Measured Chemical Compositions versus Targeted Compositions by Oxide by Glass ID (continued)

\begin{tabular}{|c|c|c|c|c|c|}
\hline Glass ID & Oxide & $\begin{array}{c}\text { Measured } \\
\text { (wt \%) }\end{array}$ & $\begin{array}{c}\text { Targeted } \\
\text { (wt \%) }\end{array}$ & $\begin{array}{c}\text { Diff of } \\
\text { Measured }\end{array}$ & $\begin{array}{l}\text { \% Diff of } \\
\text { Measured }\end{array}$ \\
\hline BS-08 & $\mathrm{Fe}_{2} \mathrm{O}_{3}$ & 8.9428 & 9.9510 & -1.0082 & $-10.1 \%$ \\
\hline BS-08 & $\mathrm{K}_{2} \mathrm{O}$ & 0.0602 & 0.0100 & 0.0502 & $502.0 \%$ \\
\hline BS-08 & $\mathrm{La}_{2} \mathrm{O}_{3}$ & 0.0399 & 0.0340 & 0.0059 & $17.4 \%$ \\
\hline BS-08 & $\mathrm{Li}_{2} \mathrm{O}$ & 5.8021 & 6.0000 & -0.1979 & $-3.3 \%$ \\
\hline BS-08 & $\mathrm{MgO}$ & 0.3346 & 0.3590 & -0.0244 & $-6.8 \%$ \\
\hline BS-08 & $\mathrm{MnO}$ & 2.9827 & 3.0300 & -0.0473 & $-1.6 \%$ \\
\hline BS-08 & $\mathrm{Na}_{2} \mathrm{O}$ & 12.6240 & 13.2070 & -0.5830 & $-4.4 \%$ \\
\hline BS-08 & $\mathrm{NiO}$ & 0.9875 & 1.1140 & -0.1265 & $-11.4 \%$ \\
\hline BS-08 & $\mathrm{PbO}$ & 0.0108 & 0.0090 & 0.0018 & $20.0 \%$ \\
\hline BS-08 & $\mathrm{SiO}_{2}$ & 44.5509 & 44.8340 & -0.2831 & $-0.6 \%$ \\
\hline BS-08 & $\mathrm{SO}_{4}$ & 1.0883 & 1.2000 & -0.1117 & $-9.3 \%$ \\
\hline BS-08 & $\mathrm{TiO}_{2}$ & 0.0083 & 0.0000 & 0.0083 & \\
\hline BS-08 & $\mathrm{ZnO}$ & 0.0293 & 0.0260 & 0.0033 & $12.7 \%$ \\
\hline BS-08 & $\mathrm{ZrO}_{2}$ & 0.1165 & 0.1240 & -0.0075 & $-6.0 \%$ \\
\hline BS-08 & Sum & 97.4224 & 100.0010 & -2.5786 & $-2.6 \%$ \\
\hline BS-09 & $\mathrm{Al}_{2} \mathrm{O}_{3}$ & 11.6960 & 11.9930 & -0.2970 & $-2.5 \%$ \\
\hline BS-09 & $\mathrm{B}_{2} \mathrm{O}_{3}$ & 7.2367 & 7.2000 & 0.0367 & $0.5 \%$ \\
\hline BS-09 & $\mathrm{BaO}$ & 0.0360 & 0.0470 & -0.0110 & $-23.4 \%$ \\
\hline BS-09 & $\mathrm{CaO}$ & 3.6169 & 3.6300 & -0.0131 & $-0.4 \%$ \\
\hline BS-09 & $\mathrm{Ce}_{2} \mathrm{O}_{3}$ & 0.0442 & 0.0390 & 0.0052 & $13.3 \%$ \\
\hline BS-09 & $\mathrm{Cr}_{2} \mathrm{O}_{3}$ & 0.1560 & 0.1520 & 0.0040 & $2.6 \%$ \\
\hline BS-09 & $\mathrm{CuO}$ & 0.0448 & 0.0420 & 0.0028 & $6.7 \%$ \\
\hline BS-09 & $\mathrm{Fe}_{2} \mathrm{O}_{3}$ & 9.5897 & 9.9510 & -0.3613 & $-3.6 \%$ \\
\hline BS-09 & $\mathrm{K}_{2} \mathrm{O}$ & 0.0602 & 0.0100 & 0.0502 & $502.0 \%$ \\
\hline BS-09 & $\mathrm{La}_{2} \mathrm{O}_{3}$ & 0.0340 & 0.0340 & 0.0000 & $0.0 \%$ \\
\hline BS-09 & $\mathrm{Li}_{2} \mathrm{O}$ & 5.0862 & 5.4000 & -0.3138 & $-5.8 \%$ \\
\hline BS-09 & $\mathrm{MgO}$ & 0.3155 & 0.3590 & -0.0435 & $-12.1 \%$ \\
\hline BS-09 & $\mathrm{MnO}$ & 2.9891 & 3.0300 & -0.0409 & $-1.3 \%$ \\
\hline BS-09 & $\mathrm{Na}_{2} \mathrm{O}$ & 12.7588 & 13.2070 & -0.4482 & $-3.4 \%$ \\
\hline BS-09 & $\mathrm{NiO}$ & 1.0097 & 1.1140 & -0.1043 & $-9.4 \%$ \\
\hline BS-09 & $\mathrm{PbO}$ & 0.0108 & 0.0090 & 0.0018 & $20.0 \%$ \\
\hline BS-09 & $\mathrm{SiO}_{2}$ & 41.8768 & 42.4340 & -0.5572 & $-1.3 \%$ \\
\hline BS-09 & $\mathrm{SO}_{4}$ & 1.1040 & 1.2000 & -0.0960 & $-8.0 \%$ \\
\hline BS-09 & $\mathrm{TiO}_{2}$ & 0.0083 & 0.0000 & 0.0083 & \\
\hline BS-09 & $\mathrm{ZnO}$ & 0.0293 & 0.0260 & 0.0033 & $12.7 \%$ \\
\hline BS-09 & $\mathrm{ZrO}_{2}$ & 0.1212 & 0.1240 & -0.0028 & $-2.3 \%$ \\
\hline BS-09 & Sum & 97.8244 & 100.0010 & -2.1766 & $-2.2 \%$ \\
\hline LL-03 & $\mathrm{Al}_{2} \mathrm{O}_{3}$ & 8.9279 & 8.9950 & -0.0671 & $-0.7 \%$ \\
\hline LL-03 & $\mathrm{B}_{2} \mathrm{O}_{3}$ & 10.4808 & 10.3050 & 0.1758 & $1.7 \%$ \\
\hline LL-03 & $\mathrm{BaO}$ & 0.0229 & 0.0350 & -0.0121 & $-34.6 \%$ \\
\hline LL-03 & $\mathrm{CaO}$ & 6.0445 & 6.0060 & 0.0385 & $0.6 \%$ \\
\hline LL-03 & $\mathrm{Ce}_{2} \mathrm{O}_{3}$ & 0.0337 & 0.0290 & 0.0047 & $16.2 \%$ \\
\hline LL-03 & $\mathrm{Cr}_{2} \mathrm{O}_{3}$ & 0.1133 & 0.1140 & -0.0007 & $-0.6 \%$ \\
\hline LL-03 & $\mathrm{CuO}$ & 0.0410 & 0.0310 & 0.0100 & $32.3 \%$ \\
\hline LL-03 & $\mathrm{Fe}_{2} \mathrm{O}_{3}$ & 7.2486 & 7.4630 & -0.2144 & $-2.9 \%$ \\
\hline LL-03 & $\mathrm{K}_{2} \mathrm{O}$ & 0.0602 & 0.0080 & 0.0522 & $652.5 \%$ \\
\hline LL-03 & $\mathrm{La}_{2} \mathrm{O}_{3}$ & 0.0290 & 0.0260 & 0.0030 & $11.5 \%$ \\
\hline LL-03 & $\mathrm{Li}_{2} \mathrm{O}$ & 7.3844 & 7.4110 & -0.0266 & $-0.4 \%$ \\
\hline LL-03 & $\mathrm{MgO}$ & 0.2467 & 0.2700 & -0.0233 & $-8.6 \%$ \\
\hline LL-03 & $\mathrm{MnO}$ & 2.2661 & 2.2720 & -0.0059 & $-0.3 \%$ \\
\hline LL-03 & $\mathrm{Na}_{2} \mathrm{O}$ & 11.9467 & 12.2420 & -0.2953 & $-2.4 \%$ \\
\hline LL-03 & $\mathrm{NiO}$ & 0.7600 & 0.8350 & -0.0750 & $-9.0 \%$ \\
\hline LL-03 & $\mathrm{PbO}$ & 0.0108 & 0.0070 & 0.0038 & $54.3 \%$ \\
\hline LL-03 & $\mathrm{SiO}_{2}$ & 43.5882 & 42.9390 & 0.6492 & $1.5 \%$ \\
\hline LL-03 & $\mathrm{SO}_{4}$ & 0.8531 & 0.9000 & -0.0469 & $-5.2 \%$ \\
\hline LL-03 & $\mathrm{TiO}_{2}$ & 0.0083 & 0.0000 & 0.0083 & \\
\hline LL-03 & $\mathrm{ZnO}$ & 0.0237 & 0.0190 & 0.0047 & $24.7 \%$ \\
\hline
\end{tabular}


SRNL-STI-2012-00152

Revision 0

Table A-4. Average Measured Chemical Compositions versus Targeted Compositions by Oxide by Glass ID (continued)

\begin{tabular}{|c|c|c|c|c|c|}
\hline Glass ID & Oxide & $\begin{array}{c}\text { Measured } \\
\text { (wt \%) }\end{array}$ & $\begin{array}{c}\text { Targeted } \\
\text { (wt \%) }\end{array}$ & $\begin{array}{c}\text { Diff of } \\
\text { Measured }\end{array}$ & $\begin{array}{l}\text { \% Diff of } \\
\text { Measured }\end{array}$ \\
\hline LL-03 & $\mathrm{ZrO}_{2}$ & 0.0932 & 0.0930 & 0.0002 & $0.2 \%$ \\
\hline LL-03 & Sum & 100.1830 & 100.0000 & 0.1830 & $0.2 \%$ \\
\hline LL-05 & $\mathrm{Al}_{2} \mathrm{O}_{3}$ & 12.1117 & 12.2370 & -0.1253 & $-1.0 \%$ \\
\hline LL-05 & $\mathrm{B}_{2} \mathrm{O}_{3}$ & 6.8101 & 6.8550 & -0.0449 & $-0.7 \%$ \\
\hline LL-05 & $\mathrm{BaO}$ & 0.0363 & 0.0420 & -0.0057 & $-13.6 \%$ \\
\hline LL-05 & $\mathrm{CaO}$ & 3.0118 & 2.9840 & 0.0278 & $0.9 \%$ \\
\hline LL-05 & $\mathrm{Ce}_{2} \mathrm{O}_{3}$ & 0.0392 & 0.0350 & 0.0042 & $12.0 \%$ \\
\hline LL-05 & $\mathrm{Cr}_{2} \mathrm{O}_{3}$ & 0.1180 & 0.1370 & -0.0190 & $-13.9 \%$ \\
\hline LL-05 & $\mathrm{CuO}$ & 0.0394 & 0.0380 & 0.0014 & $3.7 \%$ \\
\hline LL-05 & $\mathrm{Fe}_{2} \mathrm{O}_{3}$ & 9.8149 & 10.1540 & -0.3391 & $-3.3 \%$ \\
\hline LL-05 & $\mathrm{K}_{2} \mathrm{O}$ & 0.0602 & 0.0090 & 0.0512 & $568.9 \%$ \\
\hline LL-05 & $\mathrm{La}_{2} \mathrm{O}_{3}$ & 0.0293 & 0.0310 & -0.0017 & $-5.5 \%$ \\
\hline LL-05 & $\mathrm{Li}_{2} \mathrm{O}$ & 6.1465 & 6.4730 & -0.3265 & $-5.0 \%$ \\
\hline LL-05 & $\mathrm{MgO}$ & 0.2943 & 0.3240 & -0.0297 & $-9.2 \%$ \\
\hline LL-05 & $\mathrm{MnO}$ & 2.7470 & 2.7310 & 0.0160 & $0.6 \%$ \\
\hline LL-05 & $\mathrm{Na}_{2} \mathrm{O}$ & 12.6881 & 13.0060 & -0.3179 & $-2.4 \%$ \\
\hline LL-05 & $\mathrm{NiO}$ & 0.8831 & 1.0040 & -0.1209 & $-12.0 \%$ \\
\hline LL-05 & $\mathrm{PbO}$ & 0.0108 & 0.0080 & 0.0028 & $35.0 \%$ \\
\hline LL-05 & $\mathrm{SiO}_{2}$ & 41.8233 & 42.5980 & -0.7747 & $-1.8 \%$ \\
\hline LL-05 & $\mathrm{SO}_{4}$ & 1.1100 & 1.2000 & -0.0900 & $-7.5 \%$ \\
\hline LL-05 & $\mathrm{TiO}_{2}$ & 0.0083 & 0.0000 & 0.0083 & \\
\hline LL-05 & $\mathrm{ZnO}$ & 0.0271 & 0.0230 & 0.0041 & $17.8 \%$ \\
\hline LL-05 & $\mathrm{ZrO}_{2}$ & 0.1067 & 0.1120 & -0.0053 & $-4.7 \%$ \\
\hline LL-05 & Sum & 97.9162 & 100.0010 & -2.0848 & $-2.1 \%$ \\
\hline LL-06 & $\mathrm{Al}_{2} \mathrm{O}_{3}$ & 11.8708 & 12.2370 & -0.3662 & $-3.0 \%$ \\
\hline LL-06 & $\mathrm{B}_{2} \mathrm{O}_{3}$ & 8.8306 & 8.8330 & -0.0024 & $0.0 \%$ \\
\hline LL-06 & $\mathrm{BaO}$ & 0.0327 & 0.0420 & -0.0093 & $-22.1 \%$ \\
\hline LL-06 & $\mathrm{CaO}$ & 5.2470 & 5.3110 & -0.0640 & $-1.2 \%$ \\
\hline LL-06 & $\mathrm{Ce}_{2} \mathrm{O}_{3}$ & 0.0366 & 0.0350 & 0.0016 & $4.6 \%$ \\
\hline LL-06 & $\mathrm{Cr}_{2} \mathrm{O}_{3}$ & 0.1283 & 0.1370 & -0.0087 & $-6.4 \%$ \\
\hline LL-06 & $\mathrm{CuO}$ & 0.0454 & 0.0380 & 0.0074 & $19.5 \%$ \\
\hline LL-06 & $\mathrm{Fe}_{2} \mathrm{O}_{3}$ & 9.6719 & 10.1540 & -0.4821 & $-4.7 \%$ \\
\hline LL-06 & $\mathrm{K}_{2} \mathrm{O}$ & 0.0602 & 0.0090 & 0.0512 & $568.9 \%$ \\
\hline LL-06 & $\mathrm{La}_{2} \mathrm{O}_{3}$ & 0.0293 & 0.0310 & -0.0017 & $-5.5 \%$ \\
\hline LL-06 & $\mathrm{Li}_{2} \mathrm{O}$ & 6.2111 & 6.3520 & -0.1409 & $-2.2 \%$ \\
\hline LL-06 & $\mathrm{MgO}$ & 0.3715 & 0.3240 & 0.0475 & $14.7 \%$ \\
\hline LL-06 & $\mathrm{MnO}$ & 2.7051 & 2.7310 & -0.0259 & $-0.9 \%$ \\
\hline LL-06 & $\mathrm{Na}_{2} \mathrm{O}$ & 13.7361 & 14.3470 & -0.6109 & $-4.3 \%$ \\
\hline LL-06 & $\mathrm{NiO}$ & 0.8860 & 1.0040 & -0.1180 & $-11.8 \%$ \\
\hline LL-06 & $\mathrm{PbO}$ & 0.0108 & 0.0080 & 0.0028 & $35.0 \%$ \\
\hline LL-06 & $\mathrm{SiO}_{2}$ & 37.4378 & 37.0720 & 0.3658 & $1.0 \%$ \\
\hline LL-06 & $\mathrm{SO}_{4}$ & 1.1295 & 1.2000 & -0.0705 & $-5.9 \%$ \\
\hline LL-06 & $\mathrm{TiO}_{2}$ & 0.0083 & 0.0000 & 0.0083 & \\
\hline LL-06 & $\mathrm{ZnO}$ & 0.0293 & 0.0230 & 0.0063 & $27.4 \%$ \\
\hline LL-06 & $\mathrm{ZrO}_{2}$ & 0.1060 & 0.1120 & -0.0060 & $-5.4 \%$ \\
\hline LL-06 & Sum & 98.5841 & 100.0000 & -1.4159 & $-1.4 \%$ \\
\hline LL-10 & $\mathrm{Al}_{2} \mathrm{O}_{3}$ & 11.7149 & 12.2370 & -0.5221 & $-4.3 \%$ \\
\hline LL-10 & $\mathrm{B}_{2} \mathrm{O}_{3}$ & 8.9111 & 9.0830 & -0.1719 & $-1.9 \%$ \\
\hline LL-10 & $\mathrm{BaO}$ & 0.0329 & 0.0420 & -0.0091 & $-21.7 \%$ \\
\hline LL-10 & $\mathrm{CaO}$ & 0.5954 & 0.5680 & 0.0274 & $4.8 \%$ \\
\hline LL-10 & $\mathrm{Ce}_{2} \mathrm{O}_{3}$ & 0.0387 & 0.0350 & 0.0037 & $10.6 \%$ \\
\hline LL-10 & $\mathrm{Cr}_{2} \mathrm{O}_{3}$ & 0.1253 & 0.1370 & -0.0117 & $-8.5 \%$ \\
\hline LL-10 & $\mathrm{CuO}$ & 0.0376 & 0.0380 & -0.0004 & $-1.1 \%$ \\
\hline LL-10 & $\mathrm{Fe}_{2} \mathrm{O}_{3}$ & 9.4467 & 10.1540 & -0.7073 & $-7.0 \%$ \\
\hline LL-10 & $\mathrm{K}_{2} \mathrm{O}$ & 0.0602 & 0.0090 & 0.0512 & $568.9 \%$ \\
\hline LL-10 & $\mathrm{La}_{2} \mathrm{O}_{3}$ & 0.0287 & 0.0310 & -0.0023 & $-7.4 \%$ \\
\hline LL-10 & $\mathrm{Li}_{2} \mathrm{O}$ & 6.2811 & 6.5320 & -0.2509 & $-3.8 \%$ \\
\hline
\end{tabular}


SRNL-STI-2012-00152

Revision 0

Table A-4. Average Measured Chemical Compositions versus Targeted Compositions by Oxide by Glass ID (continued)

\begin{tabular}{|c|c|c|c|c|c|}
\hline Glass ID & Oxide & $\begin{array}{c}\text { Measured } \\
\text { (wt \%) }\end{array}$ & $\begin{array}{c}\text { Targeted } \\
\text { (wt \%) }\end{array}$ & $\begin{array}{c}\text { Diff of } \\
\text { Measured }\end{array}$ & $\begin{array}{l}\text { \% Diff of } \\
\text { Measured }\end{array}$ \\
\hline LL-10 & $\mathrm{MgO}$ & 0.2715 & 0.3240 & -0.0525 & $-16.2 \%$ \\
\hline LL-10 & $\mathrm{MnO}$ & 2.6534 & 2.7310 & -0.0776 & $-2.8 \%$ \\
\hline LL-10 & $\mathrm{Na}_{2} \mathrm{O}$ & 10.7773 & 11.6010 & -0.8237 & $-7.1 \%$ \\
\hline LL-10 & $\mathrm{NiO}$ & 0.8962 & 1.0040 & -0.1078 & $-10.7 \%$ \\
\hline LL-10 & $\mathrm{PbO}$ & 0.0108 & 0.0080 & 0.0028 & $35.0 \%$ \\
\hline LL-10 & $\mathrm{SiO}_{2}$ & 45.2462 & 44.1310 & 1.1152 & $2.5 \%$ \\
\hline LL-10 & $\mathrm{SO}_{4}$ & 1.0628 & 1.2000 & -0.1372 & $-11.4 \%$ \\
\hline LL-10 & $\mathrm{TiO}_{2}$ & 0.0083 & 0.0000 & 0.0083 & \\
\hline LL-10 & $\mathrm{ZnO}$ & 0.0261 & 0.0230 & 0.0031 & $13.5 \%$ \\
\hline LL-10 & $\mathrm{ZrO}_{2}$ & 0.1212 & 0.1120 & 0.0092 & $8.2 \%$ \\
\hline LL-10 & Sum & 98.3465 & 100.0000 & -1.6535 & $-1.7 \%$ \\
\hline LL-11 & $\mathrm{Al}_{2} \mathrm{O}_{3}$ & 11.9983 & 12.2370 & -0.2387 & $-2.0 \%$ \\
\hline LL-11 & $\mathrm{B}_{2} \mathrm{O}_{3}$ & 4.7735 & 4.6740 & 0.0995 & $2.1 \%$ \\
\hline LL-11 & $\mathrm{BaO}$ & 0.0363 & 0.0420 & -0.0057 & $-13.6 \%$ \\
\hline LL-11 & $\mathrm{CaO}$ & 5.3729 & 5.3640 & 0.0089 & $0.2 \%$ \\
\hline LL-11 & $\mathrm{Ce}_{2} \mathrm{O}_{3}$ & 0.0395 & 0.0350 & 0.0045 & $12.9 \%$ \\
\hline LL-11 & $\mathrm{Cr}_{2} \mathrm{O}_{3}$ & 0.1436 & 0.1370 & 0.0066 & $4.8 \%$ \\
\hline LL-11 & $\mathrm{CuO}$ & 0.0357 & 0.0380 & -0.0023 & $-6.1 \%$ \\
\hline LL-11 & $\mathrm{Fe}_{2} \mathrm{O}_{3}$ & 9.9650 & 10.1540 & -0.1890 & $-1.9 \%$ \\
\hline LL-11 & $\mathrm{K}_{2} \mathrm{O}$ & 0.0602 & 0.0090 & 0.0512 & $568.9 \%$ \\
\hline LL-11 & $\mathrm{La}_{2} \mathrm{O}_{3}$ & 0.0334 & 0.0310 & 0.0024 & $7.7 \%$ \\
\hline LL-11 & $\mathrm{Li}_{2} \mathrm{O}$ & 6.1842 & 6.4230 & -0.2388 & $-3.7 \%$ \\
\hline LL-11 & $\mathrm{MgO}$ & 0.2898 & 0.3240 & -0.0342 & $-10.6 \%$ \\
\hline LL-11 & $\mathrm{MnO}$ & 2.7147 & 2.7310 & -0.0163 & $-0.6 \%$ \\
\hline LL-11 & $\mathrm{Na}_{2} \mathrm{O}$ & 11.2895 & 11.5820 & -0.2925 & $-2.5 \%$ \\
\hline LL-11 & $\mathrm{NiO}$ & 0.9086 & 1.0040 & -0.0954 & $-9.5 \%$ \\
\hline LL-11 & $\mathrm{PbO}$ & 0.0108 & 0.0080 & 0.0028 & $35.0 \%$ \\
\hline LL-11 & $\mathrm{SiO}_{2}$ & 43.6417 & 43.8730 & -0.2313 & $-0.5 \%$ \\
\hline LL-11 & $\mathrm{SO}_{4}$ & 1.1939 & 1.2000 & -0.0061 & $-0.5 \%$ \\
\hline LL-11 & $\mathrm{TiO}_{2}$ & 0.0083 & 0.0000 & 0.0083 & \\
\hline LL-11 & $\mathrm{ZnO}$ & 0.0268 & 0.0230 & 0.0038 & $16.5 \%$ \\
\hline LL-11 & $\mathrm{ZrO}_{2}$ & 0.1209 & 0.1120 & 0.0089 & $7.9 \%$ \\
\hline LL-11 & Sum & 98.8477 & 100.0010 & -1.1533 & $-1.2 \%$ \\
\hline LL-12 & $\mathrm{Al}_{2} \mathrm{O}_{3}$ & 11.9039 & 12.2370 & -0.3331 & $-2.7 \%$ \\
\hline LL-12 & $\mathrm{B}_{2} \mathrm{O}_{3}$ & 8.8467 & 8.8450 & 0.0017 & $0.0 \%$ \\
\hline LL-12 & $\mathrm{BaO}$ & 0.0301 & 0.0420 & -0.0119 & $-28.3 \%$ \\
\hline LL-12 & $\mathrm{CaO}$ & 5.3135 & 5.3180 & -0.0045 & $-0.1 \%$ \\
\hline LL-12 & $\mathrm{Ce}_{2} \mathrm{O}_{3}$ & 0.0384 & 0.0350 & 0.0034 & $9.7 \%$ \\
\hline LL-12 & $\mathrm{Cr}_{2} \mathrm{O}_{3}$ & 0.1173 & 0.1370 & -0.0197 & $-14.4 \%$ \\
\hline LL-12 & $\mathrm{CuO}$ & 0.0341 & 0.0380 & -0.0039 & $-10.3 \%$ \\
\hline LL-12 & $\mathrm{Fe}_{2} \mathrm{O}_{3}$ & 9.5826 & 10.1540 & -0.5714 & $-5.6 \%$ \\
\hline LL-12 & $\mathrm{K}_{2} \mathrm{O}$ & 0.0602 & 0.0090 & 0.0512 & $568.9 \%$ \\
\hline LL-12 & $\mathrm{La}_{2} \mathrm{O}_{3}$ & 0.0299 & 0.0310 & -0.0011 & $-3.5 \%$ \\
\hline LL-12 & $\mathrm{Li}_{2} \mathrm{O}$ & 6.2057 & 6.3610 & -0.1553 & $-2.4 \%$ \\
\hline LL-12 & $\mathrm{MgO}$ & 0.2852 & 0.3240 & -0.0388 & $-12.0 \%$ \\
\hline LL-12 & $\mathrm{MnO}$ & 2.6889 & 2.7310 & -0.0421 & $-1.5 \%$ \\
\hline LL-12 & $\mathrm{Na}_{2} \mathrm{O}$ & 11.1749 & 11.5700 & -0.3951 & $-3.4 \%$ \\
\hline LL-12 & $\mathrm{NiO}$ & 0.8809 & 1.0040 & -0.1231 & $-12.3 \%$ \\
\hline LL-12 & $\mathrm{PbO}$ & 0.0108 & 0.0080 & 0.0028 & $35.0 \%$ \\
\hline LL-12 & $\mathrm{SiO}_{2}$ & 40.0049 & 39.8210 & 0.1839 & $0.5 \%$ \\
\hline LL-12 & $\mathrm{SO}_{4}$ & 1.1250 & 1.2000 & -0.0750 & $-6.3 \%$ \\
\hline LL-12 & $\mathrm{TiO}_{2}$ & 0.0083 & 0.0000 & 0.0083 & \\
\hline LL-12 & $\mathrm{ZnO}$ & 0.0280 & 0.0230 & 0.0050 & $21.7 \%$ \\
\hline LL-12 & $\mathrm{ZrO}_{2}$ & 0.1216 & 0.1120 & 0.0096 & $8.6 \%$ \\
\hline LL-12 & Sum & 98.4909 & 100.0000 & -1.5091 & $-1.5 \%$ \\
\hline LOL-12 & $\mathrm{Al}_{2} \mathrm{O}_{3}$ & 8.5405 & 8.7388 & -0.1983 & $-2.3 \%$ \\
\hline LOL-12 & $\mathrm{B}_{2} \mathrm{O}_{3}$ & 5.2323 & 5.4400 & -0.2077 & $-3.8 \%$ \\
\hline
\end{tabular}


SRNL-STI-2012-00152

Revision 0

Table A-4. Average Measured Chemical Compositions versus Targeted Compositions by Oxide by Glass ID (continued)

\begin{tabular}{|c|c|c|c|c|c|}
\hline Glass ID & Oxide & $\begin{array}{c}\text { Measured } \\
\text { (wt \%) }\end{array}$ & $\begin{array}{c}\text { Targeted } \\
\text { (wt \%) }\end{array}$ & $\begin{array}{c}\text { Diff of } \\
\text { Measured }\end{array}$ & $\begin{array}{r}\text { \% Diff of } \\
\text { Measured }\end{array}$ \\
\hline LOL-12 & $\mathrm{BaO}$ & 0.0324 & 0.0448 & -0.0124 & $-27.7 \%$ \\
\hline LOL-12 & $\mathrm{CaO}$ & 0.4827 & 0.4259 & 0.0568 & $13.3 \%$ \\
\hline LOL-12 & $\mathrm{Ce}_{2} \mathrm{O}_{3}$ & 0.0697 & 0.0705 & -0.0008 & $-1.1 \%$ \\
\hline LOL-12 & $\mathrm{Cr}_{2} \mathrm{O}_{3}$ & 0.0533 & 0.0372 & 0.0161 & $43.3 \%$ \\
\hline LOL-12 & $\mathrm{CuO}$ & 0.0479 & 0.0366 & 0.0113 & $30.9 \%$ \\
\hline LOL-12 & $\mathrm{Fe}_{2} \mathrm{O}_{3}$ & 7.6417 & 7.8280 & -0.1863 & $-2.4 \%$ \\
\hline LOL-12 & $\mathrm{K}_{2} \mathrm{O}$ & 0.0602 & 0.0302 & 0.0300 & $99.3 \%$ \\
\hline LOL-12 & $\mathrm{La}_{2} \mathrm{O}_{3}$ & 0.0287 & 0.0359 & -0.0072 & $-20.1 \%$ \\
\hline LOL-12 & $\mathrm{Li}_{2} \mathrm{O}$ & 5.1131 & 5.4400 & -0.3269 & $-6.0 \%$ \\
\hline LOL-12 & $\mathrm{MgO}$ & 0.2156 & 0.2240 & -0.0084 & $-3.8 \%$ \\
\hline LOL-12 & $\mathrm{MnO}$ & 2.2757 & 2.3131 & -0.0374 & $-1.6 \%$ \\
\hline LOL-12 & $\mathrm{Na}_{2} \mathrm{O}$ & 14.1540 & 14.6170 & -0.4630 & $-3.2 \%$ \\
\hline LOL-12 & $\mathrm{NiO}$ & 0.8850 & 0.9653 & -0.0803 & $-8.3 \%$ \\
\hline LOL-12 & $\mathrm{PbO}$ & 0.0108 & 0.0100 & 0.0008 & $8.0 \%$ \\
\hline LOL-12 & $\mathrm{SiO}_{2}$ & 51.1828 & 52.3775 & -1.1947 & $-2.3 \%$ \\
\hline LOL-12 & $\mathrm{SO}_{4}$ & 0.5932 & 0.6000 & -0.0068 & $-1.1 \%$ \\
\hline LOL-12 & $\mathrm{TiO}_{2}$ & 0.4320 & 0.4457 & -0.0137 & $-3.1 \%$ \\
\hline LOL-12 & $\mathrm{ZnO}$ & 0.0305 & 0.0235 & 0.0070 & $29.8 \%$ \\
\hline LOL-12 & $\mathrm{ZrO}_{2}$ & 0.0834 & 0.0826 & 0.0008 & $1.0 \%$ \\
\hline LOL-12 & Sum & 97.1657 & 99.7867 & -2.6210 & $-2.6 \%$ \\
\hline LRM & $\mathrm{Al}_{2} \mathrm{O}_{3}$ & 9.7643 & 10.0000 & -0.2357 & $-2.4 \%$ \\
\hline LRM & $\mathrm{B}_{2} \mathrm{O}_{3}$ & 7.6972 & 8.0000 & -0.3028 & $-3.8 \%$ \\
\hline LRM & $\mathrm{BaO}$ & 0.0056 & 0.0050 & 0.0006 & $12.0 \%$ \\
\hline LRM & $\mathrm{CaO}$ & 0.5683 & 0.5000 & 0.0683 & $13.7 \%$ \\
\hline LRM & $\mathrm{Ce}_{2} \mathrm{O}_{3}$ & 0.0059 & 0.0000 & 0.0059 & \\
\hline LRM & $\mathrm{Cr}_{2} \mathrm{O}_{3}$ & 0.1974 & 0.2000 & -0.0026 & $-1.3 \%$ \\
\hline LRM & $\mathrm{CuO}$ & 0.0063 &. & & \\
\hline LRM & $\mathrm{Fe}_{2} \mathrm{O}_{3}$ & 1.5609 & 1.0000 & 0.5609 & $56.1 \%$ \\
\hline LRM & $\mathrm{K}_{2} \mathrm{O}$ & 1.5112 & 1.5000 & 0.0112 & $0.7 \%$ \\
\hline LRM & $\mathrm{La}_{2} \mathrm{O}_{3}$ & 0.0059 &. & & \\
\hline LRM & $\mathrm{Li}_{2} \mathrm{O}$ & 0.1041 & 0.1000 & 0.0041 & $4.1 \%$ \\
\hline LRM & $\mathrm{MgO}$ & 0.0992 & 0.1000 & -0.0008 & $-0.8 \%$ \\
\hline LRM & $\mathrm{MnO}$ & 0.0739 & 0.1000 & -0.0261 & $-26.1 \%$ \\
\hline LRM & $\mathrm{Na}_{2} \mathrm{O}$ & 20.9306 & 20.0000 & 0.9306 & $4.7 \%$ \\
\hline LRM & $\mathrm{NiO}$ & 0.1695 & 0.1000 & 0.0695 & $69.5 \%$ \\
\hline LRM & $\mathrm{PbO}$ & 0.0855 & 0.1000 & -0.0145 & $-14.5 \%$ \\
\hline LRM & $\mathrm{SiO}_{2}$ & 53.5146 & 55.0000 & -1.4854 & $-2.7 \%$ \\
\hline LRM & $\mathrm{SO}_{4}$ & 0.2464 & 0.2400 & 0.0064 & $2.7 \%$ \\
\hline LRM & $\mathrm{TiO}_{2}$ & 0.0984 & 0.1000 & -0.0016 & $-1.6 \%$ \\
\hline LRM & $\mathrm{ZnO}$ & 0.0062 & 0.0000 & 0.0062 & \\
\hline LRM & $\mathrm{ZrO}_{2}$ & 0.8611 & 1.0000 & -0.1389 & $-13.9 \%$ \\
\hline LRM & Sum & 97.5124 & 98.0450 & -0.5326 & $-0.5 \%$ \\
\hline LT-01 & $\mathrm{Al}_{2} \mathrm{O}_{3}$ & 8.9326 & 8.9950 & -0.0624 & $-0.7 \%$ \\
\hline LT-01 & $\mathrm{B}_{2} \mathrm{O}_{3}$ & 5.5221 & 5.6000 & -0.0779 & $-1.4 \%$ \\
\hline LT-01 & $\mathrm{BaO}$ & 0.0338 & 0.0350 & -0.0012 & $-3.4 \%$ \\
\hline LT-01 & $\mathrm{CaO}$ & 0.5093 & 0.4720 & 0.0373 & $7.9 \%$ \\
\hline LT-01 & $\mathrm{Ce}_{2} \mathrm{O}_{3}$ & 0.0322 & 0.0290 & 0.0032 & $11.0 \%$ \\
\hline LT-01 & $\mathrm{Cr}_{2} \mathrm{O}_{3}$ & 0.1027 & 0.1140 & -0.0113 & $-9.9 \%$ \\
\hline LT-01 & $\mathrm{CuO}$ & 0.0363 & 0.0310 & 0.0053 & $17.1 \%$ \\
\hline LT-01 & $\mathrm{Fe}_{2} \mathrm{O}_{3}$ & 7.0448 & 7.4630 & -0.4182 & $-5.6 \%$ \\
\hline LT-01 & $\mathrm{K}_{2} \mathrm{O}$ & 0.0602 & 0.0080 & 0.0522 & $652.5 \%$ \\
\hline LT-01 & $\mathrm{La}_{2} \mathrm{O}_{3}$ & 0.0237 & 0.0260 & -0.0023 & $-8.8 \%$ \\
\hline LT-01 & $\mathrm{Li}_{2} \mathrm{O}$ & 7.3199 & 7.7000 & -0.3801 & $-4.9 \%$ \\
\hline LT-01 & $\mathrm{MgO}$ & 0.2048 & 0.2700 & -0.0652 & $-24.1 \%$ \\
\hline LT-01 & $\mathrm{MnO}$ & 2.2338 & 2.2720 & -0.0382 & $-1.7 \%$ \\
\hline LT-01 & $\mathrm{Na}_{2} \mathrm{O}$ & 8.6474 & 9.0550 & -0.4076 & $-4.5 \%$ \\
\hline LT-01 & $\mathrm{NiO}$ & 0.7419 & 0.8350 & -0.0931 & $-11.1 \%$ \\
\hline
\end{tabular}


SRNL-STI-2012-00152

Revision 0

Table A-4. Average Measured Chemical Compositions versus Targeted Compositions by Oxide by Glass ID (continued)

\begin{tabular}{|c|c|c|c|c|c|}
\hline Glass ID & Oxide & $\begin{array}{c}\text { Measured } \\
\text { (wt \%) }\end{array}$ & $\begin{array}{c}\text { Targeted } \\
\text { (wt \%) }\end{array}$ & $\begin{array}{c}\text { Diff of } \\
\text { Measured }\end{array}$ & $\begin{array}{l}\text { \% Diff of } \\
\text { Measured }\end{array}$ \\
\hline LT-01 & $\mathrm{PbO}$ & 0.0108 & 0.0070 & 0.0038 & $54.3 \%$ \\
\hline LT-01 & $\mathrm{SiO}_{2}$ & 55.6753 & 56.0760 & -0.4007 & $-0.7 \%$ \\
\hline LT-01 & $\mathrm{SO}_{4}$ & 0.8321 & 0.9000 & -0.0679 & $-7.5 \%$ \\
\hline LT-01 & $\mathrm{TiO}_{2}$ & 0.0083 & 0.0000 & 0.0083 & \\
\hline LT-01 & $\mathrm{ZnO}$ & 0.0258 & 0.0190 & 0.0068 & $35.8 \%$ \\
\hline LT-01 & $\mathrm{ZrO}_{2}$ & 0.0929 & 0.0930 & -0.0001 & $-0.1 \%$ \\
\hline LT-01 & Sum & 98.0908 & 100.0000 & -1.9092 & $-1.9 \%$ \\
\hline LT-04 & $\mathrm{Al}_{2} \mathrm{O}_{3}$ & 11.9700 & 12.2370 & -0.2670 & $-2.2 \%$ \\
\hline LT-04 & $\mathrm{B}_{2} \mathrm{O}_{3}$ & 4.8138 & 4.8000 & 0.0138 & $0.3 \%$ \\
\hline LT-04 & $\mathrm{BaO}$ & 0.0299 & 0.0420 & -0.0121 & $-28.8 \%$ \\
\hline LT-04 & $\mathrm{CaO}$ & 0.6254 & 0.5680 & 0.0574 & $10.1 \%$ \\
\hline LT-04 & $\mathrm{Ce}_{2} \mathrm{O}_{3}$ & 0.0366 & 0.0350 & 0.0016 & $4.6 \%$ \\
\hline LT-04 & $\mathrm{Cr}_{2} \mathrm{O}_{3}$ & 0.1615 & 0.1370 & 0.0245 & $17.9 \%$ \\
\hline LT-04 & $\mathrm{CuO}$ & 0.0541 & 0.0380 & 0.0161 & $42.4 \%$ \\
\hline LT-04 & $\mathrm{Fe}_{2} \mathrm{O}_{3}$ & 9.4825 & 10.1540 & -0.6715 & $-6.6 \%$ \\
\hline LT-04 & $\mathrm{K}_{2} \mathrm{O}$ & 0.0602 & 0.0090 & 0.0512 & $568.9 \%$ \\
\hline LT-04 & $\mathrm{La}_{2} \mathrm{O}_{3}$ & 0.0270 & 0.0310 & -0.0040 & $-12.9 \%$ \\
\hline LT-04 & $\mathrm{Li}_{2} \mathrm{O}$ & 6.2488 & 6.6000 & -0.3512 & $-5.3 \%$ \\
\hline LT-04 & $\mathrm{MgO}$ & 0.2848 & 0.3240 & -0.0392 & $-12.1 \%$ \\
\hline LT-04 & $\mathrm{MnO}$ & 2.6889 & 2.7310 & -0.0421 & $-1.5 \%$ \\
\hline LT-04 & $\mathrm{Na}_{2} \mathrm{O}$ & 11.1749 & 11.6150 & -0.4401 & $-3.8 \%$ \\
\hline LT-04 & $\mathrm{NiO}$ & 0.9372 & 1.0040 & -0.0668 & $-6.7 \%$ \\
\hline LT-04 & $\mathrm{PbO}$ & 0.0108 & 0.0080 & 0.0028 & $35.0 \%$ \\
\hline LT-04 & $\mathrm{SiO}_{2}$ & 46.7972 & 48.3320 & -1.5348 & $-3.2 \%$ \\
\hline LT-04 & $\mathrm{SO}_{4}$ & 1.0486 & 1.2000 & -0.1514 & $-12.6 \%$ \\
\hline LT-04 & $\mathrm{TiO}_{2}$ & 0.0083 & 0.0000 & 0.0083 & \\
\hline LT-04 & $\mathrm{ZnO}$ & 0.0265 & 0.0230 & 0.0035 & $15.2 \%$ \\
\hline LT-04 & $\mathrm{ZrO}_{2}$ & 0.1141 & 0.1120 & 0.0021 & $1.9 \%$ \\
\hline LT-04 & Sum & 96.6011 & 100.0000 & -3.3989 & $-3.4 \%$ \\
\hline LT-05 & $\mathrm{Al}_{2} \mathrm{O}_{3}$ & 11.7999 & 12.2370 & -0.4371 & $-3.6 \%$ \\
\hline LT-05 & $\mathrm{B}_{2} \mathrm{O}_{3}$ & 5.7797 & 6.0000 & -0.2203 & $-3.7 \%$ \\
\hline LT-05 & $\mathrm{BaO}$ & 0.0310 & 0.0420 & -0.0110 & $-26.2 \%$ \\
\hline LT-05 & $\mathrm{CaO}$ & 1.7602 & 1.7680 & -0.0078 & $-0.4 \%$ \\
\hline LT-05 & $\mathrm{Ce}_{2} \mathrm{O}_{3}$ & 0.0404 & 0.0350 & 0.0054 & $15.4 \%$ \\
\hline LT-05 & $\mathrm{Cr}_{2} \mathrm{O}_{3}$ & 0.1399 & 0.1370 & 0.0029 & $2.1 \%$ \\
\hline LT-05 & $\mathrm{CuO}$ & 0.0416 & 0.0380 & 0.0036 & $9.5 \%$ \\
\hline LT-05 & $\mathrm{Fe}_{2} \mathrm{O}_{3}$ & 9.4682 & 10.1540 & -0.6858 & $-6.8 \%$ \\
\hline LT-05 & $\mathrm{K}_{2} \mathrm{O}$ & 0.0602 & 0.0090 & 0.0512 & $568.9 \%$ \\
\hline LT-05 & $\mathrm{La}_{2} \mathrm{O}_{3}$ & 0.0276 & 0.0310 & -0.0034 & $-11.0 \%$ \\
\hline LT-05 & $\mathrm{Li}_{2} \mathrm{O}$ & 5.6137 & 6.0000 & -0.3863 & $-6.4 \%$ \\
\hline LT-05 & $\mathrm{MgO}$ & 0.3105 & 0.3240 & -0.0135 & $-4.2 \%$ \\
\hline LT-05 & $\mathrm{MnO}$ & 2.6728 & 2.7310 & -0.0582 & $-2.1 \%$ \\
\hline LT-05 & $\mathrm{Na}_{2} \mathrm{O}$ & 12.1758 & 12.8150 & -0.6392 & $-5.0 \%$ \\
\hline LT-05 & $\mathrm{NiO}$ & 0.9261 & 1.0040 & -0.0779 & $-7.8 \%$ \\
\hline LT-05 & $\mathrm{PbO}$ & 0.0108 & 0.0080 & 0.0028 & $35.0 \%$ \\
\hline LT-05 & $\mathrm{SiO}_{2}$ & 44.1765 & 45.3320 & -1.1555 & $-2.5 \%$ \\
\hline LT-05 & $\mathrm{SO}_{4}$ & 1.0860 & 1.2000 & -0.1140 & $-9.5 \%$ \\
\hline LT-05 & $\mathrm{TiO}_{2}$ & 0.0083 & 0.0000 & 0.0083 & \\
\hline LT-05 & $\mathrm{ZnO}$ & 0.0218 & 0.0230 & -0.0012 & $-5.2 \%$ \\
\hline LT-05 & $\mathrm{ZrO}_{2}$ & 0.1304 & 0.1120 & 0.0184 & $16.4 \%$ \\
\hline LT-05 & Sum & 96.2815 & 100.0000 & -3.7185 & $-3.7 \%$ \\
\hline LT-06 & $\mathrm{Al}_{2} \mathrm{O}_{3}$ & 11.9086 & 12.2370 & -0.3284 & $-2.7 \%$ \\
\hline LT-06 & $\mathrm{B}_{2} \mathrm{O}_{3}$ & 6.7698 & 7.2000 & -0.4302 & $-6.0 \%$ \\
\hline LT-06 & $\mathrm{BaO}$ & 0.0318 & 0.0420 & -0.0102 & $-24.3 \%$ \\
\hline LT-06 & $\mathrm{CaO}$ & 3.5610 & 3.5680 & -0.0070 & $-0.2 \%$ \\
\hline LT-06 & $\mathrm{Ce}_{2} \mathrm{O}_{3}$ & 0.0357 & 0.0350 & 0.0007 & $2.0 \%$ \\
\hline LT-06 & $\mathrm{Cr}_{2} \mathrm{O}_{3}$ & 0.1484 & 0.1370 & 0.0114 & $8.3 \%$ \\
\hline
\end{tabular}


SRNL-STI-2012-00152

Revision 0

Table A-4. Average Measured Chemical Compositions versus Targeted Compositions by Oxide by Glass ID (continued)

\begin{tabular}{|c|c|c|c|c|c|}
\hline Glass ID & Oxide & $\begin{array}{c}\text { Measured } \\
\text { (wt \%) }\end{array}$ & $\begin{array}{c}\text { Targeted } \\
\text { (wt \%) }\end{array}$ & $\begin{array}{c}\text { Diff of } \\
\text { Measured }\end{array}$ & $\begin{array}{l}\text { \% Diff of } \\
\text { Measured }\end{array}$ \\
\hline LT-06 & $\mathrm{CuO}$ & 0.0401 & 0.0380 & 0.0021 & $5.5 \%$ \\
\hline LT-06 & $\mathrm{Fe}_{2} \mathrm{O}_{3}$ & 9.9293 & 10.1540 & -0.2247 & $-2.2 \%$ \\
\hline LT-06 & $\mathrm{K}_{2} \mathrm{O}$ & 0.0602 & 0.0090 & 0.0512 & $568.9 \%$ \\
\hline LT-06 & $\mathrm{La}_{2} \mathrm{O}_{3}$ & 0.0311 & 0.0310 & 0.0001 & $0.3 \%$ \\
\hline LT-06 & $\mathrm{Li}_{2} \mathrm{O}$ & 4.9571 & 5.4000 & -0.4429 & $-8.2 \%$ \\
\hline LT-06 & $\mathrm{MgO}$ & 0.2981 & 0.3240 & -0.0259 & $-8.0 \%$ \\
\hline LT-06 & $\mathrm{MnO}$ & 2.7051 & 2.7310 & -0.0259 & $-0.9 \%$ \\
\hline LT-06 & $\mathrm{Na}_{2} \mathrm{O}$ & 13.0385 & 13.4150 & -0.3765 & $-2.8 \%$ \\
\hline LT-06 & $\mathrm{NiO}$ & 0.9506 & 1.0040 & -0.0534 & $-5.3 \%$ \\
\hline LT-06 & $\mathrm{PbO}$ & 0.0108 & 0.0080 & 0.0028 & $35.0 \%$ \\
\hline LT-06 & $\mathrm{SiO}_{2}$ & 41.5559 & 42.3320 & -0.7761 & $-1.8 \%$ \\
\hline LT-06 & $\mathrm{SO}_{4}$ & 1.1220 & 1.2000 & -0.0780 & $-6.5 \%$ \\
\hline LT-06 & $\mathrm{TiO}_{2}$ & 0.0083 & 0.0000 & 0.0083 & \\
\hline LT-06 & $\mathrm{ZnO}$ & 0.0243 & 0.0230 & 0.0013 & $5.7 \%$ \\
\hline LT-06 & $\mathrm{ZrO}_{2}$ & 0.1114 & 0.1120 & -0.0006 & $-0.5 \%$ \\
\hline LT-06 & Sum & 97.2979 & 100.0000 & -2.7021 & $-2.7 \%$ \\
\hline LT-10 & $\mathrm{Al}_{2} \mathrm{O}_{3}$ & 11.9416 & 12.2370 & -0.2954 & $-2.4 \%$ \\
\hline LT-10 & $\mathrm{B}_{2} \mathrm{O}_{3}$ & 4.5803 & 4.8000 & -0.2197 & $-4.6 \%$ \\
\hline LT-10 & $\mathrm{BaO}$ & 0.0293 & 0.0420 & -0.0127 & $-30.2 \%$ \\
\hline LT-10 & $\mathrm{CaO}$ & 3.5750 & 3.5680 & 0.0070 & $0.2 \%$ \\
\hline LT-10 & $\mathrm{Ce}_{2} \mathrm{O}_{3}$ & 0.0392 & 0.0350 & 0.0042 & $12.0 \%$ \\
\hline LT-10 & $\mathrm{Cr}_{2} \mathrm{O}_{3}$ & 0.2065 & 0.1370 & 0.0695 & $50.7 \%$ \\
\hline LT-10 & $\mathrm{CuO}$ & 0.0391 & 0.0380 & 0.0011 & $2.9 \%$ \\
\hline LT-10 & $\mathrm{Fe}_{2} \mathrm{O}_{3}$ & 9.6398 & 10.1540 & -0.5142 & $-5.1 \%$ \\
\hline LT-10 & $\mathrm{K}_{2} \mathrm{O}$ & 0.0602 & 0.0090 & 0.0512 & $568.9 \%$ \\
\hline LT-10 & $\mathrm{La}_{2} \mathrm{O}_{3}$ & 0.0302 & 0.0310 & -0.0008 & $-2.6 \%$ \\
\hline LT-10 & $\mathrm{Li}_{2} \mathrm{O}$ & 6.7493 & 7.2000 & -0.4507 & $-6.3 \%$ \\
\hline LT-10 & $\mathrm{MgO}$ & 0.2740 & 0.3240 & -0.0500 & $-15.4 \%$ \\
\hline LT-10 & $\mathrm{MnO}$ & 2.7051 & 2.7310 & -0.0259 & $-0.9 \%$ \\
\hline LT-10 & $\mathrm{Na}_{2} \mathrm{O}$ & 11.1884 & 11.6150 & -0.4266 & $-3.7 \%$ \\
\hline LT-10 & $\mathrm{NiO}$ & 0.9051 & 1.0040 & -0.0989 & $-9.9 \%$ \\
\hline LT-10 & $\mathrm{PbO}$ & 0.0108 & 0.0080 & 0.0028 & $35.0 \%$ \\
\hline LT-10 & $\mathrm{SiO}_{2}$ & 44.3905 & 44.7320 & -0.3415 & $-0.8 \%$ \\
\hline LT-10 & $\mathrm{SO}_{4}$ & 1.1647 & 1.2000 & -0.0353 & $-2.9 \%$ \\
\hline LT-10 & $\mathrm{TiO}_{2}$ & 0.0083 & 0.0000 & 0.0083 & \\
\hline LT-10 & $\mathrm{ZnO}$ & 0.0274 & 0.0230 & 0.0044 & $19.1 \%$ \\
\hline LT-10 & $\mathrm{ZrO}_{2}$ & 0.1023 & 0.1120 & -0.0097 & $-8.7 \%$ \\
\hline LT-10 & Sum & 97.6671 & 100.0000 & -2.3329 & $-2.3 \%$ \\
\hline LT-11 & $\mathrm{Al}_{2} \mathrm{O}_{3}$ & 12.0219 & 12.2370 & -0.2151 & $-1.8 \%$ \\
\hline LT-11 & $\mathrm{B}_{2} \mathrm{O}_{3}$ & 7.1321 & 7.2000 & -0.0679 & $-0.9 \%$ \\
\hline LT-11 & $\mathrm{BaO}$ & 0.0321 & 0.0420 & -0.0099 & $-23.6 \%$ \\
\hline LT-11 & $\mathrm{CaO}$ & 0.6202 & 0.5680 & 0.0522 & $9.2 \%$ \\
\hline LT-11 & $\mathrm{Ce}_{2} \mathrm{O}_{3}$ & 0.0407 & 0.0350 & 0.0057 & $16.3 \%$ \\
\hline LT-11 & $\mathrm{Cr}_{2} \mathrm{O}_{3}$ & 0.1264 & 0.1370 & -0.0106 & $-7.7 \%$ \\
\hline LT-11 & $\mathrm{CuO}$ & 0.0435 & 0.0380 & 0.0055 & $14.5 \%$ \\
\hline LT-11 & $\mathrm{Fe}_{2} \mathrm{O}_{3}$ & 9.9829 & 10.1540 & -0.1711 & $-1.7 \%$ \\
\hline LT-11 & $\mathrm{K}_{2} \mathrm{O}$ & 0.0602 & 0.0090 & 0.0512 & $568.9 \%$ \\
\hline LT-11 & $\mathrm{La}_{2} \mathrm{O}_{3}$ & 0.0287 & 0.0310 & -0.0023 & $-7.4 \%$ \\
\hline LT-11 & $\mathrm{Li}_{2} \mathrm{O}$ & 6.8516 & 7.2000 & -0.3484 & $-4.8 \%$ \\
\hline LT-11 & $\mathrm{MgO}$ & 0.2981 & 0.3240 & -0.0259 & $-8.0 \%$ \\
\hline LT-11 & $\mathrm{MnO}$ & 2.7244 & 2.7310 & -0.0066 & $-0.2 \%$ \\
\hline LT-11 & $\mathrm{Na}_{2} \mathrm{O}$ & 11.3064 & 11.6150 & -0.3086 & $-2.7 \%$ \\
\hline LT-11 & $\mathrm{NiO}$ & 0.9108 & 1.0040 & -0.0932 & $-9.3 \%$ \\
\hline LT-11 & $\mathrm{PbO}$ & 0.0108 & 0.0080 & 0.0028 & $35.0 \%$ \\
\hline LT-11 & $\mathrm{SiO}_{2}$ & 45.2997 & 45.3320 & -0.0323 & $-0.1 \%$ \\
\hline LT-11 & $\mathrm{SO}_{4}$ & 1.1295 & 1.2000 & -0.0705 & $-5.9 \%$ \\
\hline LT-11 & $\mathrm{TiO}_{2}$ & 0.0083 & 0.0000 & 0.0083 & \\
\hline
\end{tabular}


SRNL-STI-2012-00152

Revision 0

Table A-4. Average Measured Chemical Compositions versus Targeted Compositions by Oxide by Glass ID (continued)

\begin{tabular}{|c|c|c|c|c|c|}
\hline Glass ID & Oxide & $\begin{array}{c}\text { Measured } \\
\text { (wt \%) }\end{array}$ & $\begin{array}{c}\text { Targeted } \\
\text { (wt \%) }\end{array}$ & $\begin{array}{c}\text { Diff of } \\
\text { Measured }\end{array}$ & $\begin{array}{l}\text { \% Diff of } \\
\text { Measured }\end{array}$ \\
\hline LT-11 & $\mathrm{ZnO}$ & 0.0255 & 0.0230 & 0.0025 & $10.9 \%$ \\
\hline LT-11 & $\mathrm{ZrO}_{2}$ & 0.1114 & 0.1120 & -0.0006 & $-0.5 \%$ \\
\hline LT-11 & Sum & 98.7653 & 100.0000 & -1.2347 & $-1.2 \%$ \\
\hline LT-12 & $\mathrm{Al}_{2} \mathrm{O}_{3}$ & 12.0692 & 12.2370 & -0.1678 & $-1.4 \%$ \\
\hline LT-12 & $\mathrm{B}_{2} \mathrm{O}_{3}$ & 7.2367 & 7.2000 & 0.0367 & $0.5 \%$ \\
\hline LT-12 & $\mathrm{BaO}$ & 0.0338 & 0.0420 & -0.0082 & $-19.5 \%$ \\
\hline LT-12 & $\mathrm{CaO}$ & 3.6029 & 3.5680 & 0.0349 & $1.0 \%$ \\
\hline LT-12 & $\mathrm{Ce}_{2} \mathrm{O}_{3}$ & 0.0363 & 0.0350 & 0.0013 & $3.7 \%$ \\
\hline LT-12 & $\mathrm{Cr}_{2} \mathrm{O}_{3}$ & 0.1374 & 0.1370 & 0.0004 & $0.3 \%$ \\
\hline LT-12 & $\mathrm{CuO}$ & 0.0432 & 0.0380 & 0.0052 & $13.7 \%$ \\
\hline LT-12 & $\mathrm{Fe}_{2} \mathrm{O}_{3}$ & 9.6719 & 10.1540 & -0.4821 & $-4.7 \%$ \\
\hline LT-12 & $\mathrm{K}_{2} \mathrm{O}$ & 0.0602 & 0.0090 & 0.0512 & $568.9 \%$ \\
\hline LT-12 & $\mathrm{La}_{2} \mathrm{O}_{3}$ & 0.0311 & 0.0310 & 0.0001 & $0.3 \%$ \\
\hline LT-12 & $\mathrm{Li}_{2} \mathrm{O}$ & 6.2919 & 6.6000 & -0.3081 & $-4.7 \%$ \\
\hline LT-12 & $\mathrm{MgO}$ & 0.3060 & 0.3240 & -0.0180 & $-5.6 \%$ \\
\hline LT-12 & $\mathrm{MnO}$ & 2.7406 & 2.7310 & 0.0096 & $0.4 \%$ \\
\hline LT-12 & $\mathrm{Na}_{2} \mathrm{O}$ & 11.3401 & 11.6150 & -0.2749 & $-2.4 \%$ \\
\hline LT-12 & $\mathrm{NiO}$ & 0.9089 & 1.0040 & -0.0951 & $-9.5 \%$ \\
\hline LT-12 & $\mathrm{PbO}$ & 0.0108 & 0.0080 & 0.0028 & $35.0 \%$ \\
\hline LT-12 & $\mathrm{SiO}_{2}$ & 43.0534 & 42.9320 & 0.1214 & $0.3 \%$ \\
\hline LT-12 & $\mathrm{SO}_{4}$ & 1.1429 & 1.2000 & -0.0571 & $-4.8 \%$ \\
\hline LT-12 & $\mathrm{TiO}_{2}$ & 0.0083 & 0.0000 & 0.0083 & \\
\hline LT-12 & $\mathrm{ZnO}$ & 0.0271 & 0.0230 & 0.0041 & $17.8 \%$ \\
\hline LT-12 & $\mathrm{ZrO}_{2}$ & 0.1162 & 0.1120 & 0.0042 & $3.8 \%$ \\
\hline LT-12 & Sum & 98.8688 & 100.0000 & -1.1312 & $-1.1 \%$ \\
\hline QB-03 & $\mathrm{Al}_{2} \mathrm{O}_{3}$ & 8.9326 & 9.1312 & -0.1986 & $-2.2 \%$ \\
\hline QB-03 & $\mathrm{B}_{2} \mathrm{O}_{3}$ & 4.9586 & 5.1200 & -0.1614 & $-3.2 \%$ \\
\hline QB-03 & $\mathrm{BaO}$ & 0.0285 & 0.0369 & -0.0084 & $-22.8 \%$ \\
\hline QB-03 & $\mathrm{CaO}$ & 6.8071 & 6.9087 & -0.1016 & $-1.5 \%$ \\
\hline QB-03 & $\mathrm{Ce}_{2} \mathrm{O}_{3}$ & 0.0346 & 0.0301 & 0.0045 & $15.0 \%$ \\
\hline QB-03 & $\mathrm{Cr}_{2} \mathrm{O}_{3}$ & 0.2463 & 0.1395 & 0.1068 & $76.6 \%$ \\
\hline QB-03 & $\mathrm{CuO}$ & 0.0460 & 0.0322 & 0.0138 & $42.9 \%$ \\
\hline QB-03 & $\mathrm{Fe}_{2} \mathrm{O}_{3}$ & 7.8705 & 8.2116 & -0.3411 & $-4.2 \%$ \\
\hline QB-03 & $\mathrm{K}_{2} \mathrm{O}$ & 0.2436 & 0.2477 & -0.0041 & $-1.7 \%$ \\
\hline QB-03 & $\mathrm{La}_{2} \mathrm{O}_{3}$ & 0.0276 & 0.0258 & 0.0018 & $7.0 \%$ \\
\hline QB-03 & $\mathrm{Li}_{2} \mathrm{O}$ & 4.8440 & 5.1200 & -0.2760 & $-5.4 \%$ \\
\hline QB-03 & $\mathrm{MgO}$ & 0.2724 & 0.2801 & -0.0077 & $-2.7 \%$ \\
\hline QB-03 & $\mathrm{MnO}$ & 2.3371 & 2.3235 & 0.0136 & $0.6 \%$ \\
\hline QB-03 & $\mathrm{Na}_{2} \mathrm{O}$ & 13.0183 & 13.3731 & -0.3548 & $-2.7 \%$ \\
\hline QB-03 & $\mathrm{NiO}$ & 0.8869 & 0.9767 & -0.0898 & $-9.2 \%$ \\
\hline QB-03 & $\mathrm{PbO}$ & 0.0108 & 0.0079 & 0.0029 & $36.7 \%$ \\
\hline QB-03 & $\mathrm{SiO}_{2}$ & 46.7972 & 46.9128 & -0.1156 & $-0.2 \%$ \\
\hline QB-03 & $\mathrm{SO}_{4}$ & 0.9654 & 1.0000 & -0.0346 & $-3.5 \%$ \\
\hline QB-03 & $\mathrm{TiO}_{2}$ & 0.0083 & 0.0000 & 0.0083 & \\
\hline QB-03 & $\mathrm{ZnO}$ & 0.0296 & 0.0229 & 0.0067 & $29.3 \%$ \\
\hline QB-03 & $\mathrm{ZrO}_{2}$ & 0.1084 & 0.0992 & 0.0092 & $9.3 \%$ \\
\hline QB-03 & Sum & 98.4738 & 100.0000 & -1.5262 & $-1.5 \%$ \\
\hline QB-04 & $\mathrm{Al}_{2} \mathrm{O}_{3}$ & 8.9043 & 9.1312 & -0.2269 & $-2.5 \%$ \\
\hline QB-04 & $\mathrm{B}_{2} \mathrm{O}_{3}$ & 4.9425 & 5.1200 & -0.1775 & $-3.5 \%$ \\
\hline QB-04 & $\mathrm{BaO}$ & 0.0346 & 0.0369 & -0.0023 & $-6.2 \%$ \\
\hline QB-04 & $\mathrm{CaO}$ & 6.6742 & 6.9087 & -0.2345 & $-3.4 \%$ \\
\hline QB-04 & $\mathrm{Ce}_{2} \mathrm{O}_{3}$ & 0.0275 & 0.0301 & -0.0026 & $-8.6 \%$ \\
\hline QB-04 & $\mathrm{Cr}_{2} \mathrm{O}_{3}$ & 0.1389 & 0.1395 & -0.0006 & $-0.4 \%$ \\
\hline QB-04 & $\mathrm{CuO}$ & 0.0294 & 0.0322 & -0.0028 & $-8.7 \%$ \\
\hline QB-04 & $\mathrm{Fe}_{2} \mathrm{O}_{3}$ & 7.8491 & 8.2116 & -0.3625 & $-4.4 \%$ \\
\hline QB-04 & $\mathrm{K}_{2} \mathrm{O}$ & 0.2349 & 0.2477 & -0.0128 & $-5.2 \%$ \\
\hline QB-04 & $\mathrm{La}_{2} \mathrm{O}_{3}$ & 0.0243 & 0.0258 & -0.0015 & $-5.8 \%$ \\
\hline
\end{tabular}


SRNL-STI-2012-00152

Revision 0

Table A-4. Average Measured Chemical Compositions versus Targeted Compositions by Oxide by Glass ID (continued)

\begin{tabular}{|c|c|c|c|c|c|}
\hline Glass ID & Oxide & $\begin{array}{c}\text { Measured } \\
\text { (wt \%) }\end{array}$ & $\begin{array}{c}\text { Targeted } \\
(\text { wt \%) }\end{array}$ & $\begin{array}{c}\text { Diff of } \\
\text { Measured }\end{array}$ & $\begin{array}{l}\text { \% Diff of } \\
\text { Measured }\end{array}$ \\
\hline QB-04 & $\mathrm{Li}_{2} \mathrm{O}$ & 4.2843 & 4.4800 & -0.1957 & $-4.4 \%$ \\
\hline QB-04 & $\mathrm{MgO}$ & 0.2744 & 0.2801 & -0.0057 & $-2.0 \%$ \\
\hline QB-04 & $\mathrm{MnO}$ & 2.2887 & 2.3235 & -0.0348 & $-1.5 \%$ \\
\hline QB-04 & $\mathrm{Na}_{2} \mathrm{O}$ & 14.9291 & 15.2931 & -0.3640 & $-2.4 \%$ \\
\hline QB-04 & $\mathrm{NiO}$ & 0.9022 & 0.9767 & -0.0745 & $-7.6 \%$ \\
\hline QB-04 & $\mathrm{PbO}$ & 0.0108 & 0.0079 & 0.0029 & $36.7 \%$ \\
\hline QB-04 & $\mathrm{SiO}_{2}$ & 44.6579 & 45.6328 & -0.9749 & $-2.1 \%$ \\
\hline QB-04 & $\mathrm{SO}_{4}$ & 0.9542 & 1.0000 & -0.0458 & $-4.6 \%$ \\
\hline QB-04 & $\mathrm{TiO}_{2}$ & 0.0083 & 0.0000 & 0.0083 & \\
\hline QB-04 & $\mathrm{ZnO}$ & 0.0268 & 0.0229 & 0.0039 & $17.0 \%$ \\
\hline QB-04 & $\mathrm{ZrO}_{2}$ & 0.1236 & 0.0992 & 0.0244 & $24.6 \%$ \\
\hline QB-04 & Sum & 97.3199 & 100.0000 & -2.6801 & $-2.7 \%$ \\
\hline QB-19 & $\mathrm{Al}_{2} \mathrm{O}_{3}$ & 9.7971 & 10.1748 & -0.3777 & $-3.7 \%$ \\
\hline QB-19 & $\mathrm{B}_{2} \mathrm{O}_{3}$ & 4.7172 & 4.8000 & -0.0828 & $-1.7 \%$ \\
\hline QB-19 & $\mathrm{BaO}$ & 0.0318 & 0.0411 & -0.0093 & $-22.6 \%$ \\
\hline QB-19 & $\mathrm{CaO}$ & 0.5789 & 0.5668 & 0.0121 & $2.1 \%$ \\
\hline QB-19 & $\mathrm{Ce}_{2} \mathrm{O}_{3}$ & 0.0366 & 0.0335 & 0.0031 & $9.3 \%$ \\
\hline QB-19 & $\mathrm{Cr}_{2} \mathrm{O}_{3}$ & 0.1608 & 0.1555 & 0.0053 & $3.4 \%$ \\
\hline QB-19 & $\mathrm{CuO}$ & 0.0376 & 0.0359 & 0.0017 & $4.7 \%$ \\
\hline QB-19 & $\mathrm{Fe}_{2} \mathrm{O}_{3}$ & 8.5996 & 9.1501 & -0.5505 & $-6.0 \%$ \\
\hline QB-19 & $\mathrm{K}_{2} \mathrm{O}$ & 0.2716 & 0.2760 & -0.0044 & $-1.6 \%$ \\
\hline QB-19 & $\mathrm{La}_{2} \mathrm{O}_{3}$ & 0.0252 & 0.0288 & -0.0036 & $-12.5 \%$ \\
\hline QB-19 & $\mathrm{Li}_{2} \mathrm{O}$ & 4.5534 & 4.8000 & -0.2466 & $-5.1 \%$ \\
\hline QB-19 & $\mathrm{MgO}$ & 0.3051 & 0.3121 & -0.0070 & $-2.2 \%$ \\
\hline QB-19 & $\mathrm{MnO}$ & 2.5017 & 2.5890 & -0.0873 & $-3.4 \%$ \\
\hline QB-19 & $\mathrm{Na}_{2} \mathrm{O}$ & 17.6925 & 18.2752 & -0.5827 & $-3.2 \%$ \\
\hline QB-19 & $\mathrm{NiO}$ & 1.0078 & 1.0883 & -0.0805 & $-7.4 \%$ \\
\hline QB-19 & $\mathrm{PbO}$ & 0.0108 & 0.0088 & 0.0020 & $22.7 \%$ \\
\hline QB-19 & $\mathrm{SiO}_{2}$ & 45.1927 & 46.5279 & -1.3352 & $-2.9 \%$ \\
\hline QB-19 & $\mathrm{SO}_{4}$ & 0.9827 & 1.0000 & -0.0173 & $-1.7 \%$ \\
\hline QB-19 & $\mathrm{TiO}_{2}$ & 0.0083 & 0.0000 & 0.0083 & \\
\hline QB-19 & $\mathrm{ZnO}$ & 0.0299 & 0.0255 & 0.0044 & $17.3 \%$ \\
\hline QB-19 & $\mathrm{ZrO}_{2}$ & 0.1148 & 0.1105 & 0.0043 & $3.9 \%$ \\
\hline QB-19 & Sum & 96.6561 & 100.0000 & -3.3439 & $-3.3 \%$ \\
\hline QB-20 & $\mathrm{Al}_{2} \mathrm{O}_{3}$ & 9.7782 & 10.1748 & -0.3966 & $-3.9 \%$ \\
\hline QB-20 & $\mathrm{B}_{2} \mathrm{O}_{3}$ & 7.8485 & 8.4000 & -0.5515 & $-6.6 \%$ \\
\hline QB-20 & $\mathrm{BaO}$ & 0.0299 & 0.0411 & -0.0112 & $-27.3 \%$ \\
\hline QB-20 & $\mathrm{CaO}$ & 5.2190 & 5.3668 & -0.1478 & $-2.8 \%$ \\
\hline QB-20 & $\mathrm{Ce}_{2} \mathrm{O}_{3}$ & 0.0351 & 0.0335 & 0.0016 & $4.8 \%$ \\
\hline QB-20 & $\mathrm{Cr}_{2} \mathrm{O}_{3}$ & 0.1666 & 0.1555 & 0.0111 & $7.1 \%$ \\
\hline QB-20 & $\mathrm{CuO}$ & 0.0388 & 0.0359 & 0.0029 & $8.1 \%$ \\
\hline QB-20 & $\mathrm{Fe}_{2} \mathrm{O}_{3}$ & 8.6461 & 9.1501 & -0.5040 & $-5.5 \%$ \\
\hline QB-20 & $\mathrm{K}_{2} \mathrm{O}$ & 0.2710 & 0.2760 & -0.0050 & $-1.8 \%$ \\
\hline QB-20 & $\mathrm{La}_{2} \mathrm{O}_{3}$ & 0.0308 & 0.0288 & 0.0020 & $6.9 \%$ \\
\hline QB-20 & $\mathrm{Li}_{2} \mathrm{O}$ & 3.7891 & 4.2000 & -0.4109 & $-9.8 \%$ \\
\hline QB-20 & $\mathrm{MgO}$ & 0.2558 & 0.3121 & -0.0563 & $-18.0 \%$ \\
\hline QB-20 & $\mathrm{MnO}$ & 2.4597 & 2.5890 & -0.1293 & $-5.0 \%$ \\
\hline QB-20 & $\mathrm{Na}_{2} \mathrm{O}$ & 14.6932 & 15.2752 & -0.5820 & $-3.8 \%$ \\
\hline QB-20 & $\mathrm{NiO}$ & 0.9798 & 1.0883 & -0.1085 & $-10.0 \%$ \\
\hline QB-20 & $\mathrm{PbO}$ & 0.0108 & 0.0088 & 0.0020 & $22.7 \%$ \\
\hline QB-20 & $\mathrm{SiO}_{2}$ & 40.2188 & 41.7279 & -1.5091 & $-3.6 \%$ \\
\hline QB-20 & $\mathrm{SO}_{4}$ & 0.9400 & 1.0000 & -0.0600 & $-6.0 \%$ \\
\hline QB-20 & $\mathrm{TiO}_{2}$ & 0.0083 & 0.0000 & 0.0083 & \\
\hline QB-20 & $\mathrm{ZnO}$ & 0.0296 & 0.0255 & 0.0041 & $16.1 \%$ \\
\hline QB-20 & $\mathrm{ZrO}_{2}$ & 0.1331 & 0.1105 & 0.0226 & $20.5 \%$ \\
\hline QB-20 & Sum & 95.5822 & 100.0000 & -4.4178 & $-4.4 \%$ \\
\hline QB-22 & $\mathrm{Al}_{2} \mathrm{O}_{3}$ & 10.2411 & 10.1748 & 0.0663 & $0.7 \%$ \\
\hline
\end{tabular}


SRNL-STI-2012-00152

Revision 0

Table A-4. Average Measured Chemical Compositions versus Targeted Compositions by Oxide by Glass ID (continued)

\begin{tabular}{|c|c|c|c|c|c|}
\hline Glass ID & Oxide & $\begin{array}{c}\text { Measured } \\
\text { (wt \%) }\end{array}$ & $\begin{array}{c}\text { Targeted } \\
(\text { wt \%) }\end{array}$ & $\begin{array}{c}\text { Diff of } \\
\text { Measured }\end{array}$ & $\begin{array}{l}\text { \% Diff of } \\
\text { Measured }\end{array}$ \\
\hline QB-22 & $\mathrm{B}_{2} \mathrm{O}_{3}$ & 4.5562 & 4.8000 & -0.2438 & $-5.1 \%$ \\
\hline QB-22 & $\mathrm{BaO}$ & 0.0332 & 0.0411 & -0.0079 & $-19.2 \%$ \\
\hline QB-22 & $\mathrm{CaO}$ & 6.5902 & 6.5668 & 0.0234 & $0.4 \%$ \\
\hline QB-22 & $\mathrm{Ce}_{2} \mathrm{O}_{3}$ & 0.0351 & 0.0335 & 0.0016 & $4.8 \%$ \\
\hline QB-22 & $\mathrm{Cr}_{2} \mathrm{O}_{3}$ & 0.1736 & 0.1555 & 0.0181 & $11.6 \%$ \\
\hline QB-22 & $\mathrm{CuO}$ & 0.0363 & 0.0359 & 0.0004 & $1.1 \%$ \\
\hline QB-22 & $\mathrm{Fe}_{2} \mathrm{O}_{3}$ & 8.9392 & 9.1501 & -0.2109 & $-2.3 \%$ \\
\hline QB-22 & $\mathrm{K}_{2} \mathrm{O}$ & 0.2701 & 0.2760 & -0.0059 & $-2.1 \%$ \\
\hline QB-22 & $\mathrm{La}_{2} \mathrm{O}_{3}$ & 0.0317 & 0.0288 & 0.0029 & $10.1 \%$ \\
\hline QB-22 & $\mathrm{Li}_{2} \mathrm{O}$ & 3.8483 & 4.2000 & -0.3517 & $-8.4 \%$ \\
\hline QB-22 & $\mathrm{MgO}$ & 0.2516 & 0.3121 & -0.0605 & $-19.4 \%$ \\
\hline QB-22 & $\mathrm{MnO}$ & 2.6373 & 2.5890 & 0.0483 & $1.9 \%$ \\
\hline QB-22 & $\mathrm{Na}_{2} \mathrm{O}$ & 16.6815 & 16.4752 & 0.2063 & $1.3 \%$ \\
\hline QB-22 & $\mathrm{NiO}$ & 1.0037 & 1.0883 & -0.0846 & $-7.8 \%$ \\
\hline QB-22 & $\mathrm{PbO}$ & 0.0108 & 0.0088 & 0.0020 & $22.7 \%$ \\
\hline QB-22 & $\mathrm{SiO}_{2}$ & 41.1815 & 42.9279 & -1.7464 & $-4.1 \%$ \\
\hline QB-22 & $\mathrm{SO}_{4}$ & 0.9594 & 1.0000 & -0.0406 & $-4.1 \%$ \\
\hline QB-22 & $\mathrm{TiO}_{2}$ & 0.0083 & 0.0000 & 0.0083 & \\
\hline QB-22 & $\mathrm{ZnO}$ & 0.0299 & 0.0255 & 0.0044 & $17.3 \%$ \\
\hline QB-22 & $\mathrm{ZrO}_{2}$ & 0.1104 & 0.1105 & -0.0001 & $-0.1 \%$ \\
\hline QB-22 & Sum & 97.6295 & 100.0000 & -2.3705 & $-2.4 \%$ \\
\hline QB-32 & $\mathrm{Al}_{2} \mathrm{O}_{3}$ & 8.9043 & 9.0791 & -0.1748 & $-1.9 \%$ \\
\hline QB-32 & $\mathrm{B}_{2} \mathrm{O}_{3}$ & 5.0069 & 5.1200 & -0.1131 & $-2.2 \%$ \\
\hline QB-32 & $\mathrm{BaO}$ & 0.0287 & 0.0367 & -0.0080 & $-21.8 \%$ \\
\hline QB-32 & $\mathrm{CaO}$ & 0.5530 & 0.5058 & 0.0472 & $9.3 \%$ \\
\hline QB-32 & $\mathrm{Ce}_{2} \mathrm{O}_{3}$ & 0.0322 & 0.0299 & 0.0023 & $7.7 \%$ \\
\hline QB-32 & $\mathrm{Cr}_{2} \mathrm{O}_{3}$ & 0.1476 & 0.1388 & 0.0088 & $6.3 \%$ \\
\hline QB-32 & $\mathrm{CuO}$ & 0.0329 & 0.0320 & 0.0009 & $2.8 \%$ \\
\hline QB-32 & $\mathrm{Fe}_{2} \mathrm{O}_{3}$ & 7.9420 & 8.1647 & -0.2227 & $-2.7 \%$ \\
\hline QB-32 & $\mathrm{K}_{2} \mathrm{O}$ & 0.2439 & 0.2463 & -0.0024 & $-1.0 \%$ \\
\hline QB-32 & $\mathrm{La}_{2} \mathrm{O}_{3}$ & 0.0194 & 0.0257 & -0.0063 & $-24.5 \%$ \\
\hline QB-32 & $\mathrm{Li}_{2} \mathrm{O}$ & 4.8171 & 5.1200 & -0.3029 & $-5.9 \%$ \\
\hline QB-32 & $\mathrm{MgO}$ & 0.3420 & 0.2785 & 0.0635 & $22.8 \%$ \\
\hline QB-32 & $\mathrm{MnO}$ & 2.2919 & 2.3102 & -0.0183 & $-0.8 \%$ \\
\hline QB-32 & $\mathrm{Na}_{2} \mathrm{O}$ & 16.8837 & 17.1440 & -0.2603 & $-1.5 \%$ \\
\hline QB-32 & $\mathrm{NiO}$ & 0.8962 & 0.9711 & -0.0749 & $-7.7 \%$ \\
\hline QB-32 & $\mathrm{PbO}$ & 0.0108 & 0.0079 & 0.0029 & $36.7 \%$ \\
\hline QB-32 & $\mathrm{SiO}_{2}$ & 48.8830 & 49.4680 & -0.5850 & $-1.2 \%$ \\
\hline QB-32 & $\mathrm{SO}_{4}$ & 1.1250 & 1.2000 & -0.0750 & $-6.3 \%$ \\
\hline QB-32 & $\mathrm{TiO}_{2}$ & 0.0083 & 0.0000 & 0.0083 & \\
\hline QB-32 & $\mathrm{ZnO}$ & 0.0233 & 0.0227 & 0.0006 & $2.6 \%$ \\
\hline QB-32 & $\mathrm{ZrO}_{2}$ & 0.0935 & 0.0986 & -0.0051 & $-5.2 \%$ \\
\hline QB-32 & Sum & 98.2858 & 100.0000 & -1.7142 & $-1.7 \%$ \\
\hline QB-33 & $\mathrm{Al}_{2} \mathrm{O}_{3}$ & 8.6114 & 9.0791 & -0.4677 & $-5.2 \%$ \\
\hline QB-33 & $\mathrm{B}_{2} \mathrm{O}_{3}$ & 8.5086 & 8.9600 & -0.4514 & $-5.0 \%$ \\
\hline QB-33 & $\mathrm{BaO}$ & 0.0268 & 0.0367 & -0.0099 & $-27.0 \%$ \\
\hline QB-33 & $\mathrm{CaO}$ & 5.4800 & 5.6258 & -0.1458 & $-2.6 \%$ \\
\hline QB-33 & $\mathrm{Ce}_{2} \mathrm{O}_{3}$ & 0.0325 & 0.0299 & 0.0026 & $8.7 \%$ \\
\hline QB-33 & $\mathrm{Cr}_{2} \mathrm{O}_{3}$ & 0.1235 & 0.1388 & -0.0153 & $-11.0 \%$ \\
\hline QB-33 & $\mathrm{CuO}$ & 0.0285 & 0.0320 & -0.0035 & $-10.9 \%$ \\
\hline QB-33 & $\mathrm{Fe}_{2} \mathrm{O}_{3}$ & 7.4452 & 8.1647 & -0.7195 & $-8.8 \%$ \\
\hline QB-33 & $\mathrm{K}_{2} \mathrm{O}$ & 0.2590 & 0.2463 & 0.0127 & $5.2 \%$ \\
\hline QB-33 & $\mathrm{La}_{2} \mathrm{O}_{3}$ & 0.0273 & 0.0257 & 0.0016 & $6.2 \%$ \\
\hline QB-33 & $\mathrm{Li}_{2} \mathrm{O}$ & 4.2251 & 4.4800 & -0.2549 & $-5.7 \%$ \\
\hline QB-33 & $\mathrm{MgO}$ & 0.2405 & 0.2785 & -0.0380 & $-13.6 \%$ \\
\hline QB-33 & $\mathrm{MnO}$ & 2.1950 & 2.3102 & -0.1152 & $-5.0 \%$ \\
\hline QB-33 & $\mathrm{Na}_{2} \mathrm{O}$ & 12.6004 & 13.9440 & -1.3436 & $-9.6 \%$ \\
\hline
\end{tabular}


SRNL-STI-2012-00152

Revision 0

Table A-4. Average Measured Chemical Compositions versus Targeted Compositions by Oxide by Glass ID (continued)

\begin{tabular}{|c|c|c|c|c|c|}
\hline Glass ID & Oxide & $\begin{array}{c}\text { Measured } \\
\text { (wt \%) }\end{array}$ & $\begin{array}{c}\text { Targeted } \\
\text { (wt \%) }\end{array}$ & $\begin{array}{c}\text { Diff of } \\
\text { Measured }\end{array}$ & $\begin{array}{l}\text { \% Diff of } \\
\text { Measured }\end{array}$ \\
\hline QB-33 & $\mathrm{NiO}$ & 0.8726 & 0.9711 & -0.0985 & $-10.1 \%$ \\
\hline QB-33 & $\mathrm{PbO}$ & 0.0108 & 0.0079 & 0.0029 & $36.7 \%$ \\
\hline QB-33 & $\mathrm{SiO}_{2}$ & 47.3320 & 44.3480 & 2.9840 & $6.7 \%$ \\
\hline QB-33 & $\mathrm{SO}_{4}$ & 1.1032 & 1.2000 & -0.0968 & $-8.1 \%$ \\
\hline QB-33 & $\mathrm{TiO}_{2}$ & 0.0083 & 0.0000 & 0.0083 & \\
\hline QB-33 & $\mathrm{ZnO}$ & 0.0283 & 0.0227 & 0.0056 & $24.7 \%$ \\
\hline QB-33 & $\mathrm{ZrO}_{2}$ & 0.0976 & 0.0986 & -0.0010 & $-1.0 \%$ \\
\hline QB-33 & Sum & 99.2566 & 100.0000 & -0.7434 & $-0.7 \%$ \\
\hline QB-34 & $\mathrm{Al}_{2} \mathrm{O}_{3}$ & 8.9468 & 9.0791 & -0.1323 & $-1.5 \%$ \\
\hline QB-34 & $\mathrm{B}_{2} \mathrm{O}_{3}$ & 4.9908 & 5.1200 & -0.1292 & $-2.5 \%$ \\
\hline QB-34 & $\mathrm{BaO}$ & 0.0279 & 0.0367 & -0.0088 & $-24.0 \%$ \\
\hline QB-34 & $\mathrm{CaO}$ & 6.7511 & 6.9058 & -0.1547 & $-2.2 \%$ \\
\hline QB-34 & $\mathrm{Ce}_{2} \mathrm{O}_{3}$ & 0.0334 & 0.0299 & 0.0035 & $11.7 \%$ \\
\hline QB-34 & $\mathrm{Cr}_{2} \mathrm{O}_{3}$ & 0.1494 & 0.1388 & 0.0106 & $7.6 \%$ \\
\hline QB-34 & $\mathrm{CuO}$ & 0.0357 & 0.0320 & 0.0037 & $11.6 \%$ \\
\hline QB-34 & $\mathrm{Fe}_{2} \mathrm{O}_{3}$ & 8.4388 & 8.1647 & 0.2741 & $3.4 \%$ \\
\hline QB-34 & $\mathrm{K}_{2} \mathrm{O}$ & 0.2349 & 0.2463 & -0.0114 & $-4.6 \%$ \\
\hline QB-34 & $\mathrm{La}_{2} \mathrm{O}_{3}$ & 0.0264 & 0.0257 & 0.0007 & $2.7 \%$ \\
\hline QB-34 & $\mathrm{Li}_{2} \mathrm{O}$ & 4.8386 & 5.1200 & -0.2814 & $-5.5 \%$ \\
\hline QB-34 & $\mathrm{MgO}$ & 0.2570 & 0.2785 & -0.0215 & $-7.7 \%$ \\
\hline QB-34 & $\mathrm{MnO}$ & 2.2693 & 2.3102 & -0.0409 & $-1.8 \%$ \\
\hline QB-34 & $\mathrm{Na}_{2} \mathrm{O}$ & 13.0352 & 13.3040 & -0.2688 & $-2.0 \%$ \\
\hline QB-34 & $\mathrm{NiO}$ & 0.8720 & 0.9711 & -0.0991 & $-10.2 \%$ \\
\hline QB-34 & $\mathrm{PbO}$ & 0.0108 & 0.0079 & 0.0029 & $36.7 \%$ \\
\hline QB-34 & $\mathrm{SiO}_{2}$ & 46.2624 & 46.9080 & -0.6456 & $-1.4 \%$ \\
\hline QB-34 & $\mathrm{SO}_{4}$ & 1.0890 & 1.2000 & -0.1110 & $-9.3 \%$ \\
\hline QB-34 & $\mathrm{TiO}_{2}$ & 0.0083 & 0.0000 & 0.0083 & \\
\hline QB-34 & $\mathrm{ZnO}$ & 0.0249 & 0.0227 & 0.0022 & $9.7 \%$ \\
\hline QB-34 & $\mathrm{ZrO}_{2}$ & 0.0912 & 0.0986 & -0.0074 & $-7.5 \%$ \\
\hline QB-34 & Sum & 98.3939 & 100.0000 & -1.6061 & $-1.6 \%$ \\
\hline QB-35 & $\mathrm{Al}_{2} \mathrm{O}_{3}$ & 8.8287 & 9.0791 & -0.2504 & $-2.8 \%$ \\
\hline QB-35 & $\mathrm{B}_{2} \mathrm{O}_{3}$ & 5.0391 & 5.1200 & -0.0809 & $-1.6 \%$ \\
\hline QB-35 & $\mathrm{BaO}$ & 0.0290 & 0.0367 & -0.0077 & $-21.0 \%$ \\
\hline QB-35 & $\mathrm{CaO}$ & 6.6742 & 6.9058 & -0.2316 & $-3.4 \%$ \\
\hline QB-35 & $\mathrm{Ce}_{2} \mathrm{O}_{3}$ & 0.0495 & 0.0299 & 0.0196 & $65.6 \%$ \\
\hline QB-35 & $\mathrm{Cr}_{2} \mathrm{O}_{3}$ & 0.1275 & 0.1388 & -0.0113 & $-8.1 \%$ \\
\hline QB-35 & $\mathrm{CuO}$ & 0.0322 & 0.0320 & 0.0002 & $0.6 \%$ \\
\hline QB-35 & $\mathrm{Fe}_{2} \mathrm{O}_{3}$ & 7.7990 & 8.1647 & -0.3657 & $-4.5 \%$ \\
\hline QB-35 & $\mathrm{K}_{2} \mathrm{O}$ & 0.2364 & 0.2463 & -0.0099 & $-4.0 \%$ \\
\hline QB-35 & $\mathrm{La}_{2} \mathrm{O}_{3}$ & 0.0443 & 0.0257 & 0.0186 & $72.4 \%$ \\
\hline QB-35 & $\mathrm{Li}_{2} \mathrm{O}$ & 4.3058 & 4.4800 & -0.1742 & $-3.9 \%$ \\
\hline QB-35 & $\mathrm{MgO}$ & 0.2516 & 0.2785 & -0.0269 & $-9.7 \%$ \\
\hline QB-35 & $\mathrm{MnO}$ & 2.2725 & 2.3102 & -0.0377 & $-1.6 \%$ \\
\hline QB-35 & $\mathrm{Na}_{2} \mathrm{O}$ & 14.7269 & 15.2240 & -0.4971 & $-3.3 \%$ \\
\hline QB-35 & $\mathrm{NiO}$ & 0.8675 & 0.9711 & -0.1036 & $-10.7 \%$ \\
\hline QB-35 & $\mathrm{PbO}$ & 0.0108 & 0.0079 & 0.0029 & $36.7 \%$ \\
\hline QB-35 & $\mathrm{SiO}_{2}$ & 44.6044 & 45.6280 & -1.0236 & $-2.2 \%$ \\
\hline QB-35 & $\mathrm{SO}_{4}$ & 1.1250 & 1.2000 & -0.0750 & $-6.3 \%$ \\
\hline QB-35 & $\mathrm{TiO}_{2}$ & 0.0083 & 0.0000 & 0.0083 & \\
\hline QB-35 & $\mathrm{ZnO}$ & 0.0230 & 0.0227 & 0.0003 & $1.3 \%$ \\
\hline QB-35 & $\mathrm{ZrO}_{2}$ & 0.1020 & 0.0986 & 0.0034 & $3.4 \%$ \\
\hline QB-35 & Sum & 97.1579 & 100.0000 & -2.8421 & $-2.8 \%$ \\
\hline QB-36 & $\mathrm{Al}_{2} \mathrm{O}_{3}$ & 8.7295 & 9.0791 & -0.3496 & $-3.9 \%$ \\
\hline QB-36 & $\mathrm{B}_{2} \mathrm{O}_{3}$ & 4.8701 & 5.1200 & -0.2499 & $-4.9 \%$ \\
\hline QB-36 & $\mathrm{BaO}$ & 0.0240 & 0.0367 & -0.0127 & $-34.6 \%$ \\
\hline QB-36 & $\mathrm{CaO}$ & 3.6239 & 3.7058 & -0.0819 & $-2.2 \%$ \\
\hline QB-36 & $\mathrm{Ce}_{2} \mathrm{O}_{3}$ & 0.0296 & 0.0299 & -0.0003 & $-1.0 \%$ \\
\hline
\end{tabular}


SRNL-STI-2012-00152

Revision 0

Table A-4. Average Measured Chemical Compositions versus Targeted Compositions by Oxide by Glass ID (continued)

\begin{tabular}{|c|c|c|c|c|c|}
\hline Glass ID & Oxide & $\begin{array}{c}\text { Measured } \\
\text { (wt \%) }\end{array}$ & $\begin{array}{c}\text { Targeted } \\
(\text { wt \%) }\end{array}$ & $\begin{array}{c}\text { Diff of } \\
\text { Measured }\end{array}$ & $\begin{array}{l}\text { \% Diff of } \\
\text { Measured }\end{array}$ \\
\hline QB-36 & $\mathrm{Cr}_{2} \mathrm{O}_{3}$ & 0.1469 & 0.1388 & 0.0081 & $5.8 \%$ \\
\hline QB-36 & $\mathrm{CuO}$ & 0.0469 & 0.0320 & 0.0149 & $46.6 \%$ \\
\hline QB-36 & $\mathrm{Fe}_{2} \mathrm{O}_{3}$ & 7.7454 & 8.1647 & -0.4193 & $-5.1 \%$ \\
\hline QB-36 & $\mathrm{K}_{2} \mathrm{O}$ & 0.2454 & 0.2463 & -0.0009 & $-0.4 \%$ \\
\hline QB-36 & $\mathrm{La}_{2} \mathrm{O}_{3}$ & 0.0258 & 0.0257 & 0.0001 & $0.4 \%$ \\
\hline QB-36 & $\mathrm{Li}_{2} \mathrm{O}$ & 4.7687 & 5.1200 & -0.3513 & $-6.9 \%$ \\
\hline QB-36 & $\mathrm{MgO}$ & 0.2537 & 0.2785 & -0.0248 & $-8.9 \%$ \\
\hline QB-36 & $\mathrm{MnO}$ & 2.2370 & 2.3102 & -0.0732 & $-3.2 \%$ \\
\hline QB-36 & $\mathrm{Na}_{2} \mathrm{O}$ & 14.5247 & 15.2240 & -0.6993 & $-4.6 \%$ \\
\hline QB-36 & $\mathrm{NiO}$ & 0.8958 & 0.9711 & -0.0753 & $-7.8 \%$ \\
\hline QB-36 & $\mathrm{PbO}$ & 0.0108 & 0.0079 & 0.0029 & $36.7 \%$ \\
\hline QB-36 & $\mathrm{SiO}_{2}$ & 46.8507 & 48.1880 & -1.3373 & $-2.8 \%$ \\
\hline QB-36 & $\mathrm{SO}_{4}$ & 1.1429 & 1.2000 & -0.0571 & $-4.8 \%$ \\
\hline QB-36 & $\mathrm{TiO}_{2}$ & 0.0083 & 0.0000 & 0.0083 & \\
\hline QB-36 & $\mathrm{ZnO}$ & 0.0280 & 0.0227 & 0.0053 & $23.3 \%$ \\
\hline QB-36 & $\mathrm{ZrO}_{2}$ & 0.0976 & 0.0986 & -0.0010 & $-1.0 \%$ \\
\hline QB-36 & Sum & 96.3058 & 100.0000 & -3.6942 & $-3.7 \%$ \\
\hline QB-37 & $\mathrm{Al}_{2} \mathrm{O}_{3}$ & 8.8429 & 9.0791 & -0.2362 & $-2.6 \%$ \\
\hline QB-37 & $\mathrm{B}_{2} \mathrm{O}_{3}$ & 4.9103 & 5.1200 & -0.2097 & $-4.1 \%$ \\
\hline QB-37 & $\mathrm{BaO}$ & 0.0287 & 0.0367 & -0.0080 & $-21.8 \%$ \\
\hline QB-37 & $\mathrm{CaO}$ & 3.6650 & 3.7058 & -0.0408 & $-1.1 \%$ \\
\hline QB-37 & $\mathrm{Ce}_{2} \mathrm{O}_{3}$ & 0.0319 & 0.0299 & 0.0020 & $6.7 \%$ \\
\hline QB-37 & $\mathrm{Cr}_{2} \mathrm{O}_{3}$ & 0.1484 & 0.1388 & 0.0096 & $6.9 \%$ \\
\hline QB-37 & $\mathrm{CuO}$ & 0.0335 & 0.0320 & 0.0015 & $4.7 \%$ \\
\hline QB-37 & $\mathrm{Fe}_{2} \mathrm{O}_{3}$ & 7.8812 & 8.1647 & -0.2835 & $-3.5 \%$ \\
\hline QB-37 & $\mathrm{K}_{2} \mathrm{O}$ & 0.2463 & 0.2463 & 0.0000 & $0.0 \%$ \\
\hline QB-37 & $\mathrm{La}_{2} \mathrm{O}_{3}$ & 0.0191 & 0.0257 & -0.0066 & $-25.7 \%$ \\
\hline QB-37 & $\mathrm{Li}_{2} \mathrm{O}$ & 5.4845 & 5.7600 & -0.2755 & $-4.8 \%$ \\
\hline QB-37 & $\mathrm{MgO}$ & 0.3387 & 0.2785 & 0.0602 & $21.6 \%$ \\
\hline QB-37 & $\mathrm{MnO}$ & 2.2725 & 2.3102 & -0.0377 & $-1.6 \%$ \\
\hline QB-37 & $\mathrm{Na}_{2} \mathrm{O}$ & 16.7489 & 13.3040 & 3.4449 & $25.9 \%$ \\
\hline QB-37 & $\mathrm{NiO}$ & 0.8923 & 0.9711 & -0.0788 & $-8.1 \%$ \\
\hline QB-37 & $\mathrm{PbO}$ & 0.0108 & 0.0079 & 0.0029 & $36.7 \%$ \\
\hline QB-37 & $\mathrm{SiO}_{2}$ & 48.1343 & 49.4680 & -1.3337 & $-2.7 \%$ \\
\hline QB-37 & $\mathrm{SO}_{4}$ & 1.1257 & 1.2000 & -0.0743 & $-6.2 \%$ \\
\hline QB-37 & $\mathrm{TiO}_{2}$ & 0.0083 & 0.0000 & 0.0083 & \\
\hline QB-37 & $\mathrm{ZnO}$ & 0.0233 & 0.0227 & 0.0006 & $2.6 \%$ \\
\hline QB-37 & $\mathrm{ZrO}_{2}$ & 0.0942 & 0.0986 & -0.0044 & $-4.5 \%$ \\
\hline QB-37 & Sum & 100.9409 & 100.0000 & 0.9409 & $0.9 \%$ \\
\hline SPS-02 & $\mathrm{Al}_{2} \mathrm{O}_{3}$ & 11.7243 & 12.1846 & -0.4603 & $-3.8 \%$ \\
\hline SPS-02 & $\mathrm{B}_{2} \mathrm{O}_{3}$ & 4.6850 & 4.8000 & -0.1150 & $-2.4 \%$ \\
\hline SPS-02 & $\mathrm{BaO}$ & 0.0500 & 0.0625 & -0.0125 & $-20.0 \%$ \\
\hline SPS-02 & $\mathrm{CaO}$ & 0.8518 & 0.8517 & 0.0001 & $0.0 \%$ \\
\hline SPS-02 & $\mathrm{Ce}_{2} \mathrm{O}_{3}$ & 0.1086 & 0.1082 & 0.0004 & $0.4 \%$ \\
\hline SPS-02 & $\mathrm{Cr}_{2} \mathrm{O}_{3}$ & 0.0760 & 0.0675 & 0.0085 & $12.6 \%$ \\
\hline SPS-02 & $\mathrm{CuO}$ & 0.0635 & 0.0522 & 0.0113 & $21.6 \%$ \\
\hline SPS-02 & $\mathrm{Fe}_{2} \mathrm{O}_{3}$ & 10.4154 & 11.5766 & -1.1612 & $-10.0 \%$ \\
\hline SPS-02 & $\mathrm{K}_{2} \mathrm{O}$ & 0.0602 & 0.0267 & 0.0335 & $125.5 \%$ \\
\hline SPS-02 & $\mathrm{La}_{2} \mathrm{O}_{3}$ & 0.0369 & 0.0465 & -0.0096 & $-20.6 \%$ \\
\hline SPS-02 & $\mathrm{Li}_{2} \mathrm{O}$ & 4.4188 & 4.8000 & -0.3812 & $-7.9 \%$ \\
\hline SPS-02 & $\mathrm{MgO}$ & 0.2807 & 0.3190 & -0.0383 & $-12.0 \%$ \\
\hline SPS-02 & $\mathrm{MnO}$ & 3.0731 & 3.1475 & -0.0744 & $-2.4 \%$ \\
\hline SPS-02 & $\mathrm{Na}_{2} \mathrm{O}$ & 12.3106 & 12.9211 & -0.6105 & $-4.7 \%$ \\
\hline SPS-02 & $\mathrm{NiO}$ & 1.3034 & 1.4531 & -0.1497 & $-10.3 \%$ \\
\hline SPS-02 & $\mathrm{PbO}$ & 0.0108 & 0.0135 & -0.0027 & $-20.0 \%$ \\
\hline SPS-02 & $\mathrm{SiO}_{2}$ & 45.4601 & 46.6858 & -1.2257 & $-2.6 \%$ \\
\hline SPS-02 & $\mathrm{SO}_{4}$ & 0.5602 & 0.6000 & -0.0398 & $-6.6 \%$ \\
\hline
\end{tabular}


SRNL-STI-2012-00152

Revision 0

Table A-4. Average Measured Chemical Compositions versus Targeted Compositions by Oxide by Glass ID (continued)

\begin{tabular}{||c|c|c|c|c|c||}
\hline \hline Glass ID & Oxide & $\begin{array}{c}\text { Measured } \\
\text { (wt \%) }\end{array}$ & $\begin{array}{c}\text { Targeted } \\
\text { (wt \%) }\end{array}$ & $\begin{array}{c}\text { Diff of } \\
\text { Measured }\end{array}$ & $\begin{array}{c}\text { \% Diff of } \\
\text { Measured }\end{array}$ \\
\hline SPS-02 & $\mathrm{TiO}_{2}$ & 0.0083 & 0.0069 & 0.0014 & $20.3 \%$ \\
\hline $\mathrm{SPS}-02$ & $\mathrm{ZnO}$ & 0.0330 & 0.0279 & 0.0051 & $18.3 \%$ \\
\hline $\mathrm{SPS}-02$ & $\mathrm{ZrO}_{2}$ & 0.1405 & 0.1473 & -0.0068 & $-4.6 \%$ \\
\hline SPS-02 & $\mathrm{Sum}^{2}$ & 95.6712 & 99.8986 & -4.2274 & $-4.2 \%$ \\
\hline
\end{tabular}


Exhibit A-1. Measurements of Glasses in Analytical Sequence by Oxide within Preparation Method and by Analytical Series Series $=$ T, Oxide $=A l 2 O 3$ ( $w t \%)$, Prep Method $=$ LM

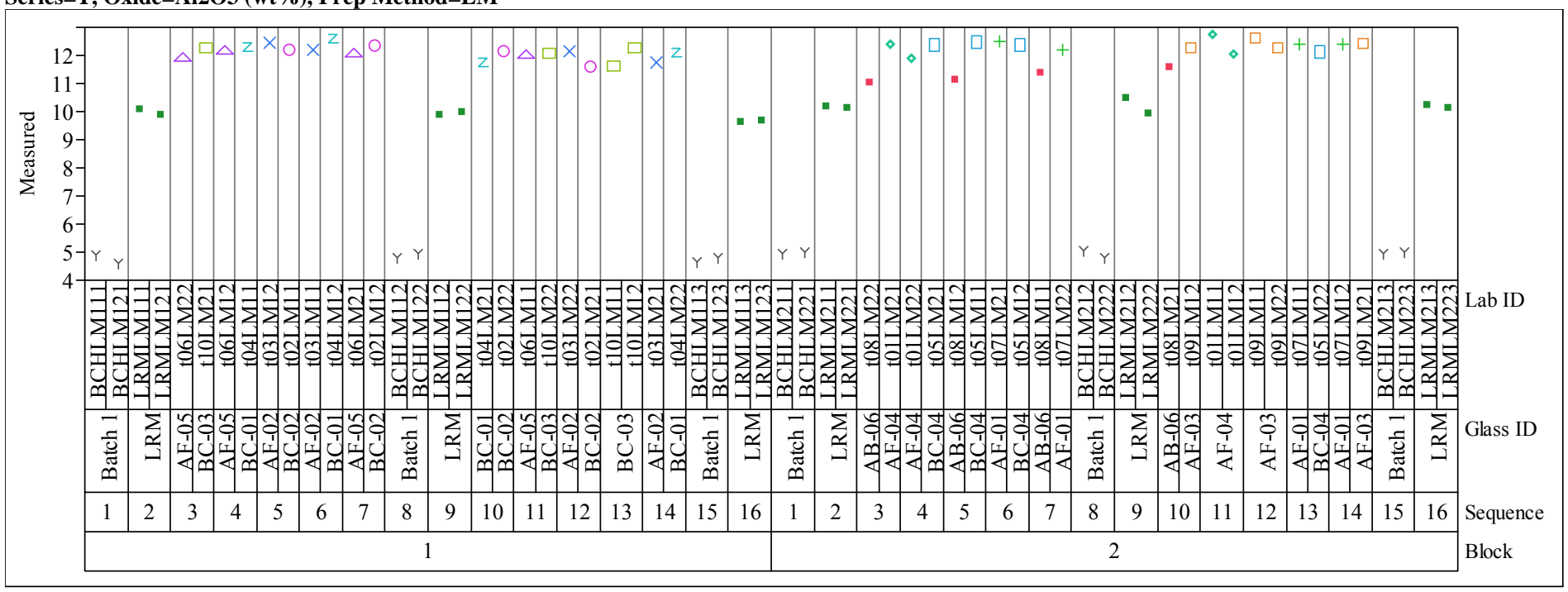

Series $=$ T, Oxide $=\mathbf{B} 203$ (wt $\%)$, Prep Method $=$ PF

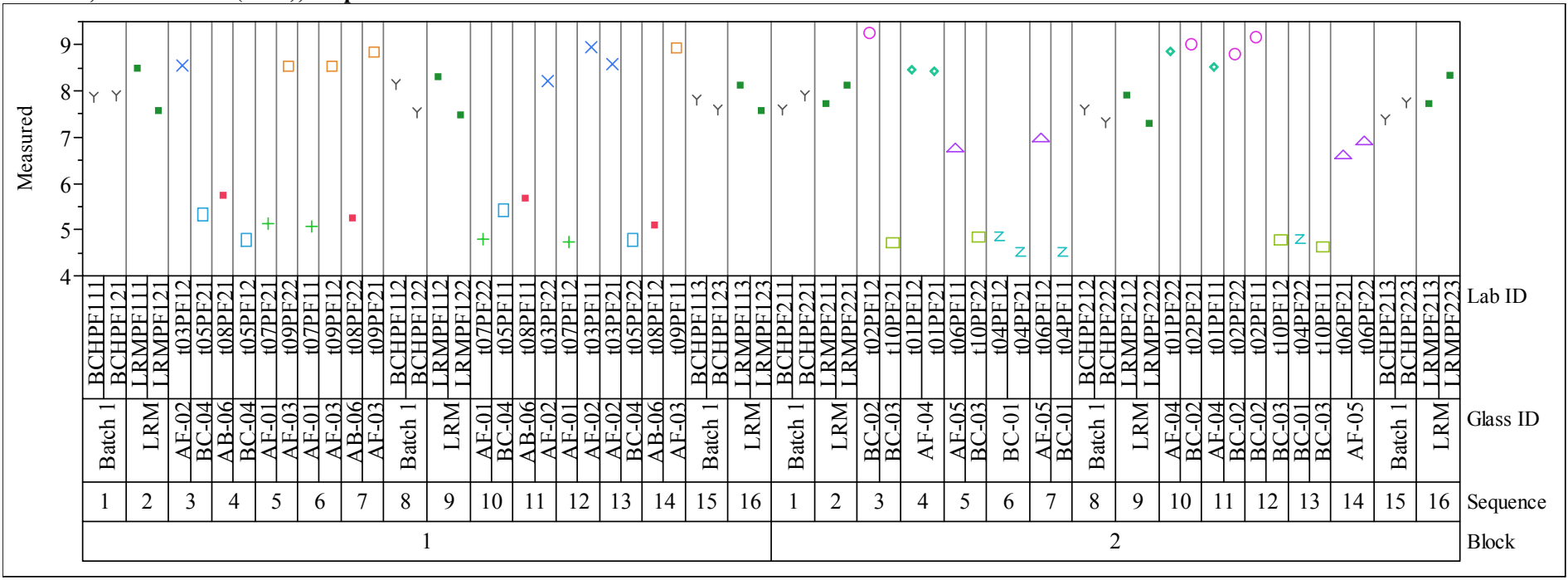


Exhibit A-1. Measurements of Glasses in Analytical Sequence by Oxide within Preparation Method and by Analytical Series (continued) Series $=T$, Oxide $=B a O(w t \%)$, Prep Method $=L M$

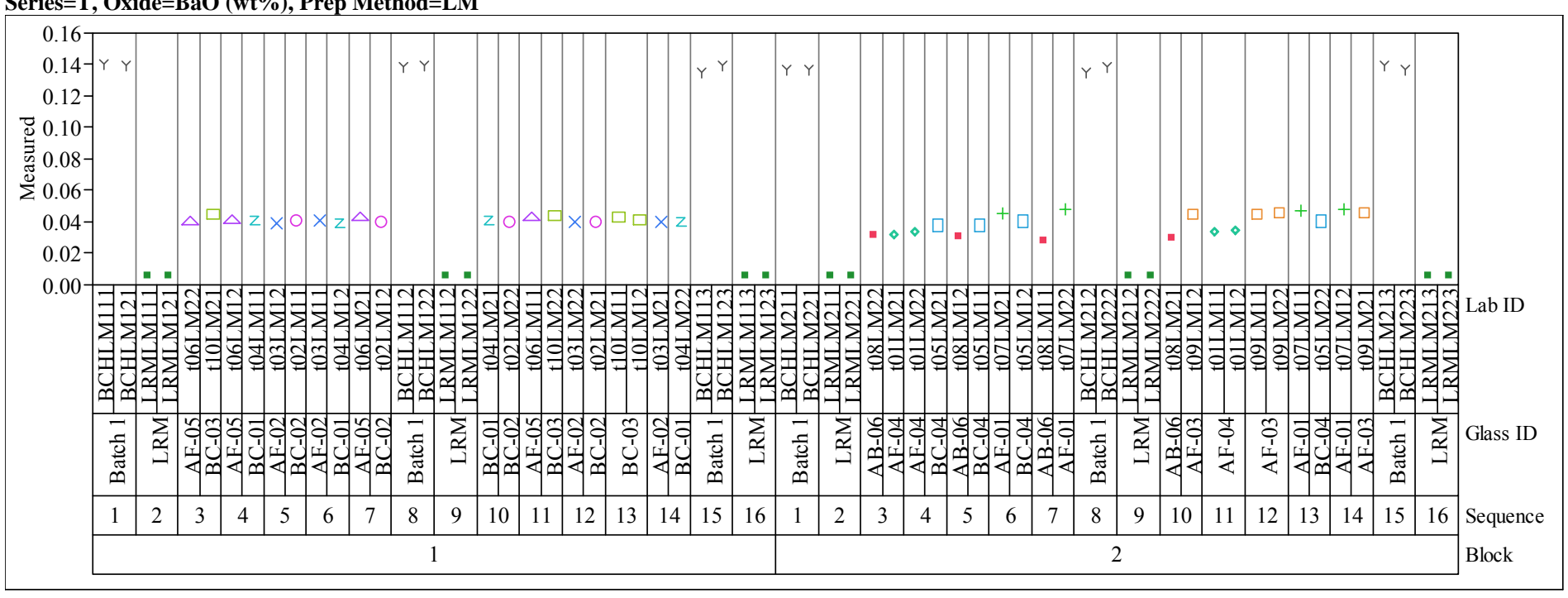

Series $=$ T, Oxide $=\mathbf{C a O}(w t \%)$, Prep Method $=\mathbf{L M}$

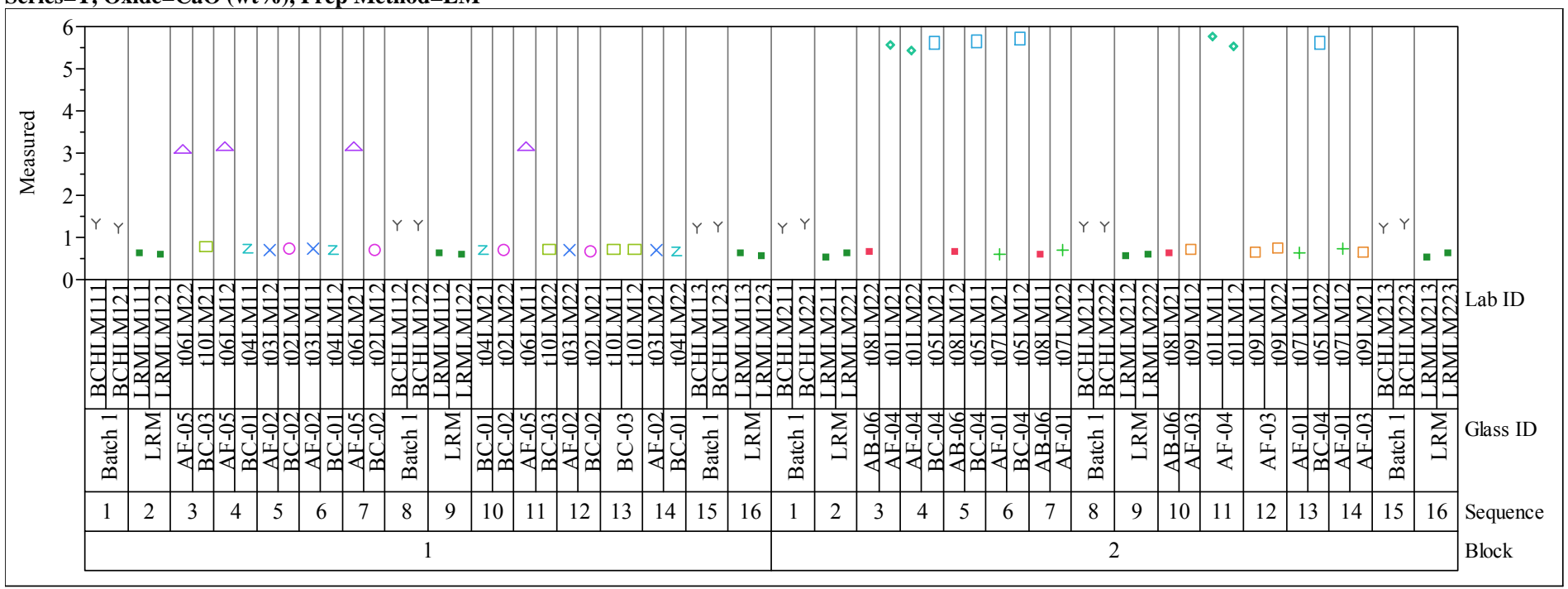


Exhibit A-1. Measurements of Glasses in Analytical Sequence by Oxide within Preparation Method and by Analytical Series (continued) Series $=$ T, Oxide=Ce2O3 (wt\%), Prep Method=LM

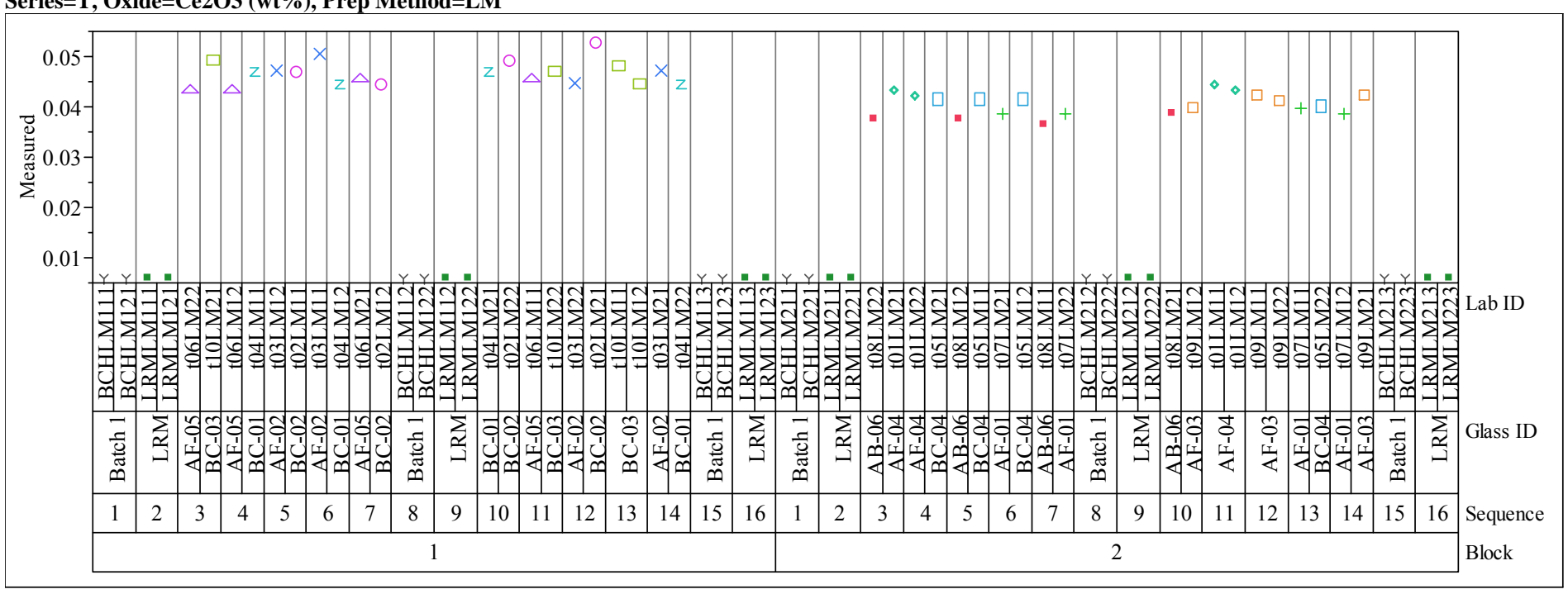

Series=T, Oxide=Cr2O3 (wt \%), Prep Method=LM

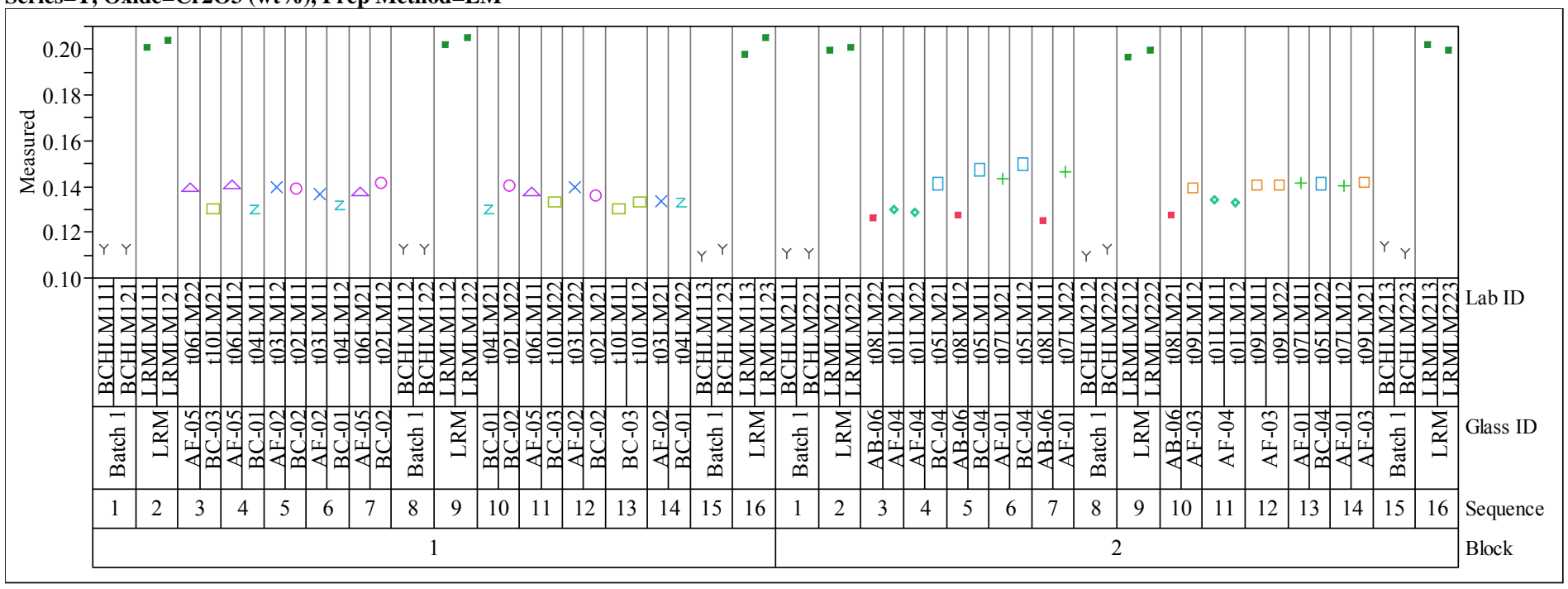




\section{Exhibit A-1. Measurements of Glasses in Analytical Sequence by Oxide within Preparation Method and by Analytical Series (continued)}

Series $=$ T, Oxide $=\mathrm{CuO}(\mathrm{wt} \%)$, Prep Method $=\mathbf{L M}$

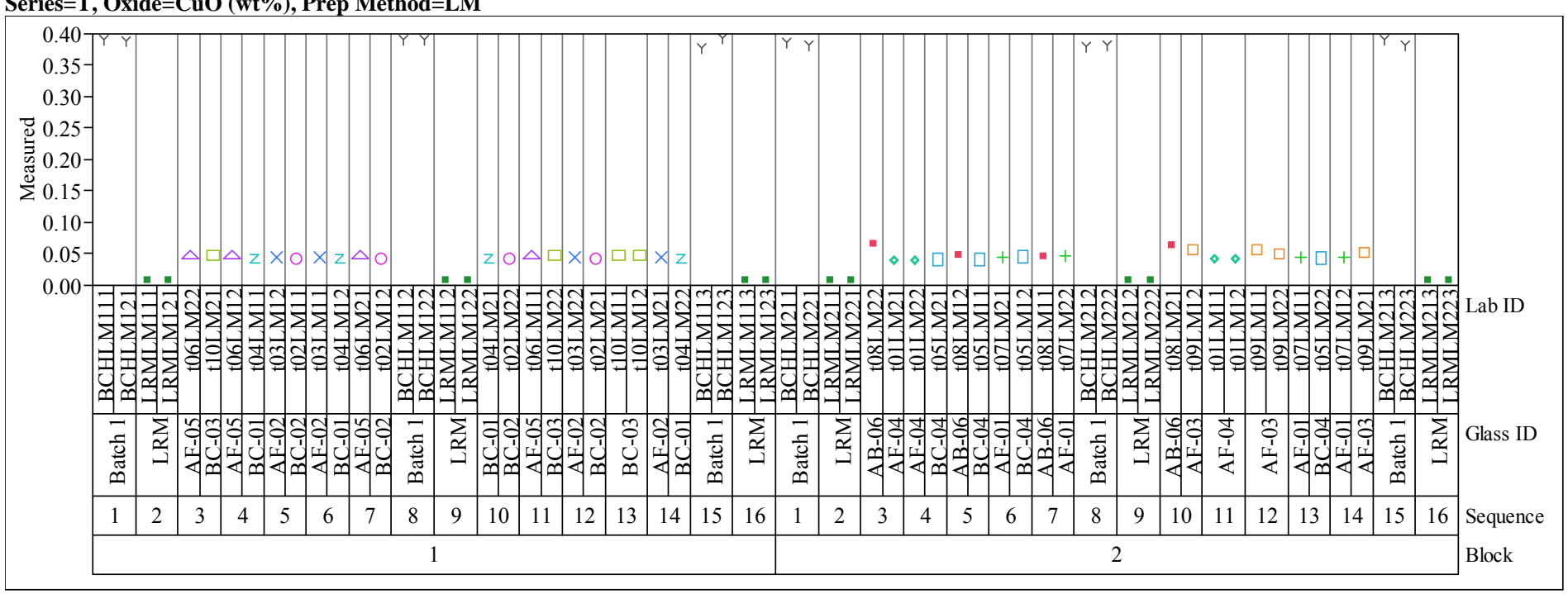

Series=T, Oxide=Fe2O3 (wt \%), Prep Method=LM

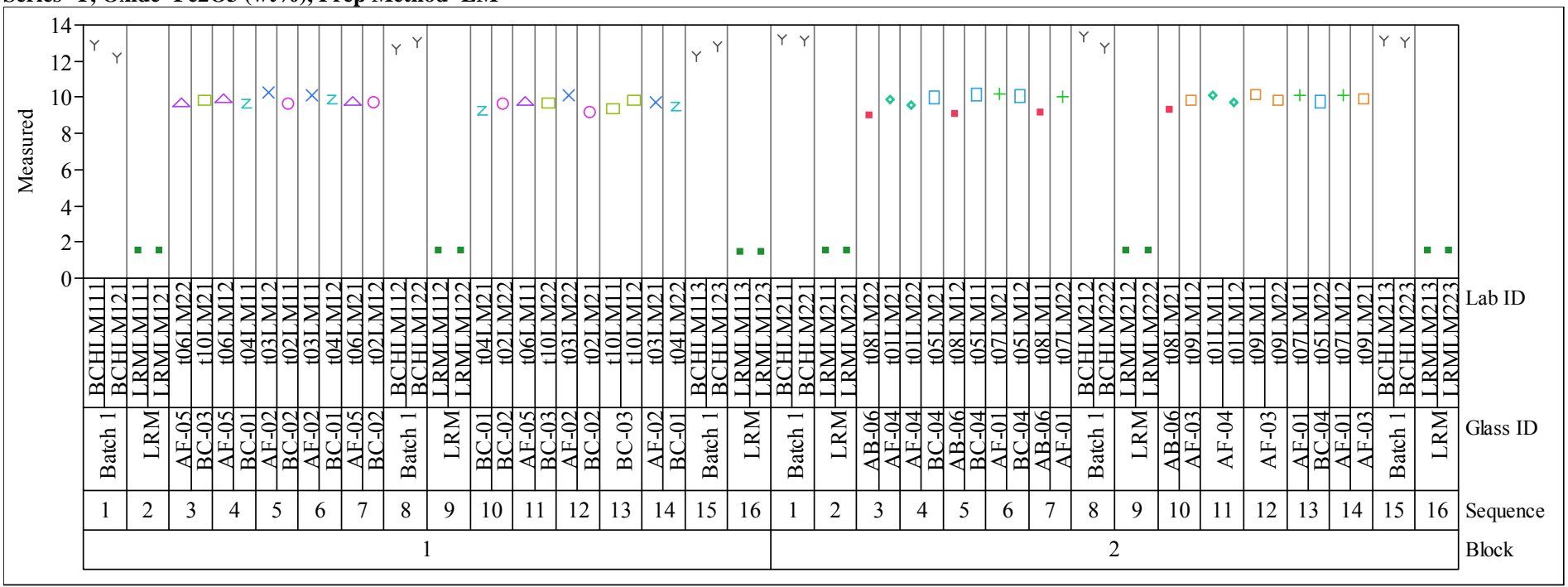




\section{Exhibit A-1. Measurements of Glasses in Analytical Sequence by Oxide within Preparation Method and by Analytical Series (continued)} Series=T, Oxide=K2O (wt \%), Prep Method $=\mathbf{L M}$

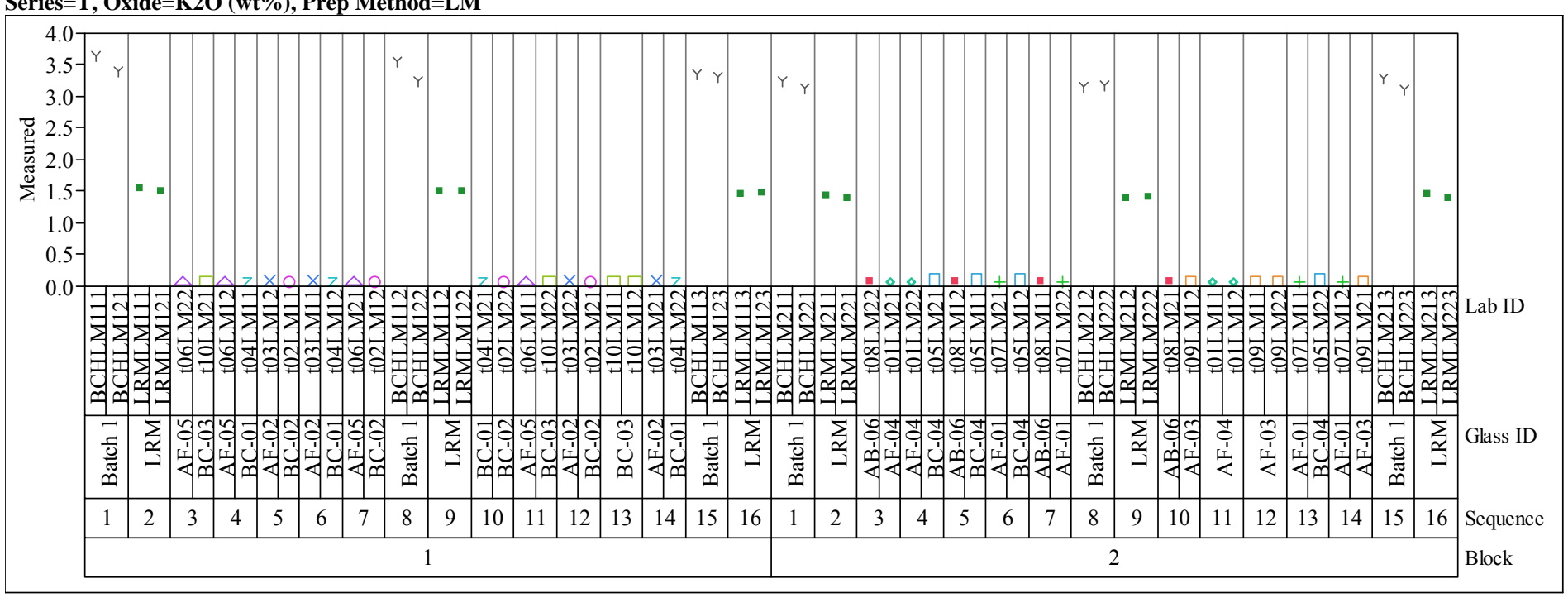

Series=T, Oxide=La2O3 (wt\%), Prep Method=LM

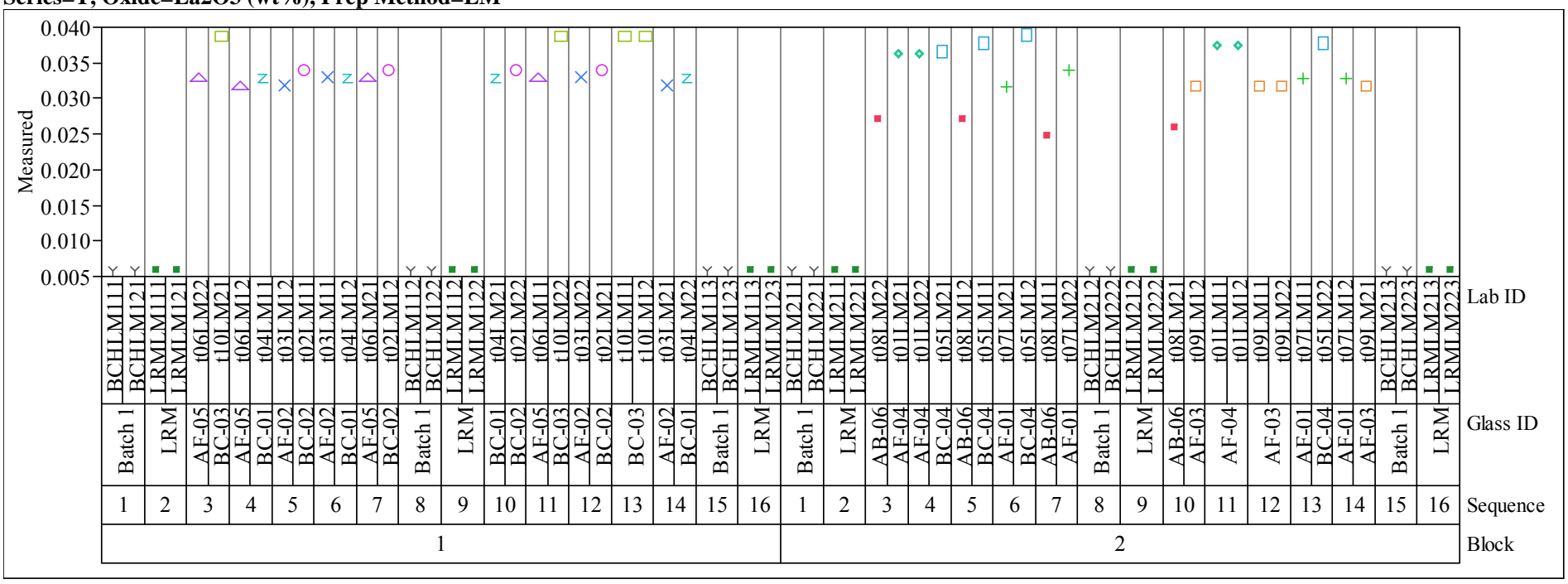




\section{Exhibit A-1. Measurements of Glasses in Analytical Sequence by Oxide within Preparation Method and by Analytical Series (continued)} Series=T, Oxide=Li2O (wt \%), Prep Method=PF

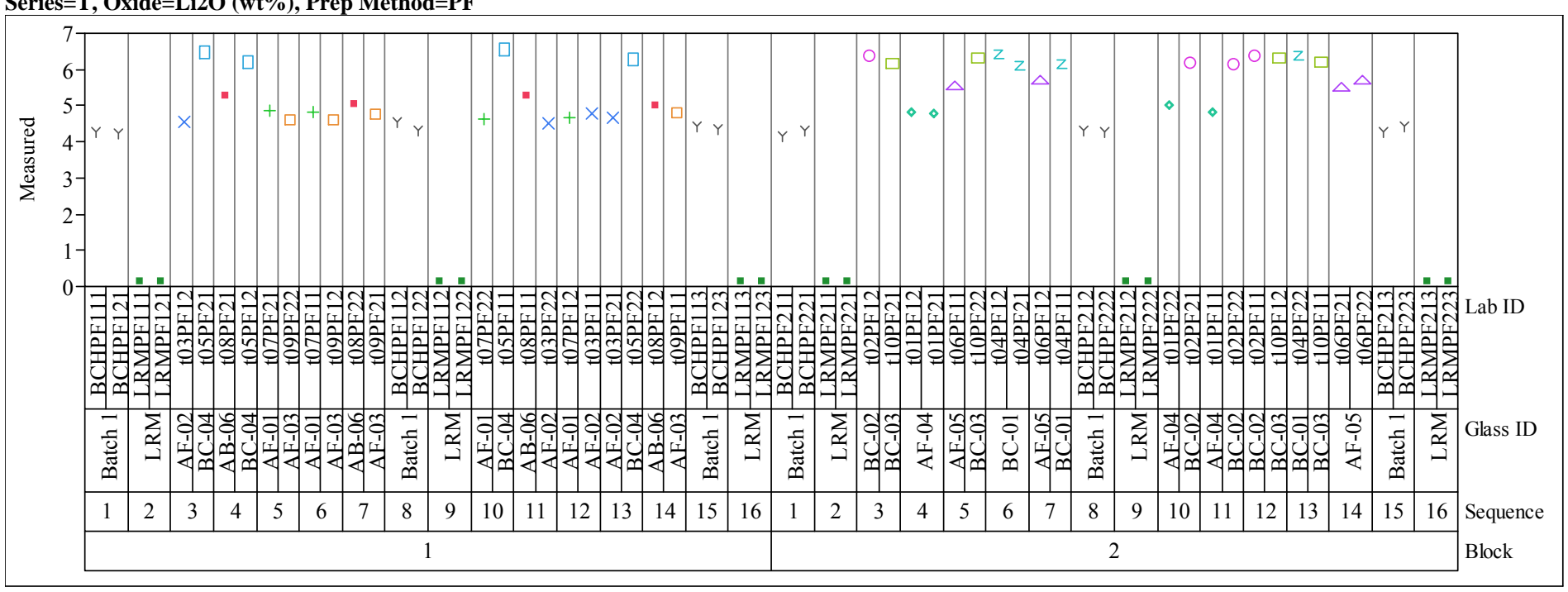

Series $=\mathrm{T}$, Oxide $=$ MgO $($ wt \%), Prep Method $=$ LM

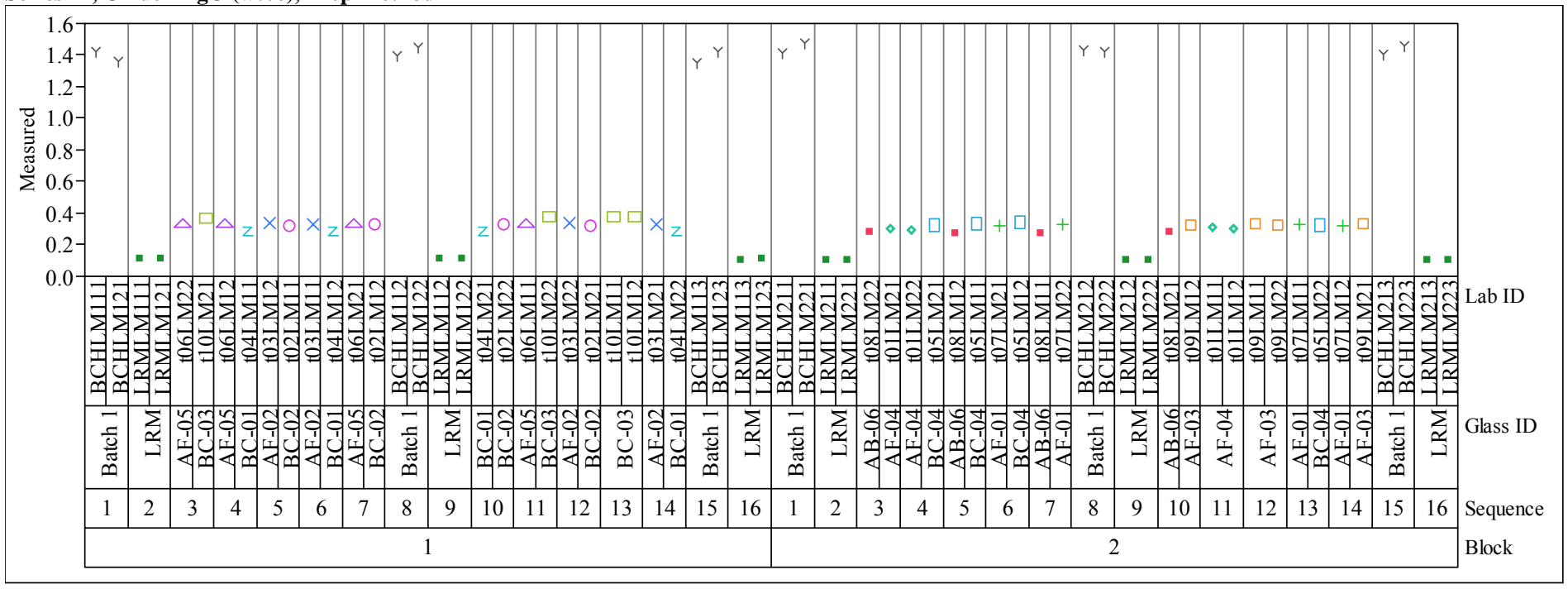




\section{Exhibit A-1. Measurements of Glasses in Analytical Sequence by Oxide within Preparation Method and by Analytical Series (continued)}

Series $=T$, Oxide $=\mathrm{MnO}(\mathrm{wt} \%)$, Prep Method $=\mathrm{LM}$

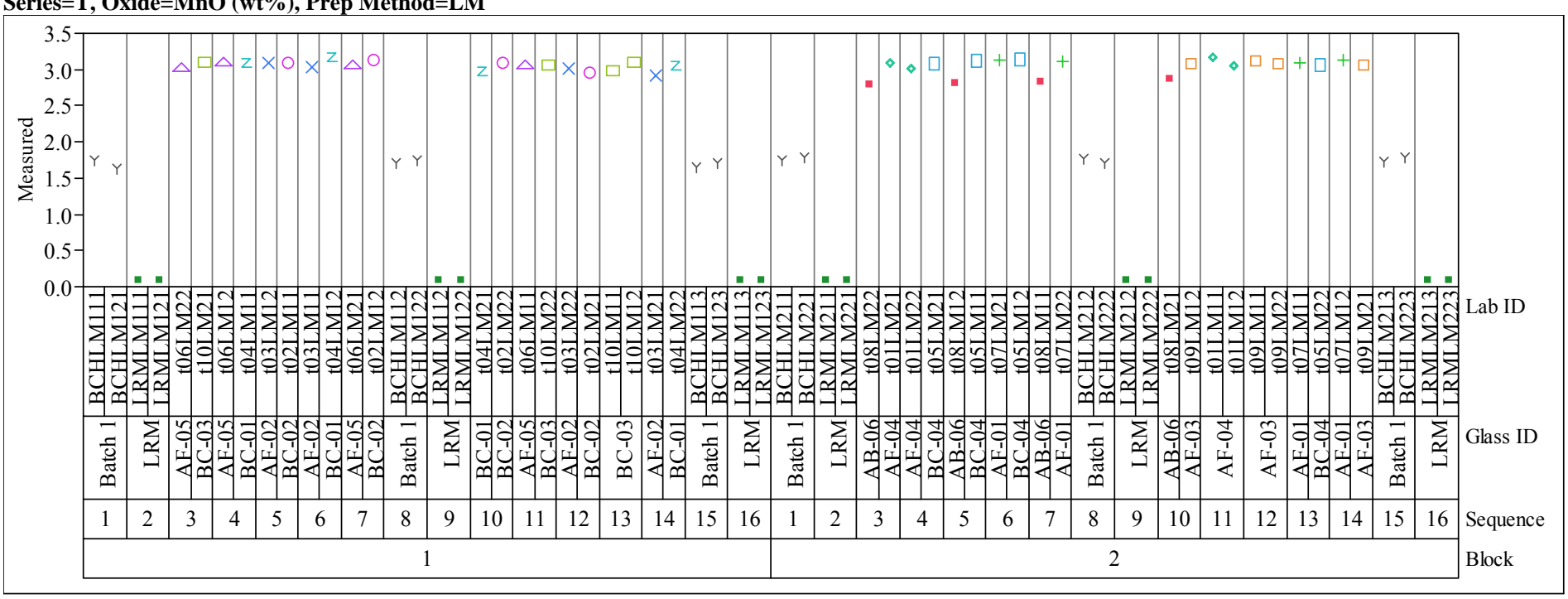

Series=T, Oxide=Na2O (wt\%), Prep Method $=$ LM

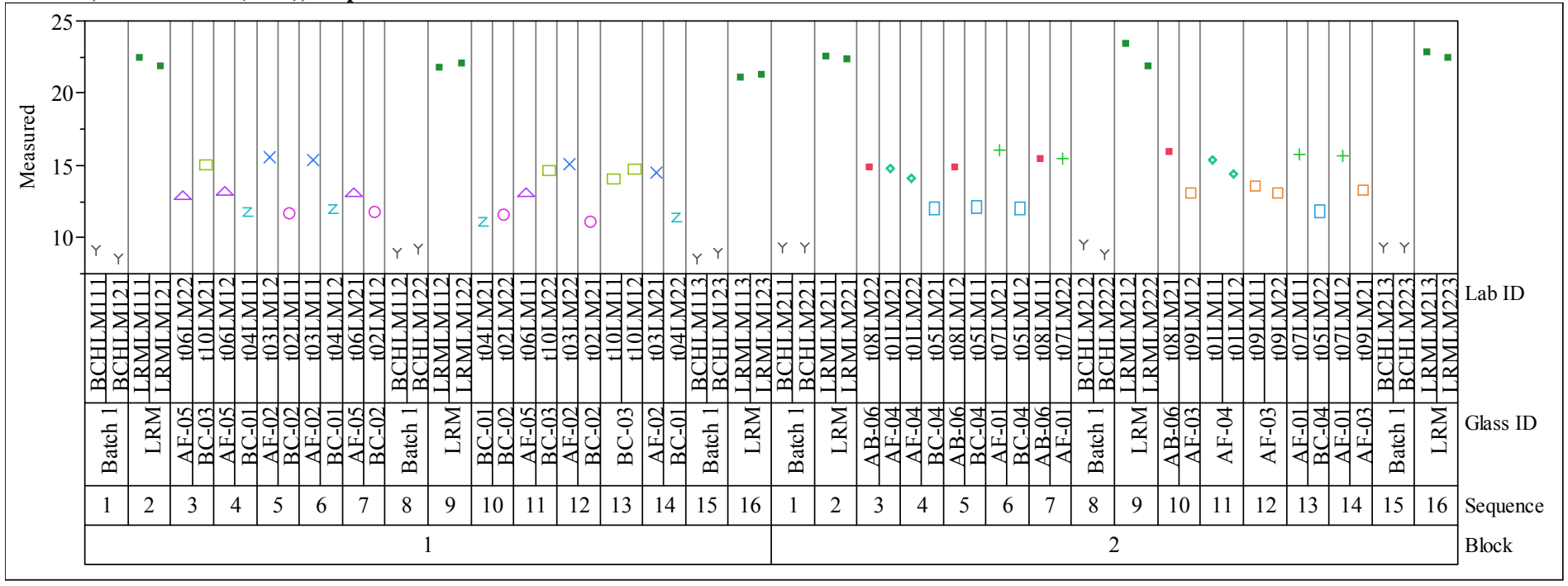




\section{Exhibit A-1. Measurements of Glasses in Analytical Sequence by Oxide within Preparation Method and by Analytical Series (continued)} Series $=\mathrm{T}$, Oxide $=\mathrm{NiO}(\mathrm{wt} \%)$, Prep Method $=\mathrm{LM}$

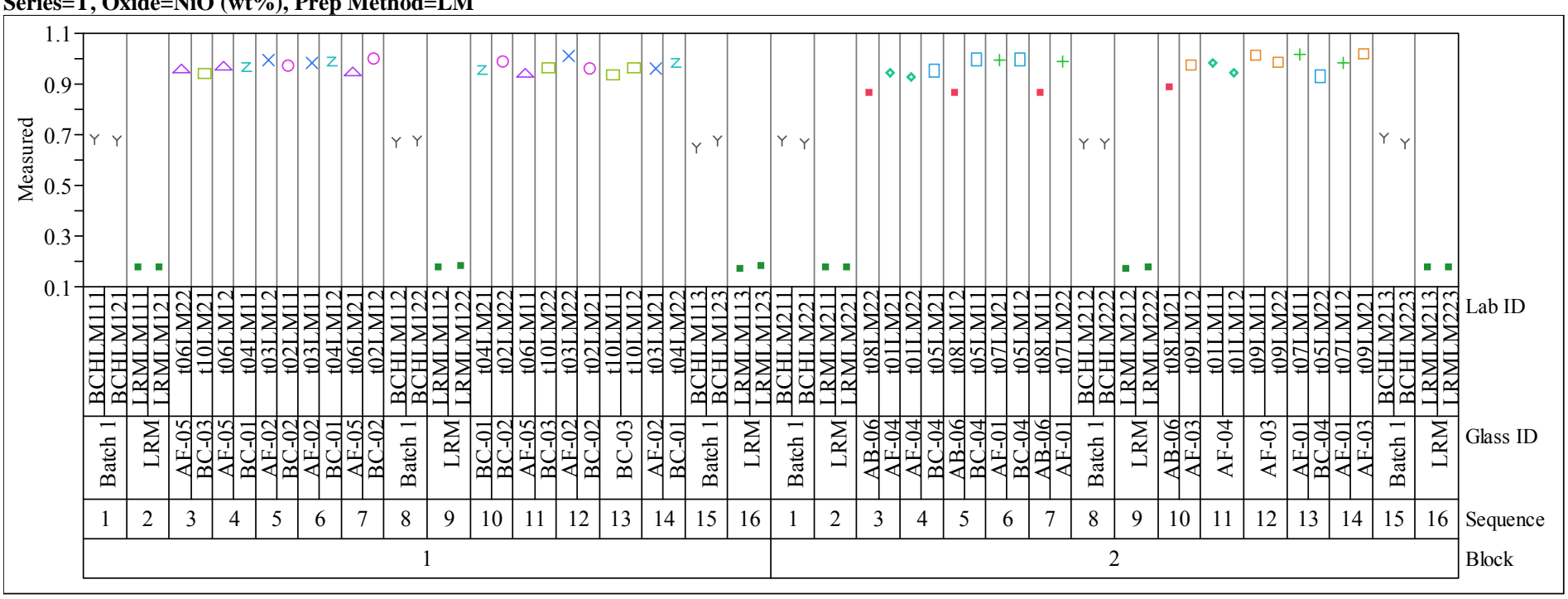

Series=T, Oxide=PbO (wt\%), Prep Method=LM

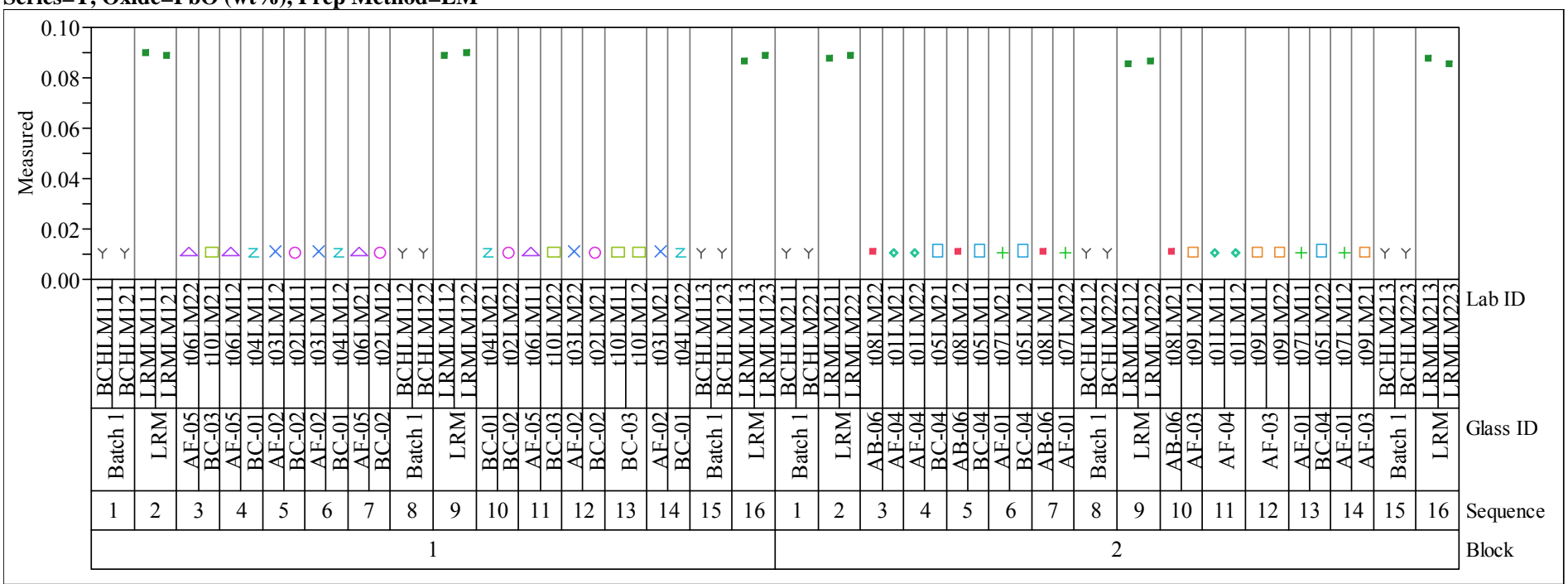




\section{Exhibit A-1. Measurements of Glasses in Analytical Sequence by Oxide within Preparation Method and by Analytical Series (continued)} Series $=$ T, Oxide=SiO2 (wt \%), Prep Method=PF

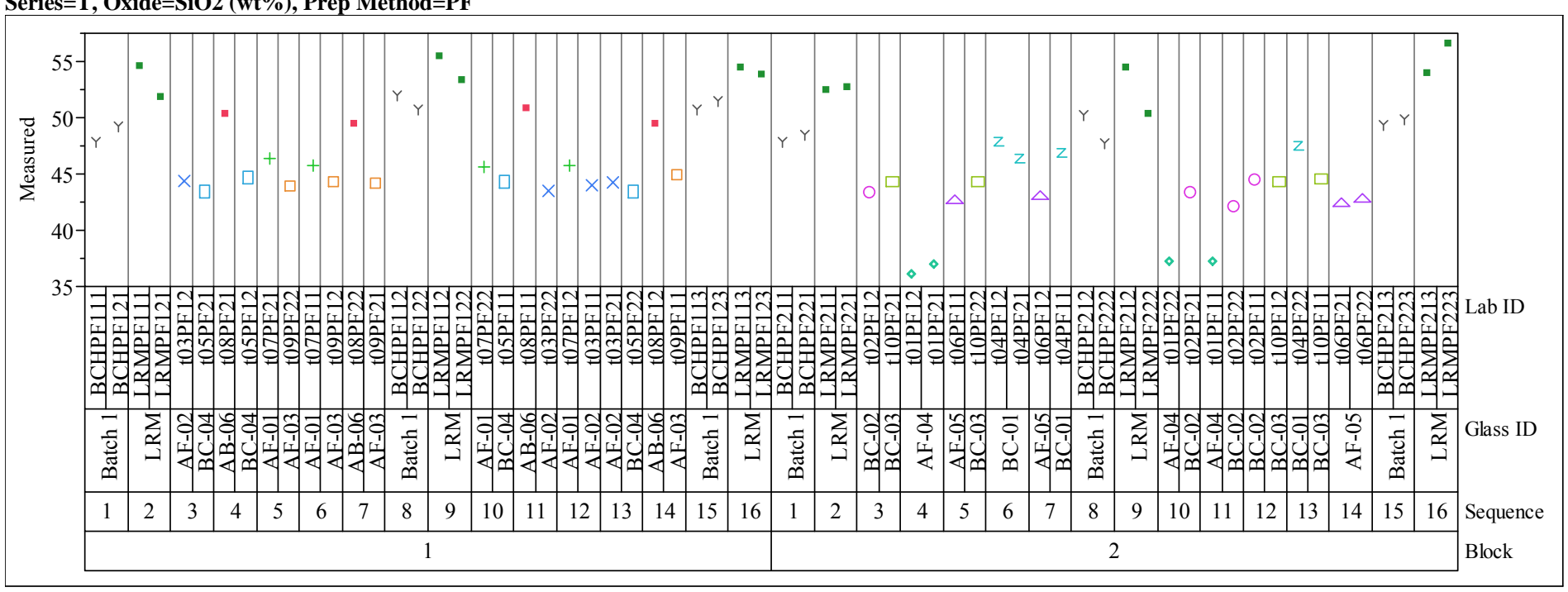

Series=T, Oxide=SO4 (wt\%), Prep Method=LM

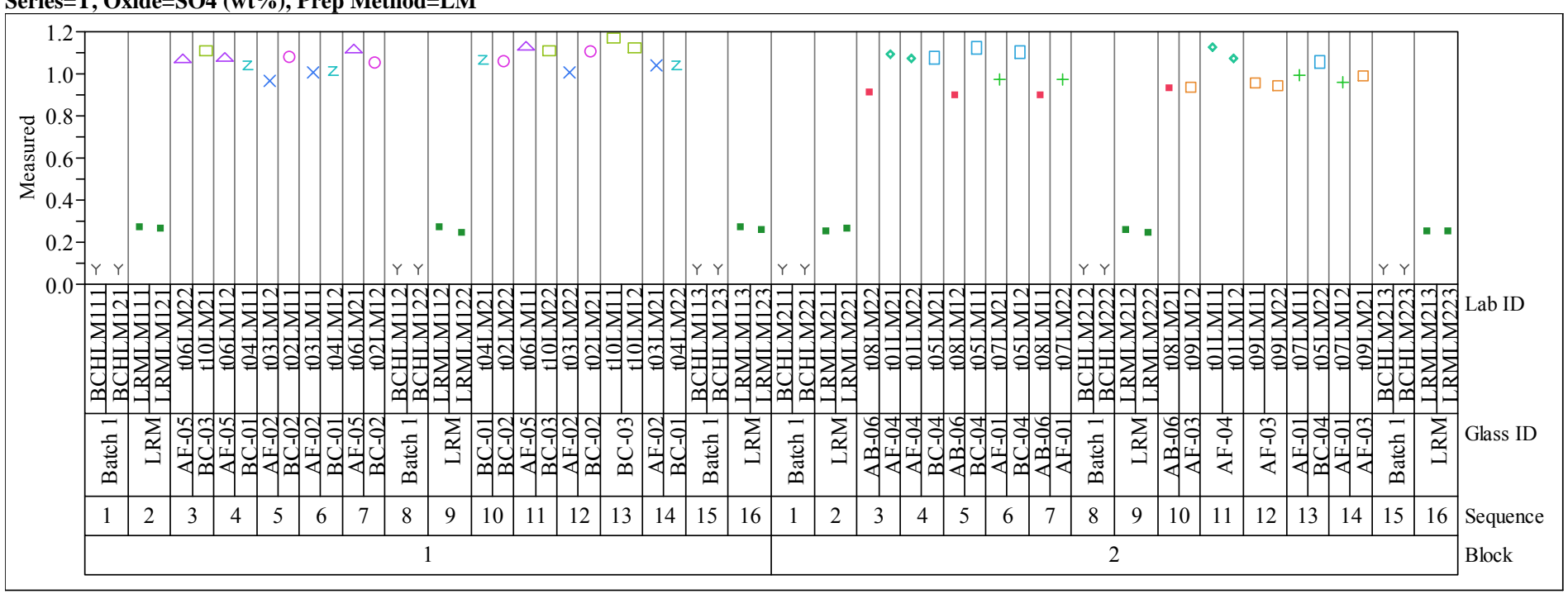




\section{Exhibit A-1. Measurements of Glasses in Analytical Sequence by Oxide within Preparation Method and by Analytical Series (continued)}

Series $=$ T, Oxide $=$ TiO2 $(w t \%)$, Prep Method $=$ LM

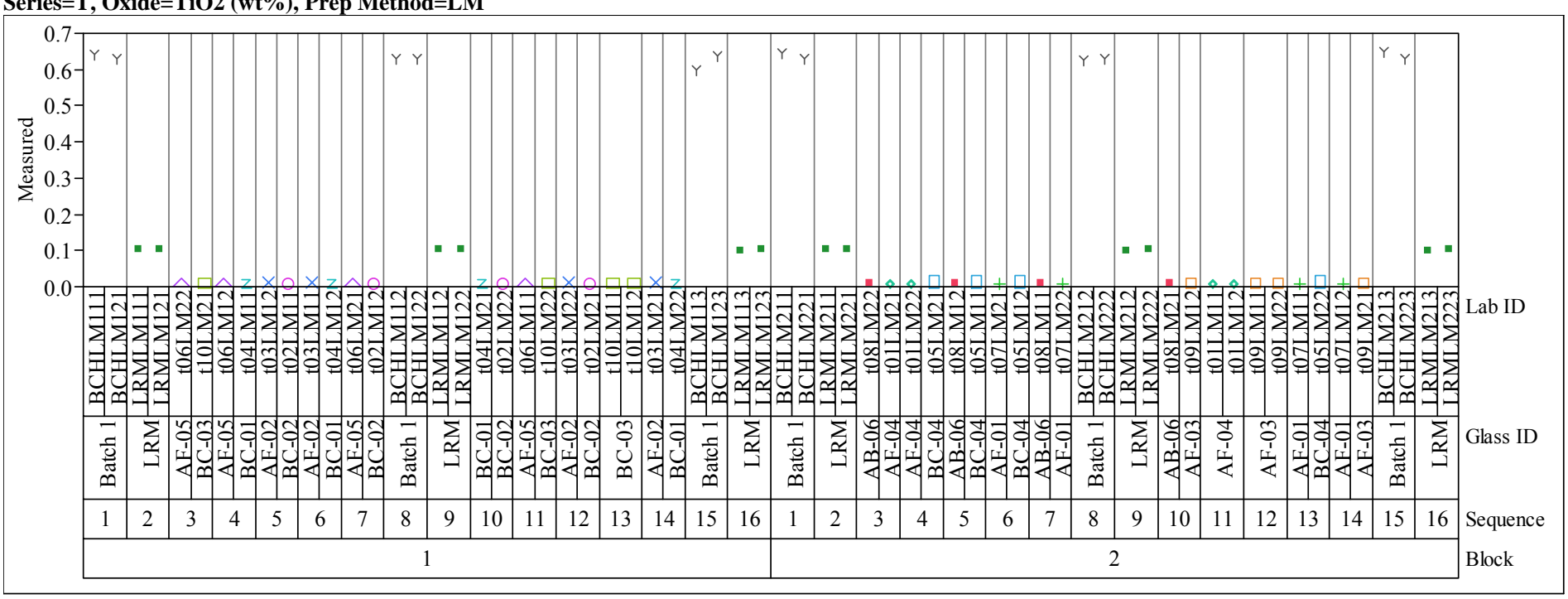

Series=T, Oxide=ZnO (wt\%), Prep Method=LM

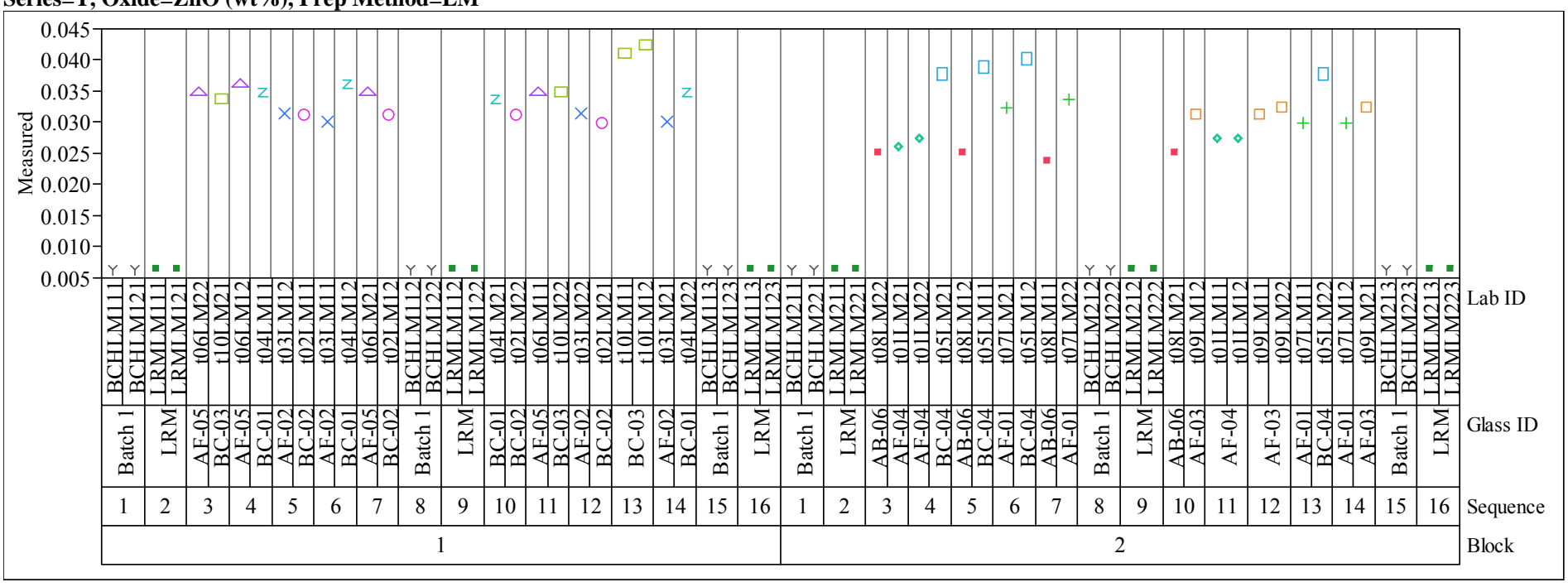




\section{Exhibit A-1. Measurements of Glasses in Analytical Sequence by Oxide within Preparation Method and by Analytical Series (continued)} Series $=\mathrm{T}$, Oxide $=\mathrm{ZrO} 2(\mathrm{wt} \%)$, Prep Method $=\mathbf{L M}$

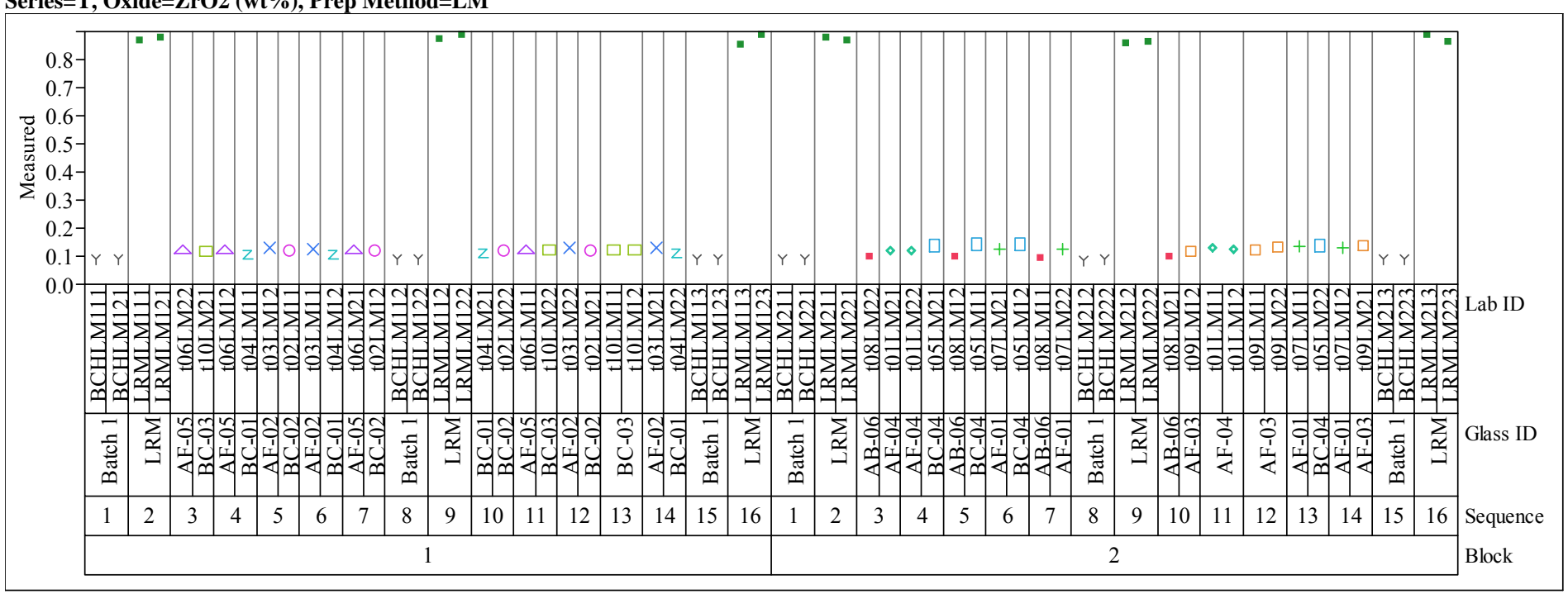

Series=U, Oxide=Al2O3 (wt\%), Prep Method=LM

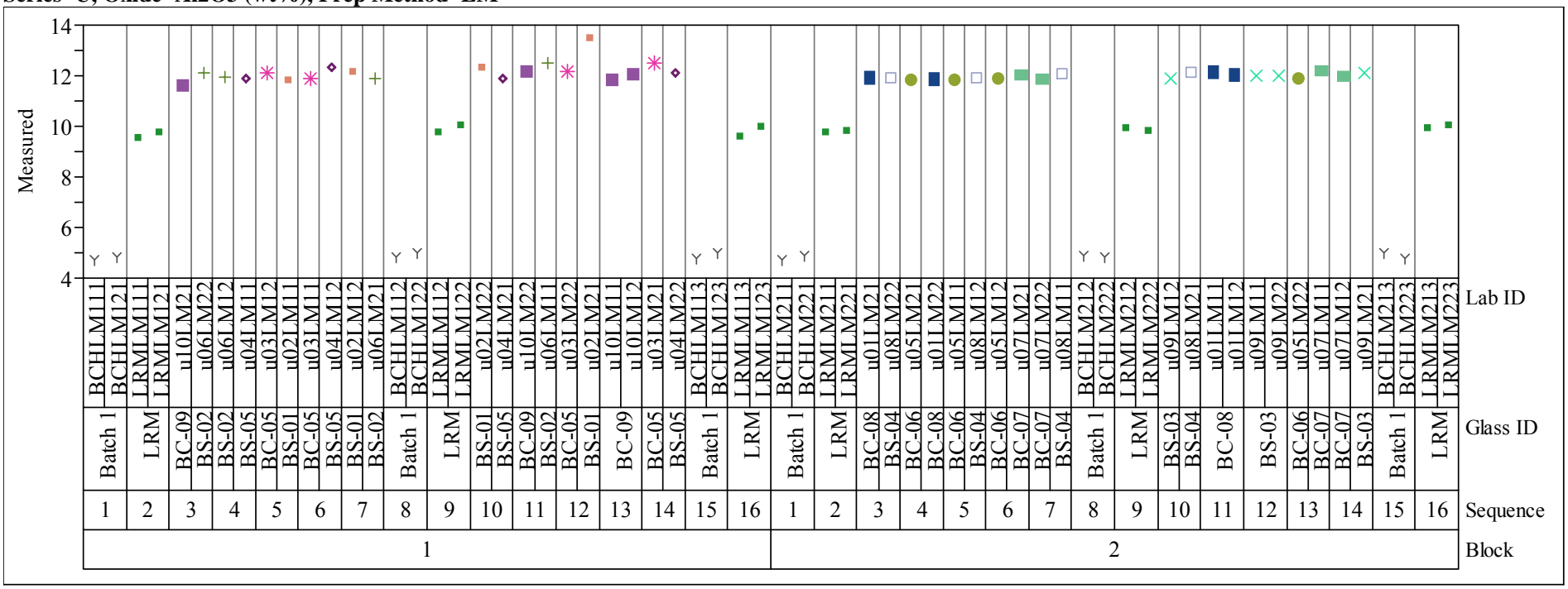




\section{Exhibit A-1. Measurements of Glasses in Analytical Sequence by Oxide within Preparation Method and by Analytical Series (continued)} Series $=\mathrm{U}$, Oxide $=\mathrm{B} 2 \mathrm{O} 3$ ( $\mathrm{wt} \%)$, Prep Method $=\mathrm{PF}$
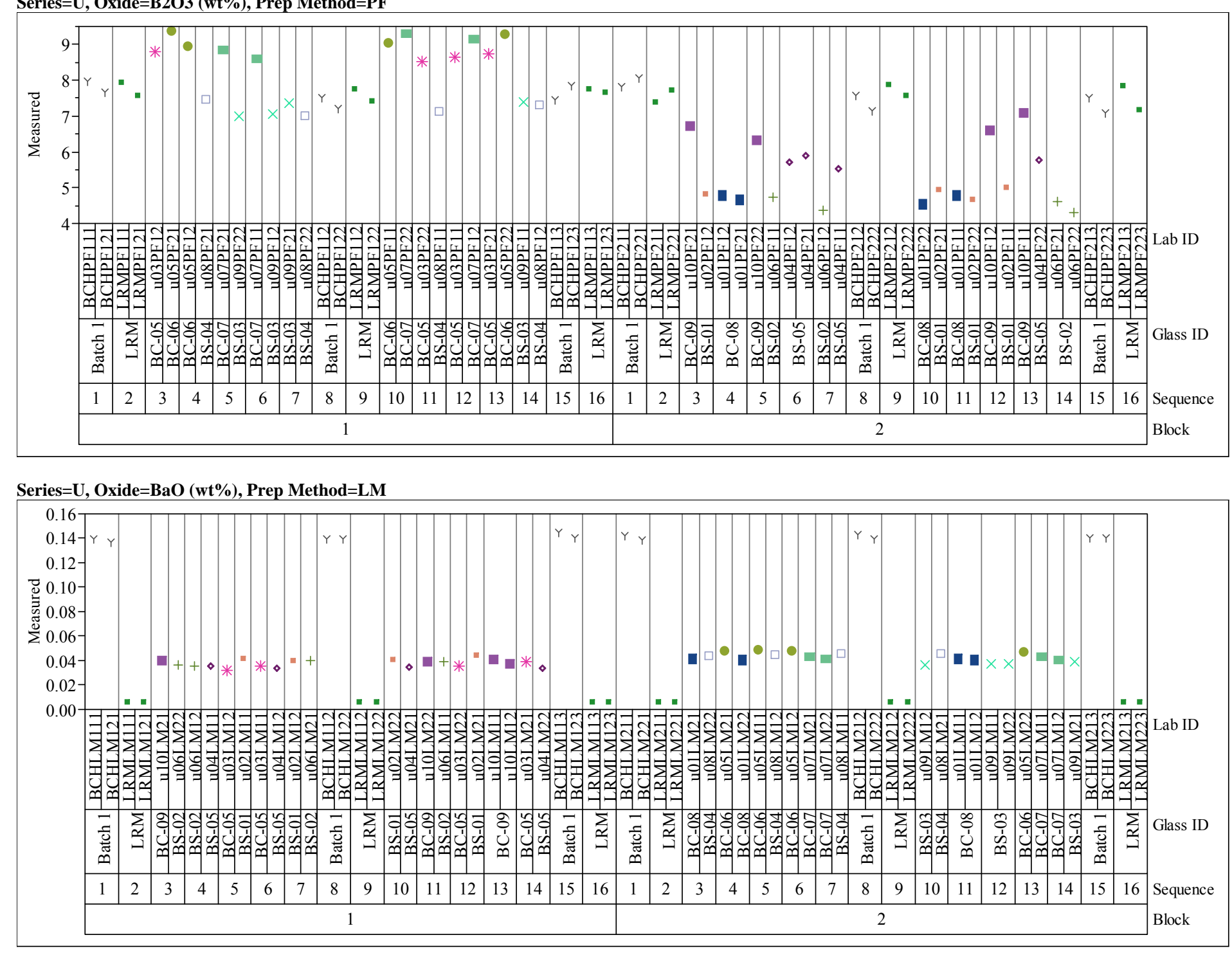
Exhibit A-1. Measurements of Glasses in Analytical Sequence by Oxide within Preparation Method and by Analytical Series (continued) Series $=\mathrm{U}$, Oxide $=\mathrm{CaO}(\mathrm{wt} \%)$, Prep Method $=\mathrm{LM}$

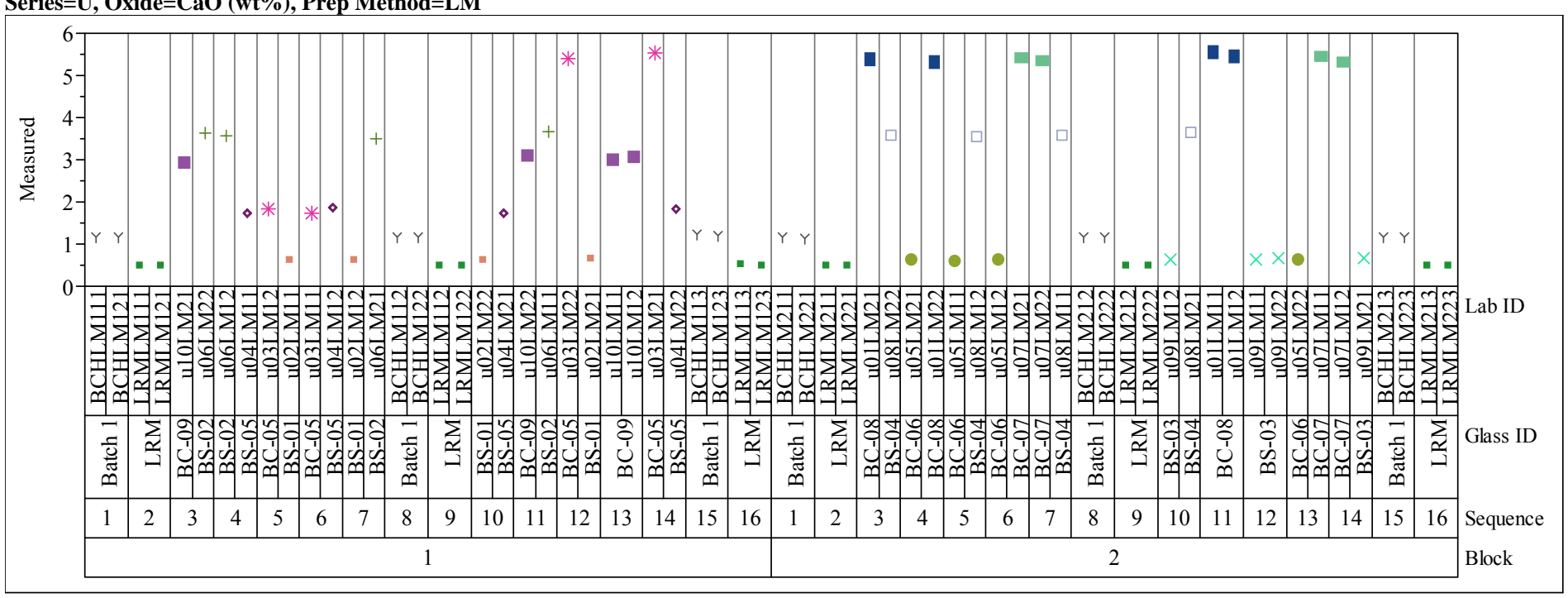

Series=U, Oxide=Ce2O3 (wt\%), Prep Method=LM

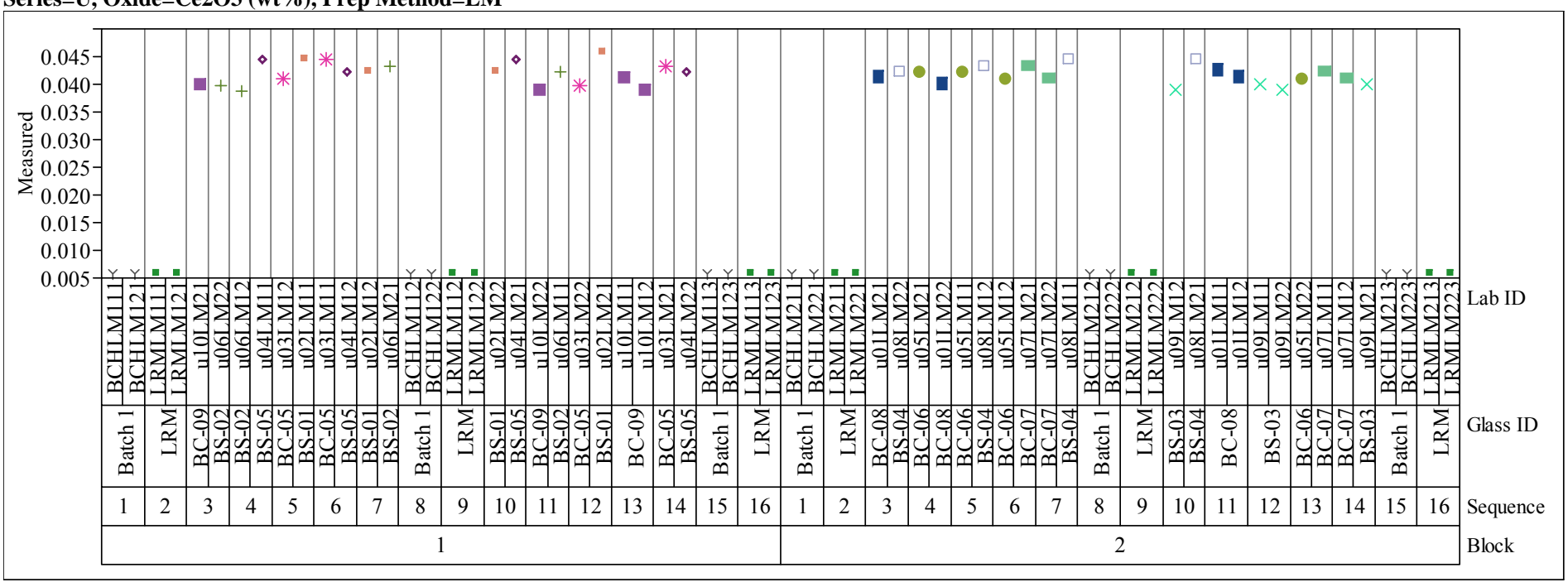


Exhibit A-1. Measurements of Glasses in Analytical Sequence by Oxide within Preparation Method and by Analytical Series (continued) Series $=\mathrm{U}$, Oxide $=\mathrm{Cr} 2 \mathrm{O} 3(\mathrm{wt} \%)$, Prep Method $=\mathrm{LM}$

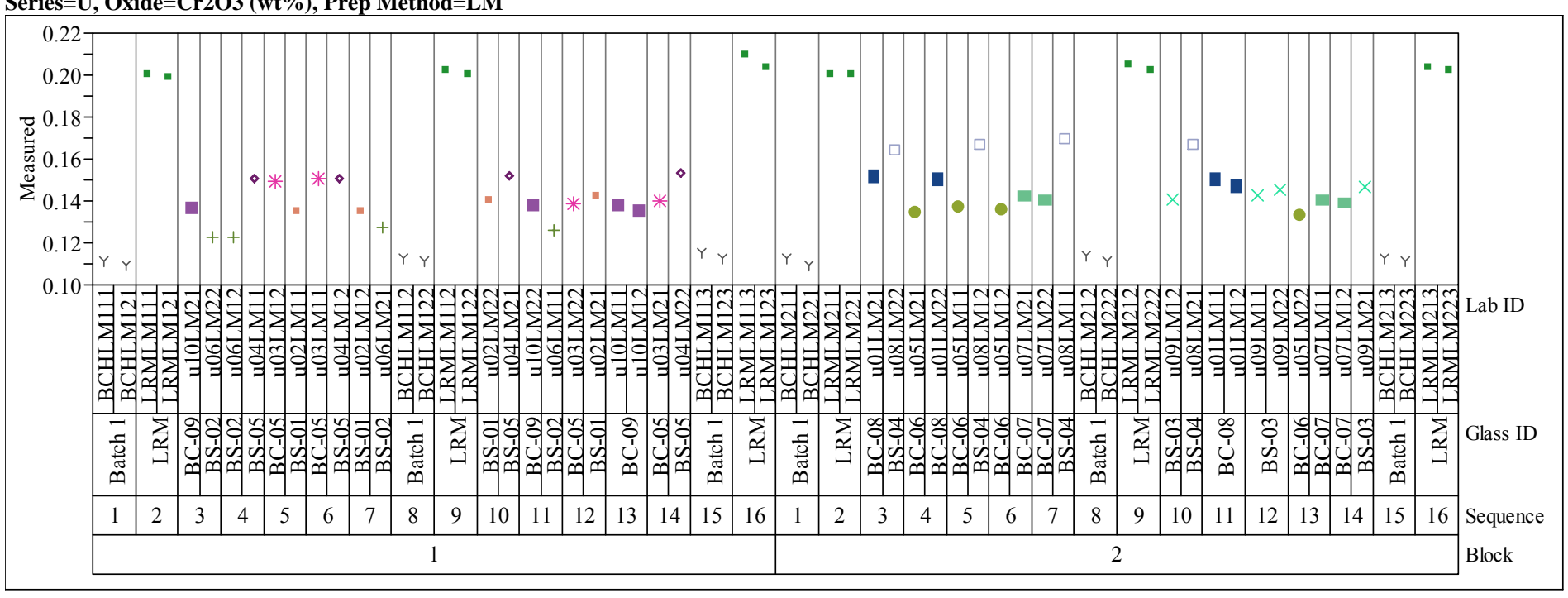

Series $=\mathrm{U}$, Oxide $=\mathrm{CuO}(\mathrm{wt} \%)$, Prep Method $=\mathrm{LM}$

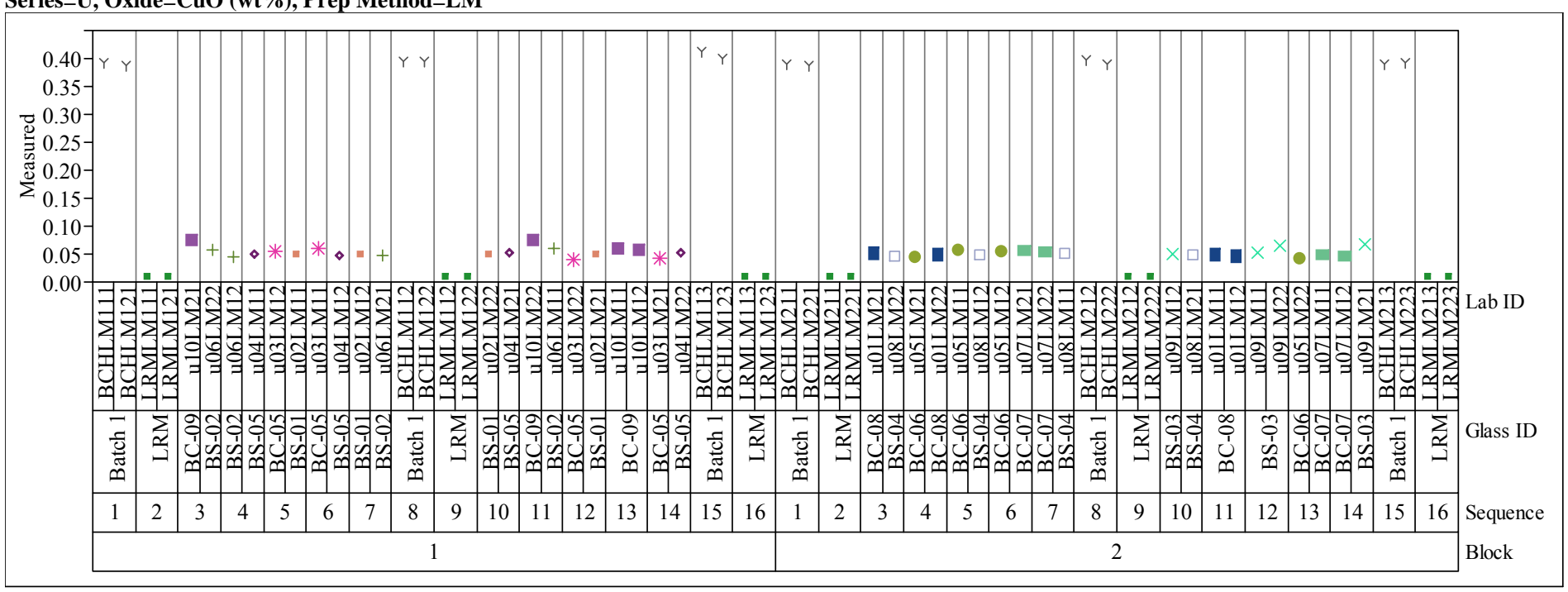




\section{Exhibit A-1. Measurements of Glasses in Analytical Sequence by Oxide within Preparation Method and by Analytical Series (continued)} Series=U, Oxide=Fe2O3 (wt\%), Prep Method $=$ LM

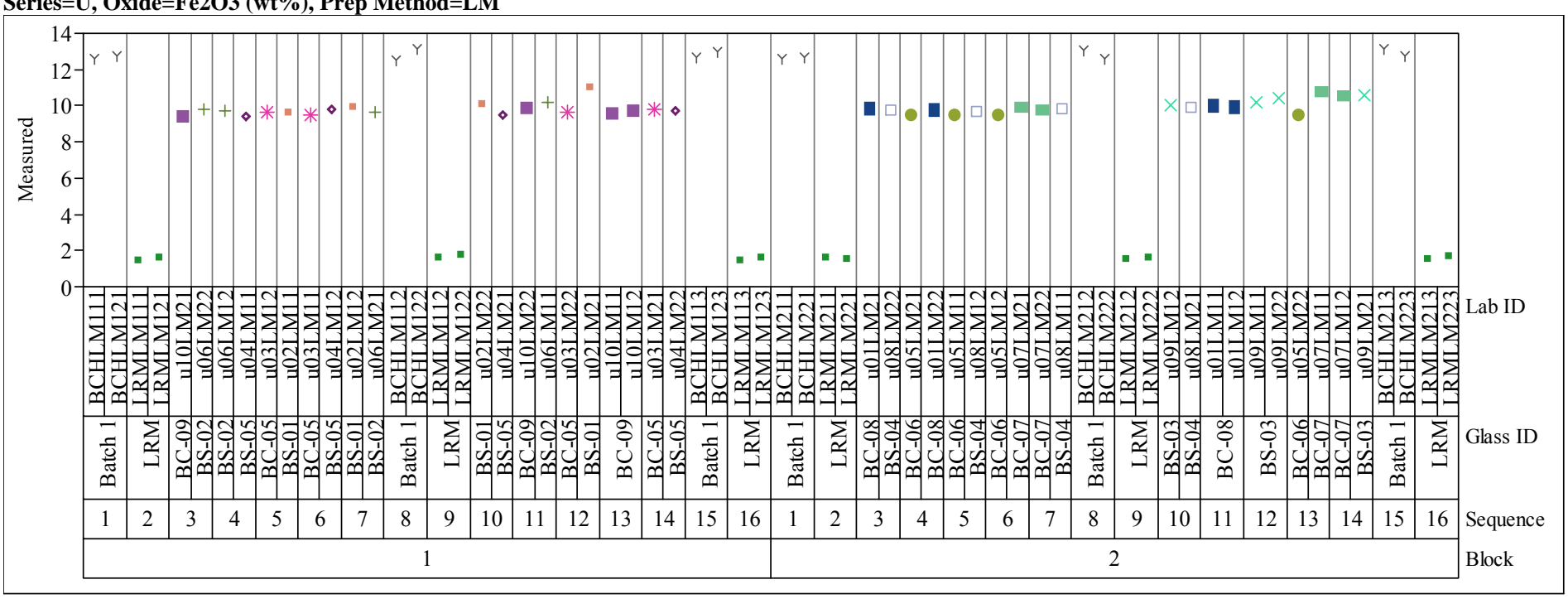

Series=U, Oxide=K2O (wt \%), Prep Method=LM

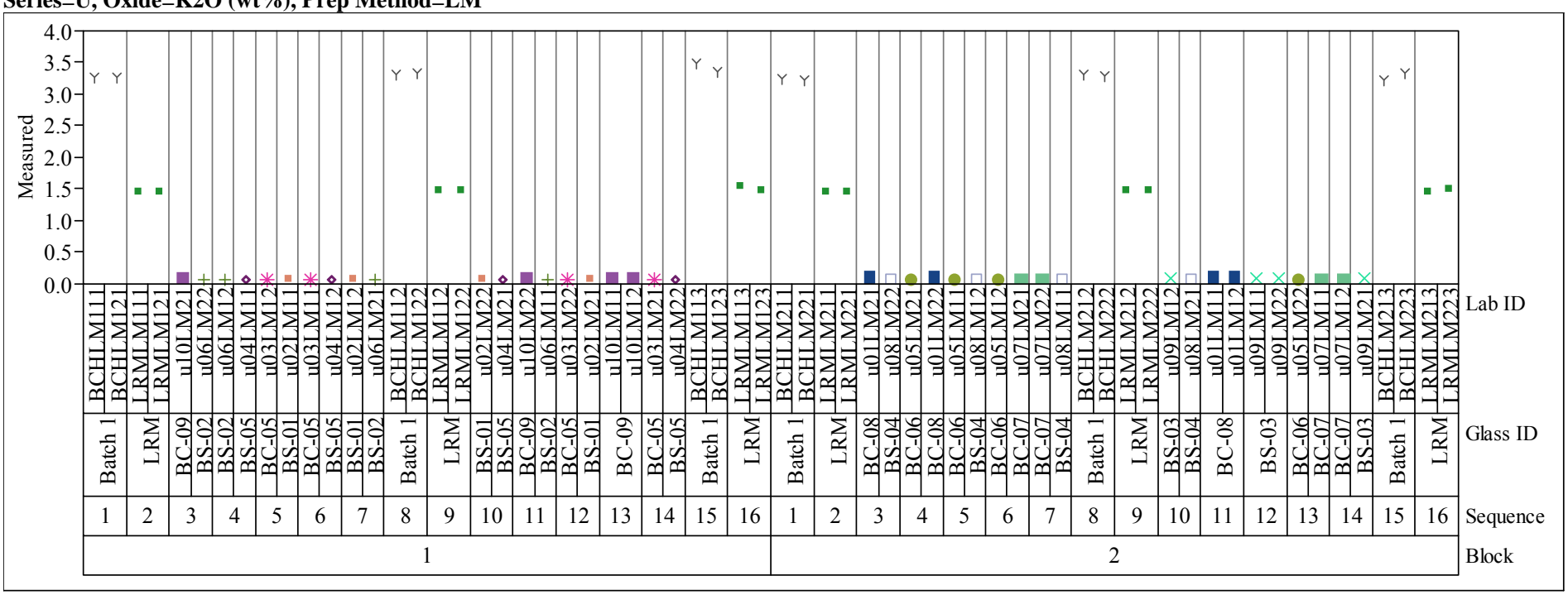


Exhibit A-1. Measurements of Glasses in Analytical Sequence by Oxide within Preparation Method and by Analytical Series (continued)

Series $=\mathrm{U}$, OXide $=\mathrm{La} 2 \mathrm{O} 3$ ( $\mathrm{wt} \%$ ), Prep Method $=\mathrm{LM}$

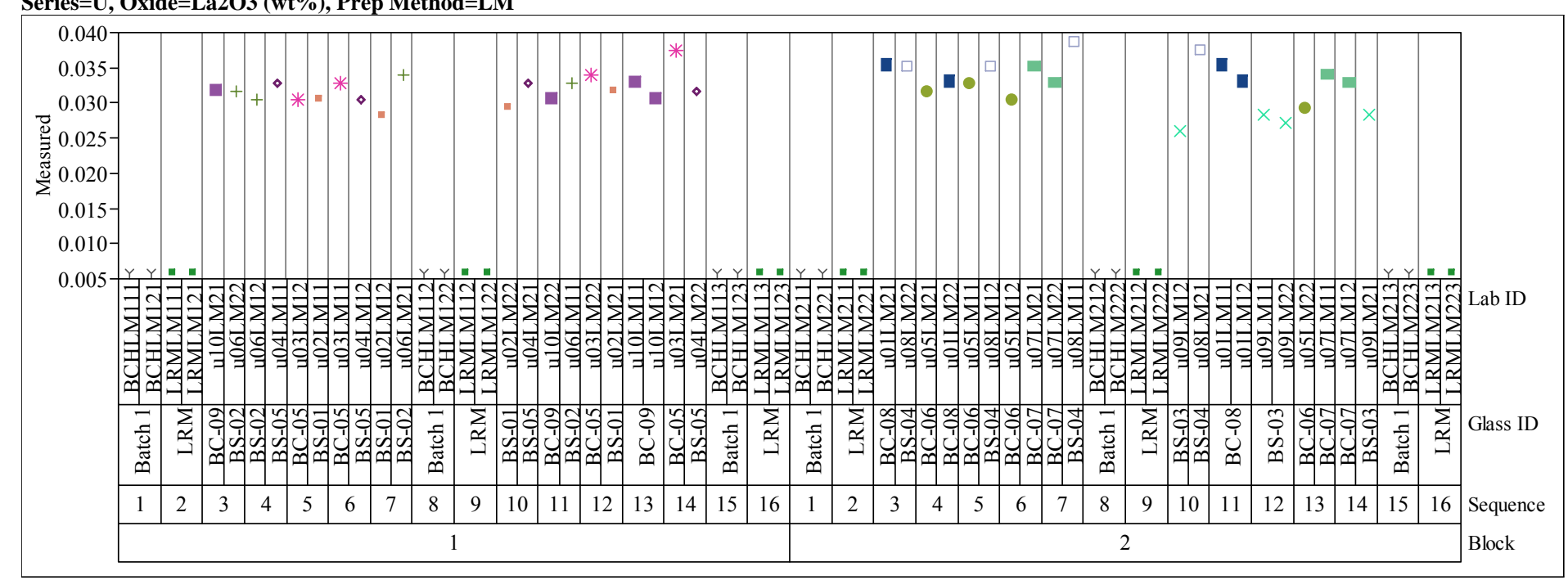

Series $=U$, Oxide=Li2O (wt \%), Prep Method $=$ PF

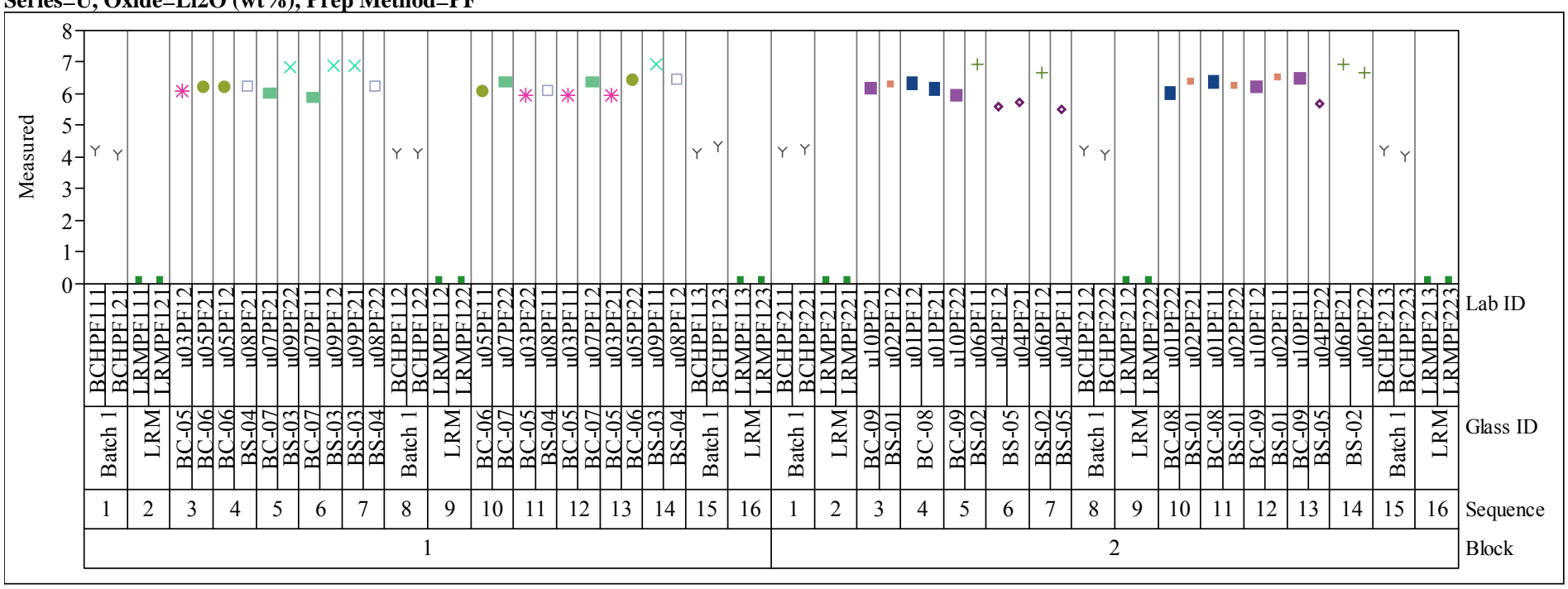




\section{Exhibit A-1. Measurements of Glasses in Analytical Sequence by Oxide within Preparation Method and by Analytical Series (continued)} Series $=\mathrm{U}$, Oxide $=\mathrm{MgO}(\mathrm{wt} \%)$, Prep Method $=\mathrm{LM}$

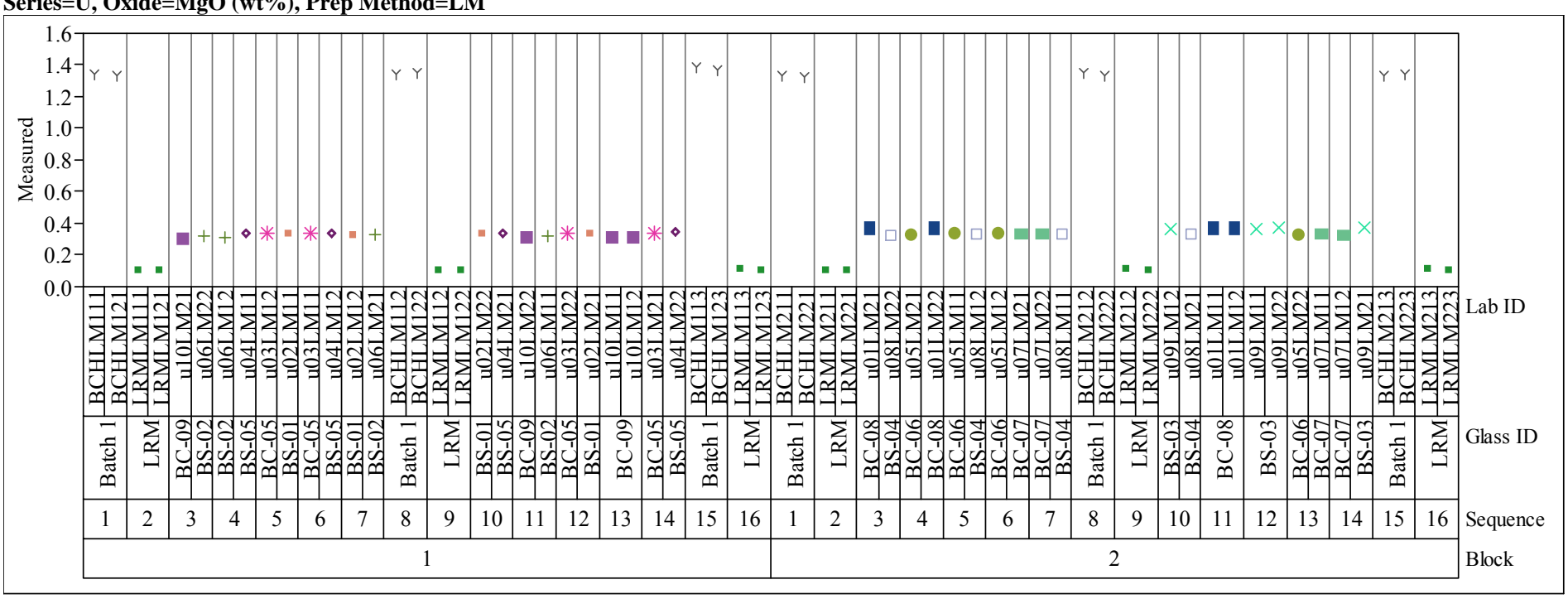

Series=U, Oxide=MnO (wt\%), Prep Method=LM

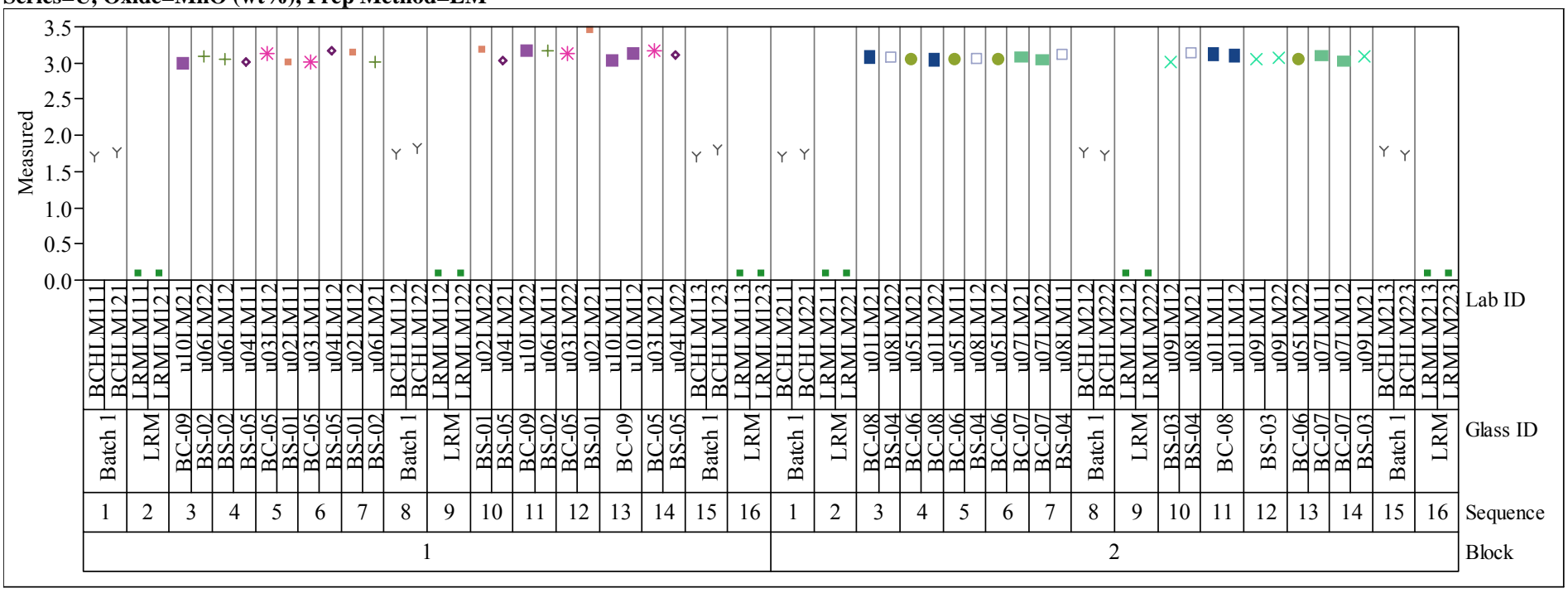




\section{Exhibit A-1. Measurements of Glasses in Analytical Sequence by Oxide within Preparation Method and by Analytical Series (continued)} Series=U, Oxide=Na2O (wt\%), Prep Method=LM

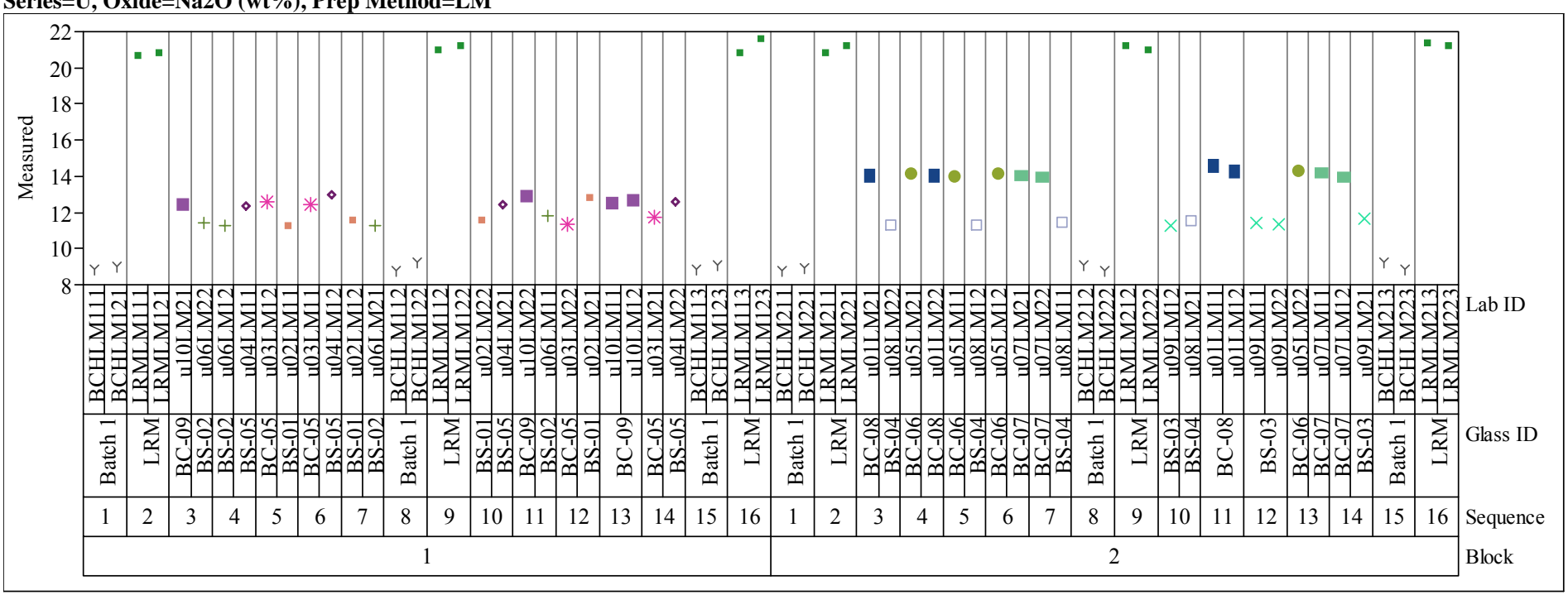

Series=U, Oxide=NiO (wt\%), Prep Method=LM

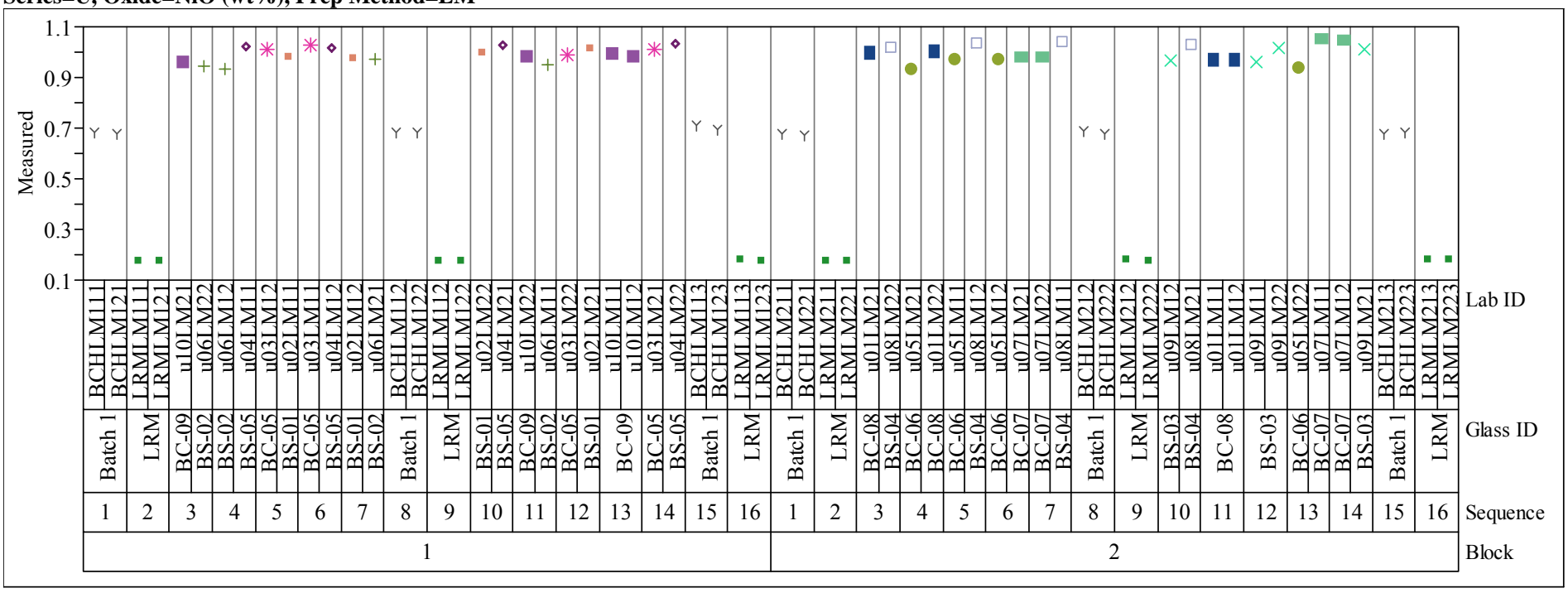




\section{Exhibit A-1. Measurements of Glasses in Analytical Sequence by Oxide within Preparation Method and by Analytical Series (continued)}

Series $=\mathrm{U}$, Oxide $=\mathbf{P b O}(\mathrm{wt} \%)$, Prep Method $=$ LM

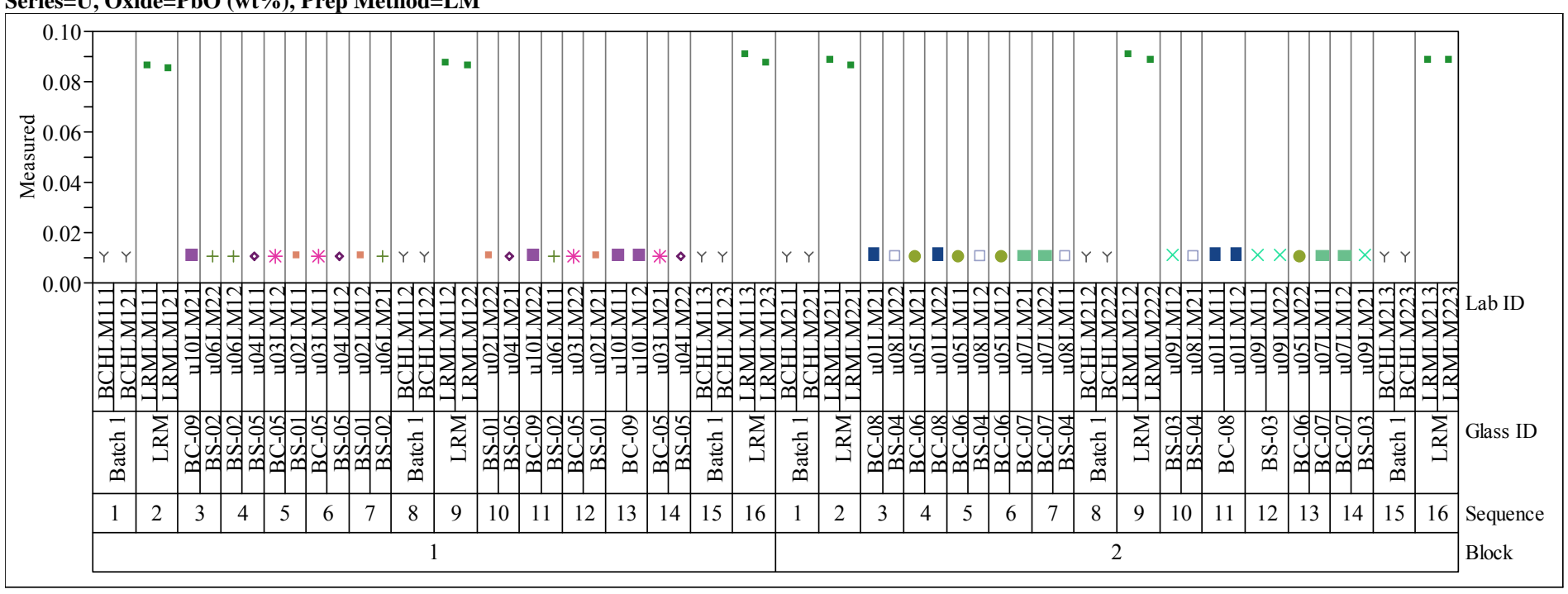

Series=U, Oxide=SiO2 (wt\%), Prep Method=PF

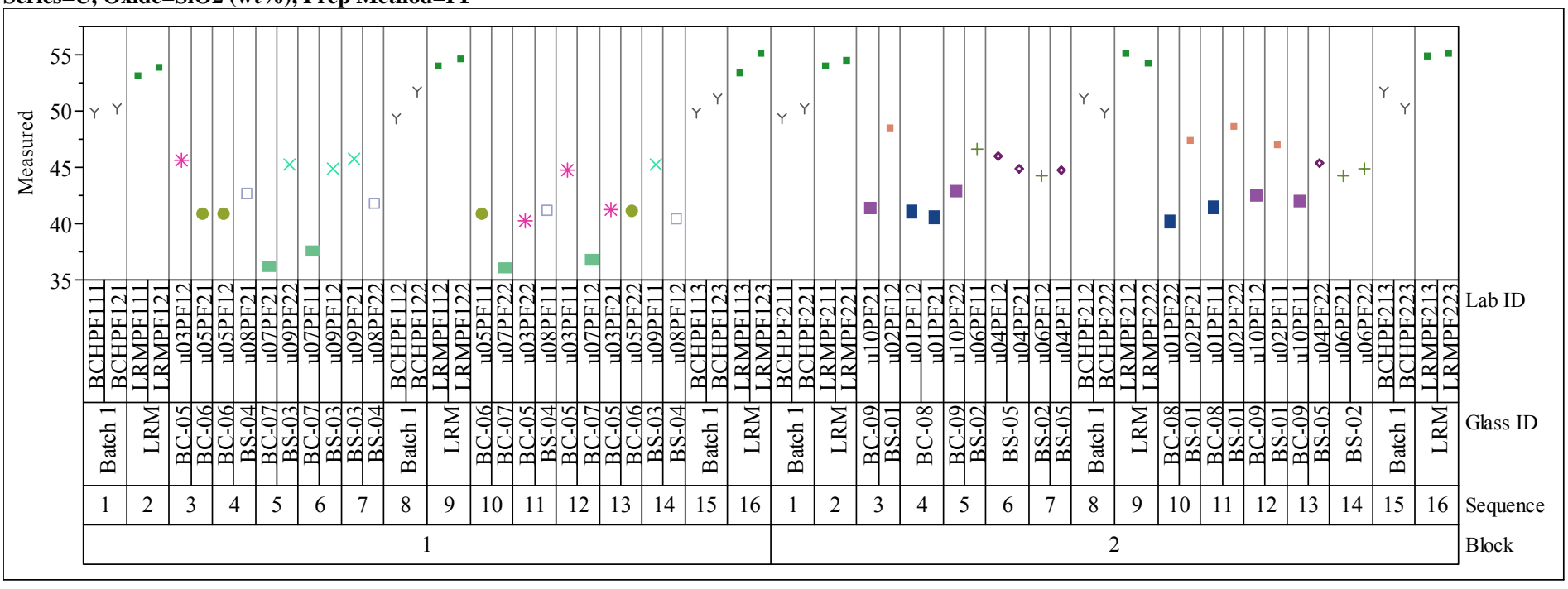




\section{Exhibit A-1. Measurements of Glasses in Analytical Sequence by Oxide within Preparation Method and by Analytical Series (continued)} Series=U, Oxide=SO4 (wt\%), Prep Method=LM

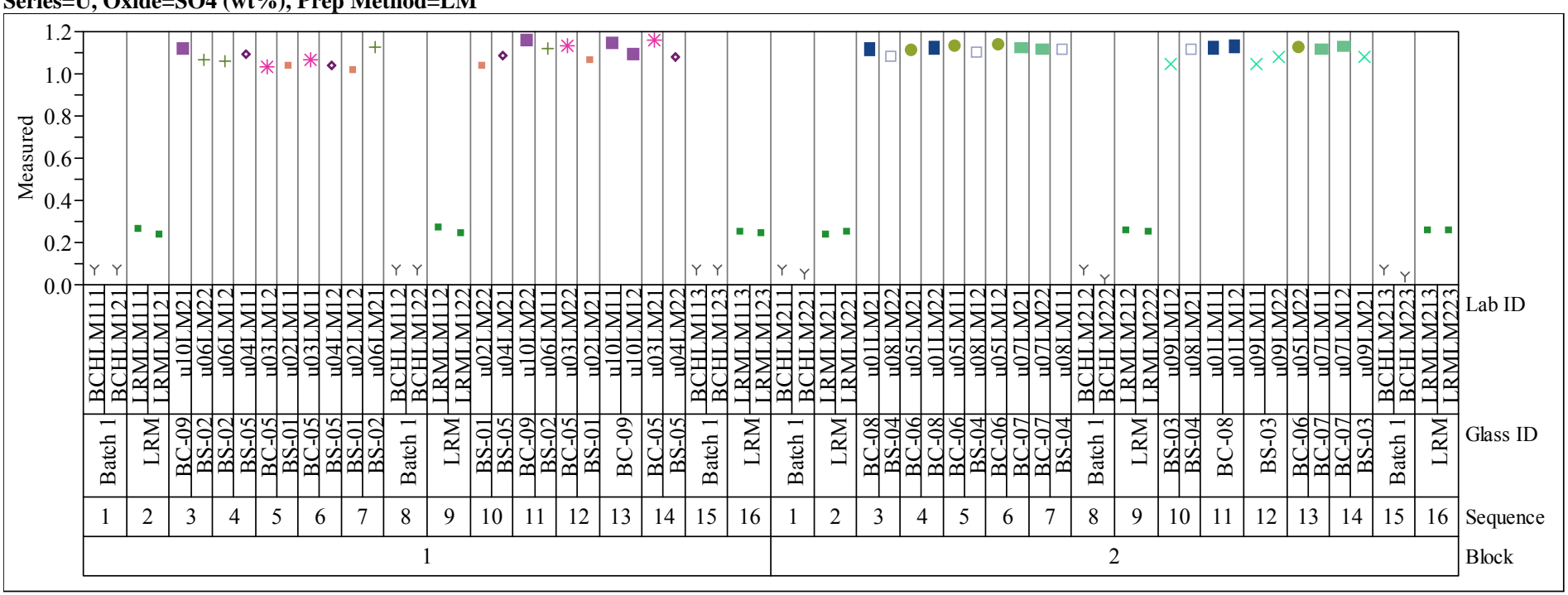

Series=U, Oxide=TiO2 (wt\%), Prep Method=LM

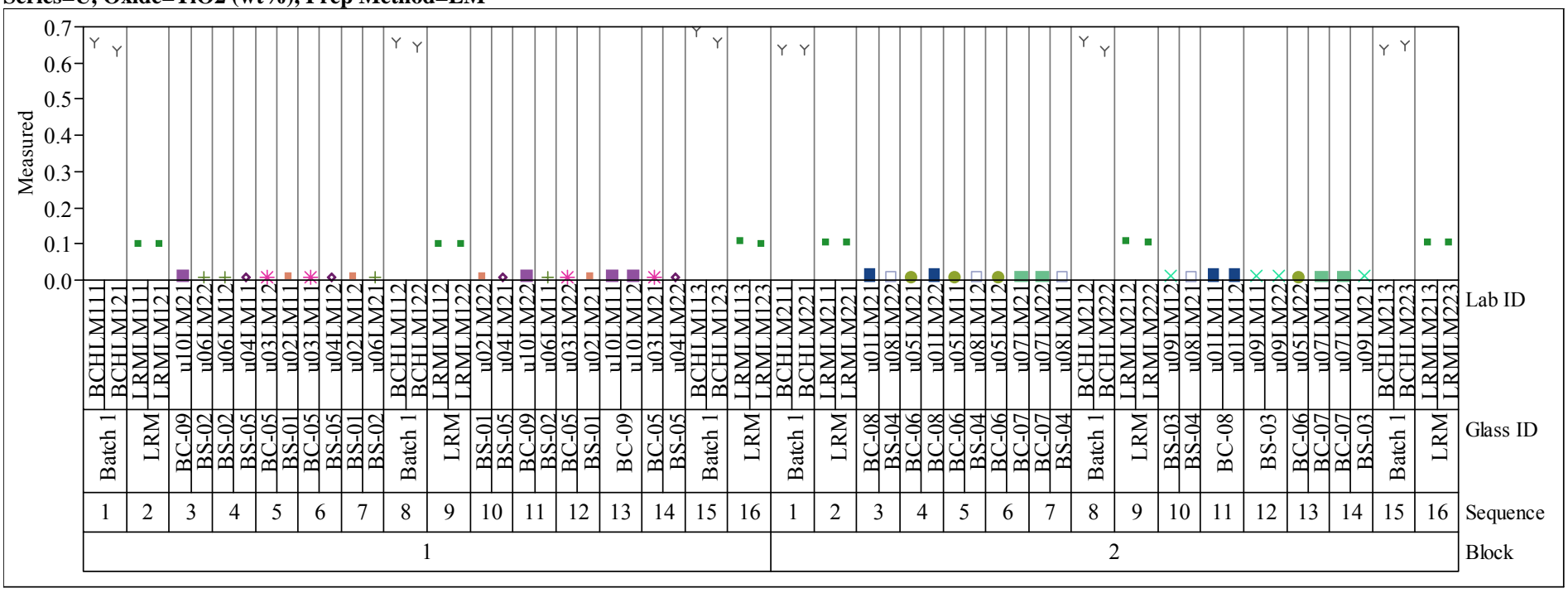


Exhibit A-1. Measurements of Glasses in Analytical Sequence by Oxide within Preparation Method and by Analytical Series (continued) Series $=\mathrm{U}$, Oxide $=\mathrm{ZnO}$ (wt\%), Prep Method $=\mathrm{LM}$

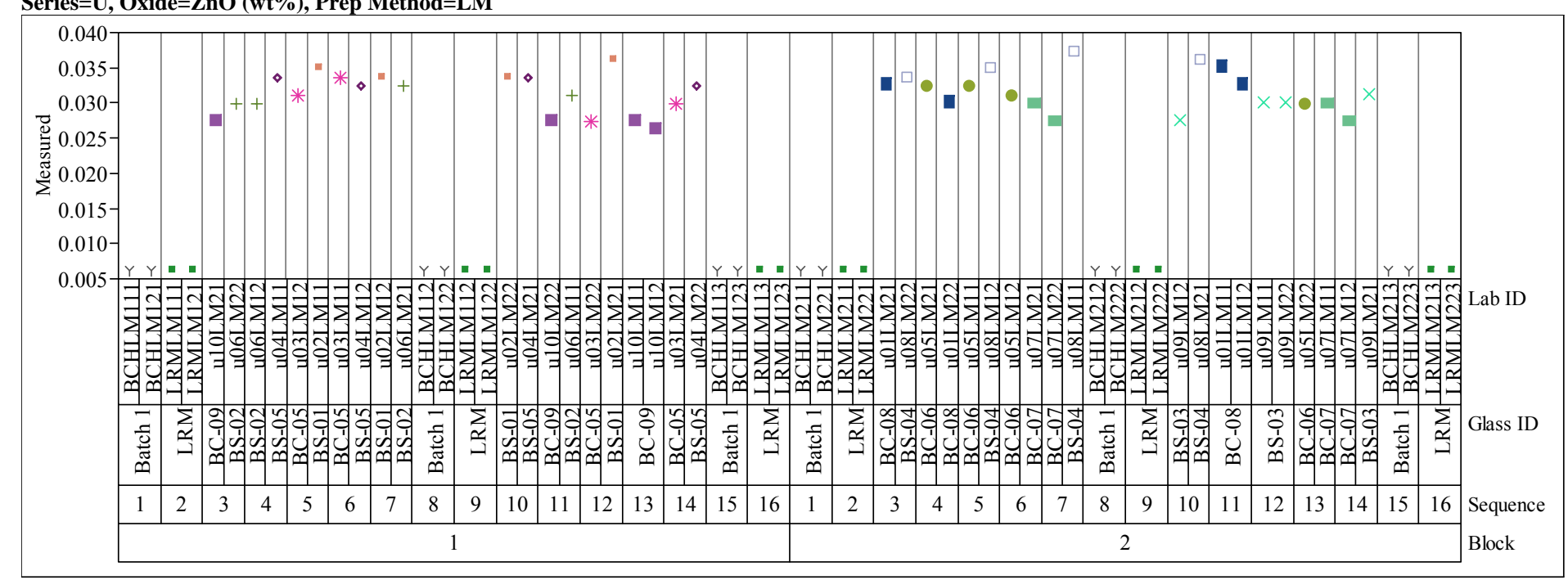

Series $=\mathrm{U}$, Oxide $=\mathrm{ZrO2}(\mathrm{wt} \%)$, Prep Method $=\mathrm{LM}$

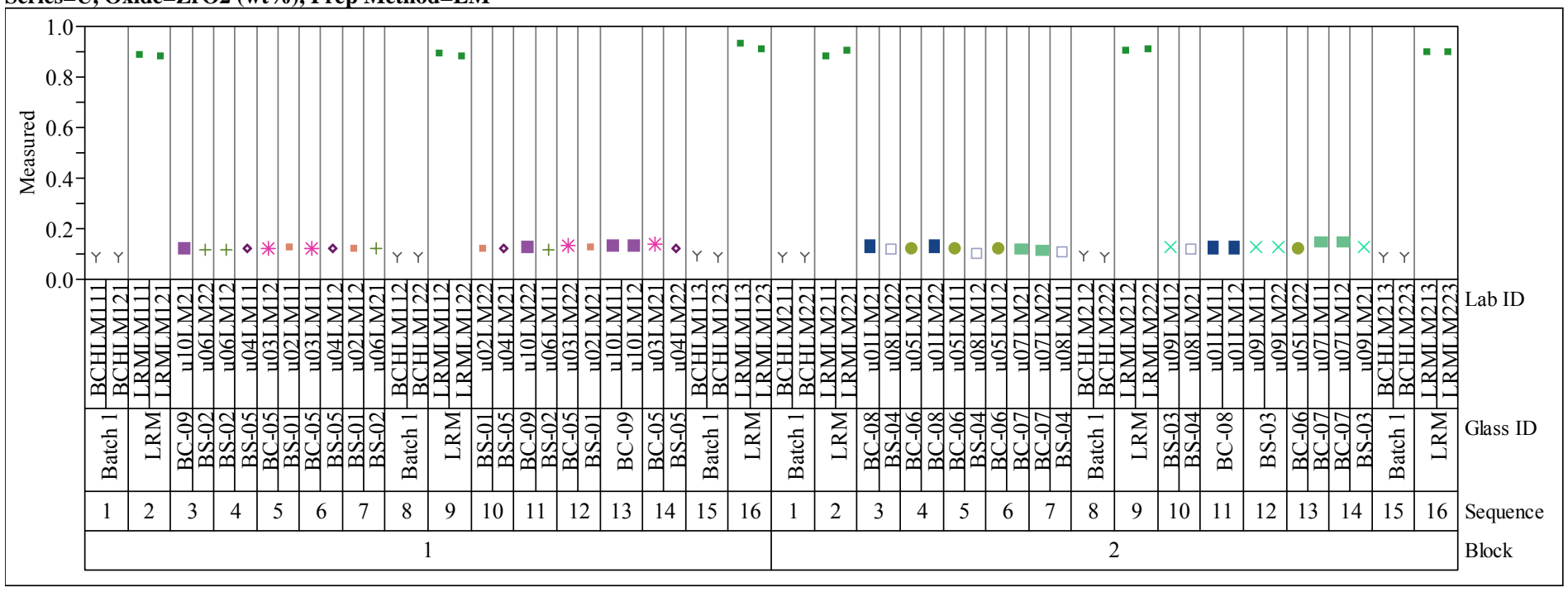


Exhibit A-1. Measurements of Glasses in Analytical Sequence by Oxide within Preparation Method and by Analytical Series (continued) Series $=V$, Oxide $=$ Al2O3 (wt\%), Prep Method $=$ LM

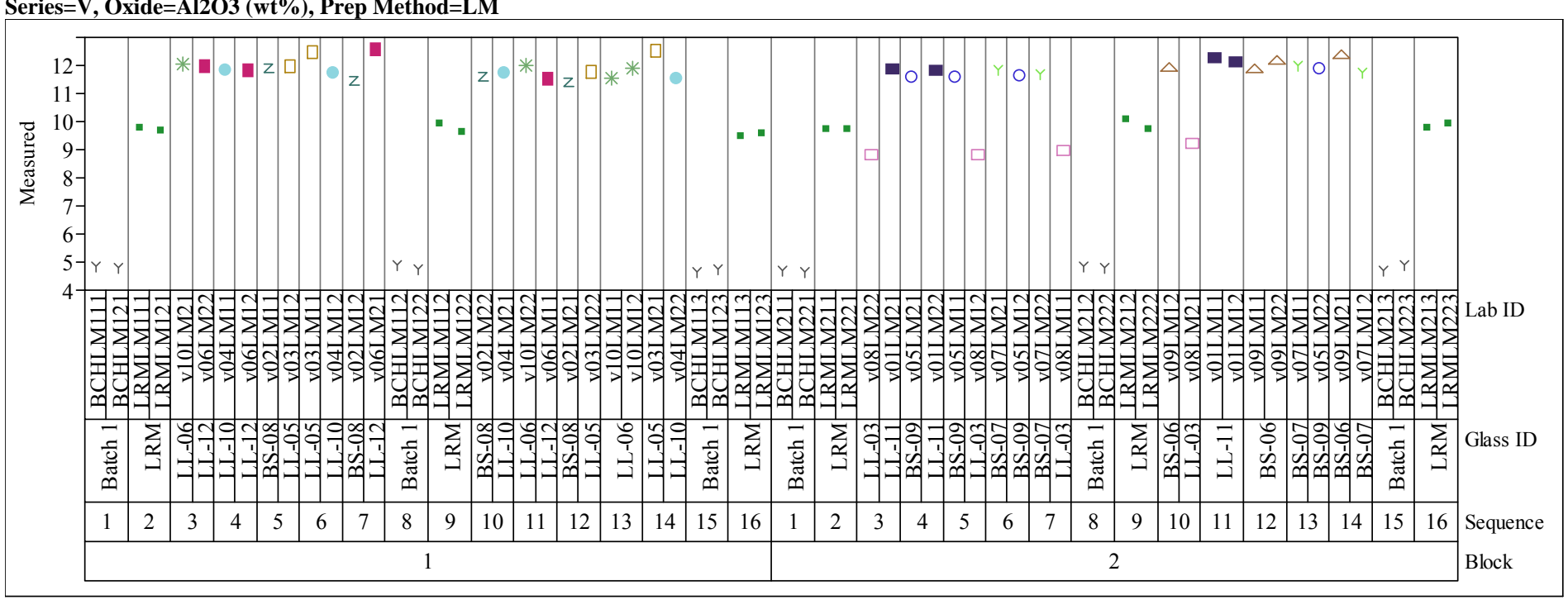

Series $=$ V, Oxide=B2O3 (wt\%), Prep Method=PF

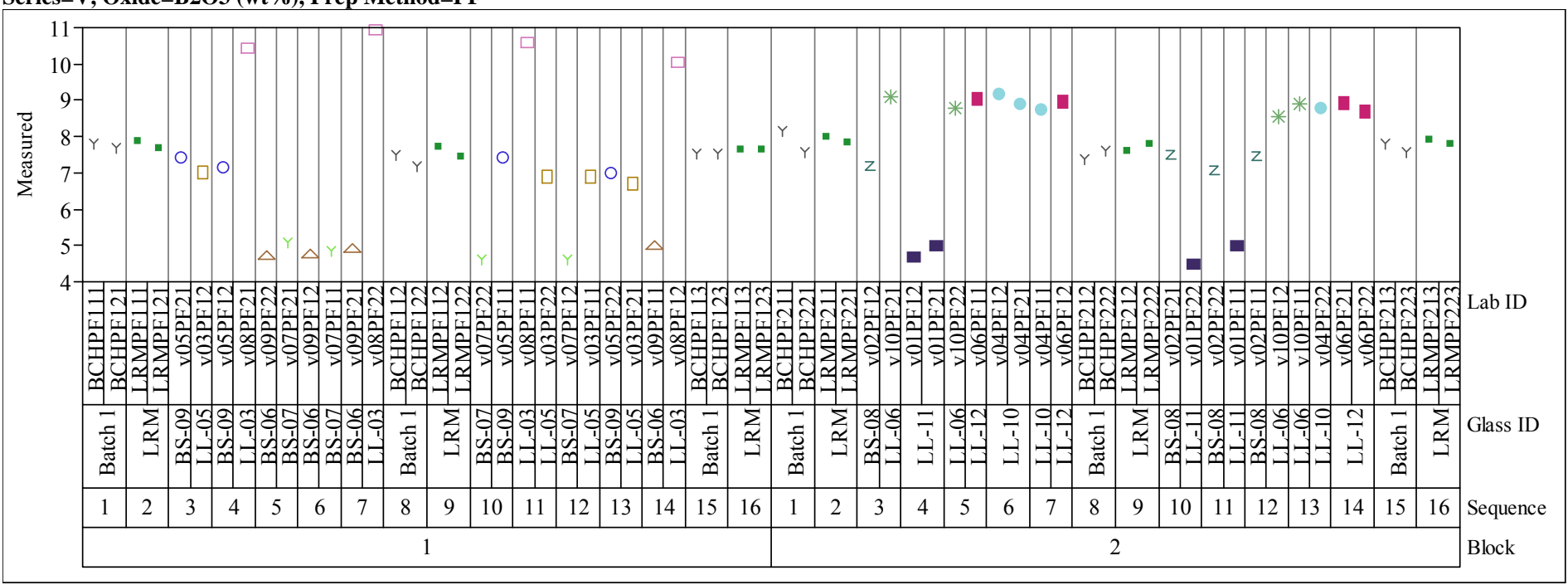




\section{Exhibit A-1. Measurements of Glasses in Analytical Sequence by Oxide within Preparation Method and by Analytical Series (continued)}

Series $=V$, Oxide $=$ BaO $(w t \%)$, Prep Method $=$ LM

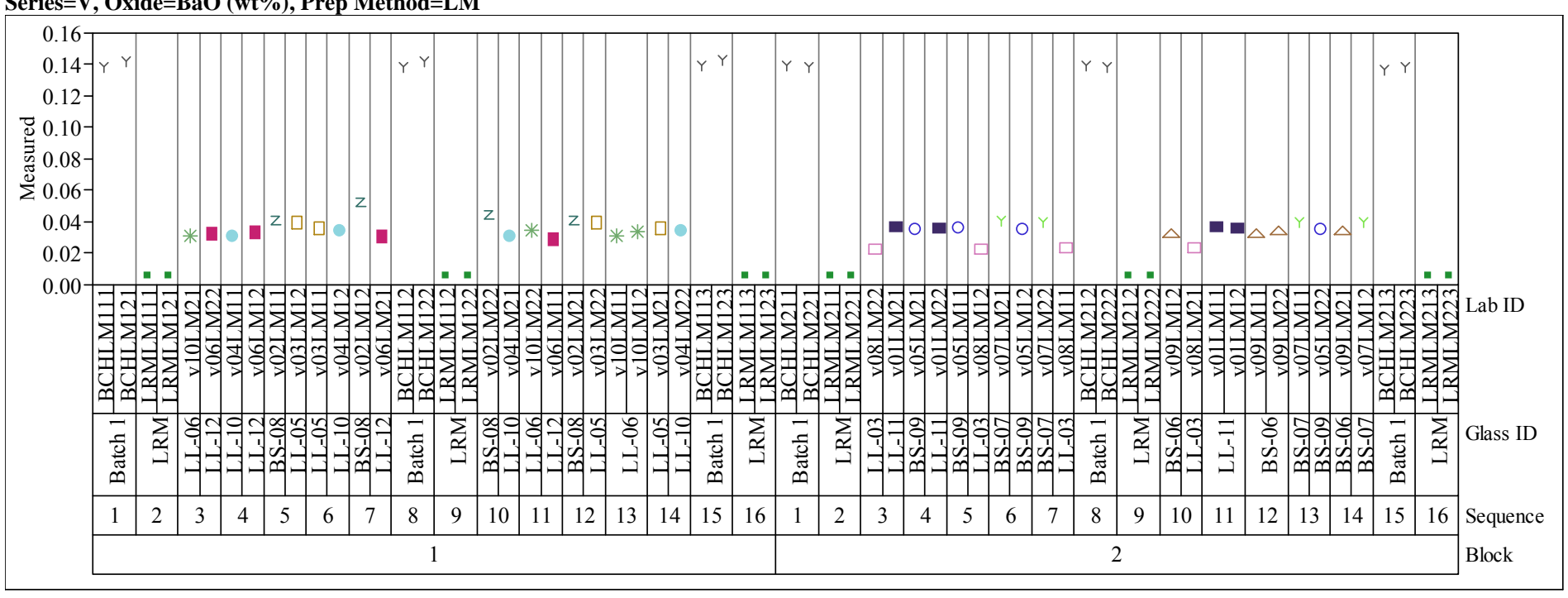

Series $=\mathrm{V}$, Oxide $=\mathrm{CaO}(\mathrm{wt} \%)$, Prep Method $=\mathrm{LM}$

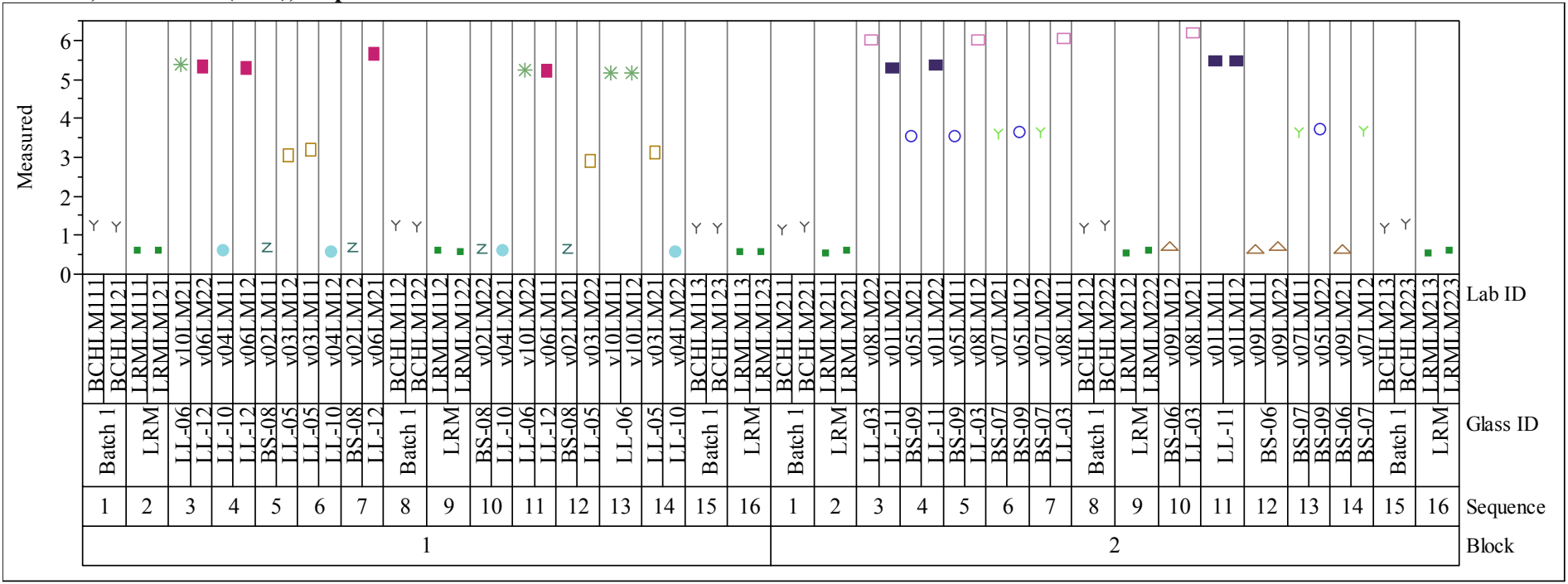




\section{Exhibit A-1. Measurements of Glasses in Analytical Sequence by Oxide within Preparation Method and by Analytical Series (continued)}

Series $=\mathrm{V}$, Oxide $=\mathrm{Ce} 2 \mathrm{O} 3$ ( $w \mathrm{t} \%$ ), Prep Method $=\mathrm{LM}$

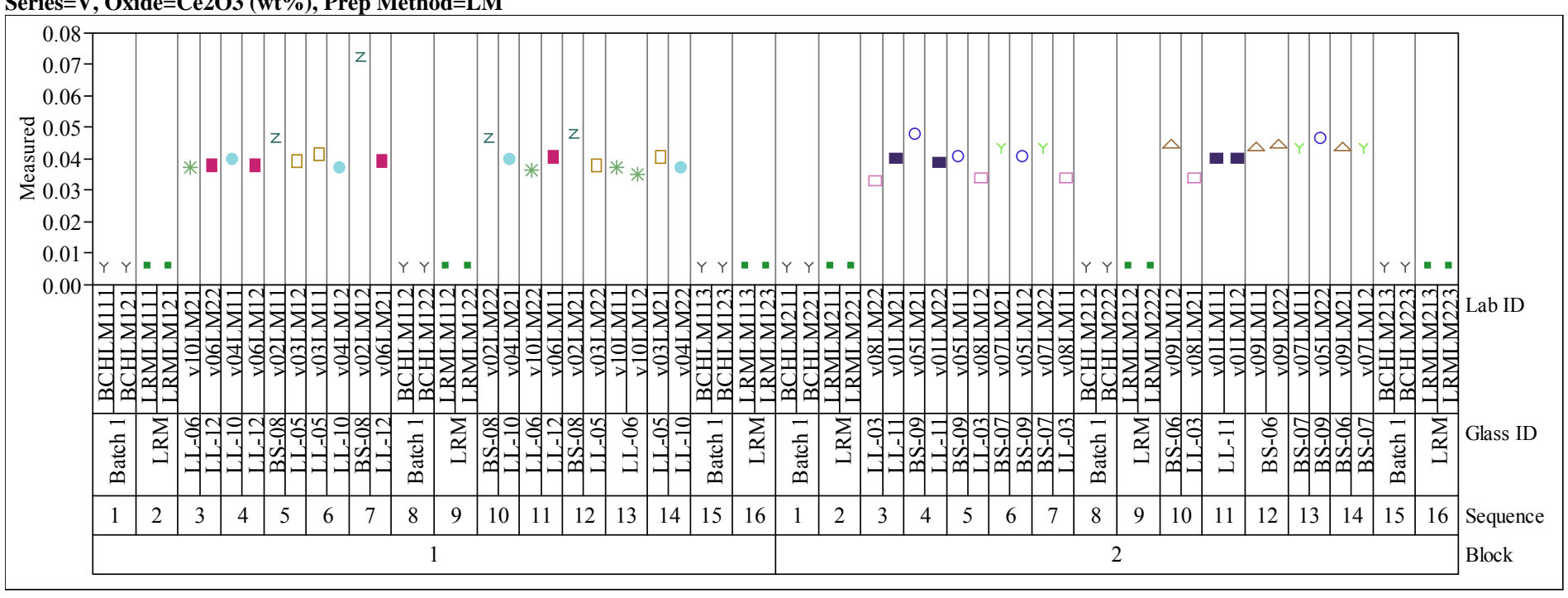

Series $=\mathrm{V}$, Oxide $=\mathrm{Cr} 2 \mathrm{O} 3(\mathrm{wt} \%)$, Prep Method $=\mathrm{LM}$

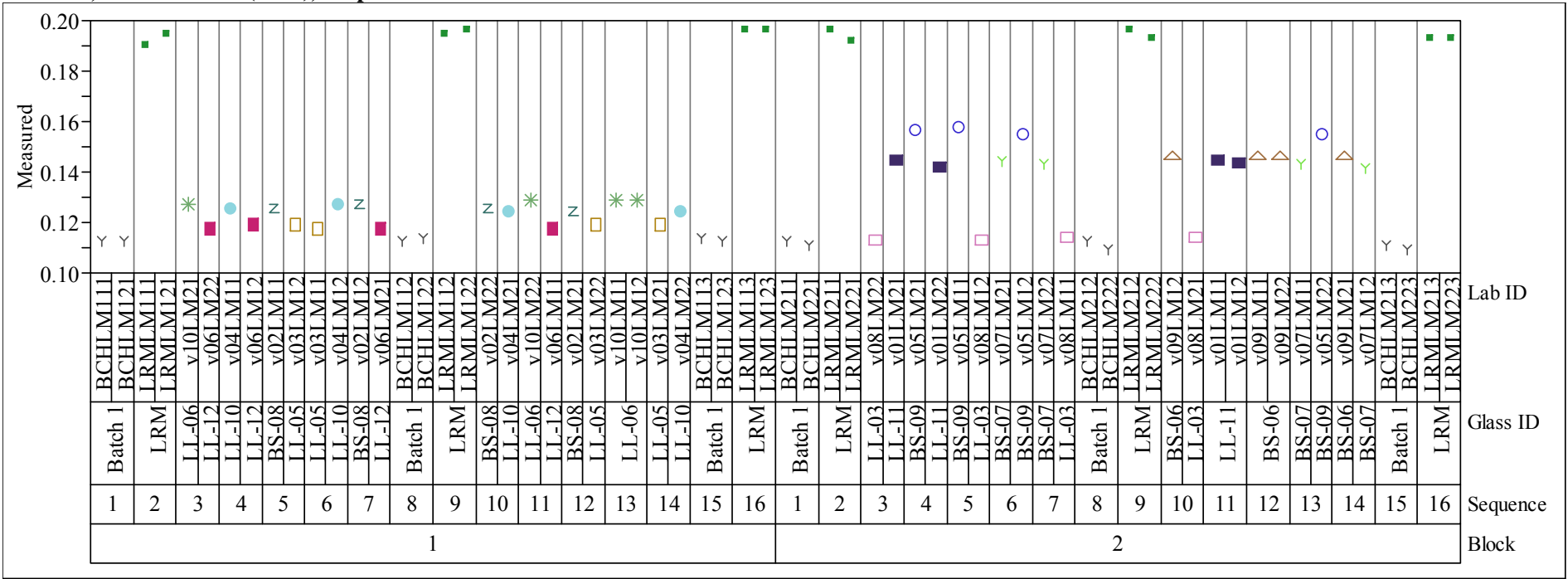




\section{Exhibit A-1. Measurements of Glasses in Analytical Sequence by Oxide within Preparation Method and by Analytical Series (continued)}

Series $=V$, Oxide $=\mathrm{CuO}$ (wt\%), Prep Method $=$ LM

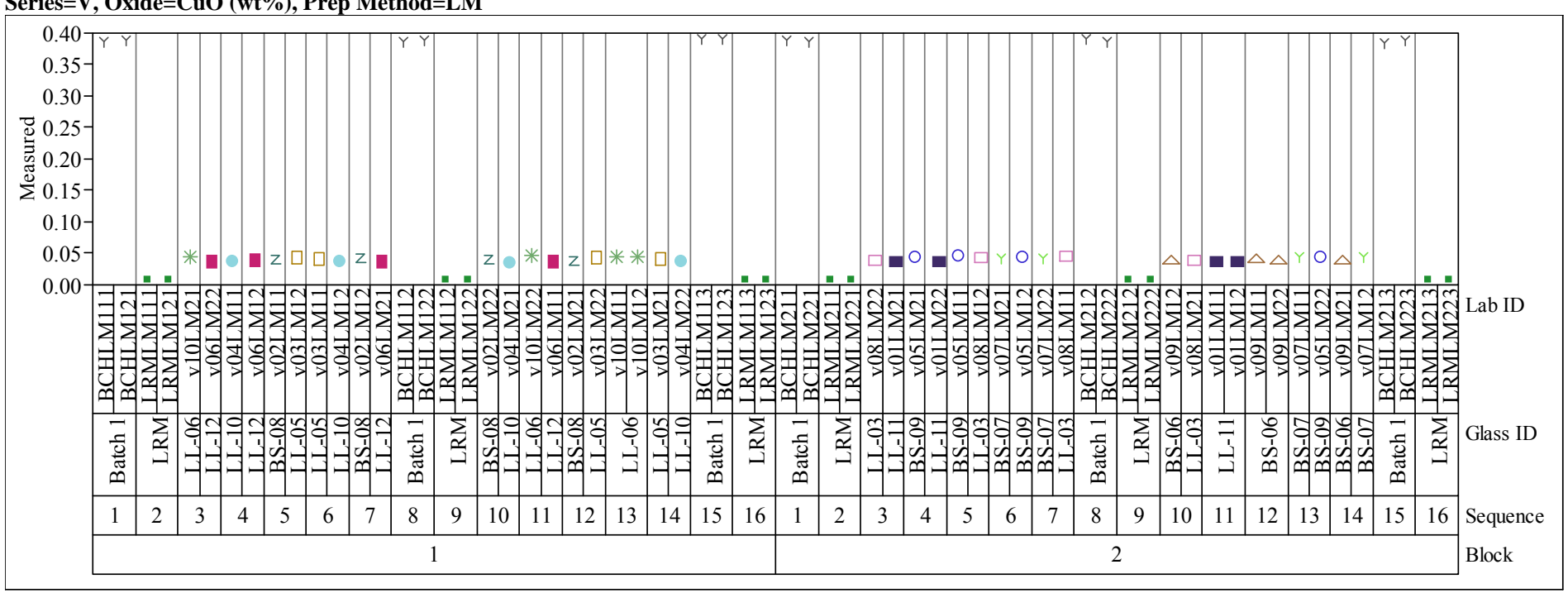

Series=V, Oxide=Fe2O3 (wt \%), Prep Method=LM

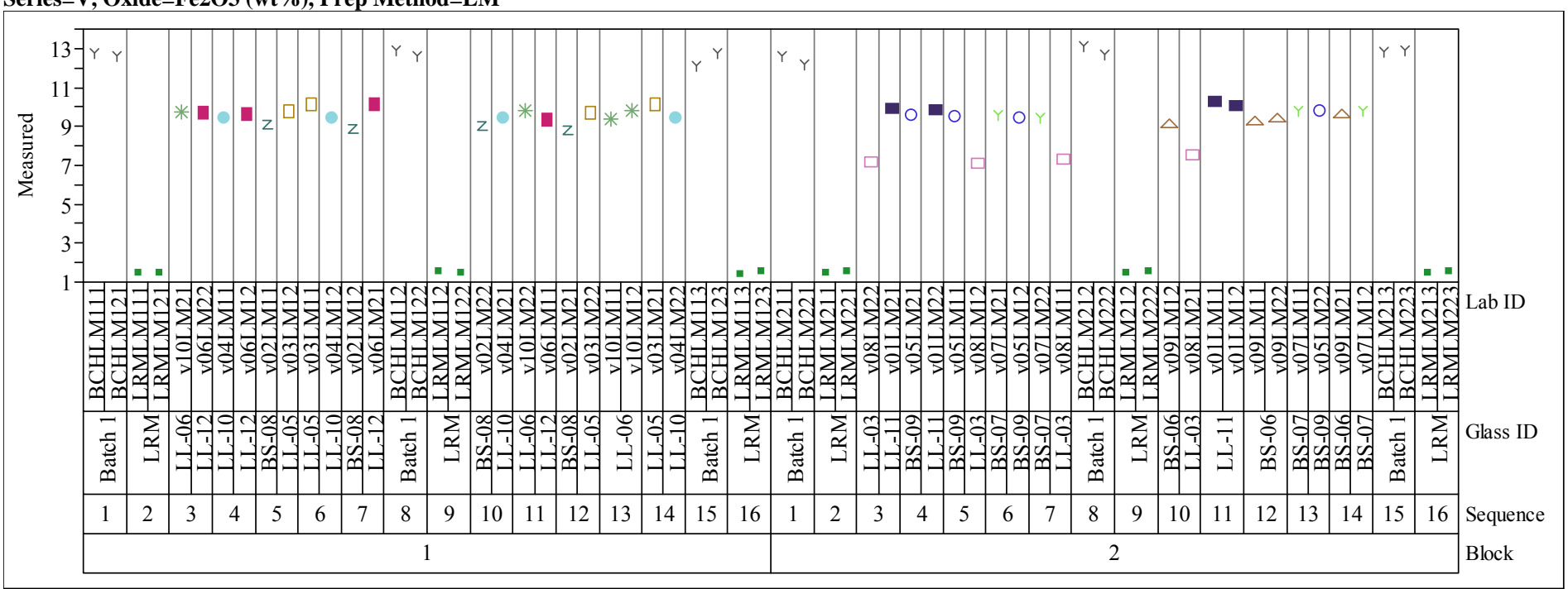




\section{Exhibit A-1. Measurements of Glasses in Analytical Sequence by Oxide within Preparation Method and by Analytical Series (continued)}

Series $=V$, Oxide $=K 2 O(w t \%)$, Prep Method $=L M$

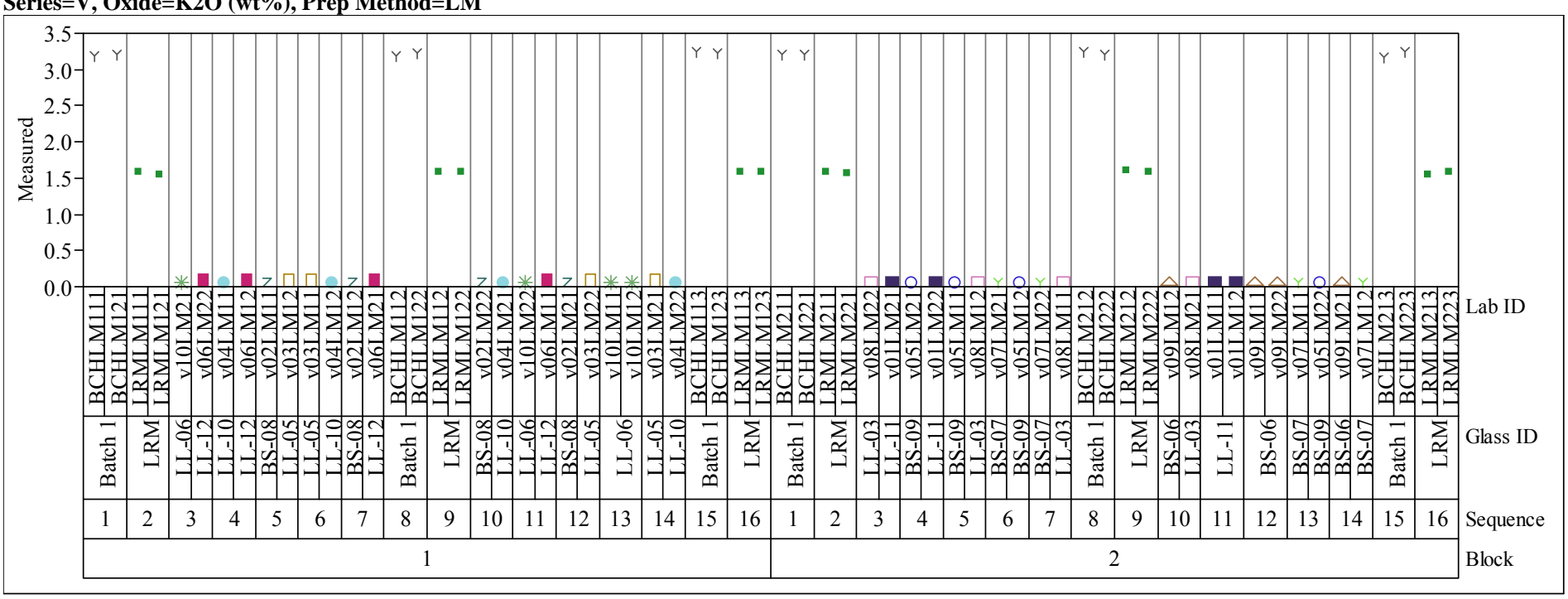

Series=V, Oxide=La2O3 (wt\%), Prep Method=LM

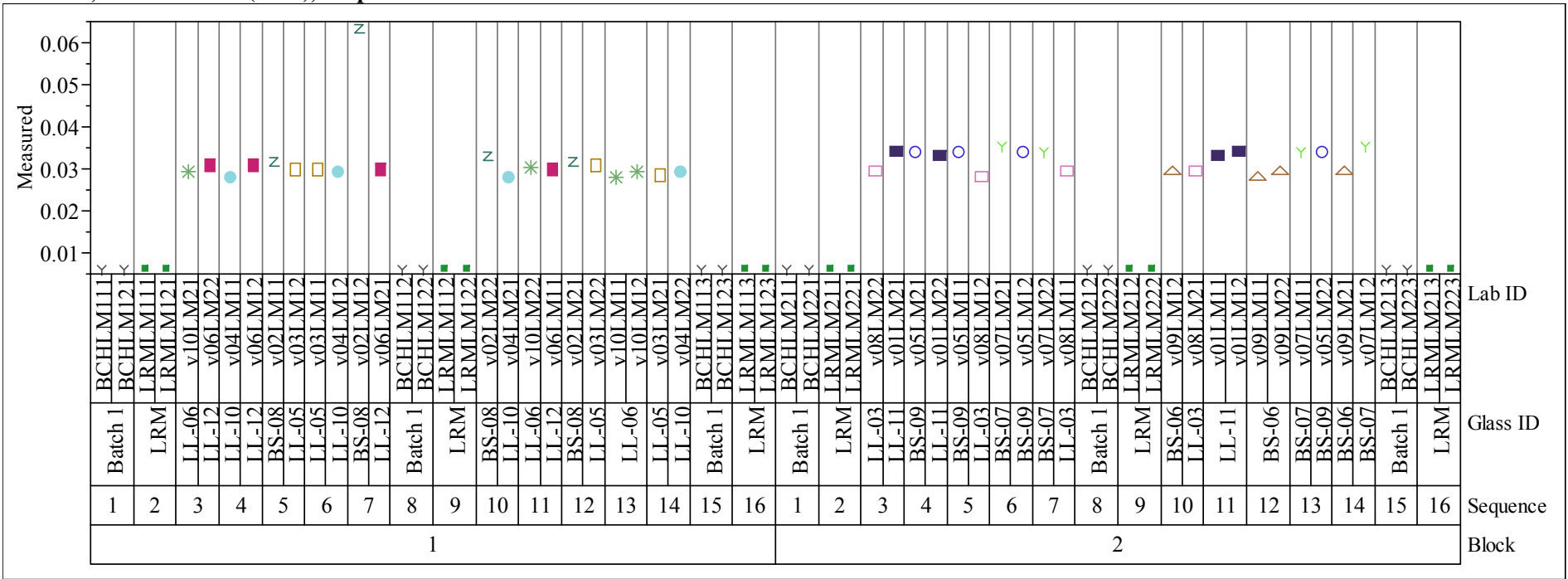




\section{Exhibit A-1. Measurements of Glasses in Analytical Sequence by Oxide within Preparation Method and by Analytical Series (continued)} Series $=\mathrm{V}$, Oxide $=\mathrm{Li} 2 \mathrm{O}(\mathrm{wt} \%)$, Prep Method $=$ PF

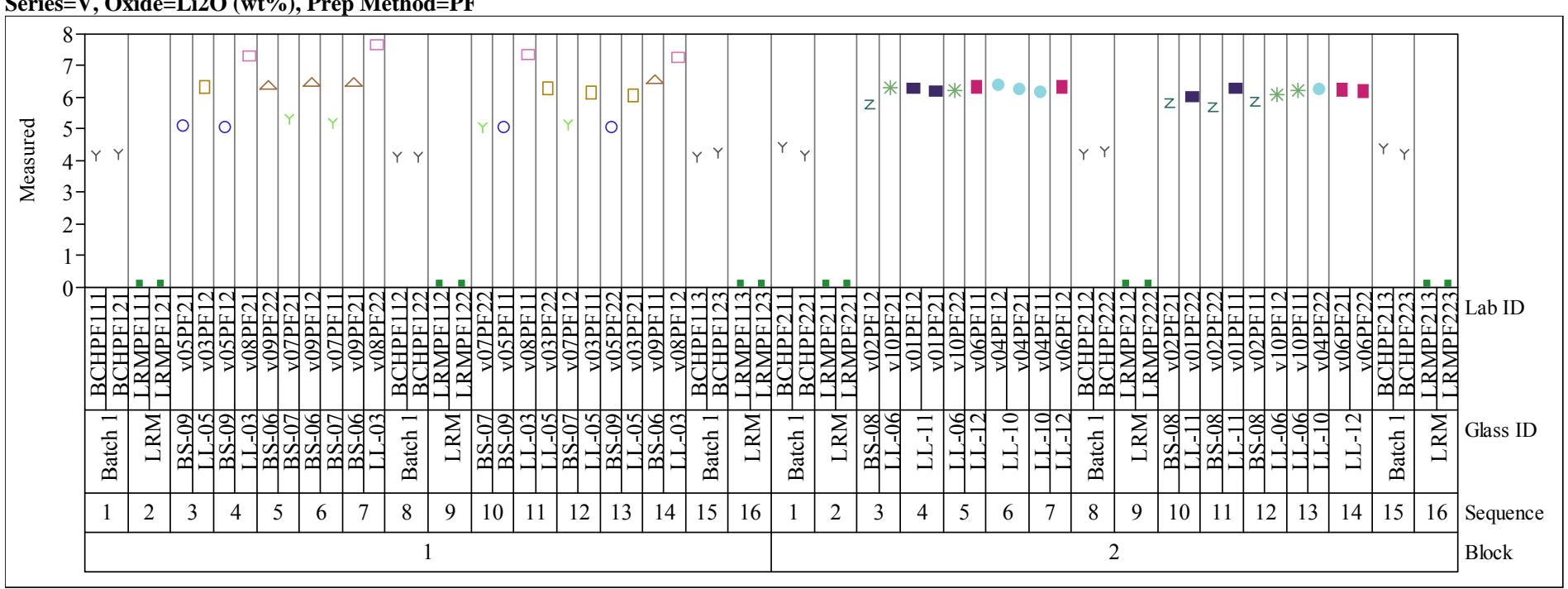

Series=V, Oxide=MgO (wt \%), Prep Method $=$ LM

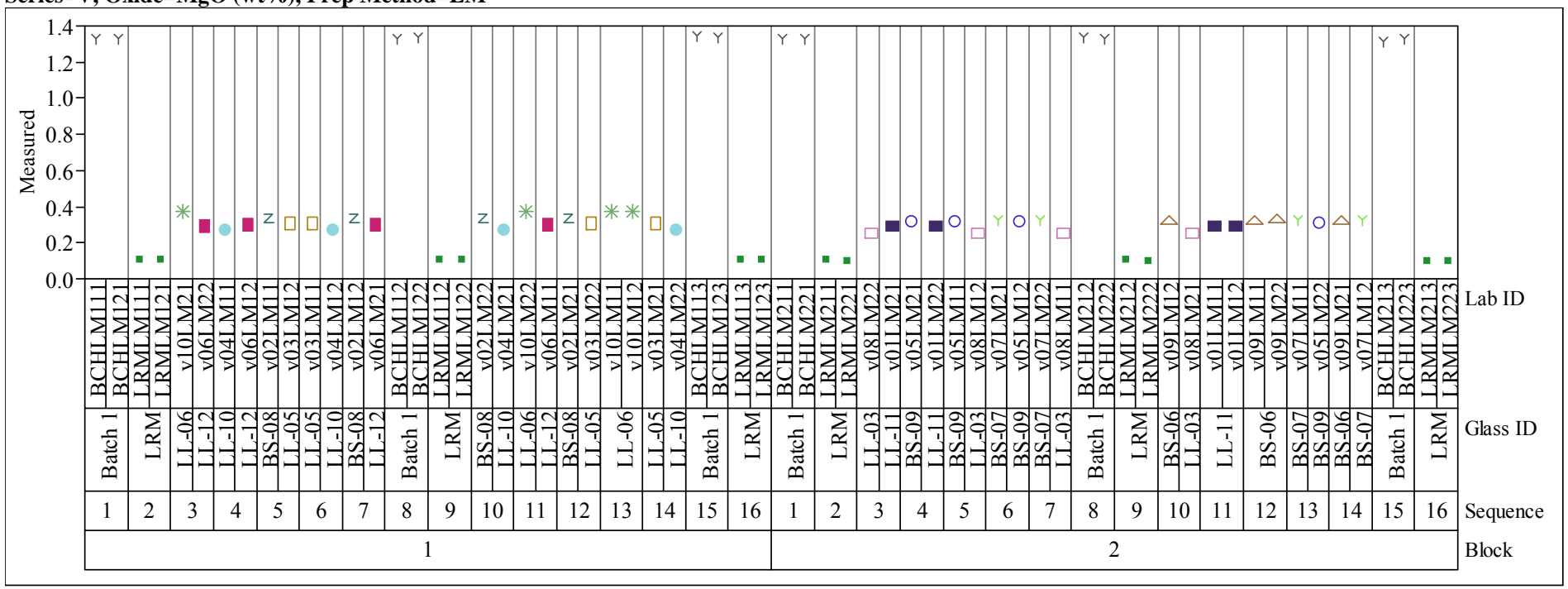




\section{Exhibit A-1. Measurements of Glasses in Analytical Sequence by Oxide within Preparation Method and by Analytical Series (continued)}

Series $=V$, Oxide $=$ MnO (wt \%), Prep Method $=$ LM

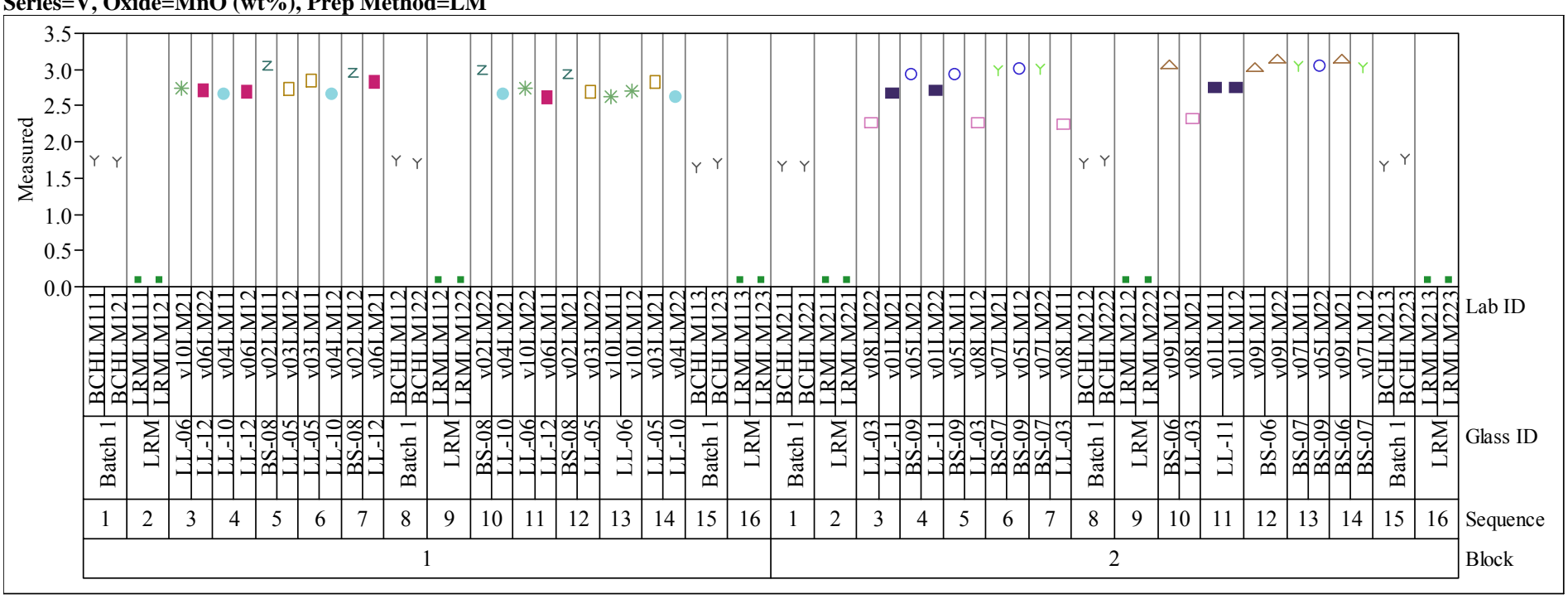

Series $=$ V, Oxide $=$ Na2O (wt \%), Prep Method $=$ LM

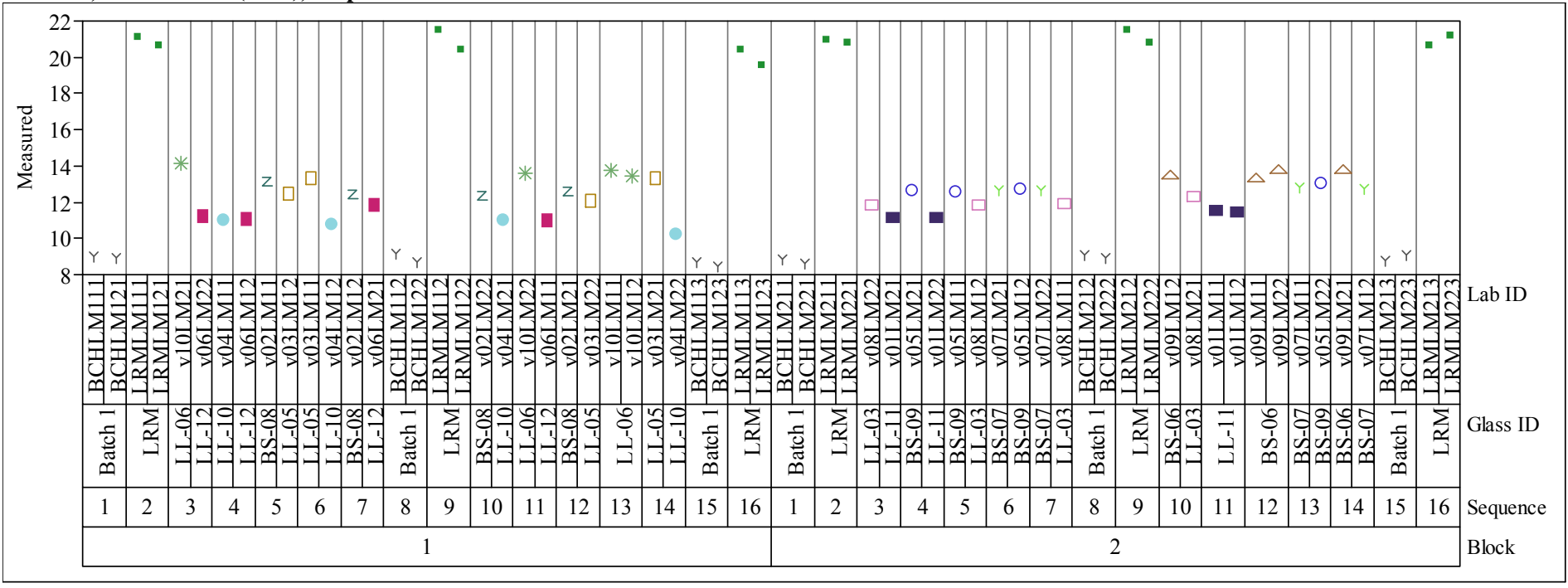




\section{Exhibit A-1. Measurements of Glasses in Analytical Sequence by Oxide within Preparation Method and by Analytical Series (continued)} Series $=\mathrm{V}$, Oxide $=\mathrm{NiO}(\mathrm{wt} \%)$, Prep Method $=\mathrm{LM}$

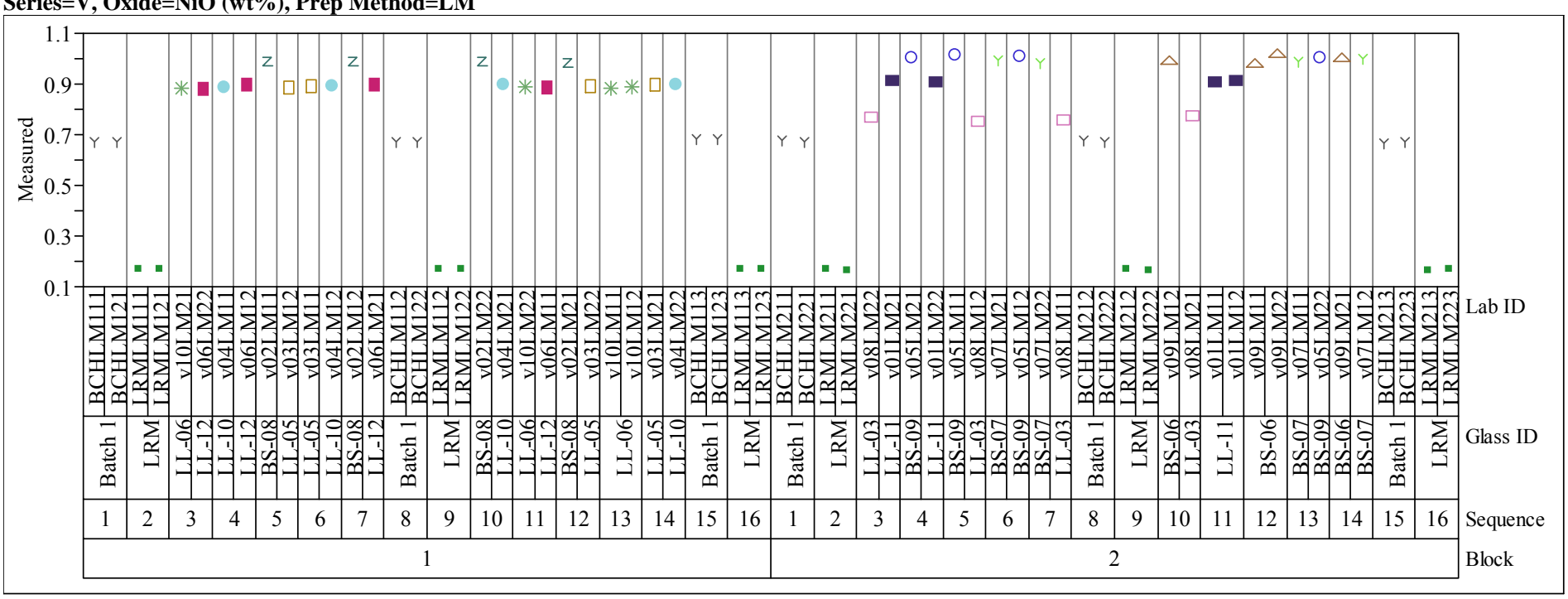

Series $=$ V, Oxide $=$ PbO (wt\%), Prep Method=LM

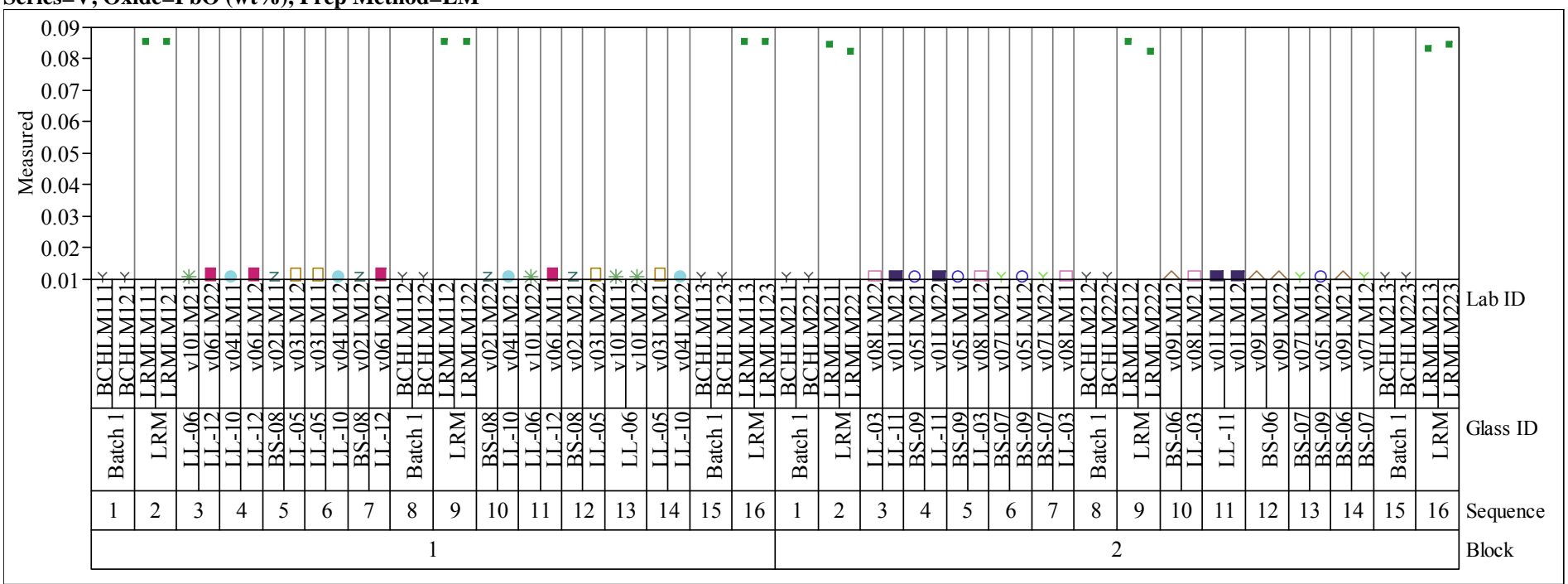




\section{Exhibit A-1. Measurements of Glasses in Analytical Sequence by Oxide within Preparation Method and by Analytical Series (continued)} Series $=\mathrm{V}$, Oxide=SiO2 (wt \%), Prep Method $=$ PF

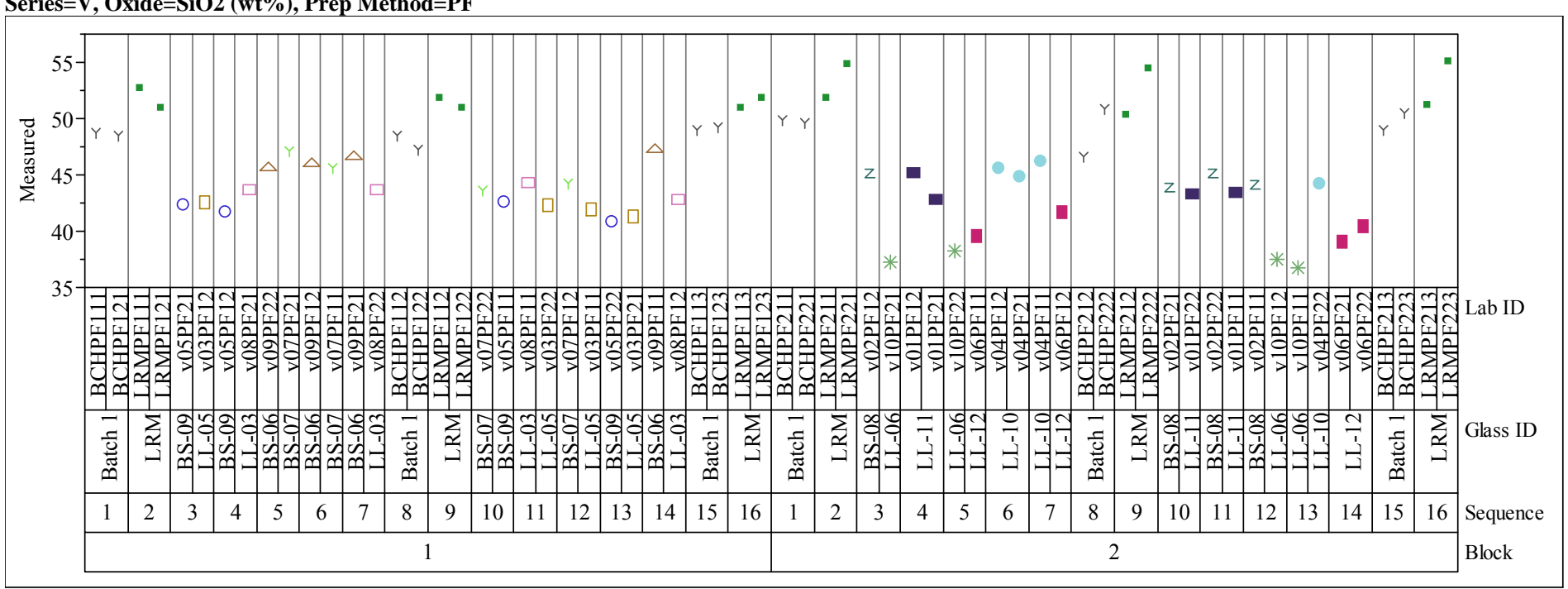

Series=V, Oxide=SO4 (wt\%), Prep Method=LM

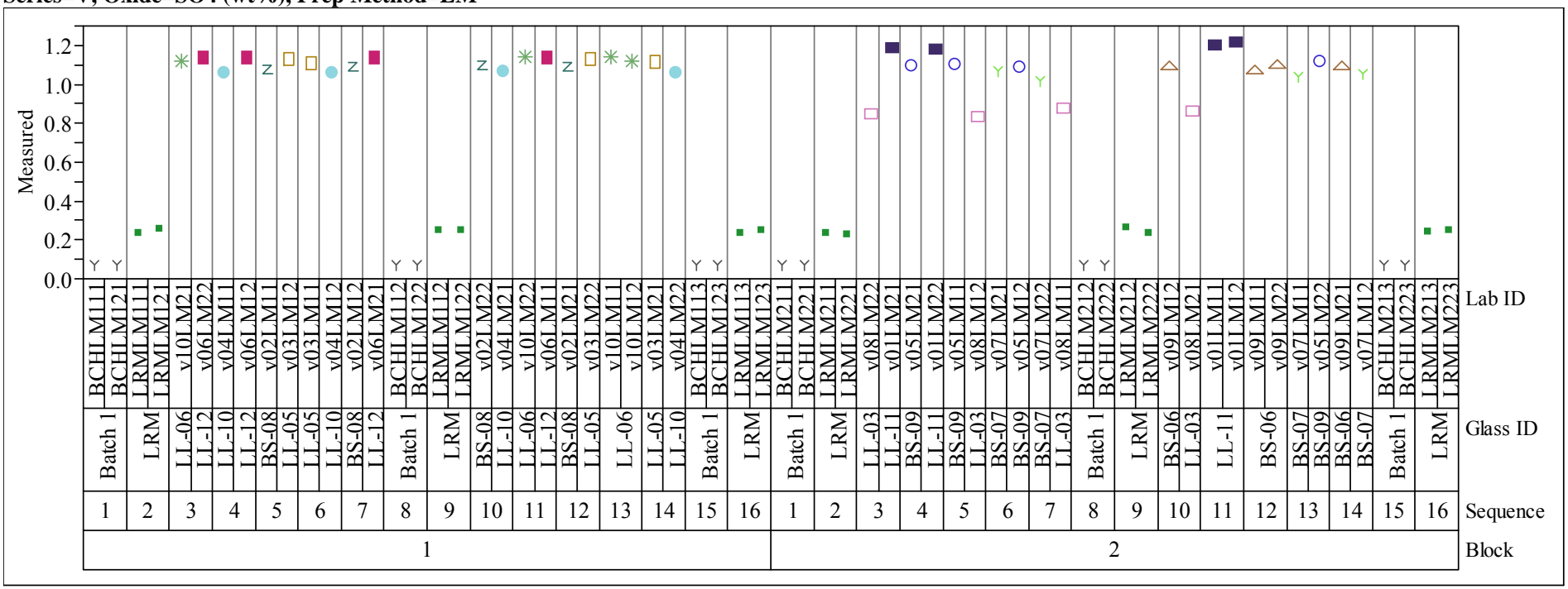




\section{Exhibit A-1. Measurements of Glasses in Analytical Sequence by Oxide within Preparation Method and by Analytical Series (continued)}

Series $=V$, Oxide $=$ TiO2 $(w t \%)$, Prep Method $=$ LM

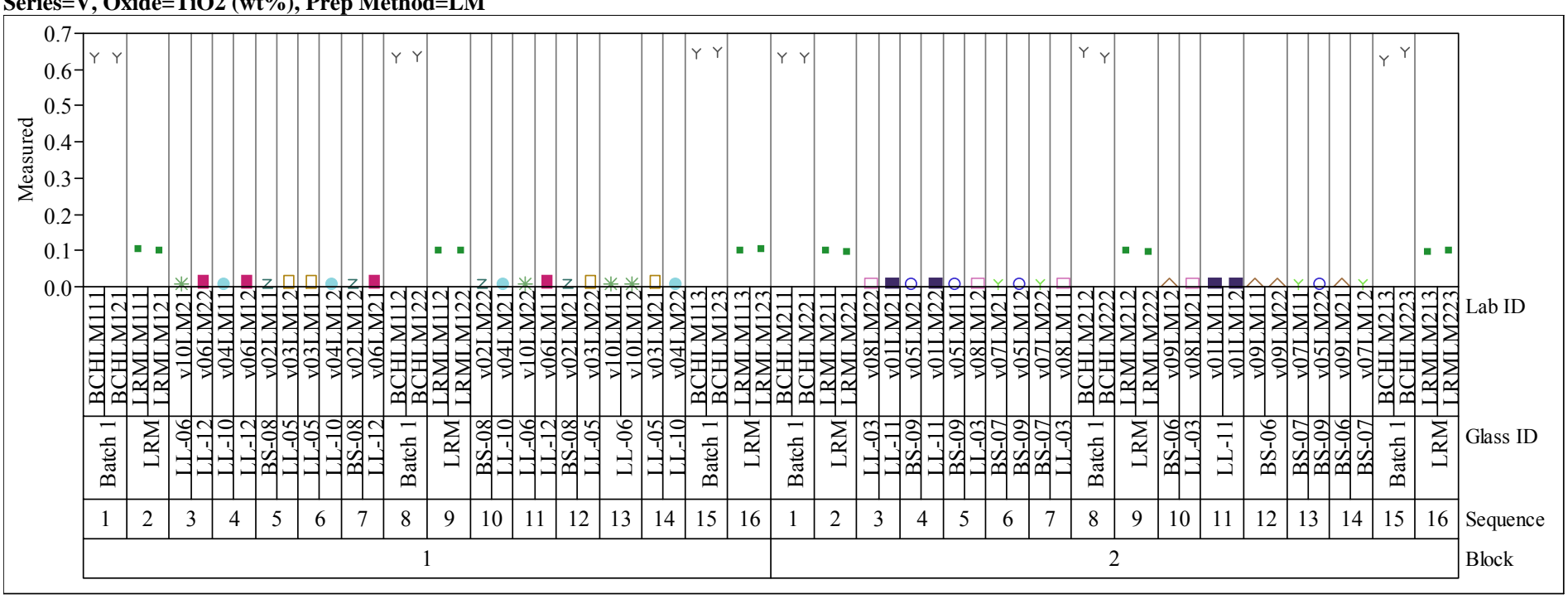

Series $=$ V, Oxide $=\mathrm{ZnO}($ wt $\%)$, Prep Method=LM

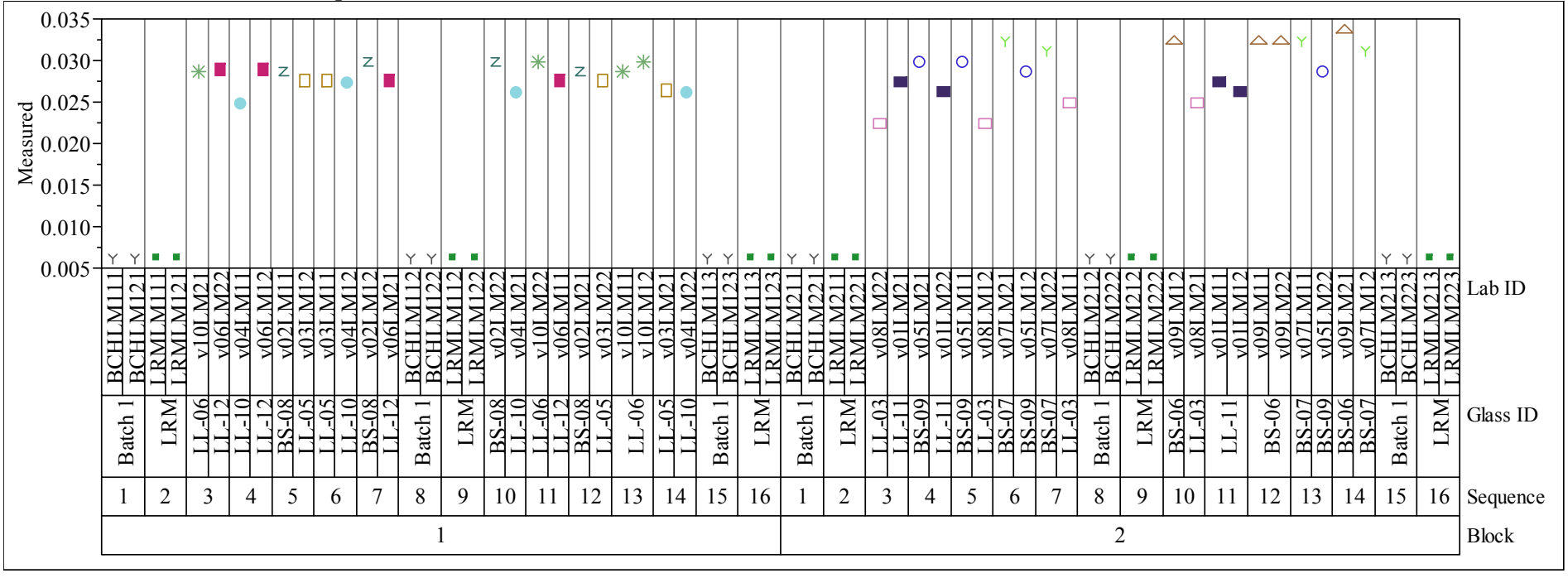


Exhibit A-1. Measurements of Glasses in Analytical Sequence by Oxide within Preparation Method and by Analytical Series (continued) Series $=\mathrm{V}$, Oxide $=\mathrm{ZrO} 2$ (wt\%), Prep Method $=\mathbf{L M}$

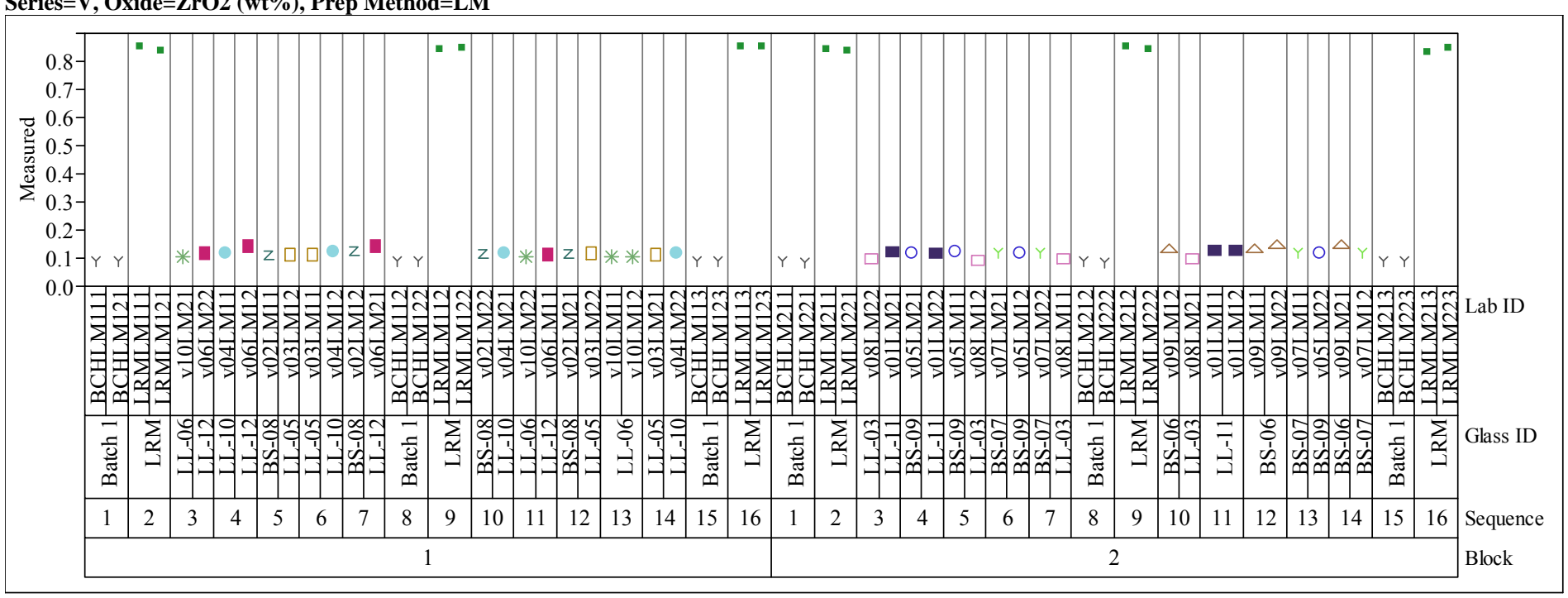

Series=W, Oxide=Al2O3 (wt\%), Prep Method $=$ LM

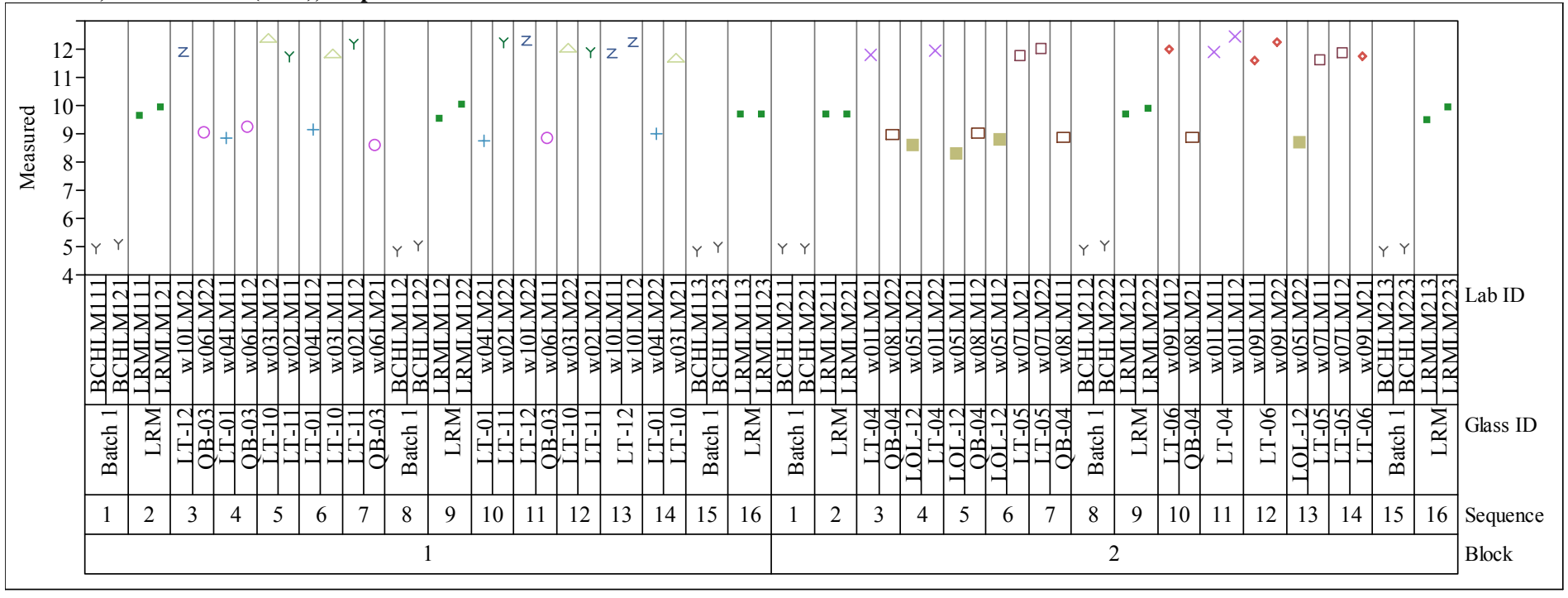




\section{Exhibit A-1. Measurements of Glasses in Analytical Sequence by Oxide within Preparation Method and by Analytical Series (continued)} Series $=$ W, Oxide=B2O3 (wt \%), Prep Method=PF

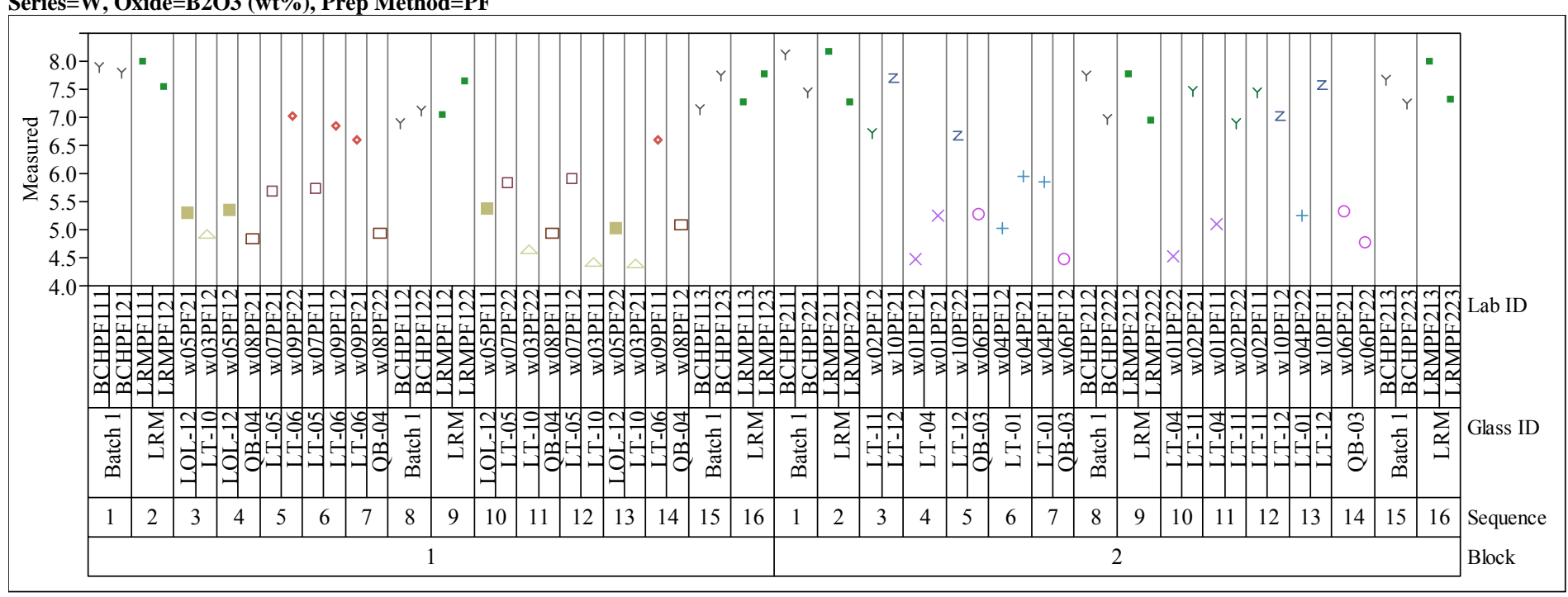

Series $=W$, Oxide=BaO (wt \%), Prep Method=LM

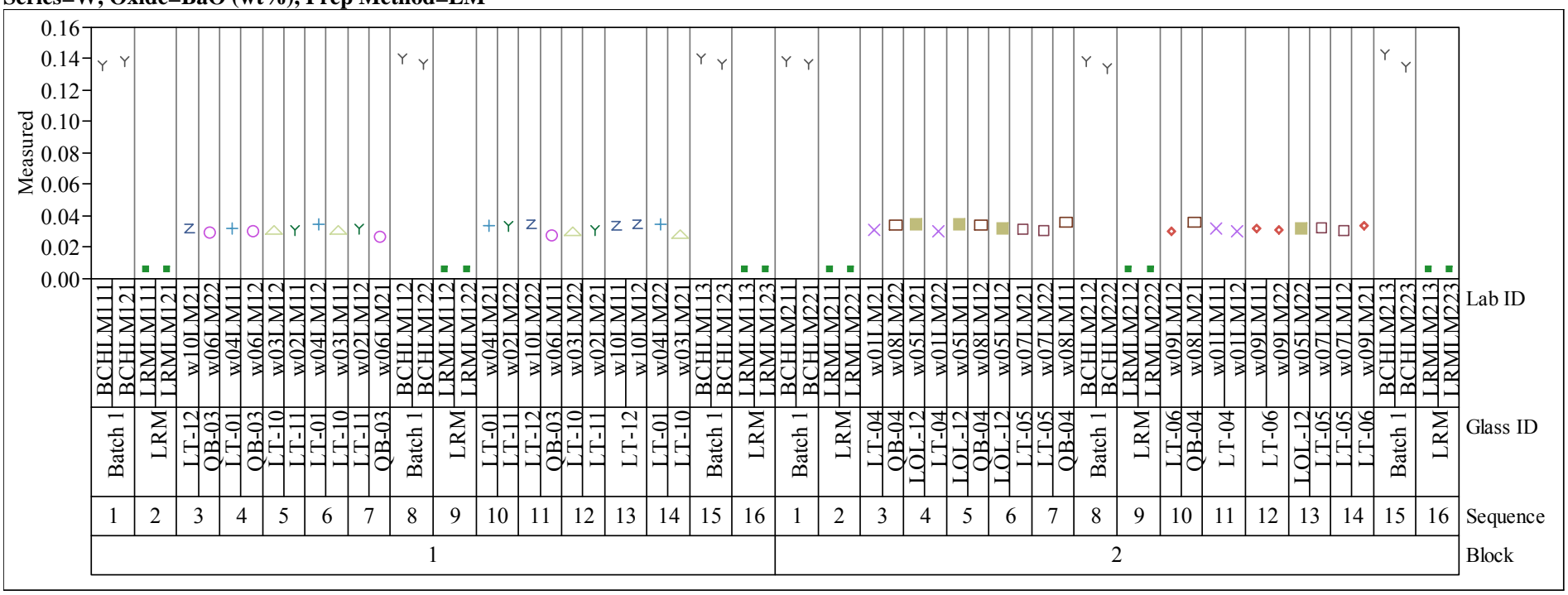




\section{Exhibit A-1. Measurements of Glasses in Analytical Sequence by Oxide within Preparation Method and by Analytical Series (continued)} Series $=\mathrm{W}$, Oxide $=\mathbf{C a O}(\mathrm{wt} \%)$, Prep Method $=\mathbf{L M}$

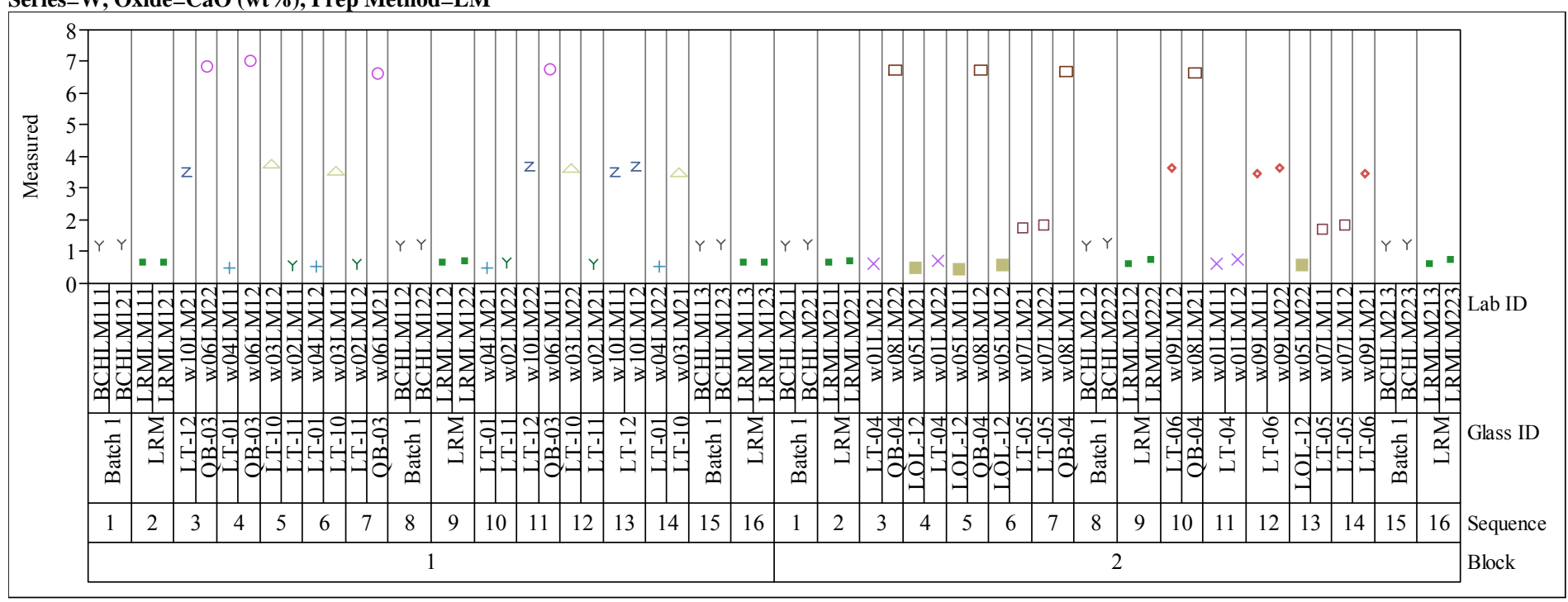

\section{Series $=\mathrm{W}$, Oxide $=\mathrm{Ce} 2 \mathrm{O} 3(\mathrm{wt} \%)$, Prep Method $=\mathrm{LM}$}

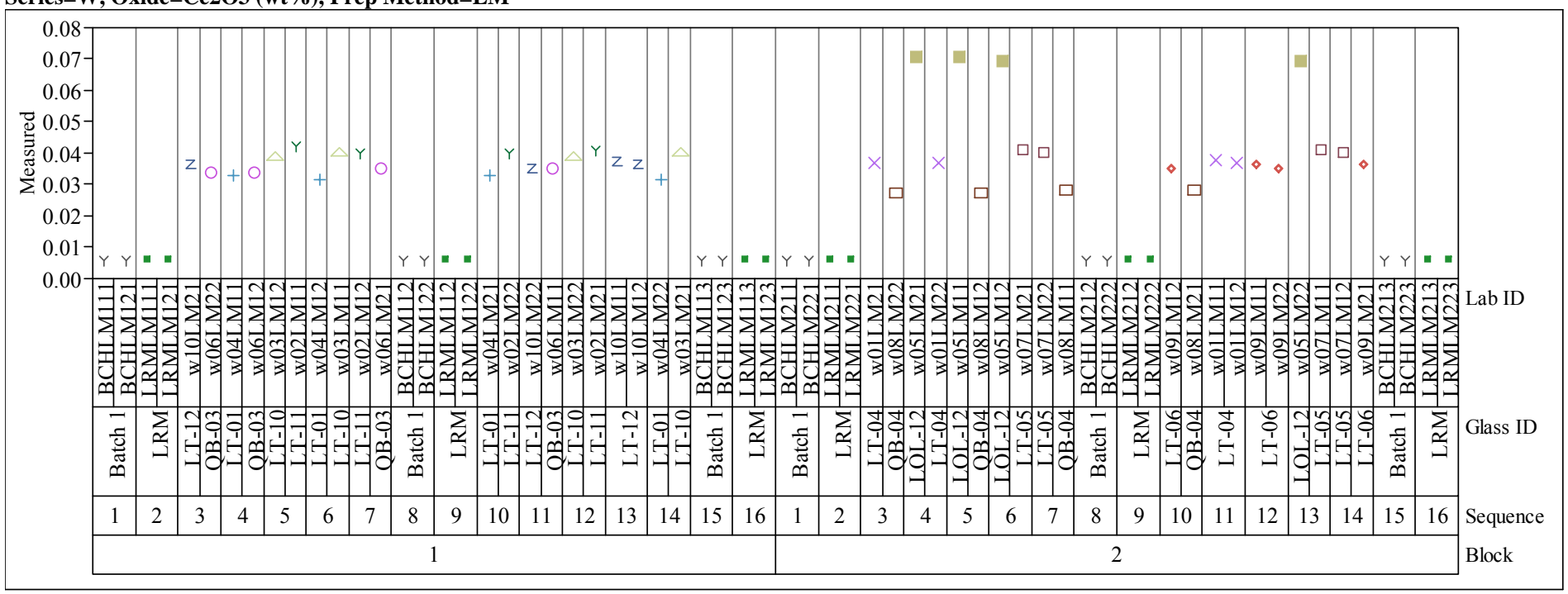




\section{Exhibit A-1. Measurements of Glasses in Analytical Sequence by Oxide within Preparation Method and by Analytical Series (continued)} Series $=$ W, Oxide $=\mathrm{Cr} 2 \mathrm{O} 3(\mathrm{wt} \%)$, Prep Method $=$ LM

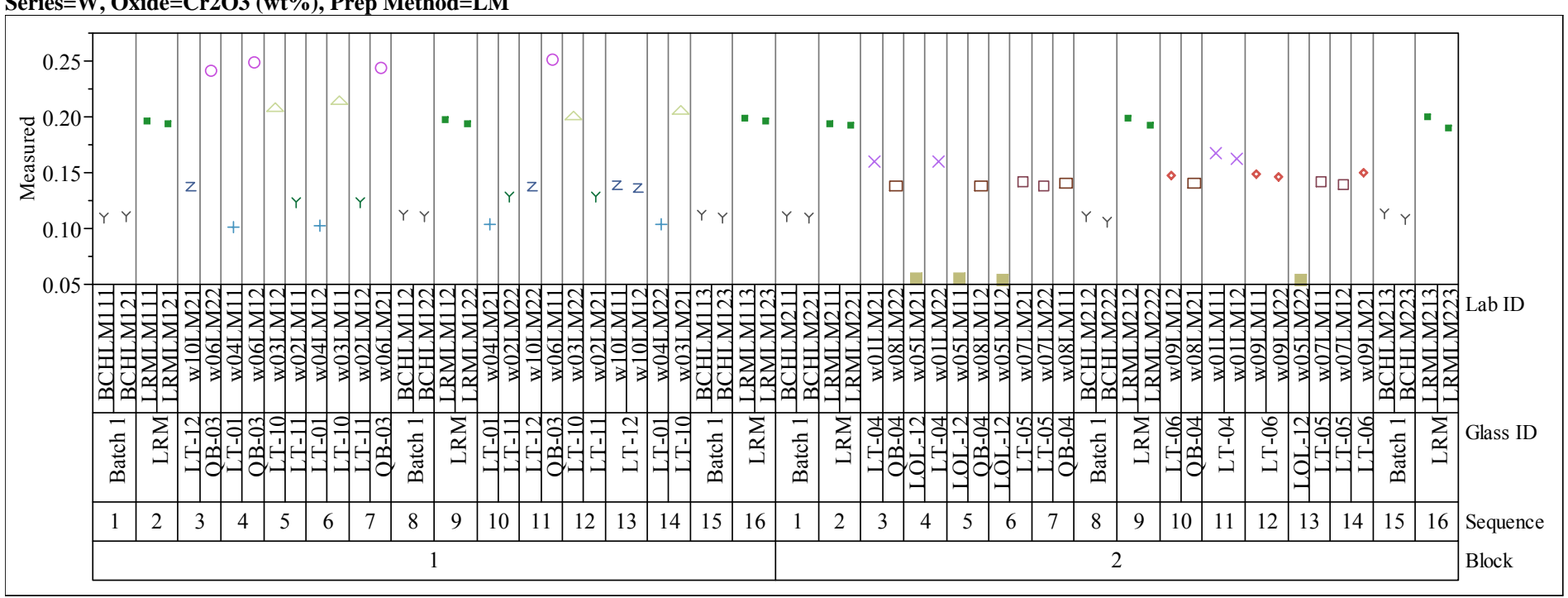

Series $=\mathrm{W}$, Oxide $=\mathrm{CuO}(\mathrm{wt} \%)$, Prep Method $=\mathrm{LM}$

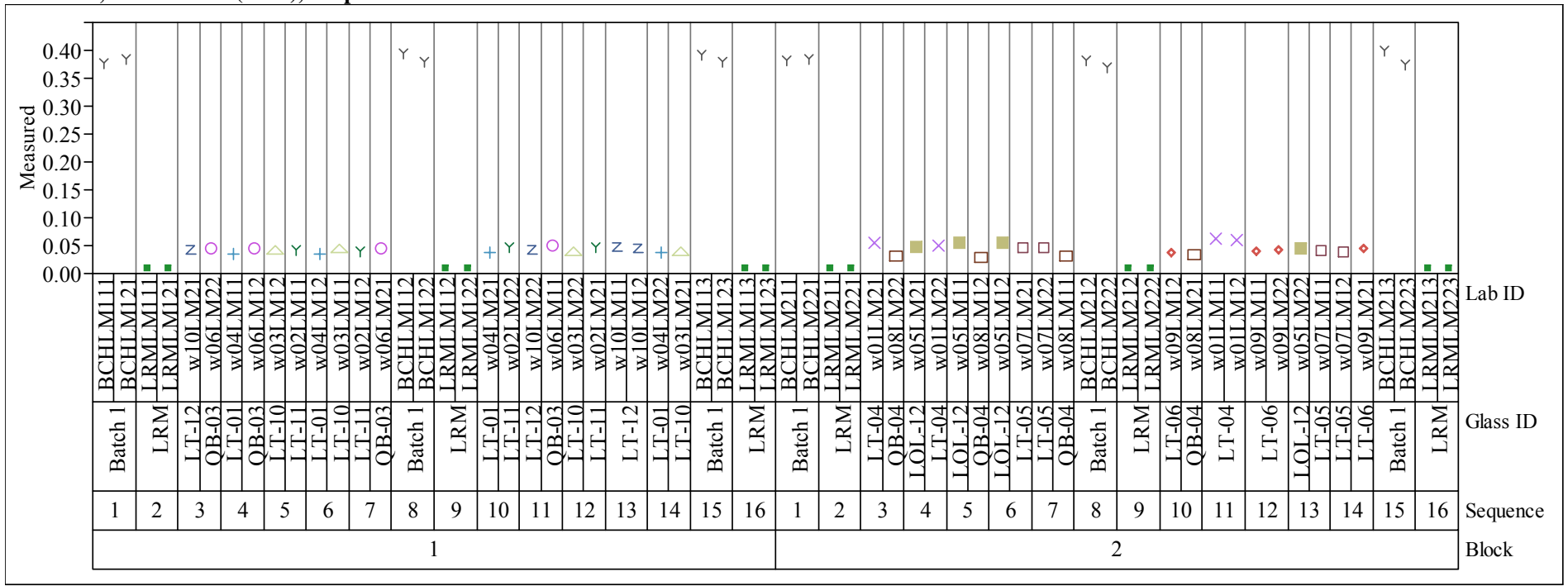




\section{Exhibit A-1. Measurements of Glasses in Analytical Sequence by Oxide within Preparation Method and by Analytical Series (continued)} Series=W, Oxide=Fe2O3 (wt\%), Prep Method=LM

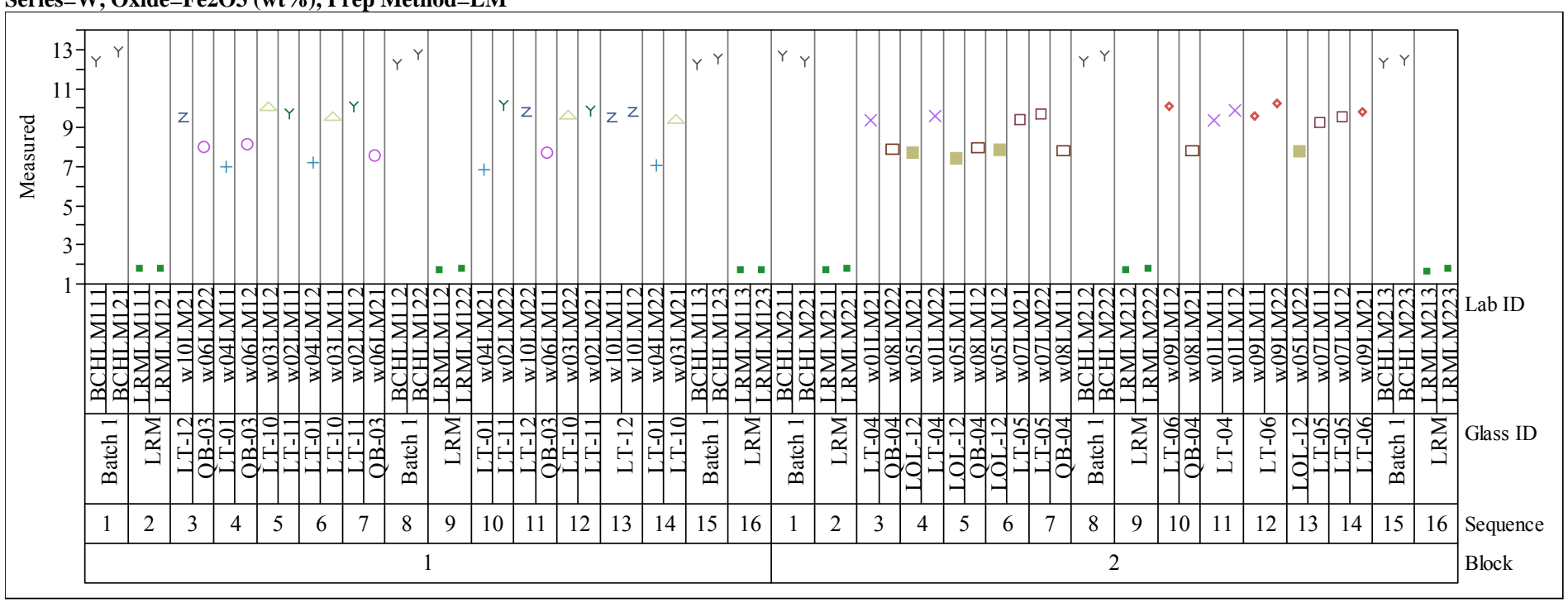

Series $=W$, Oxide $=$ K2O (wt \%), Prep Method $=$ LM

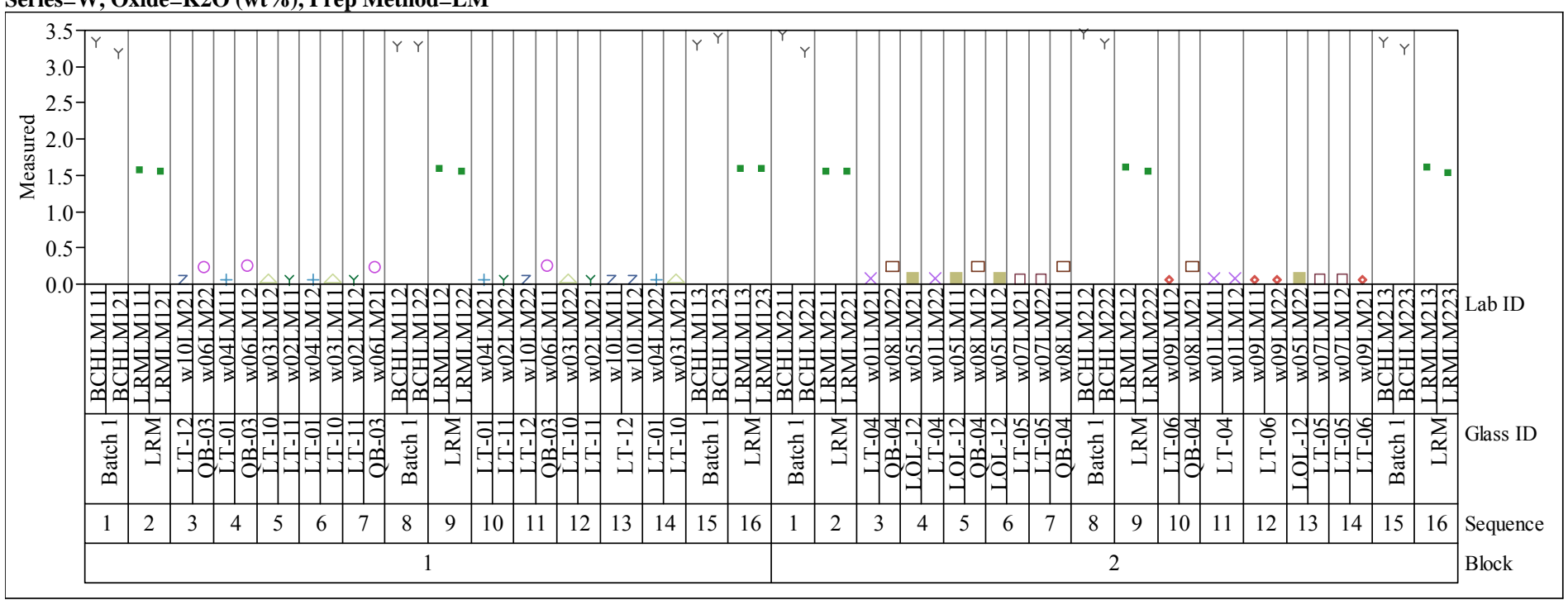


Exhibit A-1. Measurements of Glasses in Analytical Sequence by Oxide within Preparation Method and by Analytical Series (continued) Series=W, Oxide=La2O3 (wt\%), Prep Method $=$ LM

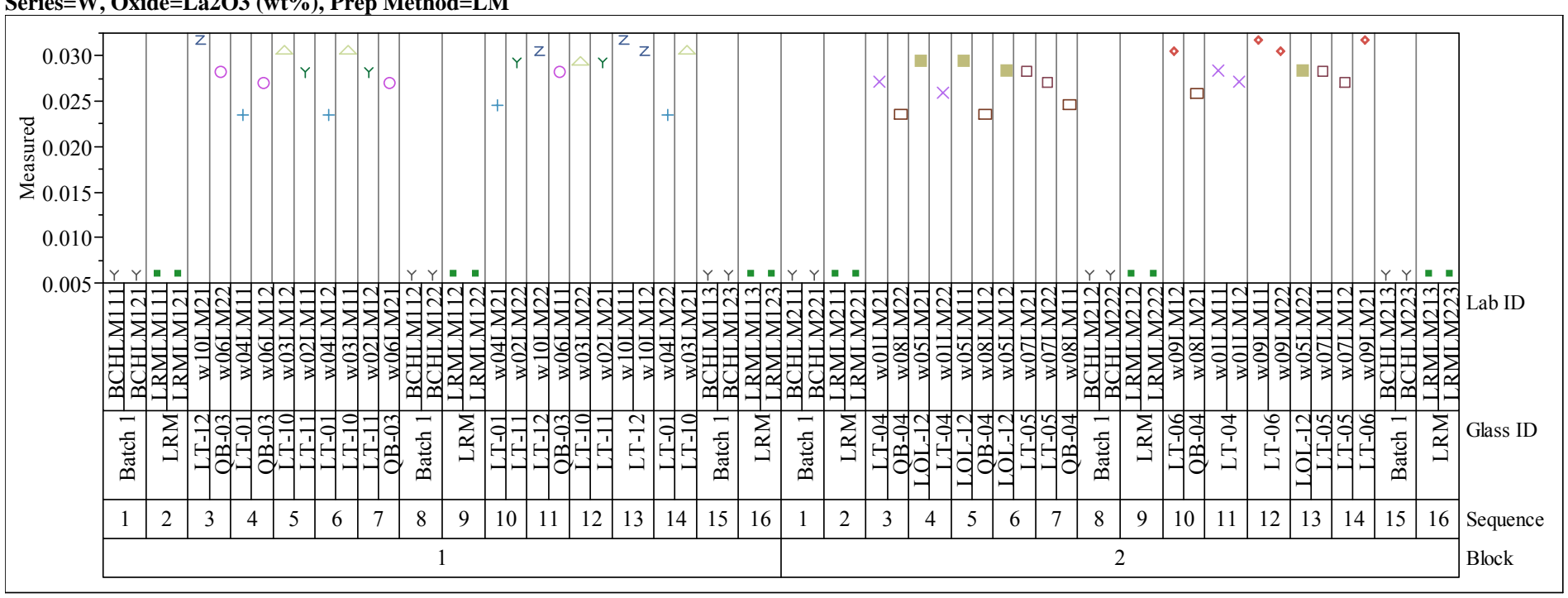

Series $=W$, Oxide $=$ Li2O (wt\%), Prep Method $=$ PF

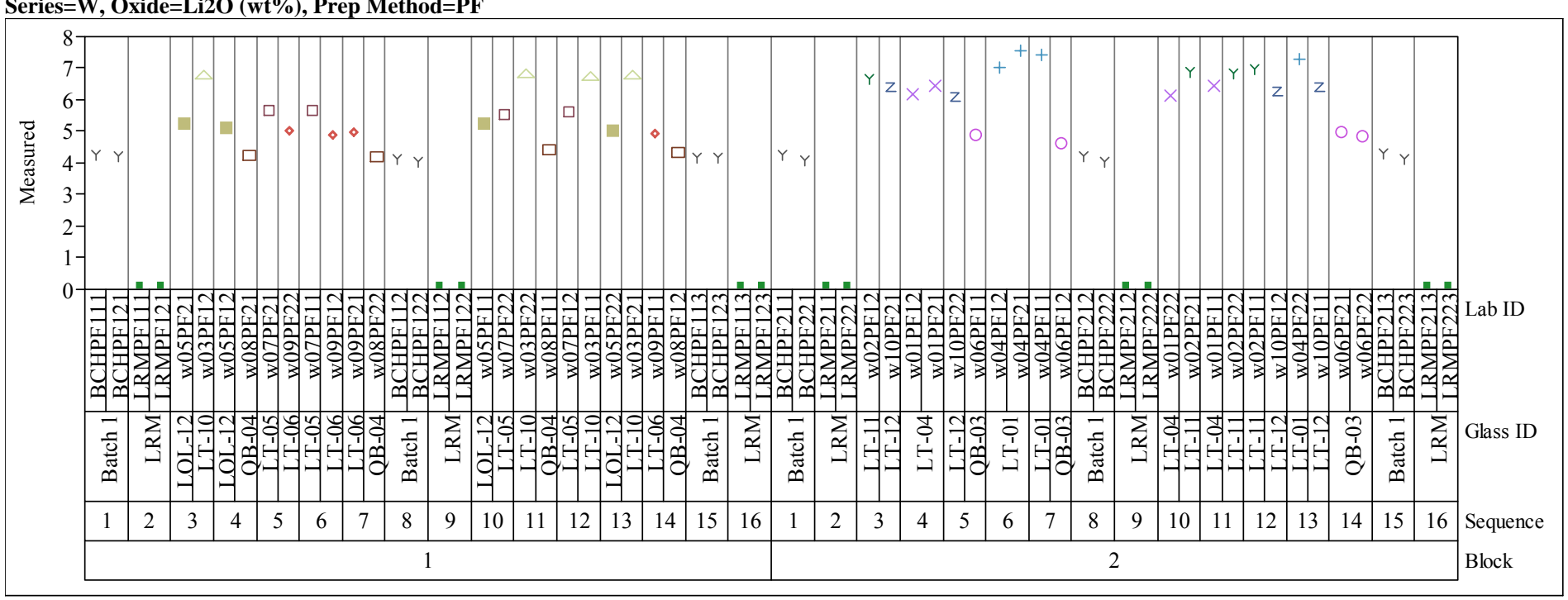




\section{Exhibit A-1. Measurements of Glasses in Analytical Sequence by Oxide within Preparation Method and by Analytical Series (continued)} Series=W, Oxide=MgO (wt \%), Prep Method=LM

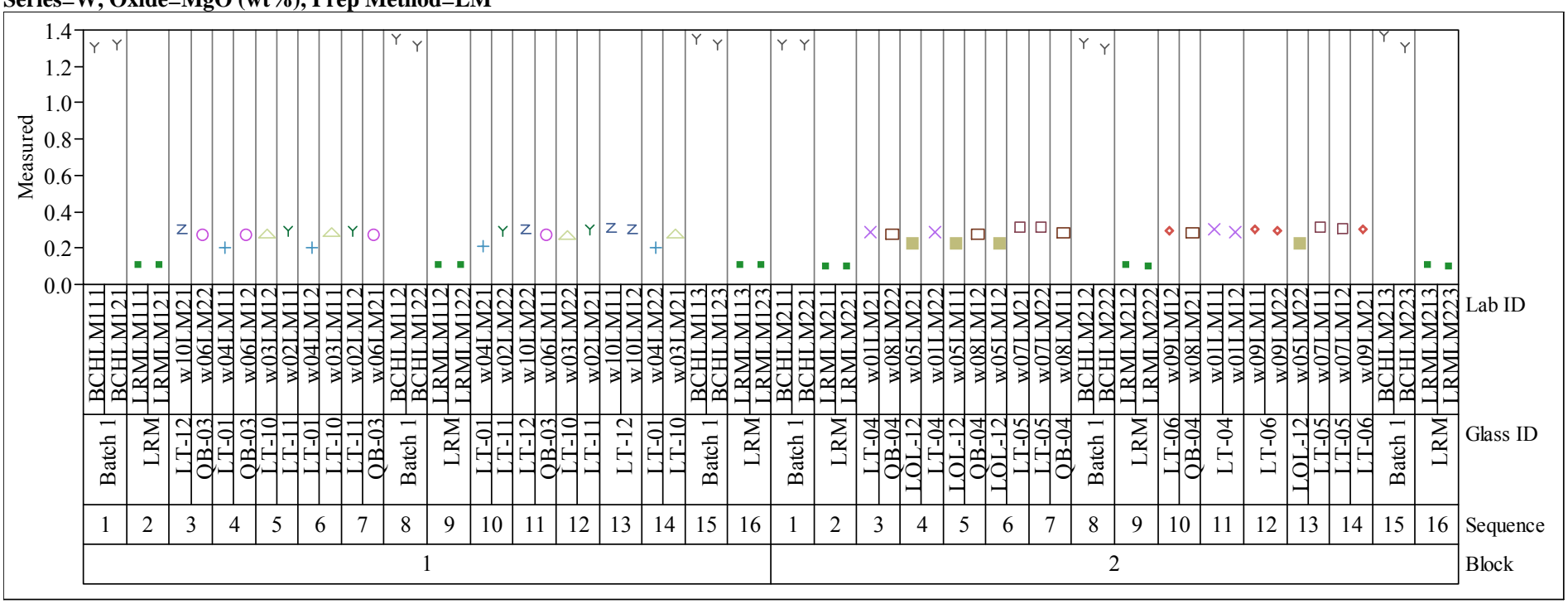

Series=W, Oxide=MnO (wt\%), Prep Method=LM

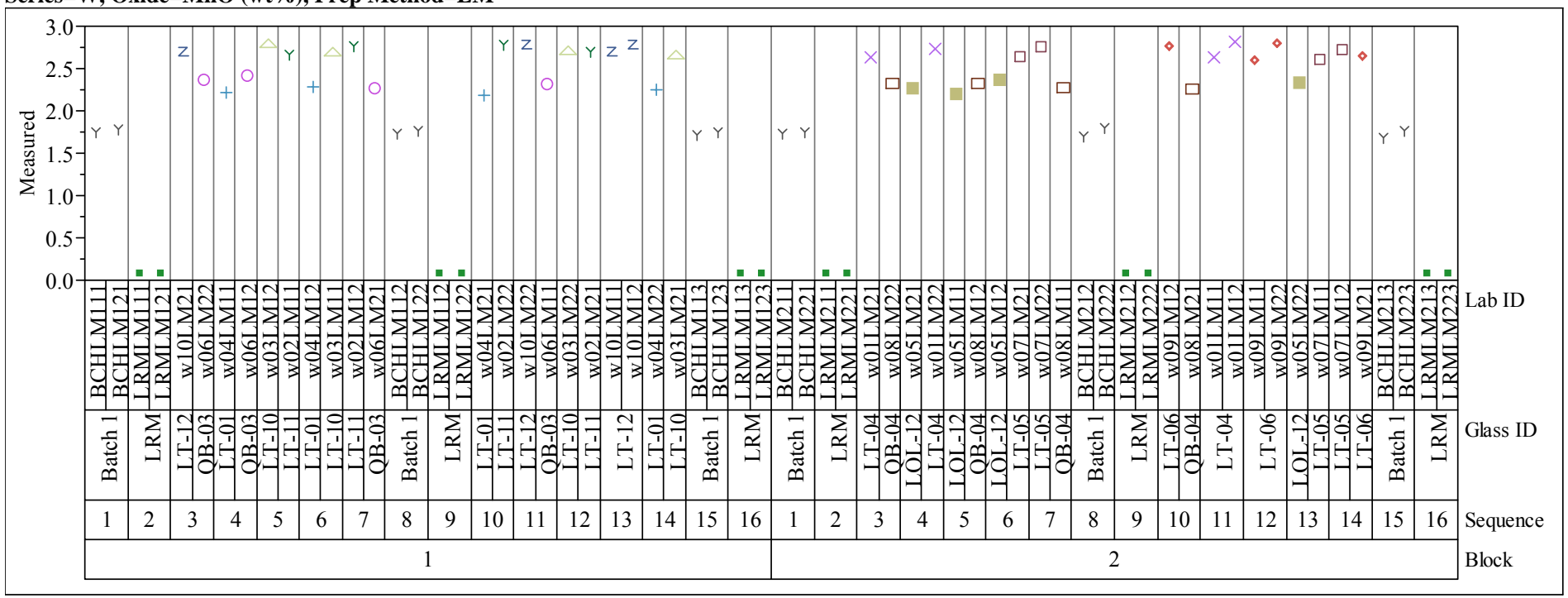




\section{Exhibit A-1. Measurements of Glasses in Analytical Sequence by Oxide within Preparation Method and by Analytical Series (continued)}

Series $=\mathrm{W}$, Oxide $=\mathrm{Na} 2 \mathrm{O}(\mathrm{wt} \%)$, Prep Method $=\mathrm{LM}$

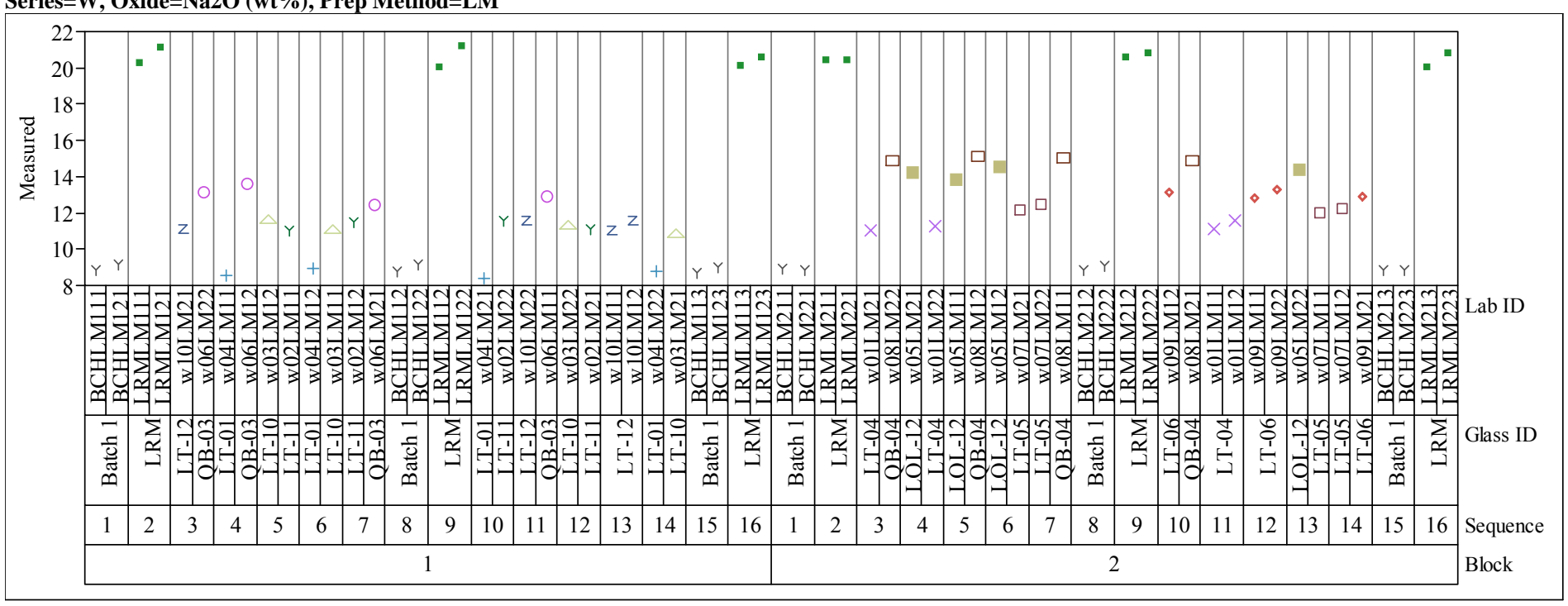

Series $=W$, Oxide $=$ NiO (wt \%), Prep Method $=$ LM

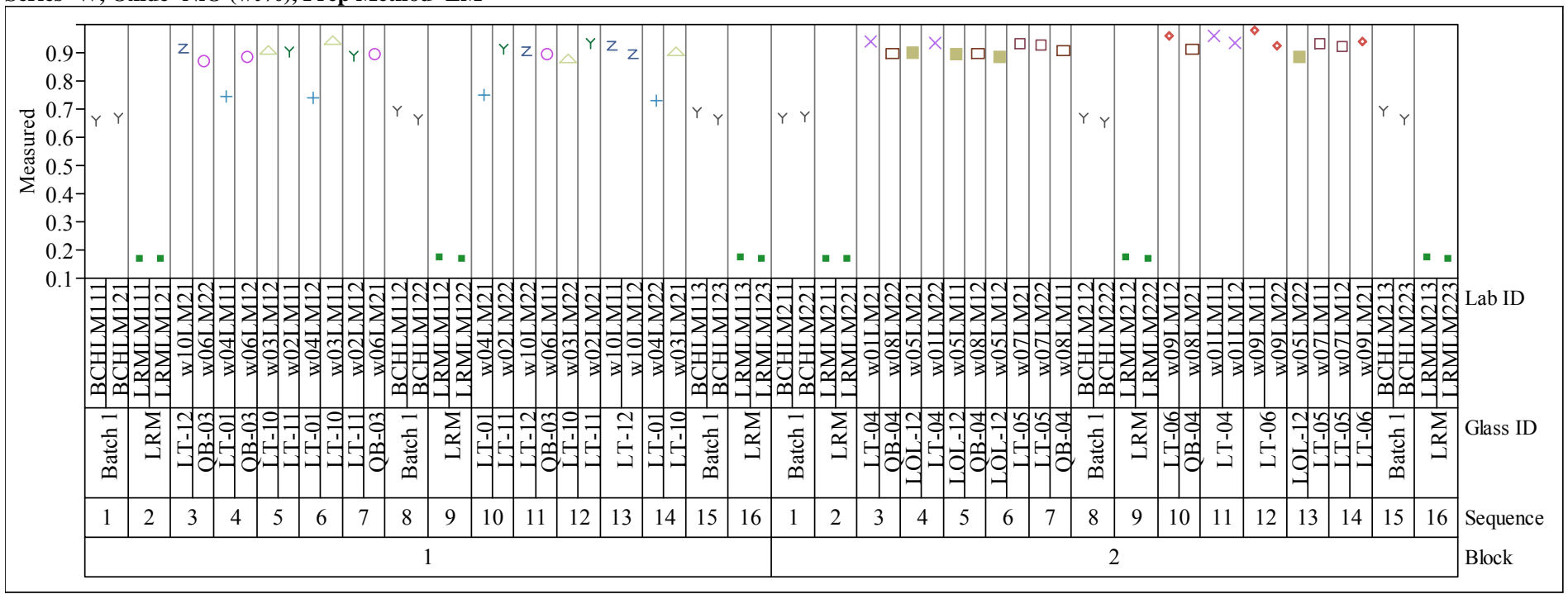




\section{Exhibit A-1. Measurements of Glasses in Analytical Sequence by Oxide within Preparation Method and by Analytical Series (continued)}

Series $=W$, Oxide=PbO (wt \%), Prep Method $=$ LM

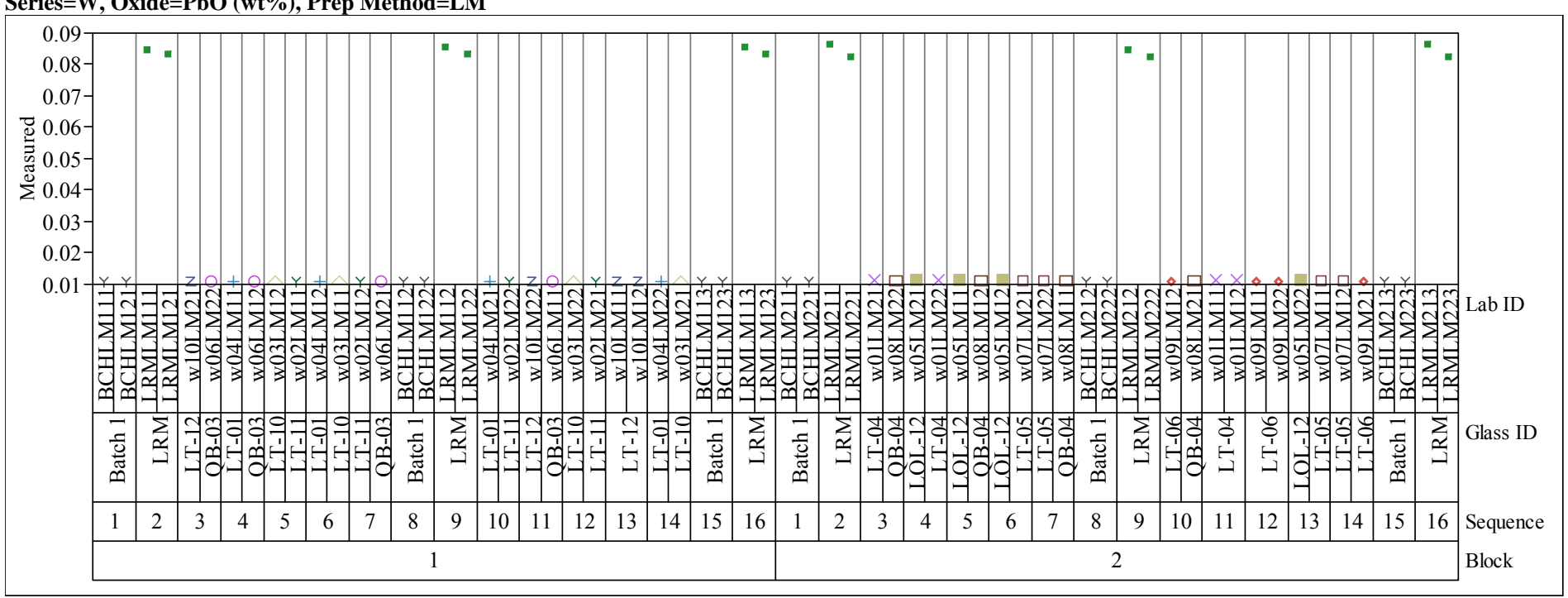

Series $=\mathrm{W}$, Oxide=SiO2 (wt \%), Prep Method $=$ PF

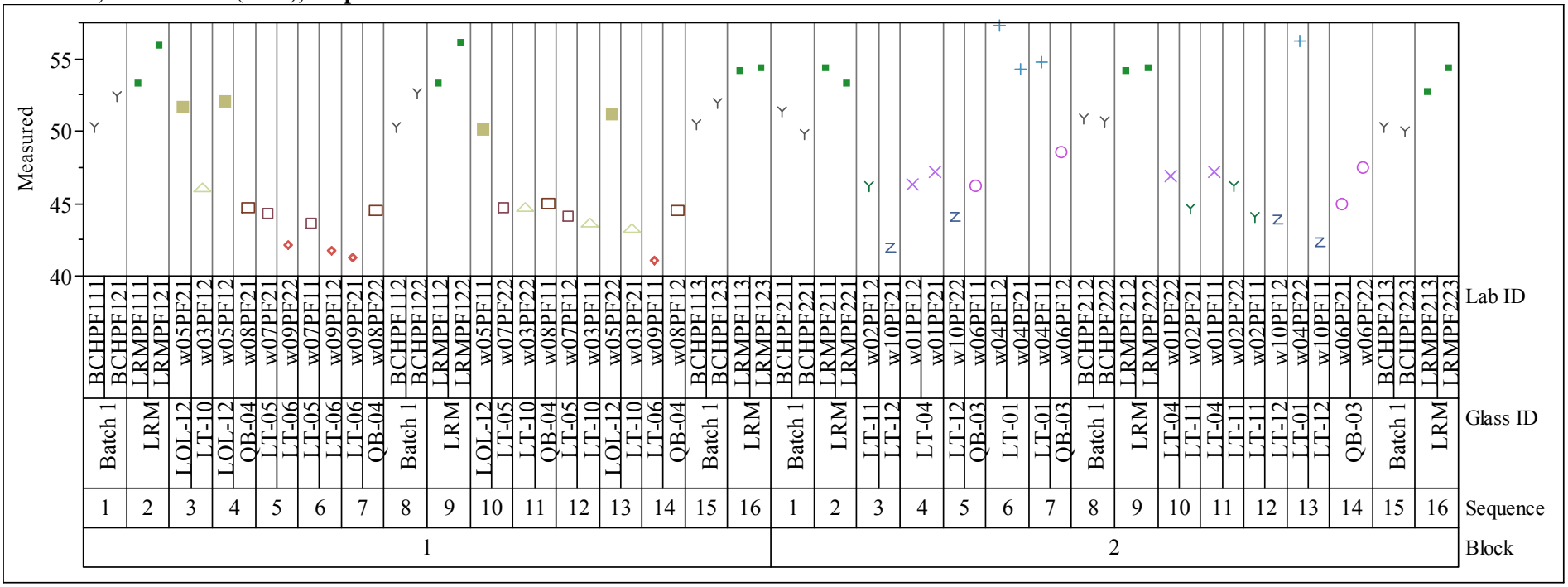




\section{Exhibit A-1. Measurements of Glasses in Analytical Sequence by Oxide within Preparation Method and by Analytical Series (continued)} Series=W, Oxide=SO4 (wt\%), Prep Method=LM

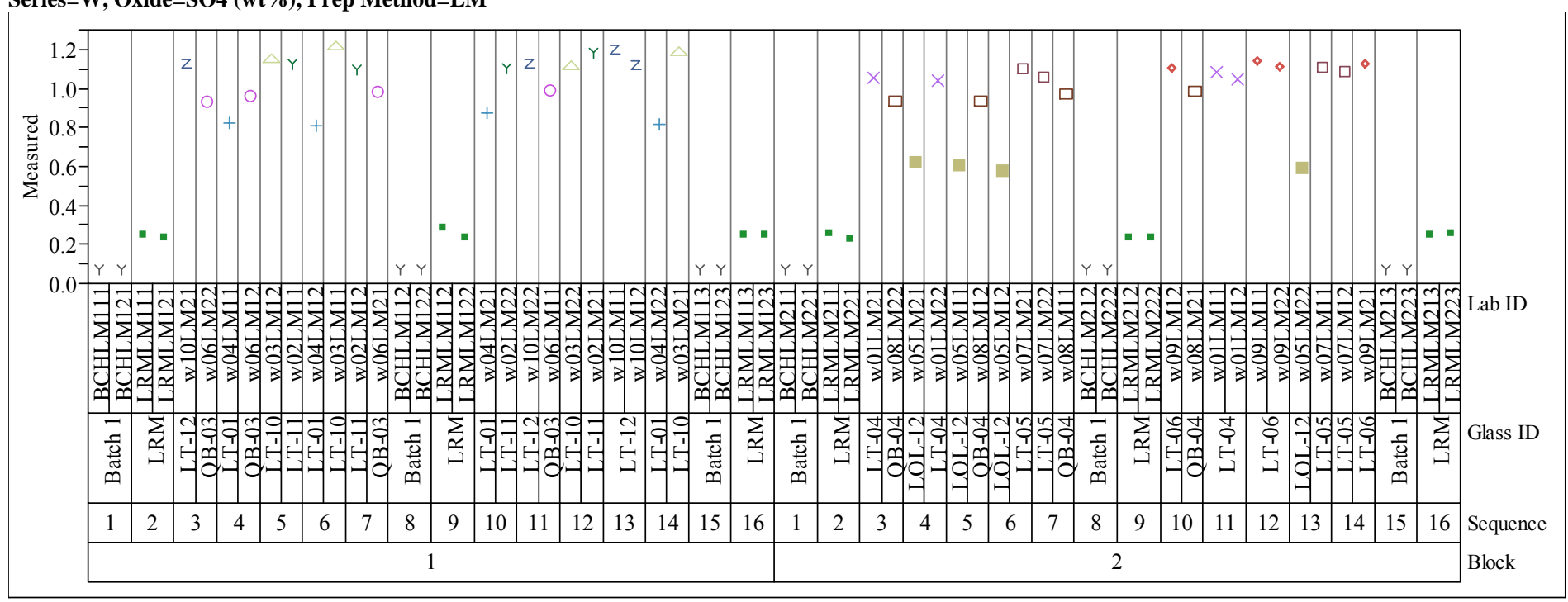

Series=W, Oxide=TiO2 (wt \%), Prep Method=LM

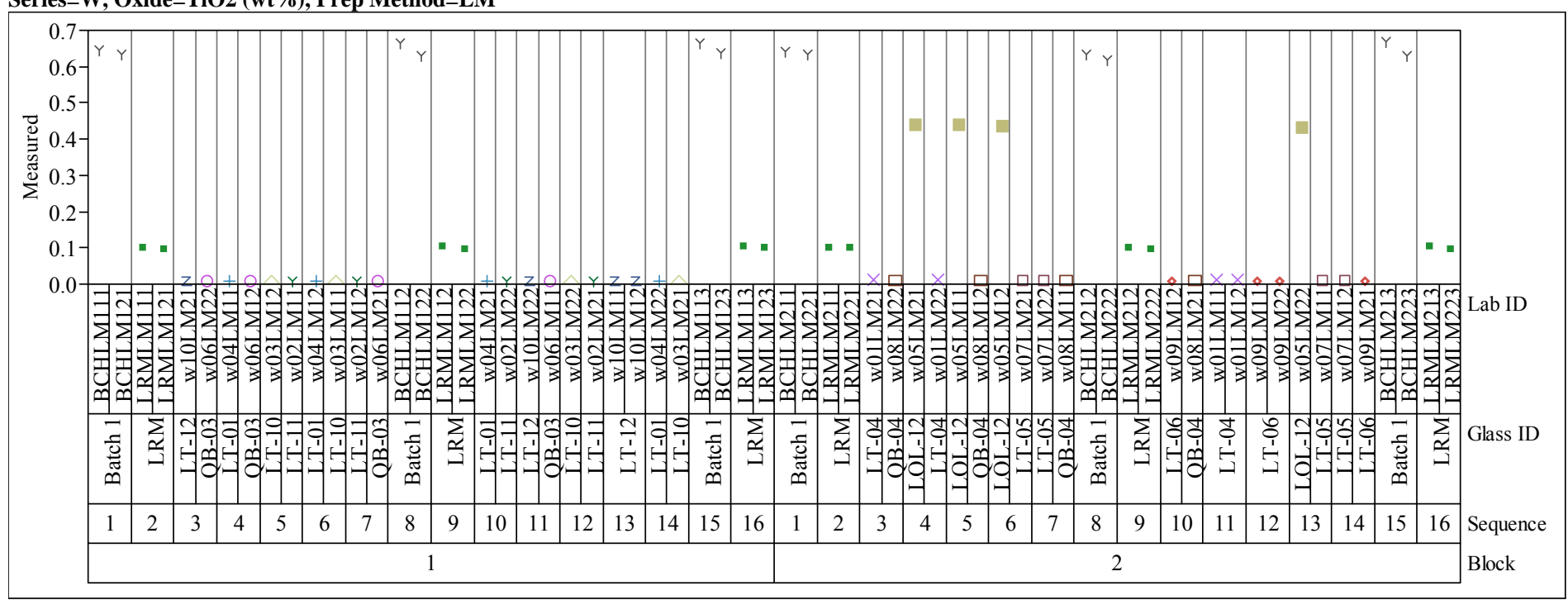


Exhibit A-1. Measurements of Glasses in Analytical Sequence by Oxide within Preparation Method and by Analytical Series (continued) Series $=W$, Oxide $=\mathrm{ZnO}(\mathrm{wt} \%)$, Prep Method $=\mathrm{LM}$

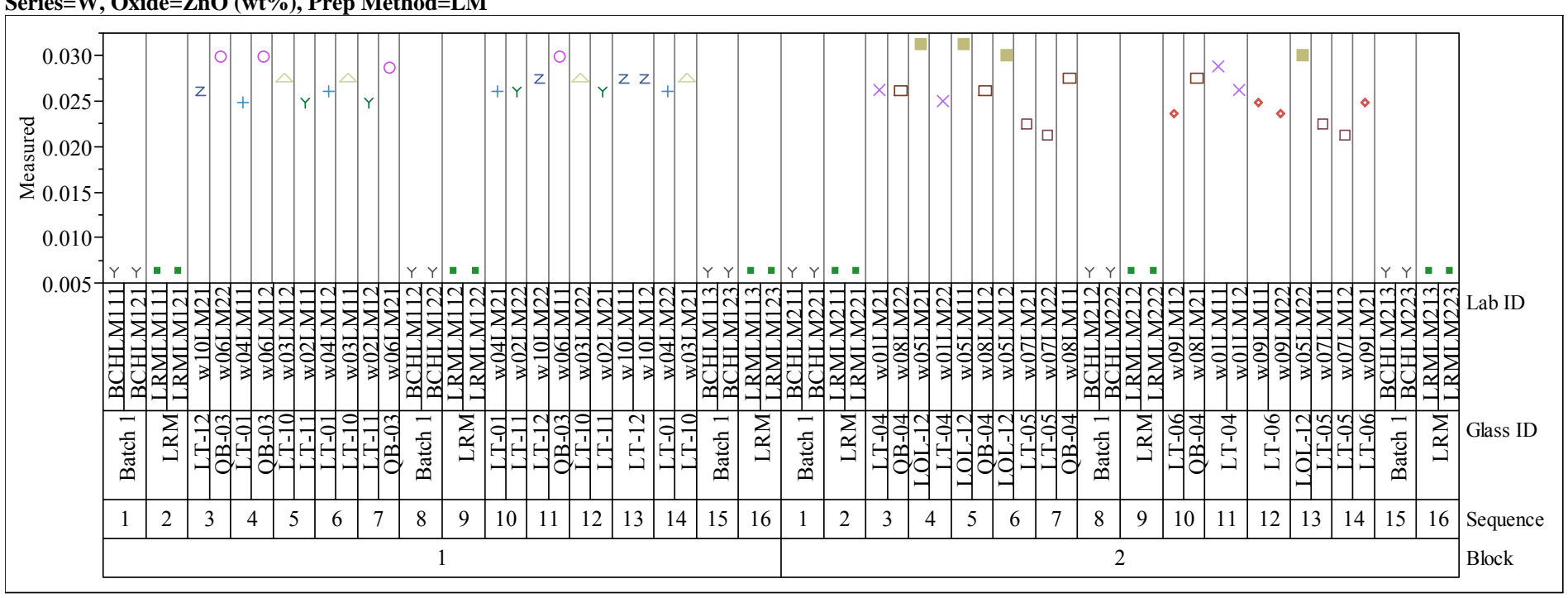

Series=W, Oxide=ZrO2 (wt\%), Prep Method=LM

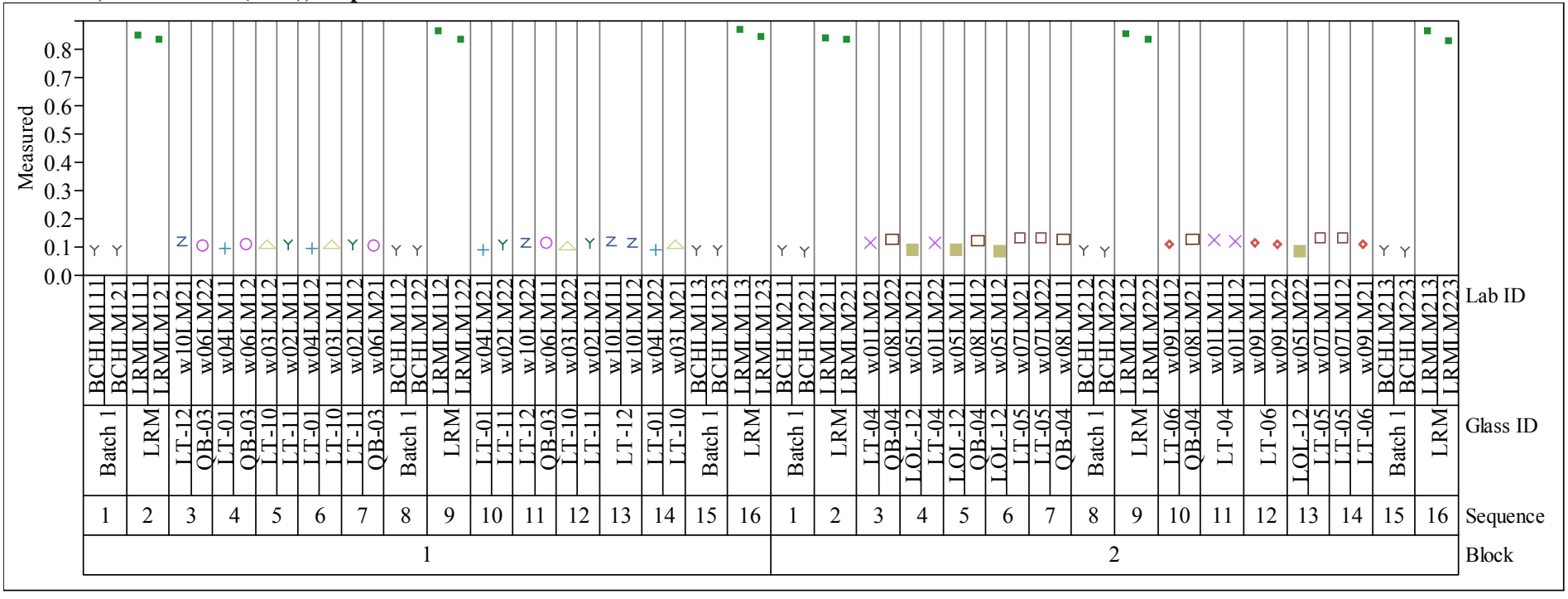




\section{Exhibit A-1. Measurements of Glasses in Analytical Sequence by Oxide within Preparation Method and by Analytical Series (continued)}

Series $=\mathrm{X}$, Oxide $=\mathrm{Al} 2 \mathrm{O} 3$ ( $\mathrm{wt} \%$ ), Prep Method $=\mathrm{LM}$

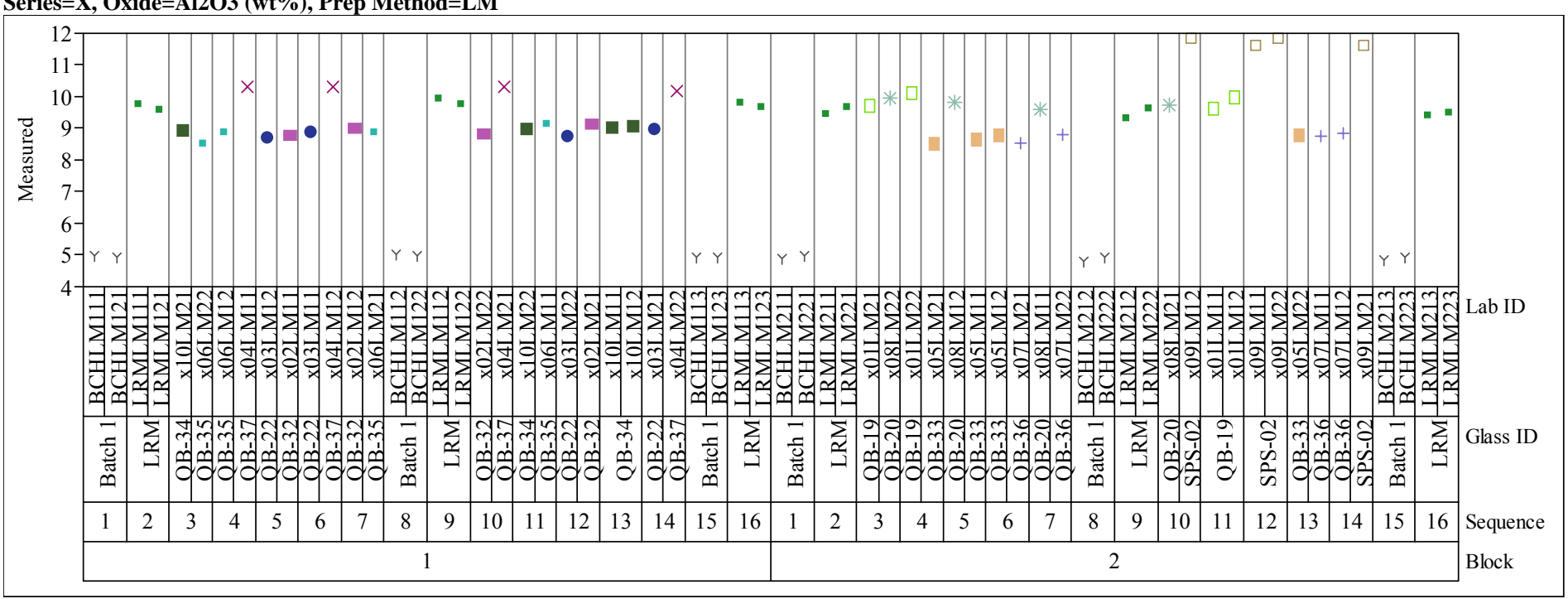

Series=X, Oxide=B2O3 (wt \%), Prep Method=PF

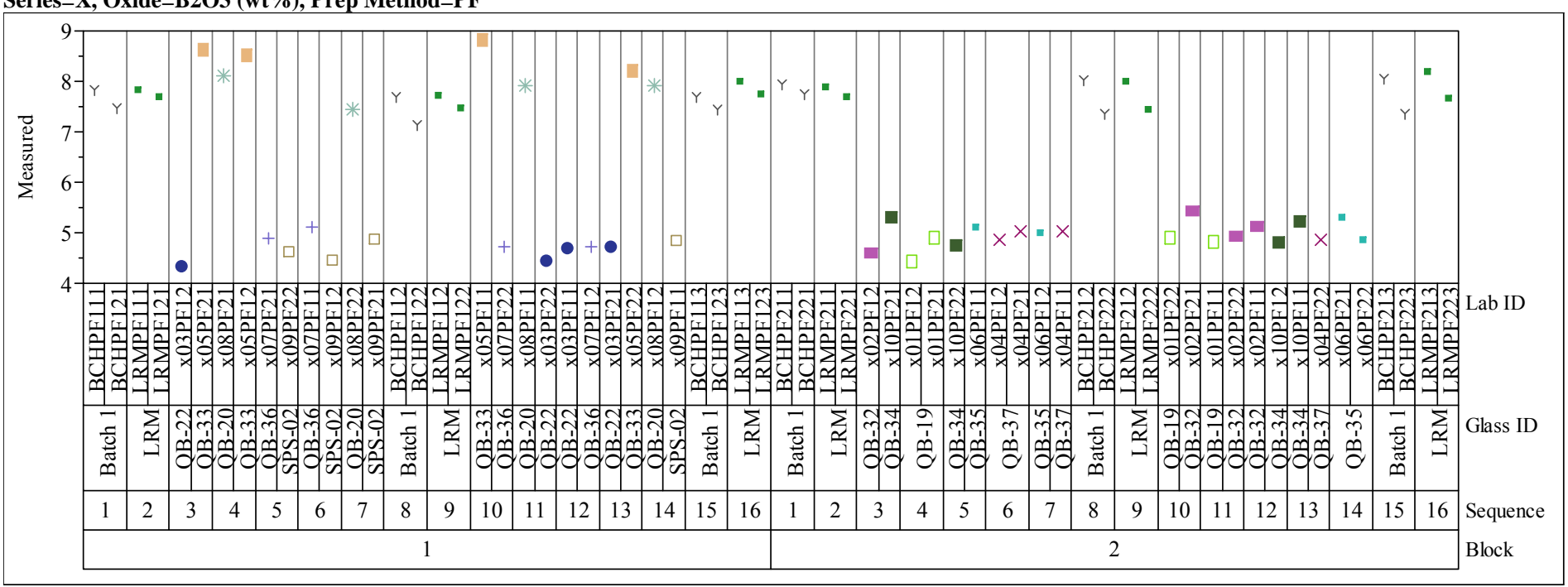




\section{Exhibit A-1. Measurements of Glasses in Analytical Sequence by Oxide within Preparation Method and by Analytical Series (continued)}

Series=X, Oxide=BaO (wt\%), Prep Method=LM

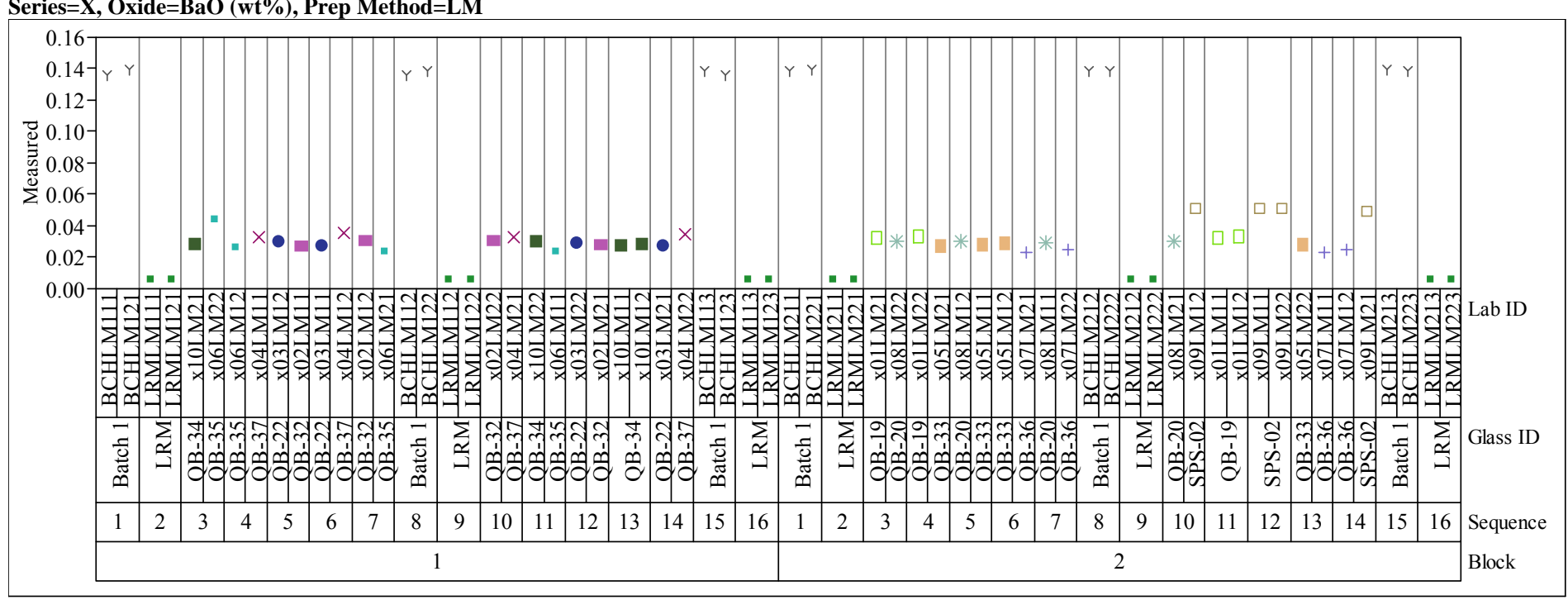

Series=X, Oxide=CaO (wt \%), Prep Method=LM
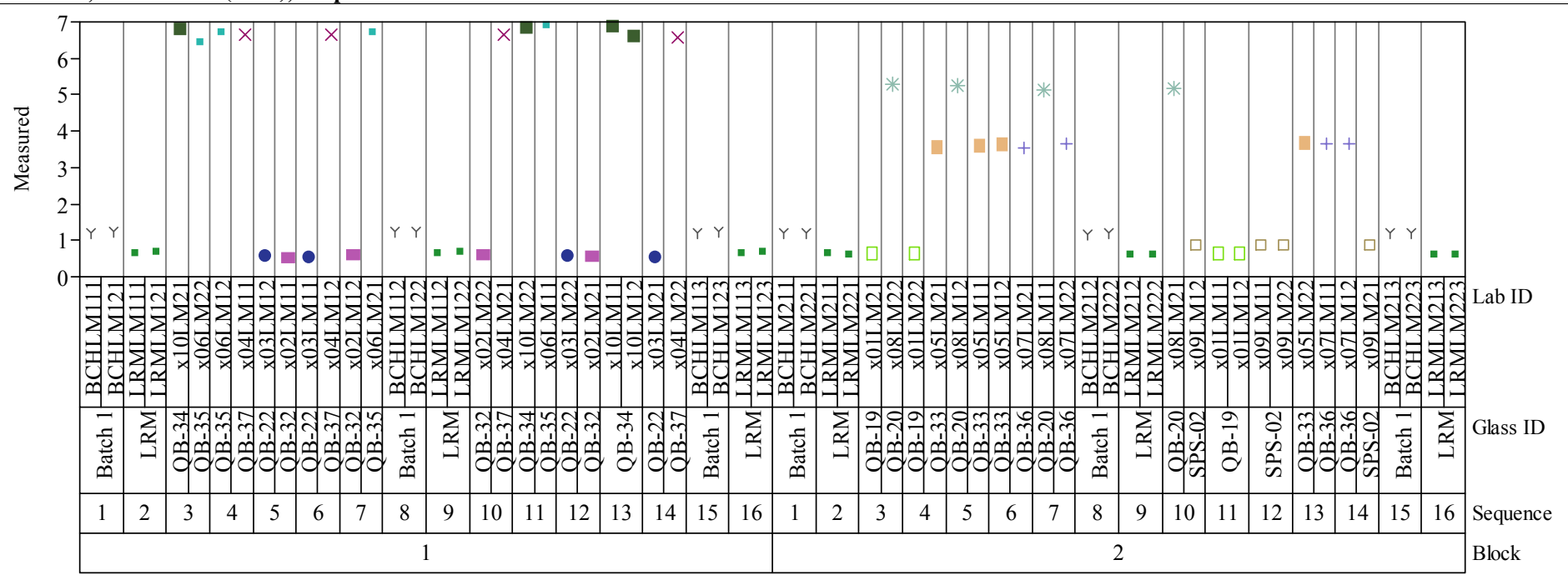
Exhibit A-1. Measurements of Glasses in Analytical Sequence by Oxide within Preparation Method and by Analytical Series (continued) Series $=X$, Oxide $=\mathrm{Ce} 2 \mathrm{O} 3(\mathrm{wt} \%)$, Prep Method $=\mathrm{LM}$

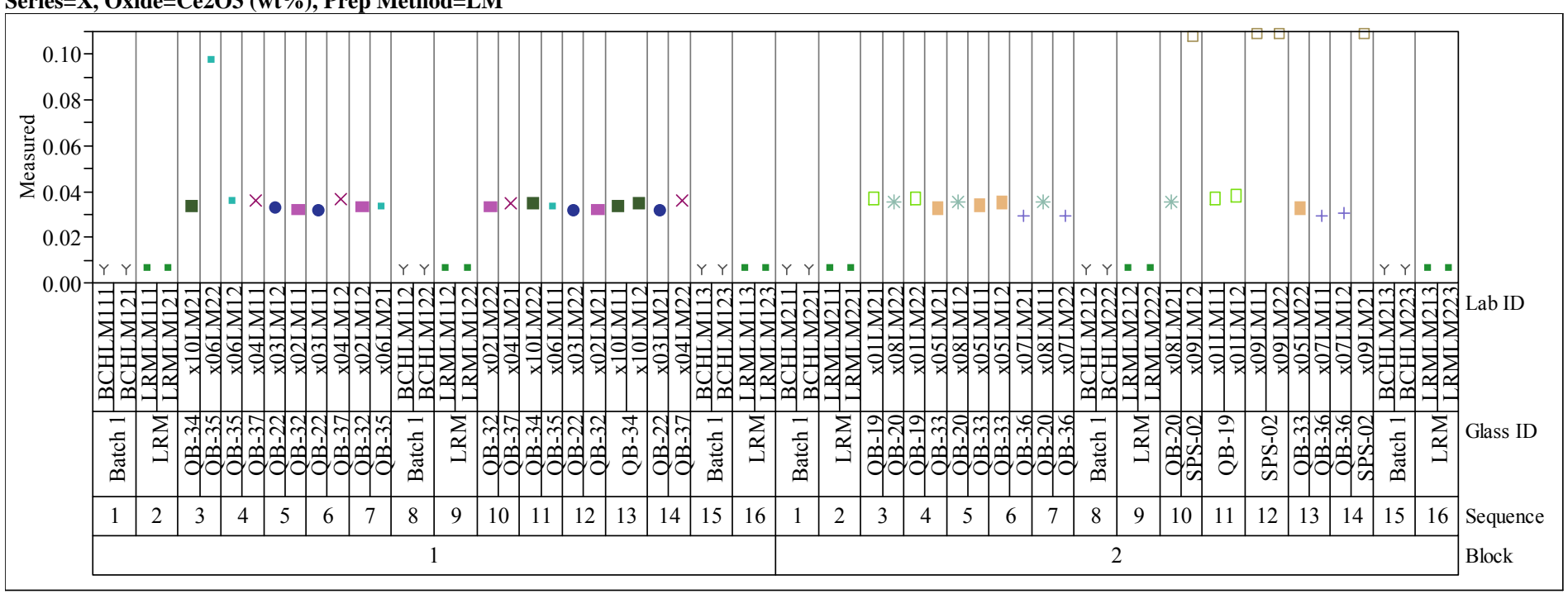

Series $=\mathrm{X}$, Oxide $=\mathrm{Cr} 2 \mathrm{O} 3(\mathrm{wt} \%)$, Prep Method $=\mathrm{LM}$

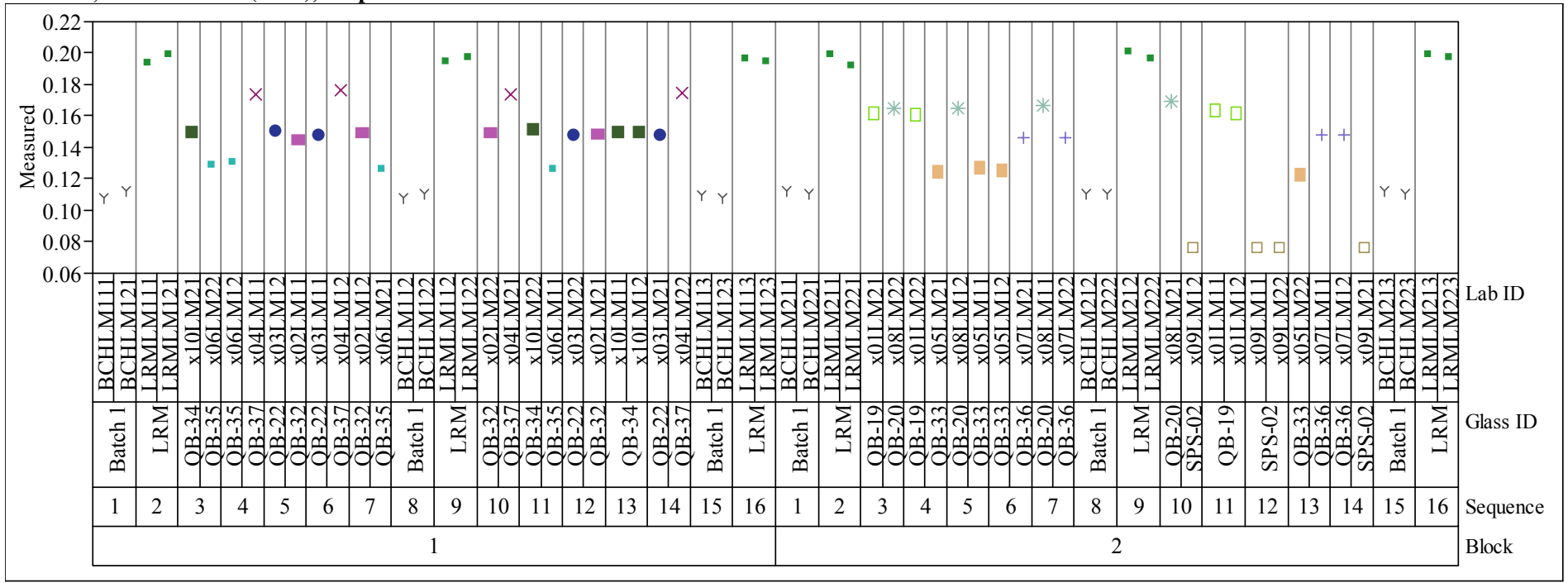




\section{Exhibit A-1. Measurements of Glasses in Analytical Sequence by Oxide within Preparation Method and by Analytical Series (continued)}

Series $=X$, Oxide $=\mathrm{CuO}(w t \%)$, Prep Method $=L M$

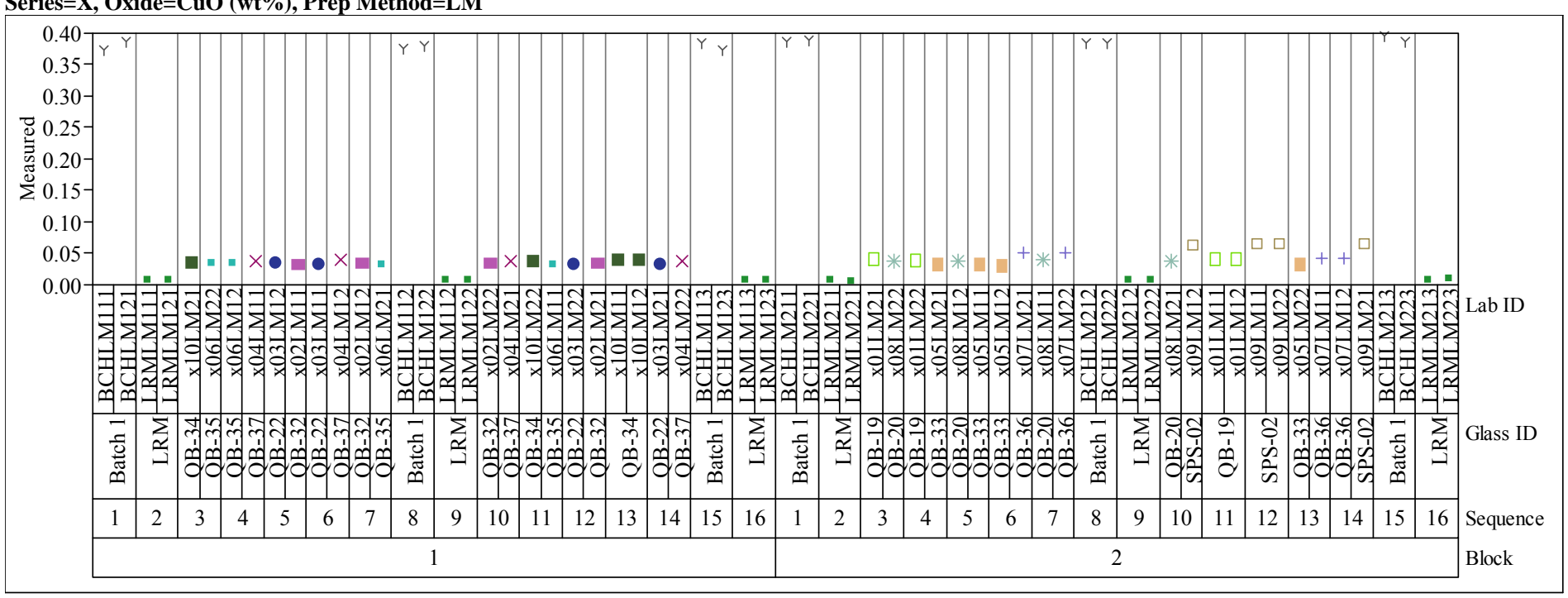

Series $=$ X, Oxide=Fe2O3 (wt\%), Prep Method=LM

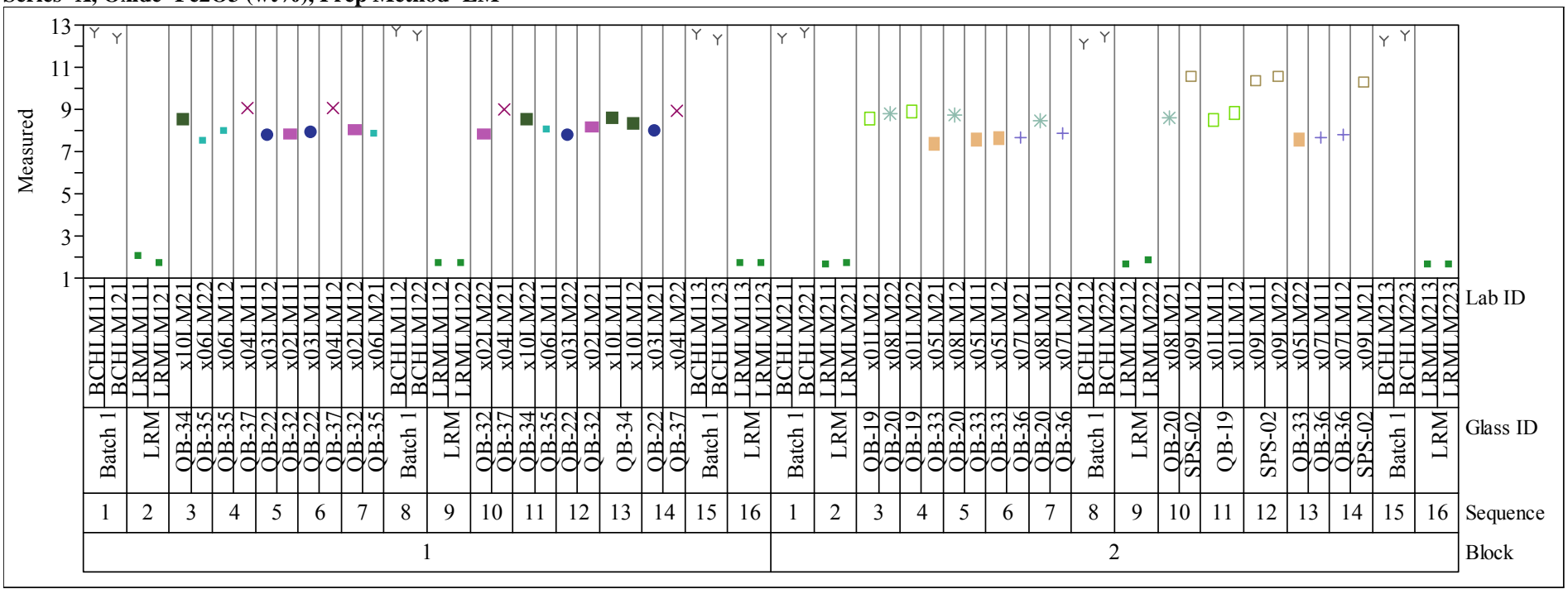




\section{Exhibit A-1. Measurements of Glasses in Analytical Sequence by Oxide within Preparation Method and by Analytical Series (continued)}

Series $=\mathrm{X}$, Oxide $=\mathrm{K} 20$ (wt\%), Prep Method $=\mathbf{L M}$

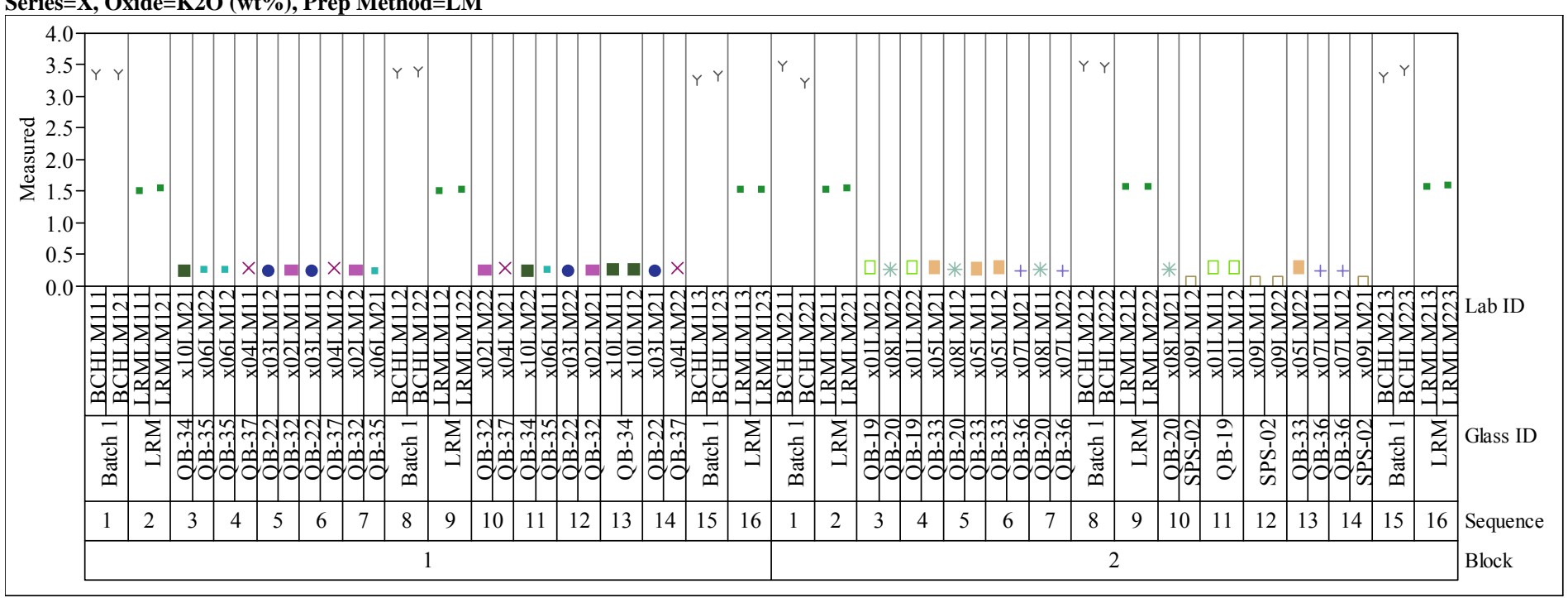

Series $=$ X, Oxide=La2O3 (wt \%), Prep Method $=$ LM

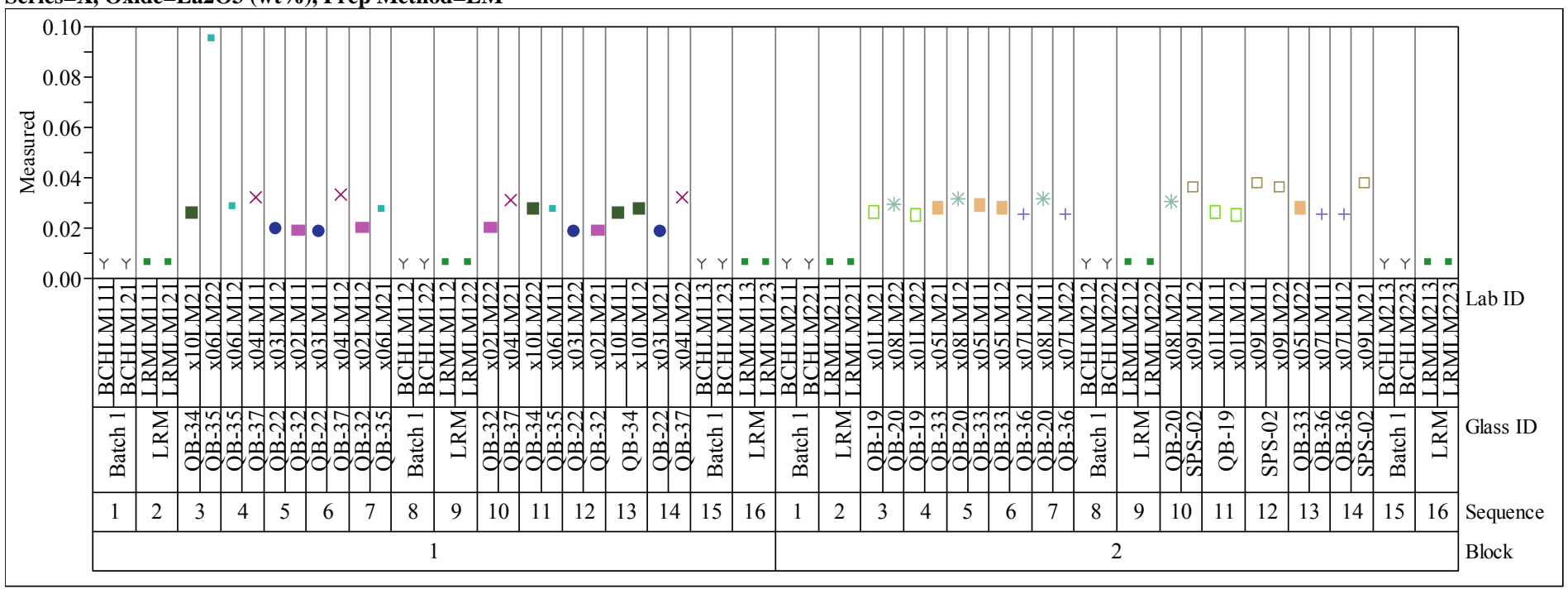




\section{Exhibit A-1. Measurements of Glasses in Analytical Sequence by Oxide within Preparation Method and by Analytical Series (continued)} Series $=X$, Oxide $=$ Li2O (wt \%), Prep Method $=$ PF

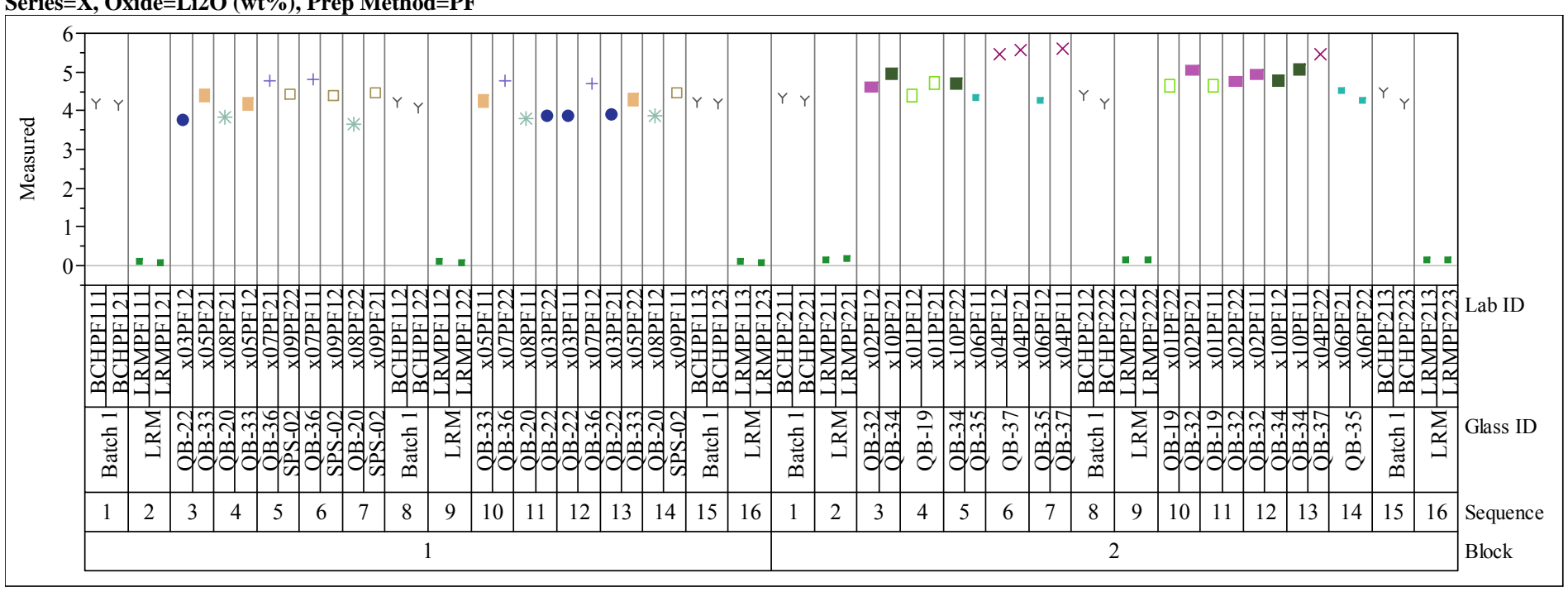

\section{Series=X, Oxide=MgO (wt \%), Prep Method $=$ LM}

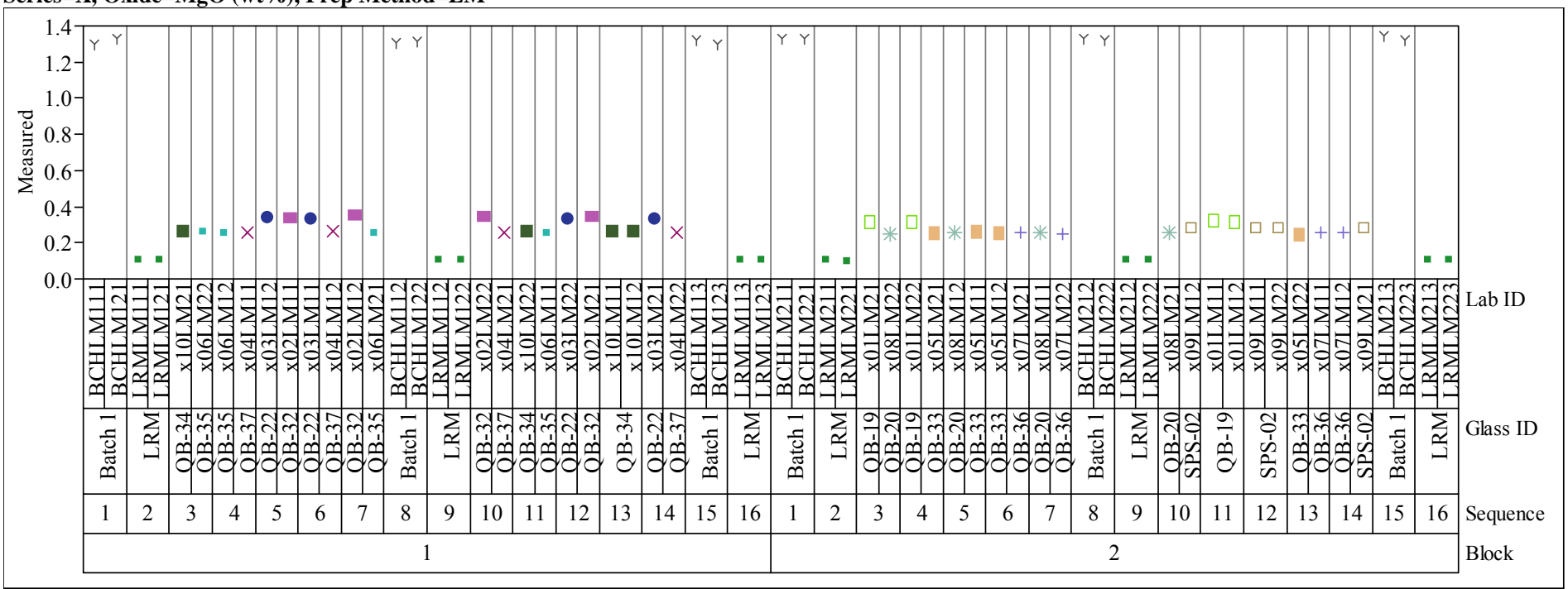




\section{Exhibit A-1. Measurements of Glasses in Analytical Sequence by Oxide within Preparation Method and by Analytical Series (continued)}

Series $=X$, Oxide $=\mathrm{MnO}(\mathrm{wt} \%)$, Prep Method $=\mathrm{LM}$

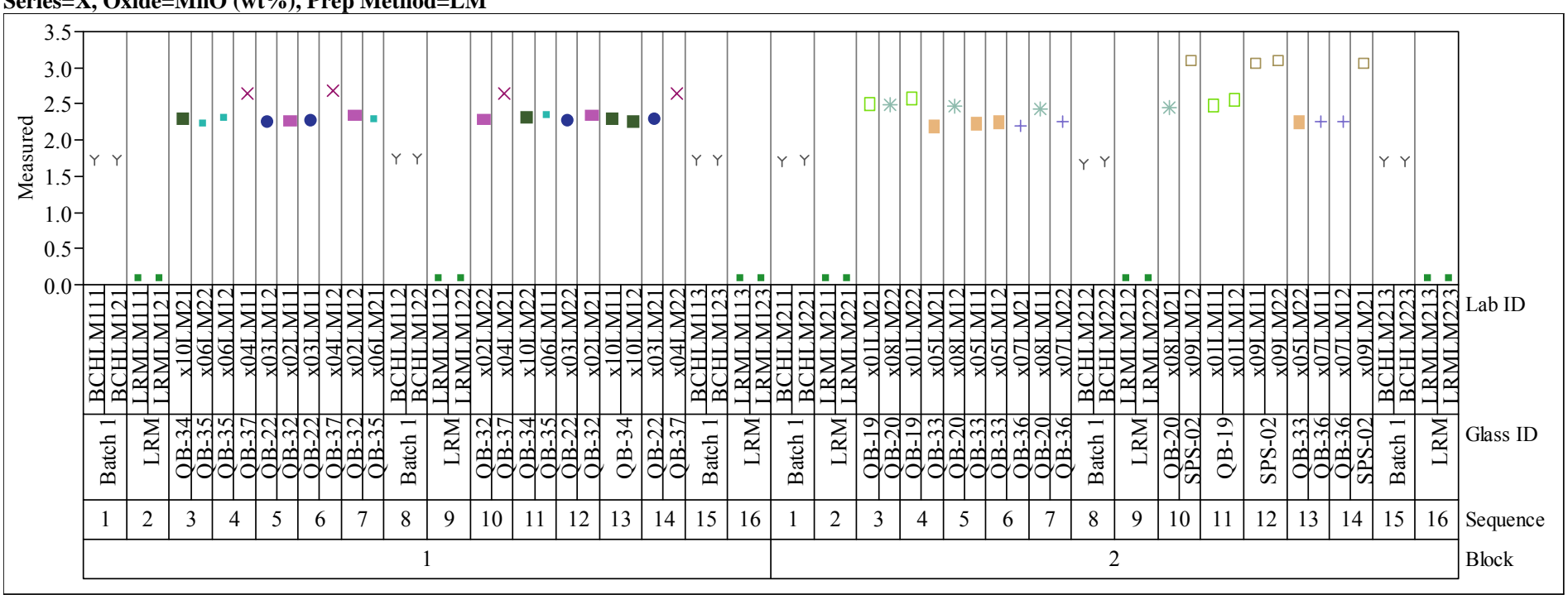

Series $=X$, Oxide=Na2O (wt \%), Prep Method=LM

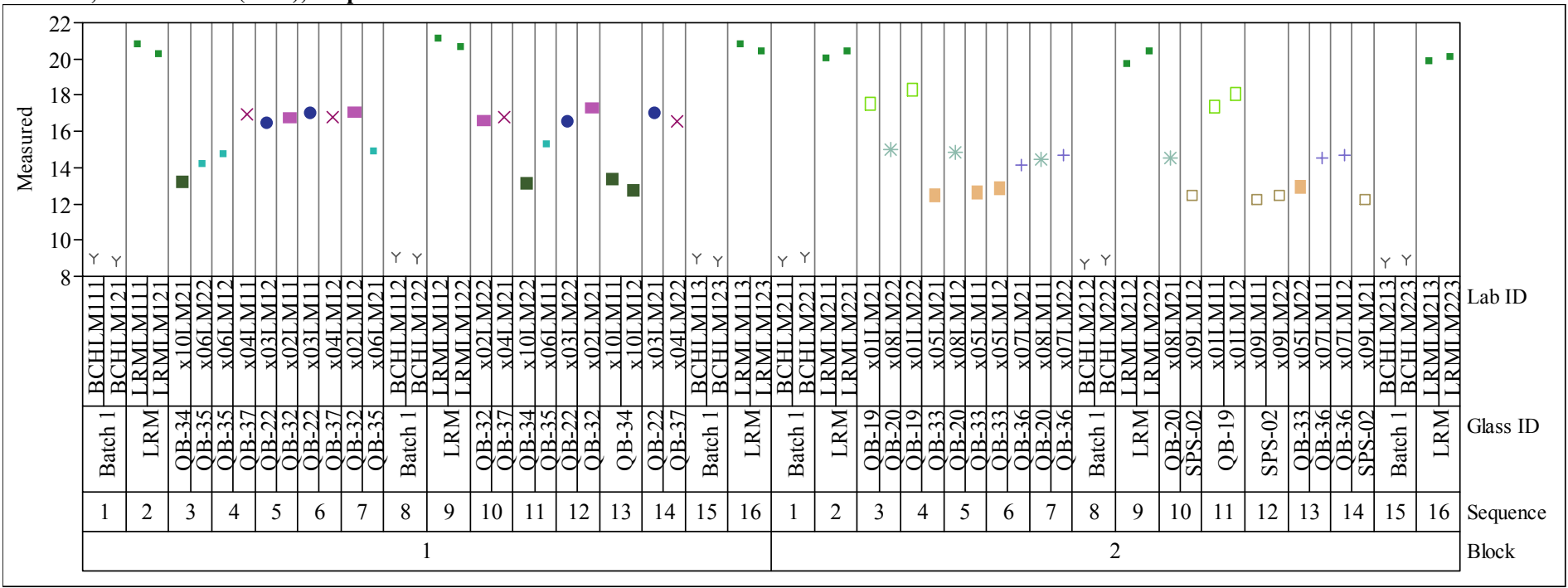




\section{Exhibit A-1. Measurements of Glasses in Analytical Sequence by Oxide within Preparation Method and by Analytical Series (continued)} Series $=\mathrm{X}$, Oxide $=\mathrm{NiO}(\mathrm{wt} \%)$, Prep Method $=\mathrm{LM}$

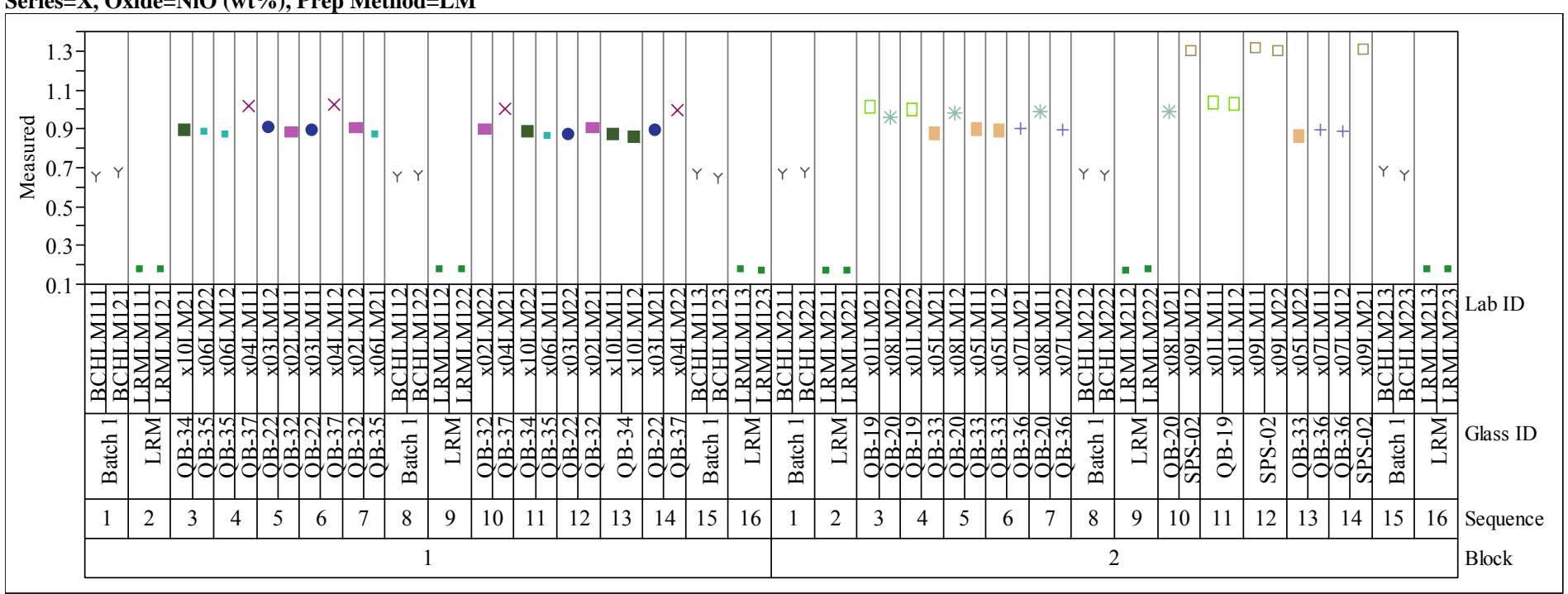

Series $=$ X, Oxide $=$ PbO (wt \%), Prep Method $=$ LM

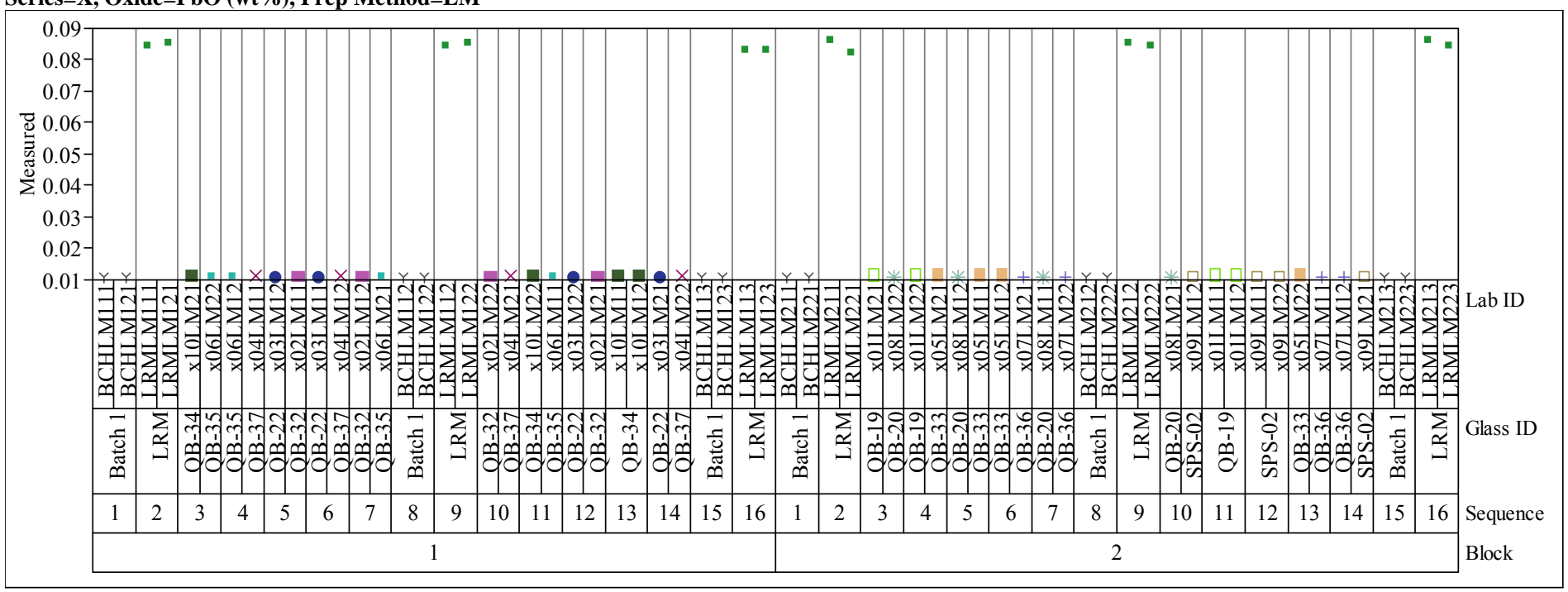




\section{Exhibit A-1. Measurements of Glasses in Analytical Sequence by Oxide within Preparation Method and by Analytical Series (continued)} Series $=X$, Oxide $=$ SiO2 $(w t \%)$, Prep Method $=$ PF

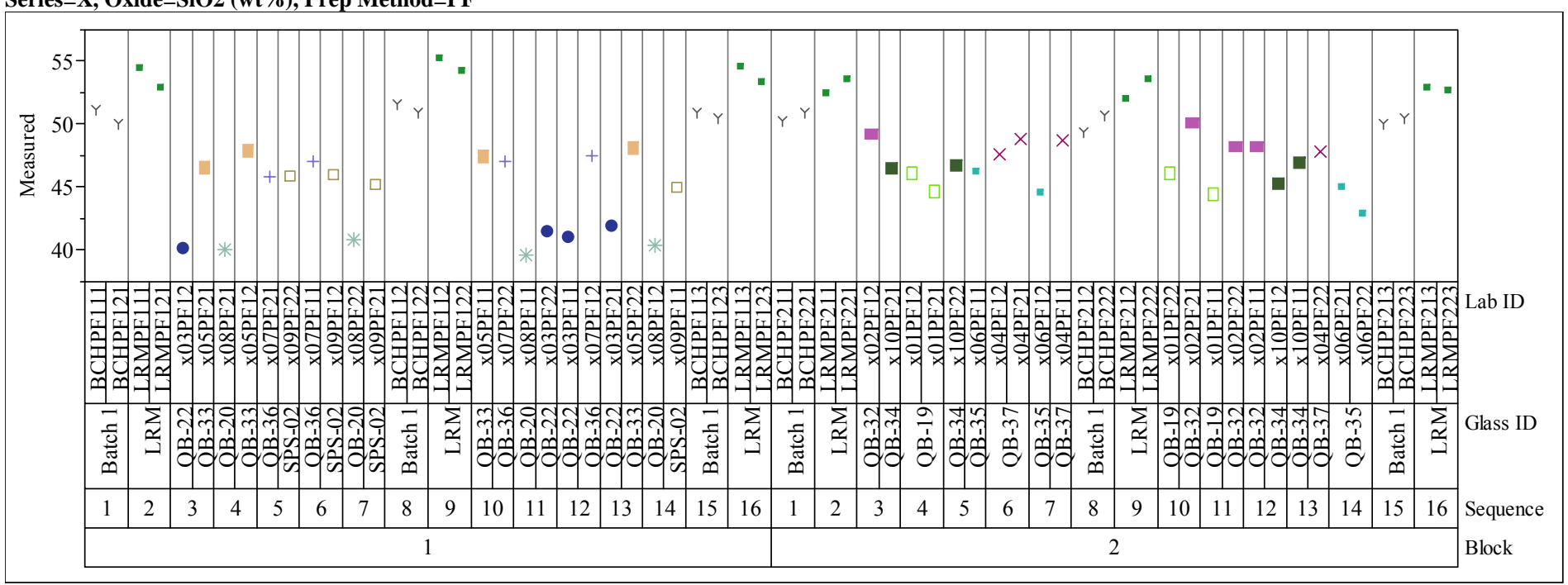

Series=X, Oxide=SO4 (wt\%), Prep Method=LM

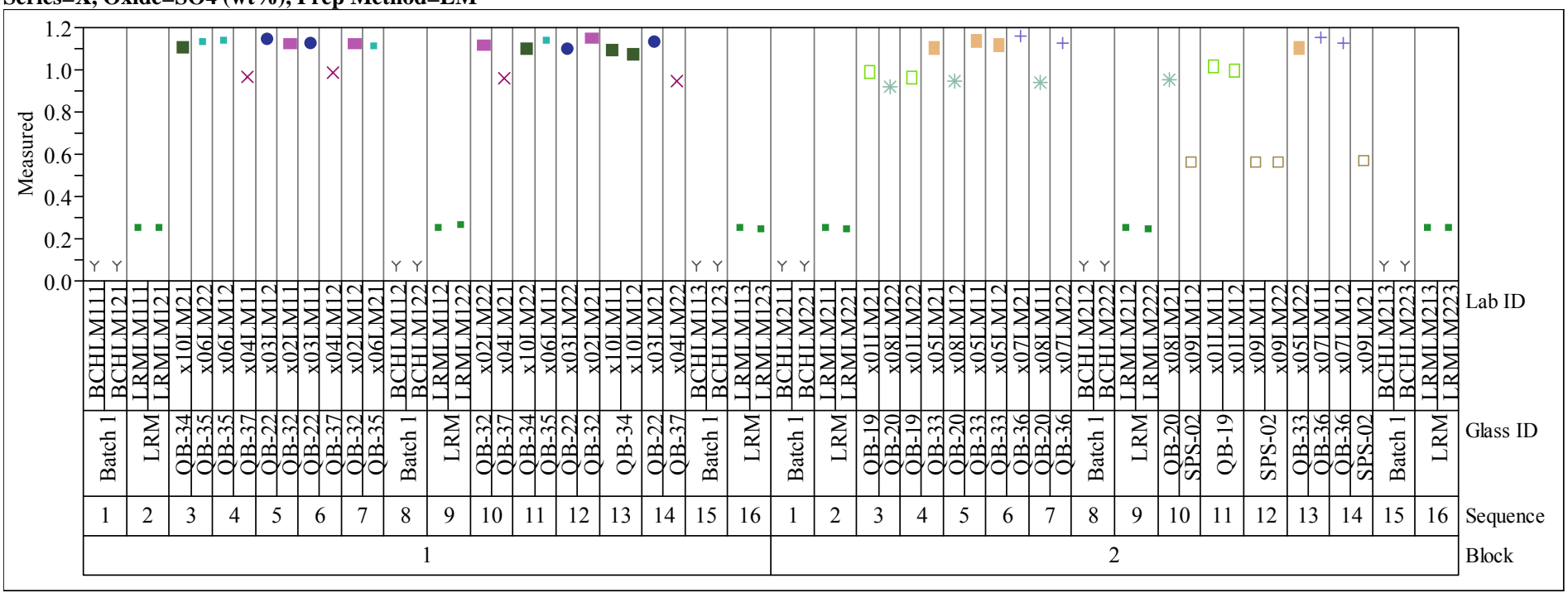




\section{Exhibit A-1. Measurements of Glasses in Analytical Sequence by Oxide within Preparation Method and by Analytical Series (continued)}

Series $=X$, Oxide $=$ TiO2 $(w t \%)$, Prep Method $=L M$

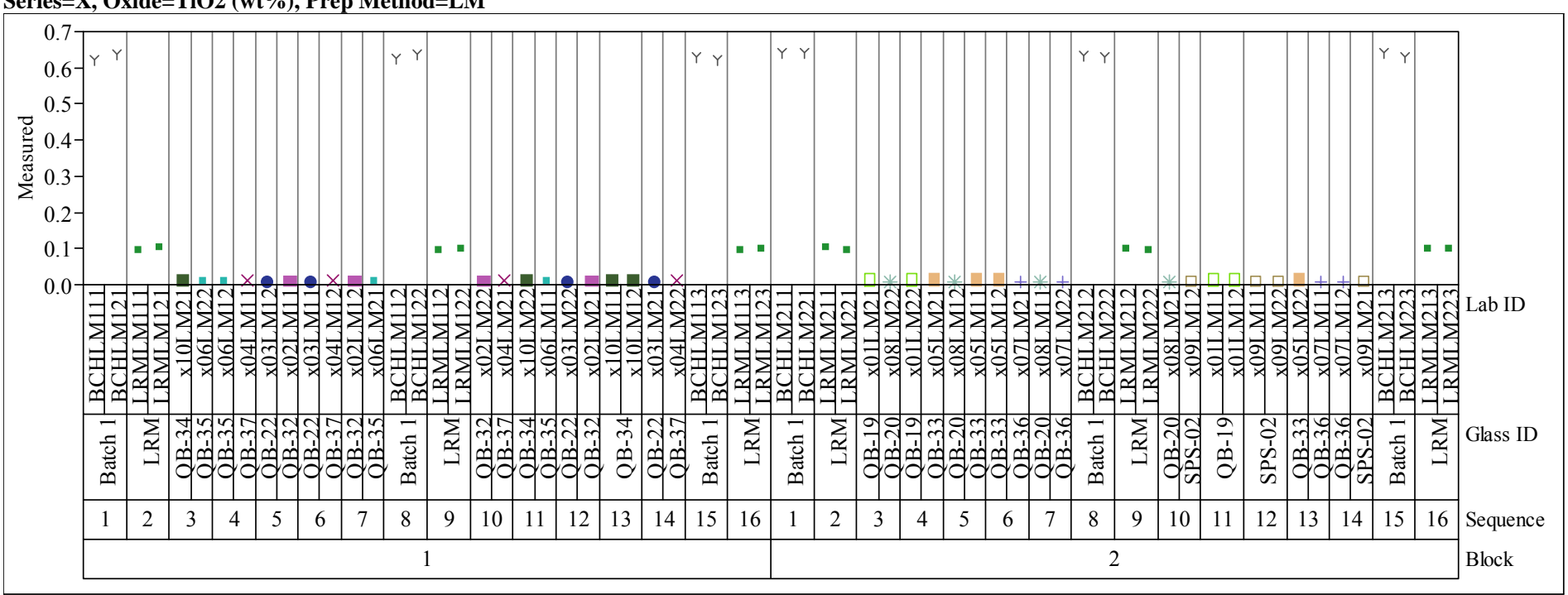

Series $=\mathrm{X}$, Oxide $=\mathrm{ZnO}(\mathrm{wt} \%)$, Prep Method $=\mathrm{LM}$

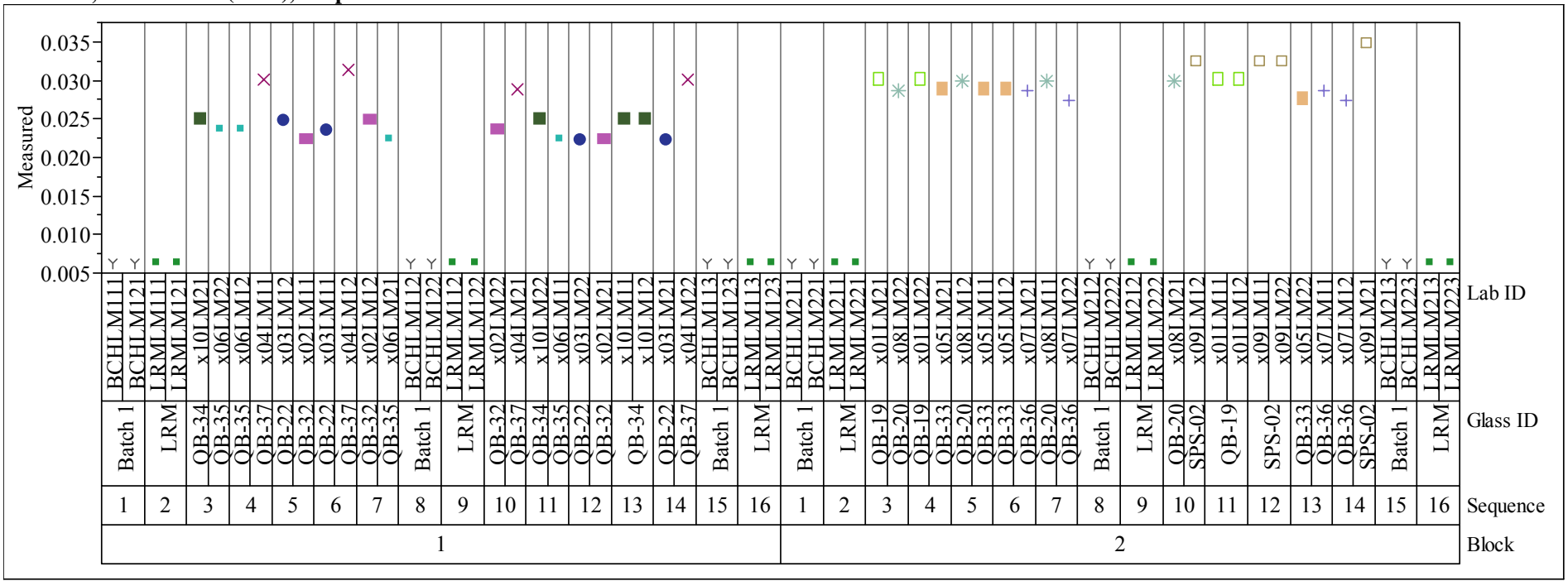


Exhibit A-1. Measurements of Glasses in Analytical Sequence by Oxide within Preparation Method and by Analytical Series (continued) Series $=\mathrm{X}$, Oxide $=\mathrm{ZrO} 2$ ( $w t \%)$, Prep Method $=$ LM

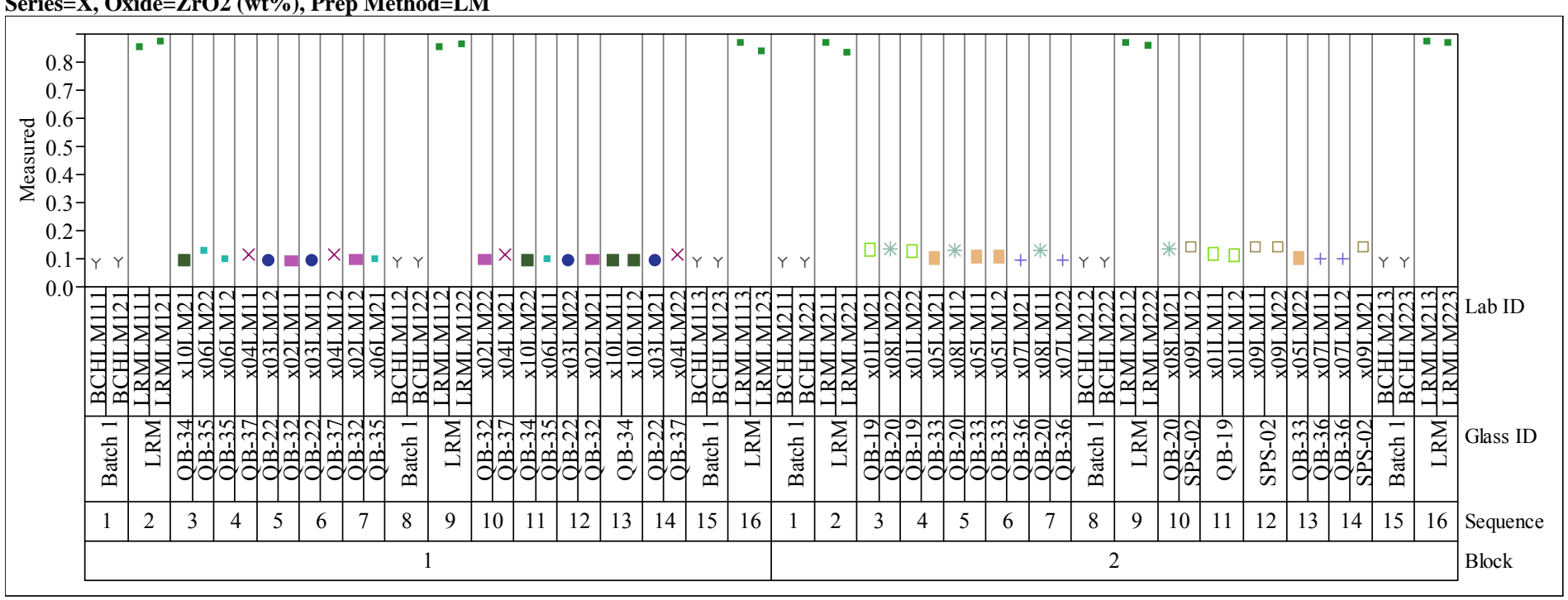


Exhibit A-2. Measurements by Lab ID within Glass ID by Targeted Concentration for Each Oxide for Each Prep by Analytical Series

Series=T, Oxide=Al2O3 (wt \%), Prep Method=LM

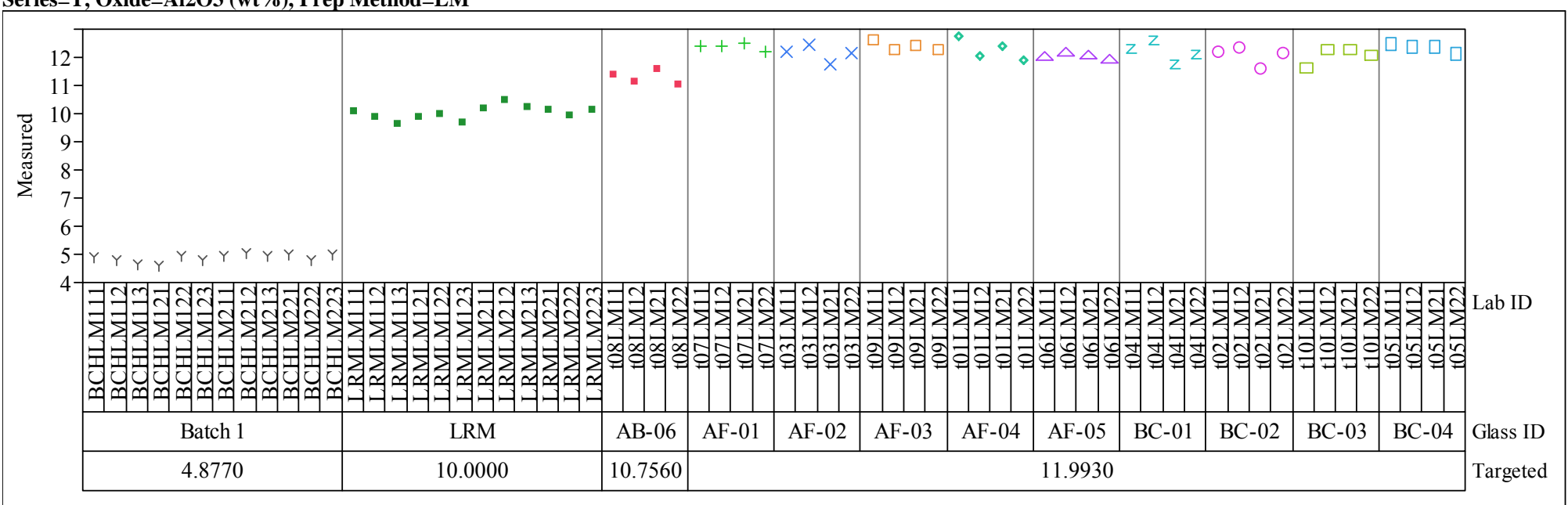

Series=T, Oxide=B2O3 (wt \%), Prep Method $=$ PF

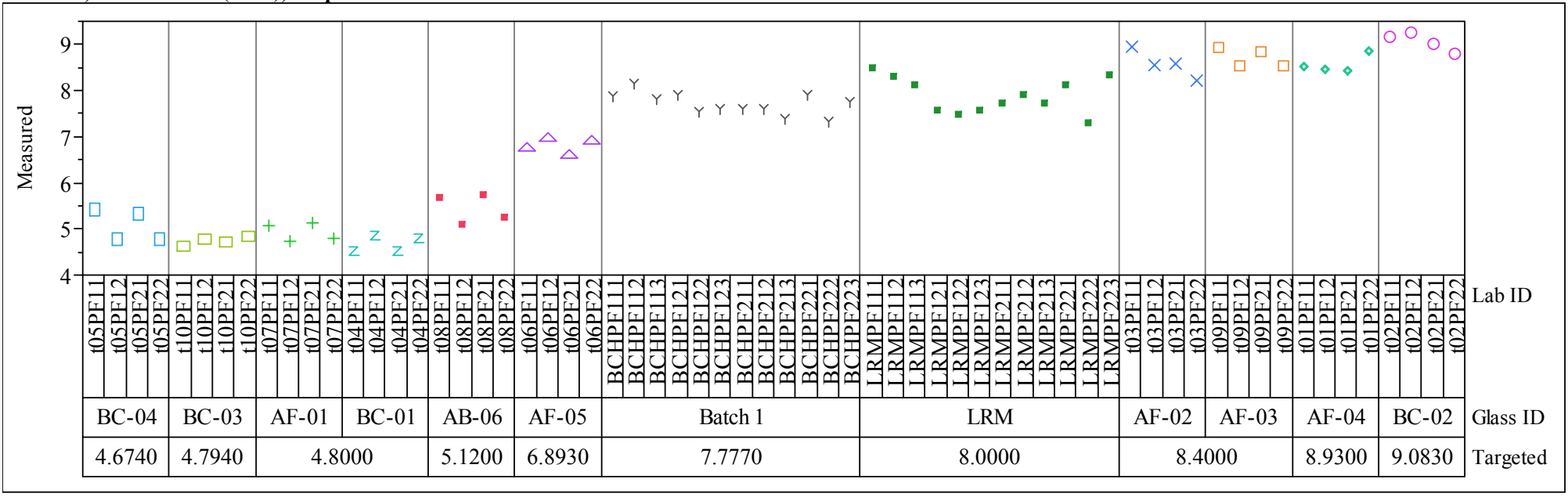


Exhibit A-2. Measurements by Lab ID within Glass ID by Targeted Concentration for Each Oxide for Each Prep by Analytical Series (continued)

Series=T, Oxide=BaO (wt \%), Prep Method $=$ LM

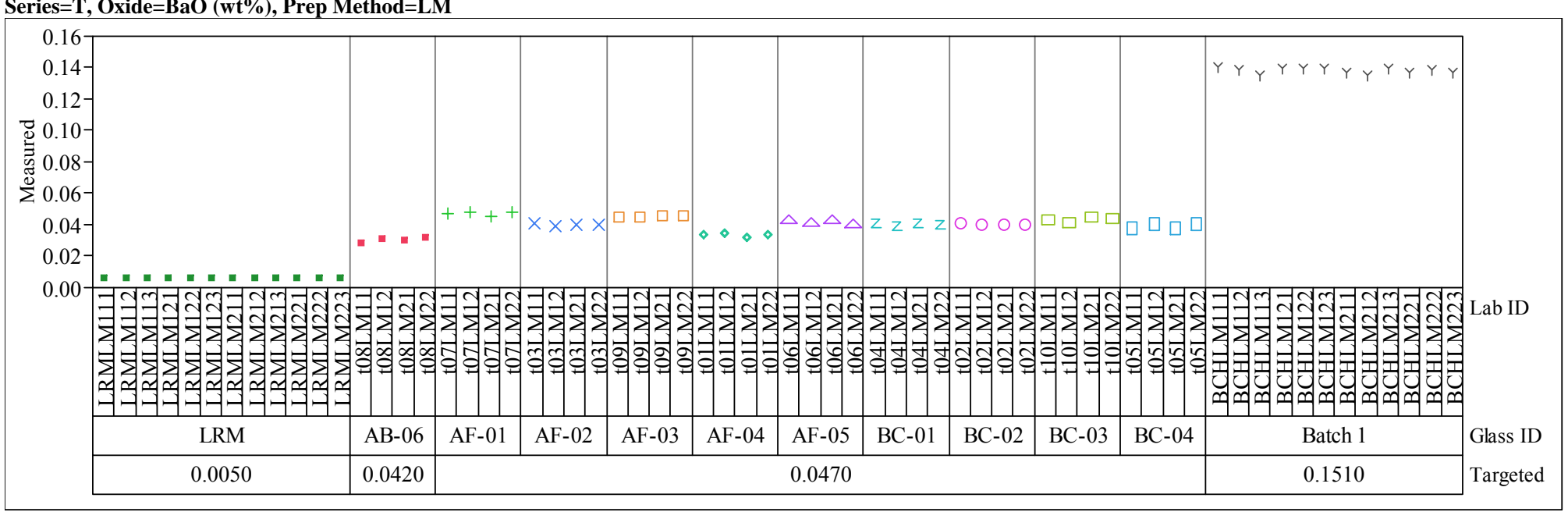

Series $=\mathrm{T}$, Oxide $=\mathrm{CaO}(\mathrm{wt} \%)$, Prep Method $=\mathrm{LM}$

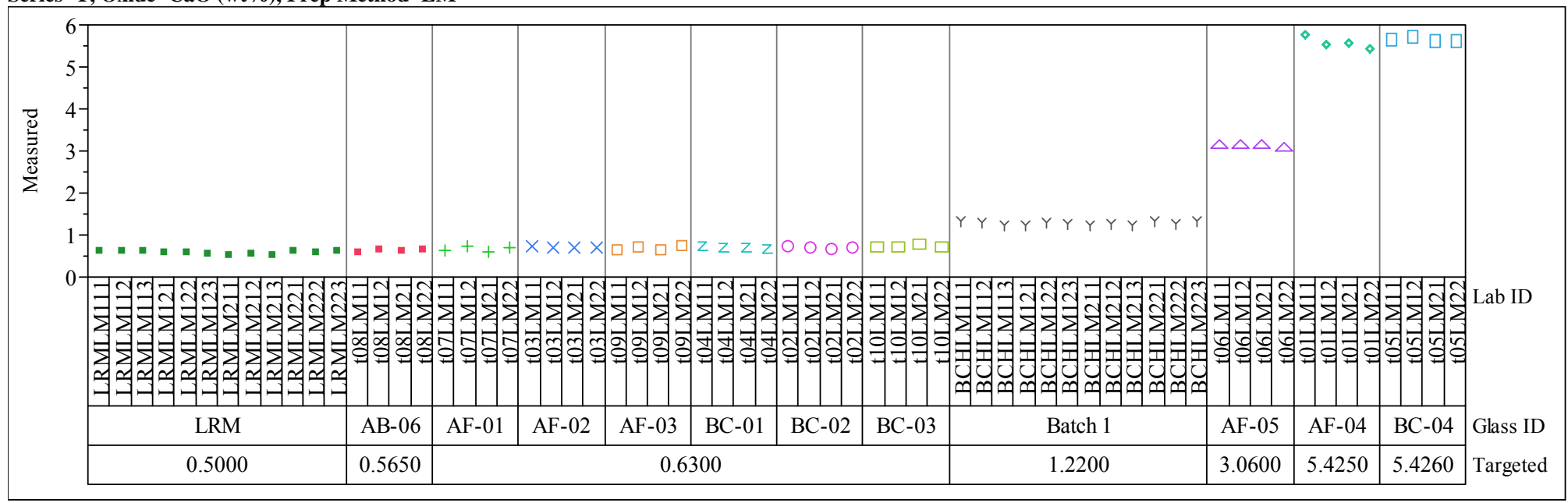


Exhibit A-2. Measurements by Lab ID within Glass ID by Targeted Concentration for Each Oxide for Each Prep by Analytical Series (continued)

Series $=$ T, Oxide $=$ Ce2O3 $($ wt $\%)$, Prep Method $=$ LM

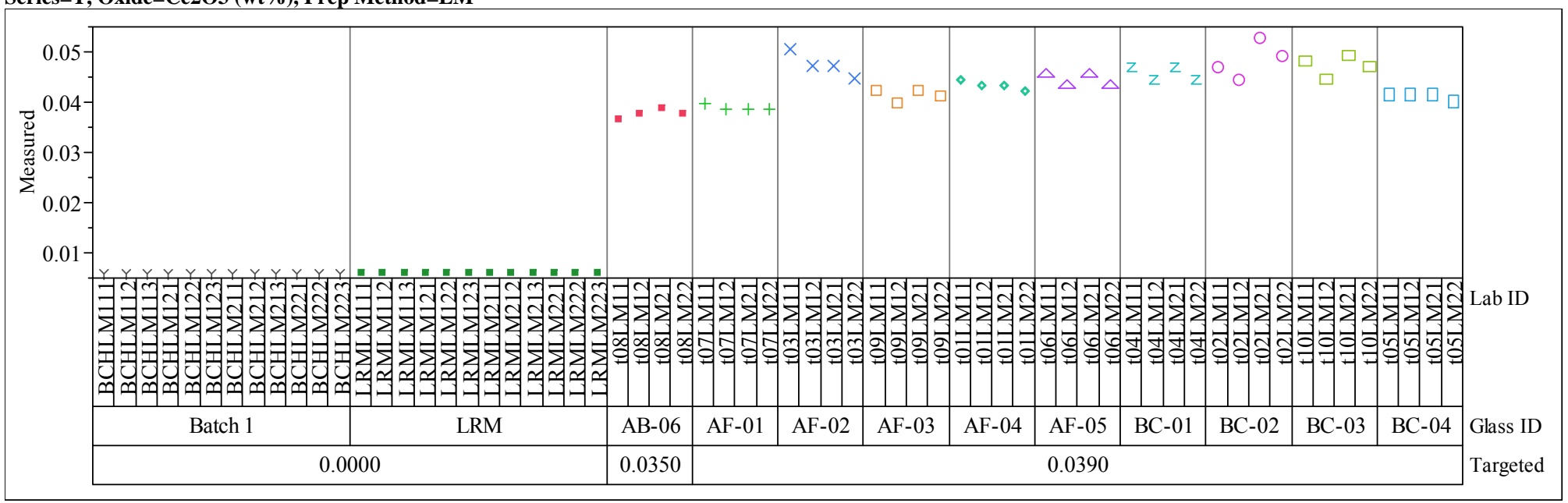

Series=T, Oxide=Cr2O3 (wt\%), Prep Method=LM

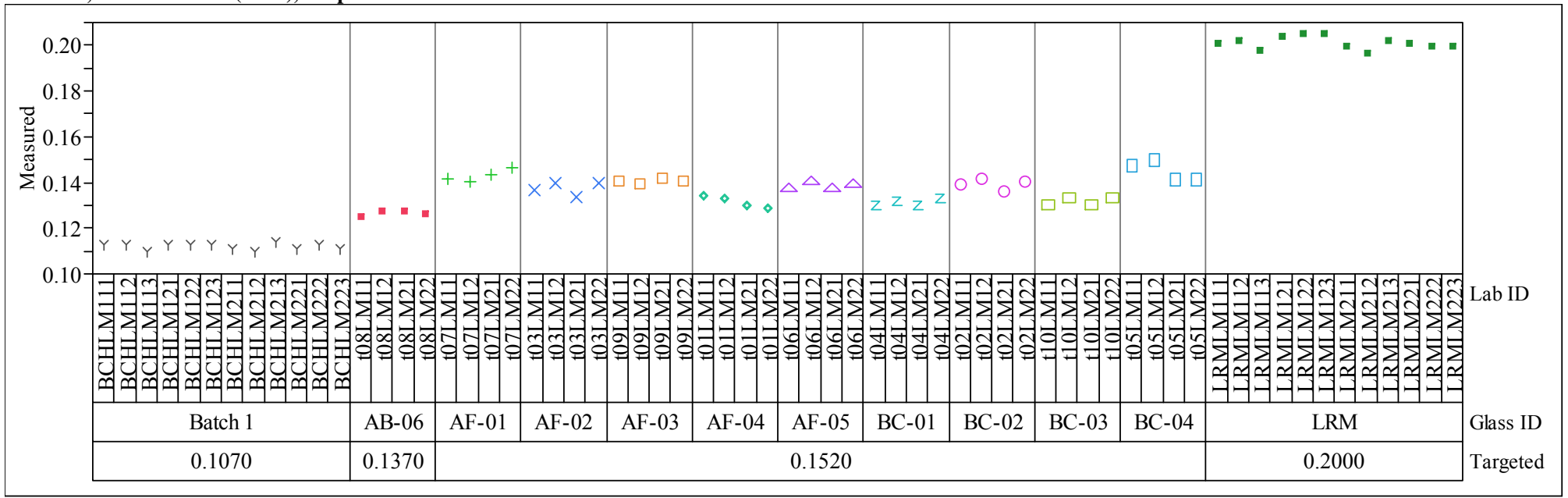


Exhibit A-2. Measurements by Lab ID within Glass ID by Targeted Concentration for Each Oxide for Each Prep by Analytical Series (continued)

Series $=\mathrm{T}$, Oxide $=\mathrm{CuO}(\mathrm{wt} \%)$, Prep Method $=\mathrm{LM}$

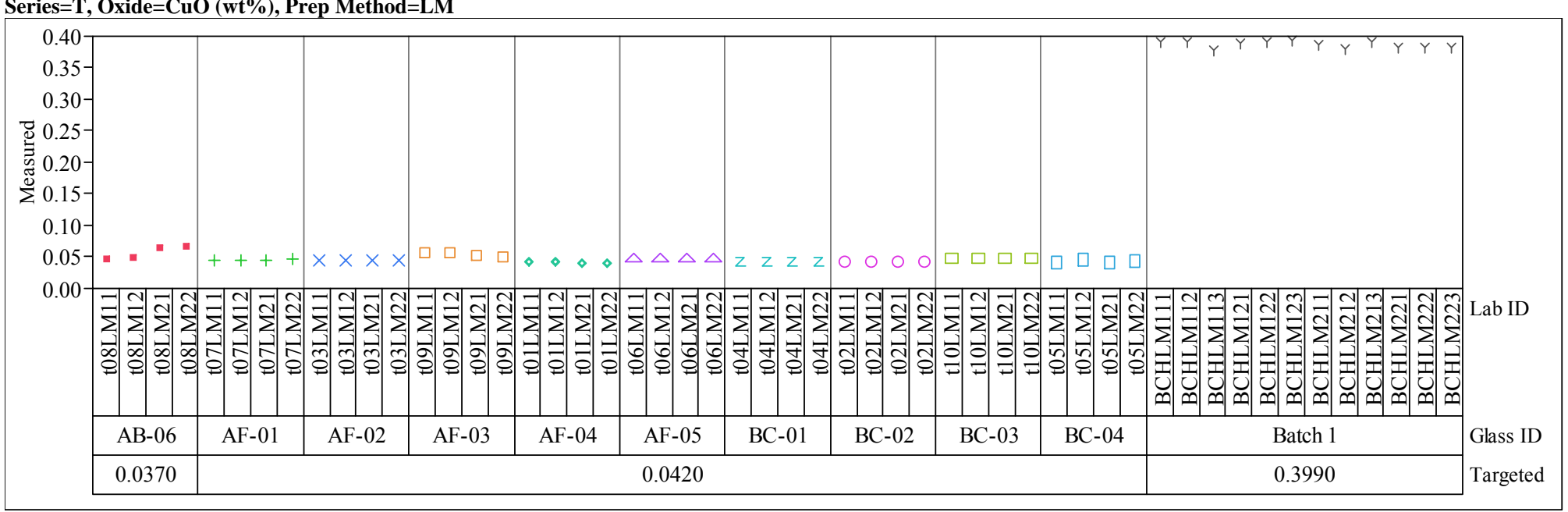

Series=T, Oxide=Fe2O3 (wt\%), Prep Method=LM

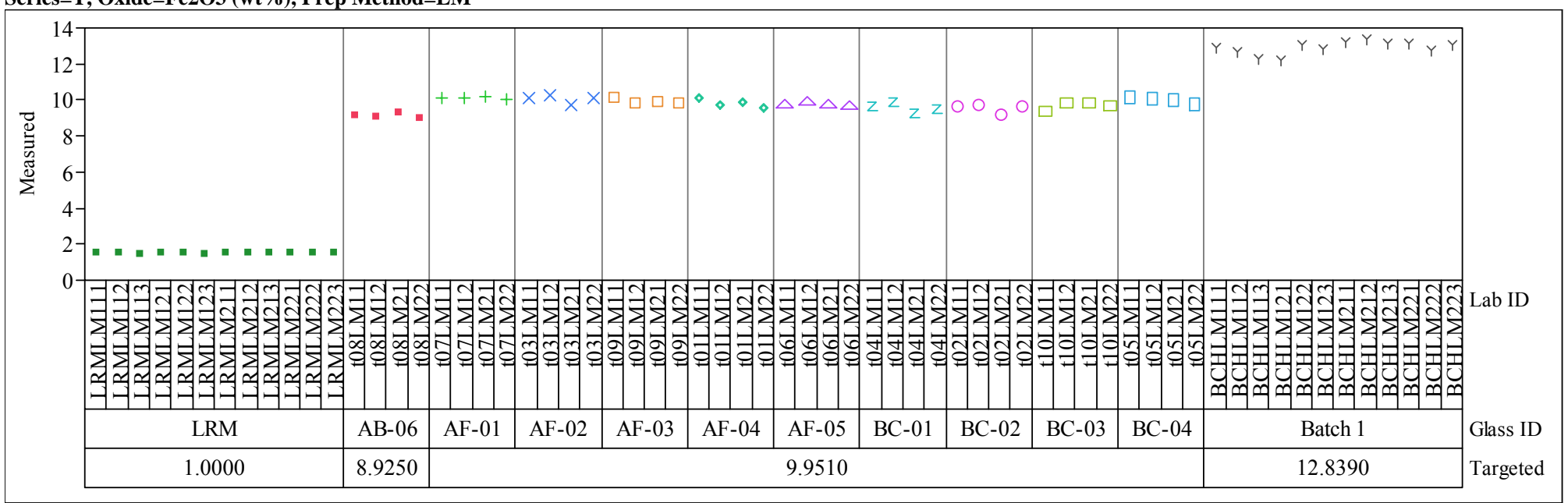


Exhibit A-2. Measurements by Lab ID within Glass ID by Targeted Concentration for Each Oxide for Each Prep by Analytical Series (continued)

Series $=$ T, Oxide=K2O (wt\%), Prep Method $=$ LM

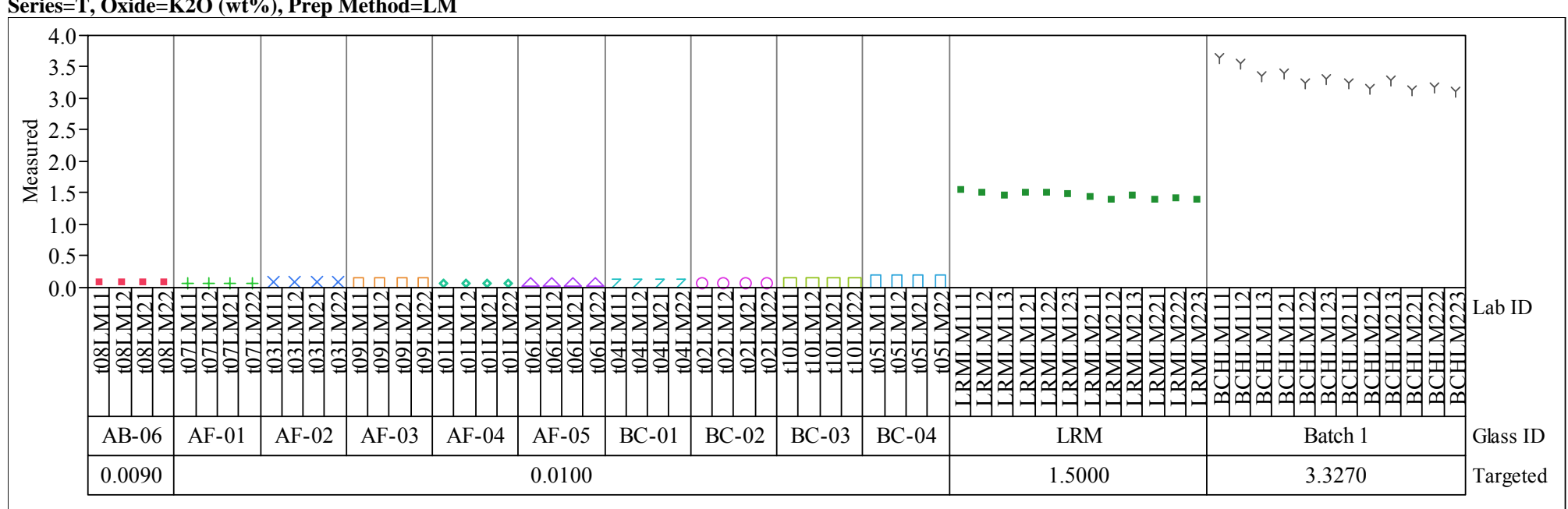

Series=T, Oxide=La2O3 (wt\%), Prep Method=LM

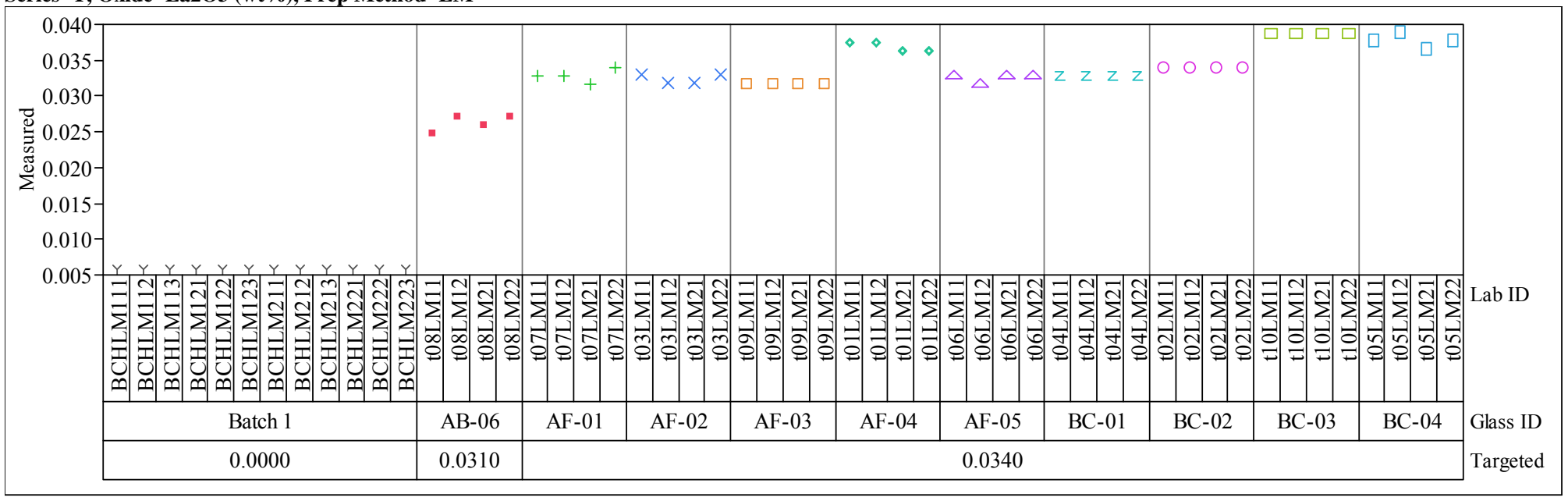


Exhibit A-2. Measurements by Lab ID within Glass ID by Targeted Concentration for Each Oxide for Each Prep by Analytical Series (continued)

Series $=$ T, Oxide $=\mathbf{L i 2 O}($ wt $\%)$, Prep Method $=$ PF

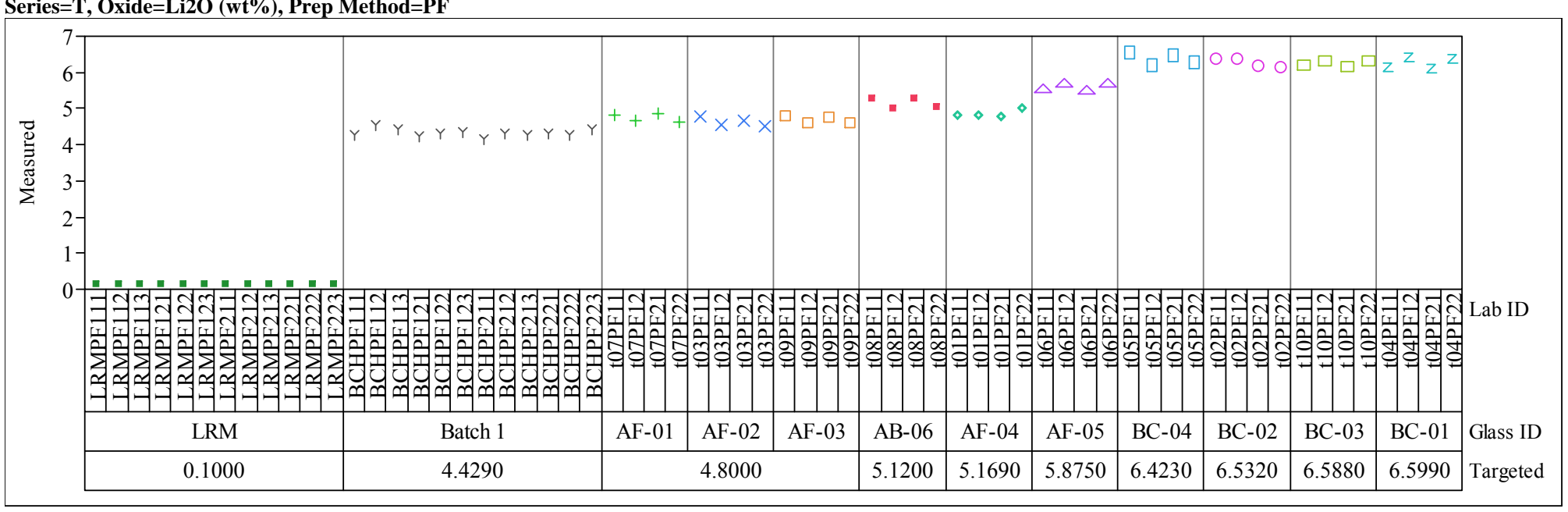

Series=T, Oxide $=\mathrm{MgO}(\mathrm{wt} \%)$, Prep Method $=\mathrm{LM}$

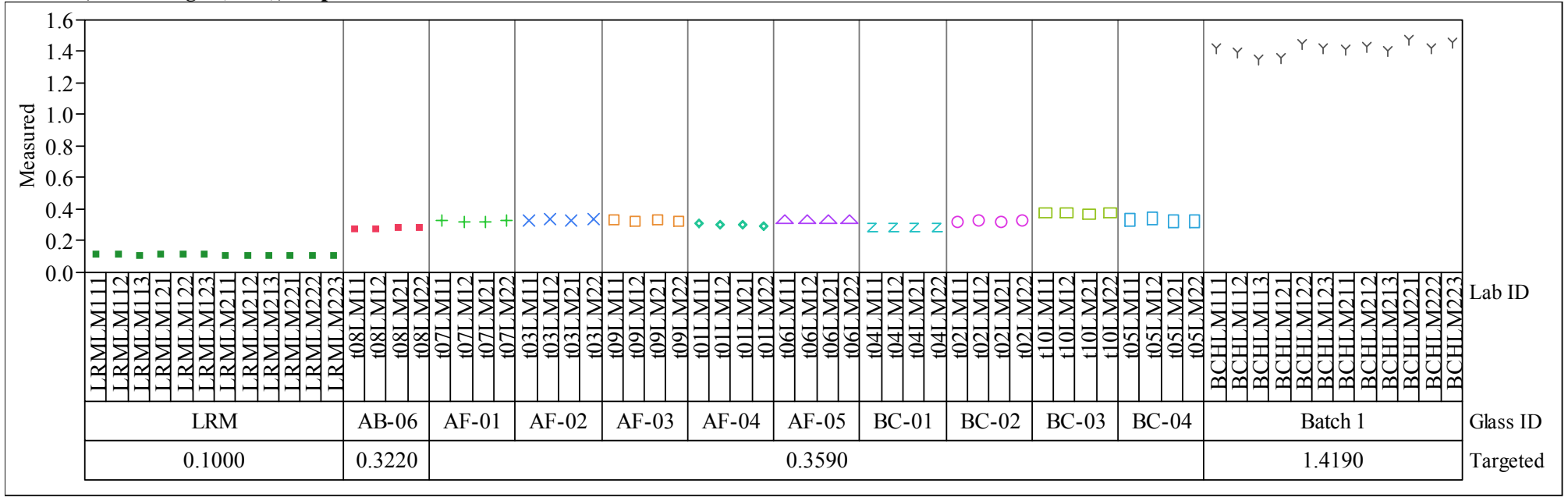


Exhibit A-2. Measurements by Lab ID within Glass ID by Targeted Concentration for Each Oxide for Each Prep by Analytical Series (continued)

Series=T, Oxide=MnO (wt\%), Prep Method=LM

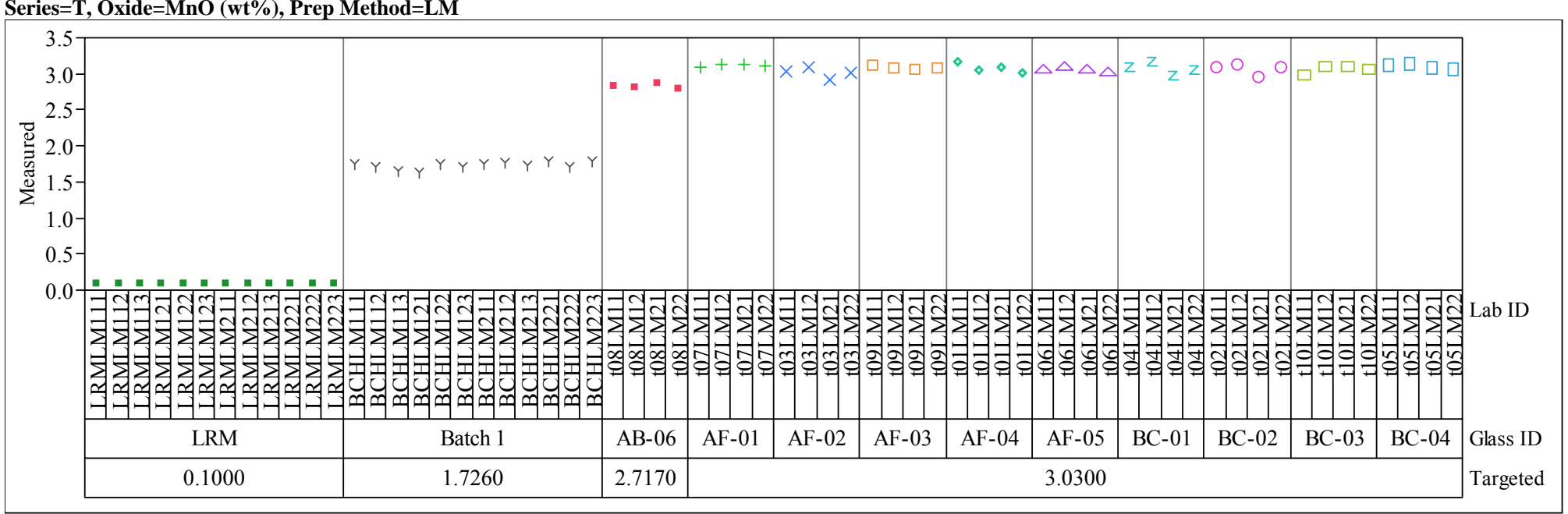

Series $=$ T, Oxide=Na2O (wt\%), Prep Method=LM

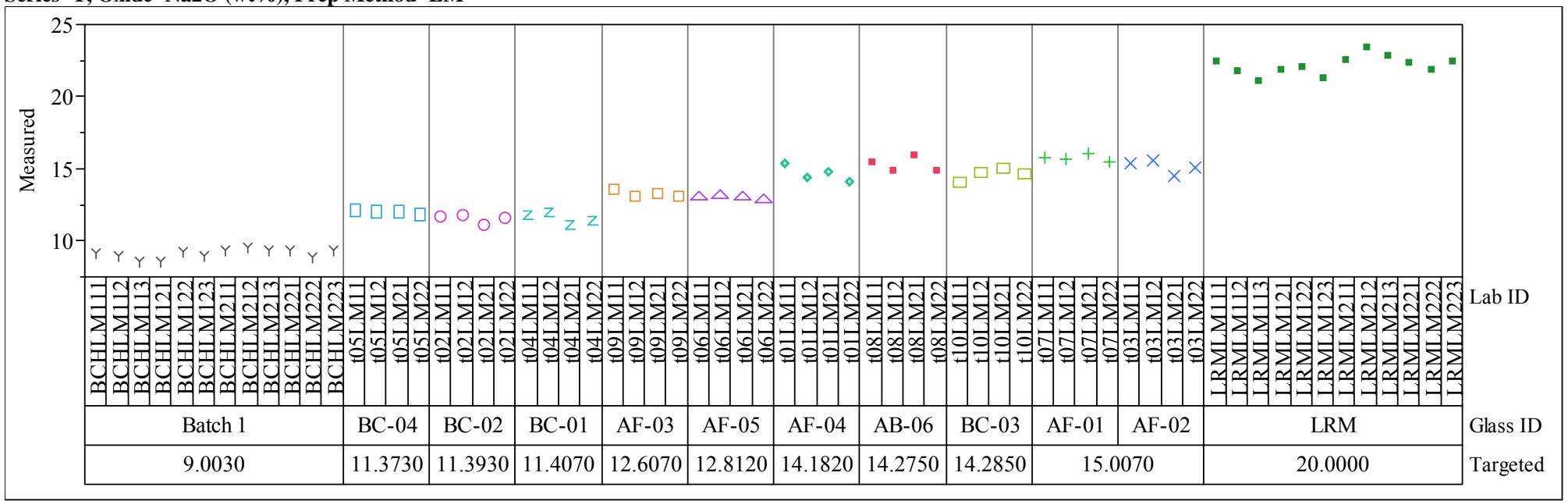


Exhibit A-2. Measurements by Lab ID within Glass ID by Targeted Concentration for Each Oxide for Each Prep by Analytical Series (continued)

Series $=\mathrm{T}$, Oxide $=\mathrm{NiO}(\mathrm{wt} \%)$, Prep Method $=\mathrm{LM}$

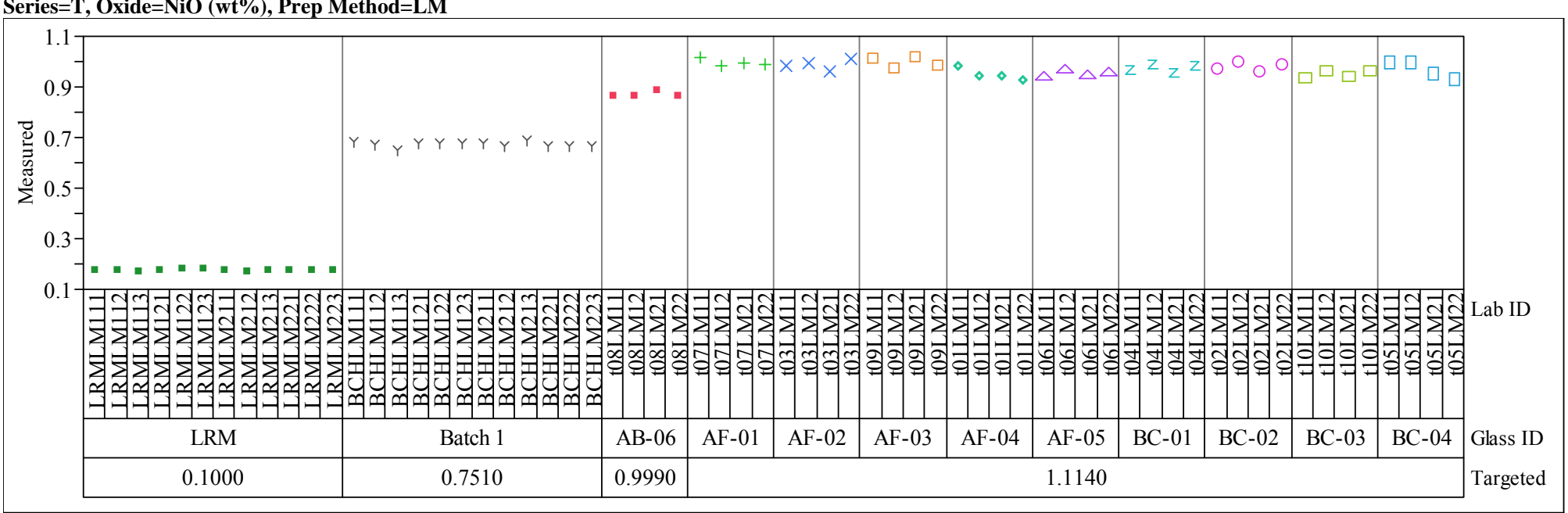

Series $=\mathbf{T}$, Oxide $=$ PbO $(w t \%)$, Prep Method $=L M$

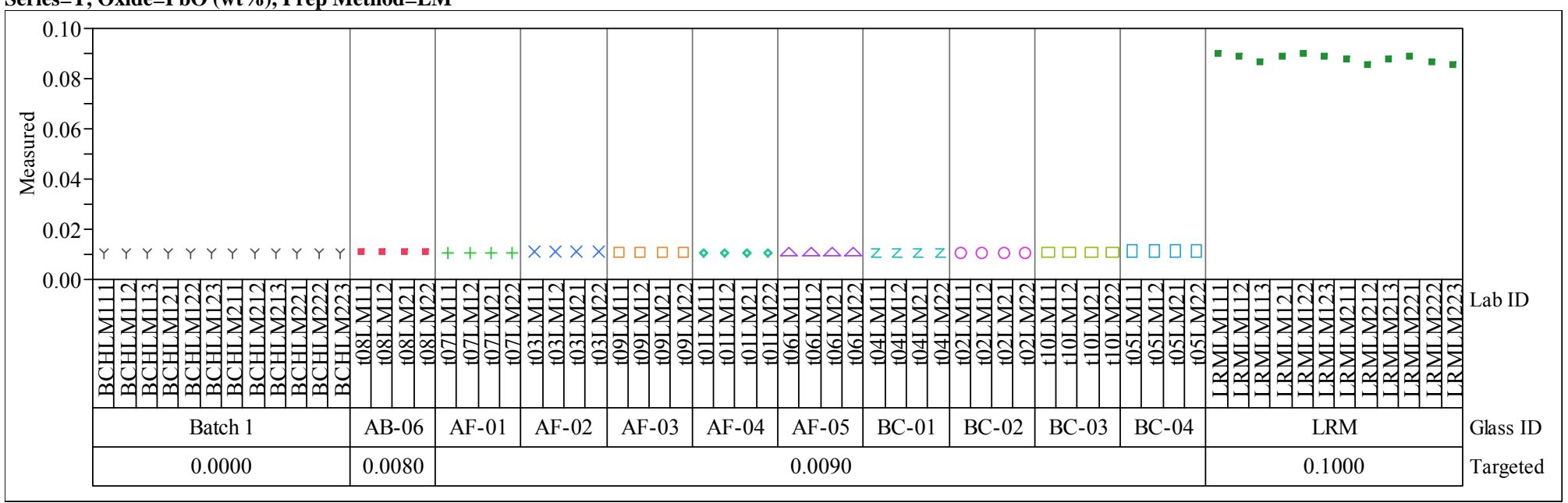


Exhibit A-2. Measurements by Lab ID within Glass ID by Targeted Concentration for Each Oxide for Each Prep by Analytical Series (continued)

Series $=\mathrm{T}$, Oxide $=\mathrm{SiO} 2(\mathrm{wt} \%)$, Prep Method $=\mathrm{PF}$

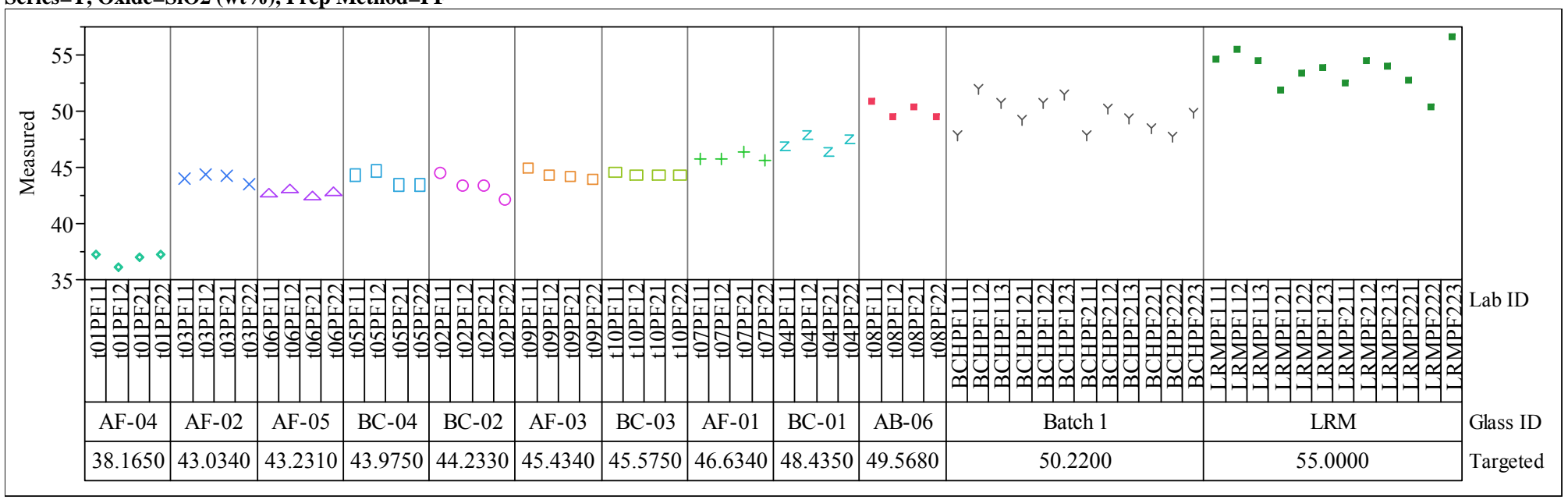

Series=T, Oxide=SO4 (wt \%), Prep Method=LM

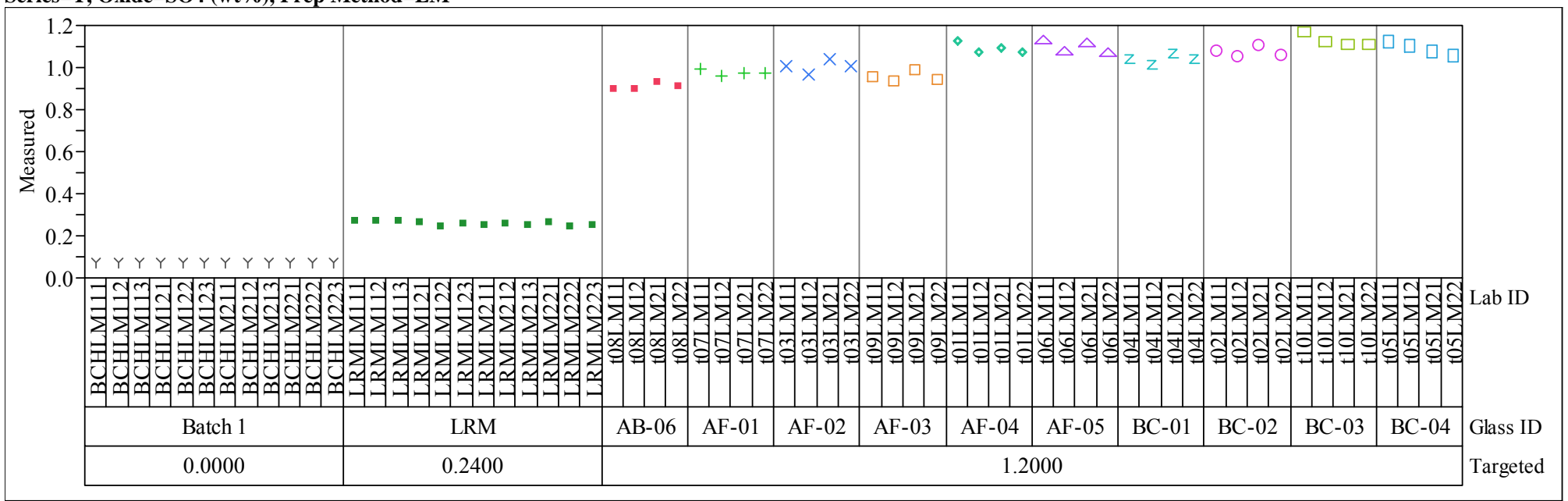


Exhibit A-2. Measurements by Lab ID within Glass ID by Targeted Concentration for Each Oxide for Each Prep by Analytical Series (continued)

Series $=\mathrm{T}$, Oxide $=\mathrm{TiO} 2(\mathrm{wt} \%)$, Prep Method $=\mathrm{LM}$

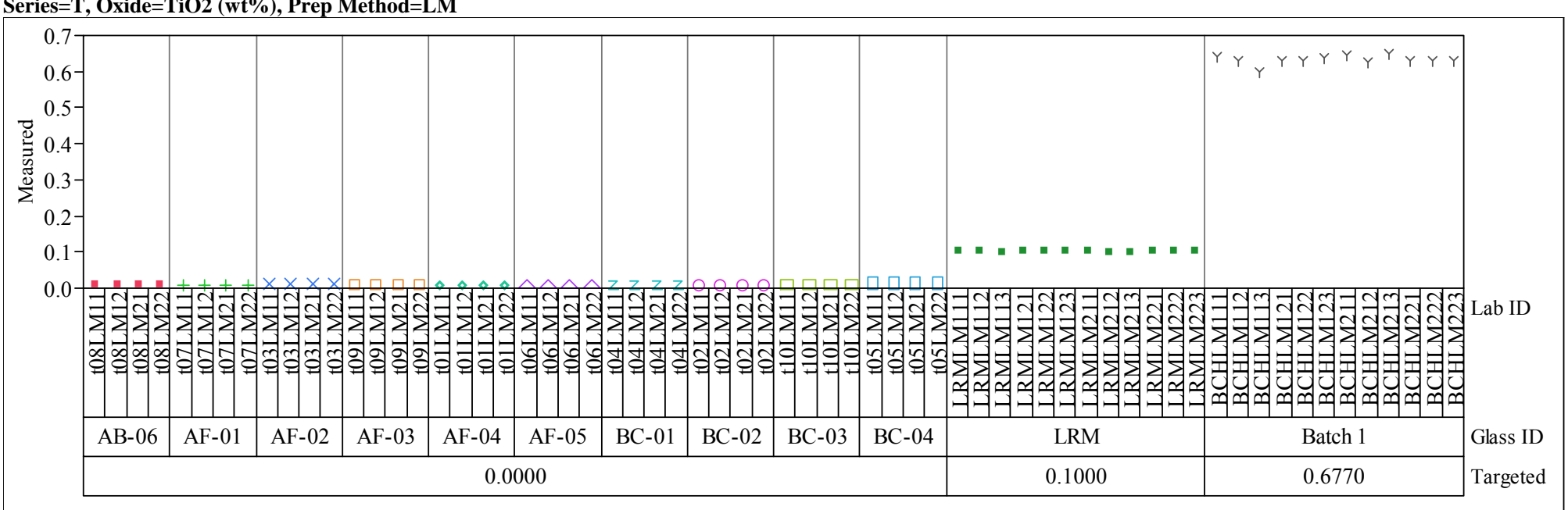

Series $=\mathrm{T}$, Oxide $=\mathrm{ZnO}(\mathrm{wt} \%)$, Prep Method $=\mathrm{LM}$

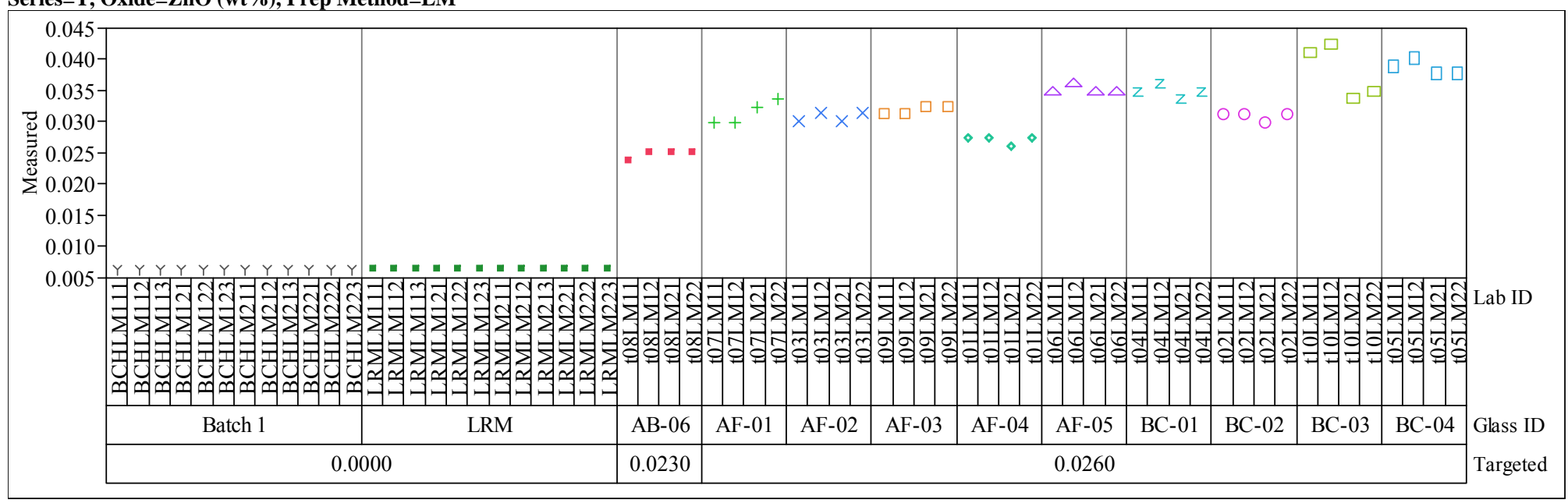


Exhibit A-2. Measurements by Lab ID within Glass ID by Targeted Concentration for Each Oxide for Each Prep by Analytical Series (continued)

Series $=$ T, Oxide $=\mathrm{ZrO} 2(\mathrm{wt} \%)$, Prep Method $=\mathrm{LM}$

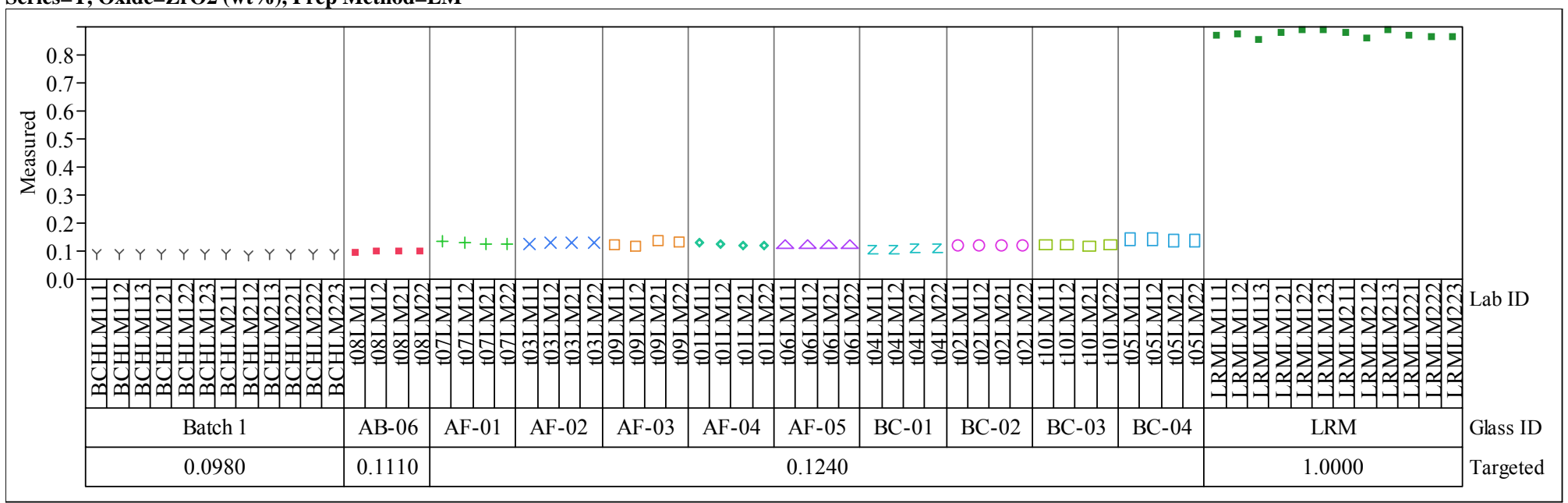

Series=U, Oxide=Al2O3 (wt\%), Prep Method $=$ LM

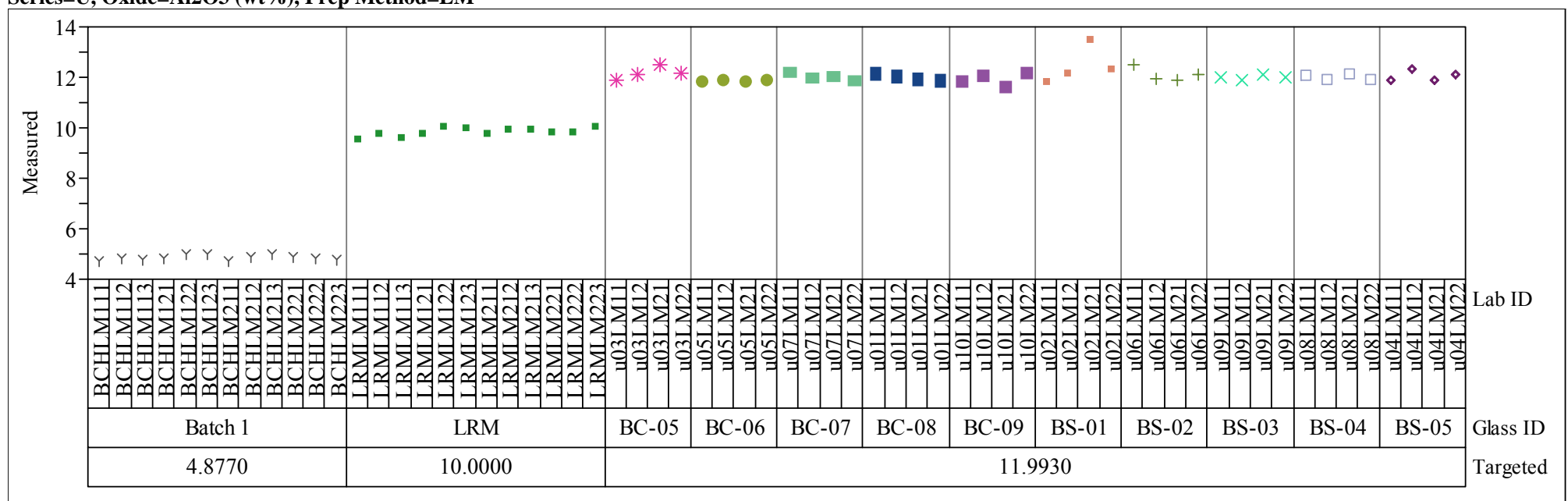


Exhibit A-2. Measurements by Lab ID within Glass ID by Targeted Concentration for Each Oxide for Each Prep by Analytical Series (continued)

Series $=$ U, Oxide=B2O3 (wt \%), Prep Method $=$ PF

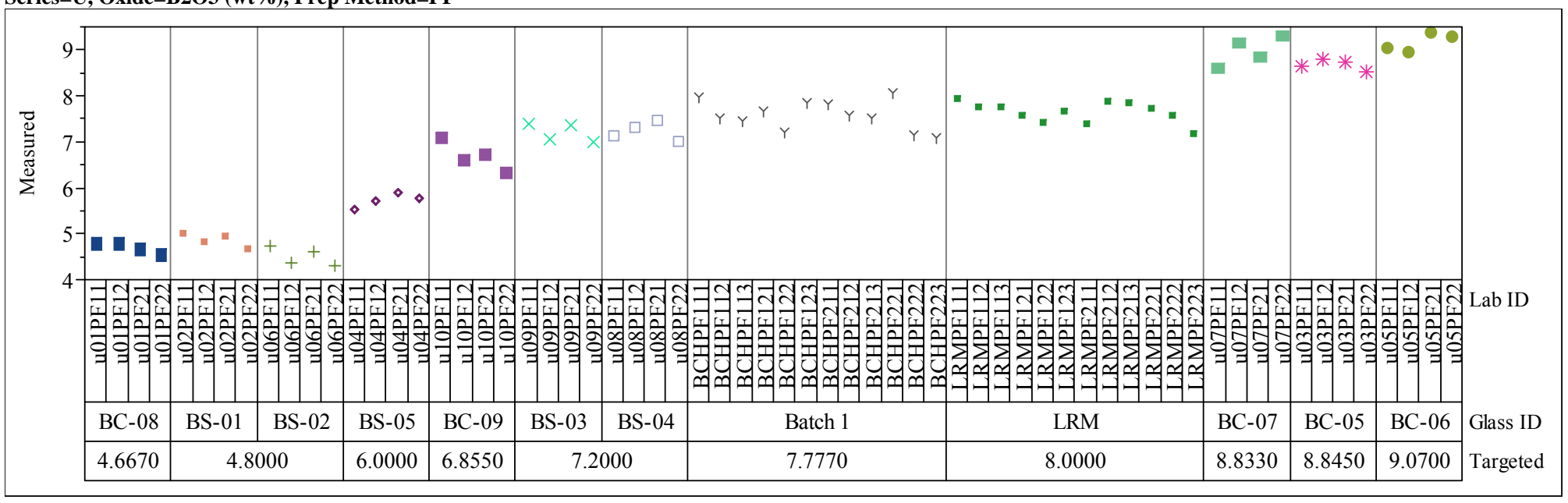

Series $=U$, Oxide $=$ BaO (wt \%), Prep Method $=L M$

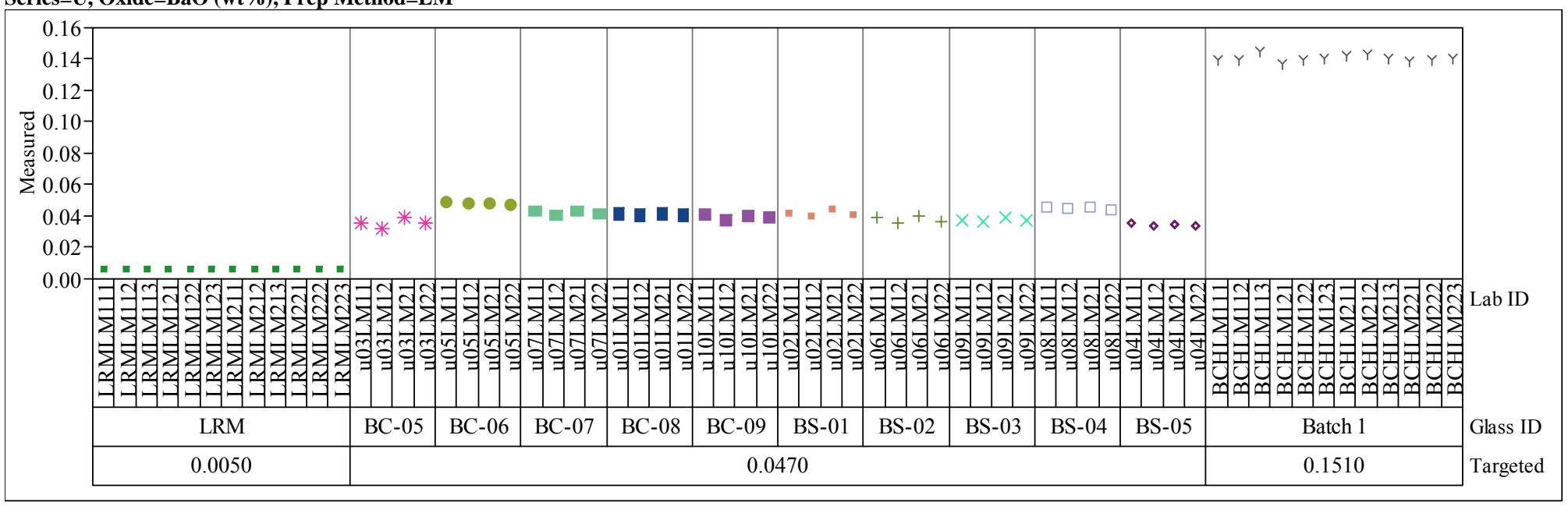


Exhibit A-2. Measurements by Lab ID within Glass ID by Targeted Concentration for Each Oxide for Each Prep by Analytical Series (continued)

Series $=\mathrm{U}$, Oxide $=\mathrm{CaO}(\mathrm{wt} \%)$, Prep Method $=\mathrm{LM}$

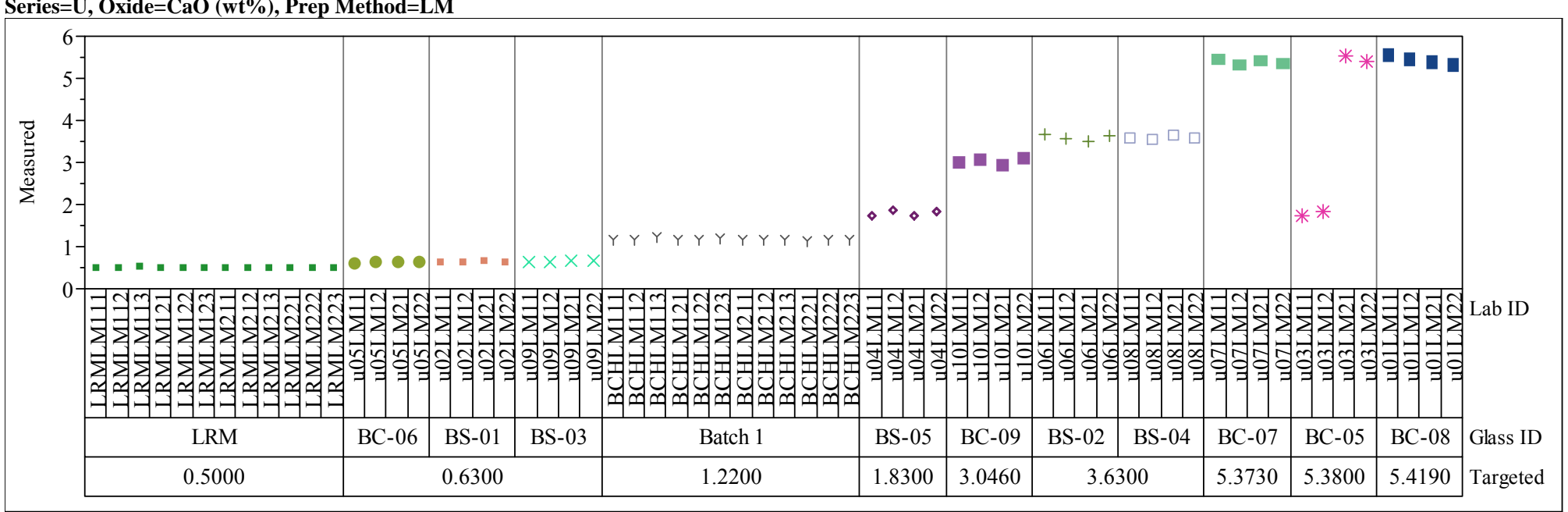

Series=U, Oxide=Ce2O3 (wt \%), Prep Method=LM

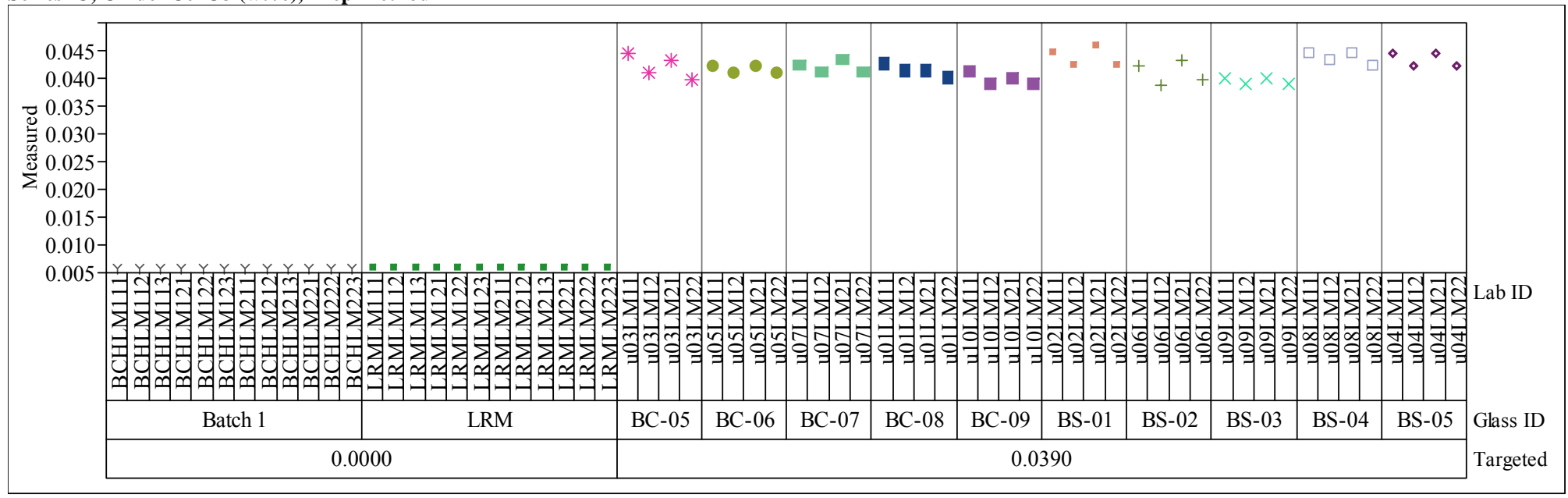


Exhibit A-2. Measurements by Lab ID within Glass ID by Targeted Concentration for Each Oxide for Each Prep by Analytical Series (continued)

Series $=\mathrm{U}$, Oxide $=\mathrm{Cr} 2 \mathrm{O} 3(\mathrm{wt} \%)$, Prep Method $=\mathrm{LM}$

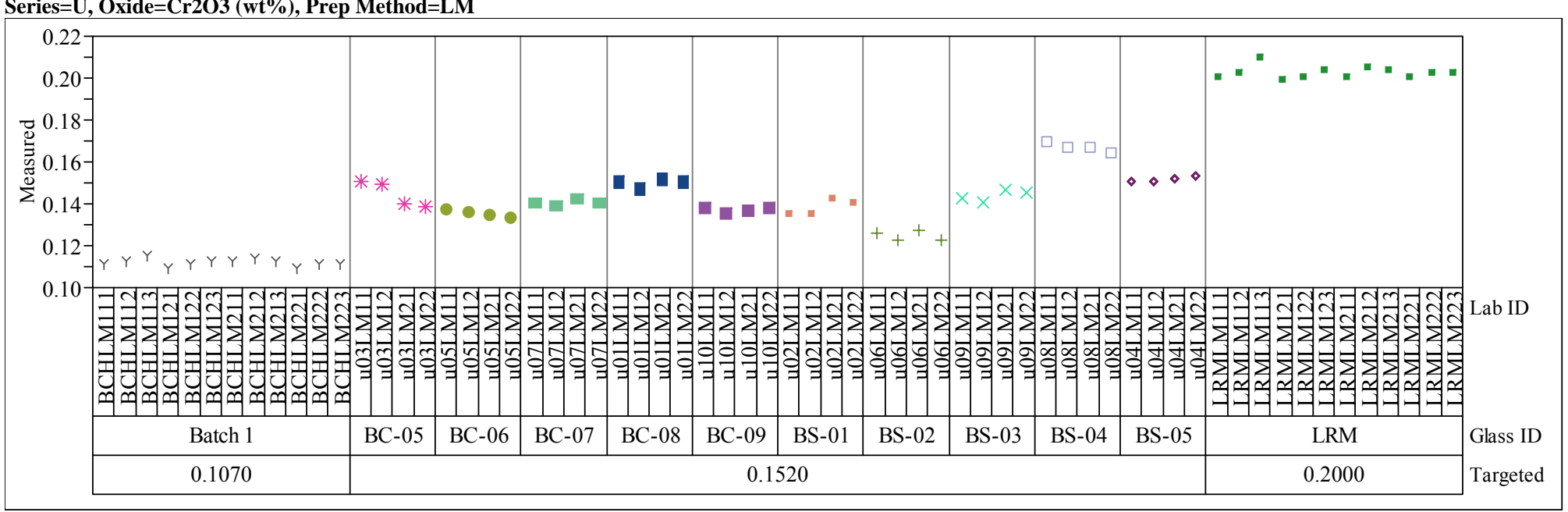

Series $=$ U, Oxide $=\mathrm{CuO}(\mathrm{wt} \%)$, Prep Method $=\mathrm{LM}$

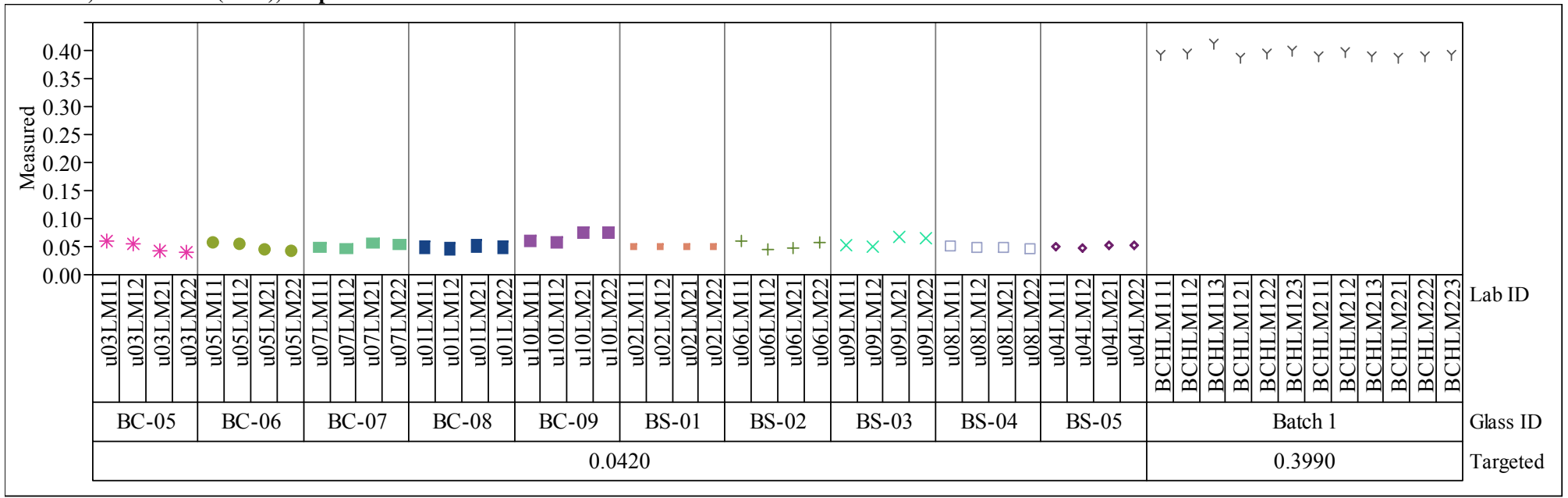


Exhibit A-2. Measurements by Lab ID within Glass ID by Targeted Concentration for Each Oxide for Each Prep by Analytical Series (continued)

Series=U, Oxide=Fe2O3 (wt\%), Prep Method=LM

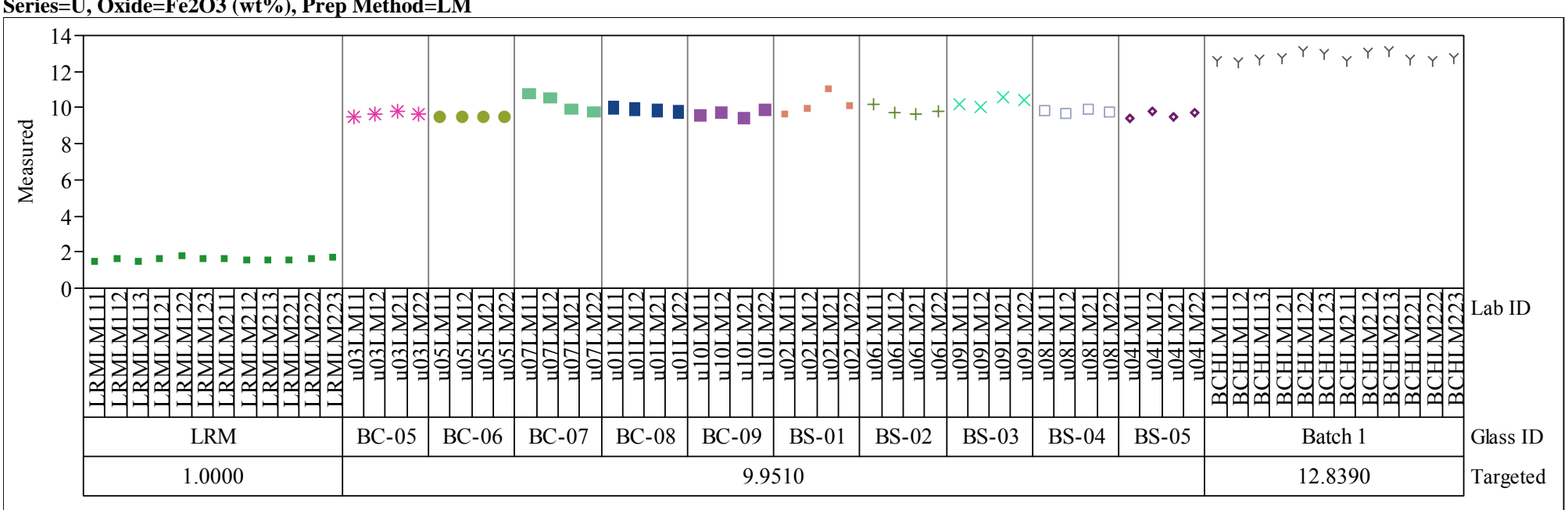

Series $=U$, Oxide=K2O (wt\%), Prep Method $=$ LM

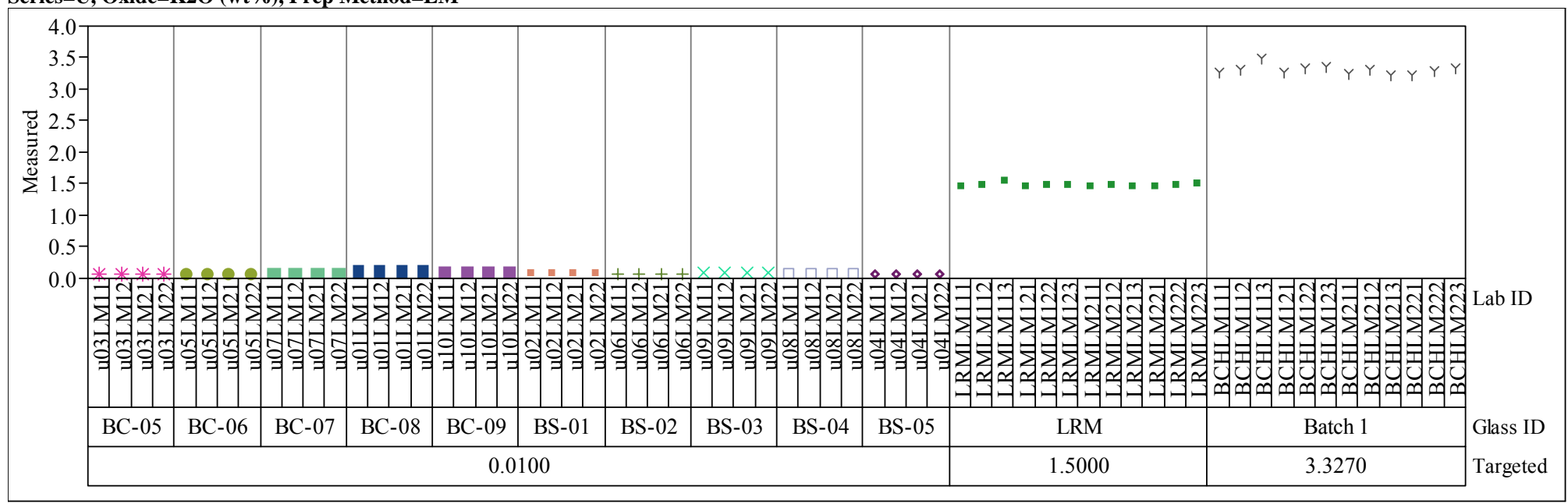


Exhibit A-2. Measurements by Lab ID within Glass ID by Targeted Concentration for Each Oxide for Each Prep by Analytical Series (continued)

Series $=$ U, Oxide $=$ La2O3 $(w t \%)$, Prep Method $=$ LM

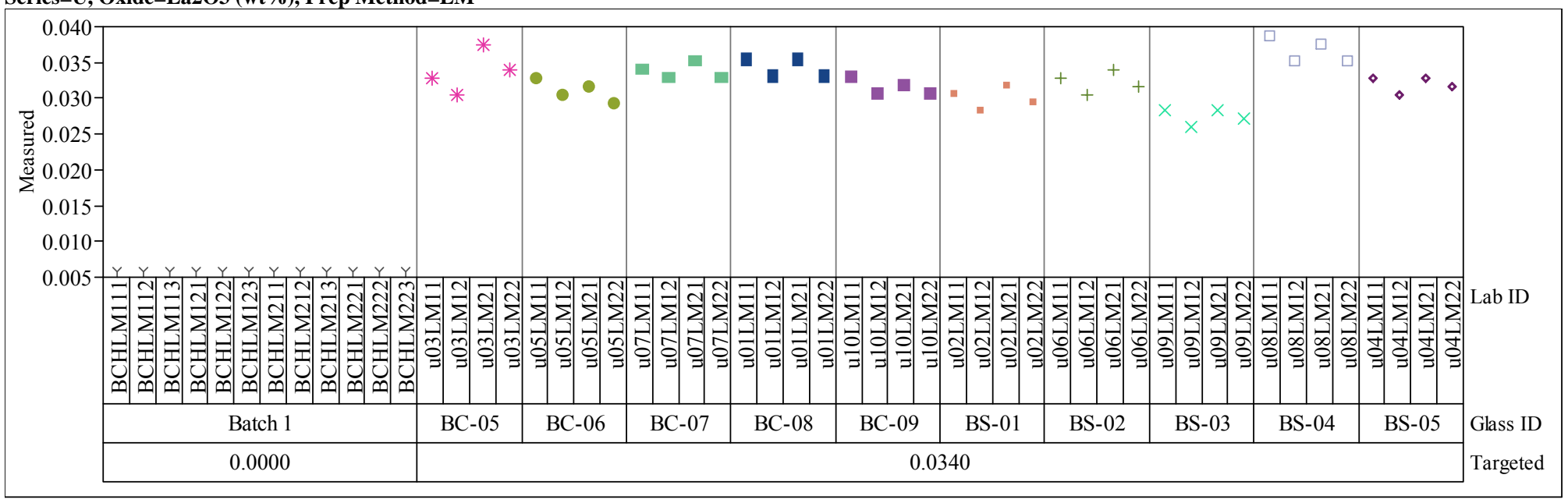

Series=U, Oxide=Li2O (wt\%), Prep Method=PF

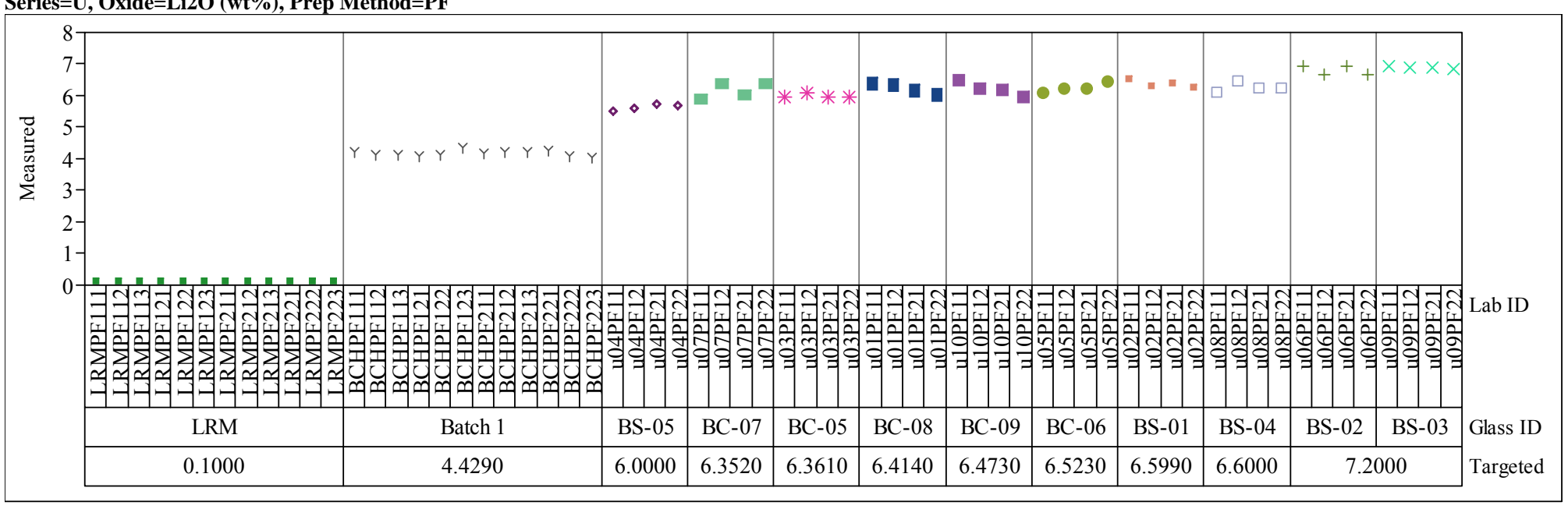


Exhibit A-2. Measurements by Lab ID within Glass ID by Targeted Concentration for Each Oxide for Each Prep by Analytical Series (continued)

Series $=\mathrm{U}$, Oxide $=\mathrm{MgO}(\mathrm{wt} \%)$, Prep Method $=\mathbf{L M}$

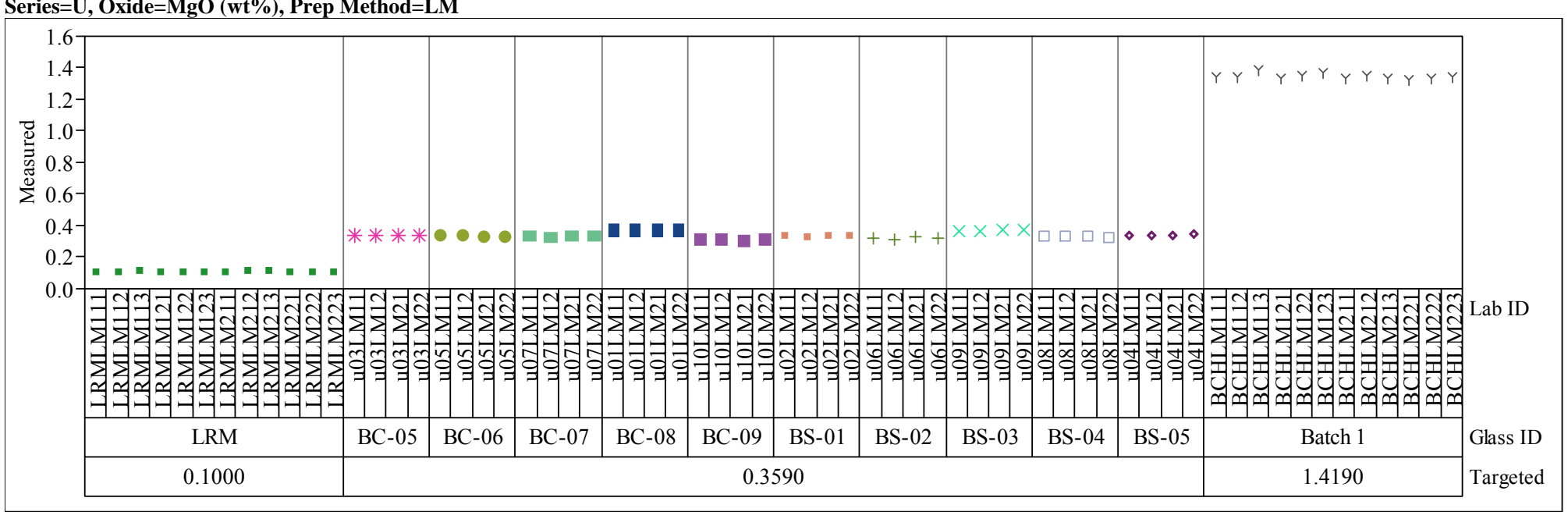

Series $=$ U, Oxide $=$ MnO (wt \%), Prep Method $=$ LM

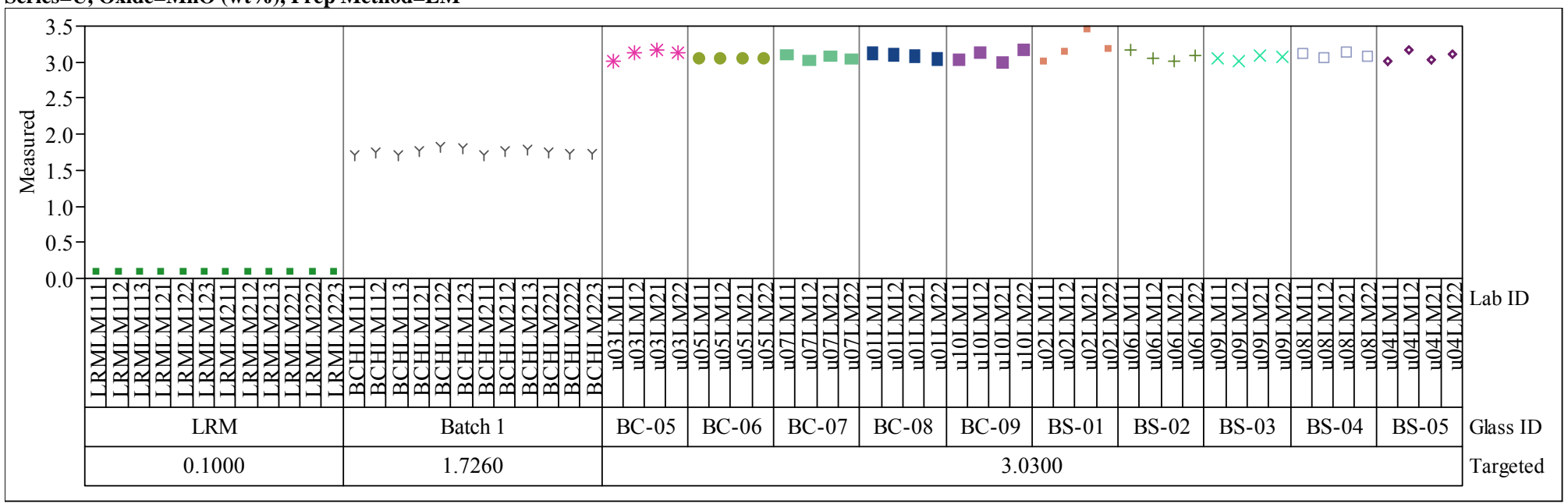


Exhibit A-2. Measurements by Lab ID within Glass ID by Targeted Concentration for Each Oxide for Each Prep by Analytical Series (continued)

Series $=\mathrm{U}$, Oxide=Na2O (wt\%), Prep Method $=$ LM

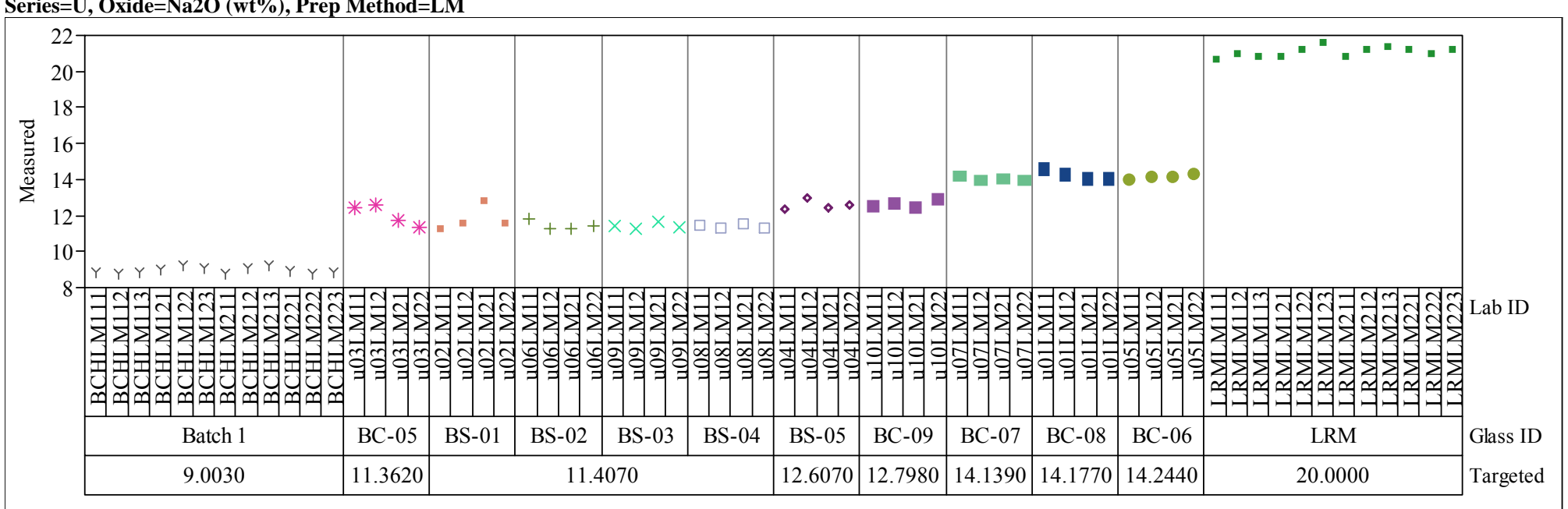

Series $=\mathrm{U}$, Oxide $=\mathrm{NiO}(\mathrm{wt} \%)$, Prep Method $=\mathrm{LM}$

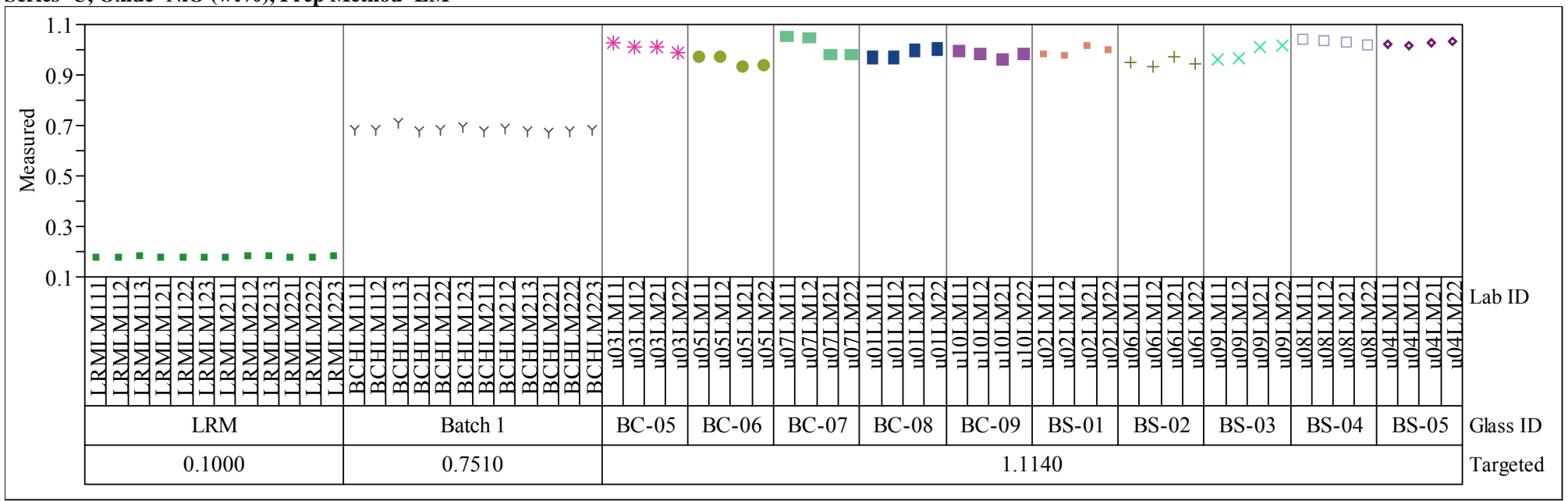


Exhibit A-2. Measurements by Lab ID within Glass ID by Targeted Concentration for Each Oxide for Each Prep by Analytical Series (continued)

Series $=\mathbf{U}$, Oxide $=\mathbf{P b O}(\mathbf{w t} \%)$, Prep Method $=\mathbf{L M}$

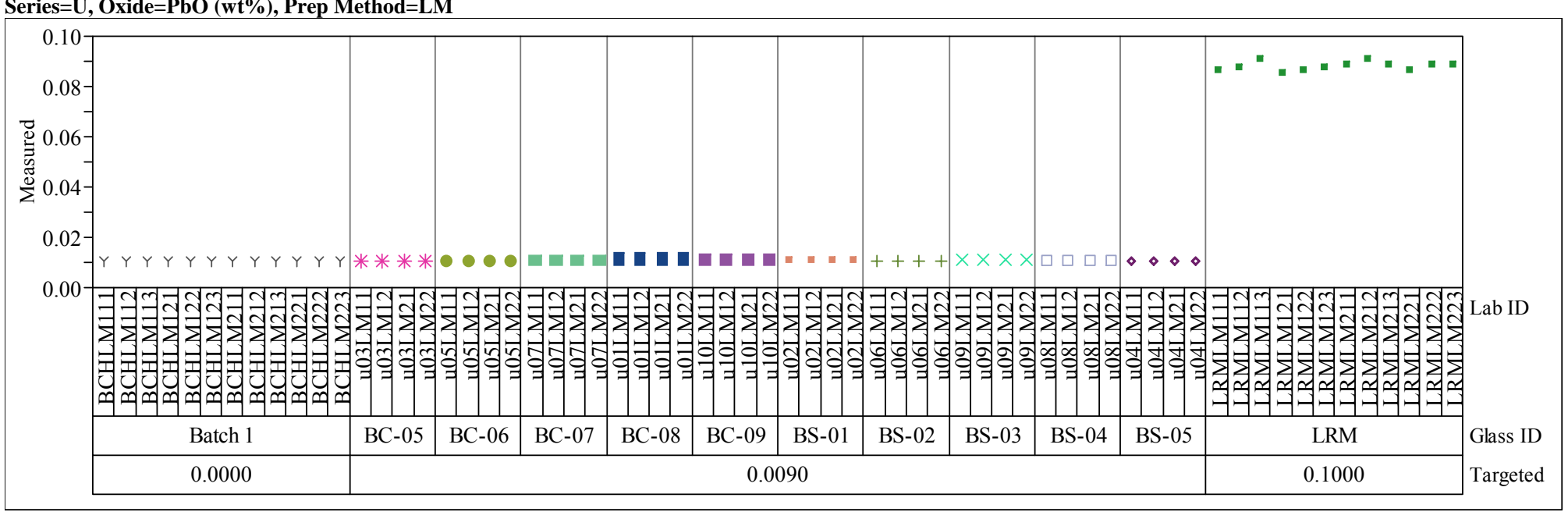

Series $=\mathrm{U}$, Oxide=SiO2 (wt\%), Prep Method=PF

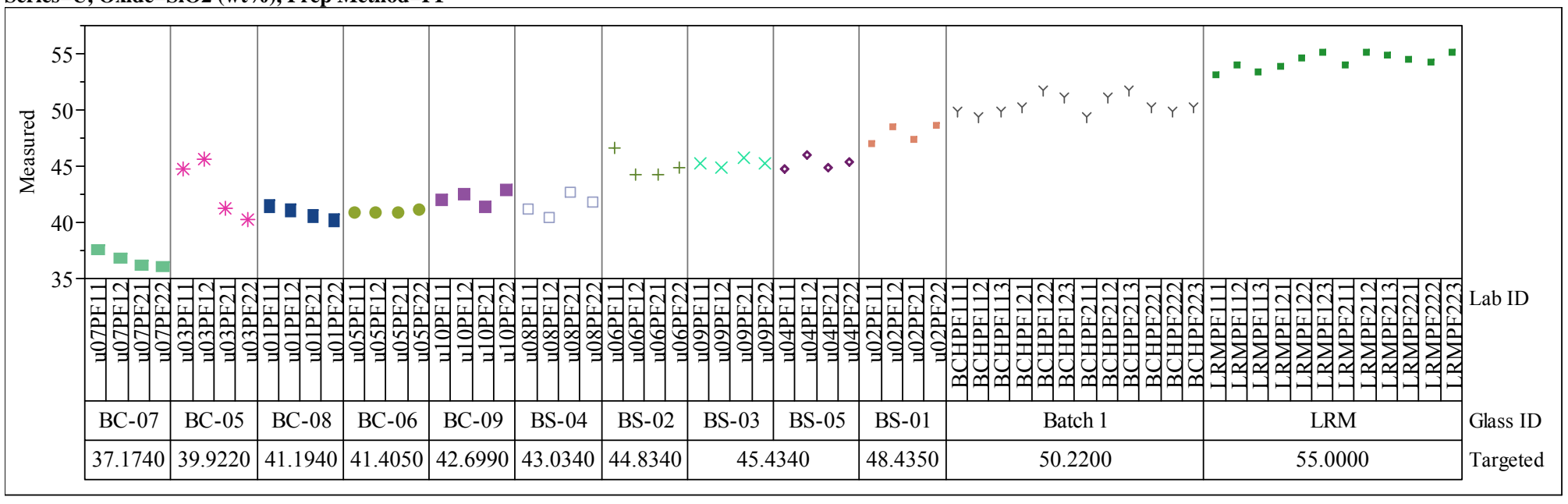


Exhibit A-2. Measurements by Lab ID within Glass ID by Targeted Concentration for Each Oxide for Each Prep by Analytical Series (continued)

Series $=\mathrm{U}$, Oxide=SO4 (wt\%), Prep Method=LM

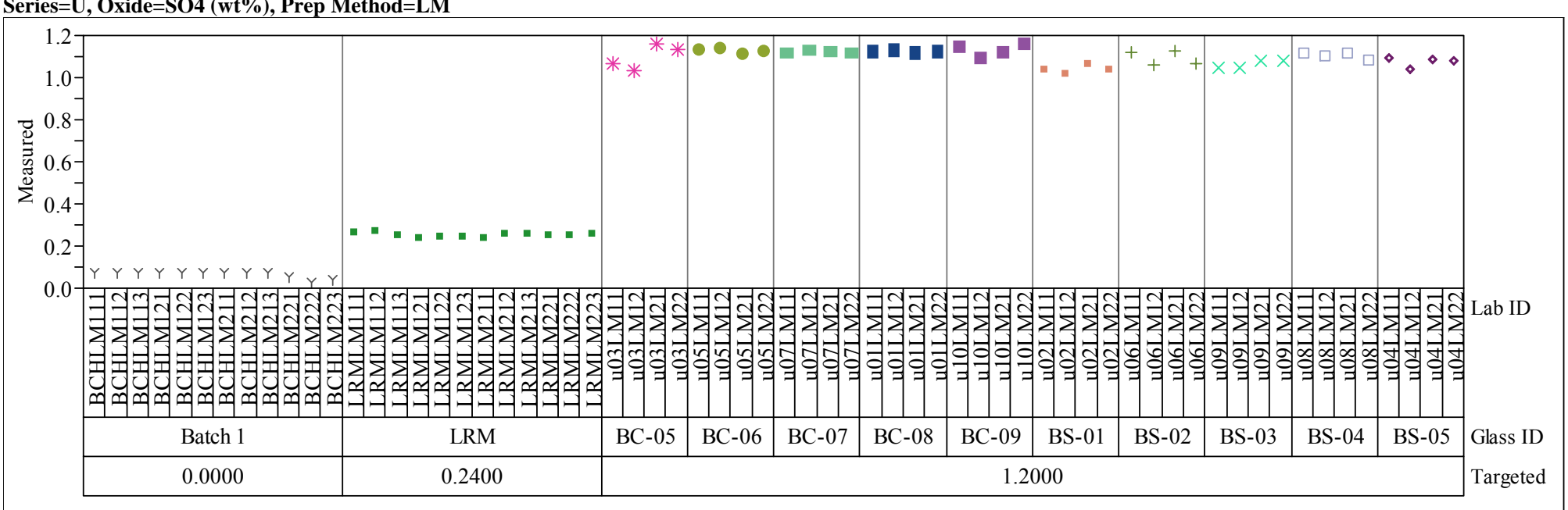

Series $=$ U, Oxide=TiO2 (wt\%), Prep Method $=$ LM

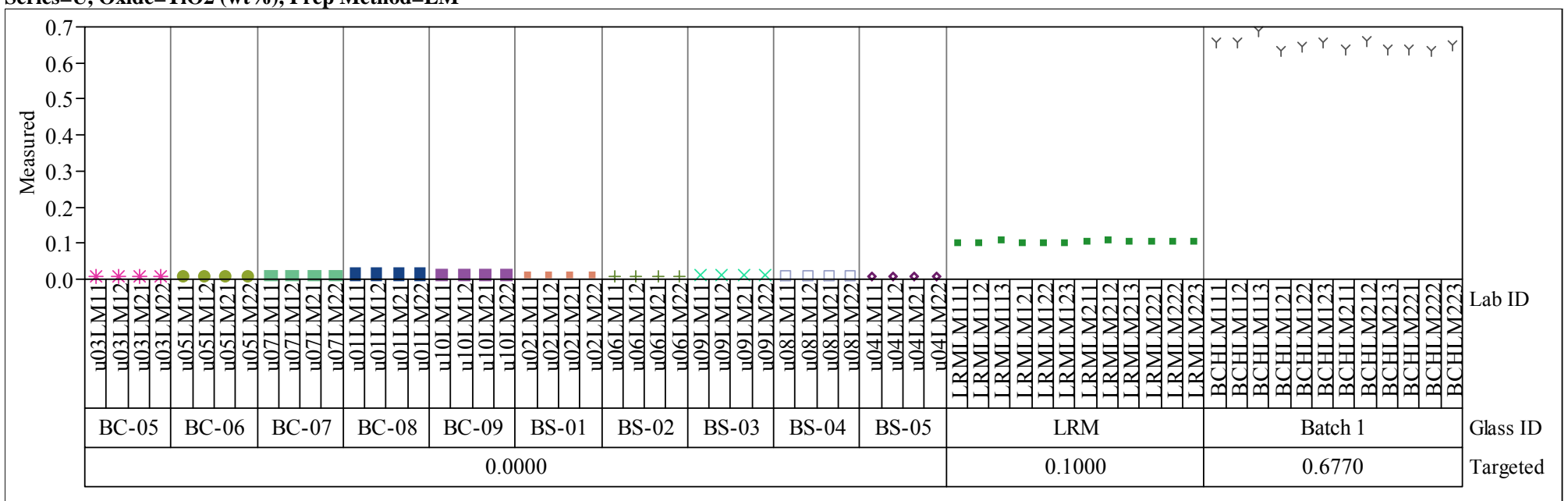


Exhibit A-2. Measurements by Lab ID within Glass ID by Targeted Concentration for Each Oxide for Each Prep by Analytical Series (continued)

Series $=\mathrm{U}$, Oxide $=\mathrm{ZnO}$ ( $w \mathrm{t} \%$ ), Prep Method $=\mathrm{LM}$

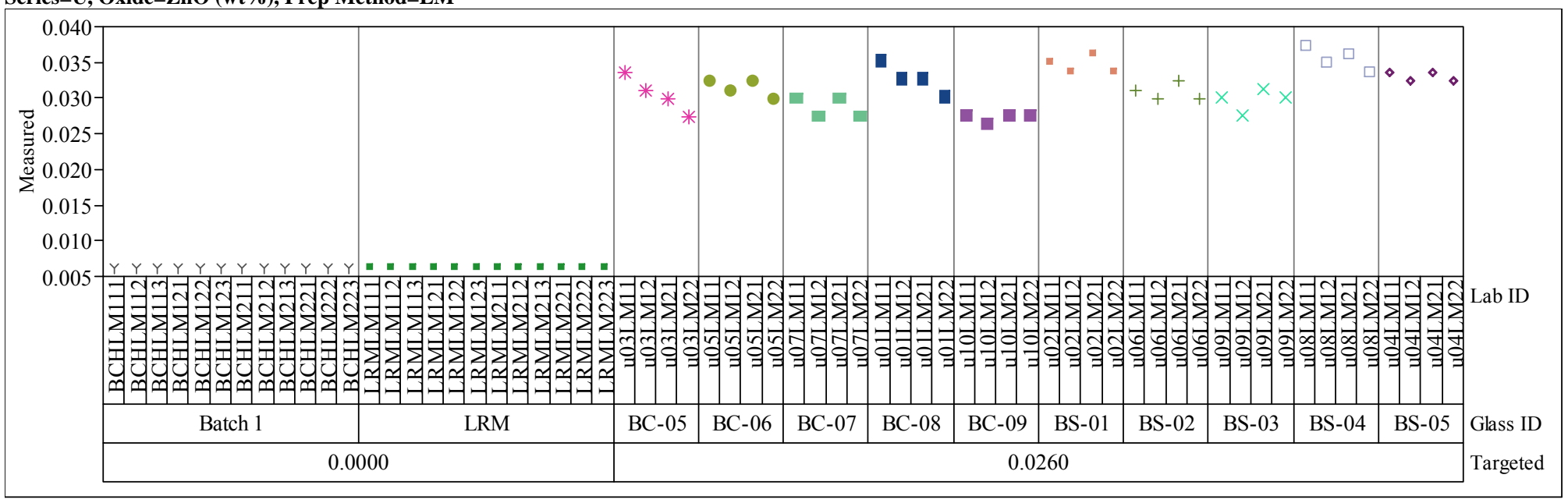

Series $=\mathrm{U}$, Oxide $=\mathrm{ZrO} 2$ (wt \%), Prep Method $=\mathrm{LM}$

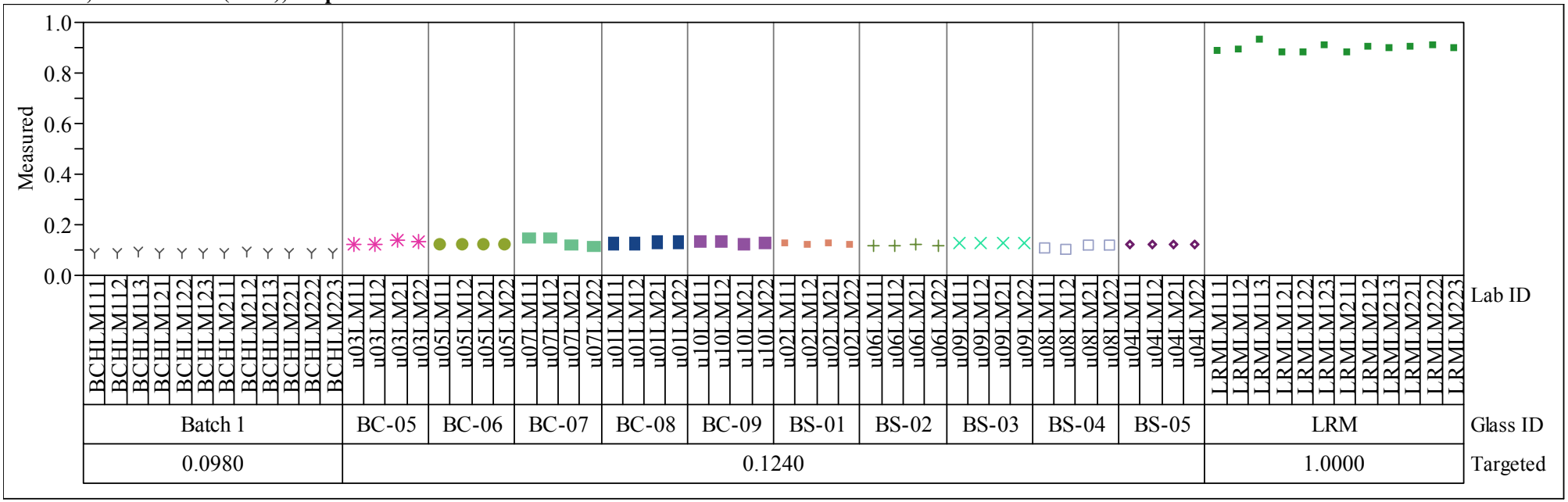


Exhibit A-2. Measurements by Lab ID within Glass ID by Targeted Concentration for Each Oxide for Each Prep by Analytical Series (continued)

Series $=$ V, Oxide=Al2O3 (wt $\%)$, Prep Method $=$ LM

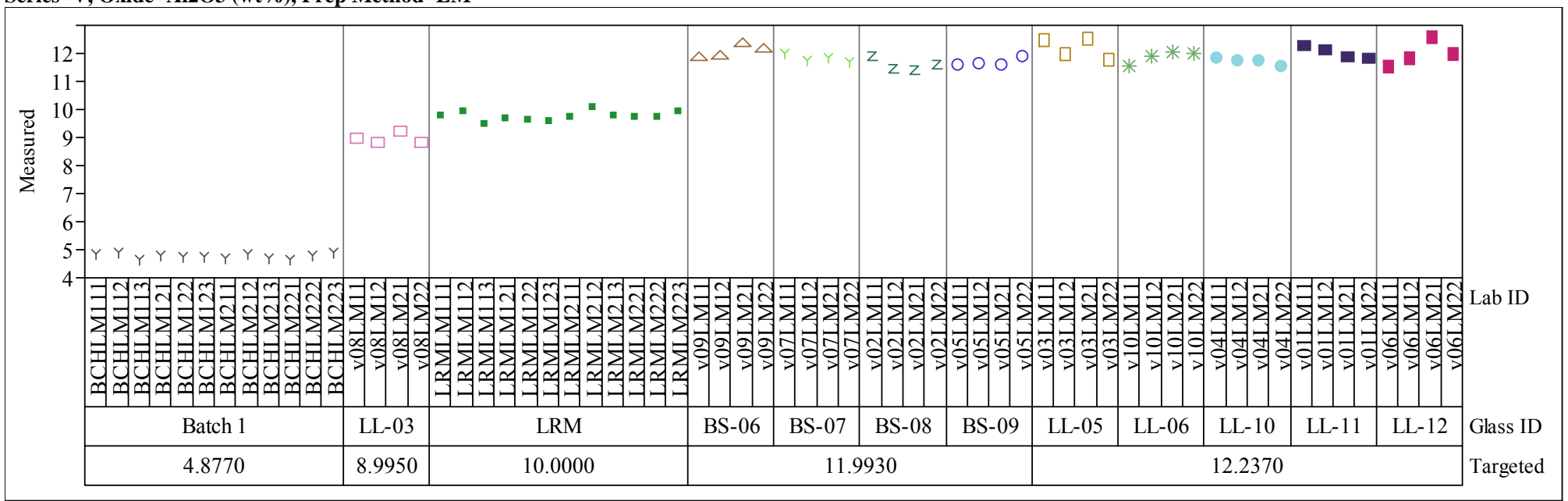

Series=V, Oxide=B2O3 (wt \%), Prep Method=PF

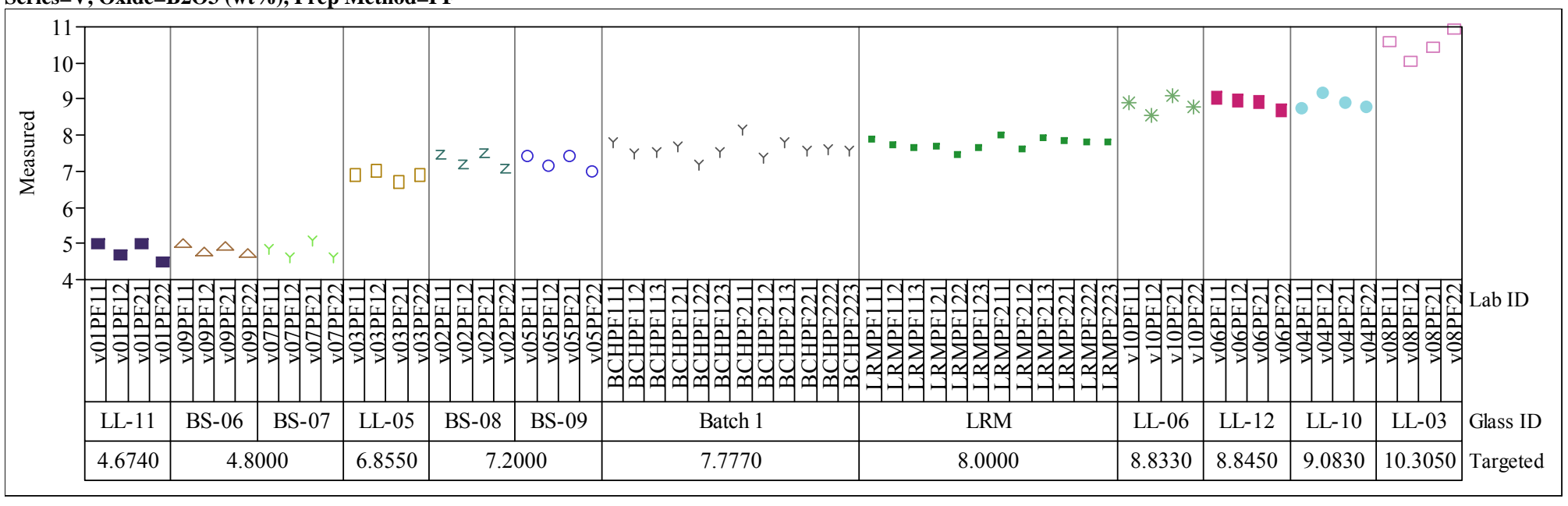


Exhibit A-2. Measurements by Lab ID within Glass ID by Targeted Concentration for Each Oxide for Each Prep by Analytical Series (continued)

Series $=\mathrm{V}$, Oxide $=\mathrm{BaO}(\mathrm{wt} \%)$, Prep Method $=\mathrm{LM}$

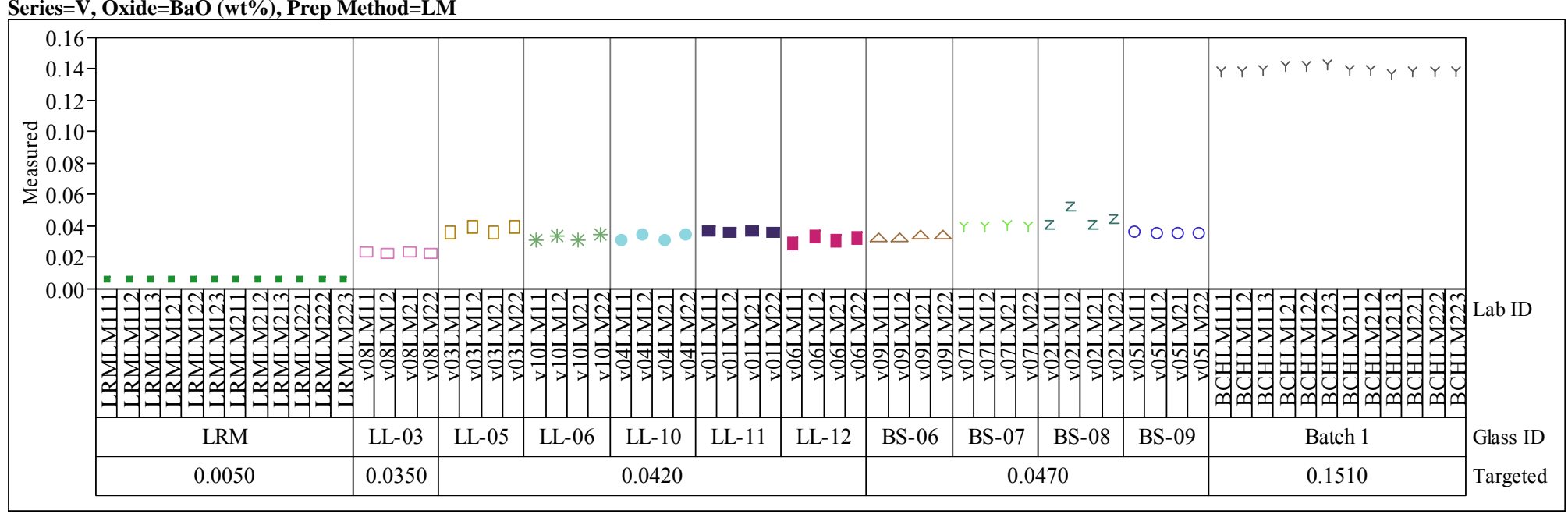

Series $=V$, Oxide $=\mathrm{CaO}(w t \%)$, Prep Method $=\mathrm{LM}$

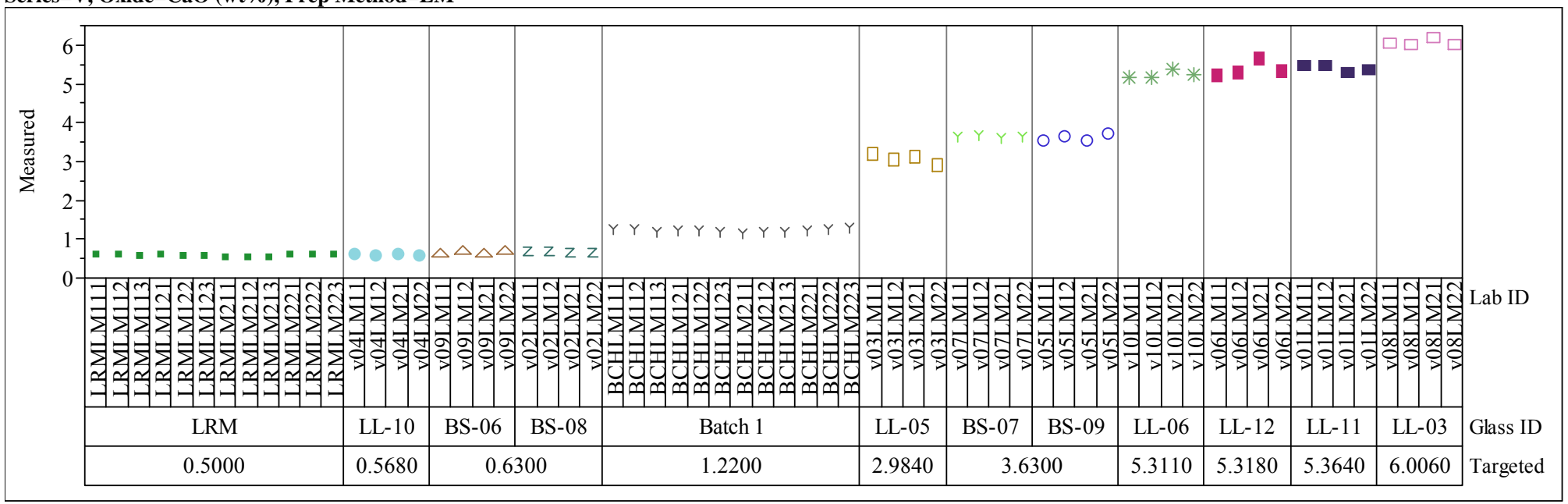


Exhibit A-2. Measurements by Lab ID within Glass ID by Targeted Concentration for Each Oxide for Each Prep by Analytical Series (continued)

Series $=$ V, Oxide $=\mathrm{Ce} 2 \mathrm{O} 3(\mathrm{wt} \%)$, Prep Method $=$ LM

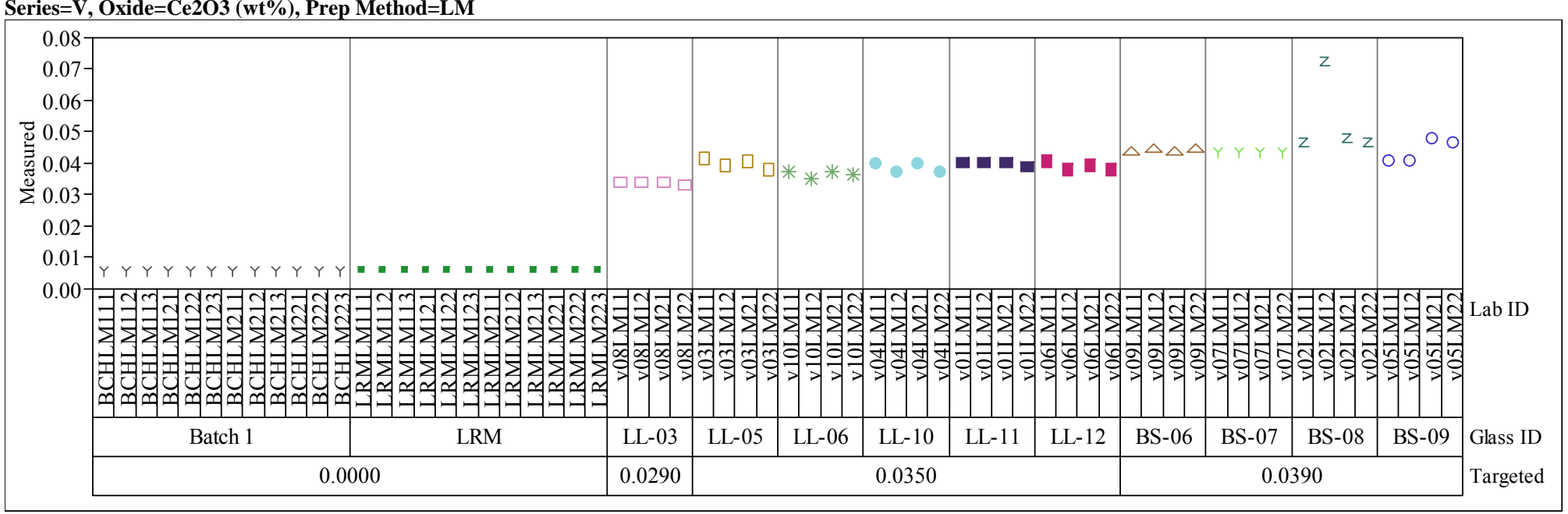

Series $=$ V, Oxide $=$ Cr2O3 (wt \%), Prep Method $=$ LM

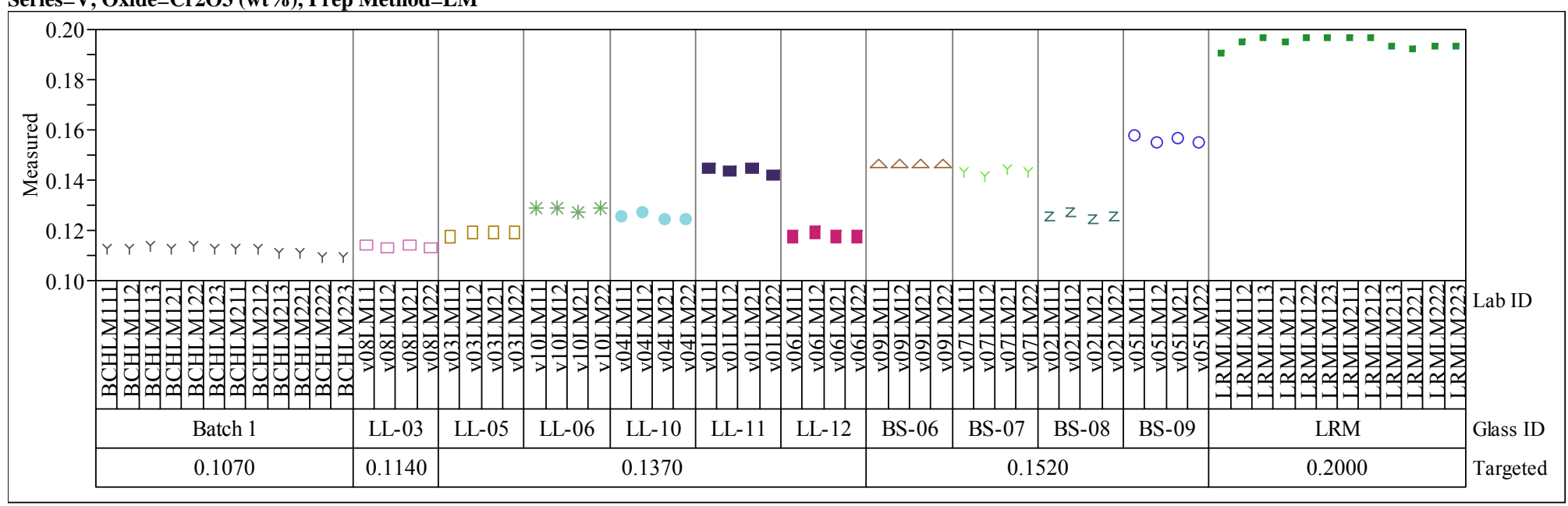


Exhibit A-2. Measurements by Lab ID within Glass ID by Targeted Concentration for Each Oxide for Each Prep by Analytical Series (continued)

Series $=$ V, Oxide $=\mathrm{CuO}(\mathrm{wt} \%)$, Prep Method $=\mathrm{LM}$

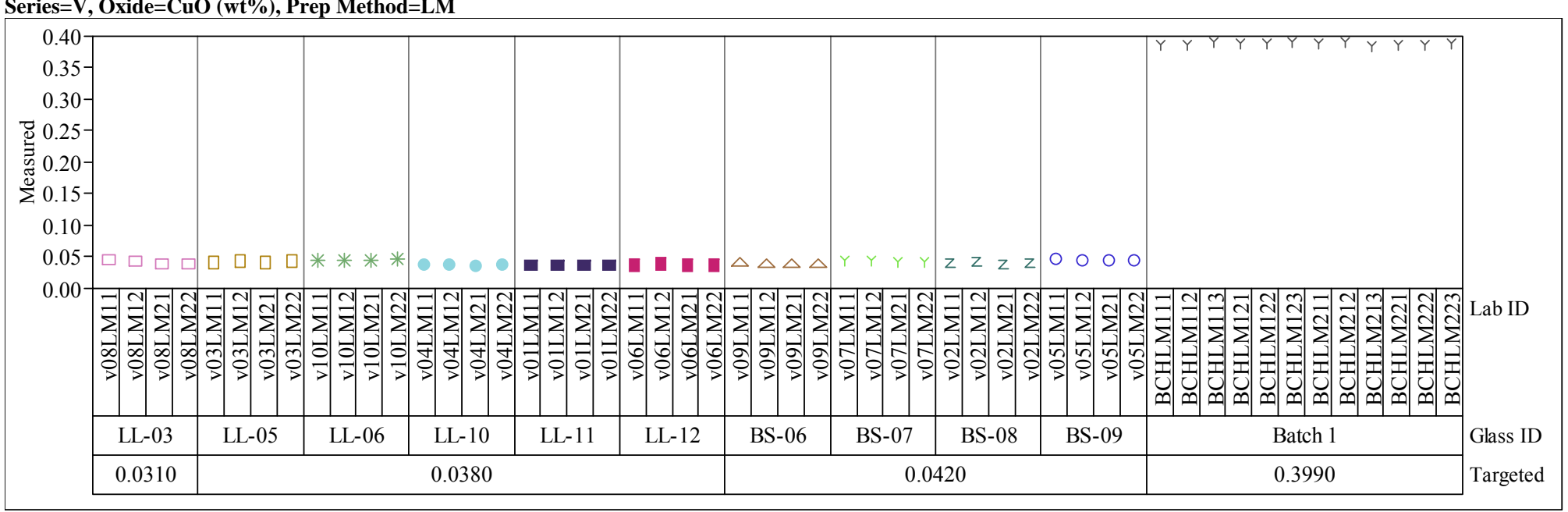

Series=V, Oxide=Fe2O3 (wt \%), Prep Method $=$ LM

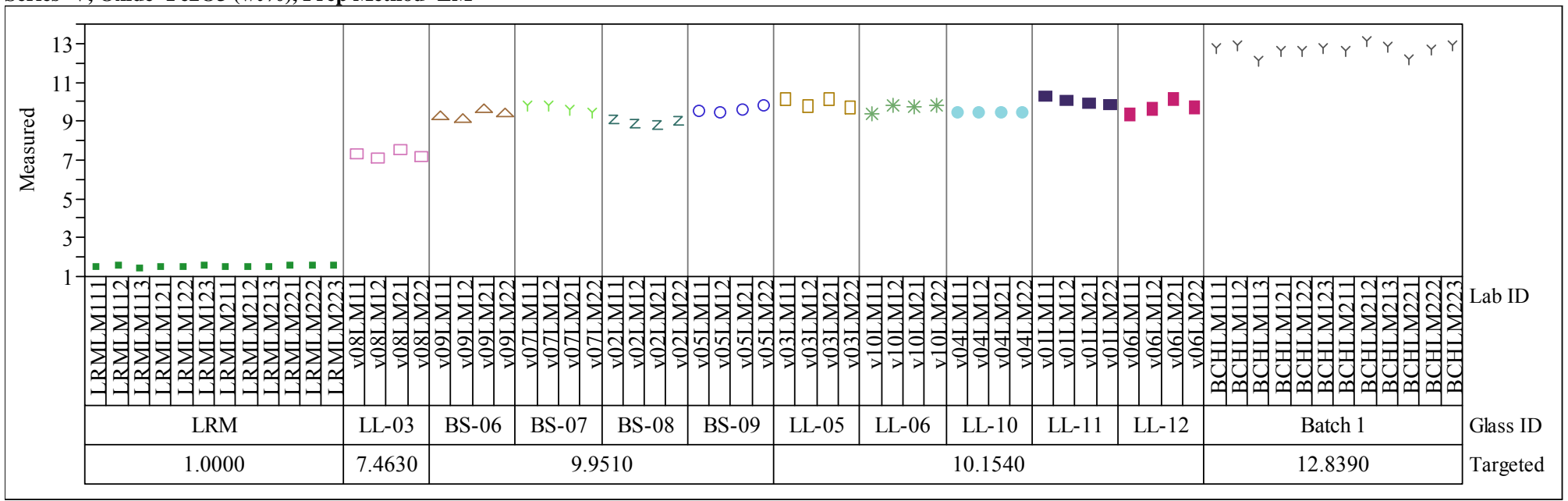


Exhibit A-2. Measurements by Lab ID within Glass ID by Targeted Concentration for Each Oxide for Each Prep by Analytical Series (continued)

Series $=$ V, Oxide $=$ K2O (wt \%), Prep Method $=$ LM

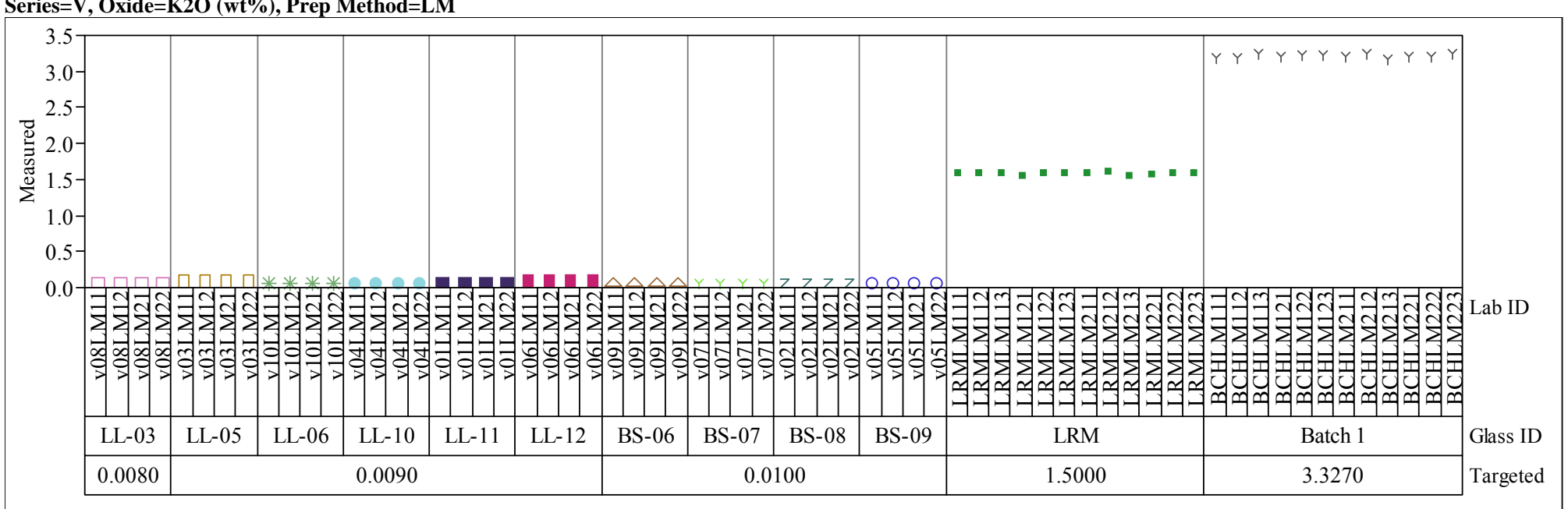

Series=V, Oxide=La2O3 (wt \%), Prep Method=LM

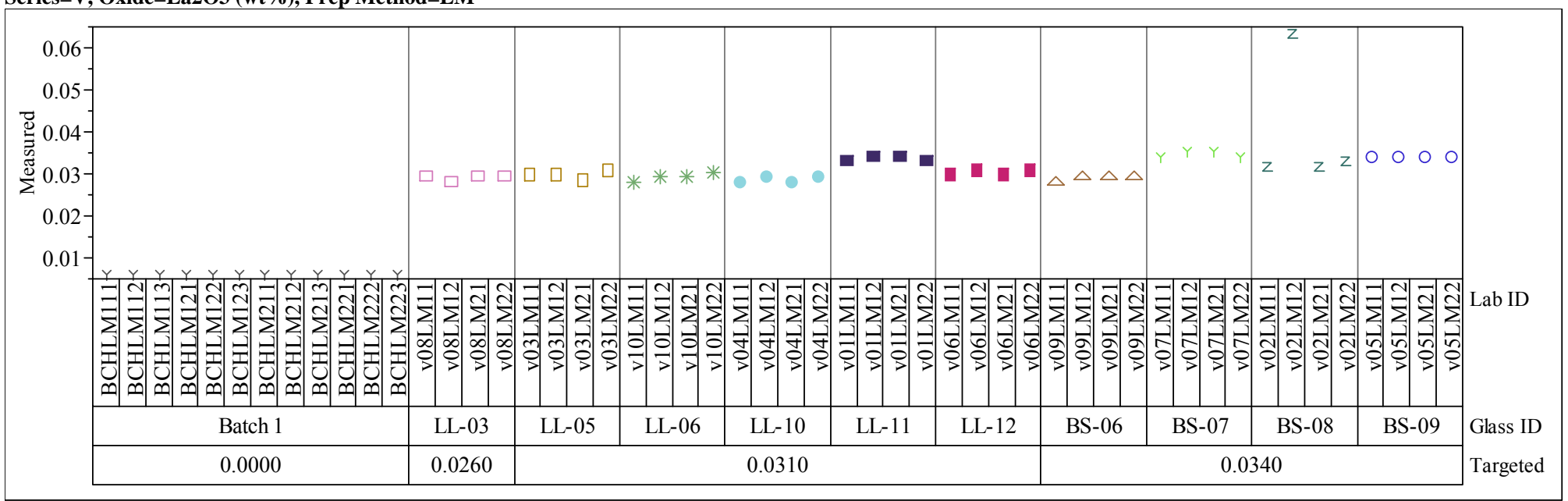


Exhibit A-2. Measurements by Lab ID within Glass ID by Targeted Concentration for Each Oxide for Each Prep by Analytical Series (continued)

Series $=$ V, Oxide $=$ Li2O (wt \%), Prep Method $=$ PF

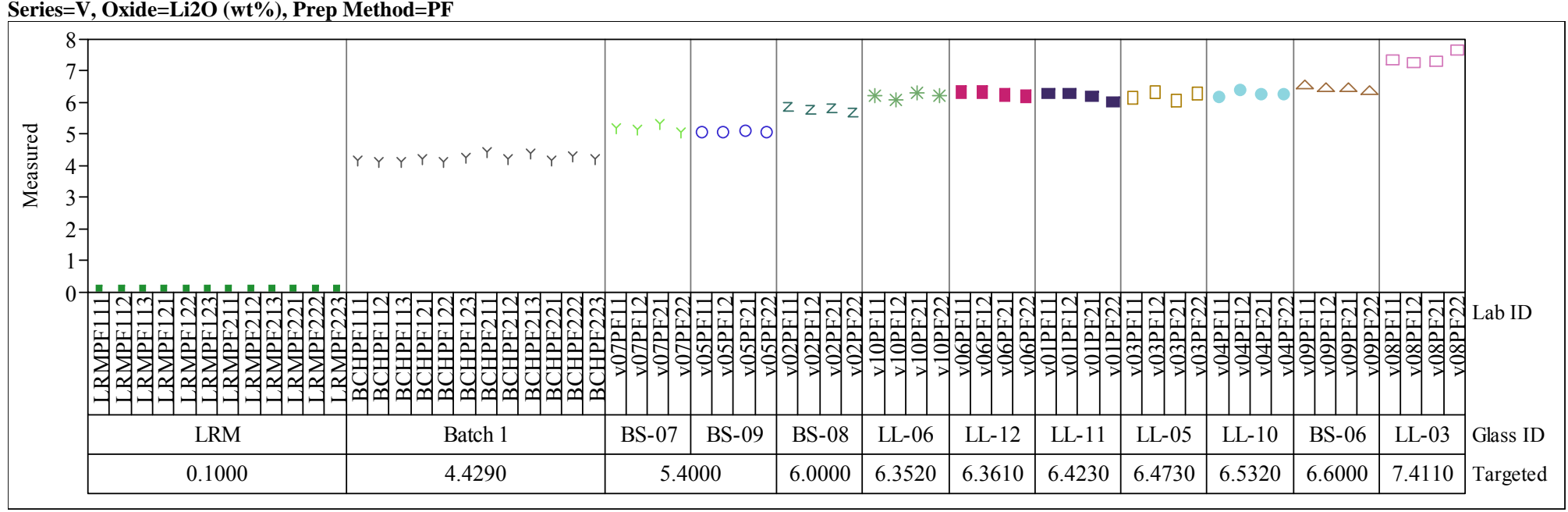

Series $=$ V, Oxide=MgO (wt \%), Prep Method $=$ LM

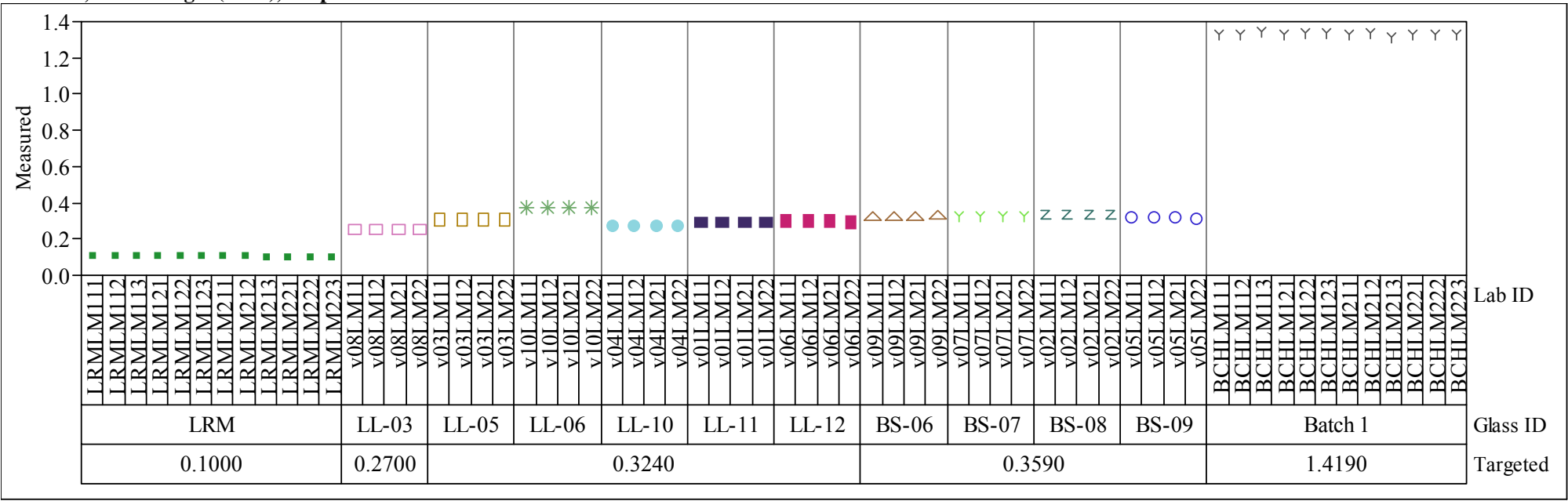


Exhibit A-2. Measurements by Lab ID within Glass ID by Targeted Concentration for Each Oxide for Each Prep by Analytical Series (continued)

Series $=\mathrm{V}$, Oxide $=\mathrm{MnO}$ (wt\%), Prep Method=LM

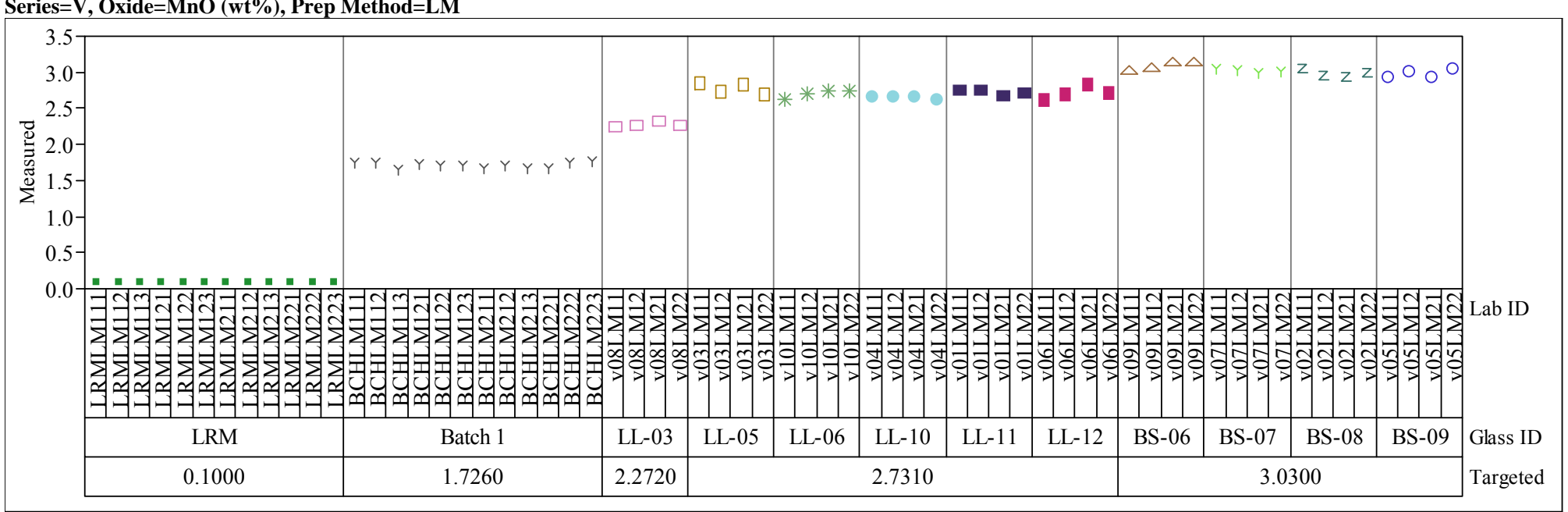

Series $=\mathrm{V}$, Oxide $=\mathrm{Na} 2 \mathrm{O}$ (wt\%), Prep Method $=\mathrm{LM}$

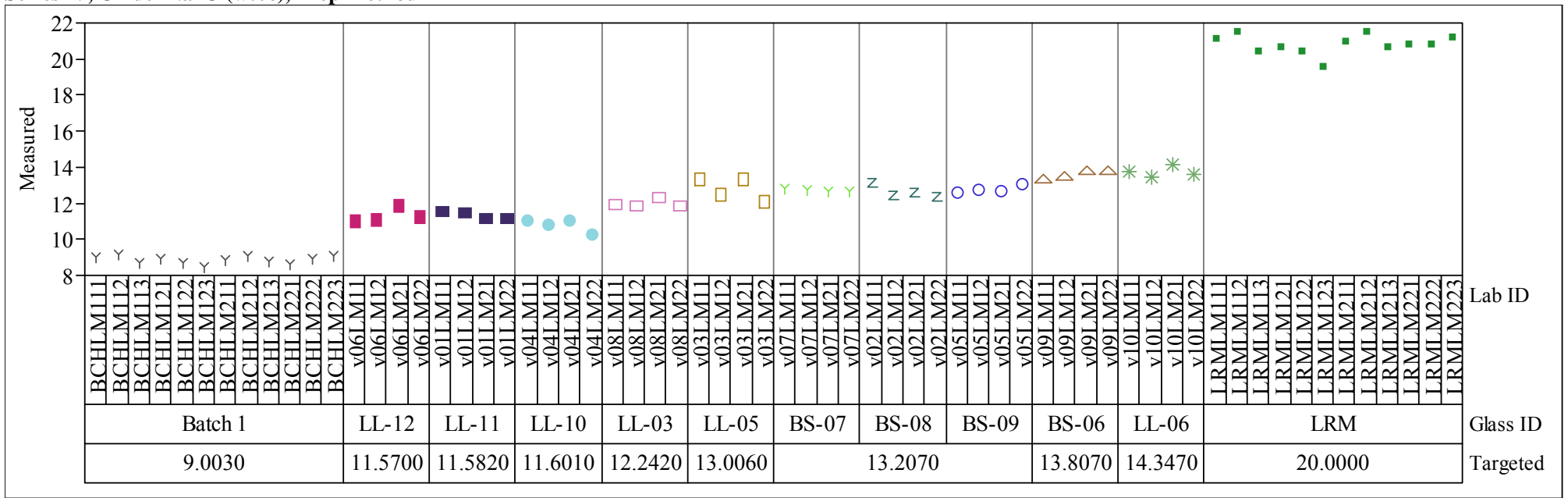


Exhibit A-2. Measurements by Lab ID within Glass ID by Targeted Concentration for Each Oxide for Each Prep by Analytical Series (continued)

Series $=\mathrm{V}$, Oxide $=\mathrm{NiO}$ (wt \%), Prep Method $=\mathrm{LM}$

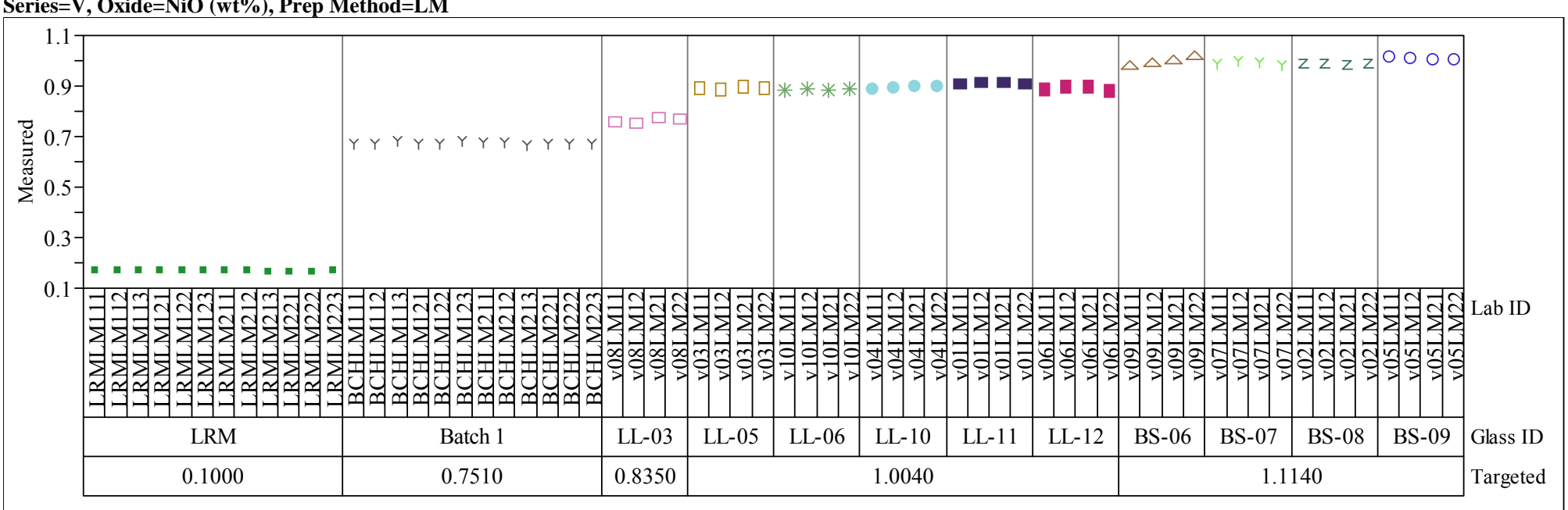

Series $=$ V, Oxide $=$ PbO (wt \%), Prep Method $=$ LM

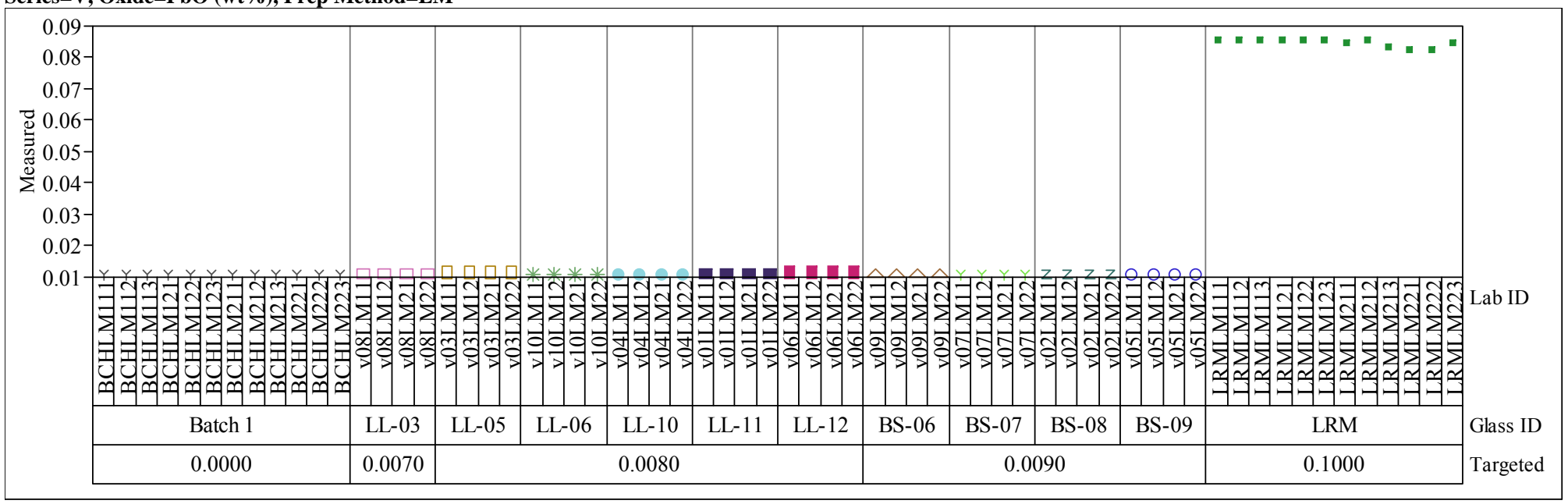


Exhibit A-2. Measurements by Lab ID within Glass ID by Targeted Concentration for Each Oxide for Each Prep by Analytical Series (continued)

Series $=\mathrm{V}$, Oxide $=\mathrm{SiO} 2$ (wt \%), Prep Method $=\mathbf{P F}$

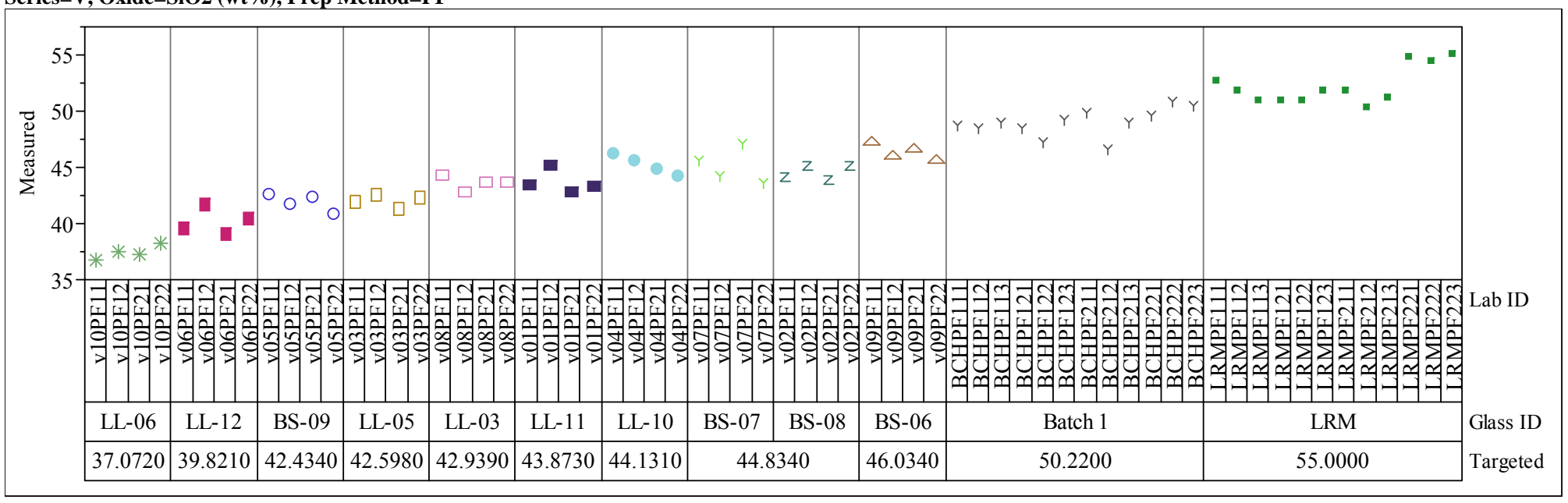

Series=V, Oxide=SO4 (wt\%), Prep Method=LM

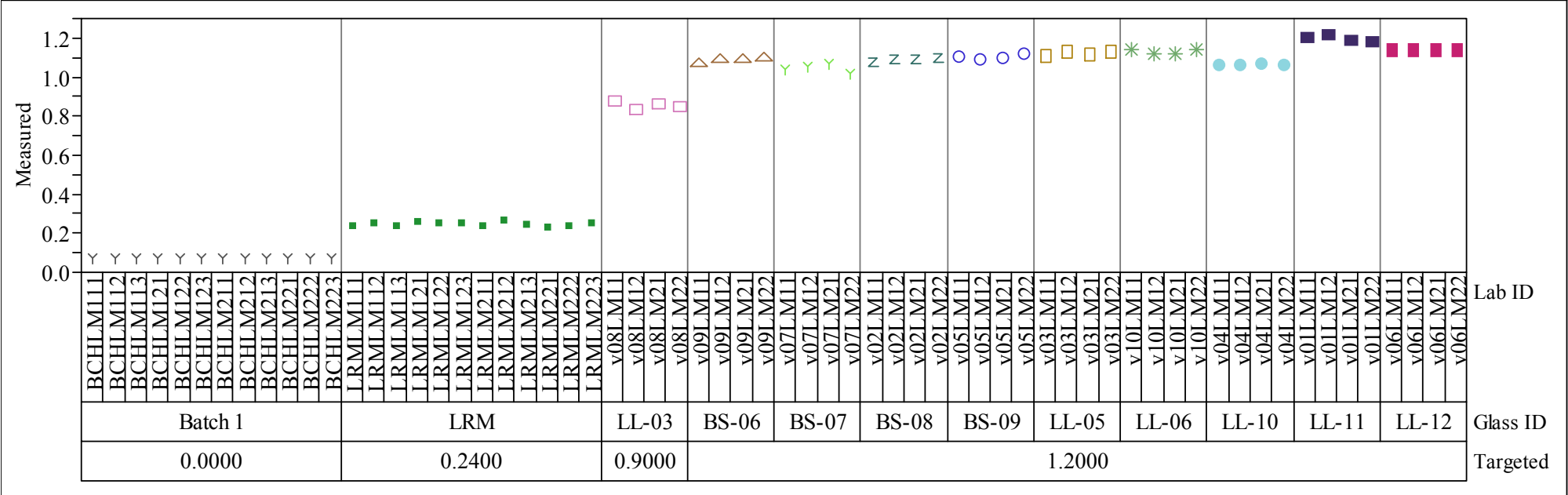


Exhibit A-2. Measurements by Lab ID within Glass ID by Targeted Concentration for Each Oxide for Each Prep by Analytical Series (continued)

Series $=$ V, Oxide=TiO2 ( $w t \%)$, Prep Method $=$ LM

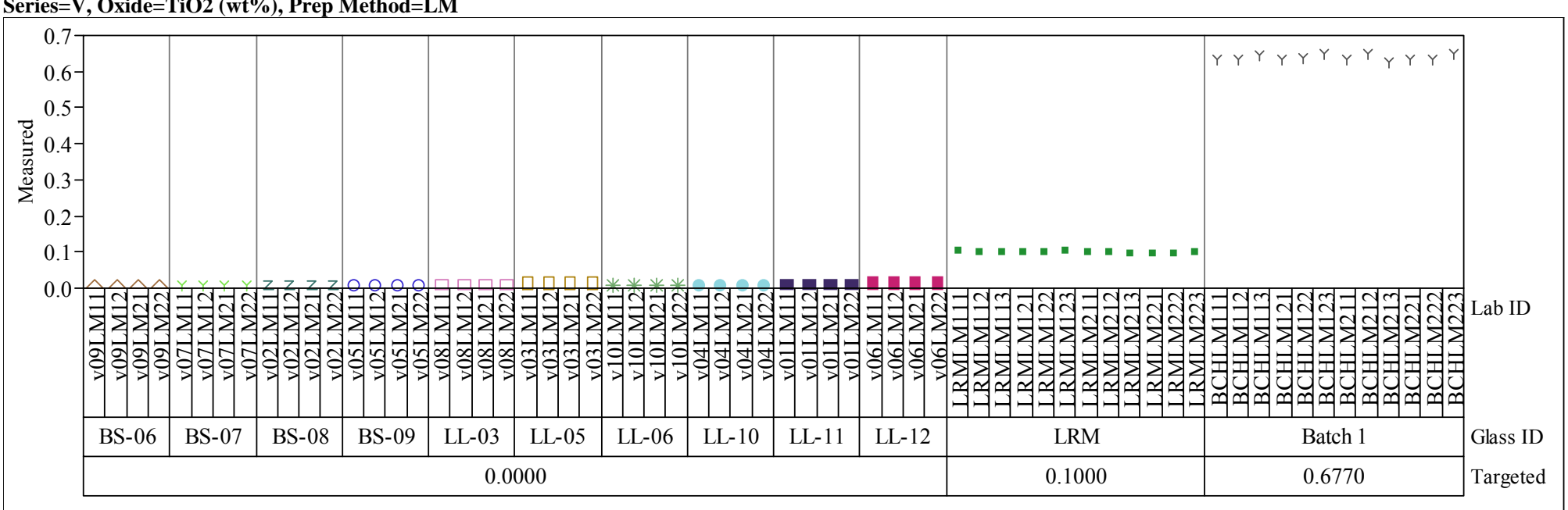

Series $=V$, Oxide $=\mathrm{ZnO}(\mathrm{wt} \%)$, Prep Method $=\mathrm{LM}$

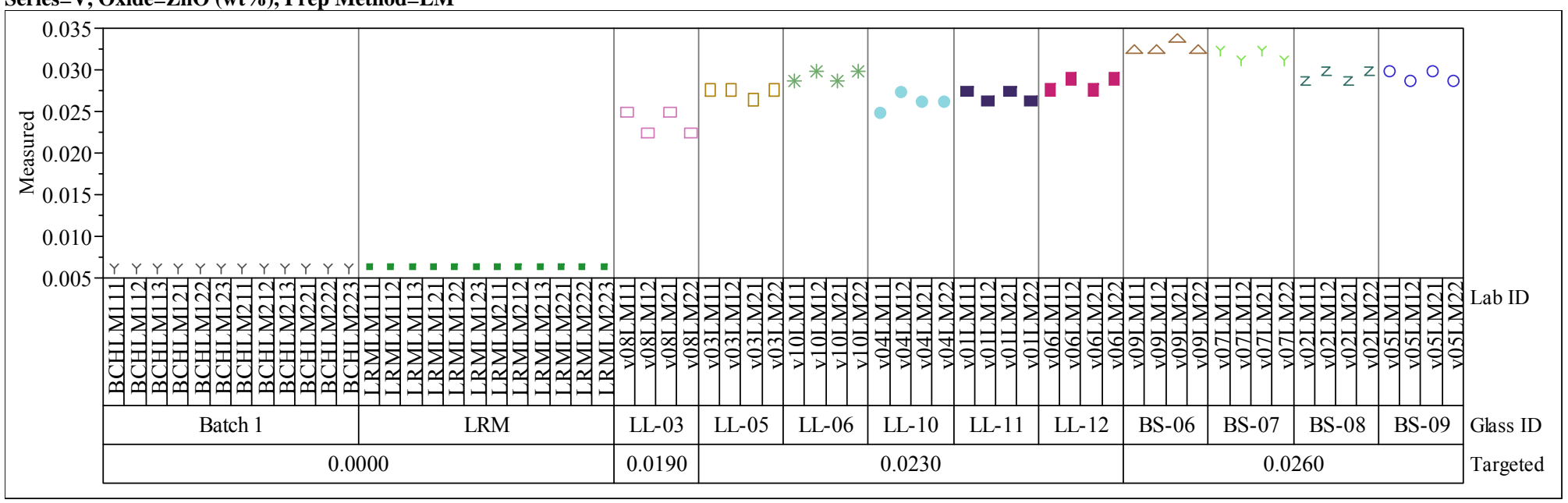


Exhibit A-2. Measurements by Lab ID within Glass ID by Targeted Concentration for Each Oxide for Each Prep by Analytical Series (continued)

Series $=$ V, Oxide $=\mathrm{ZrO} 2$ (wt \%), Prep Method $=$ LM

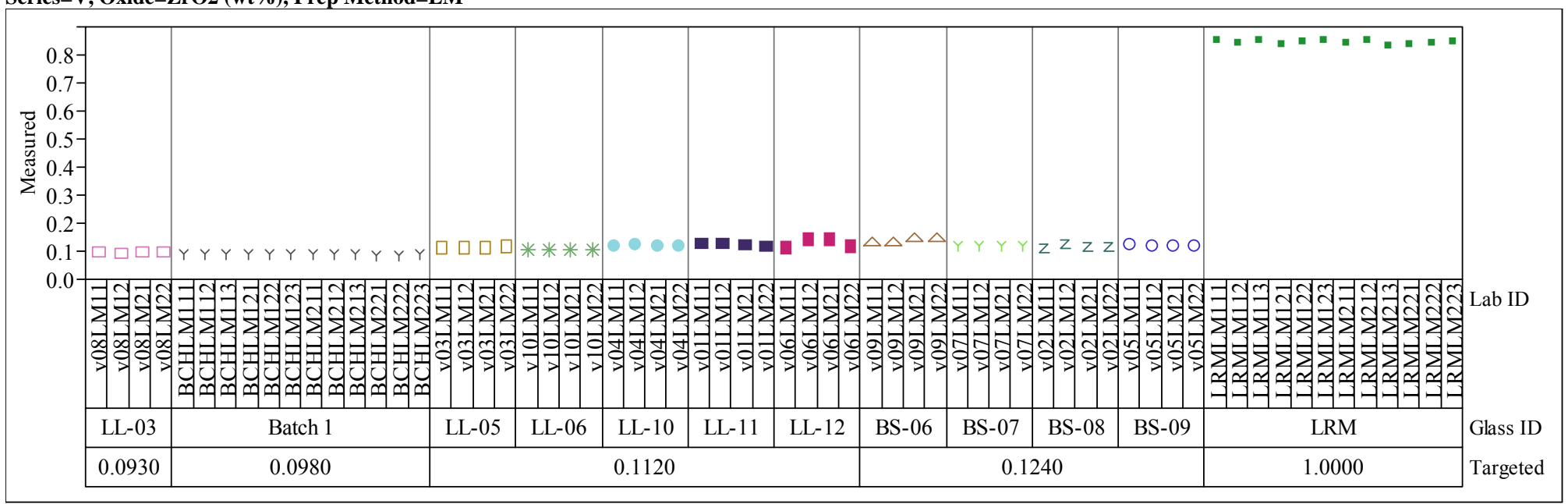

Series $=$ W, Oxide $=A 12 O 3$ (wt\%), Prep Method $=$ LM

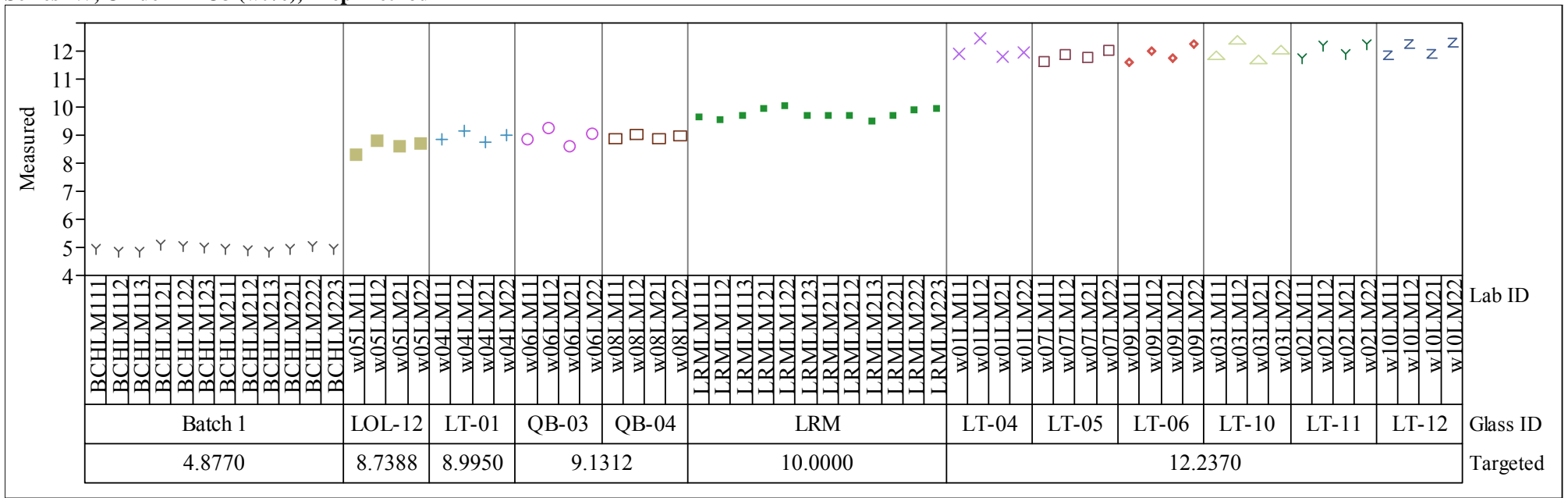


Exhibit A-2. Measurements by Lab ID within Glass ID by Targeted Concentration for Each Oxide for Each Prep by Analytical Series (continued)

Series $=W$, Oxide $=B 2 O 3$ (wt \%), Prep Method $=$ PF

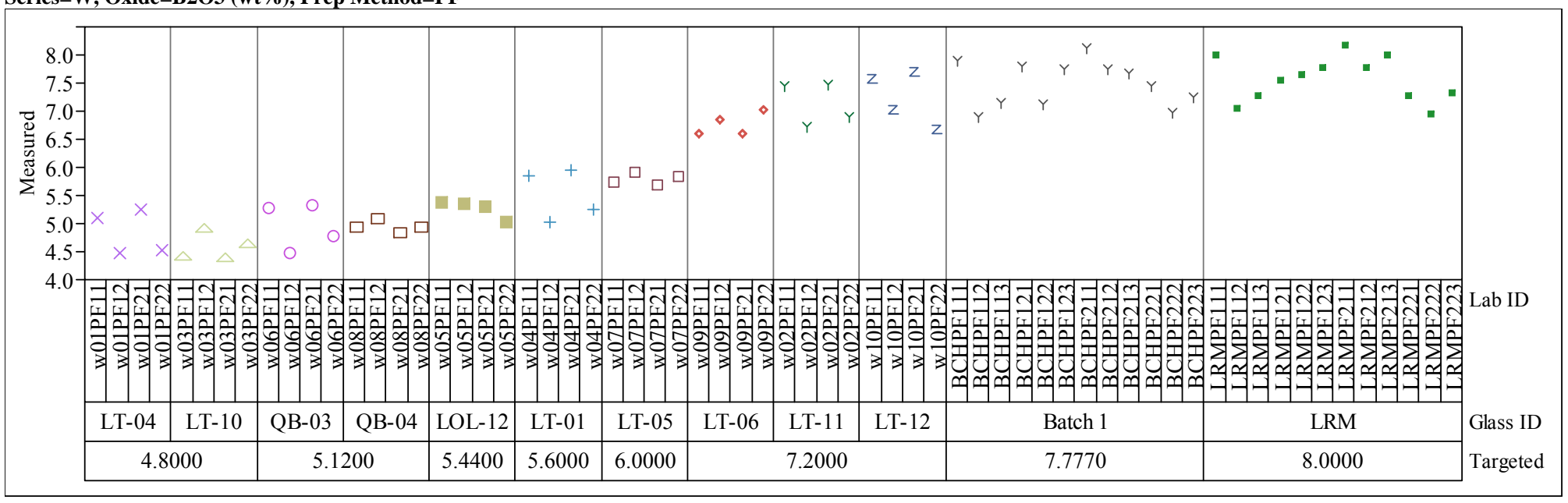

Series $=W$, Oxide $=B a O(w t \%)$, Prep Method $=L M$

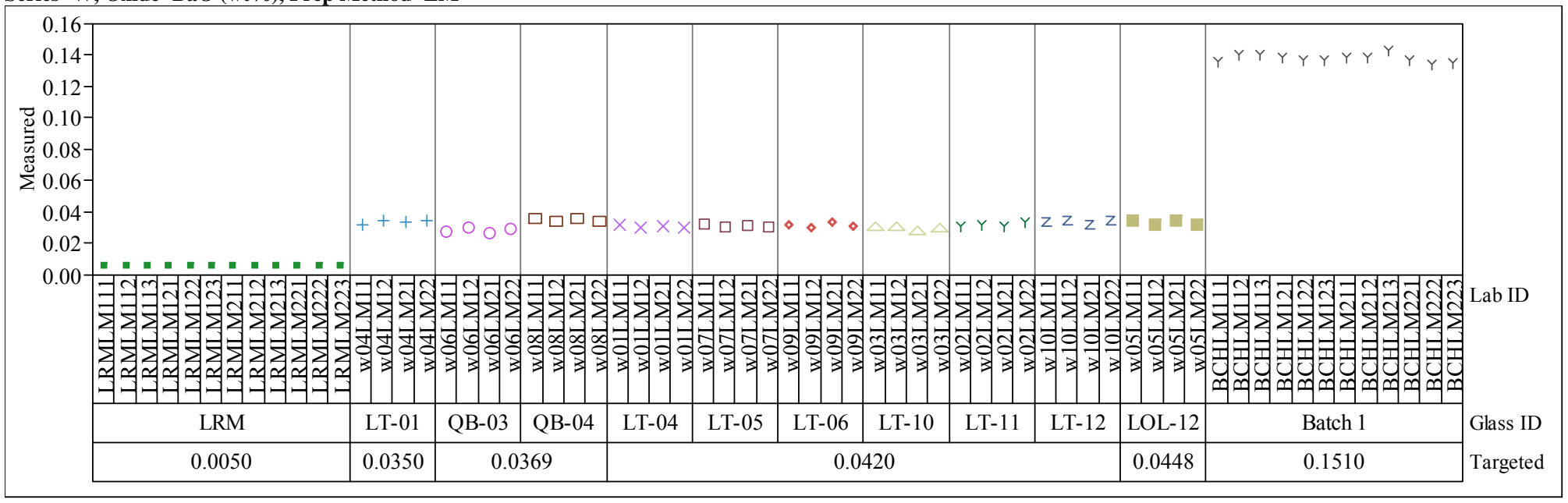


Exhibit A-2. Measurements by Lab ID within Glass ID by Targeted Concentration for Each Oxide for Each Prep by Analytical Series (continued)

Series $=$ W, Oxide $=\mathrm{CaO}$ (wt\%), Prep Method=LM

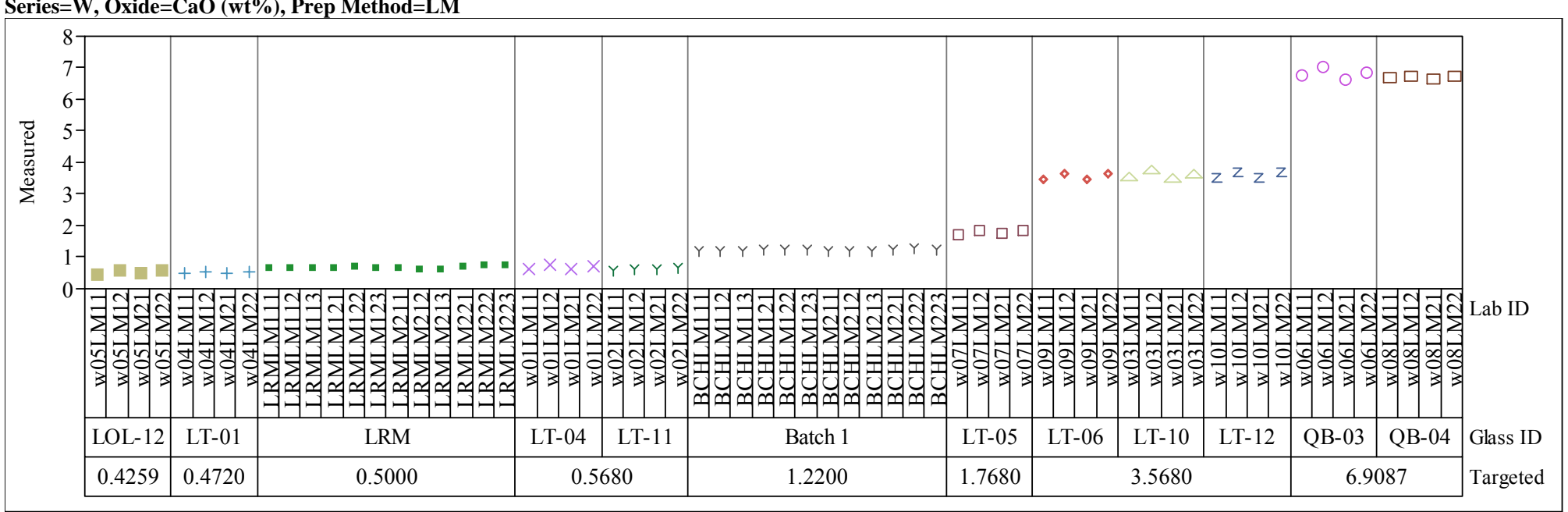

Series $=W$, Oxide $=$ Ce2O3 (wt \%), Prep Method $=$ LM

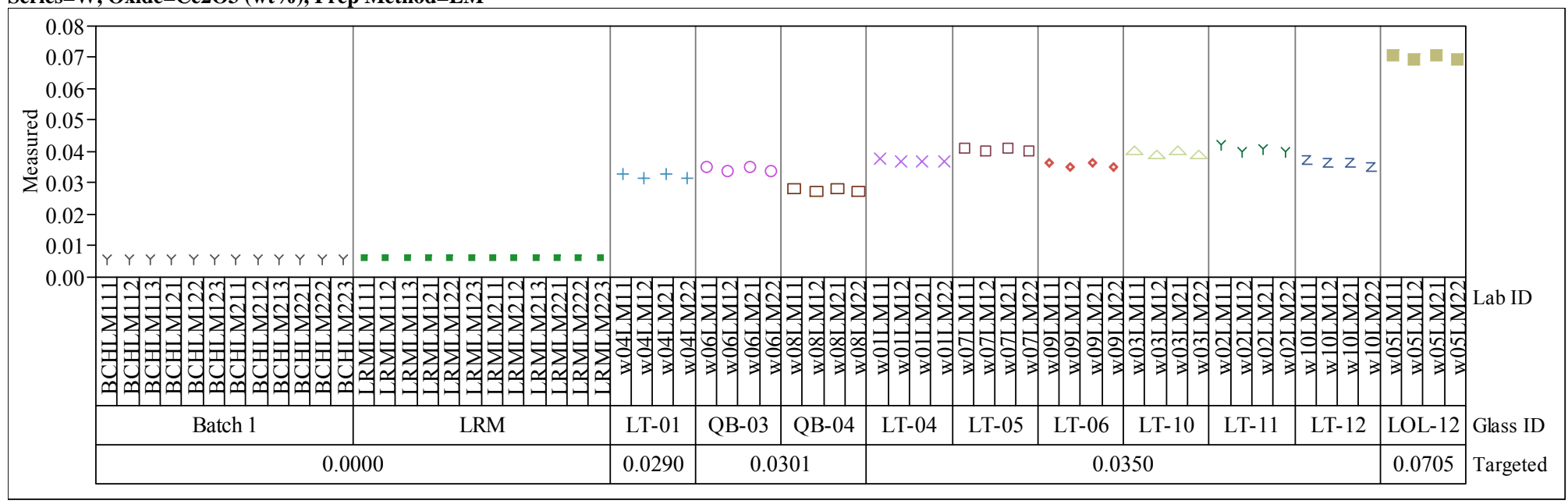


Exhibit A-2. Measurements by Lab ID within Glass ID by Targeted Concentration for Each Oxide for Each Prep by Analytical Series (continued)

Series $=$ W, Oxide $=\mathrm{Cr} 2 \mathrm{O} 3$ (wt \%), Prep Method $=$ LM

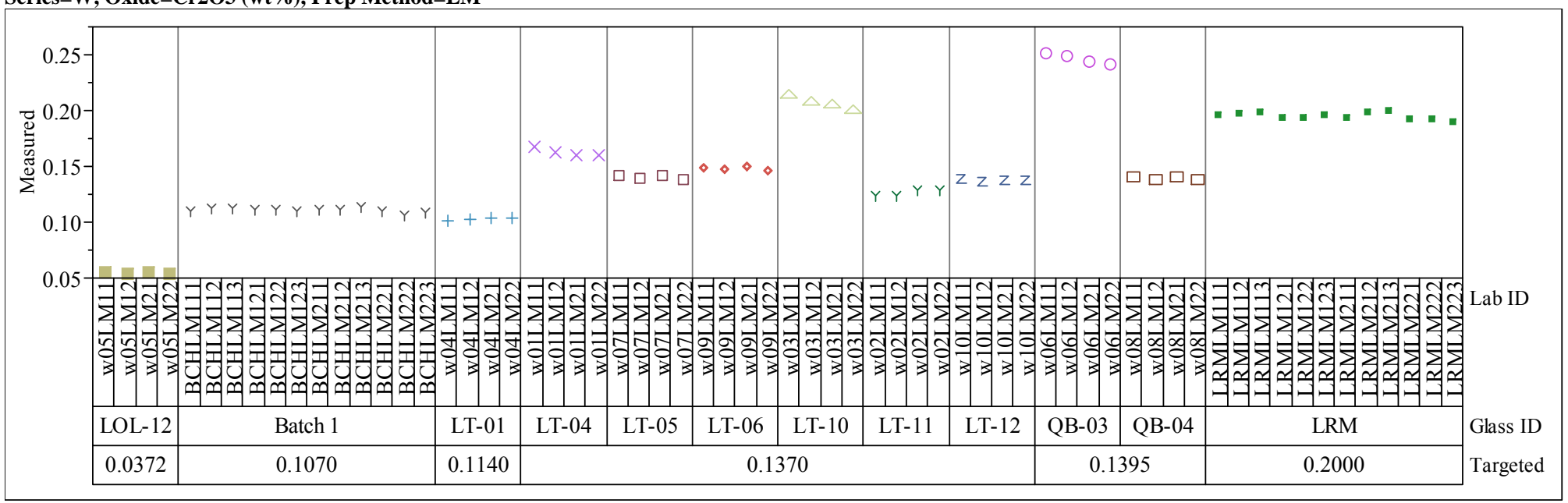

Series $=$ W, Oxide $=\mathrm{CuO}$ (wt\%), Prep Method=LM

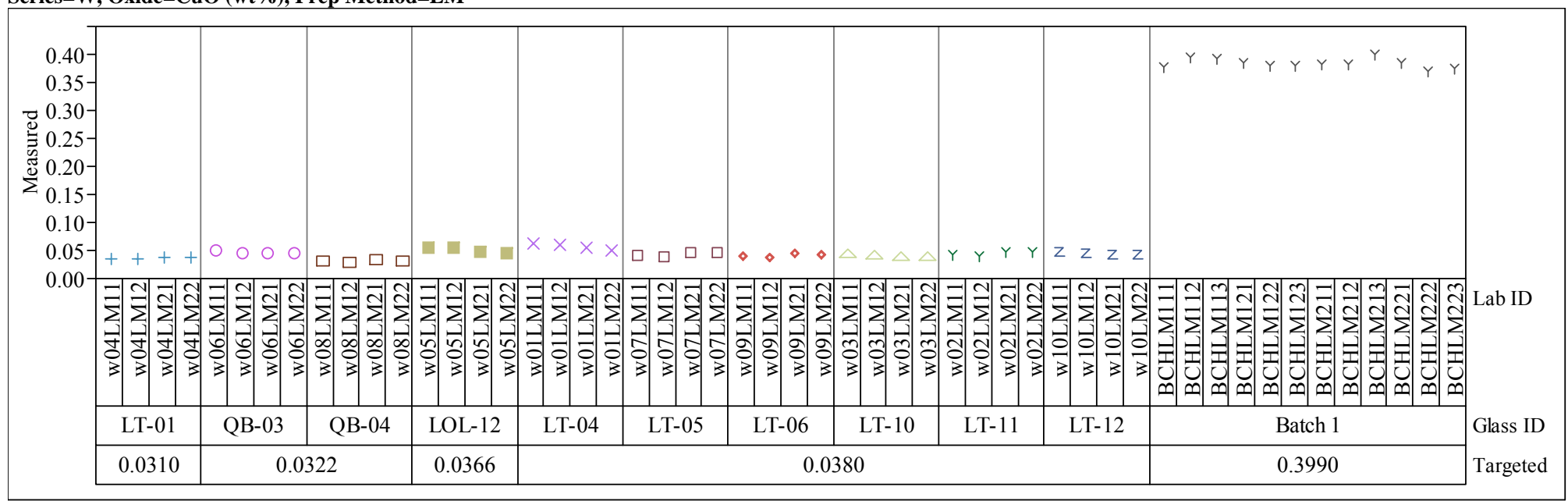


Exhibit A-2. Measurements by Lab ID within Glass ID by Targeted Concentration for Each Oxide for Each Prep by Analytical Series (continued)

Series $=$ W, Oxide $=F e 2 O 3$ (wt \%), Prep Method $=$ LM

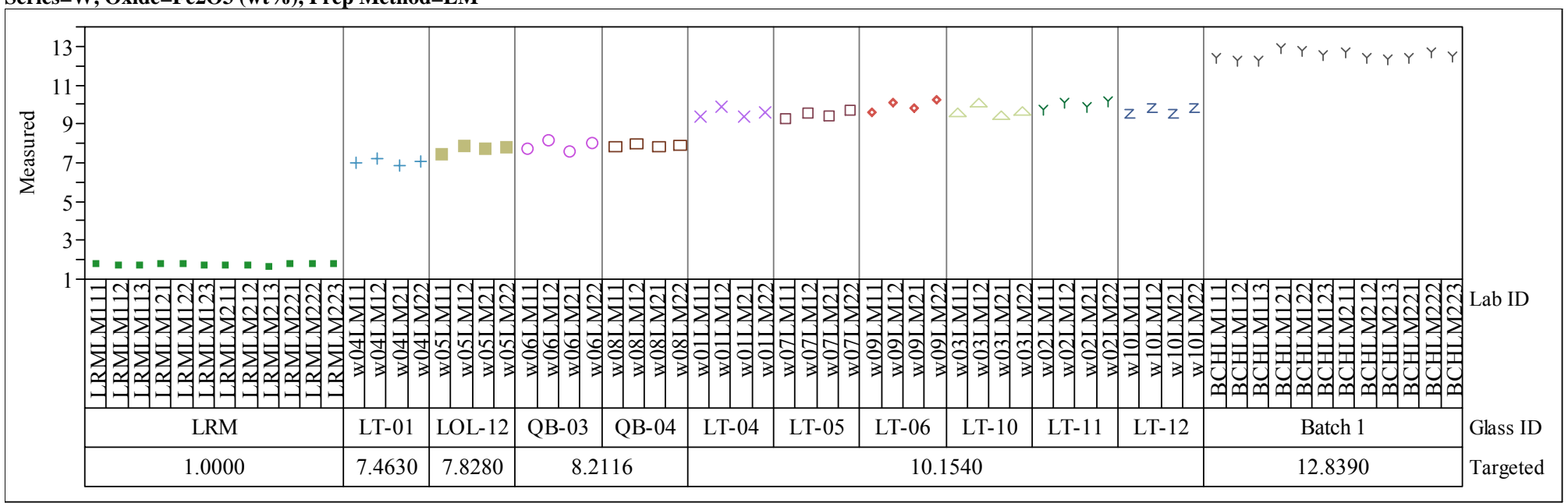

Series $=W$, Oxide $=$ K2O (wt\%), Prep Method=LM

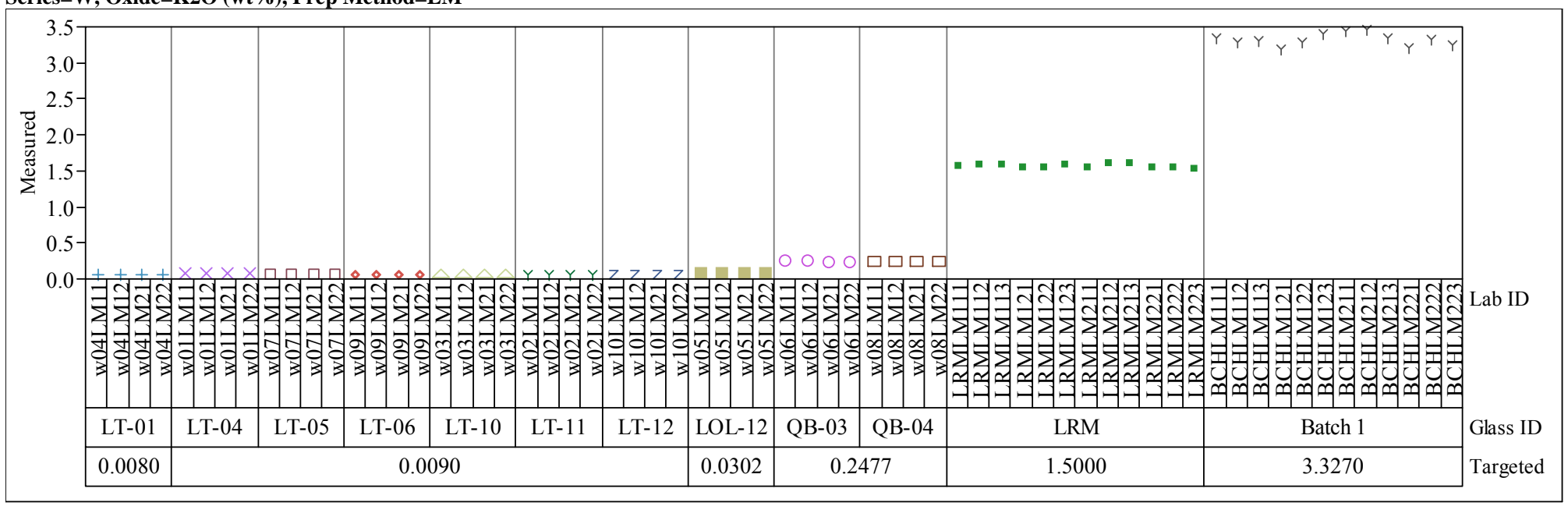


Exhibit A-2. Measurements by Lab ID within Glass ID by Targeted Concentration for Each Oxide for Each Prep by Analytical Series (continued)

Series=W, Oxide $=$ La2O3 (wt \%), Prep Method $=$ LM

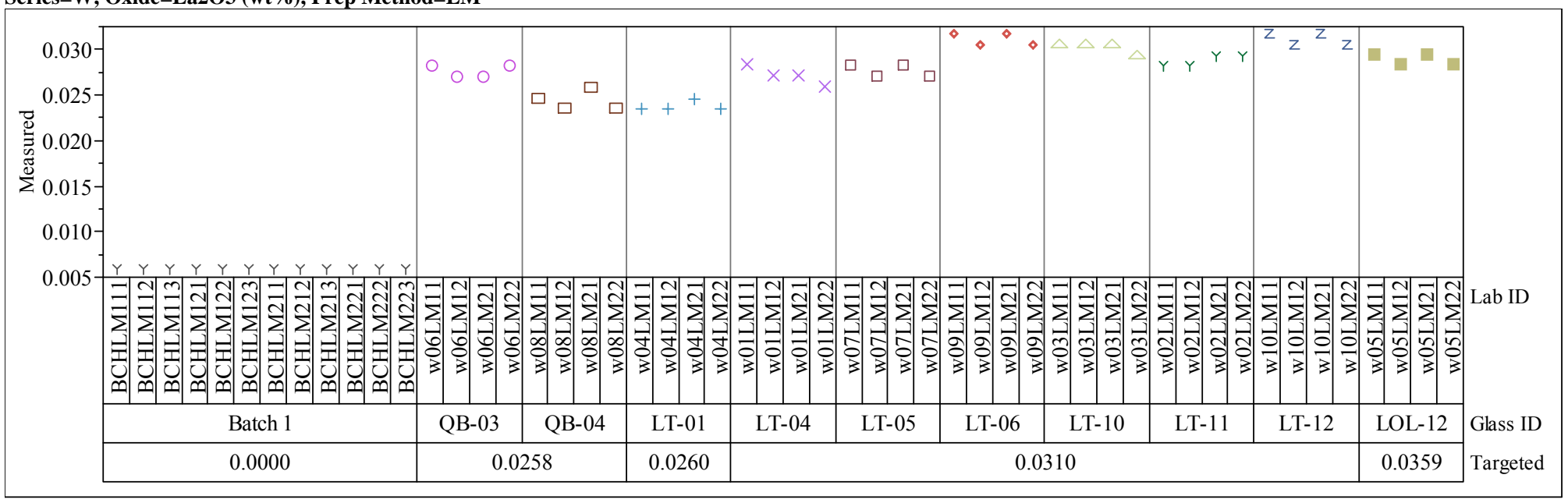

Series $=W$, Oxide $=$ Li2O (wt $\%)$, Prep Method $=$ PF

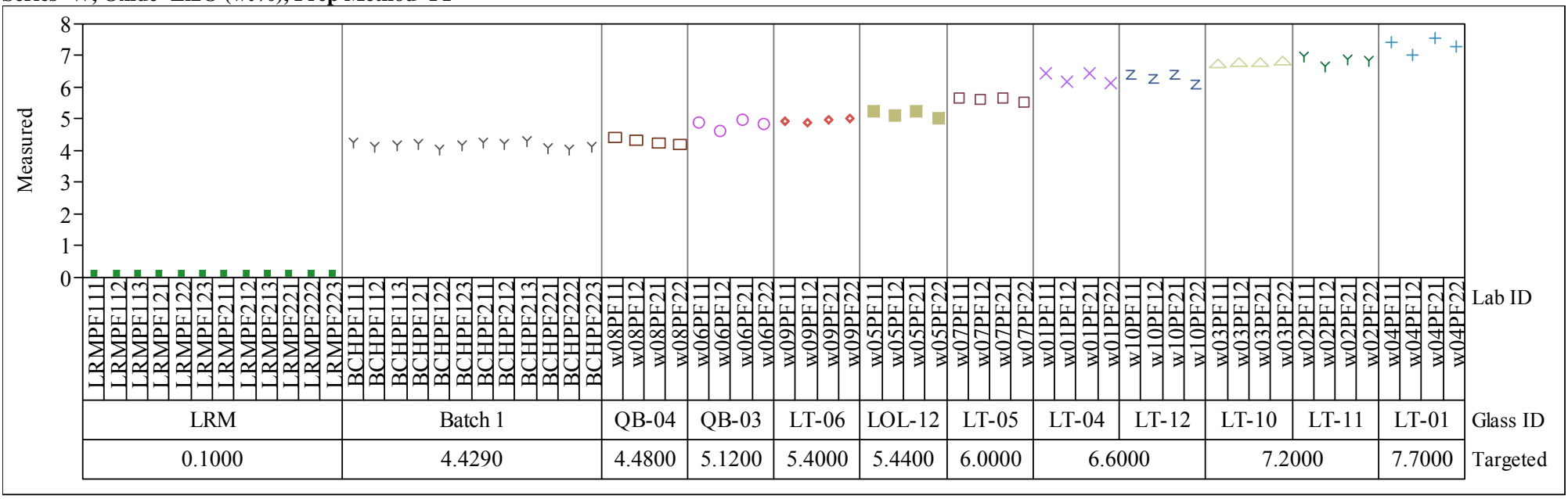


Exhibit A-2. Measurements by Lab ID within Glass ID by Targeted Concentration for Each Oxide for Each Prep by Analytical Series (continued)

Series $=\mathrm{W}$, Oxide=MgO (wt \%), Prep Method $=$ LM

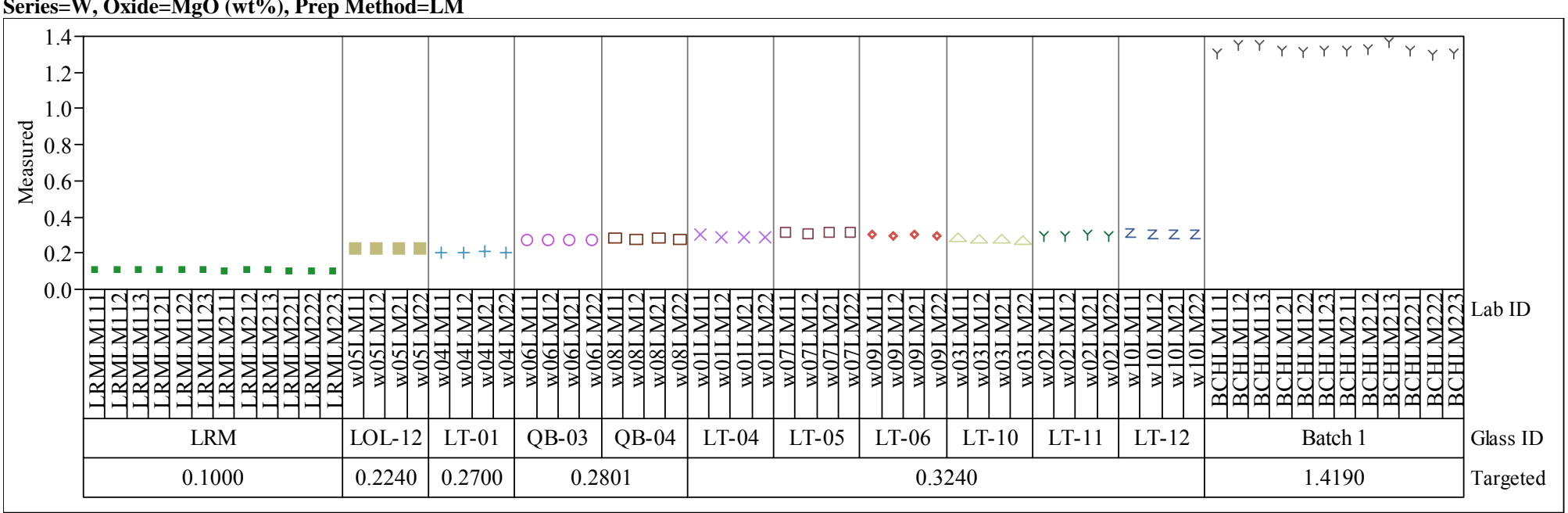

Series $=\mathrm{W}$, Oxide $=\mathrm{MnO}(\mathrm{wt} \%)$, Prep Method $=\mathrm{LM}$

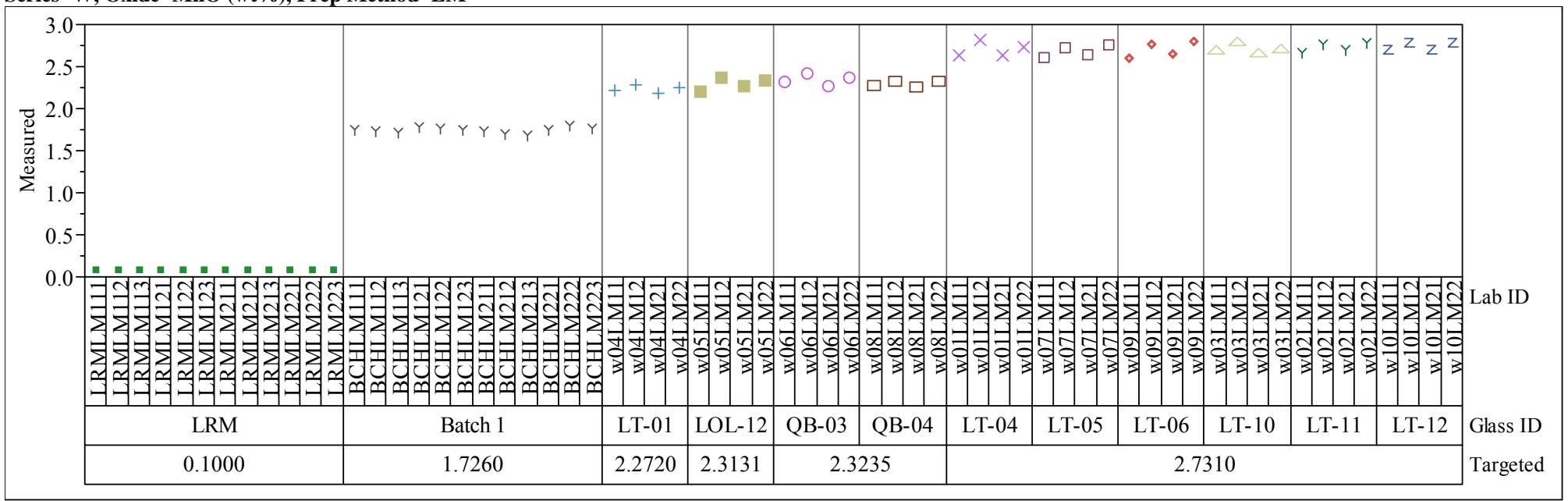


Exhibit A-2. Measurements by Lab ID within Glass ID by Targeted Concentration for Each Oxide for Each Prep by Analytical Series (continued)

Series $=W$, Oxide $=$ Na2O $(w t \%)$, Prep Method $=L M$

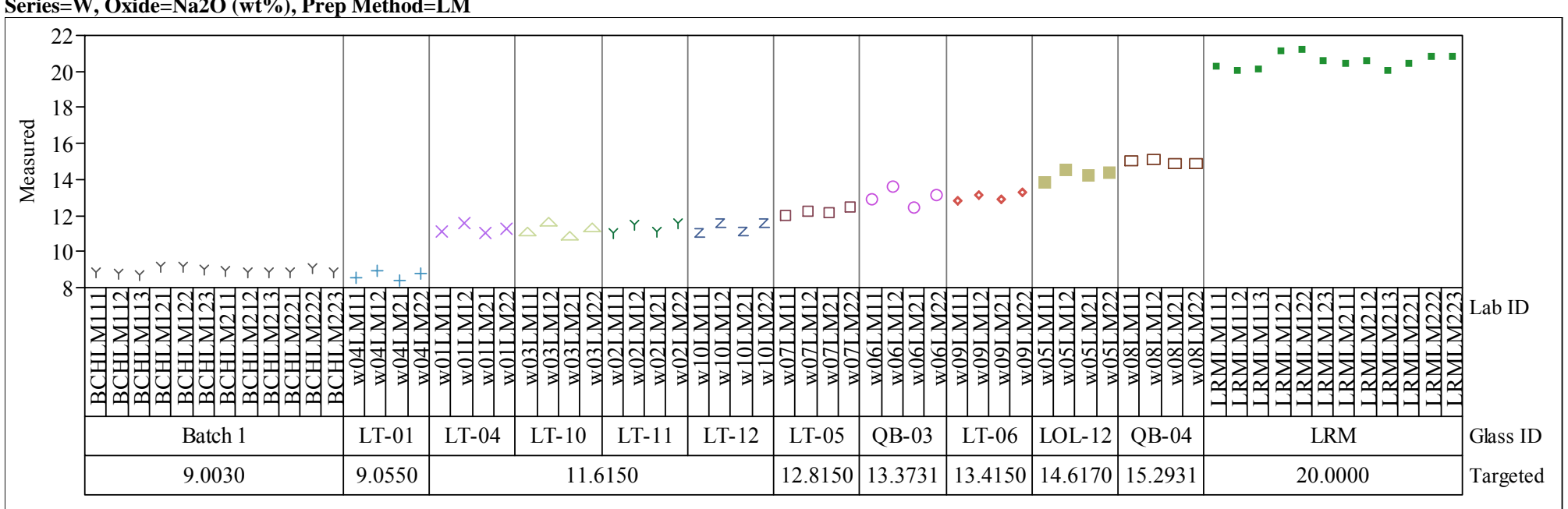

Series $=$ W, Oxide $=$ NiO (wt \%), Prep Method $=$ LM

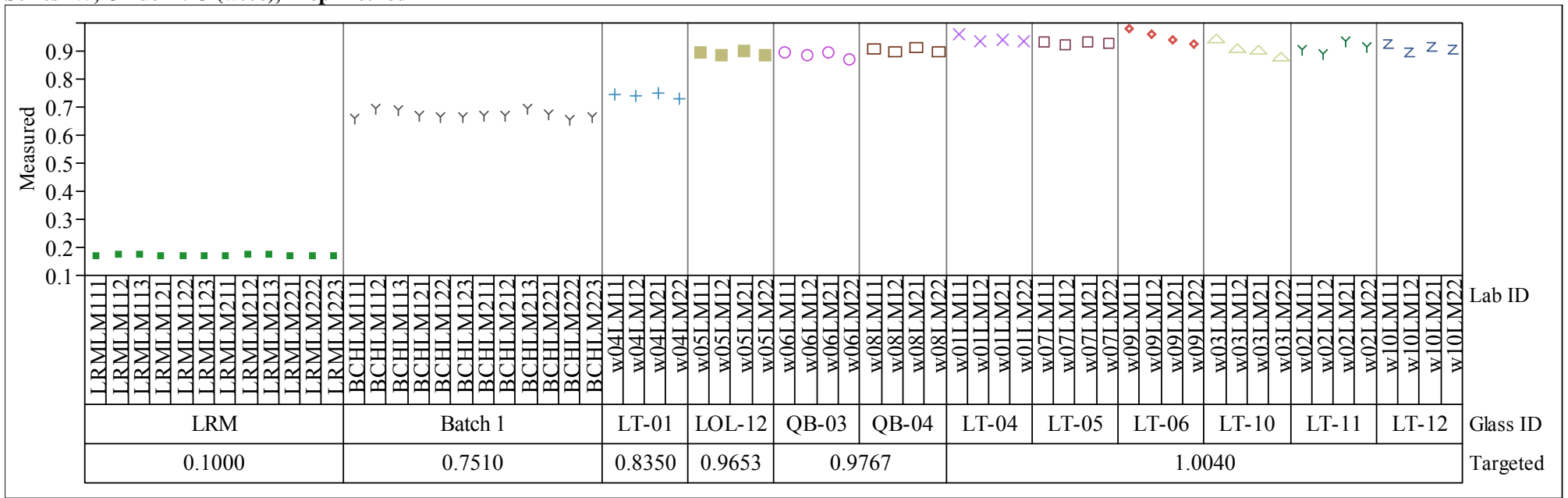


Exhibit A-2. Measurements by Lab ID within Glass ID by Targeted Concentration for Each Oxide for Each Prep by Analytical Series (continued)

Series $=W$, Oxide $=$ PbO ( $w t \%)$, Prep Method $=L M$

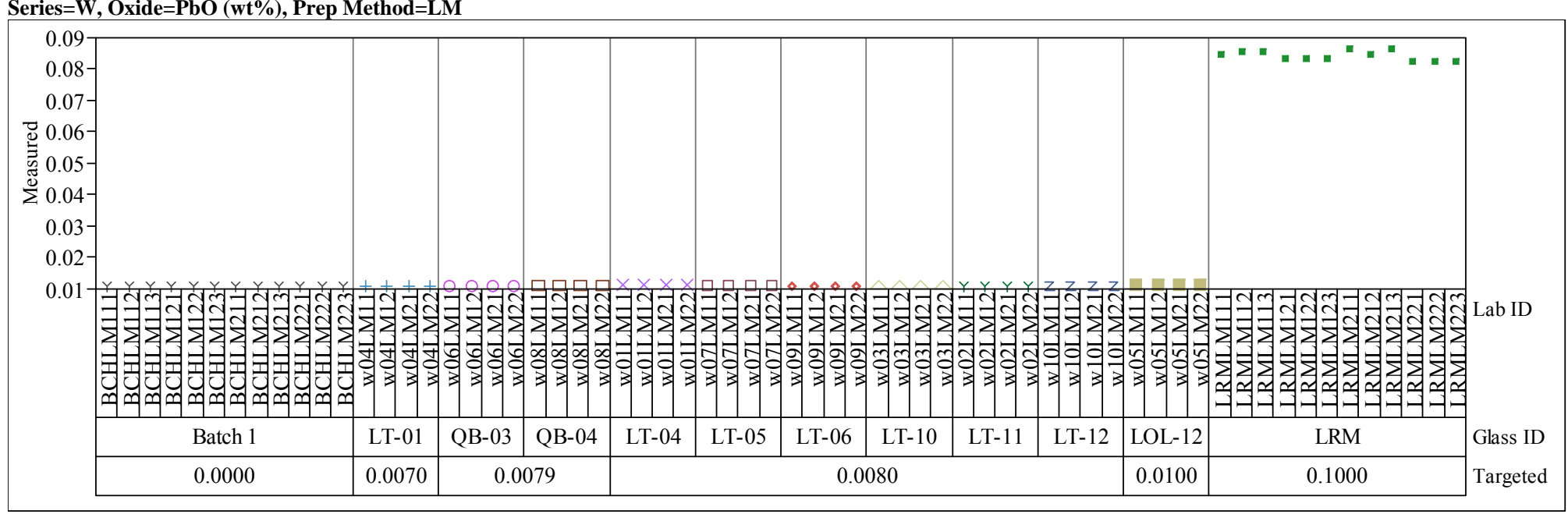

Series $=\mathrm{W}$, Oxide $=\mathrm{SiO} 2$ (wt\%), Prep Method $=\mathrm{PF}$

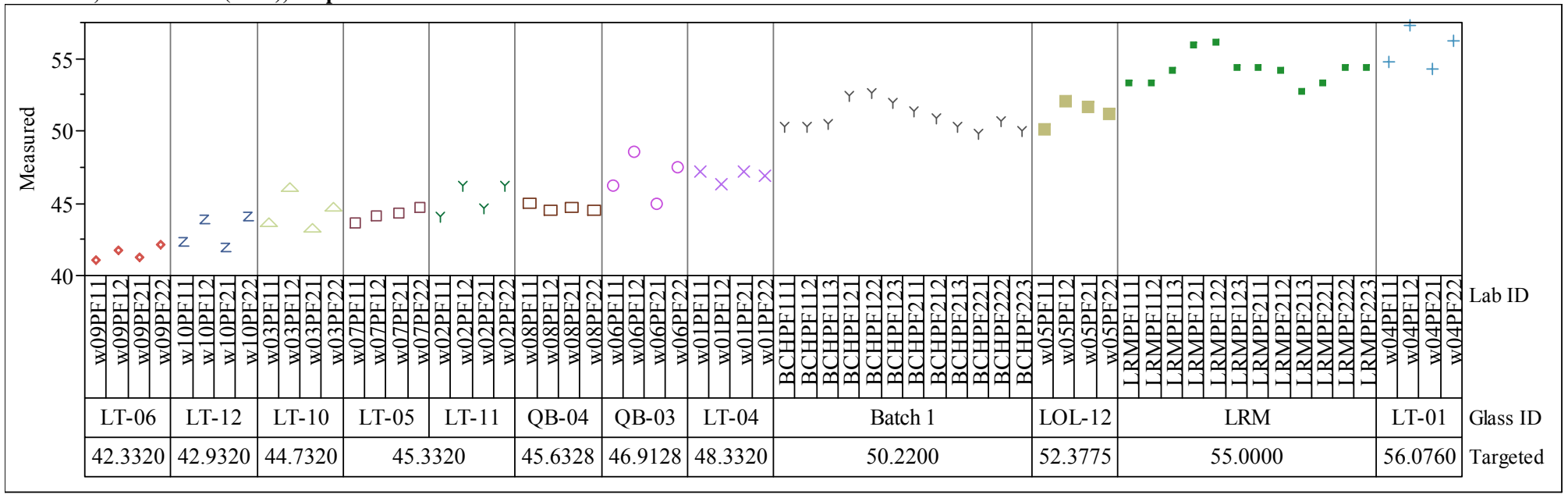


Exhibit A-2. Measurements by Lab ID within Glass ID by Targeted Concentration for Each Oxide for Each Prep by Analytical Series (continued)

Series $=$ W, Oxide $=\mathrm{SO} 4$ (wt \%), Prep Method $=$ LM

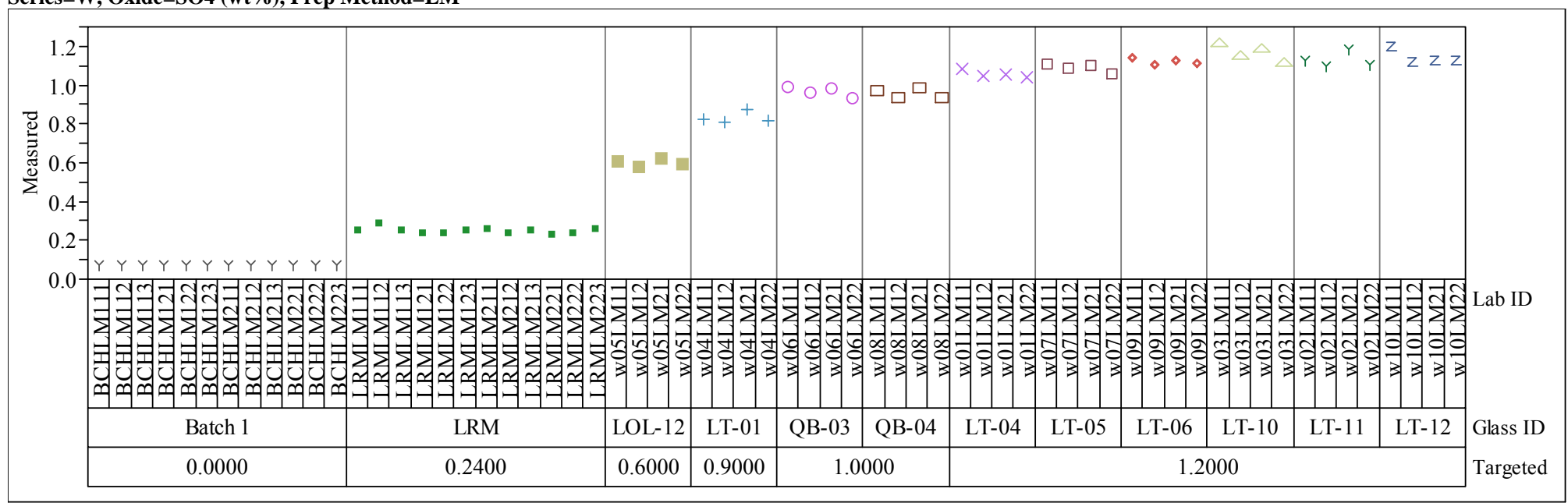

Series $=W$, Oxide=TiO2 $(w t \%)$, Prep Method $=$ LM

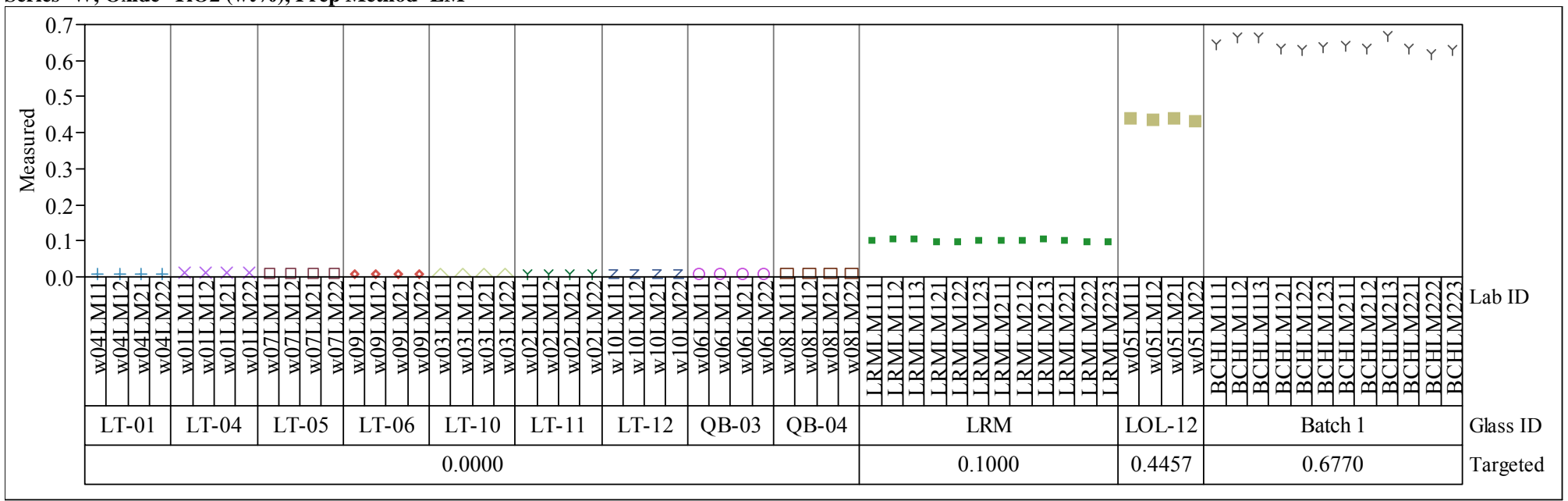


Exhibit A-2. Measurements by Lab ID within Glass ID by Targeted Concentration for Each Oxide for Each Prep by Analytical Series (continued)

Series $=W$, Oxide $=\mathrm{ZnO}($ wt $\%)$, Prep Method $=$ LM

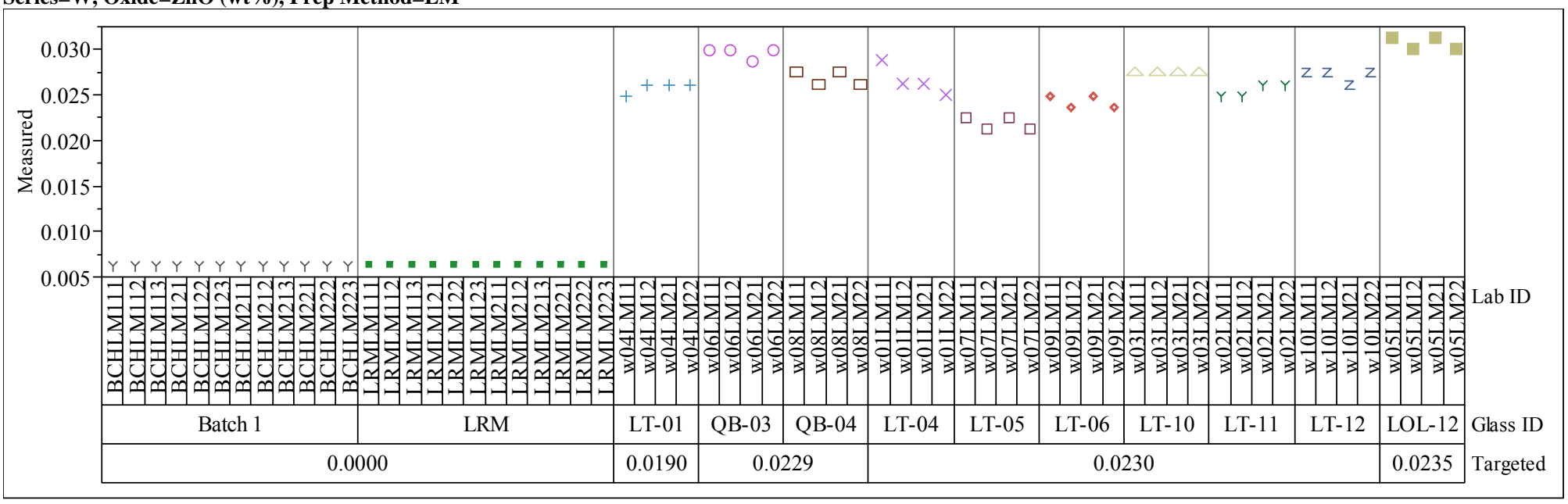

Series=W, Oxide=ZrO2 (wt\%), Prep Method=LM

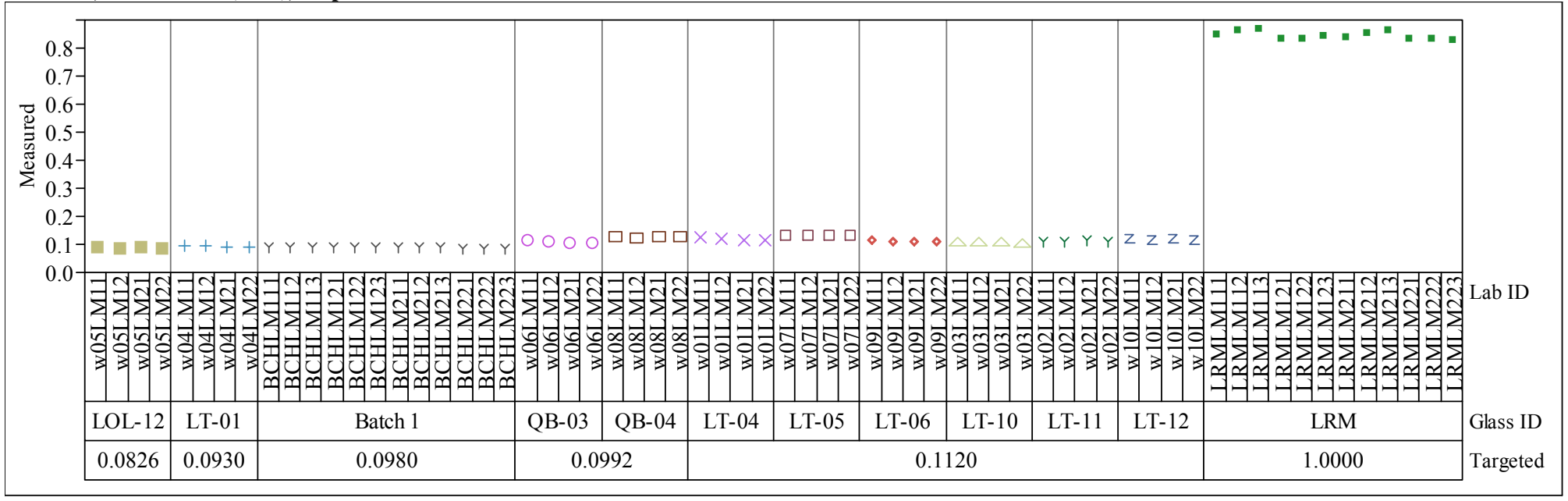


Exhibit A-2. Measurements by Lab ID within Glass ID by Targeted Concentration for Each Oxide for Each Prep by Analytical Series (continued)

Series $=$ X, Oxide=Al2O3 (wt \%), Prep Method $=$ LM

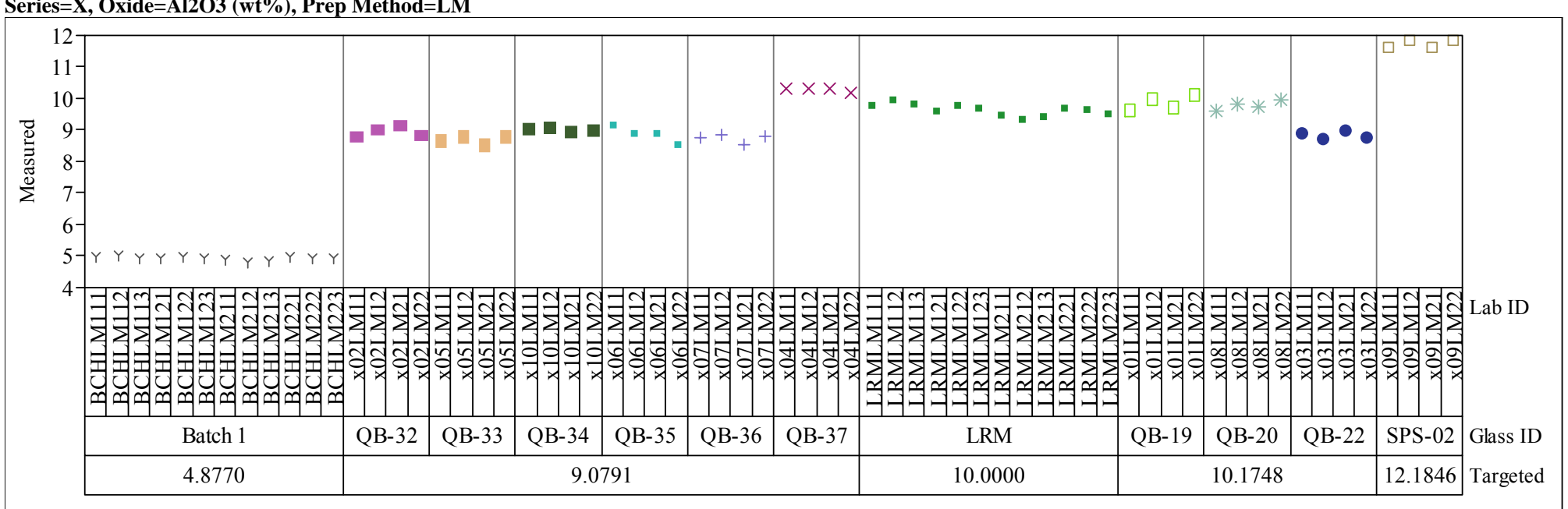

Series $=$ X, Oxide $=$ B2O3 (wt \%), Prep Method $=$ PF

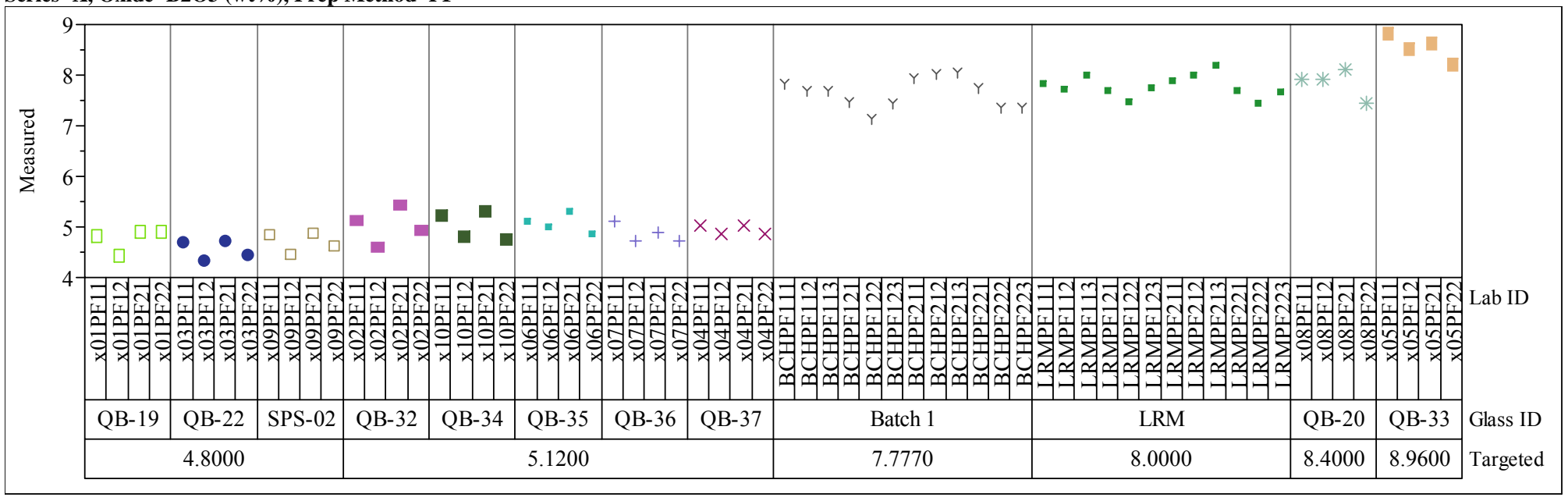


Exhibit A-2. Measurements by Lab ID within Glass ID by Targeted Concentration for Each Oxide for Each Prep by Analytical Series (continued)

Series $=X$, Oxide $=\mathrm{BaO}(\mathrm{wt} \%)$, Prep Method $=\mathrm{LM}$

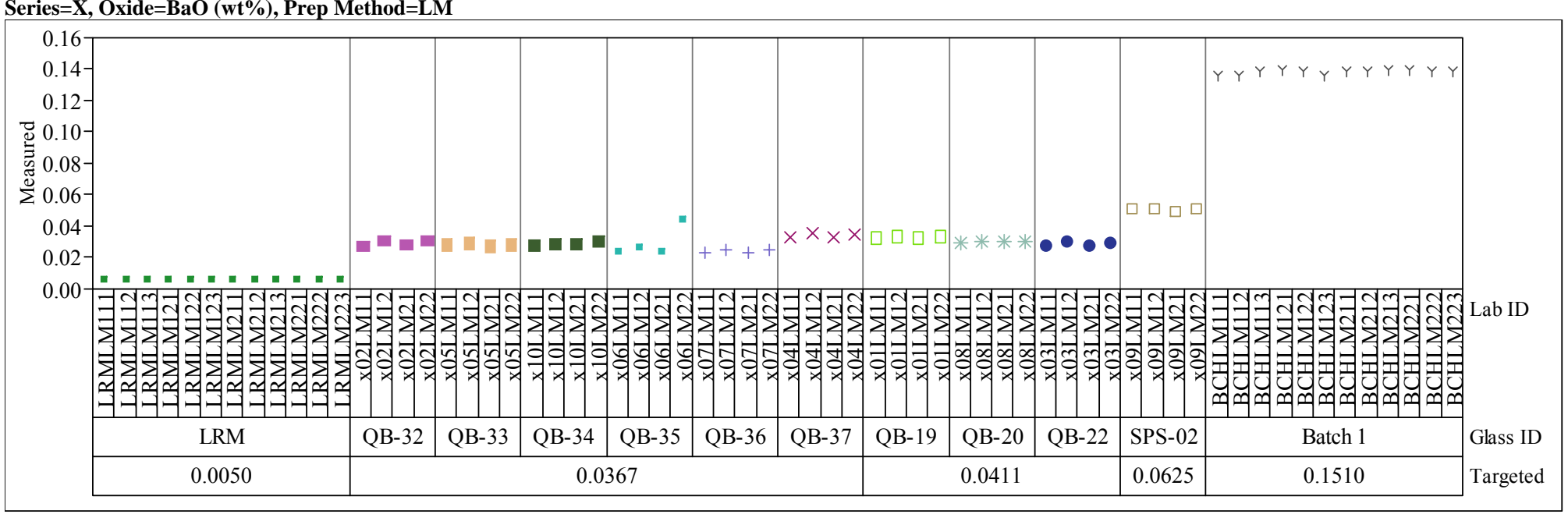

Series $=$ X, Oxide $=\mathbf{C a O}(w t \%)$, Prep Method $=\mathbf{L M}$

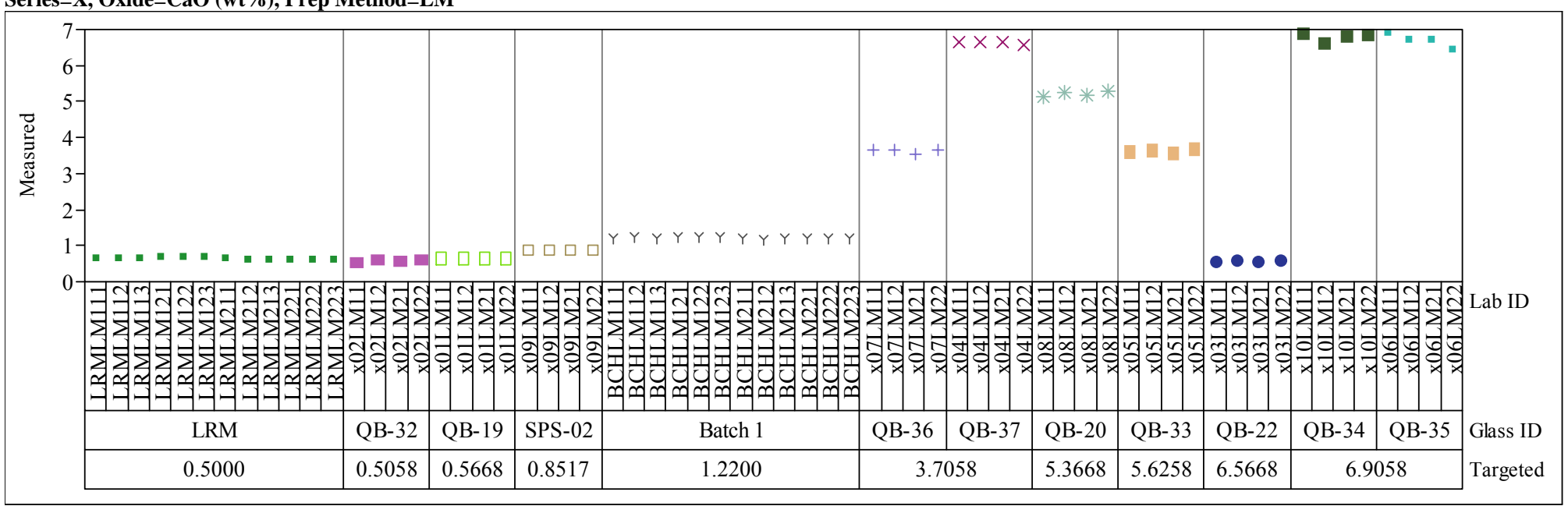


Exhibit A-2. Measurements by Lab ID within Glass ID by Targeted Concentration for Each Oxide for Each Prep by Analytical Series (continued)

Series $=$ X, Oxide $=\mathrm{Ce} 2 \mathrm{O} 3(\mathrm{wt} \%)$, Prep Method $=$ LM

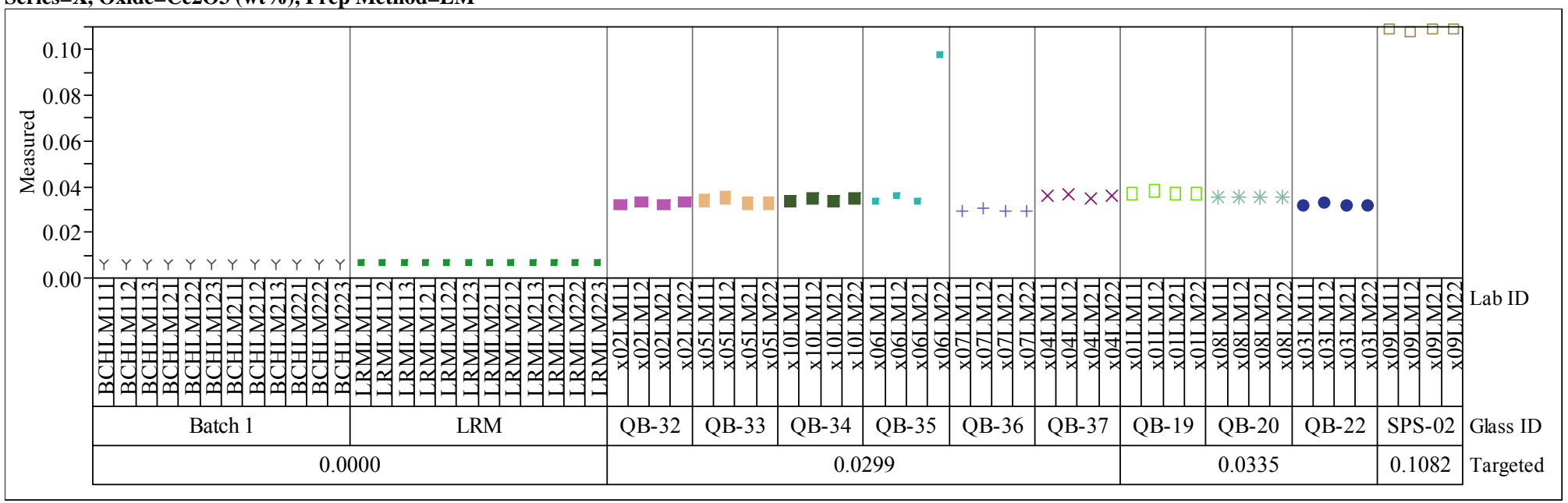

Series $=\mathrm{X}$, Oxide $=\mathrm{Cr} 2 \mathrm{O} 3(\mathrm{wt} \%)$, Prep Method $=\mathrm{LM}$

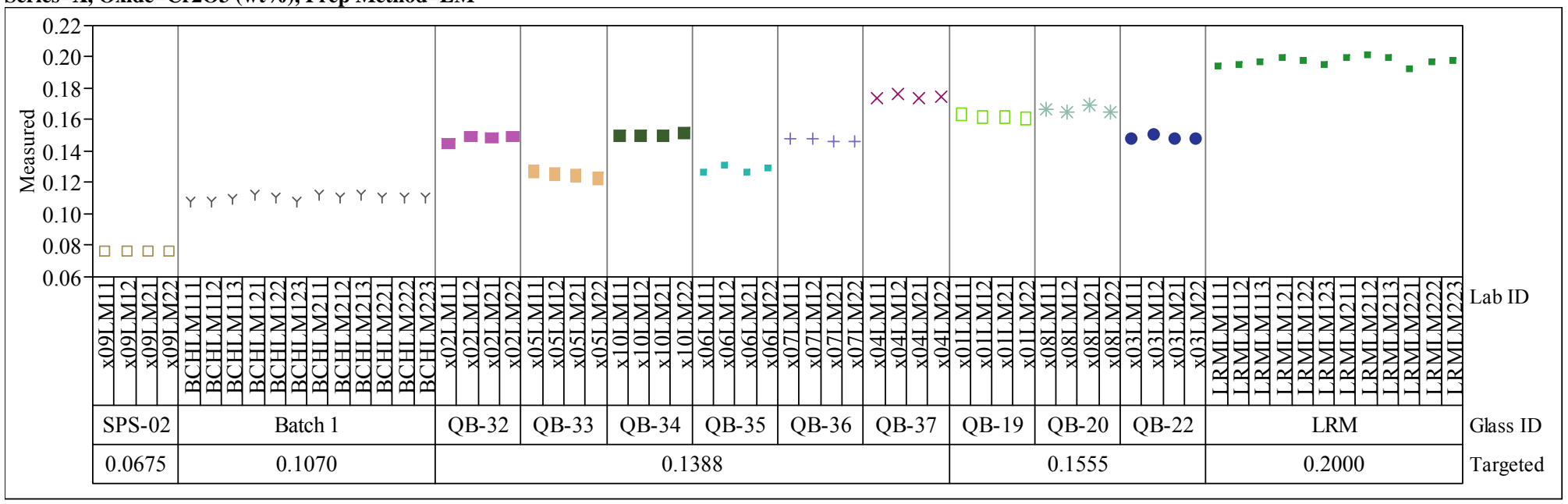


Exhibit A-2. Measurements by Lab ID within Glass ID by Targeted Concentration for Each Oxide for Each Prep by Analytical Series (continued)

Series $=\mathbf{X}$, Oxide $=\mathrm{CuO}(\mathrm{wt} \%)$, Prep Method $=\mathrm{LM}$

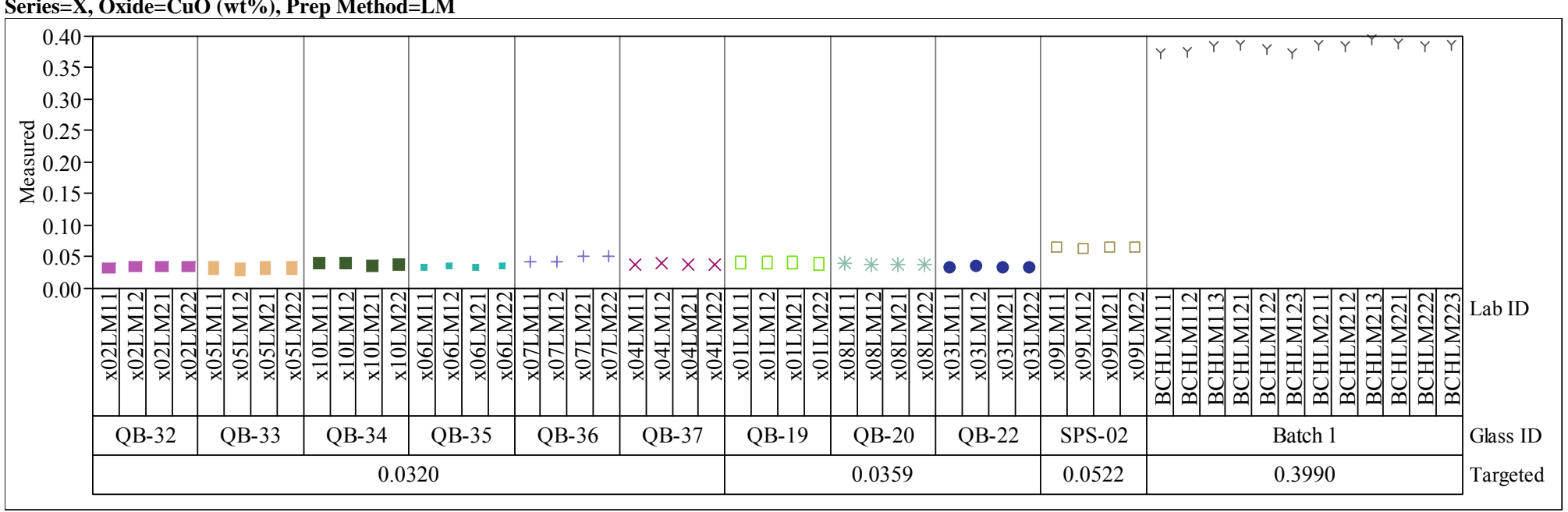

Series $=\mathrm{X}$, Oxide=Fe2O3 (wt\%), Prep Method=LM

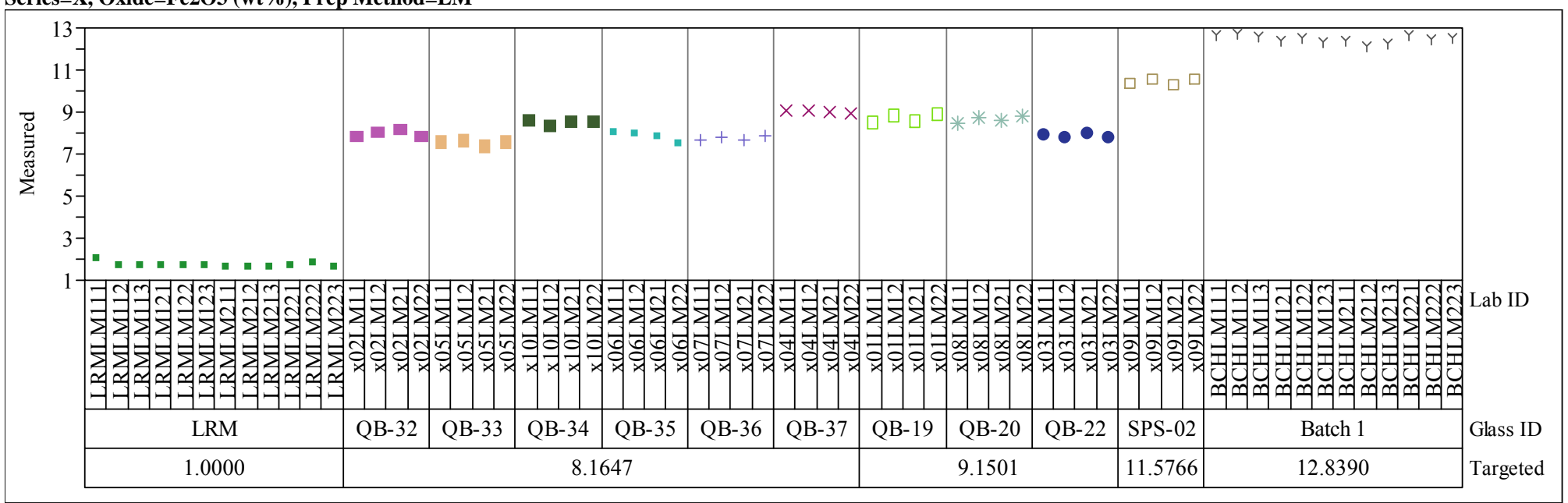


Exhibit A-2. Measurements by Lab ID within Glass ID by Targeted Concentration for Each Oxide for Each Prep by Analytical Series (continued)

Series $=$ X, Oxide $=$ K2O (wt \%), Prep Method $=$ LM

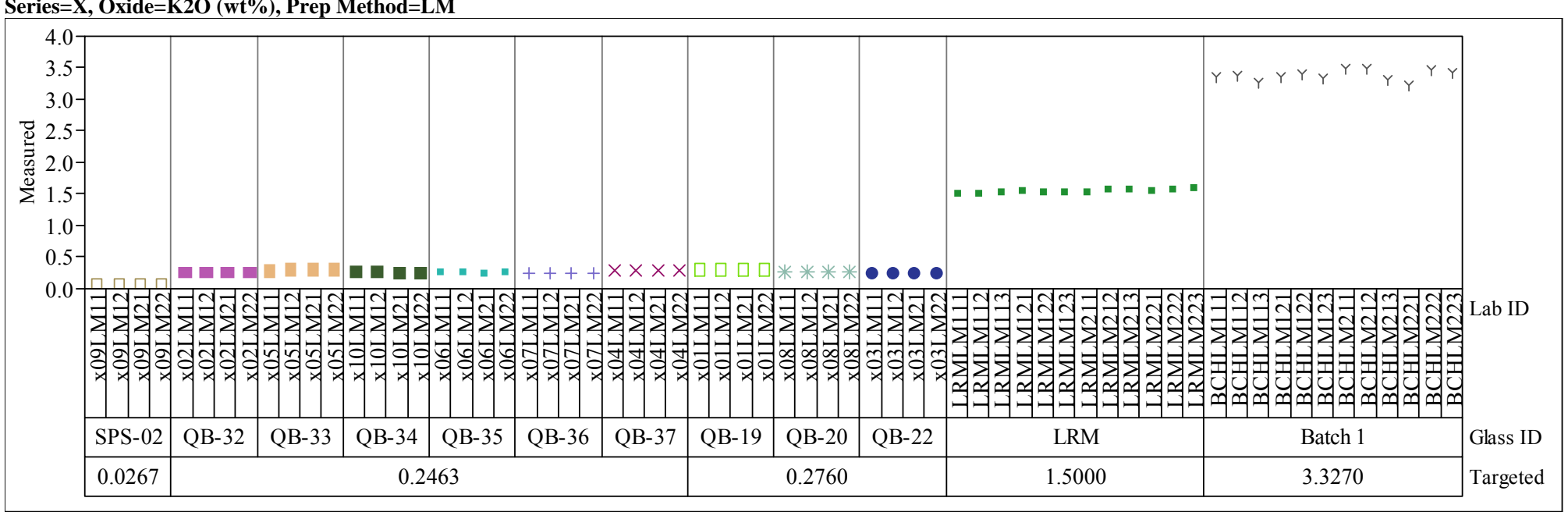

Series $=$ X, Oxide $=$ La2O3 (wt \%), Prep Method $=$ LM

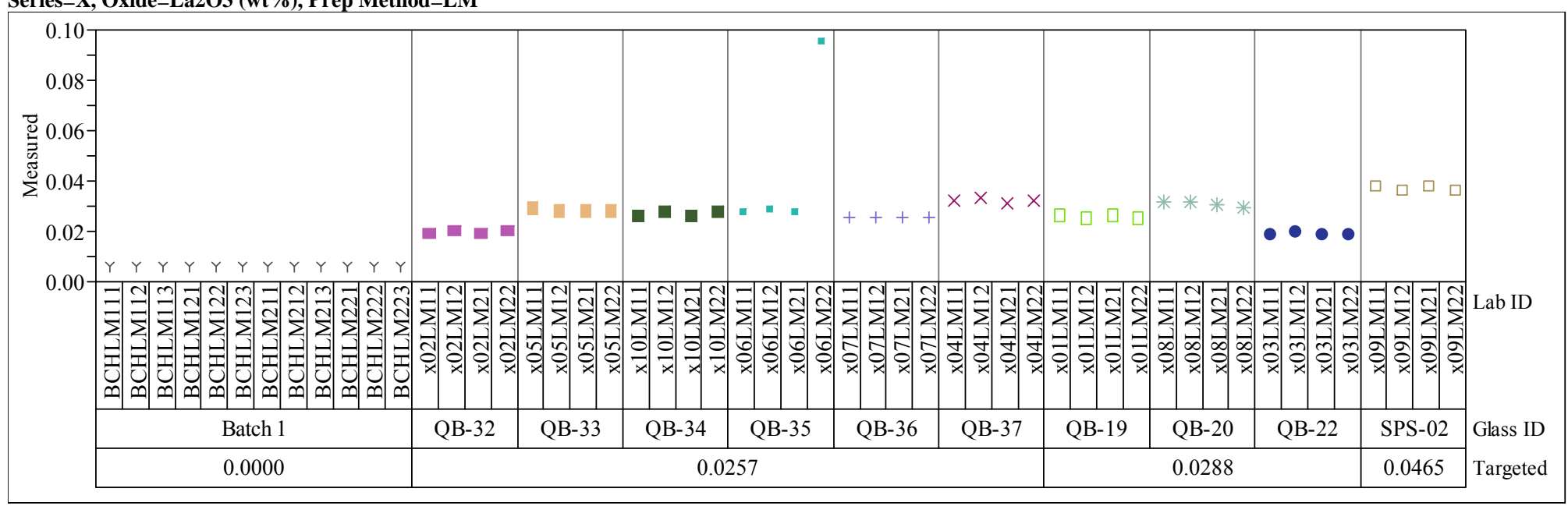


Exhibit A-2. Measurements by Lab ID within Glass ID by Targeted Concentration for Each Oxide for Each Prep by Analytical Series (continued)

Series $=X$, Oxide $=$ Li2O (wt \%), Prep Method $=$ PF

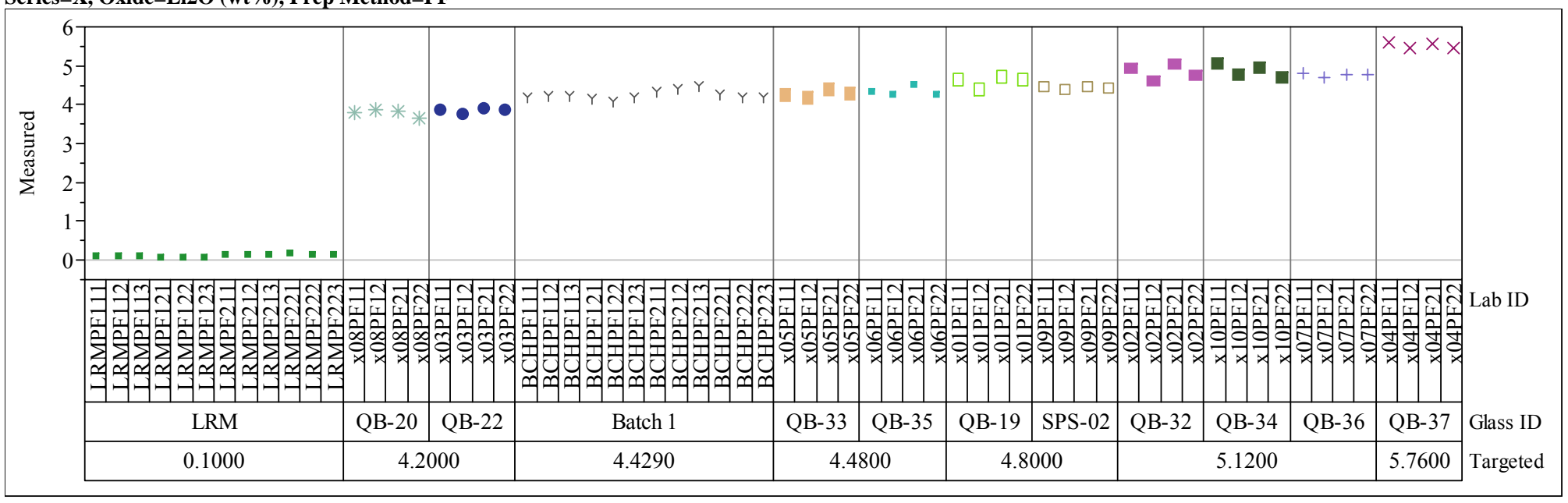

Series $=$ X, Oxide $=$ MgO (wt\%), Prep Method $=$ LM

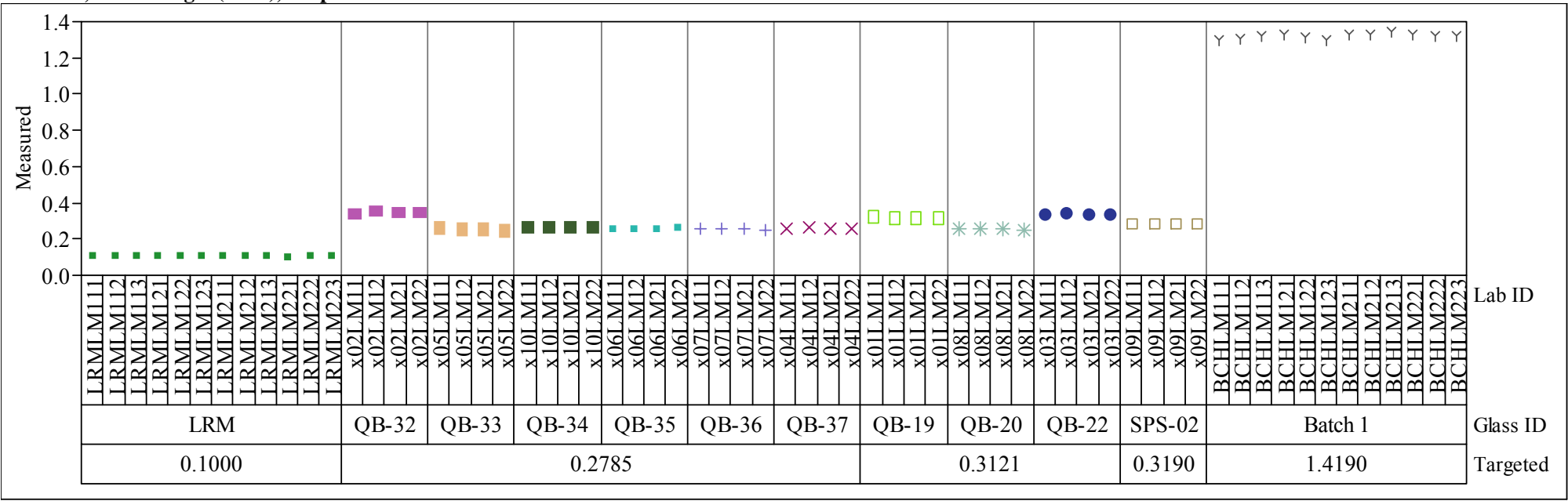


Exhibit A-2. Measurements by Lab ID within Glass ID by Targeted Concentration for Each Oxide for Each Prep by Analytical Series (continued)

Series $=\mathrm{X}$, Oxide $=\mathrm{MnO}$ (wt\%), Prep Method $=\mathrm{LM}$

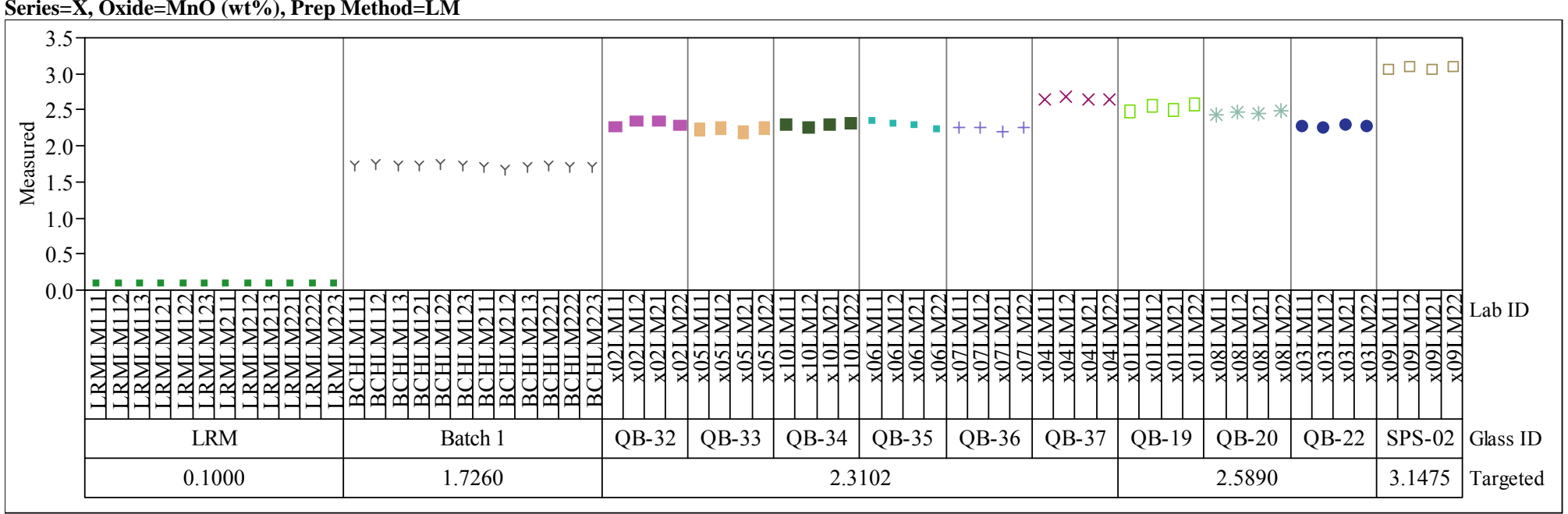

Series $=X$, Oxide $=$ Na2O (wt\%), Prep Method $=$ LM

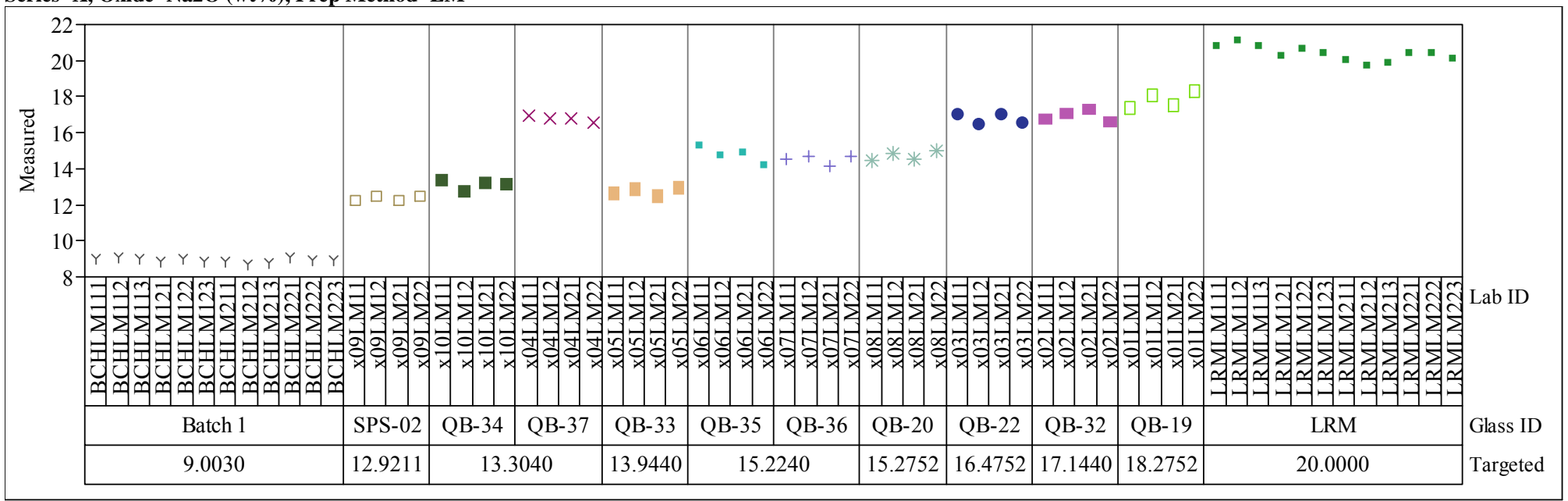


Exhibit A-2. Measurements by Lab ID within Glass ID by Targeted Concentration for Each Oxide for Each Prep by Analytical Series (continued)

Series $=$ X, Oxide $=\mathrm{NiO}(\mathrm{wt} \%$ ), Prep Method $=\mathrm{LM}$

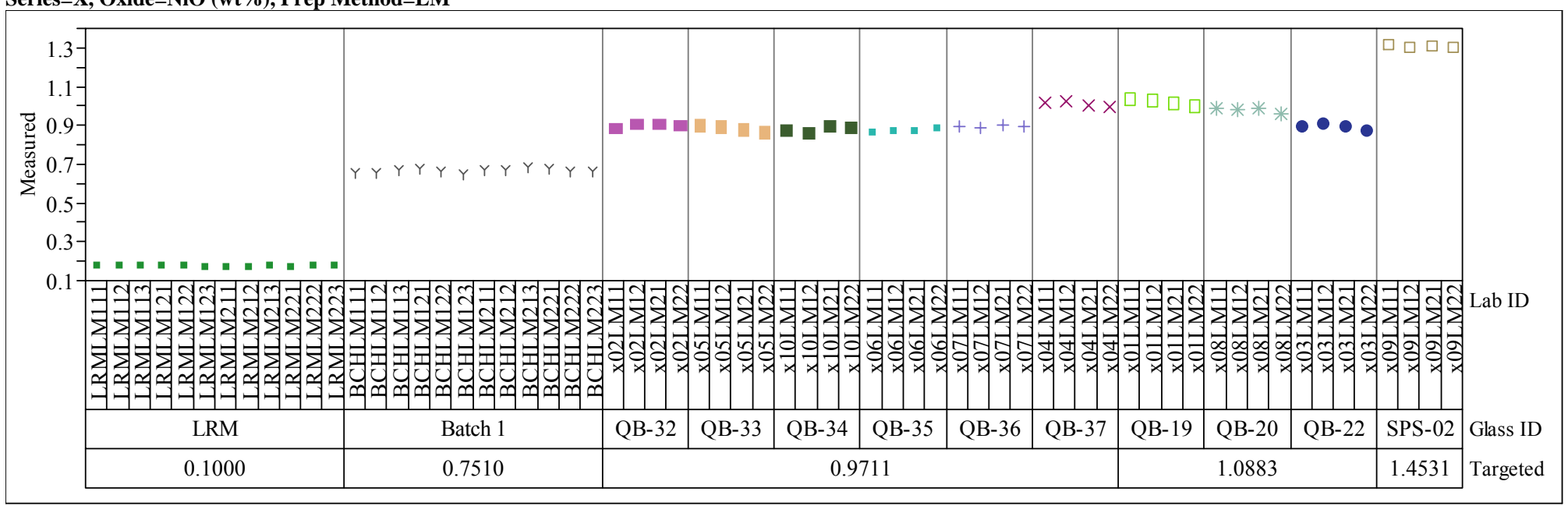

Series $=X$, Oxide $=$ PbO $(w t \%)$, Prep Method $=$ LM

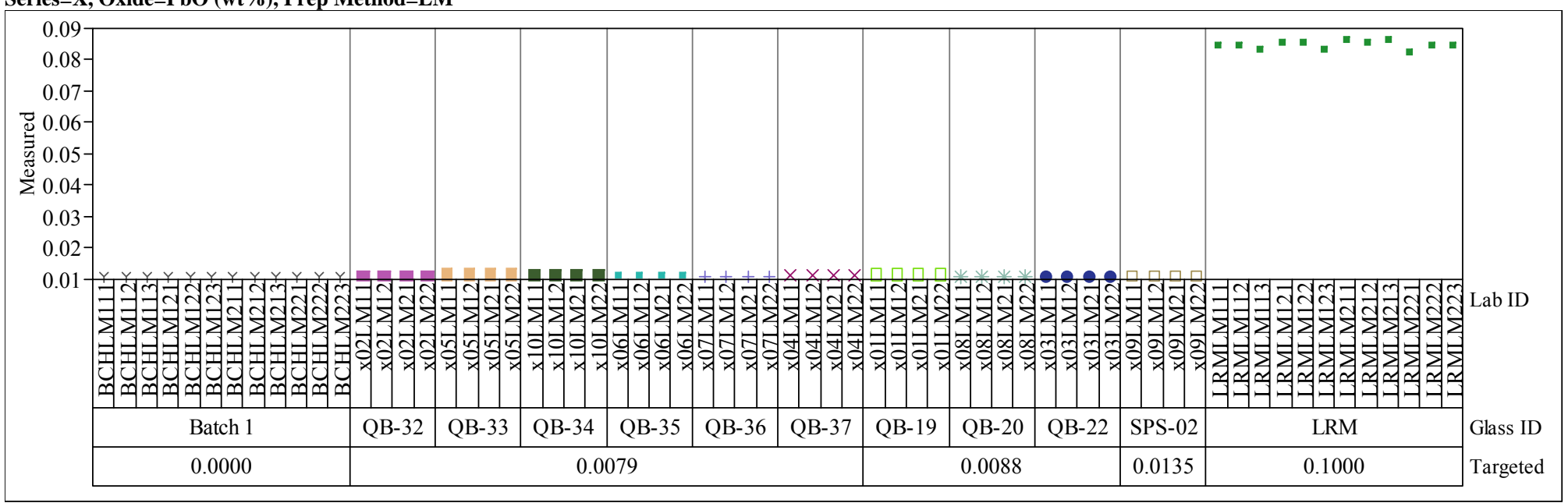


Exhibit A-2. Measurements by Lab ID within Glass ID by Targeted Concentration for Each Oxide for Each Prep by Analytical Series (continued)

Series $=\mathrm{X}$, Oxide $=\mathrm{SiO} 2$ (wt \%), Prep Method $=\mathbf{P F}$

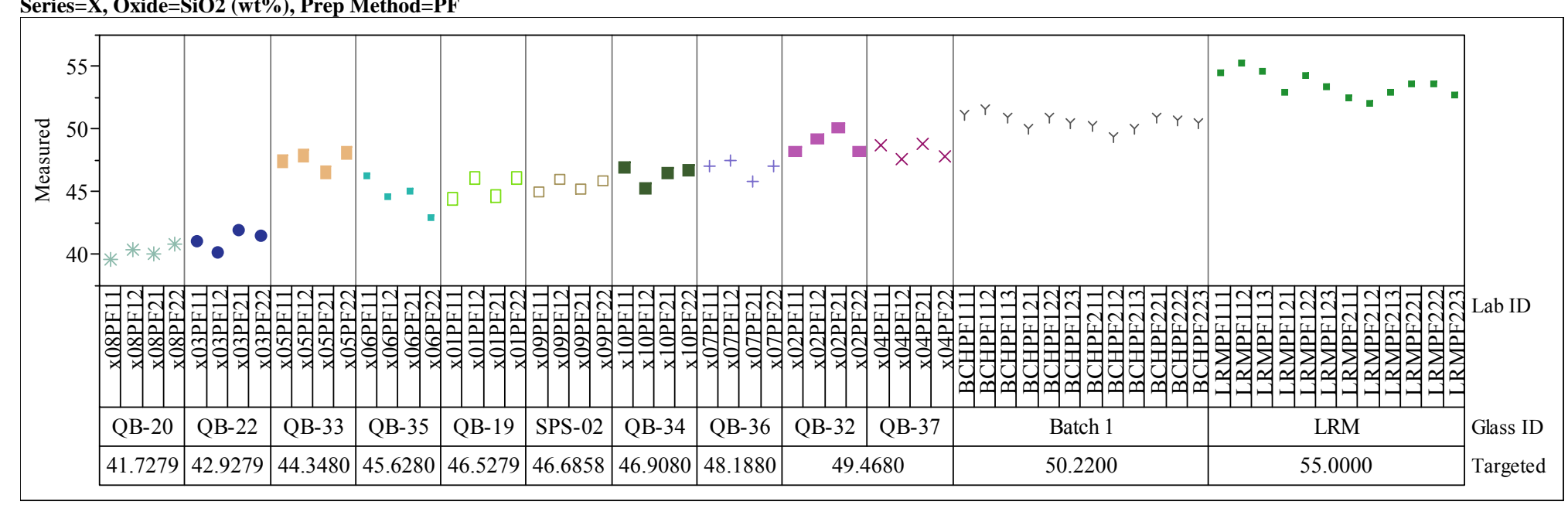

Series=X, Oxide=SO4 (wt\%), Prep Method=LM

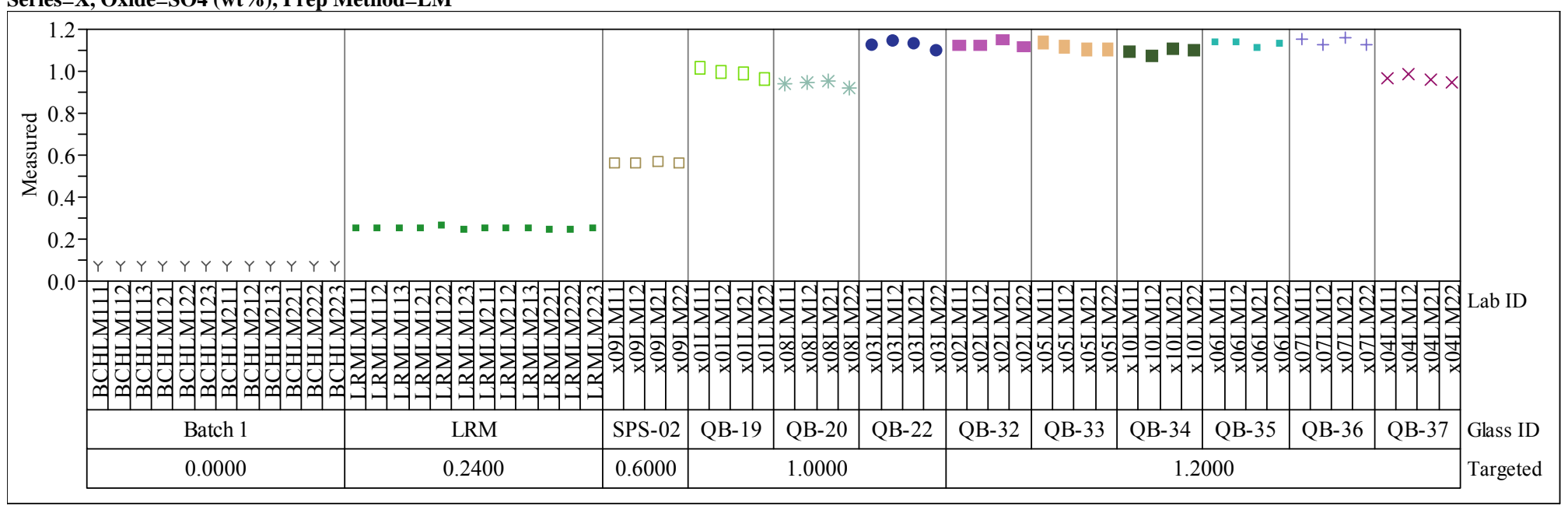


Exhibit A-2. Measurements by Lab ID within Glass ID by Targeted Concentration for Each Oxide for Each Prep by Analytical Series (continued)

Series $=\mathrm{X}$, Oxide $=$ TiO2 $(w t \%)$, Prep Method $=$ LM

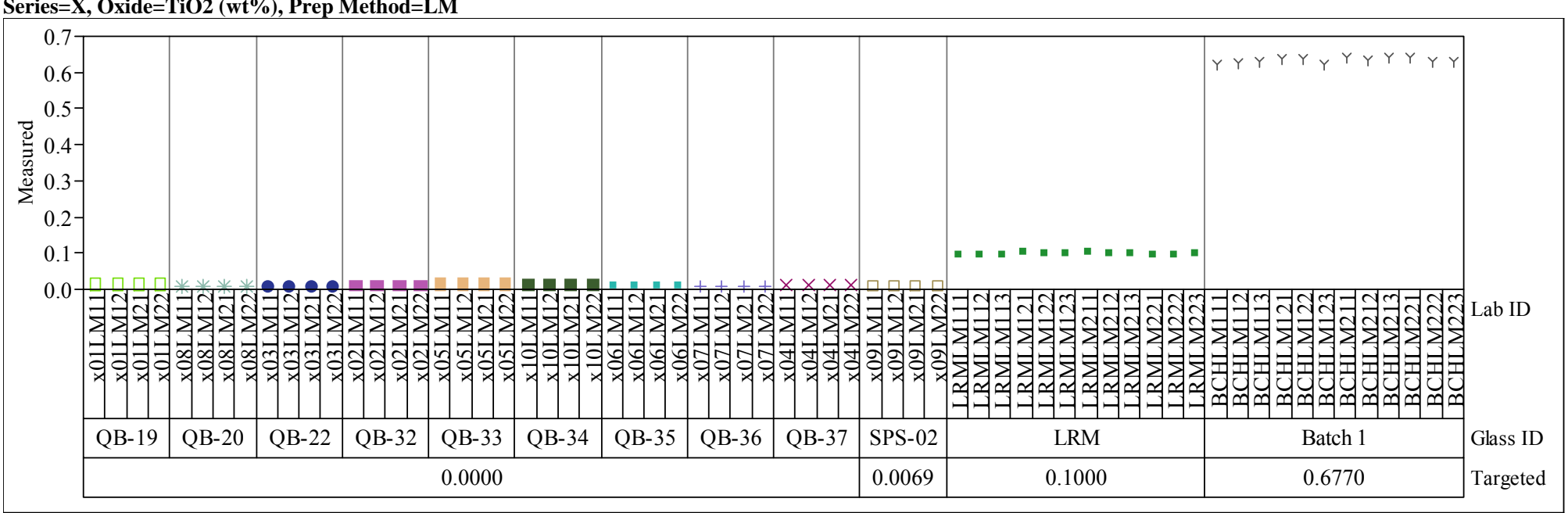

Series $=X$, Oxide $=Z n O(w t \%)$, Prep Method $=L M$

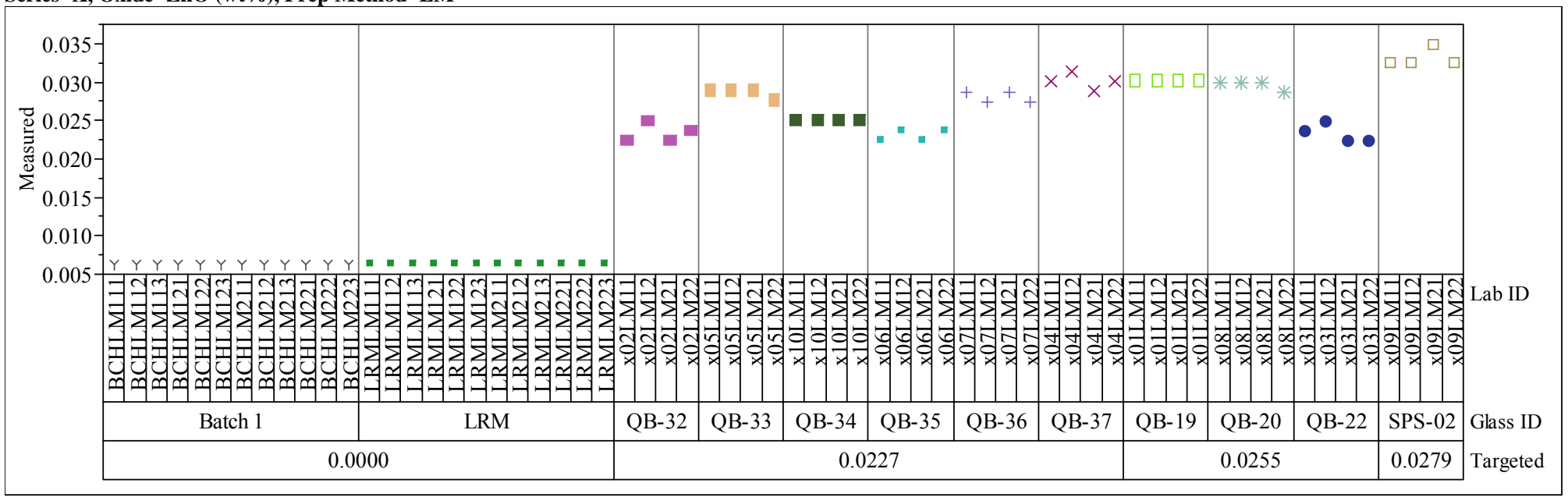


Exhibit A-2. Measurements by Lab ID within Glass ID by Targeted Concentration for Each Oxide for Each Prep by Analytical Series (continued)

Series $=\mathrm{X}$, Oxide $=\mathrm{ZrO} 2$ (wt\%), Prep Method $=$ LM

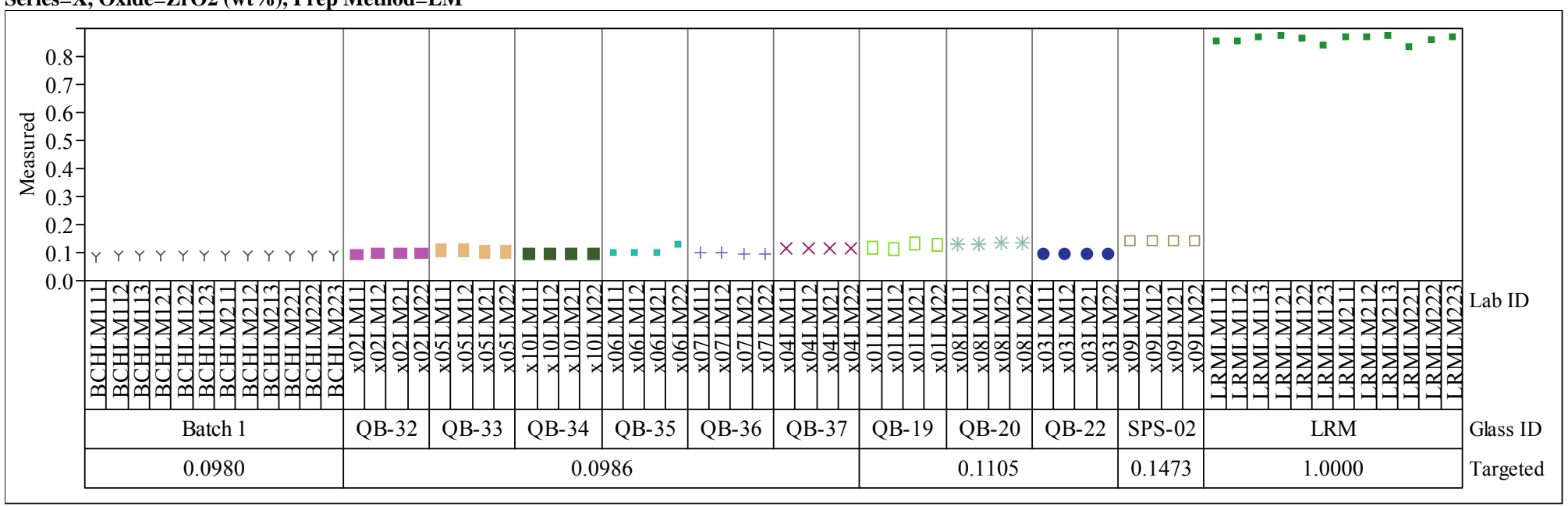




\section{Exhibit A-3. Measurements by Analytical Series, Block and Sub-Block for the Batch 1 and LRM Standards by Oxide by Prep}

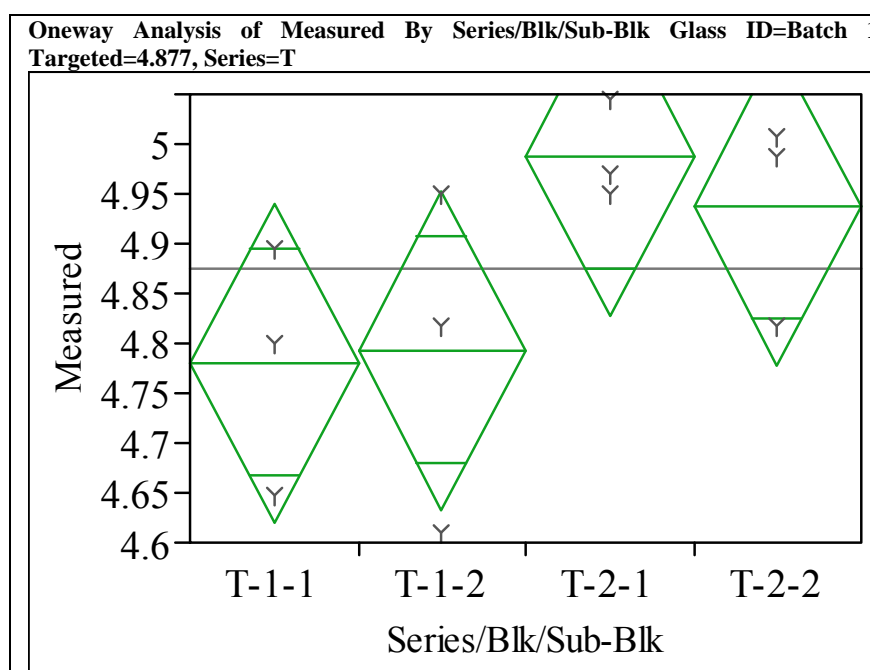

\section{Oneway Anova}

Oneway Anova

$\begin{array}{ll}\text { Rsquare } & 0.455964\end{array}$

$\begin{array}{ll} & 0.455964 \\ \text { Root Mean Square Error } \quad 0.251951 \\ \end{array}$

$\begin{array}{lr}\text { Root of Response } & 4.87491 \\ \text { Observations (or Sum Wgts) } & 12\end{array}$

Analysis of Variance

Source DF Sum of Squares Mean Square F Ratio Prob $>$ F

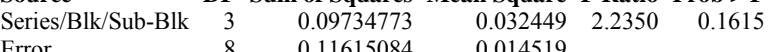

Error

0.014519

Means for Oneway Anova

Level Number Mean Std Error Lower 95\% Upper 95\%

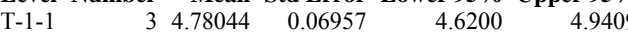

$\begin{array}{llllll}\mathrm{T}-1-2 & 3 & 4.79303 & 0.06957 & 4.6326 & 4.9535\end{array}$

$\begin{array}{llllll}\mathrm{T}-1-2 & 3 & 4.79303 & 0.06957 & 4.6326 & 4.9535 \\ \mathrm{~T}-2-1 & 3 & 4.98828 & 0.06957 & 4.8279 & 5.1487\end{array}$

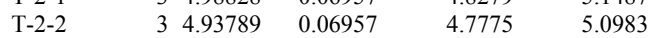

Std Error uses a pooled estimate of error variance

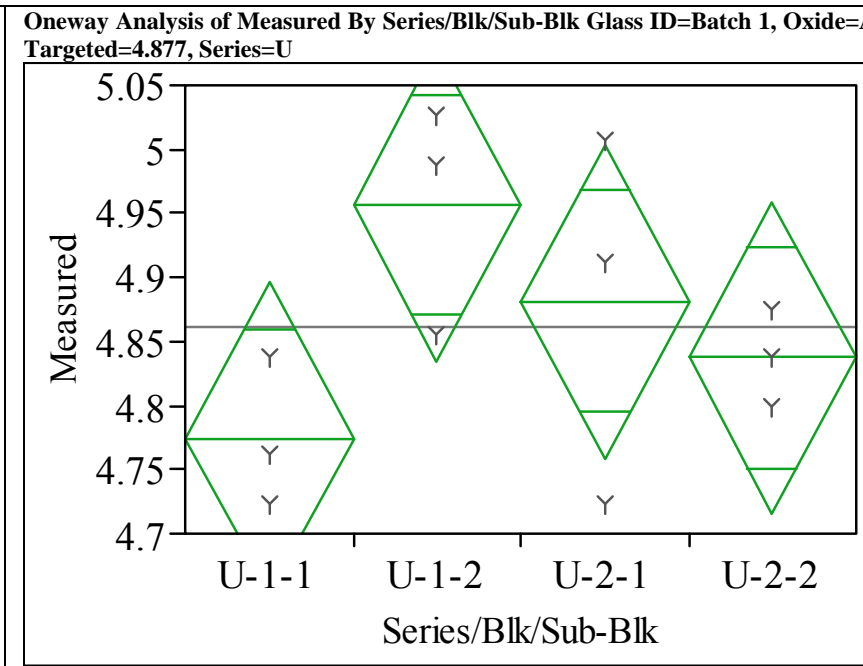

\section{Oneway Anova}

Summary of Fit

$\begin{array}{ll}\text { Rsquare } & 0.441584 \\ \text { Adj Rsquare } & 0.232178\end{array}$

Root Mean Square Error $\quad 0.091597$

$\begin{array}{lr}\text { Mean of Response } & 4.862313 \\ \text { Observations (or Sum Wgts) } & 12\end{array}$

Analysis of Variance

Source DF Sum of Squares Mean Square F Ratio Prob > F

$\begin{array}{llllll}\text { Series/Blk/Sub-Blk } & 3 & 0.05307713 & 0.017692 & 2.1087 & 0.1774\end{array}$

$\begin{array}{lrrr}\text { Error } & 8 & 0.06711995 & 0.008390 \\ \text { C. Total } & 11 & 0.12019708 & \end{array}$

Means for Oneway Anova

Level Number Mean Std Error Lower 95\% Upper 95\%

$\begin{array}{llllll}\text { U-1-1 } & 3 & 4.77414 & 0.05288 & 4.6522 & 4.896\end{array}$

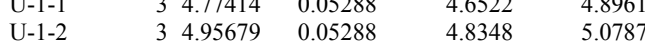

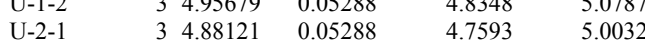

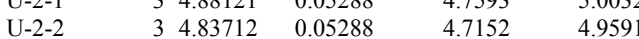

Std Error uses a pooled estimate of error variance 


\section{Exhibit A-3. Measurements by Analytical Series, Block and Sub-Block for the Batch 1 and LRM Standards by Oxide by Prep (continued)}

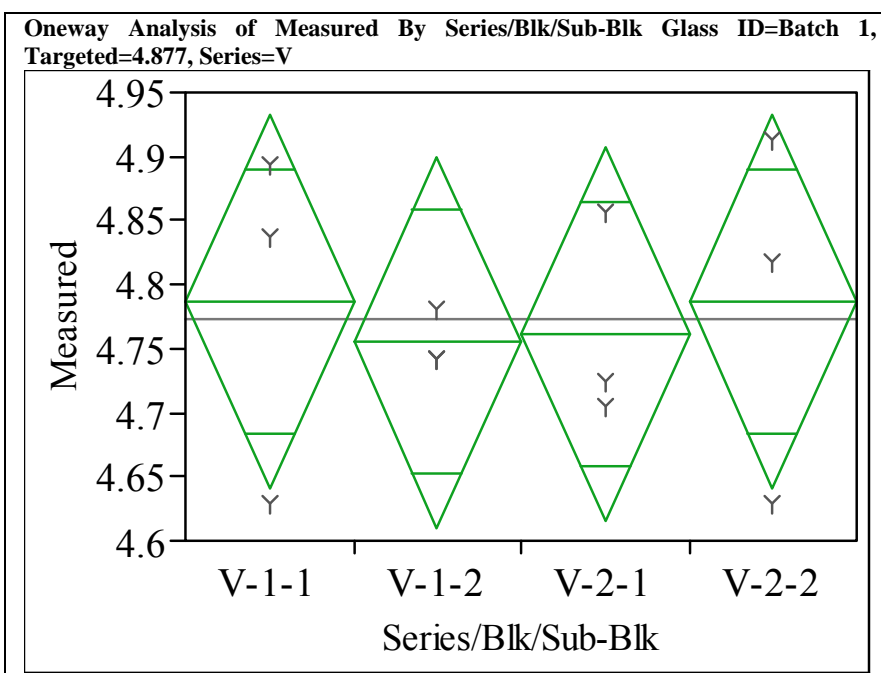

\section{Oneway Anova}

Summary of Fit

$$
\begin{array}{lr}
\text { Rsquare } & 0.025344 \\
\text { Adj Rsquare } & -0.34015 \\
\text { Root Mean Square Error } & 0.108954 \\
\text { Mean of Response } & 4.772562 \\
\text { Observations (or Sum Wgts) } & 12
\end{array}
$$

Analysis of Variance

$\begin{array}{lrrrrr}\text { Source } & \text { DF } & \text { Sum of Squares } & \text { Mean Square } & \text { F Ratio } & \text { Prob }>\text { F } \\ \text { Series/Blk/Sub-Blk } & 3 & 0.00246940 & 0.000823 & 0.0693 & 0.9747\end{array}$

$\begin{array}{lrrr}\text { Series/Blk/Sub-Blk } & 3 & 0.00246940 & 0.000823 \\ \text { Error } & 8 & 0.09496759 & 0.011871\end{array}$

0.09743699

Means for Oneway Anova

Level Number Mean Std Error Lower 95\% Upper 95\%

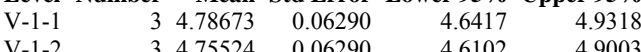

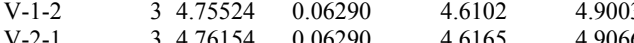

$\begin{array}{llllll}\mathrm{V}-2-1 & 3 & 4.76154 & 0.06290 & 4.6165 & 4.9066 \\ \mathrm{~V}-2-2 & 3 & 4.78673 & 0.06290 & 4.6417 & 4.9318\end{array}$

Std Error uses a pooled estimate of error variance

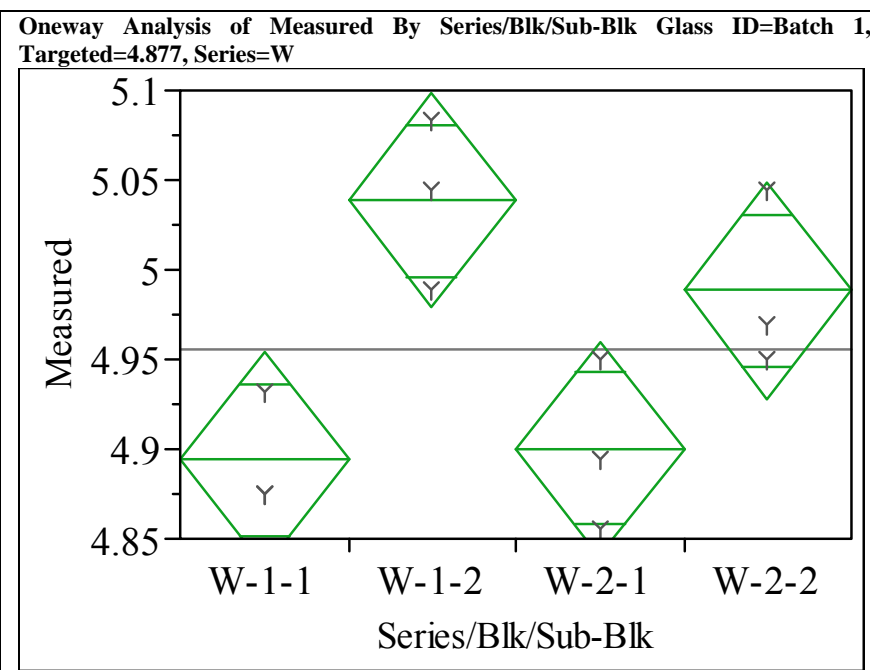

Oneway Anova

Summary of Fit

$\begin{array}{lr}\text { Rsquare } & 0.733725 \\ \text { Adj Rsquare } & 0.633872 \\ \text { Root Mean Square Error } & 0.044979 \\ \text { Mean of Response } & 4.955214 \\ \text { Observations (or Sum Wgts) } & 12\end{array}$

Observations (or Sum Wgts) 4.955214

Analysis of Variance

Source DF Sum of Squares Mean Square F Ratio Prob $>$ F

$\begin{array}{lrrrrr}\text { Series/Blk/Sub-Blk } & 3 & 0.04459788 & 0.014866 & 7.3480 & 0.0110\end{array}$

$\begin{array}{ll}0.04459788 & 0.014866 \\ 0.01618495 & 0.002023\end{array}$

C. Total

$\begin{array}{rr}8 & 0.01618495 \\ 11 & 0.06078283\end{array}$

Means for Oneway Anova

Level Number Mean Std Error Lower 95\% Upper 95\%

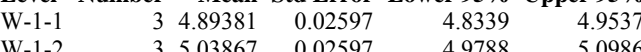

$\begin{array}{llllll}W-1-1 & 3 & 4.89381 & 0.02597 & 4.8339 & 4.9537 \\ W-1-2 & 3 & 5.03867 & 0.02597 & 4.9788 & 5.0986 \\ W-2-1 & 3 & 4.90010 & 0.02597 & 4.8402 & 4.9600\end{array}$

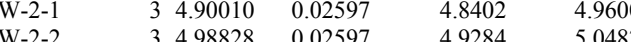

Std Error uses a pooled estimate of error variance 


\section{Exhibit A-3. Measurements by Analytical Series, Block and Sub-Block for the Batch 1 and LRM Standards by Oxide by Prep (continued)}

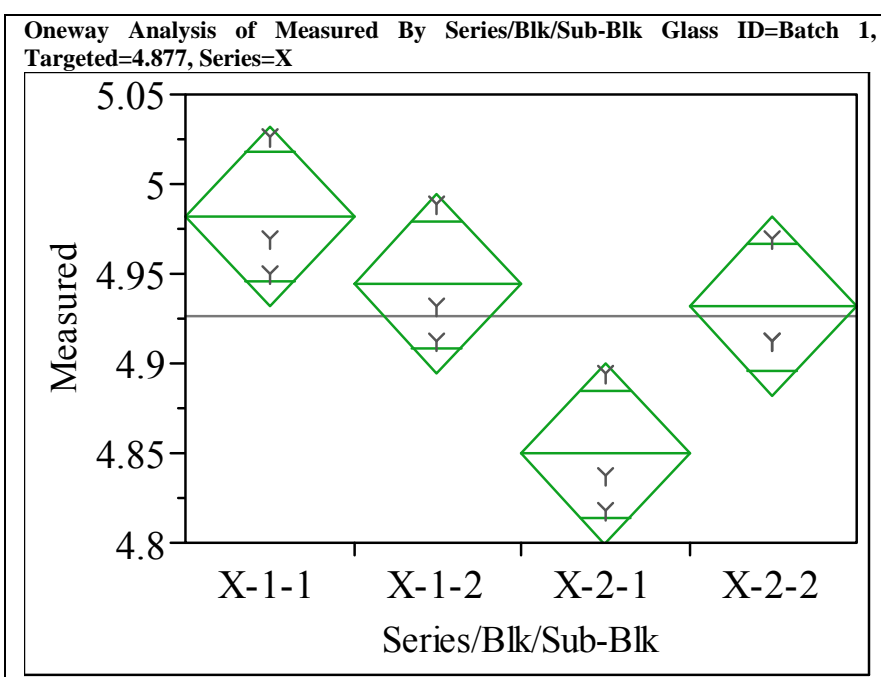

\section{Oneway Anova}

Summary of Fit

$\begin{array}{lr}\text { Rsquare } & 0.709751 \\ \text { Adj Rsquare } & 0.600907 \\ \text { Root Mean Square Error } & 0.03779 \\ \text { Mean of Response } & 4.926871 \\ \text { Observations (or Sum Wgts) } & 12\end{array}$

Analysis of Variance

Source DF Sum of Squares Mean Square F Ratio Prob > F

$\begin{array}{lrrrrr}\text { Series/Blk/Sub-Blk } & 3 & 0.02793690 & 0.009312 & 6.5208 & 0.0153\end{array}$

Error

C. Total

0.03936157

Means for Oneway Anova

Level Number Mean Std Error Lower 95\% Upper 95\%

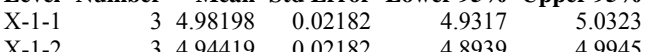

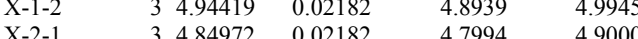

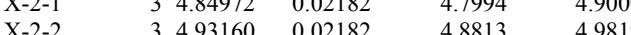

Std Error uses a pooled estimate of error variance

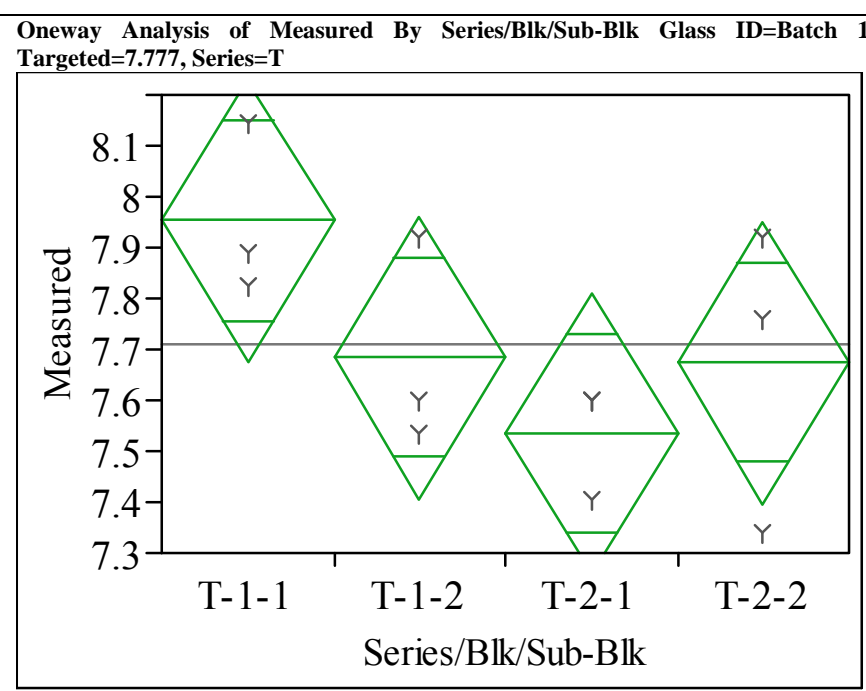

Oneway Anova

Summary of Fit

Rsquare

$\begin{array}{ll} & 0.442041 \\ \text { Adj Rsquare } & 0.232806\end{array}$

Root Mean Square Error $\quad 0.208466$

$\begin{array}{lr}\text { Mean of Response } & 7.711661 \\ \text { Observations (or Sum Wgts) } & 12\end{array}$

Analysis of Variance

Source DF Sum of Squares Mean Square F Ratio Prob >

$\begin{array}{llllll}\text { Series/Blk/Sub-Blk } & 3 & 0.27543672 & 0.091812 & 2.1127 & 0.1769\end{array}$

Error $\quad 8 \quad 0.34766542$

$\begin{array}{lll}\text { C. Total } & 11 \quad 0.62310214\end{array}$

Means for Oneway Anova

Level Number Mean Std Error Lower 95\% Upper 95\%

$\begin{array}{llrrrr}\text { T-1-1 } & 3 & 7.95315 & 0.12036 & 7.6756 & 8.2307\end{array}$

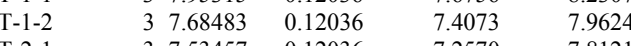

$\begin{array}{llllll}\mathrm{T}-2-1 & 3 & 7.53457 & 0.12036 & 7.2570 & 7.8121\end{array}$

$\begin{array}{llllll}\mathrm{T}-2-2 & 3 & 7.67410 & 0.12036 & 7.3965 & 7.9516\end{array}$

Std Error uses a pooled estimate of error variance 
Exhibit A-3. Measurements by Analytical Series, Block and Sub-Block for the Batch 1 and LRM Standards by Oxide by Prep (continued)

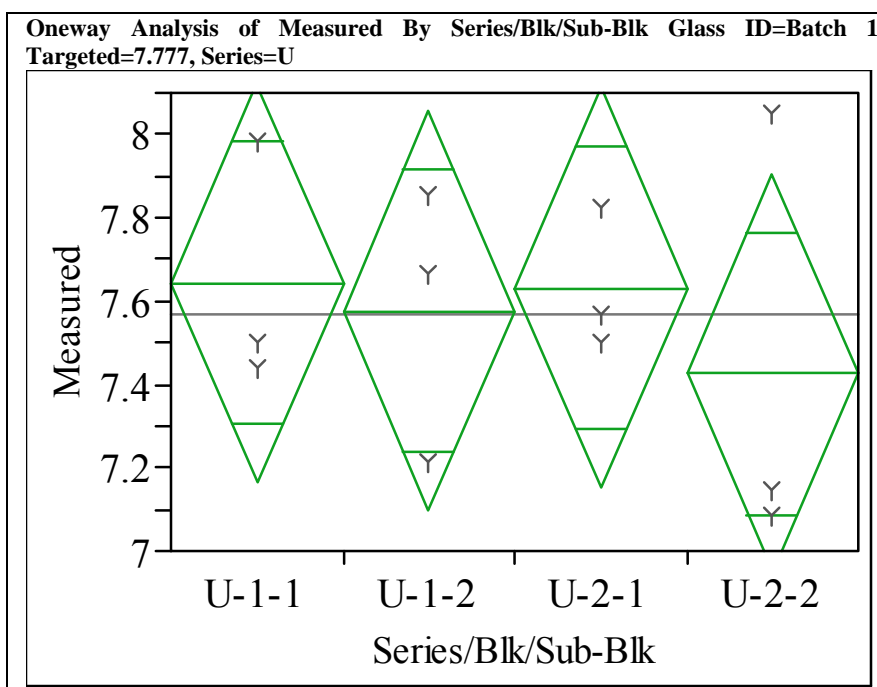

\section{Oneway Anova}

Summary of Fit

Rsquare $\quad 0.078126$

Adj Rsquare $\quad-0.26758$

Root Mean Square Error $\quad 0.360356$

$\begin{array}{lr}\text { Mean of Response } & 7.569448 \\ \text { Observations (or Sum Wgts) } & 12\end{array}$

Analysis of Variance

$\begin{array}{lrrrrr}\text { Source } & \text { DF } & \text { Sum of Squares } & \text { Mean Square } & \text { F Ratio } & \text { Prob }>\text { F } \\ \text { Series/Blk/Sub-Blk } & 3 & 0.0880395 & 0.029347 & 0.2260 & 0.8757 \\ \text { Error } & 8 & 1.0388492 & 0.129856 & & \\ \text { C. Total } & 11 & 1.1268887 & & & \\ & & & & & \end{array}$

Means for Oneway Anova

Level Number Mean Std Error Lower 95\% Upper 95\%

\begin{tabular}{llrrrr} 
U-1-1 & 3 & 7.64190 & 0.20805 & 7.1621 & 8.1217 \\
\hline & & 7.57750 & 0.20805 & 7.0977 & 8.0573
\end{tabular}

$\begin{array}{llllll}\text { U-1-2 } & 3 & 7.57750 & 0.20805 & 7.0977 & 8.0573\end{array}$

$\begin{array}{llllll}\text { U-2-1 } & 3 & 7.63116 & 0.20805 & 7.1514 & 8.1109\end{array}$

$\begin{array}{llllll}\text { U-2-2 } & 3 & 7.42724 & 0.20805 & 6.9475 & 7.9070\end{array}$

Std Error uses a pooled estimate of error variance

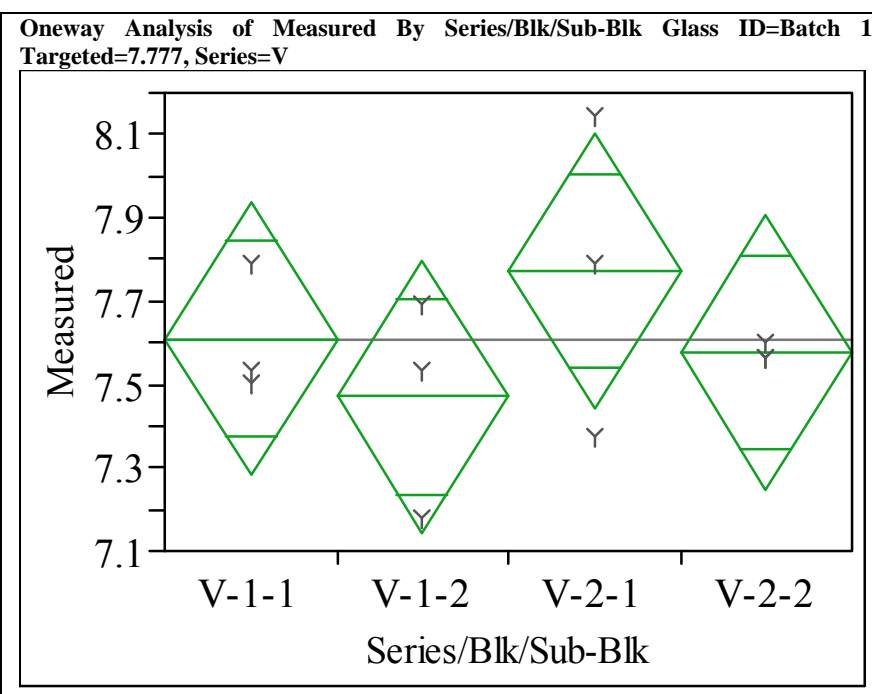

\section{Oneway Anova}

Summary of Fit

Adj Rsquace 0.221443

$\quad-0.07052$

Root Mean Square Error $\quad 0.247325$

$\begin{array}{lr}\text { Mean of Response } & 7.607014 \\ \text { Observations (or Sum Wgts) } & 12\end{array}$

Analysis of Variance

Source DF Sum of Squares Mean Square F Ratio Prob >

$\begin{array}{llllll}\text { Series/Blk/Sub-Blk } & 3 & 0.13918712 & 0.046396 & 0.7585 & 0.548\end{array}$

$\begin{array}{lll}\text { Error } & 8 & 0.48935808\end{array}$ 0.061170

Means for Oneway Anova

Level Number Mean Std Error Lower 95\% Upper 95\%

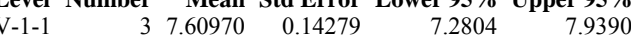

$\begin{array}{llllll}\mathrm{V}-1-1 & 3 & 7.47017 & 0.14279 & 7.1409 & 7.7994\end{array}$

$\begin{array}{llllll}\mathrm{V}-2-1 & 3 & 7.77069 & 0.14279 & 7.4414 & 8.1000\end{array}$

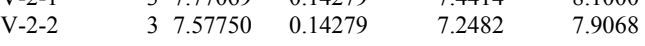

Std Error uses a pooled estimate of error variance 
Exhibit A-3. Measurements by Analytical Series, Block and Sub-Block for the Batch 1 and LRM Standards by Oxide by Prep (continued)

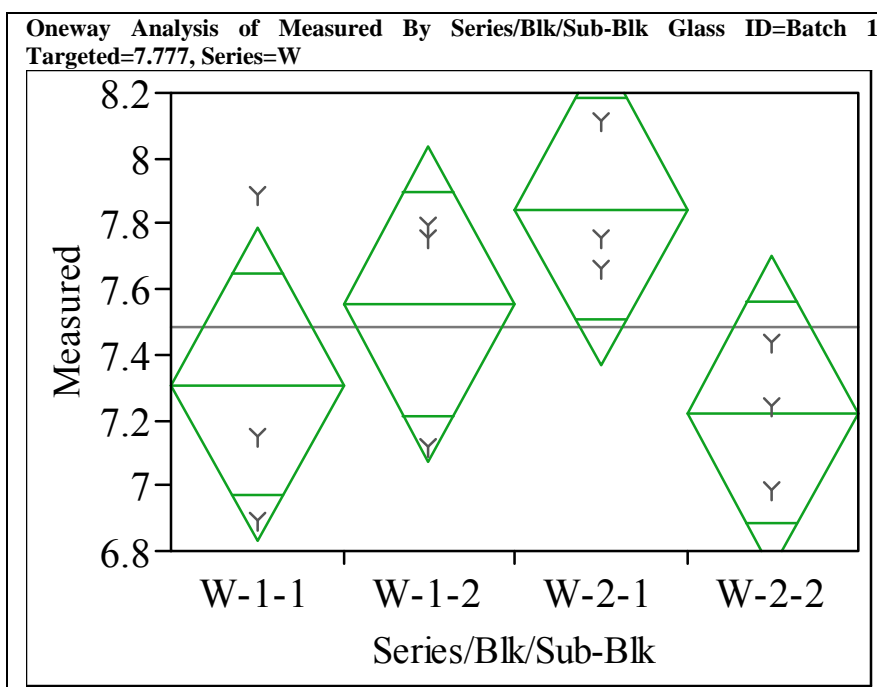

\section{Oneway Anova}

Summary of Fi

Rsquare $\quad 0.402938$

Adj Rsquare $\quad 0.17904$

Root Mean Square Error $\quad 0.361074$

$\begin{array}{lr}\text { Mean of Response } & 7.483584 \\ \text { Observations (or Sum Wgts) } & 12\end{array}$

Analysis of Variance

$\begin{array}{lrrrrr}\text { Source } & \text { DF } & \text { Sum of Squares } & \text { Mean Square } & \text { F Ratio } & \text { Prob }>\text { F } \\ \text { Series/Blk/Sub-Blk } & 3 & 0.7038842 & 0.234628 & 1.7996 & 0.2251 \\ \text { Error } & 8 & 1.0429963 & 0.130375 & & \\ \text { C. Total } & 11 & 1.7468805 & & & \\ & & & & & \end{array}$

Means for Oneway Anova

Level Number Mean Std Error Lower 95\% Upper 95\%

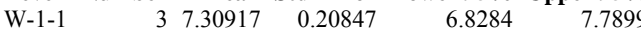

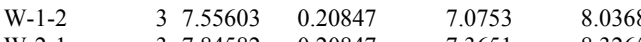

$\begin{array}{llllll}\text { W-2-1 } & 3 & 7.84582 & 0.20847 & 7.3651 & 8.326\end{array}$

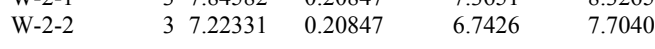

Std Error uses a pooled estimate of error variance

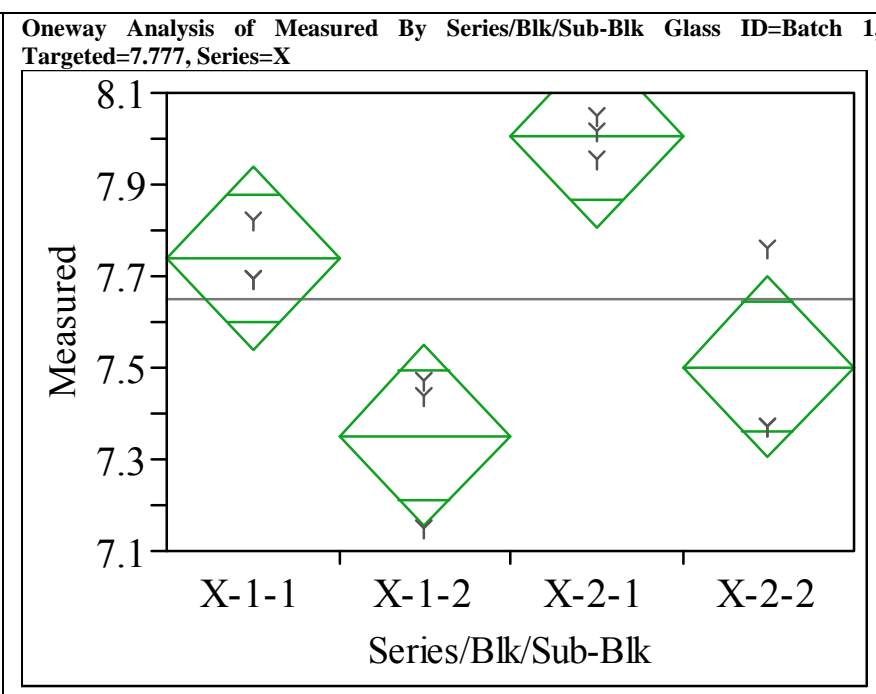

\section{Oneway Anova}

Summary of Fit

Rsquare

$\begin{array}{ll} & 0.805191 \\ \text { Adj Rsquare } & 0.732138\end{array}$

Root Mean Square Error $\quad 0.149301$

$\begin{array}{lr}\text { Mean of Response } & 7.649946 \\ \text { Observations (or Sum Wgts) } & 12\end{array}$

Analysis of Variance

\begin{tabular}{lrrrrr}
\multicolumn{2}{l}{ Analysis of Variance } & & & & \\
Source & DF & Sum of Squares & Mean Square & F Ratio & Prob $>$ F \\
Series/Blk/Sub-Blk & 3 & 0.73706105 & 0.245687 & 11.0220 & 0.0033 \\
Error & 8 & 0.17832540 & 0.022291 & & \\
C. Total & 11 & 0.91538646 & & &
\end{tabular}

Means for Oneway Anova

Level Number Mean Std Error Lower 95\% Upper 95\%

$\begin{array}{llrrrr}\text { X-1-1 } & 3 & 7.73849 & 0.08620 & 7.5397 & 7.9373 \\ \text { X-1-2 } & 3 & 7.35211 & 0.08620 & 7.1533 & 7.5509\end{array}$

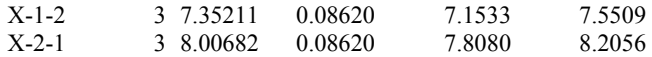

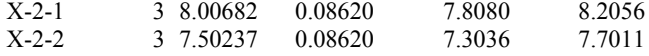

Std Error uses a pooled estimate of error variance 
Exhibit A-3. Measurements by Analytical Series, Block and Sub-Block for the Batch 1 and LRM Standards by Oxide by Prep (continued)

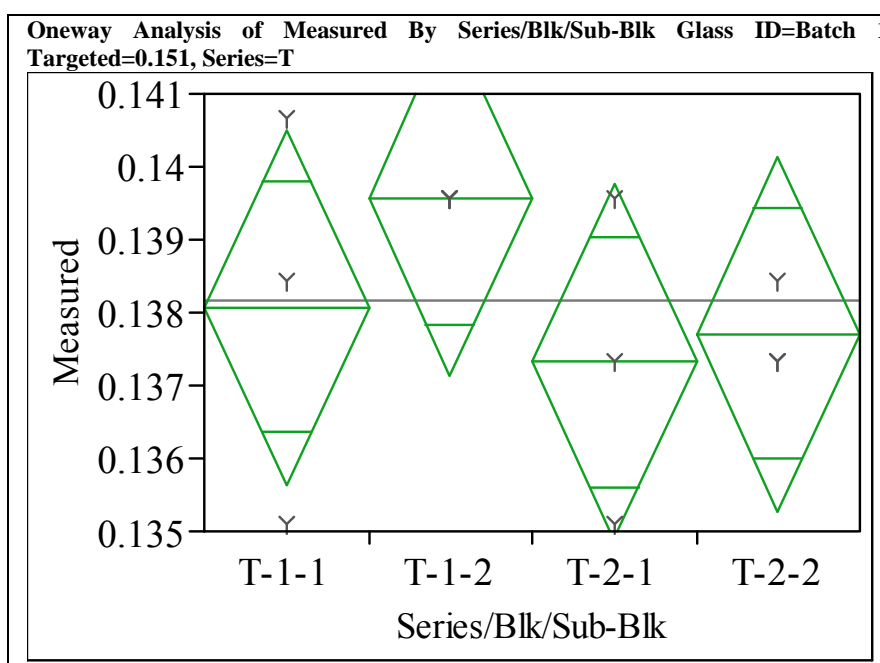

Oneway Anova

Summary of Fit

$\begin{array}{lr}\text { Rsquare } & 0.244838 \\ \text { Adj Rsquare } & -0.03835 \\ \text { Root Mean Square Error } & 0.001823 \\ \text { Mean of Response } & 0.138167 \\ \text { Observations (or Sum Wgts) } & 12\end{array}$

Analysis of Variance

Source DF Sum of Squares Mean Square F Ratio Prob $>$ F

$\begin{array}{lrrrrr}\text { Series/Blk/Sub-Blk } & 3 & 0.00000862 & 2.874 \mathrm{e}-6 & 0.8646 & 0.4980 \\ \text { Error } & 8 & 0.00002659 & 3.3242 \mathrm{e}-6 & & \end{array}$

$3.3242 \mathrm{e}-6 \quad 0.8646 \quad 0.4980$

C. Total

0.00003522

Means for Oneway Anova

Level Number Mean Std Error Lower 95\% Upper 95\%

$\begin{array}{lllrrr}\text { T-1-1 } & 3 & 0.138074 & 0.00105 & 0.13565 & 0.14050\end{array}$

$\begin{array}{llllll}\mathrm{T}-1-2 & 3 & 0.139563 & 0.00105 & 0.13714 & 0.14199\end{array}$

$\begin{array}{lllllll}\text { T-2-1 } & & 3 & 0.137330 & 0.00105 & 0.13490 & 0.13976 \\ \mathrm{~T}-2-2 & & 3 & 0.137702 & 0.00105 & 0.13527 & 0.14013\end{array}$

Std Error uses a pooled estimate of error variance

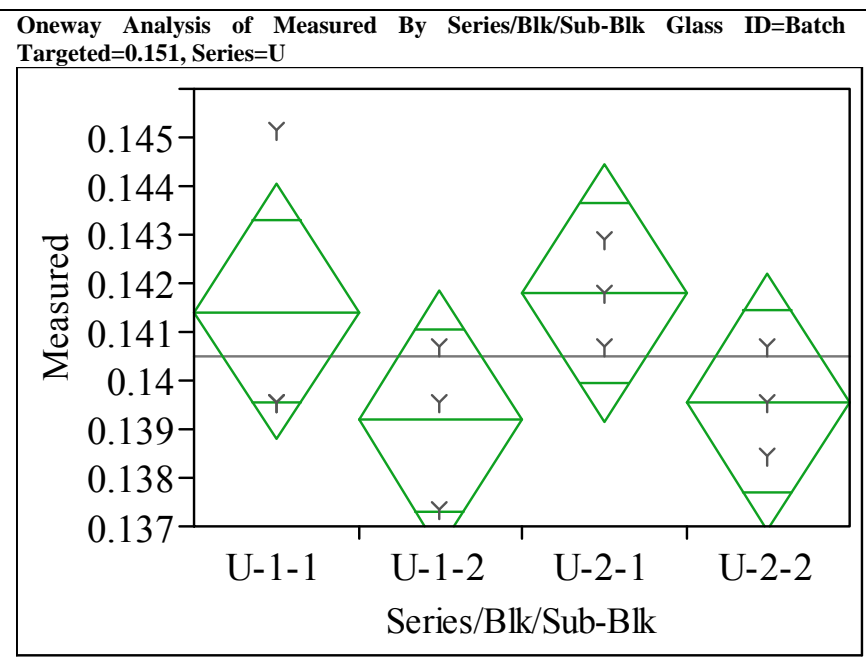

Oneway Anova

Summary of Fit

\section{Rsquare}

$\begin{array}{ll} & 0.075221 \\ \text { Root Mean Square Error } \quad 0.001987\end{array}$

Mean of Response $\quad 0.140493$

Observations (or Sum Wgts) 12

Analysis of Variance

Source DF Sum of Squares Mean Square F Ratio Prob > F

$\begin{array}{llllll}\text { Series/Blk/Sub-Blk } & 3 & 0.00001537 & 5.1248 \mathrm{e}-6 & 1.2982 & 0.3400\end{array}$

$\begin{array}{lll}\text { Error } & 8 & 0.00003158 \\ \text { C. } & 11 & 0.0000695\end{array}$

$\begin{array}{lll}\text { C. Total } & 11 \quad 0.00004695\end{array}$

Means for Oneway Anova

Level Number Mean Std Error Lower 95\% Upper 95\%

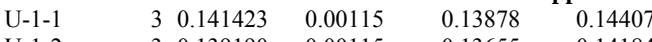

$\begin{array}{llllll}\text { U-1-2 } & 3 & 0.139190 & 0.00115 & 0.13655 & 0.14184\end{array}$

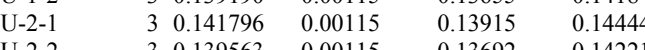

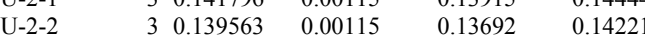

Std Error uses a pooled estimate of error variance 
Exhibit A-3. Measurements by Analytical Series, Block and Sub-Block for the Batch 1 and LRM Standards by Oxide by Prep (continued)

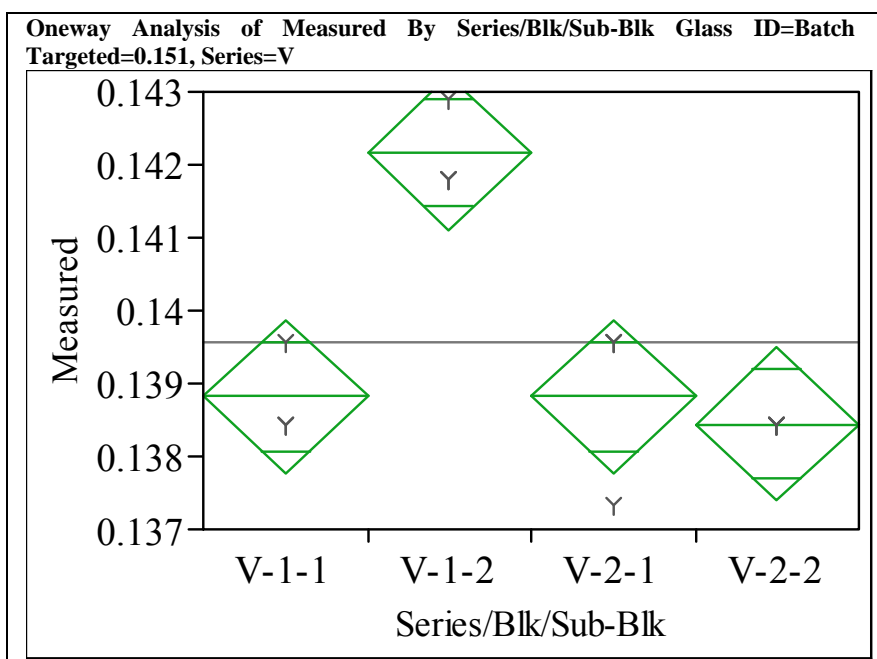

Oneway Anova

Summary of Fit

Rsquare

0.846154

Adj Rsquare $\quad 0.788462$

Root Mean Square Error $\quad 0.000789$

$\begin{array}{lr}\text { Mean of Response } & 0.139563 \\ \text { Observations (or Sum Wgts) } & 12\end{array}$

Analysis of Variance

$\begin{array}{lrrrrr}\text { Source } & \text { DF } & \text { Sum of Squares } & \text { Mean Square } & \text { F Ratio } & \text { Prob }>\text { F } \\ \text { Series/Blk/Sub-Blk } & 3 & 0.00002742 & 9.1415 \mathrm{e}-6 & 14.6667 & 0.0013\end{array}$

$\begin{array}{rrrrr}3 & 0.00002742 & 9.1415 \mathrm{e}-6 & 14.6667 & 0.0013 \\ 8 & 0.00000499 & 6.2329 \mathrm{e}-7 & & \end{array}$

C. Total

0.00003241

Means for Oneway Anova

Level Number Mean Std Error Lower 95\% Upper 95\%

$\begin{array}{lllllr}\text { V-1-1 } & 3 & 0.138818 & 0.00046 & 0.13777 & 0.13987\end{array}$

$\begin{array}{llllll}V-1-2 & 3 & 0.142168 & 0.00046 & 0.14112 & 0.14322\end{array}$

$\begin{array}{lllllll}\text { V-2-1 } & & 3 & 0.138818 & 0.00046 & 0.13777 & 0.13987 \\ \text { V-2-2 } & & 3 & 0.138446 & 0.00046 & 0.13739 & 0.13950\end{array}$

Std Error uses a pooled estimate of error variance

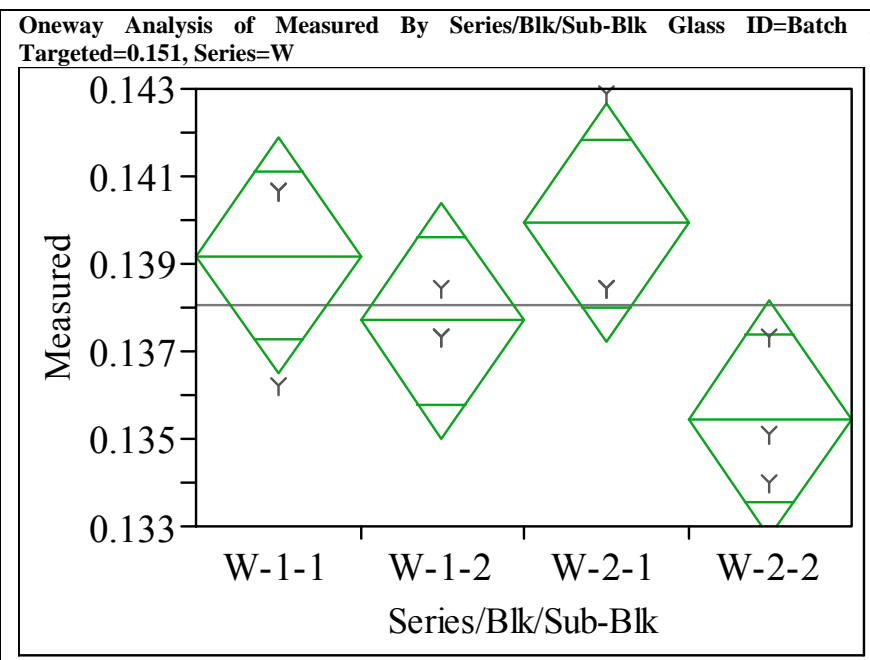

Oneway Anova

Summary of Fit

\section{Rsquare}

$\begin{array}{ll} & 0.31295 \\ \text { Root Mean Square Error } \quad 0.002038\end{array}$

Mean of Response

Observations (or Sum Wgts)

0.002038
0.138074

Analysis of Variance

Source DF Sum of Squares Mean Square F Ratio Prob > F

$\begin{array}{llllll}\text { Series/Blk/Sub-Blk } & 3 & 0.00003490 & 0.000012 & 2.8000 & 0.1086\end{array}$

$\begin{array}{lll}\text { Error } & 8 & 0.00003324\end{array}$ $4.155 \mathrm{e}-6$

Means for Oneway Anova

Level Number Mean Std Error Lower

$\begin{array}{llllll}\text { W-1-1 } & 3 & 0.139190 & 0.00118 & 0.13648 & 0.14190\end{array}$

$\begin{array}{llllll}W-1-2 & 3 & 0.137702 & 0.00118 & 0.13499 & 0.14042\end{array}$

$\begin{array}{llllll}\text { W-2-1 } & 3 & 0.139935 & 0.00118 & 0.13722 & 0.14265\end{array}$

$\begin{array}{llllll}\mathrm{W}-2-2 & 3 & 0.135469 & 0.00118 & 0.13275 & 0.13818\end{array}$

Std Error uses a pooled estimate of error variance 


\section{Exhibit A-3. Measurements by Analytical Series, Block and Sub-Block for the Batch 1 and LRM Standards by Oxide by Prep (continued)}

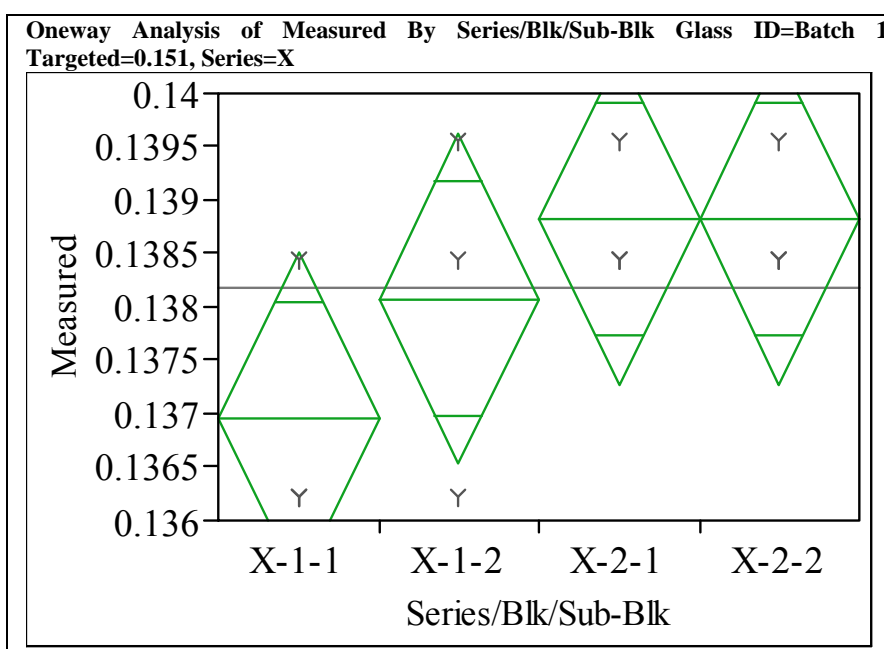

\section{Oneway Anova}

Summary of Fit

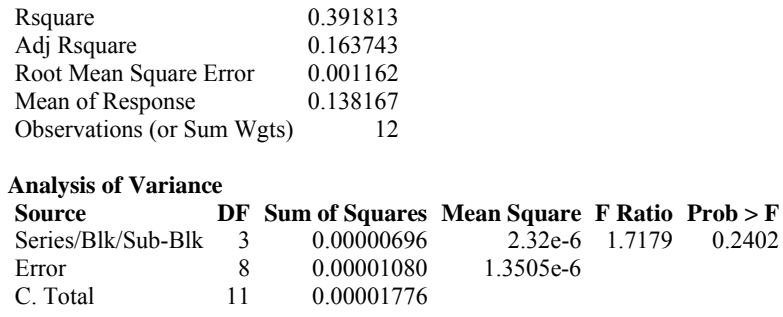

Means for Oneway Anova

Level Number Mean Std Error Lower 95\% Upper 95\%

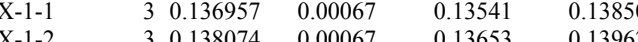

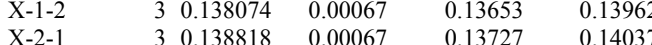

$\begin{array}{llllll}\mathrm{X}-2-1 & 3 & 0.138818 & 0.0067 & 0.13653 & 0.13962 \\ \mathrm{X}-2-2 & 3 & 0.138818 & 0.00067 & 0.13727 & 0.14037\end{array}$

Std Error uses a pooled estimate of error variance

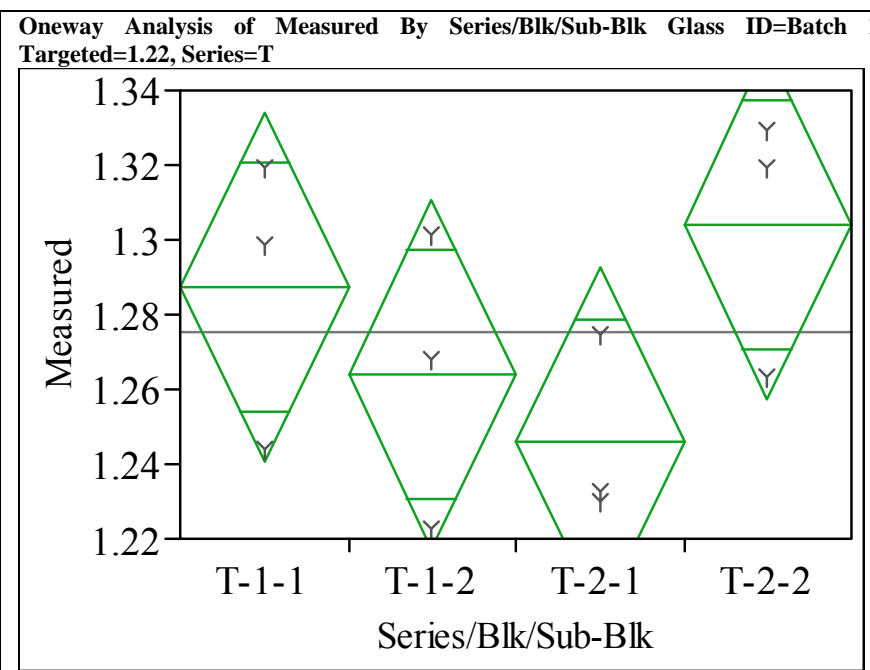

Oneway Anova

Summary of Fit

$\begin{array}{lr}\text { Rsquare } & 0.373823 \\ \text { Adj Rsquare } & 0.139007 \\ \text { Root Mean Square Error } & 0.035194 \\ \text { Mean of Response } & 1.275254 \\ \text { Observations (or Sum Wgts) } & 12\end{array}$

1.275254
12

Analysis of Variance

Source DF Sum of Squares Mean Square F Ratio Prob $>$ F $\begin{array}{lrrrrr}\text { Series/Blk/Sub-Blk } & 3 & 0.00591554 & 0.001972 & 1.5920 & 0.2660\end{array}$

Error

C. Total

80.00990888

Means for Oneway Anova

Level Number Mean Std Error Lower 95\% Upper 95\%

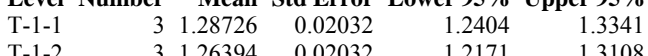

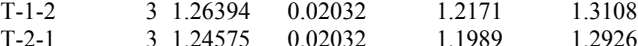

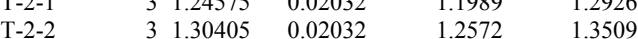

Std Error uses a pooled estimate of error variance 


\section{Exhibit A-3. Measurements by Analytical Series, Block and Sub-Block for the Batch 1 and LRM Standards by Oxide by Prep (continued)}

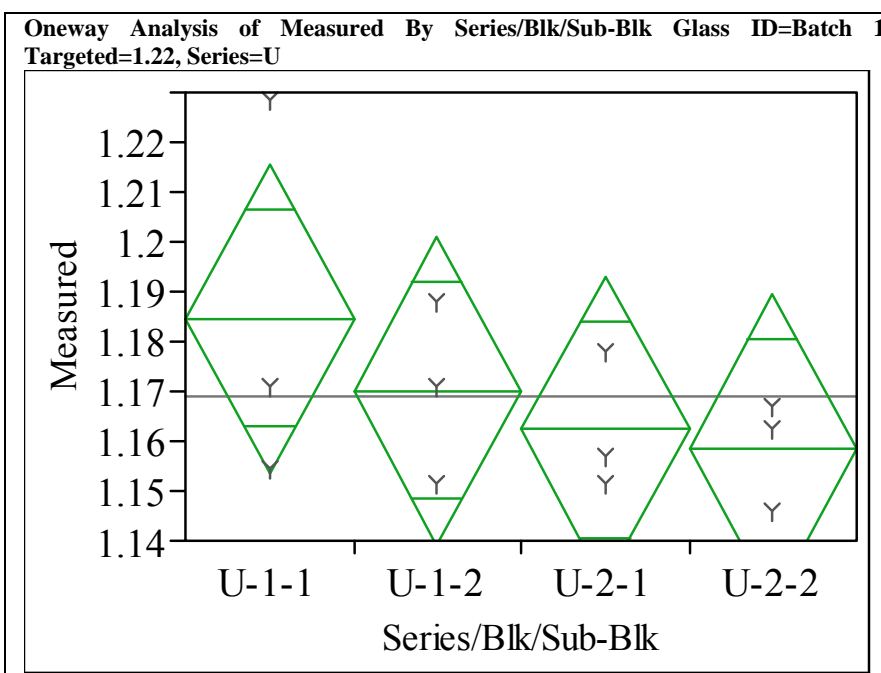

\section{Oneway Anova}

Summary of Fit

$$
\begin{array}{lr}
\text { Rsquare } & 0.217676 \\
\text { Adj Rsquare } & -0.0757 \\
\text { Root Mean Square Error } & 0.023256 \\
\text { Mean of Response } & 1.168915 \\
\text { Obe }
\end{array}
$$

$\begin{array}{lr}\text { Mean of Response } & 1.168915 \\ \text { Observations (or Sum Wgts) } & 12\end{array}$

Analysis of Variance

Source DF Sum of Squares Mean Square F Ratio Prob $>$ F

$\begin{array}{lrrrrr}\text { Series/Blk/Sub-Blk } & 3 & 0.00120386 & 0.000401 & 0.7420 & 0.5563\end{array}$

Error

$\begin{array}{lll}0.000401 & 0.7420 & 0.5563\end{array}$

C. Total

0.00553051

Means for Oneway Anova

Level Number Mean Std Error Lower 95\% Upper 95\%

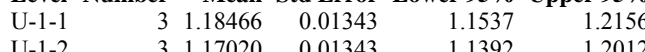

$\begin{array}{llllll}\text { U-1-2 } & 3 & 1.17020 & 0.01343 & 1.1392 & 1.2012 \\ \mathrm{U}-2-1 & 3 & 1.16227 & 0.01343 & 1.1313 & 1.1932\end{array}$

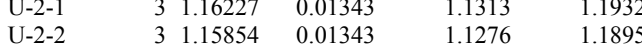

Std Error uses a pooled estimate of error variance

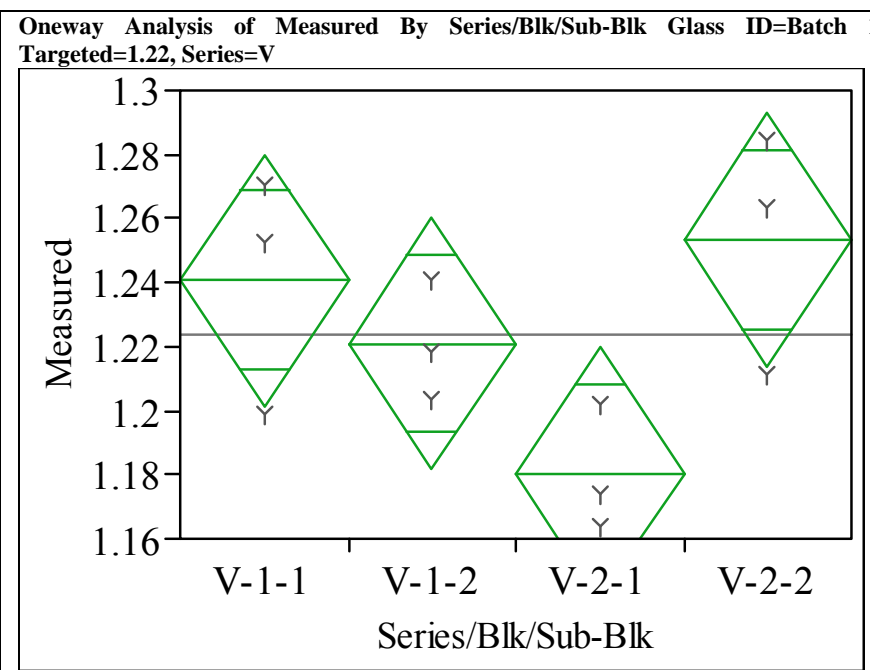

Oneway Anova

Summary of Fit

$\begin{array}{lr}\text { Rsquare } & 0.566995 \\ \text { Adj Rsquare } & 0.404618 \\ \text { Root Mean Square Error } & 0.029676 \\ \text { Mean of Response } & 1.223717 \\ \text { Observations (or Sum Wgts) } & 12\end{array}$

Observations (or Sum Wgts) 1.223717

Analysis of Variance

Source DF Sum of Squares Mean Square F Ratio Prob $>$ F

$\begin{array}{llllll}\text { Series/Blk/Sub-Blk } & 3 & 0.00922546 & 0.003075 & 3.4918 & 0.0698\end{array}$

$\begin{array}{lll}\text { Error } & 8 & 0.00704533\end{array}$

0.00088

Means for Oneway Anova

Level Number Mean Std Error Lower 95\% Upper 95\%

$\begin{array}{rrrrrr}\text { L-1-1 } & 3 & 1.24062 & 0.01713 & 1.2011 & 1.2801\end{array}$

$\begin{array}{llllll}\mathrm{V}-1-1 & 3 & 1.24062 & 0.01713 & 1.2011 & 1.2801 \\ \mathrm{~V}-1-2 & 3 & 1.22104 & 0.01713 & 1.1815 & 1.2605\end{array}$

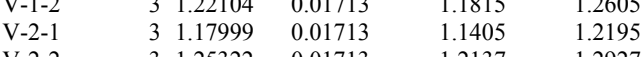

Std Error uses a pooled estimate of error variance 


\section{Exhibit A-3. Measurements by Analytical Series, Block and Sub-Block for the Batch 1 and LRM Standards by Oxide by Prep (continued)}

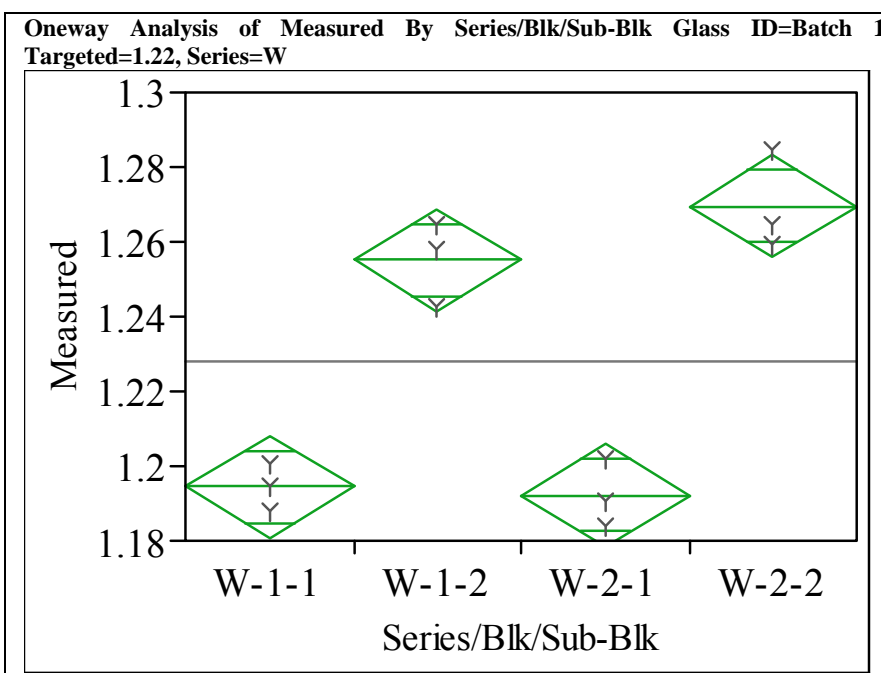

\section{Oneway Anova}

Summary of Fit

$\begin{array}{lr}\text { Rsquare } & 0.944423 \\ \text { Adj Rsquare } & 0.923582 \\ \text { Root Mean Square Error } & 0.010369 \\ \text { Mean of Response } & 1.227798 \\ \text { Observations (or Sum Wgts) } & 12\end{array}$

Analysis of Variance

Source DF Sum of Squares Mean Square F Ratio Prob > F

$\begin{array}{llllll}\text { Series/Blk/Sub-Blk } & 3 & 0.01461599 & 0.004872 & 45.3151 & <.0001\end{array}$

$\begin{array}{lll}\text { Error } & 8 & 0.00086011\end{array}$

Means for Oneway Anova
Level Number Mean Std Error Lower 95\% Upper 95\%

$\begin{array}{lrrrrr}\text { Level } & \text { Number } & \text { Mean } & \text { Std Error } & \text { Lower 95\% } & \text { Upper 95\% } \\ \text { W-1-1 } & 3 & 1.19445 & 0.00599 & 1.1806 & 1.2083\end{array}$

$\begin{array}{llllll}\text { W-1-2 } & 3 & 1.25508 & 0.00599 & 1.2413 & 1.2689 \\ \text { W-2-1 } & 3 & 1.19212 & 0.00599 & 1.1783 & 1.2059\end{array}$

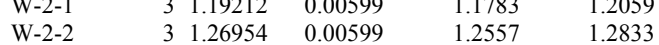

Std Error uses a pooled estimate of error variance

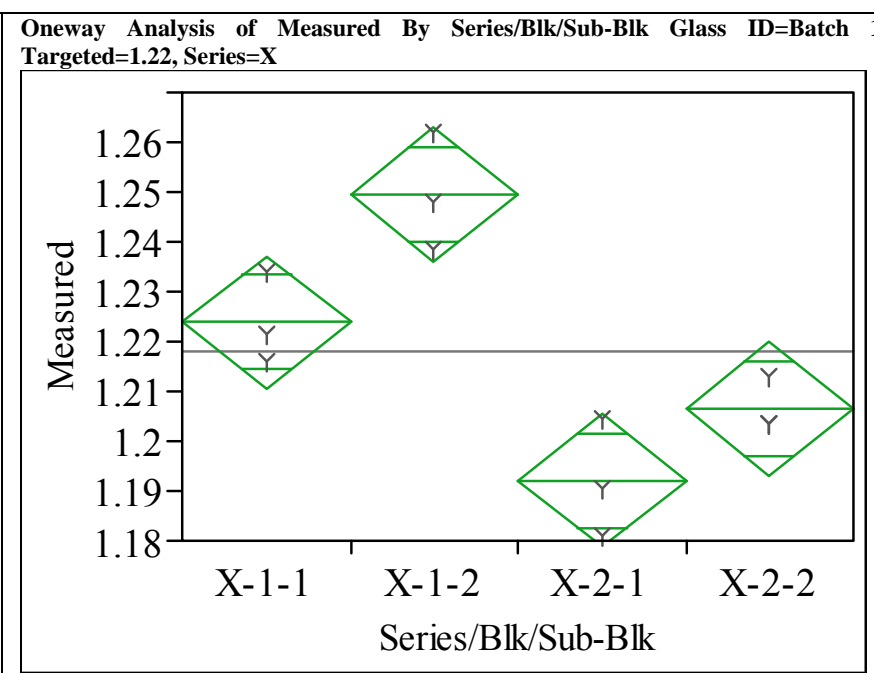

Oneway Anova

Summary of Fit

$\begin{array}{lr}\text { Rsquare } & 0.871276 \\ \text { Adj Rsquare } & 0.823004 \\ \text { Root Mean Square Error } & 0.010057 \\ \text { Mean of Response } & 1.218004 \\ \text { Observations (or Sum Wgts) } & 12\end{array}$

Observations (or Sum Wets) 1.218004

Analysis of Variance

Source DF Sum of Squares Mean Square F Ratio Prob $>$ F

$\begin{array}{lrrrrr}\text { Series/Blk/Sub-Blk } & 3 & 0.00547716 & 0.001826 & 18.0495 & 0.0006\end{array}$

$\begin{array}{lll}\text { Error } & 8 & 0.00080921\end{array}$

0.000101

0.00628637

Means for Oneway Anova

Level Number Mean Std Error Lower 95\% Upper 95\%

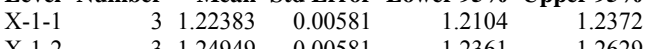

$\begin{array}{llllll}\mathrm{X}-1-1 & 3 & 1.22383 & 0.00581 & 1.2104 & 1.2372 \\ \mathrm{X}-1-2 & 3 & 1.24949 & 0.00581 & 1.2361 & 1.2629 \\ \mathrm{X}-2-1 & 3 & 1.19212 & 0.00581 & 1.1787 & 1.2055\end{array}$

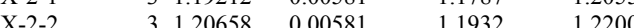

Std Error uses a pooled estimate of error variance 
Exhibit A-3. Measurements by Analytical Series, Block and Sub-Block for the Batch 1 and LRM Standards by Oxide by Prep (continued)

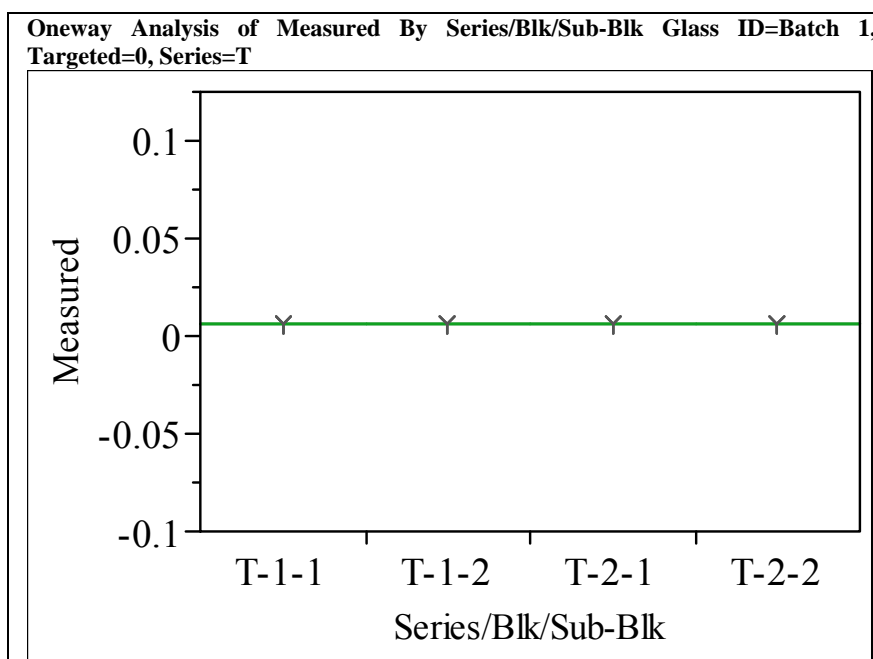

Oneway Anova

Summary of Fit

Rsquare

Adj Rsquare

$\begin{array}{lr}\text { Root Mean Square Error } & 0 \\ \text { Mean of Response } & 0.005857\end{array}$

Observations (or Sum Wgts)

Analysis of Variance

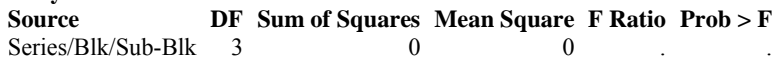

$\begin{array}{lr}\text { Error } & 8 \\ \text { C. Total } & 11\end{array}$

$\begin{array}{lll}0 & 0 \\ 0 & 0\end{array}$

Means for Oneway Anova

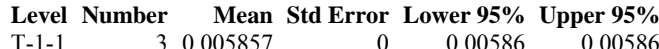

$\begin{array}{llllrr}\text { T-1-1 } & 3 & 0.005857 & 0 & 0.00586 & 0.00586 \\ \mathrm{~T}-1-2 & 3 & 0.005857 & 0 & 0.00586 & 0.00586\end{array}$

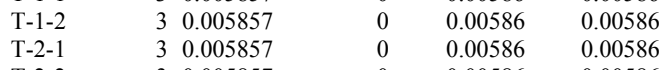

$\begin{array}{llllll}\mathrm{T}-2-2 & 3 & 0.005857 & 0 & 0.00586 & 0.00586\end{array}$

Std Error uses a pooled estimate of error variance

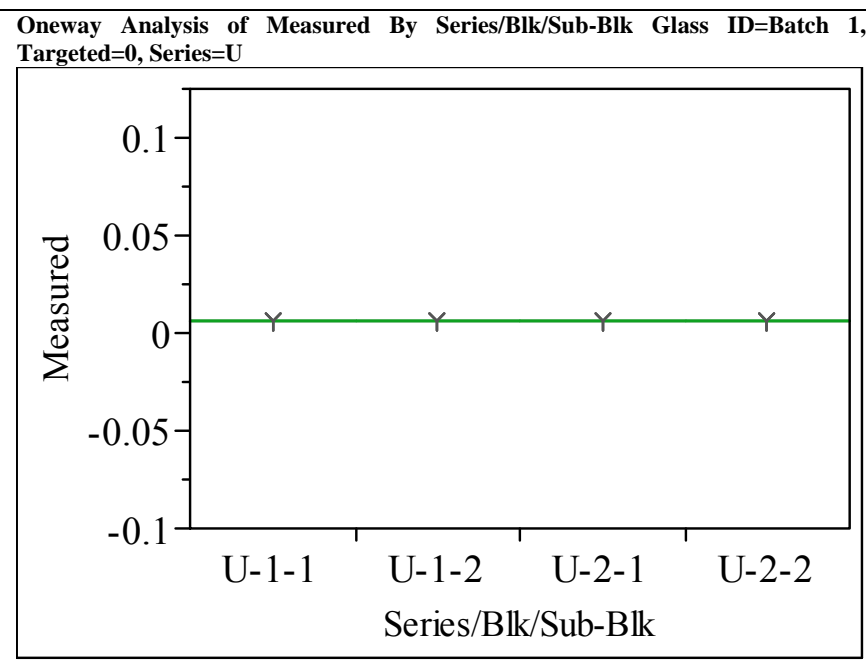

Oneway Anova

Summary of Fit

Rsquare

Adj Rsquare

$\begin{array}{lr}\text { Root Mean Square Error } & 0 \\ \text { Mean of Response } & 0.005857\end{array}$

Observations (or Sum Wgts) 12

Analysis of Variance

Sum of Squares Mean Square F Ratio Prob > F

$\begin{array}{llll}\text { Series/Blk/Sub-Blk } & 3 & 0 & 0 \\ \text { Error } & 8 & 0 & 0\end{array}$

$\begin{array}{lrr}\text { Error } & 8 & 0 \\ \text { C. Total } & 11 & 0\end{array}$

Means for Oneway Anova

Level Number Mean Std Error Lower 95\% Upper 95\%

$\begin{array}{llllll}\text { U-1-1 } & 3 & 0.005857 & 0 & 0.00586 & 0.00586\end{array}$

$\begin{array}{llllll}\mathrm{U}-1-2 & 3 & 0.005857 & 0 & 0.00586 & 0.00586\end{array}$

$\begin{array}{llllll}\text { U-2-1 } & 3 & 0.005857 & 0 & 0.00586 & 0.00586\end{array}$

$\begin{array}{llllll}\text { U-2-2 } & 3 & 0.005857 & 0 & 0.00586 & 0.00586\end{array}$

Std Error uses a pooled estimate of error variance 
Exhibit A-3. Measurements by Analytical Series, Block and Sub-Block for the Batch 1 and LRM Standards by Oxide by Prep (continued)

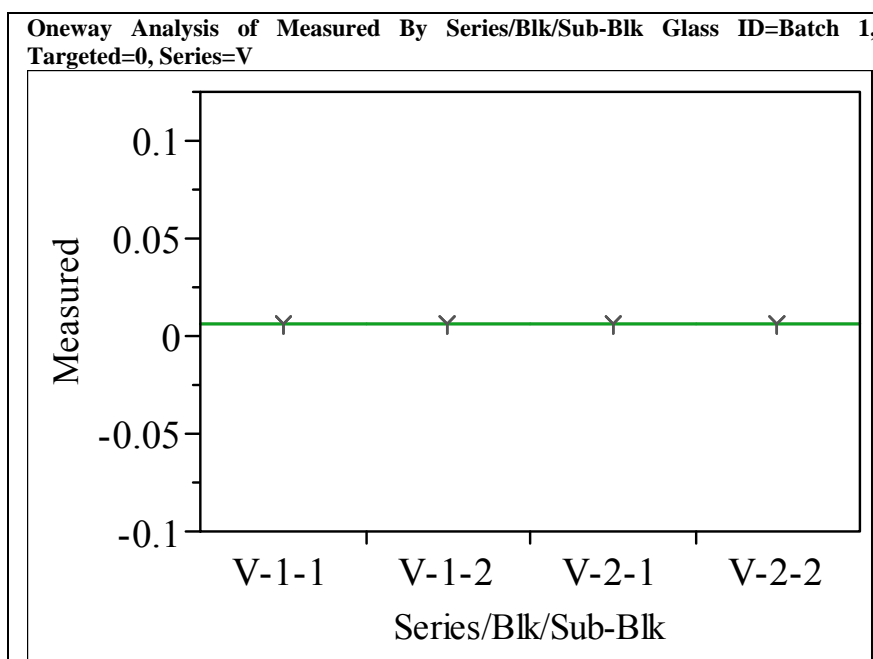

Oneway Anova

Summary of Fit

Rsquare

Adj Rsquare

$\begin{array}{lr}\text { Root Mean Square Error } & 0 \\ \text { Mean of Response } & 0.005857\end{array}$

Observations (or Sum Wgts)

Analysis of Variance

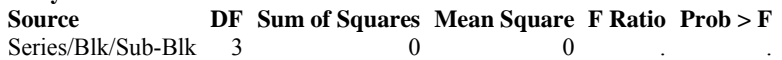

$\begin{array}{lr}\text { Error } & 8 \\ \text { C. Total } & 11\end{array}$

$\begin{array}{lll}0 & 0 \\ 0 & 0\end{array}$

0

Means for Oneway Anova

Level Number Mean Std Error Lower 95\% Upper 95\%

$\begin{array}{llllll}\text { V-1-1 } & 3 & 0.005857 & 0 & 0.00586 & 0.00586\end{array}$

$\begin{array}{llllll}\mathrm{V}-1-2 & 3 & 0.005857 & 0 & 0.00586 & 0.00586\end{array}$

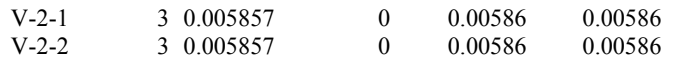

Std Error uses a pooled estimate of error variance

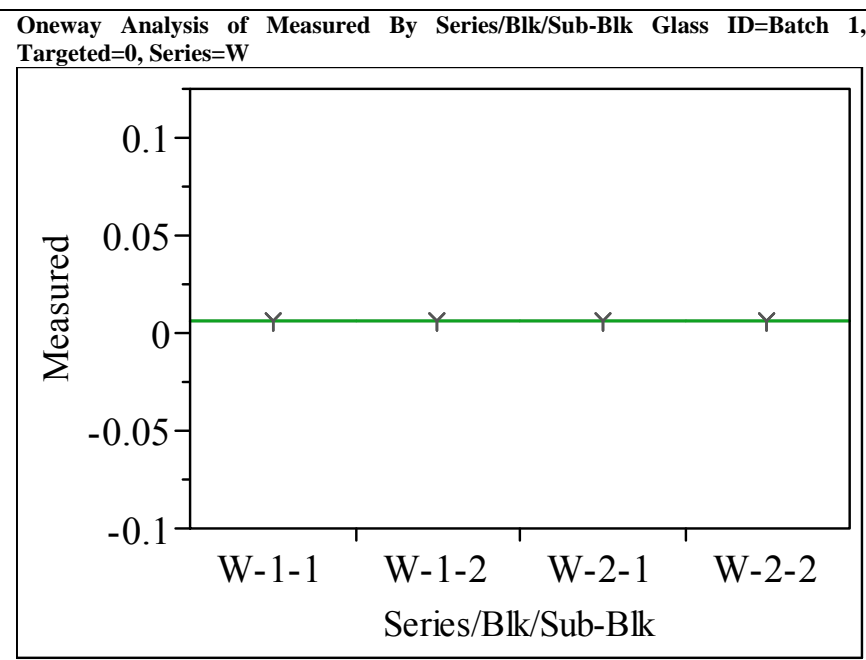

Oneway Anova

Summary of Fit

Rsquare

Adj Rsquare

$\begin{array}{lr}\text { Root Mean Square Error } & 0 \\ \text { Mean of Response } & 0.005857\end{array}$

Observations (or Sum Wgts)

Analysis of Variance

Sum of Squares Mean Square F Ratio Prob > F

$\begin{array}{llll}\text { Series/Blk/Sub-Blk } & 3 & 0 & 0 \\ \text { Error } & 8 & 0 & 0\end{array}$

$\begin{array}{lrr}\text { Error } & 8 & 0 \\ \text { C. Total } & 11 & 0\end{array}$

Means for Oneway Anova

Level Number Mean Std Error Lower 95\% Upper 95\%

$\begin{array}{llllll}\text { W-1-1 } & 3 & 0.005857 & 0 & 0.00586 & 0.00586\end{array}$

$\begin{array}{llllll}\mathrm{W}-1-2 & 3 & 0.005857 & 0 & 0.00586 & 0.00586\end{array}$

$\begin{array}{llllll}W-2-1 & 3 & 0.005857 & 0 & 0.00586 & 0.00586\end{array}$

$\begin{array}{llllll}\mathrm{W}-2-2 & 3 & 0.005857 & 0 & 0.00586 & 0.00586\end{array}$

Std Error uses a pooled estimate of error variance 


\section{Exhibit A-3. Measurements by Analytical Series, Block and Sub-Block for the Batch 1 and LRM Standards by Oxide by Prep (continued)}

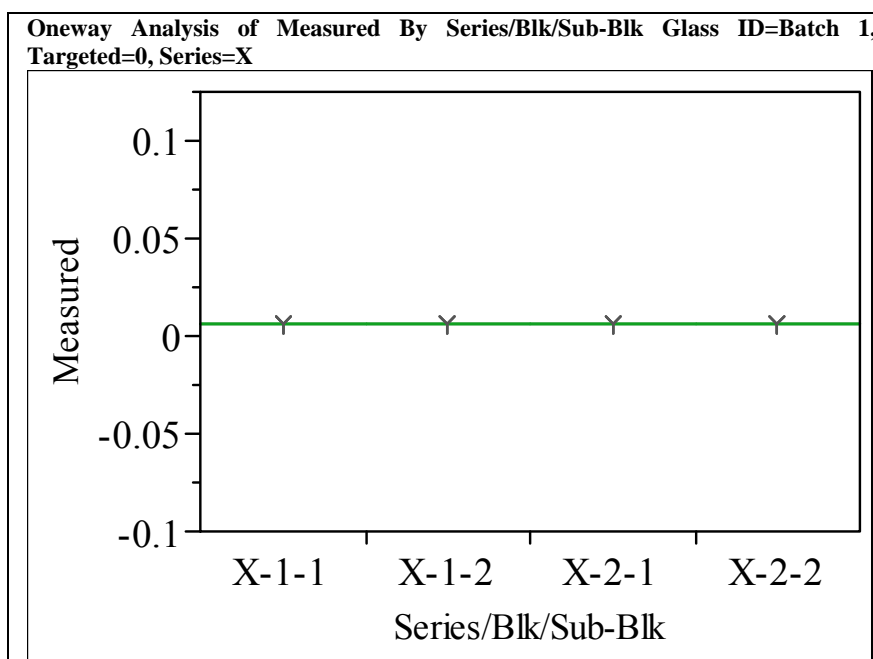

Oneway Anova

Summary of Fit

Rsquare

Adj Rsquare

$\begin{array}{lr}\text { Root Mean Square Error } & 0 \\ \text { Mean of Response } & 0.005857\end{array}$

Observations (or Sum Wgts)

Analysis of Variance

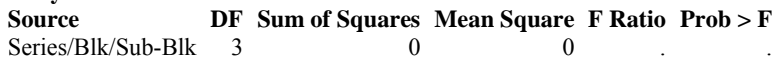

$\begin{array}{lr}\text { Error } & 8 \\ \text { C. Total } & 11\end{array}$

$\begin{array}{lll}0 & 0 \\ 0 & 0\end{array}$

0

Means for Oneway Anova

Level Number Mean Std Error Lower 95\% Upper 95\%

$\begin{array}{lllllr}\mathrm{X}-1-1 & 3 & 0.005857 & 0 & 0.00586 & 0.00586 \\ \mathrm{X}-1-2 & 3 & 0.005857 & 0 & 0.00586 & 0.00586\end{array}$

$\begin{array}{llllll}\mathrm{X}-1-2 & 3 & 0.005857 & 0 & 0.00586 & 0.00586\end{array}$

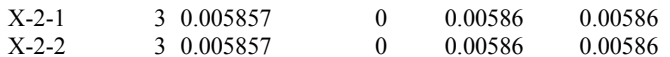

Std Error uses a pooled estimate of error variance

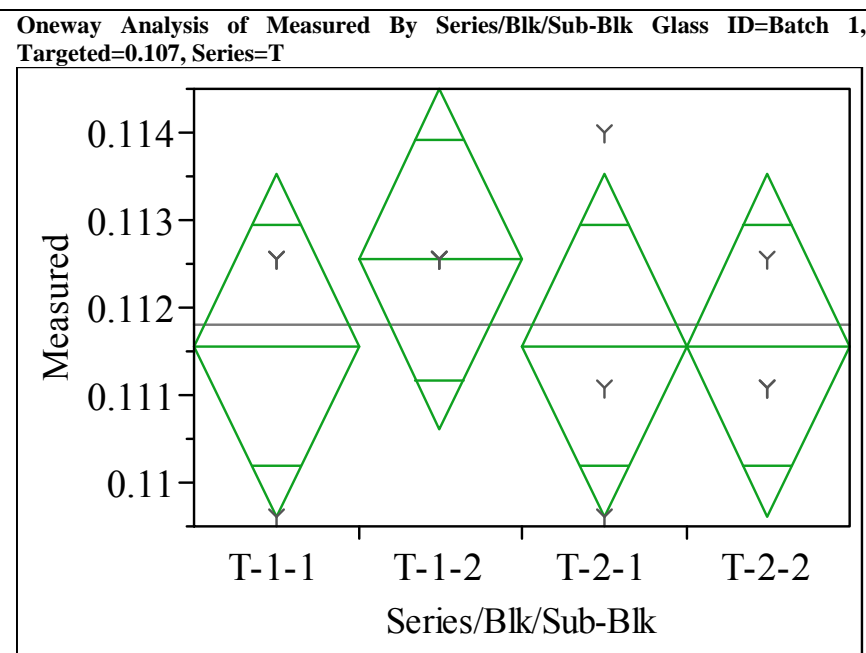

Oneway Anova

Summary of Fit

Rsquare

$\begin{array}{ll}\text { Adj Rsquare } & 0.111111 \\ -0.22222\end{array}$

Root Mean Square Error $\quad 0.001462$

$\begin{array}{lr}\text { Mean of Response } & 0.111812 \\ \text { Observations (or Sum Wgts) } & 12\end{array}$

Analysis of Variance

Source DF Sum of Squares Mean Square F Ratio Prob $>$ F

$\begin{array}{lllllll}\text { Series/Blk/Sub-Blk } & 3 & 0.00000214 & 7.1209 \mathrm{e}-7 & 0.3333 & 0.8018\end{array}$

$\begin{array}{lrr}\text { Error } & 8 & 0.00001709 \\ \text { C. } & 11 & 0.00001923\end{array}$

$2.1363 \mathrm{e}-6$

Means for Oneway Anova

Level Number Mean Std Error Lower 95\% Upper 95\%

$\begin{array}{llllrr}\text { T-1-1 } & 3 & 0.111569 & 0.00084 & 0.10962 & 0.1135\end{array}$

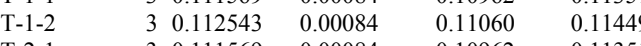

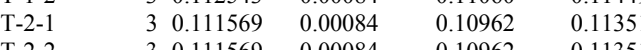

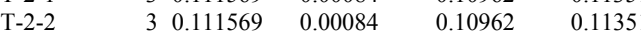

Std Error uses a pooled estimate of error variance 


\section{Exhibit A-3. Measurements by Analytical Series, Block and Sub-Block for the Batch 1 and LRM Standards by Oxide by Prep (continued)}

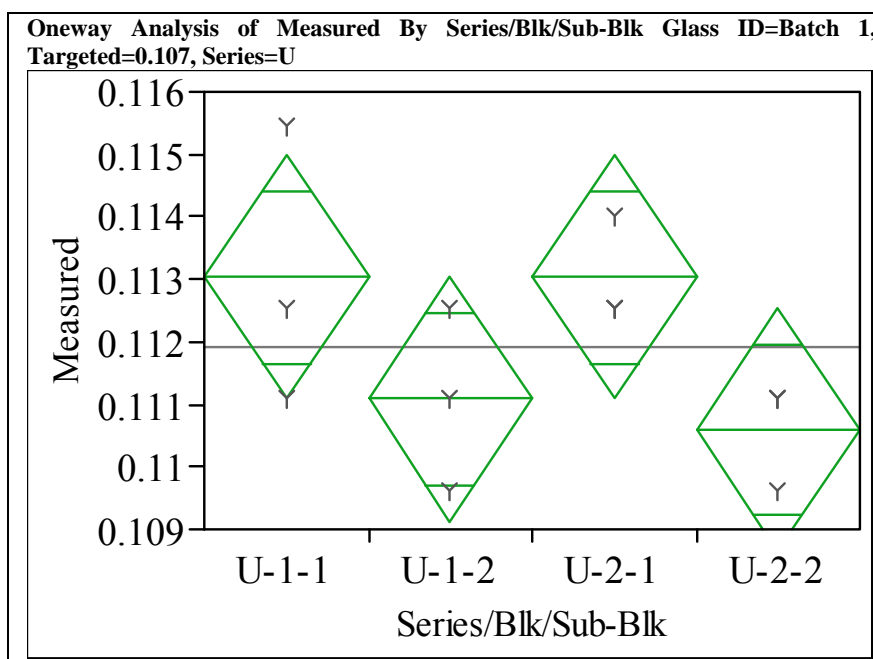

Oneway Anova

Summary of Fit

Adj Rsquare $\quad 0.26257$

Root Mean Square Error $\quad 0.001462$

$\begin{array}{lr}\text { Mean of Response } & 0.111934 \\ \text { Observations (or Sum Wgts) } & 12\end{array}$

Analysis of Variance

$\begin{array}{lrrrrr}\text { Source } & \text { DF Sum of Squares } & \text { Mean Square F Ratio Prob }>\text { F } \\ \text { Series/Blk/Sub-Blk } & 3 & 0.00001478 & 4.9253 \mathrm{e}-6 & 2.3056 & 0.1534\end{array}$

$\begin{array}{llllll}\text { Series/Blk/Sub-Blk } & 3 & 0.00001478 & 4.9253 \mathrm{e}-6 & 2.3056 & 0.1534 \\ \text { Error } & 8 & 0.00001709 & 2.1363 \mathrm{e}-6 & & \end{array}$

C. Total

0.00003187

Means for Oneway Anova

Level Number Mean Std Error Lower 95\% Upper 95\%

$\begin{array}{lrrrrr}\text { U-1-1 } & 3 & 0.113030 & 0.00084 & 0.11108 & 0.11498\end{array}$

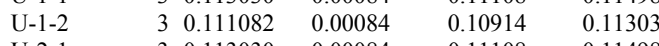

$\begin{array}{lllllll}\text { U-2-1 } & & 3 & 0.113030 & 0.00084 & 0.11108 & 0.11498 \\ \text { U-2-2 } & & 3 & 0.110594 & 0.00084 & 0.10865 & 0.11254\end{array}$

Std Error uses a pooled estimate of error variance

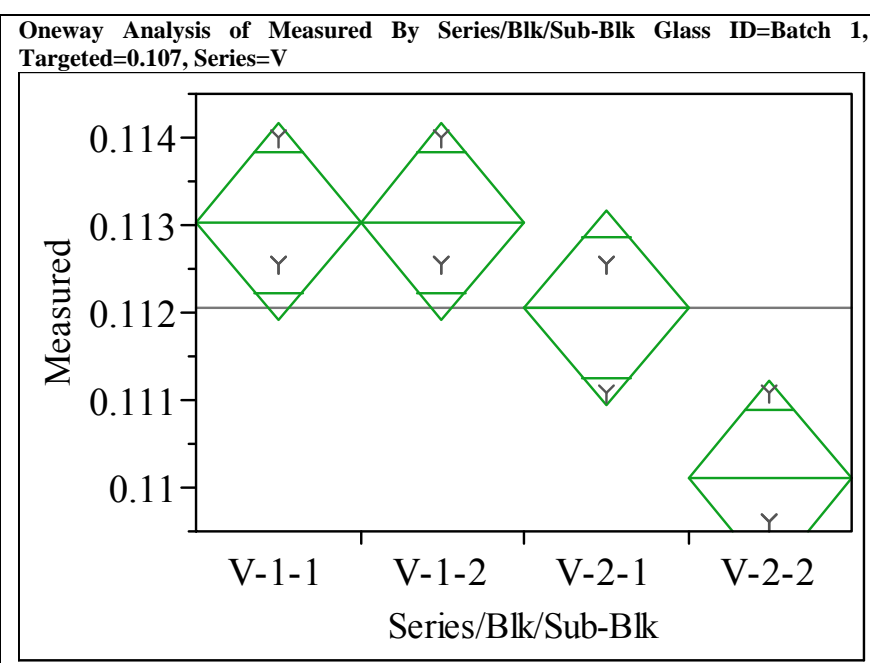

Oneway Anova

Summary of Fit

Rsquare

$\begin{array}{lr} & 0.75 \\ \text { Adj Rsquare } & 0.65625\end{array}$

Root Mean Square Error $\quad 0.000844$

$\begin{array}{lr}\text { Mean of Response } & 0.112056 \\ \text { Observations (or Sum Wgts) } & 12\end{array}$

Analysis of Variance

Source DF Sum of Squares Mean Square F Ratio Prob $>$ F

$\begin{array}{llllll}\text { Series/Blk/Sub-Blk } & 3 & 0.00001709 & 5.6967 \mathrm{e}-6 & 8.0000 & 0.0086\end{array}$

$\begin{array}{lll}\text { Error } & 8 & 0.00000570\end{array}$ $7.1209 \mathrm{e}-7$

Means for Oneway Anova

Level Number Mean Std Error Lower

$\begin{array}{llllrr}\text { V-1-1 } & 3 & 0.113030 & 0.00049 & 0.11191 & 0.11415\end{array}$

$\begin{array}{llllll}V-1-2 & 3 & 0.113030 & 0.00049 & 0.11191 & 0.11415\end{array}$

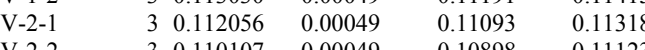

$\begin{array}{llllll}\mathrm{V}-2-2 & 3 & 0.110107 & 0.00049 & 0.10898 & 0.11123\end{array}$

Std Error uses a pooled estimate of error variance 
Exhibit A-3. Measurements by Analytical Series, Block and Sub-Block for the Batch 1 and LRM Standards by Oxide by Prep (continued)

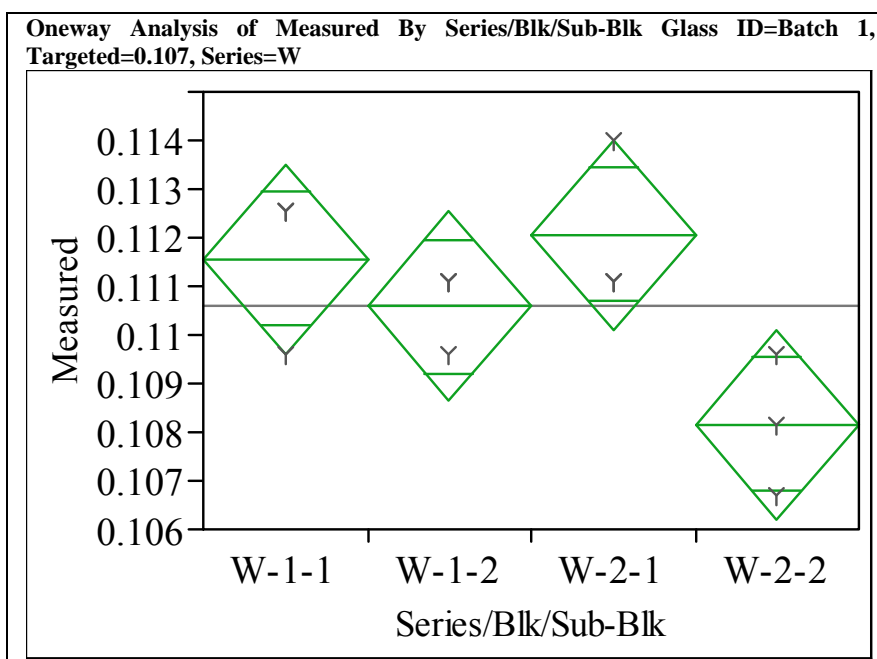

Oneway Anova

Summary of Fit

0.612903

Adj Rsquare $\quad 0.467742$

Root Mean Square Error $\quad 0.001462$

$\begin{array}{lr}\text { Mean of Response } & 0.110594 \\ \text { Observations (or Sum Wgts) } & 12\end{array}$

Analysis of Variance

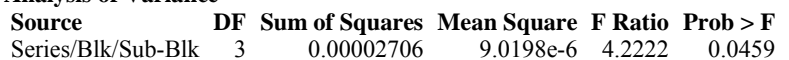

$\begin{array}{llllll}\text { Series/Blk/Sub-Blk } & 3 & 0.00002706 & 9.0198 \mathrm{e}-6 & 4.2222 & 0.0459 \\ \text { Error } & 8 & 0.00001709 & 2.1363 \mathrm{e}-6 & & \end{array}$

C. Total

0.00004415

Means for Oneway Anova

Level Number Mean Std Error Lower 95\% Upper 95\%

$\begin{array}{lllllr}\text { W-1-1 } & 3 & 0.111569 & 0.00084 & 0.10962 & 0.11351\end{array}$

$\begin{array}{llllll}\text { W-1-2 } & 3 & 0.110594 & 0.00084 & 0.10865 & 0.11254\end{array}$

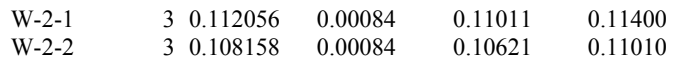

Std Error uses a pooled estimate of error variance

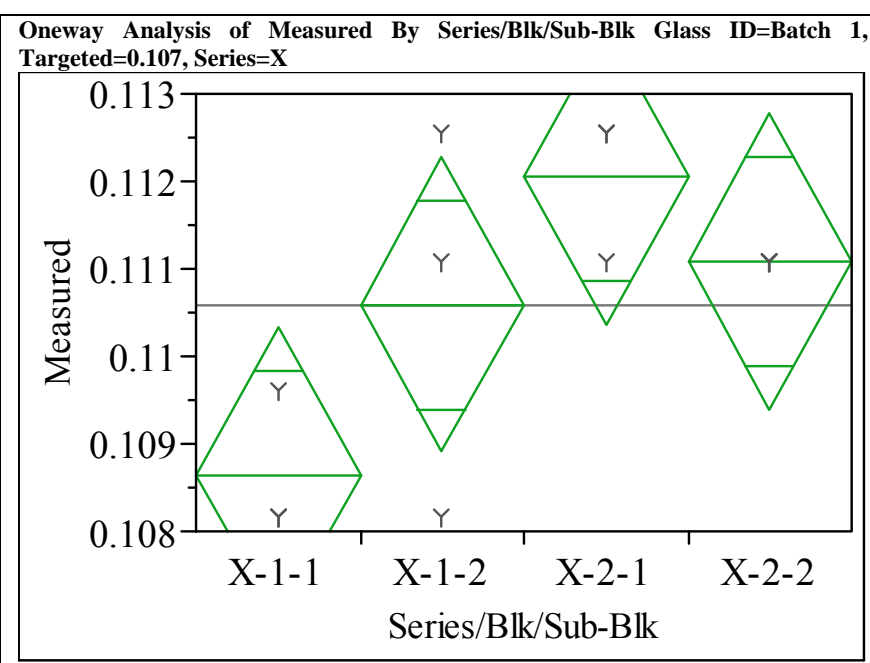

\section{Oneway Anova}

Summary of Fit

$\begin{array}{lr}\text { Rsquare } & 0.590909 \\ \text { Adj Rsquare } & 0.4375 \\ \text { Root Mean Square Error } & 0.001266 \\ \text { Mean of Response } & 0.110594 \\ \text { Observations (or Sum Wgts) } & 12\end{array}$

Analysis of Variance

Source DF Sum of Squares Mean Square F Ratio Prob > F

$\begin{array}{llllll}\text { Series/Blk/Sub-Blk } & 3 & 0.00001851 & 6.1715 \mathrm{e}-6 & 3.8519 & 0.0565\end{array}$

$\begin{array}{lll}\text { Error } & 8 & 0.00001282 \\ \text { C. Tota } & 11 & 0.0003133\end{array}$

$1.6022 \mathrm{e}-6$

Means for Oneway Anova

Level Number Mean Std Error Lower 95\% Upper 95\%

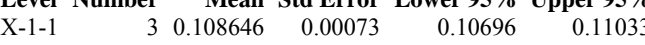

$\begin{array}{llllll}\mathrm{X}-1-2 & 3 & 0.110594 & 0.00073 & 0.10891 & 0.11228\end{array}$

$\begin{array}{llllll}\mathrm{X}-2-1 & 3 & 0.112056 & 0.00073 & 0.11037 & 0.11374\end{array}$

$\begin{array}{llllll}\mathrm{X}-2-2 & 3 & 0.111082 & 0.00073 & 0.10940 & 0.11277\end{array}$

Std Error uses a pooled estimate of error variance 
Exhibit A-3. Measurements by Analytical Series, Block and Sub-Block for the Batch 1 and LRM Standards by Oxide by Prep (continued)

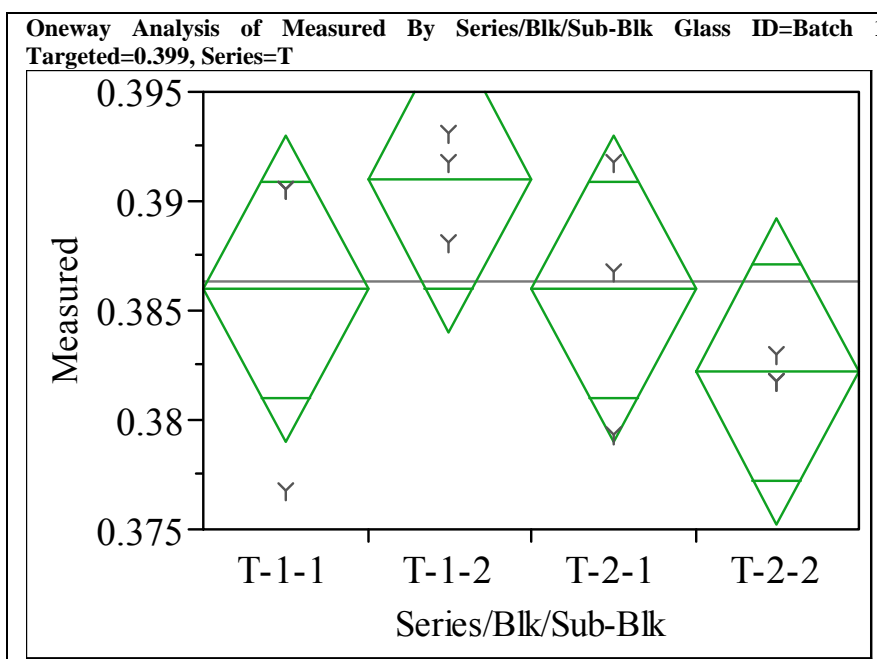

Oneway Anova

Summary of Fit

$\begin{array}{lr}\text { Rsquare } & 0.345483 \\ \text { Adj Rsquare } & 0.100039 \\ \text { Root Mean Square Error } & 0.005249 \\ \text { Mean of Response } & 0.386285 \\ \text { Observations (or Sum Wgts) } & 12\end{array}$

Analysis of Variance

$\begin{array}{lrrrrr}\text { Source } & \text { DF } & \text { Sum of Squares } & \text { Mean Square } & \text { F Ratio } & \text { Prob }>\text { F } \\ \text { Series/Blk/Sub-Blk } & 3 & 0.00011635 & 0.000039 & 1.4076 & 0.3099\end{array}$

Eeries/Bly/

Error

0.00011635
0.00022043

0.000028

Means for Oneway Anova

Level Number Mean Std Error Lower 95\% Upper 95\%

$\begin{array}{llrrrr}\text { T-1-1 } & 3 & 0.385972 & 0.00303 & 0.37898 & 0.39296\end{array}$

$\begin{array}{llllll}\mathrm{T}-1-2 & 3 & 0.390979 & 0.00303 & 0.38399 & 0.39797\end{array}$

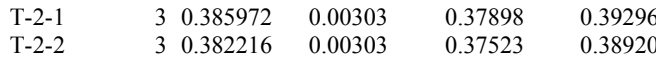

Std Error uses a pooled estimate of error variance

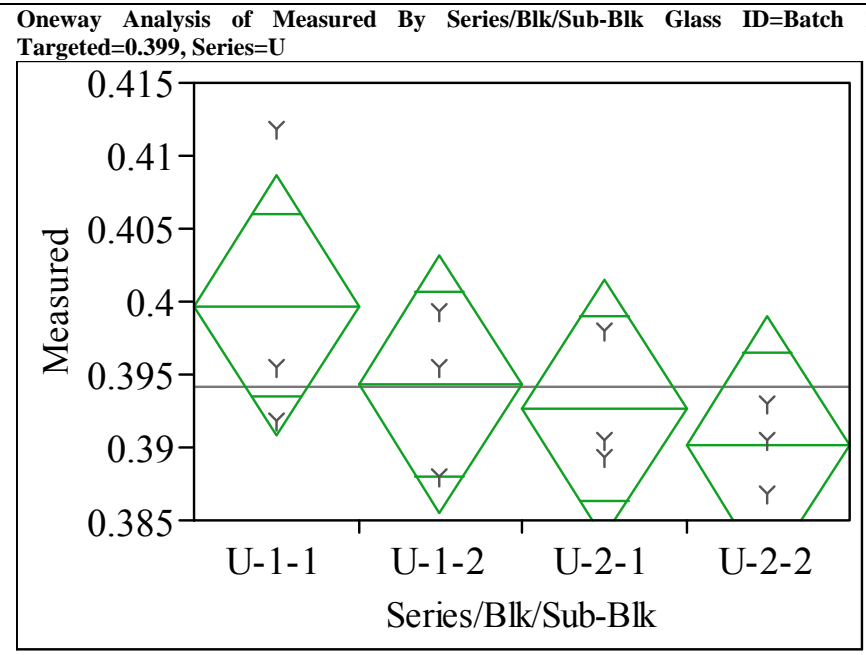

Oneway Anova

Summary of Fit

\section{Rsquare}

$\begin{array}{ll} & 0.029161 \\ \text { Root Mean Square Error } & 0.006683\end{array}$

$\begin{array}{lr}\text { Mean of Response } & 0.394213 \\ \text { Observations (or Sum Wgts) } & 12\end{array}$

Analysis of Variance

Source DF Sum of Squares Mean Square F Ratio Prob $>$ F

Series/Blk/Sub-Blk $3 \quad 0.00014873$

$\begin{array}{lrrr}\text { Error } & 8 & 0.00035728 & 0.000045 \\ \text { C. Total } & 11 & 0.00050601 & \end{array}$

Means for Oneway Anova

Level Number Mean Std Error Lower 95\% Upper 95\%

$\begin{array}{llllrr}\text { U-1-1 } & 3 & 0.399741 & 0.00386 & 0.39084 & 0.4086\end{array}$

$\begin{array}{llllll}\text { U-1-2 } & 3 & 0.394317 & 0.00386 & 0.38542 & 0.40321\end{array}$

$\begin{array}{llllll}\text { U-2-1 } & 3 & 0.392648 & 0.00386 & 0.38375 & 0.40155\end{array}$

$\begin{array}{llllll}\text { U-2-2 } & 3 & 0.390144 & 0.00386 & 0.38125 & 0.39904\end{array}$

Std Error uses a pooled estimate of error variance 
Exhibit A-3. Measurements by Analytical Series, Block and Sub-Block for the Batch 1 and LRM Standards by Oxide by Prep (continued)

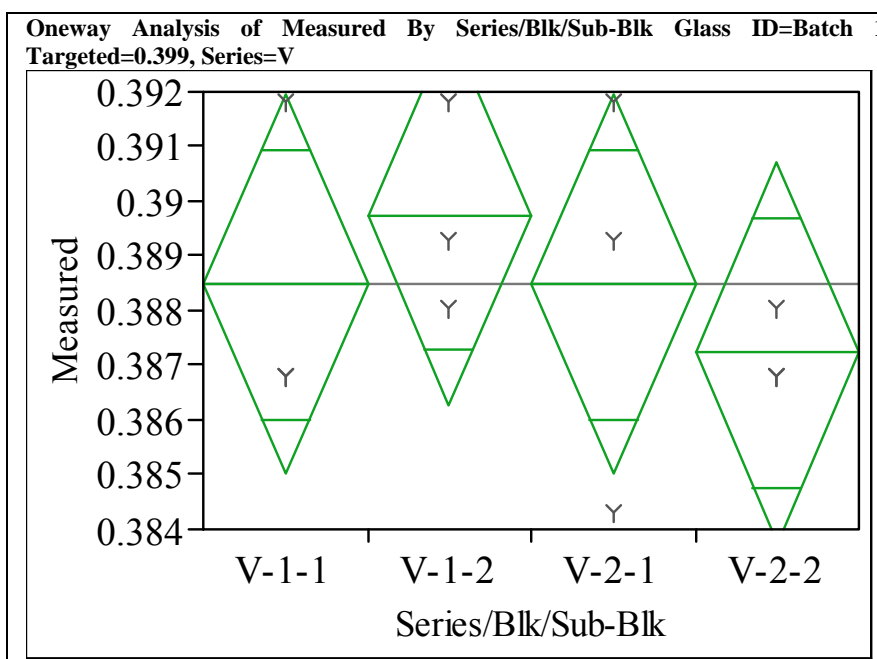

Oneway Anova

Summary of Fit

$\begin{array}{lr}\text { Rsquare } & 0.147541 \\ \text { Adj Rsquare } & -0.17213 \\ \text { Root Mean Square Error } & 0.002606 \\ \text { Mean of Response } & 0.388475 \\ \text { Observations (or Sum Wgts) } & 12\end{array}$

Analysis of Variance

Source DF Sum of Squares Mean Square F Ratio Prob > F

$\begin{array}{lrrrrr}\text { Series/Blk/Sub-Blk } & 3 & 0.00000940 & 3.134 \mathrm{e}-6 & 0.4615 & 0.7168 \\ \text { Error } & 8 & 0.00005432 & 6.7903 \mathrm{e}-6 & & \end{array}$

Error

C. Total

0.00006372

Means for Oneway Anova

Level Number Mean Std Error Lower 95\% Upper 95\%

$\begin{array}{llllll}\text { V-1-1 } & 3 & 0.388475 & 0.00150 & 0.38501 & 0.39194\end{array}$

$\begin{array}{llllll}V-1-2 & 3 & 0.389727 & 0.00150 & 0.38626 & 0.39320\end{array}$

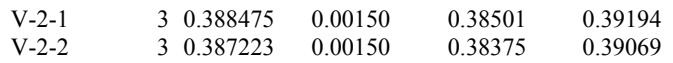

Std Error uses a pooled estimate of error variance

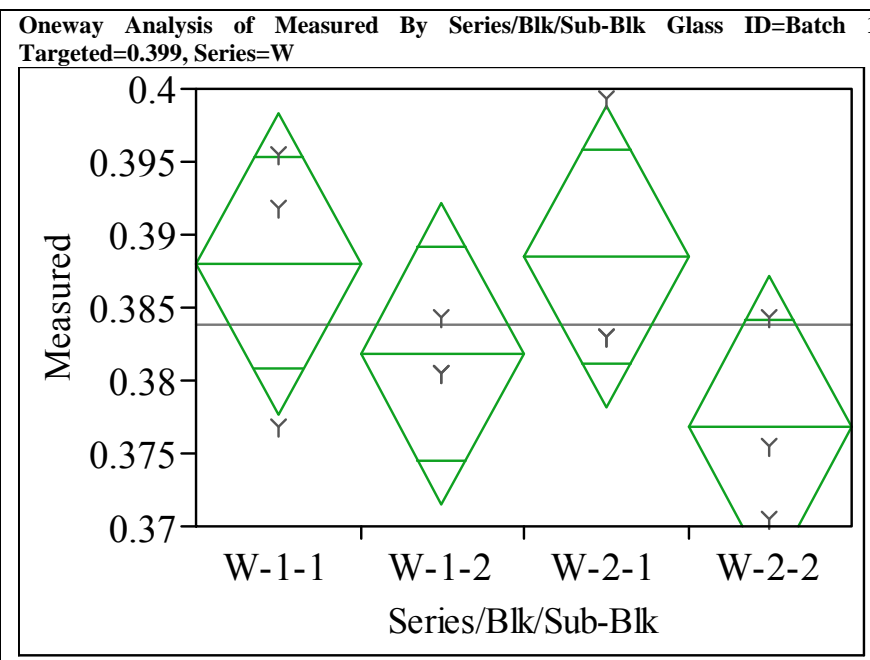

\section{Oneway Anova}

Summary of Fit

Rsquare

0.367589

0.130435
Root Mean Square Error $\quad 0.00775$

Mean of Response

Observations (or Sum Wgts)

0.00775
0.383781

Analysis of Variance

Source DF Sum of Squares Mean Square F Ratio Prob > F

$\begin{array}{lllllll}\text { Series/Blk/Sub-Blk } & 3 & 0.00027932 & 0.000093 & 1.5500 & 0.2753\end{array}$

\begin{tabular}{lll} 
Error & 8 & 0.00048055 \\
\hline
\end{tabular} 0.000060

Means for Oneway Anova

Level Number Mean Std Error Lower 95\% Upper 95\%

$\begin{array}{llllll}\text { W-1-1 } & 3 & 0.388058 & 0.00447 & 0.37774 & 0.39838\end{array}$

$\begin{array}{llllll}\mathrm{W}-1-2 & 3 & 0.381799 & 0.00447 & 0.37148 & 0.39212\end{array}$

$\begin{array}{llllll}\mathrm{W}-2-1 & 3 & 0.388475 & 0.00447 & 0.37816 & 0.39879\end{array}$

$\begin{array}{llllll}\mathrm{W}-2-2 & 3 & 0.376792 & 0.00447 & 0.36647 & 0.38711\end{array}$

Std Error uses a pooled estimate of error variance 


\section{Exhibit A-3. Measurements by Analytical Series, Block and Sub-Block for the Batch 1 and LRM Standards by Oxide by Prep (continued)}

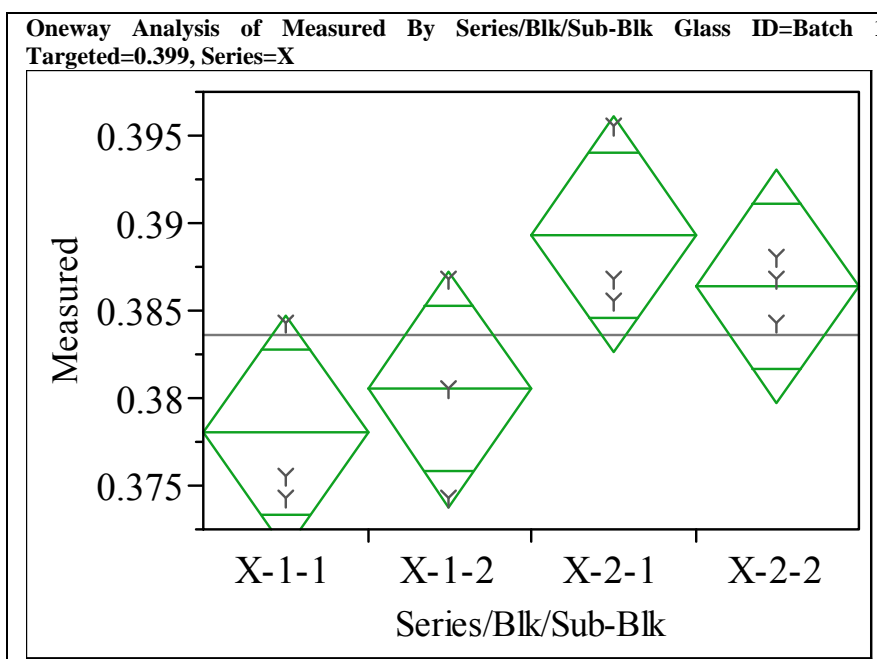

\section{Oneway Anova}

Summary of Fit

Rsquare $\quad 0.541386$

Adj Rsquare $\quad 0.369406$

Root Mean Square Error $\quad 0.005059$

$\begin{array}{lr}\text { Mean of Response } & 0.383572 \\ \text { Observations (or Sum Wgts) } & 12\end{array}$

Analysis of Variance

Source DF Sum of Squares Mean Square F Ratio Prob $>$ F

$\begin{array}{llllll}\text { Series/Blk/Sub-Blk } & 3 & 0.00024171 & 0.000081 & 3.1480 & 0.0865\end{array}$

Error

0.00020476

0.000026

Means for Oneway Anova

Level Number Mean Std Error Lower 95\% Upper 95\%

$\begin{array}{rrrrrr}\text { X-1-1 } & 3 & 0.378044 & 0.00292 & 0.37131 & 0.38478\end{array}$

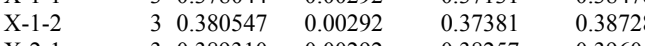

$\begin{array}{llllll}\mathrm{X}-2-1 & 3 & 0.389310 & 0.00292 & 0.38257 & 0.39605\end{array}$

$\begin{array}{llllll}\mathrm{X}-2-2 & 3 & 0.386389 & 0.00292 & 0.37965 & 0.39312\end{array}$

Std Error uses a pooled estimate of error variance

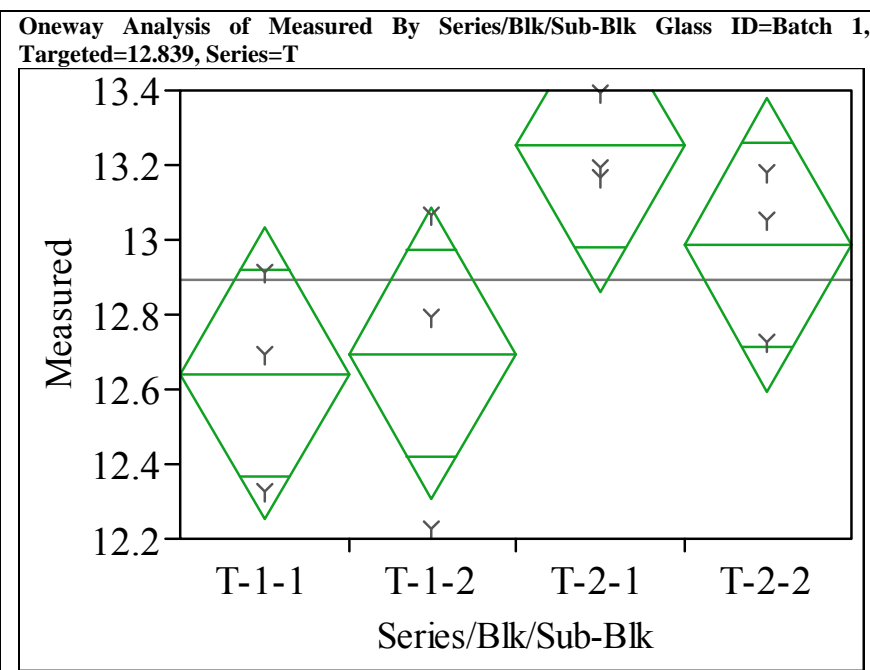

Oneway Anova

Summary of Fit

$\begin{array}{lr}\text { Rsquare } & 0.510757 \\ \text { Adj Rsquare } & 0.327291 \\ \text { Root Mean Square Error } & 0.293495 \\ \text { Mean of Response } & 12.8947 \\ \text { Observations (or Sum Wgts) } & 12\end{array}$

Analysis of Variance

Source DF Sum of Squares Mean Square F Ratio Prob $>$ F

$\begin{array}{lrrrrr}\text { Series/Blk/Sub-Blk } & 3 & 0.7194176 & 0.239806 & 2.7839 & 0.1098 \\ \text { Error } & 8 & 0.6891147 & 0.086139 & & \end{array}$

Error

C. Total

0.086139

Means for Oneway Anova

Means for Oneway Anova
Level Number Mean Std Error Lower 95\% Upper 95\%

$\begin{array}{rrrrrr}\text { Level } & \text { Number } & \text { Mean } & \text { Std Error } & \text { Lower 95\% } & \text { Upper 95\% } \\ \text { T-1-1 } & 3 & 12.6433 & 0.16945 & 12.253 & 13.034\end{array}$

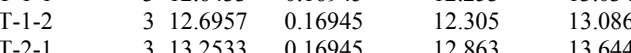

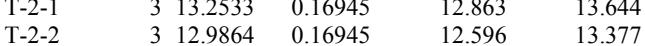

Std Error uses a pooled estimate of error variance 


\section{Exhibit A-3. Measurements by Analytical Series, Block and Sub-Block for the Batch 1 and LRM Standards by Oxide by Prep (continued)}

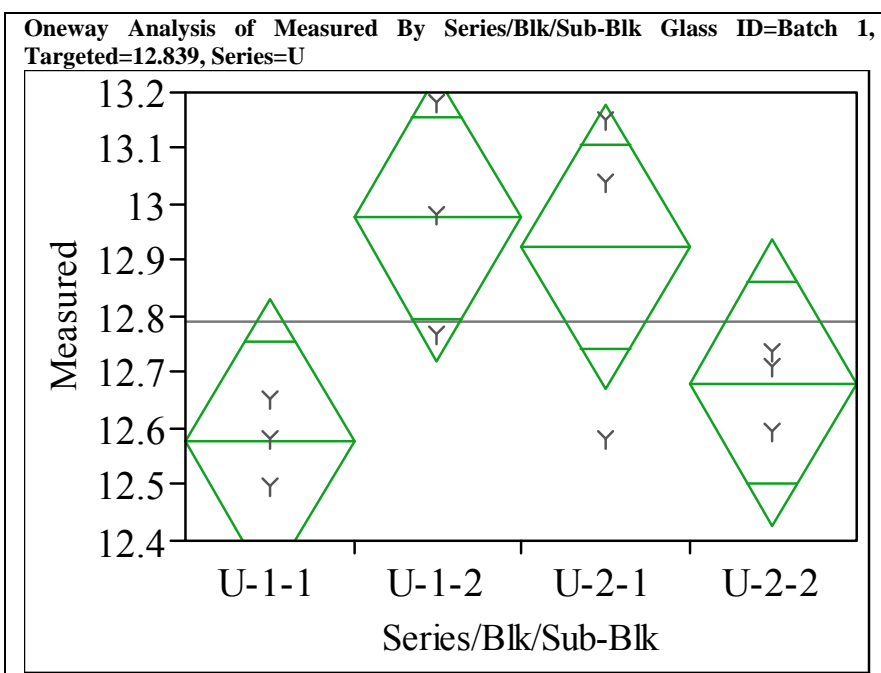

\section{Oneway Anova}

Summary of Fit

$\begin{array}{lr}\text { Rsquare } & 0.530504 \\ \text { Adj Rsquare } & 0.354442 \\ \text { Root Mean Square Error } & 0.19137 \\ \text { Mean of Response } & 12.78986 \\ \text { Observations (or Sum Wgts) } & 12\end{array}$

Analysis of Variance

Source DF Sum of Squares Mean Square F Ratio Prob > F

$\begin{array}{lrrrrr}\text { Series/Blk/Sub-Blk } & 3 & 0.33104965 & 0.110350 & 3.0132 & 0.0943\end{array}$

\begin{tabular}{lll} 
Error & 8 & 0.29297937 \\
\hline & 11 & 0.6202902
\end{tabular}

C. Total

0.62402902

Means for Oneway Anova

Level Number Mean Std Error Lower 95\% Upper 95\%

$\begin{array}{lllllr}\text { U-1-1 } & 3 & 12.5766 & 0.11049 & 12.322 & 12.831 \\ \text { U-1-2 } & 3 & 12.9769 & 0.11049 & 12.722 & 13.23\end{array}$

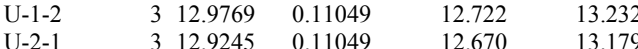

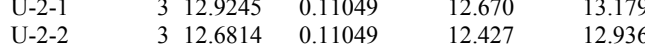

Std Error uses a pooled estimate of error variance

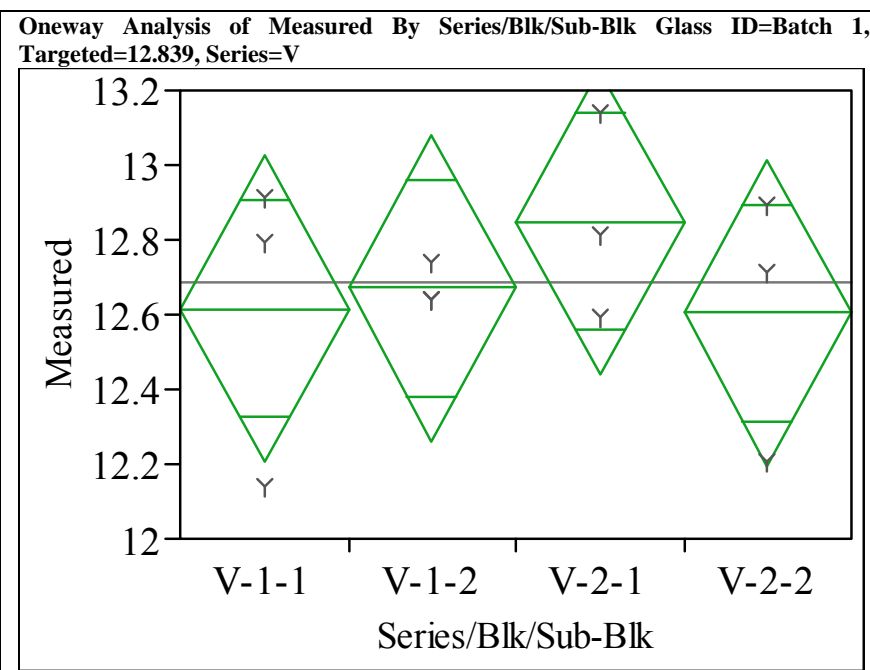

Oneway Anova

Summary of Fit

$\begin{array}{lr}\text { Rsquare } & 0.131473 \\ \text { Adj Rsquare } & -0.19422 \\ \text { Root Mean Square Error } & 0.30733 \\ \text { Mean of Response } & 12.68501 \\ \text { Observations (or Sum Wgts) } & 12\end{array}$

12.68501

Analysis of Variance

Source DF Sum of Squares Mean Square F Ratio Prob $>$ F

$\begin{array}{llrrrr}\text { Series/Blk/Sub-Blk } & 3 & 0.11438119 & 0.038127 & 0.4037 & 0.7545\end{array}$

$\begin{array}{lll}\text { Error } & 8 & 0.75561423\end{array}$

0.094452

Means for Oneway Anova

Level Number Mean Std Error Lower 95\% Upper 95\%

$\begin{array}{rrrrrr}\text { Level Number } & \text { Mean } & \text { Std Error } & \text { Lower 95\% } & \text { Upper 95\% } \\ \text { V-1 } 1 & 3 & 12.6147 & 0.17744 & 12.206 & 13.024\end{array}$

$\begin{array}{llllll}\mathrm{V}-1-1 & 3 & 12.6147 & 0.17744 & 12.206 & 13.024 \\ \mathrm{~V}-1-2 & 3 & 12.6719 & 0.17744 & 12.263 & 13.081\end{array}$

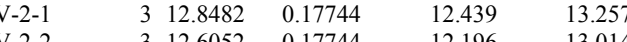

Std Error uses a pooled estimate of error variance 


\section{Exhibit A-3. Measurements by Analytical Series, Block and Sub-Block for the Batch 1 and LRM Standards by Oxide by Prep (continued)}

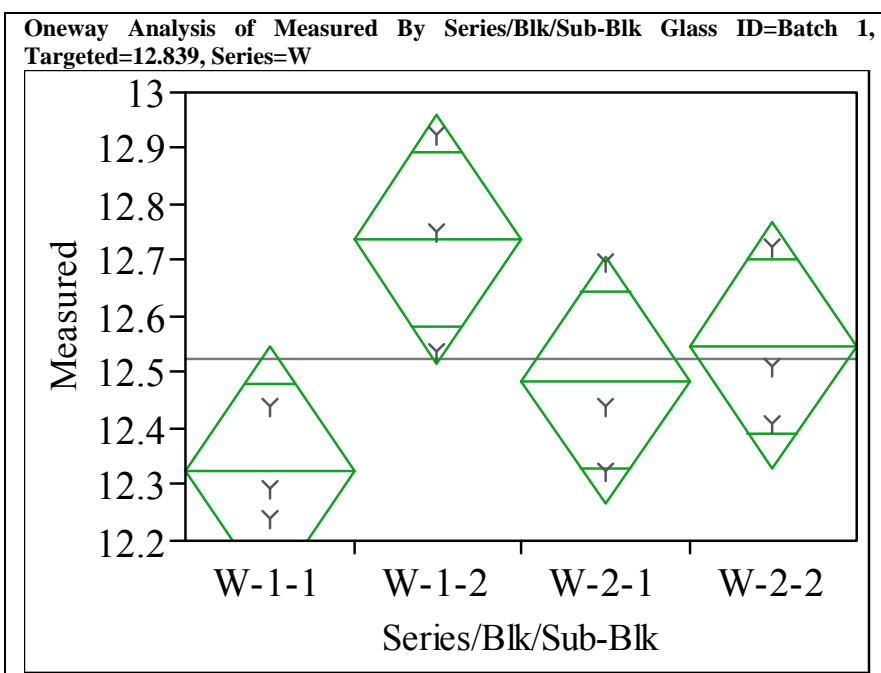

\section{Oneway Anova}

Summary of Fit

$$
\begin{array}{lr}
\text { Rsquare } & 0.545429 \\
\text { Adj Rsquare } & 0.374965 \\
\text { Root Mean Square Error } & 0.165911 \\
\text { Mean of Response } & 12.52417 \\
\text { Observations (or Sum Wgts) } & 12
\end{array}
$$

Analysis of Variance

$\begin{array}{lrrrrr}\text { Source } & \text { DF } & \text { Sum of Squares } & \text { Mean Square } & \text { F Ratio } & \text { Prob }>\text { F } \\ \text { Series/Blk/Sub-Blk } & 3 & 0.26422651 & 0.088076 & 3.1997 & 0.0837\end{array}$

$\begin{array}{lrrr}\text { Series/Blk/Sub-Blk } & 3 & 0.26422651 & 0.088076 \\ \text { Error } & 8 & 0.22021147 & 0.027526\end{array}$

C. Total

0.48443798

Means for Oneway Anova

Level Number Mean Std Error Lower 95\% Upper 95\%

$\begin{array}{llllrr}\text { W-1-1 } & 3 & 12.3240 & 0.09579 & 12.103 & 12.545 \\ \text { W-1-2 } & 3 & 12.7386 & 0.09579 & 12.518 & 12.960\end{array}$

$\begin{array}{llllll}\text { W-1-2 } & 3 & 12.7386 & 0.09579 & 12.518 & 12.960 \\ \text { W-2-1 } & 3 & 12.4860 & 0.09579 & 12.265 & 12.707\end{array}$

$\begin{array}{llllll}\text { W-2-1 } & 3 & 12.4860 & 0.09579 & 12.265 & 12.707 \\ \text { W-2-2 } & 3 & 12.5480 & 0.09579 & 12.327 & 12.769\end{array}$

Std Error uses a pooled estimate of error variance

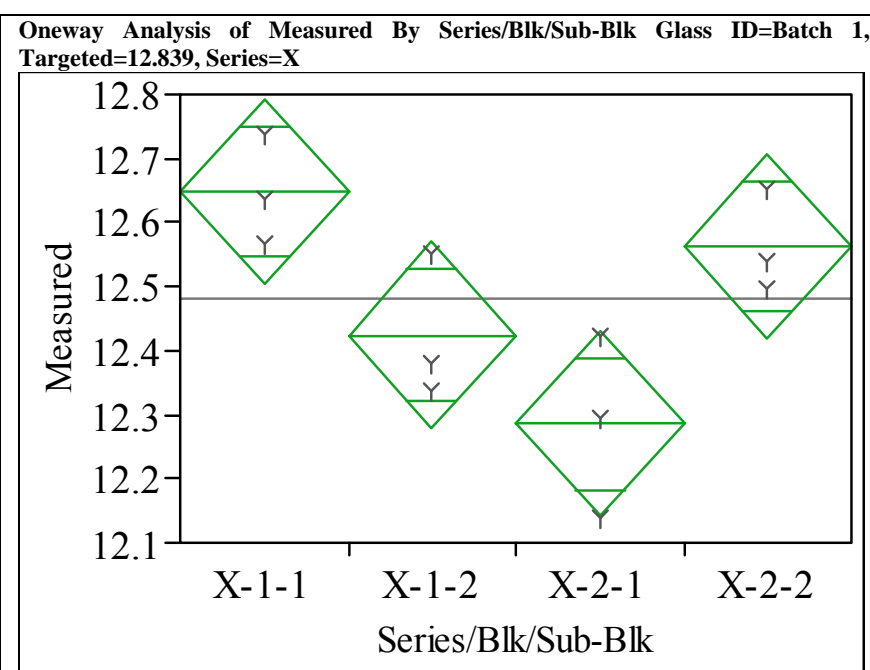

Oneway Anova

Summary of Fit

$\begin{array}{lr}\text { Rsquare } & 0.705755 \\ \text { Adj Rsquare } & 0.595413 \\ \text { Root Mean Square Error } & 0.108883 \\ \text { Mean of Response } & 12.48009 \\ \text { Observations (or Sum Wgts) } & 12\end{array}$

Analysis of Variance

Source DF Sum of Squares Mean Square F Ratio Prob $>$ F

$\begin{array}{llrrrr}\text { Series/Blk/Sub-Blk } & 3 & 0.22748485 & 0.075828 & 6.3961 & 0.0161\end{array}$

$\begin{array}{lcc}\text { Error } & 8 & 0.09484355 \\ \text { C } & 11 & 0.32232840\end{array}$

0.011855

$11 \quad 0.32232840$

Means for Oneway Anova

Level Number Mean Std Error Lower 95\% Upper 95\%

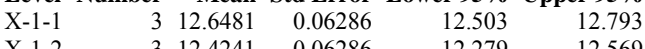

$\begin{array}{llllll}\mathrm{X}-1-1 & 3 & 12.6481 & 0.06286 & 12.503 & 12.793 \\ \mathrm{X}-1-2 & 3 & 12.42859 & 0.06286 & 12.279 & 12.569 \\ \mathrm{X}-2-2 & 3 & 12.2562 & 0.06286 & 12.141 & 12.431\end{array}$

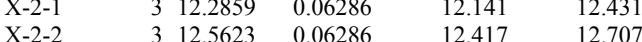

Std Error uses a pooled estimate of error variance 


\section{Exhibit A-3. Measurements by Analytical Series, Block and Sub-Block for the Batch 1 and LRM Standards by Oxide by Prep (continued)}

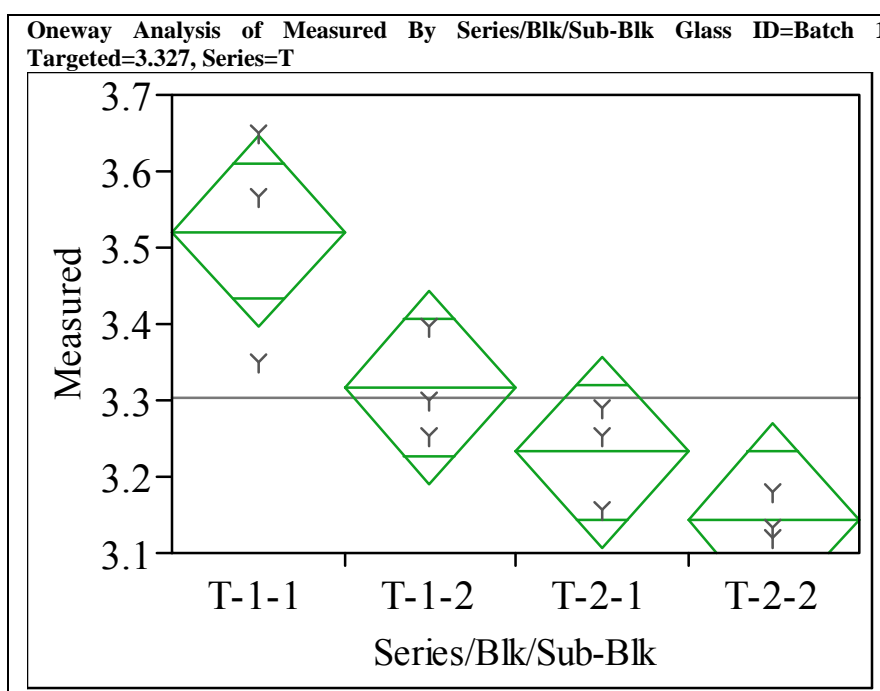

\section{Oneway Anova}

Summary of Fit

Adj Rsquare $\quad 0.682126$

Root Mean Square Error $\quad 0.093889$

$\begin{array}{lr}\text { Mean of Response } & 3.303616 \\ \text { Observations (or Sum Wgts) } & 12\end{array}$

Analysis of Variance

$\begin{array}{lrrrrr}\text { Source } & \text { DF } & \text { Sum of Squares } & \text { Mean Square } & \text { F Ratio } & \text { Prob }>\text { F } \\ \text { Series/Blk/Sub-Blk } & 3 & 0.23452776 & 0.078176 & 8.8683 & 0.0063 \\ \text { Error } & 8 & 0.07052157 & 0.008815 & & \\ \text { C. Total } & 11 & 0.30504933 & & & \\ & & & & & \end{array}$

Means for Oneway Anova

Level Number Mean Std Error Lower 95\% Upper 95\%

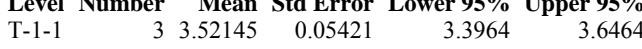

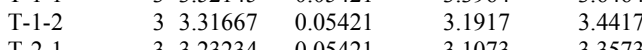

$\begin{array}{llllll}\mathrm{T}-2-1 & 3 & 3.23234 & 0.05421 & 3.1073 & 3.3573\end{array}$

$\begin{array}{llllll}\mathrm{T}-2-2 & 3 & 3.14401 & 0.05421 & 3.0190 & 3.2690\end{array}$

Std Error uses a pooled estimate of error variance

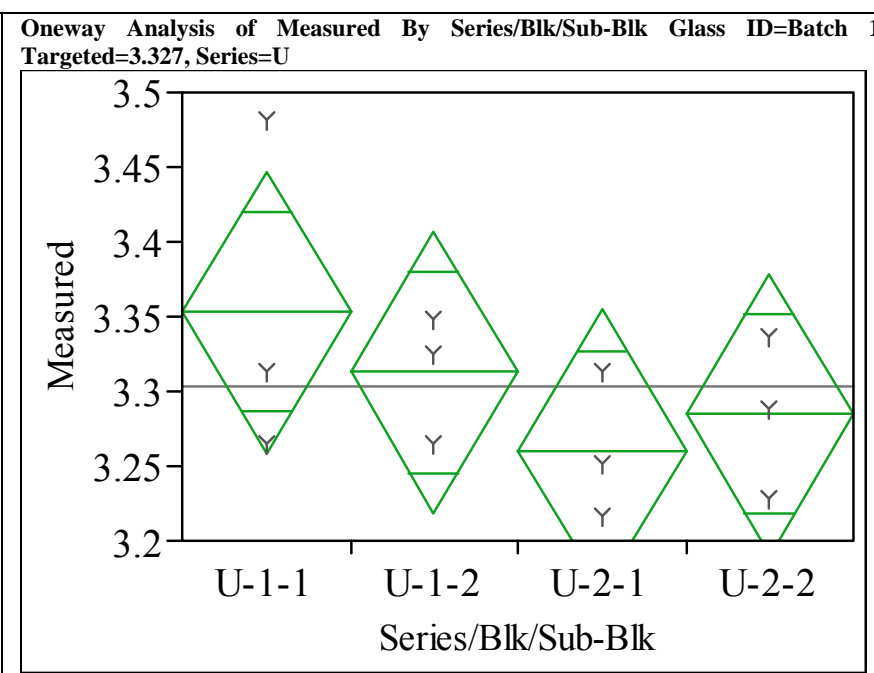

Oneway Anova

Summary of Fit

$\begin{array}{lr}\text { Rsquare } & 0.259982 \\ \text { Adj Rsquare } & -0.01752 \\ \text { Root Mean Square Error } & 0.07101 \\ \text { Mean of Response } & 3.302612 \\ \text { Observations (or Sum Wgts) } & 12\end{array}$

Observations (or Sum Wgts)

Analysis of Variance

Source DF Sum of Squares Mean Square F Ratio Prob > F $\begin{array}{lrrrrr}\text { Series/Blk/Sub-Blk } & 3 & 0.01417203 & 0.004724 & 0.9369 & 0.4667\end{array}$

$\begin{array}{lll}\text { Error } & 8 & 0.04033950\end{array}$

C. Total

$11 \quad 0.05451153$

Means for Oneway Anova

Level Number Mean Std Error Lower 95\% Upper 95\%

$\begin{array}{llllrr}\text { U-1-1 } & 3 & 3.35280 & 0.04100 & 3.2583 & 3.4473\end{array}$

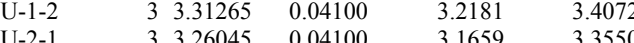

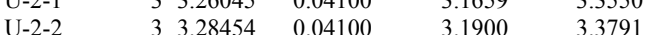

Std Error uses a pooled estimate of error variance 
Exhibit A-3. Measurements by Analytical Series, Block and Sub-Block for the Batch 1 and LRM Standards by Oxide by Prep (continued)

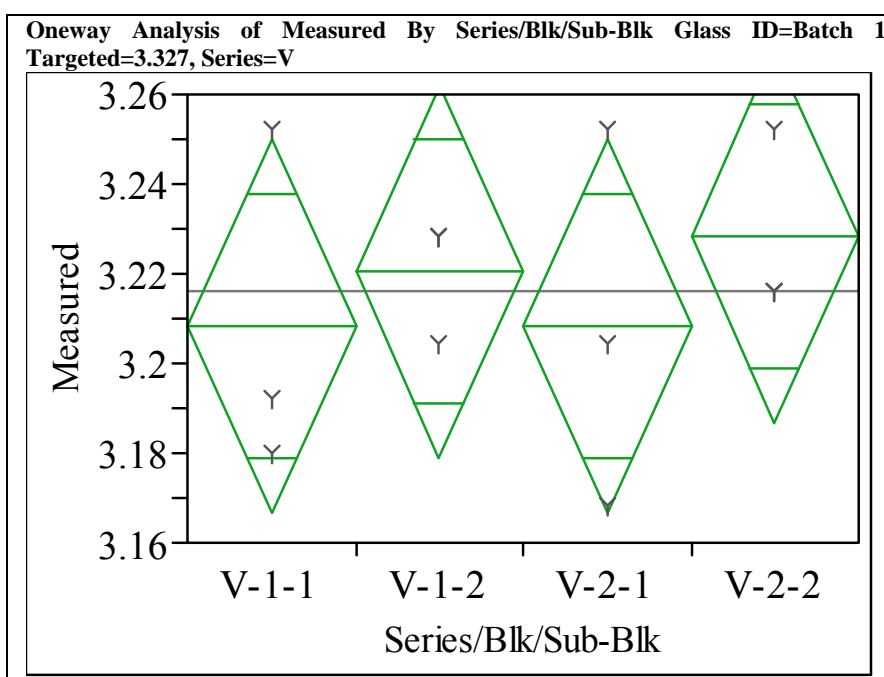

Oneway Anova

Summary of Fit

$\begin{array}{lr}\text { Rsquare } & 0.1 \\ \text { Adj Rsquare } & -0.2375 \\ \text { Root Mean Square Error } & 0.031296 \\ \text { Mean of Response } & 3.216282 \\ \text { Observations (or Sum Wgts) } & 12\end{array}$

Analysis of Variance

Source DF Sum of Squares Mean Square F Ratio Prob > F

Series/Blk/Sub-Blk $3 \begin{array}{lllll}3 & 0.00087064 & 0.000290 & 0.2963 & 0.8272\end{array}$

$\begin{array}{lrrr}\text { Error } & 8 & 0.00783573 & 0.000979\end{array}$

0.00870637

Means for Oneway Anova

Level Number Mean Std Error Lower 95\% Upper 95\%

$\begin{array}{llllll}\text { V-1-1 } & 3 & 3.20825 & 0.01807 & 3.1666 & 3.2499 \\ \text { V-1-2 } & 3 & 3.22030 & 0.01807 & 3.1786 & 3.2620 \\ \text { V-2-1 } & 3 & 3.20825 & 0.01807 & 3.1666 & 3.2499\end{array}$

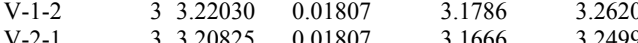

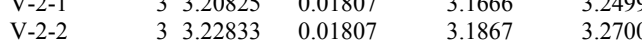

Std Error uses a pooled estimate of error variance

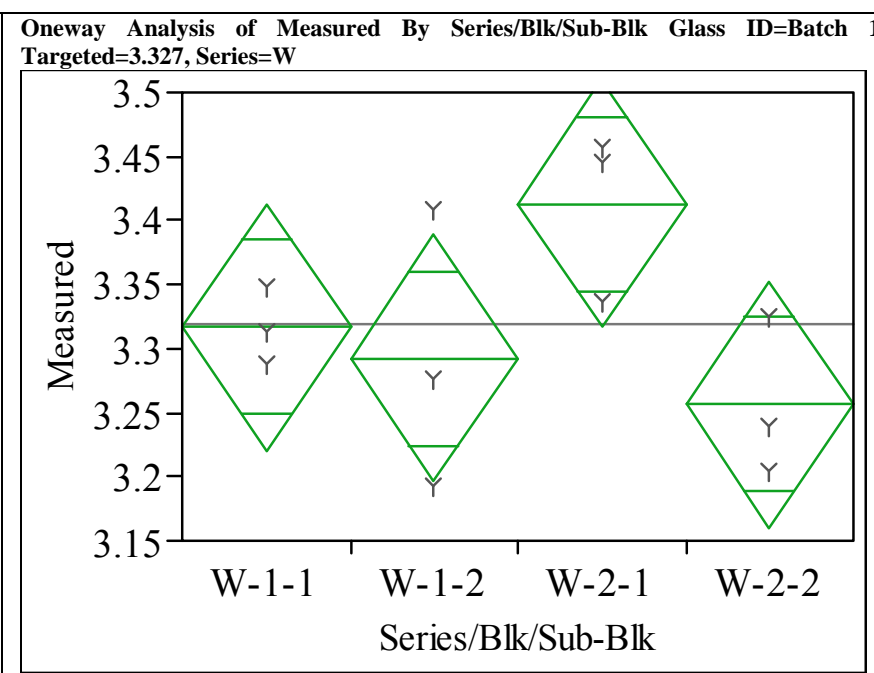

Oneway Anova

Summary of Fit

$\begin{array}{lr}\text { Rsquare } & 0.489087 \\ \text { Adj Rsquare } & 0.297495 \\ \text { Root Mean Square Error } & 0.07261 \\ \text { Mean of Response } & 3.319677 \\ \text { Observations (or Sum Wgts) } & 12\end{array}$

Analysis of Variance

Source DF Sum of Squares Mean Square F Ratio Prob > F

$\begin{array}{lrrrrr}\text { Series/Blk/Sub-Blk } & 3 & 0.04037578 & 0.013459 & 2.5528 & 0.1286\end{array}$

$\begin{array}{llll}\text { Error } & 8 & 0.04217751 & 0.005272\end{array}$

0.08255329

Means for Oneway Anova

Level Number Mean Std Error Lower 95\% Upper 95\%

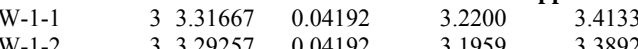

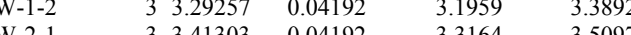

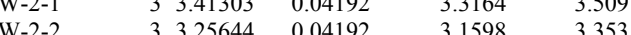

Std Error uses a pooled estimate of error variance 


\section{Exhibit A-3. Measurements by Analytical Series, Block and Sub-Block for the Batch 1 and LRM Standards by Oxide by Prep (continued)}

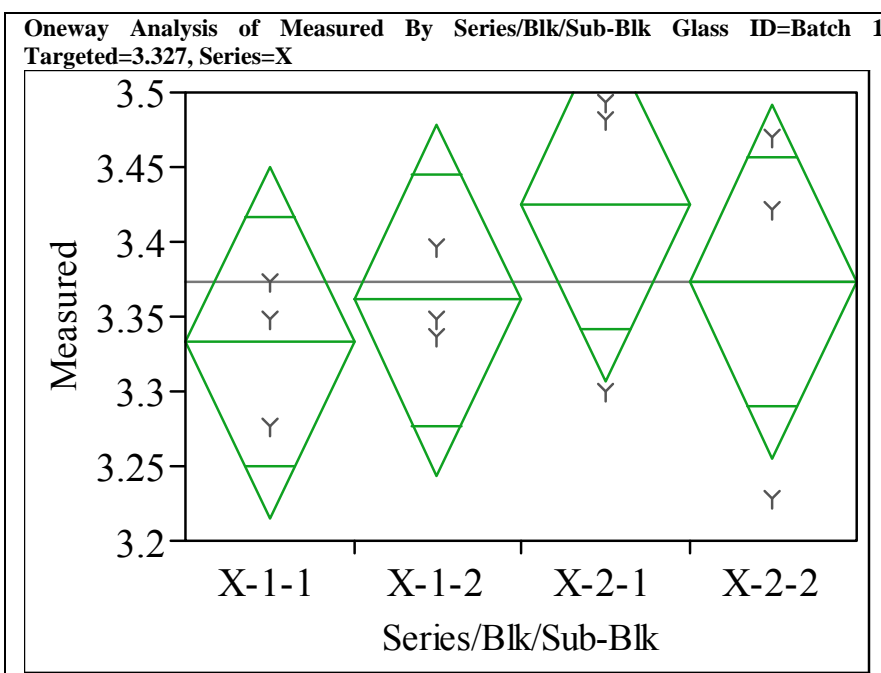

\section{Oneway Anova}

Summary of Fit

$$
\begin{array}{lr}
\text { Rsquare } & 0.176172 \\
\text { Adj Rsquare } & -0.13276 \\
\text { Root Mean Square Error } & 0.088656 \\
\text { Mean of Response } & 3.37288
\end{array}
$$

$\begin{array}{lr}\text { Mean of Response } & 3.37288 \\ \text { Observations (or Sum Wgts) } & 12\end{array}$

Analysis of Variance

Source DF Sum of Squares Mean Square F Ratio Prob $>$ F

$\begin{array}{lrrrrr}\text { Series/Blk/Sub-Blk } & 3 & 0.01344650 & 0.004482 & 0.5703 & 0.6501\end{array}$

Error

$\begin{array}{lll}0.004882 & 0.5703 & 0.650 \\ 0.007860 & & \end{array}$

C. Total

0.07632582

Means for Oneway Anova

Level Number Mean Std Error Lower 95\% Upper 95\%

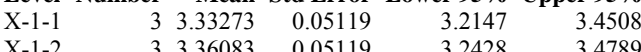

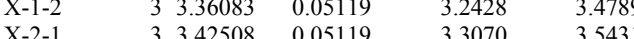

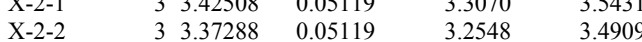

Std Error uses a pooled estimate of error variance

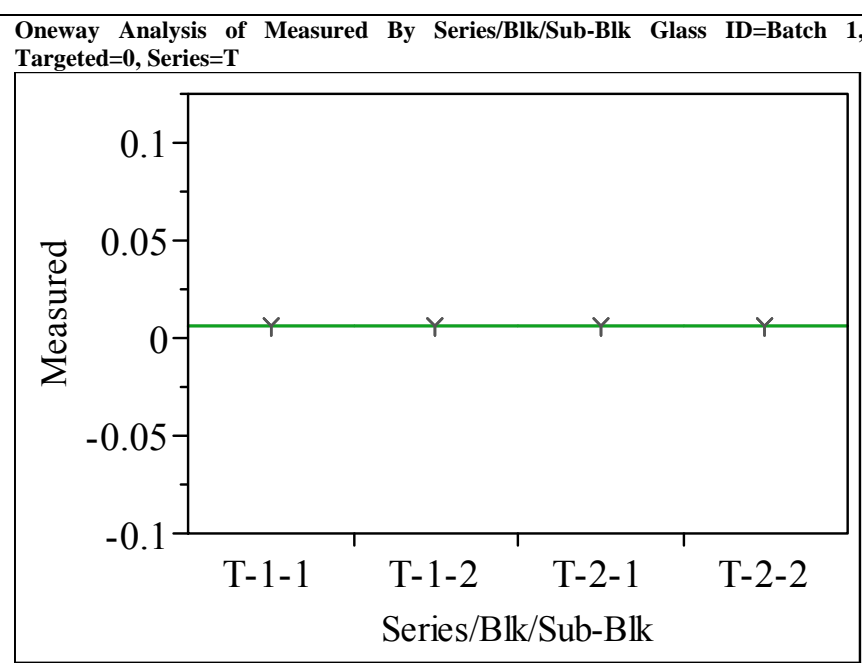

\section{Oneway Anova}

Rsquare

Rsquare
Adj Rsquare

$\begin{array}{lr}\text { Root Mean Square Error } & 0 \\ \text { Mean of Response } & 0.005864\end{array}$

Observations (or Sum Wgts) 12

Analysis of Variance

Sum of Squares Mean Square F Ratio Prob > F

$\begin{array}{llll}\text { Series/Blk/Sub-Blk } & 3 & 0 & 0 \\ \text { Error } & 8 & 0 & 0\end{array}$

$\begin{array}{lrr}\text { Error } & 8 & 0 \\ \text { C. Total } & 11 & 0\end{array}$

Means for Oneway Anova

Level Number Mean Std Error Lower 95\% Upper 95\%

$\begin{array}{llllll}\text { T-1-1 } & 3 & 0.005864 & 0 & 0.00586 & 0.00586\end{array}$

$\begin{array}{llllll}\mathrm{T}-1-2 & 3 & 0.005864 & 0 & 0.00586 & 0.00586\end{array}$

$\begin{array}{llllll}\mathrm{T}-2-1 & 3 & 0.005864 & 0 & 0.00586 & 0.00586\end{array}$

$\begin{array}{llllll}\text { T-2-2 } & 3 & 0.005864 & 0 & 0.00586 & 0.00586\end{array}$

Std Error uses a pooled estimate of error variance 
Exhibit A-3. Measurements by Analytical Series, Block and Sub-Block for the Batch 1 and LRM Standards by Oxide by Prep (continued)

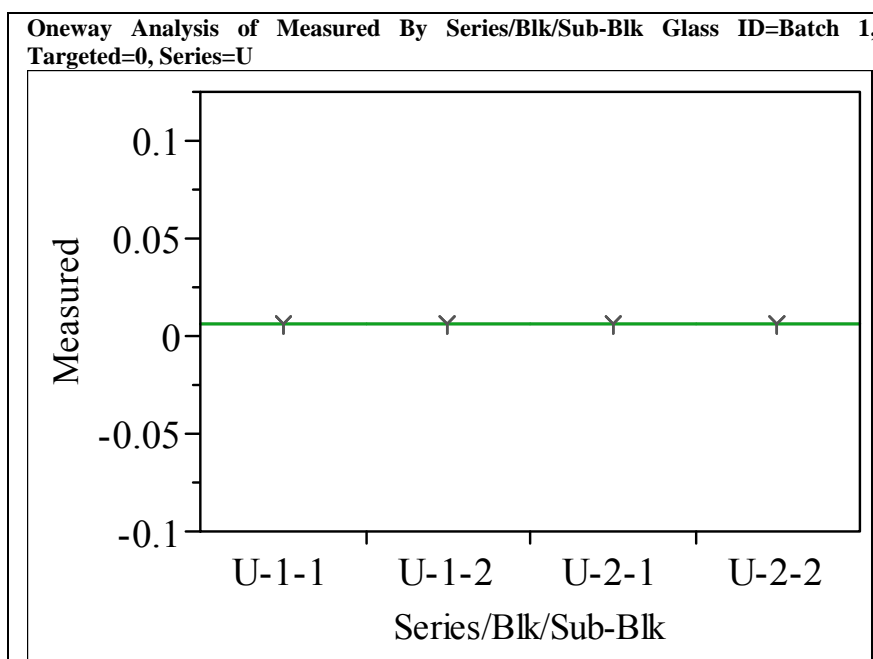

Oneway Anova

Summary of Fit

Rsquare

Adj Rsquare

$\begin{array}{lr}\text { Root Mean Square Error } & 0 \\ \text { Mean of Response } & 0.005864\end{array}$

Observations (or Sum Wgts)

Analysis of Variance

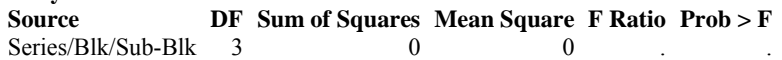

$\begin{array}{lr}\text { Error } & 8 \\ \text { C. Total } & 11\end{array}$

$\begin{array}{lll}0 & 0 \\ 0 & 0\end{array}$

0

Means for Oneway Anova

Level Number Mean Std Error Lower 95\% Upper 95\%

$\begin{array}{llllll}\text { U-1-1 } & 3 & 0.005864 & 0 & 0.00586 & 0.00586\end{array}$

$\begin{array}{llllll}\text { U-1-2 } & 3 & 0.005864 & 0 & 0.00586 & 0.00586\end{array}$

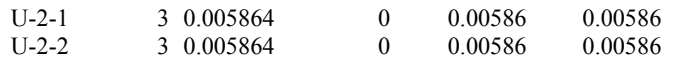

Std Error uses a pooled estimate of error variance

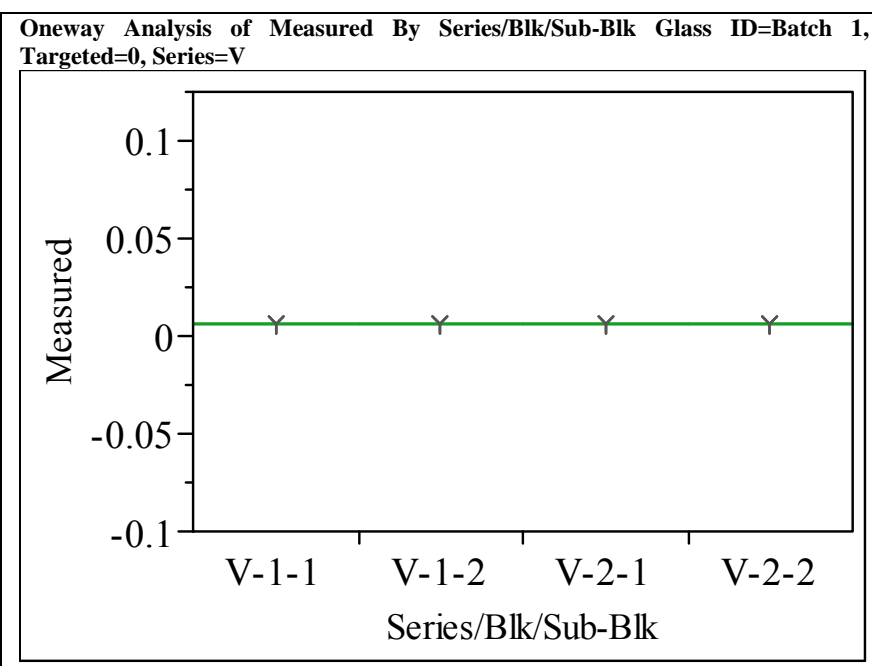

Oneway Anova

Summary of Fit

Rsquare

Adj Rsquare

$\begin{array}{lr}\text { Root Mean Square Error } & 0 \\ \text { Mean of Response } & 0.005864\end{array}$

Observations (or Sum Wgts) 12

Analysis of Variance

Sum of Squares Mean Square F Ratio Prob > F

Series/Blk/Sub-Blk

$\begin{array}{lr}\text { Error } & 8 \\ \text { C. Total } & 11\end{array}$

0
0

Means for Oneway Anova

Level Number Mean Std Error Lower 95\% Upper 95\%

$\begin{array}{llllll}\mathrm{V}-1-1 & 3 & 0.005864 & 0 & 0.00586 & 0.00586\end{array}$

$\begin{array}{llllll}V-1-2 & 3 & 0.005864 & 0 & 0.00586 & 0.00586\end{array}$

$\begin{array}{llllll}V-2-1 & 3 & 0.005864 & 0 & 0.00586 & 0.00586\end{array}$

$\begin{array}{llllll}\mathrm{V}-2-2 & 3 & 0.005864 & 0 & 0.00586 & 0.00586\end{array}$

Std Error uses a pooled estimate of error variance 
Exhibit A-3. Measurements by Analytical Series, Block and Sub-Block for the Batch 1 and LRM Standards by Oxide by Prep (continued)

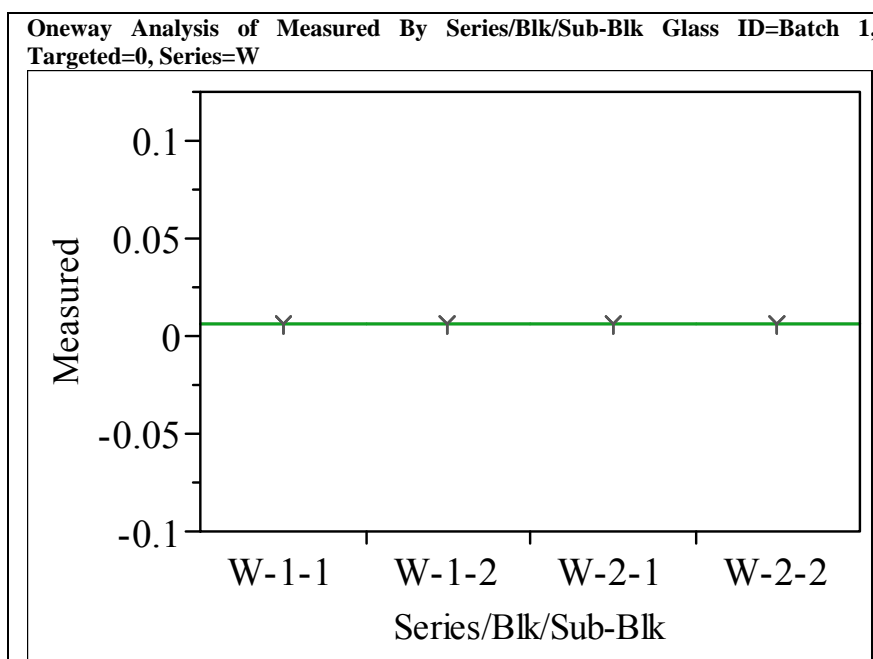

Oneway Anova

Summary of Fit

Rsquare

Adj Rsquare

$\begin{array}{lr}\text { Root Mean Square Error } & 0 \\ \text { Mean of Response } & 0.005864\end{array}$

Observations (or Sum Wgts)

Analysis of Variance

Source DF Sum of Squares Mean Square F Ratio Prob $>$ F

Series/Blk/Sub-Blk

3 Sum of Squares

Mean Square

C. Total

11

Means for Oneway Anova

Level Number Mean Std Error Lower 95\% Upper 95\%

$\begin{array}{lllllr}\text { W-1-1 } & 3 & 0.005864 & 0 & 0.00586 & 0.00586\end{array}$

$\begin{array}{llllll}\text { W-1-2 } & 3 & 0.005864 & 0 & 0.00586 & 0.00586\end{array}$

$\begin{array}{llllll}\text { W-2-1 } & 3 & 0.005864 & 0 & 0.00586 & 0.00586 \\ \text { W-2-2 } & 3 & 0.005864 & 0 & 0.00586 & 0.00586\end{array}$

Std Error uses a pooled estimate of error variance

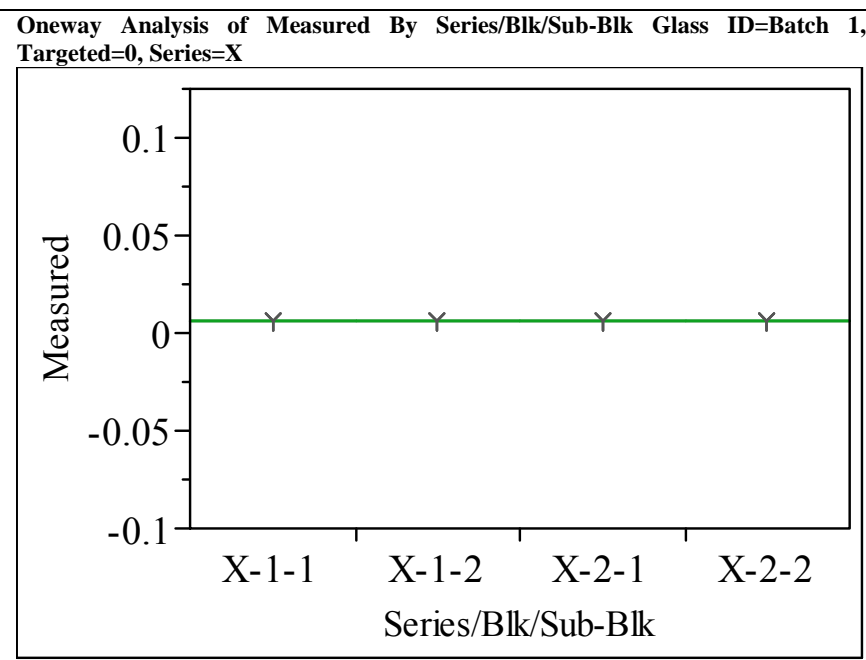

Oneway Anova

Summary of Fit

Rsquare

Adj Rsquare

$\begin{array}{lr}\text { Root Mean Square Error } & 0 \\ \text { Mean of Response } & 0.005864\end{array}$

Observations (or Sum Wgts) 12

Analysis of Variance

Sum of Squares Mean Square F Ratio Prob > F

Series/Blk/Sub-Blk

$\begin{array}{ll}\text { Error } & 8 \\ \text { C. Total } & 11\end{array}$

0

Means for Oneway Anova

Level Number Mean Std Error Lower 95\% Upper 95\%

$\begin{array}{llllll}\mathrm{X}-1-1 & 3 & 0.005864 & 0 & 0.00586 & 0.00586\end{array}$

$\begin{array}{llllll}\mathrm{X}-1-1 & 3 & 0.005864 & 0 & 0.00586 & 0.00586\end{array}$

$\begin{array}{llllll}\mathrm{X}-2-1 & 3 & 0.005864 & 0 & 0.00586 & 0.00586\end{array}$

$\begin{array}{llllll}\mathrm{X}-2-2 & 3 & 0.005864 & 0 & 0.00586 & 0.00586\end{array}$

Std Error uses a pooled estimate of error variance 
Exhibit A-3. Measurements by Analytical Series, Block and Sub-Block for the Batch 1 and LRM Standards by Oxide by Prep (continued)

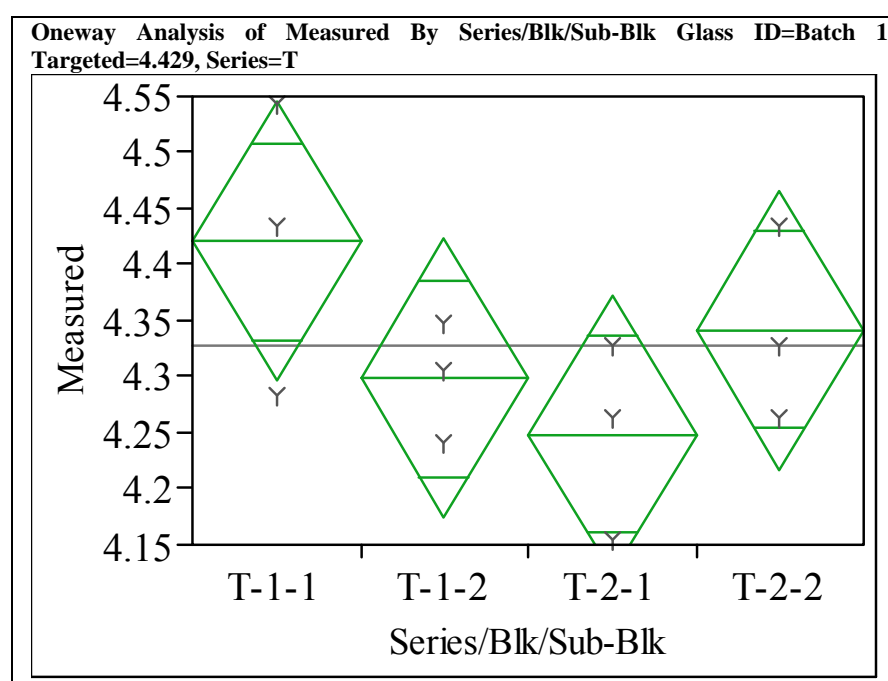

Oneway Anova

Summary of Fit

$\begin{array}{lr}\text { Rsquare } & 0.406824 \\ \text { Adj Rsquare } & 0.184383 \\ \text { Root Mean Square Error } & 0.09343 \\ \text { Mean of Response } & 4.327329 \\ \text { Observations (or Sum Wgts) } & 12\end{array}$

Analysis of Variance

Source DF Sum of Squares Mean Square F Ratio Prob $>$ F

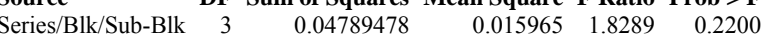

$\begin{array}{lll}\text { Error } & 8 & 0.06983367\end{array}$

$\begin{array}{lll}0.015965 & 1.8289 & 0.2200 \\ 0.008729 & & \end{array}$

C. Total

0.11772845

Means for Oneway Anova

Level Number Mean Std Error Lower 95\% Upper 95\%

$\begin{array}{rrrrrr}\text { Level Number } & \text { Mean } & \text { Std Error } & \text { Lower 95\% } & \text { Upper 95\% } \\ \mathrm{T}-1-1 & 3 & 4.42062 & 0.05394 & 4.2962 & 4.5450\end{array}$

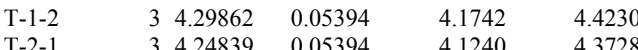

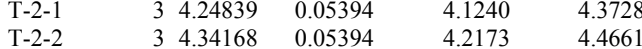

Std Error uses a pooled estimate of error variance

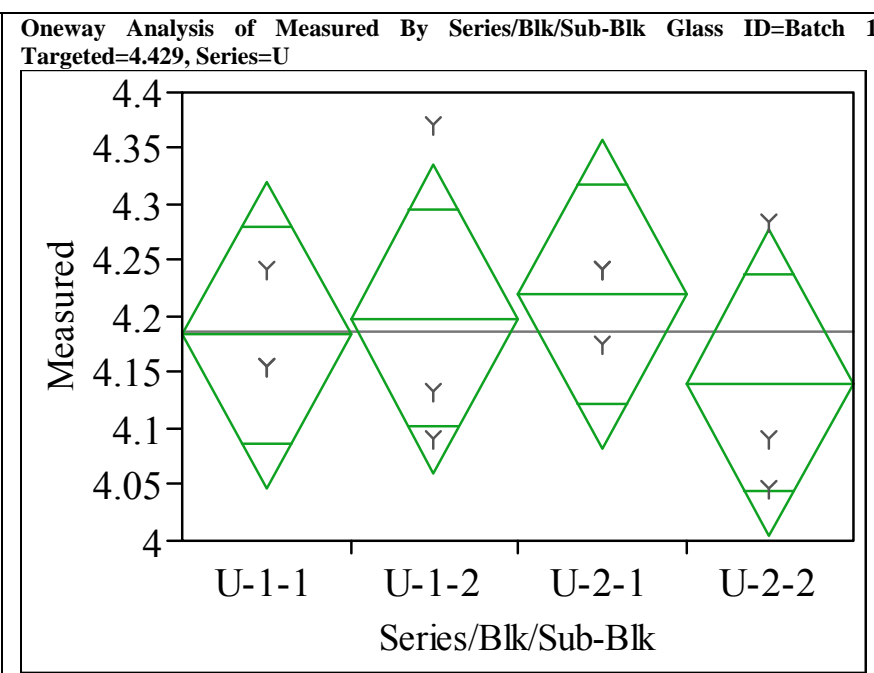

Oneway Anova

Summary of Fit

$\begin{array}{lr}\text { Rsquare } & 0.105327 \\ \text { Adj Rsquare } & -0.23017 \\ \text { Root Mean Square Error } & 0.103062 \\ \text { Mean of Response } & 4.185596 \\ \text { Observations (or Sum Wgts) } & 12\end{array}$

Analysis of Variance

Source DF Sum of Squares Mean Square F Ratio Prob $>$ F

$\begin{array}{llrrrr}\text { Series/Blk/Sub-Blk } & 3 & 0.01000383 & 0.003335 & 0.3139 & 0.8151\end{array}$

$\begin{array}{lll}\text { Error } & 8 & 0.08497460 \\ \text { C. } & 11 & 0.094984\end{array}$ 0.010622

0.09497843

Means for Oneway Anova

Level Number Mean Std Error Lower 95\% Upper 95\%

$\begin{array}{llllrr}\text { U-1-1 } & 3 & 4.18380 & 0.05950 & 4.0466 & 4.3210 \\ \text { U-1-2 } & & 3 & 4.19815 & 0.05950 & 4.0609\end{array}$

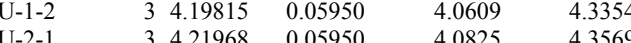

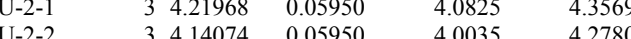

Std Error uses a pooled estimate of error variance 
Exhibit A-3. Measurements by Analytical Series, Block and Sub-Block for the Batch 1 and LRM Standards by Oxide by Prep (continued)

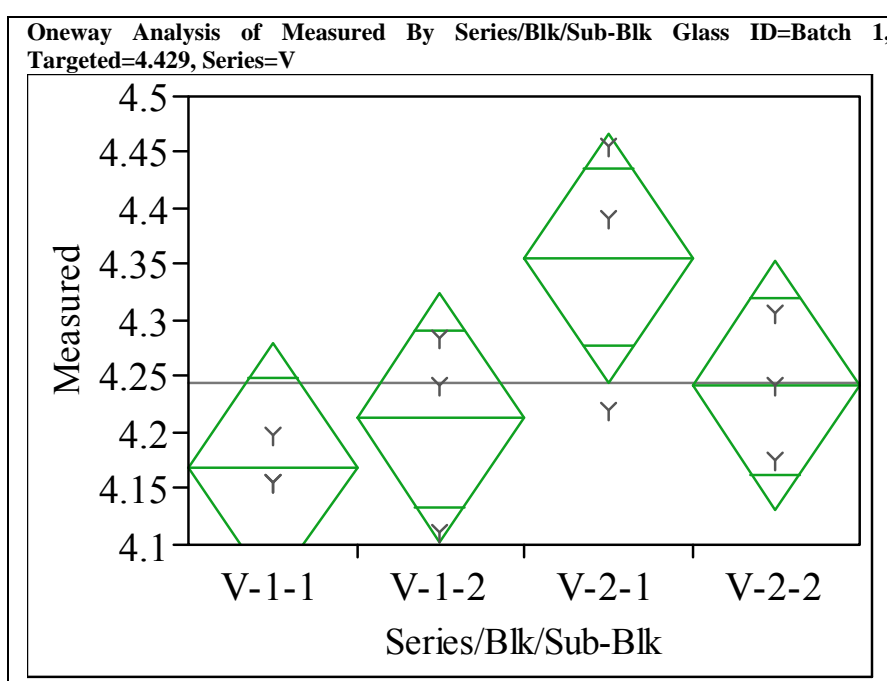

Oneway Anova

Summary of Fit

$\begin{array}{lr}\text { Rsquare } & 0.507524 \\ \text { Adj Rsquare } & 0.322845 \\ \text { Root Mean Square Error } & 0.083381 \\ \text { Mean of Response } & 4.244801 \\ \text { Observations (or Sum Wgts) } & 12\end{array}$

Analysis of Variance

Source DF Sum of Squares Mean Square F Ratio Prob > F

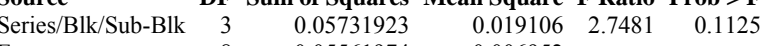

$\begin{array}{lll}\text { Error } & 8 & 0.05561974\end{array}$

C. Total

0.11293897

Means for Oneway Anova

Level Number Mean Std Error Lower 95\% Upper 95\%

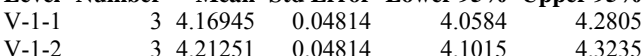

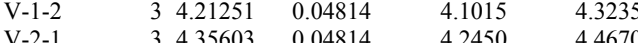

$\begin{array}{llllll}\text { V-2-1 } & 3 & 4.35603 & 0.04814 & 4.2450 & 4.4670 \\ \text { V-2-2 } & 3 & 4.24121 & 0.04814 & 4.1302 & 4.3522\end{array}$

Std Error uses a pooled estimate of error variance

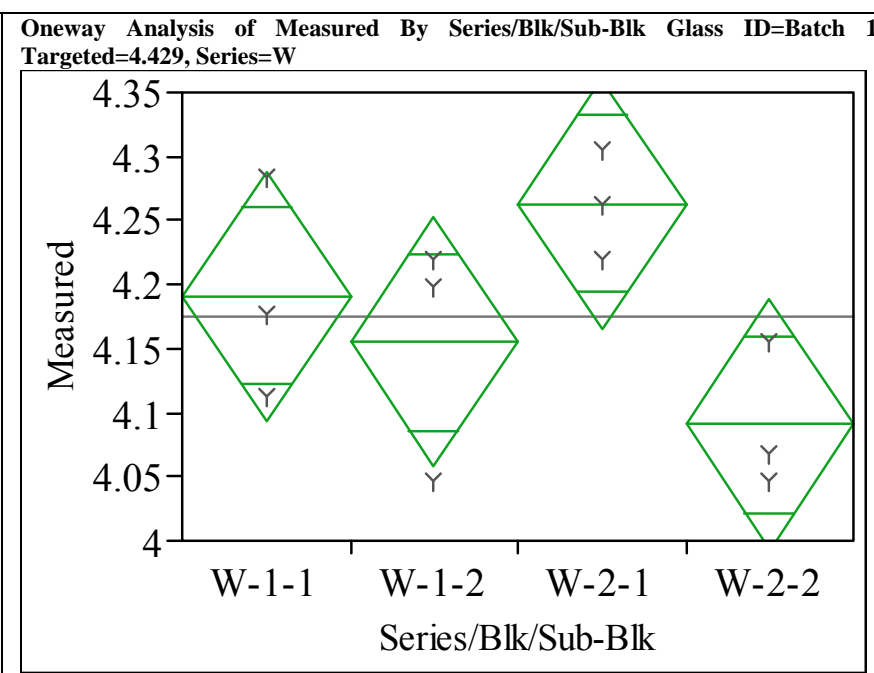

Oneway Anova

Summary of Fit

$\begin{array}{lr}\text { Rsquare } & 0.519654 \\ \text { Adj Rsquare } & 0.339525 \\ \text { Root Mean Square Error } & 0.073272 \\ \text { Mean of Response } & 4.174832 \\ \text { Observations (or Sum Wgts) } & 12\end{array}$

Analysis of Variance

Source DF Sum of Squares Mean Square F Ratio Prob $>$ F

$\begin{array}{lrrrrr}\text { Series/Blk/Sub-Blk } & 3 & 0.04646566 & 0.015489 & 2.8849 & 0.1026\end{array}$

0.005369

C. Total

$11 \quad 0.08941646$

Means for Oneway Anova

Level Number Mean Std Error Lower 95\% Upper 95\%

$\begin{array}{llllrr}\text { W-1-1 } & 3 & 4.19098 & 0.04230 & 4.0934 & 4.2885\end{array}$

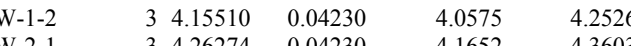

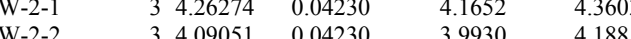

Std Error uses a pooled estimate of error variance 


\section{Exhibit A-3. Measurements by Analytical Series, Block and Sub-Block for the Batch 1 and LRM Standards by Oxide by Prep (continued)}

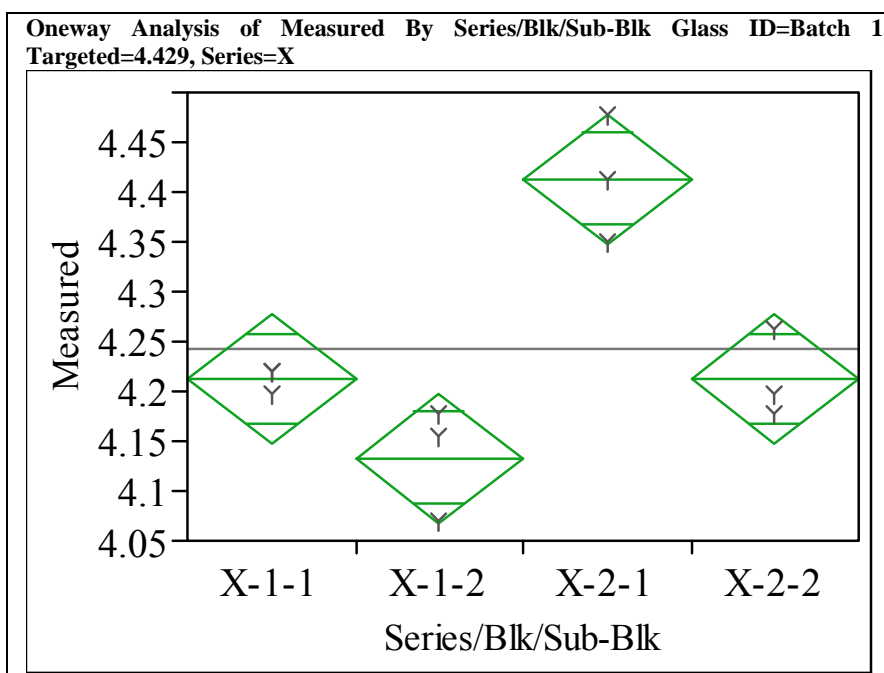

\section{Oneway Anova}

Summary of Fit

$\begin{array}{lr}\text { Rsquare } & 0.870395 \\ \text { Adj Rsquare } & 0.821793 \\ \text { Root Mean Square Error } & 0.048936 \\ \text { Mean of Response } & 4.243007 \\ \text { Observations (or Sum Wgts) } & 12\end{array}$

Analysis of Variance

Analysis of Variance
Source DF Sum of Squares Mean Square F Ratio Prob $>$ F

$\begin{array}{lrrrrr}\text { Source } & \text { DF } & \text { Sum of Squares } & \text { Mean Square } & \text { F Ratio } & \text { Prob }>\text { F } \\ \text { Series/Blk/Sub-Blk } & 3 & 0.12865928 & 0.042886 & 17.9086 & 0.0007\end{array}$

$\begin{array}{lrrr}\text { Series/Blk/Sub-Blk } & 3 & 0.12865928 & 0.042886 \\ \text { Error } & 8 & 0.01915791 & 0.002395\end{array}$

C. Total

0.14781719

Means for Oneway Anova

Level Number Mean Std Error Lower 95\% Upper 95\%

$\begin{array}{rrrrrr}\text { X-1-1 } & 3 & 4.21251 & 0.02825 & 4.1474 & 4.2777 \\ \mathrm{X}-1-2 & 3 & 4.13357 & 0.02825 & 4.0684 & 4.1987 \\ \mathrm{X}-2-1 & & 3 & 4.41345 & 0.02825 & 4.3483\end{array}$

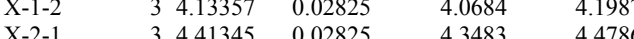

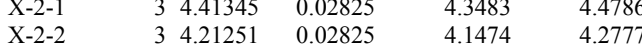

Std Error uses a pooled estimate of error variance

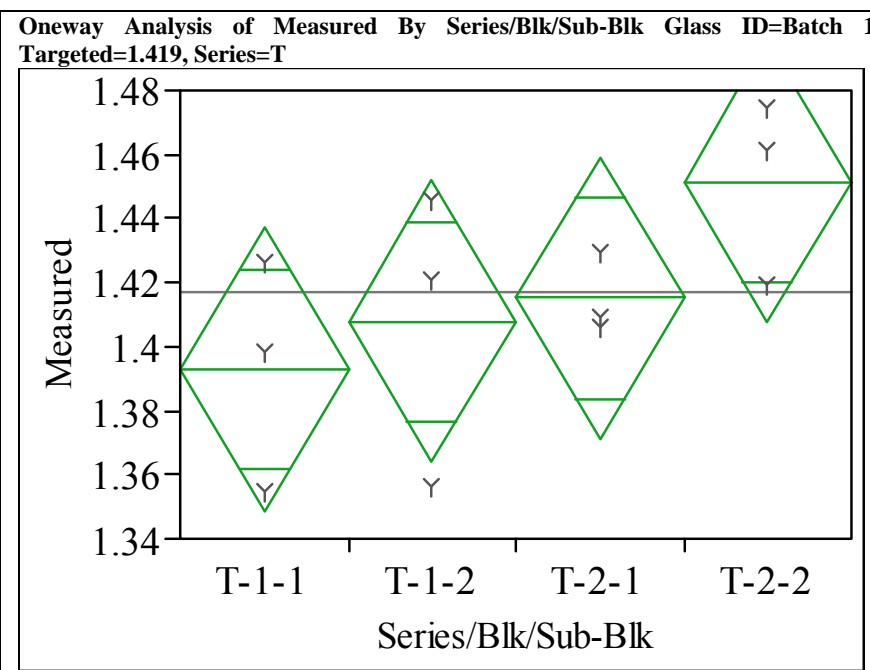

Oneway Anova

Summary of Fit

$\begin{array}{lr}\text { Rsquare } & 0.387912 \\ \text { Adj Rsquare } & 0.158379 \\ \text { Root Mean Square Error } & 0.033163 \\ \text { Mean of Response } & 1.416879 \\ \text { Observations (or Sum Wgts) } & 12\end{array}$

1.416879

Analysis of Variance

Source DF Sum of Squares Mean Square F Ratio Prob $>$ F

$\begin{array}{lrrrrr}\text { Series/Blk/Sub-Blk } & 3 & 0.00557577 & 0.001859 & 1.6900 & 0.2457 \\ \text { Error } & 8 & 0.00879804 & 0.001100 & & \end{array}$

0.001100

C. Total

$11-0.01437381$

Means for Oneway Anova

Level Number Mean Std Error Lower 95\% Upper 95\%

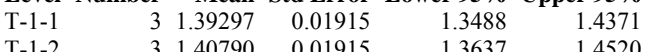

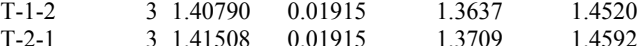

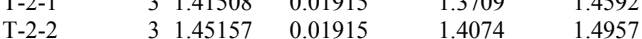

Std Error uses a pooled estimate of error variance 
Exhibit A-3. Measurements by Analytical Series, Block and Sub-Block for the Batch 1 and LRM Standards by Oxide by Prep (continued)

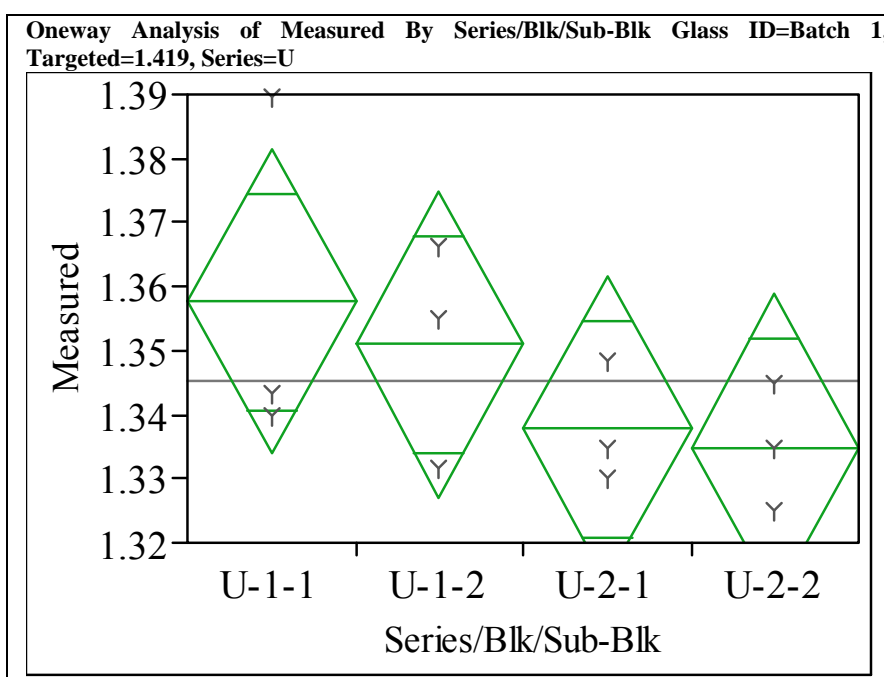

Oneway Anova

Summary of Fit

$\begin{array}{lr}\text { Rsquare } & 0.290655 \\ \text { Adj Rsquare } & 0.024651 \\ \text { Root Mean Square Error } & 0.01786 \\ \text { Mean of Response } & 1.345296 \\ \text { Observations (or Sum Wgts) } & 12\end{array}$

Analysis of Variance

Source DF Sum of Squares Mean Square F Ratio Prob > F

$\begin{array}{lrrrrr}\text { Series/Blk/Sub-Blk } & 3 & 0.00104567 & 0.000349 & 1.0927 & 0.4064 \\ \text { Error } & 8 & 0.00255196 & 0.000319 & & \end{array}$

Error

$\begin{array}{lll}0.000349 & 1.0927 & 0.4064\end{array}$

C. Total

0.00359763

Means for Oneway Anova

Level Number Mean Std Error Lower 95\% Upper 95\%

$\begin{array}{lrrrrr}\text { U-1-1 } & 3 & 1.35759 & 0.01031 & 1.3338 & 1.3814 \\ \text { U-1-2 } & 3 & 1.35096 & 0.01031 & 1.3272 & 1.3747\end{array}$

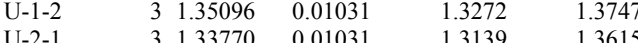

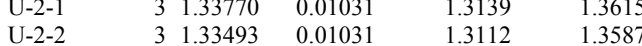

Std Error uses a pooled estimate of error variance

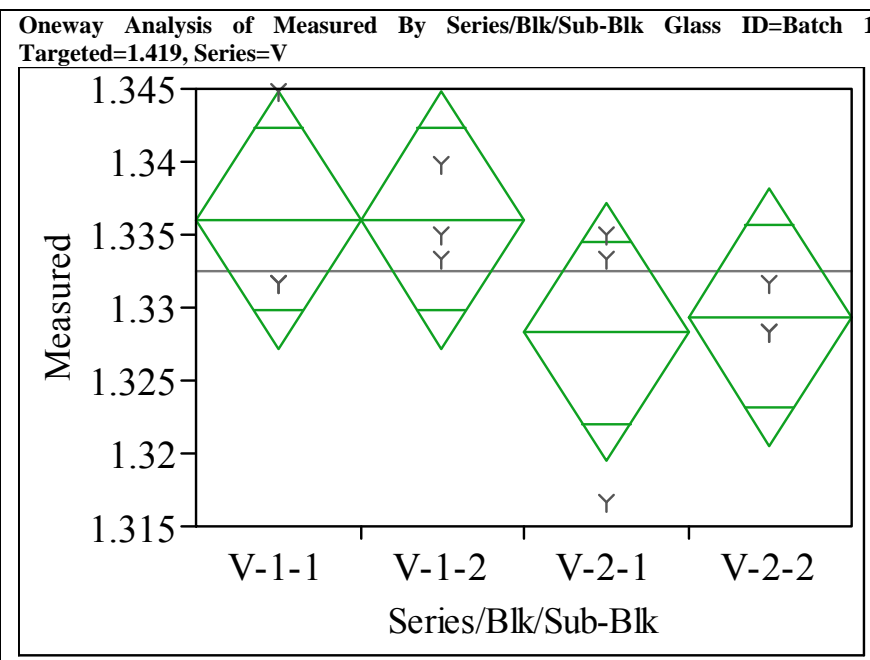

Oneway Anova

Summary of Fit

Rsquare

$\begin{array}{ll}0.308108 \\ \text { Adj Rsquare } & 0.048649\end{array}$

Root Mean Square Error $\quad 0.006633$

Mean of Response

Observations (or Sum Wgts)

12

Analysis of Variance

Source DF Sum of Squares Mean Square F Ratio Prob > F

$\begin{array}{llllll}\text { Series/Blk/Sub-Blk } & 3 & 0.00015675 & 0.000052 & 1.1875 & 0.374\end{array}$

$\begin{array}{lll}\text { Error } & 8 & 0.00035199 \\ \text { C. } & 11 & 0.0005087\end{array}$ 0.000044

Means for Oneway Anova

Level Number Mean Std

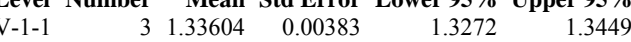

$\begin{array}{llllll}\mathrm{V}-1-2 & 3 & 1.33604 & 0.00383 & 1.3272 & 1.3449\end{array}$

$\begin{array}{llllll}\mathrm{V}-2-1 & 3 & 1.32830 & 0.00383 & 1.3195 & 1.3371\end{array}$

$\begin{array}{llllll}\mathrm{V}-2-2 & 3 & 1.32940 & 0.00383 & 1.3206 & 1.3382\end{array}$

Std Error uses a pooled estimate of error variance 
Exhibit A-3. Measurements by Analytical Series, Block and Sub-Block for the Batch 1 and LRM Standards by Oxide by Prep (continued)

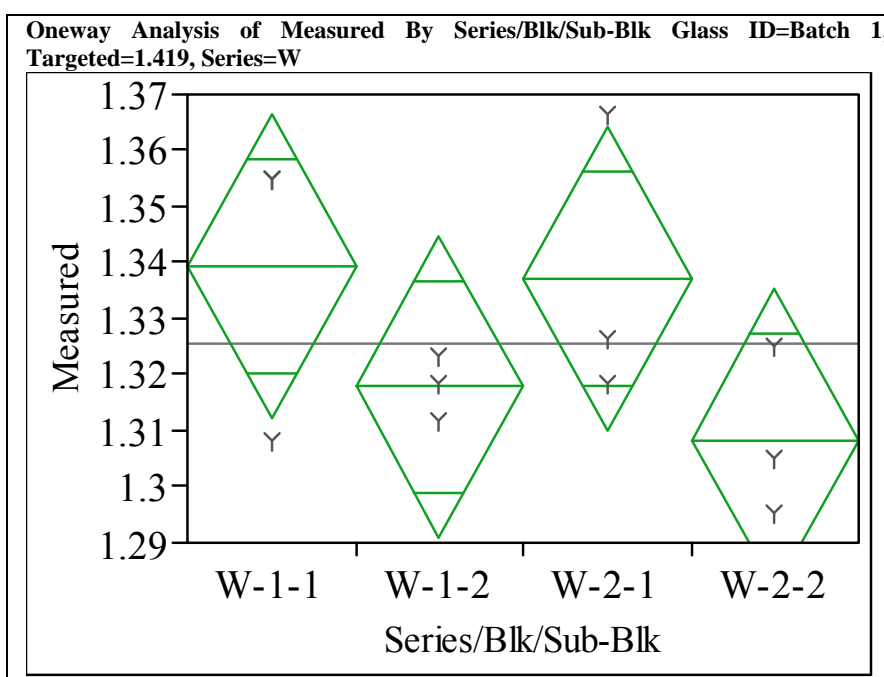

Oneway Anova

Summary of Fit

$\begin{array}{lr}\text { Rsquare } & 0.382524 \\ \text { Adj Rsquare } & 0.15097 \\ \text { Root Mean Square Error } & 0.020276 \\ \text { Mean of Response } & 1.325673 \\ \text { Observations (or Sum Wgts) } & 12\end{array}$

Analysis of Variance

Source DF Sum of Squares Mean Square F Ratio Prob > F

$\begin{array}{llllll}\text { Series/Blk/Sub-Blk } & 3 & 0.00203749 & 0.000679 & 1.6520 & 0.2533\end{array}$

\begin{tabular}{lll} 
Error & 8 & 0.00328895 \\
\hline & 1 & 0.053264
\end{tabular}

$\begin{array}{lll}0.000679 & 1.6520 & 0.2533 \\ 0.000411 & \end{array}$

C. Total

0.00532644

Means for Oneway Anova

Level Number Mean Std Error Lower 95\% Upper 95\%

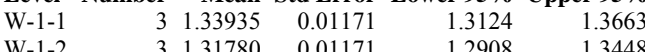

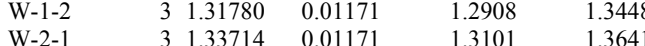

$\begin{array}{llllll}\text { W-2-1 } & 3 & 1.33714 & 0.01171 & 1.3101 & 1.3641 \\ \text { W-2-2 } & 3 & 1.30840 & 0.01171 & 1.2814 & 1.3354\end{array}$

Std Error uses a pooled estimate of error variance

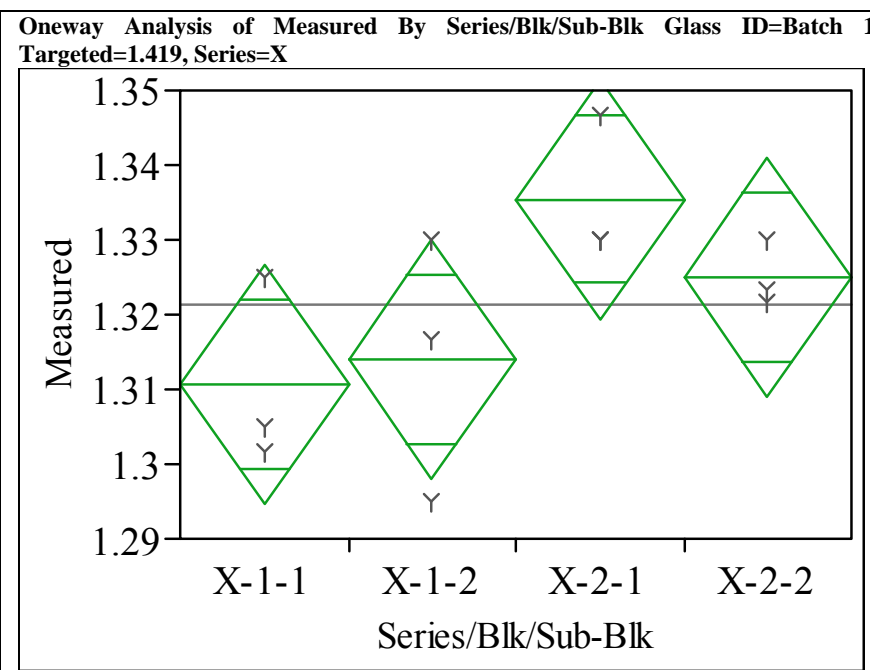

Oneway Anova

Summary of Fit

$\begin{array}{lr}\text { Rsquare } & 0.498956 \\ \text { Adj Rsquare } & 0.311065 \\ \text { Root Mean Square Error } & 0.012016 \\ \text { Mean of Response } & 1.321251 \\ \text { Observations (or Sum Wgts) } & 12\end{array}$

Observations (or Sum Wgts) 1.321251

Analysis of Variance

Source DF Sum of Squares Mean Square F Ratio Prob $>$ F

$\begin{array}{lrrrrr}\text { Series/Blk/Sub-Blk } & 3 & 0.00115017 & 0.000383 & 2.6556 & 0.1198\end{array}$

$\begin{array}{lcc}\text { Error } & 8 & 0.00115498 \\ \text { C. } & 11 & 0.00230515\end{array}$

0.000144

C. Total

$11 \quad 0.00230515$

Means for Oneway Anova

Level Number Mean Std Error Lower 95\% Upper 95\%

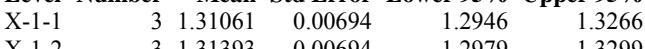

$\begin{array}{llllll}\mathrm{X}-1-1 & 3 & 1.31061 & 0.00694 & 1.2946 & 1.3266 \\ \mathrm{X}-1-2 & 3 & 1.31393 & 0.00694 & 1.2979 & 1.3299 \\ \mathrm{X}-2-1 & 3 & 1.33548 & 0.00694 & 1.3195 & 1.3515\end{array}$

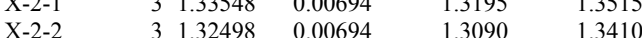

Std Error uses a pooled estimate of error variance 


\section{Exhibit A-3. Measurements by Analytical Series, Block and Sub-Block for the Batch 1 and LRM Standards by Oxide by Prep (continued)}

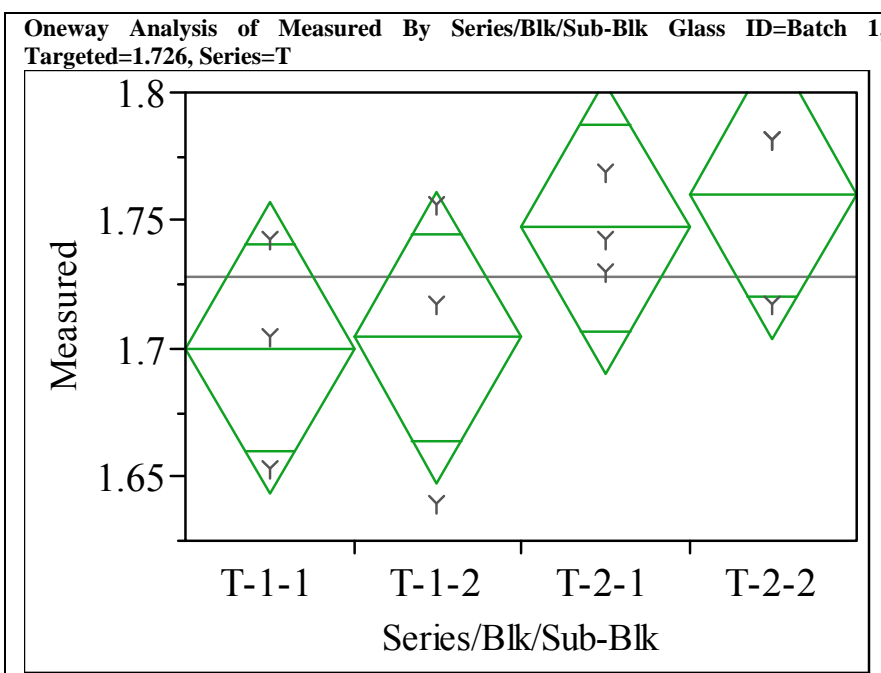

\section{Oneway Anova}

Summary of Fit

$\begin{array}{lr}\text { Rsquare } & 0.360775 \\ \text { Adj Rsquare } & 0.121065 \\ \text { Root Mean Square Error } & 0.042824 \\ \text { Mean of Response } & 1.728056 \\ \text { Observations (or Sum Wgts) } & 12\end{array}$

Analysis of Variance

Source DF Sum of Squares Mean Square F Ratio Prob > F

$\begin{array}{llllll}\text { Series/Blk/Sub-Blk } & 3 & 0.00828041 & 0.002760 & 1.5051 & 0.2857\end{array}$

Error $\quad 8 \quad 0.01467134$

$\begin{array}{lll}0.002760 & 1.5051 & 0.285 \\ 0.001834 & & \end{array}$

C. Total

0.02295175

Means for Oneway Anova

Level Number Mean Std Error Lower 95\% Upper 95\%

$\begin{array}{rrrrrr}\text { Level Number } & \text { Mean } & \text { Std Error } & \text { Lower 95\% } & \text { Upper 95\% } \\ \mathrm{T}-1-1 & 3 & 1.70008 & 0.02472 & 1.6431 & 1.7571 \\ \mathrm{~T}-1-2 & 3 & 1.70438 & 0.02472 & 1.6474 & 1.7614\end{array}$

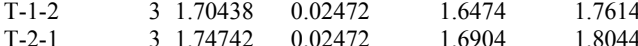

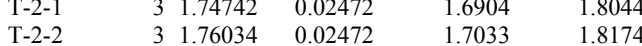

Std Error uses a pooled estimate of error variance

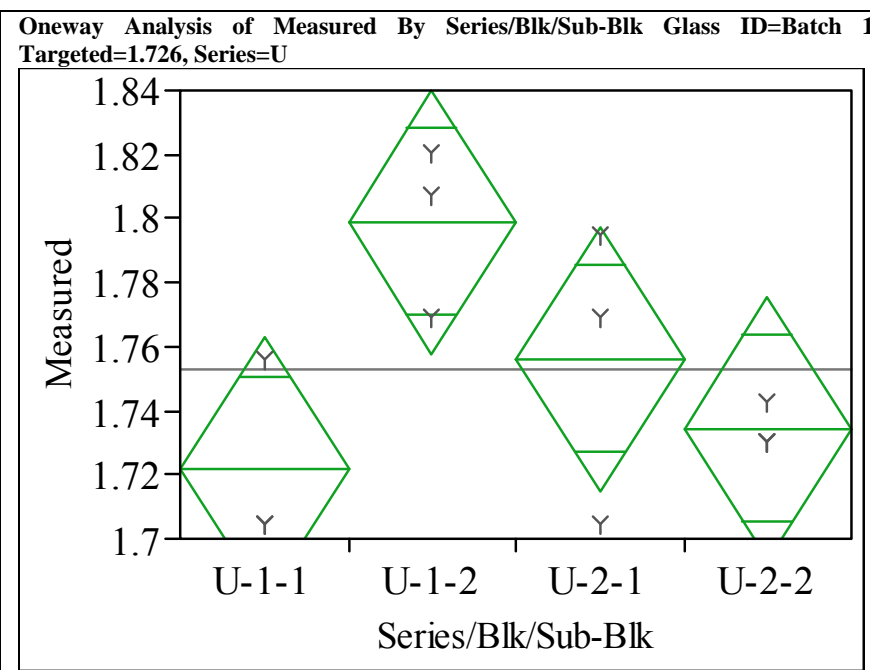

Oneway Anova

Summary of Fit

$\begin{array}{lr}\text { Rsquare } & 0.575058 \\ \text { Adj Rsquare } & 0.415704 \\ \text { Root Mean Square Error } & 0.030962 \\ \text { Mean of Response } & 1.752804 \\ \text { Observations (or Sum Wgts) } & 12\end{array}$

Observations (or Sum Wgts) 1.752804

Analysis of Variance

Source DF Sum of Squares Mean Square F Ratio Prob $>$ F

$\begin{array}{llrrrr}\text { Series/Blk/Sub-Blk } & 3 & 0.01037830 & 0.003459 & 3.6087 & 0.0651\end{array}$

$\begin{array}{lcc}\text { Error } & 8 & 0.00766911 \\ \text { C. } & 11 & 0.0180741\end{array}$

0.000959

0.01804741

Means for Oneway Anova

Level Number Mean Std Error Lower 95\% Upper 95\%

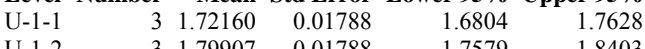

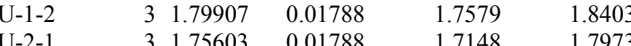

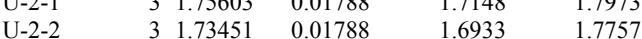

Std Error uses a pooled estimate of error variance 


\section{Exhibit A-3. Measurements by Analytical Series, Block and Sub-Block for the Batch 1 and LRM Standards by Oxide by Prep (continued)}

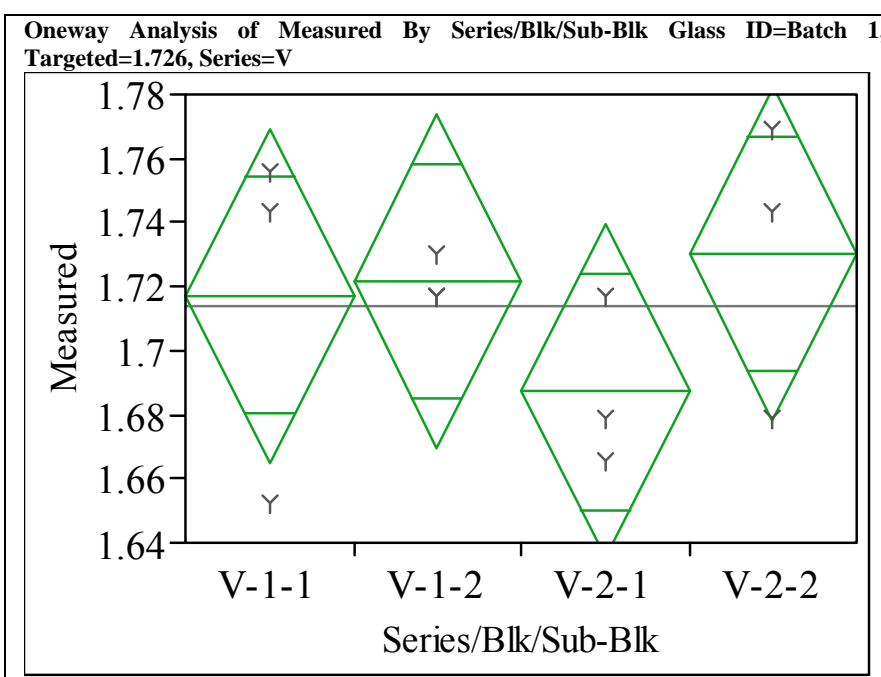

\section{Oneway Anova}

Summary of Fit

$\begin{array}{ll}\text { Rsquare } & 0.205059 \\ \text { Adj Rsquare } & -0.09304 \\ \text { Root Mean Square Error } & 0.039093 \\ \text { Mean of Response } & 1.714068\end{array}$

Mean of Response 1.714068

Analysis of Variance

Source DF Sum of Squares Mean Square F Ratio Prob > F

$\begin{array}{lrrrrr}\text { Series/Blk/Sub-Blk } & 3 & 0.00315378 & 0.001051 & 0.6879 & 0.5844 \\ \text { Error } & 8 & 0.01222611 & 0.001528 & & \\ \text { C. } & \text { Tota } & 0.01537990 & & & \end{array}$

Error

0.001528

C. Total

$\begin{array}{rr}11 & 0.01537990\end{array}$

Means for Oneway Anova

Level Number Mean Std Error Lower 95\% Upper 95\%

$\begin{array}{lrrrrr}\text { V-1-1 } & 3 & 1.71730 & 0.02257 & 1.6652 & 1.7693 \\ \text { V-1-2 } & 3 & 1.72160 & 0.02257 & 1.6696 & 1.7736\end{array}$

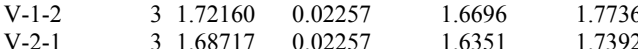

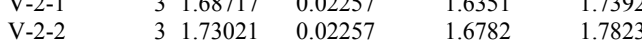

Std Error uses a pooled estimate of error variance

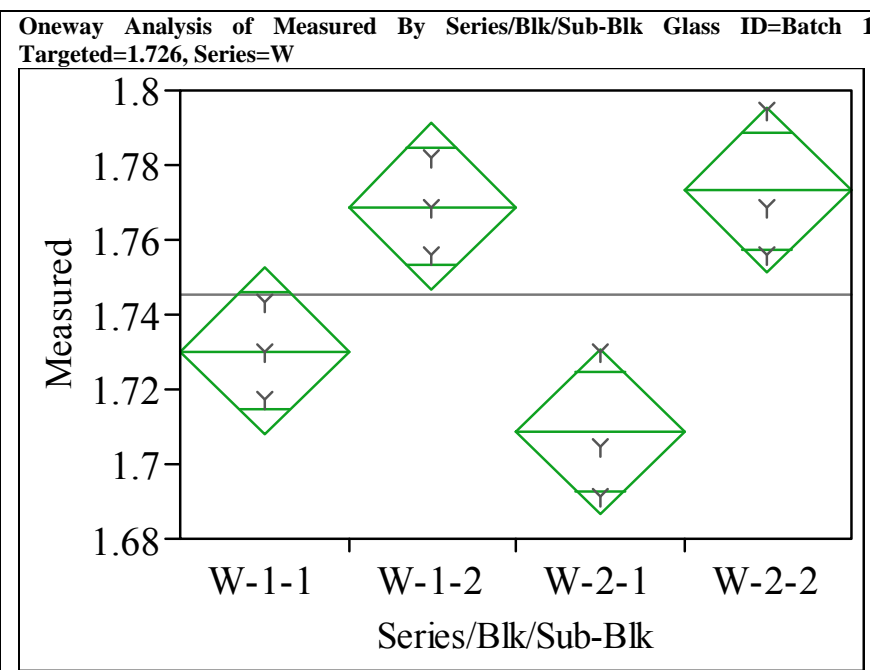

Oneway Anova

Summary of Fit

$\begin{array}{lr}\text { Rsquare } & 0.796954 \\ \text { Adj Rsquare } & 0.720812 \\ \text { Root Mean Square Error } & 0.016669 \\ \text { Mean of Response } & 1.745272 \\ \text { Observations (or Sum Wgts) } & 12\end{array}$

Observations (or Sum Wgts) 1.745272

Analysis of Variance

Source DF Sum of Squares Mean Square F Ratio Prob $>$ F

$\begin{array}{lrrrrr}\text { Series/Blk/Sub-Blk } & 3 & 0.00872500 & 0.002908 & 10.4667 & 0.0038\end{array}$

0.00872500

0.000278

C. Total

110.01094793

Means for Oneway Anova

Level Number Mean Std Error Lower 95\% Upper 95\%

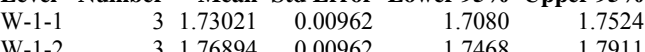

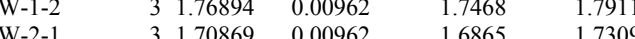

$\begin{array}{llllll}W-1-2 & 3 & 1.76894 & 0.00962 & 1.7468 & 1.7911 \\ W-2-1 & 3 & 1.70869 & 0.00962 & 1.6865 & 1.7309 \\ \text { W-2-2 } & 3 & 1.77325 & 0.00962 & 1.7511 & 1.795\end{array}$

Std Error uses a pooled estimate of error variance 


\section{Exhibit A-3. Measurements by Analytical Series, Block and Sub-Block for the Batch 1 and LRM Standards by Oxide by Prep (continued)}

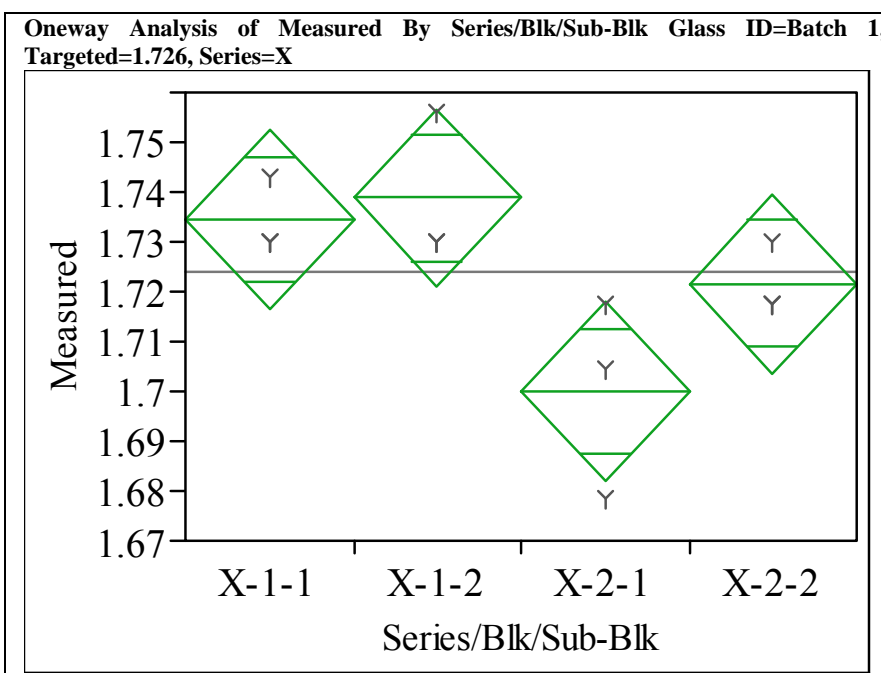

Oneway Anova

Summary of Fit

$\begin{array}{lr}\text { Rsquare } & 0.653333 \\ \text { Adj Rsquare } & 0.523333 \\ \text { Root Mean Square Error } & 0.013439 \\ \text { Mean of Response } & 1.723752 \\ \text { Observations (or Sum Wgts) } & 12\end{array}$

Analysis of Variance

Source DF Sum of Squares Mean Square F Ratio Prob > F

$\begin{array}{lrrrrr}\text { Series/Blk/Sub-Blk } & 3 & 0.00272309 & 0.000908 & 5.0256 & 0.0302 \\ \text { Error } & 8 & 0.00144490 & 0.000181 & & \\ \text { C. } & \text { Tota } & 0.0046799 & & & \end{array}$

$\begin{array}{lll}\text { Error } & 8 & 0.00144490\end{array}$

C. Total

0.00416799

Means for Oneway Anova

Level Number Mean Std Error Lower 95\% Upper 95\%

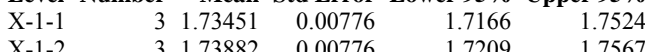

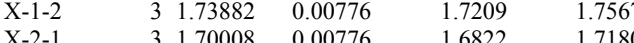

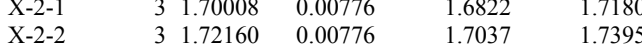

Std Error uses a pooled estimate of error variance

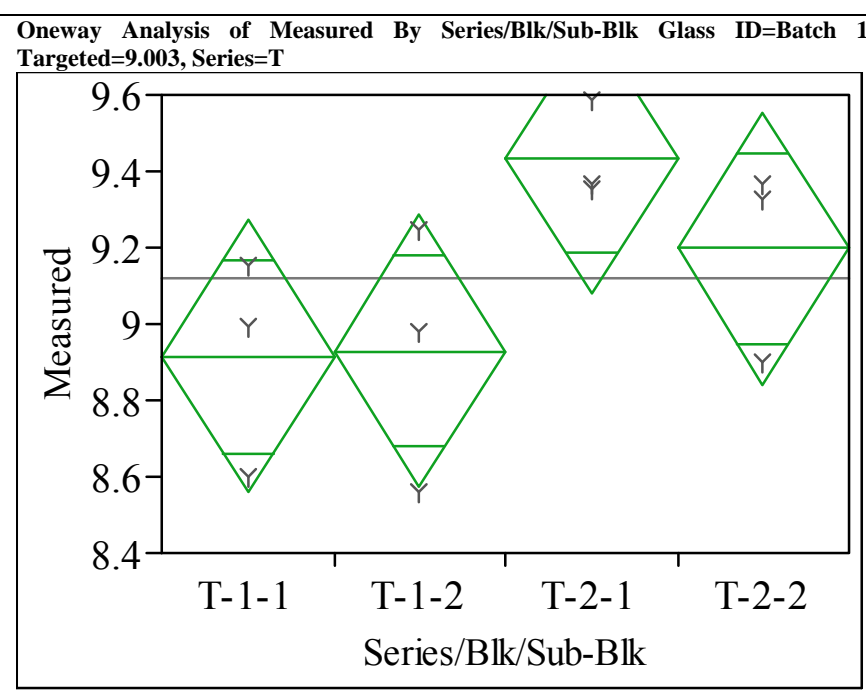

\section{Oneway Anova}

Summary of Fit

Rsquare

Adj Rsquare

Root Mean Square E 0.302179

Error $\quad 0.267231$

\begin{tabular}{lr} 
Observations (or Sum Wgts) & 9.11922 \\
\hline
\end{tabular}

Analysis of Variance

Source DF Sum of Squares Mean Square F Ratio Prob >

$\begin{array}{llllll}\text { Series/Blk/Sub-Blk } & 3 & 0.5543984 & 0.184799 & 2.5878 & 0.1255\end{array}$

$\begin{array}{lrr}\text { Error } & 8 & 0.5712975 \\ \text { C. } & 11 & 1.125659\end{array}$ 0.071412

Means for Oneway Anova

Level Number Mean Std Error Lower 95\% Upper 95\%

$\begin{array}{lrrrrr}\text { T-1-1 } & 3 & 8.91477 & 0.15429 & 8.5590 & 9.2706\end{array}$

$\begin{array}{llllll}\mathrm{T}-1-2 & 3 & 8.92825 & 0.15429 & 8.5725 & 9.2840\end{array}$

$\begin{array}{llllll}\mathrm{T}-2-1 & 3 & 9.43600 & 0.15429 & 9.0802 & 9.7918\end{array}$

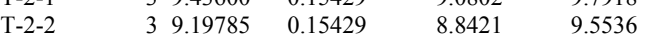

Std Error uses a pooled estimate of error variance 
Exhibit A-3. Measurements by Analytical Series, Block and Sub-Block for the Batch 1 and LRM Standards by Oxide by Prep (continued)

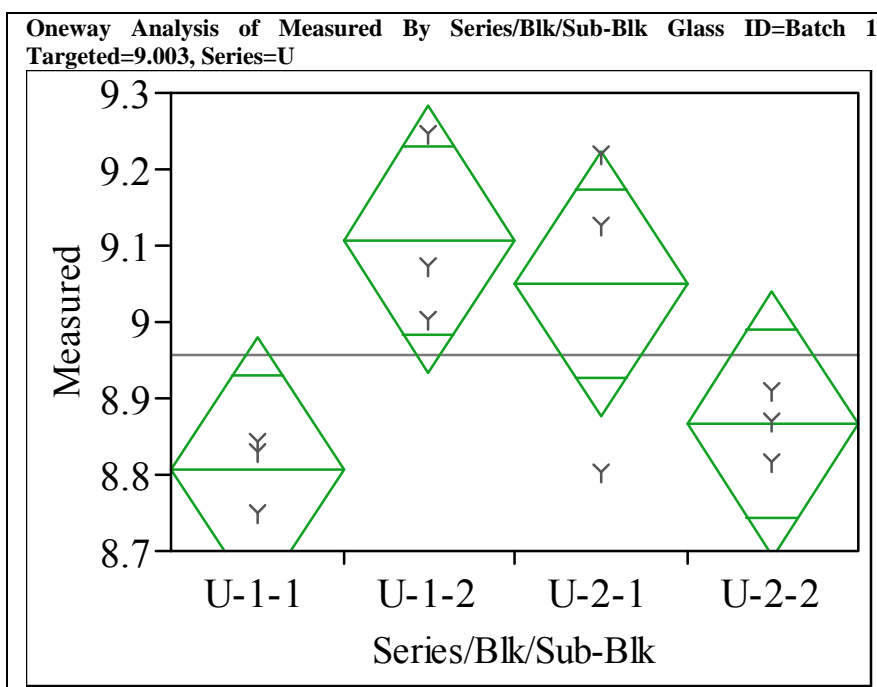

\section{Oneway Anova}

Summary of Fit

$\begin{array}{lr}\text { Rsquare } & 0.576743 \\ \text { Adj Rsquare } & 0.418022 \\ \text { Root Mean Square Error } & 0.130925 \\ \text { Mean of Response } & 8.95746 \\ \text { Observations (or Sum Wgts) } & 12\end{array}$

Analysis of Variance

$\begin{array}{lrrrrr}\text { Source } & \text { DF } & \text { Sum of Squares } & \text { Mean Square } & \text { F Ratio } & \text { Prob }>\text { F } \\ \text { Series/Blk/Sub-Blk } & 3 & 0.18685886 & 0.062286 & 3.6337 & 0.0641 \\ \text { Error } & 8 & 0.13713078 & 0.017141 & & \\ \text { C. Total } & 11 & 0.32398964 & & & \\ & & & & & \end{array}$

Means for Oneway Anova

Level Number Mean Std Error Lower 95\% Upper 95\%

$\begin{array}{llllrr}\text { U-1-1 } & 3 & 8.80693 & 0.07559 & 8.6326 & 8.9812\end{array}$

$\begin{array}{llllll}\text { U-1-2 } & 3 & 9.10799 & 0.07559 & 8.9337 & 9.2823\end{array}$

$\begin{array}{llllll}\text { U-2-1 } & 3 & 9.04957 & 0.07559 & 8.8753 & 9.2239\end{array}$

Std Error uses a pooled estimate of error variance

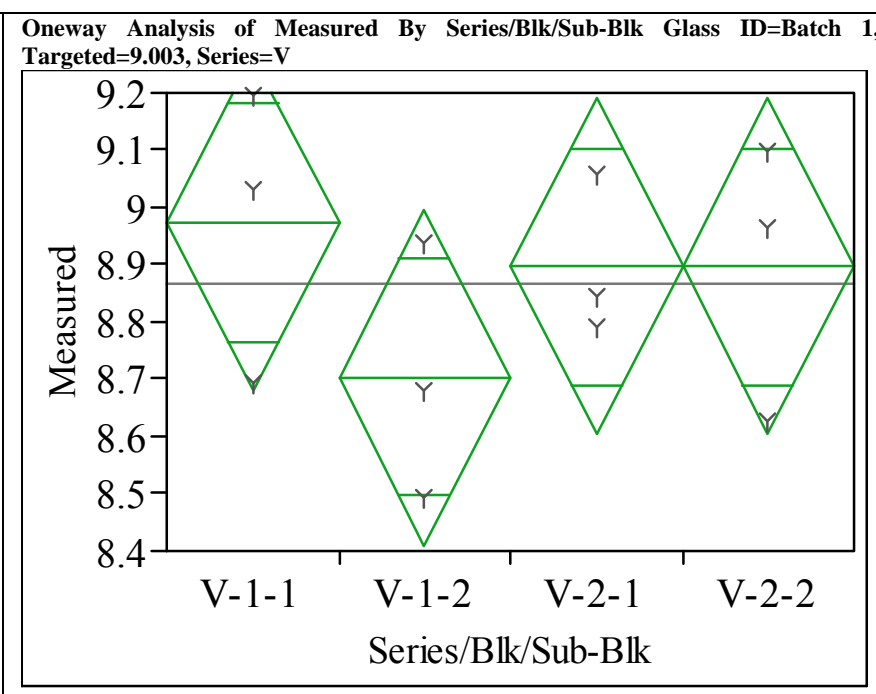

Oneway Anova

Summary of Fit

0.235104

Adj Rsquare $\quad-0.05173$

Root Mean Square Error $\quad 0.220231$

$\begin{array}{lr}\text { Mean of Response } & 8.867593 \\ \text { Observations (or Sum Wgts) } & 12\end{array}$

Analysis of Variance

Source DF Sum of Squares Mean Square F Ratio Prob >

$\begin{array}{llllll}\text { Series/Blk/Sub-Blk } & 3 & 0.11926259 & 0.039754 & 0.8196 & 0.5186\end{array}$

Error $\quad 8 \quad 0.38801227$

0.048502

Means for Oneway Anova

Level Number Mean Std Error Lower 95\% Upper 95\%

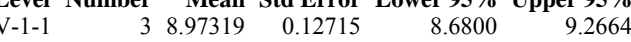

$\begin{array}{llllll}\mathrm{V}-1-2 & 3 & 8.70359 & 0.12715 & 8.4104 & 8.9968\end{array}$

$\begin{array}{llllll}\text { V-2-1 } & 3 & 8.89680 & 0.12715 & 8.6036 & 9.1900\end{array}$

$\begin{array}{llllll}\mathrm{V}-2-2 & 3 & 8.89680 & 0.12715 & 8.6036 & 9.1900\end{array}$

Std Error uses a pooled estimate of error variance 
Exhibit A-3. Measurements by Analytical Series, Block and Sub-Block for the Batch 1 and LRM Standards by Oxide by Prep (continued)

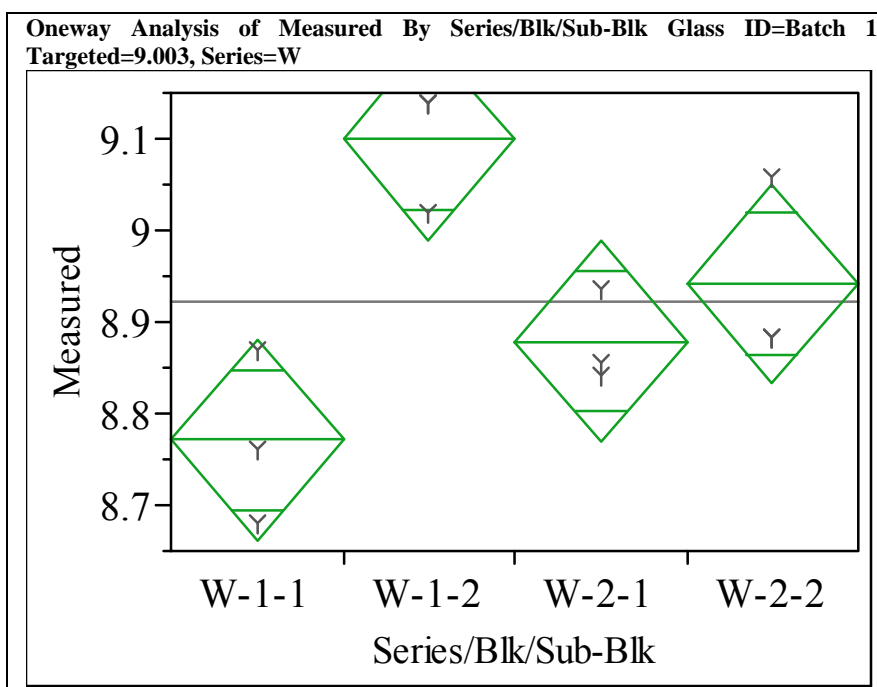

\section{Oneway Anova}

Summary of Fi

Rsquare $\quad 0.759984$

Adj Rsquare $\quad 0.669978$

Root Mean Square Error $\quad 0.081718$

$\begin{array}{lr}\text { Mean of Response } & 8.922637 \\ \text { Observations (or Sum Wgts) } & 12\end{array}$

Analysis of Variance

$\begin{array}{lrrrrr}\text { Source } & \text { DF } & \text { Sum of Squares } & \text { Mean Square } & \text { F Ratio } & \text { Prob }>\text { F } \\ \text { Series/Blk/Sub-Blk } & 3 & 0.16915724 & 0.056386 & 8.4437 & 0.0073 \\ \text { Error } & 8 & 0.05342286 & 0.006678 & & \\ \text { C. Total } & 11 & 0.22258010 & & & \end{array}$

Means for Oneway Anova

Level Number Mean Std Error Lower 95\% Upper 95\%

$\begin{array}{llrrrr}\text { W-1-1 } & 3 & 8.77099 & 0.04718 & 8.6622 & 8.8798\end{array}$

$\begin{array}{llllll}W-1-2 & 3 & 9.09900 & 0.04718 & 8.9902 & 9.2078\end{array}$

$\begin{array}{llllll}\text { W-2-1 } & 3 & 8.87883 & 0.04718 & 8.7700 & 8.9876\end{array}$

Std Error uses a pooled estimate of error variance

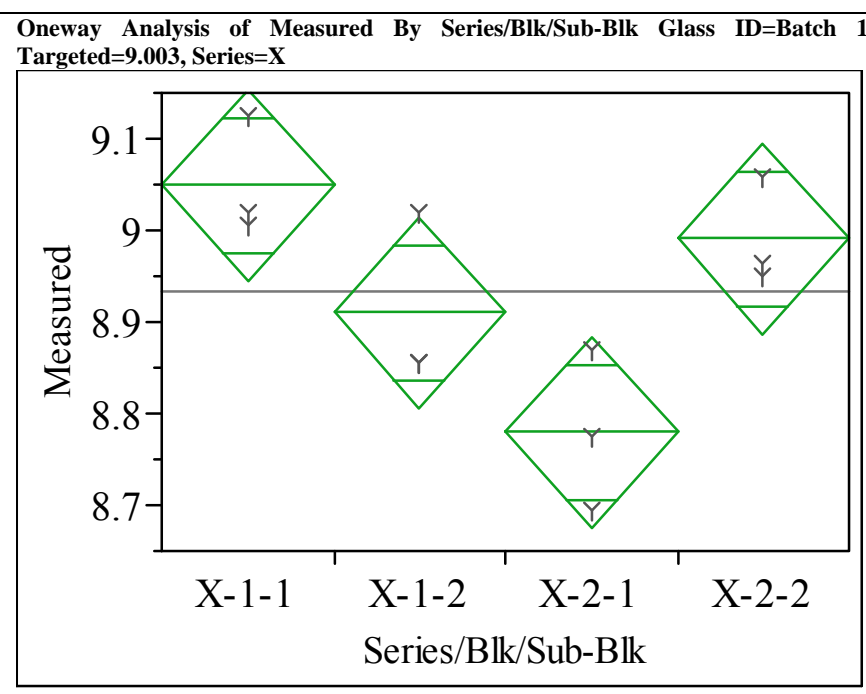

Oneway Anova

Summary of Fit

$\begin{array}{lr}0.716407 \\ \text { Adj Rsquare } & 0.61006\end{array}$

Root Mean Square Error $\quad 0.077924$

$\begin{array}{lr}\text { Mean of Response } & 8.932747 \\ \text { Observations (or Sum Wgts) } & 12\end{array}$

Analysis of Variance

Source DF Sum of Squares Mean Square F Ratio Prob >

$\begin{array}{lllllll}\text { Series/Blk/Sub-Blk } & 3 & 0.12271509 & 0.040905 & 6.7365 & 0.0140\end{array}$

Error $\quad 8 \quad 0.04857725$ 0.006072

Means for Oneway Anova

Level Number Mean Std Error Lower 95\% Upper 95\%

$\begin{array}{llrrrr}\mathrm{X}-1-1 & 3 & 9.04957 & 0.04499 & 8.9458 & 9.1533\end{array}$

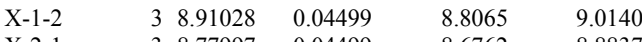

$\begin{array}{llllll}\mathrm{X}-2-1 & 3 & 8.77997 & 0.04499 & 8.6762 & 8.8837\end{array}$

$\begin{array}{llllll}\mathrm{X}-2-2 & 3 & 8.99116 & 0.04499 & 8.8874 & 9.0949\end{array}$

Std Error uses a pooled estimate of error variance 
Exhibit A-3. Measurements by Analytical Series, Block and Sub-Block for the Batch 1 and LRM Standards by Oxide by Prep (continued)

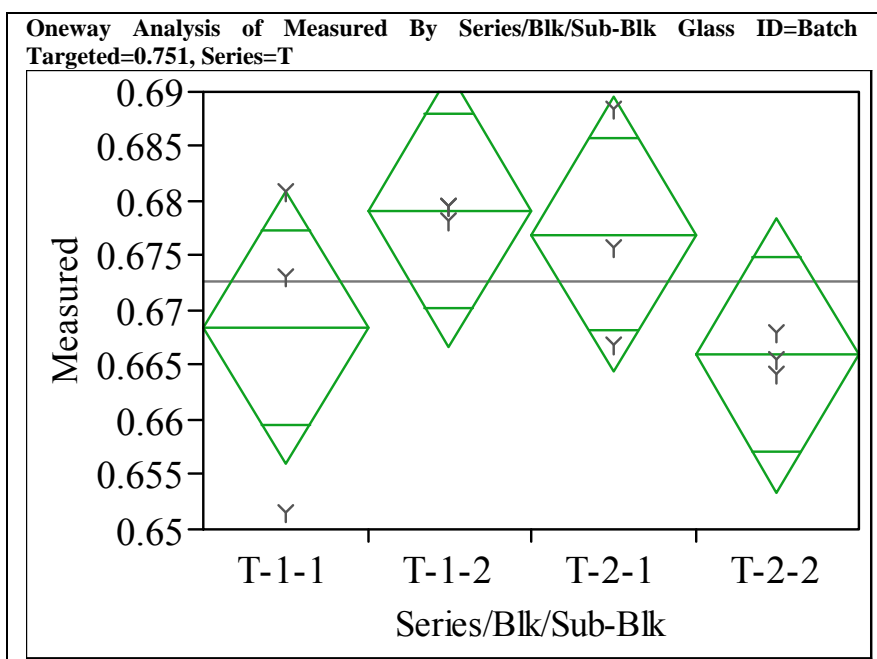

Oneway Anova

Summary of Fit

0.3423

Adj Rsquare $\quad 0.095663$

Root Mean Square Error $\quad 0.009394$

$\begin{array}{lr}\text { Mean of Response } & 0.672622 \\ \text { Observations (or Sum Wgts) } & 12\end{array}$

Analysis of Variance

$\begin{array}{lrrrrr}\text { Source } & \text { DF } & \text { Sum of Squares } & \text { Mean Square } & \text { F Ratio } & \text { Prob }>\text { F } \\ \text { Series/Blk/Sub-Blk } & 3 & 0.00036744 & 0.000122 & 1.3879 & 0.3151\end{array}$

$\begin{array}{lrrrrr}\text { Series/Blk/Sub-Blk } & 3 & 0.00036744 & 0.000122 & 1.3879 & 0.3151 \\ \text { Error } & 8 & 0.00070600 & 0.000088 & & \end{array}$

C. Total

$11 \quad 0.00107343$

Means for Oneway Anova

Level Number Mean Std Error Lower 95\% Upper 95\%

$\begin{array}{lllrrr}\mathrm{T}-1-1 & 3 & 0.668487 & 0.00542 & 0.65598 & 0.68099\end{array}$

$\begin{array}{llllll}\mathrm{T}-1-2 & 3 & 0.679091 & 0.00542 & 0.66658 & 0.69160\end{array}$

$\begin{array}{llllll}\mathrm{T}-2-1 & 3 & 0.676970 & 0.00542 & 0.66446 & 0.68948\end{array}$

$\begin{array}{llllll}\mathrm{T}-2-2 & 3 & 0.665942 & 0.00542 & 0.65343 & 0.67845\end{array}$

Std Error uses a pooled estimate of error variance

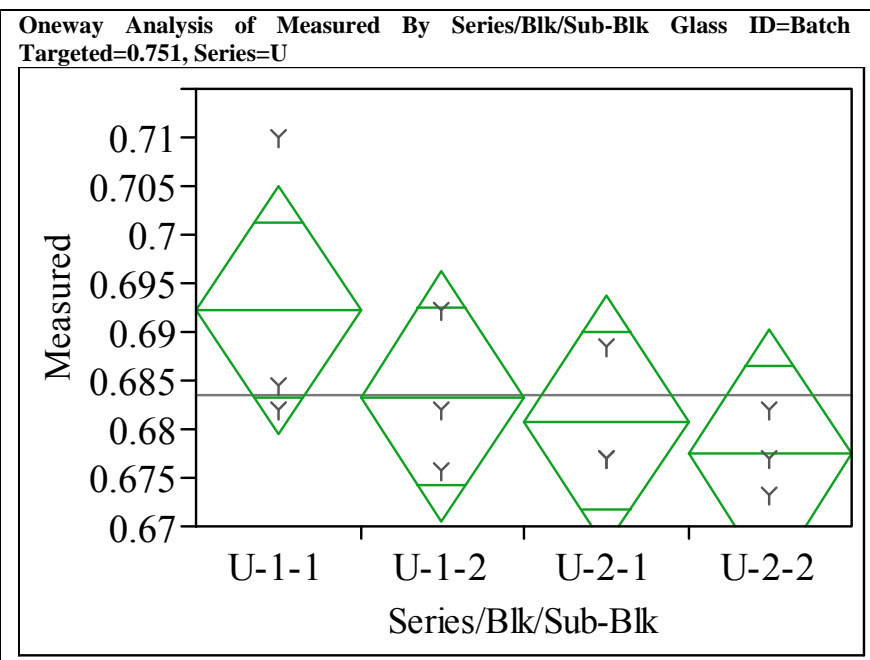

\section{Oneway Anova}

Summary of Fit

$\begin{array}{lr}\text { Rsquare } & 0.327412 \\ \text { Adj Rsquare } & 0.075192 \\ \text { Root Mean Square Error } & 0.009656 \\ \text { Mean of Response } & 0.683439 \\ \text { Observations (or Sum Wgts) } & 12\end{array}$

Analysis of Variance

Source DF Sum of Squares Mean Square F Ratio Prob > F

$\begin{array}{llllll}\text { Series/Blk/Sub-Blk } & 3 & 0.00036312 & 0.000121 & 1.2981 & 0.3400\end{array}$

$\begin{array}{lrr}\text { Error } & 8 & 0.00074594\end{array}$ 0.000093

Means for Oneway Anova

Level Number Mean Std Error Lower

$\begin{array}{llllrr}\text { U-1-1 } & 3 & 0.692240 & 0.00558 & 0.67938 & 0.70510\end{array}$

$\begin{array}{llllll}\text { U-1-2 } & 3 & 0.683333 & 0.00558 & 0.67048 & 0.69619\end{array}$

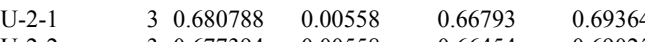

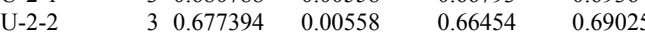

Std Error uses a pooled estimate of error variance 


\section{Exhibit A-3. Measurements by Analytical Series, Block and Sub-Block for the Batch 1 and LRM Standards by Oxide by Prep (continued)}

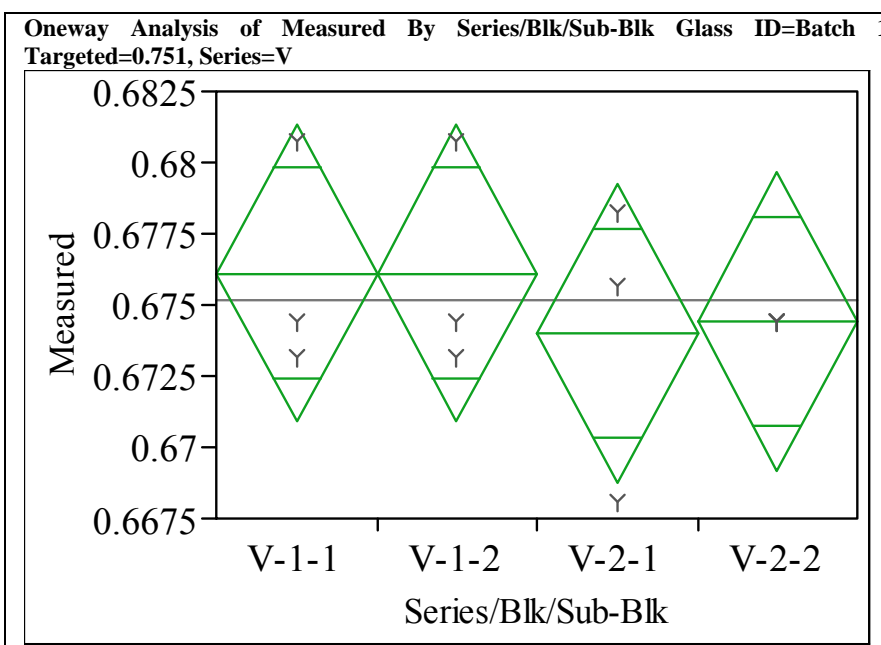

\section{Oneway Anova}

Summary of Fit

$$
\begin{aligned}
& \begin{array}{ll}
\text { Rsquare } & 0.083417
\end{array} \\
& \text { Adj Rsquare } \quad-0.2603 \\
& \text { Root Mean Square Error } \quad 0.003922 \\
& \text { Mean of Response } \quad 0.67516
\end{aligned}
$$

Means for Oneway Anova

Level Number Mean Std Error Lower 95\% Upper 95\%

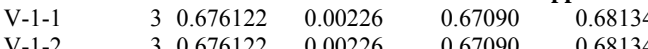

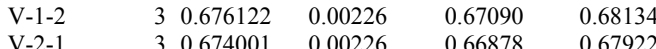

$\begin{array}{llllll}\mathrm{V}-2-1 & 3 & 0.674001 & 0.00226 & 0.66878 & 0.67922 \\ \mathrm{~V}-2-2 & 3 & 0.674425 & 0.00226 & 0.66920 & 0.67965\end{array}$

Std Error uses a pooled estimate of error variance

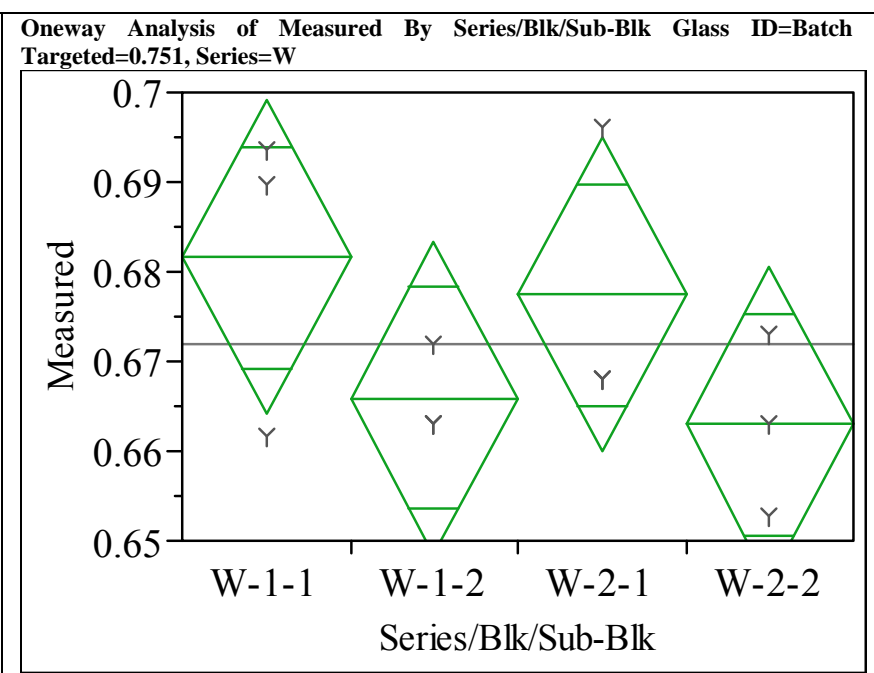

Oneway Anova

Summary of Fit

$\begin{array}{lr}\text { Rsquare } & 0.342002 \\ \text { Adj Rsquare } & 0.095253 \\ \text { Root Mean Square Error } & 0.013163 \\ \text { Mean of Response } & 0.671986 \\ \text { Observations (or Sum Wgts) } & 12\end{array}$

Observations (or Sum Wgts) 0.671986

Analysis of Variance

Source DF Sum of Squares Mean Square F Ratio Prob $>$ F

$\begin{array}{lrrrrr}\text { Series/Blk/Sub-Blk } & 3 & 0.00072043 & 0.000240 & 1.3860 & 0.3156\end{array}$

$\begin{array}{lll}\text { Error } & 8 & 0.00138608\end{array}$

0.000173

C. Total

0.00210652

Means for Oneway Anova

Level Number Mean Std Error Lower 95\% Upper 95\%

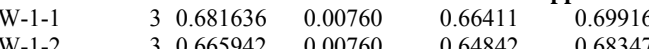

$\begin{array}{llllll}W-1-1 & 3 & 0.681636 & 0.00760 & 0.66411 & 0.69916 \\ W-2-1 & 3 & 0.665942 & 0.00760 & 0.64842 & 0.68347 \\ W-2 & & 0.677394 & 0.00760 & 0.65987 & 0.69492\end{array}$

$\begin{array}{llllll}\mathrm{W}-1-2 & 3 & 0.665942 & 0.00760 & 0.64842 & 0.68347 \\ \mathrm{~W}-2-2 & 3 & 0.662973 & 0.00760 & 0.65987 & 0.69492\end{array}$

Std Error uses a pooled estimate of error variance 


\section{Exhibit A-3. Measurements by Analytical Series, Block and Sub-Block for the Batch 1 and LRM Standards by Oxide by Prep (continued)}

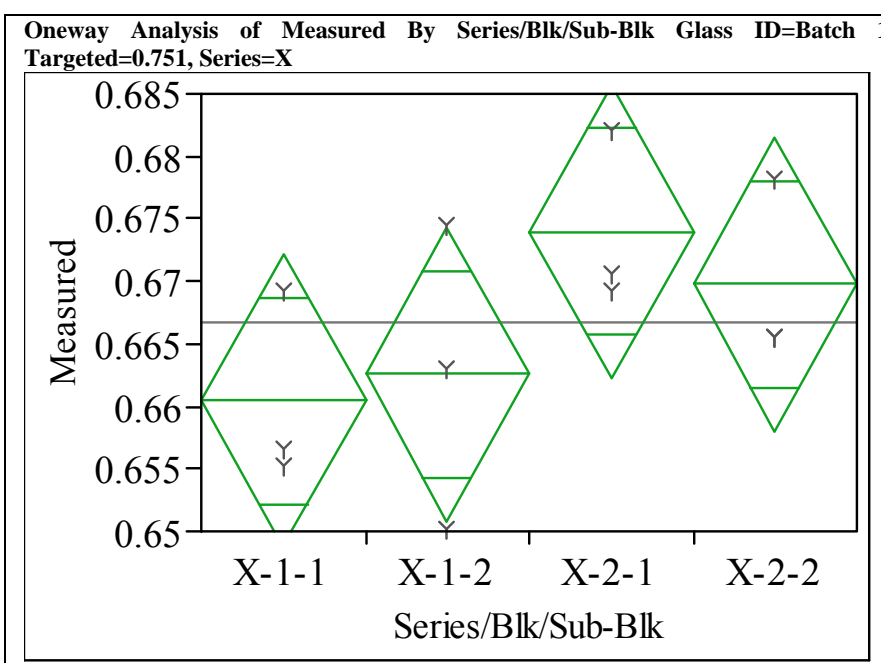

\section{Oneway Anova}

Oneway Anova
Summary of Fit

0.366413
Rsquare

$\begin{array}{ll}\text { Adj Rsquare } & 0.128818 \\ \text { Root Mean Square Error } & 0.008793\end{array}$

\begin{tabular}{ll} 
Mean of Response & 0.666684 \\
\hline
\end{tabular}

Observations (or Sum Wgts) $\quad 12$

Analysis of Variance

Source DF Sum of Squares Mean Square F Ratio Prob $>$ F

$\begin{array}{llllll}\text { Series/Blk/Sub-Blk } & 3 & 0.00035772 & 0.000119 & 1.5422 & 0.2771\end{array}$

Error

0.00061856

0.00007

Means for Oneway Anova

Level Number Mean Std Error Lower 95\% Upper 95\%

$\begin{array}{lrrrrr}\text { X-1-1 } & 3 & 0.660428 & 0.00508 & 0.64872 & 0.67213\end{array}$

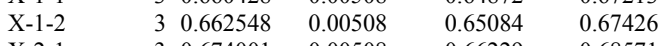

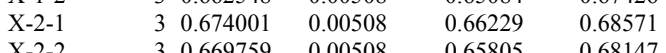

$\begin{array}{llllll}\mathrm{X}-2-2 & 3 & 0.669759 & 0.00508 & 0.65805 & 0.68147\end{array}$

Std Error uses a pooled estimate of error variance

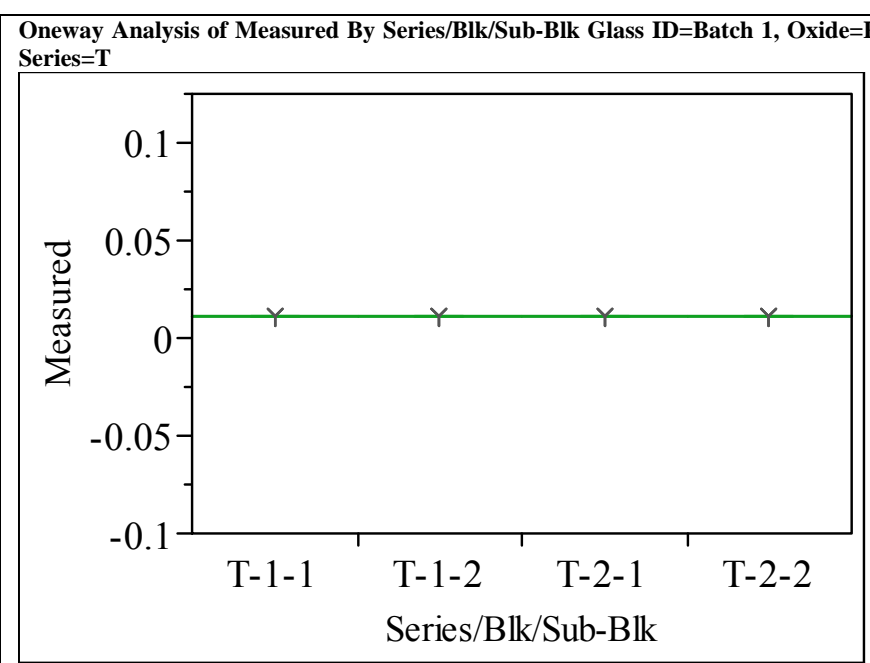

Oneway Anova

Summary of Fit

Adj Rsquare $\quad-0.375$

Root Mean Square Error $\quad 2.12 \mathrm{e}-18$

$\begin{array}{lr}\text { Mean of Response } & 0.010772 \\ \text { Observations (or Sum Wgts) } & 12\end{array}$

Analysis of Variance

Source $\quad$ DF Sum of Squares Mean Square F Ratio Prob > F

$\begin{array}{llllll}\text { Series/Blk/Sub-Blk } & 3 & 0 & 0 & 0.0000 & 1.0000\end{array}$

$\begin{array}{lrr}\text { Error } & 8 & 3.6111 \mathrm{e}-35 \\ \text { C. Th } & 11 & 3.611 \mathrm{e}-35\end{array}$

C. Total

$3.6111 \mathrm{e}-35$

$4.514 \mathrm{e}-36$

Means for Oneway Anova

Level Number Mean Std Error Lower 95\% Upper 95\%

$\begin{array}{llllll}\text { T-1-1 } & 3 & 0.010772 & 1.227 \mathrm{e}-18 & 0.01077 & 0.01077\end{array}$

$\begin{array}{llllll}\text { T-1-2 } & 3 & 0.010772 & 1.227 \mathrm{e}-18 & 0.01077 & 0.01077\end{array}$

$\begin{array}{llllll}\mathrm{T}-2-1 & 3 & 0.010772 & 1.227 \mathrm{e}-18 & 0.01077 & 0.01077\end{array}$

$\begin{array}{llllll}\text { T-2-2 } & 3 & 0.010772 & 1.227 \mathrm{e}-18 & 0.01077 & 0.01077\end{array}$

Std Error uses a pooled estimate of error variance 
Exhibit A-3. Measurements by Analytical Series, Block and Sub-Block for the Batch 1 and LRM Standards by Oxide by Prep (continued)

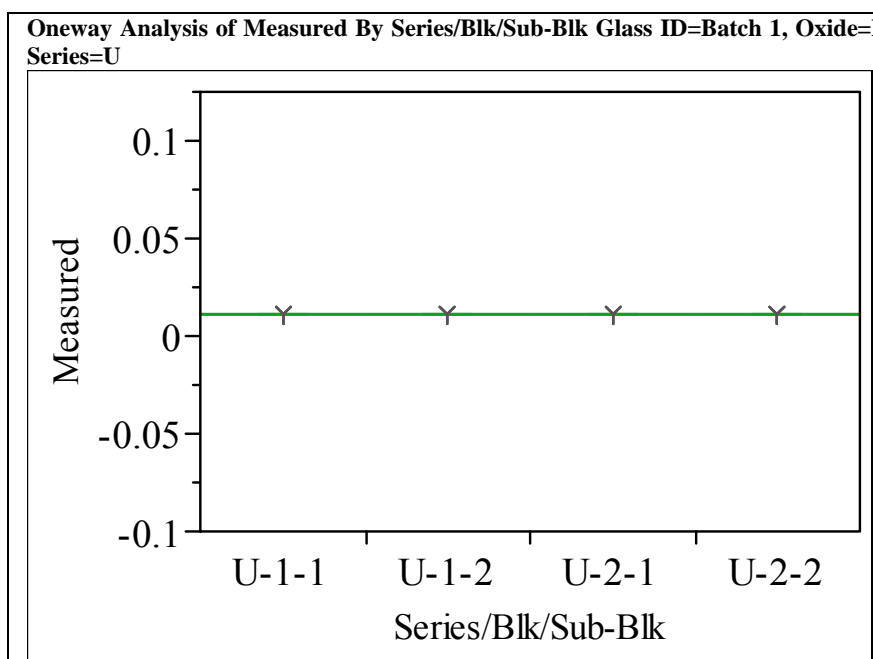

Oneway Anova

Summary of Fit

Rsquare

Adj Rsquare $\quad-0.375$

Root Mean Square Error $\quad 2.12 \mathrm{e}-18$

$\begin{array}{lr}\text { Mean of Response } & 0.010772 \\ \text { Observations (or Sum Wots) } & 12\end{array}$

Observations (or Sun

Analysis of Variance

Source DF Sum of Squares Mean Square F Ratio Prob $>$ F

$\begin{array}{llllll}\text { Series/Blk/Sub-Blk } & 3 & 0 & 0 & 0.0000 & 1.0000\end{array}$

Error
C. Total

$8 \quad 3.6111 \mathrm{e}-35$

$4.514 \mathrm{e}-36$

Means for Oneway Anova

Level Number Mean Std Error Lower 95\% Upper 95\%

$\begin{array}{llllrr}\text { U-1-1 } & 3 & 0.010772 & 1.227 \mathrm{e}-18 & 0.01077 & 0.01077\end{array}$

$\begin{array}{llllll}\text { U-1-2 } & 3 & 0.010772 & 1.227 \mathrm{e}-18 & 0.01877 & 0.01077 \\ \mathrm{U}-2-1 & & 3 & 0.01072 & 1.22078 & 0.01077\end{array}$

$\begin{array}{llllll}\text { U-2-1 } & 3 & 0.010772 & 1.227 \mathrm{e}-18 & 0.01077 & 0.01077\end{array}$

$\begin{array}{llllll}\text { U-2-2 } & 3 & 0.010772 & 1.227 \mathrm{e}-18 & 0.01077 & 0.01077\end{array}$

Std Error uses a pooled estimate of error variance

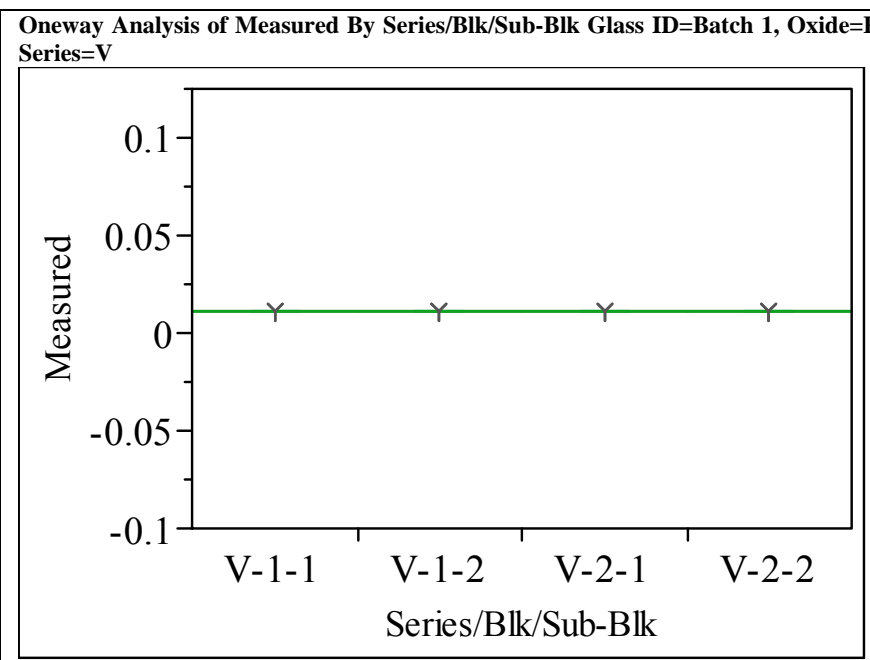

Oneway Anova

Summary of Fit

Rsquare

Adj Rsquare $\quad-0.375$

Root Mean Square Error $\quad 2.12 \mathrm{e}-18$

$\begin{array}{lr}\text { Mean of Response } & 0.010772 \\ \text { Observations (or Sum Wgts) } & 12\end{array}$

Analysis of Variance

Sum of Squares Mean Square F Ratio Prob $>$ F

$\begin{array}{llllll}\text { Series/Blk/Sub-Blk } & 3 & 0 & 0 & 0.0000 & 1.0000\end{array}$

$\begin{array}{lrr}\text { Error } & 8 & 3.6111 \mathrm{e}-35 \\ \text { C. T } & 11 & 3.611 \mathrm{e}-35\end{array}$

C. Total

$3.6111 \mathrm{e}-35$

$4.514 \mathrm{e}-36$

Means for Oneway Anova

Level Number Mean Std Error Lower 95\% Upper 95\%

$\begin{array}{llllll}\mathrm{V}-1-1 & 3 & 0.010772 & 1.227 \mathrm{e}-18 & 0.01077 & 0.01077\end{array}$

$\begin{array}{llllll}V-1-2 & 3 & 0.010772 & 1.227 \mathrm{e}-18 & 0.01077 & 0.01077\end{array}$

$\begin{array}{llllll}\mathrm{V}-2-1 & 3 & 0.010772 & 1.227 \mathrm{e}-18 & 0.01077 & 0.01077\end{array}$

$\begin{array}{llllll}\mathrm{V}-2-2 & 3 & 0.010772 & 1.227 \mathrm{e}-18 & 0.01077 & 0.01077\end{array}$

Std Error uses a pooled estimate of error variance 
Exhibit A-3. Measurements by Analytical Series, Block and Sub-Block for the Batch 1 and LRM Standards by Oxide by Prep (continued)

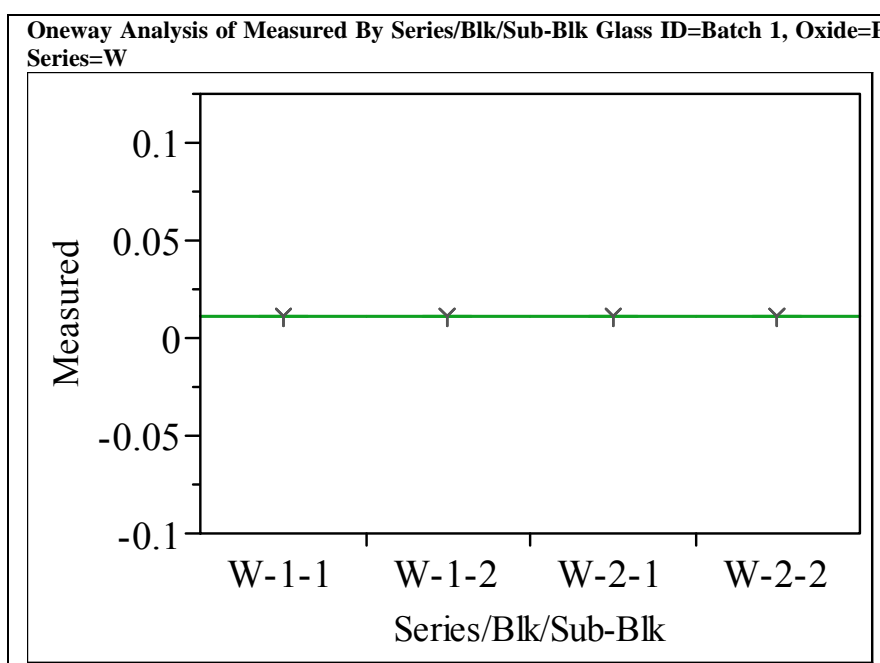

Oneway Anova

Summary of Fit

Rsquare

Adj Rsquare $\quad-0.375$

Root Mean Square Error $\quad 2.12 \mathrm{e}-18$

$\begin{array}{lr}\text { Mean of Response } & 0.010772 \\ \text { Observations (or Sum Wgts) } & 12\end{array}$

Observations (or Sun

Analysis of Variance

Source DF Sum of Squares Mean Square F Ratio Prob $>$ F

$\begin{array}{lrrrrr} & & & \\ \text { Source } & & & \\ \text { Series/Blk/Sub-Blk } & 3 & 0 & 0 & 0.0000 & 1.0000 \\ \text { Error } & 8 & 3.6111 \mathrm{e}-35 & 4.514 \mathrm{e}-36 & & \\ \text { C } & 8 & 36 & & & \end{array}$

Error

C. Total

$3.6111 \mathrm{e}-35$

Means for Oneway Anova

Level Number Mean Std Error Lower 95\% Upper 95\%

$\begin{array}{lllllr}\text { W-1-1 } & 3 & 0.010772 & 1.227 \mathrm{e}-18 & 0.01077 & 0.01077\end{array}$

$\begin{array}{llllll}\text { W-1-2 } & 3 & 0.010772 & 1.227 \mathrm{e}-18 & 0.01077 & 0.01077 \\ \mathrm{~W}-2-1 & 3 & 0.010772 & 1.227 \mathrm{e}-18 & 0.01077 & 0.01077\end{array}$

$\begin{array}{llllll}\mathrm{W}-2-1 & 3 & 0.010772 & 1.227 \mathrm{e}-18 & 0.01077 & 0.01077 \\ \mathrm{~W}-2-2 & 3 & 0.010772 & 1.227 \mathrm{e}-18 & 0.01077 & 0.01077\end{array}$

Std Error uses a pooled estimate of error variance

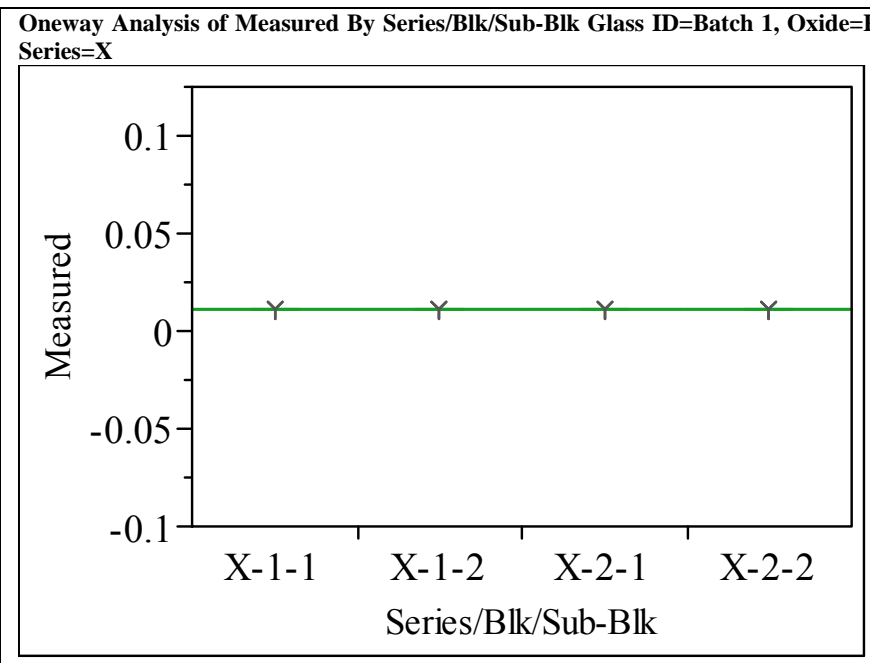

Oneway Anova

Summary of Fit

Rsquare

Adj Rsquare $\quad-0.375$

Root Mean Square Error $\quad$ 2.12e-18

$\begin{array}{lr}\text { Mean of Response } & 0.010772 \\ \text { Observations (or Sum Wgts) } & 12\end{array}$

Analysis of Variance

Source

$\begin{array}{lrrrrr}\text { Series/Blk/Sub-Blk } & 3 & 0 & 0 & 0.0000 & 1.0000\end{array}$

$\begin{array}{lrr}\text { Error } & 8 & 3.6111 \mathrm{e}-35 \\ \text { C. T } & 11 & 3.611 \mathrm{e}-35\end{array}$

C. Total

$3.6111 \mathrm{e}-35$

$4.514 \mathrm{e}-36$

Means for Oneway Anova

Level Number Mean Std Error Lower 95\% Upper 95\%

$\begin{array}{llllll}\mathrm{X}-1-1 & 3 & 0.010772 & 1.227 \mathrm{e}-18 & 0.01077 & 0.01077\end{array}$

$\begin{array}{llllll}\mathrm{X}-1-1 & 3 & 0.010772 & 1.227 \mathrm{e}-18 & 0.01077 & 0.01077 \\ \mathrm{X}-2-1 & 3 & 0.010772 & 1.227 \mathrm{e}-18 & 0.01077 & 0.01077\end{array}$

$\begin{array}{llllll}\mathrm{X}-2-1 & 3 & 0.010772 & 1.227 \mathrm{e}-18 & 0.01077 & 0.01077\end{array}$

$\begin{array}{llllll}\mathrm{X}-2-2 & 3 & 0.010772 & 1.227 \mathrm{e}-18 & 0.01077 & 0.01077\end{array}$

Std Error uses a pooled estimate of error variance 


\section{Exhibit A-3. Measurements by Analytical Series, Block and Sub-Block for the Batch 1 and LRM Standards by Oxide by Prep (continued)}

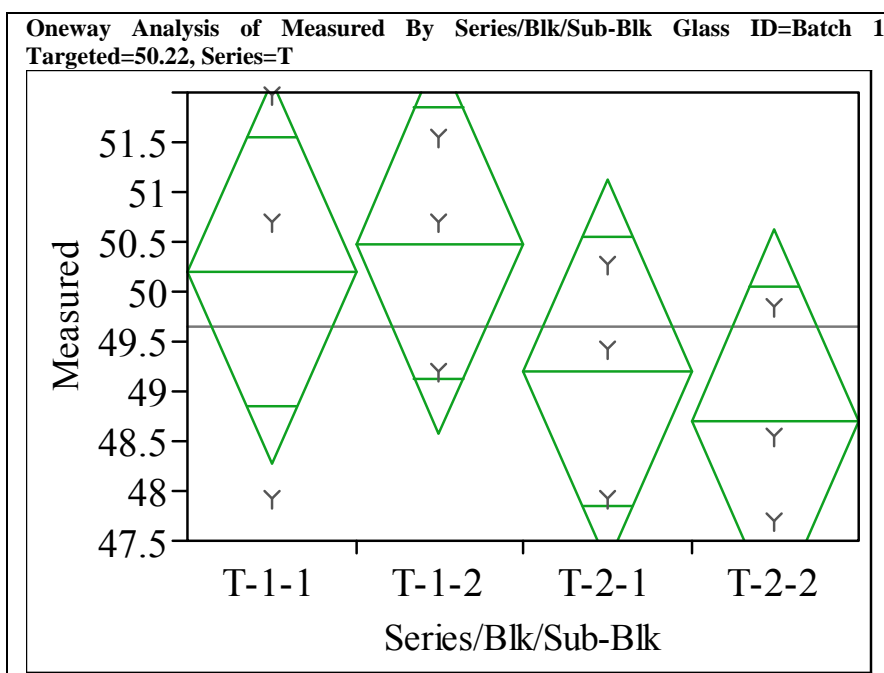

\section{Oneway Anova}

Summary of Fit

$$
\begin{array}{lr}
\text { Rsquare } & 0.274663 \\
\text { Adj Rsquare } & 0.002662 \\
\text { Root Mean Square Error } & 1.441715 \\
\text { Mean of Response } & 49.64959 \\
\text { Observations (or Sum Wgts) } & 12
\end{array}
$$

Analysis of Variance

Source DF Sum of Squares Mean Square F Ratio Prob $>$ F

$\begin{array}{lrrrrr}\text { Series/Blk/Sub-Blk } & 3 & 6.296645 & 2.09888 & 1.0098 & 0.4373\end{array}$

80.628330

$\begin{array}{lll}2.09888 & 1.0098 & 0.4373 \\ 2.07854 & & \end{array}$

C. Total

22.924975

Means for Oneway Anova

Level Number Mean Std Error Lower 95\% Upper 95\%

$\begin{array}{rrrrrr}\text { T-1-1 } & 3 & 50.2022 & 0.83237 & 48.283 & 52.122 \\ \text { T- } 1-2 & 3 & 50.4875 & 0.83237 & 48.568 & 52.407\end{array}$

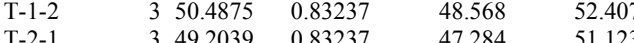

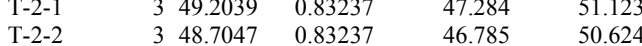

Std Error uses a pooled estimate of error variance

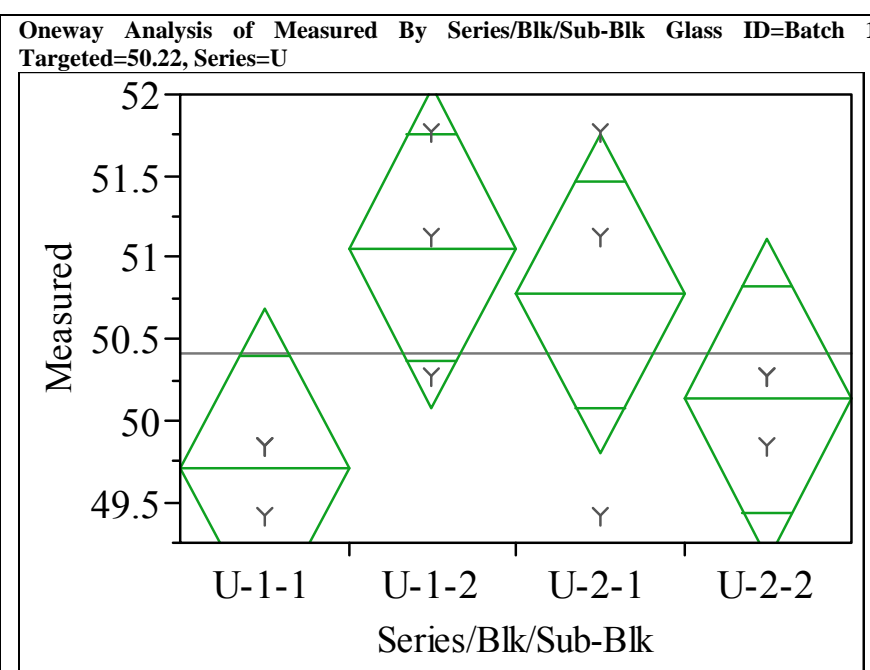

Oneway Anova

Summary of Fit

$\begin{array}{lr}\text { Rsquare } & 0.438735 \\ \text { Adj Rsquare } & 0.228261 \\ \text { Root Mean Square Error } & 0.735911 \\ \text { Mean of Response } & 50.41617 \\ \text { Observations (or Sum Wgts) } & 12\end{array}$

Analysis of Variance

Source DF Sum of Squares Mean Square F Ratio Prob > F

$\begin{array}{llrrrr}\text { Source } & \text { DF Sum of Squares } & \text { Mean Square } & \text { F Ratio } & \text { Prob }>\text { F } \\ \text { Series/Blk/Sub-Blk } & 3 & 3.3866873 & 1.12890 & 2.0845 & 0.1807\end{array}$

$\begin{array}{llll}\text { Series/Blk/Sub-Blk } & 3 & 3.3866873 & 1.12890 \\ \text { Error } & 8 & 4.3325189 & 0.54156\end{array}$

C. Tota

7.7192062

Means for Oneway Anova
Level Number Mean Std Error Lower 95\% Upper 95\%

\begin{tabular}{rrrrrr} 
U-1-1 & 3 & 49.7031 & 0.42488 & 48.723 & 50.683 \\
\hline & 3 & 51.0580 & 0.42488 & 50.078 & 52.038
\end{tabular}

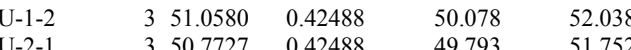

$\begin{array}{lllllll}\mathrm{U}-1-2 & & 51.0580 & 0.42488 & 50.078 & 52.038 \\ \mathrm{U}-2-2 & & 3 & 50.7727 & 0.42488 & 49.793 & 51.752 \\ & & 5 & 50.1309 & 0.42488 & 49.151 & 51.111\end{array}$

Std Error uses a pooled estimate of error variance 


\section{Exhibit A-3. Measurements by Analytical Series, Block and Sub-Block for the Batch 1 and LRM Standards by Oxide by Prep (continued)}

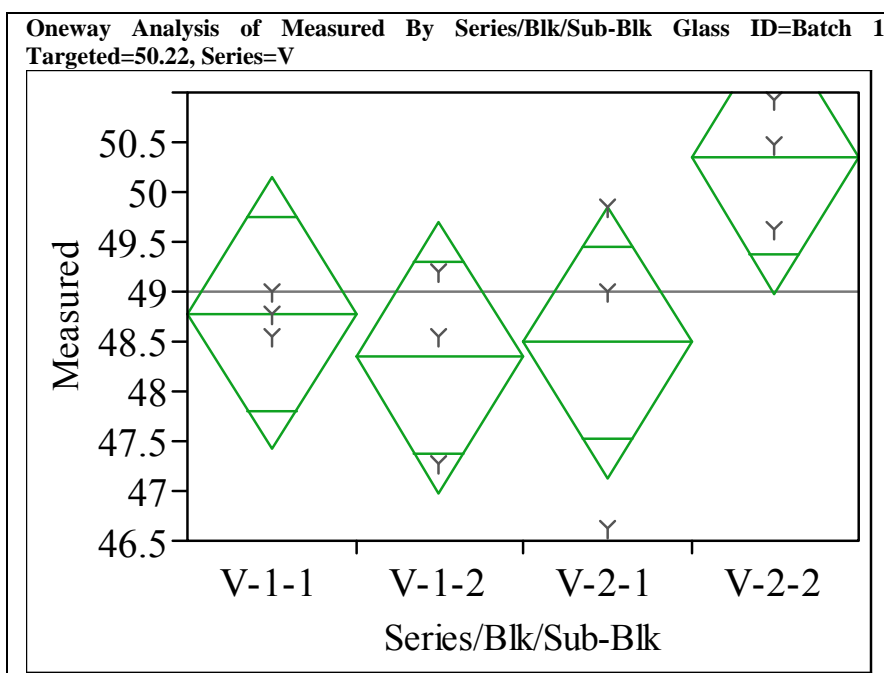

\section{Oneway Anova}

Summary of Fit

$$
\begin{array}{lr}
\text { Rsquare } & 0.47619 \\
\text { Adj Rsquare } & 0.279762 \\
\text { Root Mean Square Error } & 1.024112 \\
\text { Mean of Response } & 48.98997 \\
\text { Observations (or Sum Wgts) } & 12
\end{array}
$$

Analysis of Variance

$\begin{array}{lrrrrr}\text { Source } & \text { DF } & \text { Sum of Squares } & \text { Mean Square } & \text { F Ratio } & \text { Prob > F } \\ \text { Series/Blk/Sub-Blk } & 3 & 7.627674 & 2.54256 & 2.4242 & 0.1408\end{array}$

Error$$
8.390442
$$$$
1.04881
$$

C. Total

16.018116

Means for Oneway Anova

Level Number Mean Std Error Lower 95\% Upper 95\%

$\begin{array}{llllrr}\text { V-1-1 } & 3 & 48.7760 & 0.59127 & 47.413 & 50.140 \\ \text { V-1-2 } & 3 & 48.3482 & 0.59127 & 46.985 & 49.712\end{array}$

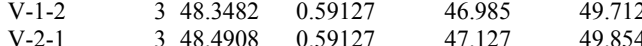

$\begin{array}{llllll}V-2-1 & 3 & 48.4908 & 0.59127 & 47.127 & 49.854 \\ \text { V-2-2 } & 3 & 50.3449 & 0.59127 & 48.981 & 51.708\end{array}$

Std Error uses a pooled estimate of error variance

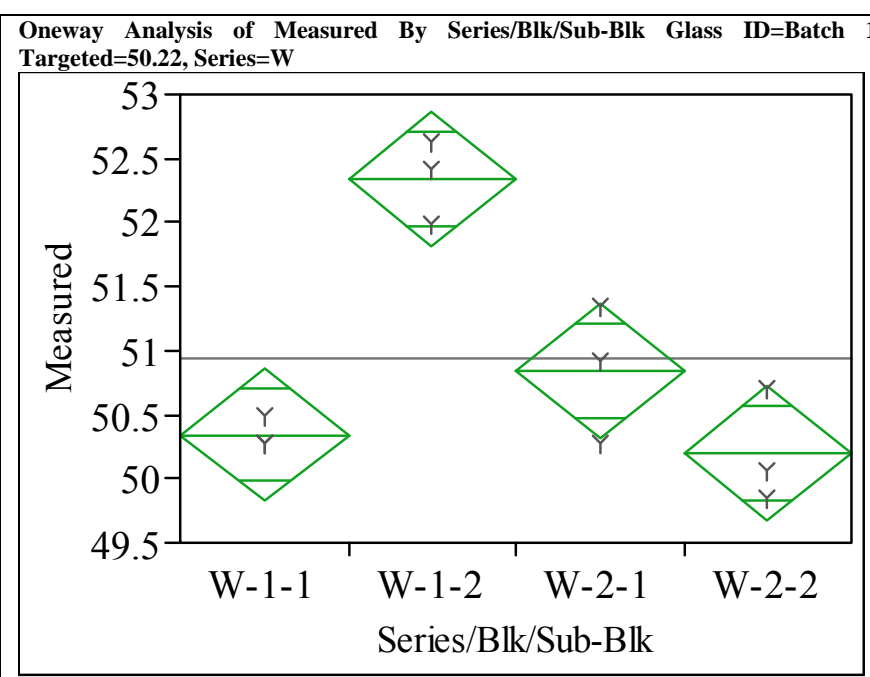

Oneway Anova

Summary of Fit

$\begin{array}{lr}\text { Rsquare } & 0.875921 \\ \text { Adj Rsquare } & 0.829391 \\ \text { Root Mean Square Error } & 0.390581 \\ \text { Mean of Response } & 50.93317 \\ \text { Observations (or Sum Wgts) } & 12\end{array}$

Analysis of Variance

$\begin{array}{lrrrrr}\text { Source } & \text { DF } & \text { Sum of Squares } & \text { Mean Square } & \text { F Ratio } & \text { Prob }>\text { F } \\ \text { Series/Blk/Sub-Blk } & 3 & 8.6154580 & 2.87182 & 18.8250 & 0.0006\end{array}$

$\begin{array}{lrrr}\text { Series/Blk/Sub-Blk } & 3 & 8.6154580 & 2.87182 \\ \text { Error } & 8 & 1.2204279 & 0.15255 \\ \text { C. Total } & 11 & 9.8358858 & \\ \end{array}$

Means for Oneway Anova

Level Number Mean Std Error Lower 95\% Upper 95\%

$\begin{array}{llllll}\text { W-1-1 } & 3 & 50.3449 & 0.22550 & 49.825 & 50.865 \\ \text { W-1-2 } & 3 & 52.3415 & 0.22550 & 51.822 & 52.862\end{array}$

$\begin{array}{llllll}W-1-1 & 3 & 50.3449 & 0.22550 & 49.825 & 50.865 \\ W-12 & 3 & 52.3415 & 0.22550 & 51.822 & 52.862 \\ W-2-1 & 3 & 50.8440 & 0.22550 & 50.324 & 51.364\end{array}$

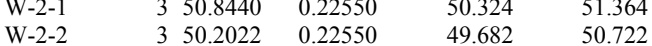

Std Error uses a pooled estimate of error variance 


\section{Exhibit A-3. Measurements by Analytical Series, Block and Sub-Block for the Batch 1 and LRM Standards by Oxide by Prep (continued)}

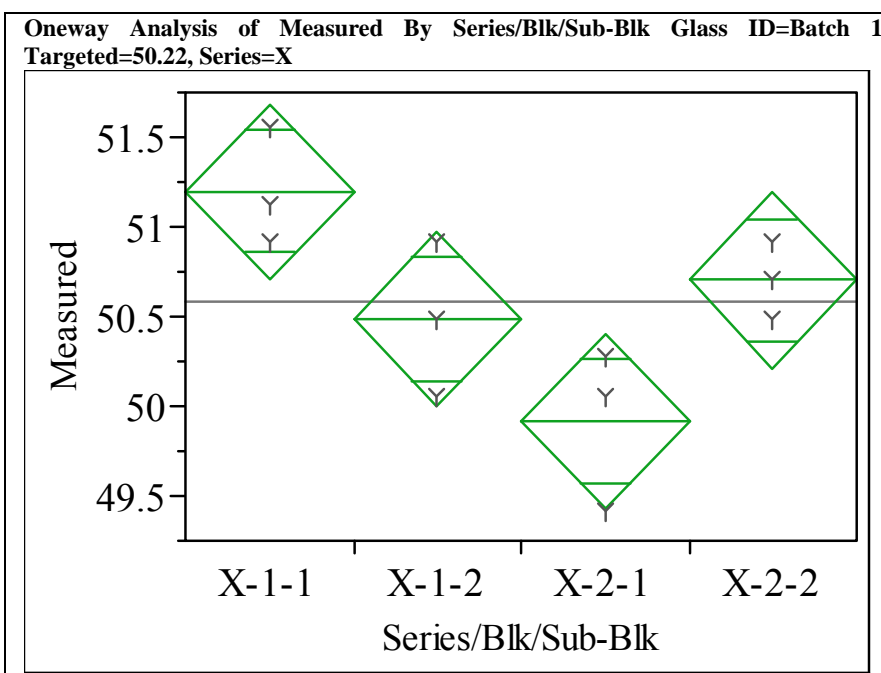

\section{Oneway Anova}

Summary of Fit

$$
\begin{array}{lr}
\text { Rsquare } & 0.704329 \\
\text { Adj Rsquare } & 0.593453 \\
\text { Root Mean Square Error } & 0.365355 \\
\text { Mean of Response } & 50.57662 \\
\text { Observations (or Sum Wgts) } & 12
\end{array}
$$

Analysis of Variance

Source DF Sum of Squares Mean Square F Ratio Prob > F

$\begin{array}{lrrrrr}\text { Series/Blk/Sub-Blk } & 3 & 2.5438293 & 0.847943 & 6.3524 & 0.0164\end{array}$

Error

$\begin{array}{lll}0.847943 & 6.3524 & 0.0164 \\ 0.133484 & & \end{array}$

C. Total

3.6117037

Means for Oneway Anova

Level Number Mean Std Error Lower 95\% Upper 95\%

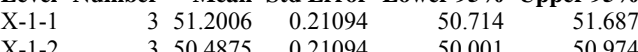

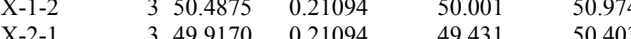

$\begin{array}{llllll}\mathrm{X}-2-1 & 3 & 4.9170 & 0.21094 & 49.431 & 50.403 \\ \mathrm{X}-2-2 & 3 & 50.7014 & 0.21094 & 50.215 & 51.188\end{array}$

Std Error uses a pooled estimate of error variance

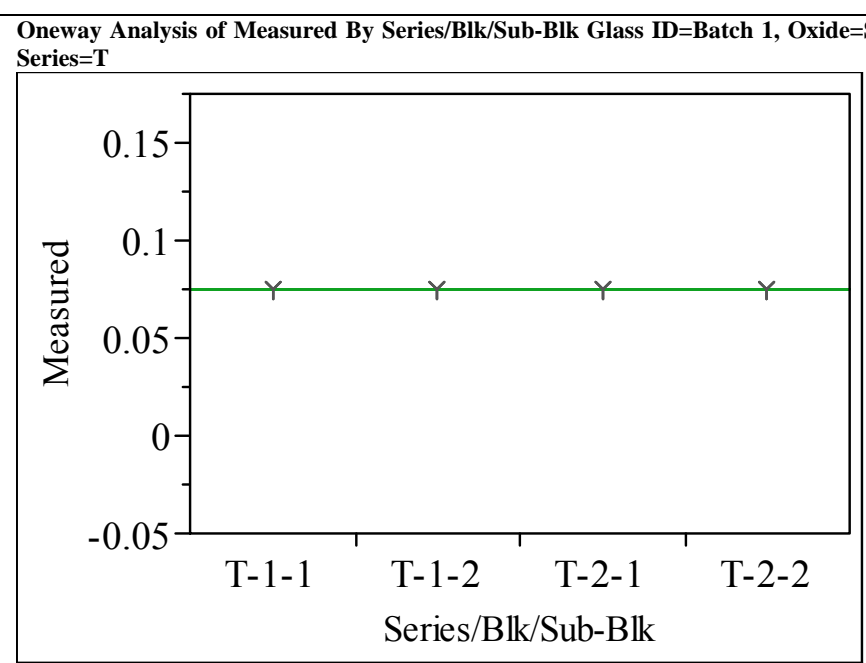

\section{Oneway Anova}

Rsquare

Adj Rsquare

$\begin{array}{lr}\text { Root Mean Square Error } & 0 \\ \text { Mean of Response } & 0.074898\end{array}$

Observations (or Sum Wgts) 12

Analysis of Variance

Sum of Squares Mean Square F Ratio Prob > F

$\begin{array}{lrrr}\text { Series/Blk/Sub-Blk } & 3 & 0 & 0 \\ \text { Error } & 8 & 0 & 0 \\ \text { C. } & 11 & 0 & \end{array}$

$\begin{array}{lrr}\text { Error } & 8 & 0 \\ \text { C. Total } & 11 & 0\end{array}$

Means for Oneway Anova

Level Number Mean Std Error Lower 95\% Upper 95\%

$\begin{array}{lllllr}\text { T-1-1 } & 3 & 0.074898 & 0 & 0.07490 & 0.07490\end{array}$

$\begin{array}{llllll}\mathrm{T}-1-2 & 3 & 0.074898 & 0 & 0.07490 & 0.07490\end{array}$

$\begin{array}{llllll}\mathrm{T}-2-1 & 3 & 0.074898 & 0 & 0.07490 & 0.07490\end{array}$

$\begin{array}{llllll}\mathrm{T}-2-2 & 3 & 0.074898 & 0 & 0.07490 & 0.07490\end{array}$

Std Error uses a pooled estimate of error variance 
Exhibit A-3. Measurements by Analytical Series, Block and Sub-Block for the Batch 1 and LRM Standards by Oxide by Prep (continued)

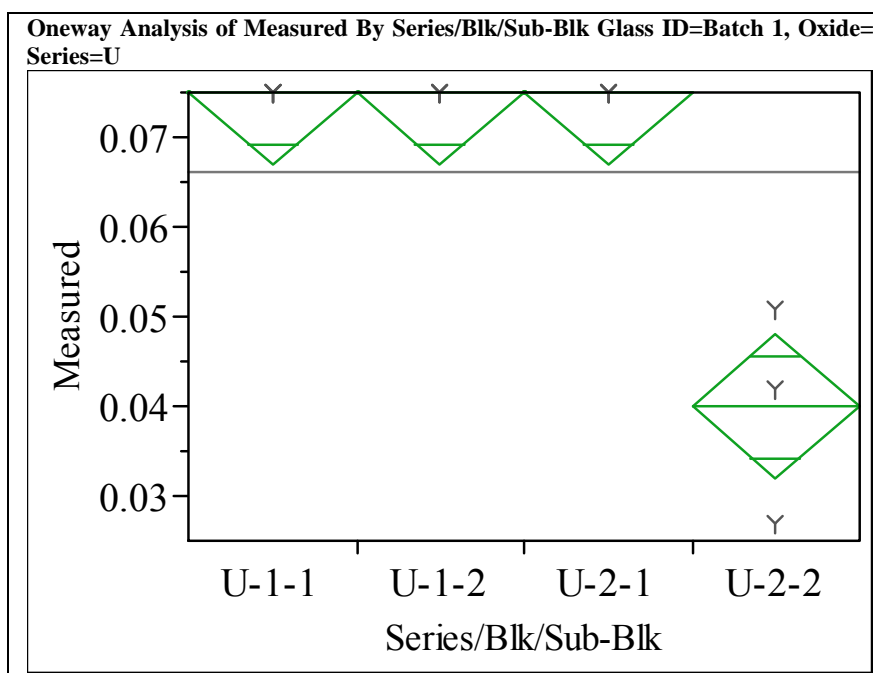

Oneway Anova

Summary of Fit

$\begin{array}{lr}\text { Rsquare } & 0.903614 \\ \text { Adj Rsquare } & 0.86747 \\ \text { Root Mean Square Error } & 0.006054 \\ \text { Mean of Response } & 0.066159 \\ \text { Observations (or Sum Wgts) } & \end{array}$

Analysis of Variance

Source DF Sum of Squares Mean Square F Ratio Prob > F

$\begin{array}{lllllll}\text { Series/Blk/Sub-Blk } & 3 & 0.00274872 & 0.000916 & 25.0000 & 0.0002\end{array}$

Error $\quad 8 \quad 0.00029320$

Means for Oneway Anova

Level Number Mean Std Error Lower 95\% Upper 95\%

$\begin{array}{lrrrrr}\text { Level Number } & \text { Mean } & \text { Std Error } & \text { Lower 95\% } & \text { Upper 95\% } \\ \text { U-1-1 } & 3 & 0.074898 & 0.00350 & 0.06684 & 0.08296\end{array}$

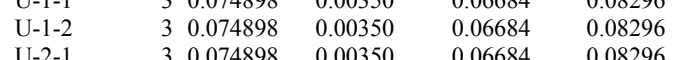

$\begin{array}{llllll}\mathrm{U}-2-1 & 3 & 0.074898 & 0.00350 & 0.06684 & 0.08296\end{array}$

Std Error uses a pooled estimate of error variance

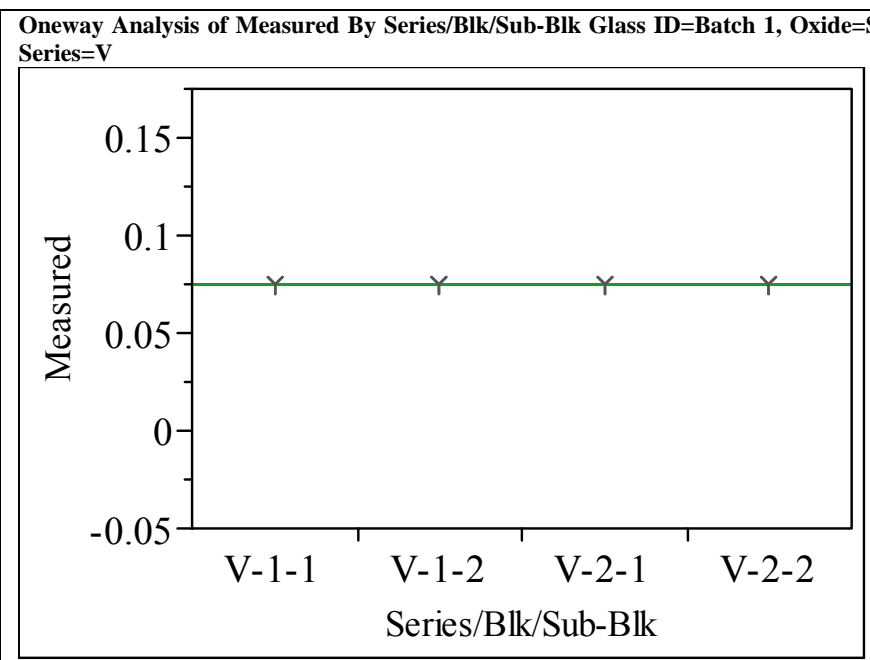

Oneway Anova

Summary of Fit

Rsquare

Adj Rsquare

$\begin{array}{lr}\text { Root Mean Square Error } & 0 \\ \text { Mean of Response } & 0.074898\end{array}$

Observations (or Sum Wgts) 12

Analysis of Variance

Sum of Squares Mean Square F Ratio Prob > F

$\begin{array}{llll}\text { Series/Blk/Sub-Blk } & 3 & 0 & 0 \\ \text { Error } & 8 & 0 & 0 \\ \text { C. } & 1 & 0 & \end{array}$

$\begin{array}{lrr}\text { Error } & 8 & 0 \\ \text { C. Total } & 11 & 0\end{array}$

Means for Oneway Anova

Level Number Mean Std Error Lower 95\% Upper 95\%

$\begin{array}{llllll}V-1-1 & 3 & 0.074898 & 0 & 0.07490 & 0.07490\end{array}$

$\begin{array}{llllll}V-1-2 & 3 & 0.074898 & 0 & 0.07490 & 0.07490\end{array}$

$\begin{array}{llllll}\mathrm{V}-2-1 & 3 & 0.074898 & 0 & 0.07490 & 0.07490\end{array}$

$\begin{array}{llllll}\mathrm{V}-2-2 & 3 & 0.074898 & 0 & 0.07490 & 0.07490\end{array}$

Std Error uses a pooled estimate of error variance 
Exhibit A-3. Measurements by Analytical Series, Block and Sub-Block for the Batch 1 and LRM Standards by Oxide by Prep (continued)

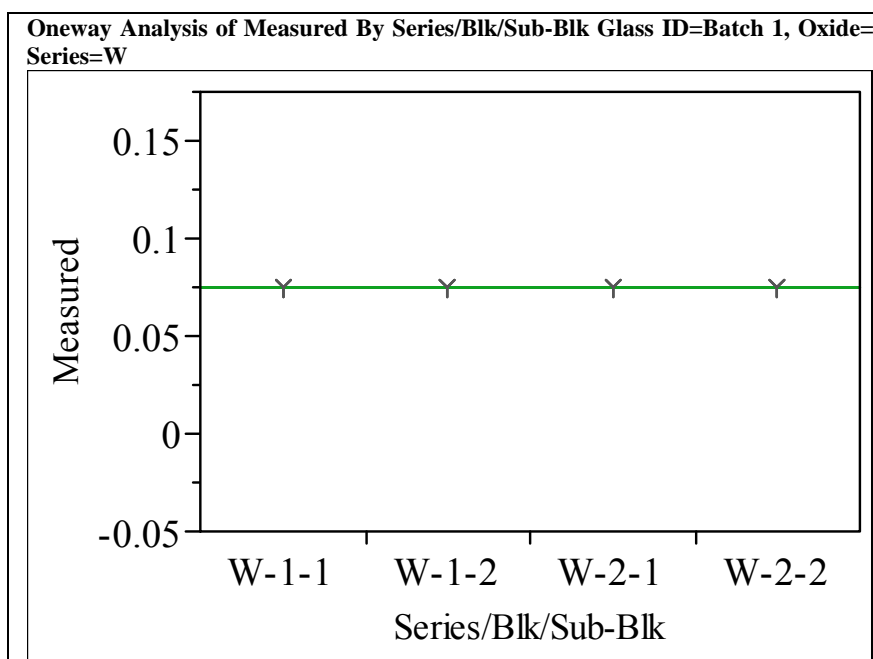

Oneway Anova

Summary of Fit

Rsquare

Adj Rsquare

$\begin{array}{lr}\text { Root Mean Square Error } & 0 \\ \text { Mean of Response } & 0.074898\end{array}$

Observations (or Sum Wgts)

Analysis of Variance

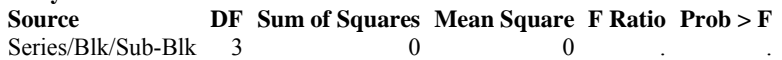

$3 \begin{array}{lr}3 & 0\end{array}$

$\begin{array}{lrl}\text { Error } & \\ \text { C. Total } & 11\end{array}$

0

Means for Oneway Anova

Level Number Mean Std Error Lower 95\% Upper 95\%

$\begin{array}{lllllr}\text { W-1-1 } & 3 & 0.074898 & 0 & 0.07490 & 0.07490\end{array}$

$\begin{array}{llllll}\text { W-1-2 } & 3 & 0.074898 & 0 & 0.07490 & 0.07490\end{array}$

$\begin{array}{llllll}\text { W-2-1 } & 3 & 0.074898 & 0 & 0.07490 & 0.07490 \\ \text { W-2-2 } & 3 & 0.074898 & 0 & 0.07490 & 0.07490\end{array}$

Std Error uses a pooled estimate of error variance

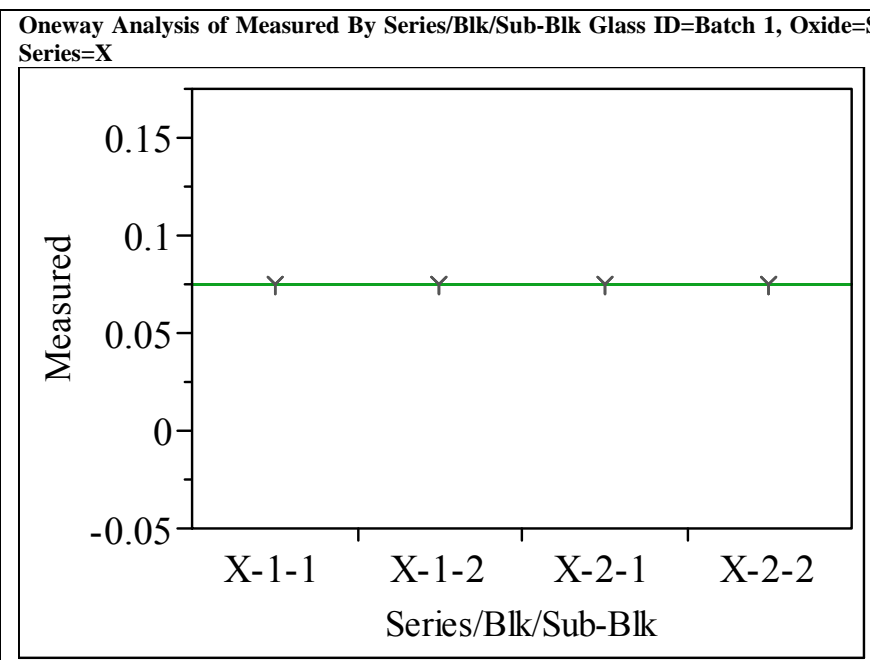

Oneway Anova

Summary of Fit

Rsquare

Adj Rsquare

$\begin{array}{lr}\text { Root Mean Square Error } & 0 \\ \text { Mean of Response } & 0.074898\end{array}$

Observations (or Sum Wgts) 12

Analysis of Variance

Sum of Squares Mean Square F Ratio Prob > F

Series/Blk/Sub-Blk

$\begin{array}{lr}\text { Error } & 8 \\ \text { C. Total } & 11\end{array}$

0
0

Means for Oneway Anova

Level Number Mean Std Error Lower 95\% Upper 95\%

$\begin{array}{llllll}\mathrm{X}-1-1 & 3 & 0.074898 & 0 & 0.07490 & 0.07490 \\ \mathrm{X}-1-2 & 3 & 0.074898 & 0 & 0.0740 & 0.0790\end{array}$

$\begin{array}{llllll}\mathrm{X}-1-2 & 3 & 0.074898 & 0 & 0.07490 & 0.07490\end{array}$

$\begin{array}{llllll}\mathrm{X}-2-1 & 3 & 0.074898 & 0 & 0.07490 & 0.07490\end{array}$

$\begin{array}{llllll}\mathrm{X}-2-2 & 3 & 0.074898 & 0 & 0.07490 & 0.07490\end{array}$

Std Error uses a pooled estimate of error variance 


\section{Exhibit A-3. Measurements by Analytical Series, Block and Sub-Block for the Batch 1 and LRM Standards by Oxide by Prep (continued)}

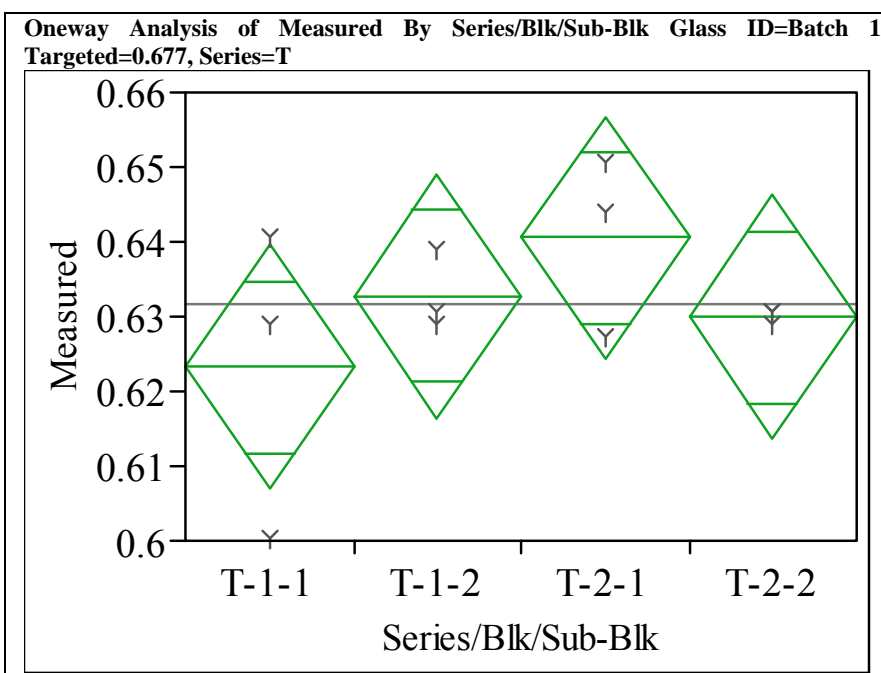

\section{Oneway Anova}

Summary of Fit

$\begin{array}{lr}\text { Rsquare } & 0.276906 \\ \text { Adj Rsquare } & 0.005746 \\ \text { Root Mean Square Error } & 0.012229 \\ \text { Mean of Response } & 0.631616 \\ \text { Observations (or Sum Wgts) } & 12\end{array}$

Analysis of Variance

Source DF Sum of Squares Mean Square F Ratio Prob > F

$\begin{array}{llllll}\text { Series/Blk/Sub-Blk } & 3 & 0.00045814 & 0.000153 & 1.0212 & 0.4329\end{array}$

Error $\quad 8 \quad 0.00110636$

$\begin{array}{lll}0.000153 & 1.0212 & 0.4329 \\ 0.000150 & & \end{array}$

C. Total

0.001165450

Means for Oneway Anova

Level Number Mean Std Error Lower 95\% Upper 95\%

$\begin{array}{llllll}\text { T-1-1 } & 3 & 0.623276 & 0.00706 & 0.60699 & 0.63956\end{array}$

$\begin{array}{llllll}\mathrm{T}-1-1 & 3 & 0.6232728 & 0.00706 & 0.61645 & 0.6490 \\ \mathrm{~T}-1-2 & 3 & 0.6320512 & 0.00706 & 0.62423 & 0.65679 \\ \mathrm{~T}-2-1 & 3 & 0.6405948 & 0.00706 & 0.61367 & 0.64623\end{array}$

$\begin{array}{llllll}T-1-2 & 3 & 0.632728 & 0.00706 & 0.61645 & 0.64901 \\ T-2-1 & 3 & 0.64512 & 0.00706 & 0.62423 & 0.65679 \\ \mathrm{~T}-2-2 & 3 & 0.629948 & 0.00706 & 0.61367 & 0.64623\end{array}$

Std Error uses a pooled estimate of error variance

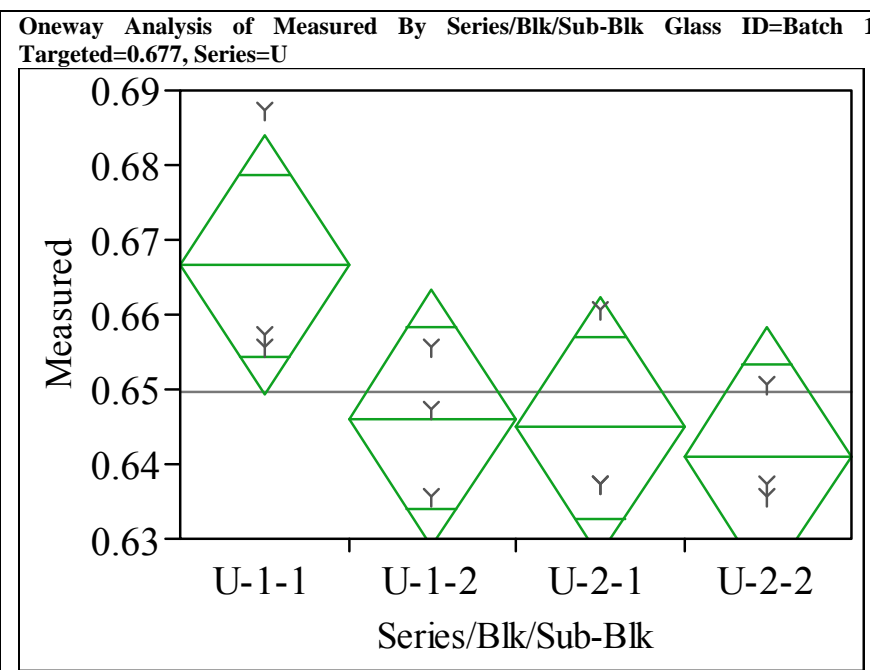

Oneway Anova

Summary of Fit

$\begin{array}{lr}\text { Rsquare } & 0.471214 \\ \text { Adj Rsquare } & 0.272919 \\ \text { Root Mean Square Error } & 0.012929 \\ \text { Mean of Response } & 0.649686 \\ \text { Observations (or Sum Wgts) } & 12\end{array}$

Analysis of Variance

$\begin{array}{lrrrrr}\text { Source } & \text { DF } & \text { Sum of Squares } & \text { Mean Square } & \text { F Ratio } & \text { Prob }>\text { F } \\ \text { Series/Blk/Sub-Blk } & 3 & 0.00119172 & 0.000397 & 2.3763 & 0.1458\end{array}$

$\begin{array}{lrrr}\text { Series/Blk/Sub-Blk } & 3 & 0.00119172 & 0.000397 \\ \text { Error } & 8 & 0.00133732 & 0.000167 \\ \text { C. Total } & 11 & 0.00252904 & \\ \end{array}$

0.00252904

Means for Oneway Anova

Level Number Mean Std Error Lower 95\% Upper 95\%

$\begin{array}{rrrrrr}\text { U-1-1 } & 3 & 0.666644 & 0.00746 & 0.64943 & 0.68386\end{array}$

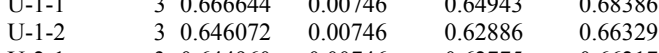

$\begin{array}{lllllll} & 3 & 0.644960 & 0.00746 & 0.62775 & 0.66217\end{array}$

Std Error uses a pooled estimate of error variance 
Exhibit A-3. Measurements by Analytical Series, Block and Sub-Block for the Batch 1 and LRM Standards by Oxide by Prep (continued)

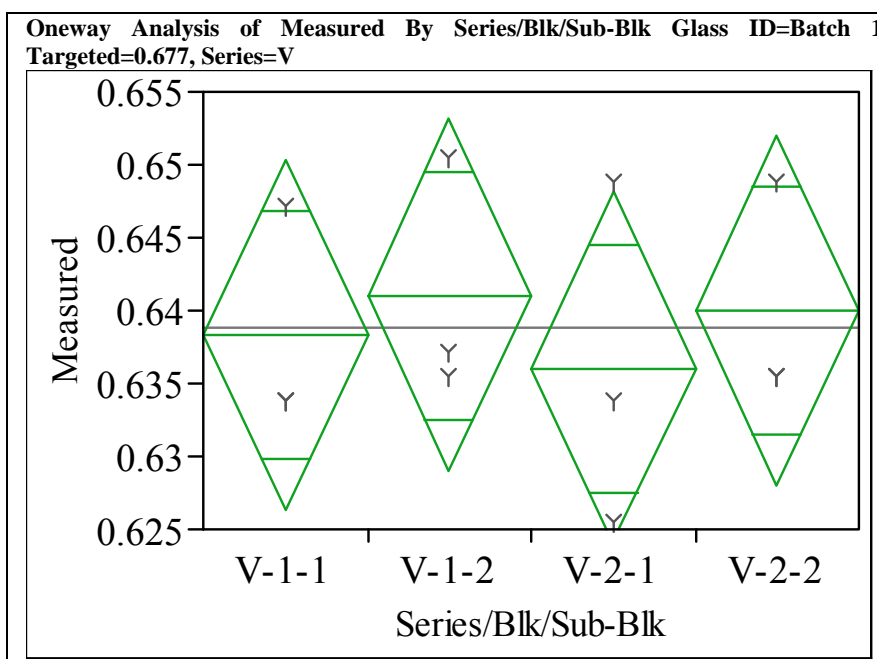

Oneway Anova

Summary of Fit

Rsquare $\quad 0.061333$

Adj Rsquare $\quad-0.29067$

Root Mean Square Error $\quad 0.009034$

$\begin{array}{lr}\text { Mean of Response } & 0.638844 \\ \text { Observations (or Sum Wgts) } & 12\end{array}$

Analysis of Variance

$\begin{array}{lcrrrrr}\text { Source } & \text { DF } & \text { Sum of Squares } & \text { Mean Square } & \text { F Ratio } & \text { Prob }>\text { F } \\ \text { Series/Blk/Sub-Blk } & 3 & 0.00004266 & 0.000014 & 0.1742 & 0.9108\end{array}$

Error

0.00065290

0.000082

Means for Oneway Anova

Level Number Mean Std Error Lower 95\% Upper 95\%

$\begin{array}{lllrrr}\mathrm{V}-1-1 & 3 & 0.638288 & 0.00522 & 0.62626 & 0.65032\end{array}$

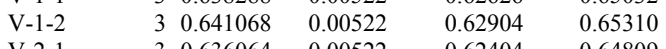

$\begin{array}{lllllll}\text { V-2-1 } & & 3 & 0.636064 & 0.00522 & 0.62404 & 0.64809 \\ \text { V-2-2 } & & 3 & 0.639956 & 0.00522 & 0.62793 & 0.65198\end{array}$

Std Error uses a pooled estimate of error variance

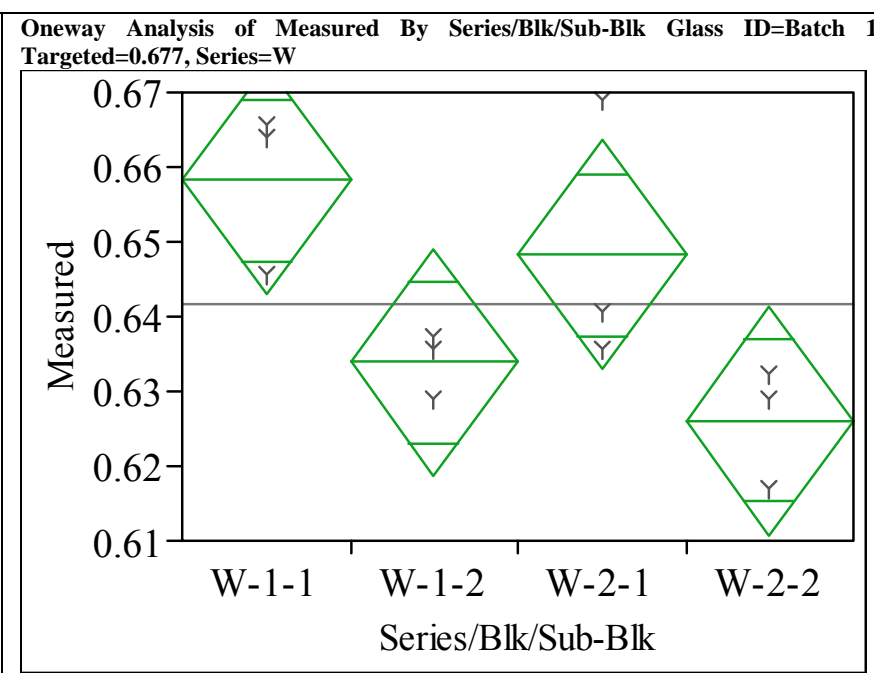

Oneway Anova

Summary of Fit

$\begin{array}{lr}\text { Rsquare } & 0.639697 \\ \text { Adj Rsquare } & 0.504583 \\ \text { Root Mean Square Error } & 0.011496 \\ \text { Mean of Response } & 0.641624 \\ \text { Observations (or Sum Wgts) } & 12\end{array}$

Observations (or Sum Wots) 0.641624

Analysis of Variance

Source DF Sum of Squares Mean Square F Ratio Prob $>$ F

$\begin{array}{lrrrrr}\text { Series/Blk/Sub-Blk } & 3 & 0.00187707 & 0.000626 & 4.7345 & 0.0350\end{array}$

$\begin{array}{lll}\text { Error } & 8 & 0.00105725\end{array}$

0.000132

C. Total

$11 \quad 0.00293432$

Means for Oneway Anova

Level Number Mean Std Error Lower 95\% Upper 95\%

$\begin{array}{llllll}\text { W-1-1 } & 3 & 0.658304 & 0.00664 & 0.64300 & 0.67361\end{array}$

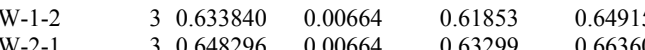

$\begin{array}{llllll}W-1-2 & 3 & 0.633840 & 0.00664 & 0.61853 & 0.64915 \\ W-2-2 & 3 & 0.648296 & 0.00664 & 0.63299 & 0.66360\end{array}$

Std Error uses a pooled estimate of error variance 


\section{Exhibit A-3. Measurements by Analytical Series, Block and Sub-Block for the Batch 1 and LRM Standards by Oxide by Prep (continued)}

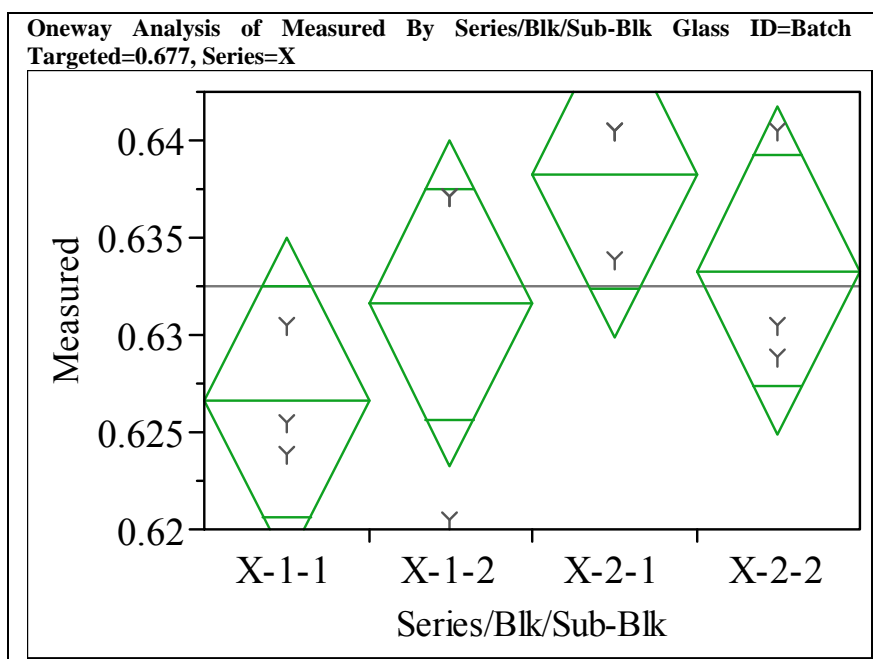

Oneway Anova

Summary of Fit

0.395431

Adj Rsquare $\quad 0.168717$

Root Mean Square Error $\quad 0.006315$

$\begin{array}{lr}\text { Mean of Response } & 0.63245 \\ \text { Observations (or Sum Wgts) } & 12\end{array}$

Analysis of Variance

$\begin{array}{lrrrrr}\text { Source } & \text { DF } & \text { Sum of Squares } & \text { Mean Square } & \text { F Ratio } & \text { Prob > F } \\ \text { Series/Blk/Sub-Blk } & 3 & 0.00020867 & 0.000070 & 1.7442 & 0.2352 \\ \text { Error } & 8 & 0.00031903 & 0.000040 & & \\ \text { C. Total } & 11 & 0.00052770 & & & \\ & & & & & \end{array}$

Means for Oneway Anova

Level Number Mean Std Error Lower 95\% Upper 95\%

$\begin{array}{llllll}\text { X-1-1 } & 3 & 0.626612 & 0.00365 & 0.61820 & 0.63502 \\ \text { X-1-2 } & 3 & 0.631616 & 0.0365 & 0.62321 & 0.6402\end{array}$

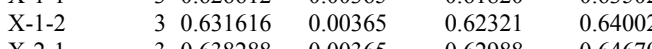

$\begin{array}{lllllll}\mathrm{X}-2-1 & & 3 & 0.638288 & 0.00365 & 0.62988 & 0.64670\end{array}$

$\begin{array}{llllll}\mathrm{X}-2-2 & 3 & 0.633284 & 0.00365 & 0.62488 & 0.64169\end{array}$

Std Error uses a pooled estimate of error variance

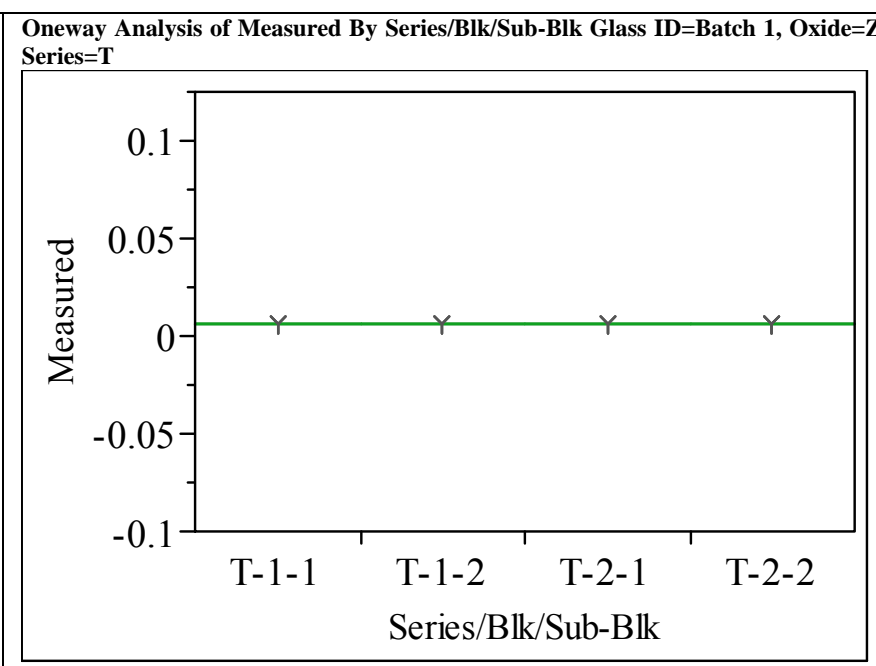

Oneway Anova

Summary of Fit

Rsquare

Adj Rsquare

$\begin{array}{lr}\text { Root Mean Square Error } & 0 \\ \text { Mean of Response } & 0.006224\end{array}$

Observations (or Sum Wgts) 12

Analysis of Variance

Sum of Squares Mean Square F Ratio Prob > F

$\begin{array}{llll}\text { Series/Blk/Sub-Blk } & 3 & 0 & 0 \\ \text { Error } & 8 & 0 & 0\end{array}$

$\begin{array}{lrr}\text { Error } & 8 & 0 \\ \text { C. Total } & 11 & 0\end{array}$

Means for Oneway Anova

Level Number Mean Std Error Lower 95\% Upper 95\%

$\begin{array}{llllll}\text { T-1-1 } & 3 & 0.006224 & 0 & 0.00622 & 0.00622\end{array}$

$\begin{array}{llllll}\mathrm{T}-1-2 & 3 & 0.006224 & 0 & 0.00622 & 0.00622\end{array}$

$\begin{array}{llllll}\mathrm{T}-2-1 & 3 & 0.006224 & 0 & 0.00622 & 0.00622\end{array}$

$\begin{array}{llllll}\text { T-2-2 } & 3 & 0.006224 & 0 & 0.00622 & 0.00622\end{array}$

Std Error uses a pooled estimate of error variance 
Exhibit A-3. Measurements by Analytical Series, Block and Sub-Block for the Batch 1 and LRM Standards by Oxide by Prep (continued)

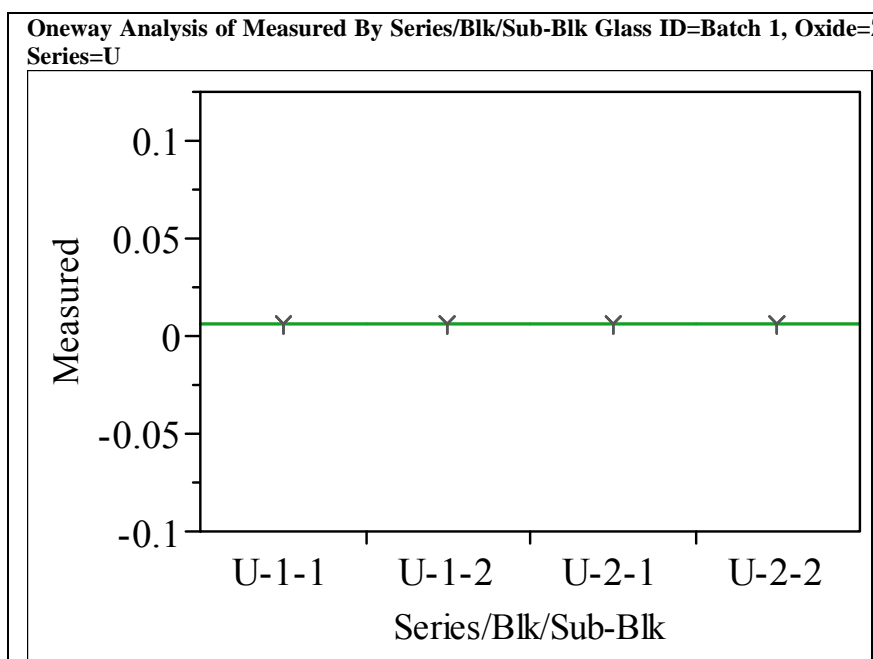

Oneway Anova

Summary of Fit

Rsquare

Adj Rsquare

$\begin{array}{lr}\text { Root Mean Square Error } & 0 \\ \text { Mean of Response } & 0.006224\end{array}$

Observations (or Sum Wgts)

Analysis of Variance

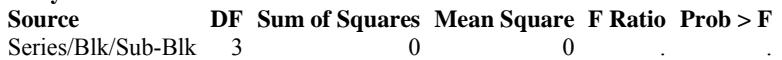

$\begin{array}{lr}\text { Error } & 8 \\ \text { C. Total } & 11\end{array}$

$\begin{array}{lll}0 & 0 \\ 0 & 0\end{array}$

0

Means for Oneway Anova

Level Number Mean Std Error Lower 95\% Upper 95\%

$\begin{array}{llllll}\text { U-1-1 } & 3 & 0.006224 & 0 & 0.00622 & 0.00622\end{array}$

$\begin{array}{llllll}\text { U-1-2 } & 3 & 0.006224 & 0 & 0.00622 & 0.00622\end{array}$

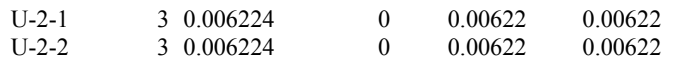

Std Error uses a pooled estimate of error variance

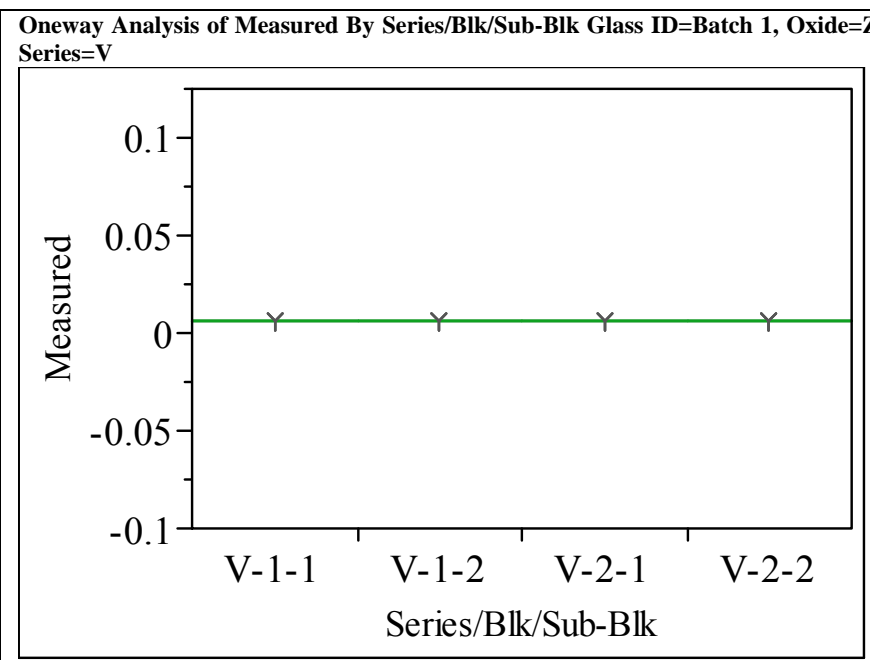

Oneway Anova

Summary of Fit

Rsquare

Adj Rsquare

$\begin{array}{lr}\text { Root Mean Square Error } & 0 \\ \text { Mean of Response } & 0.006224\end{array}$

Observations (or Sum Wgts) 12

Analysis of Variance

Sum of Squares Mean Square F Ratio Prob > F

Series/Blk/Sub-Blk

$\begin{array}{lr}\text { Error } & 8 \\ \text { C. Total } & 11\end{array}$

0

Means for Oneway Anova

Level Number Mean Std Error Lower 95\% Upper 95\%

$\begin{array}{llllll}\mathrm{V}-1-1 & 3 & 0.006224 & 0 & 0.00622 & 0.00622\end{array}$

$\begin{array}{llllll}V-1-2 & 3 & 0.006224 & 0 & 0.00622 & 0.00622\end{array}$

$\begin{array}{llllll}V-2-1 & 3 & 0.006224 & 0 & 0.00622 & 0.00622\end{array}$

$\begin{array}{llllll}\mathrm{V}-2-2 & 3 & 0.006224 & 0 & 0.00622 & 0.00622\end{array}$

Std Error uses a pooled estimate of error variance 
Exhibit A-3. Measurements by Analytical Series, Block and Sub-Block for the Batch 1 and LRM Standards by Oxide by Prep (continued)

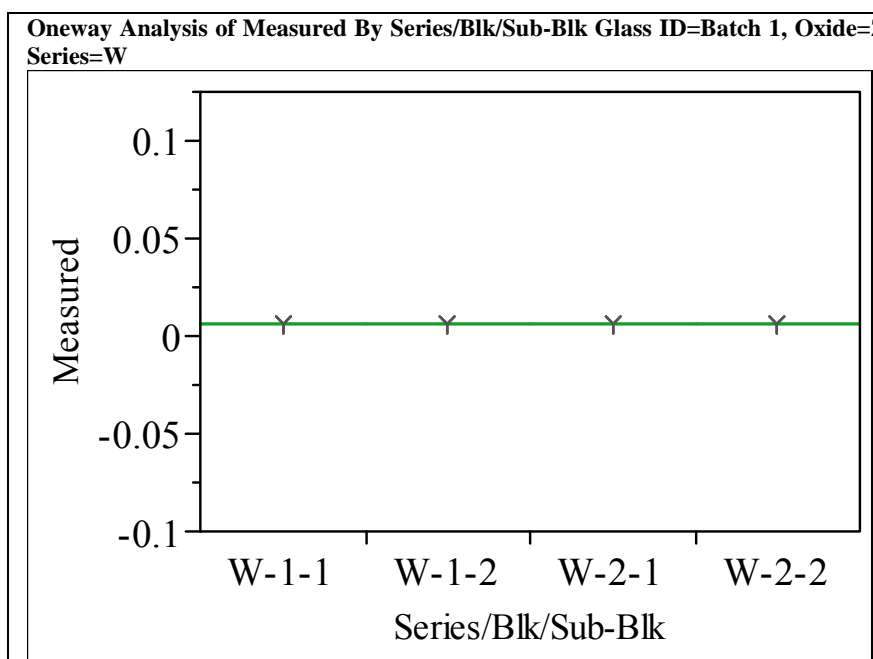

Oneway Anova

Summary of Fit

Rsquare

Adj Rsquare

$\begin{array}{lr}\text { Root Mean Square Error } & 0 \\ \text { Mean of Response } & 0.006224\end{array}$

Observations (or Sum Wgts)

Analysis of Variance

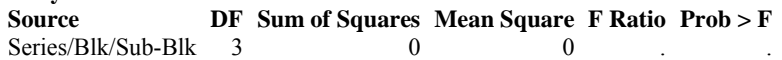

$3 \begin{array}{lr}3 & 0 \\ 8 & 0\end{array}$

Mean Square

C. Total

11

Means for Oneway Anova

Level Number Mean Std Error Lower 95\% Upper 95\%

$\begin{array}{rrrrrr}\text { W-1-1 } & 3 & 0.006224 & 0 & 0.00622 & 0.00622 \\ \text { W-12 } & 3 & 0.006224 & 0 & 0.00622 & 0.0022\end{array}$

$\begin{array}{llllll}\text { W-1-2 } & 3 & 0.006224 & 0 & 0.00622 & 0.00622\end{array}$

$\begin{array}{llllll}\text { W-2-1 } & 3 & 0.006224 & 0 & 0.00622 & 0.00622\end{array}$

$\begin{array}{llllll}\text { W-2-2 } & 3 & 0.006224 & 0 & 0.00622 & 0.00622\end{array}$

Std Error uses a pooled estimate of error variance

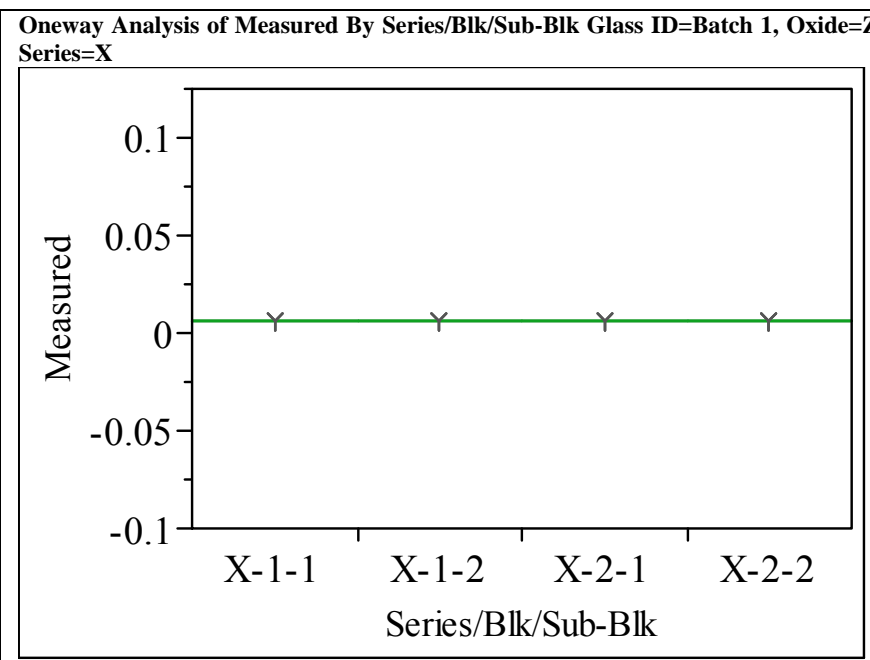

Oneway Anova

Summary of Fit

Rsquare

Adj Rsquare

$\begin{array}{lr}\text { Root Mean Square Error } & 0 \\ \text { Mean of Response } & 0.006224\end{array}$

Observations (or Sum Wgts) 12

Analysis of Variance

Sum of Squares Mean Square F Ratio Prob > F

$\begin{array}{llll}\text { Series/Blk/Sub-Blk } & 3 & 0 & 0 \\ \text { Error } & 8 & 0 & 0\end{array}$

$\begin{array}{lrr}\text { Error } & 8 & 0 \\ \text { C. Total } & 11 & 0\end{array}$

Means for Oneway Anova

Level Number Mean Std Error Lower 95\% Upper 95\%

$\begin{array}{llllll}\mathrm{X}-1-1 & 3 & 0.006224 & 0 & 0.00622 & 0.00622\end{array}$

$\begin{array}{llllll}\mathrm{X}-1-2 & 3 & 0.006224 & 0 & 0.00622 & 0.00622\end{array}$

$\begin{array}{llllll}\mathrm{X}-2-1 & 3 & 0.006224 & 0 & 0.00622 & 0.00622\end{array}$

$\begin{array}{llllll}\mathrm{X}-2-2 & 3 & 0.006224 & 0 & 0.00622 & 0.00622\end{array}$

Std Error uses a pooled estimate of error variance 
Exhibit A-3. Measurements by Analytical Series, Block and Sub-Block for the Batch 1 and LRM Standards by Oxide by Prep (continued)

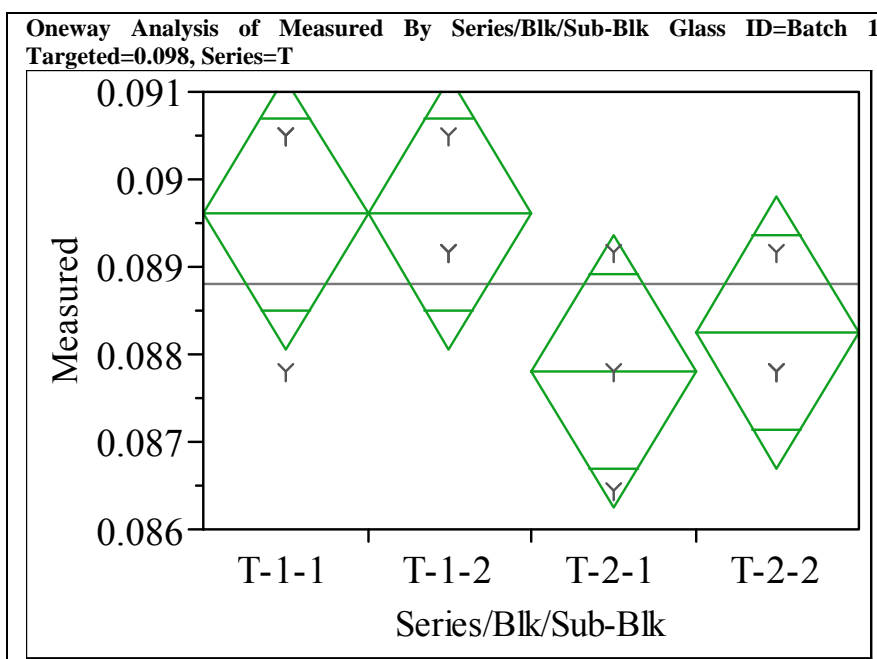

Oneway Anova

Summary of Fit

$\begin{array}{lr}\text { Rsquare } & 0.414634 \\ \text { Adj Rsquare } & 0.195122 \\ \text { Root Mean Square Error } & 0.00117 \\ \text { Mean of Response } & 0.088815 \\ \text { Observations (or Sum Wgts) } & 12\end{array}$

Analysis of Variance

Source DF Sum of Squares Mean Square F Ratio Prob > F

$\begin{array}{llllll}\text { Series/Blk/Sub-Blk } & 3 & 0.00000775 & 2.5849 \mathrm{e}-6 & 1.8889 & 0.2099 \\ \text { Error } & 8 & 0.00001095 & 1.3685 \mathrm{e}-6 & & \end{array}$

$1.3685 \mathrm{e}-6$

Means for Oneway Anova

Level Number Mean Std

\begin{tabular}{llllrr} 
T-1-1 & 3 & 0.089603 & 0.00068 & 0.08805 & 0.09116 \\
\hline & 3 & 0.00963 & 0.0068 & 0.08805 & 0.0916
\end{tabular}

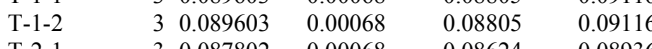

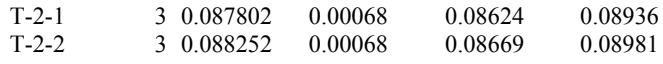

Std Error uses a pooled estimate of error variance

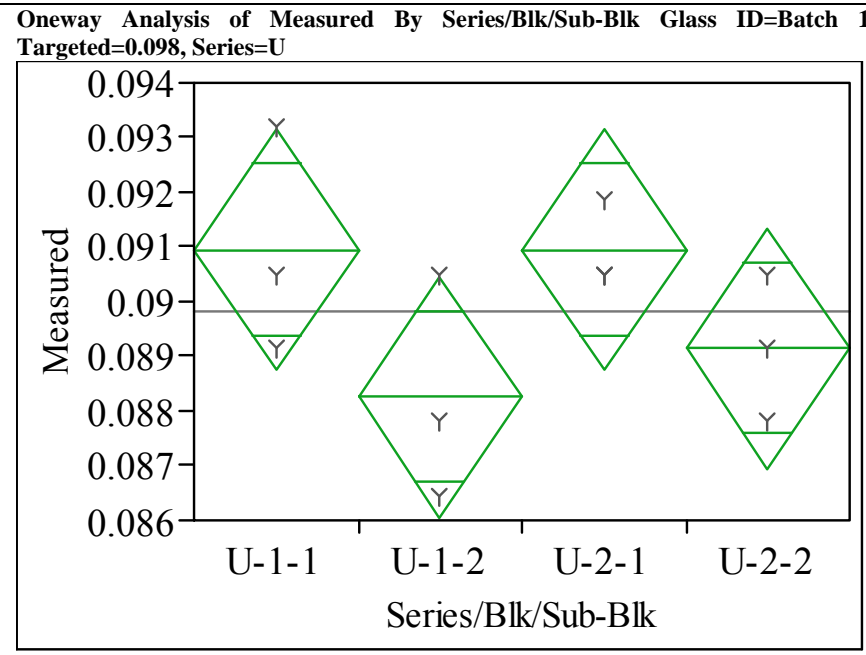

\section{Oneway Anova}

Summary of Fit

$\begin{array}{lr}\text { Rsquare } & 0.428571 \\ \text { Adj Rsquare } & 0.214286 \\ \text { Root Mean Square Error } & 0.001654 \\ \text { Mean of Response } & 0.089828 \\ \text { Observations (or Sum Wgts) } & 12\end{array}$

Analysis of Variance

Source DF Sum of Squares Mean Square F Ratio Prob > F

$\begin{array}{llllll}\text { Series/Blk/Sub-Blk } & 3 & 0.00001642 & 5.474 \mathrm{e}-6 & 2.0000 & 0.1927\end{array}$

$\begin{array}{lll}\text { Error } & 8 & 0.00002190 \\ \text { C. } & 11 & 0.00003832\end{array}$ $2.737 \mathrm{e}-6$

Means for Oneway Anova

Level Number Mean Std Error Lower 95\% Upper 95\%

$\begin{array}{llllll}\text { U-1-1 } & 3 & 0.090954 & 0.00096 & 0.08875 & 0.09316\end{array}$

$\begin{array}{llllll}\mathrm{U}-1-2 & 3 & 0.088252 & 0.00096 & 0.08605 & 0.09045\end{array}$

$\begin{array}{llllll}\mathrm{U}-2-1 & 3 & 0.090954 & 0.00096 & 0.08875 & 0.09316\end{array}$

$\begin{array}{llllll}\text { U-2-2 } & 3 & 0.089153 & 0.00096 & 0.08695 & 0.09136\end{array}$

Std Error uses a pooled estimate of error variance 
Exhibit A-3. Measurements by Analytical Series, Block and Sub-Block for the Batch 1 and LRM Standards by Oxide by Prep (continued)

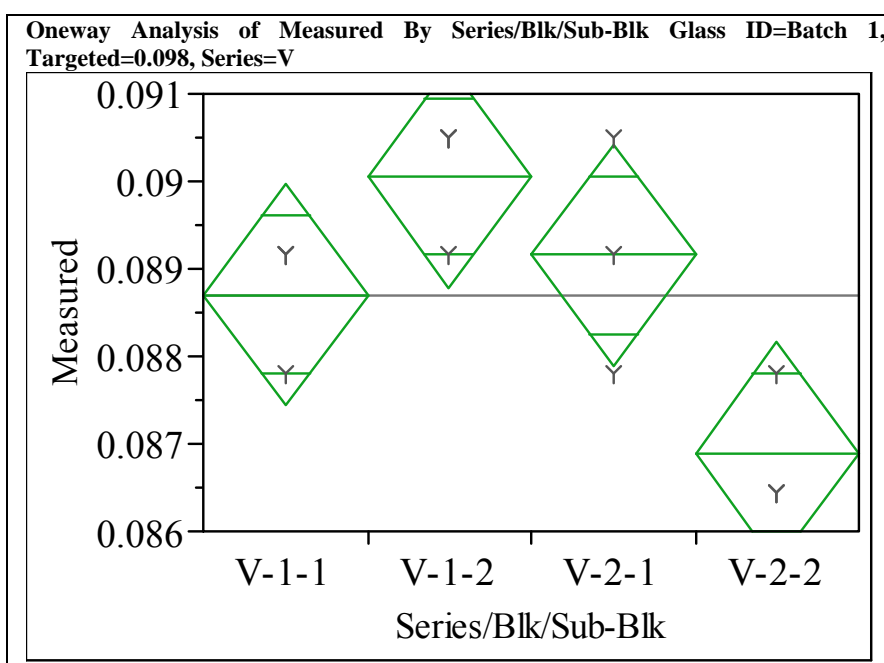

Oneway Anova

Summary of Fit

0.684211

Adj Rsquare $\quad 0.565789$

Root Mean Square Error $\quad 0.00095$

$\begin{array}{lr}\text { Mean of Response } & 0.088703 \\ \text { Observations (or Sum Wgts) } & 12\end{array}$

Analysis of Variance

Source DF Sum of Squares Mean Square F Ratio Prob $>$ F

$\begin{array}{lrrrrr}\text { Series/Blk/Sub-Blk } & 3 & 0.00001581 & 5.2712 \mathrm{e}-6 & 5.7778 & 0.0211 \\ \text { Error } & 8 & 0.00000730 & 9.1233 \mathrm{e}-7 & & \end{array}$

Error

$9.1233 \mathrm{e}-7$

Means for Oneway Anova

Level Number Mean Std Error Lower 95\% Upper 95\%

$\begin{array}{lllrrr}\mathrm{V}-1-1 & 3 & 0.088703 & 0.00055 & 0.08743 & 0.08997\end{array}$

$\begin{array}{llllll}\mathrm{V}-1-2 & 3 & 0.090053 & 0.00055 & 0.08878 & 0.09133\end{array}$

$\begin{array}{llllll}\text { V-2-1 } & 3 & 0.089153 & 0.00055 & 0.08788 & 0.09042\end{array}$

$\begin{array}{llllll}\mathrm{V}-2-2 & 3 & 0.086901 & 0.00055 & 0.08563 & 0.08817\end{array}$

Std Error uses a pooled estimate of error variance

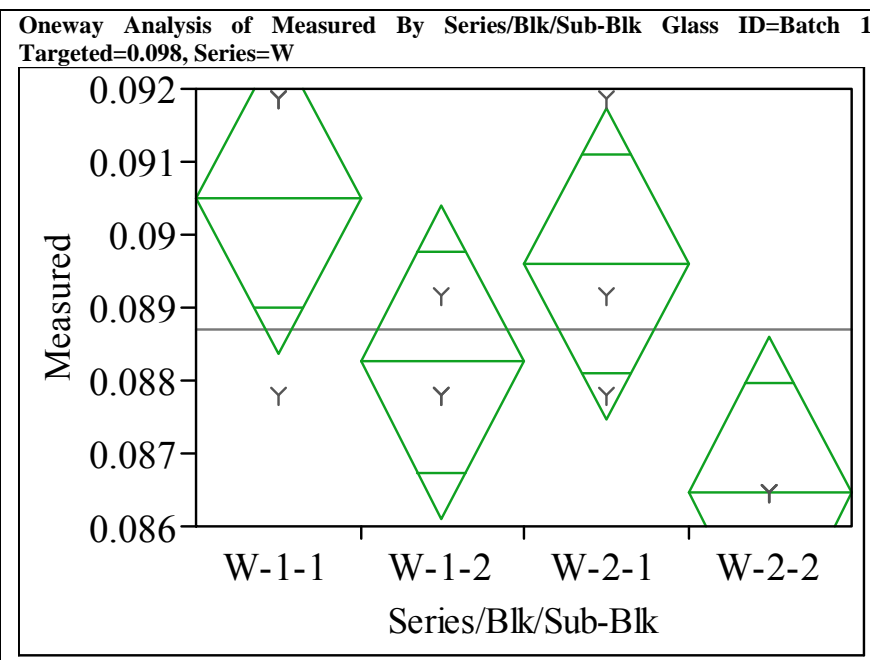

\section{Oneway Anova}

Summary of Fit

Rsquare

$\begin{array}{lr} & 0.575 \\ \text { Adj Rsquare } & 0.415625\end{array}$

Root Mean Square Error $\quad 0.001608$

Mean of Response

Observations (or Sum Wgts) $\begin{array}{r}0.088703 \\ \hline\end{array}$

Analysis of Variance

Source DF Sum of Squares Mean Square F Ratio Prob > F

$\begin{array}{llllll}\text { Series/Blk/Sub-Blk } & 3 & 0.00002798 & 9.326 \mathrm{e}-6 & 3.6078 & 0.0651\end{array}$

$\begin{array}{lll}\text { Error } & 8 & 0.00002068\end{array}$

$2.5849 \mathrm{e}-6$

Means for Oneway Anova

Level Number Mean Std Error Lower 95\% Upper 95\%

$\begin{array}{llllll}\mathrm{W}-1-1 & 3 & 0.090504 & 0.00093 & 0.08836 & 0.09264\end{array}$

$\begin{array}{llllll}\mathrm{W}-1-2 & 3 & 0.088252 & 0.00093 & 0.08611 & 0.09039\end{array}$

$\begin{array}{llllll}\text { W-2-1 } & 3 & 0.089603 & 0.00093 & 0.08746 & 0.09174\end{array}$

$\begin{array}{llllll}\text { W-2-2 } & 3 & 0.086451 & 0.00093 & 0.08431 & 0.08859\end{array}$

Std Error uses a pooled estimate of error variance 
Exhibit A-3. Measurements by Analytical Series, Block and Sub-Block for the Batch 1 and LRM Standards by Oxide by Prep (continued)

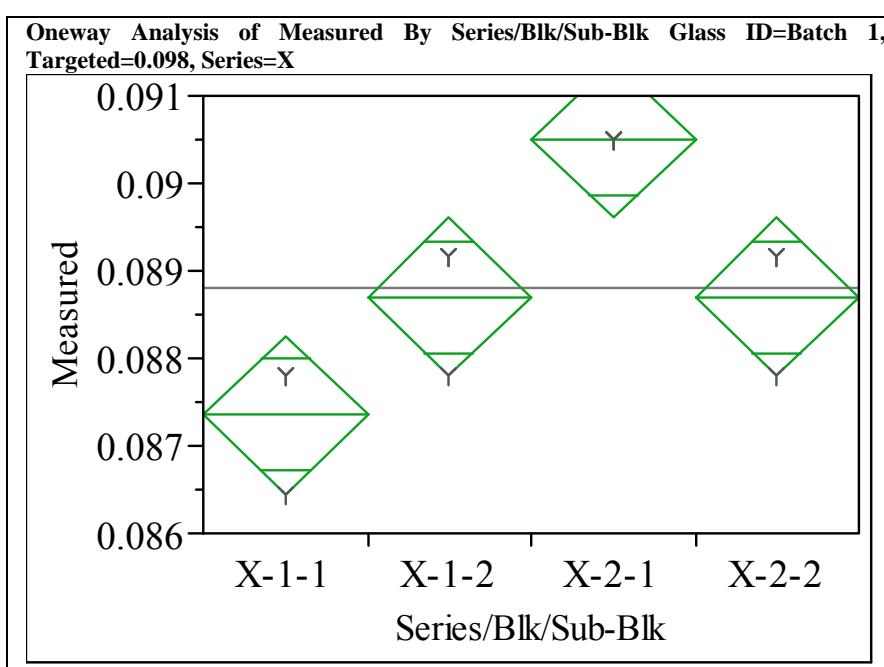

Oneway Anova

Summary of Fit

Rsquare $\quad 0.804878$

Adj Rsquare $\quad 0.731707$
Ret

Root Mean Square Error $\quad 0.000675$

$\begin{array}{lr}\text { Mean of Response } & 0.088815 \\ \text { Observations (or Sum Wgts) } & 12\end{array}$

Analysis of Variance

Source DF Sum of Squares Mean Square F Ratio Prob $>$ F

$\begin{array}{llllll}\text { Series/Blk/Sub-Blk } & 3 & 0.00001505 & 5.0178 \mathrm{e}-6 & 11.0000 & 0.0033\end{array}$

Error

0.00000365 $4.5617 \mathrm{e}-7$

Means for Oneway Anova

Level Number Mean Std Error Lower 95\% Upper 95\%

$\begin{array}{rrrrrr}\mathrm{X}-1-1 & 3 & 0.087352 & 0.00039 & 0.08645 & 0.08825\end{array}$

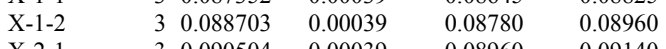

$\begin{array}{llllll}\mathrm{X}-2-1 & 3 & 0.090504 & 0.00039 & 0.08960 & 0.09140\end{array}$

$\begin{array}{llllll}\mathrm{X}-2-2 & 3 & 0.088703 & 0.00039 & 0.08780 & 0.08960\end{array}$

Std Error uses a pooled estimate of error variance

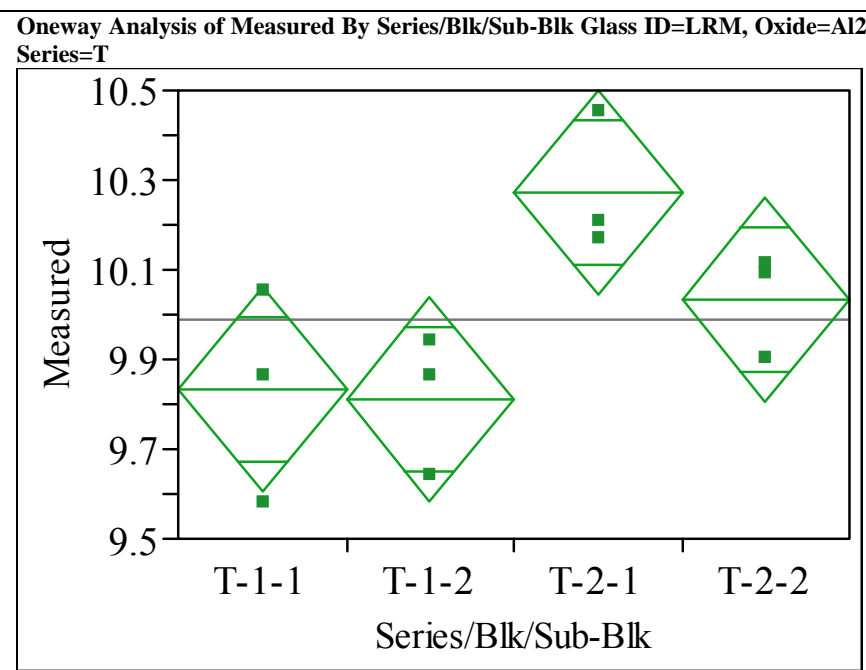

Oneway Anova

Summary of Fit

$\begin{array}{lr}\text { Rsquare } & 0.636846 \\ \text { Adj Rsquare } & 0.500663 \\ \text { Root Mean Square Error } & 0.171882 \\ \text { Mean of Response } & 9.987582 \\ \text { Observations (or Sum Wgts) } & 12\end{array}$

Observations (or Sum Wgts) 9.987582

Analysis of Variance

Source DF Sum of Squares Mean Square F Ratio Prob > F

$\begin{array}{lrrrrr}\text { Series/Blk/Sub-Blk } & 3 & 0.41447166 & 0.138157 & 4.6764 & 0.0360\end{array}$

C. Total

8
11

.029543

Means for Oneway Anova

Level Number Mean Std Error Lower 95\% Upper 95\%

$\begin{array}{lllllr}\text { T-1-1 } & 3 & 9.8317 & 0.09924 & 9.603 & 10.061 \\ \mathrm{~T}-1-2 & 3 & 9.8128 & 0.09924 & 9.584 & 10.042\end{array}$

$\begin{array}{rrrrrr}T-1-1 & 3 & 9.8128 & 0.09924 & 9.584 & 10.042 \\ \mathrm{~T}-1-2 & 3 & 10.2726 & 0.09924 & 10.044 & 10.501\end{array}$

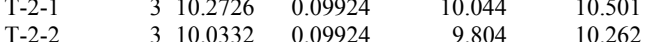

Std Error uses a pooled estimate of error variance 
Exhibit A-3. Measurements by Analytical Series, Block and Sub-Block for the Batch 1 and LRM Standards by Oxide by Prep (continued)

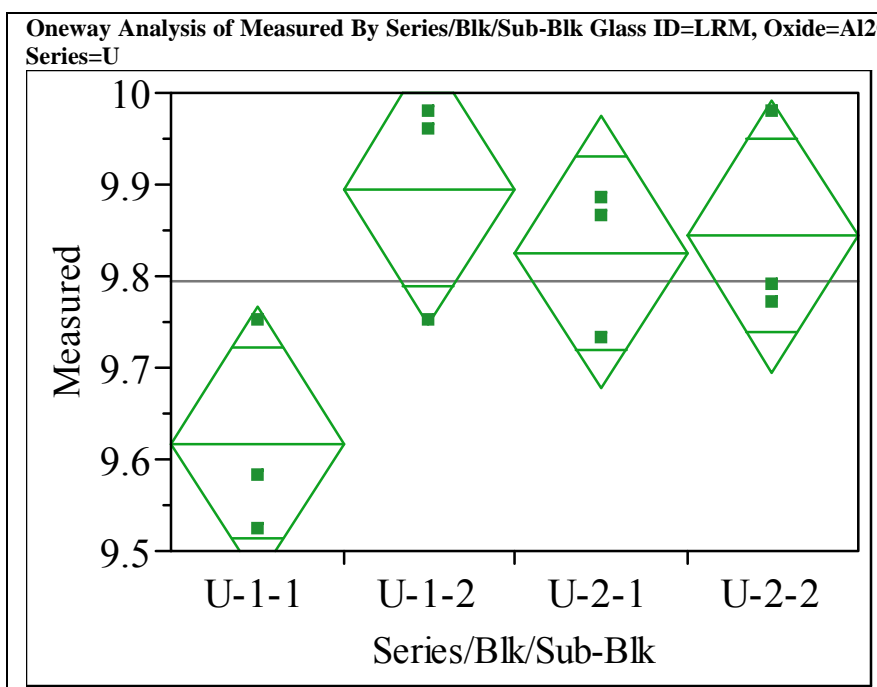

\section{Oneway Anova}

Summary of Fit

Rsquare $\quad 0.574501$

Adj Rsquare $\quad 0.414938$

Root Mean Square Error $\quad 0.111518$

$\begin{array}{lr}\text { Mean of Response } & 9.795483 \\ \text { Observations (or Sum Wgts) } & 12\end{array}$

Analysis of Variance

$\begin{array}{lrrrrrr}\text { Source } & \text { DF } & \text { Sum of Squares } & \text { Mean Square } & \text { F Ratio } & \text { Prob }>\text { F } \\ \text { Series/Blk/Sub-Blk } & 3 & 0.13432916 & 0.044776 & 3.6005 & 0.0654 \\ \text { Error } & 8 & 0.09948986 & 0.012436 & & \\ \text { C. Total } & 11 & 0.23381902 & & & \\ & & & & & & \end{array}$

Means for Oneway Anova

Level Number Mean Std Error Lower 95\% Upper 95\%

$\begin{array}{lrrrrr}\text { U-1-1 } & 3 & 9.61756 & 0.06438 & 9.4691 & 9.766\end{array}$

$\begin{array}{lllllr}\text { U-1-2 } & 3 & 9.89468 & 0.06438 & 9.7462 & 10.043\end{array}$

$\begin{array}{llllll}\text { U-2-1 } & 3 & 9.82540 & 0.06438 & 9.6769 & 9.974 \\ \text { U-2-2 } & 3 & 9.84430 & 0.06438 & 9.6958 & 9.993\end{array}$

Std Error uses a pooled estimate of error variance

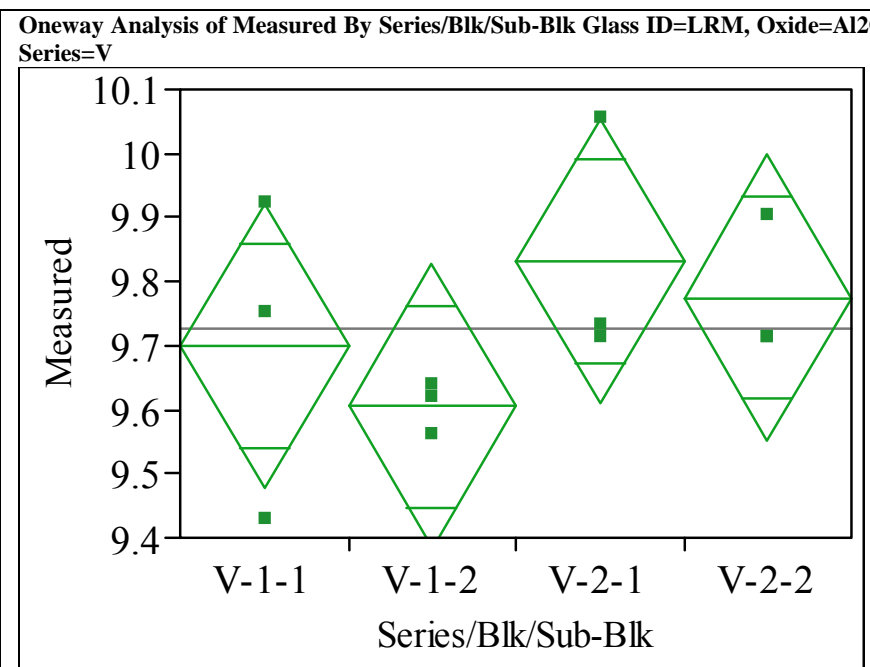

Oneway Anova

Summary of Fit

$\begin{array}{lr}\text { Rsquare } & 0.278776 \\ \text { Adj Rsquare } & 0.008317 \\ \text { Root Mean Square Error } & 0.167499 \\ \text { Mean of Response } & 9.727776 \\ \text { Observations (or Sum Wgts) } & 12\end{array}$

Analysis of Variance

$\begin{array}{lrrrrr}\text { Source } & \text { DF } & \text { Sum of Squares } & \text { Mean Square } & \text { F Ratio } & \text { Prob }>\text { F } \\ \text { Series/Blk/Sub-Blk } & 3 & 0.08675611 & 0.028919 & 1.0308 & 0.4292\end{array}$

$\begin{array}{lrrr}\text { Series/Blk/Sub-Blk } & 3 & 0.08675611 & 0.028919 \\ \text { Error } & 8 & 0.22444722 & 0.028056 \\ \text { C. Total } & 11 & 0.31120333 & \\ \end{array}$

Means for Oneway Anova

Means for Oneway Anova
Level Number Mean Std Error Lower 95\% Upper 95\%

$\begin{array}{rrrrrr}\text { Level } & \text { Number } & \text { Mean } & \text { Std Error } & \text { Lower 95\% } & \text { Upper 95\% } \\ \text { V-1-1 } & 3 & 9.69943 & 0.09671 & 9.4764 & 9.922\end{array}$

$\begin{array}{llllll}\mathrm{V}-1-1 & 3 & 9.69943 & 0.09671 & 9.4764 & 9.922 \\ \mathrm{~V}-1-2 & 3 & 9.60496 & 0.09671 & 9.3820 & 9.828\end{array}$

$\begin{array}{lllllr}V-1-2 & 3 & 9.60496 & 0.09671 & 9.3820 & 9.828 \\ V-2-1 & 3 & 9.83170 & 0.09671 & 9.6087 & 10.055\end{array}$

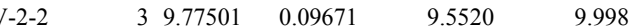

Std Error uses a pooled estimate of error variance 
Exhibit A-3. Measurements by Analytical Series, Block and Sub-Block for the Batch 1 and LRM Standards by Oxide by Prep (continued)

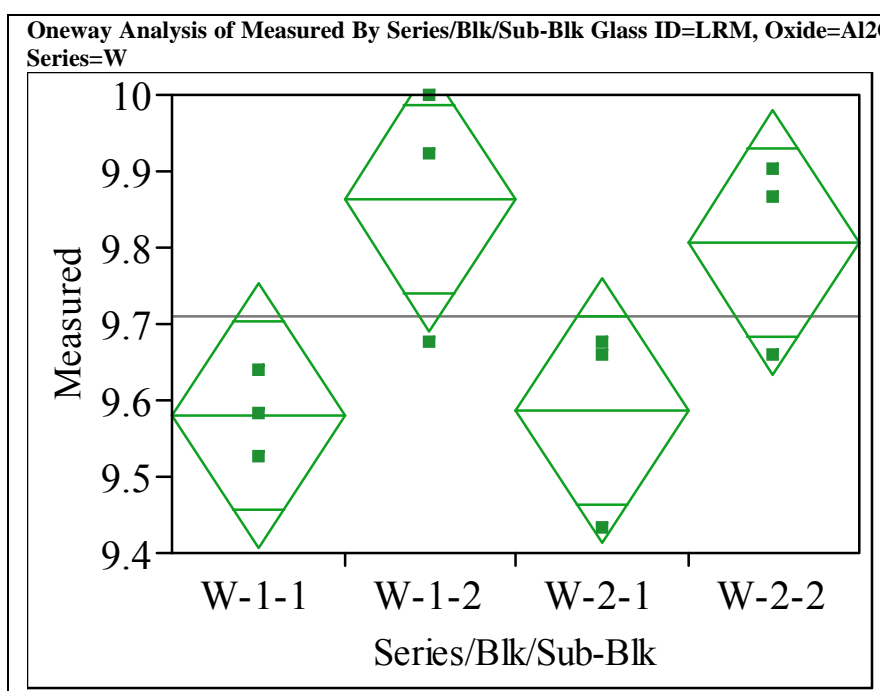

\section{Oneway Anova}

Summary of Fit

Rsquare $\quad 0.590925$

Adj Rsquare $\quad 0.437523$

Root Mean Square Error $\quad 0.129996$

$\begin{array}{lr}\text { Mean of Response } & 9.708881 \\ \text { Observations (or Sum Wgts) } & 12\end{array}$

Analysis of Variance

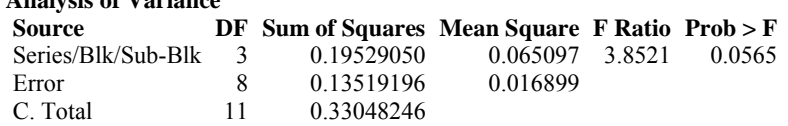

Means for Oneway Anova

Level Number Mean Std Error Lower 95\% Upper 95\%

$\begin{array}{lrrrrr}\text { W-1-1 } & 3 & 9.57977 & 0.07505 & 9.4067 & 9.753\end{array}$

$\begin{array}{llllll}\text { W-1-2 } & 3 & 9.86319 & 0.07505 & 9.6901 & 10.036\end{array}$

$\begin{array}{llllll}\text { W-2-1 } & 3 & 9.58606 & 0.07505 & 9.4130 & 9.759 \\ \text { W-2-2 } & 3 & 9.80651 & 0.07505 & 9.6334 & 9.980\end{array}$

Std Error uses a pooled estimate of error variance

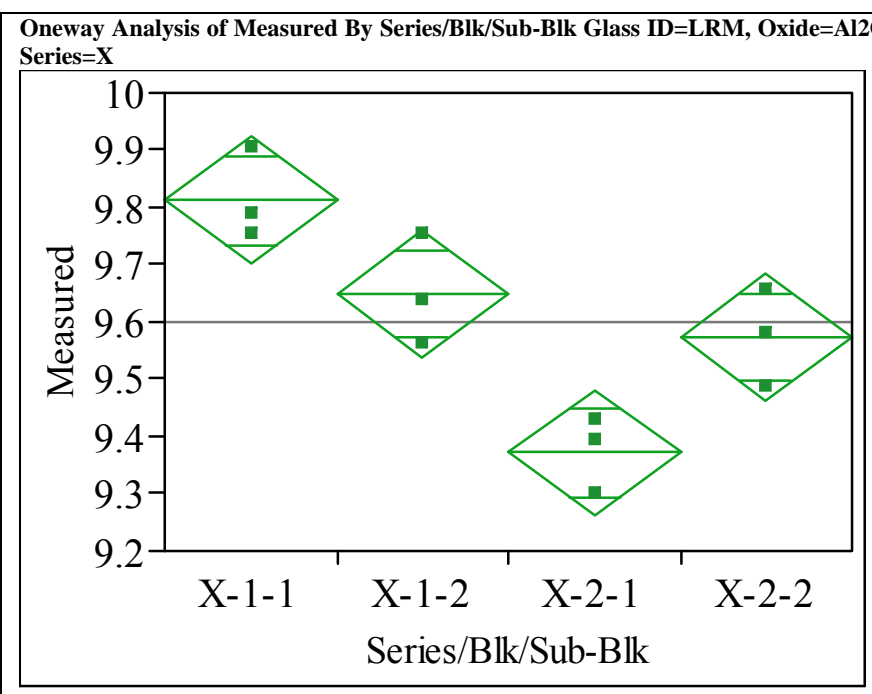

\section{Oneway Anova}

Summary of Fit

\section{Rsquare}

Adj Rsquare $\quad 0.847338$

Root Mean Square Error $\quad 0.082361$

Mean of Response $\quad 9.601809$

$\begin{array}{lr}\text { Observations (or Sum Wgts) } & 12\end{array}$

Analysis of Variance

\begin{tabular}{lrrrrr}
\multicolumn{2}{l}{ Analysis of Variance } & & & & \\
Source & DF & Sum of Squares & Mean Square & F Ratio Prob > F \\
Series/Blk/Sub-Blk & 3 & 0.30120674 & 0.100402 & 14.8012 & 0.0013 \\
Error & 8 & 0.05426720 & 0.006783 & & \\
C. Total & 11 & 0.35547393 & & &
\end{tabular}

Means for Oneway Anova

Level Number Mean Std Error Lower 95\% Upper 95\%

$\begin{array}{rrrrrr}\mathrm{X}-1-1 & 3 & 9.81280 & 0.04755 & 9.7031 & 9.9225\end{array}$

$\begin{array}{llllll}\mathrm{X}-1-2 & 3 & 9.64905 & 0.04755 & 9.5394 & 9.7587 \\ \mathrm{X}-2-1 & & 3 & 9.37192 & 0.04755 & 9.2623\end{array}$

$\begin{array}{llllll}\mathrm{X}-2-1 & 3 & 9.37192 & 0.04755 & 9.2623 & 9.4816\end{array}$

$\begin{array}{llllll}\mathrm{X}-2-2 & 3 & 9.57347 & 0.04755 & 9.4638 & 9.6831\end{array}$

Std Error uses a pooled estimate of error variance 
Exhibit A-3. Measurements by Analytical Series, Block and Sub-Block for the Batch 1 and LRM Standards by Oxide by Prep (continued)

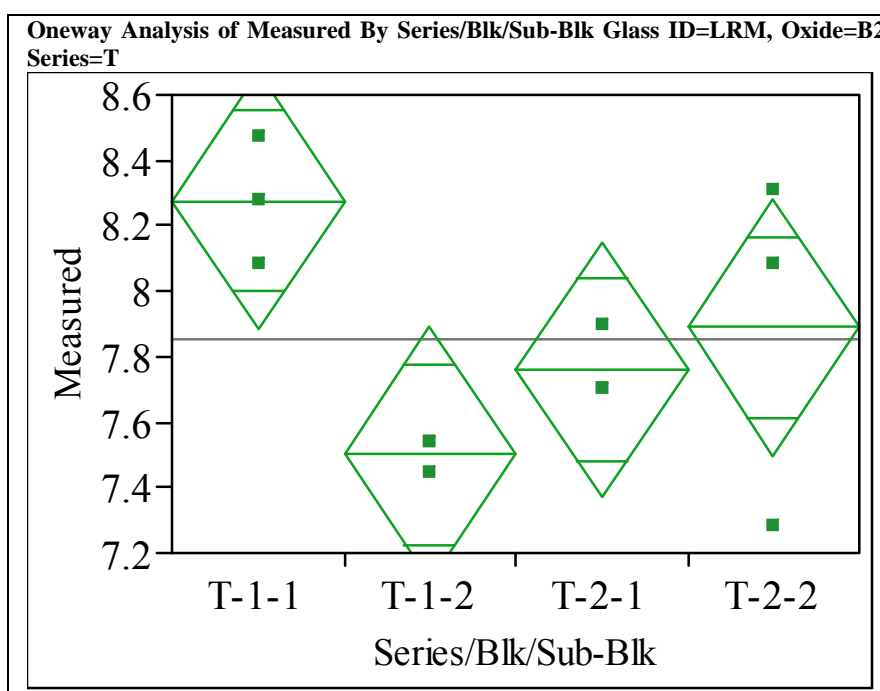

\section{Oneway Anova}

Summary of Fit

Adj Rsquare $\quad 0.414222$

Root Mean Square Error $\quad 0.294229$

$\begin{array}{lr}\text { Mean of Response } & 7.856556 \\ \text { Observations (or Sum Wgts) } & 12\end{array}$

Analysis of Variance

$\begin{array}{lrrrrr}\text { Source } & \text { DF } & \text { Sum of Squares } & \text { Mean Square } & \text { F Ratio } & \text { Prob }>\text { F } \\ \text { Series/Blk/Sub-Blk } & 3 & 0.9330980 & 0.311033 & 3.5928 & 0.0657 \\ \text { Error } & 8 & 0.6925661 & 0.086571 & & \\ \text { C. Total } & 11 & 1.6256641 & & & \end{array}$

Means for Oneway Anova

Level Number Mean Std Error Lower 95\% Upper 95\%

$\begin{array}{lrrrrr}\text { T-1-1 } & 3 & 8.27514 & 0.16987 & 7.8834 & 8.6669\end{array}$

\begin{tabular}{llllll}
$\mathrm{T}-1-2$ & 3 & 7.50237 & 0.16987 & 7.1106 & 7.8941 \\
\hline
\end{tabular}

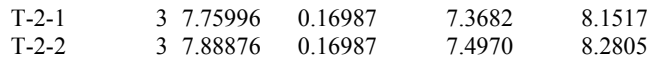

Std Error uses a pooled estimate of error variance

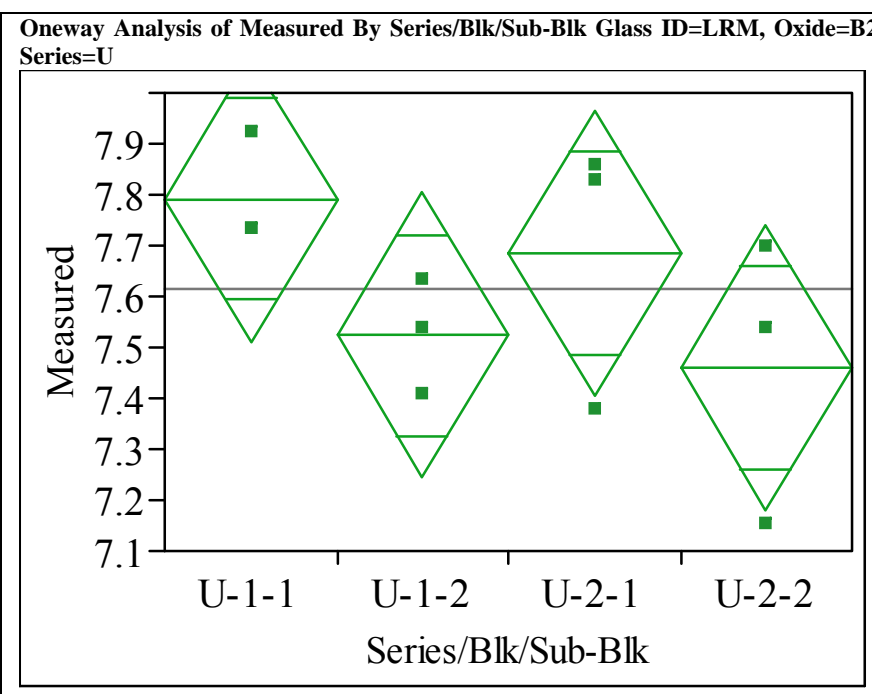

\section{Oneway Anova}

Summary of Fit

Rsquare

Adj Rsquare $\quad 0.367837$

Root Mean Square Error $\quad 0.210528$

$\begin{array}{lr}\text { Mean of Response } & 7.615064 \\ \text { Observations (or Sum Wgts) } & 12\end{array}$

Analysis of Variance

$\begin{array}{lrrrrr}\begin{array}{l}\text { Analysis of Variance } \\ \text { Source }\end{array} & \text { DF } & \text { Sum of Squares } & \text { Mean Square } & \text { F Ratio } & \text { Prob }>\text { F } \\ \text { Series/Blk/Sub-Blk } & 3 & 0.20631834 & 0.068773 & 1.5517 & 0.2749 \\ \text { Error } & 8 & 0.35457726 & 0.044322 & & \\ \text { C. Total } & 11 & 0.56089560 & & & \end{array}$

Means for Oneway Anova

Level Number Mean Std Error Lower 95\% Upper 95\%

$\begin{array}{llrrrr}\text { U-1-1 } & 3 & 7.79216 & 0.12155 & 7.5119 & 8.0724\end{array}$

$\begin{array}{llllll}\text { U-1-2 } & 3 & 7.52383 & 0.12155 & 7.2435 & 7.8041\end{array}$

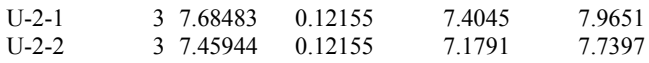

Std Error uses a pooled estimate of error variance 
Exhibit A-3. Measurements by Analytical Series, Block and Sub-Block for the Batch 1 and LRM Standards by Oxide by Prep (continued)

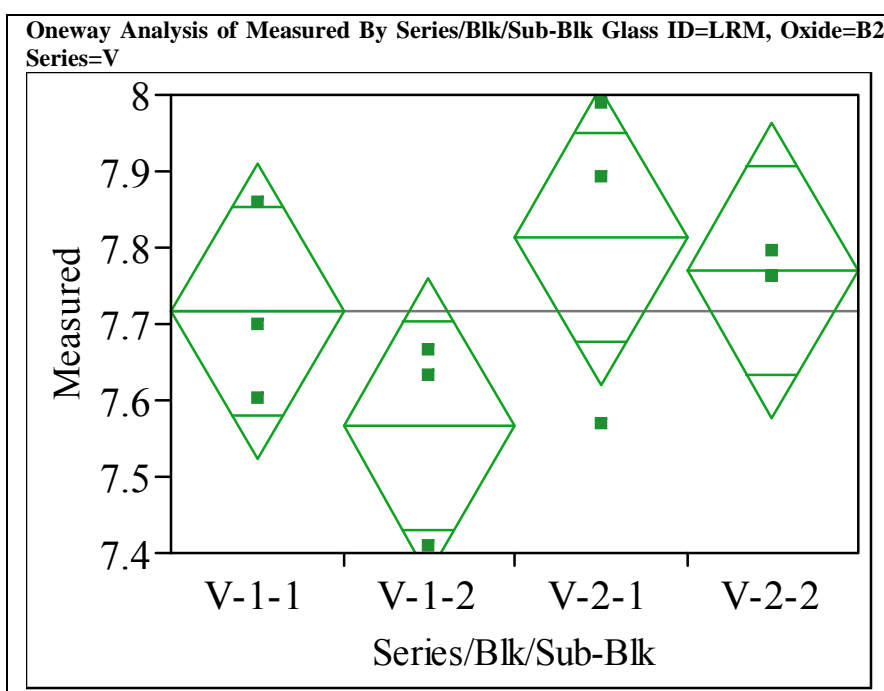

Oneway Anova

Summary of Fit

Rsquare $\quad 0.380353$

Adj Rsquare $\quad 0.147985$

Root Mean Square Error $\quad 0.145787$

$\begin{array}{lr}\text { Mean of Response } & 7.717027 \\ \text { Observations (or Sum Wgts) } & 12\end{array}$

Analysis of Variance

$\begin{array}{lrrrrr}\text { Source } & \text { DF } & \text { Sum of Squares } & \text { Mean Square } & \text { F Ratio } & \text { Prob }>\text { F } \\ \text { Series/Blk/Sub-Blk } & 3 & 0.10436874 & 0.034790 & 1.6369 & 0.2564 \\ \text { Error } & 8 & 0.17003120 & 0.021254 & & \\ \text { C. Total } & 11 & 0.27439994 & & & \\ & & & & & \end{array}$

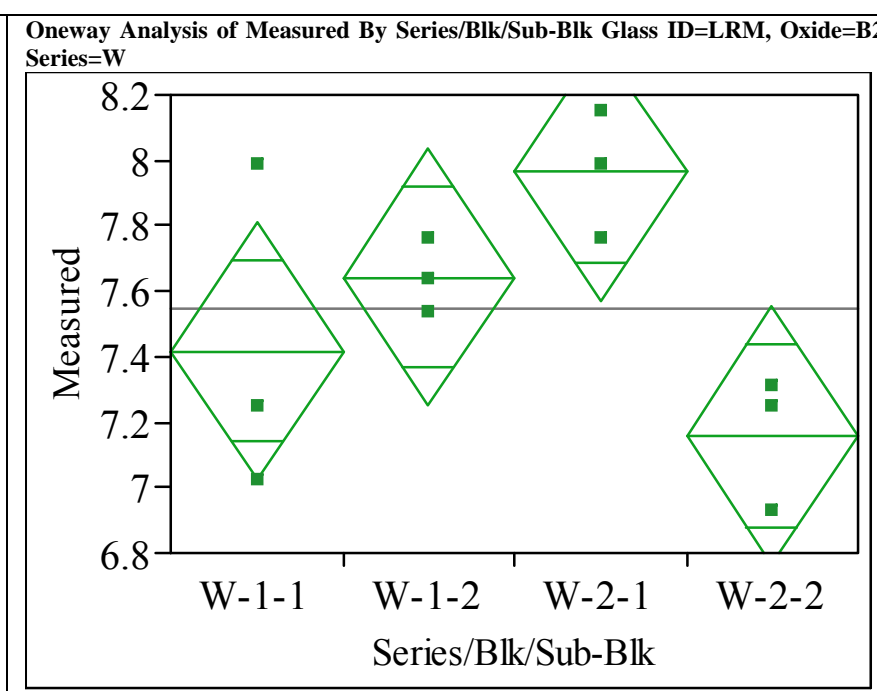

Means for Oneway Anova

Level Number Mean Std Error Lower 95\% Upper 95\%

$\begin{array}{llllrr}\text { V-1-1 } & 3 & 7.71703 & 0.08417 & 7.5229 & 7.9111 \\ \text { V-1-2 } & 3 & 7.56677 & 0.08417 & 7.3727 & 7.7609\end{array}$

$\begin{array}{llllll}\mathrm{V}-1-2 & 3 & 7.56677 & 0.08417 & 7.3727 & 7.7609 \\ \mathrm{~V}-2-1 & 3 & 7.81362 & 0.08417 & 7.6195 & 8.0077\end{array}$

$\begin{array}{llllll}\text { V-2-1 } & 3 & 7.81362 & 0.08417 & 7.6195 & 8.0077 \\ \text { V-2-2 } & 3 & 7.77069 & 0.08417 & 7.5766 & 7.9648\end{array}$

Std Error uses a pooled estimate of error variance

Oneway Anova

Summary of Fit

Rsquare

0.45163

Root Mean Square Error $\quad 0.295255$

$\begin{array}{lr}\text { Mean of Response } & 7.545299 \\ \text { Observations (or Sum Wgts) } & 12\end{array}$

Analysis of Variance

Source DF Sum of Squares Mean Square F Ratio Prob $>$

$\begin{array}{llllll}\text { Series/Blk/Sub-Blk } & 3 & 1.0512905 & 0.350430 & 4.0198 & 0.0513\end{array}$

$\begin{array}{lrr}\text { Error } & 8 & 0.6974044\end{array}$ 0.087176

Means for Oneway Anova

Level Number Mean Std Error Lower 95\% Upper 95\%

$\begin{array}{llllrr}\text { W-1-1 } & 3 & 7.41650 & 0.17047 & 7.0234 & 7.8096\end{array}$

$\begin{array}{llllll}\mathrm{W}-1-2 & 3 & 7.64190 & 0.17047 & 7.2488 & 8.0350\end{array}$

$\begin{array}{llllll}\text { W-2-1 } & 3 & 7.96389 & 0.17047 & 7.5708 & 8.3570\end{array}$

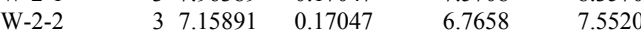

Std Error uses a pooled estimate of error variance 
Exhibit A-3. Measurements by Analytical Series, Block and Sub-Block for the Batch 1 and LRM Standards by Oxide by Prep (continued)

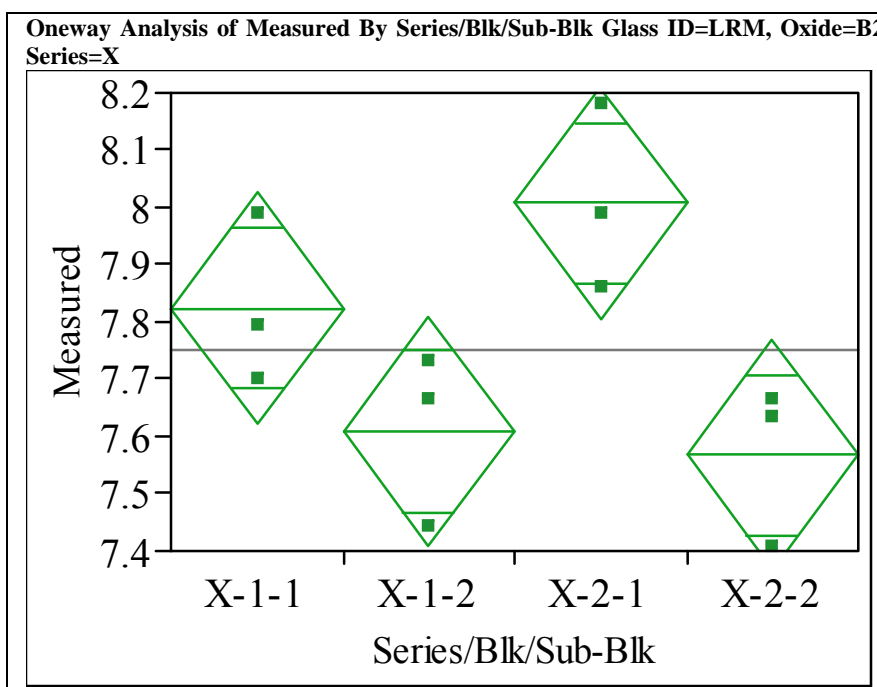

Oneway Anova

Summary of Fit

Rsquare $\quad 0.673038$

Adj Rsquare $\quad 0.550427$

Root Mean Square Error $\quad 0.15074$

$\begin{array}{lr}\text { Mean of Response } & 7.751909 \\ \text { Observations (or Sum Wgts) } & 12\end{array}$

Analysis of Variance

$\begin{array}{lrrrrr}\text { Source } & \text { DF } & \text { Sum of Squares } & \text { Mean Square } & \text { F Ratio } & \text { Prob }>\text { F } \\ \text { Series/Blk/Sub-Blk } & 3 & 0.37418959 & 0.124730 & 5.4892 & 0.0242 \\ \text { Error } & 8 & 0.18178132 & 0.022723 & & \\ \text { C. Total } & 11 & 0.55597092 & & & \\ & & & & & \end{array}$

Means for Oneway Anova

Level Number Mean Std Error Lower 95\% Upper 95\%

$\begin{array}{lrrrrr}\mathrm{X}-1-1 & 3 & 7.82436 & 0.08703 & 7.6237 & 8.0250 \\ \mathrm{X}-1-2 & 3 & 7.60970 & 0.08703 & 7.409 & 7.8104\end{array}$

$\begin{array}{llllll}\mathrm{X}-1-2 & 3 & 7.60970 & 0.08703 & 7.4090 & 7.8104 \\ \mathrm{X}-2-1 & 3 & 8.00682 & 0.08703 & 7.8061 & 8.2075\end{array}$

$\begin{array}{llllll}\mathrm{X}-1-2 & 3 & 8.00682 & 0.08703 & 7.8061 & 8.2075 \\ \mathrm{X}-2-1 & 3 & 8.0682 & 0.08703 & 7.3661 & 7.7675\end{array}$

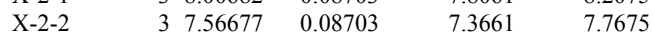

Std Error uses a pooled estimate of error variance

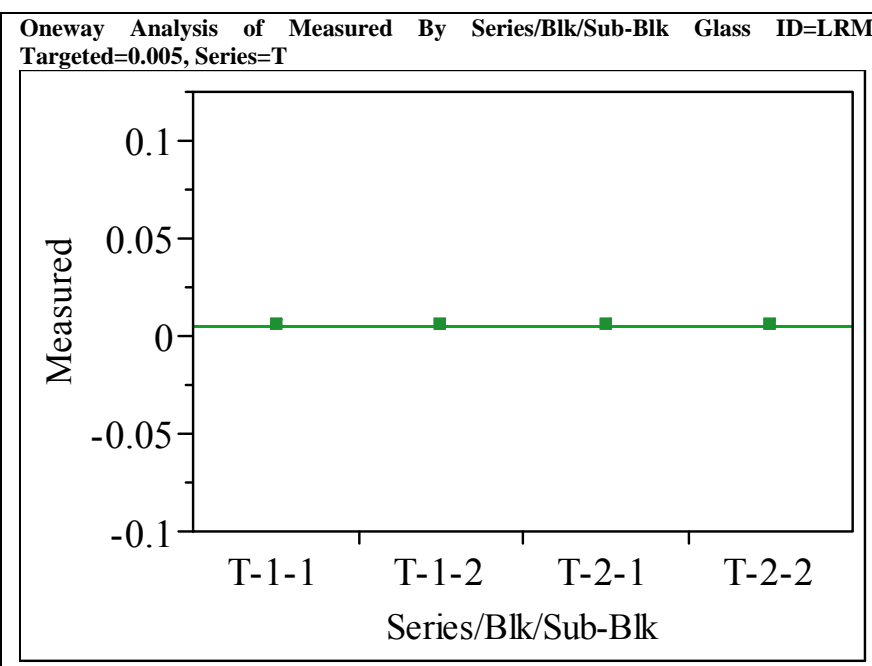

Oneway Anova

Summary of Fit

Rsquare

Adj Rsquare

$\begin{array}{lr}\text { Root Mean Square Error } & 0 \\ \text { Mean of Response } & 0.005583\end{array}$

Observations (or Sum Wgts) 12

Analysis of Variance

Sum of Squares Mean Square F Ratio Prob > F

$\begin{array}{llll}\text { Series/Blk/Sub-Blk } & 3 & 0 & 0 \\ \text { Error } & 8 & 0 & 0 \\ \text { C. } & 11 & 0 & \end{array}$

$\begin{array}{lrr}\text { Error } & 8 & 0 \\ \text { C. Total } & 11 & 0\end{array}$

Means for Oneway Anova

Level Number Mean Std Error Lower 95\% Upper 95\%

$\begin{array}{llllll}\mathrm{T}-1-1 & 3 & 0.005583 & 0 & 0.00558 & 0.00558\end{array}$

$\begin{array}{llllll}\mathrm{T}-1-2 & 3 & 0.005583 & 0 & 0.00558 & 0.00558\end{array}$

$\begin{array}{llllll}\mathrm{T}-2-1 & 3 & 0.005583 & 0 & 0.00558 & 0.00558\end{array}$

$\begin{array}{llllll}\text { T-2-2 } & 3 & 0.005583 & 0 & 0.00558 & 0.00558\end{array}$

Std Error uses a pooled estimate of error variance 


\section{Exhibit A-3. Measurements by Analytical Series, Block and Sub-Block for the Batch 1 and LRM Standards by Oxide by Prep (continued)}

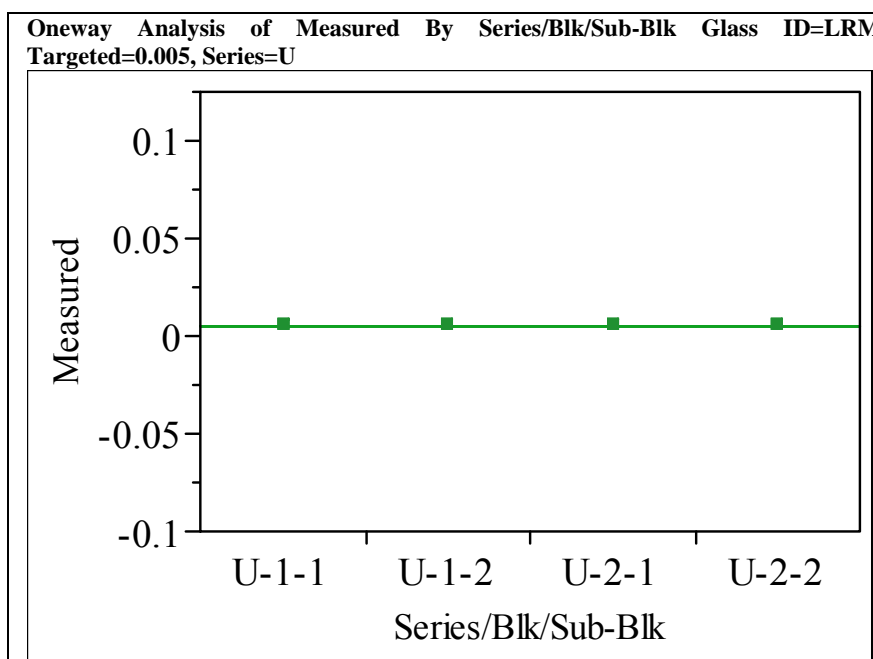

Oneway Anova

Summary of Fit

Rsquare

Adj Rsquare

$\begin{array}{lr}\text { Root Mean Square Error } & 0 \\ \text { Mean of Response } & 0.005583\end{array}$

Observations (or Sum Wgts)

Analysis of Variance

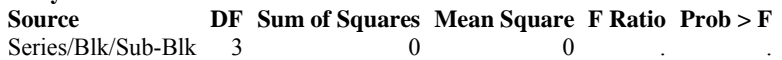

$\begin{array}{lll}\text { Error } & \text { Total } \\ \text { C. } & 11\end{array}$

$\begin{array}{lll}0 & 0 \\ 0 & 0\end{array}$

0

Means for Oneway Anova

Level Number Mean Std Error Lower 95\% Upper 95\%

$\begin{array}{llllrr}\mathrm{U}-1-1 & 3 & 0.005583 & 0 & 0.00558 & 0.00558\end{array}$

$\begin{array}{llllll}\text { U-1-2 } & 3 & 0.005583 & 0 & 0.00558 & 0.00558 \\ \mathrm{U}-2-1 & 3 & 0.005583 & 0 & 0.00558 & 0.00558\end{array}$

$\begin{array}{llllll}\mathrm{U}-2-2 & 3 & 0.005583 & 0 & 0.00558 & 0.00558\end{array}$

Std Error uses a pooled estimate of error variance

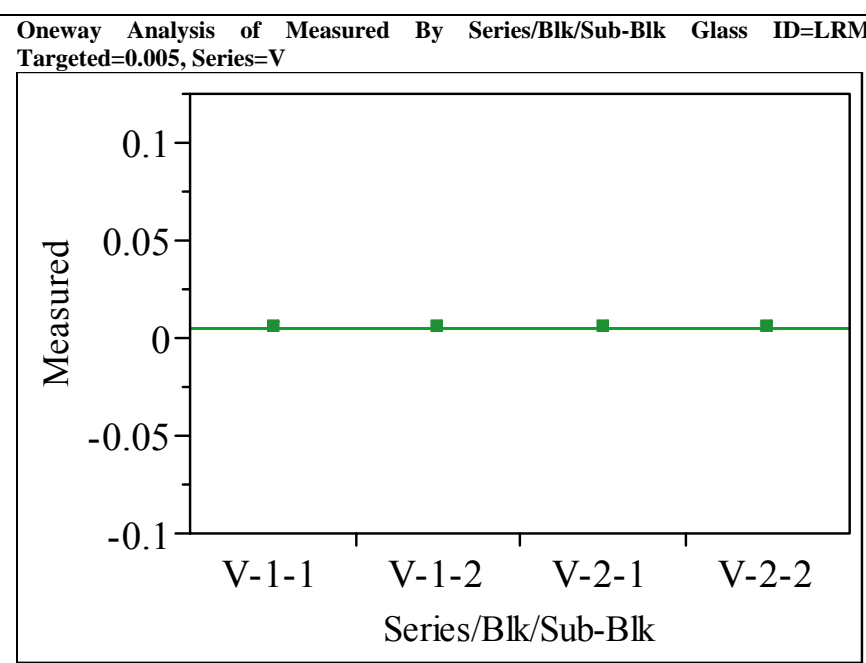

Oneway Anova

Summary of Fit

Rsquare

Adj Rsquare

$\begin{array}{lr}\text { Root Mean Square Error } & 0 \\ \text { Mean of Response } & 0.005583\end{array}$

Observations (or Sum Wgts) 12

Analysis of Variance

Sum of Squares Mean Square F Ratio Prob > F

Series/Blk/Sub-Blk

$\begin{array}{lr}\text { Error } & 8 \\ \text { C. Total } & 11\end{array}$

0
0

Means for Oneway Anova

Level Number Mean Std Error Lower 95\% Upper 95\%

$\begin{array}{llllll}V-1-1 & 3 & 0.005583 & 0 & 0.00558 & 0.00558\end{array}$

$\begin{array}{llllll}V-1-2 & 3 & 0.005583 & 0 & 0.00558 & 0.00558\end{array}$

$\begin{array}{llllll}\mathrm{V}-2-1 & 3 & 0.005583 & 0 & 0.00558 & 0.00558\end{array}$

$\begin{array}{llllll}\mathrm{V}-2-2 & 3 & 0.005583 & 0 & 0.00558 & 0.00558\end{array}$

Std Error uses a pooled estimate of error variance 


\section{Exhibit A-3. Measurements by Analytical Series, Block and Sub-Block for the Batch 1 and LRM Standards by Oxide by Prep (continued)}

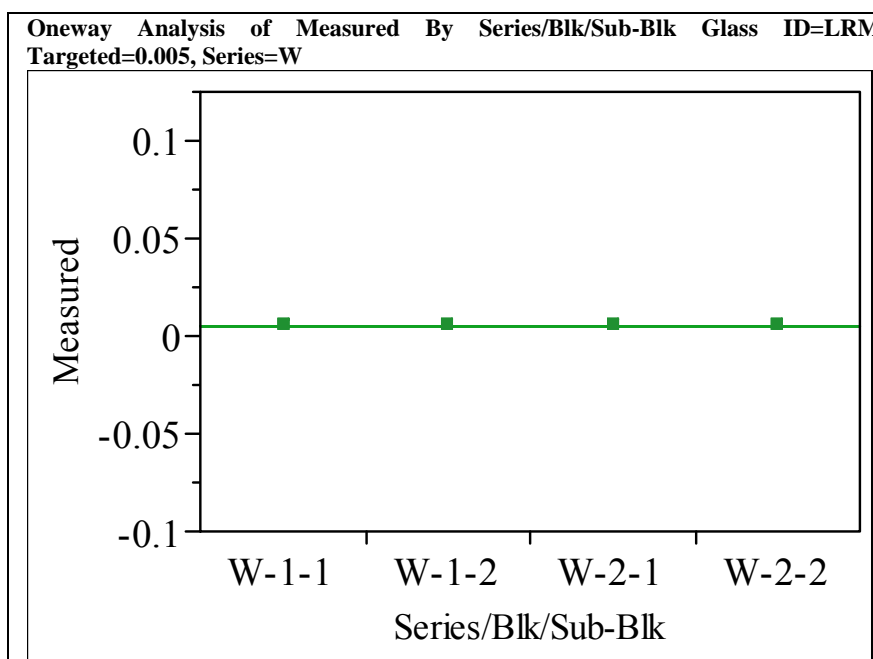

Oneway Anova

Summary of Fit

Rsquare

Adj Rsquare

$\begin{array}{lr}\text { Root Mean Square Error } & 0 \\ \text { Mean of Response } & 0.005583\end{array}$

Observations (or Sum Wgts)

Analysis of Variance

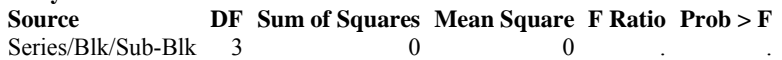

$\begin{array}{lr}\text { Error } & 8 \\ \text { C. Total } & 11\end{array}$

0

Means for Oneway Anova

Level Number Mean Std Error Lower 95\% Upper 95\%

$\begin{array}{lrrrrr}\mathrm{W}-1-1 & 3 & 0.005583 & 0 & 0.00558 & 0.00558\end{array}$

$\begin{array}{llllll}\mathrm{W}-1-1 & 3 & 0.005583 & 0 & 0.00558 & 0.00558 \\ \mathrm{~W}-2 & 3 & 0.005583 & 0 & 0.00558 & 0.00558\end{array}$

$\begin{array}{llllll}\text { W-2-1 } & 3 & 0.005583 & 0 & 0.00558 & 0.00558 \\ \text { W-2-2 } & 3 & 0.005583 & 0 & 0.00558 & 0.00558\end{array}$

Std Error uses a pooled estimate of error variance

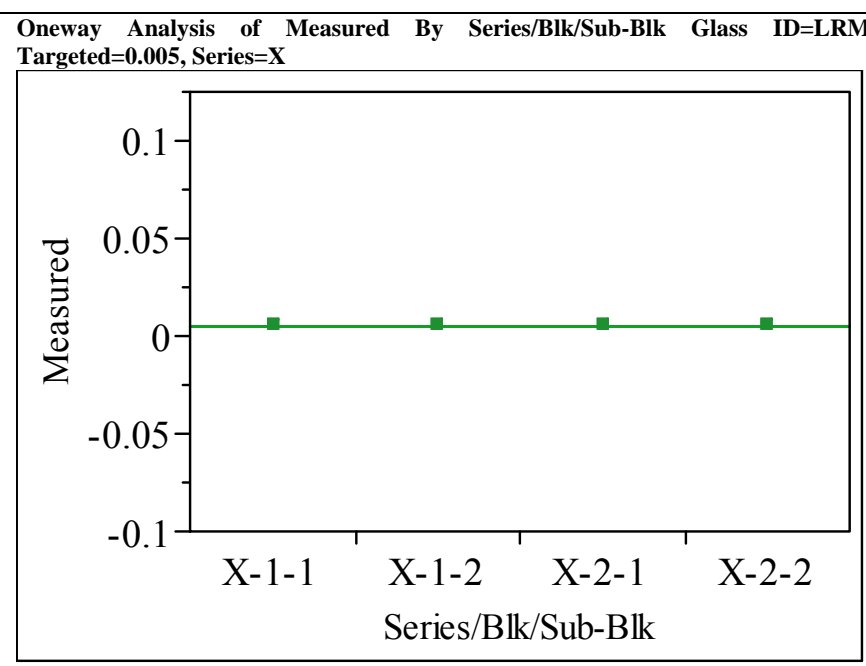

Oneway Anova

Summary of Fit

Rsquare

Adj Rsquare

$\begin{array}{lr}\text { Root Mean Square Error } & 0 \\ \text { Mean of Response } & 0.005583\end{array}$

Observations (or Sum Wgts) 12

Analysis of Variance

Sum of Squares Mean Square F Ratio Prob >

Series/Blk/Sub-Blk

$\begin{array}{lr}\text { Error } & 8 \\ \text { C. Total } & 11\end{array}$

$\begin{array}{ll}0 & 0 \\ 0 & 0 \\ 0 & 0\end{array}$

Means for Oneway Anova

Level Number Mean Std Error Lower 95\% Upper 95\%

$\begin{array}{llllll}\mathrm{X}-1-1 & 3 & 0.005583 & 0 & 0.00558 & 0.00558\end{array}$

$\begin{array}{llllll}\mathrm{X}-1-2 & 3 & 0.005583 & 0 & 0.00558 & 0.00558\end{array}$

$\begin{array}{llllll}\mathrm{X}-2-1 & 3 & 0.005583 & 0 & 0.00558 & 0.00558\end{array}$

$\begin{array}{llllll}\mathrm{X}-2-2 & 3 & 0.005583 & 0 & 0.00558 & 0.00558\end{array}$

Std Error uses a pooled estimate of error variance 
Exhibit A-3. Measurements by Analytical Series, Block and Sub-Block for the Batch 1 and LRM Standards by Oxide by Prep (continued)

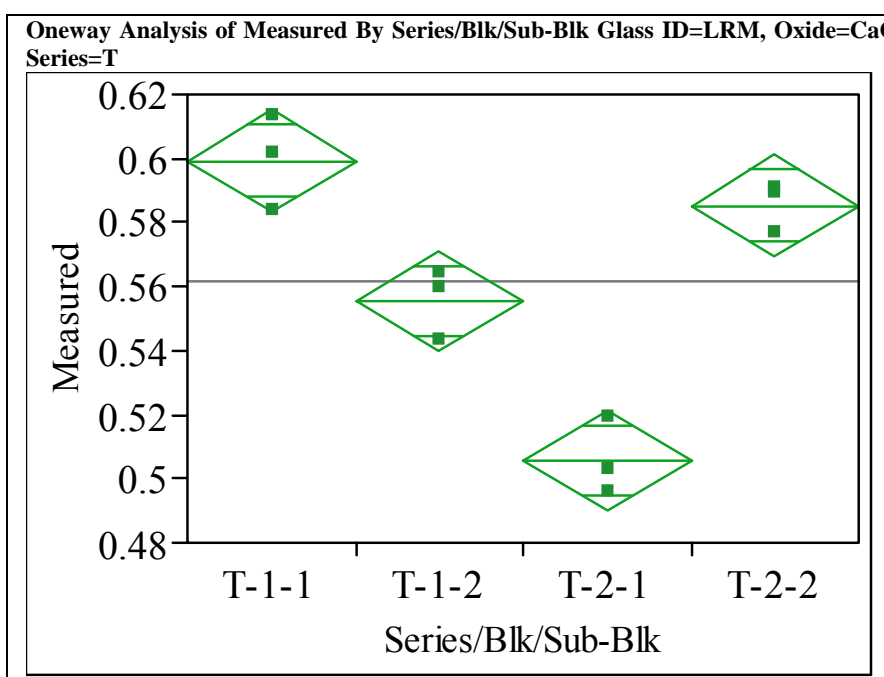

Oneway Anova

Summary of Fit

$\begin{array}{lr}\text { Rsquare } & 0.933445 \\ \text { Adj Rsquare } & 0.908487 \\ \text { Root Mean Square Error } & 0.011748 \\ \text { Mean of Response } & 0.561429 \\ \text { Observations (or Sum Wgts) } & 12\end{array}$

Analysis of Variance

$\begin{array}{lrrrrr}\text { Source } & \text { DF } & \text { Sum of Squares } & \text { Mean Square } & \text { F Ratio } & \text { Prob }>\text { F } \\ \text { Series/Blk/Sub-Blk } & 3 & 0.01548638 & 0.005162 & 37.4007 & <.0001\end{array}$

Error

0.01659055

Means for Oneway Anova

Level Number Mean Std Error Lower 95\% Upper 95\%

$\begin{array}{rrrrrr}\text { Level } & \text { Number } & \text { Mean } & \text { Std Error } & \text { Lower 95\% } & \text { Upper 95\% } \\ \text { T-1-1 } & 3 & 0.599324 & 0.00678 & 0.58368 & 0.61497\end{array}$

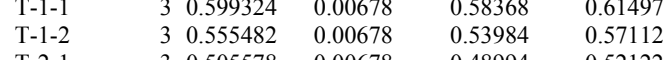

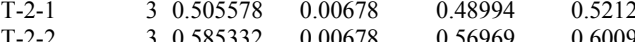

Std Error uses a pooled estimate of error variance

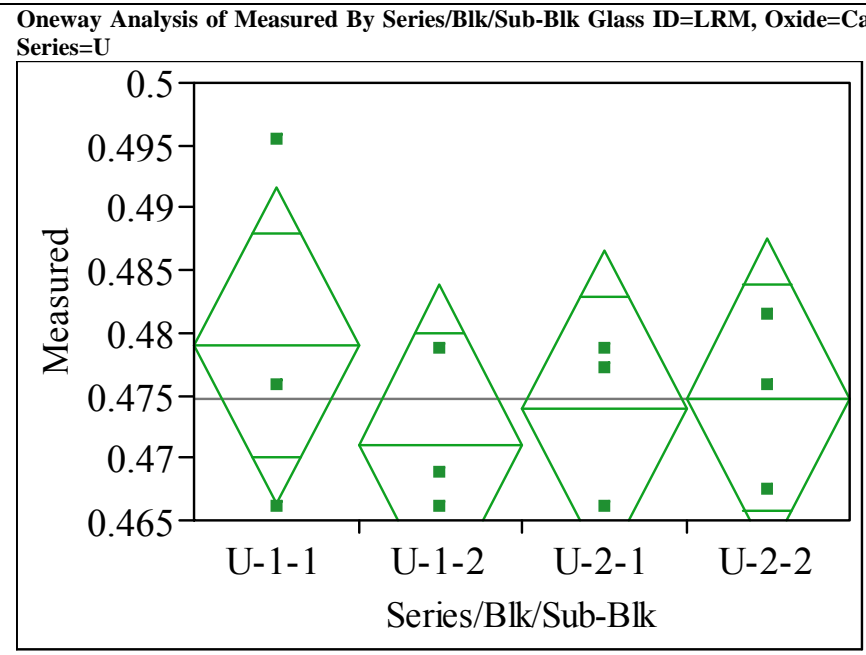

Oneway Anova

Summary of Fit

Rsquare

$\begin{array}{ll}0.117426 \\ \text { Adj Rsquare } & -0.21354\end{array}$

Root Mean Square Error $\quad 0.00955$

$\begin{array}{lr}\text { Mean of Response } & 0.474679 \\ \text { Observations (or Sum Wgts) } & 12\end{array}$

Analysis of Variance

Source DF Sum of Squares Mean Square F Ratio Prob $>$ F

$\begin{array}{lll}0.000032 & 0.3548 & 0.787\end{array}$

$\begin{array}{lrr}\text { Error } & 8 & 0.00072959\end{array}$ 0.000091

Means for Oneway Anova

Level Number Mean Std Error Lower 95\% Upper 95\%

$\begin{array}{lllrrr}\text { U-1-1 } & 3 & 0.478993 & 0.00551 & 0.46628 & 0.4917\end{array}$

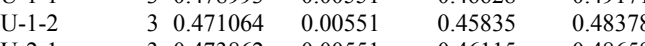

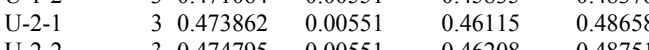

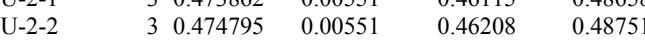

Std Error uses a pooled estimate of error variance 
Exhibit A-3. Measurements by Analytical Series, Block and Sub-Block for the Batch 1 and LRM Standards by Oxide by Prep (continued)

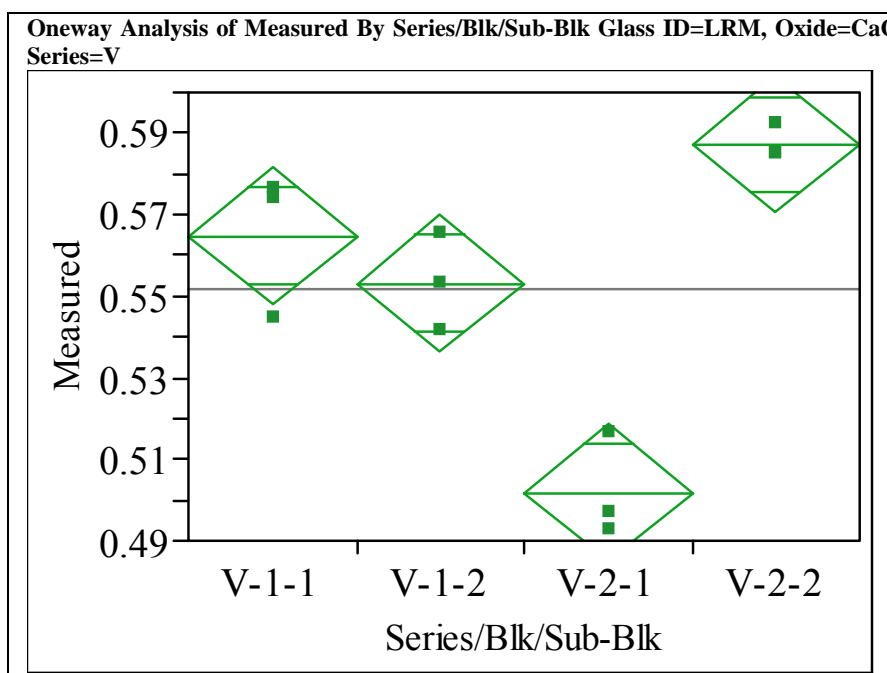

Oneway Anova

Summary of Fit

$\begin{array}{lr}\text { Rsquare } & 0.902253 \\ \text { Adj Rsquare } & 0.865598 \\ \text { Root Mean Square Error } & 0.012619 \\ \text { Mean of Response } & 0.551751 \\ \text { Observations (or Sum Wgts) } & 12\end{array}$

Analysis of Variance

Source DF Sum of Squares Mean Square F Ratio Prob > F

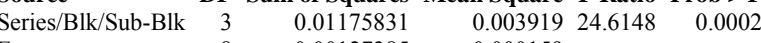

$\begin{array}{lll}\text { Error } & 8 & 0.0012738 \\ \text { C. } & & \end{array}$

C. Total

0.01303216

Means for Oneway Anova

Level Number Mean Std Error Lower 95\% Upper 95\%

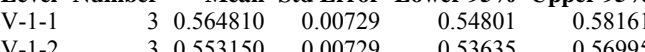

$\begin{array}{llllll}V-1-1 & & 0.564810 & 0.00729 & 0.5481 & 0.58161 \\ V-2 & 3 & 0.553150 & 0.00729 & 0.53635 & 0.56995 \\ V-2-1 & 3 & 0.501846 & 0.00729 & 0.48505 & 0.51865\end{array}$

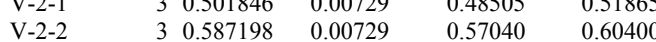

Std Error uses a pooled estimate of error variance

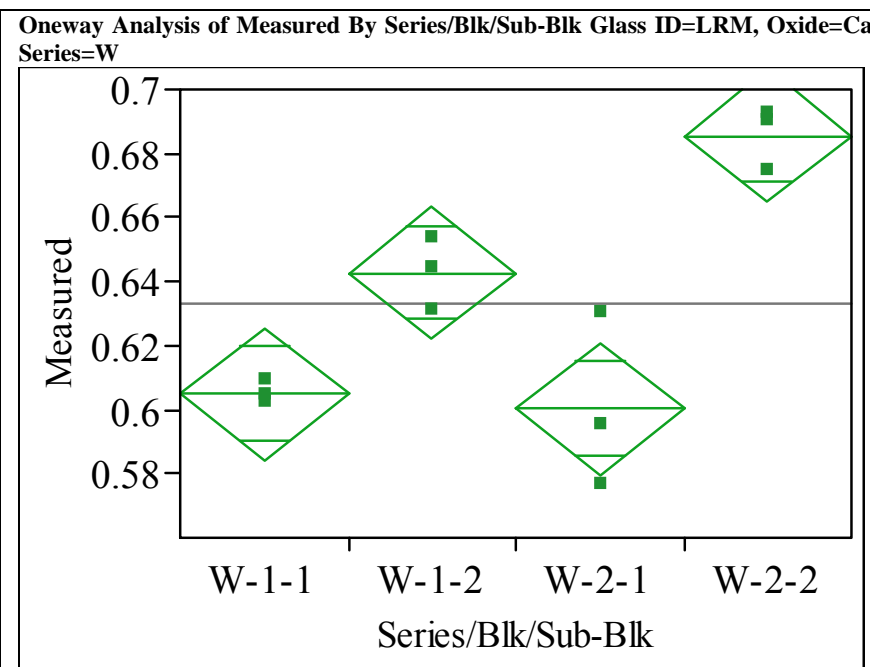

Oneway Anova

Summary of Fit

$\begin{array}{lr}\text { Rsquare } & 0.880139 \\ \text { Adj Rsquare } & 0.835192 \\ \text { Root Mean Square Error } & 0.015528 \\ \text { Mean of Response } & 0.633371 \\ \text { Observations (or Sum Wgts) } & 12\end{array}$

Observations (or Sum Wgts) 0.633371

Analysis of Variance

Source DF Sum of Squares Mean Square F Ratio Prob $>$ F

$\begin{array}{lllrlr}\text { Series/Blk/Sub-Blk } & 3 & 0.01416505 & 0.004722 & 19.5814 & 0.0005\end{array}$

$\begin{array}{lll}\text { Error } & 8 & 0.00192905 \\ \text { C. } & 11 & 0.0169410\end{array}$

0.000241

Means for Oneway Anova

Level Number Mean Std Error Lower 95\% Upper 95\%

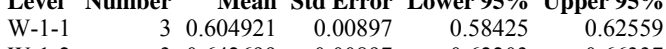

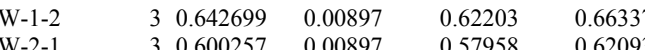

$\begin{array}{lllllll}W-1-2 & 3 & 0.642699 & 0.00897 & 0.62203 & 0.66337 \\ W-2-1 & & 3 & 0.600257 & 0.00897 & 0.57958 & 0.62093\end{array}$

Std Error uses a pooled estimate of error variance 
Exhibit A-3. Measurements by Analytical Series, Block and Sub-Block for the Batch 1 and LRM Standards by Oxide by Prep (continued)

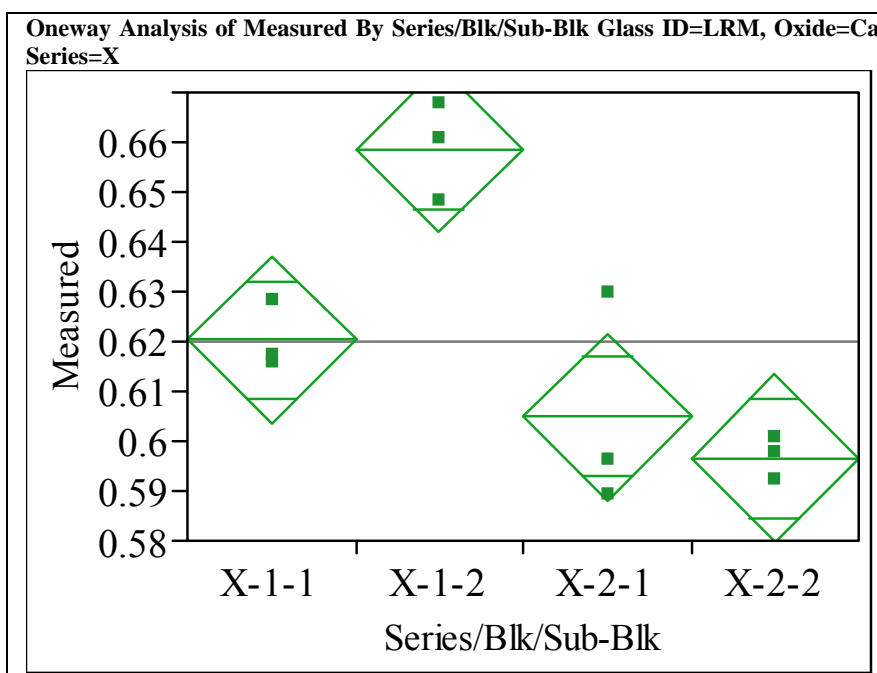

Oneway Anova

Summary of Fit

$\begin{array}{lr}\text { Rsquare } & 0.842544 \\ \text { Adj Rsquare } & 0.783498 \\ \text { Root Mean Square Error } & 0.012599 \\ \text { Mean of Response } & 0.620079 \\ \text { Observations (or Sum Wgts) } & 12\end{array}$

Analysis of Variance

Source DF Sum of Squares Mean Square F Ratio Prob > F

Series/Blk/Sub-Blk $3 \quad 3 \quad 0.00679539 \quad 0.002265 \quad 14.2693 \quad 0.0014$

$\begin{array}{lrr}\text { Error } & 8 & 0.00126993 \\ \text { C. } & 11 & 0.0080532\end{array}$

Means for Oneway Anova
Level Number Mean Std Error Lower 95\% Upper 95\%

$\begin{array}{rrrrrr}\text { Level } & \text { Number } & \text { Mean } & \text { Std Error } & \text { Lower 95\% } & \text { Upper 95\% } \\ \text { X-1-1 } & 3 & 0.620312 & 0.00727 & 0.60354 & 0.63709\end{array}$

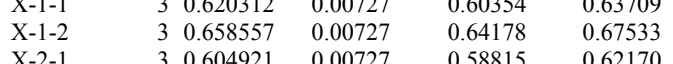

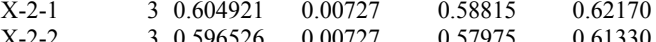

Std Error uses a pooled estimate of error variance

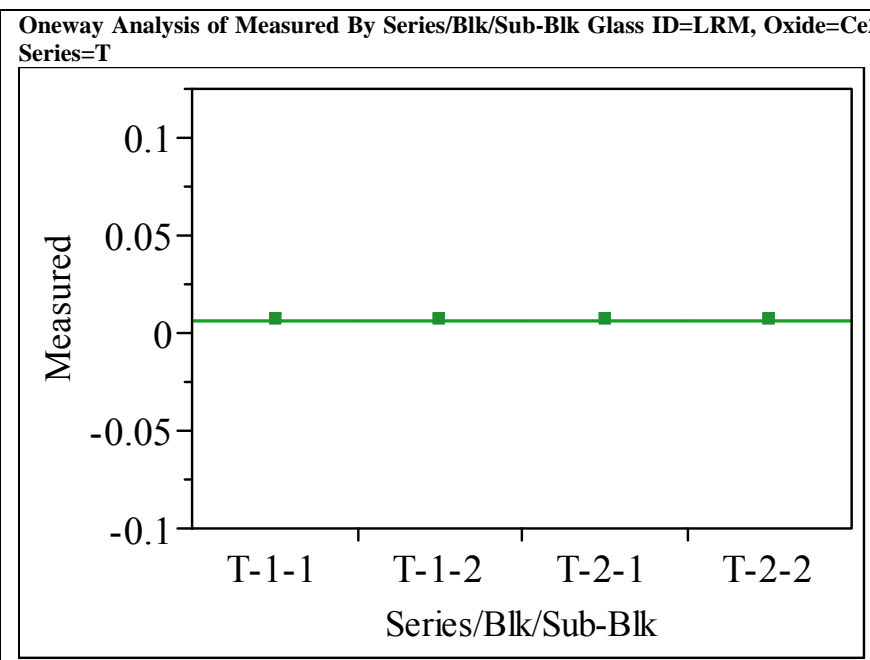

Oneway Anova

Summary of Fit

Rsquare

Adj Rsquare

$\begin{array}{lr}\text { Root Mean Square Error } & 0 \\ \text { Mean of Response } & 0.005857\end{array}$

Observations (or Sum Wgts) 12

Analysis of Variance

Sum of Squares Mean Square F Ratio Prob > F

$\begin{array}{llll}\text { Series/Blk/Sub-Blk } & 3 & 0 & 0 \\ \text { Error } & 8 & 0 & 0\end{array}$

$\begin{array}{lrr}\text { Error } & 8 & 0 \\ \text { C. Total } & 11 & 0\end{array}$

Means for Oneway Anova

Level Number Mean Std Error Lower 95\% Upper 95\%

$\begin{array}{llllll}\text { T-1-1 } & 3 & 0.005857 & 0 & 0.00586 & 0.00586\end{array}$

$\begin{array}{llllll}\text { T-1-2 } & 3 & 0.005857 & 0 & 0.00586 & 0.00586\end{array}$

$\begin{array}{llllll}\mathrm{T}-2-1 & 3 & 0.005857 & 0 & 0.00586 & 0.00586\end{array}$

$\begin{array}{llllll}\text { T-2-2 } & 3 & 0.005857 & 0 & 0.00586 & 0.00586\end{array}$

Std Error uses a pooled estimate of error variance 
Exhibit A-3. Measurements by Analytical Series, Block and Sub-Block for the Batch 1 and LRM Standards by Oxide by Prep (continued)

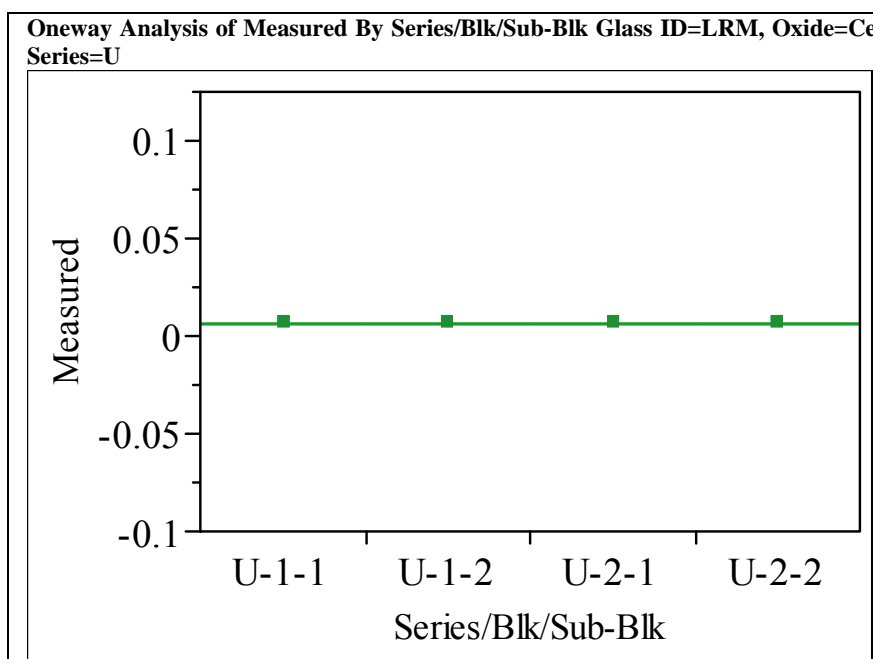

Oneway Anova

Summary of Fit

Rsquare

Adj Rsquare

$\begin{array}{lr}\text { Root Mean Square Error } & 0 \\ \text { Mean of Response } & 0.005857\end{array}$

Observations (or Sum Wgts)

Analysis of Variance

$\begin{array}{lrrr}\text { Source } & \text { DF Sum of Squares Mean Square F Ratio Prob }>\text { F } \\ \text { Series/Blk/Sub-Blk } & 3 & 0 & 0\end{array}$

$\begin{array}{lr}\text { Error } & 8 \\ \text { C. Total } & 11\end{array}$

0

Means for Oneway Anova

Level Number Mean Std Error Lower 95\% Upper 95\%

$\begin{array}{llllrr}\text { U-1-1 } & 3 & 0.005857 & 0 & 0.00586 & 0.00586\end{array}$

$\begin{array}{llllll}\text { U-1-2 } & 3 & 0.005857 & 0 & 0.00586 & 0.00586\end{array}$

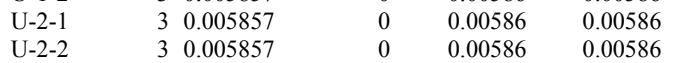

Std Error uses a pooled estimate of error variance

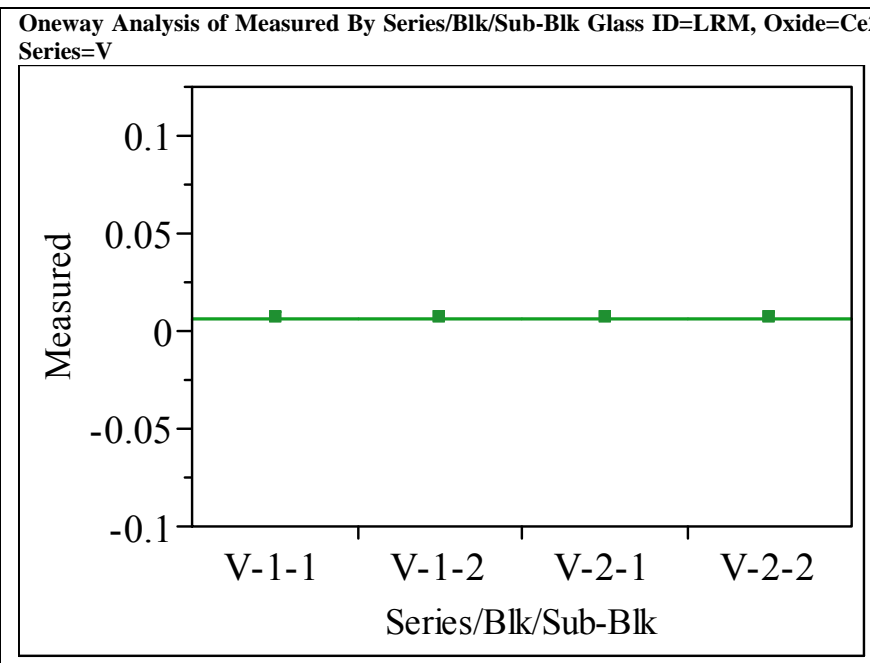

Oneway Anova

Summary of Fit

Rsquare

Adj Rsquare

$\begin{array}{lr}\text { Root Mean Square Error } & 0 \\ \text { Mean of Response } & 0.005857\end{array}$

Observations (or Sum Wgts) 12

Analysis of Variance

Sum of Squares Mean Square F Ratio Prob > F

Series/Blk/Sub-Blk

$\begin{array}{lr}\text { Error } & 8 \\ \text { C. Total } & 11\end{array}$

$\begin{array}{ll}0 & 0 \\ 0 & 0 \\ 0 & 0\end{array}$

Means for Oneway Anova

Level Number Mean Std Error Lower 95\% Upper 95\%

$\begin{array}{llllll}\mathrm{V}-1-1 & 3 & 0.005857 & 0 & 0.00586 & 0.00586\end{array}$

$\begin{array}{llllll}V-1-2 & 3 & 0.005857 & 0 & 0.00586 & 0.00586\end{array}$

$\begin{array}{llllll}\mathrm{V}-2-1 & 3 & 0.005857 & 0 & 0.00586 & 0.00586\end{array}$

$\begin{array}{llllll}\mathrm{V}-2-2 & 3 & 0.005857 & 0 & 0.00586 & 0.00586\end{array}$

Std Error uses a pooled estimate of error variance 
Exhibit A-3. Measurements by Analytical Series, Block and Sub-Block for the Batch 1 and LRM Standards by Oxide by Prep (continued)

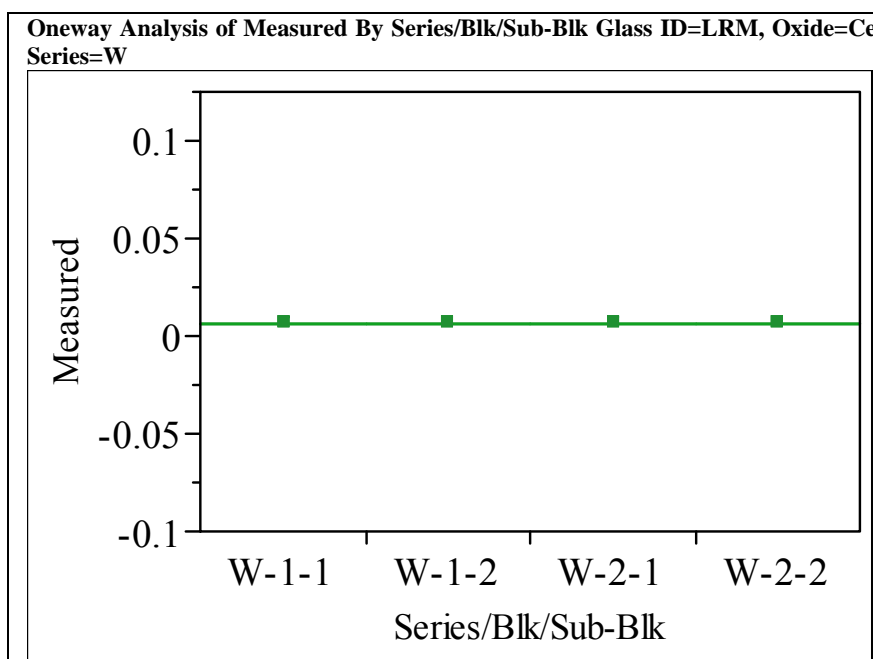

Oneway Anova

Summary of Fit

Rsquare

Adj Rsquare

$\begin{array}{lr}\text { Root Mean Square Error } & 0 \\ \text { Mean of Response } & 0.005857\end{array}$

Observations (or Sum Wgts)

Analysis of Variance

$\begin{array}{lrrr}\text { Source } & \text { DF Sum of Squares Mean Square } & \text { F Ratio Prob }>\text { F } \\ \text { Series/Blk/Sub-Blk } & 3 & 0 & 0\end{array}$

$\begin{array}{lr}\text { Error } & 8 \\ \text { C. Total } & 11\end{array}$

0

Means for Oneway Anova

Level Number Mean Std Error Lower 95\% Upper 95\%

$\begin{array}{rrrrrr}\text { W-1 }-1 & 3 & 0.005857 & 0 & 0.00586 & 0.00586\end{array}$

$\begin{array}{llllll}\mathrm{W}-1-1 & 3 & 0.005857 & 0 & 0.00586 & 0.00586 \\ \mathrm{~W}-1-2 & 3 & 0.005857 & 0 & 0.00586 & 0.00586\end{array}$

$\begin{array}{llllll}\text { W-2-1 } & 3 & 0.005857 & 0 & 0.00586 & 0.00586 \\ \text { W-2-2 } & 3 & 0.005857 & 0 & 0.00586 & 0.00586\end{array}$

Std Error uses a pooled estimate of error variance

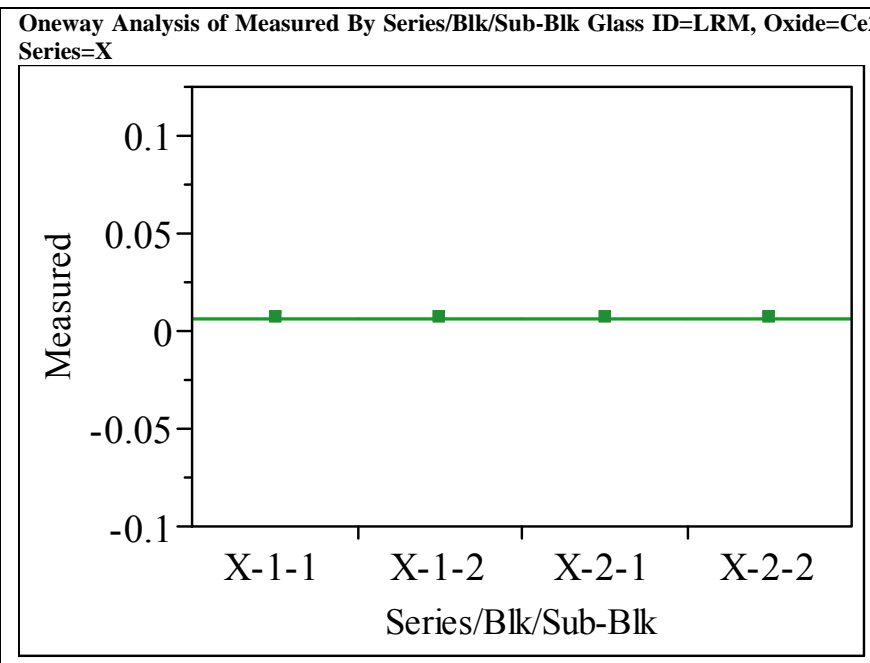

Oneway Anova

Summary of Fit

Rsquare

Adj Rsquare

$\begin{array}{lr}\text { Root Mean Square Error } & 0 \\ \text { Mean of Response } & 0.005857\end{array}$

Observations (or Sum Wgts) 12

Analysis of Variance

Sum of Squares Mean Square F Ratio Prob > F

Series/Blk/Sub-Blk

$\begin{array}{lr}\text { Error } & 8 \\ \text { C. Total } & 11\end{array}$

$\begin{array}{ll}0 & 0 \\ 0 & 0 \\ 0 & 0\end{array}$

Means for Oneway Anova

Level Number Mean Std Error Lower 95\% Upper 95\%

$\begin{array}{llllll}\mathrm{X}-1-1 & 3 & 0.005857 & 0 & 0.00586 & 0.00586\end{array}$

$\begin{array}{llllll}\mathrm{X}-1-2 & 3 & 0.005857 & 0 & 0.00586 & 0.00586\end{array}$

$\begin{array}{llllll}\mathrm{X}-2-1 & 3 & 0.005857 & 0 & 0.00586 & 0.00586\end{array}$

$\begin{array}{llllll}\mathrm{X}-2-2 & 3 & 0.005857 & 0 & 0.00586 & 0.00586\end{array}$

Std Error uses a pooled estimate of error variance 
Exhibit A-3. Measurements by Analytical Series, Block and Sub-Block for the Batch 1 and LRM Standards by Oxide by Prep (continued)

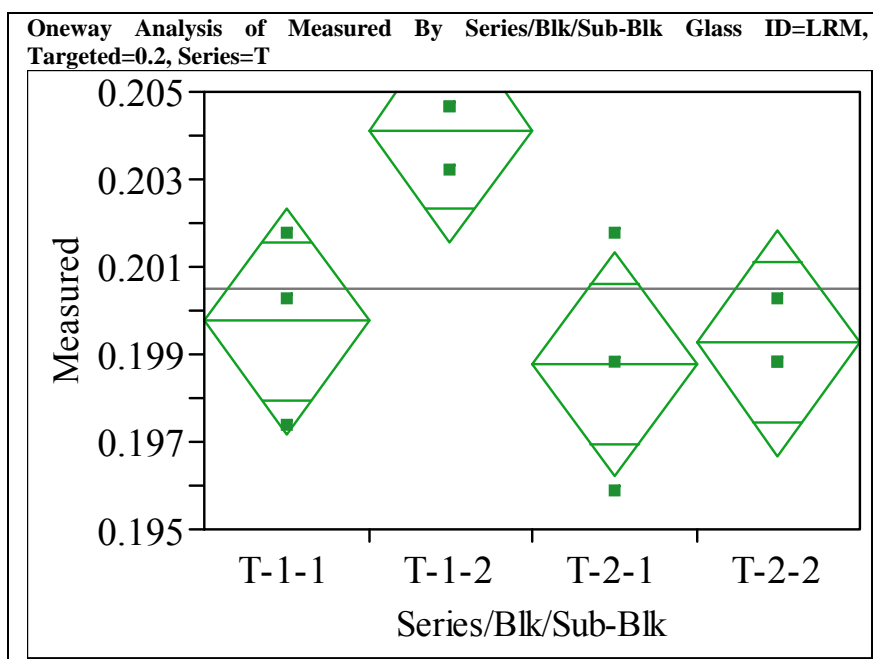

Oneway Anova

Summary of Fit

$\begin{array}{ll} & 0.647059 \\ \text { Adj Rsquare } & 0.514706\end{array}$

Root Mean Square Error $\quad 0.001934$

$\begin{array}{lr}\text { Mean of Response } & 0.200483 \\ \text { Observations (or Sum Wgts) } & 12\end{array}$

Analysis of Variance

Source DF Sum of Squares Mean Square F Ratio Prob $>$ F

$\begin{array}{lrrrrr}\text { Series/Blk/Sub-Blk } & 3 & 0.00005483 & 0.000018 & 4.8889 & 0.0323 \\ \text { Error } & 8 & 0.00002991 & 3.738 \mathrm{e}-6 & & \end{array}$

Error

$3.738 \mathrm{e}-6$

Means for Oneway Anova

Level Number Mean Std Error Lower 95\% Upper 95\%

$\begin{array}{llllrr}\text { T-1-1 } & 3 & 0.199752 & 0.00112 & 0.19718 & 0.2023\end{array}$

$\begin{array}{llllll}\mathrm{T}-1-2 & 3 & 0.204137 & 0.00112 & 0.20156 & 0.2067\end{array}$

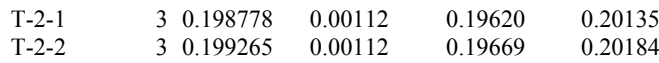

Std Error uses a pooled estimate of error variance

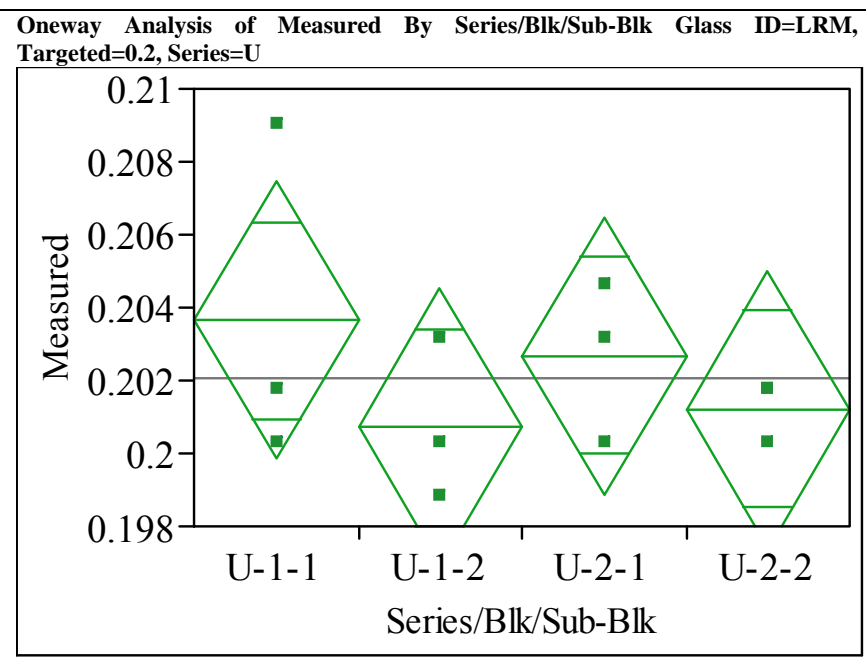

\section{Oneway Anova}

Summary of Fit

$\begin{array}{lr}\text { Rsquare } & 0.198257 \\ \text { Adj Rsquare } & -0.1024 \\ \text { Root Mean Square Error } & 0.002862 \\ \text { Mean of Response } & 0.202066 \\ \text { Observations (or Sum Wgts) } & 12\end{array}$

Analysis of Variance

Source DF Sum of Squares Mean Square F Ratio Prob > F

$\begin{array}{lllllll}\text { Series/Blk/Sub-Blk } & 3 & 0.00001620 & 0.0000054 & 0.6594 & 0.5997\end{array}$

$\begin{array}{lll}\text { Error } & 8 & 0.00006551\end{array}$ $8.1891 \mathrm{e}-6$

Means for Oneway Anova

Level Number Mean Std Error Lower

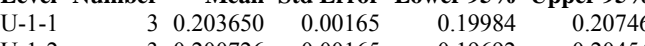

$\begin{array}{llllll}\text { U-1-2 } & 3 & 0.200726 & 0.00165 & 0.19692 & 0.20454\end{array}$

$\begin{array}{lllllll}\text { U-2-1 } & 3 & 0.202675 & 0.00165 & 0.19887 & 0.20649\end{array}$

$\begin{array}{llllll}\text { U-2-2 } & 3 & 0.201214 & 0.00165 & 0.19740 & 0.20502\end{array}$

Std Error uses a pooled estimate of error variance 
Exhibit A-3. Measurements by Analytical Series, Block and Sub-Block for the Batch 1 and LRM Standards by Oxide by Prep (continued)

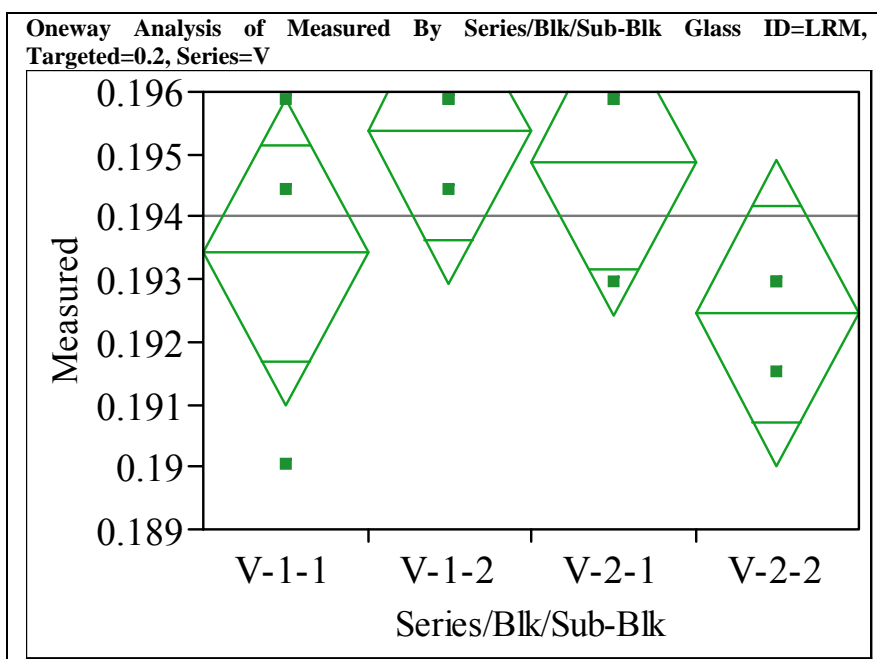

Oneway Anova

Summary of Fit

$\begin{array}{lr}\text { Rsquare } & 0.374486 \\ \text { Adj Rsquare } & 0.13918 \\ \text { Root Mean Square Error } & 0.001839 \\ \text { Mean of Response } & 0.194027 \\ \text { Observations (or Sum Wgts) } & 12\end{array}$

Analysis of Variance

Source DF Sum of Squares Mean Square F Ratio Prob > F

$\begin{array}{llllll}\text { Series/Blk/Sub-Blk } & 3 & 0.00001620 & 0.0000054 & 1.5965 & 0.2650 \\ \text { Error } & 8 & 0.00002706 & 3.3824 \mathrm{e}-6 & & \end{array}$

Error

$3.3824 \mathrm{e}-6$

Means for Oneway Anova

Level Number Mean Std Error Lower 95\% Upper 95\%

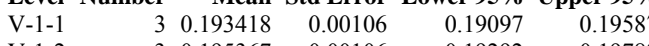

$\begin{array}{llllll}\text { V-1-2 } & 3 & 0.195367 & 0.00106 & 0.19292 & 0.19782\end{array}$

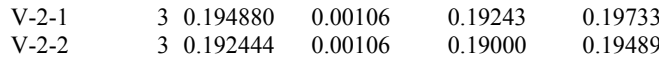

Std Error uses a pooled estimate of error variance

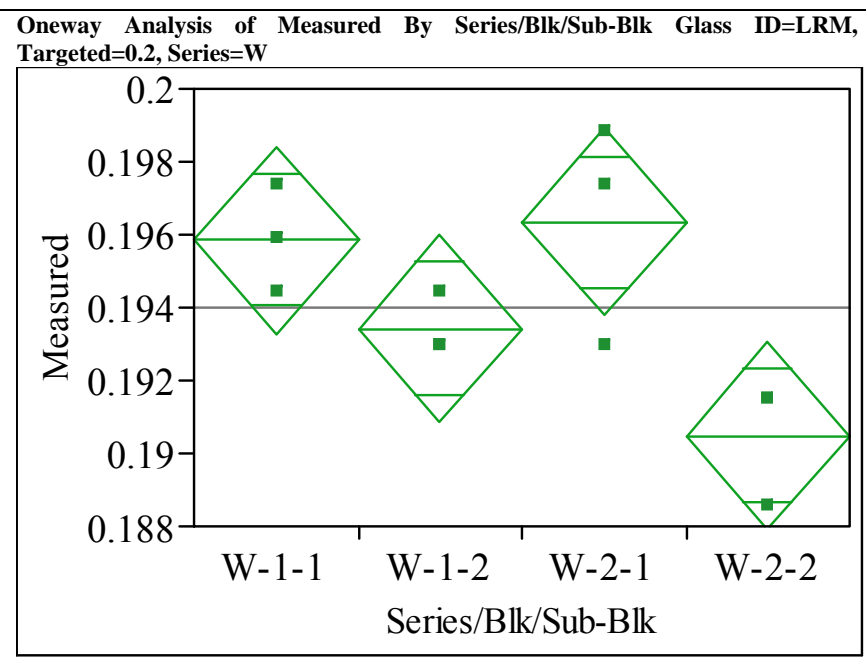

Oneway Anova

Summary of Fit

Rsquare

$\begin{array}{ll} & 0.5864972 \\ 0 & 0.001934\end{array}$

Mean of Response

0.194027
12

Analysis of Variance

Source DF Sum of Squares Mean Square F Ratio Prob > F

$\begin{array}{llllll}\text { Series/Blk/Sub-Blk } & 3 & 0.00006462 & 0.000022 & 5.7619 & 0.0213\end{array}$

$\begin{array}{lrr}\text { Error } & 8 & 0.00002991 \\ \text { C. } & 11 & 0.0009453\end{array}$

$3.738 \mathrm{e}-6$

Means for Oneway Anova

Level Number Mean Std Error Lower 95\% Upper 95\%

$\begin{array}{llllll}\text { W-1-1 } & 3 & 0.195854 & 0.00112 & 0.19328 & 0.19843\end{array}$

$\begin{array}{llllll}W-1-2 & 3 & 0.193418 & 0.00112 & 0.19084 & 0.19599\end{array}$

$\begin{array}{lllllll}\mathrm{W}-2-1 & 3 & 0.196342 & 0.00112 & 0.19377 & 0.19892\end{array}$

$\begin{array}{llllll}W-2-2 & 3 & 0.190495 & 0.00112 & 0.18792 & 0.19307\end{array}$

Std Error uses a pooled estimate of error variance 
Exhibit A-3. Measurements by Analytical Series, Block and Sub-Block for the Batch 1 and LRM Standards by Oxide by Prep (continued)

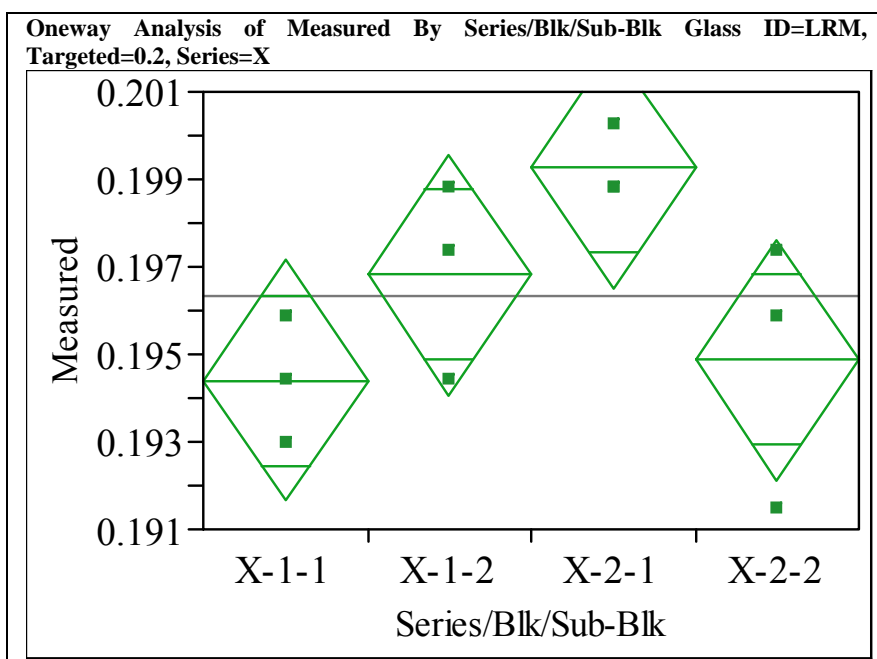

Oneway Anova

Summary of Fit

$\begin{array}{lr}\text { Rsquare } & 0.563636 \\ \text { Adj Rsquare } & 0.4 \\ \text { Root Mean Square Error } & 0.002067 \\ \text { Mean of Response } & 0.196342 \\ \text { Observations (or Sum Wgts) } & 12\end{array}$

Analysis of Variance

Source DF Sum of Squares Mean Square F Ratio Prob $>$ F

$\begin{array}{lrrrrr}\text { Series/Blk/Sub-Blk } & 3 & 0.00004415 & 0.000015 & 3.4444 & 0.0719 \\ \text { Error } & 8 & 0.00003418 & 4.273 \mathrm{e}-6 & & \end{array}$

Error

$4.273 \mathrm{e}-6$

Means for Oneway Anova

Level Number Mean Std Error Lower 95\% Upper 95\%

$\begin{array}{rrrrrr}\text { X-1-1 } & 3 & 0.194393 & 0.00119 & 0.19164 & 0.19714\end{array}$

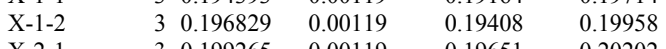

$\begin{array}{llllll}\mathrm{X}-2-1 & 3 & 0.199265 & 0.00119 & 0.19651 & 0.20202\end{array}$

$\begin{array}{llllll}\mathrm{X}-2-2 & 3 & 0.194880 & 0.00119 & 0.19213 & 0.19763\end{array}$

Std Error uses a pooled estimate of error variance

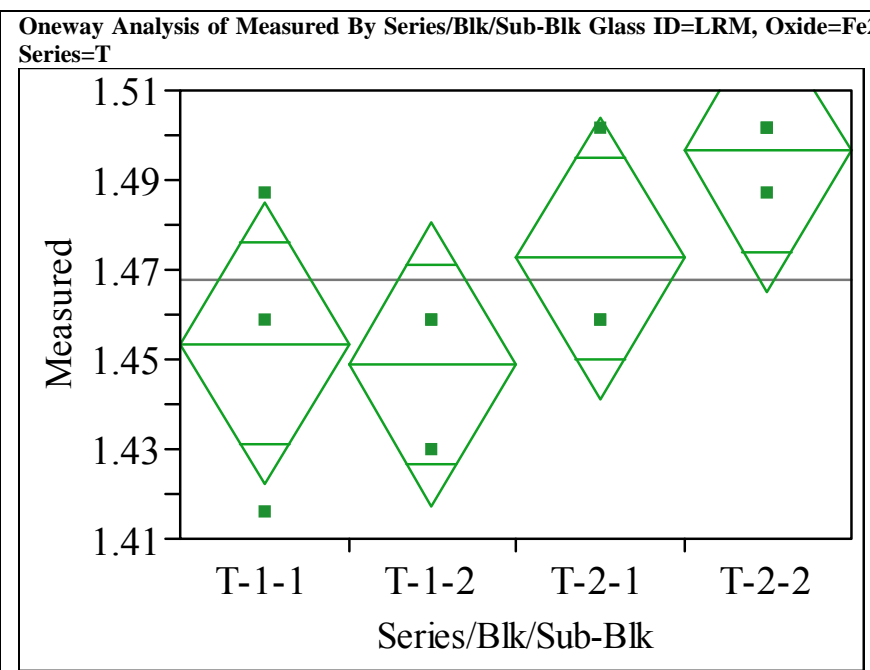

Oneway Anova

Summary of Fit

$\begin{array}{lr}\text { Rsquare } & 0.484375 \\ \text { Adj Rsquare } & 0.291016 \\ \text { Root Mean Square Error } & 0.023709 \\ \text { Mean of Response } & 1.467825 \\ \text { Observations (or Sum Wgts) } & 12\end{array}$

Observations (or Sum Wots) 1.467825

Analysis of Variance

Source DF Sum of Squares Mean Square F Ratio Prob $>$ F $\begin{array}{lrrrrr}\text { Series/Blk/Sub-Blk } & 3 & 0.00422435 & 0.001408 & 2.5051 & 0.1330\end{array}$

Error

C. Total

0.00872125

Means for Oneway Anova

Level Number Mean Std Error Lower 95\% Upper 95\%

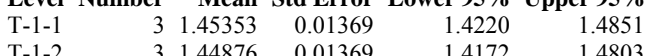

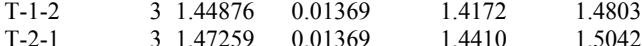

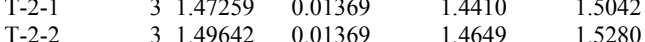

Std Error uses a pooled estimate of error variance 
Exhibit A-3. Measurements by Analytical Series, Block and Sub-Block for the Batch 1 and LRM Standards by Oxide by Prep (continued)

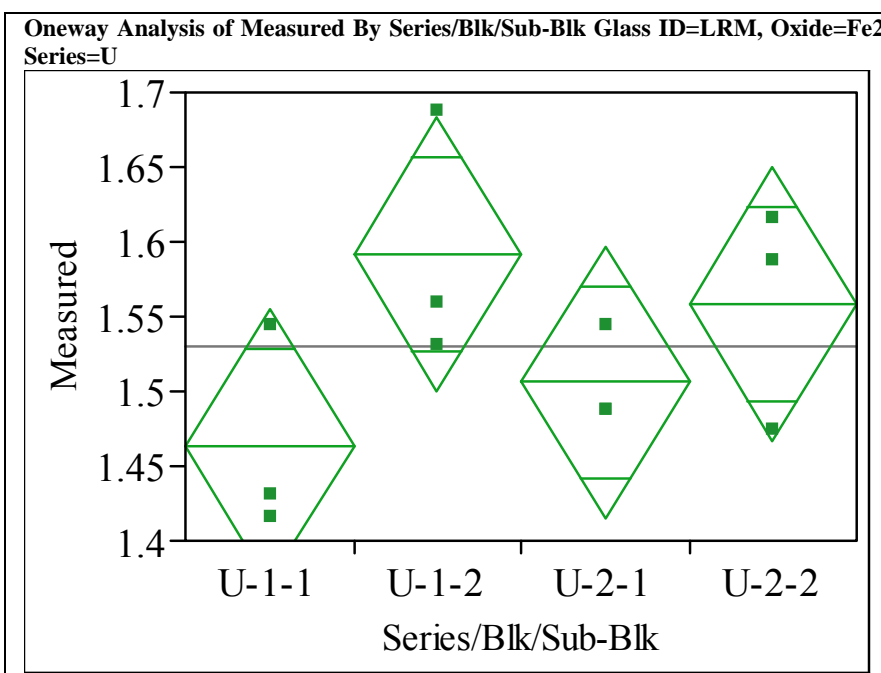

Oneway Anova

Summary of Fit

$\begin{array}{lr}\text { Rsquare } & 0.435583 \\ \text { Adj Rsquare } & 0.223926 \\ \text { Root Mean Square Error } & 0.068566 \\ \text { Mean of Response } & 1.529779 \\ \text { Observations (or Sum Wgts) } & 12\end{array}$

Analysis of Variance

Source DF Sum of Squares Mean Square F Ratio Prob $>$ F

$\begin{array}{lrrrrr}\text { Source } & \text { DF } & \text { Sum of Squares } & \text { Mean Square } & \text { F Ratio } & \text { Prob }>\text { F } \\ \text { Series/Blk/Sub-Blk } & 3 & 0.02902540 & 0.009675 & 2.0580 & 0.1843\end{array}$

$\begin{array}{lrrr}\text { Series/Blk/Sub-Blk } & 3 & 0.02902540 & 0.009675 \\ \text { Error } & 8 & 0.03761037 & 0.004701 \\ \text { C. Total } & 11 & 0.06663577 & \\ & & \end{array}$

0.06663577

Means for Oneway Anova

Level Number Mean Std Error Lower 95\% Upper 95\%

$\begin{array}{lllllr}\text { U-1-1 } & 3 & 1.46306 & 0.03959 & 1.3718 & 1.5543 \\ \mathrm{U}-1-2 & 3 & 1.59173 & 0.03959 & 1.5004 & 1.6830\end{array}$

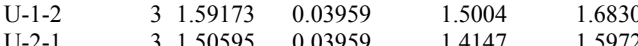

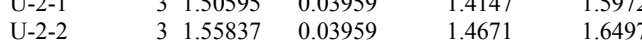

Std Error uses a pooled estimate of error variance

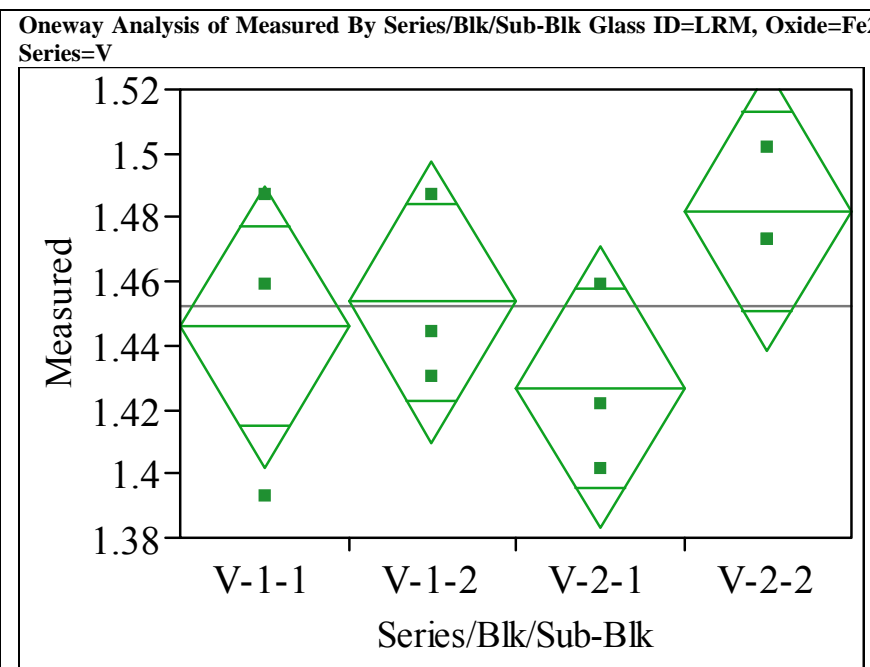

Oneway Anova

Summary of Fit

$\begin{array}{lr}\text { Rsquare } & 0.353096 \\ \text { Adj Rsquare } & 0.110508 \\ \text { Root Mean Square Error } & 0.032945 \\ \text { Mean of Response } & 1.452099 \\ \text { Observations (or Sum Wgts) } & 12\end{array}$

Analysis of Variance

$\begin{array}{lrrrrr}\text { Source } & \text { DF } & \text { Sum of Squares } & \text { Mean Square } & \text { F Ratio } & \text { Prob }>\text { F } \\ \text { Series/Blk/Sub-Blk } & 3 & 0.00473945 & 0.001580 & 1.4555 & 0.2977\end{array}$

$\begin{array}{llll}\text { Series/Blk/Sub-Blk } & 3 & 0.00473945 & 0.001580 \\ \text { Error } & 8 & 0.00868309 & 0.001085\end{array}$

C. Total

0.01342254

Means for Oneway Anova

Level Number Mean Std Error Lower 95\% Upper 95\%

$\begin{array}{rrrrrr}\text { Level } & \text { Number } & \text { Mean } & \text { Std Error } & \text { Lower } 95 \% & \text { Upper 95\% } \\ \text { V-1-1 } & 3 & 1.44590 & 0.01902 & 1.4020 & 1.4898\end{array}$

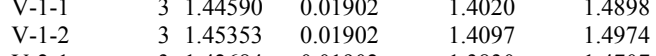

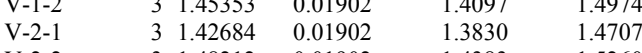

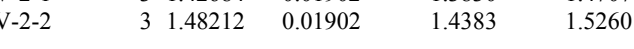

Std Error uses a pooled estimate of error variance 
Exhibit A-3. Measurements by Analytical Series, Block and Sub-Block for the Batch 1 and LRM Standards by Oxide by Prep (continued)

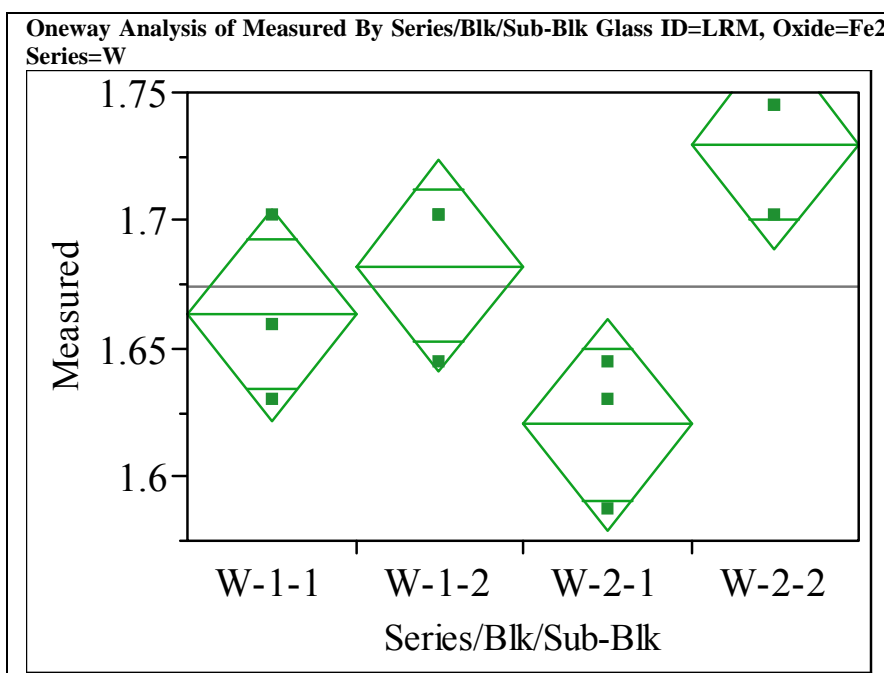

Oneway Anova

Summary of Fit

$\begin{array}{lr}\text { Rsquare } & 0.705236 \\ \text { Adj Rsquare } & 0.594699 \\ \text { Root Mean Square Error } & 0.03116 \\ \text { Mean of Response } & 1.67394 \\ \text { Observations (or Sum Wgts) } & 12\end{array}$

Analysis of Variance

Source DF Sum of Squares Mean Square F Ratio Prob > F

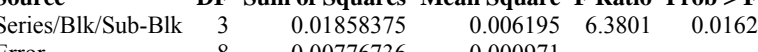

Error

$0.000971 \quad 6.3801 \quad 0.0162$

C. Total

0.02635111

Means for Oneway Anova

Level Number Mean Std Error Lower 95\% Upper 95\%

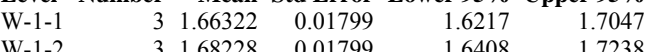

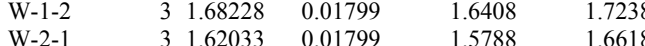

$\begin{array}{llllll}\text { W-2-1 } & 3 & 1.62033 & 0.01799 & 1.5788 & 1.6618 \\ \text { W-2-2 } & 3 & 1.72994 & 0.01799 & 1.6885 & 1.7714\end{array}$

Std Error uses a pooled estimate of error variance

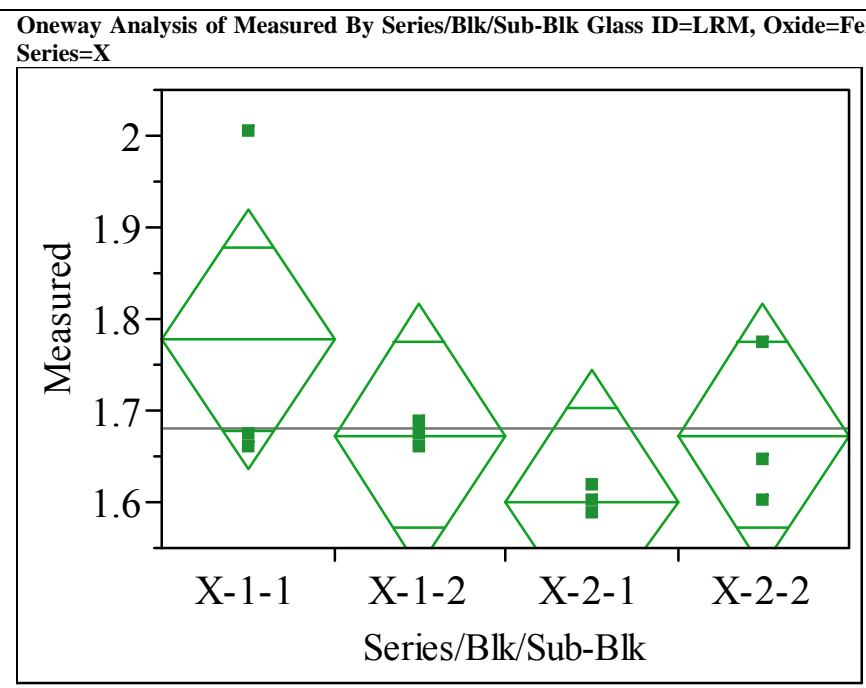

Oneway Anova

Summary of Fit

Rsquare

$\begin{array}{ll}\text { Adj Rsquare } & 0.340085 \\ & 0.092617\end{array}$

Root Mean Square Error $\quad 0.107307$

Mean of Response $\quad 1.681089$

$\begin{array}{rr}\text { Observations (or Sum Wgts) } & 12\end{array}$

Analysis of Variance

Source DF Sum of Squares Mean Square F Ratio Prob $>$

$\begin{array}{llllll}\text { Series/Blk/Sub-Blk } & 3 & 0.04747288 & 0.015824 & 1.3743 & 0.3187\end{array}$

$\begin{array}{lrr}\text { Error } & 8 & 0.09211816 \\ \text { C. } & 11 & 0.13959104\end{array}$

$\begin{array}{lrr}\text { C. Total } & 11 \quad 0.13959104\end{array}$

Means for Oneway Anova

evel Number Mean Std Error Lower 95\% Upper 95\%

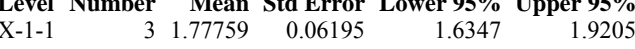

$\begin{array}{llllll}\mathrm{X}-1-2 & 3 & 1.67275 & 0.06195 & 1.63299 & 1.8156\end{array}$

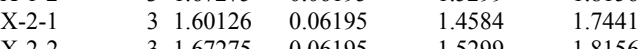

$\begin{array}{llllll}\mathrm{X}-2-2 & 3 & 1.67275 & 0.06195 & 1.5299 & 1.8156\end{array}$

Std Error uses a pooled estimate of error variance 
Exhibit A-3. Measurements by Analytical Series, Block and Sub-Block for the Batch 1 and LRM Standards by Oxide by Prep (continued)

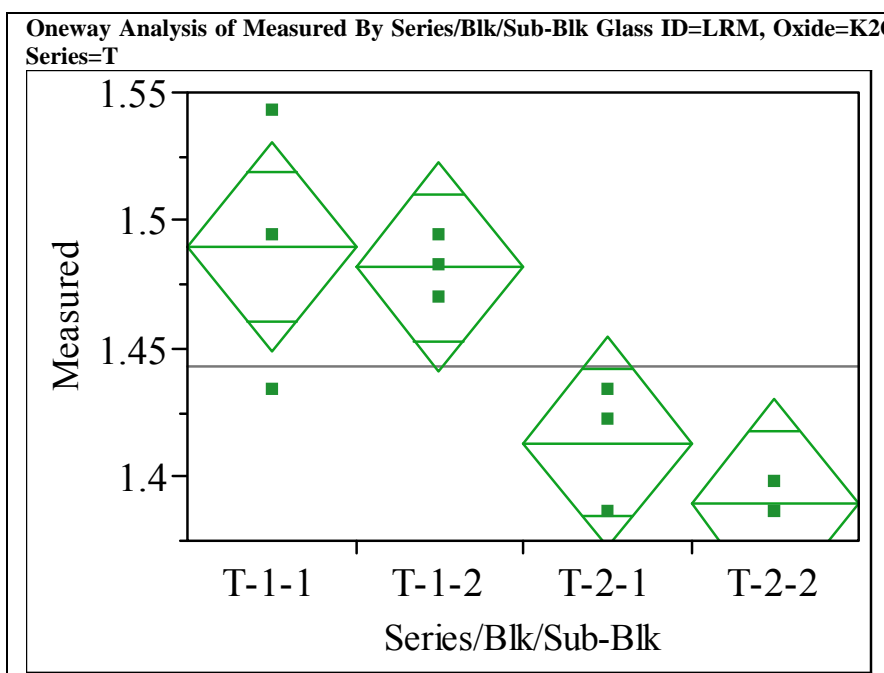

Oneway Anova

Summary of Fit

$\begin{array}{lr}\text { Rsquare } & 0.747164 \\ \text { Adj Rsquare } & 0.65235 \\ \text { Root Mean Square Error } & 0.030711 \\ \text { Mean of Response } & 1.443512 \\ \text { Observations (or Sum Wgts) } & 12\end{array}$

Analysis of Variance

Source DF Sum of Squares Mean Square F Ratio Prob > F

Series/Blk/Sub-Blk $\quad 3 \quad r \begin{array}{lrrr}0.02229797 & 0.007433 & 7.8803 & 0.0090\end{array}$

$\begin{array}{lcl}\text { Error } & 8 & 0.00754552\end{array}$

C. Total

0.02984349

Means for Oneway Anova

Level Number Mean Std Error Lower 95\% Upper 95\%

$\begin{array}{lrrrrr}\text { Level Number } & \text { Mean } & \text { Std Error } & \text { Lower } & \text { 95\% } & \text { Upper 95\% } \\ \text { T-1-1 } & 3 & 1.48969 & 0.01773 & 1.4488 & 1.5306\end{array}$

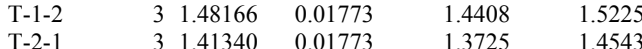

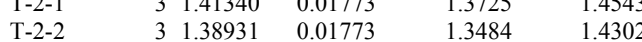

Std Error uses a pooled estimate of error variance

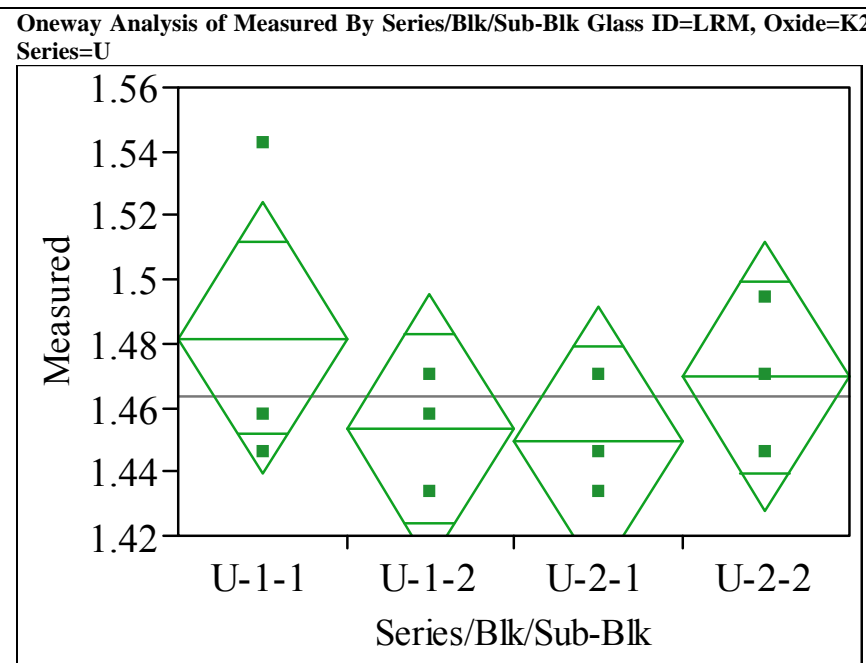

Oneway Anova

Summary of Fit

$\begin{array}{lr}\text { Rsquare } & 0.198068 \\ \text { Adj Rsquare } & -0.10266 \\ \text { Root Mean Square Error } & 0.03168 \\ \text { Mean of Response } & 1.463589 \\ \text { Observations (or Sum Wgts) } & 12\end{array}$

Analysis of Variance

$\begin{array}{lrrrrr}\text { Source } & \text { DF } & \text { Sum of Squares } & \text { Mean Square } & \text { F Ratio } & \text { Prob }>\text { F } \\ \text { Series/Blk/Sub-Blk } & 3 & 0.00198312 & 0.000661 & 0.6586 & 0.6001\end{array}$

$\begin{array}{llll}\text { Series/Blk/Sub-Blk } & 3 & 0.00198312 & 0.000661 \\ \text { Error } & 8 & 0.00802921 & 0.001004\end{array}$

C. Total

0.01001232

Means for Oneway Anova

Level Number Mean Std Error Lower 95\% Upper 95\%

\begin{tabular}{lrrrrr} 
U-1-1 & 3 & 1.48166 & 0.01829 & 1.4395 & 1.5238 \\
\hline & 3 & 1.5355 & 0.0182 & 1.414 & 1.4957
\end{tabular}

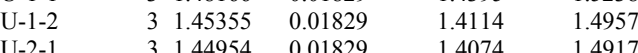

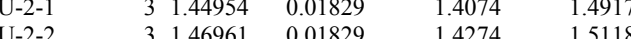

Std Error uses a pooled estimate of error variance 
Exhibit A-3. Measurements by Analytical Series, Block and Sub-Block for the Batch 1 and LRM Standards by Oxide by Prep (continued)

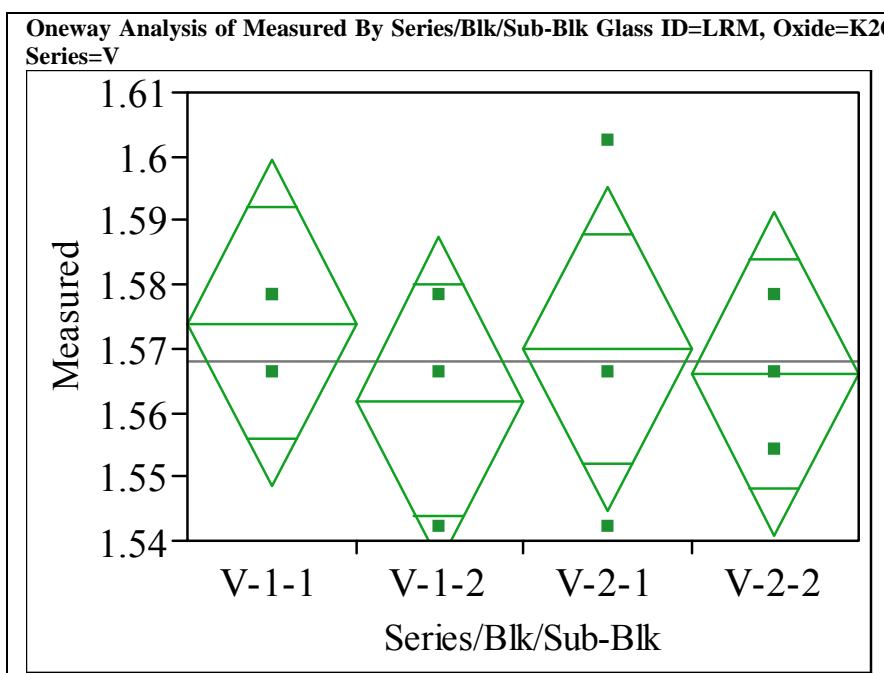

Oneway Anova

Summary of Fit

$\begin{array}{lr}\text { Rsquare } & 0.076923 \\ \text { Adj Rsquare } & -0.26923 \\ \text { Root Mean Square Error } & 0.019046 \\ \text { Mean of Response } & 1.567988 \\ \text { Observations (or Sum Wgts) } & 12\end{array}$

Analysis of Variance

Source DF Sum of Squares Mean Square F Ratio Prob > F

$\begin{array}{lrrrrr}\text { Series/Blk/Sub-Blk } & 3 & 0.00024184 & 0.000081 & 0.2222 & 0.8783 \\ \text { Error } & 8 & 0.00290212 & 0.000363 & & \\ \text { C. } & 11 & 0.00314397 & & \end{array}$

Error

$\begin{array}{lll}0.000081 & 0.2222 & 0.8783 \\ 0.000363 & & \end{array}$

C. Total

0.00314397

Means for Oneway Anova

Level Number Mean Std Error Lower 95\% Upper 95\%

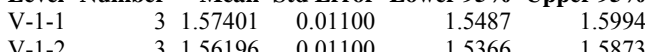

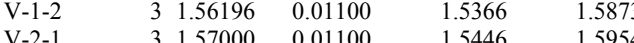

$\begin{array}{llllll}\mathrm{V}-2-1 & 3 & 1.57000 & 0.01100 & 1.5446 & 1.5954 \\ \mathrm{~V}-2-2 & 3 & 1.56598 & 0.01100 & 1.5406 & 1.5913\end{array}$

Std Error uses a pooled estimate of error variance

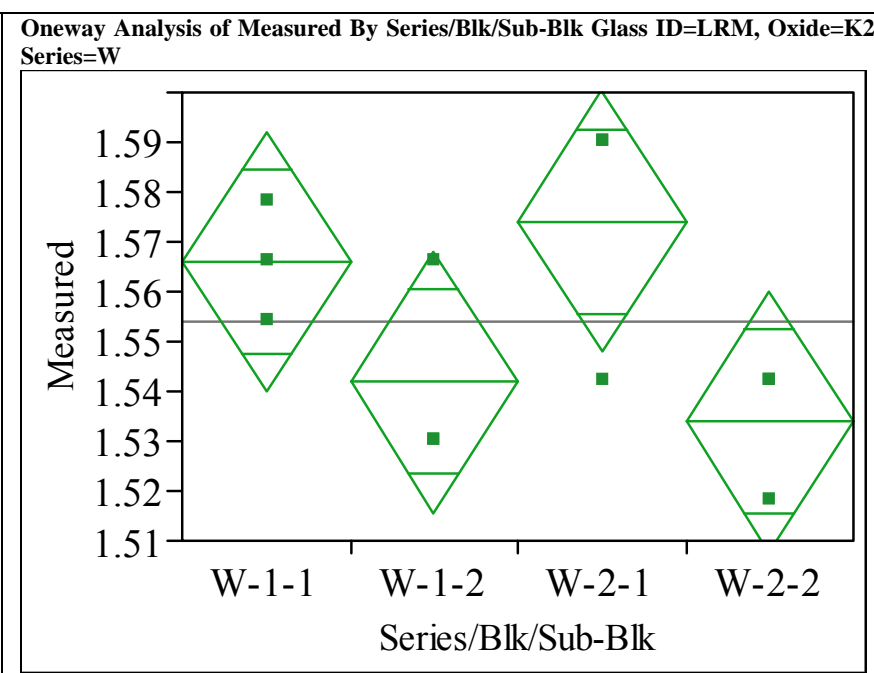

Oneway Anova

Summary of Fit

$\begin{array}{lr}\text { Rsquare } & 0.515152 \\ \text { Adj Rsquare } & 0.333333 \\ \text { Root Mean Square Error } & 0.019671 \\ \text { Mean of Response } & 1.553934 \\ \text { Observations (or Sum Wgts) } & 12\end{array}$

1.553934

Analysis of Variance

Source DF Sum of Squares Mean Square F Ratio Prob $>$ F

$\begin{array}{lrrrrr}\text { Series/Blk/Sub-Blk } & 3 & 0.00328907 & 0.001096 & 2.8333 & 0.1062 \\ \text { Error } & 8 & 0.00309560 & 0.000387 & & \end{array}$

0.000387

C. Total

$\begin{array}{ll}11 & 0.00638467\end{array}$

Means for Oneway Anova

Level Number Mean Std Error Lower 95\% Upper 95\%

$\begin{array}{lrrrrr}\text { Level } & \text { Number } & \text { Mean } & & & \\ \text { Wt Error } & \text { Lower } & \mathbf{9 5 \%} & \text { Upper 95\% } \\ \text { W-1-2 } & 3 & 1.56598 & 0.01136 & 1.5398 & 1.5922\end{array}$

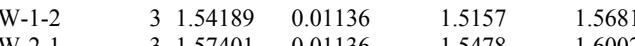

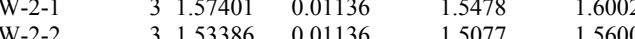

Std Error uses a pooled estimate of error variance 
Exhibit A-3. Measurements by Analytical Series, Block and Sub-Block for the Batch 1 and LRM Standards by Oxide by Prep (continued)

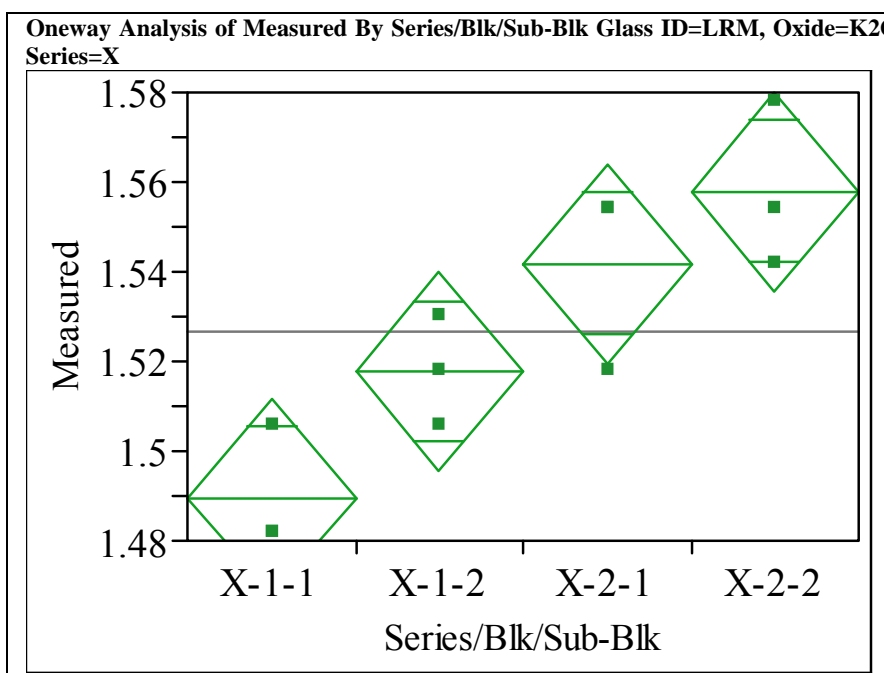

Oneway Anova

Summary of Fit

$\begin{array}{lr}\text { Rsquare } & 0.781732 \\ \text { Adj Rsquare } & 0.699881 \\ \text { Root Mean Square Error } & 0.016677 \\ \text { Mean of Response } & 1.526831 \\ \text { Observations (or Sum Wgts) } & 12\end{array}$

Analysis of Variance

Source DF Sum of Squares Mean Square F Ratio Prob > F

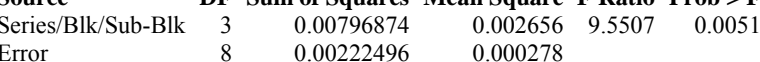

Error $\quad 8 \quad 0.00222496$

C. Total

0.01019370

Means for Oneway Anova

Level Number Mean Std Error Lower 95\% Upper 95\%

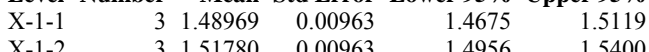

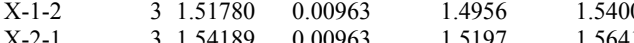

$\begin{array}{llllll}\mathrm{X}-2-1 & 3 & 1.54189 & 0.00963 & 1.5197 & 1.5641 \\ \mathrm{X}-2-2 & 3 & 1.55795 & 0.00963 & 1.5357 & 1.5802 \\ & & & & & \end{array}$

Std Error uses a pooled estimate of error variance

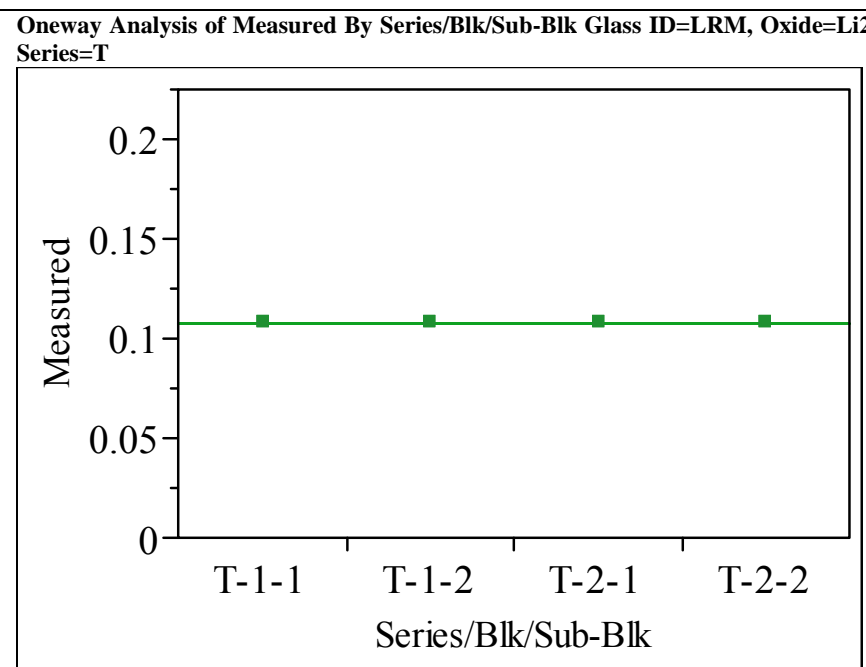

\section{Oneway Anova}

Summary of Fit

Rsquare

Adj Rsquare

Root Mean Square Error $\quad 0$

0.107645

Observations (or Sum Wg

Analysis of Variance

Source DF Sum of Squares Mean Square F Ratio Prob > F

Series/Blk/Sub-Blk

Error

C. Total

8

0

(1)

Means for Oneway Anova

Level Number Mean Std Error Lower 95\% Upper 95\%

$\begin{array}{rrrrrr}\text { Level Number } & \text { Mean } & \text { Std Error Lower } & \text { 95\% } & \text { Upper 95\% } \\ \text { T-1-1 } & 3 & 0.107645 & 0 & 0.10765 & 0.10765\end{array}$

$\begin{array}{llllll}\mathrm{T}-1-2 & 3 & 0.107645 & 0 & 0.10765 & 0.10765 \\ \mathrm{~T}-2-1 & 3 & 0.107645 & & 0.10765 & 0.10765\end{array}$

$\begin{array}{llllll}T-1-2 & 3 & 0.107645 & 0 & 0.10765 & 0.10765 \\ \mathrm{~T}-2-1 & 3 & 0.107645 & 0 & 0.10765 & 0.10765 \\ \mathrm{~T}-2-2 & 3 & 0.107645 & 0 & 0.10765 & 0.10765\end{array}$

Std Error uses a pooled estimate of error variance 
Exhibit A-3. Measurements by Analytical Series, Block and Sub-Block for the Batch 1 and LRM Standards by Oxide by Prep (continued)

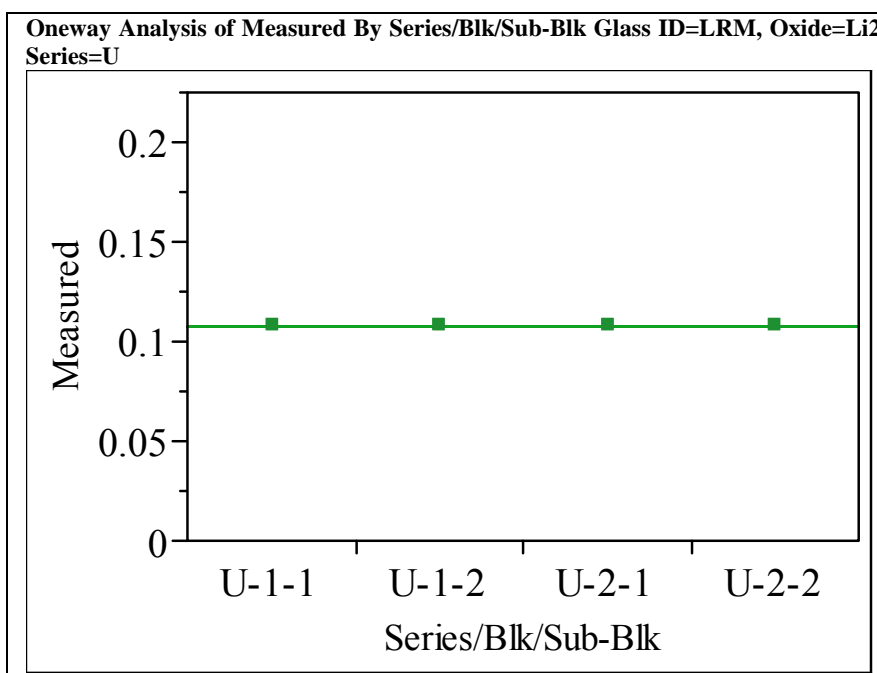

Oneway Anova

Summary of Fit

Rsquare

Adj Rsquare

Root Mean Square Error $\quad 0$

$\begin{array}{lr}\text { Mean of Response } & 0.107645 \\ \text { Observations (or Sum Wgts) } & 12\end{array}$

Analysis of Variance

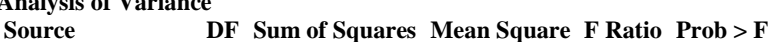

$\begin{array}{lrrr}\text { Series/Blk/Sub-Blk } & 3 & 0 & 0 \\ \text { Error } & 8 & 0 & 0\end{array}$

Error

C. Total

Means for Oneway Anova

Level Number Mean Std Error Lower 95\% Upper 95\%

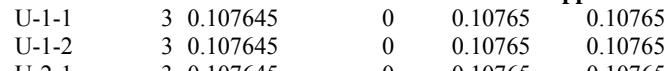

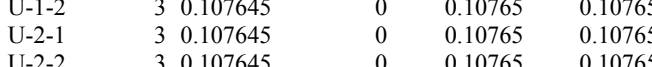

Std Error uses a pooled estimate of error variance

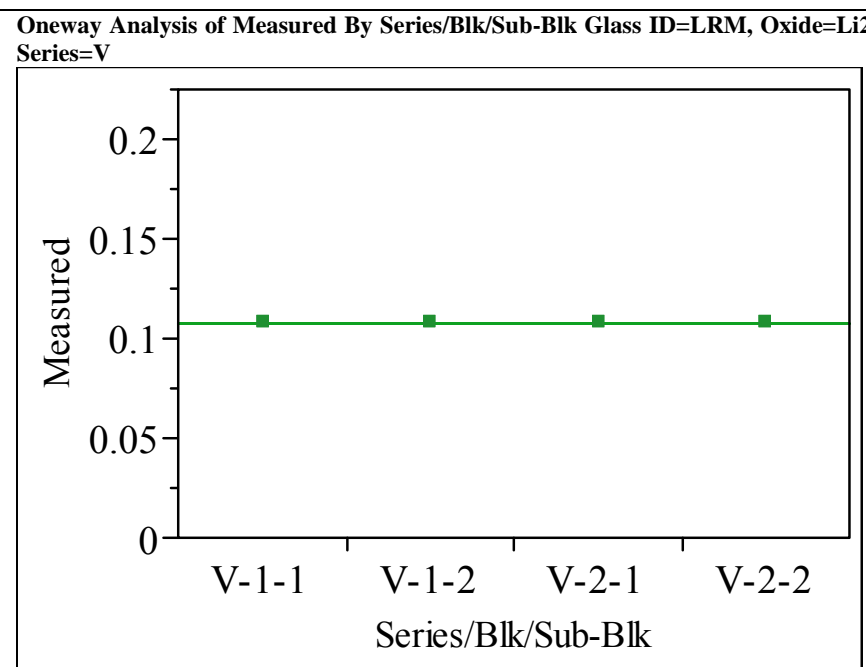

Oneway Anova

Summary of Fit

Rsquare

Adj Rsquare

Root Mean Square Error $\quad 0$

0.107645

Observations (or Sum Wg

Source DF Sum of Squares Mean Square F Ratio Prob > F

Series/Blk/Sub-Blk

Error

$\begin{array}{lll}3 & 0 & 0 \\ 8 & 0 & 0\end{array}$

C. Total

Means for Oneway Anova

Level Number Mean Std Error Lower 95\% Upper 95\%

$\begin{array}{llllll}\text { V-1-1 } & 3 & 0.107645 & 0 & 0.10765 & 0.10765 \\ V-1-2 & 3 & 0.107645 & 0 & 0.10765 & 0.10765\end{array}$

$\begin{array}{llllll}V-1-1 & 3 & 0.107645 & 0 & 0.10765 & 0.10765 \\ V-1-2 & 3 & 0.107645 & 0 & 0.10765 & 0.10765 \\ V-2-1 & 3 & 0.107645 & 0 & 0.10765 & 0.10765\end{array}$

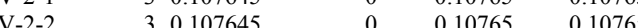

Std Error uses a pooled estimate of error variance 
Exhibit A-3. Measurements by Analytical Series, Block and Sub-Block for the Batch 1 and LRM Standards by Oxide by Prep (continued)

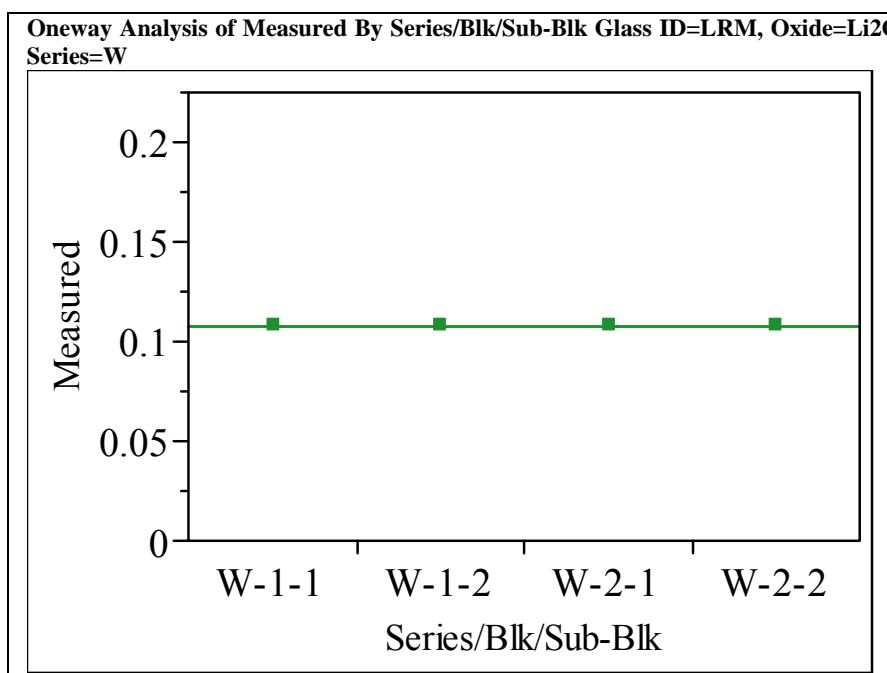

Oneway Anova

Summary of Fit

Rsquare

Adj Rsquare

Root Mean Square Error $\quad 0$

$\begin{array}{lr}\text { Mean of Response } & 0.107645 \\ \text { Observations (or Sum Wgts) } & 12\end{array}$

Analysis of Variance

Source DF Sum of Squares Mean Square F Ratio Prob > F

$\begin{array}{lrrr}\text { Source } & \text { DF Sum of Squares Mean Square } & \text { F } \\ \text { Series/Blk/Sub-Blk } & 3 & 0 & 0\end{array}$

$8 \quad 0$

C. Total

Means for Oneway Anova

Level Number Mean Std Error Lower 95\% Upper 95\%

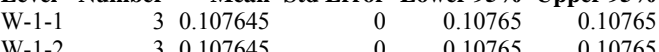

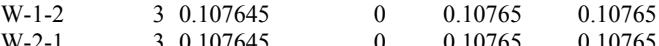

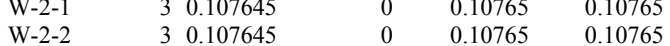

Std Error uses a pooled estimate of error variance

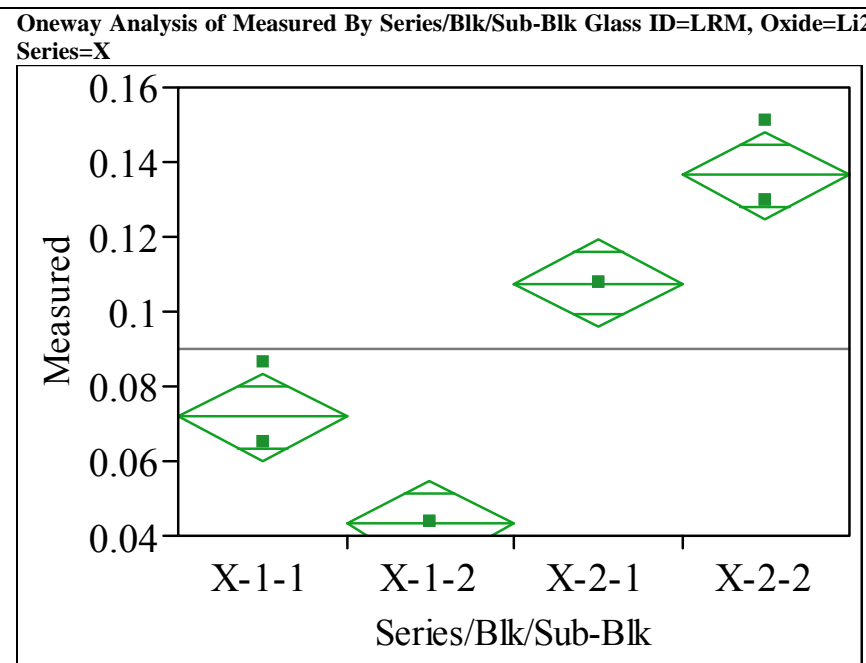

Oneway Anova

Summary of Fit

$\begin{array}{lr}\text { Rsquare } & 0.960396 \\ \text { Adj Rsquare } & 0.945545 \\ \text { Root Mean Square Error } & 0.008789 \\ \text { Mean of Response } & 0.089704\end{array}$

Analysis of Variance

Source DF Sum of Squares Mean Square F Ratio Prob > F

$\begin{array}{lrrrrr}\text { Source } & \text { DF } & \text { Sum of Squares } & \text { Mean Square } & \text { F Ratio } & \text { Prob }>\text { F } \\ \text { Series/Blk/Sub-Blk } & 3 & 0.01498643 & 0.004995 & 64.6667 & <.0001\end{array}$

$\begin{array}{llll}\text { Series/Blk/Sub-Blk } & 3 & 0.01498643 & 0.004995 \\ \text { Error } & 8 & 0.00061800 & 0.000077\end{array}$

C. Total

0.01560443

Means for Oneway Anova

Level Number Mean Std Error Lower 95\% Upper 95\%

$\begin{array}{rrrrrr}\text { L-1-1 } & 3 & 0.071763 & 0.00507 & 0.06006 & 0.08346\end{array}$

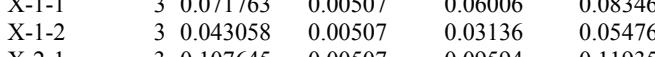

$\begin{array}{llllll}\mathrm{X}-2-1 & 3 & 0.107645 & 0.00507 & 0.09594 & 0.11935\end{array}$

$\begin{array}{llllll}\mathrm{X}-2-2 & 3 & 0.136350 & 0.00507 & 0.12465 & 0.1480\end{array}$

Std Error uses a pooled estimate of error variance 
Exhibit A-3. Measurements by Analytical Series, Block and Sub-Block for the Batch 1 and LRM Standards by Oxide by Prep (continued)

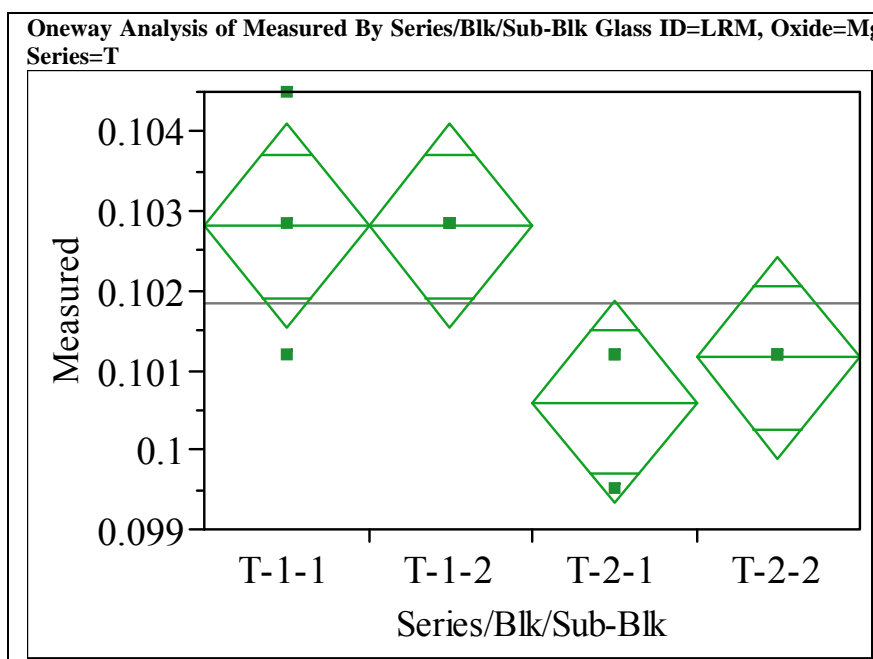

Oneway Anova

Summary of Fit

Rsquare $\quad 0.614458$

0.614488
Adj Rsquare $\quad 0.46988$

Root Mean Square Error $\quad 0.000957$

$\begin{array}{lr}\text { Mean of Response } & 0.101847 \\ \text { Observations (or Sum Wgts) } & 12\end{array}$

Analysis of Variance

$\begin{array}{lrrrrr}\text { Source } & \text { DF } & \text { Sum of Squares } & \text { Mean Square } & \text { F Ratio } & \text { Prob }>\text { F } \\ \text { Series/Blk/Sub-Blk } & 3 & 0.00001169 & 3.8958 \mathrm{e}-6 & 4.2500 & 0.0452 \\ \text { Error } & 8 & 0.00000733 & 9.1665 \mathrm{e}-7 & & \\ \text { C. } & 1 & 0.0001902 & & & \end{array}$

C. Total

0.00001902

Means for Oneway Anova

Level Number Mean Std Error Lower 95\% Upper 95\%

$\begin{array}{lllllr}\text { T-1-1 } & 3 & 0.102815 & 0.00055 & 0.10154 & 0.10409\end{array}$

$\begin{array}{llllll}\mathrm{T}-1-2 & 3 & 0.102815 & 0.00055 & 0.10154 & 0.10409\end{array}$

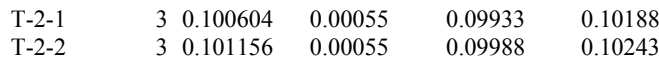

Std Error uses a pooled estimate of error variance

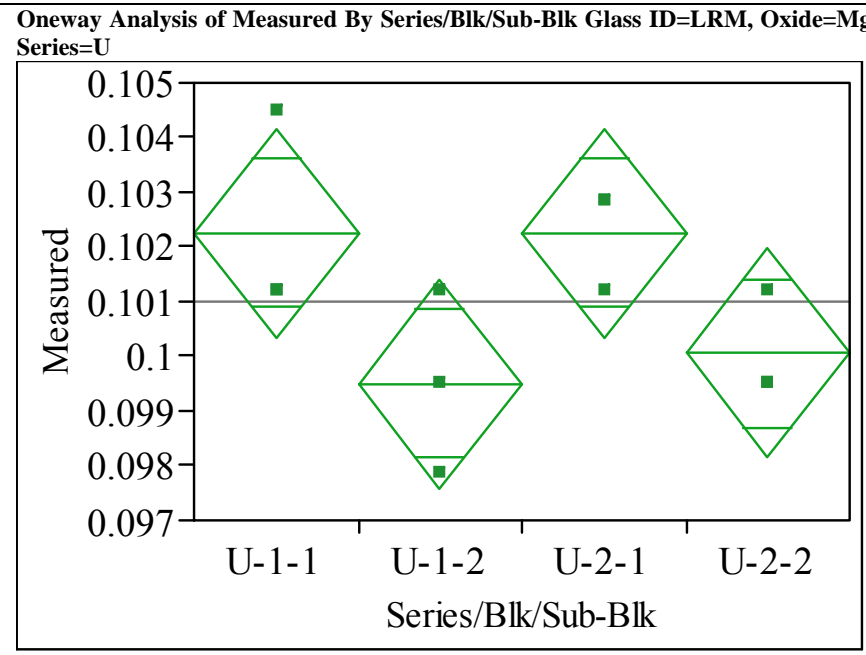

Oneway Anova

Summary of Fit

Rsquare

0.36129
0.001436

Mean of Response

$\begin{array}{rr}0.101018 \\ \text { Observations (or Sum Wgts) } & 12\end{array}$

Analysis of Variance

Source DF Sum of Squares Mean Square F Ratio Prob > F

$\begin{array}{llllll}\text { Series/Blk/Sub-Blk } & 3 & 0.00001902 & 6.3402 \mathrm{e}-6 & 3.0741 & 0.0907\end{array}$

Error $\quad 8 \quad 0.00001650$ $2.0625 \mathrm{e}-6$

Means for Oneway Anova

Level Number Mean Std Error Lower 95\% Upper 95\%

$\begin{array}{llllrr}\text { U-1-1 } & 3 & 0.102262 & 0.00083 & 0.10035 & 0.10417\end{array}$

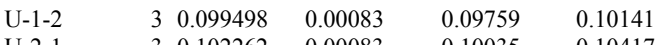

$\begin{array}{llllll}\text { U-2-1 } & 3 & 0.102262 & 0.00083 & 0.10035 & 0.10417\end{array}$

$\begin{array}{llllll}\mathrm{U}-2-2 & 3 & 0.100051 & 0.00083 & 0.09814 & 0.10196\end{array}$

Std Error uses a pooled estimate of error variance 
Exhibit A-3. Measurements by Analytical Series, Block and Sub-Block for the Batch 1 and LRM Standards by Oxide by Prep (continued)

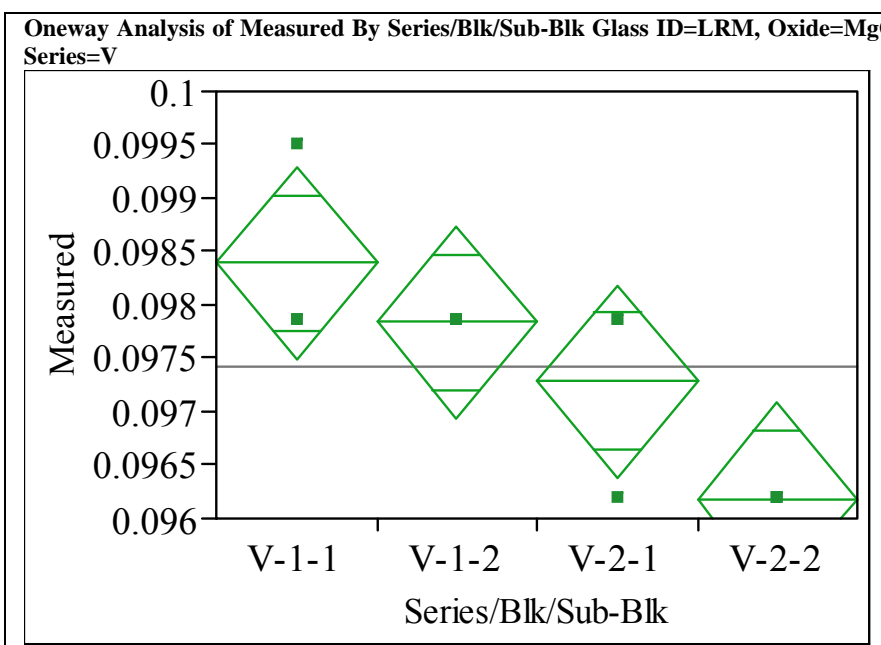

\section{Oneway Anova}

Summary of Fit

$\begin{array}{ll}\text { Rsquare } & 0.686275 \\ \text { Adj Rsquare } & 0.568627\end{array}$

Adj Rsquare $\quad 0.568627$

Root Mean Square Error $\quad 0.000677$

$\begin{array}{lr}\text { Mean of Response } & 0.097425 \\ \text { Observations (or Sum Wgts) } & 12\end{array}$

Analysis of Variance

Source DF Sum of Squares Mean Square F Ratio Prob > F

$\begin{array}{lrrrrr}\text { Source } & \text { DF } & \text { Sum of Squares } & \text { Mean Square F Ratio } & \text { Prob > F } \\ \text { Series/Blk/Sub-Blk } & 3 & 0.00000802 & 2.6736 \mathrm{e}-6 & 5.8333 & 0.0206 \\ \text { Error } & 8 & 0.00000367 & 4.5833 \mathrm{e}-7 & & \end{array}$

$4.5833 \mathrm{e}-7$

C. Total

$\begin{array}{rr}8 & 0.00000367 \\ 11 & 0.00001169\end{array}$

Means for Oneway Anova

Level Number Mean Std Error Lower 95\% Upper 95\%

$\begin{array}{lrrrrr}\text { Level Number } & \text { Mean } & \text { Std Error } & \text { Lower 95\% } & \text { Upper 95\% } \\ \text { V-1-1 } & 3 & 0.098392 & 0.00039 & 0.09749 & 0.09929 \\ V-1-2 & 3 & 0.097840 & 0.00039 & 0.09694 & 0.09874\end{array}$

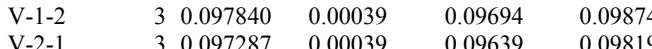

$\begin{array}{llllll}\mathrm{V}-2-1 & 3 & 0.097287 & 0.00039 & 0.09639 & 0.09819 \\ \mathrm{~V}-2-2 & 3 & 0.096181 & 0.00039 & 0.09528 & 0.09708\end{array}$

Std Error uses a pooled estimate of error variance

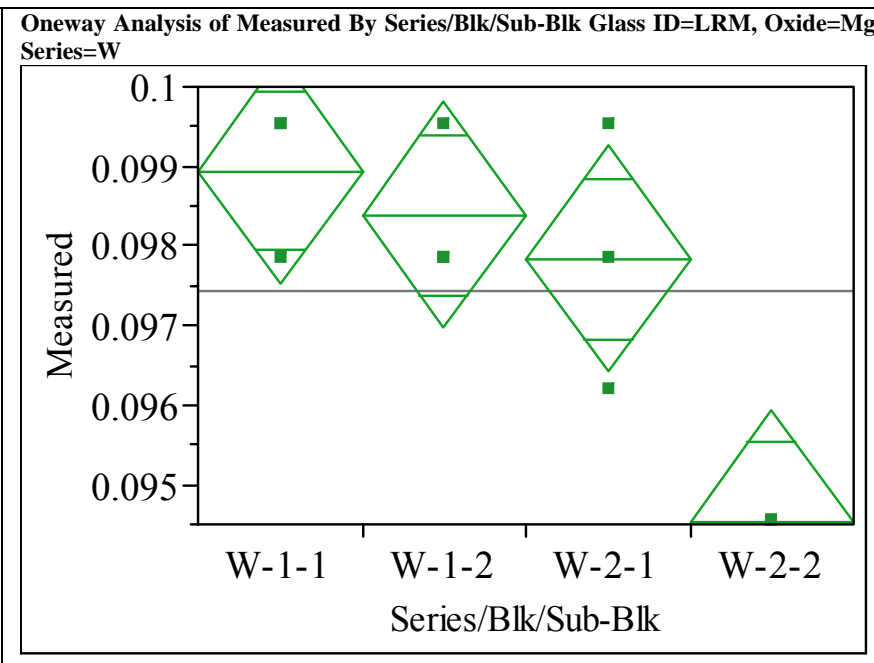

Oneway Anova

Summary of Fit

Adj Rsquare $\quad 0.717949$

Root Mean Square Error $\quad 0.00107$

$\begin{array}{lr}\text { Mean of Response } & 0.097425 \\ \text { Observations (or Sum Wgts) } & 12\end{array}$

Analysis of Variance

Source DF Sum of Squares Mean Square F Ratio Prob $>$ F

$\begin{array}{llllll}\text { Series/Blk/Sub-Blk } 3 & 0.00003552 & 0.000012 & 10.3333 & 0.0040\end{array}$

$\begin{array}{lrrr}\text { Error } & 8 & 0.00000917 & 1.146 \mathrm{e}-6 \\ \text { C. Total } & 11 & 0.00004469 & \end{array}$

Means for Oneway Anova

Level Number Mean Std Error Lower 95\% Upper 95\%

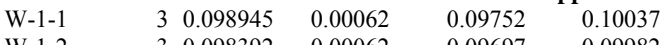

$\begin{array}{llllll}\mathrm{W}-1-2 & 3 & 0.098392 & 0.00062 & 0.09697 & 0.09982\end{array}$

$\begin{array}{llllll}\text { W-2-1 } & 3 & 0.097840 & 0.00062 & 0.09641 & 0.09926\end{array}$

$\begin{array}{llllll}\text { W-2-2 } & 3 & 0.094523 & 0.00062 & 0.09310 & 0.09595\end{array}$

Std Error uses a pooled estimate of error variance 
Exhibit A-3. Measurements by Analytical Series, Block and Sub-Block for the Batch 1 and LRM Standards by Oxide by Prep (continued)

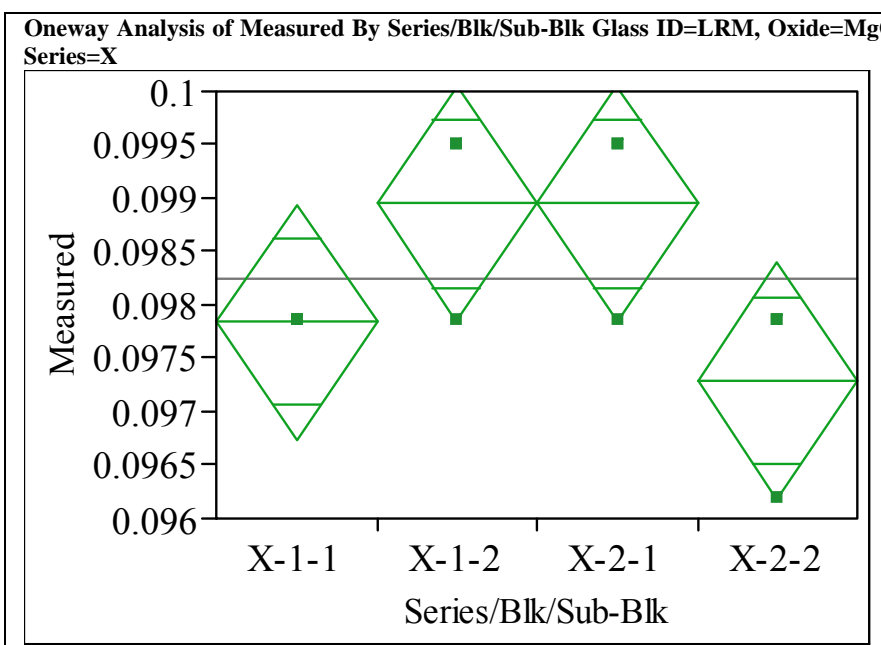

\section{Oneway Anova}

Summary of Fit

$\begin{array}{ll}\text { Rsquare } & 0.529412 \\ \text { Adj Rsquare } & 0.352941\end{array}$

Adj Rsquare $\quad 0.35294$

Root Mean Square Error $\quad 0.000829$

Mean of Response 0.09825

Analysis of Variance

Source DF Sum of Squares Mean Square F Ratio Prob > F

$\begin{array}{lrrrrrr}\text { Source } & \text { DF } & \text { Sum of Squares } & \text { Mean Square F Ratio } & \text { Prob > F } \\ \text { Series/Blk/Sub-Blk } & 3 & 0.00000619 & 2.0625 \mathrm{e}-6 & 3.0000 & 0.0951 \\ \text { Error } & 8 & 0.00000550 & 6.8749 \mathrm{e}-7 & & \end{array}$

$\begin{array}{lrr}\text { Error } & 8 & 0.00000550 \\ \text { C. Total } & 11 & 0.00001169\end{array}$

$6.8749 \mathrm{e}-7$

Means for Oneway Anova

Level Number Mean Std Error Lower 95\% Upper 95\%

$\begin{array}{rrrrrr}\text { Level } & \text { Number } & \text { Mean } & \text { Std Error } & \text { Lower 95\% } & \text { Upper 95\% } \\ \mathrm{X}-1-1 & 3 & 0.097840 & 0.00048 & 0.09674 & 0.09894\end{array}$

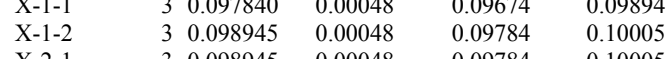

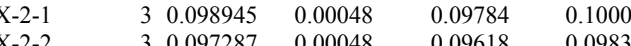

Std Error uses a pooled estimate of error variance

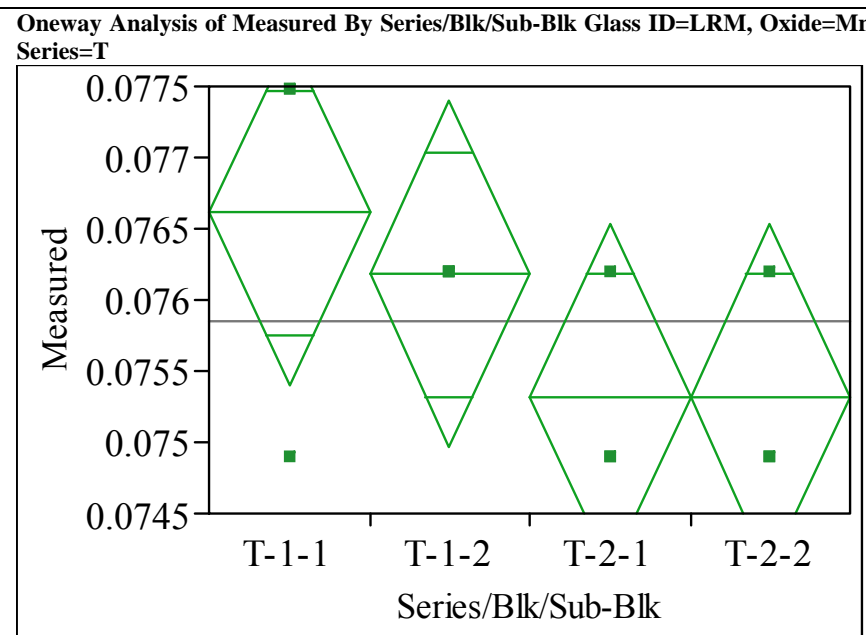

\section{Oneway Anova}

Summary of Fit

Rsquare

0.12
Root Mean Square Error $\quad 0.000913$

Root Mean Square Error $\quad 0.000913$

Observations (or Sum Wgts)

Source DF Sum of Squares Mean Square F Ratio Prob > F

$\begin{array}{lrrrrr}\text { Series/Blk/Sub-Blk } & 3 & 0.00000375 & 1.2504 \mathrm{e}-6 & 1.5000 & 0.2869\end{array}$

$\begin{array}{lll}\text { Error } & 8 & 0.00000667\end{array}$

0.00001042

$8.336 \mathrm{e}-7$

Means for Oneway Anova

Level Number Mean Std Error Lower 95\% Upper 95\%

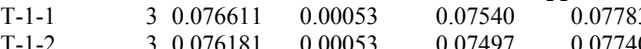

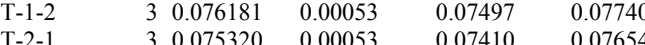

$\begin{array}{llllll} & 3 & 0.075320 & 0.00053 & 0.07410 & 0.07654\end{array}$

Std Error uses a pooled estimate of error variance 
Exhibit A-3. Measurements by Analytical Series, Block and Sub-Block for the Batch 1 and LRM Standards by Oxide by Prep (continued)

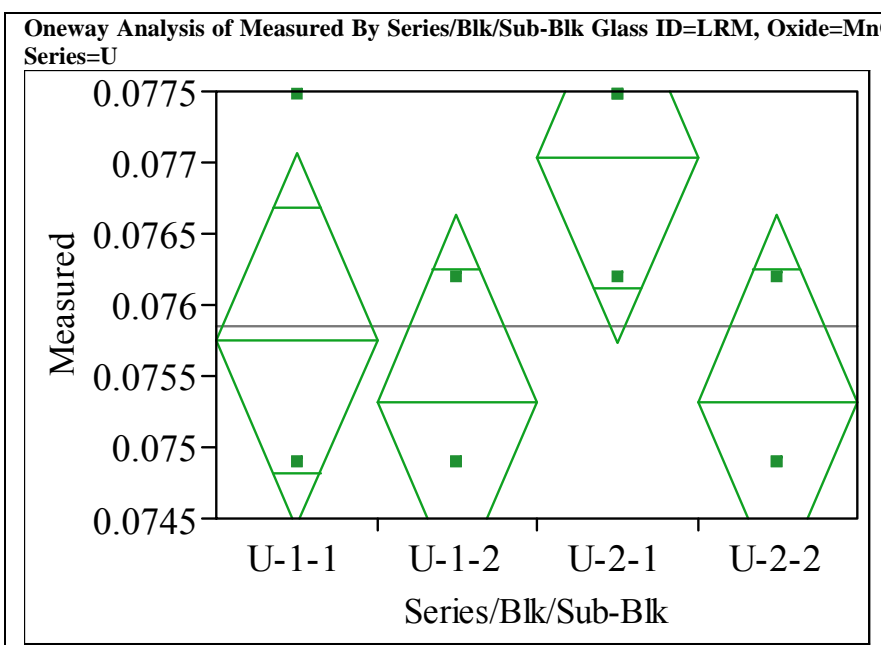

\section{Oneway Anova}

Summary of Fit

Rsquare

Adj Rsquare

0.222222

Root Mean Square Error $\quad 0.000986$

Mean of Resp

0.075858

Observations (or Sum Wgts)

Analysis of Variance

Source DF Sum of Squares Mean Square F Ratio Prob $>$ F

$\begin{array}{lrrrrr}\text { Series/Blk/Sub-Blk } & 3 & 0.00000597 & 1.9914 \mathrm{e}-6 & 2.0476 & 0.1858\end{array}$

$\begin{array}{lll}\text { Error } & 8 & 0.00000778 \\ \text { C. } & 11 & 0.00001375\end{array}$

$\begin{array}{lll}1.9914 \mathrm{e}-6 & 2.0476 & 0.1858 \\ 9.7253 \mathrm{e}-7 & & \end{array}$

C. Total

$11 \quad 0.00001375$

Means for Oneway Anova

Level Number Mean Std Error Lower 95\% Upper 95\%

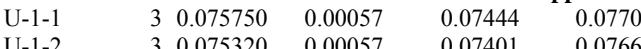

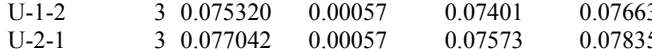

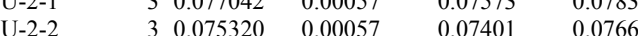

Std Error uses a pooled estimate of error variance

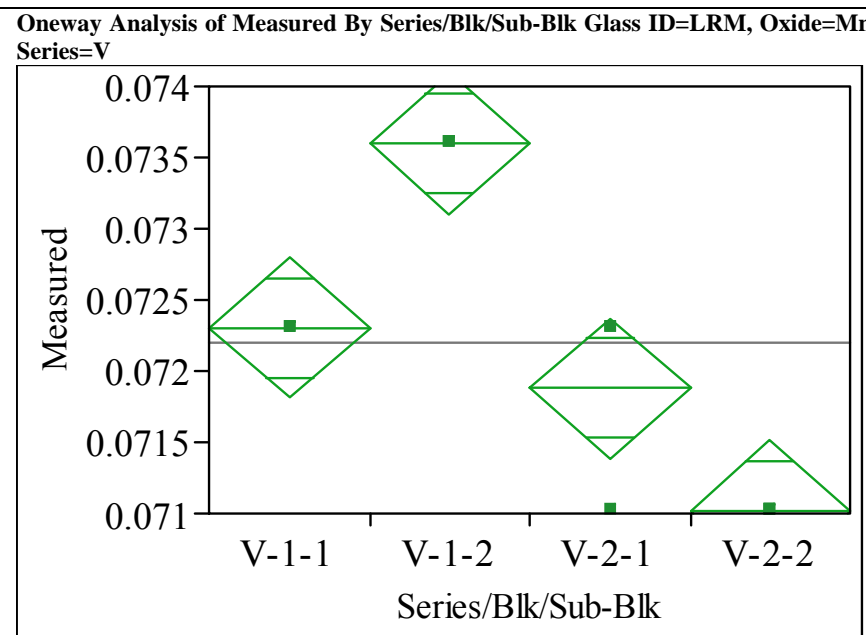

\section{Oneway Anova}

Summary of Fit

Rsquare $\quad 0.903614$

0.86747
Adj Rsquare

Root Mean Square Error $\quad 0.000373$

Observations (or Sum Wgts) $\quad 0.0722$

Analysis of Variance

Source DF Sum of Squares Mean Square F Ratio Prob $>$ F

$\begin{array}{lrrrrr} & & & \\ \text { Series/Blk/Sub-Blk } & 3 & 0.00001042 & 3.4733 \mathrm{e}-6 & 25.0000 & 0.0002\end{array}$

$\begin{array}{llll}\text { Error } & 8 & 0.00000111 & 1.3893 \mathrm{e}-7\end{array}$

$\begin{array}{lrr}\text { C. Total } & 11 & 0.00001153\end{array}$

Means for Oneway Anova

Level Number Mean Std Error Lower 95\% Upper 95\%

$\begin{array}{lllllll}\text { V-1-1 } & 3 & 0.072307 & 0.00022 & 0.07181 & 0.07280\end{array}$

$\begin{array}{lllllll}V-1 & & 3 & 0.073598 & 0.00022 & 0.07310 & 0.07409 \\ \text { V-2-1 } & & 3 & 0.071877 & 0.00022 & 0.07138 & 0.07237 \\ V-2-2 & & 3 & 0.071016 & 0.00022 & 0.07052 & 0.07151\end{array}$

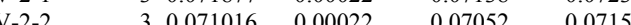

Std Error uses a pooled estimate of error variance 
Exhibit A-3. Measurements by Analytical Series, Block and Sub-Block for the Batch 1 and LRM Standards by Oxide by Prep (continued)

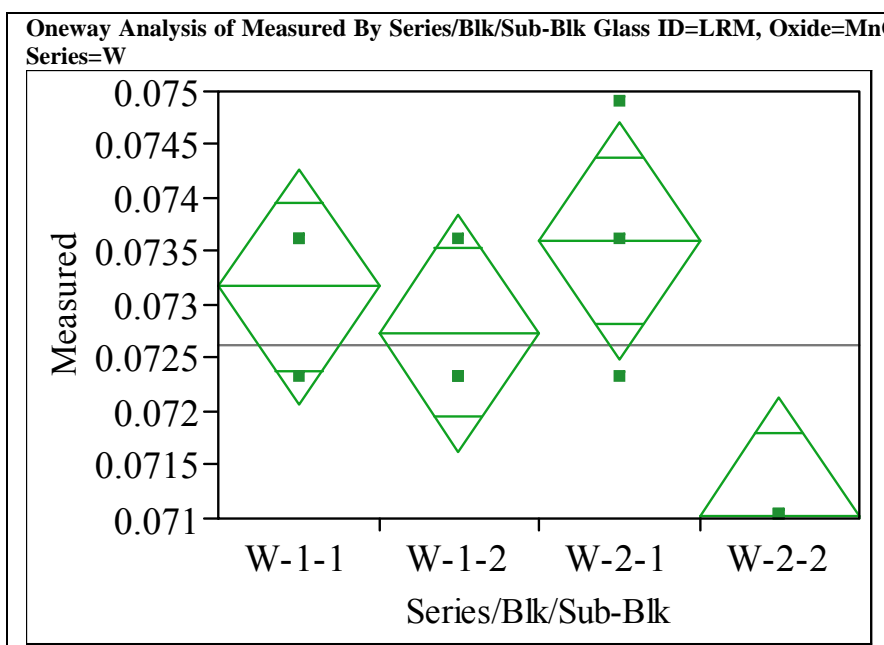

\section{Oneway Anova}

Summary of Fit

Rsquare $\quad 0.674797$

Adj Rsquare $\quad 0.552846$

Root Mean Square Error $\quad 0.000833$

Mean of Response 0.07263

Analysis of Variance

Source DF Sum of Squares Mean Square F Ratio Prob $>$ F

$\begin{array}{lrrrrrr}\text { Source } & \text { DF } & \text { Sum of Squares } & \text { Mean Square } & \text { F Ratio } & \text { Prob }>\text { F } \\ \text { Series/Blk/Sub-Blk } & 3 & 0.00001153 & 3.8438 \mathrm{e}-6 & 5.5333 & 0.0237\end{array}$

$\begin{array}{lrrrr}\text { Series/Blk/Sub-Blk } & 3 & 0.00001153 & 3.8438 \mathrm{e}-6 & 5.53 \\ \text { Error } & 8 & 0.00000556 & 6.9467 \mathrm{e}-7\end{array}$

Means for Oneway Anova
Level Number Mean Std Error Lower 95\% Upper 95\%

$\begin{array}{lrrrrr}\text { Level } & 3 & \text { Mean } & \text { Std Error } & \text { Lower 95\% } & \text { Upper 95\% } \\ \text { W-1 } & 0.073168 & 0.00048 & 0.07206 & 0.07428 \\ \text { W } 1-2 & 3 & 0.072738 & 0.00048 & 0.07163 & 0.07385\end{array}$

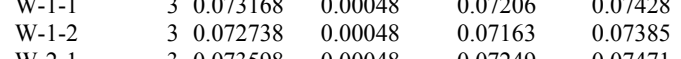

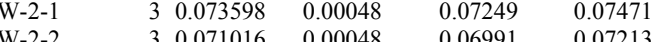

Std Error uses a pooled estimate of error variance

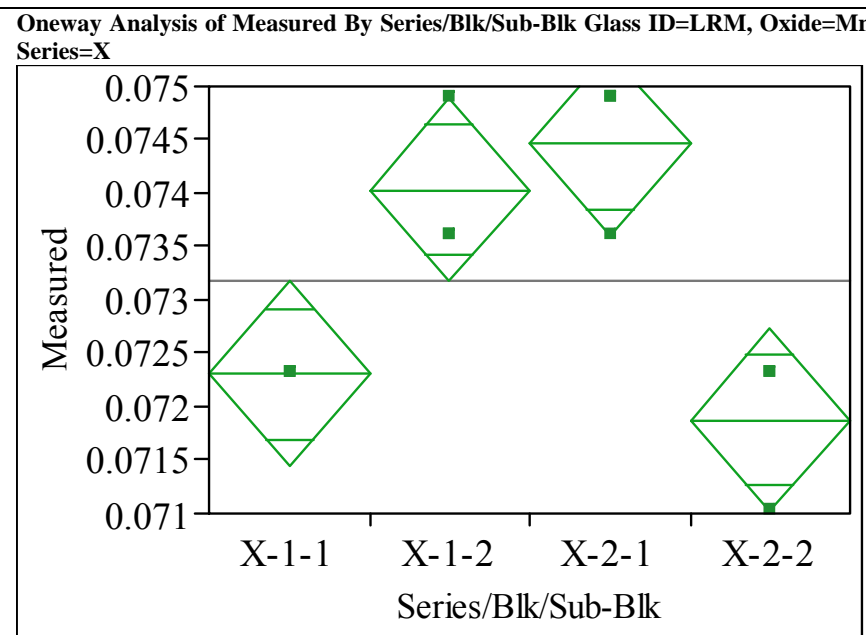

\section{Oneway Anova}

Summary of Fit

$\begin{array}{lr}\text { Rsquare } & 0.8125 \\ \text { Adj Rsquare } & 0.742188 \\ \text { Root Mean Square Error } & 0.000646 \\ \text { Mean of Response } & 0.073168 \\ \text { Observations (or Sum Wgts) } & 12\end{array}$

Mean of Response $\quad 0.073168$

Analysis of Variance

Source DF Sum of Squares Mean Square F Ratio Prob $>$ F

$\begin{array}{lrrrrr}\text { Source } & \text { DF } & \text { Sum of Squares } & \text { Mean Square } & \text { F Ratio } & \text { Prob }>\text { F } \\ \text { Series/Blk/Sub-Blk } & 3 & 0.00001445 & 4.8163 \mathrm{e}-6 & 11.5556 & 0.0028\end{array}$

$\begin{array}{lllr}\text { Series/Blk/Sub-Blk } & 3 & 0.00001445 & 4.8163 \mathrm{e}-6 \\ \text { Error } & 8 & 0.00000333 & 4.168 \mathrm{e}-7\end{array}$

$\begin{array}{lrr}\text { Error } & 8 & 0.00000333 \\ \text { C. Total } & 11 & 0.00001778\end{array}$

Means for Oneway Anova

Level Number Mean Std Error Lower 95\% Upper 95\%

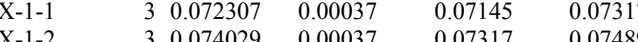

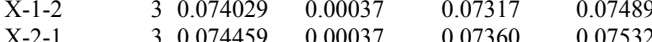

$\begin{array}{llllll}\mathrm{X}-1-2 & 3 & 0.074029 & 0.0037 & 0.07317 & 0.07489 \\ \mathrm{X}-2-2 & 3 & 0.071877 & 0.00037 & 0.07102 & 0.07532\end{array}$

Std Error uses a pooled estimate of error variance 
Exhibit A-3. Measurements by Analytical Series, Block and Sub-Block for the Batch 1 and LRM Standards by Oxide by Prep (continued)

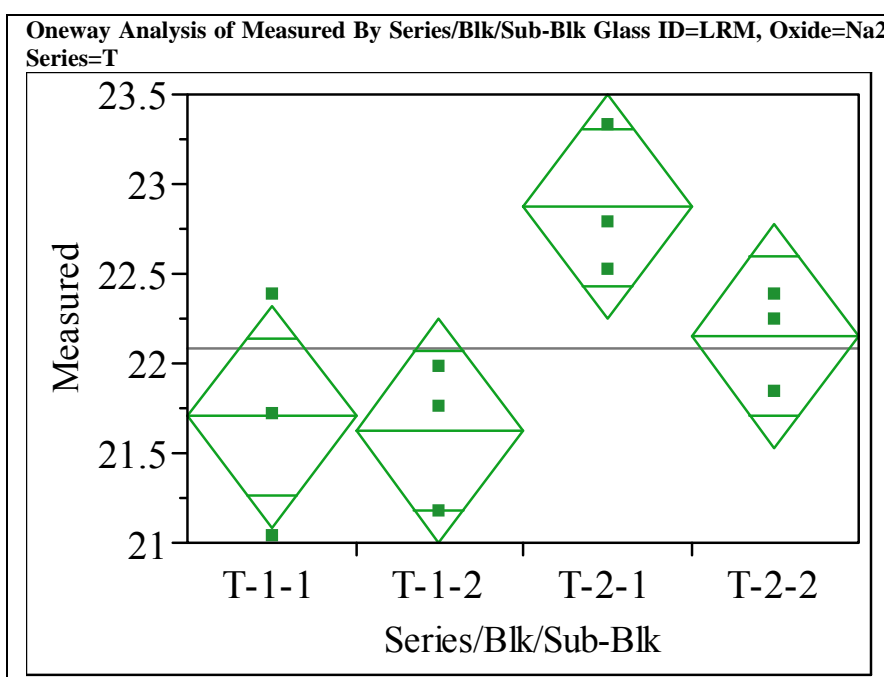

Oneway Anova

Summary of Fit

$\begin{array}{lr}\text { Rsquare } & 0.626195 \\ \text { Adj Rsquare } & 0.486018 \\ \text { Root Mean Square Error } & 0.468078 \\ \text { Mean of Response } & 22.0881 \\ \text { Observations (or Sum Wgts) } & 12\end{array}$

Analysis of Variance

Source DF Sum of Squares Mean Square F Ratio Prob > F

$\begin{array}{lrrrrrr}\text { Source } & \text { DF Sum of Squares } & \text { Mean Square } & \text { F Ratio } & \text { Prob }>\text { F } \\ \text { Series/Blk/Sub-Blk } & 3 & 2.9362432 & 0.978748 & 4.4672 & 0.0402\end{array}$

$\begin{array}{lrrr}\text { Series/Blk/Sub-Blk } & 3 & 2.9362432 & 0.978748 \\ \text { Error } & 8 & 1.7527785 & 0.219097\end{array}$

C. Total

4.6890217

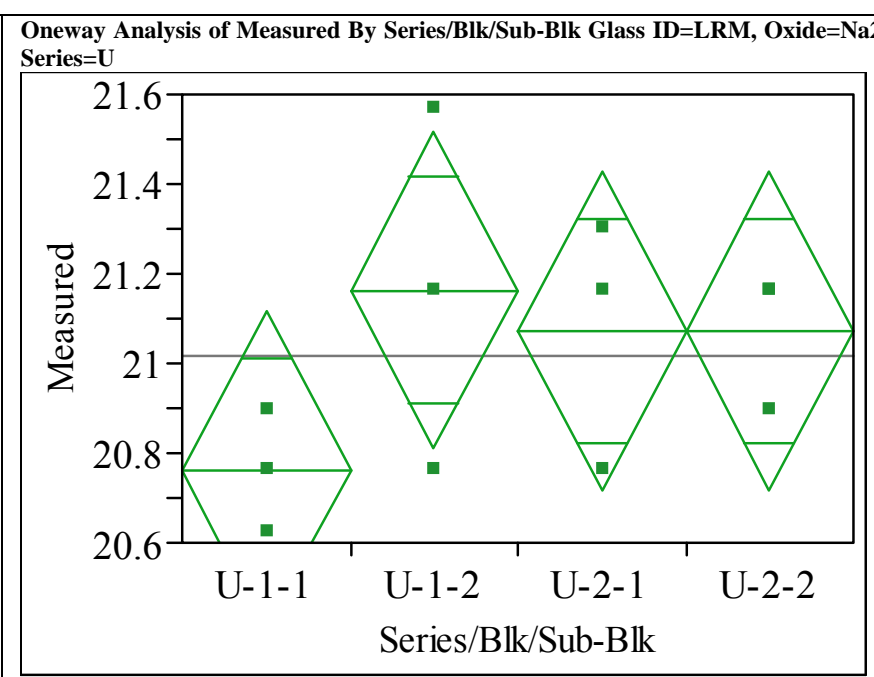

Oneway Anova

Summary of Fit

$\begin{array}{lr}\text { Rsquare } & 0.332149 \\ \text { Adj Rsquare } & 0.081705 \\ \text { Root Mean Square Error } & 0.266777 \\ \text { Mean of Response } & 21.01757 \\ \text { Observations (or Sum Wgts) } & 12\end{array}$

Analysis of Variance

Source DF Sum of Squares Mean Square F Ratio Prob $>$ F

$\begin{array}{lrrrrr}\text { Source } & \text { DF } & \text { Sum of Squares } & \text { Mean Square } & \text { F Ratio } & \text { Prob }>\mathbf{F} \\ \text { Series/Blk/Sub-Blk } & 3 & 0.28316537 & 0.094388 & 1.3262 & 0.3320\end{array}$

$\begin{array}{lrrr}\text { Series/Blk/Sub-Blk } & 3 & 0.28316537 & 0.094388 \\ \text { Error } & 8 & 0.56935925 & 0.071170\end{array}$

Means for Oneway Anova

Level Number Mean Std Error Lower 95\% Upper 95\%

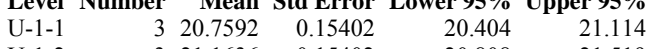

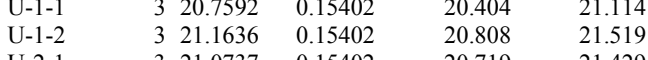

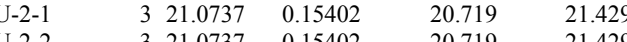

$\begin{array}{rrrrrr}\mathrm{T}-1-1 & 3 & 21.7028 & 0.27025 & 21.080 & 22.326\end{array}$

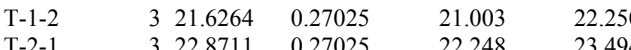

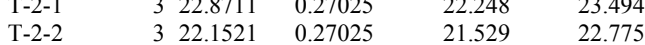

Std Error uses a pooled estimate of error variance

Std Error uses a pooled estimate of error variance 
Exhibit A-3. Measurements by Analytical Series, Block and Sub-Block for the Batch 1 and LRM Standards by Oxide by Prep (continued)

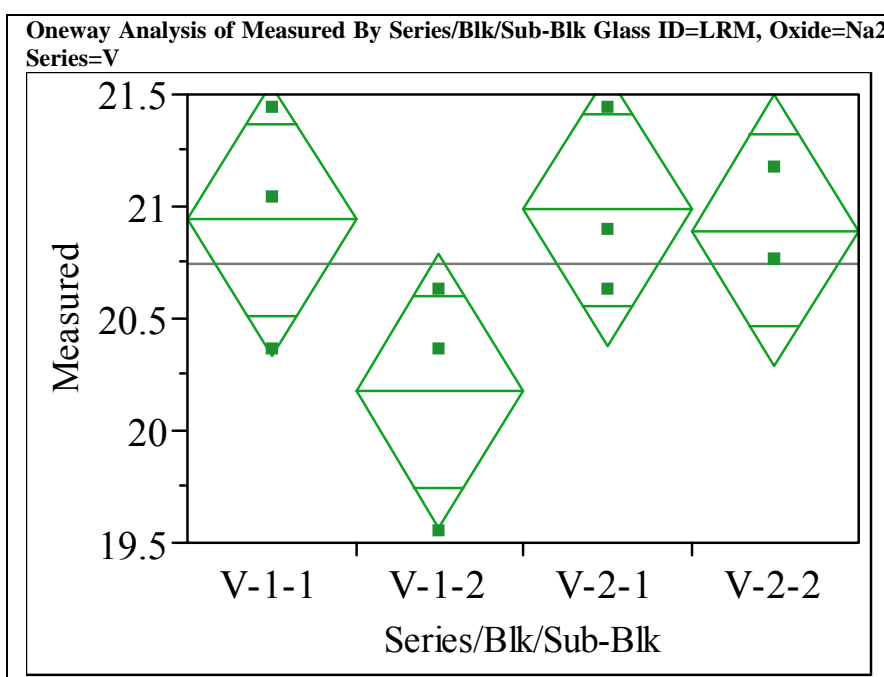

Oneway Anova

Summary of Fit

$\begin{array}{lr}\text { Rsquare } & 0.442142 \\ \text { Adj Rsquare } & 0.232946 \\ \text { Root Mean Square Error } & 0.457129 \\ \text { Mean of Response } & 20.74797 \\ \text { Observations (or Sum Wgts) } & 12\end{array}$

Analysis of Variance

Source DF Sum of Squares Mean Square F Ratio Prob > F

$\begin{array}{llllll}\text { Series/Blk/Sub-Blk } & 3 & 1.3249717 & 0.441657 & 2.1135 & 0.1768\end{array}$

$\begin{array}{lll}\text { Error } & 8 & 1.6717357\end{array}$

0.208967

C. Total

2.9967073

Means for Oneway Anova

Level Number Mean Std Error Lower 95\% Upper 95\%

$\begin{array}{llllrr}\text { V-1-1 } & 3 & 20.9389 & 0.26392 & 20.330 & 21.548 \\ \text { V-1-2 } & 3 & 20.1751 & 0.26392 & 19.566 & 20.784\end{array}$

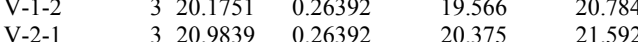

$\begin{array}{llllll}V-2-1 & 3 & 20.9839 & 0.26392 & 20.375 & 21.592 \\ \text { V-2-2 } & 3 & 20.8940 & 0.26392 & 20.285 & 21.503\end{array}$

Std Error uses a pooled estimate of error variance

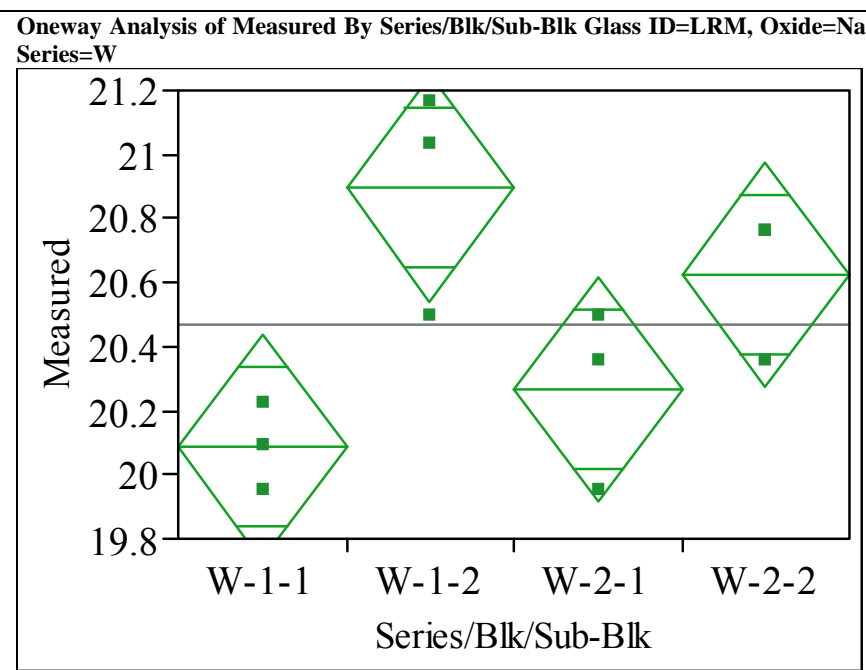

Oneway Anova

Summary of Fit

\begin{tabular}{lr} 
Rsquare & 0.679443 \\
Adj Rsquare & 0.559233 \\
Root Mean Square Error & 0.263924 \\
Mean of Response & 20.46713 \\
\hline
\end{tabular}

Analysis of Variance

Source DF Sum of Squares Mean Square F Ratio Prob > F

$\begin{array}{lrrrrrr}\text { Source } & \text { DF Sum of Squares } & \text { Mean Square } & \text { F Ratio } & \text { Prob }>\text { F } \\ \text { Series/Blk/Sub-Blk } & 3 & 1.1811176 & 0.393706 & 5.6522 & 0.0224\end{array}$

$\begin{array}{llll}\text { Series/Blk/Sub-Blk } & 3 & 1.1811176 & 0.393706 \\ \text { Error } & 8 & 0.5572452 & 0.069656\end{array}$

C. Total

17383628

Means for Oneway Anova

Level Number Mean Std Error Lower 95\% Upper 95\%

$\begin{array}{lrrrrr} & \\ \text { W-1-1 } & 3 & 20.0852 & 0.15238 & 19.734 & 20.437\end{array}$

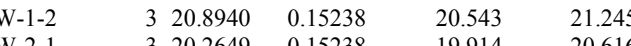

$\begin{array}{llllll}\text { W-2-1 } & 3 & 20.2649 & 0.15238 & 19.914 & 20.616 \\ \text { W-2-2 } & 3 & 20.6244 & 0.15238 & 20.273 & 20.976\end{array}$

Std Error uses a pooled estimate of error variance 
Exhibit A-3. Measurements by Analytical Series, Block and Sub-Block for the Batch 1 and LRM Standards by Oxide by Prep (continued)

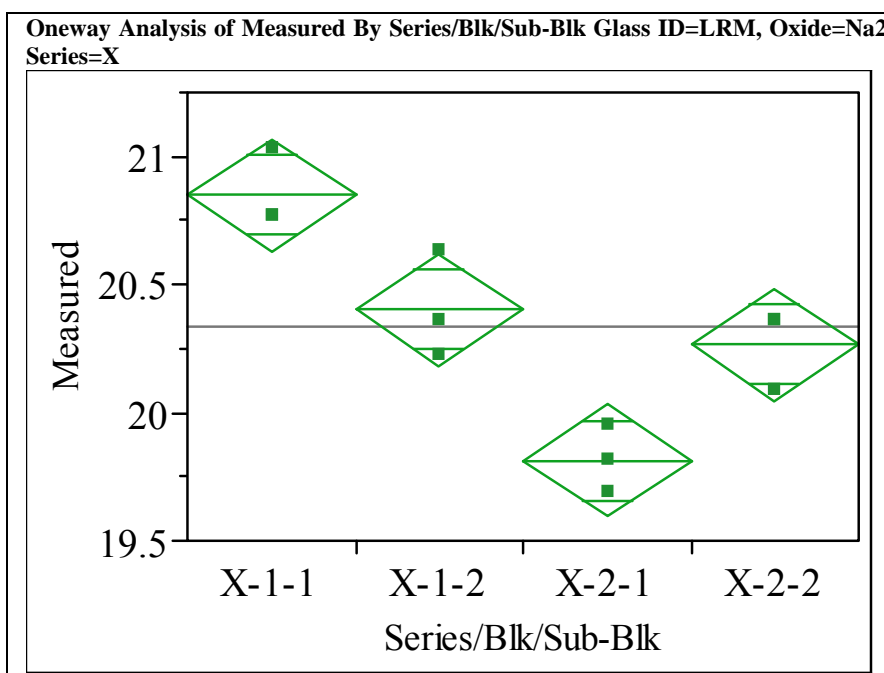

Oneway Anova

Summary of Fit

$\begin{array}{lr}\text { Rsquare } & 0.881967 \\ \text { Adj Rsquare } & 0.837705 \\ \text { Root Mean Square Error } & 0.165096 \\ \text { Mean of Response } & 20.33233 \\ \text { Observations (or Sum Wgts) } & 12\end{array}$

Analysis of Variance

Source DF Sum of Squares Mean Square F Ratio Prob > F

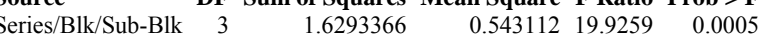

Error $\quad 8 \quad 0.2180525$

$\begin{array}{lll}0.543112 & 19.9259 & 0.0005 \\ 0.027257 & \end{array}$

C. Total

1.8473891

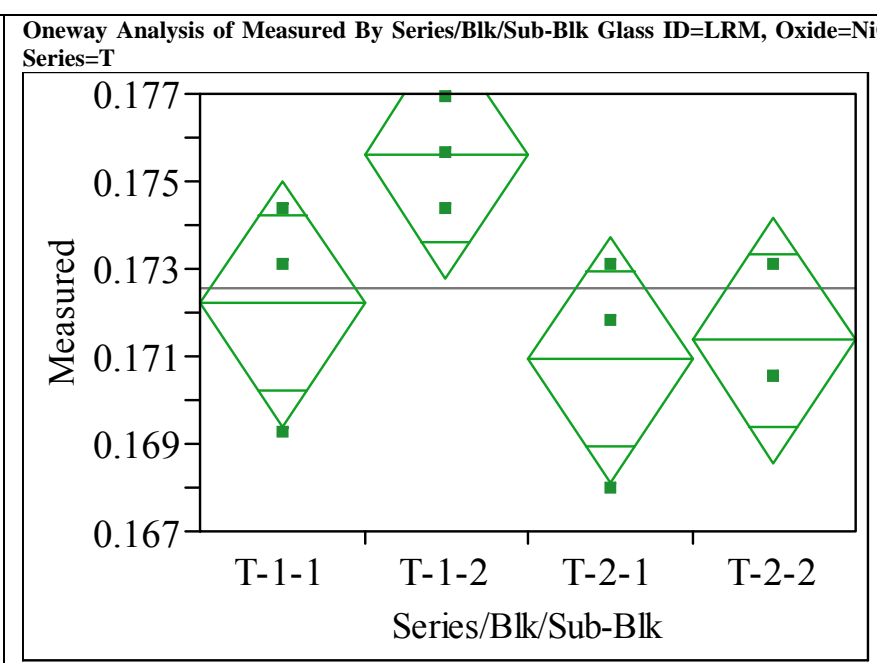

Oneway Anova

Summary of Fit

Rsquare

0.531083

Adj Rsquare $\quad 0.35524$

$\begin{array}{ll}\text { Root Mean Square Error } & 0.00211 \\ \text { Mean of Response } & 0.17253\end{array}$

Observations (or Sum Wgts) $\quad 12$

Analysis of Variance

Source DF Sum of Squares Mean Square F Ratio Prob > F

$\begin{array}{llllll}\text { Series/Blk/Sub-Blk } & 3 & 0.00004035 & 0.000013 & 3.0202 & 0.0939\end{array}$

$\begin{array}{lrr}\text { Error } & 8 & 0.00003562\end{array}$ $4.453 \mathrm{e}-6$

Means for Oneway Anova

Level Number Mean Std Error Lower 95\% Upper 95\%

$\begin{array}{llllrr}\text { X-1-1 } & 3 & 20.8491 & 0.09532 & 20.629 & 21.069 \\ \text { X-1-2 } & 3 & 20.3997 & 0.09532 & 20.180 & 20.620 \\ \text { X-2-1 } & 3 & 19.8156 & 0.09532 & 19.596 & 20.035\end{array}$

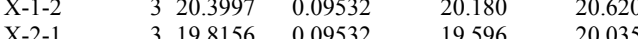

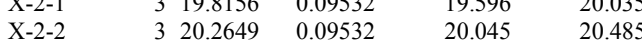

Std Error uses a pooled estimate of error variance

Means for Oneway Anova

Level Number Mean Std Error Lower 95\% Upper 95\%

$\begin{array}{lllllr}\text { T-1-1 } & 3 & 0.172212 & 0.00122 & 0.16940 & 0.17502\end{array}$

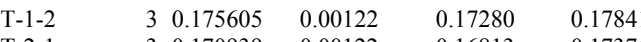

$\begin{array}{llllll}T-2-1 & 3 & 0.170939 & 0.00122 & 0.16813 & 0.17375\end{array}$

$\begin{array}{llllll}\text { T-2-2 } & 3 & 0.171363 & 0.00122 & 0.16855 & 0.17417\end{array}$

Std Error uses a pooled estimate of error variance 
Exhibit A-3. Measurements by Analytical Series, Block and Sub-Block for the Batch 1 and LRM Standards by Oxide by Prep (continued)

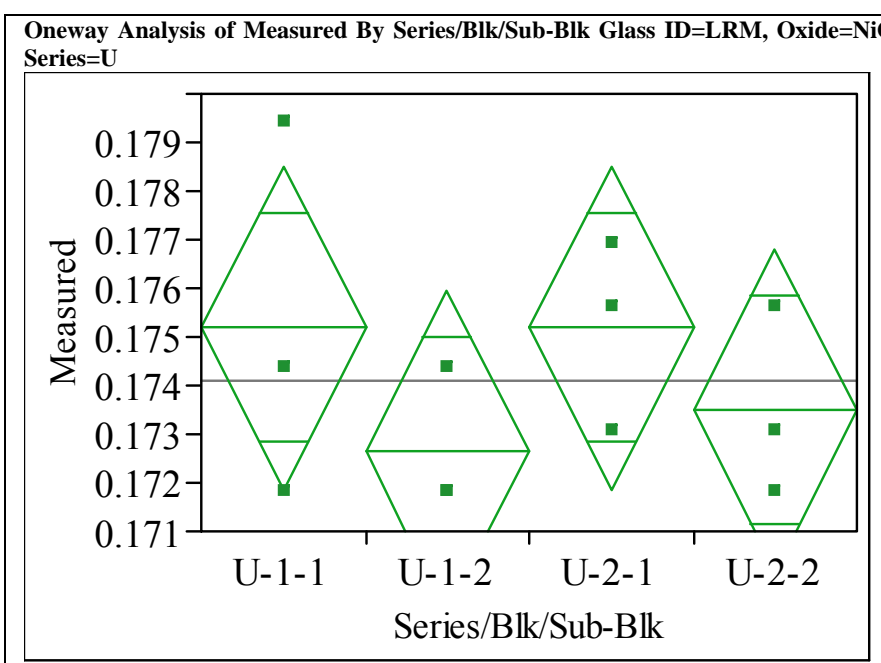

Oneway Anova

Summary of Fit

Rsquare $\quad 0.226891$

Adj Rsquare $\quad-0.06303$

Root Mean Square Error $\quad 0.002491$

$\begin{array}{lr}\text { Mean of Response } & 0.17412 \\ \text { Observations (or Sum Wgts) } & 12\end{array}$

Analysis of Variance

$\begin{array}{lrrrrr}\text { Source } & \text { DF } & \text { Sum of Squares } & \text { Mean Square } & \text { F Ratio } & \text { Prob }>\text { F } \\ \text { Series/Blk/Sub-Blk } & 3 & 0.00001457 & 4.8578 \mathrm{e}-6 & 0.7826 & 0.5362\end{array}$

$\begin{array}{lrrrrr}\text { Series/Blk/Sub-Blk } & 3 & 0.00001457 & 4.8578 \mathrm{e}-6 & 0.7826 & 0.5362 \\ \text { Error } & 8 & 0.00004966 & 6.2071 \mathrm{e}-6 & & \end{array}$

C. Total

0.00006423

Means for Oneway Anova

Level Number Mean Std Error Lower 95\% Upper 95\%

$\begin{array}{llllrr}\text { U-1-1 } & 3 & 0.175181 & 0.00144 & 0.17186 & 0.17850\end{array}$

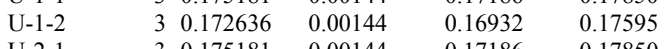

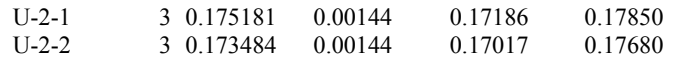

Std Error uses a pooled estimate of error variance

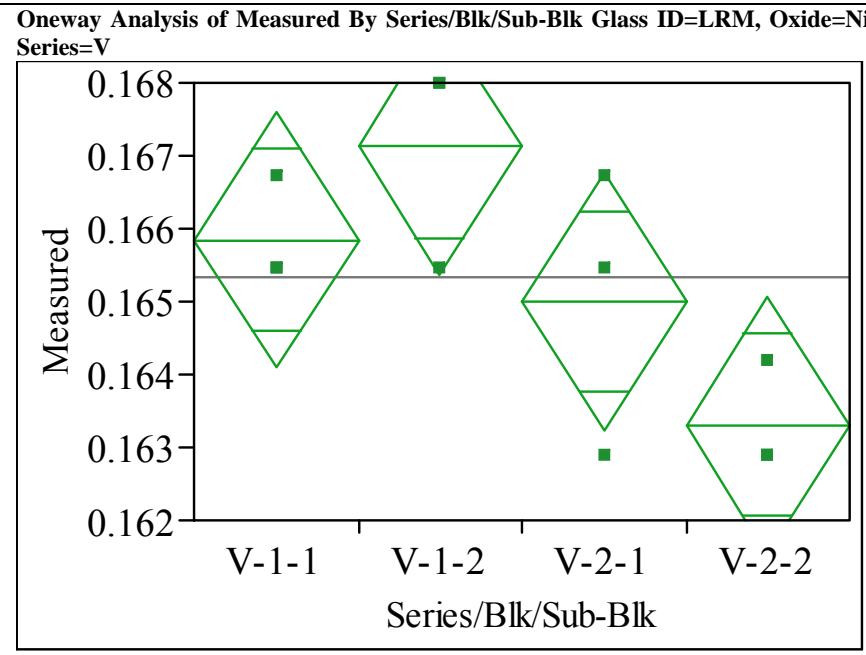

Oneway Anova

Summary of Fit

Rsquare

0.48
Root Mean Square Error $\quad 0.001324$

Mean of Response $\quad 0.165319$

Observations (or Sum Wgts) $\quad 12$

Analysis of Variance

Source DF Sum of Squares Mean Square F Ratio Prob > F

$\begin{array}{llllll}\text { Series/Blk/Sub-Blk } & 3 & 0.00002307 & 7.6915 \mathrm{e}-6 & 4.3846 & 0.0420\end{array}$

$\begin{array}{lll}\text { Error } & 8 & 0.00001403\end{array}$

$1.7542 \mathrm{e}-6$

Means for Oneway Anova

Level Number Mean Std Error Lowe

$\begin{array}{llllrr}\text { V-1-1 } & 3 & 0.165849 & 0.00076 & 0.16409 & 0.1676\end{array}$

$\begin{array}{llllll}V-1-2 & 3 & 0.167122 & 0.00076 & 0.16536 & 0.16889\end{array}$

$\begin{array}{lllllll}\mathrm{V}-2-1 & & 3 & 0.165001 & 0.00076 & 0.16324 & 0.16676\end{array}$

$\begin{array}{llllll}\mathrm{V}-2-2 & 3 & 0.163304 & 0.00076 & 0.16154 & 0.16507\end{array}$

Std Error uses a pooled estimate of error variance 
Exhibit A-3. Measurements by Analytical Series, Block and Sub-Block for the Batch 1 and LRM Standards by Oxide by Prep (continued)

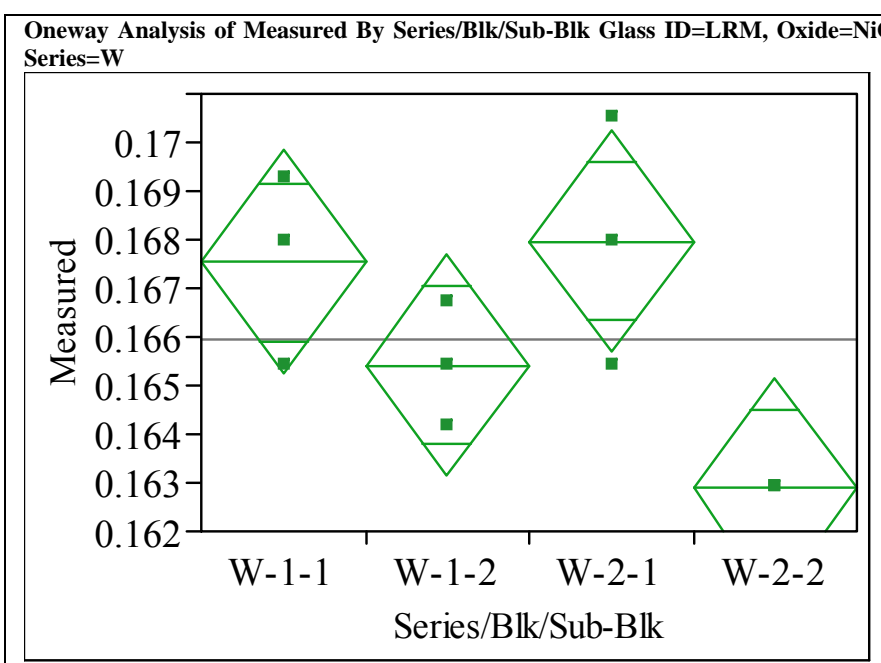

Oneway Anova

Summary of Fit

Rsquare $\quad 0.673469$

Adj Rsquare $\quad 0.55102$

Root Mean Square Error $\quad 0.001723$

$\begin{array}{lr}\text { Mean of Response } & 0.165955 \\ \text { Observations (or Sum Wgts) } & 12\end{array}$

Analysis of Variance

$\begin{array}{lrrrrr}\text { Source } & \text { DF Sum of Squares Mean Square F Ratio Prob > F } \\ \text { Series/Blk/Sub-Blk } & 3 & 0.00004898 & 0.000016 & 5.5000 & 0.0240\end{array}$

$\begin{array}{llllll}\text { Series/Blk/Sub-Blk } & 3 & 0.00004898 & 0.000016 & 5.5000 & 0.0240 \\ \text { Error } & 8 & 0.00002375 & 2.969 \mathrm{e}-6 & & \end{array}$

C. Total

0.00007273

Means for Oneway Anova

Level Number Mean Std Error Lower 95\% Upper 95\%

$\begin{array}{lllrrr}\text { W-1-1 } & 3 & 0.167546 & 0.00099 & 0.16525 & 0.16984\end{array}$

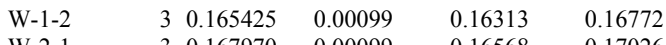

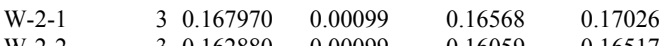

$\begin{array}{llllll}\text { W-2-2 } & 3 & 0.162880 & 0.00099 & 0.16059 & 0.16517\end{array}$

Std Error uses a pooled estimate of error variance

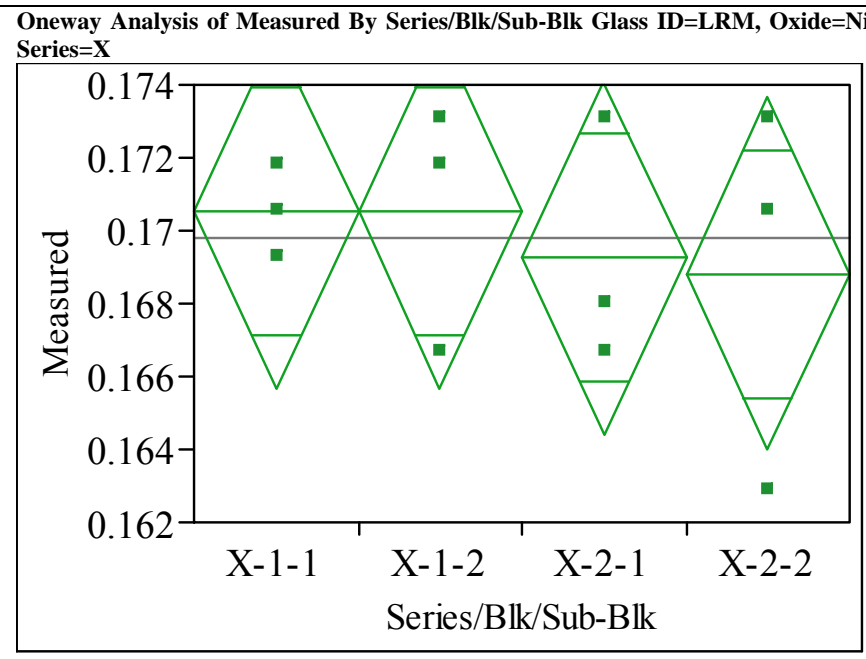

\section{Oneway Anova}

Summary of Fit

Rsquare

$\begin{array}{ll} & -0.29021 \\ \text { Root Mean Square Error } & 0.003618\end{array}$

Mean of Response

Observations (or Sum Wgts)

Analysis of Variance

Source DF Sum of Squares Mean Square F Ratio Prob $>$ F

$\begin{array}{llllll}\text { Series/Blk/Sub-Blk } & 3 & 0.00000688 & 2.294 \mathrm{e}-6 & 0.1753 & 0.9102\end{array}$

$\begin{array}{lrr}\text { Error } & 8 & 0.00010471 \\ \text { C. Tota } & 11 & 0.0001159\end{array}$ 0.000013

Means for Oneway Anova

Level Number Mean Std Error Lower

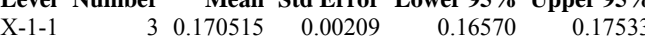

$\begin{array}{llllll}\mathrm{X}-1-2 & 3 & 0.170515 & 0.00209 & 0.16570 & 0.17533 \\ \mathrm{X}-2-1 & 3 & 0.169243 & 0.00209 & 0.1643 & 0.17406\end{array}$

$\begin{array}{llllll}\mathrm{X}-2-1 & 3 & 0.169243 & 0.00209 & 0.16443 & 0.17406\end{array}$

$\begin{array}{llllll}\mathrm{X}-2-2 & 3 & 0.168818 & 0.00209 & 0.16400 & 0.17364\end{array}$

Std Error uses a pooled estimate of error variance 
Exhibit A-3. Measurements by Analytical Series, Block and Sub-Block for the Batch 1 and LRM Standards by Oxide by Prep (continued)

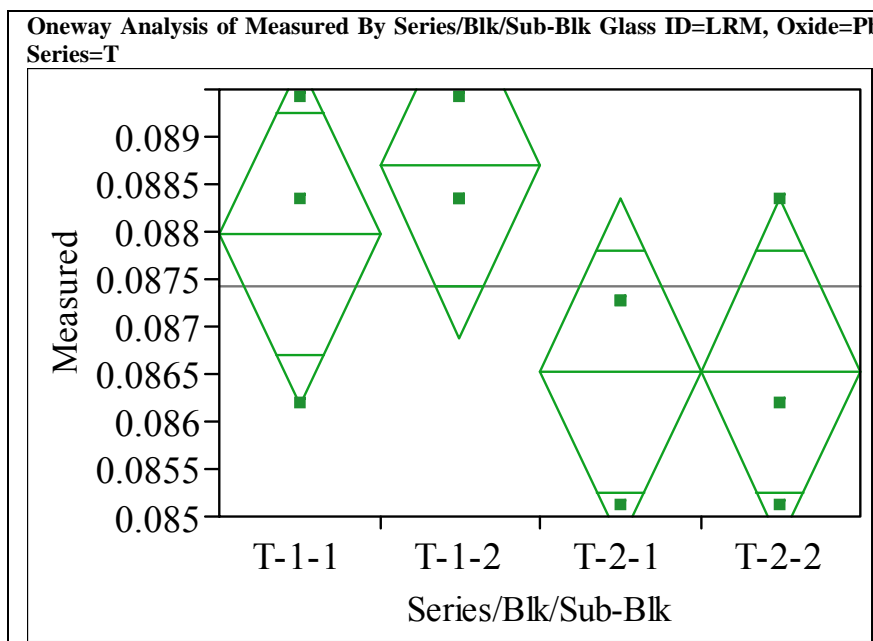

\section{Oneway Anova}

Summary of Fit

$\begin{array}{ll}\text { Rsquare } & 0.415385 \\ \text { Adj Rsquare } & 0.196154\end{array}$

Adj Rsquare $\quad 0.19615$

Root Mean Square Error $\quad 0.001355$

0.087433

Analysis of Variance

Source DF Sum of Squares Mean Square F Ratio Prob $>$ F

Series/Blk/Sub-Blk 3 Sum o Squares Mean Square $\mathbf{F}^{2}$ Ratio Prob > F

$\begin{array}{lrrr}\text { Series/Blk/Sub-Blk } & 3 & 0.00001044 & 3.4811 \mathrm{e}-6 \\ \text { Error } & 8 & 0.00001470 & 1.8372 \mathrm{e}-6\end{array}$

0.00002514

Means for Oneway Anova

Level Number Mean Std Error Lower 95\% Upper 95\%

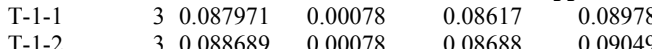

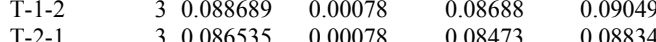

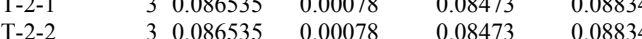

Std Error uses a pooled estimate of error variance

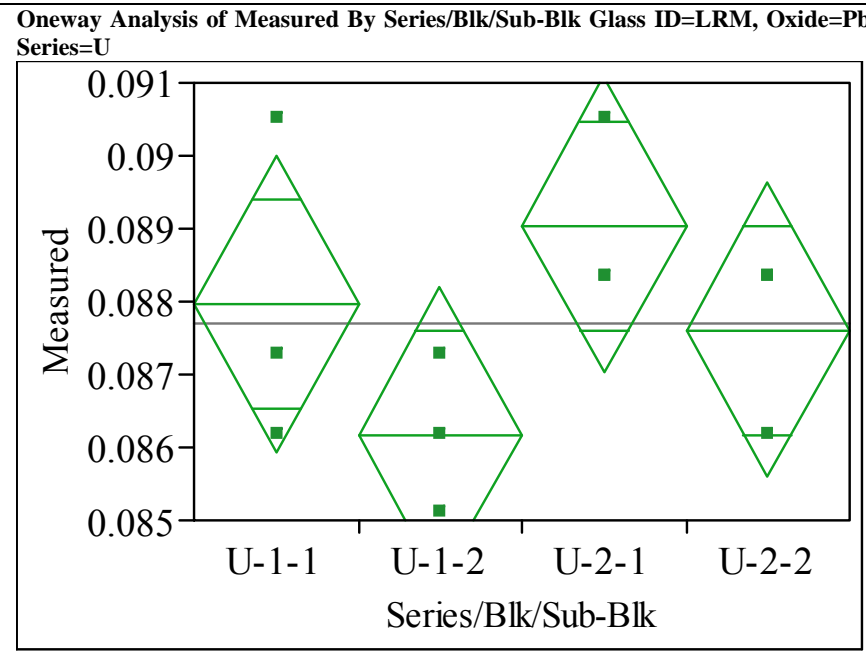

Oneway Anova

Summary of Fit

Rsquare

$\begin{array}{ll} & 0.182663 \\ \text { Root Mean Square Error } \quad 0.001523\end{array}$

Mean of Response $\quad 0.087702$

Observations (or Sum Wgts) 12

Analysis of Variance

Source DF Sum of Squares Mean Square F Ratio Prob > F

$\begin{array}{llllll}\text { Series/Blk/Sub-Blk } & 3 & 0.00001267 & 4.2224 \mathrm{e}-6 & 1.8194 & 0.2216\end{array}$

$\begin{array}{lrr}\text { Error } & 8 & 0.00001857\end{array}$

$2.3207 \mathrm{e}-6$

Means for Oneway Anova

Level Number Mean Std Error Lower 95

$\begin{array}{llllll}\text { U-1-1 } & 3 & 0.087971 & 0.00088 & 0.08594 & 0.09000\end{array}$

$\begin{array}{llllll}\text { U-1-2 } & 3 & 0.086176 & 0.00088 & 0.08415 & 0.08820\end{array}$

$\begin{array}{llllll}\text { U-2-1 } & 3 & 0.089049 & 0.00088 & 0.08702 & 0.09108\end{array}$

$\begin{array}{llllll}\text { U-2-2 } & 3 & 0.087612 & 0.00088 & 0.08558 & 0.08964\end{array}$

Std Error uses a pooled estimate of error variance 
Exhibit A-3. Measurements by Analytical Series, Block and Sub-Block for the Batch 1 and LRM Standards by Oxide by Prep (continued)

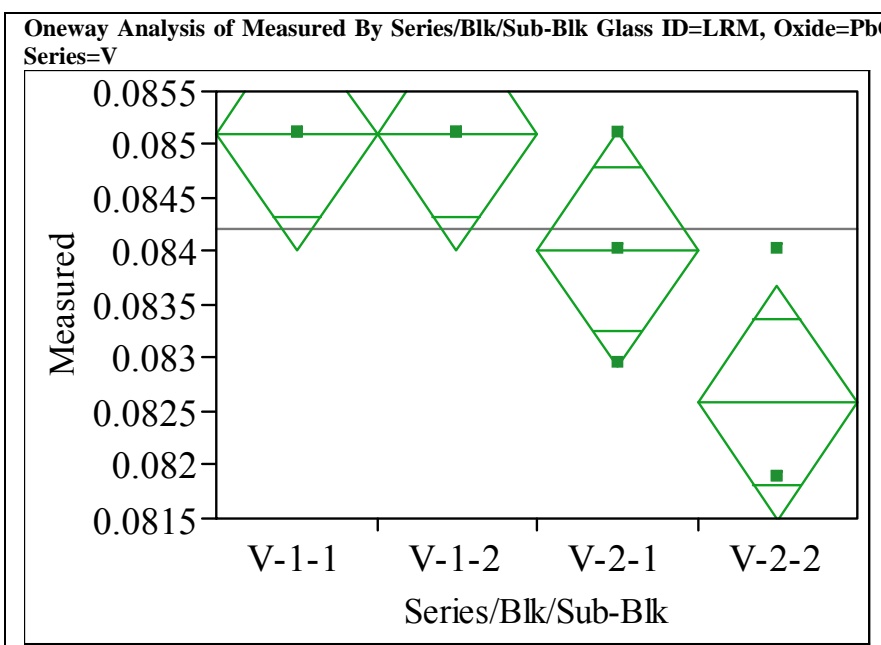

\section{Oneway Anova}

Summary of Fit

$\begin{array}{ll}\text { Rsquare } & 0.702128 \\ \text { Adj Rsquare } & 0.590426\end{array}$

Adj Rsquare $\quad 0.590426$

Root Mean Square Error $\quad 0.000823$

Mean of Response $\quad 0.08420$

Analysis of Variance

Source DF Sum of Squares Mean Square F Ratio Prob > F

$\begin{array}{lrrrrr}\text { Source } & \text { DF } & \text { Sum of Squares } & \text { Mean Square } & \text { Fatio } & \text { Prob }>\text { F } \\ \text { Series/Blk/Sub-Blk } & 3 & 0.00001276 & 4.2547 \mathrm{e}-6 & 6.2857 & 0.0169\end{array}$

$\begin{array}{lrrr}\text { Series/Blk/Sub-Blk } & 3 & 0.00001276 & 4.2547 \mathrm{e}-6 \\ \text { Error } & 8 & 0.00000542 & 6.7688 \mathrm{e}-7\end{array}$

0.00001818

Means for Oneway Anova

Level Number Mean Std Error Lower 95\% Upper 95\%

$\begin{array}{llrrrr}\text { Level Number } & \text { Mean } & \text { Std Error } & \text { Lower } & & \\ \text { V-1-1 } & 3 & 0.085099 & 0.00048 & 0.08400 & 0.08619 \\ V-1-2 & 3 & 0.085099 & 0.00048 & 0.08400 & 0.08619\end{array}$

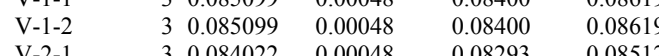

$\begin{array}{llllll}\mathrm{V}-1-2 & 3 & 0.08509 & 0.0008 & 0.08400 & 0.08619 \\ \mathrm{~V}-2-1 & 3 & 0.084022 & 0.00048 & 0.08293 & 0.08512\end{array}$

Std Error uses a pooled estimate of error variance

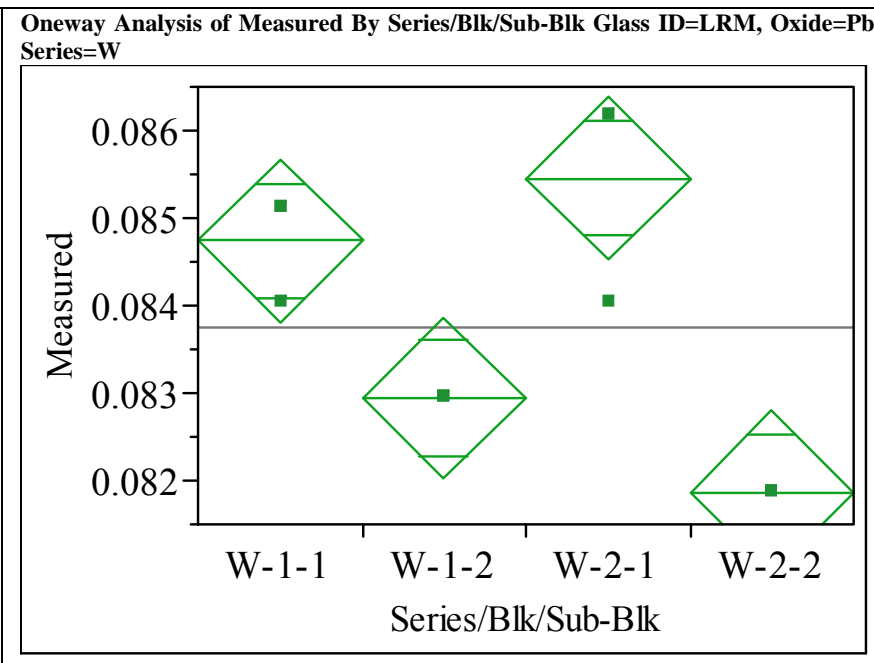

Oneway Anova

Summary of Fit

Rsquare

Adj Rsquare $\quad 0.810997$

Root Mean Square Error $\quad 0.000695$

$\begin{array}{lr}\text { Mean of Response } & 0.083752 \\ \text { Observations (or Sum Wgts) } & 12\end{array}$

Analysis of Variance

Source DF Sum of Squares Mean Square F Ratio Prob $>$ F

$\begin{array}{lllllll}\text { Series/Blk/Sub-Blk } & 3 & 0.00002427 & 8.0903 \mathrm{e}-6 & 16.7333 & 0.0008\end{array}$

$\begin{array}{lrrr}\text { Error } & 8 & 0.00000387 & 4.8348 \mathrm{e}-7 \\ \text { C. Total } & 11 & 0.00002814 & \end{array}$

Means for Oneway Anova

Level Number Mean Std Error Lower 95\% Upper 95\%

$\begin{array}{lllllll}\text { W-1-1 } & 3 & 0.084740 & 0.00040 & 0.08381 & 0.08567\end{array}$

$\begin{array}{llllll}\text { W-1-2 } & 3 & 0.082944 & 0.00040 & 0.08202 & 0.08387\end{array}$

$\begin{array}{llllll}\mathrm{W}-2-1 & 3 & 0.085458 & 0.00040 & 0.08453 & 0.08638\end{array}$

$\begin{array}{llllll}\text { W-2-2 } & 3 & 0.081867 & 0.00040 & 0.08094 & 0.08279\end{array}$

Std Error uses a pooled estimate of error variance 
Exhibit A-3. Measurements by Analytical Series, Block and Sub-Block for the Batch 1 and LRM Standards by Oxide by Prep (continued)

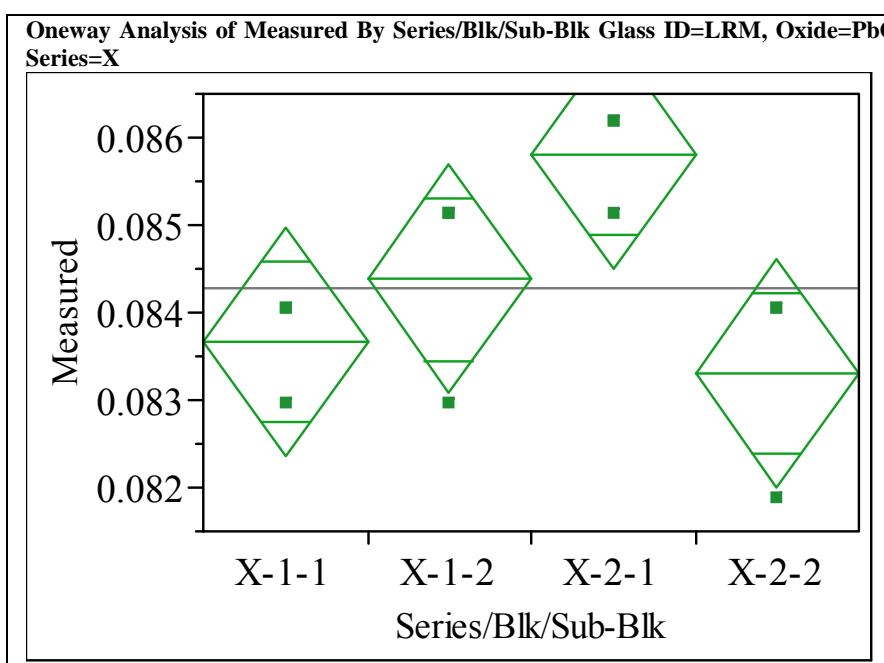

Oneway Anova

Summary of Fit

$\begin{array}{lr}\text { Rsquare } & 0.589744 \\ \text { Adj Rsquare } & 0.435897 \\ \text { Root Mean Square Error } & 0.000983 \\ \text { Mean of Response } & 0.084291 \\ \text { Observations (or Sum Wgts) } & 12\end{array}$

Analysis of Variance

Source DF Sum of Squares Mean Square F Ratio Prob > F

$\begin{array}{lrrrrr}\text { Series/Blk/Sub-Blk } & 3 & 0.00001112 & 3.7067 \mathrm{e}-6 & 3.8333 & 0.0571 \\ \text { Error } & 8 & 0.00000774 & 9.6697 \mathrm{e}-7 & & \\ \text { C. } & 11 & 0.00007886 & & & \end{array}$

Error

$9.6697 \mathrm{e}-7$

Means for Oneway Anova

0.00001886

Level Number Mean Std Error Lower 95\% Upper 95\%

$\begin{array}{rrrrrr}\text { X-1-1 } & 3 & 0.083663 & 0.00057 & 0.08235 & 0.08497\end{array}$

$\begin{array}{llllll}\mathrm{X}-1-1 & 3 & 0.083663 & 0.0057 & 0.08235 & 0.08497 \\ \mathrm{X}-2-1 & 3 & 0.084381 & 0.00057 & 0.08307 & 0.0856\end{array}$

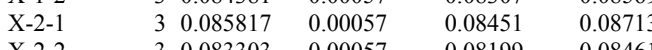

$\begin{array}{llllll}\mathrm{X}-2-2 & 3 & 0.083303 & 0.00057 & 0.08199 & 0.08461\end{array}$

Std Error uses a pooled estimate of error variance

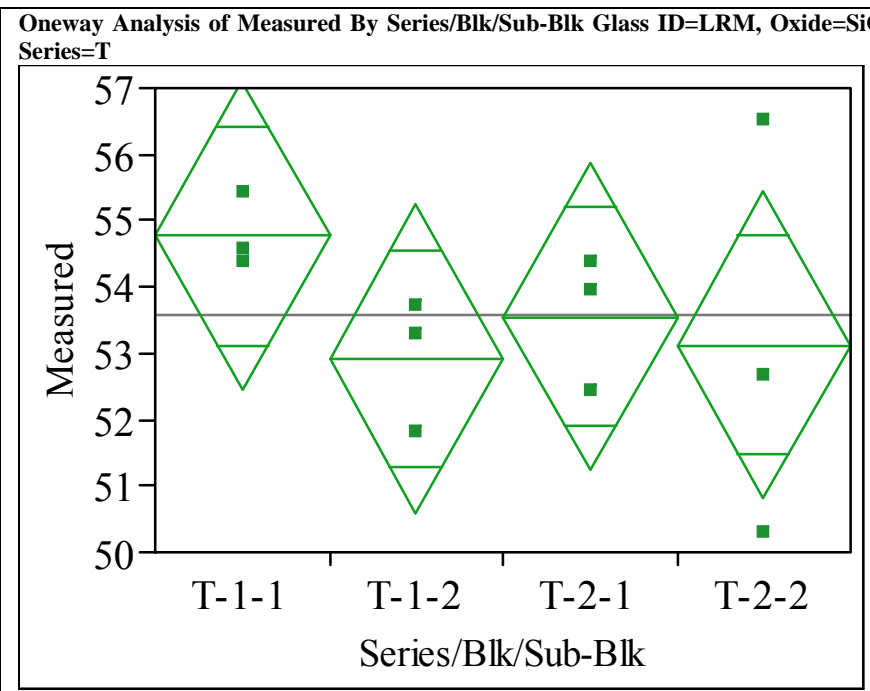

\section{Oneway Anova}

Summary of $F$

$\begin{array}{lr}\text { Rsquare } & 0.202399 \\ \text { Adj Rsquare } & -0.0967 \\ \text { Root Mean Square Error } & 1.744546 \\ \text { Mean of Response } & 53.58947 \\ \text { Observations (or Sum Wgts) } & 12\end{array}$

Observations (or Sum W

Analysis of Variance

Source DF Sum of Squares Mean Square F Ratio Prob $>$ F

$\begin{array}{lllllll}\text { Series/Blk/Sub-Blk } & 3 & 6.178416 & 2.05947 & 0.6767 & 0.5903\end{array}$

$\begin{array}{lrr}\text { Error } & 8 & 24.347536 \\ \text { C. Total } & 11 & 30.525952\end{array}$

Means for Oneway Anova

Level Number Mean Std Error Lower 95\% Upper 95\%

$\begin{array}{llllll}\text { T-1-1 } & 3 & 54.7661 & 1.0072 & 52.443 & 57.089\end{array}$

$\begin{array}{llllll}\mathrm{T}-1-1 & 3 & 54.7661 & 1.0072 & 52.443 & 57.089 \\ \mathrm{~T}-1-2 & 3 & 52.9120 & 1.0072 & 50.589 & 55.235\end{array}$

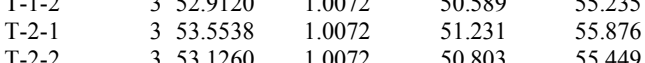

Std Error uses a pooled estimate of error variance 
Exhibit A-3. Measurements by Analytical Series, Block and Sub-Block for the Batch 1 and LRM Standards by Oxide by Prep (continued)

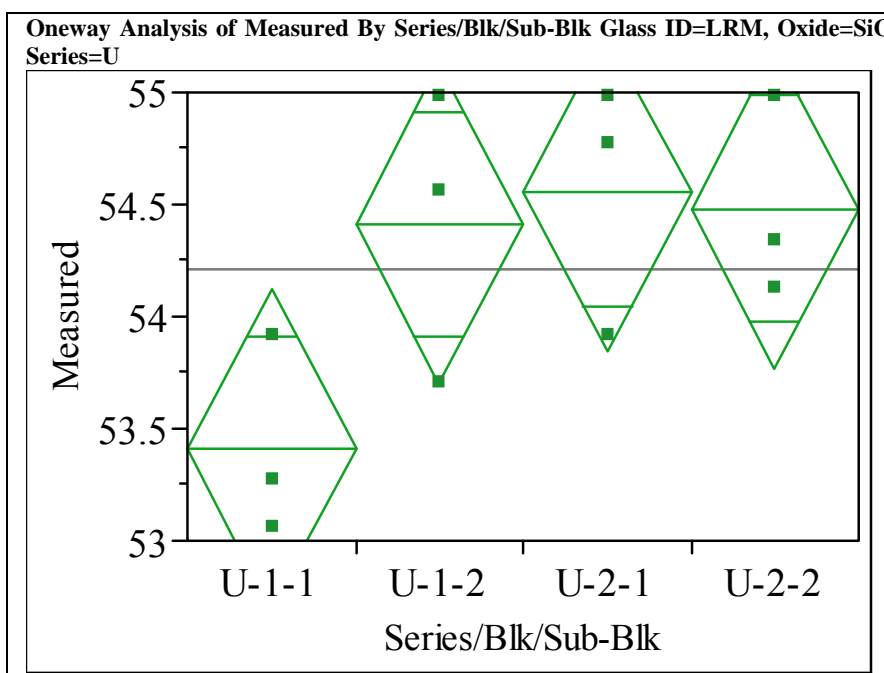

Oneway Anova

Summary of Fit

$\begin{array}{lr}\text { Rsquare } & 0.532346 \\ \text { Adj Rsquare } & 0.356976 \\ \text { Root Mean Square Error } & 0.534825 \\ \text { Mean of Response } & 54.21343 \\ \text { Observations (or Sum Wgts) } & 12\end{array}$

Analysis of Variance

Analysis of Variance
Source SF Sum of Squares Mean Square F Ratio Prob $>$ F

$\begin{array}{lrrrrr}\text { Source } & \text { DF } & \text { Sum of Squares } & \text { Mean Square } & \text { F Ratio } & \text { Prob }>\text { F } \\ \text { Series/Blk/Sub-Blk } & 3 & 2.6048507 & 0.868284 & 3.0356 & 0.0930\end{array}$

$\begin{array}{lrrr}\text { Series/Blk/Sub-Blk } & 3 & 2.6048507 & 0.868284 \\ \text { Error } & 8 & 2.2883022 & 0.286038 \\ \text { C. Total } & 11 & 4.8931530 & \\ & \end{array}$

Means for Oneway Anova

Level Number Mean Std Error Lower 95\% Upper 95\%

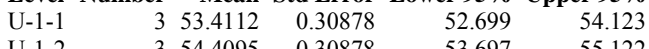

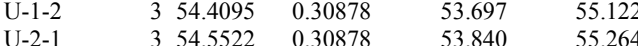

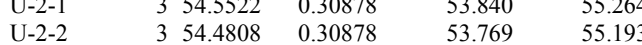

Std Error uses a pooled estimate of error variance

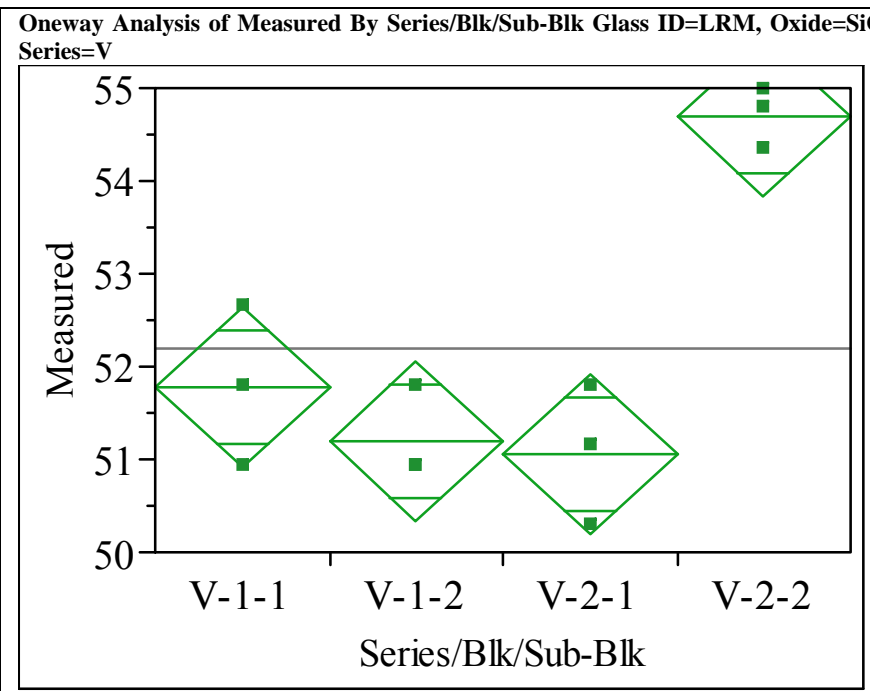

\section{Oneway Anova}

$\begin{array}{lr}\text { Rsquare } & 0.88801 \\ \text { Adj Rsquare } & 0.846014 \\ \text { Root Mean Square Error } & 0.64179 \\ \text { Mean of Response } & 52.18109 \\ \text { Observations (or Sum Wgts) } & 12\end{array}$

Observations (or Sum W

Analysis of Variance

Source DF Sum of Squares Mean Square F Ratio Prob $>$ F

$\begin{array}{lllllll}\text { Series/Blk/Sub-Blk } & 3 & 26.128598 & 8.70953 & 21.1451 & 0.0004\end{array}$

$\begin{array}{lrrr}\text { Error } & 8 & 3.295155 & 0.41189 \\ \text { C. Total } & 11 & 29.423753 & \end{array}$

Means for Oneway Anova

Level Number Mean Std Error Lower 95\% Upper 95\%

$\begin{array}{llllll}V-1-1 & 3 & 51.7711 & 0.37054 & 50.917 & 52.626\end{array}$

$\begin{array}{llllll}V-1-2 & 3 & 51.2006 & 0.37054 & 50.346 & 52.055\end{array}$

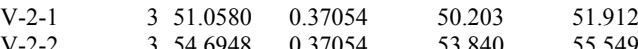

Std Error uses a pooled estimate of error variance 
Exhibit A-3. Measurements by Analytical Series, Block and Sub-Block for the Batch 1 and LRM Standards by Oxide by Prep (continued)

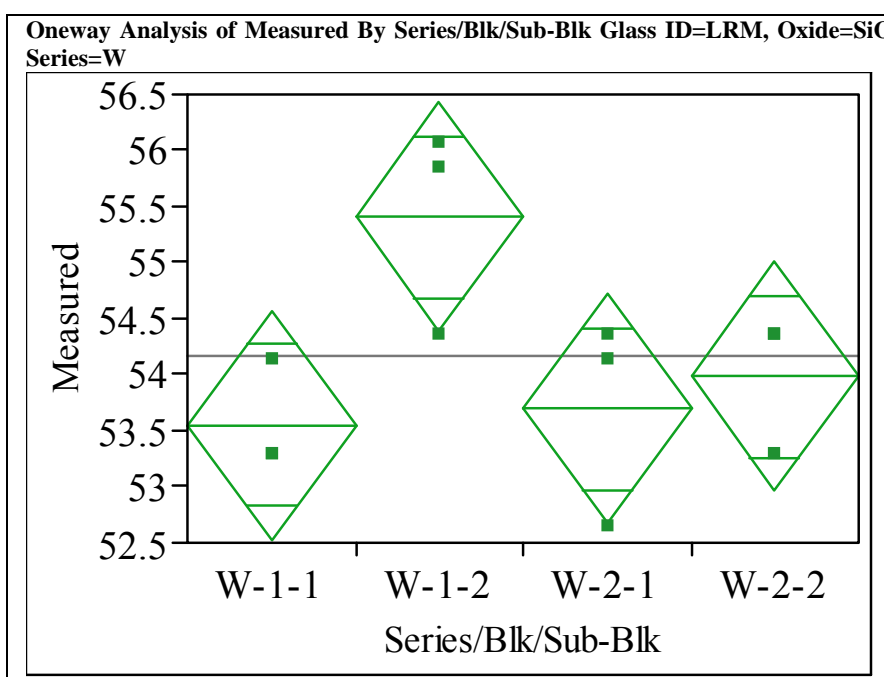

Oneway Anova

Summary of Fit

$\begin{array}{lr}\text { Rsquare } & 0.579376 \\ \text { Adj Rsquare } & 0.421642 \\ \text { Root Mean Square Error } & 0.768859 \\ \text { Mean of Response } & 54.15995 \\ \text { Observations (or Sum Wgts) } & 12\end{array}$

Observations (or Sum

Analysis of Variance
Source

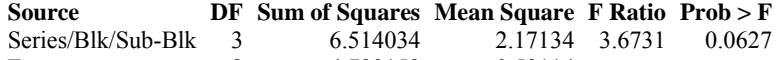

$\begin{array}{lrrr}\text { Series/Blk/Sub-Blk } & 3 & 6.514034 & 2.17134 \\ \text { Error } & 8 & 4.729158 & 0.59114\end{array}$

C. Total

11.243192

Means for Oneway Anova

Level Number Mean Std Error Lower 95\% Upper 95\%

$\begin{array}{llllrr}\text { W-1-1 } & 3 & 53.5538 & 0.44390 & 52.530 & 54.577 \\ \text { W-1-2 } & 3 & 55.4079 & 0.44390 & 54.384 & 56.372\end{array}$

$\begin{array}{llllll}\text { W-1-2 } & 3 & 55.4079 & 0.44390 & 54.384 & 56.432 \\ \text { W-2-1 } & 3 & 53.6964 & 0.44390 & 52.673 & 54.720\end{array}$

$\begin{array}{llllll}W-2-1 & 3 & 53.6964 & 0.44390 & 52.673 & 54.720 \\ \mathrm{~W}-2-2 & 3 & 53.9817 & 0.44390 & 52.958 & 55.005\end{array}$

Std Error uses a pooled estimate of error variance

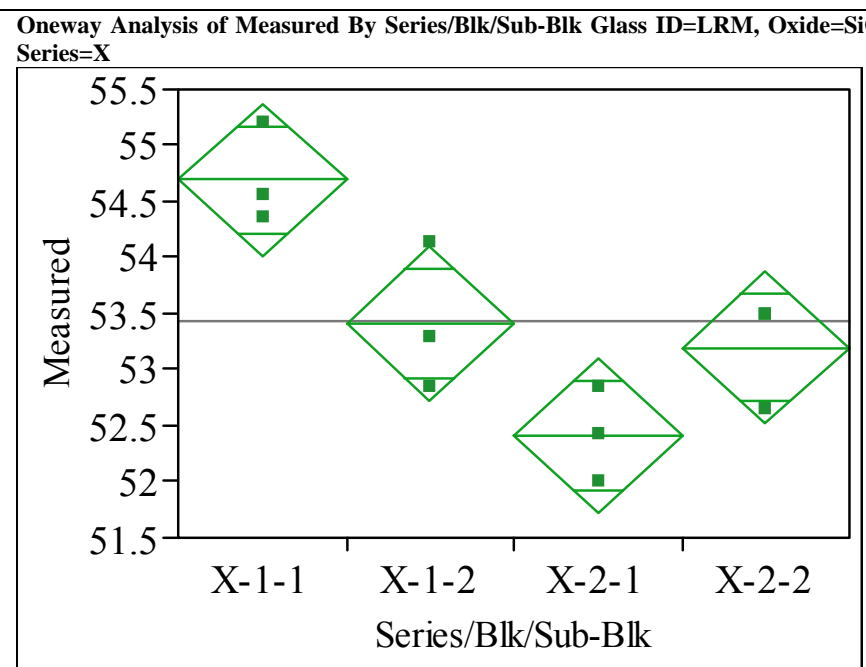

Oneway Anova

Summary of Fit

$\begin{array}{lr}\text { Rsquare } & 0.793026 \\ \text { Adj Rsquare } & 0.715411 \\ \text { Root Mean Square Error } & 0.512986 \\ \text { Mean of Response } & 53.42902 \\ \text { Observations (or Sum Wgts) } & 12\end{array}$

Analysis of Variance

Source DF Sum of Squares Mean Square F Ratio Prob > F

$\begin{array}{lrrrrr}\text { Source } & \text { DF Sum of Squares } & \text { Mean Square } & \text { F Ratio } & \text { Prob }>\text { F } \\ \text { Series/Blk/Sub-Blk } & 3 & 8.066265 & 2.68876 & 10.2174 & 0.0041\end{array}$

$\begin{array}{lrrr}\text { Series/Blk/Sub-Blk } & 3 & 8.066265 & 2.68876 \\ \text { Error } & 8 & 2.105238 & 0.26315 \\ \text { C. Total } & 11 & 10.171503 & \end{array}$

Means for Oneway Anova

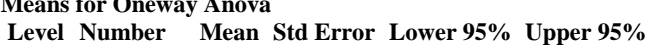

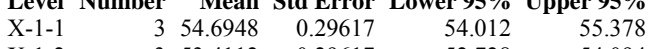

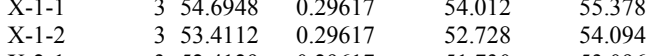

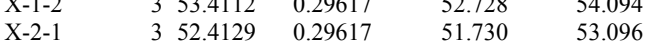

$\begin{array}{llllll}\mathrm{X}-2-1 & 3 & 52.4129 & 0.29617 & 51.730 & 53.096 \\ \mathrm{X}-2-2 & 3 & 53.1973 & 0.29617 & 52.514 & 53.880\end{array}$

Std Error uses a pooled estimate of error variance 
Exhibit A-3. Measurements by Analytical Series, Block and Sub-Block for the Batch 1 and LRM Standards by Oxide by Prep (continued)

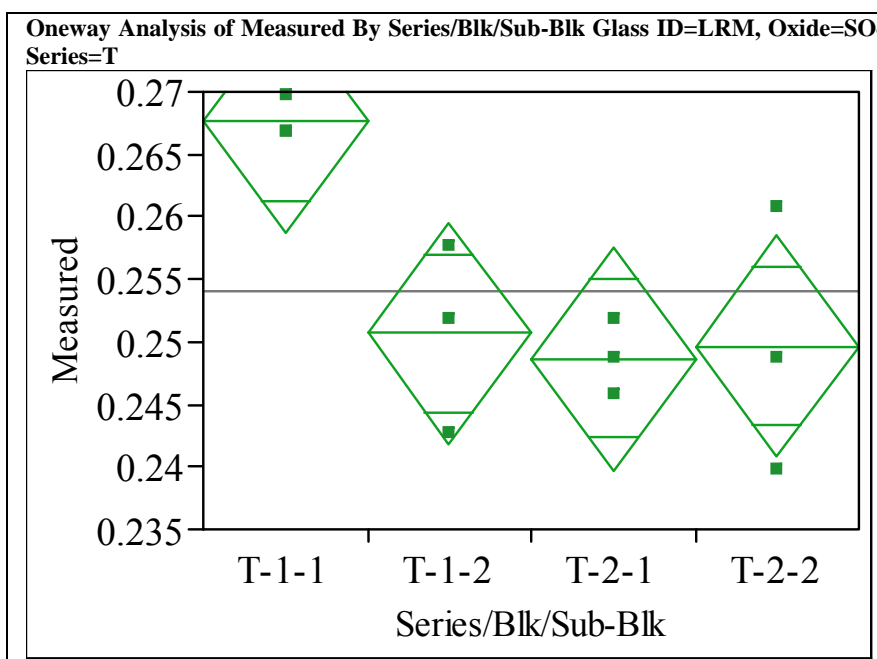

Oneway Anova

Summary of Fit

0.671233

Adj Rsquare $\quad 0.547945$

Root Mean Square Error $\quad 0.006699$

$\begin{array}{lr}\text { Mean of Response } & 0.254152 \\ \text { Observations (or Sum Wgts) } & 12\end{array}$

Analysis of Variance

$\begin{array}{lrrrrr} & \text { DF } & \text { Sum of Squares } & \text { Mean Square } & \text { F Ratio } & \text { Prob }>\text { F } \\ \text { Source } & 3 & 0.00073299 & 0.000244 & 5.4444 & 0.0247 \\ \text { Series/Blk/Sub-Blk } & 8 & 0.00035902 & 0.000045 & & \\ \text { Error } & 11 & 0.00109201 & & & \\ \text { C. Total } & 1 & & & & \end{array}$

Means for Oneway Anova

Level Number Mean Std Error Lower 95\% Upper 95\%

$\begin{array}{llllll}\text { T-1-1 } & 3 & 0.267634 & 0.00387 & 0.25871 & 0.27655\end{array}$

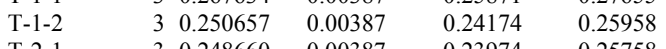

$\begin{array}{lllllll}\mathrm{T}-2-1 & & 3 & 0.248660 & 0.00387 & 0.23974 & 0.25758 \\ \mathrm{~T}-2-2 & & 3 & 0.249658 & 0.00387 & 0.24074 & 0.25858\end{array}$

Std Error uses a pooled estimate of error variance

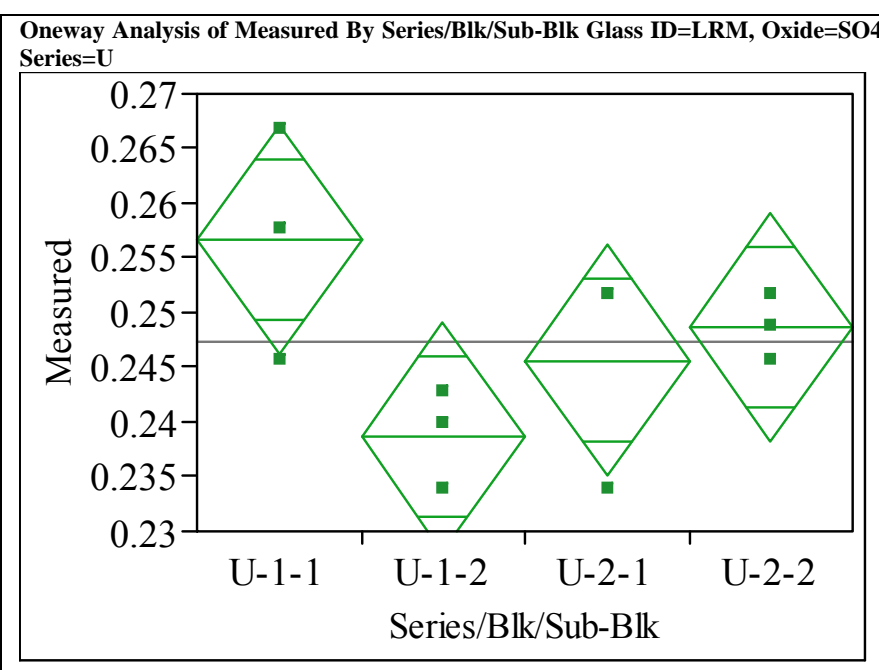

Oneway Anova

Summary of Fit

$\begin{array}{lr}\text { Rsquare } & 0.501127 \\ \text { Adj Rsquare } & 0.31405 \\ \text { Root Mean Square Error } & 0.007879 \\ \text { Mean of Response } & 0.247411 \\ \text { Observations (or Sum Wgts) } & 12\end{array}$

Analysis of Variance

Source DF Sum of Squares Mean Square F Ratio Prob $>$ F

$\begin{array}{llllll}\text { Series/Blk/Sub-Blk } & 3 & 0.00049888 & 0.000166 & 2.6787 & 0.1179\end{array}$

$\begin{array}{lrrr}\text { Error } & 8 & 0.00049664 & 0.000062 \\ \text { C. Total } & 11 & 0.00099552 & \end{array}$

Means for Oneway Anova

Level Number Mean Std Error Lower 95\% Upper 95\%

$\begin{array}{llllrr}\text { U-1-1 } & 3 & 0.256649 & 0.00455 & 0.24616 & 0.26714\end{array}$

$\begin{array}{llllll}\text { U-1-2 } & 3 & 0.238673 & 0.00455 & 0.22818 & 0.24916\end{array}$

$\begin{array}{llllll}\text { U-2-1 } & 3 & 0.245664 & 0.00455 & 0.23517 & 0.25615\end{array}$

$\begin{array}{llllll}\mathrm{U}-2-2 & 3 & 0.248660 & 0.00455 & 0.23817 & 0.25915\end{array}$

Std Error uses a pooled estimate of error variance 
Exhibit A-3. Measurements by Analytical Series, Block and Sub-Block for the Batch 1 and LRM Standards by Oxide by Prep (continued)

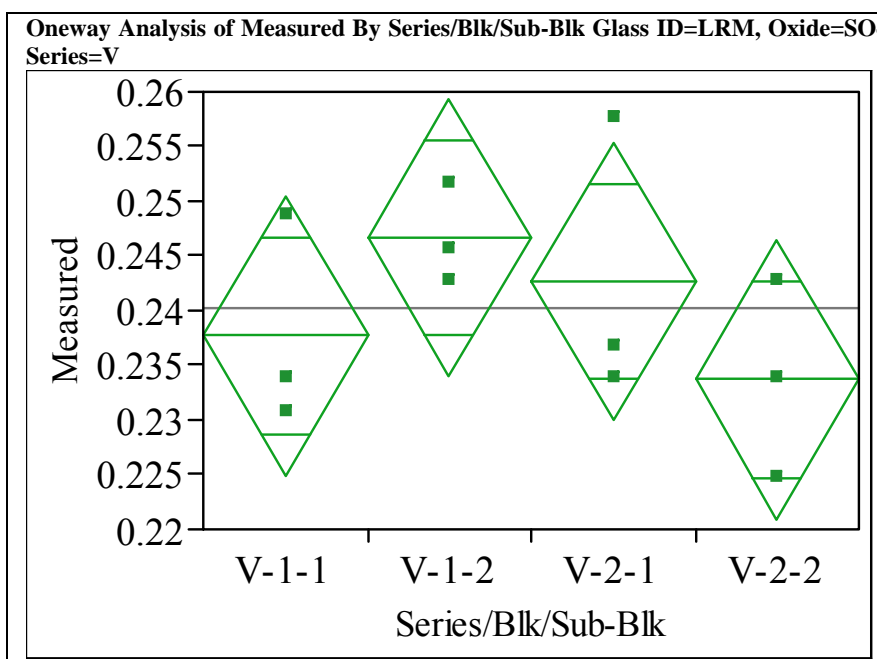

Oneway Anova

Summary of Fit

Rsquare $\quad 0.284457$

Adj Rsquare $\quad 0.016129$

Root Mean Square Error $\quad 0.009552$

$\begin{array}{lr}\text { Mean of Response } & 0.240171 \\ \text { Observations (or Sum Wgts) } & 12\end{array}$

Analysis of Variance

Source DF Sum of Squares Mean Square F Ratio Prob $>$ F

$\begin{array}{llllll}\text { Series/Blk/Sub-Blk } & 3 & 0.00029021 & 0.000097 & 1.0601 & 0.4182\end{array}$

Error

0.00073000

0.000091

Means for Oneway Anova

Level Number Mean Std Error Lower 95\% Upper 95\%

$\begin{array}{lllllr}\mathrm{V}-1-1 & 3 & 0.237675 & 0.00552 & 0.22496 & 0.25039\end{array}$

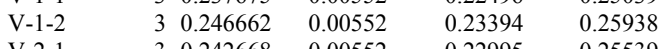

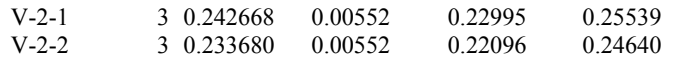

Std Error uses a pooled estimate of error variance

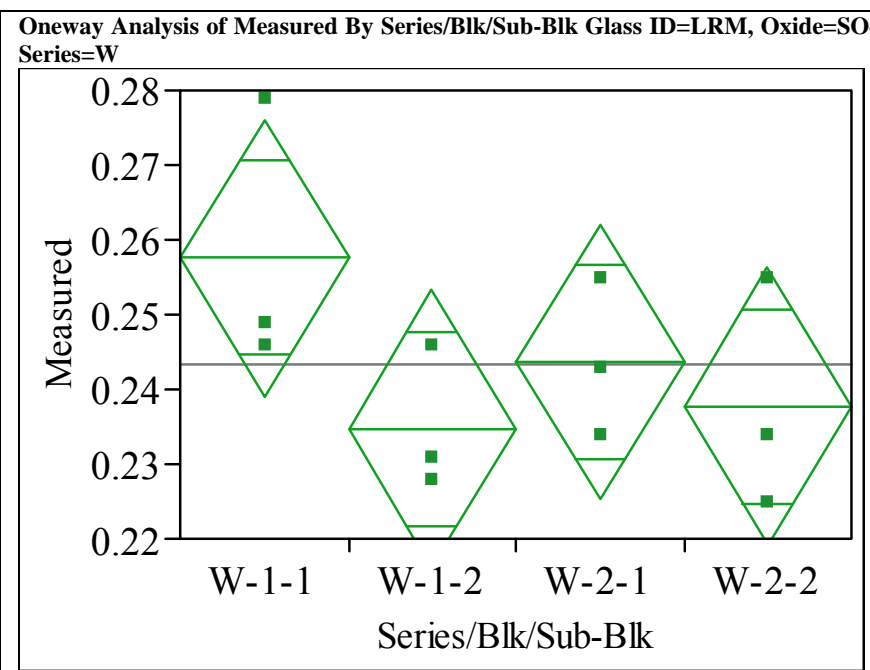

Oneway Anova

Summary of Fit

$\begin{array}{lr}\text { Rsquare } & 0.377376 \\ \text { Adj Rsquare } & 0.143891 \\ \text { Root Mean Square Error } & 0.013891 \\ \text { Mean of Response } & 0.243417 \\ \text { Observations (or Sum Wgts) } & 12\end{array}$

Analysis of Variance

Source DF Sum of Squares Mean Square F Ratio Prob $>$ F

$\begin{array}{lrrrrr}\text { Source } & \text { DF } & \text { Sum of Squares } & \text { Mean Square } & \text { F Ratio } & \text { Prob }>\mathbf{F} \\ \text { Series/Blk/Sub-Blk } & 3 & 0.00093569 & 0.000312 & 1.6163 & 0.2608\end{array}$

$\begin{array}{llll}\text { Series/Blk/Sub-Blk } & 3 & 0.00093569 & 0.000312 \\ \text { Error } & 8 & 0.00154377 & 0.000193 \\ \text { C. } & 11 & 0.00247946 & \end{array}$

C. Total

0.00247946

Means for Oneway Anova

Level Number Mean Std Error Lower 95\% Upper 95\%

$\begin{array}{llrrrr}\text { W-1-1 } & 3 & 0.257647 & 0.00802 & 0.23915 & 0.27614\end{array}$

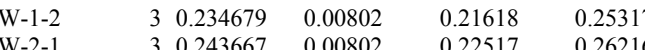

$\begin{array}{llllll}W-1-2 & 3 & 0.234679 & 0.00802 & 0.21618 & 0.25317 \\ W-2-2 & 3 & 0.243667 & 0.00802 & 0.22517 & 0.26216\end{array}$

Std Error uses a pooled estimate of error variance 
Exhibit A-3. Measurements by Analytical Series, Block and Sub-Block for the Batch 1 and LRM Standards by Oxide by Prep (continued)

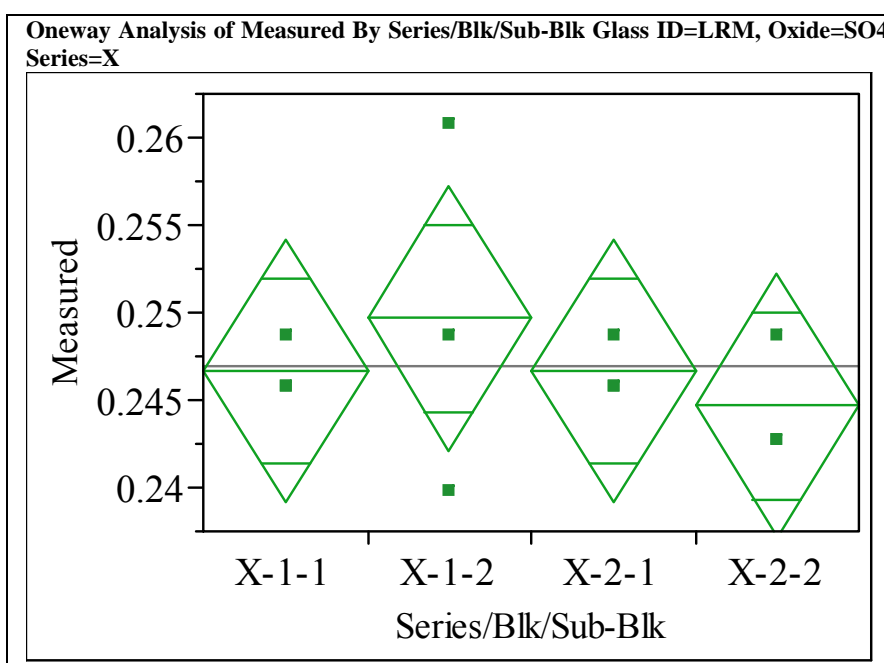

Oneway Anova

Summary of Fit

$\begin{array}{lr}\text { Rsquare } & 0.129114 \\ \text { Adj Rsquare } & -0.19747 \\ \text { Root Mean Square Error } & 0.005671 \\ \text { Mean of Response } & 0.246912 \\ \text { Observations (or Sum Wgts) } & 12\end{array}$

Analysis of Variance

Source DF Sum of Squares Mean Square F Ratio Prob $>$ F

$\begin{array}{lrrrrr}\text { Series/Blk/Sub-Blk } & 3 & 0.00003815 & 0.000013 & 0.3953 & 0.7600 \\ \text { Error } & 8 & 0.00025730 & 0.000032 & & \end{array}$

Error 0.000032

Means for Oneway Anova

Level Number Mean Std Error Lower 95\% Upper 95\%

$\begin{array}{lllrrr}\mathrm{X}-1-1 & 3 & 0.246662 & 0.00327 & 0.23911 & 0.2542\end{array}$

$\begin{array}{llllll}\mathrm{X}-1-2 & 3 & 0.249658 & 0.00327 & 0.24211 & 0.2572\end{array}$

$\begin{array}{lllllll}\text { X-2-1 } & & 3 & 0.246662 & 0.00327 & 0.23911 & 0.25421 \\ \text { X-2-2 } & & 3 & 0.244665 & 0.00327 & 0.23711 & 0.25222\end{array}$

Std Error uses a pooled estimate of error variance

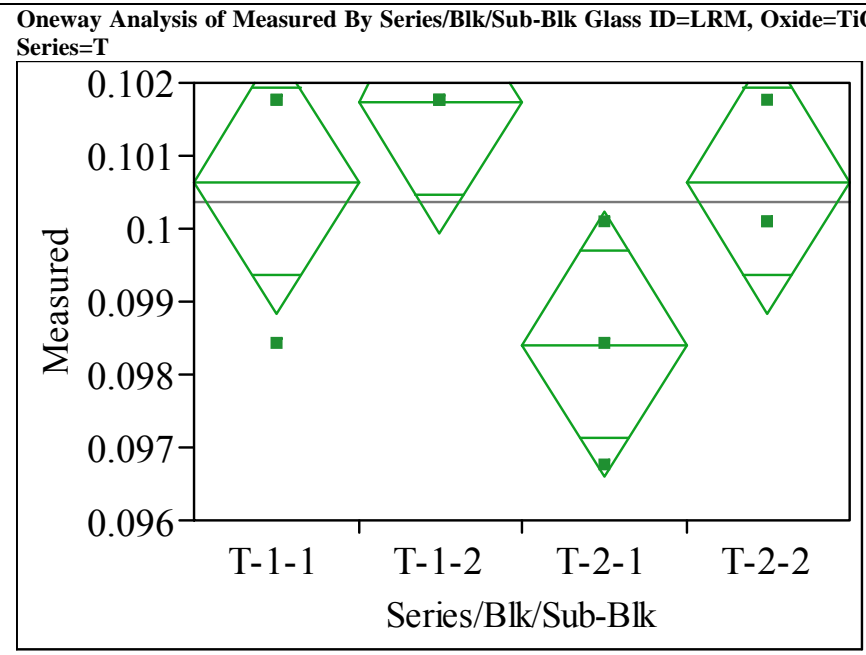

Oneway Anova

Summary of Fit

Rsquare

0.371429
Root Mean Square Error $\quad 0.001362$

$\begin{array}{ll}\text { Mean of Response } & 0.100358\end{array}$

$\begin{array}{lr}\text { Observations (or Sum Wgts) } & 12\end{array}$

Analysis of Variance

Source DF Sum of Squares Mean Square F Ratio Prob $>$ F

$\begin{array}{llllll}\text { Series/Blk/Sub-Blk } & 3 & 0.00001762 & 5.8736 \mathrm{e}-6 & 3.1667 & 0.0855\end{array}$

$\begin{array}{lrrr}\text { Error } & 8 & 0.00001484 & 1.8548 \mathrm{e}-6 \\ \text { C. Total } & 11 & 0.00003246 & \end{array}$

Means for Oneway Anova

Level Number Mean Std Error Lower 95\% Upper 95\%

$\begin{array}{llllrr}\text { T-1-1 } & 3 & 0.100636 & 0.00079 & 0.09882 & 0.10245\end{array}$

$\begin{array}{llllll}\mathrm{T}-1-1 & 3 & 0.101748 & 0.00079 & 0.09993 & 0.10356\end{array}$

$\begin{array}{llllll}\text { T-2-1 } & 3 & 0.098412 & 0.00079 & 0.09660 & 0.10023\end{array}$

$\begin{array}{llllll}\mathrm{T}-2-2 & 3 & 0.100636 & 0.00079 & 0.09882 & 0.10245\end{array}$

Std Error uses a pooled estimate of error variance 
Exhibit A-3. Measurements by Analytical Series, Block and Sub-Block for the Batch 1 and LRM Standards by Oxide by Prep (continued)

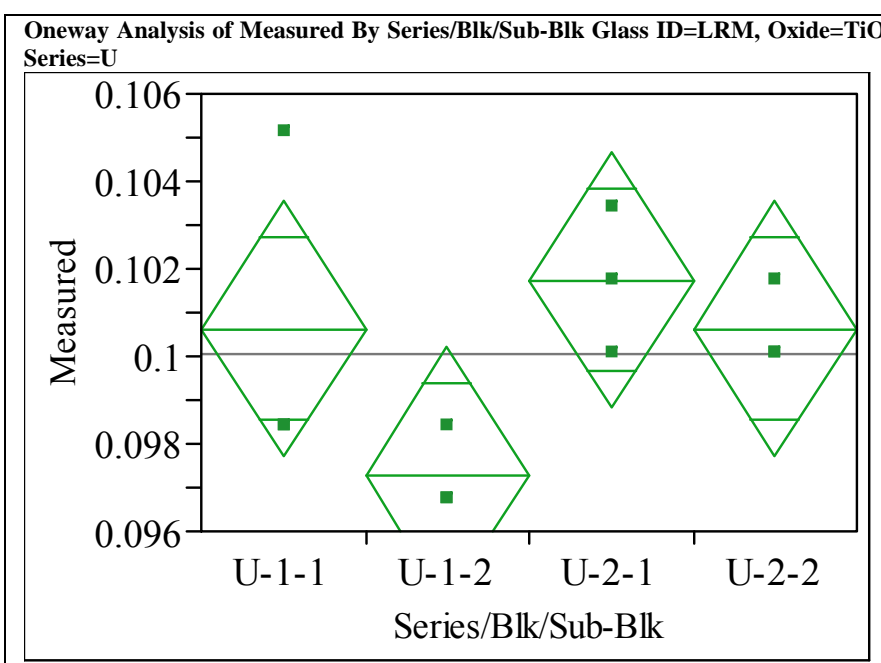

Oneway Anova

Summary of Fit

$\begin{array}{ll}\text { Rsquare } & 0.461538 \\ \text { Adj Rsquare } & 0.259615\end{array}$

Root Mean Square Error $\quad 0.002207$

$\begin{array}{lr}\text { Mean of Response } & 0.10008 \\ \text { Observations (or Sum Wgts) } & 12\end{array}$

Analysis of Variance

$\begin{array}{lrrrrr} & \text { DF } & \text { Sum of Squares } & \text { Mean Square } & \text { F Ratio } & \text { Prob }>\text { F } \\ \text { Source } & 3 & 0.00003339 & 0.000011 & 2.2857 & 0.1556 \\ \text { Series/Blk/Sub-Blk } & 8 & 0.00003895 & 4.869 \mathrm{e}-6 & & \\ \text { Error } & 11 & 0.00007234 & & & \\ \text { C. Total } & 11 & & & & \end{array}$

Means for Oneway Anova

Level Number Mean Std Error Lower 95\% Upper 95\%

$\begin{array}{lllllr}\text { U-1-1 } & 3 & 0.100636 & 0.00127 & 0.09770 & 0.10357\end{array}$

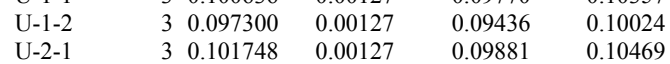

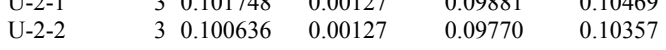

Std Error uses a pooled estimate of error variance

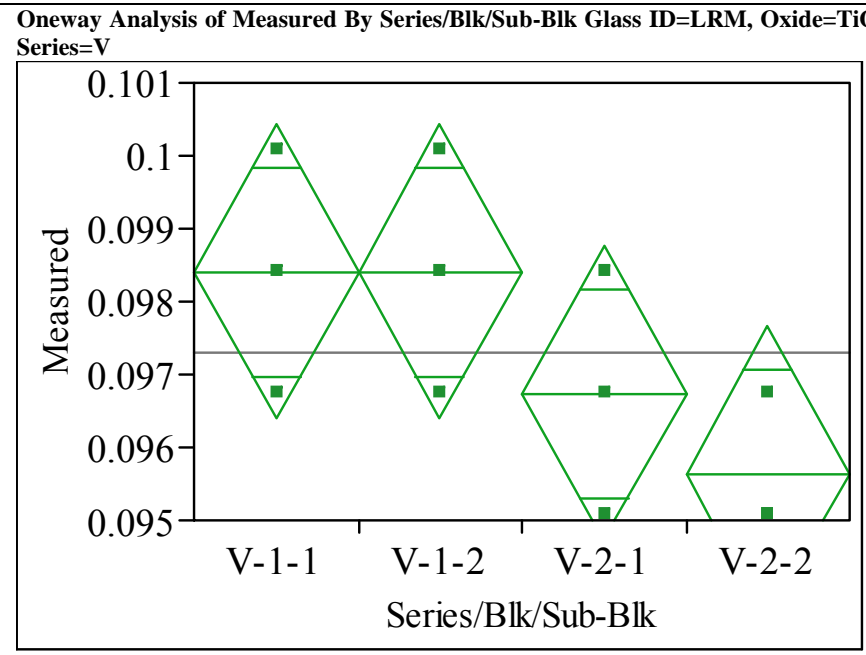

\section{Oneway Anova}

Summary of Fit

$\begin{array}{lr}\text { Rsquare } & 0.473684 \\ \text { Adj Rsquare } & 0.276316 \\ \text { Root Mean Square Error } & 0.001523 \\ \text { Mean of Response } & 0.0973 \\ \text { Observations (or Sum Wgts) } & 12\end{array}$

Analysis of Variance

Source DF Sum of Squares Mean Square F Ratio Prob > F

$\begin{array}{llllll}\text { Series/Blk/Sub-Blk } & 3 & 0.00001669 & 5.5644 \mathrm{e}-6 & 2.4000 & 0.1433\end{array}$

$\begin{array}{lrr}\text { Error } & 8 & 0.00001855 \\ \text { C. Tota } & 11 & 0.0003524\end{array}$ $2.3185 \mathrm{e}-6$

Means for Oneway Anova

Level Number Mean Std Error Lower

$\begin{array}{llllrr}\text { V-1-1 } & 3 & 0.098412 & 0.00088 & 0.09638 & 0.10044\end{array}$

$\begin{array}{llllll}V-1-2 & 3 & 0.098412 & 0.00088 & 0.09638 & 0.10044\end{array}$

$\begin{array}{llllll}V-2-1 & 3 & 0.096744 & 0.00088 & 0.09472 & 0.09877\end{array}$

$\begin{array}{llllll}\mathrm{V}-2-2 & 3 & 0.095632 & 0.00088 & 0.09360 & 0.09766\end{array}$

Std Error uses a pooled estimate of error variance 
Exhibit A-3. Measurements by Analytical Series, Block and Sub-Block for the Batch 1 and LRM Standards by Oxide by Prep (continued)

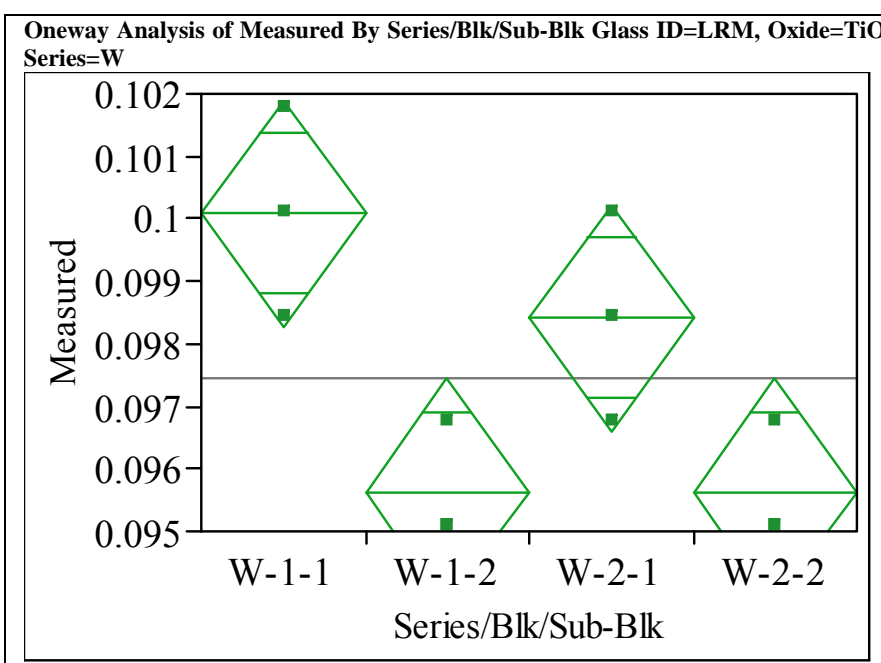

Oneway Anova

Summary of Fit

0.74502

Adj Rsquare $\quad 0.649402$

Root Mean Square Error $\quad 0.001362$

$\begin{array}{lr}\text { Mean of Response } & 0.097439 \\ \text { Observations (or Sum Wgts) } & 12\end{array}$

Analysis of Variance

$\begin{array}{lrrrrr} & \text { DF } & \text { Sum of Squares } & \text { Mean Square } & \text { F Ratio } & \text { Prob }>\text { F } \\ \text { Source } & 3 & 0.00004336 & 0.000014 & 7.7917 & 0.0093 \\ \text { Series/Blk/Sub-Blk } & 8 & 0.00001484 & 1.855 \mathrm{e}-6 & & \\ \text { Error } & 11 & 0.00005819 & & & \\ \text { C. Total } & 11 & & & & \end{array}$

Means for Oneway Anova

Level Number Mean Std Error Lower 95\% Upper 95\%

$\begin{array}{lllllr}\text { W-1-1 } & 3 & 0.100080 & 0.00079 & 0.09827 & 0.10189\end{array}$

$\begin{array}{llllll}\text { W-1-2 } & 3 & 0.095632 & 0.00079 & 0.09382 & 0.09745\end{array}$

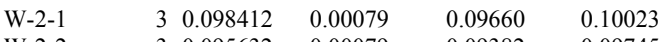

$\begin{array}{llllll}\text { W-2-2 } & 3 & 0.095632 & 0.00079 & 0.09382 & 0.09745\end{array}$

Std Error uses a pooled estimate of error variance

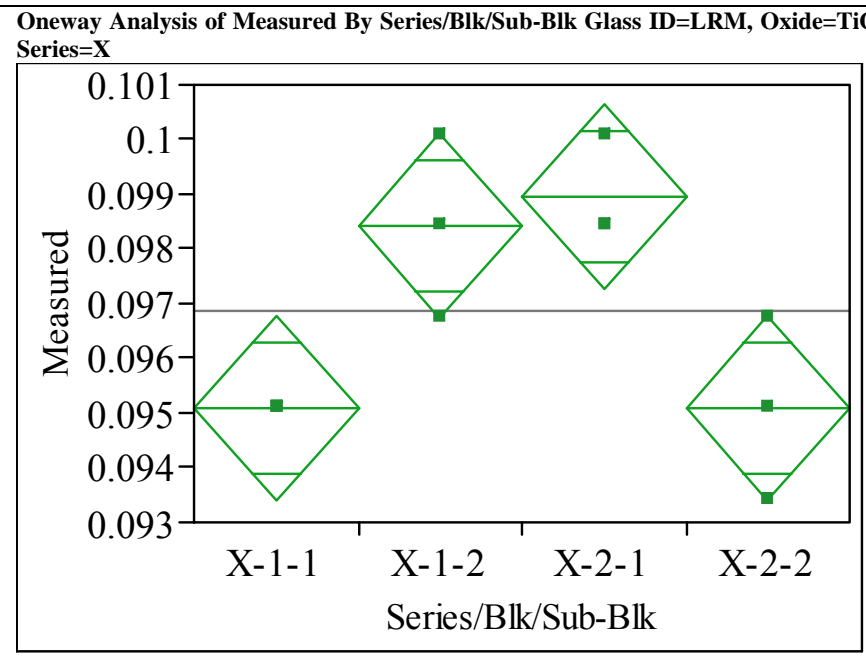

Oneway Anova

Summary of Fit

$$
\text { Rsquare }
$$

0.753304

Adj Rsquare $\quad 0.660793$

Root Mean Square Error $\quad 0.001274$

$\begin{array}{lr}\text { Mean of Response } & 0.096883 \\ \text { Observations (or Sum Wgts) } & 12\end{array}$

Analysis of Variance

$\begin{array}{lrrrrr}\text { Source } & \text { DF } & \text { Sum of Squares } & \text { Mean Square } & \text { F Ratio Prob }>\text { F } \\ \text { Series/Blk/Sub-Blk } & 3 & 0.00003965 & 0.000013 & 8.1429 & 0.0082\end{array}$

$\begin{array}{lrrr}\text { Error } & 8 & 0.00001298 & 1.623 \mathrm{e}-6\end{array}$

Means for Oneway Anova

Level Number Mean Std Error Lower 95\% Upper 95\%

$\begin{array}{llllrr}\mathrm{X}-1-1 & 3 & 0.095076 & 0.00074 & 0.09338 & 0.09677\end{array}$

$\begin{array}{llllll}\mathrm{X}-1-2 & 3 & 0.098412 & 0.00074 & 0.09672 & 0.10011\end{array}$

$\begin{array}{llllll}\mathrm{X}-2-1 & 3 & 0.098968 & 0.00074 & 0.09727 & 0.10066\end{array}$

$\begin{array}{llllll}\mathrm{X}-2-2 & 3 & 0.095076 & 0.00074 & 0.09338 & 0.09677\end{array}$

Std Error uses a pooled estimate of error variance 
Exhibit A-3. Measurements by Analytical Series, Block and Sub-Block for the Batch 1 and LRM Standards by Oxide by Prep (continued)

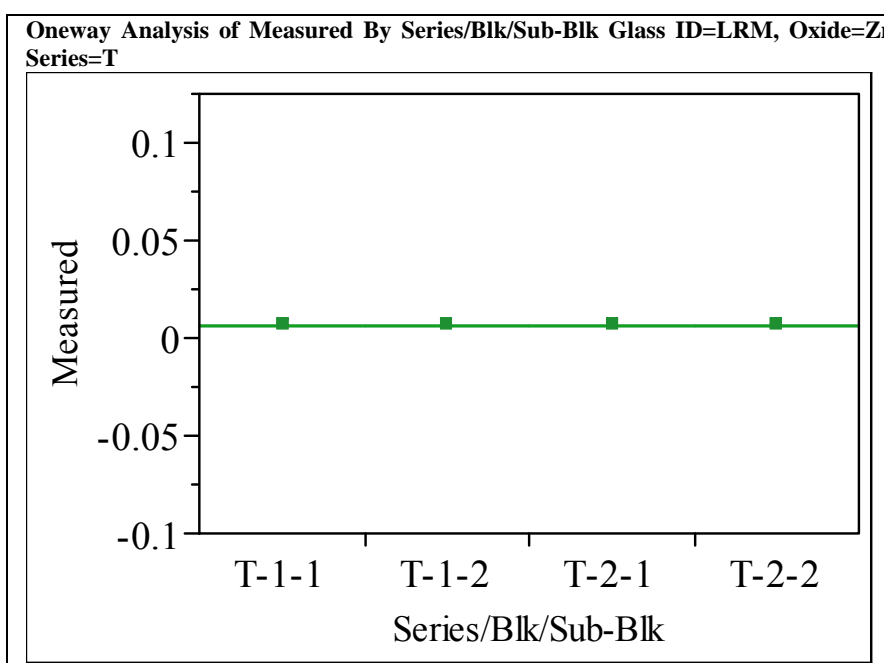

Oneway Anova
Summary of Fit

Rsquare

Adj Rsquare

$\begin{array}{lr}\text { Root Mean Square Error } & 0 \\ \text { Mean of Response } & 0.006224\end{array}$

Observations (or Sum Wgts) 12

Analysis of Variance

$\begin{array}{lrrr}\text { Source } & \text { DF Sum of Squares Mean Square } & \text { F Ratio Prob }>\text { F } \\ \text { Series/Blk/Sub-Blk } & 3 & 0 & 0\end{array}$

$\begin{array}{lr}\text { Error } & 8 \\ \text { C. Total } & 11\end{array}$

0

Means for Oneway Anova

\begin{tabular}{lrrrrr} 
Level Number & Mean & Std Error & Lower 95\% & Upper 95\% \\
T-1-1 & 3 & 0.006224 & 0 & 0.00622 & 0.00622 \\
T $-1-2$ & 3 & 0.006224 & 0 & 0.00622 & 0.00622 \\
\hline & 3 & 0.06224 & 0 & 0.00622 & 0.0622
\end{tabular}

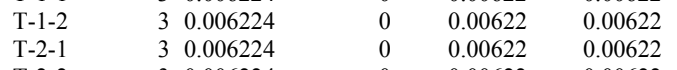

$\begin{array}{llllll}\mathrm{T}-2-2 & 3 & 0.006224 & 0 & 0.00622 & 0.00622\end{array}$

Std Error uses a pooled estimate of error variance

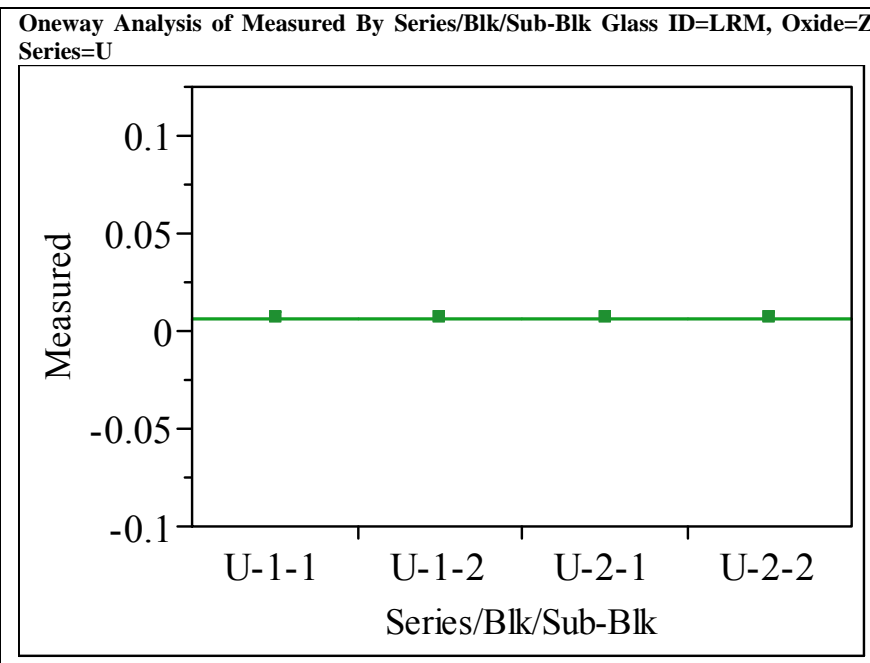

Oneway Anova

Summary of Fit

Rsquare

Adj Rsquare

$\begin{array}{lr}\text { Root Mean Square Error } & 0 \\ \text { Mean of Response } & 0.006224\end{array}$

Observations (or Sum Wgts) 12

Analysis of Variance

Sum of Squares Mean Square F Ratio Prob > F

$\begin{array}{llll}\text { Series/Blk/Sub-Blk } & 3 & 0 & 0 \\ \text { Error } & 8 & 0 & 0\end{array}$

$\begin{array}{lrr}\text { Error } & 8 & 0 \\ \text { C. Total } & 11 & 0\end{array}$

Means for Oneway Anova

Level Number Mean Std Error Lower 95\% Upper 95\%

$\begin{array}{llllll}\text { U-1-1 } & 3 & 0.006224 & 0 & 0.00622 & 0.00622\end{array}$

$\begin{array}{llllll}\text { U-1-2 } & 3 & 0.006224 & 0 & 0.00622 & 0.00622\end{array}$

$\begin{array}{llllll}\mathrm{U}-2-1 & 3 & 0.006224 & 0 & 0.00622 & 0.00622\end{array}$

$\begin{array}{llllll}\mathrm{U}-2-2 & 3 & 0.006224 & 0 & 0.00622 & 0.00622\end{array}$

Std Error uses a pooled estimate of error variance 
Exhibit A-3. Measurements by Analytical Series, Block and Sub-Block for the Batch 1 and LRM Standards by Oxide by Prep (continued)

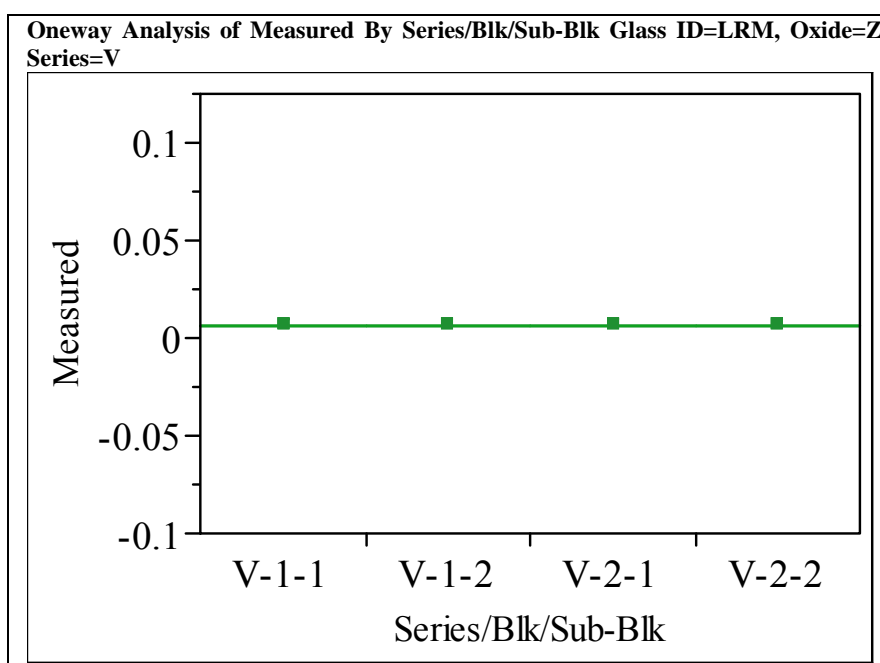

Oneway Anova

Summary of Fit

Rsquare

Adj Rsquare

$\begin{array}{lr}\text { Root Mean Square Error } & 0 \\ \text { Mean of Response } & 0.006224\end{array}$

Observations (or Sum Wgts) 12

Analysis of Variance

$\begin{array}{lrrr}\text { Source } & \text { DF Sum of Squares Mean Square } \mathbf{F} \text { Ratio Prob }>\text { F } \\ \text { Series/Blk/Sub-Blk } & 3 & 0 & 0\end{array}$

$\begin{array}{lr}\text { Error } & 8 \\ \text { C. Total } & 11\end{array}$

0

Means for Oneway Anova

Level Number Mean Std Error Lower 95\% Upper 95\%

$\begin{array}{llllll}\mathrm{V}-1-1 & 3 & 0.006224 & 0 & 0.00622 & 0.00622\end{array}$

$\begin{array}{llllll}\mathrm{V}-1-1 & 3 & 0.006224 & 0 & 0.00622 & 0.00622 \\ \mathrm{~V}-2-1 & 3 & 0.006224 & 0 & 0.00622 & 0.00622\end{array}$

$\begin{array}{llllll}\text { V-2-1 } & 3 & 0.006224 & 0 & 0.00622 & 0.00622 \\ \text { V-2-2 } & 3 & 0.006224 & 0 & 0.00622 & 0.00622\end{array}$

Std Error uses a pooled estimate of error variance

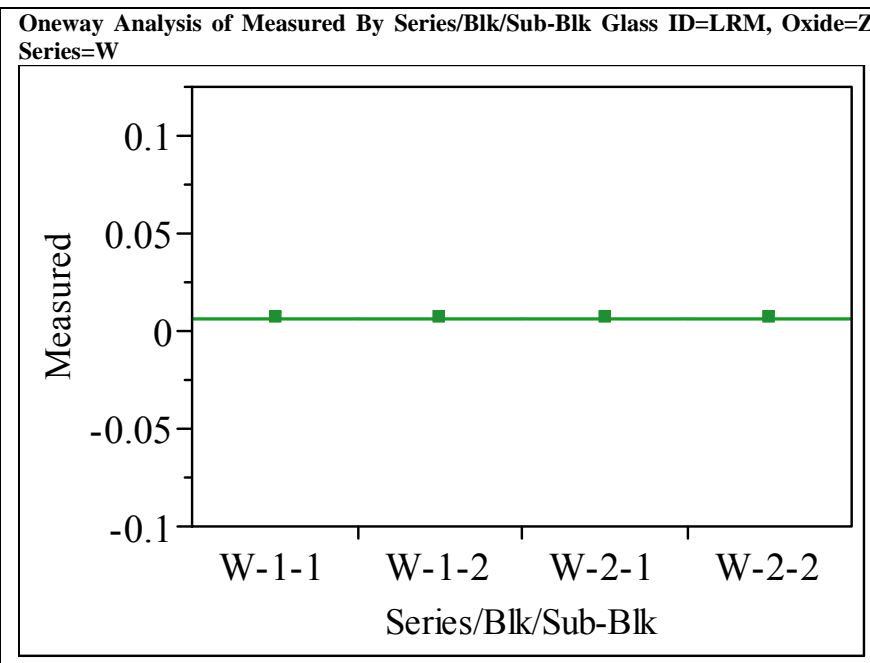

Oneway Anova

Summary of Fit

Rsquare

Adj Rsquare

$\begin{array}{lr}\text { Root Mean Square Error } & 0 \\ \text { Mean of Response } & 0.006224\end{array}$

Observations (or Sum Wgts) 12

Analysis of Variance

Sum of Squares Mean Square F Ratio Prob > F

Series/Blk/Sub-Blk

$\begin{array}{lr}\text { Error } & 8 \\ \text { C. Total } & 11\end{array}$

0
0

Means for Oneway Anova

Level Number Mean Std Error Lower 95\% Upper 95\%

$\begin{array}{llllll}\text { W-1-1 } & 3 & 0.006224 & 0 & 0.00622 & 0.00622\end{array}$

$\begin{array}{llllll}\mathrm{W}-1-2 & 3 & 0.006224 & 0 & 0.00622 & 0.00622\end{array}$

$\begin{array}{llllll}\mathrm{W}-2-1 & 3 & 0.006224 & 0 & 0.00622 & 0.00622\end{array}$

$\begin{array}{llllll}\mathrm{W}-2-2 & 3 & 0.006224 & 0 & 0.00622 & 0.00622\end{array}$

Std Error uses a pooled estimate of error variance 
Exhibit A-3. Measurements by Analytical Series, Block and Sub-Block for the Batch 1 and LRM Standards by Oxide by Prep (continued)

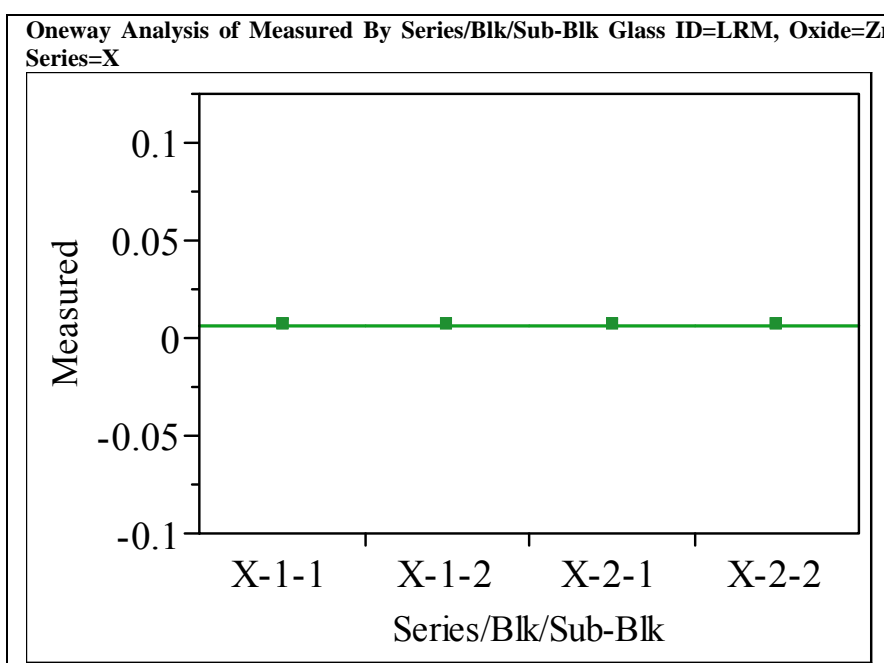

Oneway Anova

Summary of Fit

Rsquare

Adj Rsquare

$\begin{array}{lr}\text { Root Mean Square Error } & 0 \\ \text { Mean of Response } & 0.006224\end{array}$

Observations (or Sum Wgts) 12

Analysis of Variance

$\begin{array}{lrrr}\text { Source } & \text { DF Sum of Squares Mean Square } \mathbf{F} \text { Ratio Prob }>\text { F } \\ \text { Series/Blk/Sub-Blk } & 3 & 0 & 0\end{array}$

$\begin{array}{lr}\text { Error } & 8 \\ \text { C. Total } & 11\end{array}$

0

Means for Oneway Anova

Level Number Mean Std Error Lower 95\% Upper 95\%

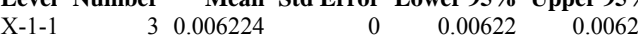

$\begin{array}{llllll}\mathrm{X}-1-1 & 3 & 0.006224 & 0 & 0.00622 & 0.00622 \\ \mathrm{X}-2-2 & 3 & 0.006224 & 0 & 0.00622 & 0.00622\end{array}$

$\begin{array}{llllll}\mathrm{X}-2-1 & 3 & 0.006224 & 0 & 0.00622 & 0.00622 \\ \mathrm{X}-2-2 & 3 & 0.006224 & 0 & 0.00622 & 0.00622\end{array}$

Std Error uses a pooled estimate of error variance

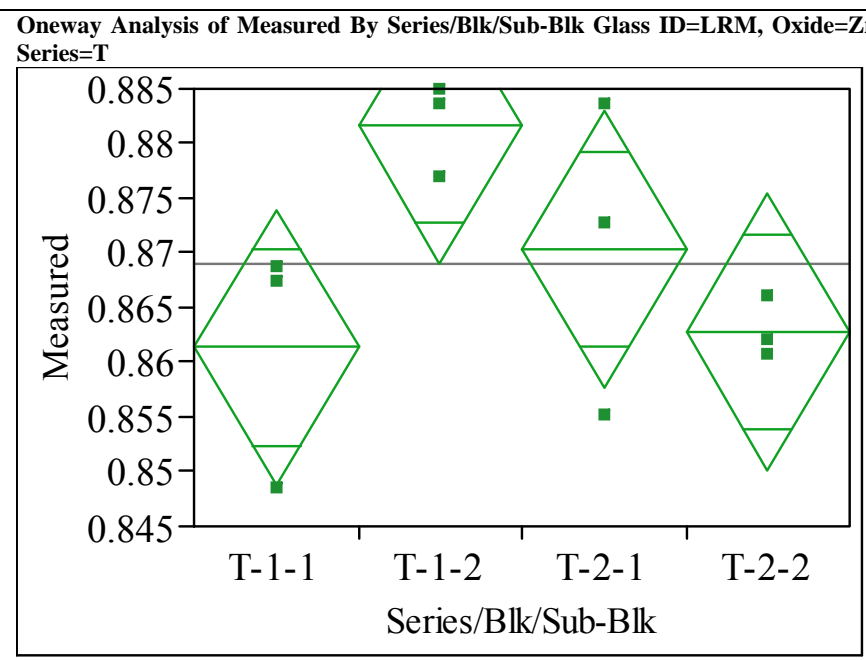

Oneway Anova

Summary of Fit

Rsquare

$\begin{array}{ll} & 0.338749 \\ \text { Root Mean Square Error } & 0.009488\end{array}$

\begin{tabular}{ll} 
Mean of Response & 0.869015 \\
\hline
\end{tabular}

$\begin{array}{lr}\text { Observations (or Sum Wgts) } & 12\end{array}$

Analysis of Variance

Source DF Sum of Squares Mean Square F Ratio Prob > F

$\begin{array}{llllll}\text { Series/Blk/Sub-Blk } & 3 & 0.00077731 & 0.000259 & 2.8784 & 0.103\end{array}$

$\begin{array}{lrr}\text { Error } & 8 & 0.00072013 \\ \text { C. Tot } & 11 & 0.0019744\end{array}$ 0.000090

Means for Oneway Anova

Level Number Mean Std Error Lower 95\% Upper 95\%

$\begin{array}{llrrrr}\text { T-1-1 } & 3 & 0.861360 & 0.00548 & 0.84873 & 0.87399\end{array}$

$\begin{array}{llllll}\mathrm{T}-1-2 & 3 & 0.881622 & 0.00548 & 0.86899 & 0.89425\end{array}$

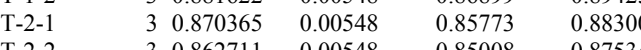

$\begin{array}{llllll}\mathrm{T}-2-2 & 3 & 0.862711 & 0.00548 & 0.85008 & 0.87534\end{array}$

Std Error uses a pooled estimate of error variance 
Exhibit A-3. Measurements by Analytical Series, Block and Sub-Block for the Batch 1 and LRM Standards by Oxide by Prep (continued)

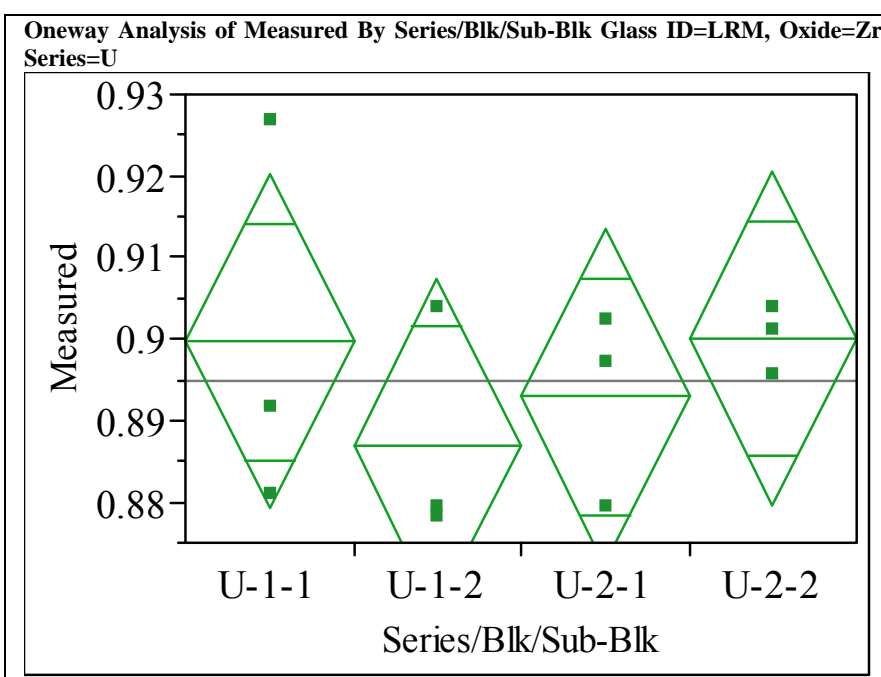

Oneway Anova

Summary of Fit

$\begin{array}{lr}\text { Rsquare } & 0.154578 \\ \text { Adj Rsquare } & -0.16246 \\ \text { Root Mean Square Error } & 0.015382 \\ \text { Mean of Response } & 0.894905 \\ \text { Observations (or Sum Wgts) } & 12\end{array}$

Analysis of Variance

Source DF Sum of Squares Mean Square F Ratio Prob > F

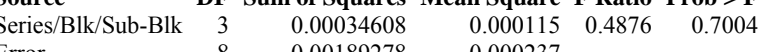

$\begin{array}{lll}\text { Error } & 8 & 0.00189278\end{array}$

C. Total

0.00223886

Means for Oneway Anova

Level Number Mean Std Error Lower 95\% Upper 95\%

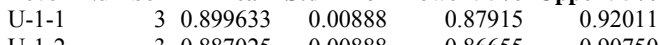

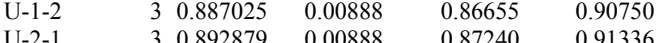

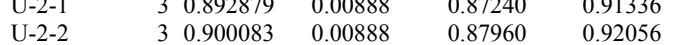

Std Error uses a pooled estimate of error variance

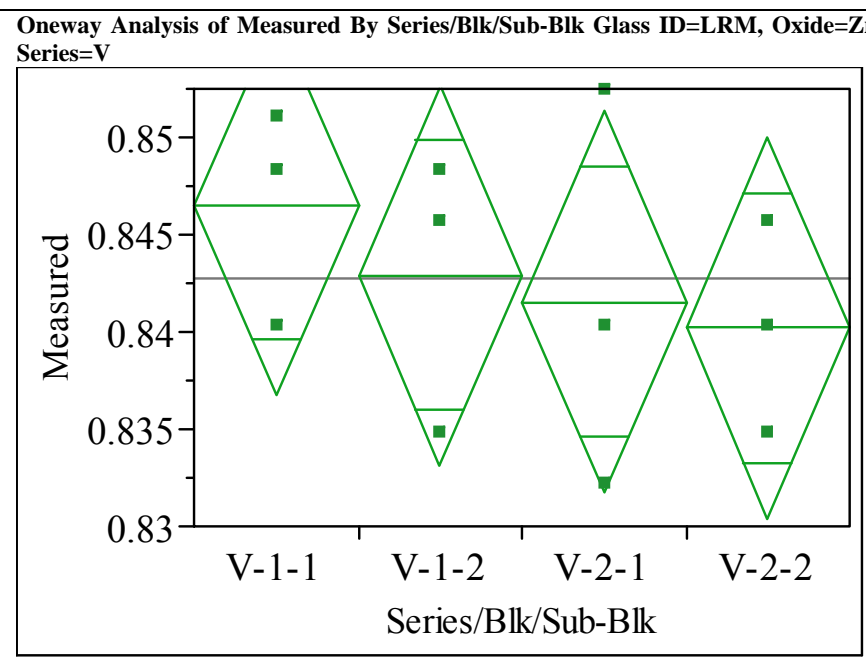

Oneway Anova

Summary of Fit

$$
\text { Rsquare }
$$

0.132824

Adj Rsquare $\quad-0.19237$

Root Mean Square Error $\quad 0.007347$

$\begin{array}{lr}\text { Mean of Response } & 0.842787 \\ \text { Observations (or Sum Wgts) } & 12\end{array}$

Analysis of Variance

Source DF Sum of Squares Mean Square F Ratio Prob > F

Series/Blk/Sub-Blk $3 \quad 0.00006614$

$\begin{array}{lrr}\text { Error } & 8 & 0.00043184 \\ \text { C. } & 11 & 0.000498\end{array}$ 0.000054

Means for Oneway Anova

Level Number Mean Std Error Lower 95\% Upper 95\%

$\begin{array}{lllrrr}\mathrm{V}-1-1 & 3 & 0.846501 & 0.00424 & 0.83672 & 0.85628\end{array}$

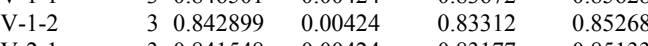

$\begin{array}{lllllll}\mathrm{V}-2-1 & & 3 & 0.841548 & 0.00424 & 0.83177 & 0.85133\end{array}$

$\begin{array}{llllll}\mathrm{V}-2-2 & 3 & 0.840198 & 0.00424 & 0.83042 & 0.84998\end{array}$

Std Error uses a pooled estimate of error variance 
Exhibit A-3. Measurements by Analytical Series, Block and Sub-Block for the Batch 1 and LRM Standards by Oxide by Prep (continued)

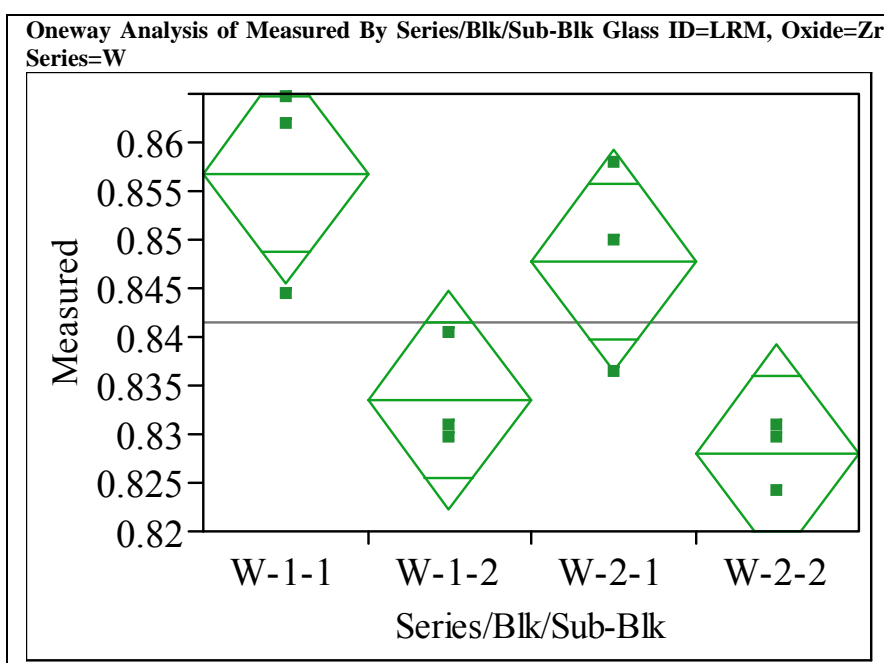

Oneway Anova

Summary of Fit

Rsquare $\quad 0.731403$

Adj Rsquare $\quad 0.630679$

Root Mean Square Error $\quad 0.00848$

$\begin{array}{lr}\text { Mean of Response } & 0.841548 \\ \text { Observations (or Sum Wgts) } & 12\end{array}$

Analysis of Variance

Source DF Sum of Squares Mean Square F Ratio Prob $>$ F

$\begin{array}{llllll}\text { Series/Blk/Sub-Blk } & 3 & 0.00156678 & 0.000522 & 7.2615 & 0.0113\end{array}$

Error

0.00057538

0.000072

Means for Oneway Anova

Level Number Mean Std Error Lower 95\% Upper 95\%

$\begin{array}{lllrrr}\mathrm{W}-1-1 & 3 & 0.856857 & 0.00490 & 0.84557 & 0.86815\end{array}$

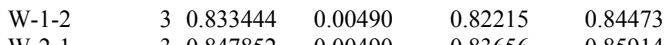

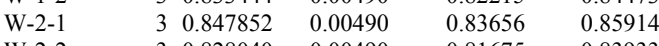

$\begin{array}{llllll}\mathrm{W}-2-2 & 3 & 0.828040 & 0.00490 & 0.81675 & 0.83933\end{array}$

Std Error uses a pooled estimate of error variance

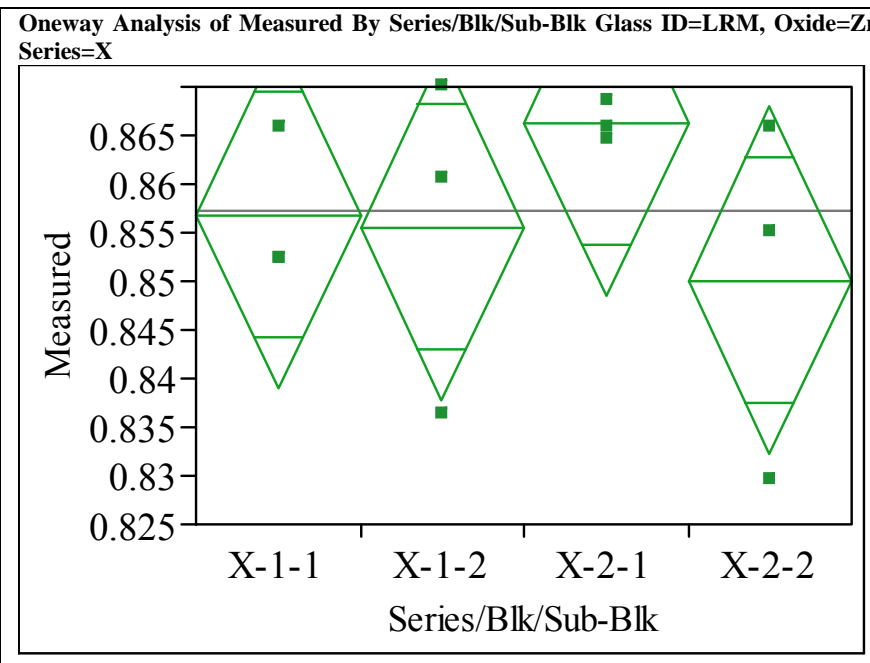

Oneway Anova

Summary of Fit

$$
\text { Rsquare }
$$

$\begin{array}{lr}\text { Rsquare } & 0.22139 \\ \text { Adj Rsquare } & -0.07059\end{array}$

Root Mean Square Error $\quad 0.013412$

$\begin{array}{lr}\text { Mean of Response } & 0.857195 \\ \text { Observations (or Sum Wgts) } & 12\end{array}$

Analysis of Variance

$\begin{array}{lrrrrrr}\text { Source } & \text { DF } & \text { Sum of Squares } & \text { Mean Square } & \text { F Ratio } & \text { Prob }>\text { F } \\ \text { Series/Blk/Sub-Blk } & 3 & 0.00040918 & 0.000136 & 0.7582 & 0.5482\end{array}$

$\begin{array}{lrr}\text { Error } & 8 & 0.00143905 \\ \text { C. } & 11 & 0.00184823\end{array}$ 0.000180

Means for Oneway Anova

Level Number Mean Std Error Lower 95\% Upper 95\%

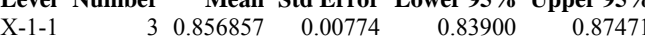

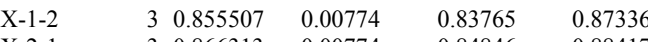

$\begin{array}{lllllll}\mathrm{X}-2-1 & 3 & 0.866313 & 0.00774 & 0.84846 & 0.88417 \\ \mathrm{X}-2-2 & & 3 & 0.850103 & 0.00774 & 0.83225 & 0.86796\end{array}$

$\begin{array}{llllll}\mathrm{X}-2-2 & 3 & 0.850103 & 0.00774 & 0.83225 & 0.86796\end{array}$

Std Error uses a pooled estimate of error variance 


\section{Exhibit A-4. Measured versus Targeted Compositions by Glass ID by Oxide.}

Overlay Plot Oxide=Al2O3 (wt\%)

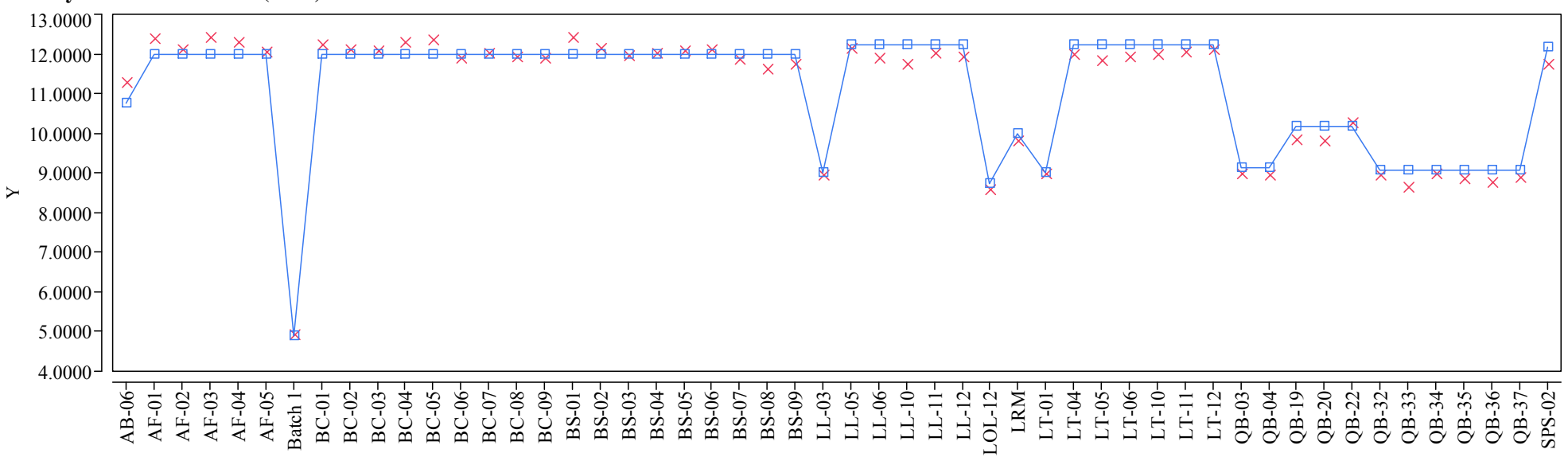

Glass ID

Overlay Plot Oxide=B2O3 (wt \%)

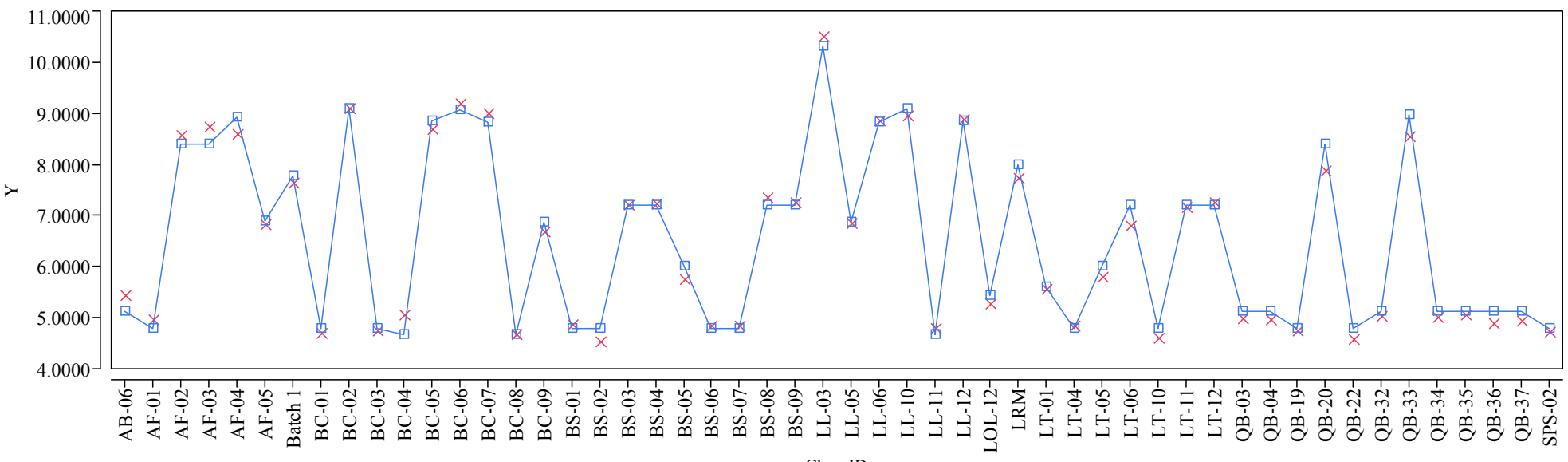

Glass ID

Y $\times$ Measured $\square$-Targeted 


\section{Exhibit A-4. Measured versus Targeted Compositions by Glass ID by Oxide. (continued)}

Overlay Plot Oxide=BaO (wt \%)

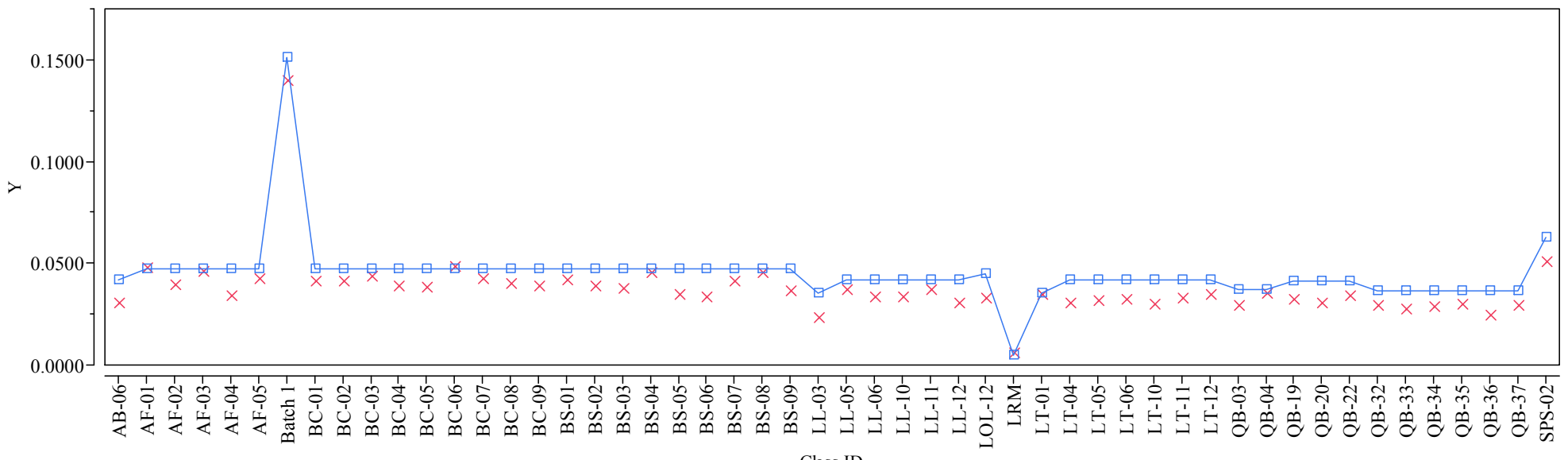

Overlay Plot Oxide $=\mathrm{CaO}(\mathrm{wt} \%)$

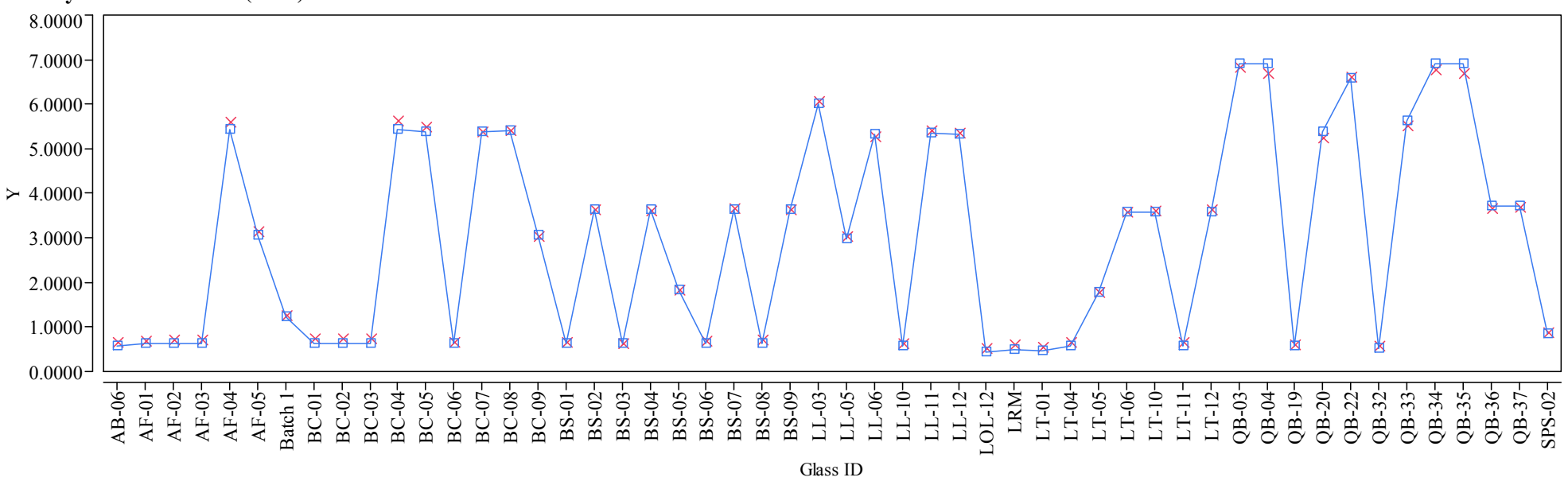

$\mathrm{Y} \times$ Measured $\square$ - Targeted 


\section{Exhibit A-4. Measured versus Targeted Compositions by Glass ID by Oxide. (continued)}

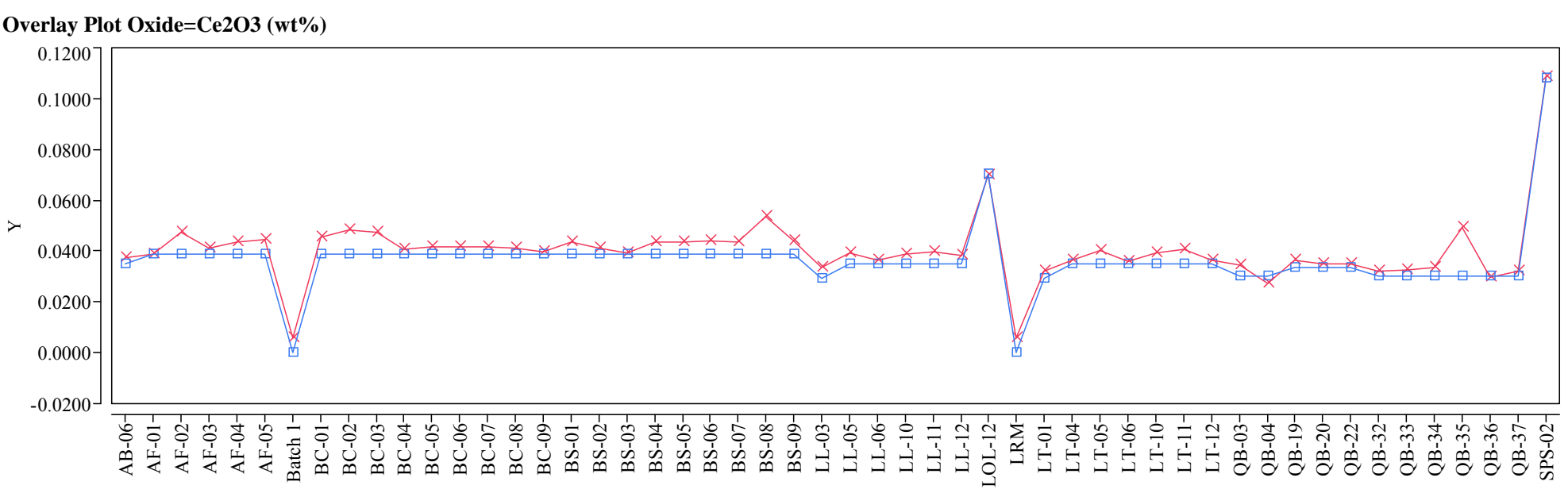

Overlay Plot Oxide=Cr2O3 (wt\%)

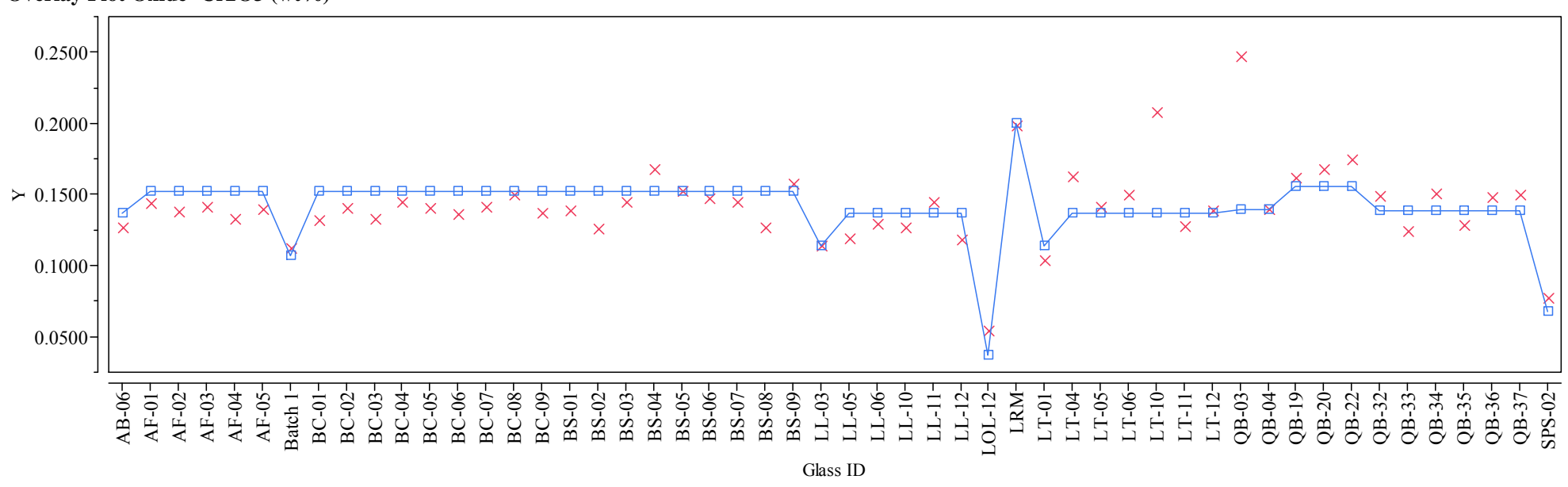

$\mathrm{Y} \times$ Measured $\square$ - Targeted 


\section{Exhibit A-4. Measured versus Targeted Compositions by Glass ID by Oxide. (continued)}

Overlay Plot Oxide $=\mathrm{CuO}(\mathrm{wt} \%)$

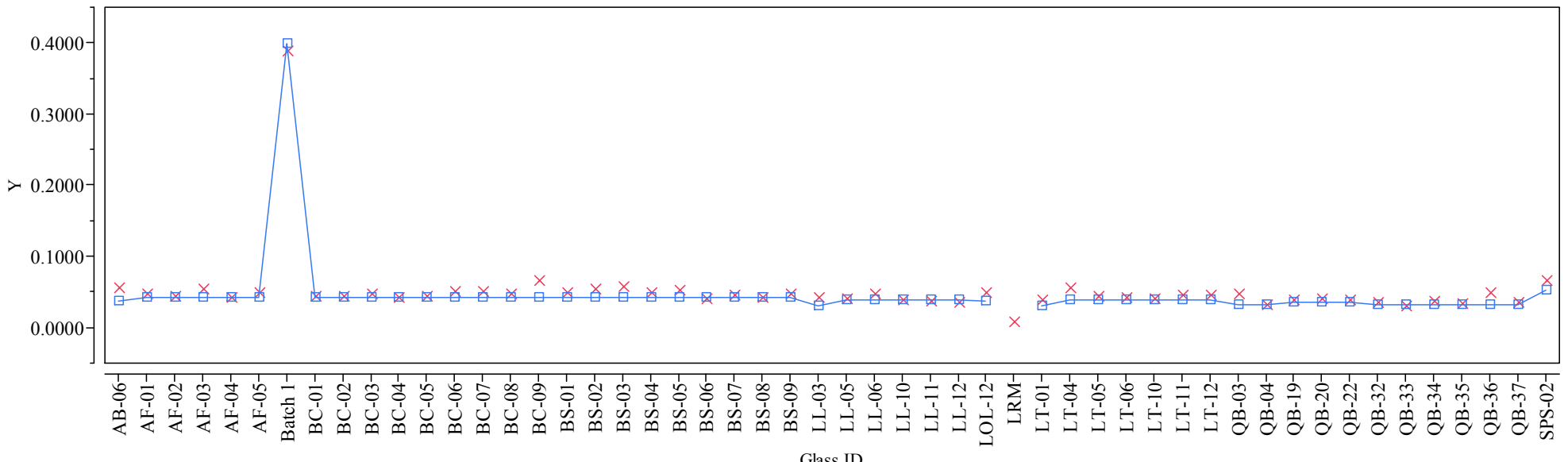

Overlay Plot Oxide=Fe2O3 (wt $\%)$

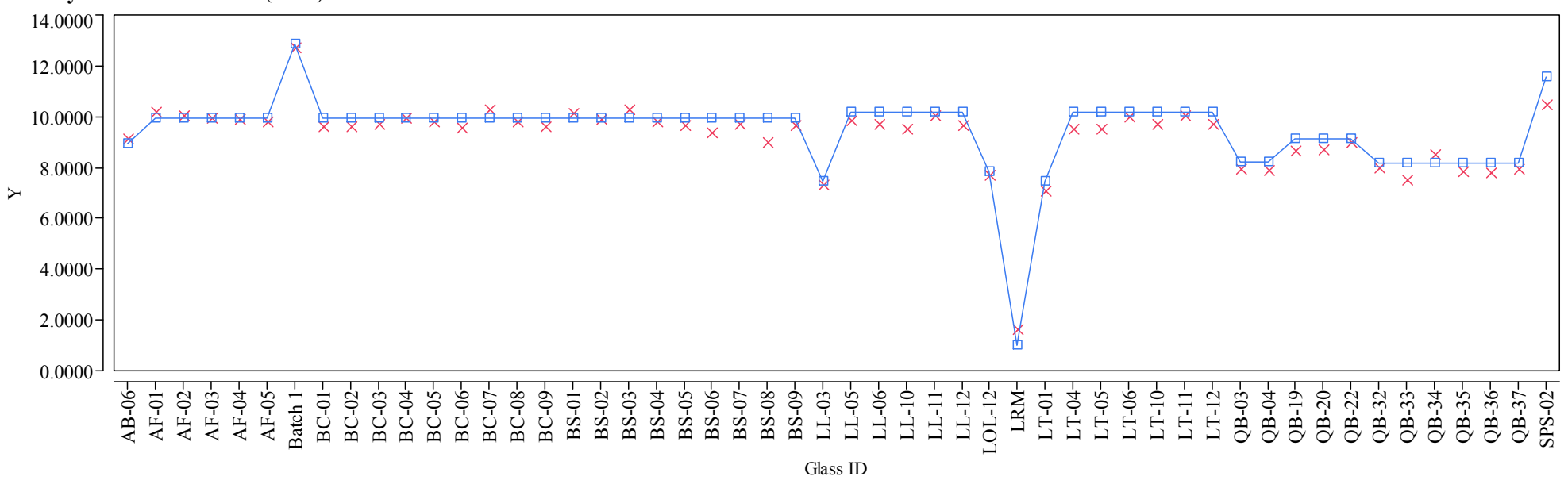

$\mathrm{Y} \times$ Measured $\square$ - Targeted 


\section{Exhibit A-4. Measured versus Targeted Compositions by Glass ID by Oxide. (continued)}

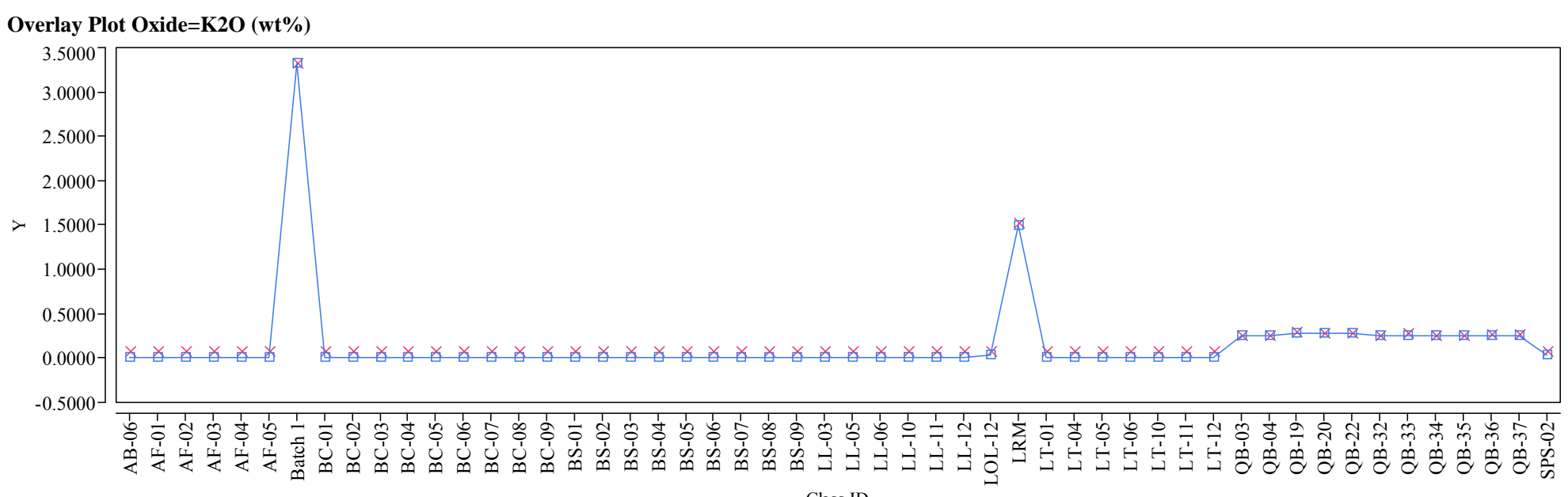

Overlay Plot Oxide=La2O3 (wt \%)

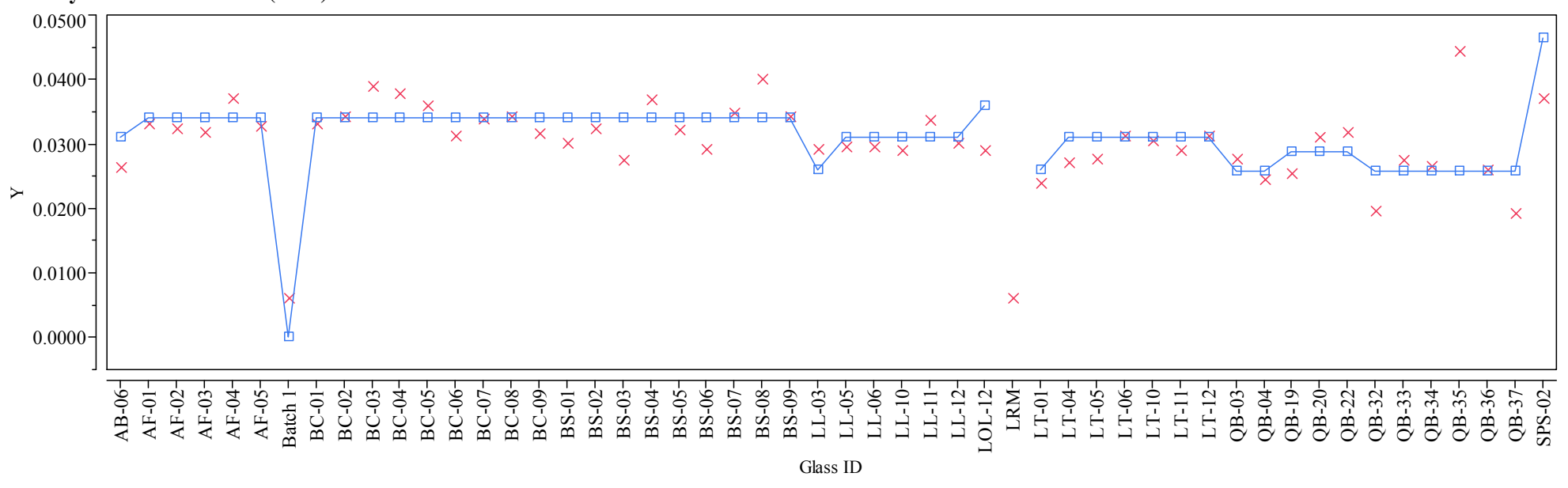

$\mathrm{Y} \times$ Measured $\square$ - Targeted 


\section{Exhibit A-4. Measured versus Targeted Compositions by Glass ID by Oxide. (continued)}

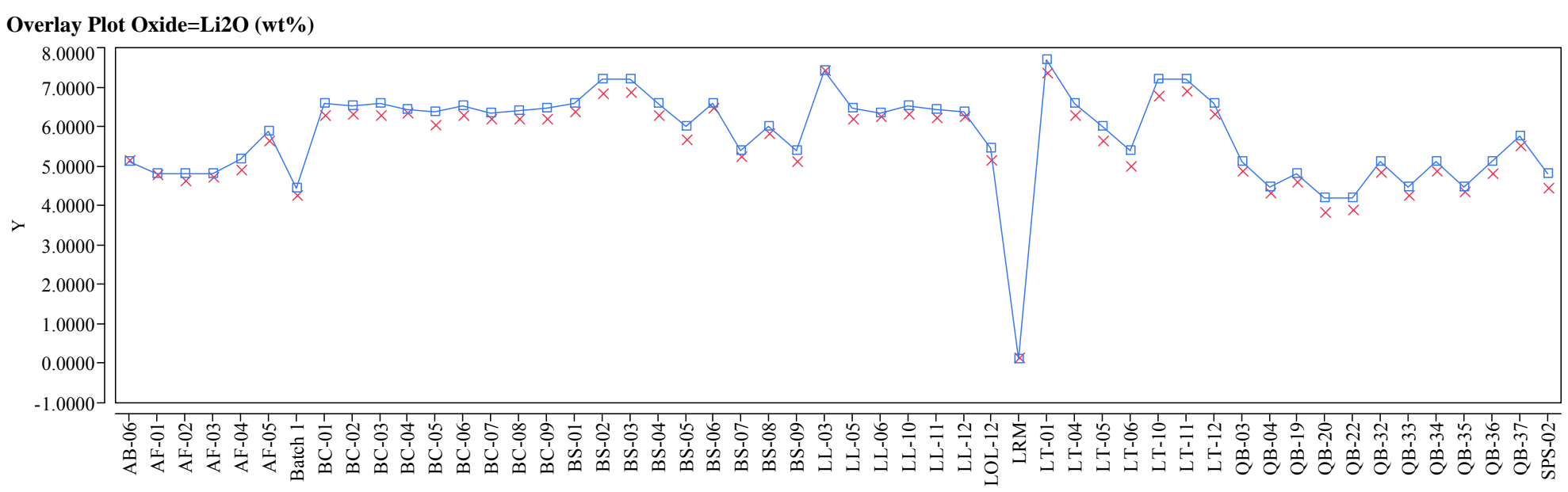

Overlay Plot Oxide=MgO (wt\%)

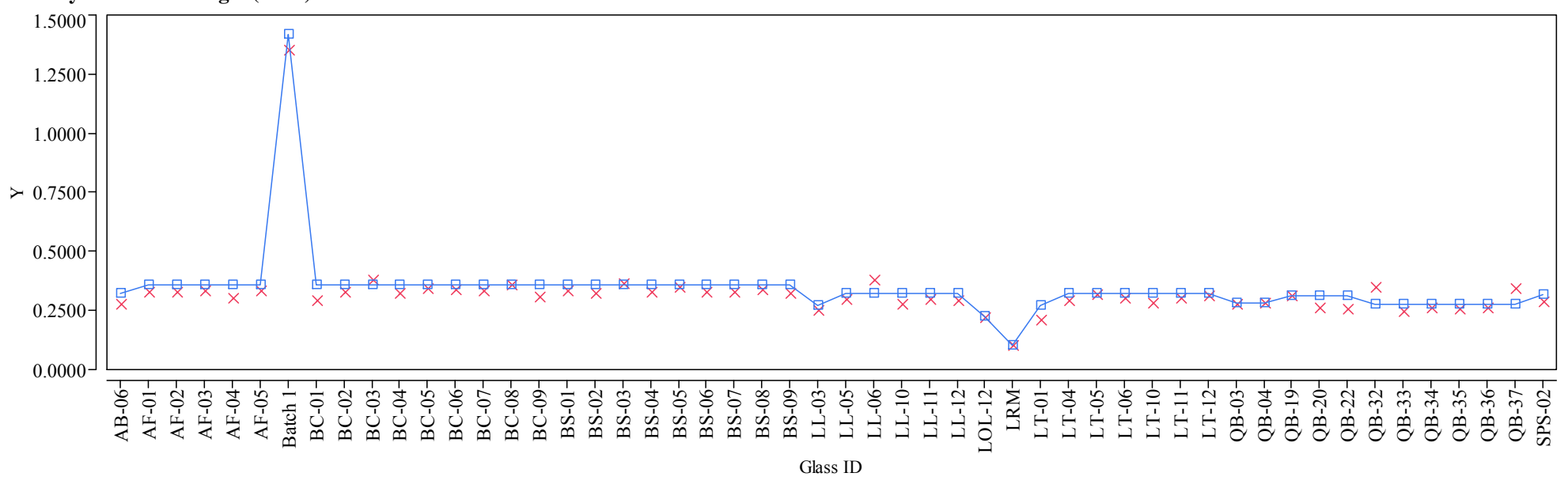

$\mathrm{Y} \times$ Measured $\square$ - Targeted 


\section{Exhibit A-4. Measured versus Targeted Compositions by Glass ID by Oxide. (continued)}

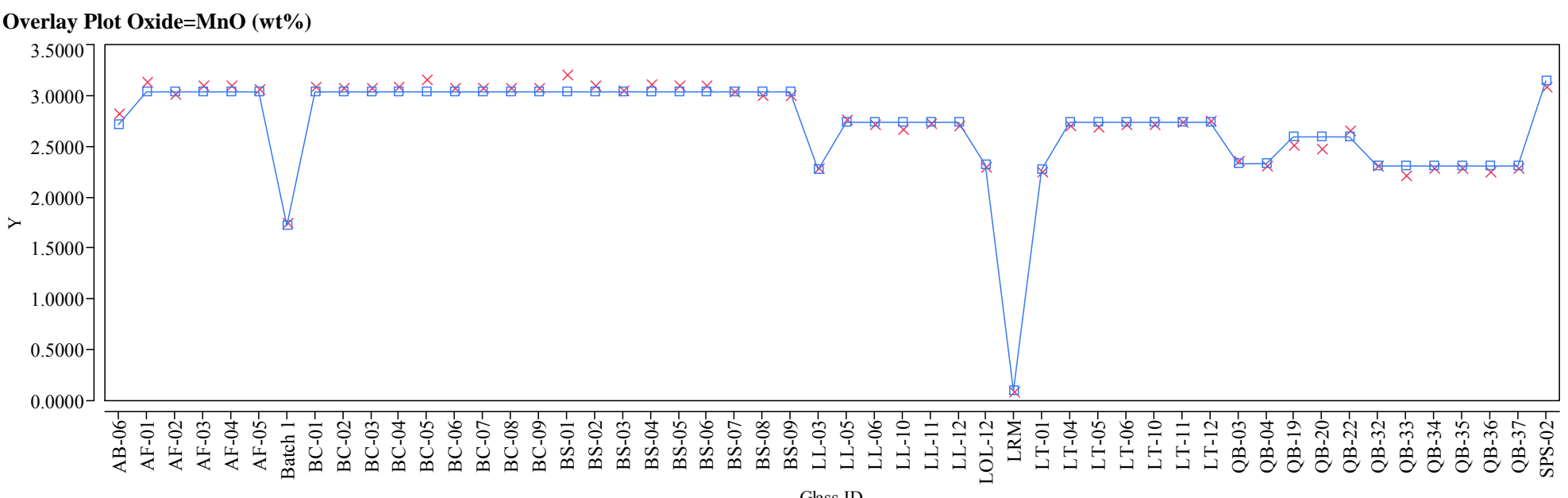

Overlay Plot Oxide=Na2O (wt \%)

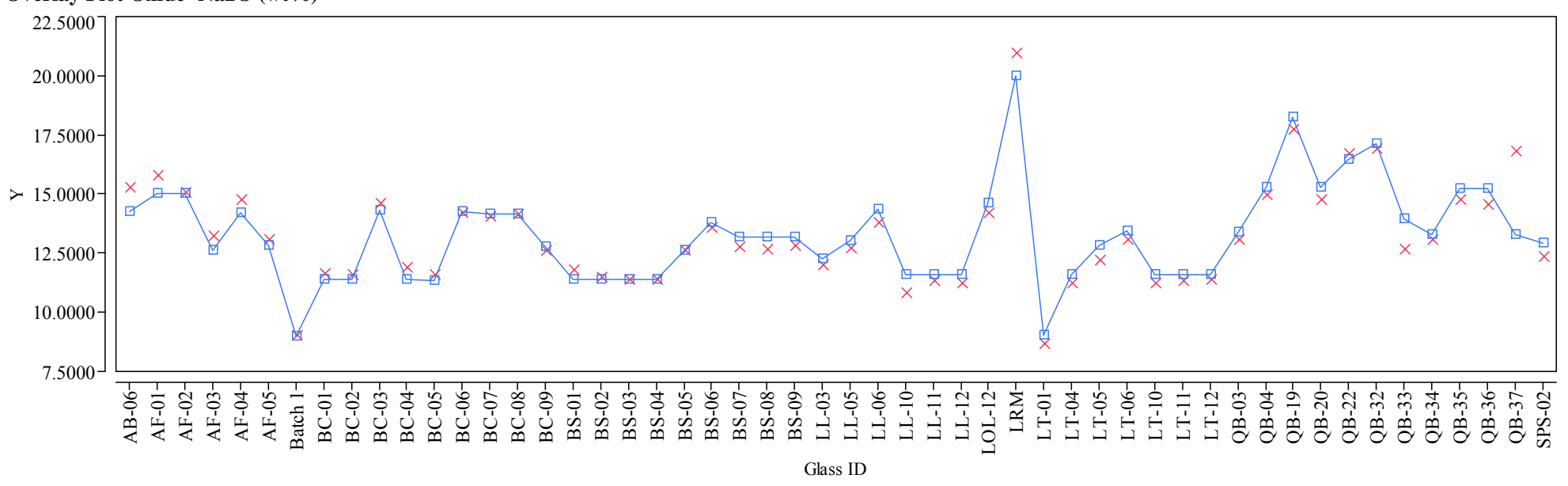

$\mathrm{Y} \times$ Measured $\square$ - Targeted 


\section{Exhibit A-4. Measured versus Targeted Compositions by Glass ID by Oxide. (continued)}

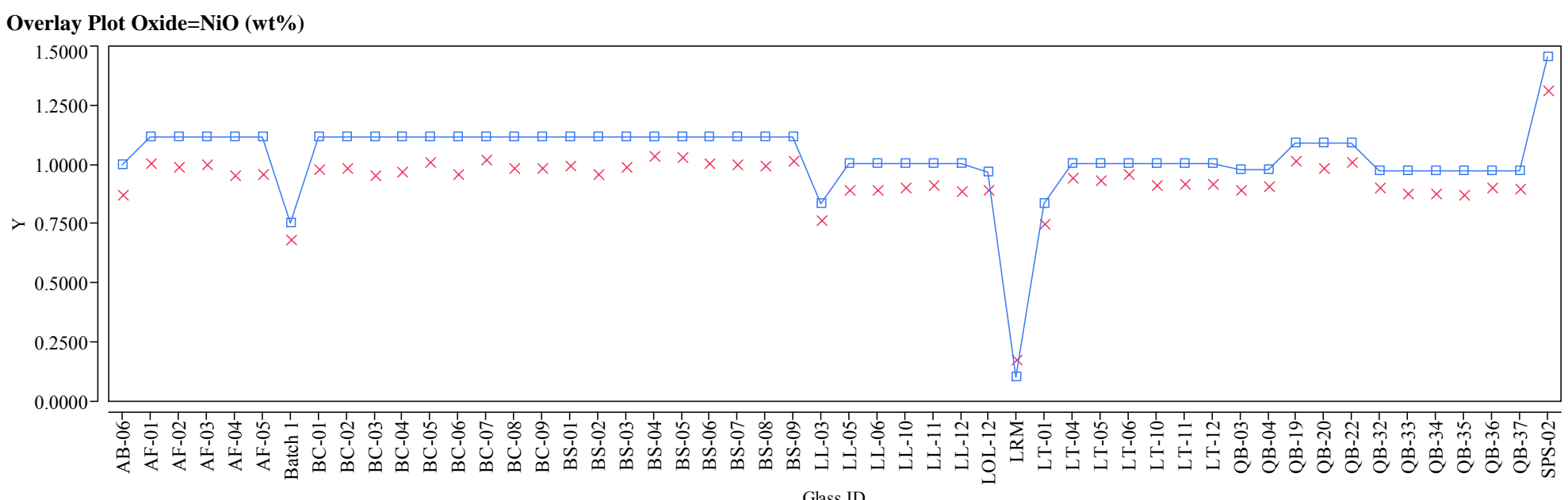

Overlay Plot Oxide=PbO (wt \%)

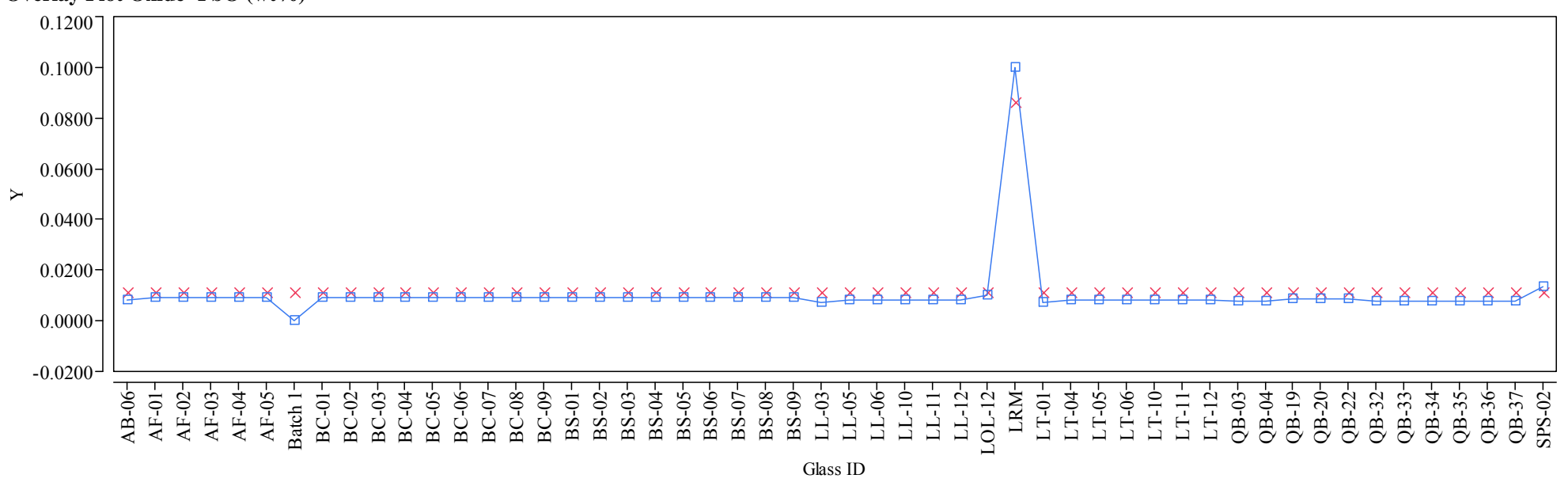

$\mathrm{Y} \times$ Measured $\square$ - Targeted 


\section{Exhibit A-4. Measured versus Targeted Compositions by Glass ID by Oxide. (continued)}

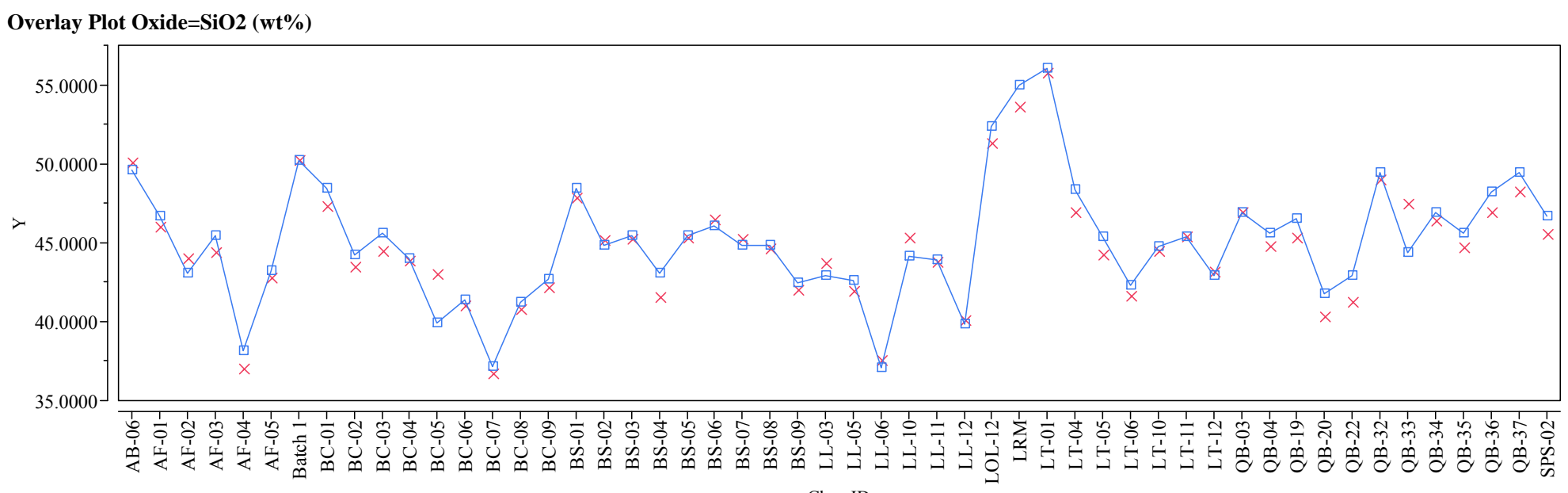

Overlay Plot Oxide=SO4 (wt $\%)$

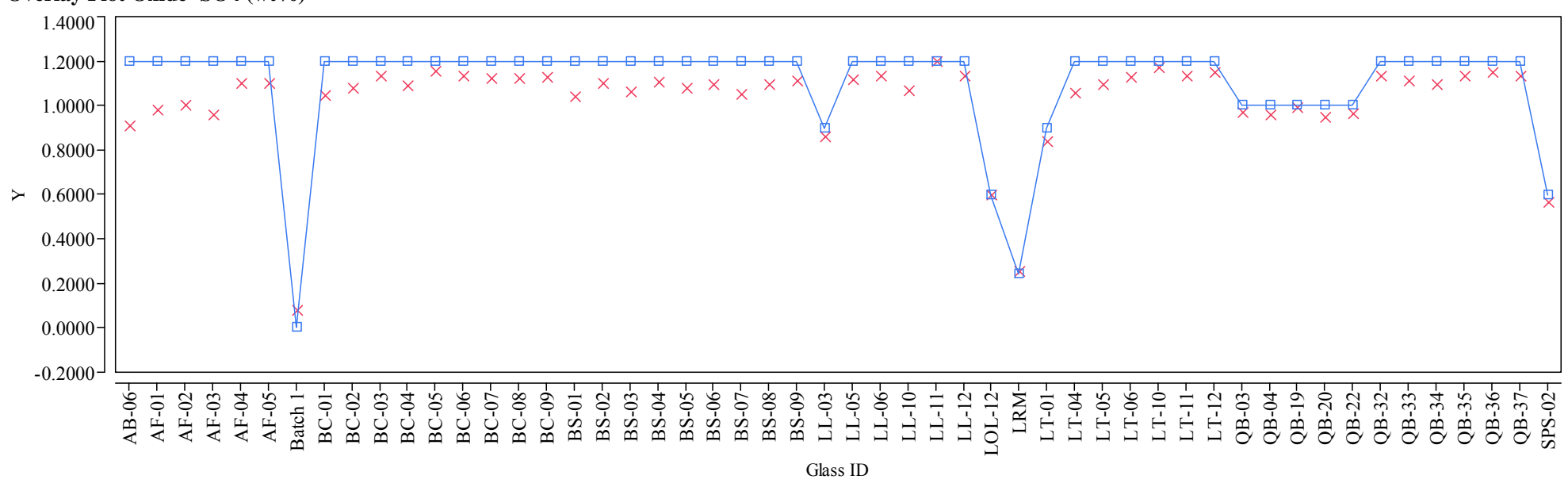

$\mathrm{Y} \times$ Measured $\square$ - Targeted 


\section{Exhibit A-4. Measured versus Targeted Compositions by Glass ID by Oxide. (continued)}

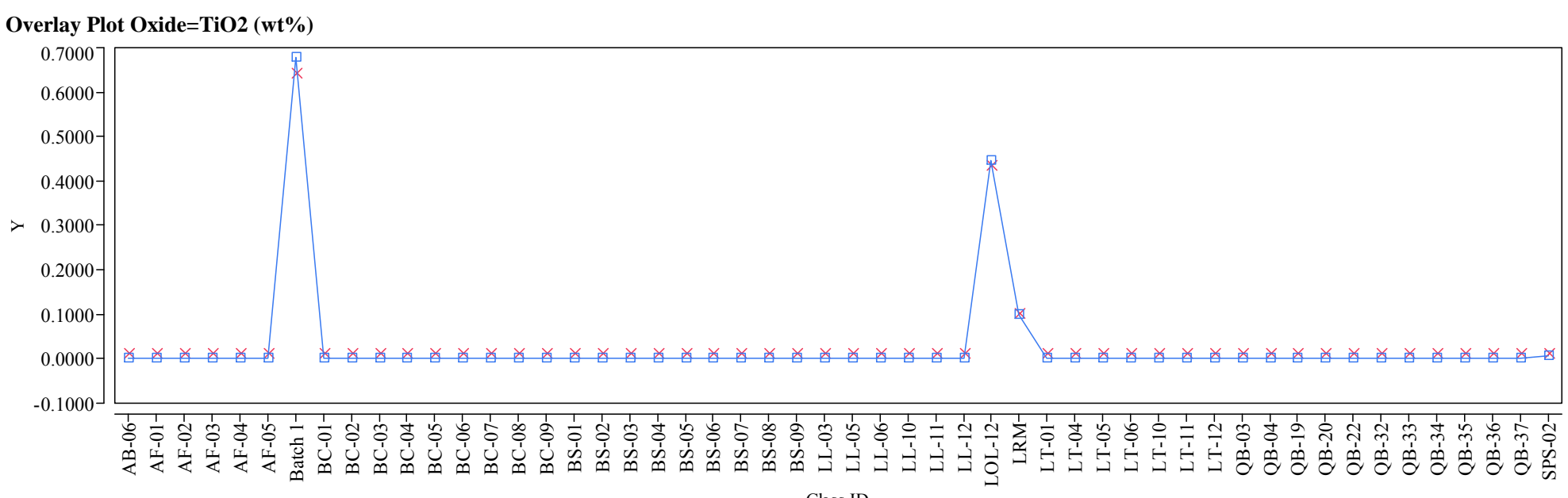

Overlay Plot Oxide=ZnO (wt \%)

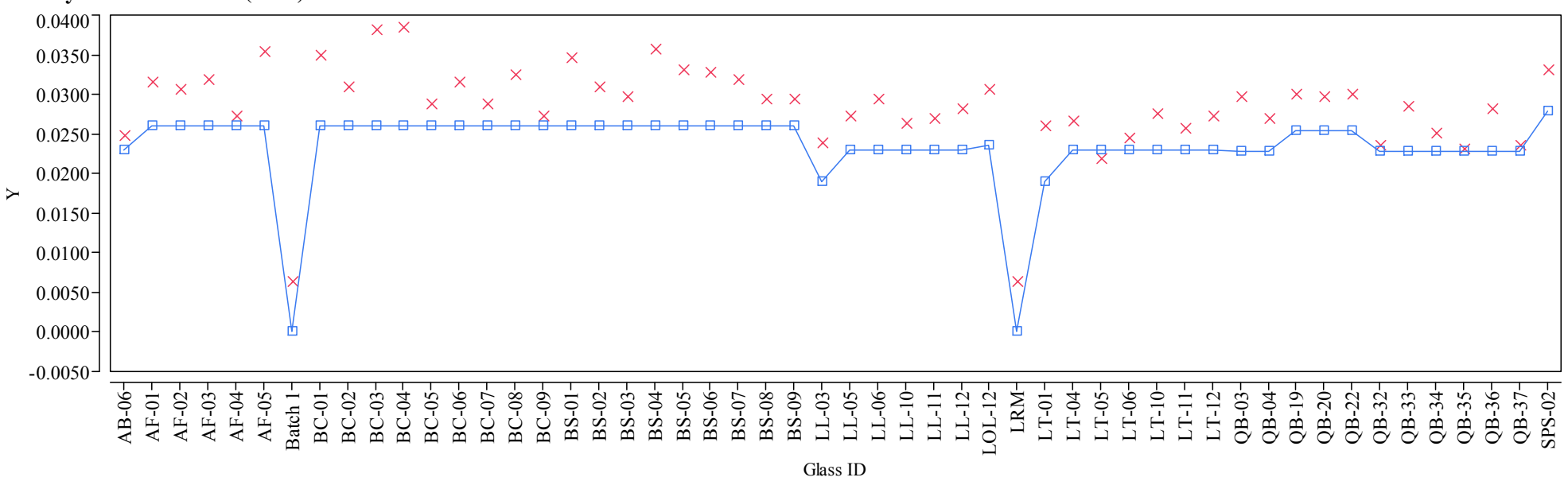

$\mathrm{Y} \times$ Measured $\square$ - Targeted 


\section{Exhibit A-4. Measured versus Targeted Compositions by Glass ID by Oxide. (continued)}

\section{Overlay Plot Oxide=ZrO2 (wt\%)}

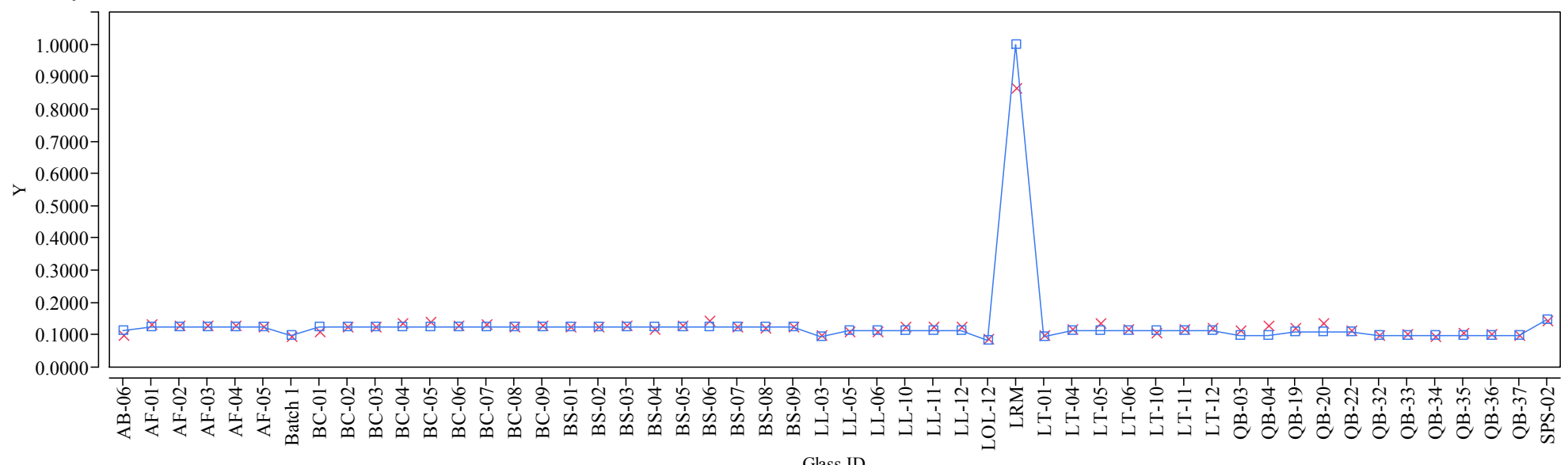

Glass ID

Overlay Plot, Sum of Oxides

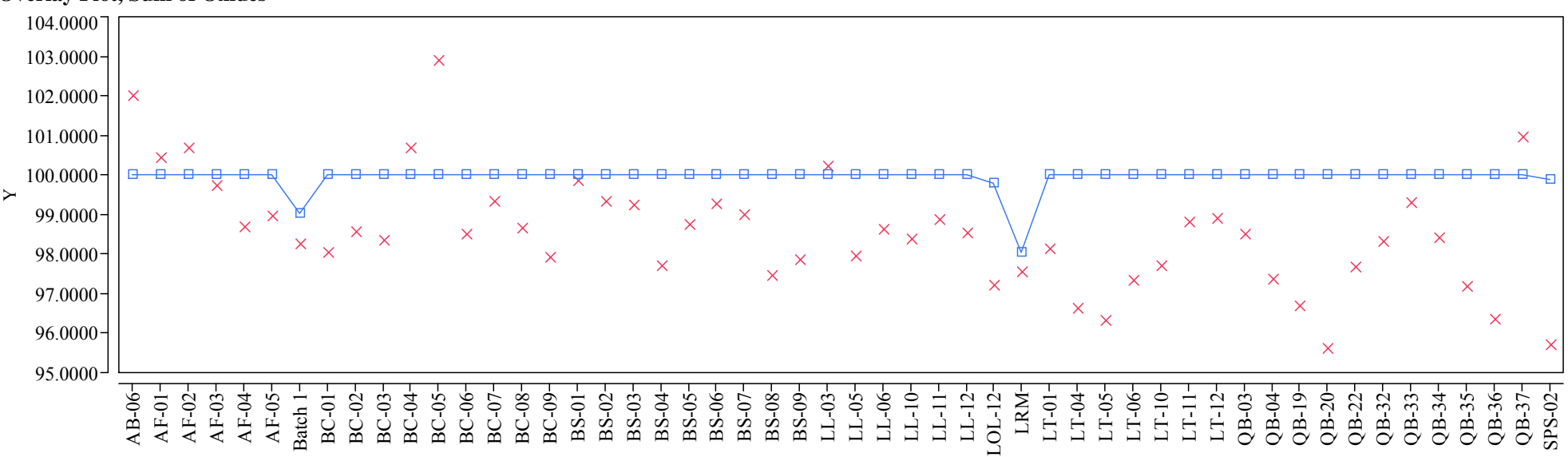

Glass ID

Y $\times$ Measured $\square$-Targeted 
SRNL-STI-2012-00152

Revision 0

Appendix B. Results from Fitting Fulcher Equations to the Viscosity Measurements for the Study Glasses 


\section{Exhibit B-1. Results of Fitting Fulcher Equations to the Measured Viscosity Data.}

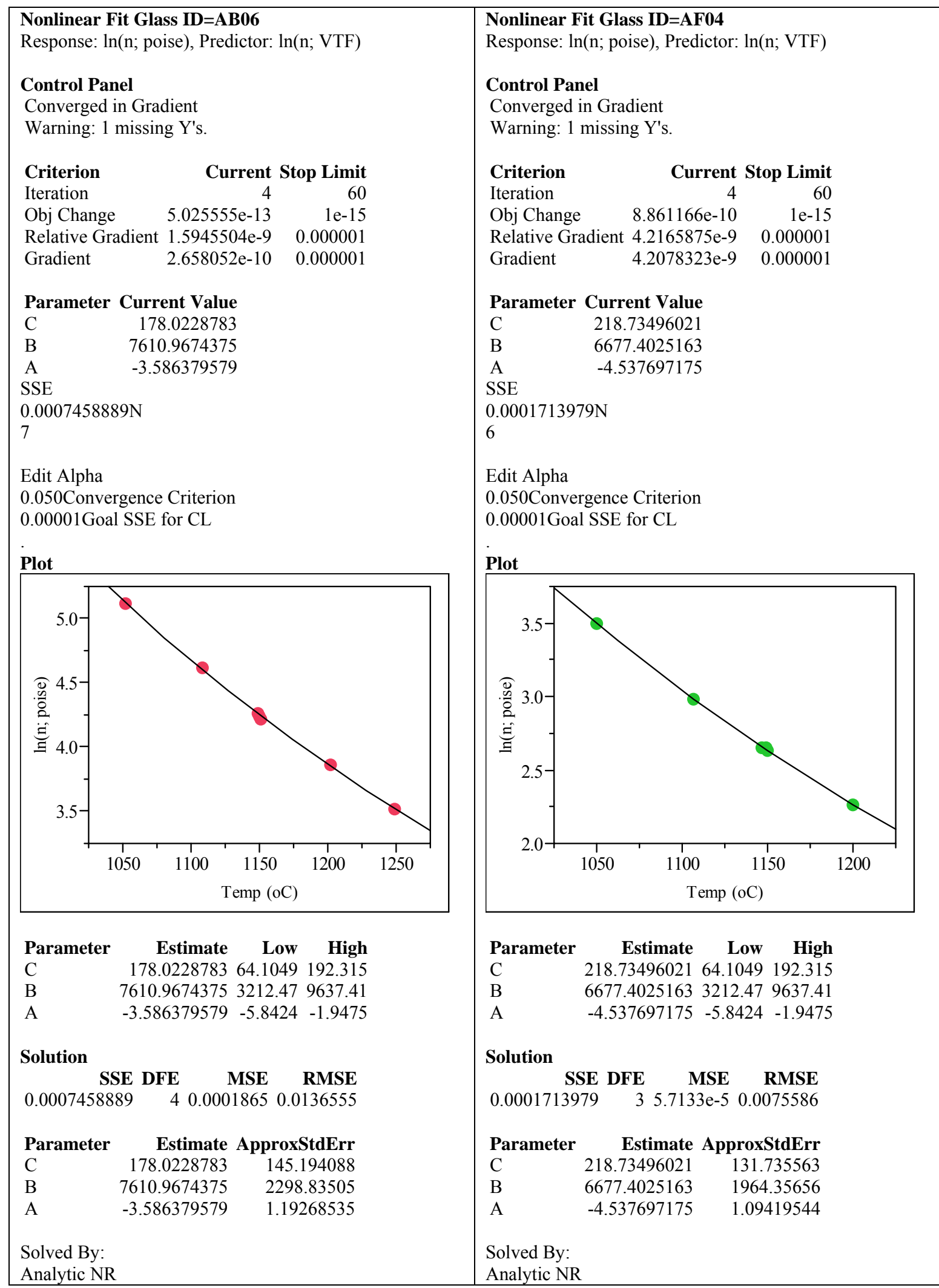


Exhibit B-1. Results of Fitting Fulcher Equations to the Measured Viscosity Data. (cont'd)

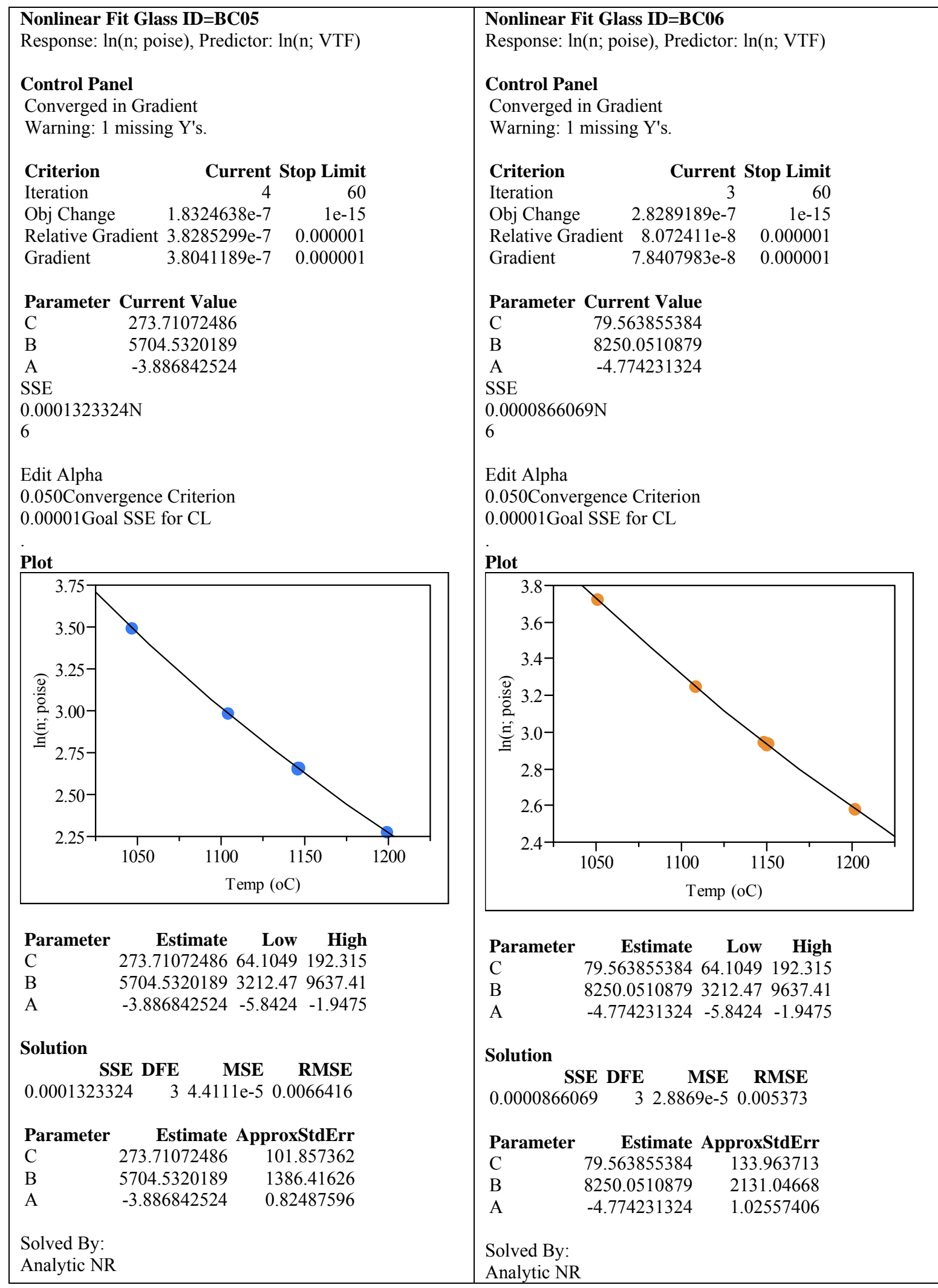


Exhibit B-1. Results of Fitting Fulcher Equations to the Measured Viscosity Data. (cont'd)

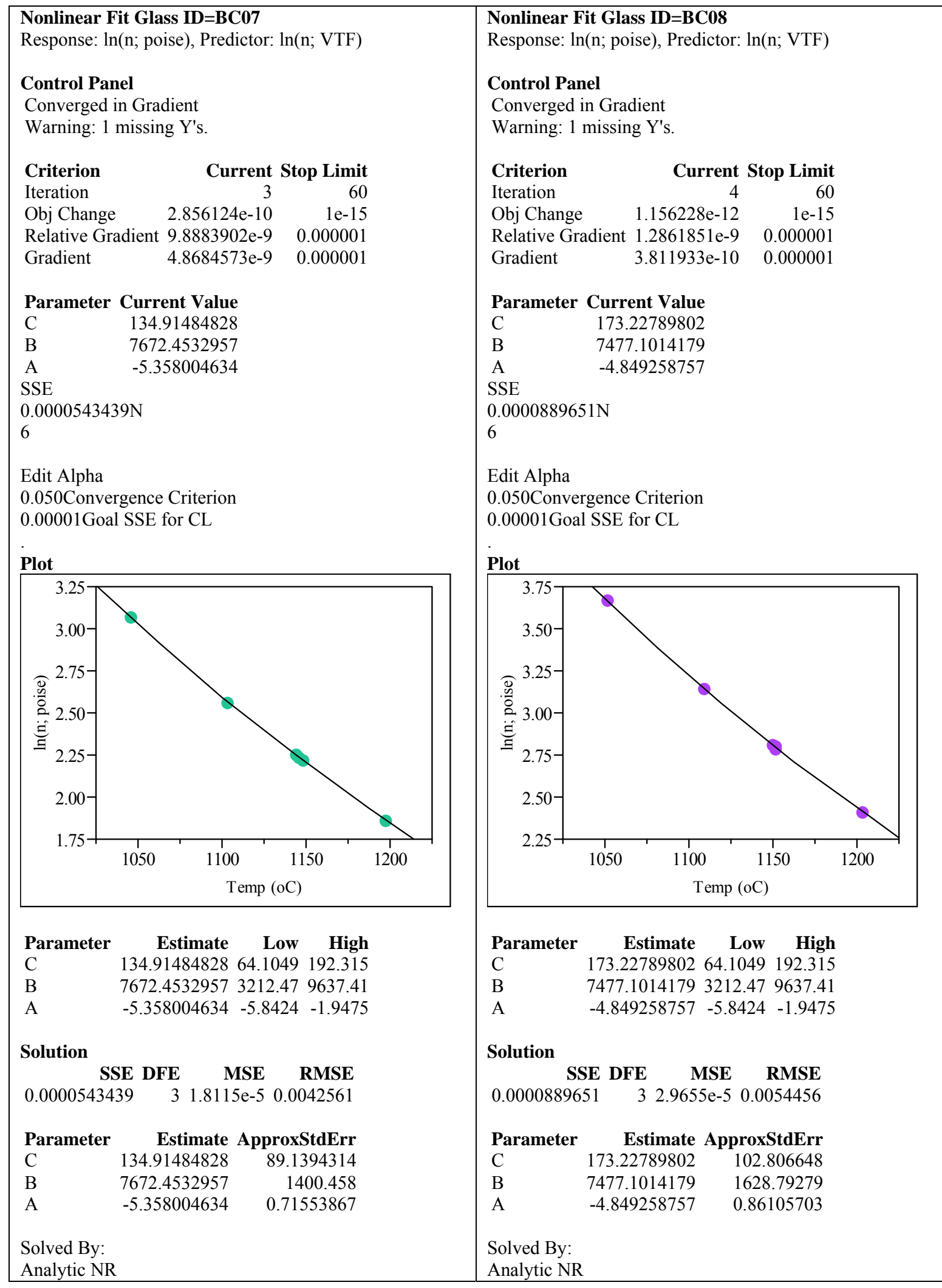


Exhibit B-1. Results of Fitting Fulcher Equations to the Measured Viscosity Data. (cont'd)

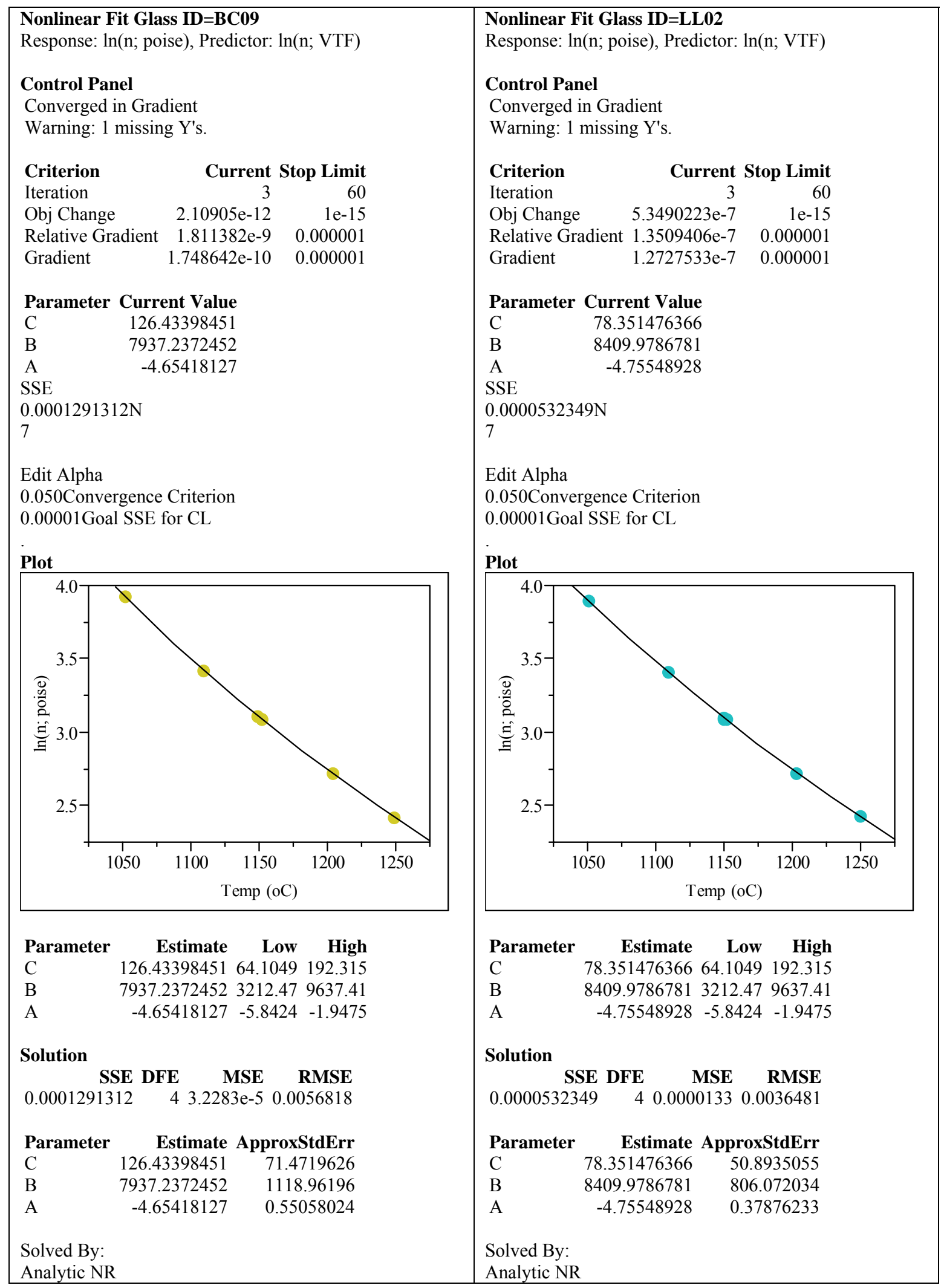


Exhibit B-1. Results of Fitting Fulcher Equations to the Measured Viscosity Data. (cont'd)

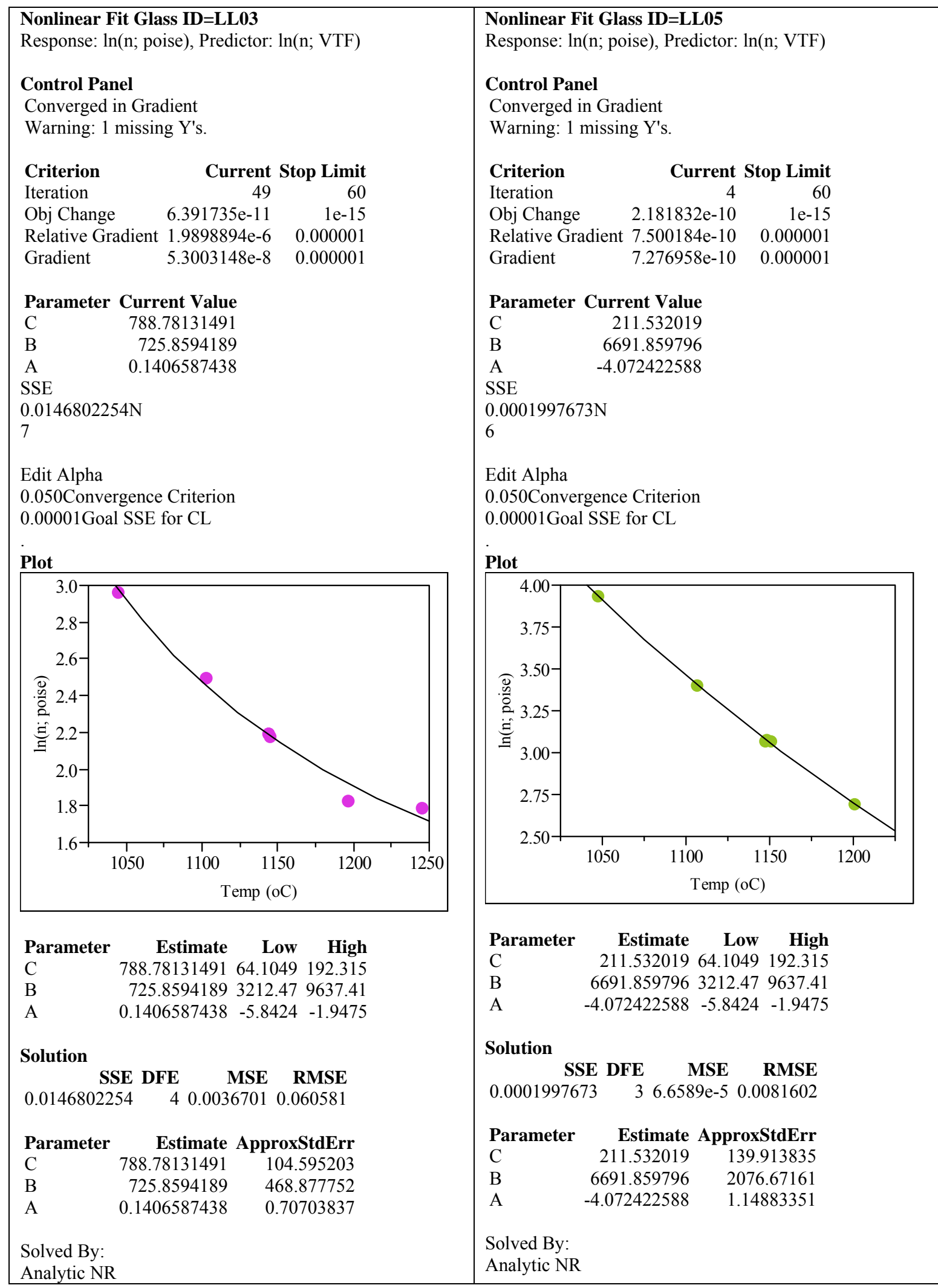


Exhibit B-1. Results of Fitting Fulcher Equations to the Measured Viscosity Data. (cont'd)

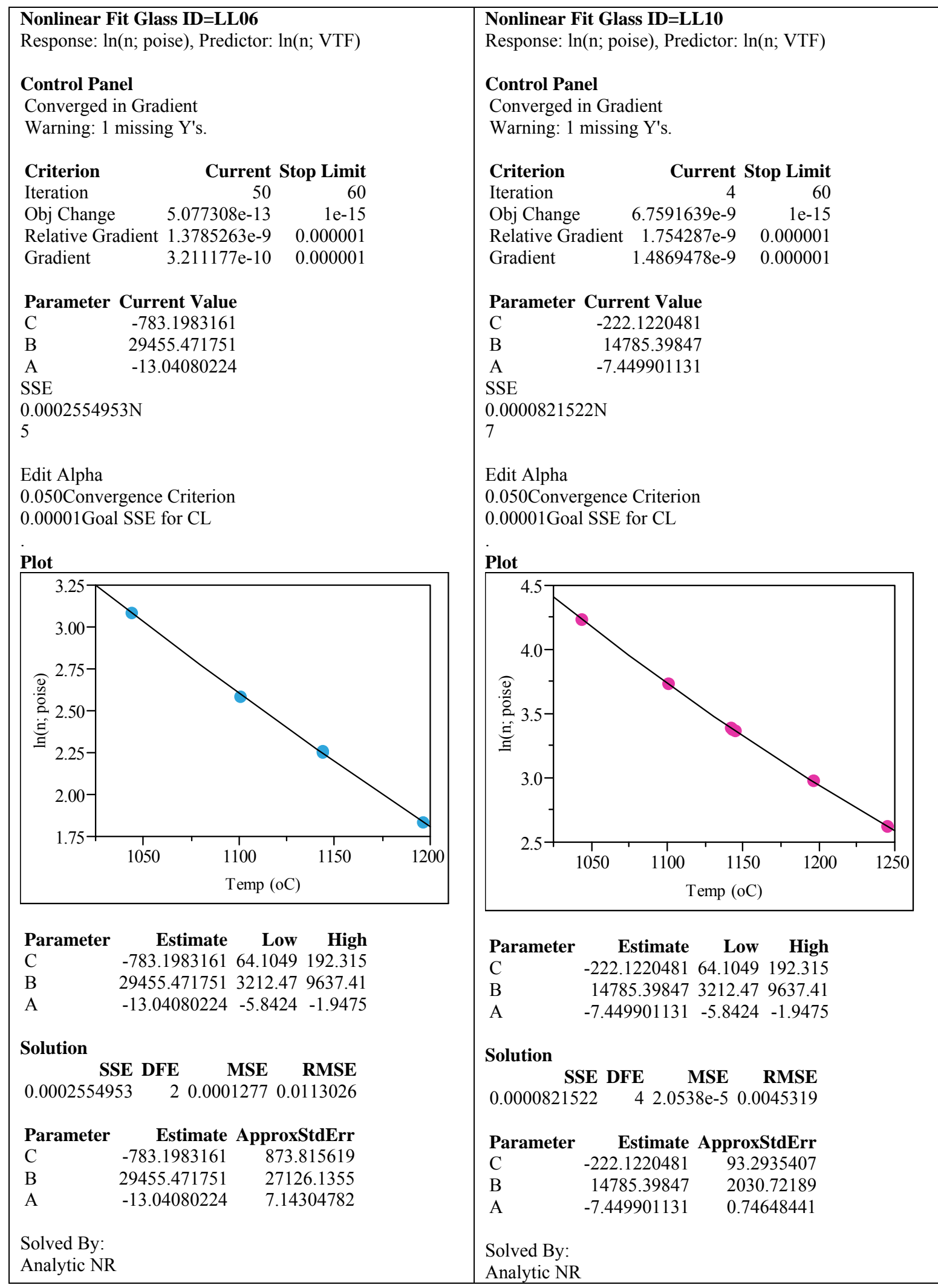


Exhibit B-1. Results of Fitting Fulcher Equations to the Measured Viscosity Data. (cont'd)

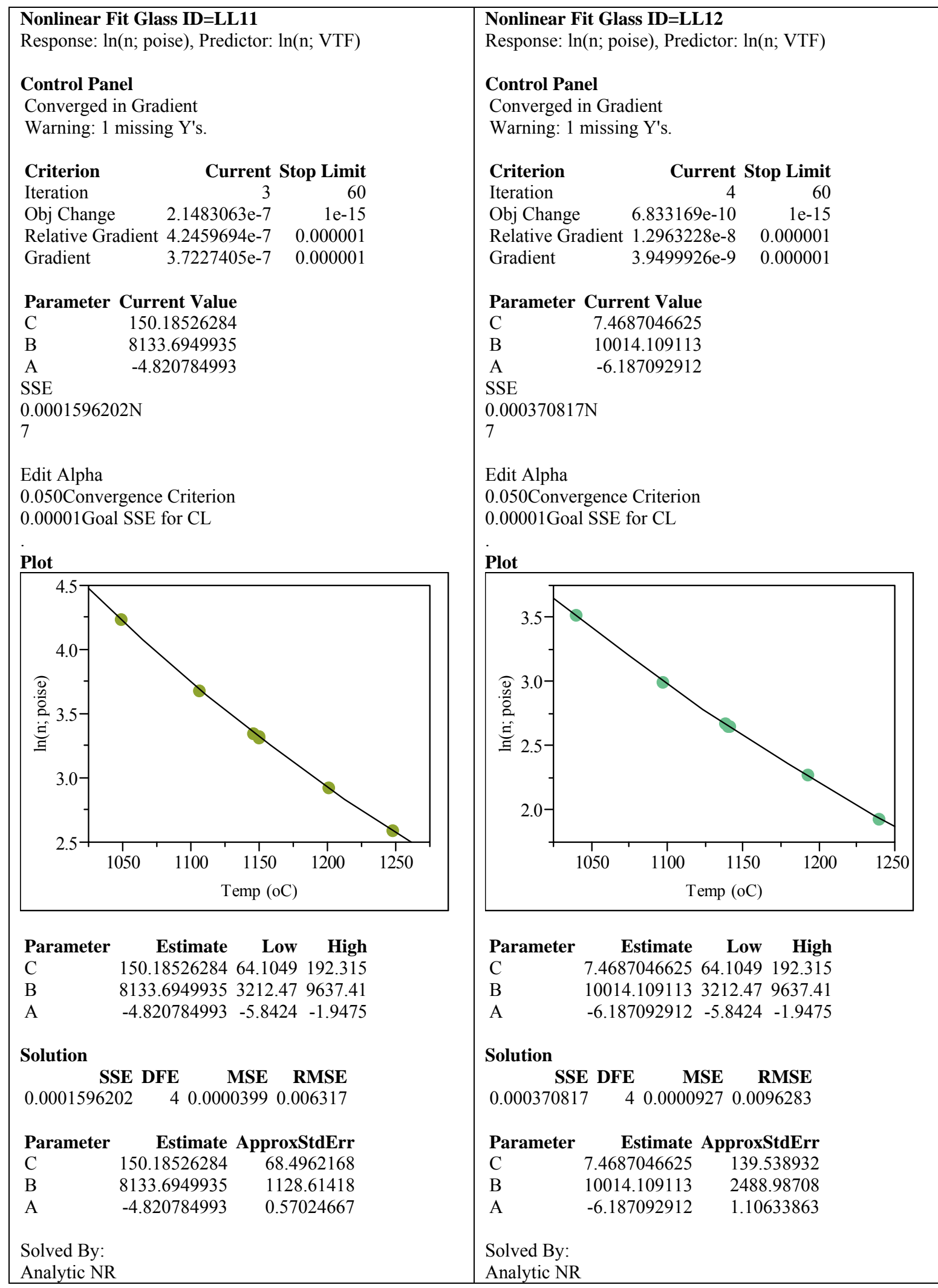


SRNL-STI-2012-00152

Revision 0

Exhibit B-1. Results of Fitting Fulcher Equations to the Measured Viscosity Data. (cont'd)

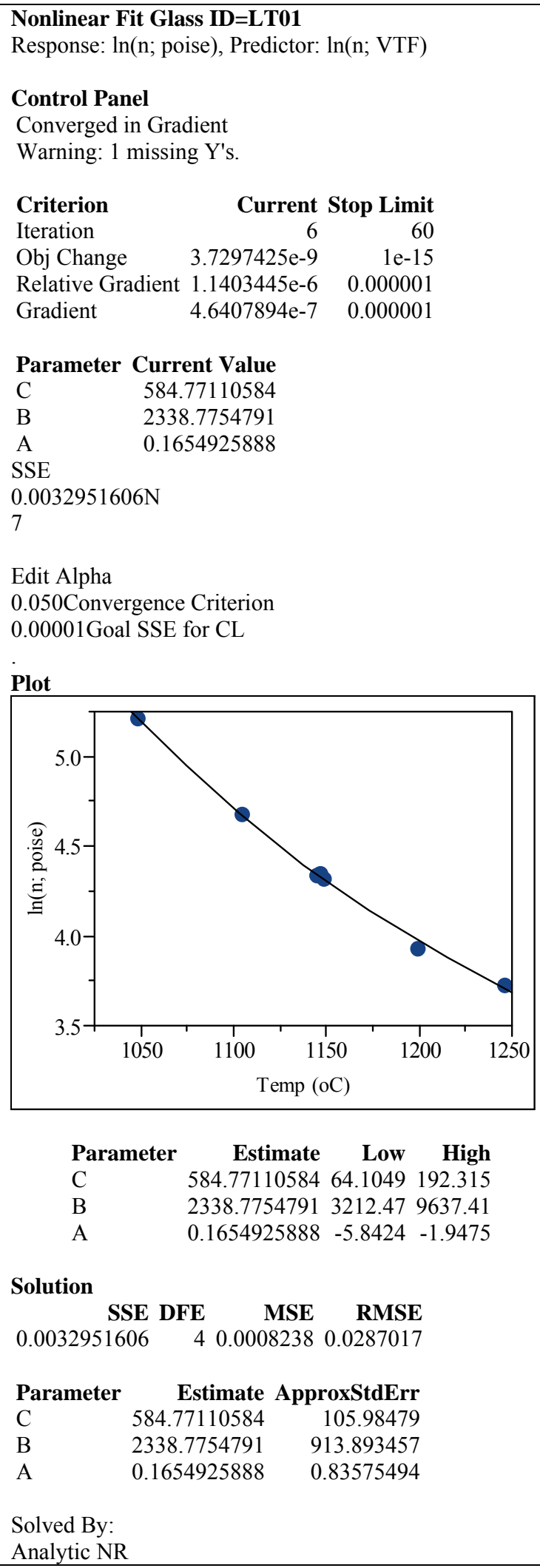


SRNL-STI-2012-00152

Revision 0

Appendix C. Final Report on the Modelling Sulfate Solubilities in US Radioactive Waste Borosilicate Glasses, Prepared by the University of Sheffield 


\section{Modelling Sulfate Solubilities in US Radioactive Waste Borosilicate Glasses}

\section{Final Project Report, Issue 01}

$22^{\text {nd }}$ December 2011

Dr. Paul A. Bingham and Dr. Russell J. Hand

Immobilisation Science Laboratory (ISL),

University of Sheffield, Dept. of Materials

Science and Engineering, Sir Robert Hadfield Building, Mappin Street, Sheffield S1 3JD, UK 


\section{Executive Summary}

The Immobilisation Science Laboratory (ISL), University of Sheffield, has developed preliminary models that can predict sulfate solubility in a given waste glass composition, based on cation field strength and other compositional relationships. A combination of these preliminary models with the extensive set of data collected at the Savannah River National Laboratory (SRNL) was undertaken with the aim of further testing and validating the model whilst providing valuable information on mechanisms of enhancing sulfate solubility in glasses of interest for US radioactive waste vitrification.

Eight $(2 \times 2 \times 2)$ different permutations of the ISL cation field strength index $(\mathrm{CFSI})$ model were utilised (retained or batched sulfate; inclusion or omission of sulfate in CFS model; and total or normalised CFSI). In addition, the concentration ratios (i) $\left[\mathrm{SiO}_{2}\right] /$ [Balance]; (ii) $\left[\mathrm{SiO}_{2}+\mathrm{B}_{2} \mathrm{O}_{3}\right] /$ [Balance]; and (iii) $\left[\mathrm{SiO}_{2}+\mathrm{B}_{2} \mathrm{O}_{3}+\mathrm{Al}_{2} \mathrm{O}_{3}\right] /$ [Balance] were plotted against retained and batched $\mathrm{SO}_{4}{ }^{2-}$ with the aim of extracting additional information from the available data. A data set containing 290 glasses was modelled.

Modelling of 290 US glasses has demonstrated that enhanced sulfate solubilities do occur at low values of CFSI, and despite considerable spread in the data there is broad support for using lower field strength glasses for the purpose of providing enhanced sulfate capacities. Application of the simplest model considered here, $\left[\mathrm{SiO}_{2}\right] /$ [Balance] in mol \%, provides the strongest support. It shows that, of the 290 glasses surveyed, yellow phase formation was not encountered in any of the 13 samples for which $\left[\mathrm{SiO}_{2}\right] /$ [Balance] < ca. 0.85. This ratio corresponds with glasses containing less than $\sim 45 \mathrm{~mol} \% \mathrm{SiO}_{2}$. Retained $\mathrm{SO}_{4}{ }^{2-}$ contents within this compositional space were consistently close to $0.75 \mathrm{~mol} \% \mathrm{SO}_{4}{ }^{2-}$ (typically $1-1.1 \mathrm{wt} \% \mathrm{SO}_{4}{ }^{2-}$ ).

It is recommended that further work should focus on providing laboratory-scale samples doped with $\mathrm{SO}_{4}{ }^{2-}$ with compositions within the region of interest (low cation field strength indices and $\mathrm{SiO}_{2}$ contents below ca. $45 \mathrm{~mol} \%$ with $\mathrm{SO}_{4}{ }^{2-}$ contents in the range $0.6-1.0 \mathrm{~mol} \%$ ). This will enable experimental validation of the conclusion of this work, namely, that higher sulfate capacities are most strongly linked, compositionally, with $\mathrm{SiO}_{2}$ content, and the additional experimental nodes will provide the necessary data for further model development and validation. 


\section{Background}

Defense nuclear wastes within the US Department of Energy (DOE) complex contain relatively high concentrations of sulfate, which has a low solubility in borosilicate glass. This dictates that the waste be blended with lower sulfate concentration waste sources or heavily washed to reduce sulfate levels prior to vitrification. High concentrations of sulfate can also impose a limit on waste loading, which in turn hinders waste throughput for a vitrification plant. It is therefore desirable to develop enhanced borosilicate glass compositions with improved sulfate solubility.

Recent studies at Savannah River National Laboratory (SRNL) in support of the Defense Waste Processing Facility (DWPF) have identified frit additives that can be used to improve sulfate solubility in simulated waste glasses. However, due to the complexity of Savannah River waste compositions, much of this work has been done on an empirical basis, making it difficult to apply the findings to future waste compositions despite the large number of glass systems studied.

The Immobilisation Science Laboratory (ISL), University of Sheffield, has developed preliminary models that can predict sulfate solubility in a given waste glass composition, based on cation field strength and other compositional relationships. A combination of these preliminary models with the extensive set of data collected at SRNL was undertaken with the aim of further testing and validating the model whilst providing valuable information on mechanisms of enhancing sulfate solubility, thus allowing for future glass compositions to be tailored for improved sulfate solubility.

ISL has experience with high sulfate containing glasses and model development of nuclear waste glass compositions containing high concentrations of sulfate. This project consisted of the further development and application by ISL of this model, specifically in order to further develop a cation field strength model for sulfate solubility in borosilicate glasses based on data collected over the course of multiple studies already conducted at SRNL. ISL also identified and established further glass composition regions for which the model is lacking.

A sulfate glass composition database was sent by SRNL to ISL, who subsequently reviewed the data and applied the previously developed cation field strength model to the SRNL glass database. Eight different permutations of the model were utilised (retained or batched 
sulfate; inclusion or omission of sulfate in CFS model; and total or normalised CFSI). In addition, the concentration ratios (i) $\left[\mathrm{SiO}_{2}\right] /$ [Balance]; (ii) $\left[\mathrm{SiO}_{2}+\mathrm{B}_{2} \mathrm{O}_{3}\right] /$ [Balance]; and (iii) $\left[\mathrm{SiO}_{2}+\right.$ $\mathrm{B}_{2} \mathrm{O}_{3}+\mathrm{Al}_{2} \mathrm{O}_{3}$ ] / [Balance] were utilised in an effort to extract the maximum information from the available data. 


\section{Experimental Methodologies}

\subsection{Data supplied}

In order to provide comparability between modelled data sets, only glasses prepared under similar or identical conditions were considered. The majority of data was provided to ISL in the Excel file "Data Summary Spreadsheet 3-1-11 for Sheffield" [3] supplied by A. Billings to P. A. Bingham on $1^{\text {st }}$ March 2011. The spreadsheet states that this data pertains to SRNL Document Number SRNL-L3100-2011-00038. Additional data was obtained from other US sources [4-6].

Glasses considered in this study were produced under similar conditions. In [3, 5 and 6] batches were 100 or 150 grams in size and were melted at $1150{ }^{\circ} \mathrm{C}$ for 1 hour in $\mathrm{Pt} / \mathrm{Au}$ crucibles with a loosely fitted lid placed over them. Glasses produced in [4] were prepared from batch sizes of 250 grams and melted at $1150{ }^{\circ} \mathrm{C}$ for 2 hours in $\mathrm{Al}_{2} \mathrm{O}_{3}$ crucibles; crucible lids were sealed with a nepheline gel. The data from the study by Peeler et al. [4] should therefore be compared carefully with the rest $[3,5,6]$ of the modelled data.

\subsection{Models used}

The cation field strength index (CFSI) predictor model [1-2] was constructed in a Microsoft Excel spreadsheet. Assumptions made in the model have been stated and supported by published data for appropriate glasses. These assumptions were appraised and approved by A. Billings and K. Fox at SRNL and are reproduced and referenced in the Appendix.

The assumptions made resulted in new values of $\left(z / a^{2}\right)$ for some oxides associated with the CFSI model. Moreover, four variants have been utilised here: Including S or Not Including S (i.e. to include the effects of sulfate in the glass on the value of the resulting CFSI); and total or normalised CFSI. Total CFSI is not normalised; it represents the total CFSI of the glass and is calculated according to (1). Normalised CFSI is the CFSI normalised to 1 mole-cation and is calculated according to (2).

$$
\text { CFSI }_{\text {Total }}=\sum_{i=1}^{n}\left(m_{i} c_{i}\left(z / a^{2}\right)_{i}\right)
$$




$$
\mathrm{CFSI}_{\text {Normalised }}=\frac{\sum_{i=1}^{n} m_{i} c_{i}\left(\frac{z}{a_{i}^{2}}\right)}{\sum_{i=1}^{n} m_{i} c_{i}}
$$

where $m_{i}=$ mol. fraction of the $i$ th oxide; $c_{i}=$ number of cations in one molecule of the $i$ th oxide; $z=$ cationic charge; and $a=$ interatomic distance in picometers. Values of $\left(z / \mathrm{a}^{2}\right)_{i}$ were calculated for the ith oxide in the glass using the constants described in the Appendix. In addition, simple models were used to calculate (mol. \%): (i) $\left[\mathrm{SiO}_{2}\right] /\left[\right.$ Balance]; (ii) $\left[\mathrm{SiO}_{2}+\mathrm{B}_{2} \mathrm{O}_{3}\right] /$ [Balance]; and (iii) $\left[\mathrm{SiO}_{2}+\mathrm{B}_{2} \mathrm{O}_{3}+\mathrm{Al}_{2} \mathrm{O}_{3}\right] /$ [Balance]. 


\section{Results and Discussion}

Modelling of the available experimental data has produced a total of 14 graphs (Figures 1-14). These are as follows:

CFSI models

1. Normalised CFSI (Sulfate Included) vs. Retained $\mathrm{SO}_{4}{ }^{2-}$ (mol \%)

1a. (Log plot) Normalised CFSI (Sulfate Included) vs. Retained $\mathrm{SO}_{4}{ }^{2-}(\mathrm{mol} \%)$

2. Normalised CFSI (Sulfate Omitted) vs. Retained $\mathrm{SO}_{4}{ }^{2-}(\mathrm{mol} \%)$

3. Total CFSI (Sulfate Included) vs. Retained $\mathrm{SO}_{4}{ }^{2-}$ (mol \%)

4. Total CFSI (Sulfate Omitted) vs. Retained $\mathrm{SO}_{4}{ }^{2-}$ (mol \%)

5. Normalised CFSI (Sulfate Included) vs. Batched $\mathrm{SO}_{4}{ }^{2-}(\mathrm{mol} \%)$

6. Normalised CFSI (Sulfate Omitted) vs. Batched $\mathrm{SO}_{4}{ }^{2-}$ (mol \%)

7. Total CFSI (Sulfate Included) vs. Batched $\mathrm{SO}_{4}{ }^{2-}(\mathrm{mol} \%)$

8. Total CFSI (Sulfate Omitted) vs. Batched $\mathrm{SO}_{4}{ }^{2-}(\mathrm{mol} \%)$

Other models

9. $\left[\mathrm{SiO}_{2}\right] /$ [Balance] (mol. \%) vs. Retained $\mathrm{SO}_{4}{ }^{2-}(\mathrm{mol} \%)$

10. $\left[\mathrm{SiO}_{2}+\mathrm{B}_{2} \mathrm{O}_{3}\right] /$ [Balance] (mol. \%) vs. Retained $\mathrm{SO}_{4}{ }^{2-}(\mathrm{mol} \%)$

11. $\left[\mathrm{SiO}_{2}+\mathrm{B}_{2} \mathrm{O}_{3}+\mathrm{Al}_{2} \mathrm{O}_{3}\right] /$ [Balance] (mol. \%) vs. Retained $\mathrm{SO}_{4}{ }^{2-}$ (mol \%)

12. $\left[\mathrm{SiO}_{2}\right] /$ [Balance] (mol. \%) vs. Batched $\mathrm{SO}_{4}{ }^{2-}(\mathrm{mol} \%)$

13. $\left[\mathrm{SiO}_{2}+\mathrm{B}_{2} \mathrm{O}_{3}\right] /$ [Balance] (mol. \%) vs. Batched $\mathrm{SO}_{4}{ }^{2-}(\mathrm{mol} \%)$

14. $\left[\mathrm{SiO}_{2}+\mathrm{B}_{2} \mathrm{O}_{3}+\mathrm{Al}_{2} \mathrm{O}_{3}\right] /$ [Balance] (mol. \%) vs. Batched $\mathrm{SO}_{4}{ }^{2-}(\mathrm{mol} \%)$

\subsection{Cation Field Strength Index Modelling}

As shown in Figures 1-8 the first comment on the results of this modelling work is that no well-defined and clear trend linking CFSI and sulfate solubility in the glasses has yet been established. However, careful visual analysis of the plots indicates a diffuse line dividing a region with higher probability of yellow sulfate phase formation and a region with a lower probability. This has been illustrated in one plot (Figure 1) for guidance purposes only. Similar lines can be drawn on all other plots. The region with lower probability corresponds to low CFSI values. This is broadly consistent with ISL's model for the effects of composition with sulfate solubility in glasses [1, 2]. As noted previously, data taken from Peeler et al. [4] describes samples produced 
under slightly different conditions to the other samples $[3,5,6]$ and therefore must be considered with care. Despite this, and were these data from Peeler et al. [4] omitted, still the resulting relationship would not be well-defined. It is worth noting that the compositions of all studied glasses actually fall within a relatively narrow range, and the observed results may be due, at least in part, to natural scatter within a tightly-controlled compositional region. Furthermore, the relationship identified in [1,2] identifies a linear relationship between the logarithm of sulfate solubility with CFSI, rather than linear sulfate solubility. If plotted on a log basis, it could be argued that the observed regions shown by the dotted line in Figure 1 become somewhat more well-defined (as shown in Figure 1a). However, given the relatively small differences in both composition and sulfate solubility (i.e. not order of magnitude difference as shown in $[1,2]$, it was felt that a linear scale was more appropriate. Indeed, as we have shown previously in [1], plotting sulfate solubility on a linear scale is sufficient to illustrate a nearly linear relationship when considering sulfate solubility differences within the same order of magnitude.

The small number of "oversaturated" melts - melts for which the sulfate solubility limit had been reached but either not exceeded or only marginally exceeded (i.e. not sufficiently to produce the yellow phase), and illustrated by white markers in all Figures, also indicate that low CFSI values favour higher sulfate capacities.

It must be concluded that, whilst broadly applicable to sulfate solubility in oxide glasses, the CFSI model does not provide a sufficiently descriptive model for sulfate capacity in US radioactive waste borosilicate glasses with a high degree of accuracy over a relatively narrow range of compositions and sulfate solubilities. This indicates that other mechanisms, which are not fully represented by cation field strength, are also at work. One possibility may be that the cation field strength scale does not directly model the interconnectedness, or degree of polymerisation, of the glass network, in this case the (boro)silicate network. It was therefore believed that some models which could more accurately represent this structural arrangement may provide clearer results. For this reason the modelling described in Section 3.2 was conducted. 


\subsection{Modelling using simple compositional models}

In addition to CFSI modelling a number of simple measures were also considered in an effort to extract further information from the data. This has been successful, inasmuch as there was clearer identification of glass compositional regions with higher sulfate solubilities. As shown in Figures 9 and 12, the simple measure of the molar content of $\mathrm{SiO}_{2}$ divided by the sum of the concentrations of all other constituents, $\left[\mathrm{SiO}_{2}\right]$ / [Balance] shows that the glasses lowest in $\mathrm{SiO}_{2}$ do not exhibit yellow phase at the levels of sulfate addition included in the data. This is shown at $\left[\mathrm{SiO}_{2}\right] /[$ Balance $]<0.85$, corresponding with approximately $<45 \mathrm{~mol} . \% \mathrm{SiO}_{2}$. This is the most convincing evidence resulting from the current study, that glasses lower in $\mathrm{SiO}_{2}$, and therefore more depolymerised with (expected) lower average values of Si Q-species (the number of bridging oxygens per $\mathrm{Si}^{4+}$ ). Literature shows that a full and detailed understanding of the structural origins of the observed behaviour has yet to be developed. It is known that $\mathrm{SO}_{4}{ }^{2-}$ environments in alkali borosilicate radioactive waste glasses are associated with network modifying cations such as $\mathrm{Na}^{+}[7,8]$ but with little or no clear link to the nature and structure of the glass-forming network. Backnaes and Deubener [9] have recently reviewed the literature on sulfur solubility as a function of melt composition, noting work by Ducea et al. [10], which has shown an approximately linear relationship, albeit with some spread, between $\mathrm{SiO}_{2}$ content and sulfur solubility of a range of magmas (data which they compiled from other literature) showing a wide range of $\mathrm{SiO}_{2}$ contents. This behaviour is consistent with the behaviour which we have observed in the US borosilicate glasses (Figures 9 and 12). We also note that the "cutoff" in yellow phase formation which we have observed in our data below ca. $45 \mathrm{~mol} \% \mathrm{SiO}_{2}$ broadly corresponds with rapidly increasing levels of (with decreasing $\mathrm{SiO}_{2}$ content) of "free" $\mathrm{O}^{2-}$ ions, i.e. oxygens not directly bonded to $\mathrm{Si}^{4+}[11,12]$ and substantial changes in $\mathrm{BO} / \mathrm{NBO}$ ratio and $\mathrm{Si} \mathrm{Q}-$ speciation with only small further decreases in $\mathrm{SiO}_{2}$ content [11-14].

We note that the inclusion of $\mathrm{B}_{2} \mathrm{O}_{3}$ (as the other network-forming species) and $\mathrm{Al}_{2} \mathrm{O}_{3}$ (which is abundant and primarily tetrahedrally coordinated in alkali borosilicate glasses) in the numerator of the simple models, do not further improve the observed trend in the data, as shown in Figures 10, 11, 13 and 14. However, they do not greatly detract from it either, indicating some involvement of $\mathrm{B}_{2} \mathrm{O}_{3}$ and $\mathrm{Al}_{2} \mathrm{O}_{3}$ in the determination of sulfate solubilities, consistent with the cation field strength approach. A French study [15] showed that variation in the ratio $\mathrm{R}=\left[\mathrm{Na}_{2} \mathrm{O}\right]$ / $\left[\mathrm{B}_{2} \mathrm{O}_{3}\right]$ strongly affected sulfate solubility; however, this is expected on the basis of the cation field strength approach, on moving through compositional space from what is essentially a $\mathrm{SiO}_{2}$ $\mathrm{B}_{2} \mathrm{O}_{3}$ glass towards what is essentially a $\mathrm{SiO}_{2}-\mathrm{Na}_{2} \mathrm{O}$ glass. Indian research also supports the view that $\mathrm{SiO}_{2}$ content has a strong deterministic effect on sulphate solubilities. Indian barium 
borosilicate glasses, developed specifically as hosts for high-sulfate wastes [16], were shown to incorporate up to $3 \mathrm{~mol}$. \% (added) $\mathrm{SO}_{4}{ }^{2-}$ before the onset of molten salt layer formation during melting. It is helpful to note here that these Indian glasses exhibit CFSI values which are considerably higher than would be expected if CFSI was the overriding determining factor in sulphate solubility in these glasses. This is largely due to the high $\left(\mathrm{SiO}_{2}+\mathrm{B}_{2} \mathrm{O}_{3}\right)$ content. However, considering $\mathrm{SiO}_{2}$ content alone - in this case $40 \mathrm{~mol}$. \%, would give a $\left[\mathrm{SiO}_{2}\right] /$ [Balance] ratio of 0.6667 , which is consistent with the data modelled here, as shown in Figure 12.

\section{Recommendations for further work}

Generation of additional data points, particularly at $\mathrm{SiO}_{2}$ contents corresponding with $\left[\mathrm{SiO}_{2}\right] /$ [Balance] < ca. 0.85 (ca. $45 \mathrm{~mol}$. \% and below), with associated $\mathrm{SO}_{4}{ }^{2-}$ additions of $0.6-$ $1.0 \mathrm{~mol}$ \%, are required to (i) fully confirm and (ii) define and expand upon, the observed reliance of sulfate solubility on $\mathrm{SiO}_{2}$ content in the US alkali borosilicate glass systems under consideration here. This would lead to the use of this model to help formulate new glass compositions with enhanced capacities for sulfate. Clearly any such formulations would also need to fulfil all of the other property criteria (durability, processing behaviour, mechanical properties, etc), and these would also need to be considered in the context of the type and level of waste loading to be undertaken. Ultimately the goal would be to develop new glass frits which provide all of the benefits of existing frits but with enhanced sulfate capacities in the resulting vitrified wastes. 
Report checked and signed, 22 ${ }^{\text {nd }}$ December 2011:

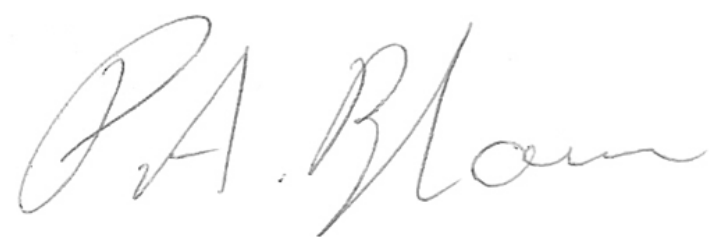

Dr. Paul A. Bingham

Immobilisation Science Laboratory

Dept. of Engineering Materials

University of Sheffield
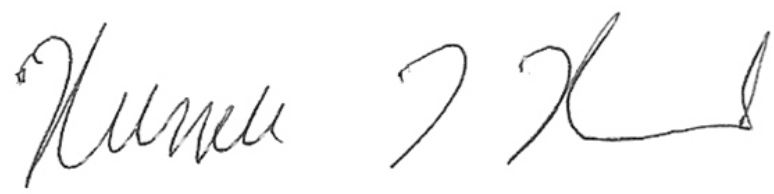

Dr. Russell J. Hand

Immobilisation Science Laboratory

Dept. of Engineering Materials

University of Sheffield 


\section{References and Data Sources}

[1] P. A. Bingham and R. J. Hand, Materials Research Bulletin 43 (2008) 1679-1693.

[2] P. A. Bingham, A. J. Connelly, R. J. Hand, N. C. Hyatt and P. A. Northrup, Glass Technol.: Eur. J. Glass Sci. Technol. A 50 (2009) 135-138.

[3] "Data Summary Spreadsheet 3-1-11 for Sheffield.xls" supplied by A. Billings (SRNL) to P. A. Bingham and R. J. Hand (ISL) by email on $1^{\text {st }}$ March 2011. Data contained within pertains to SRNL Document Number SRNL-L3100-2011-00038

[4] D. K. Peeler, C. C. Herman, M. E. Smith, T. H. Lorier, D. R. Best, T. B. Edwards and M. A. Baich, An assessment of the sulfur solubility limit for the frit 418 - sludge batch $2 / 3$ system, US DoE Report WSRC-TR-2004-00081), February 2004.

[5] A. L. Youchak-Billings, Inter-Office Memorandum: A scoping study examining the possibility of a frit additive to increase sulfur solubility in DWPF Glass, US DoE Document Number SRNL-PSE-2008-00173, August 21, 2008.

[6] A. L. Billings and K. Fox, Sulfate solubility limit verification for DWPF sludge batch 7b, US DoE Report SRNL-STI-2011-00482, September 2011. Data contained with provided by K. Fox (SRNL) to P. A. Bingham and R. J. Hand (ISL) as the file "Fox Version Sulfur Workup (no links).xls" on $28^{\text {th }}$ October 2011.

[7] D. A. McKeown, I. S. Muller, H. Gan, I. L. Pegg and C. A. Kendziora, J. Non-Cryst. Solids 288 (2001) 191-199.

[8] B. Brendebach, M. A. Denecke, G. Roth and S. Weisenburger, J. Phys: Conf. Series 190 (2009) 012186

[9] L. Backnaes and J. Deubener, Rev. Mineral. Geochem. 73 (2011) 143-165.

[10] M. N. Ducea, B. I. A. McInnes and P. J. Wyllie, Int. Geol. Rev. 36 (1994) 703-714.

[11] G. W. Toop and C. S. Samis, Trans. Metall. Soc. AIME 224 (1962) 878-887.

[12] S. Holmquist, J. Am. Ceram. Soc. 49 (1966) 467-473.

[13] J. E. Shelby, An Introduction to Glass Science and Technology, Royal Society of Chemistry Publ., 2005, Cambridge, UK.

[14] H. Maekawa, T. Maekawa, K, Kawamura and T. Yokokawa, J. Non-Cryst. Solids 127 (1991) 53-64.

[15] D. Manara, A. Grandjean, O. Pinet, J. L. Dussossoy and D. R. Neuville, J. Non-Cryst. Solids 353 (2007) 12-23.

[16] R. K. Mishra, K. V. Sudarsan, P. Sengupta, R. K. Vatsa, A. K. Tyagi, C. P. Kaushik, D. Das, and K. Raj, J. Amer. Ceram. Soc. 91 (2008) 3903-3907. 
Figure 1. Retained $\mathrm{SO}_{4}{ }^{2-}$ (mol.\%) as $f$ (Normalised Cation Field Strength Index, Sulfate Included). Data from [3]; additional data from Peeler et al 2004 [4] and Billings \& Fox 2011 [6]. Dotted line indicates regions of lower / higher probability of yellow phase formation - NB visual guide only

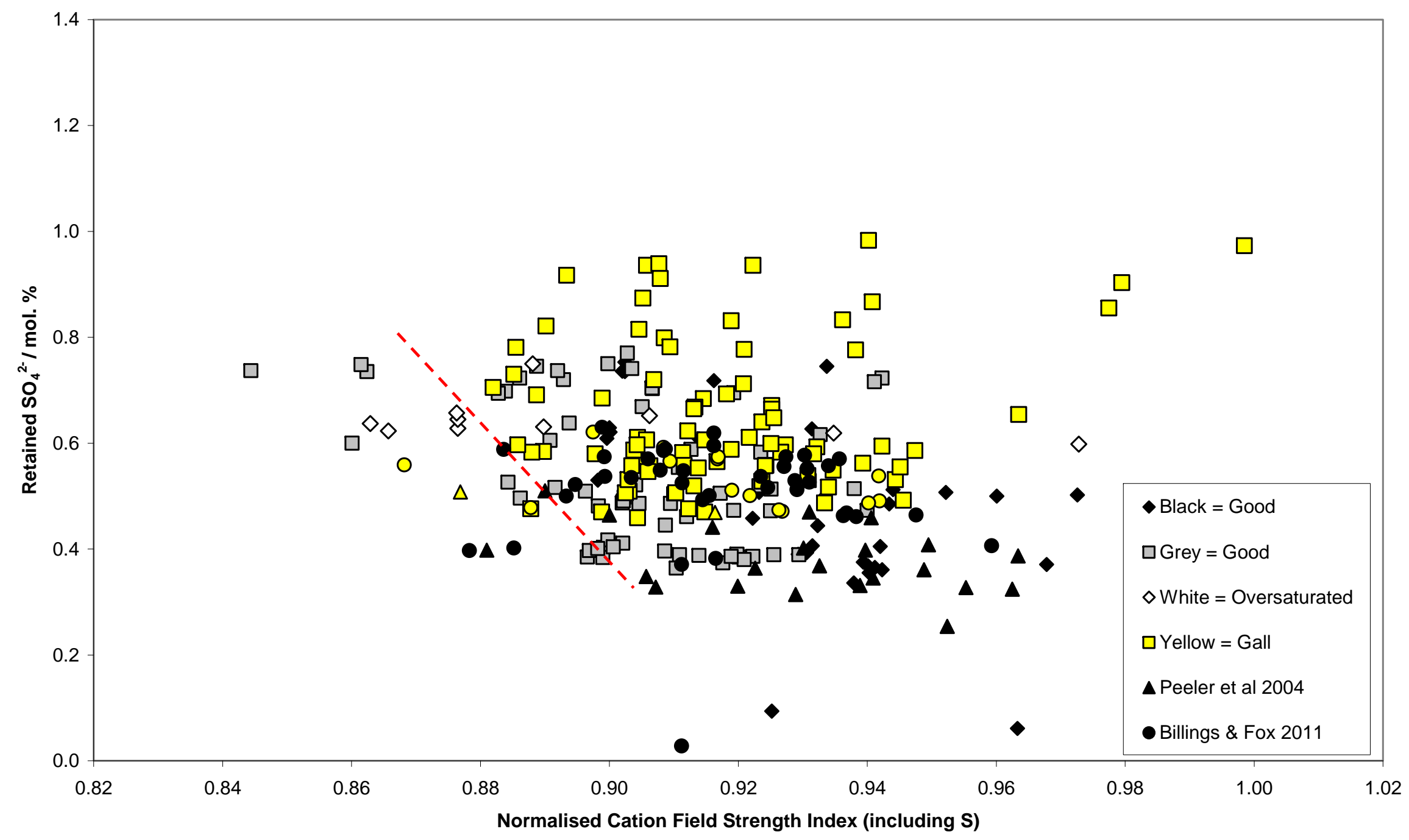


Figure 1a. Retained $\mathrm{SO}_{4}{ }^{2-}$ (mol.\%) as $f$ (Normalised Cation Field Strength Index, Sulfate Included), logarithmic plot. Data from [3]; additional data from Peeler et al 2004 [4] and Billings \& Fox 2011 [6]. Dotted line indicates regions of lower / higher probability of yellow phase, visual guide only

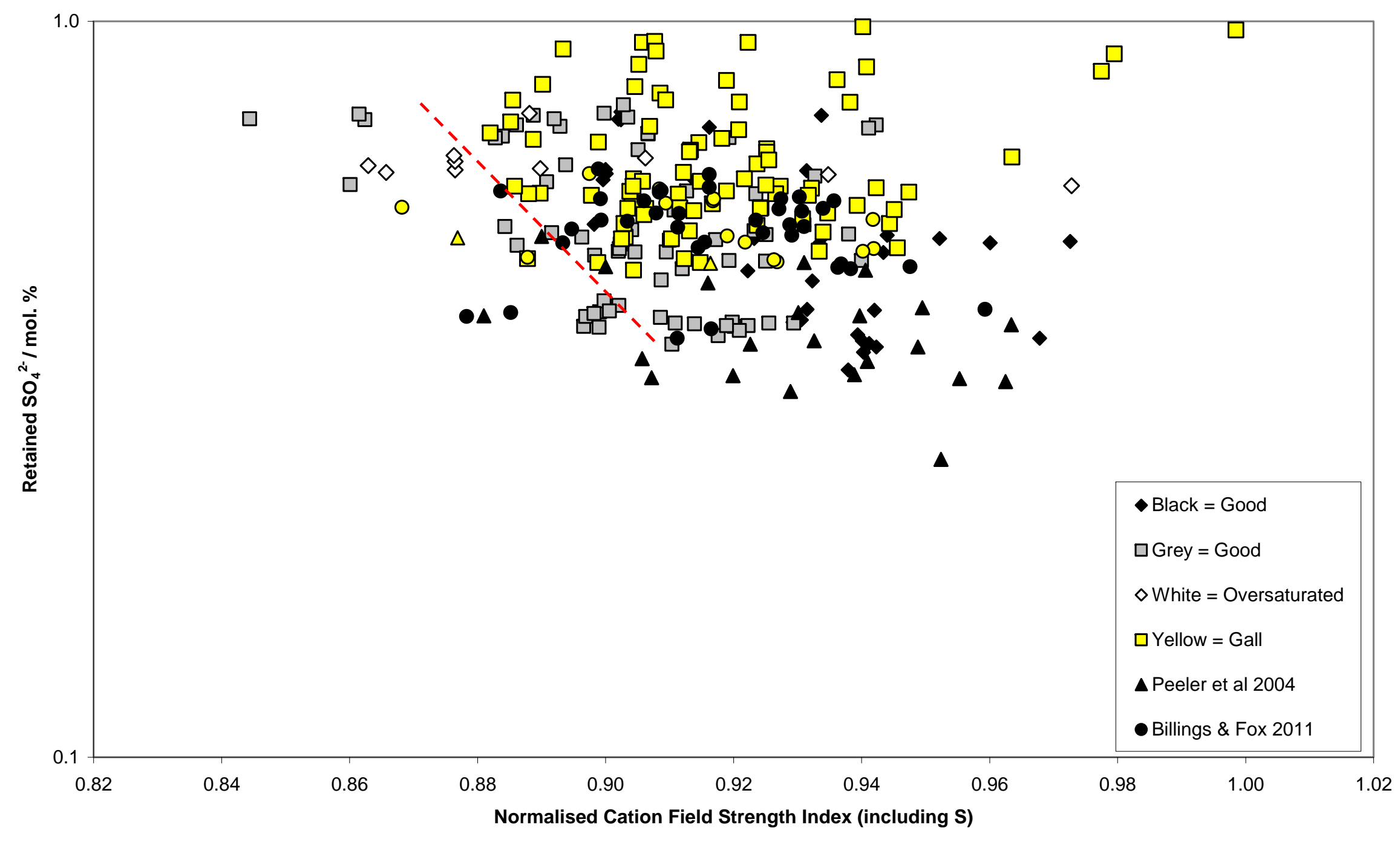


Figure 2. Retained $\mathrm{SO}_{4}{ }^{2-}$ (mol.\%) as $f$ (Normalised Cation Field Strength Index, Sulfate Omitted). Data from [3]; additional data from Peeler et al 2004 [4] and Billings \& Fox 2011 [6]

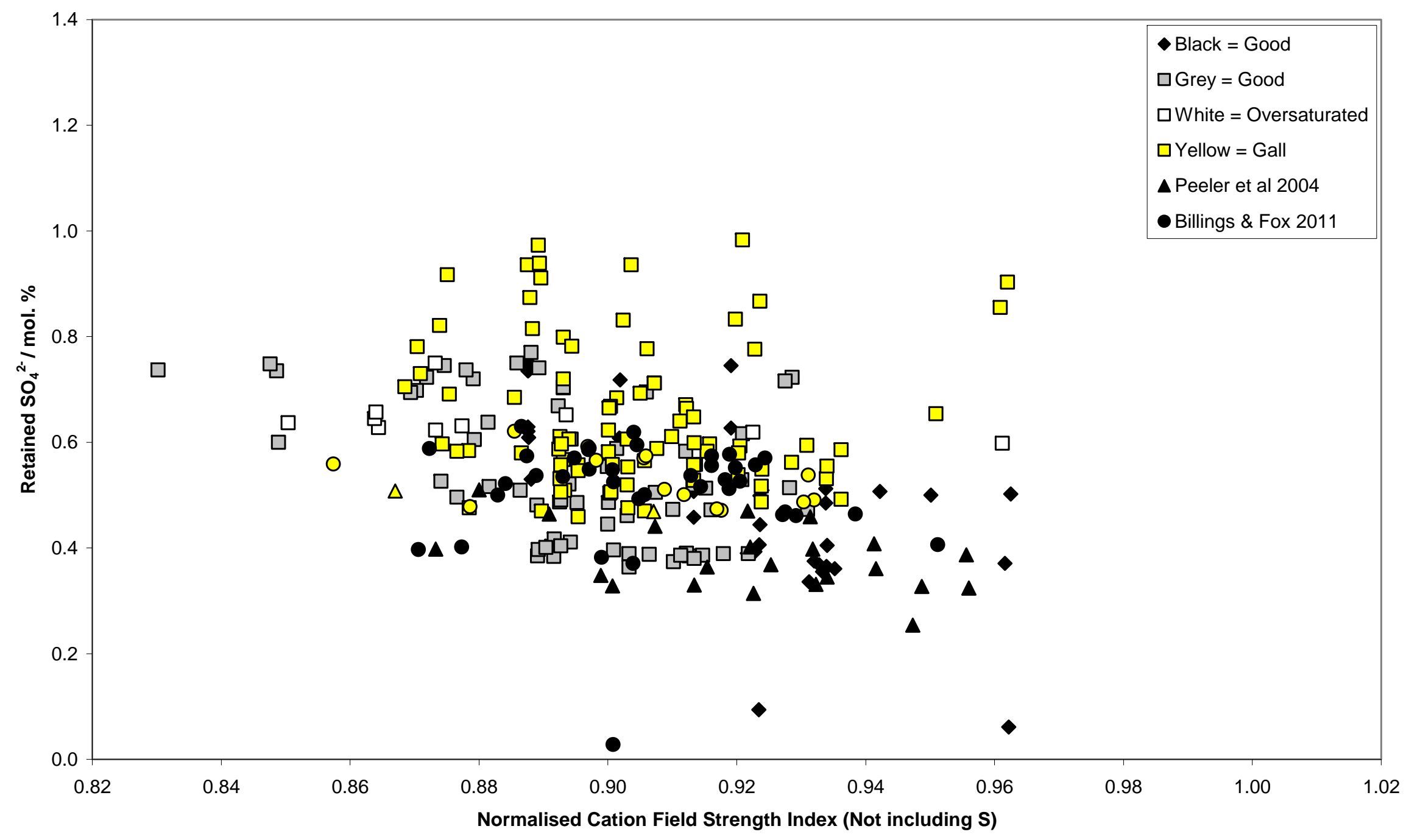


Figure 3. Retained $\mathrm{SO}_{4}{ }^{2-}$ (mol.\%) as $f$ (Total Cation Field Strength Index, Sulfate Included). Data from [3]; additional data from Peeler et al 2004 [4] and Billings \& Fox 2011 [6]

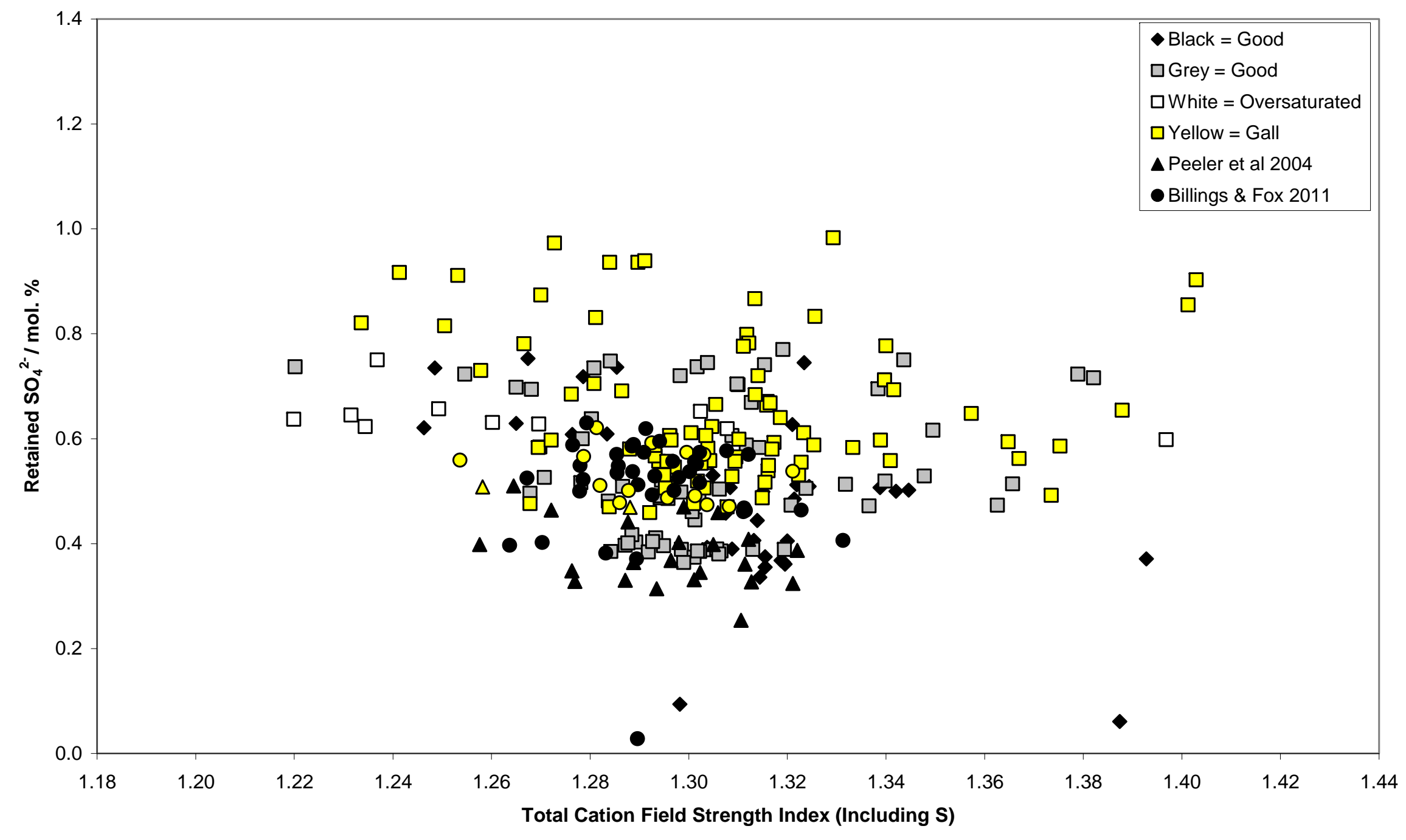


Figure 4. Retained $\mathrm{SO}_{4}{ }^{2-}$ (mol.\%) as $f$ (Total Cation Field Strength Index, Sulfate Omitted). Data from [3]; additional data from Peeler et al 2004 [4] and Billings \& Fox 2011 [6]

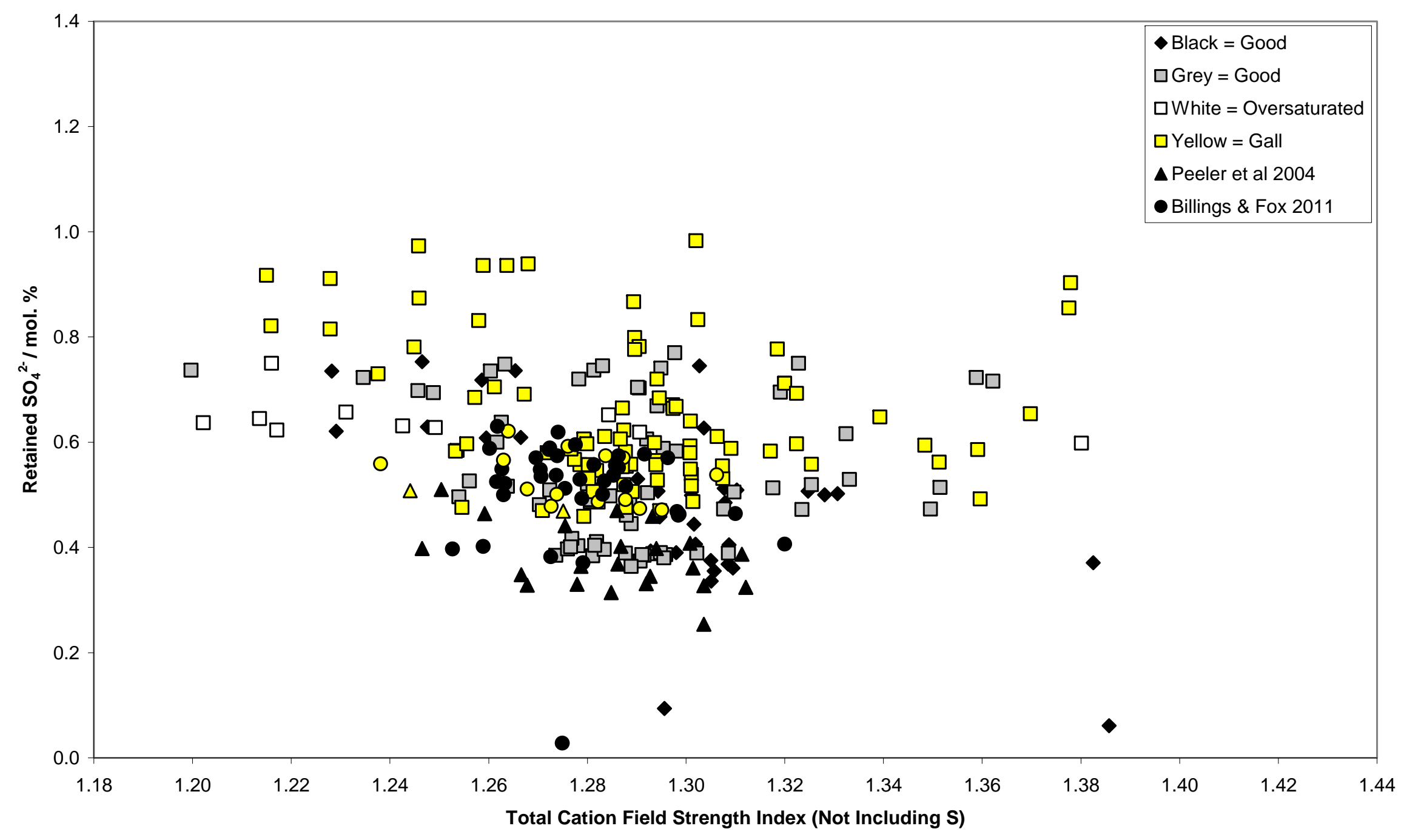


Figure 5. Added $\mathrm{SO}_{4}{ }^{2-}$ (mol.\%) as $f$ (Normalised Cation Field Strength Index, Sulfate Included). Data from [3]; additional data from Peeler et al 2004 [4], YouchakBillings 2008 [5] and Billings \& Fox 2011 [6]

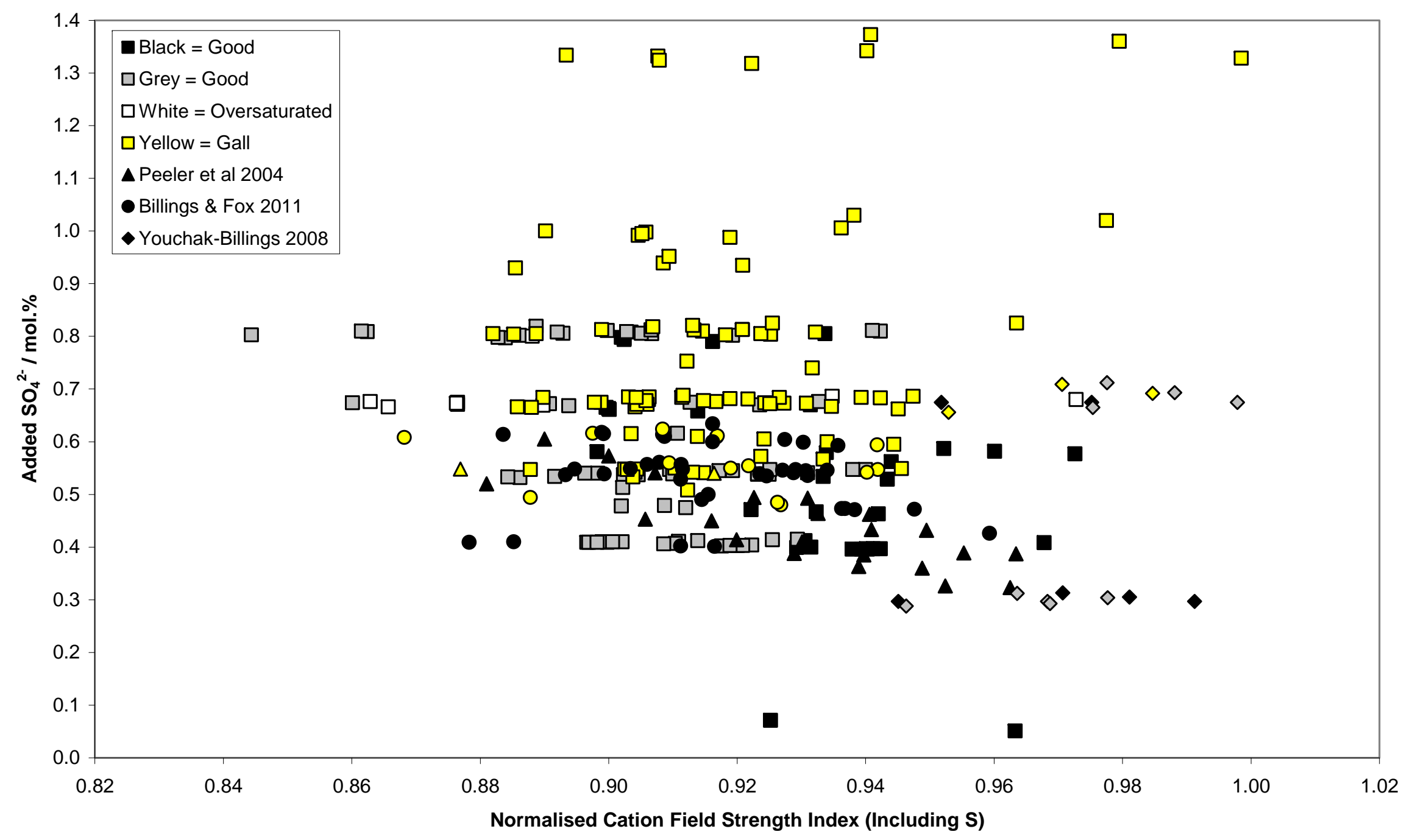


Figure 6. Added $\mathrm{SO}_{4}{ }^{2-}$ (mol.\%) as $f$ (Normalised Cation Field Strength Index, Sulfate Omitted). Data from [3]; additional data from Peeler et al 2004 [4], YouchakBillings 2008 [5] and Billings \& Fox 2011 [6]

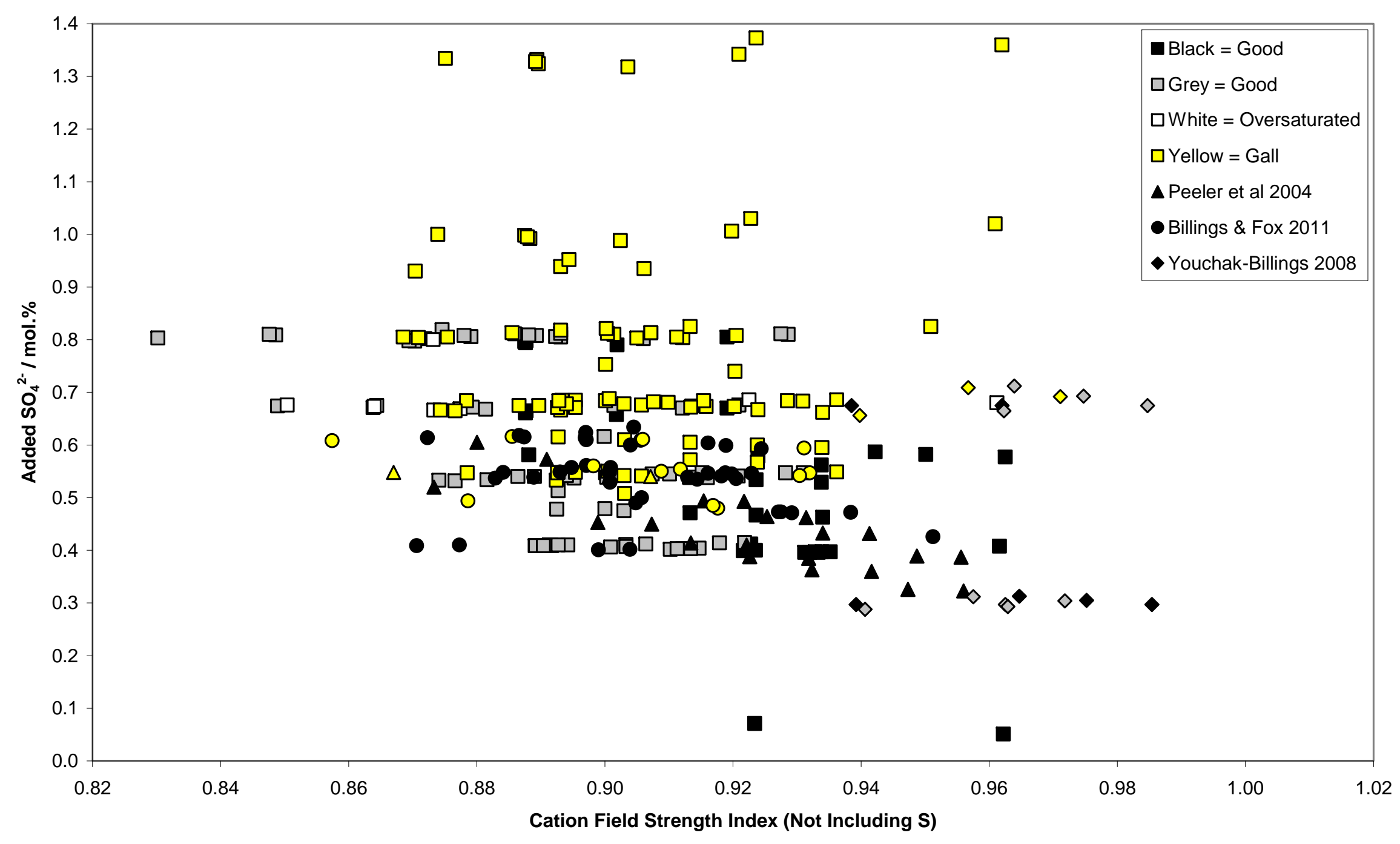


Figure 7. Added $\mathrm{SO}_{4}{ }^{2-}$ (mol.\%) as $f$ (Total Cation Field Strength Index, Sulfate Included). Data from [3]; additional data from Peeler et al 2004 [4], YouchakBillings 2008 [5] and Billings \& Fox 2011 [6]

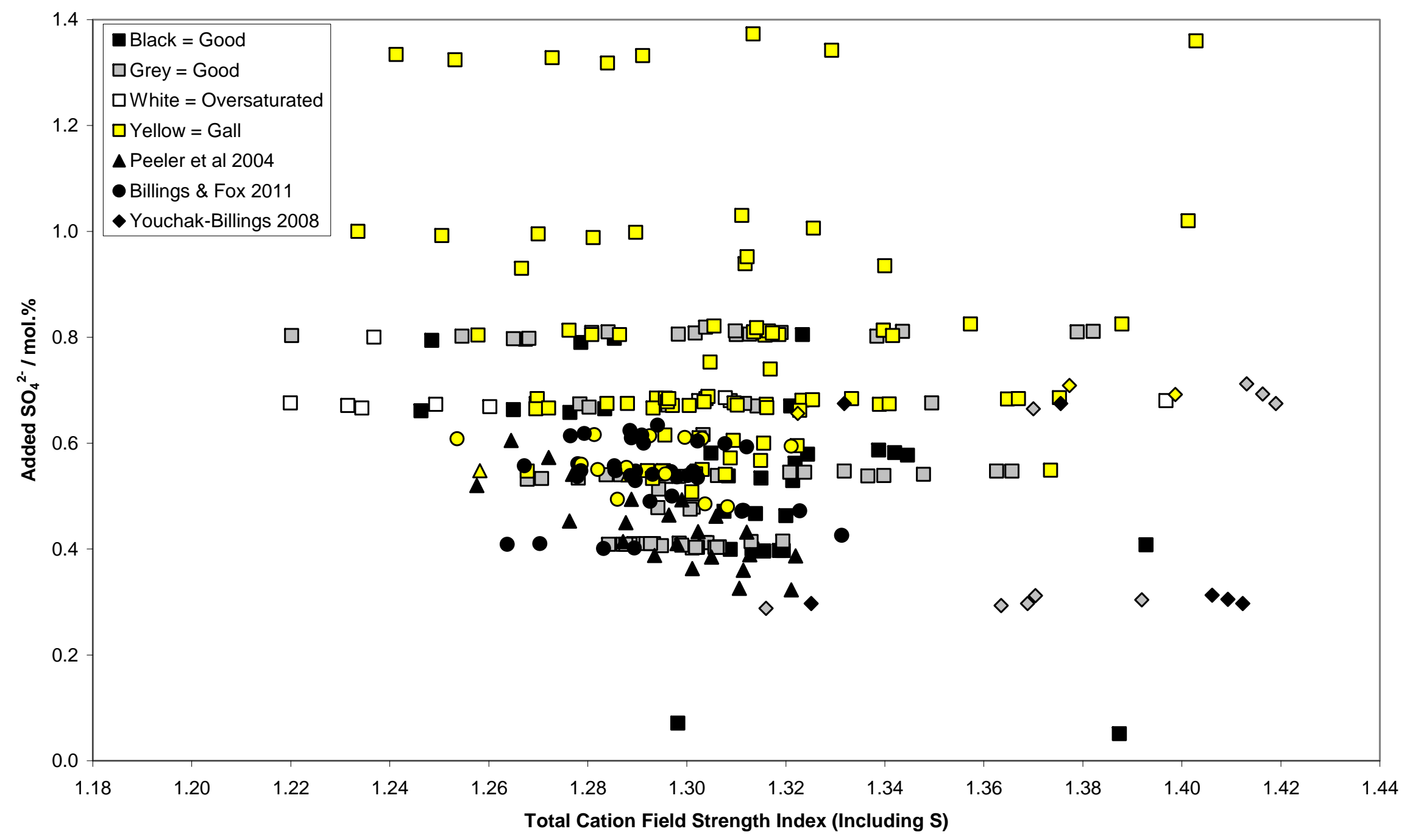


Figure 8. Added $\mathrm{SO}_{4}{ }^{2-}$ (mol.\%) as $f$ (Total Cation Field Strength Index, Sulfate Omitted). Data from [3]; additional data from Peeler et al 2004 [4], Youchak-Billings 2008 [5] and Billings \& Fox 2011 [6]

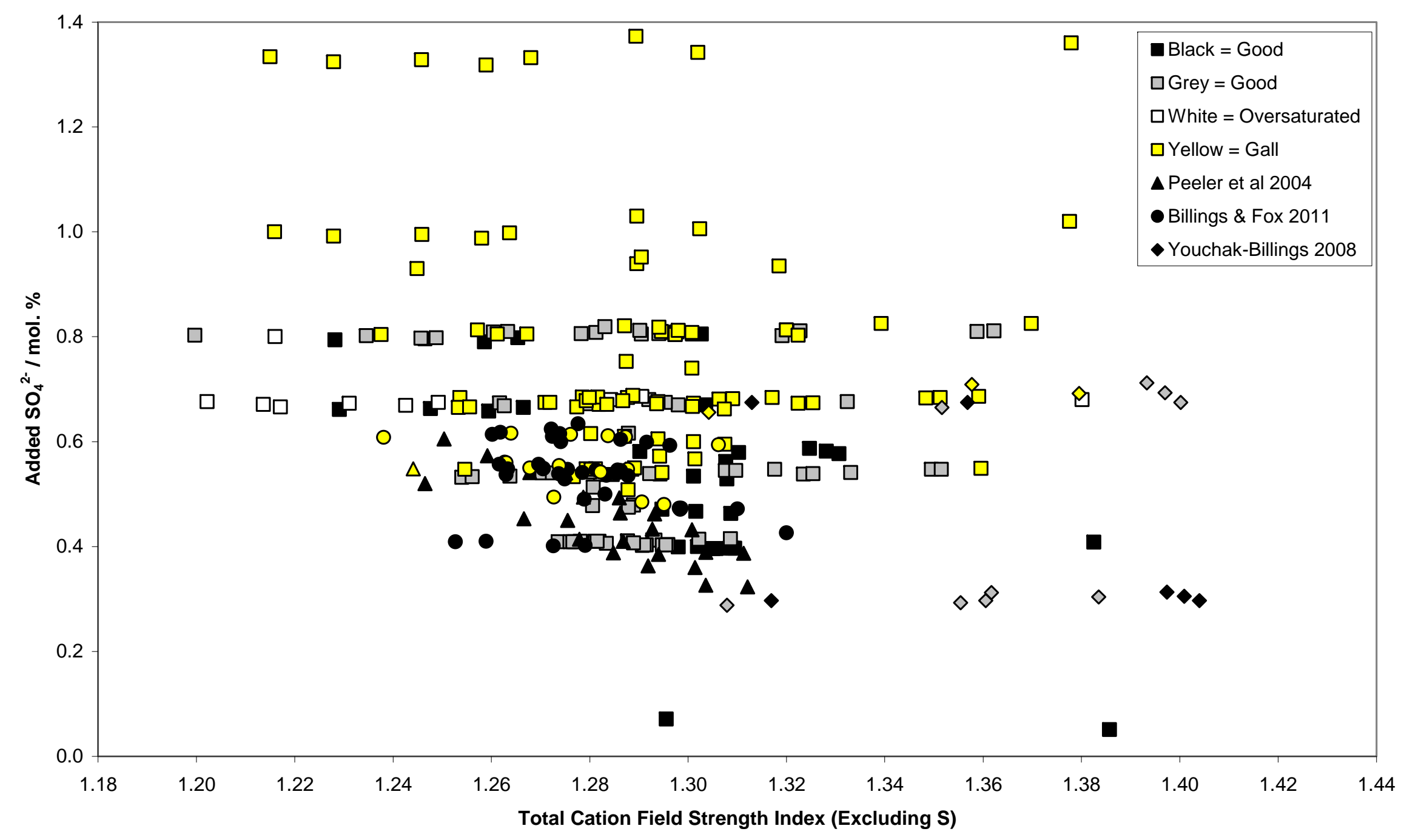


Figure 9. Retained $\mathrm{SO}_{4}{ }^{2-}(\mathrm{mol} . \%)$ as $f\left(\left[\mathrm{SiO}_{2}\right]\right.$ / [Balance]). Data from [3]; additional data from Peeler et al 2004 [4], Youchak-Billings 2008 [5] and Billings \& Fox 2011 [6]. Dotted line shows regions of high / low probability of yellow phase based on available data - visual guide only.

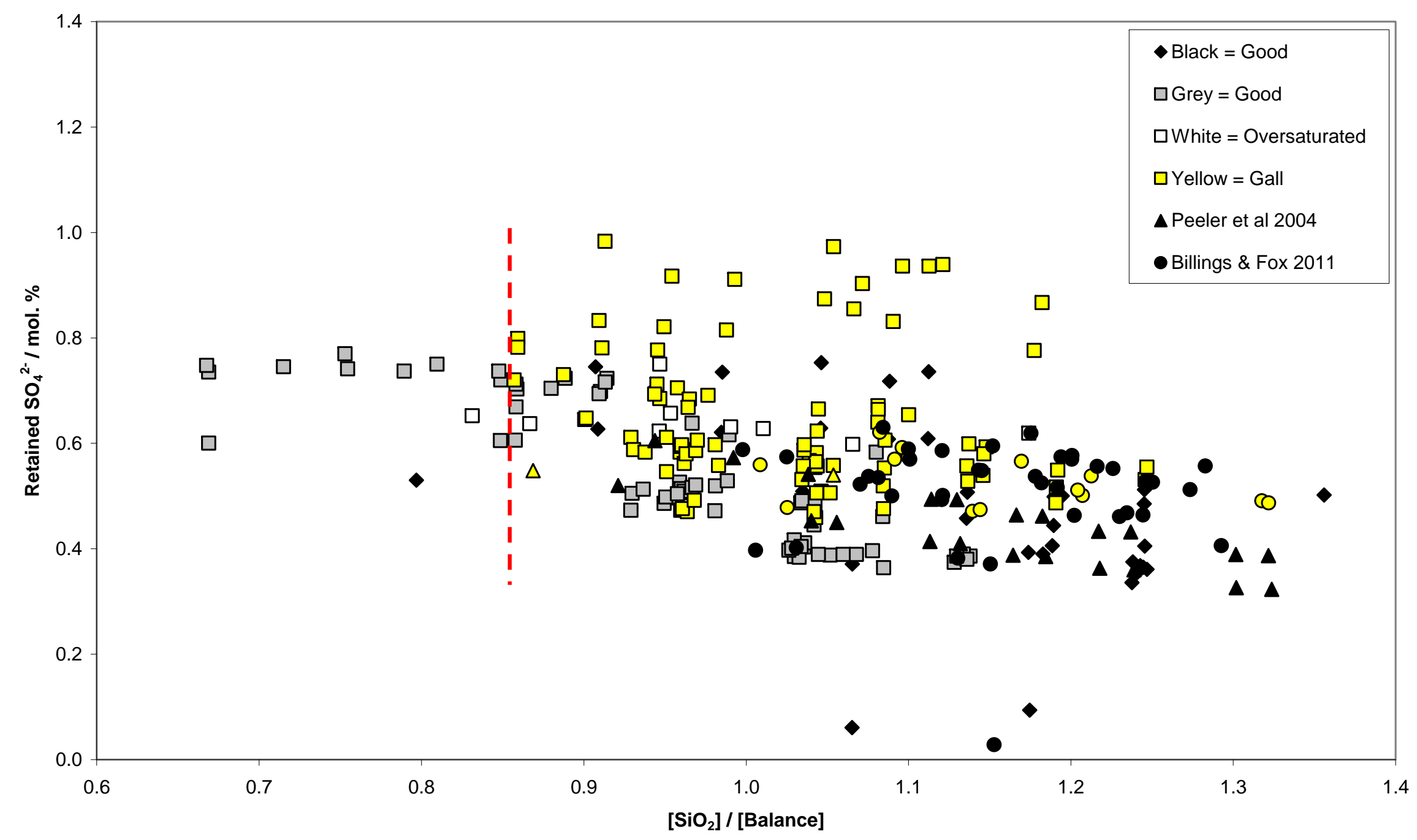



Figure 10. Retained $\mathrm{SO}_{4}{ }^{2-}(\mathrm{mol} . \%)$ as $f\left(\left[\mathrm{SiO}_{2}+\mathrm{B}_{2} \mathrm{O}_{3}\right] /\right.$ [Balance]). Data from [3]; additional data from Peeler et al 2004 [4], Youchak-Billings 2008 [5] and Billings \& Fox $2011[6]$

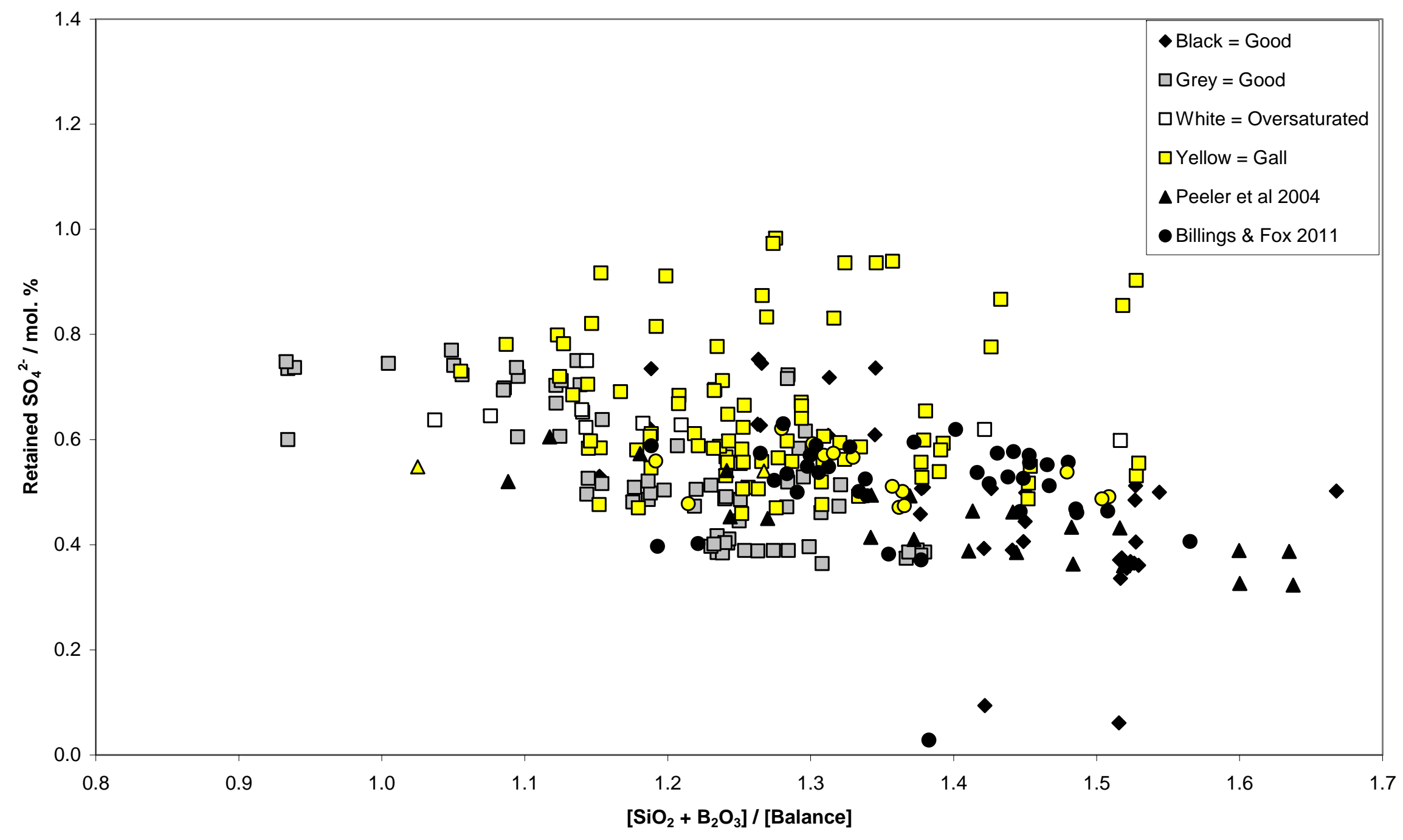


Figure 11. Retained $\mathrm{SO}_{4}{ }^{2-}(\mathrm{mol} . \%)$ as $f\left(\left[\mathrm{SiO}_{2}+\mathrm{B}_{2} \mathrm{O}_{3}+\mathrm{Al}_{2} \mathrm{O}_{3}\right] /\right.$ [Balance]). Data from [3]; additional data from Peeler et al 2004 [4], Youchak-Billings 2008 [5] and Billings \& Fox 2011 [6]

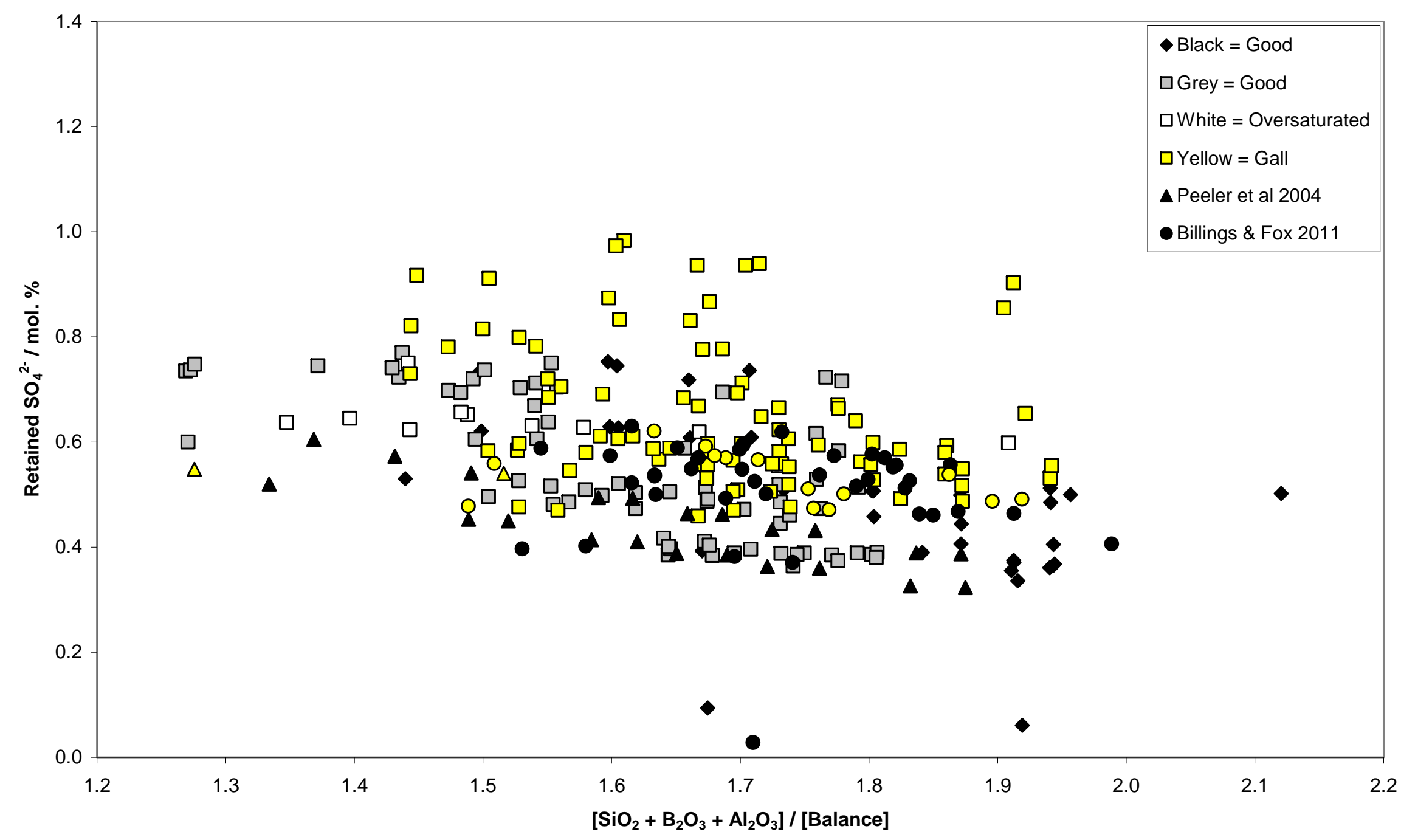


Figure 12. Added $\mathrm{SO}_{4}{ }^{2-}(\mathrm{mol} . \%)$ as $f\left(\left[\mathrm{SiO}_{2}\right] /\right.$ [Balance]). Data from [3]; additional data from Peeler et al 2004 [4], Youchak-Billings 2008 [5] and Billings \& Fox $2011[6]$

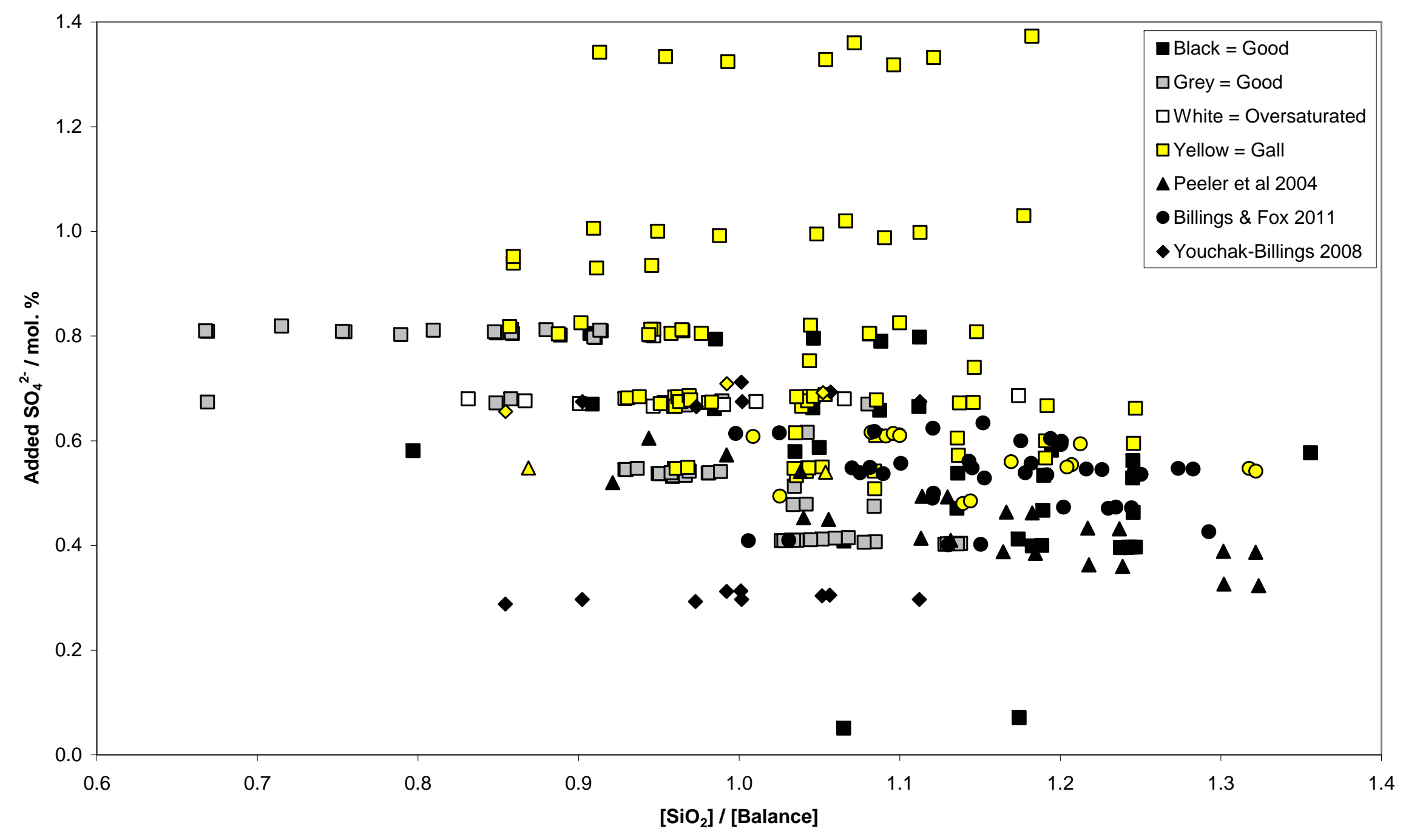


Figure 13. Added $\mathrm{SO}_{4}{ }^{2-}(\mathrm{mol} . \%)$ as $f\left(\left[\mathrm{SiO}_{2}+\mathrm{B}_{2} \mathrm{O}_{3}\right]\right.$ / [Balance]). Data from [3]; additional data from Peeler et al 2004 [4], Youchak-Billings 2008 [5] and Billings \& Fox 2011 [6]

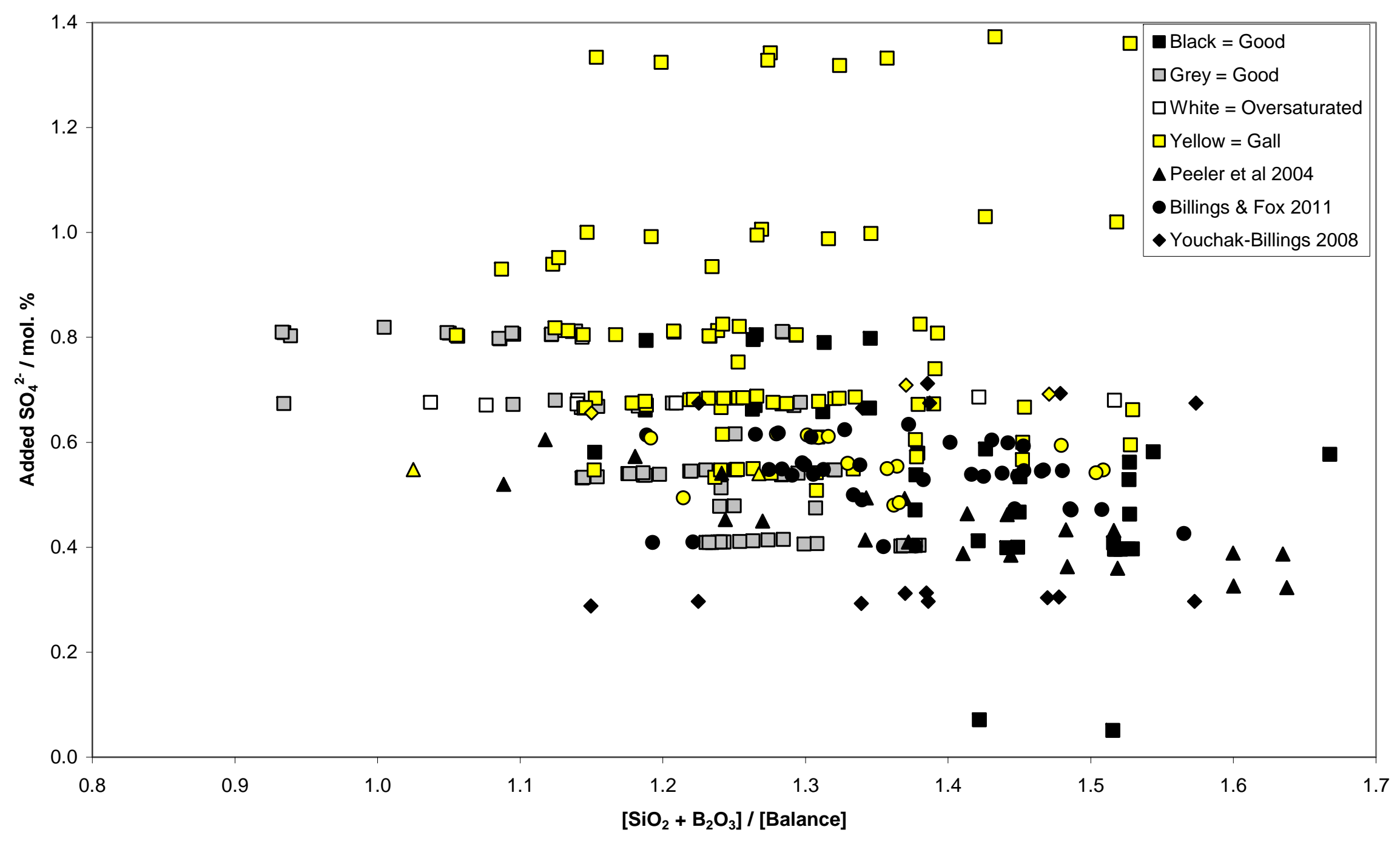


Figure 14. Added $\mathrm{SO}_{4}{ }^{2-}(\mathrm{mol} . \%)$ as $f\left(\left[\mathrm{SiO}_{2}+\mathrm{B}_{2} \mathrm{O}_{3}+\mathrm{Al}_{2} \mathrm{O}_{3}\right] /\right.$ [Balance]). Data from [3]; additional data from Peeler et al 2004 [4], Youchak-Billings 2008 [5] and Billings \& Fox $2011[6]$

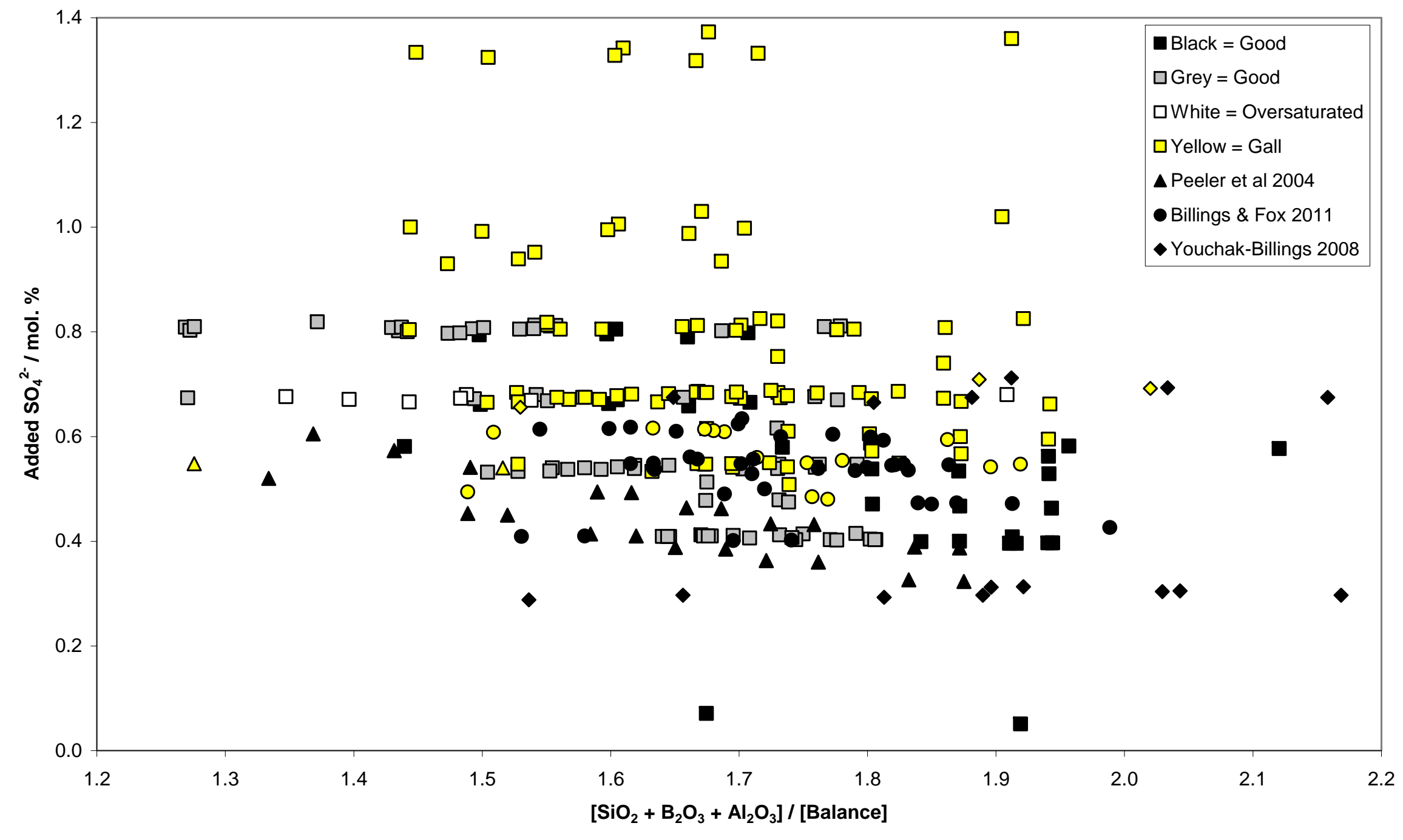




\section{$\underline{\text { Appendix }}$}

List of assumptions made in the cation field strength index model, with data sources and references listed.

Shannon ionic radii: The data was taken from: R.D. Shannon, "Revised Effective Ionic Radii and Systematic Studies of Interatomic Distances in Halides and Chalcogenides", Acta Cryst. A32 751-767 (1976).

1) Silicon

$\mathrm{Si}^{4+}$ is well-known to be coordination number $(\mathrm{CN}) 4$ in silicate matrices, crystalline and amorphous. Discussed in depth by Brown et al., Chapter 9 in: Reviews in Mineralogy Vol. 32, Mineral Soc. of America, 1995.

2) Sodium

$\mathrm{Na}^{+}$is widely held to be CN6 in oxide glasses, although with some spread in the data. As discussed in depth by Brown et al, Chapter 9 in: Reviews in Mineralogy Vol. 32, Mineral Soc. Of America, 1995, a Na CN of approx. 6 is spectroscopically proven.

3) Lithium

$\mathrm{Li}^{+}$is widely held to be CN4 in oxide glasses, although with some data spread. As discussed in depth by Brown et al, Chapter 9 in: Reviews in Mineralogy Vol. 32, Mineral Soc. Of America, 1995, a Na CN of approx. 4 is spectroscopically proven.

\section{4) Calcium}

$\mathrm{Ca}^{2+}$ is widely held to be CN6 in oxide glasses, although with some data spread. As discussed in depth by Brown et al, Chapter 9 in: Reviews in Mineralogy Vol. 32, Mineral Soc. Of America, 1995, a Na CN of approx. 6 is spectroscopically proven. Also shown by Taniguchi et al., J. NonCryst. Solids 211 (1997) 56-63.

\section{5) Boron}

Evidence from the following papers strongly indicates that an arbitrary assumption that $1 / 3$ of $\mathrm{B}^{3+}$ is $\mathrm{CN} 4$ and $2 / 3$ of $\mathrm{B}^{3+}$ is $\mathrm{CN} 3$ in all SRNL glasses considered here is a realistic assumption.

Y. Tanaka et al., Phys. Chem. Glasses: Eur. J. Glass Sci. Technol. B 50 (2009) 289-293 
A. Quintas et al., Mat. Res. Bull. 44 (2009) 1895-1898.

A. Quintas et al., Appl. Magn. Reson. 32 (2007) 613-634

E. M. Pierce et al., Geochim. Cosmochim. Acta 74 (2010) 2634-2654.

D. Holland et al., Appl. Magn. Reson. 32 (2007) 483-497

M. M. Islam et al., Phys. Chem. Glasses: Eur. J. Glass Sci. Technol. B 51 (2010) 137-145

A. Duddridge et al., Glass Technol. 44 (2003) 85-89.

6) Iron

Document SRNL-STI-2010-00191 states that a redox of $\mathrm{Fe}^{2+} /$ Total $\mathrm{Fe}=0.2$ is targeted in these glasses, although analysed samples in the report show values of $0-0.17$. However, for the present data, redox was not controlled and only loose-fitting lids were used during melting. Based on this information, plus ISL experience and literature consensus, it has been assumed that iron occurs as $20 \% \mathrm{Fe}^{2+}$ and $80 \% \mathrm{Fe}^{3+}$ in the SRNL glasses considered here. It is also assumed that all $\mathrm{Fe}^{2+}$ occurs in $\mathrm{CN} 6$ and all $\mathrm{Fe}^{3+}$ occurs in $\mathrm{CN} 4$, again based on literature and experience. For example see:

N. J. Cassingham et al., Phys. Chem. Glasses: Eur. J. Glass Sci. Technol. A 49 (2008) 2126.

T. T. Volotinen et al., Phys. Chem. Glasses: Eur. J. Glass Sci. Technol. B 49 (2008) 258270

P. A. Bingham et al., J. Non-Cryst. Solids 353 (2007) 2479-2494.

P. A. Bingham et al., C. R. Chimie 5 (2002) 787-796

P. A. Bingham et al., J. Non-Cryst. Solids 253 (1999) 203-209

X. C. Yang et al., Physica Scripta T115 (2005) 445-447

Perhaps the closest glasses, in terms of bulk composition, to the SRNL glasses under consideration here were those studied by Cassingham et al. (above) and others by G. Licheri et al., J. Non-Cryst. Solids 72 (1985) 211-220. Both studies show $\mathrm{Fe}^{3+}$ to be CN4, and over all literature $\mathrm{Fe}^{2+}$ is considered to be mainly CN6 in the most relevant glasses. Therefore these CN's have been selected in this study: $\mathrm{Fe}^{2+}=\mathrm{CN} 6$ and $\mathrm{Fe}^{3+}=\mathrm{CN} 4$.

\section{7) Aluminium}

Evidence from the following papers indicates that it is reasonable to assume all $\mathrm{Al}^{3+}$ is $\mathrm{CN} 4$ in the glasses under consideration: 
A. Quintas et al., Appl. Magn. Reson. 32 (2007) 613-634.

D. Holland et al., Appl. Magn. Reson. 32 (2007) 483-497

Wu and Stebbins, J. Non-Cryst. Solids 355 (2009) 556-562

\section{8) Manganese}

$\mathrm{Mn}$ is very easily reduced from $\mathrm{Mn}^{3+}$ to $\mathrm{Mn}^{2+}$ according to electrochemical series (e.g. H. D. Schreiber, J. Non-Cryst. Solids 84 (1986) 129-141). Also D. A. McKeown et al., J. Non-Cryst. Solids 328 (2003) demonstrated that $\mathrm{Mn}^{2+}$ is the dominant oxidation state in a wide range of US radioactive waste glasses similar in composition to those under consideration here. Therefore it is reasonable to assume that all $\mathrm{Mn}$ is present as $\mathrm{Mn}^{2+}$ in these glasses. McKeown et al. also showed that the average $\mathrm{Mn} \mathrm{CN}$ is approximately 5, therefore it is assumed for the model that $\mathrm{Mn}$ is present as $\mathrm{Mn}^{2+}$ in $50 \% \mathrm{CN} 4$ sites and 50\% CN6 sites, averaging CN5.

\section{9) Magnesium}

$\mathrm{Mg}^{2+}$ is believed to be largely CN4 in silicate glasses, as discussed by the following authors:

K. Shimoda et al., J. Phys. Chem. B 112 (2008) 6747-6752

M. B. Volf, Chemical Approach to Glasses, Elsevier, Amsterdam, 1984.

R. Roy, J. Am. Chem. Soc. 72 (1950) 3307-3308

Therefore it is assumed for the model that $\mathrm{Mg}^{2+}$ is $100 \% \mathrm{CN} 4$ in the glasses studied.

10) Titanium

Titanium will be present in most glasses, including all those considered here, as $\mathrm{Ti}^{4+}$ (see $\mathrm{H}$. Schreiber, J. Non-Cryst. Solids 84 (1986) 129-141). Coordination of $\mathrm{Ti}^{4+}$ has been studied extensively by Farges et al.:

F. Farges, J. Non-Cryst. Solids 244 (1999) 25-33

F. Farges et al., Geochim. Cosmochim. Acta 60 (1996) 3023-3038; 3039-3053; 30553065

Farges and colleagues have concluded that $\mathrm{Ti}^{4+}$ occurs predominantly in CN5 in a wide range of glasses, therefore we have assumed that $\mathrm{Ti}^{4+}$ is $50 \% \mathrm{CN} 4$ and $50 \% \mathrm{CN} 6$, averaging at $\mathrm{CN} 5$, in the SRNL glasses studied here.

11) Nickel

Nickel will be present in glass in all but extremely strongly oxidising or highly reducing conditions as $\mathrm{Ni}^{2+}$ (see H. Schreiber, J. Non-Cryst. Solids 84 (1986) 129-141). Galoisy and Calas 
(Geochim. Cosmochim. Acta 55 (1991) 1563-1574) studied the coordination of $\mathrm{Ni}^{2+}$ in oxide glasses and determined that $\mathrm{Ni}^{2+}$ occurs in a mixture of CN4 and CN5 sites. This is supported by further work by Galoisy et al. (Phys. Chem. Glasses: Eur. J. Glass Sci. Technol. B 46 (2005) 394399. On the basis of the available literature we have assumed that $\mathrm{Ni}^{2+}$ is $75 \% \mathrm{CN} 4$ and $25 \% \mathrm{CN} 6$ to give a sensible average of $\mathrm{CN} 4.5$

12) Zirconium

Based on work by F. Farges et al. (Geochim. Cosmochim. Acta 55 (1991) 1563-1574) and by A. J. Connelly et al. (J. Non-Cryst. Solids 357 (2011) 1647-1656), $\mathrm{Zr}^{4+}$ is shown to occur predominantly in $\mathrm{CN} 6$ sites in glasses broadly similar to those under consideration here.

\section{3) Cerium}

Cerium is assumed to be present in its $\mathrm{Ce}^{3+}$ form in the glasses studied as a result of its electrochemical series behaviour and mutual redox interactions with iron, as discussed by H. D. Schreiber et al., J. Non-Cryst. Solids $38 \& 39$ (1980) 785-790 and H. D. Schreiber, J. Non-Cryst. Solids 84 (1986) 129-141. The $\mathrm{CN}$ of $\mathrm{Ce}^{3+}$ in alkali borosilicate radioactive waste glasses has been shown to be CN8 by Jollivet et al. (J. Nucl. Mater. 346 (2005) 253-265) and this has been assumed in the model.

\section{4) Chromium}

Chromium is present essentially as $\mathrm{Cr}^{3+}$ under atmospheric glass melting conditions as shown by electrochemical series (H. D. Schreiber, J. Non-Cryst. Solids 84 (1986) 129-141) and also by others spectroscopically:

M. Casalboni et al., J. Phys. Condens. Matter 8 (1996) 9059-9069

O. Villain et al., J. Non-Cryst. Solids 356 (2010) 2228-2234.

D. A. McKeown et al., J. Non-Cryst. Solids 357 (2011) 2735-2743.

$\mathrm{Cr}^{3+}$ is also CN6 in oxide glasses as described in the above references. 


\section{Distribution:}

J. W. Amoroso, 999-W

A. B. Barnes, 999-W

D. R. Best, 999-W

H. M. Boyd, 704-27S

J. M. Bricker, 704-27S

C. L. Crawford, 773-42A

T. B. Edwards, 999-W

H. H. Elder, 704-24S

T. L. Fellinger, 704-26S

S. D. Fink, 773-A

K. M. Fox, 999-W

B. J. Giddings, 786-5A

J. M. Gillam, 766-H

B. A. Hamm, 766-H

C. C. Herman, 999-W

E. W. Holtzscheiter, 704-15S

J. F. Iaukea, 704-30S

P. R. Jackson, DOE-SR, 703-46A
C. M. Jantzen, 773-A

F. C. Johnson, 999-W

M. T. Keefer, 766-H

P. A. Lee, 703-41A

S. L. Marra, 773-A

D. W. Mcilmoyle, 766-H

J. E. Occhipinti, 704-S

D. K. Peeler, 999-W

F. M. Pennebaker, 773-42A

J. W. Ray, 704-S

A. R. Shafer, 704-27S

H. B. Shah, 766-H

D. C. Sherburne, 704-S

M. E. Smith, 704-30S

A. V. Staub, 704-27S

M. E. Stone, 999-W

K. H. Subramanian, 766-H

W. R. Wilmarth, 773-A 